

\section{DISCLAIMER}

This report was prepared as an account of work sponsored by an agency of the United States Government. Neither the United States Government nor any agency Thereof, nor any of their employees, makes any warranty, express or implied, or assumes any legal liability or responsibility for the accuracy, completeness, or usefulness of any information, apparatus, product, or process disclosed, or represents that its use would not infringe privately owned rights. Reference herein to any specific commercial product, process, or service by trade name, trademark, manufacturer, or otherwise does not necessarily constitute or imply its endorsement, recommendation, or favoring by the United States Government or any agency thereof. The views and opinions of authors expressed herein do not necessarily state or reflect those of the United States Government or any agency thereof. 


\section{DISCLAIMER}

Portions of this document may be illegible in electronic image products. Images are produced from the best available original document. 


\section{PART 2}

(Physics-Nuclear - TID-4500)

\title{
THE BIBLIOGRAPHY OF
}

\section{INTEGRAL CHARGED PARTICLE NUCLEAR DATA}

\author{
ARCHIVAL EDITION
}

Literature scanned from January 1, 1976, through March 15, 1980

Index of data in the international exchange format (EXFOR) received between

January 1, 1976, and March 15, 1980

T.W. BurRows and P. DEMPSEY

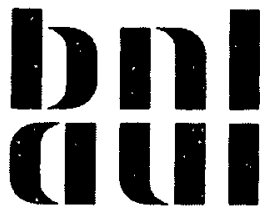

March 1980

NATIONAL NUCLEAR DATA CENTER

(in support of the International Nuclear Reaction Data Centers Network)

BROOKHAVEN NATIONAL LABORATORY ASSOCIATED UNIVERSITIES, INC.

UNOER CONIRACT NO. DE-AC02-76CHO0016 WITH THE

UNITED STATES DEPARTMENT OF ENERGY 


\section{DISCLAIMER}

This book was prepared as an account of work sponsored by an agency of the United States Government. Neither the United States Government nor any agency thereof, nor any of their employees, makes any warranty, express or implied, or assumes any legal liability or responsibility for the accuracy, completeness, or usefulness of any information, apparatus, product, or process disclosed, or represents that its use would not infringe privately owned rights. Reference herein to any specific commercial product, process, or service by trade name, trademark, manufacturer, or otherwise, does not necessarily constitute or imply its endorsement, recommendation, or favoring by the United States Government or any agency thereof. The views and opinions of authors expressed herein do not necessarily state or reflect those of the United States Government or any agency thereof.

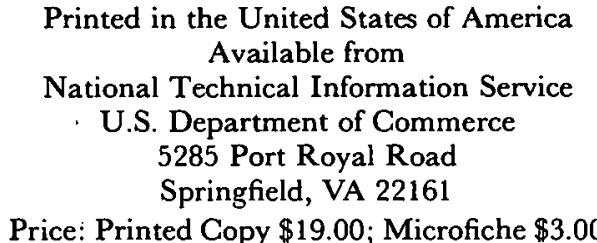


THE BIBL IOGRAPHY OF INTEGRAL CHARGED PARTICLE NUCLEAR DATA, FOURTH EDITION Table of Contents

Table of Contents

Part i

Introduction

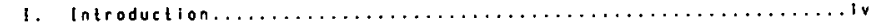

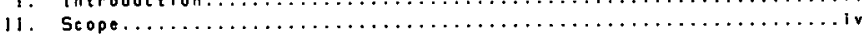

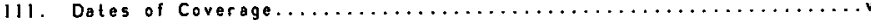

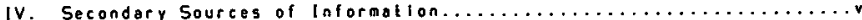

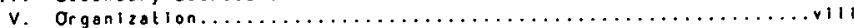

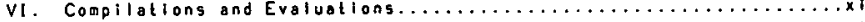

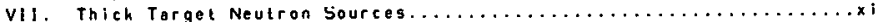

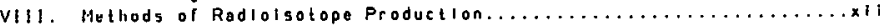

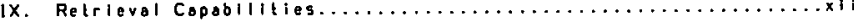

$\mathrm{x}$. Acknooledgements..........................

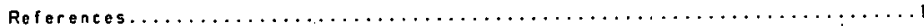

Tables

1. Other Nuclear Dato Bibliographic Sources..................

2. Centers Participaling in the Compllation and Exchange

of Charged-Particle Nuclear Data......................

3. Core Journal Coverage............................

4. Selected Evaluations, Revieus, and Compilations of

Charged-Particle Nuclear Data.......................

5. Some Recent Revieus of Thick-Target Neutron Yields...........

Figures

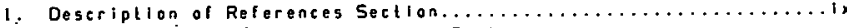

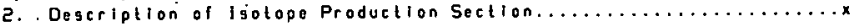

Part 2

Introduction

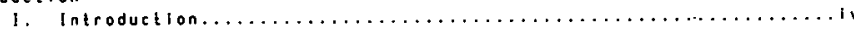

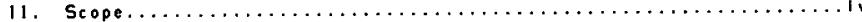

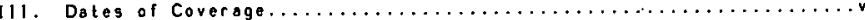

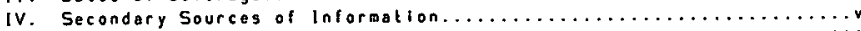

$v$. Organizotion........................

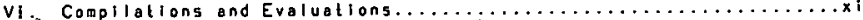

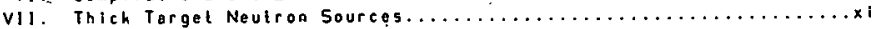

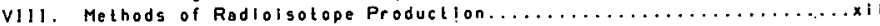

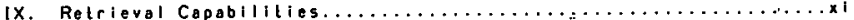

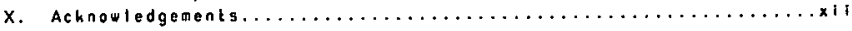

lsotope Production.............................. 554

Tables

1. Other Nuclear Data Blbllográphic Sources....................

2. Centers Partlctpating in the Compitation and Exchange

of Charged-Particle Nuclear Deta......................

3. Core Journal Coverage.............................

4. Selected Evaluations. Revieas, and Compliations of

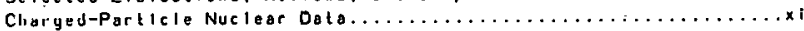

5. Some Recent Reviews of Thlek-Targel Neutron Yields............

Figures

1. Description of References Section.....................

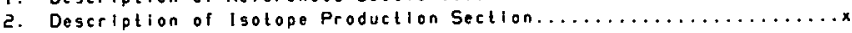

Appendices

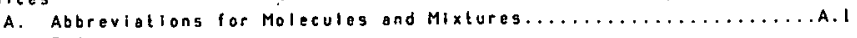

B. References

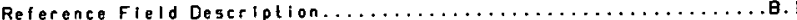

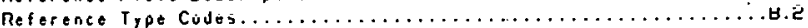

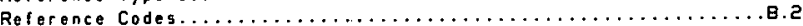

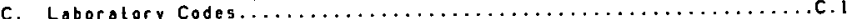

D. Some Abbreviations Used in Comments ..................

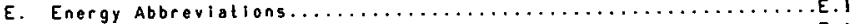

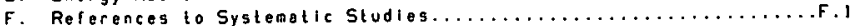


THE BIBLIOGRAPHY OF INTEGRAL CHARGED PARTICLE NUCLEAR DATA, FOURTH EOITION Introduction

\section{INTRODUCTION}

This publication is the fourth annual odition of the National Nuclear Date Center charged-parlicle bibliography. This edition is cumulative and supersedes the previous editions. The original publication (BNL-NCS-50640) was on oulgrouth of a recommendation by the newly-formed international Zharged-Particle Nuclear Data Compilation Neluorh (see Toble 2 for members of the neiteorh). The bibliography's primary oims ore to satisfy the need expressed by the netoorh for a concise and comprehensive index of integral charged-porticle cross section dato and lo provide an index of charged-partlcle data complled in the international exchange format. EXFOR. The reader is referred to Table l for a partial list of olher nuclear data bibliographies relevant to charged-particle Induced reactions ond to A Source Lisi of Nuclear Data Bibliographies. Compllations, and Evaluationsi for a more comprehenstue list.

The rapid growit in the size of this publicalion has forced a change in phllosophy. The present publication is an acebival volume covering the flist four years of scanning. Future publications will be cumulative supplements lo the Fourth Edition.

\section{Table 1}

Other Nuclear Data Bibliographic Sources

1. W.B. Ewbank, et Nuclear Structure References. 1969-1974.

Nucl. Data Sheets 16. Supplement. Academic Press. NY (1975).

- Recent References'. 3 issues/year oith lasi isgue cumulative. for year

in Nuel. Date Sheets. Academic Prass. NY.

2. INIS Atomindex. International Atomic Energy Agency, Vienna.

3. F.K. McGoosn and W.T. Milner. Reaction List for Charged-Particle-induced Reactions.' At. Deta Nucl. Data Reprints 2. Acadenic Press. NY. (1974). Nucl. Data Tables All, 1. Acodemic Press, NY. (1972).

At. Data Nucl. Dato Tables 12. 499. Academic Press. NY. (1973).

At. Data Nucl. Dala Tables 15, 189. Academic Pross, NY (1975).

At. Data Nucl. Data Tables 18. I. Academic Press. NY (1976).

\section{SCOPE}

For the purposes of this bibliography. Integral charged particte data may be defined as dato which have been integrated over the product angle and energy or deta which may be easlly converted to such a representation.

General criteria for inclusion of daba in this bibliography are the following.

1. Exclitation lunctions. Inich target. Or product yields leading to the formation of ground or metastable states. This includes such quantities os lission yieles, isomerie ratios, multiplicities. and excitation functions for gpecific particle groups where such data readily yields information on the excitation functions or thich target yields for ground or metastable states.

2. The atomic mass and charge of the incldent perticle must be greater then or equal to 1 .

3. The alomic mass of the targot mugt be greater than or equal to 1 .

1. T.W. Burrows and N.E. Holden. 'A Source List of Nuclear Data Bloliographtes. Compilations, and Evaluations, Second Edition.' Brookhaven National Laboratory Report BNL-NCS-50702, Second Edition (1978).

- Thich larget yields are defined as measurements on targets whose thicknegs is suffleient to degrade the incldent portlcle energy boloo the reaction threshold. The term product yield is used uhen in the compllers opintion there is an ombigulty in what quantlity has been meosured. 
THE BJELIOGRAPHY OF INTEGRAL CHARgED PARTICLE NUCLEAR DATA, FOURTH EDITION Scope

4. The atomic mosses of the outgoing and residual nuclei aust be greater than or equal to l wh the exception. of processes which do not lead to definite residual nucleus le.e. lotal cross sectionsl and of gamma-ray production reactions.

There may be several exceptions to these crtterla. Any charged-particle dato compiled in the EXFOR format are Indexed along with the pertinent references. If a ceference includes data satisfying the criterio noted above and addlional data satisfying all the criterta exceptenergy, these addit onal dato are also indexed.

\section{DATES OF COVERAGE}

For those journals included in the bloliograghy of the Charged Particle Dota Group, Oak Ridge National Laboratory lsee entry 3 of Toble 1 for a cumblative list of thelr blbliography). the present coverage began with the first journal issue after their nominal cui-off date of January. 1976. Coverage of all other journals beyan otth the first issue bearing a 1976 cover dale. The cul-of date for the present edition is March 15. 1980. All other types of references uhich came to our attention between January 1. 1976, and March 15. 1980, have been scanned.

In eddition. all charged-particle data complled in the EXFOR format have been indexed along vith the pertinent references. Prior work which is related directly to a current poper and which has been cited by the authors has also been included.

Table 3 lists the coverage of 27 core journals lo give a representative sample of the present coverage. Appendix $B$ lists all references contalned in this publitation. Other references have been scanned without finding pertinent entries. A complete list of the coverage may be obtalned from the outhors.

\section{SECONDARY SOURCES OF INFORMATION}

Since this blbllography is designed to serive both as an arehival and a current-aoreness bibliography, abstracts, theses. progresgreports. ele. have been included. However. they are present in this edition only if they have not been superceded by a primary source such as a journal or conference paper. Nevertheless. all references scaned ohich satisfy the criteria of sectlons Il and Ill ore indexed in the compulerized bibliographic file and may be retrleved on request (sec section IX for retrieval detalls). 
THE BIBL IOGRAPHY OF INTEGRAL CHARGEO PARTICLE NUCLEAR DATA, FOURTH EDITION

Centers Porticloaling in the Compllotion and Exchange of Charged-Particle, Nuclear Do

$$
\text { Tablo } 2
$$

Centers Particlpoting In the Compliation and Exchange of Charged-Particle Nuclear Dala.

Center ciede

A. CAJaD

B. KaChaPaG

c. NNDC

D. NDS

E.

F. Harwell

G. FIZ

H. IBJ

1. NEADB

\section{Center}

Dr. F.E. Chuhreev

Center for Nuclear Structure and Reaction Data of the USSR Stote Committe on the Utilization of Atomic Energy Mascow. USSR

(Handles disgemination for the Union of Soviet Socialist Republics ond Eastern Europel

Prof. H. Munzel

Charyed Particle Nuclear Data Group

Instilut fur Radiochemie

Kernforschungzentrud Karlsiuhe

Postfoche 3640

D-7500 Karlsruhe, Federal Republic of Germany

Dr. S. Pearlatein

Nalional Nuclear Data Center

Brookhaven National Laboratory

Upton, NY 11973, USA

(Handies disseaination for the Unitied States of Aaerica and Canada)

Dr. J.j. Schmidt

Nucloor Data Section

IAEA. P.O.B. 590

Karntnerring 1 !

101 ! Vienno. Austrio

(All countries not covered by centers A, C, O, or I should contact NDS for inforation)

Prof. Hajime Tanaha

Department of Physics

Hokhaido Unlversily

Sapoora. Hokkaido, Japan

Dr. G. Dearnaley

Nuclear Physic Division. Hanger 8

Atomic Reseorch Establishment

Haroell. Didcal. Oxll ORA

Uniled Kingdom

Dr. H. Behrens

Fachinformationszentrua

7514 Eggenstein-Lecpoldshafen?

Federal Republic of Germany

(Handleg dissemination for the Federal Republic of Germany)

Dr. A. Marcinkowshl

Nuclear Data Group

Inst. Baden Jadrouych

$\mathrm{H}_{0}<\mathrm{a}$ GS

PL-00-681 Hor 50 w, Poland

Dr. N. Tubbs

Nuclear Energy Agency Data Bank

B.P. No. 9

F-91190 Gif-sur-Yuelte

France

(Handles dissemination for Nuclear Energy Agency countries other than USA, Canada, and FRG) 
THE BIEL IOGRAPHY OF INTEGRAL CHARGED PARTICLE NUCLEAR DATA, FOURTH EOITION 'Core' Journal Coverage

1

Toble 3

'Core' Journal Coverage

\section{Joucnal}

Anneles de Physlque (Paris)

Annals of Phystes [New Yorh]

Australian Journal of Physics

Bulletin of the Acodemy of Sclences of the USSR,

Physical Series

Calladian Journal of Chemistry

Conadian Jouillal of Physics

Comples Rendus Hepdomadalres des Seances de

1 Acadenie des Sciences. Serles B. Physlque

Helvetia Phystca Acta

International Journal of Applied Radiation and lsotopes :.

lzvestia Ahadeati Nauk SSSR, Serlya Fizlcheskaya

Journal of Inorganic and Nuclear Chemistry

Journal of the Physlcal Soclety of Japon

Journal of Physics $G$

Lettere ot I Nuovo Cimento, Ser.

Nuclear Instruments and Methods

Nuclear Physics A

Nuovo Cimento A

Physica Scripto

Physical Review C

Physical Revieo Letters

Physics Letters $B$

Revue Roumaine de Physique

Soviet Alomic Energy"

Soviet Journel of Nuclear Physles.

Uhrains'hit Fizichnia Zhurnal

Yadernaya Fizika

Zeitschrift fuer Physih A
First issue

Vol (Issue)

Last Issue

Vel (issue)

$914)$

$95(2)$

ट9(1)

$4(4)$

गट4(1)

$32(4)$

$39(5) \quad 43(1)$

$53(24)$. $\quad 57(23)$

$53(23) \quad 58(2)$

ट्) (5)

289 ( 12 )

$47(2)$

$52(2)$

$27(1)$

$39(5)$

$30(12)$

$43(11)$

$38(2)$

$40(1)$

$42(1)$

$47(6)$

$5(12)$

$14(14)$

$26(18)$

$131(1)$

25611)

$167(3)$

334 (2)

54 (2)

12(6)

$13(2)$

$20(6)$

टा (3)

$44(9)$

89(4)

$60(2)$

24 (9)

$20(10)$

$46(6)$

$39(3)$

2i (6)

29 (5)

$20(10)$

$24(10)$

2) (6)

$30(9)$

275 (4)

$294(1)$ 
THE BIBLIOGRAPHY OF INTEGRAL CHARGED PARTICLE NUCLEAR DATA, FOURTH EDITION Organization

\section{ORGANIZATION}

This bibliography is divided into two sections. References and lsotope Production. The References section contains all references satisfying the criterta noted above and is organized as described in the next paragraph. The isotope Production section contains an abbeviated refecence line for all entries which contain information on dofinite residuat nuileus, on particle production, or on mass, charge. or lsotople distributions and is described betow. Entries in the References section are sequentiolly numbered. These sequence numbers serve as a link between the two sections.

The References section is ordered by increasing $Z$ and $A$ of the incldent particle: then by increasing $Z$ and $A$ of the target. Within this order. the entrles are ordered by residual nuclous ard outgoing particles or processes: then by quantity (eage cross section). All enteles having the same reaction are grouped under a common heading. Alt entrles elating to the sace experiment are blocked together under the some sequence number. Wilhin oblock the entries are ordered by inverse publication date with the exception of the matn reference which is lways titst and translations and dato index lines which are aloys last. The blocks are ordered by increasing minimum energy. The reader is referred lo figure I for a detailed desciption of this section.

The Isotope Production section is limited in the information it contains. Only those references which include data on definite residual nucleus, particle production. or mass, charge. or Isotopic digteibutions are included. The Information included ore the lsotope praduced, quantity, torget, incident particle. energy range, laboratory code. Iirst author of the firgt reference in the blach. sequence number. and data liag. The sequence number refers lo the block in the Reference section where complete bibliographic information may be found. The presence of a data flag indicates that the block contains a data index line. The entries are ordered by increasing $Z$ and $A$ of the product with oas distributions preceding all other entries and charge distribution preceding the isotope production for that charge. Within this grouping the entries ore ordered by increasing $Z$ and $A$ of the torget; then by increasing $Z$ and $A$ of the incident particle: and, tinolly, by increasing sequence number. The reader is referred to Figure 2 for a detalied description of this section. 
THEI BIBLIOGRAPHY OF INTEGRAL CHARGED PARTICLE NUCLEAR DATA, FOURTH EDITION Organization

\section{REFERENCES(cont)}

$\begin{array}{cc}\text { No. Lab Work Reference } & \text { Emin Emax Author, Comments } \\ \text { Type } & \text { (MeV) (MeV) }\end{array}$

Cu(p,x)2"Ne cumulative $\sigma(E) \times f a c t o r$ Reaction and quantify meosured

2400 KFK COMP R ZAED.M-15 2 D79 1.0+3 3:0+3 MLenzel+ TBL. FROM EXFOR.

Cu(P,x) ${ }^{2} \mathrm{Na}$ cumulative $\sigma(E)$

2401 L.MS txpt J $31 \mathrm{~N} 38 \quad 13765.9+2 \quad$ Orth+ TRI. INTERLAB COMPARISON,

Expt 4 EXFORBD096.037 679 5.9+2 2 PTS, FRM LAS+MTR. WGHTD. AVE.

$C_{U}(P, x)^{24} \mathrm{Na}$ cumulative $\sigma(E)$ Loboratory code (see Appendix $C$ for exponsions)

2402 MCG Expt I NP/A $2882919777.5+1 \quad$ HolLb+ TBL. ACTIVATION METHOD.

Expt 4 EXFORBD082.00? $1797.5+1$. (1.3+-0.1)MICRDBARNS.

2403 LAS FXPE J JIN $38 \quad 13765.9+2$ Orth+ TBL. INTERLAB CDMPARISON.

EXPt 4 EXFORBD096.039 679 5.9+2 .3 PTS. FRM LAS.MTR.BNW. HGHTD. AVE.

Cu $(P, x)^{24} \mathrm{Na}$ independent including metastable $\sigma(E)$

2404 BNL Expt J PR 17] $1301681.0+33.0+3$ Hidis + TBL. FROM NE24/NA24 RATID.

Expt 4 EXFORBD078.002 D78 $1.0+3 \quad 3.0+3$. 3 ENTRIE.5.

2405 KFK COMP R ZAED-M-J' $20791.0+33.0+3$ Muenzel+ TBL, FRDM EXFOR.

Cu $(p, x)^{24} \mathrm{Na}$ First Author and comments (see Appendix $D$ for exponsions)

2406 BNL Expt J PR 1?1 130168 1,0+3 3,0+3 HLII:+ TBL. RATIO NEZ4, CUM/NAZ4, IND EXPt 4 EXFORBD078.002 D78 $1.0+3 \quad 3.0+3$. 3 ENTRIE.5.

2407 KFK COMP A ZAED-M-15 2 D79 $1.0+33.0+3$ Muenzel+ TBL. FRDM EXFOR,

Cu $(p, x)^{2} " \mathrm{Na}$ cumulative? $\sigma(E) \quad \begin{aligned} & \text { Reference code (see Appendix } B \text { for } \\ & \text { explanation and expansions) }\end{aligned}$

2408 BNL Expt J PR 171 $1301691,0+33.0+3$ HLdis+ TBL. CORRECTE.D FOR RYSD MON 5 IG Expt J PR $110 \quad 1130 \quad 581,0+35,9+3$ Caretto+ IBL. CURV. RADIOCHEMISTRY.

EXPt 4 EXFORB0078.002 D78 $1.0+33.0+3$. 3 ENTRIES.

2409 KFK COMP R ZAED-M-IS $20791.0+3 \quad 3.0+3$ Muenzel+ TBL. FROM EXFOR.

Cu $(p, x)^{24} \mathrm{Na}$ cumulative? $\sigma(E) \times f$ actor

2410 BNL Expt J PR 1101130 58 1.0+3 5.9+3 Caretto+ TBL. CURV. RADIOCHEMISTRY.

Cu(p,x) ${ }^{2} \mathrm{Mg}$ cumulative $\sigma(E)$

2411 L.AS EXPE J JIN $38 \quad 13 \quad 76 \quad 5.9+2$

EXPt 4 EXFORBD096.035 $6795.9+2$

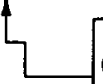

Energy range of incident particles

(in laboratory system unless otherwise

noled in comments). $1.7+1=17$.

Cu $(p+x)^{38} \mathrm{Cl}$ cumulative? $\sigma(E)$

2412 MTR Expt J NP/A $169437716,0.2$

Expt 4 EXFORBDOBS.049 $1796.0+2$

(See Appendix $E$ for expansion of abbreviation)

CIInE+ TBL. G-TECH. CFD. CALCULATE.D

- 1 data polnt.

[u $(p, x)^{39} \mathrm{Cl}$ cumulative $\sigma(E)$

2413 MTR Expt J NP/A 169 437 $716.0+2$

Expt 4 EXFORBO005.048 $1796.0+2$

Cline+ TBL, G-TECH, CFD, CALCULATE,D - I data point.

Cu(p,x) ${ }^{00} \mathrm{Cl}$ Cumulative $\sigma(E)$
414 MTR Expt J NP/A 169:437 $716.0+2$
Expt 4 EXFORBD085:047 $1796.0+2$
[u $(p, x)^{36}$ Ar o(E) Data index line. Data available from cooperating centers (see Toble 2)

CIIne+ TBL, G-TE.CH, CFD, CALCULATE.D

- j data point.

FIGURE I, DESCRIPTION OF REFERENCE SECTION 


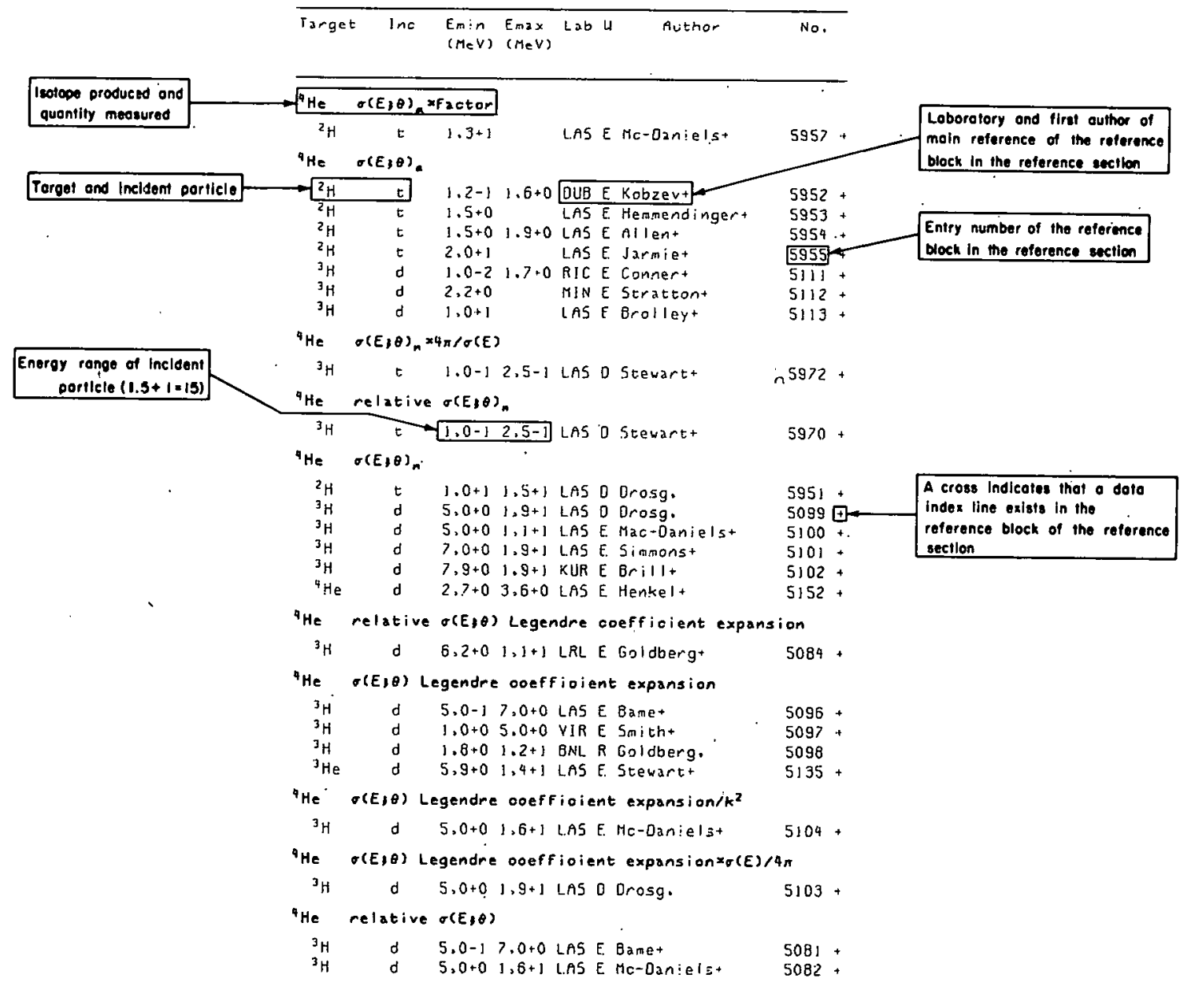


THE BIBLIOGRAPHY OF INTEGRAL CHARGED PARTICLE NUCLEAR DATA. FOURTH EDITION Compllations and Evaluations

\section{COMPILATIONS AND EVALUATIONS}

To enhance the usefulness of this publication several compilations. evaluations and revieus published prior to the present effort are given in Table 4 . -A Source List of Nuclear Dola Bibliographies, Compilations, and Evaluations"l contains more comprehensive list.

Table 4

Selected Evaluations. Revieus, and Compllations of Charged-Particle Nuclear Dala

1. K.A. Keller. al. Q-Values and Excltation Functions of Nuclear Reactions." Landhall-Rernstein, Group 1. Vol, 5 , Parts A-C. Springer-Verlag. Berlin (1973).

2. H.J. Kim, elal. Nuclear Cross Sections for Charged-Particle-laduced Reactions.' Nucl. Data Al. 203. Academic Press, NY (1965).

Nucl. Data A2, I. Academic Press. NY (1966).

Nucl. Data A3. 123. Academle Press. NY (1967).

3. H. Lishien and A. Poulsen. Neutron Production Cross Sections and Energies for the Reactions T(p,n) ${ }^{3} \mathrm{He}$. D(d,n) ${ }^{3} \mathrm{He}$, and Ttd, nit He." Nucl. Data Tables $\amalg, 569$. Acodemte Press. NY (1973).

Neutron Production Cross Sections and Energies for the Reactions ${ }^{7} \mathrm{~L}(\mathrm{p}, n)^{7} \mathrm{Be}$ and ${ }^{7} \mathrm{Li}(\mathrm{p}, n)^{7} \mathrm{Be} \mathrm{C}^{*}$. At. Data Nucl. Data Tables 15.57. Acodenic Press, NY (!975).

4. F.K. McGooan, etal. Nuclear Cross Sections for Charged-Particle Induced Reactions Mn. Fe, Co. - Oah Ridge Nalional Laboratory Report ORNL-CPX-1 (1964).

- Nuclear Cross Sections for Charged-Parlicle Induced Reactions Ni. Cu. Oak Ridge National Laboratory Report ORNL-CPX-2 (1964).

5. R.J. de Meljer, etal. "Tablas for Reaction Gama-Ray Spectroscopy." At. Data Nucl. Data Tables 15. 391. Acadenic Press. NY (1975).

6. W. Neubert. Peak Crass Sections for (HI, $x n$ ) Reactions.' Nuel. Data Tables All. 531. Academic Press. NY (1973).

7. 5. Pearlsteln. Starter Evatuated Charged Particle Data Library." Brookhaven National Leboratory Report BNL-19148 (1974).

a. E.K. Warburton, etal. GanatRays from Fusion-Evaporalion Reactions in 32 < 46 Nuclei. At. Data Nucl. Data Tables 14.147.

\section{THICK TARGET NEUTRON YIELDS}

As has been noted obove, this bibliography covers integral dato onlyi that 15. data which has been integrated over product ongle and energy. Much of tho date on thich target neutron yields are presented either os o-degree excitation functions or secondary energy distributions and: therefore. fall outside the present scope of this publication. Several recent symposta and artictes reviewing thich torget neutron yleld dato and methods ore sumarized in Table 5 for the conventence of the reader. Tables 1 and 4 and A Source List of Nuclear Dala Bibliographies, Compilations, and Evaluations'l contain addillonal references pertinent to neutron sources.

Table 5

Soge Recent Revieus of Thich Torget Neutron Sources

1. H.H. Barschall. 'Intense Sources of Fast Neutrons.' Ann. Rev. Nucl. Part. Sci. 28, 207. Annual Revieus, Inc.. Palo Alto, CA. (1978).

2. M.R. Bhat and S. Pearlstein, editors. Symposium on Neutron Cross Sections from to to $40 \mathrm{MeV}$." Brookhaven National Laboratory Report BNL-NCS-50681 119771 . 
THE BIBL IOGRAPHY OF INTEGRAL CHARGED PARTICLE NUCLEAR DATA. FOURTH EDITION Thich Target Neutron Yields

3. M.A. Lone. Lbed., p. 79

4. M.A. Lone, editor. 'International Specialists Sympasium.' Report NBSIR 77-1279 (1977).

5. Neutron Dosimetry for Biology ond Medicine." ICRU Report 26 ICRU Publications, Washington, DC. (I977).

6. H. Ullabater, editor. High Energy and H.gh intensity Nestron Sources. Nucl. Instr. Methods 145,1 . North-Holland Publishing Co.. Aasterdam. Holland. (1977).

\title{
VIII. METHODS OF RADIOISOTOPE PRODUCTION
}

While this publication should be very useful in providing the sources of cross section data for radiolsotope production, it does not index. per se. the techniques of radioisotope or labelied compound production. The Radiopharmaceutical Research Group. Chenistry Dept.. Broohbaven Netional Laboratory does index such techniques for accelerators.2 Other blbliographies dealing with such melhods any be lound in 'A Source List of Nuclear Data Bibliagraphies. Compilations, and Evaluations.l.

\section{X. RETRIEVAL CAPABilities}

Retrievals may be oblained based on targel nucleus. incident particle, outgolng particles or processes. residual nucleus, quantily. energy range. or any comblation of these. Send requests to:

\author{
Nabional Nuclear Data Center \\ Building 1970 \\ Broohhoven National Laboratory \\ Upton. NY 11973 \\ U.S.A.
}

For more detalled information. you should contact the authors.

All Information Anatysis Centers supported by the Departient of Energy are requited to conform ith a DOE plan for charging services. There is no charge lo DOE and its contractors. However, there could be nominal charges for others. Further information will be supplied on request.

\section{$\mathrm{X}$. ACKNOWLEDGEMENTS}

The authors wish to thank C.L. Dunford for his early planning of the format and scope of the compulerized charged-particle bibllogrophy. We are also grateful to G. Walle for the many hours spent scanning the literoture and lo R. Kinsey lor aid in producing the graphies program necesgary for this publication. We wish to acknowledge the timely transattals of data compiled by the Karlsruhe Charged Particle Data Graup. the Center for Nuclear Structure and Reaction Data of lhe USSR, and the Nuclear Dota Section. Internalional Alomic Energy Agency and to thank Karlsruhe for maintaining the charged-porticle Exfor library. We thanh the staff of the National Nuclear Data Center for their aid, encouragenent, and many usefust suggestions and the people who have brought our attention to errors or onissions. Finally, we gratefully acknowledge Jean Burt who coauthored lhis publication in its first three years of existione.

2. D.R. Christman and K.I. Karlstrom. Acceleralor Produced Nuclides for Use In Biology and Medicine. A Bibliography, 1939 - 1973.' Brookhaven National Laboratory Report BNL-50448 (1975)

- Acceleralor Produced Nuclides for Use in Biology and Medicine. A Bibliography. January 1974 - June 1976. Broohhaven National Laboralory Report BNL-50448-Vol. II (1978). 
ISOTOPE

PRODUCTION 


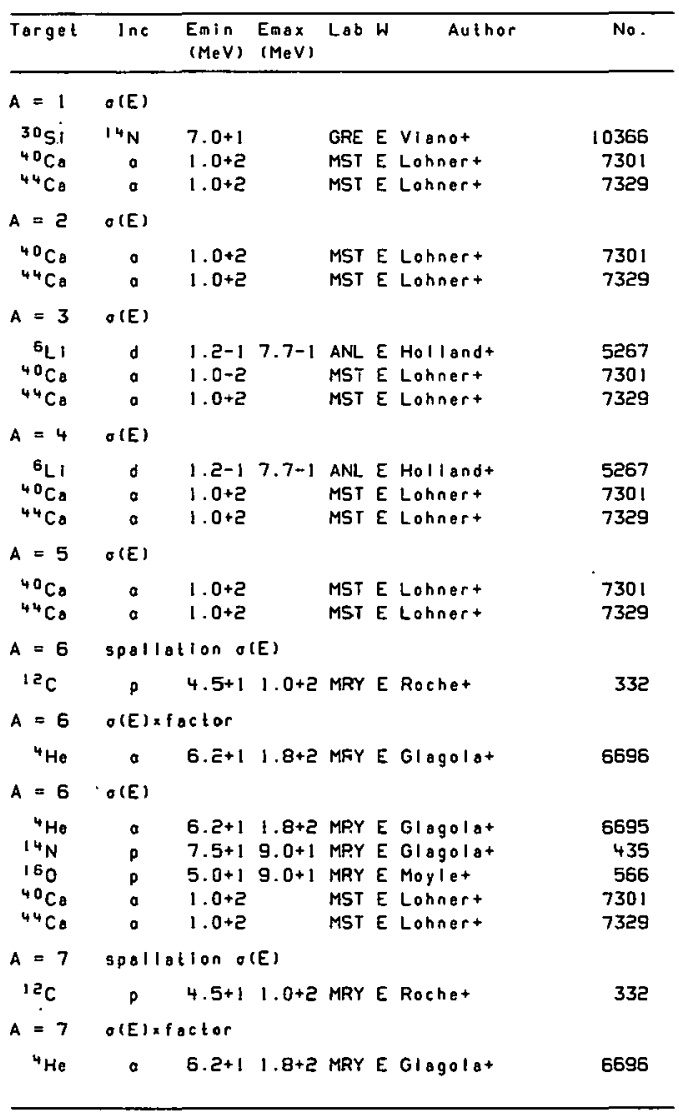

\begin{tabular}{|c|c|c|c|c|c|c|}
\hline Target & $\operatorname{lnc}$ & $\begin{array}{l}\text { Emin } \\
\text { (MeV) }\end{array}$ & $\begin{array}{l}\text { Emax } \\
(\operatorname{MeV})\end{array}$ & Lob & Author & No. \\
\hline$A=7$ & $\sigma(E)$ & & & & & \\
\hline $\begin{array}{l}14 \mathrm{~N} \\
16 \mathrm{O} \\
{ }^{40} \mathrm{Ca} \\
{ }^{4} \mathrm{Ca}^{\mathrm{Ca}}\end{array}$ & $\begin{array}{l}p \\
p \\
0 \\
0\end{array}$ & $\begin{array}{l}7.5+1 \\
5.0+1 \\
1.0+2 \\
1.0+2\end{array}$ & $\begin{array}{l}9.0+1 \\
9.0+1 \\
\end{array}$ & $\begin{array}{l}\text { MRY } \\
\text { MRY } \\
\text { MST } \\
\text { MST }\end{array}$ & $\begin{array}{l}\text { E Glagolat } \\
\text { E Moylet } \\
\text { E Lohner+ } \\
\text { E Lohnert }\end{array}$ & $\begin{array}{r}435 \\
565 \\
7301 \\
7329\end{array}$ \\
\hline$A=B$ & $\sigma(E)$ & & & & & \\
\hline $\begin{array}{l}14 \mathrm{~N} \\
16 \mathrm{O} \\
{ }^{40} \mathrm{Co} \\
{ }^{4} \mathrm{Ca}\end{array}$ & $\begin{array}{l}p \\
p \\
a \\
a\end{array}$ & $\begin{array}{l}7.5+1 \\
5.0+1 \\
1.0+2 \\
1.0+2\end{array}$ & $\begin{array}{l}9.0+1 \\
9.0+1 \\
9\end{array}$ & $\begin{array}{l}\text { MRY } \\
\text { MRY } \\
\text { MST } \\
\text { MST }\end{array}$ & $\begin{array}{l}\text { E Glagolat } \\
\text { E Moylet } \\
\text { E Lohnert } \\
\text { E Lohnert }\end{array}$ & $\begin{array}{r}435 \\
566 \\
7301 \\
7329\end{array}$ \\
\hline$A=9$ & spall & tiono & (E) & & & \\
\hline${ }^{12} \mathrm{C}$ & p & $4.5+1$ & $1.0+2$ & MRY & E Rochet & 332 \\
\hline$A=9$ & $\sigma(E)$ & & & & & \\
\hline $\begin{array}{l}1{ }^{14} \mathrm{~N} \\
16 \mathrm{O} \\
{ }^{4}{ }^{\circ} \mathrm{Ca} \\
{ }^{4}{ }^{4} \mathrm{Ca}\end{array}$ & $\begin{array}{l}p \\
p \\
o \\
a\end{array}$ & $\begin{array}{l}7.5+1 \\
5.0+1 \\
1.0+2 \\
1.0+2\end{array}$ & $\begin{array}{l}9.0+1 \\
9.0+1\end{array}$ & $\begin{array}{l}\text { MRY } \\
\text { MRY } \\
\text { MST } \\
\text { MST }\end{array}$ & $\begin{array}{l}\text { E Glagolat } \\
\text { E Moylet } \\
\text { E Lohnert } \\
\text { E Lohnert }\end{array}$ & $\begin{array}{r}435 \\
566 \\
7301 \\
7329\end{array}$ \\
\hline$A=10$ & spoll & ation & $\cdot(E)$ & & & \\
\hline${ }^{12} \mathrm{c}$ & p & $4.5+1$ & $1.0+2$ & MRY & E Rochot & 332 \\
\hline$A=10$ & $O(E)$ & & & & & \\
\hline $\begin{array}{l}14 \mathrm{~N} \\
16 \mathrm{~N}\end{array}$ & $p$ & $7.5+1$ & $9.0+1$ & MRY & E Glagolat & 435 \\
\hline $\begin{array}{l}160 \\
40 \mathrm{cg}\end{array}$ & p & $5.0+1$ & $9.0+1$ & MRY & E Moylet & 566 \\
\hline $\begin{array}{l}{ }^{40} \mathrm{C} B \\
{ }^{4} \mathrm{Cl}^{4} \mathrm{Co}\end{array}$ & a & $1.0+2$ & & MST & E Lohnert & 7301 \\
\hline${ }^{44} \mathrm{Co}$ & - & $1.0+2$ & & MST & E Lohnert & 7329 \\
\hline$A=11$ & spall & ation & o(E) & & & \\
\hline${ }^{12} \mathrm{C}$ & $p$ & $4.5+1$ & $1.0+2$ & MRY & E Rochet & 332 \\
\hline$A=11$ & diroc & $t$-inter & ractior & $n \circ C$ & & \\
\hline${ }^{12} \mathrm{c}$ & ${ }^{18} \mathrm{~F}$ & $5.0+1$ & $7.6+1$ & MBG & E Puhlhofert & 11769 \\
\hline$A=11$ & $O(E)$ & & & & & \\
\hline $\begin{array}{l}14 \mathrm{~N} \\
10_{0}\end{array}$ & p & $7.5+1$ & $9.0+1$ & MRY & E Glagolat & $\begin{array}{l}435 \\
565\end{array}$ \\
\hline $\begin{array}{l}160 \\
40 \mathrm{Ca}\end{array}$ & p & $\begin{array}{l}5.0+1 \\
1.0+2\end{array}$ & $9.0+1$ & $\begin{array}{l}\text { MRY } \\
\text { MST }\end{array}$ & $\begin{array}{l}\text { E Moylet } \\
\text { E Lohnert }\end{array}$ & $\begin{array}{r}566 \\
730 !\end{array}$ \\
\hline${ }^{44} \mathrm{Ca}$ & $a$ & $1.0+2$ & & MST & E Lohnert & 7329 \\
\hline
\end{tabular}




\begin{tabular}{|c|c|c|c|c|c|c|}
\hline Tarjet & $\operatorname{lnc}$ & $\begin{array}{l}\text { Emin } \\
(\mathrm{MeV})\end{array}$ & $\begin{array}{l}\text { Enax } \\
(\operatorname{MeV})\end{array}$ & Lab W & Author & No. \\
\hline$A=12$ & \multicolumn{6}{|c|}{ direct-Inleraction o(E) } \\
\hline $\begin{array}{l}12 \mathrm{C} \\
12 \mathrm{C}\end{array}$ & $\begin{array}{l}19_{F} \\
19 F\end{array}$ & $\begin{array}{l}5 \cdot 0+1 \\
9 \cdot 2+1\end{array}$ & $7.6+1$ & $\begin{array}{l}\text { MBG E } \\
\text { MBG M }\end{array}$ & $\begin{array}{l}\text { Puhlnofert } \\
\text { Kohlmeyert }\end{array}$ & $\begin{array}{l}11769 \\
11770\end{array}$ \\
\hline$A=12$ & \multicolumn{6}{|l|}{$\sigma(E)$} \\
\hline $\begin{array}{l}12 \mathrm{C} \\
13 \mathrm{C} \\
{ }^{40} \mathrm{Co} \\
{ }^{4}{ }^{4} \mathrm{Co}\end{array}$ & $\begin{array}{r}180 \\
180 \\
0 \\
0\end{array}$ & $\begin{array}{l}1.0+2 \\
1.0+2 \\
1.0+2 \\
1.0+2\end{array}$ & & $\begin{array}{l}\text { STR E } \\
\text { STR E } \\
\text { MST E } \\
\text { MST E }\end{array}$ & $\begin{array}{l}\text { Coffint } \\
\text { Coffint } \\
\text { Lohnert } \\
\text { Lohnert }\end{array}$ & $\begin{array}{r}11627 \\
11640 \\
7301 \\
7329\end{array}$ \\
\hline$A=13$ & \multicolumn{6}{|c|}{ diroct-lnteroction $O(E)$} \\
\hline $\begin{array}{l}{ }^{12} \mathrm{C} \\
1^{2} \mathrm{C}\end{array}$ & $\begin{array}{l}19_{F} \\
19_{F}\end{array}$ & $\begin{array}{l}5.0+1 \\
9.2+1\end{array}$ & $7.6+1$ & $\begin{array}{l}\text { MBG E } \\
\text { MBG M }\end{array}$ & $\begin{array}{l}\text { Puhlhofert } \\
\text { Kohlmeyort }\end{array}$ & $\begin{array}{l}11769 \\
11770\end{array}$ \\
\hline$A=i 3$ & $O(E)$ & & & & & \\
\hline $\begin{array}{l}12 c \\
13 c\end{array}$ & $\begin{array}{l}180 \\
100\end{array}$ & $\begin{array}{l}1.0+2 \\
1.0+2\end{array}$ & & $\begin{array}{l}\text { STR E } \\
\text { STR E }\end{array}$ & $\begin{array}{l}\text { Coffint } \\
\text { Coffint }\end{array}$ & $\begin{array}{l}11627 \\
11640\end{array}$ \\
\hline$A=14$ & \multicolumn{6}{|c|}{ direct-interactlon o(E) } \\
\hline $\begin{array}{l}12 \mathrm{c} \\
12 \mathrm{c}\end{array}$ & $\begin{array}{l}19 \mathrm{~F} \\
19 \mathrm{~F}\end{array}$ & $\begin{array}{l}5.0+1 \\
9.2+1\end{array}$ & $7.6+1$ & $\begin{array}{l}\text { MEG E } \\
\text { MBG } M\end{array}$ & $\begin{array}{l}\text { Puhlhofert } \\
\text { Kohlmeyert }\end{array}$ & $\begin{array}{l}11769 \\
1.1770\end{array}$ \\
\hline$A=: 4$ & $\sigma(E)$ & & & & & \\
\hline $\begin{array}{l}1{ }^{12} \mathrm{C} \\
1{ }^{3} \mathrm{C}\end{array}$ & $\begin{array}{l}180 \\
10_{0}\end{array}$ & $\begin{array}{l}1.0+2 \\
1.0+2\end{array}$ & & $\begin{array}{l}\text { STRE } \\
\text { STR E }\end{array}$ & $\begin{array}{l}\text { Coffint } \\
\text { Coffint }\end{array}$ & $\begin{array}{l}11627 \\
11540\end{array}$ \\
\hline$A=15$ & \multicolumn{6}{|c|}{ direct-interaction o(E) } \\
\hline $\begin{array}{l}1 z^{2} c \\
1{ }^{2} c\end{array}$ & $\begin{array}{l}19_{F} \\
19_{F}\end{array}$ & $\begin{array}{l}5 \cdot 0+1 \\
9 \cdot 2+1\end{array}$ & $7.6+1$ & $\begin{array}{l}\text { MBG E } \\
\text { MBG M }\end{array}$ & $\begin{array}{l}\text { Puhlnofert } \\
\text { Kohlmeyert }\end{array}$ & $\begin{array}{l}11769 \\
11770\end{array}$ \\
\hline$A=15$ & \multicolumn{6}{|c|}{ o(E) $\times$ foctor } \\
\hline $\begin{array}{l}12 C C \\
A=15\end{array}$ & $\begin{array}{l}19_{F} \\
O(E)\end{array}$ & $5.0+1$ & $9.2+1$ & MBG M & Kohlmeyert & 11771 \\
\hline $\begin{array}{l}120 \\
120 \\
130\end{array}$ & $\begin{array}{l}180 \\
19 \mathrm{~F} \\
180\end{array}$ & $\begin{array}{l}1.0+2 \\
9.2+1 \\
1.0+2\end{array}$ & & $\begin{array}{l}\text { STR E } \\
\text { MBG M } \\
\text { STR E }\end{array}$ & $\begin{array}{l}\text { Coffint } \\
\text { Kohlmeyert } \\
\text { Coffint }\end{array}$ & $\begin{array}{l}11627 \\
11772 \\
11640\end{array}$ \\
\hline$A=16$ & \multicolumn{6}{|c|}{ direct-Interaction o(E) } \\
\hline $\begin{array}{l}12= \\
12=\end{array}$ & $\begin{array}{l}19 \mathrm{~F} \\
19_{F}\end{array}$ & $\begin{array}{l}5 \cdot 0+1 \\
9 \cdot 2+1\end{array}$ & $7.6+1$ & $\begin{array}{l}\text { MBG E } \\
\text { MBG M }\end{array}$ & $\begin{array}{l}\text { Puhlhofert } \\
\text { Kohloeyert }\end{array}$ & $\begin{array}{l}11769 \\
11770\end{array}$ \\
\hline
\end{tabular}

\begin{tabular}{|c|c|c|c|c|c|c|}
\hline Target & $\ln e$ & $\begin{array}{l}\text { Emin } \\
(M e V)\end{array}$ & $\begin{array}{l}\text { Emax } \\
(\mathrm{MeV})\end{array}$ & $\begin{array}{ll}\text { Lob } \mathrm{H} \\
\end{array}$ & Author & No. \\
\hline$A=16$ & $O(E)$ & & & & & \\
\hline $\begin{array}{l}12 \mathrm{C} \\
13 \mathrm{C}\end{array}$ & $\begin{array}{l}180 \\
180\end{array}$ & $\begin{array}{l}1.0+2 \\
1.0+2\end{array}$ & & $\begin{array}{l}\text { STR E } \\
\text { STR E }\end{array}$ & $\begin{array}{l}\text { Corfint } \\
\text { Cofrint }\end{array}$ & $\begin{array}{l}11627 \\
11640\end{array}$ \\
\hline$A=17$ & compor & und-nuc & cleus. & $O(E)$ & & \\
\hline${ }^{12} \mathrm{c}$ & $199_{F}$ & $9.2+1$ & & MBG M & Kohlmeyert & 11768 \\
\hline$A=17$ & direc & $t$-inter & roction & in $\sigma(E)$ & & \\
\hline $\begin{array}{l}12 \mathrm{c} \\
12 \mathrm{C}\end{array}$ & $\begin{array}{l}19_{F} \\
19_{F}\end{array}$ & $\begin{array}{l}5 \cdot 0+1 \\
9 \cdot 2+1\end{array}$ & $7.6+1$ & $\begin{array}{l}\text { MBG E } \\
\text { MEG M }\end{array}$ & $\begin{array}{l}\text { Publhafert } \\
\text { Kohlmeyert }\end{array}$ & $\begin{array}{l}11769 \\
11770\end{array}$ \\
\hline$A=17$ & $O(E)$ & & & & & \\
\hline $\begin{array}{l}12 c \\
13 c\end{array}$ & $\begin{array}{l}180 \\
180\end{array}$ & $\begin{array}{l}1.0+2 \\
1.0+2\end{array}$ & & $\begin{array}{l}\text { STR E } \\
\text { STR E }\end{array}$ & $\begin{array}{l}\text { Coffint } \\
\text { Coffint }\end{array}$ & $\begin{array}{l}11627 \\
11640\end{array}$ \\
\hline$A=18$ & $\operatorname{cospo}$ & und-nue & cleuso & O(E) & & \\
\hline${ }^{12} \mathrm{C}$ & ${ }^{19} \mathrm{~F}$ & $9.2+1$ & & MBG M & Kohlmeyert & 11768 \\
\hline$A=18$ & direc & t-inter & raction & on $O(E)$ & & \\
\hline $\begin{array}{l}.12 \mathrm{C} \\
12 \mathrm{C}\end{array}$ & $\begin{array}{l}19_{F} \\
19 F\end{array}$ & $\begin{array}{l}5.0+1 \\
9.2+1\end{array}$ & $7.6+1$ & $\begin{array}{l}\text { MBG E } \\
\text { MBG M }\end{array}$ & $\begin{array}{l}\text { Puhlihofert } \\
\text { Kohlmeyert }\end{array}$ & $\begin{array}{l}11769 \\
11770\end{array}$ \\
\hline$A=18$ & $O(E)$ & & & ' & : & \\
\hline $\begin{array}{l}12 c \\
12 c \\
13 c\end{array}$ & $\begin{array}{l}180 \\
195 \\
180\end{array}$ & $\begin{array}{l}1.0+2 \\
9.2+1 \\
1.0+2\end{array}$ & & $\begin{array}{l}\text { STR E } \\
\text { MBG M } \\
\text { STR E }\end{array}$ & $\begin{array}{l}\text { Coflint } \\
\text { Kohlmeyert } \\
\text { Cofflint }\end{array}$ & $\begin{array}{r}11627 \\
11772 \\
-\quad 11640\end{array}$ \\
\hline$A=19$ & compo & und-nuc & clous. & O(E) & & \\
\hline $\begin{array}{l}12 c \\
12 c \\
13 c\end{array}$ & $\begin{array}{l}19_{F} \\
195 \\
10^{2}\end{array}$ & $\begin{array}{l}5.0+1 \\
9.2+1 \\
1.0+2\end{array}$ & $7.6+1$ & $\begin{array}{l}\text { MEG E } \\
\text { MBG M } \\
\text { STR E }\end{array}$ & $\begin{array}{l}\text { Puhlhofert } \\
\text { Kohlmeyert } \\
\text { Cofflint }\end{array}$ & $\begin{array}{l}11767 \\
11768 \\
11641\end{array}$ \\
\hline$A=19$ & direc & $=t-\ln l e r$ & rection & $O \cap O(E)$ & & \\
\hline $\begin{array}{l}12 c \mathrm{c} \\
12 \mathrm{c}\end{array}$ & $\begin{array}{l}19 \mathrm{~F} \\
19 \mathrm{~F}\end{array}$ & $\begin{array}{l}5 \cdot 0+1 \\
9 \cdot 2+1\end{array}$ & $7.6+1$ & $\begin{array}{l}\text { MBG E } \\
\text { MBG M }\end{array}$ & $\begin{array}{l}\text { Puhlnofert } \\
\text { Kohlmeyert }\end{array}$ & $\begin{array}{l}11769 \\
11770\end{array}$ \\
\hline$A=19$ & $\theta(E)$ & & & & & \\
\hline $12 \mathrm{c}$ & $10_{0}$ & $1.0+2$ & & STRE & Coffint & 11627 \\
\hline${ }^{12} \mathrm{c}$ & $.19 \mathrm{~F}$ & $5.0+1$ & $7.6+1$ & THD T & Puhlhofer. & 11766 \\
\hline${ }^{12} \mathrm{C}$ & $19 \mathrm{~F}$ & $9.2+1$ & & MBG M & 1 Kohlmeyert & 11772 \\
\hline $13 \mathrm{C}$ & 180 & $1.0+2$ & & STR E & Coffint & 11540 \\
\hline
\end{tabular}




\begin{tabular}{|c|c|c|c|c|c|c|}
\hline Target & $\operatorname{lnc}$. & $\begin{array}{l}E n \ln \\
(\mathrm{MeV})\end{array}$ & $\begin{array}{l}E \max \\
(\mathrm{MeV})\end{array}$ & Lab W & Author & No. \\
\hline$A=20$ & \multicolumn{6}{|c|}{ compound-nueleus o(E) } \\
\hline $\begin{array}{l}12 \mathrm{C} \\
{ }^{13} \mathrm{C}\end{array}$ & $\begin{array}{l}19 \mathrm{~F} \\
180\end{array}$ & $\begin{array}{l}9.2+1 \\
1.0+2\end{array}$ & & $\begin{array}{l}\text { MBG M } \\
\text { STR E }\end{array}$ & $\begin{array}{l}\text { Kohlmeyert } \\
\text { Coffint }\end{array}$ & $\begin{array}{l}11768 \\
11641\end{array}$ \\
\hline$A=20$ & \multicolumn{6}{|c|}{ diroct-interoction o[E] } \\
\hline $\begin{array}{l}1^{2} c \\
1{ }^{2} c\end{array}$ & $\begin{array}{l}198 \\
19 F\end{array}$ & $\begin{array}{l}5 \cdot 0+1 \\
9 \cdot 2+1\end{array}$ & $7.6+1$ & $\begin{array}{l}\text { MBG E } \\
\text { MBG M }\end{array}$ & $\begin{array}{l}\text { Puhlhofert } \\
\text { Kohlmeyert }\end{array}$ & $\begin{array}{l}11769 \\
11770\end{array}$ \\
\hline$A=20$ & \multicolumn{6}{|c|}{ O(E) x foctor } \\
\hline $\begin{array}{l}1{ }^{2} \mathrm{C} \\
\mathrm{A}=20\end{array}$ & \multicolumn{5}{|l|}{$\sigma(E)$} & 11771 \\
\hline $\begin{array}{l}12 \mathrm{c} \\
12 \mathrm{c} \\
13 \mathrm{c} \\
160 \\
160\end{array}$ & $\begin{array}{l}180 \\
195 \\
180 \\
160 \\
160\end{array}$ & $\begin{array}{l}1 \cdot 0+2 \\
5 \cdot 0+1 \\
1 \cdot 0+2 \\
3 \cdot 5+1 \\
4 \cdot 5+1\end{array}$ & $\begin{array}{l}7.6+1 \\
8.0+1 \\
5.4+1\end{array}$ & $\begin{array}{l}\text { STR E } \\
\text { THD T } \\
\text { STR E } \\
\text { NBI E } \\
\text { WZI E }\end{array}$ & $\begin{array}{l}\text { Cofflint } \\
\text { Puhlihofor. } \\
\text { Coffint } \\
\text { Fernondezt } \\
\text { Tserfuyot }\end{array}$ & $\begin{array}{l}11627 \\
11766 \\
11640 \\
10856 \\
10857\end{array}$ \\
\hline$A=21$ & \multicolumn{6}{|c|}{ compound-nueleus o(E) } \\
\hline $\begin{array}{l}{ }^{12} \mathrm{C} \\
{ }^{13} \mathrm{C}\end{array}$ & $\begin{array}{l}19 \mathrm{~F} \\
10_{\mathrm{O}}\end{array}$ & $\begin{array}{l}9.2+1 \\
1.0+2\end{array}$ & & $\begin{array}{l}\text { MBG M } \\
\text { STR E }\end{array}$ & $\begin{array}{l}\text { Kohldeyert } \\
\text { Cofflnt }\end{array}$ & $\begin{array}{l}11768 \\
11641\end{array}$ \\
\hline$A=21$ & \multicolumn{6}{|c|}{ direct-interaction $O(E)$} \\
\hline $\begin{array}{l}{ }^{12} \mathrm{C} \\
A=21\end{array}$ & \multicolumn{6}{|l|}{$O(E)$} \\
\hline $\begin{array}{l}12 c \\
12 c \\
12 c \\
12 c \\
13 c\end{array}$ & $\begin{array}{l}10_{0} \\
19 \mathrm{~F} \\
19_{F} \\
19_{F} \\
10_{0}\end{array}$ & $\begin{array}{l}1.0+2 \\
5 \cdot 0+1 \\
5.0+1 \\
9.2+1 \\
1.0+2\end{array}$ & $\begin{array}{l}7.0+1 \\
7.6+1\end{array}$ & $\begin{array}{l}\text { STR E } \\
\text { STR M } \\
\text { THD T } \\
\text { MEG M } \\
\text { STR E }\end{array}$ & $\begin{array}{l}\text { Coffint } \\
\text { Wagnert } \\
\text { Puhl hofor. } \\
\text { Kohlmeyort } \\
\text { Coffint }\end{array}$ & $\begin{array}{l}11627 \\
11765 \\
11766 \\
11772 \\
11640\end{array}$ \\
\hline$A=22$ & \multicolumn{6}{|c|}{ compound-nuclous $\sigma: E$ I } \\
\hline $\begin{array}{l}12 c \\
12 c \\
13 c\end{array}$ & $\begin{array}{l}19_{F} \\
19 F \\
18_{0}\end{array}$ & $\begin{array}{l}5 \cdot 0+1 \\
9 \cdot 2+1 \\
1.0+2\end{array}$ & $7.6+1$ & $\begin{array}{l}\text { MBG E } \\
\text { MBG M } \\
\text { STR E }\end{array}$ & $\begin{array}{l}\text { Puhlhofort } \\
\text { Kohlmeyert } \\
\text { Coffint }\end{array}$ & $\begin{array}{l}11767 \\
11768 \\
11641\end{array}$ \\
\hline$A=22$ & \multicolumn{6}{|c|}{ direct-interection $O(E)$} \\
\hline $1 a_{C}$ & $19^{F}$ & $9.2+1$ & & MBG M K & Kohlmeyort & 11770 \\
\hline
\end{tabular}

\begin{tabular}{|c|c|c|c|c|c|c|}
\hline Target & $\ln c$ & $\begin{array}{l}\text { Em In } \\
(\mathrm{MeV})\end{array}$ & $\begin{array}{l}\text { Emax } \\
(M \in V)\end{array}$ & Lab & Author & No. \\
\hline$A=2 \bar{E}$ & $\theta(E)$ & & & & & \\
\hline $\begin{array}{l}{ }^{12} \mathrm{C} \\
{ }^{2} \mathrm{c} \\
{ }^{2} \mathrm{C} \\
{ }^{1} \mathrm{C} \\
{ }^{1} \mathrm{C} \\
{ }^{3} \mathrm{C}\end{array}$ & $\begin{array}{l}10_{0} \\
19 \mathrm{~F} \\
19 \mathrm{~F} \\
19_{\mathrm{F}} \\
10^{\circ}\end{array}$ & $\begin{array}{l}1.0+2 \\
5.0+1 \\
5.0+1 \\
9.2+1 \\
1.0+2\end{array}$ & $\begin{array}{l}7.0+1 \\
7.6+1\end{array}$ & $\begin{array}{l}\text { STR } \\
\text { STR } \\
\text { THD } \\
\text { MBG } \\
\text { STR }\end{array}$ & $\begin{array}{l}\text { E Coffint } \\
\text { M Wagnert } \\
\text { T Puhlhofer. } \\
\text { M Kohlmeyert } \\
\text { E Coffint }\end{array}$ & $\begin{array}{l}11627 \\
11765 \\
11766 \\
11772 \\
11640\end{array}$ \\
\hline$A=23$ & comp & und-nue & cleuso & $o(E)$ & & \\
\hline $\begin{array}{l}12 \mathrm{C} \\
1{ }^{2} \mathrm{c} \\
1{ }^{3} \mathrm{c}\end{array}$ & $\begin{array}{l}19 \% \\
19 F \\
180\end{array}$ & $\begin{array}{l}5.0+1 \\
9.2+1 \\
1.0+2\end{array}$ & $7.6+1$ & $\begin{array}{l}\text { MBG } \\
\text { MBG } \\
\text { STR }\end{array}$ & $\begin{array}{l}\text { E Puhlhofert } \\
\text { M Kohlmeyert } \\
\text { E Coffint }\end{array}$ & $\begin{array}{l}11767 \\
11768 \\
11641\end{array}$ \\
\hline$A=23$ & dire & $t$ - Inter & raction & in $O C E$ & & \\
\hline $\begin{array}{l}12 c \\
A=23\end{array}$ & $\begin{array}{l}19_{\mathrm{F}} \\
\text { port }\end{array}$ & $\begin{array}{l}9.2+1 \\
01 \quad 0(E)\end{array}$ & )$\times$ facto & $\begin{array}{l}\text { MBG } \\
\text { or }\end{array}$ & M Kohlmoyert & 11770 \\
\hline $\begin{array}{l}1{ }^{2} \mathrm{C} \\
A=23\end{array}$ & $\begin{array}{l}1{ }^{2} \mathrm{C} \\
0(E)\end{array}$ & $1.2+1$ & $1.6+1$ & YAL & E Erb+ & 9774 \\
\hline $\begin{array}{l}12 c \\
12 c \\
12 c \\
12 c \\
13 c \\
1 c^{2} 0 \\
160\end{array}$ & $\begin{array}{l}180 \\
195 \\
197 \\
180 \\
160 \\
160\end{array}$ & $\begin{array}{l}1.0+2 \\
5 \cdot 0+1 \\
9 \cdot 2+1 \\
1.0+2 \\
3 \cdot 5+1 \\
4 \cdot 5+1\end{array}$ & $\begin{array}{r}7.6+1 \\
. \\
8.0+1 \\
5.4+1\end{array}$ & $\begin{array}{l}\text { STR } \\
\text { THO } \\
\text { MBG } \\
\text { STR } \\
\text { NEI } \\
\text { WZI }\end{array}$ & $\begin{array}{l}\text { E Colfint } \\
\text { T Puhlihofer. } \\
\text { M Kohlmoyert } \\
\text { E Coffint } \\
\text { E Fornandez+ } \\
\text { E Tgerruyat }\end{array}$ & $\begin{array}{l}11627 \\
11766 \\
11772 \\
11640 \\
10856 \\
10857\end{array}$ \\
\hline$A=24$ & compe & und-nuc & clous. & $\sigma(E)$ & & \\
\hline $\begin{array}{l}{ }^{12} \mathrm{c} \\
1^{12} \mathrm{c} \\
13 \mathrm{c}\end{array}$ & $\begin{array}{l}19_{\mathrm{F}} \\
19_{\mathrm{F}} \\
10_{\mathrm{O}}\end{array}$ & $\begin{array}{l}5.0+1 \\
9.2+1 \\
1.0+2\end{array}$ & $7.6+1$ & $\begin{array}{l}\text { MEG } \\
\text { MEG } \\
\text { STR }\end{array}$ & $\begin{array}{l}\text { E Puhlhofert } \\
\text { M Kohtmeyert } \\
\text { E Coffint }\end{array}$ & $\begin{array}{l}11767 \\
11768 \\
11641\end{array}$ \\
\hline$A=24$ & O(E) & & & & & \\
\hline $\begin{array}{l}12 c \\
12 c \\
12 c \\
12 c \\
13 c \\
180 \\
160\end{array}$ & $\begin{array}{l}18_{0} \\
19 \mathrm{~F} \\
19_{\mathrm{F}} \\
19_{\mathrm{F}} \\
18_{0} \\
16_{0} \\
16_{0}\end{array}$ & $\begin{array}{l}1.0+2 \\
5 \cdot 0+1 \\
5 \cdot 0+1 \\
9 \cdot 2+1 \\
1.0+2 \\
3 \cdot 5+1 \\
4.5+1\end{array}$ & $\begin{array}{l}7.0+1 \\
7.6+1\end{array}$ & $\begin{array}{l}\text { STR } \\
\text { STR } \\
\text { THD } \\
\text { MBC } \\
\text { STR } \\
\text { NBI } \\
\text { WZI }\end{array}$ & $\begin{array}{l}\text { E Coffint } \\
M \text { Wognert } \\
\text { T Puhlnofer. } \\
M \text { Kohlmeyert } \\
\text { E Coffint } \\
\text { E Fernandez+ } \\
\text { E Tsersugat }\end{array}$ & $\begin{array}{l}11627 \\
11765 \\
11766 \\
11772 \\
11640 \\
10856 \\
10857\end{array}$ \\
\hline
\end{tabular}

1.C 19 F 9.0 


\begin{tabular}{|c|c|c|c|c|c|c|}
\hline Target & $\ln c$ & $\begin{array}{l}\text { Emin } \\
(M e V)\end{array}$ & $\begin{array}{l}\text { Emax } \\
(M \in V)\end{array}$ & Lab & Author & No. \\
\hline$A=25$ & compo & und-nuc & clous & $\sigma(E)$ & & \\
\hline $\begin{array}{l}1{ }^{2} \mathrm{C} \\
1{ }^{2} \mathrm{C}\end{array}$ & $\begin{array}{l}199_{F} \\
19\end{array}$ & $\begin{array}{l}5.0+1 \\
9.2+1\end{array}$ & $7.8+1$ & $\begin{array}{l}\text { MBG } \\
\text { MEG }\end{array}$ & $\begin{array}{l}\text { E Puhlhofert } \\
\text { M Kohlmeyert }\end{array}$ & $\begin{array}{l}11767 \\
11768\end{array}$ \\
\hline$A=25$ & $\sigma(E)$ & & . & & & \\
\hline $\begin{array}{l}125 \\
120 \\
120 \\
130 \\
150\end{array}$ & $\begin{array}{l}180 \\
19 \% \\
19 \% \\
180 \\
160\end{array}$ & $\begin{array}{l}1.0+2 \\
5 \cdot 0+1 \\
9 \cdot 2+1 \\
1.0+2 \\
3 \cdot 5+1\end{array}$ & $\begin{array}{l}7.6+1 \\
8.0+1\end{array}$ & $\begin{array}{l}\text { STR } \\
\text { THD } \\
\text { MEG } \\
\text { STR } \\
\text { NEI }\end{array}$ & $\begin{array}{l}\text { E. Colfint } \\
\text { T Puhliofer. } \\
\text { M Kohlmeyert } \\
\text { E Coffint } \\
\text { E Fernandezt }\end{array}$ & $\begin{array}{l}11627 \\
11756 \\
11772 \\
11640 \\
10856\end{array}$ \\
\hline$A=26$ & compo & und-nue & clous & $\theta(E)$ & & \\
\hline $\begin{array}{l}12 \mathrm{c} \\
1{ }^{2} \mathrm{c} \\
1{ }^{3} \mathrm{c}\end{array}$ & $\begin{array}{l}19 \% \\
18 \% \\
180\end{array}$ & $\begin{array}{l}5.0+1 \\
9.2+1 \\
1.0+2\end{array}$ & $7.6+1$ & $\begin{array}{l}\text { MBG } \\
\text { MBG } \\
\text { STR }\end{array}$ & $\begin{array}{l}\text { E Puhlhofert } \\
\text { M Kohlmeyert } \\
\text { E Coffint }\end{array}$ & $\begin{array}{l}11767 \\
11768 \\
11641\end{array}$ \\
\hline$A=26$ & $O(E)$ & & & & & \\
\hline $\begin{array}{l}12 c \\
12 c \\
13 c \\
180 \\
180\end{array}$ & $\begin{array}{l}180 \\
185 \\
180 \\
160 \\
160\end{array}$ & $\begin{array}{l}1.0+2 \\
5 \cdot 0+1 \\
1.0+2 \\
3 \cdot 5+1 \\
4 \cdot 5+1\end{array}$ & $\begin{array}{l}7.6+1 \\
8.0 \div 1 \\
5.4+1\end{array}$ & $\begin{array}{l}\text { STR } \\
\text { THD } \\
\text { STR } \\
\text { NBI } \\
\text { WZI }\end{array}$ & $\begin{array}{l}\text { E Coffint } \\
\text { T Puhinofer. } \\
\text { E Coffint } \\
\text { E Fernandez+ } \\
\text { E Tserruyat }\end{array}$ & $\begin{array}{l}11627 \\
11766 \\
11640 \\
10856 \\
10857\end{array}$ \\
\hline$A=27$ & $\operatorname{compo}$ & und-nuc & clous & $\sigma(E)$ & . & \\
\hline $\begin{array}{l}12 \mathrm{c} \\
12 \mathrm{c} \\
13 \mathrm{c}\end{array}$ & $\begin{array}{l}19_{\mathrm{F}} \\
19_{\mathrm{F}} \\
1 \mathrm{~g}^{\circ}\end{array}$ & $\begin{array}{l}5.0+1 \\
9.2+1 \\
1.0+2\end{array}$ & $7.6+1$ & $\begin{array}{l}\text { MBG } \\
\text { MBG } \\
\text { STR }\end{array}$ & $\begin{array}{l}\text { E Puhlihofert } \\
\text { M Kohlmeyert } \\
\text { E Coffint }\end{array}$ & $\begin{array}{l}11767 \\
11768 \\
11641\end{array}$ \\
\hline$A=27$ & $\theta(E)$ & & & & & \\
\hline $\begin{array}{l}12 \mathrm{C} \\
12 \mathrm{C} \\
13 \mathrm{C} \\
16 \mathrm{O} \\
1 \mathrm{C}^{16} \\
27_{\mathrm{Al}}\end{array}$ & $\begin{array}{l}18_{0} \\
19 \mathrm{~F} \\
18_{0} \\
16_{0} \\
18_{0} \\
16_{0}\end{array}$ & $\begin{array}{l}1.0+2 \\
5 \cdot 0+1 \\
1: 0+2 \\
3 \cdot 5+1 \\
4 \cdot 5+1 \\
3 \cdot 0+1\end{array}$ & $\begin{array}{l}7.6+1 \\
0.0+1 \\
5.4+1 \\
9.0+1\end{array}$ & $\begin{array}{l}\text { STR } \\
\text { THD } \\
\text { STR } \\
\text { NBI } \\
\text { WZI } \\
\text { THD }\end{array}$ & $\begin{array}{l}\text { E Coffint } \\
\text { T Puhlihofer. } \\
\text { E Cofflint } \\
\text { E Fernandez+ } \\
\text { E Tserruyat } \\
\text { T Puhlhofor. }\end{array}$ & $\begin{array}{l}11627 \\
11766 \\
11640 \\
10855 \\
10857 \\
10950\end{array}$ \\
\hline$A=28$ & compo & und-nuc & clous & o(E) & & \\
\hline $\begin{array}{l}{ }^{12} \mathrm{C} \\
1{ }^{12} \mathrm{C} \\
1{ }^{3} \mathrm{C}\end{array}$ & $\begin{array}{l}18_{F} \\
19_{F} \\
18_{0}\end{array}$ & $\begin{array}{l}5.0+1 \\
9.2+1 \\
1.0+2\end{array}$ & $7.6+1$ & $\begin{array}{l}\text { MBG } \\
\text { MBG } \\
\text { STR }\end{array}$ & $\begin{array}{l}\text { E Puhlhofert } \\
\text { M Kohlmeyert } \\
\text { E Collint }\end{array}$ & $\begin{array}{l}11767 \\
11768 \\
11641\end{array}$ \\
\hline
\end{tabular}

\begin{tabular}{|c|c|c|c|c|c|c|}
\hline Target & $\ln c$ & $\begin{array}{l}\text { Emin } \\
\text { (MeV) }\end{array}$ & $\begin{array}{l}\text { Emax } \\
(\mathrm{MeV})\end{array}$ & Lab & Author & No. \\
\hline$A=28$ & $O(E)$ & & & & & \\
\hline $\begin{array}{l}12 \mathrm{C} \\
160 \\
160 \\
27 \mathrm{Al}\end{array}$ & $\begin{array}{l}195 \\
180 \\
150 \\
160\end{array}$ & $\begin{array}{l}5 \cdot 0+1 \\
3 \cdot 5+1 \\
4 \cdot 5+1 \\
3 \cdot 0+1\end{array}$ & $\begin{array}{l}7.6+1 \\
8 \cdot 0+1 \\
5 \cdot 4+1 \\
8.0+1\end{array}$ & $\begin{array}{l}\text { THO } \\
\text { NQI } \\
\text { WZI } \\
\text { THD }\end{array}$ & $\begin{array}{l}\text { T Puhliofier. } \\
\text { E Fernandezt } \\
\text { E Tserruyat. } \\
\text { T Puhlhofer. }\end{array}$ & $\begin{array}{l}11766 \\
10856 \\
10857 \\
10950\end{array}$ \\
\hline$A=29$ & compo & und-nuc & clous. & $O(E)$ & & \\
\hline $\begin{array}{l}12^{2} C \\
12 c\end{array}$ & $\begin{array}{l}19_{F} \\
19_{F}\end{array}$ & $\begin{array}{l}5.0+1 \\
9.2+1\end{array}$ & $7.6+1$ & MBG & $\begin{array}{l}\text { E Puhlinofert } \\
\text { M Kohlneyert }\end{array}$ & $\begin{array}{l}11767 \\
11768\end{array}$ \\
\hline$A=29$ & $o(E)$ & & & & & \\
\hline $\begin{array}{l}1{ }^{12} \mathrm{C} \\
160 \\
160 \\
27_{A 1} \\
27_{A 1} \\
27_{A 1} \\
27_{A 1}\end{array}$ & $\begin{array}{l}19 F \\
15^{\circ} \\
160 \\
18_{0} \\
19 \mathrm{~F} \\
19 \mathrm{~F} \\
19 \mathrm{~F}\end{array}$ & $\begin{array}{l}5 \cdot 0+1 \\
3 \cdot 5+1 \\
4 \cdot 5+1 \\
3 \cdot 0+1 \\
4 \cdot 0+1 \\
4 \cdot 0+1 \\
4 \cdot 0+1\end{array}$ & $\begin{array}{l}7 \cdot 6+1 \\
8 \cdot 0+1 \\
5 \cdot 4+1 \\
8 \cdot 0+1 \\
9 \cdot 2+1 \\
9 \cdot 2+1 \\
9 \cdot 2+1\end{array}$ & $\begin{array}{l}\text { THD } \\
\text { NBI } \\
\text { WZI } \\
\text { THD } \\
\text { ORL } \\
\text { PAR } \\
\text { THD }\end{array}$ & $\begin{array}{l}\text { T Puhliofer. } \\
\text { E Fernandez+ } \\
\text { E Tserruyat } \\
\text { T Puhlihofer. } \\
\text { R Stohsiead. } \\
\text { R Lefort } \\
\text { T Puhliofer. }\end{array}$ & $\begin{array}{l}11766 \\
10856 \\
10857 \\
10950 \\
11803 \\
11804 \\
11805\end{array}$ \\
\hline$A=30$ & compo & und-nuc & clouso & $O(E)$ & & \\
\hline $\begin{array}{l}{ }^{12} c \\
1{ }^{2} c\end{array}$ & $\begin{array}{l}195 \\
19 F\end{array}$ & $\begin{array}{l}5 \cdot 0+1 \\
9 \cdot 2+1\end{array}$ & $7.6+1$ & $\begin{array}{l}\text { MBG } \\
\text { MBG } \mathrm{F}\end{array}$ & $\begin{array}{l}\text { E Puhthofart } \\
\text { M Kohlmeyert }\end{array}$ & $\begin{array}{l}11767 \\
11768\end{array}$ \\
\hline$A=30$ & $O(E)$ & & & . & . & \\
\hline $\begin{array}{l}128 \\
160 \\
180 \\
27 \mathrm{Al} \\
27 \mathrm{Al} \\
27_{\mathrm{Al}} \\
27_{\mathrm{Al}} \\
27_{\mathrm{Al}}\end{array}$ & $\begin{array}{l}195 \\
190 \\
180 \\
180 \\
1180 \\
199 \\
199 \\
19 \%\end{array}$ & $\begin{array}{l}5 \cdot 0+1 \\
3 \cdot 5+1 \\
4 \cdot 5+1 \\
3 \cdot 0+1 \\
6 \cdot 0+1 \\
4 \cdot 0+15 \\
4 \cdot 0+15 \\
4 \cdot 0+1\end{array}$ & $\begin{array}{l}7 \cdot 6+1 \\
8 \cdot 0+1 \\
5 \cdot 4+1 \\
8 \cdot 0+1 \\
8 \cdot 0+1 \\
9 \cdot 2+1 \\
9 \cdot 2+1 \\
9 \cdot 2+1\end{array}$ & $\begin{array}{l}\text { THD } \\
\text { NBI } \\
\text { WZI } \\
\text { THD } \\
\text { NBI } \\
\text { ORL } \\
\text { PAR F } \\
\text { THD }\end{array}$ & $\begin{array}{l}\text { T Puhlhofer. } \\
\text { E Fernandezt } \\
\text { E Tserruyat } \\
\text { T Puhlhofer. } \\
\text { M Bacht } \\
\text { R Stohsleed. } \\
\text { R Lefortt } \\
\text { T Puhlhofer. }\end{array}$ & $\begin{array}{l}11766 \\
10856 \\
10857 \\
10950 \\
10951 \\
11803 \\
11804 \\
11805\end{array}$ \\
\hline$A=31$ & Spoll & ottion o & $\sigma(E)$ & & & \\
\hline $\begin{array}{c}56_{F} \\
A=31\end{array}$ & $\begin{array}{c}\rho \\
\sigma(E)\end{array}$ & $1.5+2=$ & $3.7+2$ & NRL F & R Silberberg+ & 1940 \\
\hline $\begin{array}{l}160 \\
160 \\
27 \mathrm{Al} \\
27 \mathrm{Al}\end{array}$ & $\begin{array}{l}160 \\
160 \\
160 \\
160\end{array}$ & $\begin{array}{l}3 \cdot 5+1 \\
4 \cdot 5+1 \\
3 \cdot 0+1 \\
6 \cdot 0+1\end{array}$ & $\begin{array}{l}8.0+1 \\
5 \cdot 4+1 \\
8 \cdot 0+1 \\
8.0+1\end{array}$ & $\begin{array}{l}\text { NBI } \\
\text { WZI } \\
\text { THD } \\
\text { NBI }\end{array}$ & $\begin{array}{l}\text { E Fernandez+ } \\
\text { E Tserruyot } \\
\text { T Puhlhofer. } \\
\text { M Bockt }\end{array}$ & $\begin{array}{l}10856 \\
10857 \\
10950 \\
10951\end{array}$ \\
\hline
\end{tabular}




\begin{tabular}{|c|c|c|c|c|c|c|}
\hline Target & $\ln c$ & $\begin{array}{l}\text { Emin } \\
(\mathrm{MeV})\end{array}$ & $\begin{array}{l}\text { Emax } \\
(M e V)\end{array}$ & Lab h & Author & No. \\
\hline$A=31$ & $O(E)$ & & & & & \\
\hline $\begin{array}{l}27_{A 1} \\
27_{A 1} \\
27_{A 1}\end{array}$ & $\begin{array}{l}19_{F} \\
19_{F} \\
19_{F}\end{array}$ & $\begin{array}{l}4.0+1 \\
4.0+1 \\
4.0+1\end{array}$ & $\begin{array}{l}9.2+1 \\
9.2+1 \\
9.2+1\end{array}$ & $\begin{array}{l}\text { ORL F } \\
\text { PAR } \\
\text { THD T }\end{array}$ & $\begin{array}{l}\text { R Stohstead. } \\
\text { R Lefortt } \\
\text { T Puhlhofer. }\end{array}$ & $\begin{array}{l}11803 \\
11804 \\
11805\end{array}$ \\
\hline$A=32$ & spoll & ation & $O(E)$ & & & \\
\hline $\begin{array}{l}{ }^{56} \mathrm{Fe}^{\circ} \\
\mathrm{A}=32\end{array}$ & $\begin{array}{c}p \\
\theta(E)\end{array}$ & $1.5+2$ & $3.7+2$ & NRL $P$ & R Silberbergt & 1940 \\
\hline $\begin{array}{l}27 \mathrm{A1} \\
27_{\mathrm{A1}} \\
27_{\mathrm{Al}} \\
27_{\mathrm{A1}} \\
27_{\mathrm{Al}}\end{array}$ & $\begin{array}{l}160 \\
160 \\
19_{F} \\
19 F \\
19 F\end{array}$ & $\begin{array}{l}3 \cdot 0+1 \\
6 \cdot 0+1 \\
4 \cdot 0+1 \\
4.0+1 \\
4.0+1\end{array}$ & $\begin{array}{l}8.0+1 \\
8.0+1 \\
9.2+1 \\
9.2+1 \\
9.2+1\end{array}$ & $\begin{array}{l}\text { THD I } \\
\text { NQI } \\
\text { ORL F } \\
\text { PAR F } \\
\text { THD I }\end{array}$ & $\begin{array}{l}\text { T Puhlhofer. } \\
\text { M Bacht } \\
\text { R Stohstead. } \\
\text { R Lefortt } \\
\text { T Puhlhofer. }\end{array}$ & $\begin{array}{l}10950 \\
10951 \\
11803 \\
11804 \\
11805\end{array}$ \\
\hline$A=33$ & spal & etion & $\sigma(E)$ & & & \\
\hline $\begin{array}{l}{ }^{36} \mathrm{Fe} \\
\mathrm{A}=33\end{array}$ & $\begin{array}{c}D \\
O(E)\end{array}$ & $1.5+2$ & $3.7+2$ & NRL $F$ & R Silberbergt & 1940 \\
\hline $\begin{array}{l}27 \mathrm{Al} \\
27 \mathrm{Al} \\
27 \mathrm{Al} \\
27_{\mathrm{Al}} \\
27_{\mathrm{Al}}\end{array}$ & $\begin{array}{l}160 \\
180 \\
19 \mathrm{~F} \\
19 \mathrm{~F} \\
19 \mathrm{~F}\end{array}$ & $\begin{array}{l}3 \cdot 0+1 \\
6 \cdot 0+1 \\
4 \cdot 0+1 \\
4 \cdot 0+1 \\
4.0+1\end{array}$ & $\begin{array}{l}8.0+1 \\
8.0+1 \\
9.2+1 \\
9.2+1 \\
9.2+1\end{array}$ & $\begin{array}{l}\text { THD } \\
\text { NBI } \\
\text { ORL } F \\
\text { PAR F } \\
\text { THD }\end{array}$ & $\begin{array}{l}\text { T Puhlhofer. } \\
\text { M Bacht } \\
\text { R Stohstead. } \\
\text { R Lefortt } \\
\text { T Puhlhofer. }\end{array}$ & $\begin{array}{l}10950 \\
10951 \\
11803 \\
11804 \\
11805\end{array}$ \\
\hline$A=34$ & spal & lation & $O(E)$ & & & \\
\hline $\begin{array}{l}36 \mathrm{Fe} \\
A=34\end{array}$ & $\begin{array}{c}p \\
O(E)\end{array}$ & $1.5+2$ & $23.7+2$ & NRL & R Silberbergt & 1940 \\
\hline $\begin{array}{l}27_{\mathrm{Al}} \\
27_{\mathrm{Al}} \\
27_{\mathrm{Al}} \\
27_{\mathrm{Al}} \\
27_{\mathrm{Al}}\end{array}$ & $\begin{array}{l}16_{0} \\
160 \\
19_{F} \\
19_{F} \\
19 F\end{array}$ & $\begin{array}{l}3 \cdot 0+1 \\
6 \cdot 0+1 \\
4 \cdot 0+1 \\
4 \cdot 0+1 \\
4 \cdot 0+1\end{array}$ & $\begin{array}{l}8.0+1 \\
8.0+1 \\
9.2+1 \\
9.2+1 \\
19.2+1\end{array}$ & $\begin{array}{l}\text { THD } \\
\text { NQI } \\
\text { ORL } \\
\text { PAR F } \\
\text { THD }\end{array}$ & $\begin{array}{l}\text { T Puhlhofer. } \\
\text { M Eackt } \\
\text { R Stokstead. } \\
\text { R Leforlt } \\
\text { T Puhlofer. }\end{array}$ & $\begin{array}{l}10950 \\
10951 \\
11803 \\
11804 \\
11805\end{array}$ \\
\hline$A \cdot 35$ & Spol & lotion & $O(E)$ & & & \\
\hline $\begin{array}{l}56 \mathrm{~F} \cdot \\
A=35\end{array}$ & $\begin{array}{c}p \\
\sigma(E)\end{array}$ & $1.5+2$ & $3.7+2$ & NRL F & R Silberbergt & 1940 \\
\hline $\begin{array}{l}277_{A 1} \\
27 A 1\end{array}$ & $\begin{array}{l}180 \\
160\end{array}$ & $\begin{array}{l}3.0+1 \\
6.0+1\end{array}$ & $\begin{array}{l}18.0+1 \\
18.0+1\end{array}$ & $\begin{array}{l}\text { THD } \\
\text { NBI }\end{array}$ & $\begin{array}{l}\text { T Puhlhofer. } \\
\text { M Backt }\end{array}$ & $\begin{array}{l}10950 \\
1095 !\end{array}$ \\
\hline
\end{tabular}

\begin{tabular}{|c|c|c|c|c|c|c|}
\hline Target & $\ln c$ & $\begin{array}{l}\text { Eain } \\
\text { (MeV) }\end{array}$ & $\begin{array}{l}\text { Emax } \\
(\operatorname{MeV})\end{array}$ & Lab & Author & No. \\
\hline$A=35$ & $O(E)$ & & & & & \\
\hline $\begin{array}{l}27_{A 1} \\
27_{A 1} \\
27_{A 1}\end{array}$ & $\begin{array}{l}19_{F} \\
19_{F} \\
19_{F}\end{array}$ & $\begin{array}{l}4.0+1 \\
4.0+1 \\
4.0+1\end{array}$ & $\begin{array}{l}9 \cdot 2+1 \\
9 \cdot 2+1 \\
9 \cdot 2+1\end{array}$ & $\begin{array}{l}\text { ORL } \\
\text { PAR } \\
\text { THD }\end{array}$ & $\begin{array}{l}\text { R Slohstead. } \\
\text { R Lefortt } \\
\text { T Puhlhofer. }\end{array}$ & $\begin{array}{l}11803 \\
11804 \\
11805\end{array}$ \\
\hline$A=36$ & spall & altions & $\sigma(E)$ & & & \\
\hline $\begin{array}{l}{ }^{56} \mathrm{Fe} \\
\mathrm{A}=36\end{array}$ & $\begin{array}{c}\text { D } \\
\text { ovora }\end{array}$ & $\begin{array}{l}1.5+2 \\
98 \text { o(E) }\end{array}$ & $3.7+2$ & NRL & R Silberbergt & 1940 \\
\hline $\begin{array}{l}238 U \\
A=36\end{array}$ & $\begin{array}{l}4 O_{A R} \\
\text { O(E) }\end{array}$ & $2.4+2$ & & YAL & R Bromley. & 12411 \\
\hline $\begin{array}{l}27 \mathrm{Al} \\
27 \mathrm{Al} \\
27 \mathrm{Al} \\
27_{\mathrm{Al}} \\
27_{\mathrm{Al}}\end{array}$ & $\begin{array}{l}160 \\
160 \\
19 F \\
19 \mathrm{~F} \\
19 \mathrm{~F}\end{array}$ & $\begin{array}{l}3 \cdot 0+1 \\
6 \cdot 0+1 \\
4 \cdot 0+1 \\
4 \cdot 0+1 \\
4 \cdot 0+1\end{array}$ & $\begin{array}{l}8 \cdot 0+1 \\
8 \cdot 0+1 \\
9 \cdot 2+1 \\
9 \cdot 2+1 \\
9.2+1\end{array}$ & $\begin{array}{l}\text { THD } \\
\text { NQ1 } \\
\text { ORL } \\
\text { PAR } \\
\text { THD }\end{array}$ & $\begin{array}{l}\text { T Puhlhofer. } \\
\text { M Backt } \\
\text { R Stohsiead. } \\
\text { R Lefortt } \\
\text { T Puhlhofer. }\end{array}$ & $\begin{array}{l}10950 \\
10951 \\
11803 \\
11804 \\
11805\end{array}$ \\
\hline$A=37$ & spoll & otions & $\sigma(E)$ & & & \\
\hline $\begin{array}{l}{ }^{56} \mathrm{Fe} \\
A=37\end{array}$ & $\begin{array}{c}\text { p } \\
\text { aver }\end{array}$ & $\begin{array}{l}1.5+2 \\
g \cdot \sigma(E)\end{array}$ & $1^{3.7+2}$ & NRL & R Silberbergt & 1940 \\
\hline $\begin{array}{l}23 \theta_{U} \\
A=37\end{array}$ & $\begin{array}{l}40 \mathrm{Ar} \\
\text { O(E) }\end{array}$ & $2.4+2$ & & YAL & R Bromloy. & 12411 \\
\hline $\begin{array}{l}277_{A 1} \\
277_{A 1} \\
277_{A 1} \\
277_{A 1} \\
27 A 1\end{array}$ & $\begin{array}{l}180 \\
180 \\
195 \\
19 F \\
195\end{array}$ & $\begin{array}{l}3 \cdot 0+1 \\
6 \cdot 0+1 \\
4 \cdot 0+1 \\
4 \cdot 0+1 \\
4 \cdot 0+1\end{array}$ & $\begin{array}{l}8 \cdot 0+1 \\
0.0+1 \\
9.2+1 \\
9.2+1 \\
9.2+1\end{array}$ & $\begin{array}{l}\text { THD } \\
\text { NBI } \\
\text { ORL } \\
\text { PAR } \\
\text { THD }\end{array}$ & $\begin{array}{l}\text { T Puhlhofer. } \\
\text { M Bacht } \\
\text { R Stohstead. } \\
\text { R Lofortt } \\
\text { T Puhihofer. }\end{array}$ & $\begin{array}{l}10950 \\
10951 \\
11803 \\
11804 \\
11805\end{array}$ \\
\hline$A=38$ & spalt & otion. & $o(E)$ & & & \\
\hline $\begin{array}{l}56 \mathrm{Fe} \\
\mathrm{A}=38\end{array}$ & $\begin{array}{c}p \\
\text { overe }\end{array}$ & $\begin{array}{l}1.5+2 \\
90 \quad \sigma(E)\end{array}$ & $3.7+2$ & NRL & R Silberbergt & 1940 \\
\hline $\begin{array}{l}23 \theta_{U} \\
A=38\end{array}$ & $\begin{array}{l}{ }^{40} \mathrm{Ar} \\
\text { O(E) }\end{array}$ & $2.4+2$ & & YAL & R Groaley. & 12411 \\
\hline $\begin{array}{l}27 \mathrm{Al} \\
27 \mathrm{Al} \\
27 \mathrm{Al}\end{array}$ & $\begin{array}{l}160 \\
150 \\
19 \mathrm{~F}\end{array}$ & $\begin{array}{l}3.0+1 \\
6.0+1 \\
4.0+1\end{array}$ & $\begin{array}{l}8.0+1 \\
8.0+1 \\
9.2+1\end{array}$ & $\begin{array}{l}\text { THD } \\
\text { NBI } \\
\text { ORL }\end{array}$ & $\begin{array}{l}\text { T Puhlhofer. } \\
\text { M Bacht } \\
\text { R Slohstead. }\end{array}$ & $\begin{array}{l}10950 \\
1095 ! \\
11803\end{array}$ \\
\hline
\end{tabular}




\begin{tabular}{|c|c|c|c|c|c|c|}
\hline Targel & $\ln c$ & $\begin{array}{l}\text { Emin } \\
(\mathrm{MeV})\end{array}$ & $\begin{array}{l}\text { Emax } \\
(\operatorname{MeV})\end{array}$ & Lab & Author & No. \\
\hline$A=38$ & $O(E)$ & & & & & \\
\hline $\begin{array}{l}27 \mathrm{Al} \\
27 \mathrm{Al}\end{array}$ & $\begin{array}{l}19_{F} \\
19_{F}\end{array}$ & $\begin{array}{l}4.0+1 \\
4.0+1\end{array}$ & $\begin{array}{l}9.2+1 \\
9.2+1\end{array}$ & $\begin{array}{l}\text { PAR } \\
\text { PHD }\end{array}$ & $\begin{array}{l}\text { R Lefortit } \\
\text { T Puhlhofer. }\end{array}$ & $\begin{array}{l}11804 \\
11805\end{array}$ \\
\hline$A=39$ & spoll & ation & o(E) & & & \\
\hline $\begin{array}{l}{ }^{56} \mathrm{Fe} \\
\mathrm{A}=39\end{array}$ & $\begin{array}{c}\text { p } \\
\text { avera }\end{array}$ & $\begin{array}{l}1.5+2 \\
g e \text { ote }\end{array}$ & $3.7+2$ & NRL & R Silberbergt & 1940 \\
\hline $\begin{array}{l}238 U \\
A=39\end{array}$ & $\begin{array}{l}40 \mathrm{Ar} \\
\sigma(E)\end{array}$ & $2.4+2$ & & YAL & R Bromley & $124 ! 1$ \\
\hline $\begin{array}{l}27_{\mathrm{Al}} \\
27_{\mathrm{Al}} \\
27_{\mathrm{Al}} \\
27_{\mathrm{Al}} \\
27_{\mathrm{Al}}\end{array}$ & $\begin{array}{l}160 \\
160 \\
19 \mathrm{~F} \\
19 \mathrm{~F} \\
19_{\mathrm{F}}\end{array}$ & $\begin{array}{l}3 \cdot 0+1 \\
6 \cdot 0+1 \\
4 \cdot 0+1 \\
4 \cdot 0+1 \\
4 \cdot 0+1\end{array}$ & $\begin{array}{l}8 \cdot 0+1 \\
8 \cdot 0+1 \\
9 \cdot 2+1 \\
9 \cdot 2+1 \\
9 \cdot 2+1\end{array}$ & $\begin{array}{l}\text { THD } \\
\text { NBI } \\
\text { ORL } \\
\text { PAR } \\
\text { THD }\end{array}$ & $\begin{array}{l}\text { T Puhlhofer. } \\
\text { M Bacht } \\
\text { R Stohstead. } \\
\text { R Lefort+ } \\
\text { T Puhlhofer. }\end{array}$ & $\begin{array}{l}10950 \\
10951 \\
11803 \\
11804 \\
11805\end{array}$ \\
\hline$A=4 C$ & spot & ation & $\sigma(E)$ & & & \\
\hline $\begin{array}{l}56 \mathrm{Fe} \\
A=4 \mathrm{C}\end{array}$ & $\begin{array}{c}p \\
\text { cumu }\end{array}$ & $\begin{array}{l}1.5+2 \\
\text { ative }\end{array}$ & $\begin{array}{l}3.7+2 \\
\text { fission }\end{array}$ & $\begin{array}{l}\text { NRL } \\
\text { in } y i \theta\end{array}$ & $\begin{array}{l}\text { R sllberberg+ } \\
\text { eld }\end{array}$ & 1940 \\
\hline $\begin{array}{l}170 \mathrm{Er} \\
182 \mathrm{~W}\end{array}$ & $\begin{array}{r}180 \\
0\end{array}$ & $\begin{array}{l}1.7+2 \\
1.2+2\end{array}$ & & $\begin{array}{l}\text { ORL } \\
\text { ORL }\end{array}$ & $\begin{array}{l}R \text { Plasil. } \\
\text { R Plasil. }\end{array}$ & $\begin{array}{r}11442 \\
8544\end{array}$ \\
\hline$A=40$ & aver & ge olE & & & & \\
\hline $\begin{array}{l}{ }^{238} \mathrm{U} \\
A=40\end{array}$ & $\begin{array}{l}{ }^{40} \mathrm{Ar} \\
\sigma(E)\end{array}$ & $\begin{array}{r}2.4+2 \\
.\end{array}$ & & YAL & R Bromley. & 12411 \\
\hline $\begin{array}{l}27 \mathrm{Al} \\
27_{\mathrm{Al}} \\
27 \mathrm{Al} \\
27_{\mathrm{Al}} \\
27_{\mathrm{Al}}\end{array}$ & $\begin{array}{l}15_{0} \\
15_{0} \\
19_{F} \\
19_{F} \\
19_{F}\end{array}$ & $\begin{array}{l}3 \cdot 0+1 \\
6 \cdot 0+1 \\
4 \cdot 0+1 \\
1+0+1 \\
4 \cdot 0+1\end{array}$ & $\begin{array}{l}8.0+1 \\
8.0+1 \\
9.2+1 \\
9.2+1 \\
9.2+1\end{array}$ & $\begin{array}{l}\text { THD } \\
\text { NBI } \\
\text { ORL } \\
\text { PAR } \\
\text { THD }\end{array}$ & $\begin{array}{l}\text { T Puhlhofer, } \\
\text { M Bacht } \\
\text { R Stokstead. } \\
\text { R Lefort } \\
\text { T Puhlhofer. }\end{array}$ & $\begin{array}{l}10950 \\
10951 \\
11803 \\
11804 \\
11805\end{array}$ \\
\hline$A=41$ & spol & ation & $O(E)$ & & & \\
\hline $\begin{array}{l}56 \mathrm{Fe} \\
A=41\end{array}$ & $\begin{array}{c}p \\
\text { cumu }\end{array}$ & $\begin{array}{l}1.5+2 \\
\text { ative }\end{array}$ & $\begin{array}{l}3.7+2 \\
\text { fisstort }\end{array}$ & NRL & $\begin{array}{l}\text { R Sillberbergt } \\
\text { ield }\end{array}$ & 1940 \\
\hline $\begin{array}{l}170 \mathrm{Er} \\
182 \mathrm{~W}\end{array}$ & $\begin{array}{r}160 \\
0\end{array}$ & $\begin{array}{l}1.7+2 \\
1.2+5\end{array}$ & & $\begin{array}{l}\text { ORL } \\
\text { ORL }\end{array}$ & $\begin{array}{l}\text { R Plasil. } \\
\text { R Plasil. }\end{array}$ & $\begin{array}{r}11442 \\
8644\end{array}$ \\
\hline
\end{tabular}

\begin{tabular}{|c|c|c|c|c|c|c|}
\hline Target & $\ln c$ & $\begin{array}{l}\text { Emin } \\
(\mathrm{MeV})\end{array}$ & $\begin{array}{l}\text { Emax } \\
(\operatorname{MeV})\end{array}$ & Lob $\mathrm{K}$ & Authoor & No. \\
\hline$A=41$ & overs & $g e O(E)$ & & & & \\
\hline $\begin{array}{l}230 \mathrm{U} \\
A=41\end{array}$ & $\begin{array}{l}40 \mathrm{Ar} \\
0(\varepsilon)\end{array}$ & $2.4+2$ & & YAL $F$ & R Bromley. & 12411. \\
\hline $\begin{array}{l}27_{A 1} \\
27_{A 1} \\
27 A 1 \\
27 A 1 \\
27_{A 1}\end{array}$ & $\begin{array}{l}16_{0} \\
16_{0} \\
19 \mathrm{~F} \\
19_{\mathrm{F}} \\
19_{\mathrm{F}}\end{array}$ & $\begin{array}{l}3 \cdot 0+1 \\
6 \cdot 0+1 \\
4 \cdot 0+1 \\
4 \cdot 0+1 \\
4 \cdot 0+1\end{array}$ & $\begin{array}{l}8 \cdot 0+1 \\
8 \cdot 0+1 \\
9.2+1 \\
9.2+1 \\
9.2+1\end{array}$ & $\begin{array}{l}\text { THD } \\
\text { NBI } \\
\text { ORL } \\
\text { PAR } \\
\text { THD }\end{array}$ & $\begin{array}{l}\text { T Puhlhofer. } \\
\text { M Bacht } \\
\text { R Stahstead. } \\
\text { R Lefortt } \\
\text { T Puhlhofer. }\end{array}$ & $\begin{array}{l}10950 \\
10951 \\
11803 \\
11804 \\
11805\end{array}$ \\
\hline$A=42$ & spall & otion. & $O(E)$ & & & \\
\hline $\begin{array}{c}56 \mathrm{Fe} \\
A=42\end{array}$ & cumul & $\begin{array}{l}1.5+2 \\
\text { ative f }\end{array}$ & $\begin{array}{l}3.7+2 \\
\text { fisgion }\end{array}$ & $\begin{array}{l}\text { NRL F } \\
n y \mid e\end{array}$ & $\begin{array}{l}\text { R Silberberg+ } \\
\text { id }\end{array}$ & 1940 \\
\hline $\begin{array}{l}170 \mathrm{Er} \\
182 \mathrm{~W}\end{array}$ & $\begin{array}{r}160 \\
0\end{array}$ & $\begin{array}{l}1.7+2 \\
1.2+2\end{array}$ & & $\begin{array}{l}\text { ORL } \\
\text { ORL }\end{array}$ & $\begin{array}{l}\text { R Plasil. } \\
\text { R Plasil. }\end{array}$ & $\begin{array}{r}11442 \\
8644\end{array}$ \\
\hline$A=4 e^{\circ}$ & avero & $g e$ o(E) & & & & \\
\hline $\begin{array}{l}238 U \\
A=42\end{array}$ & $\begin{array}{l}40_{A r} \\
O(E)\end{array}$ & $2.4+2$ & & YAL $F$ & R Bromley. & 12411 \\
\hline $\begin{array}{l}27 \mathrm{Al} \\
27 \mathrm{Al} \\
27_{\mathrm{Al}}\end{array}$ & $\begin{array}{l}19 \mathrm{~F} \\
19 \mathrm{~F} \\
19_{F}\end{array}$ & $\begin{array}{l}4.0+1 \\
4.0+1 \\
4.0+1\end{array}$ & $\begin{array}{l}9.2+1 \\
9.2+1 \\
9.2+1\end{array}$ & $\begin{array}{l}\text { ORL } \\
\text { PAR } \\
\text { THD }\end{array}$ & $\begin{array}{l}\text { R Stohgtead. } \\
\text { R Lefort+ } \\
\text { T Puhlhofer. }\end{array}$ & $\begin{array}{l}11803 \\
11804 \\
11805\end{array}$ \\
\hline$A=43$ & spall & lationo & $o(E)$ & & & \\
\hline $\begin{array}{c}56_{\mathrm{Fe}} \\
A=43\end{array}$ & $\begin{array}{c}p \\
\text { cumul }\end{array}$ & $\begin{array}{l}1.5+2 \\
\text { lative f }\end{array}$ & $\begin{array}{l}3.7+2 \\
\text { fission }\end{array}$ & & $\begin{array}{l}\text { R Silberberg+ } \\
\text { ald }\end{array}$ & 1940 \\
\hline $\begin{array}{l}170 \mathrm{Er} \\
182 \mathrm{~W}\end{array}$ & $\begin{array}{r}160 \\
a\end{array}$ & $\begin{array}{l}1.7+2 \\
1.2+2\end{array}$ & & $\begin{array}{l}\text { ORL } \\
\text { ORL }\end{array}$ & $\begin{array}{l}\text { R Plasil. } \\
\text { R Plasil. }\end{array}$ & $\begin{array}{r}11442 \\
8644\end{array}$ \\
\hline$A=43$ & overo & ge $O(E)$ & & & & \\
\hline $\begin{array}{l}238_{U} \\
A=43\end{array}$ & $\begin{array}{l}40_{\mathrm{Ar}} \\
\because(\mathrm{E})\end{array}$ & $2.4+2$ & & YAL & R Bromley. & 12411 \\
\hline $\begin{array}{l}277_{A 1} \\
27_{A 1} \\
27 A 1\end{array}$ & $\begin{array}{l}19_{F} \\
19_{F} \\
18_{F}\end{array}$ & $\begin{array}{l}4 \cdot 0+1 \\
4 \cdot 0+1 \\
4 \cdot 0+1\end{array}$ & $\begin{array}{l}9 \cdot 2+1 \\
9 \cdot 2+1 \\
9 \cdot 2+1\end{array}$ & $\begin{array}{l}\text { ORL } \\
\text { PAR } \\
\text { THD }\end{array}$ & $\begin{array}{l}\text { R Stohstead. } \\
\text { R Lefort+ } \\
\text { T Puhlhofer. }\end{array}$ & $\begin{array}{l}11803 \\
11804 \\
11805\end{array}$ \\
\hline
\end{tabular}




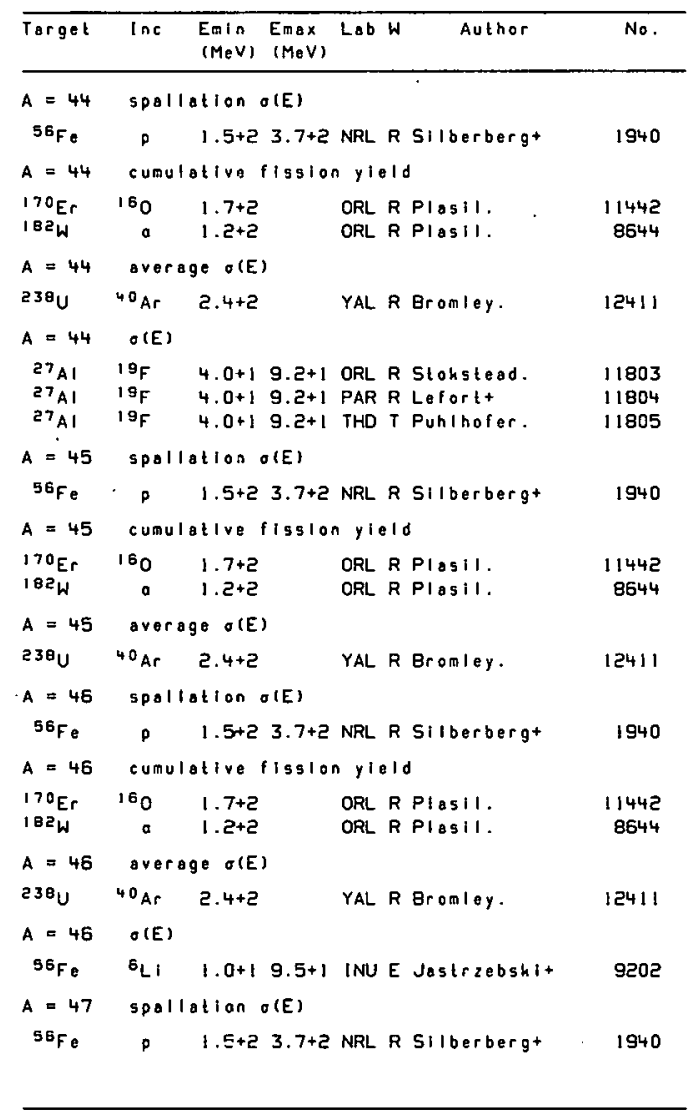

\begin{tabular}{|c|c|c|c|c|}
\hline Target & $\ln e$ & $\begin{array}{ll}\text { Emin } & \text { Emax } \\
(\operatorname{MeV}) & (\mathrm{MeV})\end{array}$ & Lab W & No. \\
\hline$A=47$ & \multicolumn{4}{|c|}{ cumulative lission yleld } \\
\hline $\begin{array}{l}170 \mathrm{Er} \\
18 \mathrm{~F}_{\mathrm{W}}\end{array}$ & $\begin{array}{r}160 \\
0\end{array}$ & $\begin{array}{l}1.7+2 \\
1.2+2\end{array}$ & $\begin{array}{l}\text { ORL R Plasil. } \\
\text { ORL R Plasil. }\end{array}$ & $\begin{array}{r}11442 \\
8644\end{array}$ \\
\hline$A=47$ & \multicolumn{4}{|c|}{ average o(E) } \\
\hline $23 \theta^{8}$ & $40 \mathrm{Ar}$ & $2.4+2$ & YAL R Bronley. & 12411 \\
\hline$A=47$ & $O(E)$ & & . & \\
\hline${ }^{56} \mathrm{Fe}_{\mathrm{B}}$ & ${ }^{6} \mathrm{Li}$ & $1.0+19.5+1$ & INU E Jastrzebshit & 9202 \\
\hline$A=48$ & \multicolumn{4}{|c|}{ spaliotion o(E) } \\
\hline $56 \mathrm{Fe}$ & p & $1.5+23.7+2$ & NRL R Silberbergt & 1940 \\
\hline$A=48$ & \multicolumn{4}{|c|}{ cumulative flasion yield } \\
\hline $\begin{array}{l}170 \mathrm{Er} \\
182_{W}\end{array}$ & 160 & $\begin{array}{l}1.7+2 \\
1.2+2\end{array}$ & $\begin{array}{l}\text { ORL R Plasil. } \\
\text { ORL R PIasil. }\end{array}$ & $\begin{array}{r}11442 \\
8644\end{array}$ \\
\hline$A=48$ & \multicolumn{4}{|c|}{ average $O(E)$} \\
\hline $238_{U}$ & ${ }^{40} \mathrm{Ar}$ & $2.4+2$ & YAL R Brombey. & 12411 \\
\hline$A=48$ & $\sigma(E)$ & & & \\
\hline $56_{\mathrm{Fe}}$ & ${ }^{6} L_{1}$ & $1.0+19.5+1$ & INU $\varepsilon$ Jastrzebskit & 9202 \\
\hline$A=49$ & \multicolumn{4}{|c|}{ spallation of(E) } \\
\hline${ }^{56} \mathrm{Fe}$ & $\rho$ & $1.5+2 \quad 3.7+2$ & NRL R Silberberg* & 1940 \\
\hline$A=49$ & \multicolumn{4}{|c|}{ cumulative lission yleld } \\
\hline $\begin{array}{l}170 \mathrm{Er} \\
182_{\mathrm{W}}\end{array}$ & $\begin{array}{r}160 \\
0\end{array}$ & $\begin{array}{l}1.7+5 \\
1.5+5\end{array}$ & $\begin{array}{l}\text { ORL R Plasil. } \\
\text { ORL R Plasil. }\end{array}$ & $\begin{array}{r}11442 \\
8644\end{array}$ \\
\hline$A=49$ & \multicolumn{4}{|c|}{ average $\sigma(E)$} \\
\hline $\begin{array}{l}230 \mathrm{U} \\
A=49\end{array}$ & $\begin{array}{l}{ }^{40} \mathrm{Ar} \\
O(E)\end{array}$ & $2.4+2$ & YAL R Bromley. & $12411^{\circ}$ \\
\hline${ }^{56} \mathrm{Fe}$ & $\sigma_{L I}$ & $1.0+19.5+1$ & INU E Jastrzebshit & 9202 \\
\hline$A=50$ & \multicolumn{4}{|c|}{ Spollotion o(E) } \\
\hline${ }^{56} \mathrm{Fe}$ & $p$ & $1.5+23.7+2$ & NRL. R Silberbergt & 1940 \\
\hline$A=50$ & \multicolumn{4}{|c|}{ cumulative fission yield } \\
\hline $170^{\circ} \mathrm{Er}$ & 150 & $1.7+2$ & ORL R Plasil. & 11442 \\
\hline
\end{tabular}




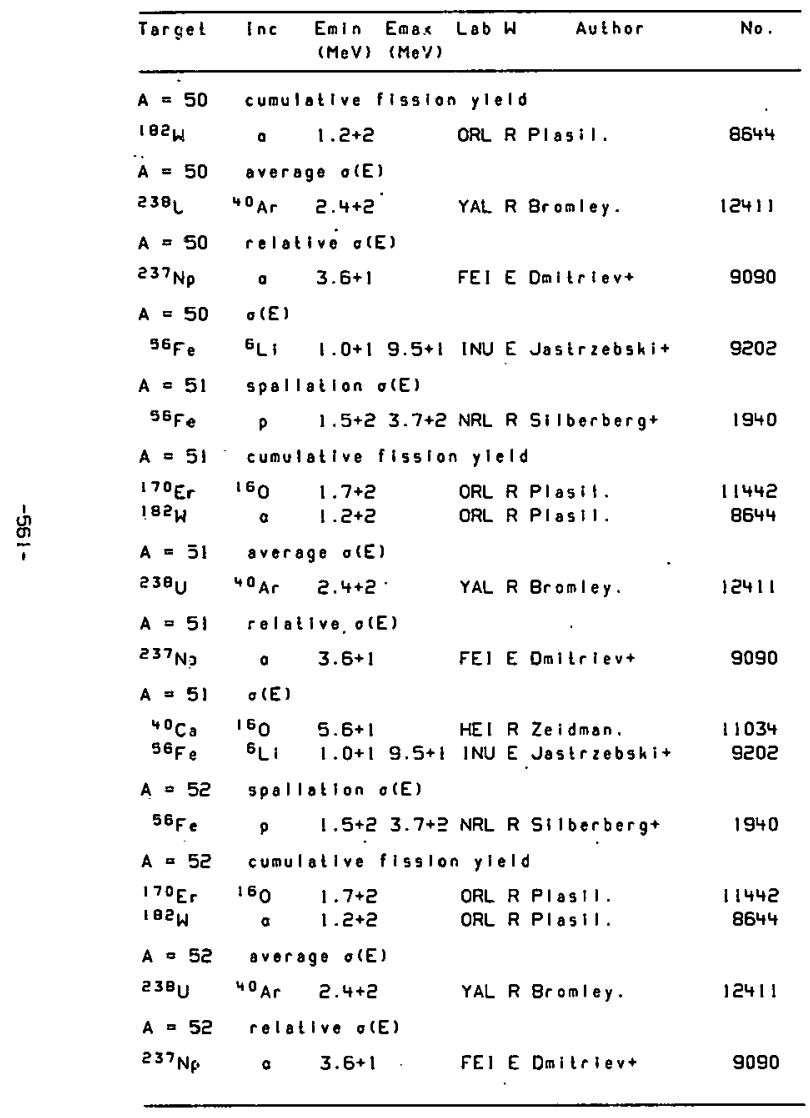

\begin{tabular}{|c|c|c|c|c|c|c|}
\hline Target & $\ln s$ & $\begin{array}{l}\text { Emin } \\
(\mathrm{MeV})\end{array}$ & $\begin{array}{l}\text { Emax } \\
(\operatorname{MeV})\end{array}$ & Lab $h$ & futhor & No. \\
\hline$A=52$ & $\sigma(E)$ & & & & & \\
\hline $\begin{array}{l}{ }^{40} \mathrm{Co} \\
{ }^{56} \mathrm{Fe}\end{array}$ & $\begin{array}{l}160 \\
{ }^{6} \mathrm{Li}\end{array}$ & $\begin{array}{l}5.6+1 \\
1.0+1\end{array}$ & $9.5+1$ & $\begin{array}{l}\text { HEI } \\
\text { INU E }\end{array}$ & $\begin{array}{l}\text { R Zeidaran. } \\
\text { E Jastrzebshit }\end{array}$ & $\begin{array}{r}11034 \\
9202\end{array}$ \\
\hline$A=53$ & spsil & ation & $O(E)$ & & & \\
\hline${ }^{56} \mathrm{Fe}$ & $\rho$ & $1.5+2$ & $3.7+2$ & NRL $F$ & R silberbergt & 1940 \\
\hline$A=53$ & cumul & lative & flasion & $\ln y 101$ & Id & \\
\hline $\begin{array}{l}170 \mathrm{Er} \\
182 \mathrm{~W}\end{array}$ & $\begin{array}{r}160 \\
0\end{array}$ & $\begin{array}{l}1.7+2 \\
1.2+2\end{array}$ & & $\begin{array}{l}\text { ORL } \\
\text { ORL }\end{array}$ & $\begin{array}{l}\text { R Plasil. } \\
\text { RPlasil. }\end{array}$ & $\begin{array}{r}11442 \\
8644\end{array}$ \\
\hline$A=53$ & overa & ge olE & & & . & \\
\hline $238_{U}$ & ${ }^{40} \mathrm{Ar}$ & $2.4+2$ & & YAL $F$ & R Bromley. & 12411 \\
\hline$A=53$ & rolat & Ire of & & & & \\
\hline $23{ }^{7} \mathrm{~Np}$ & $a$ & $3.6+1$ & & FEI E & E Omitriezt & 9090 \\
\hline$A=53$ & $\sigma(E)$ & & & & & \\
\hline $\begin{array}{l}{ }^{40} \mathrm{Ca} \\
42 \mathrm{Ca} \\
96 \mathrm{Fe}\end{array}$ & $\begin{array}{l}160 \\
150 \\
6 \mathrm{Li}\end{array}$ & $\begin{array}{l}5.6+1 \\
5.6+1 \\
1.0+1\end{array}$ & $9.5+1$ & $\begin{array}{l}\text { HEI F } \\
\text { HEI F } \\
\text { INU E }\end{array}$ & $\begin{array}{l}\text { R Zeidman. } \\
\text { R Zeidman. } \\
\text { E Jastrzebshit }\end{array}$ & $\begin{array}{r}11034 \\
11048 \\
9202\end{array}$ \\
\hline$A=54$ & spolt & lation & $\sigma(E)$ & & & \\
\hline${ }^{56} \mathrm{Fe}$ & p & $1.5+2$ & $3.7+2$ & NRL F & R Silberbergt & 1940 \\
\hline$A=54$ & cumut & lotive & fissior & in yie & eld & \\
\hline $\begin{array}{l}170 \mathrm{Er} \\
182 \mathrm{~W}\end{array}$ & $\begin{array}{r}160 \\
0\end{array}$ & $\begin{array}{l}1.7+2 \\
1.2+2\end{array}$ & & $\begin{array}{l}\text { ORL F } \\
\text { ORL F }\end{array}$ & $\begin{array}{l}\text { RPlasil. } \\
\text { RPlasil. }\end{array}$ & $\begin{array}{r}11442 \\
8544\end{array}$ \\
\hline$A=54$ & overe & $g$ e $\sigma(E$ & & & & \\
\hline $\begin{array}{l}238 U \\
A=54\end{array}$ & $\begin{array}{l}40_{\mathrm{Ar}} \\
r \dot{\mathrm{C}} \mathrm{at}\end{array}$ & $\begin{array}{l}2.4+2 \\
\text { ive oll }\end{array}$ & & YAL F & R Bromley. & 12411 \\
\hline $\begin{array}{l}2{ }^{37} \mathrm{~Np} \\
A=54\end{array}$ & $\begin{array}{c}0 \\
0(E)\end{array}$ & $3.6+1$ & & FEI E & E Doritrievt & 9090 \\
\hline $\begin{array}{l}{ }^{40} \mathrm{Ca} \\
{ }^{42} \mathrm{Ca} \\
56 \mathrm{Fe}\end{array}$ & $\begin{array}{l}160 \\
160 \\
6 L_{1}\end{array}$ & $\begin{array}{l}5.6+1 \\
5 \cdot 6+1 \\
1.0+1\end{array}$ & $9.5+1$ & $\begin{array}{l}\text { HEI F } \\
\text { HEI } \\
\text { INU }\end{array}$ & $\begin{array}{l}\text { R Zeldman. } \\
\text { R Zeidman. } \\
\text { E Jastrzebskit }\end{array}$ & $\begin{array}{r}11034 \\
11048 \\
9202\end{array}$ \\
\hline
\end{tabular}




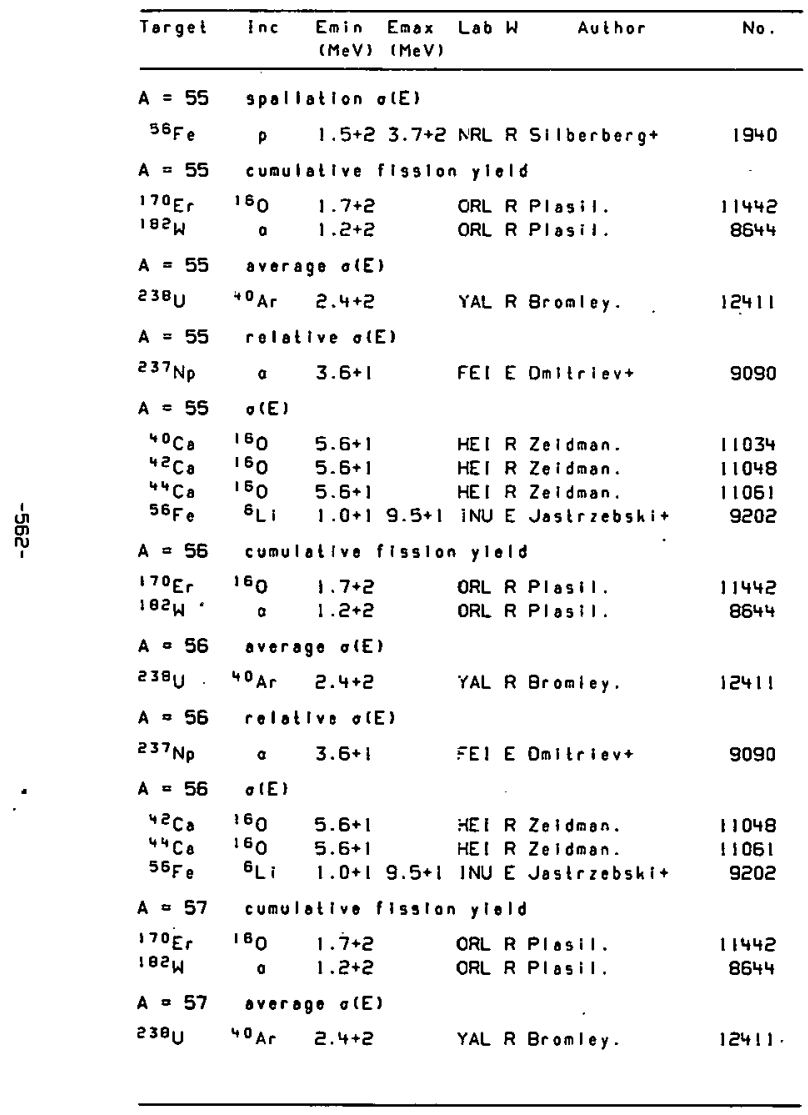

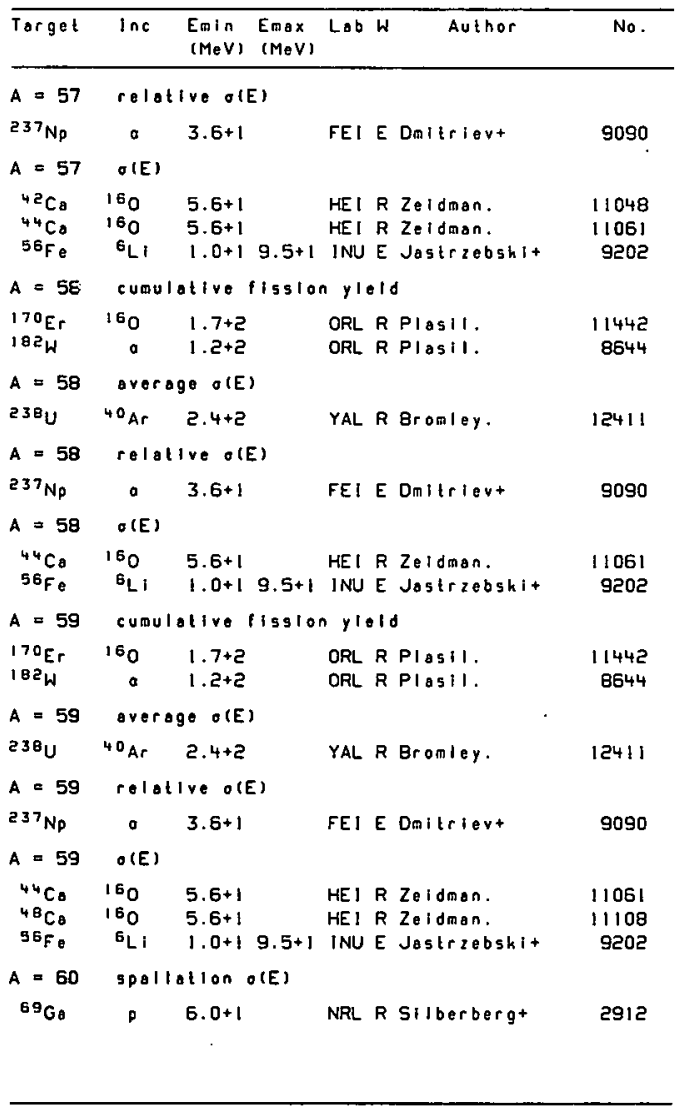




\begin{tabular}{|c|c|c|c|c|c|}
\hline Target & $\operatorname{Inc}$ & $\begin{array}{ll}E \min & \text { Emax } \\
(M e V) & (M e V)\end{array}$ & Lab & Author & No. \\
\hline$A=E 0$ & \multicolumn{5}{|c|}{ cumulative figsion yleld } \\
\hline $\begin{array}{l}170 \mathrm{Er} \\
102 \mathrm{~W}\end{array}$ & $\begin{array}{r}160 \\
0\end{array}$ & $\begin{array}{l}1.7+2 \\
1.2+2\end{array}$ & $\begin{array}{l}\text { ORL } \\
\text { ORL }\end{array}$ & $\begin{array}{l}\text { R Plasil. } \\
\text { R Plasil. }\end{array}$ & $\begin{array}{r}11442 \\
8644\end{array}$ \\
\hline$A=60$ & \multicolumn{5}{|c|}{ average o(E) } \\
\hline${ }^{23 \theta} \mathrm{U}$ & $40 \mathrm{Ar}$ & $2.4+2$ & YAL & R Bromley. & 12411 \\
\hline$A=60$ & \multicolumn{5}{|c|}{ relative o(E) } \\
\hline $\begin{array}{l}237 \mathrm{~Np} \\
242 \mathrm{Pu}\end{array}$ & a & $\begin{array}{l}3.6+1 \\
2.6+1 \quad 3.1+1\end{array}$ & $\begin{array}{l}\text { FEI } \\
\text { FEI }\end{array}$ & $\begin{array}{l}\text { E Dmitrievt } \\
\text { E Dmitrievt. }\end{array}$ & $\begin{array}{l}9090 \\
9114\end{array}$ \\
\hline$A=60$ & \multicolumn{5}{|l|}{$o(E)$} \\
\hline $\begin{array}{r}48 \mathrm{Ca} \\
56 \mathrm{Fe} \\
226 \mathrm{Ra}\end{array}$ & $\begin{array}{c}160 \\
6 \mathrm{Li} \\
1\end{array}$ & $\begin{array}{l}5.6+1 \\
1.0+1 \\
2.3+1\end{array}$ & $\begin{array}{l}\text { HEI } \\
\text { INU } \\
\text { LAS }\end{array}$ & $\begin{array}{l}\text { R Zeidman. } \\
\text { E Jastrzebskit } \\
\text { E Webert }\end{array}$ & $\begin{array}{r}11108 \\
9202 \\
6068\end{array}$ \\
\hline$A=61$ & \multicolumn{5}{|c|}{ Spallation o(E) } \\
\hline${ }^{69} \mathrm{Ga}$ & p & $6.0+1$ & NRL F & R Silberbergt & 2912 \\
\hline$A=61$ & \multicolumn{5}{|c|}{ cumulative fisslon yleld } \\
\hline $\begin{array}{l}170 \mathrm{Er} \\
182 \mathrm{~W}\end{array}$ & $\begin{array}{r}160 \\
0\end{array}$ & $\begin{array}{l}1.7+2 \\
1.2+2\end{array}$ & $\begin{array}{l}\text { ORL F } \\
\text { ORL F }\end{array}$ & $\begin{array}{l}\text { R Plasil. } \\
\text { RPlasil. }\end{array}$ & $\begin{array}{r}11442 \\
8644\end{array}$ \\
\hline$A=61$ & \multicolumn{5}{|c|}{ average o(E) } \\
\hline $\begin{array}{l}238 \mathrm{U} \\
A=61\end{array}$ & \multicolumn{3}{|c|}{ relative o(E) } & R Bromley. & 12411 \\
\hline $\begin{array}{l}237 \mathrm{~Np} \\
242 \mathrm{Pu}\end{array}$ & $:$ & $\begin{array}{l}3.6+1 \\
2.6+1 \quad 3.1+1\end{array}$ & $\begin{array}{l}\text { FEI } \\
\text { FEI }\end{array}$ & $\begin{array}{l}\text { E Doildievt } \\
\text { E Donilrievt }\end{array}$ & $\begin{array}{l}9090 \\
9114\end{array}$ \\
\hline$A=6 !$ & \multicolumn{5}{|l|}{$\sigma(E)$} \\
\hline $\begin{array}{r}48 \mathrm{Ca} \\
226 \mathrm{Ra}\end{array}$ & $\begin{array}{r}160 \\
1\end{array}$ & $\begin{array}{l}5.6+1 \\
2.3+1\end{array}$ & $\begin{array}{l}\text { HEI F } \\
\text { LAS E }\end{array}$ & $\begin{array}{l}\text { R Zeidman. } \\
\text { E Webert }\end{array}$ & $\begin{array}{r}11108 \\
6068\end{array}$ \\
\hline$A=62$ & \multicolumn{5}{|c|}{ spollotion o(E) } \\
\hline${ }^{69} \mathrm{Go}$ & $p$ & $6.0+1$ & NRI & R Silberbergt & 2912 \\
\hline$A=62$ & \multicolumn{5}{|c|}{ cumulotive flggion yield } \\
\hline $\begin{array}{l}170 \mathrm{Er} \\
182 \mathrm{~W}\end{array}$ & $\begin{array}{r}160 \\
0\end{array}$ & $\begin{array}{l}1.7+2 \\
1.2+2\end{array}$ & $\begin{array}{l}\text { ORL F } \\
\text { ORL F }\end{array}$ & $\begin{array}{l}\text { R Plasil. } \\
\text { R Plasili. }\end{array}$ & $\begin{array}{r}11442 \\
8644\end{array}$ \\
\hline
\end{tabular}

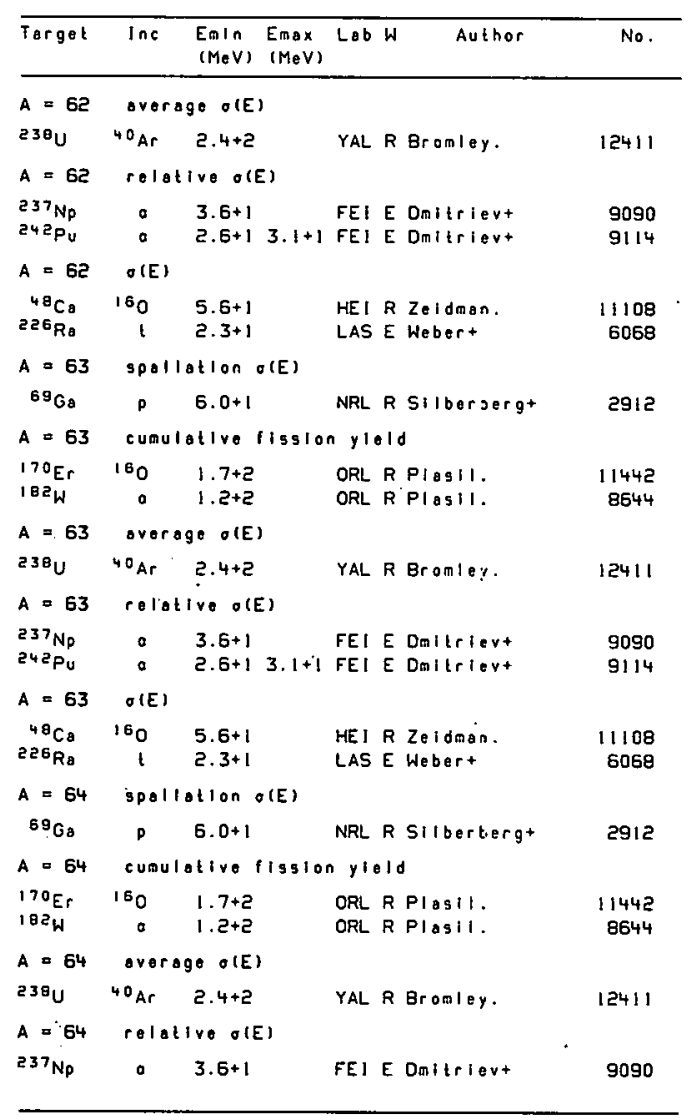




\begin{tabular}{|c|c|c|c|c|}
\hline Targat & $\operatorname{Inc}$ & $\begin{array}{ll}\text { Emin } & \text { Emax } \\
(\mathrm{MeV}) & (\mathrm{MeV})\end{array}$ & Author & No. \\
\hline$A=64$ & \multicolumn{2}{|c|}{ relative o(E) } & & \multirow{3}{*}{9114} \\
\hline $242 \mathrm{Pu}$ & 。 & $2 . E+1 \quad 3.1+1$ & \multirow[t]{2}{*}{ FEI E Dmitrievt } & \\
\hline$A=64$ & $O(E)$ & \multirow[b]{2}{*}{$2.3+1$} & & \\
\hline $226 \mathrm{Ra}$ & $t$ & & LAS E Webert & 6068 \\
\hline$A=65$ & \multicolumn{2}{|c|}{ Spallation o(E) } & \multirow[b]{2}{*}{ NRL R Silberberg+ } & \\
\hline${ }^{69} \mathrm{~GB}_{\mathrm{B}}$ & $p$ & $6.0+1$ & & 2912 \\
\hline$A=65$ & \multicolumn{3}{|c|}{ cumulative fission yleld } & \\
\hline $\begin{array}{l}170 \mathrm{Er} \\
182 \mathrm{~W}\end{array}$ & $\begin{array}{r}160 \\
0\end{array}$ & $\begin{array}{l}1.7+2 \\
1 . e+2\end{array}$ & $\begin{array}{l}\text { ORL R PIOSII, } \\
\text { ORL R PIOSII. }\end{array}$ & $\begin{array}{r}11442 \\
8644\end{array}$ \\
\hline$A=65$ & \multicolumn{2}{|c|}{ avorago a(E) } & \multirow[b]{2}{*}{ YAL R Bromley. } & \\
\hline $238 \mathrm{U}$ & ${ }^{40} \mathrm{Ar}$ & $2.4+2$ & & 12411 \\
\hline$A=65$ & \multicolumn{2}{|c|}{ relative o(E) } & \multicolumn{2}{|l|}{ 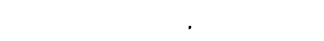 } \\
\hline $\begin{array}{l}237 \mathrm{~Np} \\
242 \mathrm{Pu}_{u}\end{array}$ & $:$ & $\begin{array}{l}3.6+1 \\
2.6+13.1+1\end{array}$ & $\begin{array}{l}\text { FEI E Omltrievt } \\
\text { FEI E Dmitriovt }\end{array}$ & $\begin{array}{l}9090 \\
9114\end{array}$ \\
\hline$A=65$ & \multicolumn{2}{|l|}{ O(E) } & \multirow{3}{*}{ LAS E Webert } & \\
\hline $226_{R B}$ & $\mathfrak{t}$ & $2.3+1$ & & 6068 \\
\hline$A=66$ & \multicolumn{2}{|c|}{ Spallaticn o(E) } & & \\
\hline${ }^{68} \mathrm{Go}_{\mathrm{O}}$ & $p$ & $6 \cdot[1+1$ & \multirow{2}{*}{$\begin{array}{l}\text { NRL R Silberberg* } \\
\text { on ylelo }\end{array}$} & 2912 \\
\hline$A=66$ & \multicolumn{2}{|c|}{ cumulative fission yleld } & & \\
\hline $\begin{array}{l}170 \mathrm{Er} \\
18 \mathrm{FW}_{\mathrm{W}}\end{array}$ & $\begin{array}{r}160 \\
0\end{array}$ & $\begin{array}{l}1.7+2 \\
1.2+2\end{array}$ & \multirow[t]{2}{*}{$\begin{array}{l}\text { ORL R PIasil. } \\
\text { ORL R PIOSI1. }\end{array}$} & $\begin{array}{r}11442 \\
8644\end{array}$ \\
\hline$A=66$ & \multicolumn{2}{|c|}{ overage $(E)$} & & \\
\hline $238 \mathrm{U}$ & ${ }^{40} \mathrm{Ar}$ & $2.4+2$ & \multirow[t]{2}{*}{ YAL R Bromley. } & 12411 \\
\hline$A=66$ & \multicolumn{2}{|c|}{ relative o(E) } & & \\
\hline $\begin{array}{l}237 \mathrm{~Np} \\
242 \mathrm{Pu}\end{array}$ & $:$ & $\begin{array}{l}3.6+1 \\
2.6+1 \quad 3.1+1\end{array}$ & \multirow[t]{2}{*}{$\begin{array}{l}\text { FEI E Omitriert } \\
\text { I FEI E Dmitrievt }\end{array}$} & $\begin{array}{l}9090 \\
9114\end{array}$ \\
\hline$A=65$ & $\sigma(E)$ & & & \\
\hline${ }^{226} \mathrm{Ro}_{\mathrm{B}}$ & $t$ & $2.3+1$ & LAS E Webert & 6068 \\
\hline
\end{tabular}

\begin{tabular}{|c|c|c|c|c|}
\hline Targat & $\operatorname{Inc}$ & $\begin{array}{ll}\text { Enin } & \text { Emax } \\
\text { (MeV) } & \text { (MeV) }\end{array}$ & Lab W & No. \\
\hline$A=67$ & \multicolumn{2}{|c|}{ Spallatlon $O(E)$} & \multirow[b]{2}{*}{ NRL R silberberg+ } & \\
\hline${ }^{69} G_{a}$ & p & $6.0+1$ & & 2912 \\
\hline$A=67$ & \multicolumn{3}{|c|}{ cumulative fission yield } & \\
\hline $\begin{array}{l}170 \mathrm{Er} \\
102 \mathrm{~W}\end{array}$ & $\begin{array}{r}160 \\
0\end{array}$ & $\begin{array}{l}1.7+2 \\
1.2+2\end{array}$ & $\begin{array}{l}\text { ORL R Plasil. } \\
\text { ORL R PI EsII. }\end{array}$ & $\begin{array}{r}11442 \\
8644\end{array}$ \\
\hline$A=67$ & \multicolumn{2}{|c|}{ average $O(E)$} & & \\
\hline $230 \mathrm{U}$ & ${ }^{40} \mathrm{Ar}$ & $2.4+2$ & YAL R Bromley. & 12411 \\
\hline$A=67$ & \multicolumn{2}{|c|}{ relative $O(E)$} & & \\
\hline $\begin{array}{l}237 \mathrm{~Np} \\
242 \mathrm{Pu}\end{array}$ & : & $\begin{array}{l}3.6+1 \\
2.6+1 \quad 3.1+1\end{array}$ & $\begin{array}{l}\text { FEI E Dmitrievt } \\
\text { FEI E Dmitrievt }\end{array}$ & $\begin{array}{l}9090 \\
9114\end{array}$ \\
\hline$A=67$ & \multicolumn{2}{|l|}{$O(E)$} & & \\
\hline $22 \epsilon_{R a}$ & $t$ & $2.3+1$ & LAS E webert & 6068 \\
\hline$A=68$ & \multicolumn{2}{|c|}{ spoltation o(E) } & & \\
\hline${ }^{69} \mathrm{Ga}$ & P & $6.0+1$ & NRL R Silberbergt & 2912 \\
\hline$A=68$ & \multicolumn{3}{|c|}{ cumulative fisgion yield } & \\
\hline $\begin{array}{l}170 \mathrm{Er} \\
18 \mathrm{~W}_{\mathrm{W}}\end{array}$ & $\begin{array}{r}160 \\
0\end{array}$ & $\begin{array}{l}1.7+2 \\
1.2+2\end{array}$ & $\begin{array}{l}\text { ORL R Plasil. } \\
\text { ORL R Plasil. }\end{array}$ & $\begin{array}{r}1144 a \\
8644\end{array}$ \\
\hline$A=68$ & \multicolumn{2}{|c|}{ averoge o(E) } & & \\
\hline $23 \theta^{2}$ & ${ }^{40} \mathrm{Ar}$ & $2.4+2$ & YAL R Bromley. & 12411 \\
\hline$A=68$ & \multicolumn{2}{|c|}{ relative $O(E)$} & & \\
\hline $\begin{array}{l}237 \mathrm{~Np} \\
242 \mathrm{Pu}\end{array}$ & : & $\begin{array}{l}3 \cdot 6+1 \\
2 \cdot 6+1 \quad 3 \cdot 1+1\end{array}$ & $\begin{array}{l}\text { FEI E Dmitrlevt } \\
\text { FEI E Doltrievt }\end{array}$ & $\begin{array}{l}9090 \\
9114\end{array}$ \\
\hline$A=68$ & \multicolumn{2}{|c|}{$O(E)$} & & \\
\hline $226 \mathrm{Rs}$ & $t$ & $2 \cdot 3+1$ & LASE Webert & 6068 \\
\hline$A=69$ & \multicolumn{2}{|c|}{ spallation o(E) } & & \\
\hline${ }^{69} \mathrm{Ga}$ & $p$ & $6.0+1$ & NRL $R$ Silberbergt & 2912 \\
\hline$A=69$ & \multicolumn{3}{|c|}{ cumulative fission yield } & \\
\hline $\begin{array}{l}170 E r \\
102 W\end{array}$ & $\begin{array}{r}150 \\
0\end{array}$ & $\begin{array}{l}1.7+2 \\
1.2+2\end{array}$ & $\begin{array}{l}\text { ORL R PIAsII. } \\
\text { ORL R PIOSII. }\end{array}$ & $\begin{array}{r}11442 \\
8644\end{array}$ \\
\hline
\end{tabular}




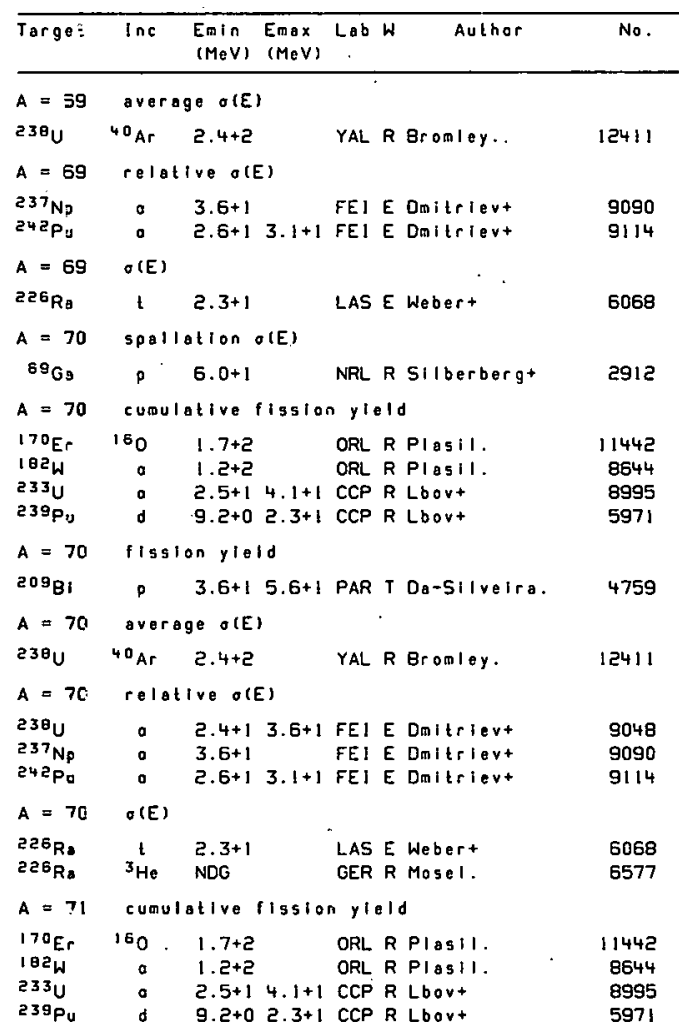

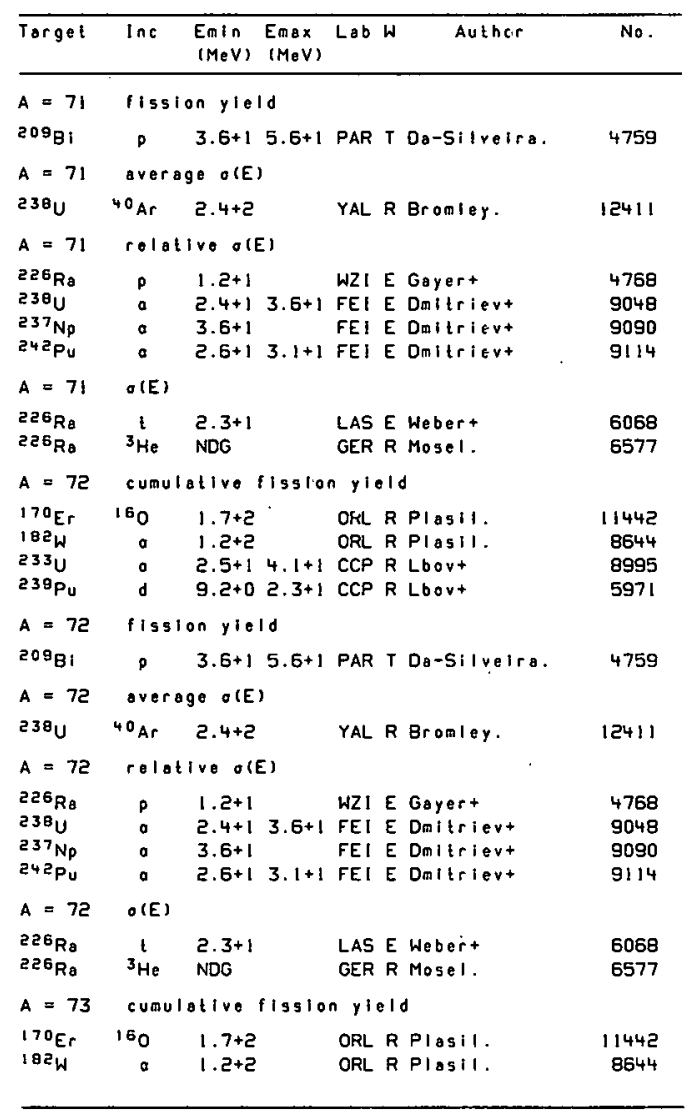




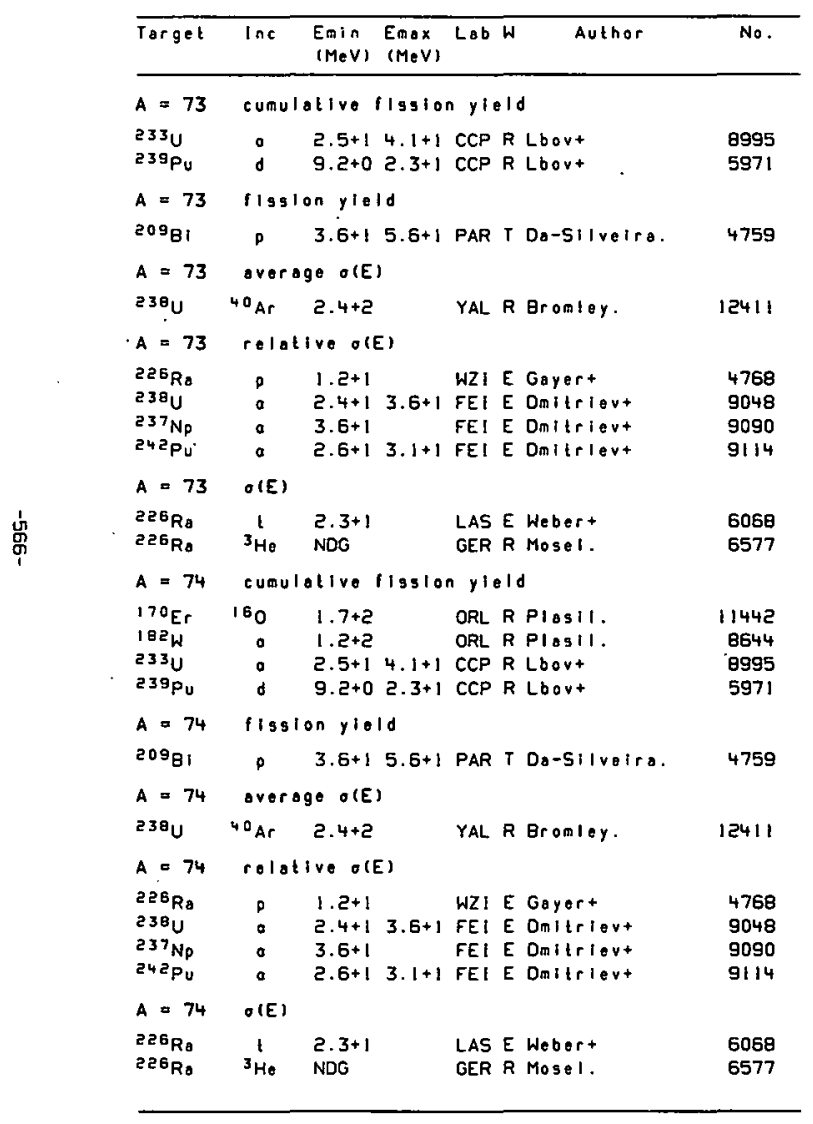

\begin{tabular}{|c|c|c|c|c|c|c|}
\hline Torget & $\operatorname{Inc}$ & $\begin{array}{l}\text { Emin } \\
(\mathrm{MeV})\end{array}$ & $\begin{array}{l}\text { Emax } \\
(\mathrm{MeV})\end{array}$ & Lab W & Author & No. \\
\hline$A=75$ & \multicolumn{6}{|c|}{ primary flasion yield } \\
\hline $209_{\mathrm{BI}}$ & $p$ & $3.2+1$ & & TUE $R$ & Q Gonnenvelnt & 4761 \\
\hline$A=75$ & \multicolumn{6}{|c|}{ cumulotive fisgion yleld } \\
\hline 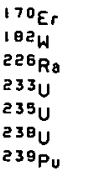 & $\begin{array}{r}160 \\
0 \\
p \\
0 \\
p \\
p \\
d\end{array}$ & $\begin{array}{l}1.7+2 \\
1.2+2 \\
1.3+1 \\
2.5+1 \\
1.2+1 \\
7.1+0 \\
9.2+0\end{array}$ & $\begin{array}{l} \\
4.1+1 \\
9.5+1 \\
1.0+2 \\
2.3+1\end{array}$ & $\begin{array}{ll}\text { ORL } & R \\
\text { ORL } & R \\
\text { ANL } & T \\
\text { CCP } & R \\
\text { CCP } & R \\
\text { CCP } & R \\
\text { CCP } & R\end{array}$ & $\begin{array}{l}\text { Plasil. } \\
\text { Plasil. } \\
\text { WIIkinst } \\
\text { Loovt } \\
\text { Lbovt } \\
\text { Lbovt } \\
\text { L Loovt }\end{array}$ & $\begin{array}{l}11442 \\
8644 \\
4770 \\
8995 \\
4849 \\
4898 \\
5971\end{array}$ \\
\hline$A=75$ & \multicolumn{6}{|c|}{ fisgion yiold } \\
\hline $\begin{array}{l}{ }^{209} B 1 \\
A=75\end{array}$ & $\begin{array}{c}\text { P } \\
\text { overo }\end{array}$ & $\begin{array}{l}3.6+1= \\
g 0 \text { o(E) }\end{array}$ & $5 \cdot 6+1$ & PAR T & Do-silvelra. & 4759 \\
\hline $\begin{array}{l}238 \mathrm{U} \\
A=75\end{array}$ & $\begin{array}{l}40 \mathrm{Ar} \\
\text { relot }\end{array}$ & $\begin{array}{l}2.4+2 \\
\text { IVo olE }\end{array}$ & & YAL $R$ & Bromley. & 12411 \\
\hline $\begin{array}{l}258 \mathrm{Ro} \\
23 \theta_{\mathrm{U}} \\
237 \mathrm{~Np} \\
242 \mathrm{Pu}\end{array}$ & $\begin{array}{l}p \\
0 \\
0 \\
0\end{array}$ & $\begin{array}{l}1 \cdot 2+1 \\
2 \cdot 4+1 \\
3 \cdot 6+1 \\
2.6+1\end{array}$ & $\begin{array}{l}3.6+1 \\
3.1+1\end{array}$ & $\begin{array}{ll}\text { WZI } & E \\
F E I & E \\
F E] & E \\
F E I & E\end{array}$ & $\begin{array}{l}\text { Gayert } \\
\text { Dmitrlevt } \\
\text { Omitrievt } \\
\text { Omitrievt }\end{array}$ & $\begin{array}{l}4768 \\
9048 \\
9090 \\
9114\end{array}$ \\
\hline$A=75$ & \multicolumn{6}{|l|}{$Q(E)$} \\
\hline $\begin{array}{l}526 \mathrm{Ra} \\
226 \mathrm{Ro} \\
23 \theta_{\mathrm{U}}\end{array}$ & $\begin{array}{c}{ }^{1} \\
3_{\mathrm{He}} \\
12_{\mathrm{C}}\end{array}$ & $\begin{array}{l}2.3+1 \\
\text { NDG } \\
\text { NDG }\end{array}$ & & $\begin{array}{l}\text { LAS E } \\
\text { GER R } \\
\text { DUB R }\end{array}$ & $\begin{array}{l}\text { Webert } \\
\text { Mosel. } \\
\text { Flerov. }\end{array}$ & $\begin{array}{r}6068 \\
6577 \\
10157\end{array}$ \\
\hline$A=76$ & \multicolumn{6}{|c|}{ primory flasion yield } \\
\hline${ }^{209} \mathrm{Bi}$ & p & $3.2+1$ & & TUE $\mathbf{R}$ & Q Gonnenveint & 4761 \\
\hline$A=76$ & \multicolumn{6}{|c|}{ cumulative fisgion yield } \\
\hline $\begin{array}{l}170 \mathrm{Er} \\
182 \mathrm{~W} \\
226 \mathrm{Ro} \\
233 \mathrm{U} \\
239 \mathrm{Pu}\end{array}$ & $\begin{array}{r}160 \\
0 \\
p \\
0 \\
0\end{array}$ & $\begin{array}{l}1.7+2 \\
1.2+2 \\
1.3+1 \\
2.5+1 \\
9.2+0\end{array}$ & $\begin{array}{c}. \\
4.1+1 \\
2.3+1\end{array}$ & $\begin{array}{ll}\text { ORL } & R \\
\text { ORL } & R \\
\text { ANL } & T \\
\text { CCP } & R \\
\text { CCP } & R\end{array}$ & $\begin{array}{l}\text { Plosil. } \\
\text { Plosil. } \\
\text { Wlikinst } \\
\text { Lbovt } \\
\text { Lbovt }\end{array}$ & $\begin{array}{l}11442 \\
8644 \\
4770 \\
8995 \\
5971\end{array}$ \\
\hline$A=76$ & \multicolumn{6}{|c|}{ fisglon yield } \\
\hline $209_{B i}$ & p & $3.6+1=$ & $5.6+1$ & PAR $T$ & Do-silvelra. & 4759 \\
\hline
\end{tabular}




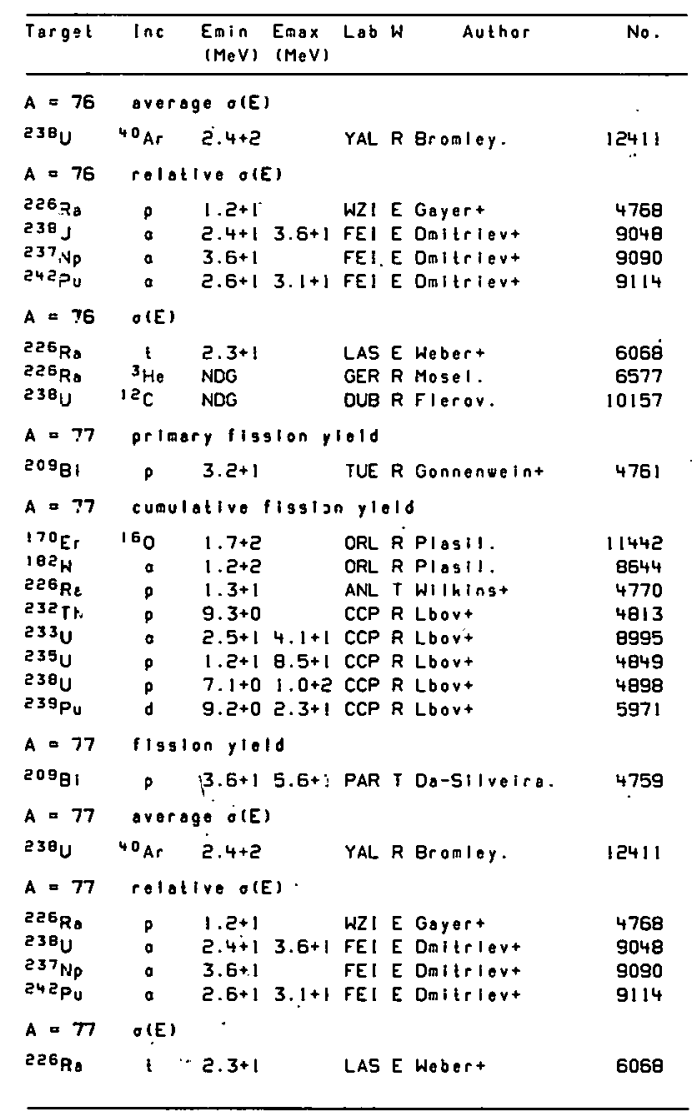

\begin{tabular}{|c|c|c|c|c|c|c|}
\hline Target & $\ln c$ & $\begin{array}{l}\text { Emin } \\
(\mathrm{MeV})\end{array}$ & $\begin{array}{l}\text { Emax } \\
\text { (MeV) }\end{array}$ & Lab & Author & No. \\
\hline$A=77$ & $O(E)$. & & & & & \\
\hline $226 \mathrm{Ra}$ & ${ }^{3} \mathrm{He}$ & NDG & & GER & R Mosel. & 6577 \\
\hline$A=78$ & \multicolumn{6}{|c|}{ primary fission yield } \\
\hline${ }^{209} \mathrm{Bi}$ & p & $3 \cdot 2+1$ & & TUE & R Gonnenweint & $476 !$ \\
\hline$A=78$ & \multicolumn{6}{|c|}{ cumulative fission yielo } \\
\hline $\begin{array}{l}170 \mathrm{Er} \\
102 \mathrm{~W} \\
226 \mathrm{Rs} \\
232 \mathrm{Th} \\
233 \mathrm{U} \\
233 \mathrm{U} \\
239 \mathrm{Pu}\end{array}$ & $\begin{array}{r}160 \\
0 \\
p \\
p \\
d \\
\alpha \\
d\end{array}$ & $\begin{array}{l}1.7+2 \\
1.2+2 \\
1.3+1 . \\
9.3+0 \\
6.0+0 \\
2.5+1 \\
9.2+0\end{array}$ & $\begin{array}{l}9.0+0 \\
4.1+1 \\
2.3+1\end{array}$ & $\begin{array}{l}\text { ORL } \\
\text { ORL } \\
\text { ANL } \\
\text { CCP } \\
\text { BRC } \\
\text { CCP } \\
\text { CCP }\end{array}$ & $\begin{array}{l}\text { R Plasil. } \\
\text { R Plasil. } \\
\text { T Wlluinst } \\
\text { R Lbovt } \\
\text { E Clerjochst } \\
\text { R Lbovt } \\
\text { R Lbovt }\end{array}$ & $\begin{array}{r}11442 \\
8644 \\
4770 \\
4813 \\
5935 \\
8995 \\
5971\end{array}$ \\
\hline$A=78$ & \multicolumn{6}{|c|}{ fisgion yield } \\
\hline $\begin{array}{l}209 B i \\
A=78\end{array}$ & $\begin{array}{c}\text { P } \\
\text { overo }\end{array}$ & $\begin{array}{l}3.6+1 \\
g \circ O(E)\end{array}$ & $5.6+1$ & PAR & T Da-silveira. & 4759 \\
\hline 2384 & ${ }^{40} \mathrm{Ar}$ & $2.4+2$ & & YAL & R Bromley. & 12411 \\
\hline$A=78$ & \multicolumn{6}{|c|}{ relative o(E) } \\
\hline $\begin{array}{l}226 \mathrm{Ra} \\
238_{\mathrm{U}} \\
237 \mathrm{~Np} \\
242 \mathrm{Pu}\end{array}$ & $\begin{array}{l}0 \\
\vdots \\
0\end{array}$ & $\begin{array}{l}1.2+1 \\
2.4+1 \\
3.6+1 \\
2.6+1\end{array}$ & $\begin{array}{l}3.6+1 \\
3.1+1\end{array}$ & $\begin{array}{l}\text { WZI } \\
\text { FEI } \\
\text { FEI } \\
\text { FEI }\end{array}$ & $\begin{array}{l}\text { E Gayert } \\
\text { E Omitrievt } \\
\text { E Dmitrievt } \\
\text { E Omitrievt }\end{array}$ & $\begin{array}{l}4768 \\
9048 \\
9090 \\
9114\end{array}$ \\
\hline$A=78$ & \multicolumn{6}{|l|}{$\sigma(E)$} \\
\hline $\begin{array}{l}226 \mathrm{Ra} \\
226 \mathrm{Ra} \\
238_{\mathrm{U}}\end{array}$ & $\begin{array}{c}{ }^{k} \\
3_{\mathrm{He}} \\
1 \mathrm{a}_{\mathrm{C}}\end{array}$ & $\begin{array}{l}2.3+1 \\
\text { NDG } \\
\text { NDG }\end{array}$ & & $\begin{array}{l}\text { LAS } \\
\text { GER } \\
\text { DUB }\end{array}$ & $\begin{array}{l}\text { E Webert } \\
R \text { Mogel. } \\
\text { R Flerov. }\end{array}$ & $\begin{array}{r}6068 \\
6577 \\
10157\end{array}$ \\
\hline$A=79$ & \multicolumn{6}{|c|}{ primary fission ylolo } \\
\hline $\begin{array}{l}{ }_{209} 09 \mathrm{gi} \\
226 \mathrm{Ra} \\
233 \mathrm{U}\end{array}$ & $\begin{array}{l}p \\
p \\
p\end{array}$ & $\begin{array}{l}3.2+1 \\
1.3+1 \\
1.3+1\end{array}$ & & $\begin{array}{l}\text { TUE } \\
\text { TUE } \\
\text { BRC }\end{array}$ & $\begin{array}{l}\text { R Gosnenveint } \\
R \text { Gonnenveint } \\
\text { E Patint }\end{array}$ & $\begin{array}{l}4761 \\
4769 \\
5933\end{array}$ \\
\hline$A=79$. & \multicolumn{6}{|c|}{ cumulative fission yield } \\
\hline $\begin{array}{l}170 \mathrm{Er} \\
182 \mathrm{~W} \\
200^{\circ}\end{array}$ & $\begin{array}{c}160 \\
0 \\
0\end{array}$ & $\begin{array}{l}1.7+2 \\
1.2+2 \\
\text { NDG }\end{array}$ & & $\begin{array}{l}\text { ORL } \\
\text { ORL } \\
\text { TNL }\end{array}$ & $\begin{array}{l}R \text { Plesil. } \\
R \text { Plesil. } \\
R \text { Newson. }\end{array}$ & $\begin{array}{r}11442 \\
8544 \\
4760\end{array}$ \\
\hline
\end{tabular}




\begin{tabular}{|c|c|c|c|c|c|c|}
\hline Target & Inc & $\begin{array}{l}\text { Emin } \\
(\operatorname{MeV})\end{array}$ & $\begin{array}{l}\text { Emax } \\
(\operatorname{MeV})\end{array}$ & Lab W & Author & No. \\
\hline$A=79$ & \multicolumn{6}{|c|}{ cumulative fission yelo } \\
\hline $\begin{array}{l}256_{R A} \\
235 T h \\
233_{U} \\
233 U \\
235 U \\
23 \theta_{U} \\
23 \theta_{U} \\
230^{2}\end{array}$ & $\begin{array}{l}p \\
p \\
d \\
a \\
p \\
p \\
p \\
d\end{array}$ & $\begin{array}{l}1 \cdot 3+1 \\
9 \cdot 3+0 \\
6 \cdot 0+0 \\
2 \cdot 5+1 \\
1 \cdot 2+1 \\
7 \cdot 1+0 \\
1 \cdot 2+1 \\
9 \cdot 2+0\end{array}$ & $\begin{array}{l}9.0+0 \\
4.1+1 \\
8.5+1 \\
1.0+2 \\
2.2+1 \\
2.3+1\end{array}$ & $\begin{array}{l}\text { ANL T } \\
\text { CCP R } \\
\text { BRC E } \\
\text { CCP R } \\
\text { CCP R } \\
\text { CCP R } \\
\text { TUE R } \\
\text { CCP R }\end{array}$ & $\begin{array}{l}T \text { Wliblinst } \\
R \text { Lbovt } \\
\text { E Clerjachst } \\
R \text { Lbovt } \\
R \text { Lbovt } \\
R \text { Lbovt } \\
R \text { Gonnenweint } \\
R \text { Lbovt }\end{array}$ & $\begin{array}{l}4770 \\
4813 \\
5935 \\
8995 \\
4849 \\
4898 \\
4899 \\
597 !\end{array}$ \\
\hline$A=79$ & \multicolumn{6}{|c|}{ fission yield } \\
\hline $\begin{array}{l}{ }^{209} \mathrm{Bi} \\
A=79\end{array}$ & \multicolumn{5}{|c|}{ average $O(E)$} & 4759 \\
\hline $23 \theta^{4}$ & $40_{A r}$ & $2.4+2$ & & YAL $R$ & R Bromloy. & 12411 \\
\hline$A=79$ & \multicolumn{6}{|c|}{ relotive $\sigma(E)$} \\
\hline $\begin{array}{l}226 \mathrm{Rg} \\
238_{\mathrm{U}} \\
237 \mathrm{~Np} \\
242 \mathrm{Pu}\end{array}$ & $\begin{array}{l}p \\
a \\
a \\
a\end{array}$ & $\begin{array}{l}1.2+1 \\
2.4+1 \\
3.6+1 \\
2.6+1\end{array}$ & $3.6+1$ & $\begin{array}{ll}\text { WZI } & E \\
\text { FEI } & E \\
\text { FEI } & E \\
\text { FEI E }\end{array}$ & $\begin{array}{l}\text { E Gayert } \\
\text { E Dalirient } \\
\text { E Dolirleyt } \\
\text { E Dolitrleyt }\end{array}$ & $\begin{array}{l}4768 \\
9048 \\
9090 \\
9114\end{array}$ \\
\hline$A=79$ & \multicolumn{6}{|l|}{$\sigma(E)$} \\
\hline $\begin{array}{l}256 R_{8} \\
256_{B} \\
238_{U}\end{array}$ & $\begin{array}{c}{ }^{t} \\
{ }^{3}{ }^{2} \mathrm{Ce}\end{array}$ & $\begin{array}{l}2.3+1 \\
\text { NDG } \\
\text { NDG }\end{array}$ & & $\begin{array}{l}\text { LAS E } \\
\text { GER R } \\
\text { DUB R }\end{array}$ & $\begin{array}{l}\text { E Webert } \\
\text { R Mosel. } \\
\text { R Flerov. }\end{array}$ & $\begin{array}{r}6068 \\
6577 \\
10157\end{array}$ \\
\hline$A=80$ & \multicolumn{6}{|c|}{ priary fission yiold } \\
\hline $\begin{array}{l}\log _{\mathrm{Bl}} \\
226 \mathrm{RB} \\
233_{\mathrm{U}}\end{array}$ & $\begin{array}{l}p \\
p \\
d\end{array}$ & $\begin{array}{l}3 \cdot 2+1 \\
1 \cdot 3+1 \\
1 \cdot 3+1\end{array}$ & & $\begin{array}{l}\text { TUE }{ }^{R} \\
\text { TUE }{ }^{\circ} \\
\text { ERC E }\end{array}$ & $\begin{array}{l}\text { R Gonnenweint } \\
\text { R Gonnonweint } \\
\text { E Patint }\end{array}$ & $\begin{array}{l}476 ! \\
4769 \\
5933\end{array}$ \\
\hline$A=00$ & \multicolumn{6}{|c|}{ cumulative fission yield } \\
\hline $\begin{array}{l}170 \mathrm{ER} \\
18 \mathrm{E}_{\mathrm{W}} \\
209_{\mathrm{BI}} \\
226 \mathrm{Ra} \\
232 \mathrm{Th} \\
233_{\mathrm{U}} \\
233_{\mathrm{U}} \\
238_{\mathrm{U}}\end{array}$ & $\begin{array}{r}160 \\
\mathbf{a} \\
p \\
p \\
p \\
d \\
\mathbf{p} \\
p\end{array}$ & $\begin{array}{l}1.7+2 \\
1.2+2 \\
N J G \\
1.3+1 \\
9.3+0 \\
6.0+0 \\
2.5+1 \\
1.2+1\end{array}$ & $\begin{array}{l} \\
9.0+0 \\
4.1+1 \\
2.2+1\end{array}$ & $\begin{array}{ll}\text { ORL } & R \\
\text { ORL } & R \\
\text { TNL } & R \\
\text { ANL } & T \\
\text { CCP } & R \\
\text { ERC } & E \\
\text { CCP } & R \\
\text { TUE } & R\end{array}$ & $\begin{array}{l}\text { R Plasil. } \\
R \text { Plasil. } \\
R \text { Newson. } \\
T \text { Wilkinst } \\
R \text { Loovt } \\
\text { E Clerjackst } \\
R \text { Lbovt } \\
\text { R Gonnenweint }\end{array}$ & $\begin{array}{r}11442 \\
8644 \\
4760 \\
4770 \\
4813 \\
5935 \\
8995 \\
4899\end{array}$ \\
\hline
\end{tabular}

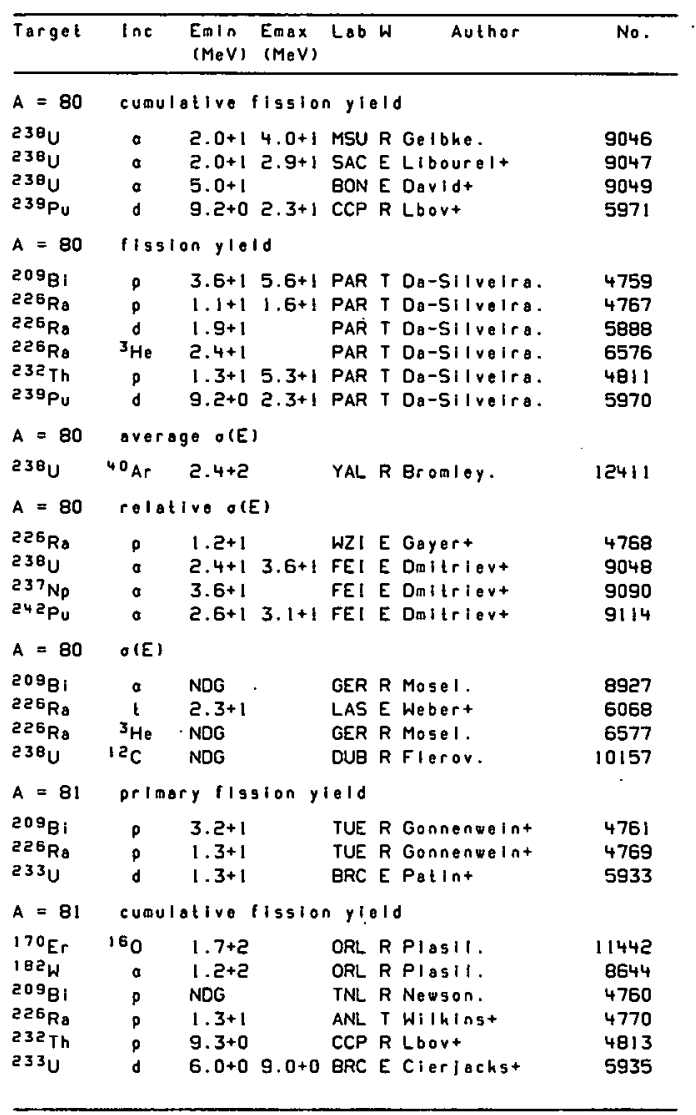




\begin{tabular}{|c|c|c|c|c|c|c|c|}
\hline Target & $\ln c$ & $\begin{array}{l}\text { Emin } \\
(M e V)\end{array}$ & $\begin{array}{l}\text { Emax } \\
\text { IMeV: }\end{array}$ & Lab & $w$ & Author & No. \\
\hline$A=81$ & cumu & ot tive $f$ & fission & n yle & eld & . & \\
\hline 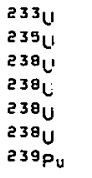 & $\begin{array}{l}a \\
p \\
p \\
p \\
a \\
a \\
d\end{array}$ & $\begin{array}{l}2 \cdot 5+1 \\
1.2+1 \\
7 \cdot 1+0 \\
1.2+1 \\
2 \cdot 0+1 \\
2.0+1 \\
9.2+0\end{array}$ & $\begin{array}{l}4.1+1 \\
8.5+1 \\
1.0+2 \\
2.2+1 \\
4 \cdot 0+1 \\
2.9+1 \\
2 \cdot 3+1\end{array}$ & $\begin{array}{l}\text { CCP } \\
\text { CCP } \\
\text { CCP } \\
\text { TUE } \\
\text { MSU } \\
\text { SAC } \\
\text { CCP }\end{array}$ & $\begin{array}{l}R \\
R \\
R \\
R \\
R \\
E \\
R\end{array}$ & $\begin{array}{l}\text { Lbovt } \\
\text { Lbovt } \\
\text { Lbovt } \\
\text { Gonnenweint } \\
\text { Gelbke. } \\
\text { Libourelt } \\
\text { Lbovt }\end{array}$ & $\begin{array}{r}8995 \\
4849 \\
4898 \\
4899 \\
: 9046 \\
9047 \\
5971\end{array}$ \\
\hline$A=81$ & flos & on $y \mid 01$ & & & & & \\
\hline $\begin{array}{l}209 \mathrm{Bi} \\
226 \mathrm{RB} \\
226 \mathrm{Rg} \\
22 \mathrm{RB}_{\mathrm{Ra}} \\
232 \mathrm{Ti} \\
239 \mathrm{Pu}\end{array}$ & $\begin{array}{c}p \\
p \\
d \\
{ }^{3} \mathrm{He} \\
p \\
d\end{array}$ & $\begin{array}{l}3.6+1 \\
1.1+1 \\
1.9+1 \\
2.4+1 \\
1.3+1 \\
9.2+0\end{array}$ & $\begin{array}{l}5.6+1 \\
1.6+1 \\
\\
5.3+1 \\
2.3+1\end{array}$ & $\begin{array}{l}\text { PAR } \\
\text { PAR } \\
\text { PAR } \\
\text { PAR } \\
\text { PAR } \\
\text { PAR }\end{array}$ & $\begin{array}{l}T \\
T \\
T \\
T \\
T \\
T\end{array}$ & $\begin{array}{l}\text { Da-Silveira. } \\
\text { Da-Sitveira. } \\
\text { Da-SIlveira. } \\
\text { Da-Silveira. } \\
\text { Do-Silveira. } \\
\text { Do-Silveira. }\end{array}$ & $\begin{array}{l}4759 \\
4767 \\
5888 \\
6576 \\
4811 \\
5970\end{array}$ \\
\hline$A=81$ & over & $g \circ . O(E)$ & & & & & \\
\hline $\begin{array}{l}238_{U} \\
A=81\end{array}$ & $\begin{array}{l}40 \mathrm{Ar} \\
\mathrm{rOl}\end{array}$ & $\begin{array}{l}2.4+2 \\
\text { Ive olE }\end{array}$ & & YAL & $R$ & Bromley. & $\{2411$ \\
\hline $\begin{array}{l}226 \mathrm{Rg} \\
23 \theta_{\mathrm{U}} \\
237 \mathrm{Ng} \\
24{ }^{2} \mathrm{Pu}\end{array}$ & $\begin{array}{l}p \\
a \\
0 \\
a\end{array}$ & $\begin{array}{l}1.2+1 \\
2.4+1 \\
3.6+1 \\
2.6+1\end{array}$ & $\begin{array}{l}3.6+1 \\
3.1+1\end{array}$ & $\begin{array}{l}\text { WZI } \\
\text { FEI } \\
\text { FEI } \\
\text { FEI }\end{array}$ & & $\begin{array}{l}\text { Gayert } \\
\text { Dmilrievt } \\
\text { Dmilitievt } \\
\text { Dmilrlevt }\end{array}$ & $\begin{array}{l}4768 \\
9048 \\
9090 \\
9114\end{array}$ \\
\hline$A=81$ & $\theta(E)$ & . & & & & & \\
\hline $\begin{array}{l}20 \theta_{11} \\
226_{R o} \\
226_{R o} \\
23 \theta_{U}\end{array}$ & $\begin{array}{c}a \\
b \\
{ }^{3} \mathrm{He} \\
{ }^{12} \mathrm{C}\end{array}$ & $\begin{array}{l}\text { NDG } \\
2.3+1 \\
\text { NDG } \\
\text { NDG }\end{array}$ & & $\begin{array}{l}\text { GER } \\
\text { LAS } \\
\text { GER } \\
\text { DUB }\end{array}$ & $\begin{array}{l}R \\
E \\
R \\
R\end{array}$ & $\begin{array}{l}\text { Mosel. } \\
\text { Webert } \\
\text { Mosel. } \\
\text { Flerov. }\end{array}$ & $\begin{array}{r}8927 \\
6068 \\
6577 \\
10157\end{array}$ \\
\hline$A=82$ & prim & ry fiss & ston $y 1$ & leld & . & . & \\
\hline $\begin{array}{l}209_{B i} \\
256 R_{0} \\
233 \mathrm{U}\end{array}$ & $\begin{array}{l}p \\
p \\
d\end{array}$ & $\begin{array}{l}3 \cdot 2+1 \\
1 \cdot 3+1 \\
1.3+1\end{array}$ & & $\begin{array}{l}\text { TUE } \\
\text { TUE } \\
\text { BRC }\end{array}$ & $\begin{array}{l}R \\
R \\
E\end{array}$ & $\begin{array}{l}\text { Gonnenweint } \\
\text { Gonnenweint } \\
\text { Patlint }\end{array}$ & $\begin{array}{l}4761 \\
4769 \\
5933\end{array}$ \\
\hline$A=82$ & cunu & ative & fission & in yio & & & \\
\hline $\begin{array}{l}170 E_{r} \\
10 e_{W} \\
209_{B I}\end{array}$ & $\begin{array}{r}160 \\
0 \\
p\end{array}$ & $\begin{array}{l}1.7+2 \\
1.2+2 \\
\text { NDG }\end{array}$ & & $\begin{array}{l}\text { ORL } \\
\text { ORL } \\
\text { iNL }\end{array}$ & $\begin{array}{l}R \\
R \\
R\end{array}$ & $\begin{array}{l}\text { Plosil. } \\
\text { Plasil. } \\
\text { Newson. }\end{array}$ & $\begin{array}{r}11442 \\
8644 \\
4760\end{array}$ \\
\hline
\end{tabular}

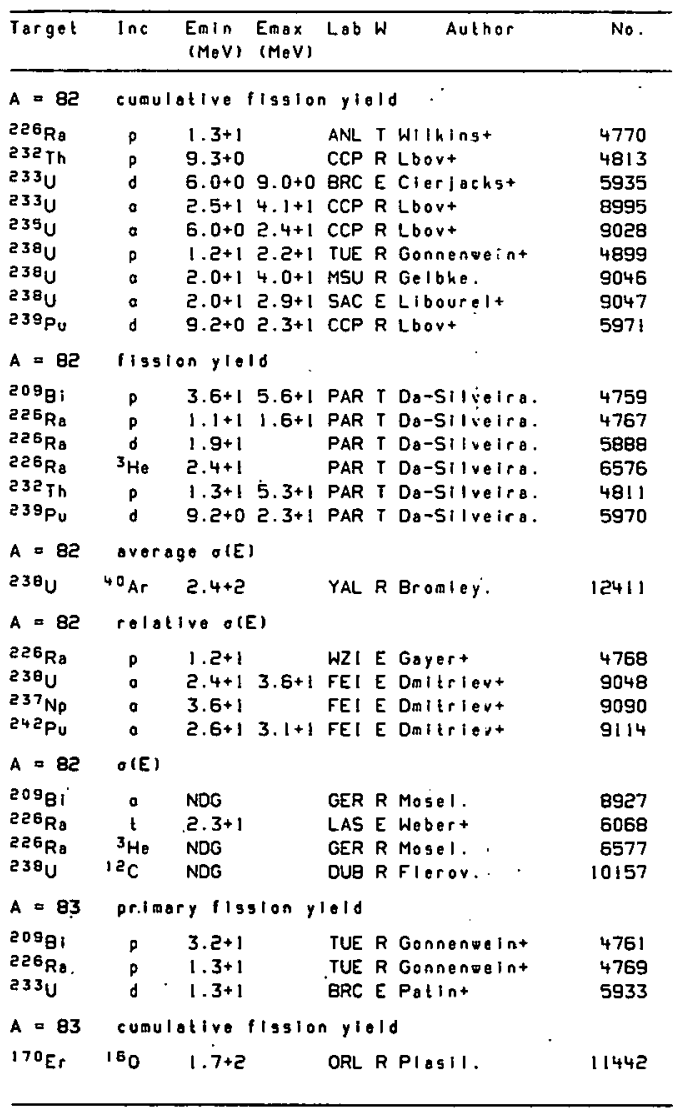




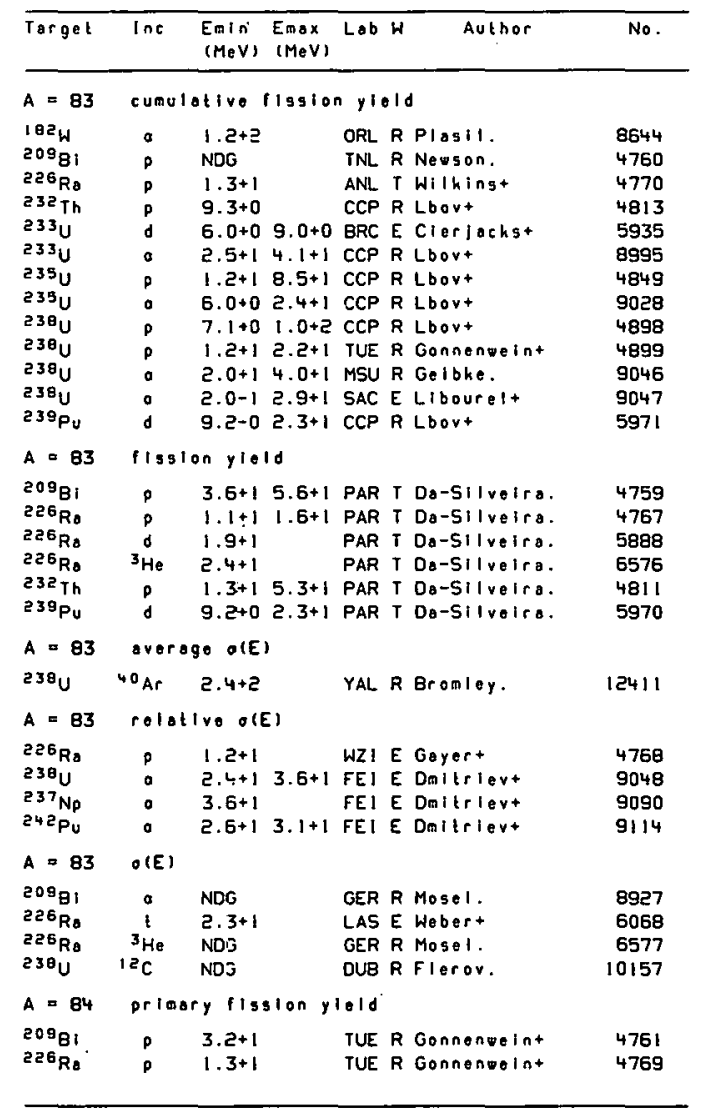

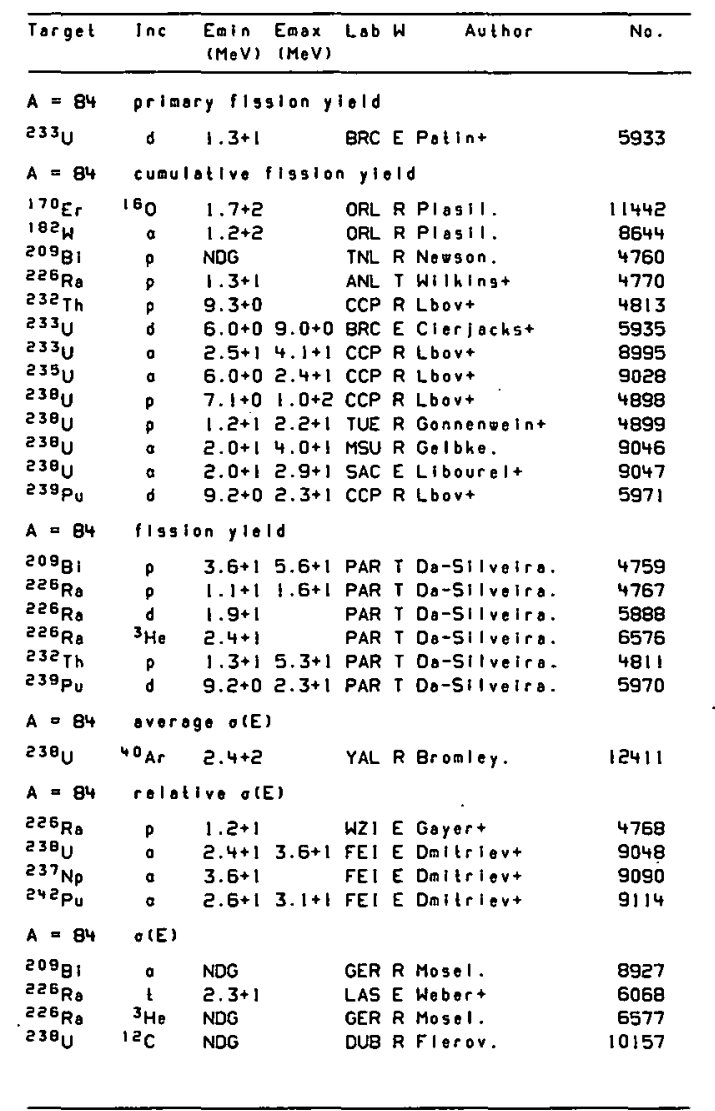




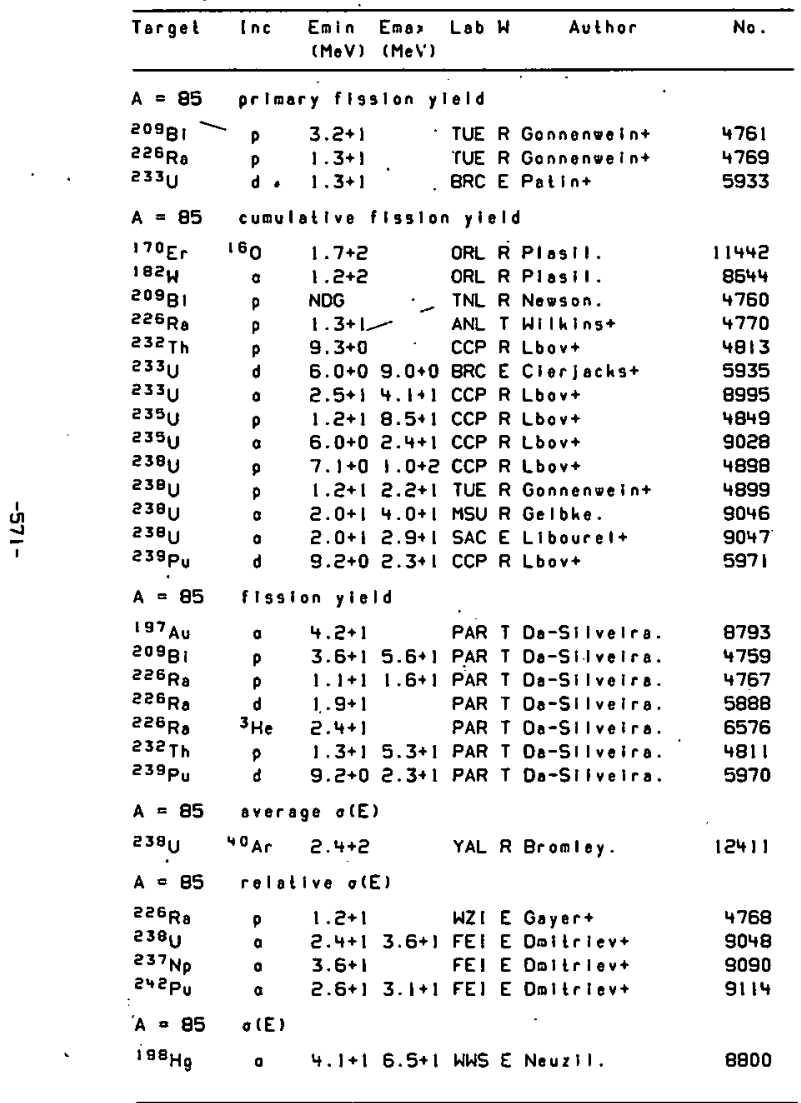

\begin{tabular}{|c|c|c|c|c|c|c|}
\hline Target & $\operatorname{lnc}$ & $\begin{array}{l}\text { Emin } \\
\text { (Mev) }\end{array}$ & $\begin{array}{l}\text { EqBX } \\
(\mathrm{MeV})\end{array}$ & Lab H & Ajthor & No. \\
\hline$A=85$ & $\theta(E)$ & & & & & \\
\hline $\begin{array}{l}199 \mathrm{Hg} \\
200 \mathrm{Hg} \\
201 \mathrm{Hg} \\
202 \mathrm{Hg} \\
204 \mathrm{Hg} \\
209 \mathrm{Hi}^{\circ} \\
226 \mathrm{Ra} \\
226 \mathrm{Ra}^{2} \\
230_{\mathrm{U}} \\
230_{\mathrm{U}}\end{array}$ & $\begin{array}{c}a \\
a \\
a \\
a \\
0 \\
0 \\
\vdots \\
3^{3} \mathrm{He} \\
12 \mathrm{C} \\
12 \mathrm{C}\end{array}$ & $\begin{array}{l}4.1+1 \\
4.1+1 \\
4.1+1 \\
4.1+1 \\
4.1+1 \\
\text { NDG } \\
2.3+1 \\
\text { NDG } \\
\text { NDG } \\
\text { NDG }\end{array}$ & $\begin{array}{r}6.5+1 \\
\cdot \\
5 \\
5\end{array}$ & $\begin{array}{l}\text { WWS E } \\
\text { WWS E } \\
\text { WWS E } \\
\text { WWS E } \\
\text { WWS E } \\
\text { GER R } \\
\text { LAS E } \\
\text { GER R } \\
\text { OUB R } \\
\text { OUB R }\end{array}$ & $\begin{array}{l}\text { E Neuzil. } \\
\text { E Neuzil. } \\
\text { E Neuzil. } \\
\text { E Neuzil. } \\
\text { E Neuzil. } \\
\text { R Mosel. } \\
\text { E Webert } \\
\text { R Mosel. } \\
\text { R Flerov. } \\
\text { R Flerov. }\end{array}$ & $\begin{array}{r}8805 \\
8810 \\
8814 \\
8821 \\
8828 \\
8927 \\
6068 \\
6577 \\
10157 \\
10157\end{array}$ \\
\hline$A=86$ & pritas & ry fiss & sion yie & iold & & 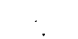 \\
\hline $\begin{array}{l}209_{\mathrm{gi}} \\
226_{\mathrm{Ra}} \\
233_{\mathrm{U}} \mathrm{U}\end{array}$ & $\begin{array}{l}p \\
p \\
d\end{array}$ & $\begin{array}{l}3.2+1 \\
1.3+1 \\
1.3+1\end{array}$ & & $\begin{array}{l}\text { TUE R } \\
\text { TUE R } \\
\text { BRC E }\end{array}$ & $\begin{array}{l}\text { R Gonnenvelnt } \\
\text { R Gonnenwelnt } \\
\text { E Patint }\end{array}$ & $\begin{array}{l}4761 \\
4769 \\
5933\end{array}$ \\
\hline$A=86$ & cumul & allve f & fission & 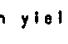 & 10 & \\
\hline 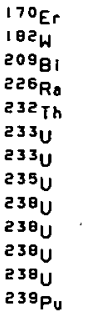 & $\begin{array}{c}160 \\
0 \\
p \\
0 \\
0 \\
0 \\
0 \\
0 \\
0 \\
0 \\
0 \\
.0 \\
0\end{array}$ & $\begin{array}{l}1.7+2 \\
1.2+2 \\
N D G \\
1.3+1 \\
9.3+0 \\
6.0+0 \\
2.5+1 \\
6.0+0 \\
7.1+0 \\
1.2+1 \\
2.0+1 \\
2.0+1 \\
9.2+0\end{array}$ & $\begin{array}{l}9.0+0 \\
4.1+1 \\
2.4+1 \\
1.0+2 \\
2.2+1 \\
4.0+1 \\
2.9+1 \\
2.3+1\end{array}$ & 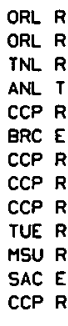 & $\begin{array}{l}\text { R Plasil. } \\
R \text { Plasil. } \\
R \text { Newson. } \\
\text { T Hilkins- } \\
R \text { Lbovt } \\
\text { E Clerjackst } \\
R \text { Lbovt } \\
R \text { Lbovt } \\
\text { R Lbovt } \\
\text { R Gonnenweint } \\
\text { R Gelbks. } \\
\text { E Liboute:t } \\
\text { R Lbovt }\end{array}$ & $\begin{array}{r}11442 \\
8644 \\
4760 \\
4770 \\
4813 \\
5935 \\
8995 \\
9028 \\
4898 \\
4899 \\
9046 \\
9047 \\
5971\end{array}$ \\
\hline$A=B E$ & flgsl & on yiel & & & ' & \\
\hline 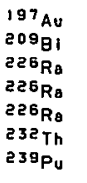 & $\begin{array}{c}a \\
p \\
p \\
0 \\
3^{\mathrm{He}} \\
p \\
d\end{array}$ & $\begin{array}{l}4.2+1 \\
3.6+1 \\
1.1+1 \\
1.9+1 \\
2.4+1 \\
1.3+1 \\
9.2+0\end{array}$ & $\begin{array}{l}5.6+1 \\
1.6+1 \\
5.3+1 \\
2.3+1\end{array}$ & $\begin{array}{l}\text { PAR T } \\
\text { PAR T } \\
\text { PAR T } \\
\text { PAR T } \\
\text { PAR T } \\
\text { PAR T } \\
\text { PAR T }\end{array}$ & 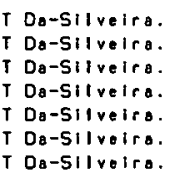 & $\begin{array}{l}8793 \\
4759 \\
4767 \\
5888 \\
6576 \\
4811 \\
5970\end{array}$ \\
\hline
\end{tabular}




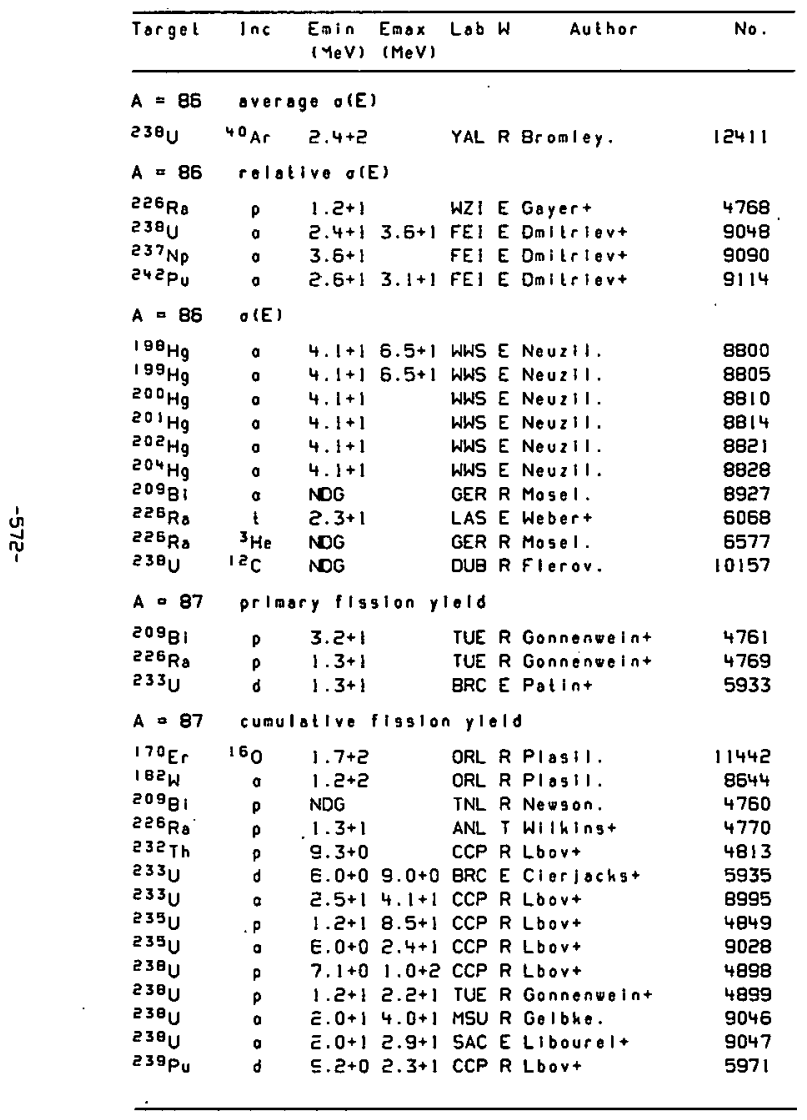

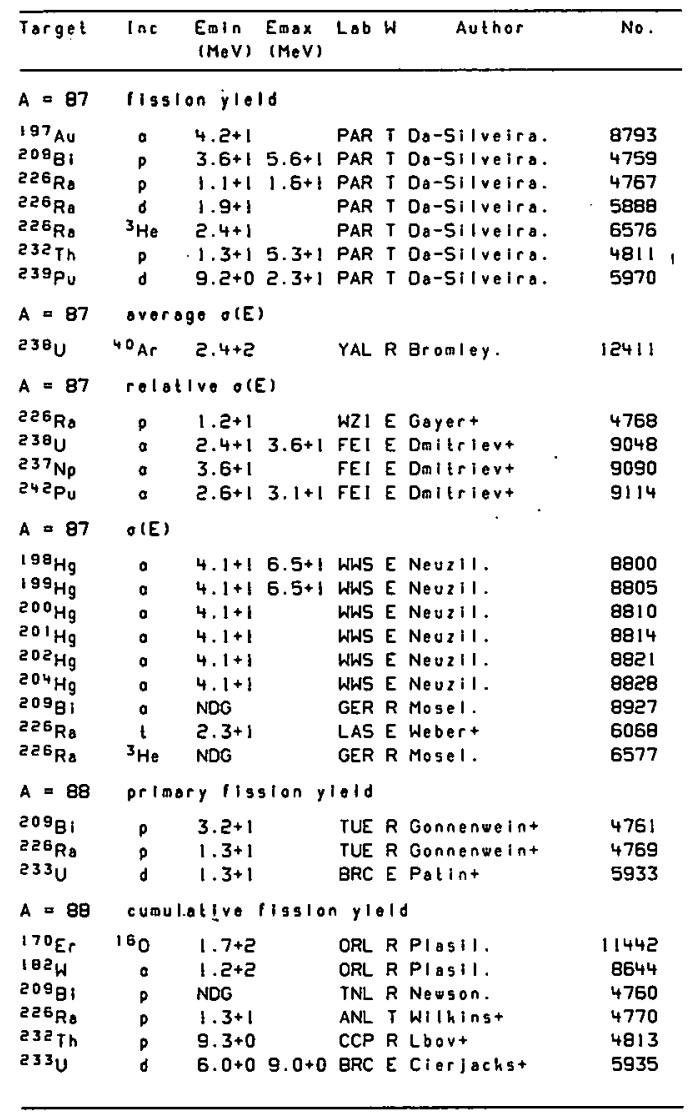




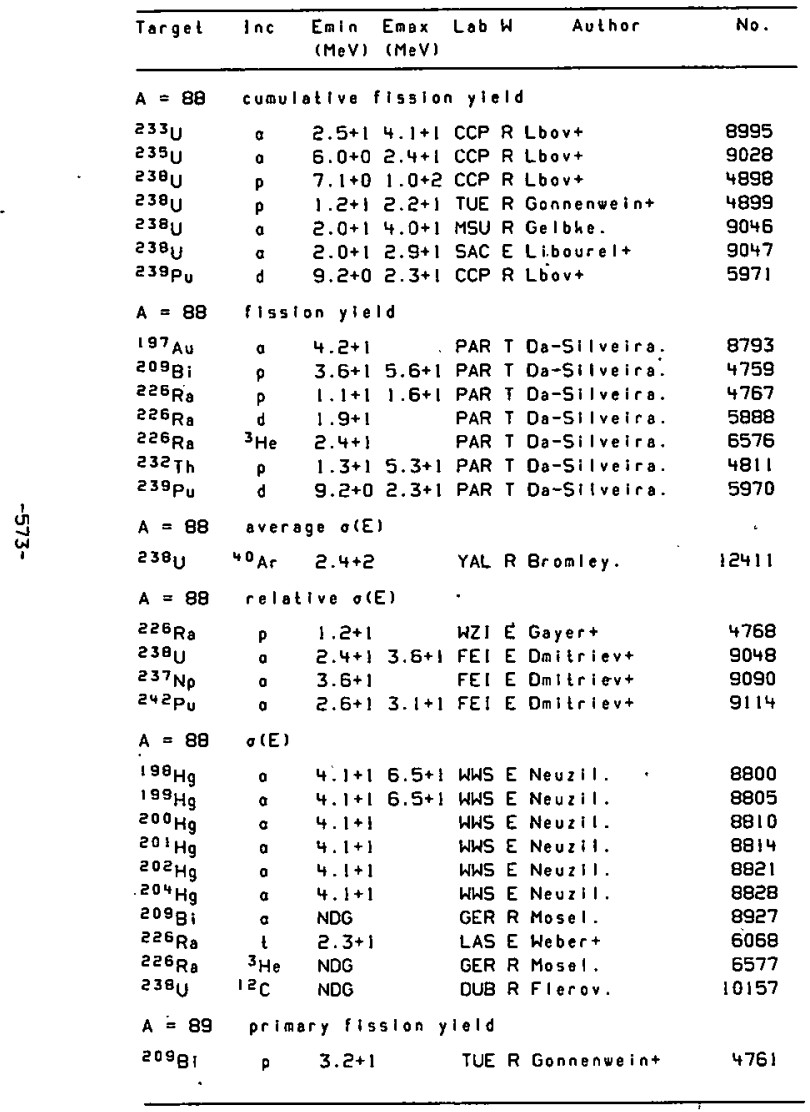

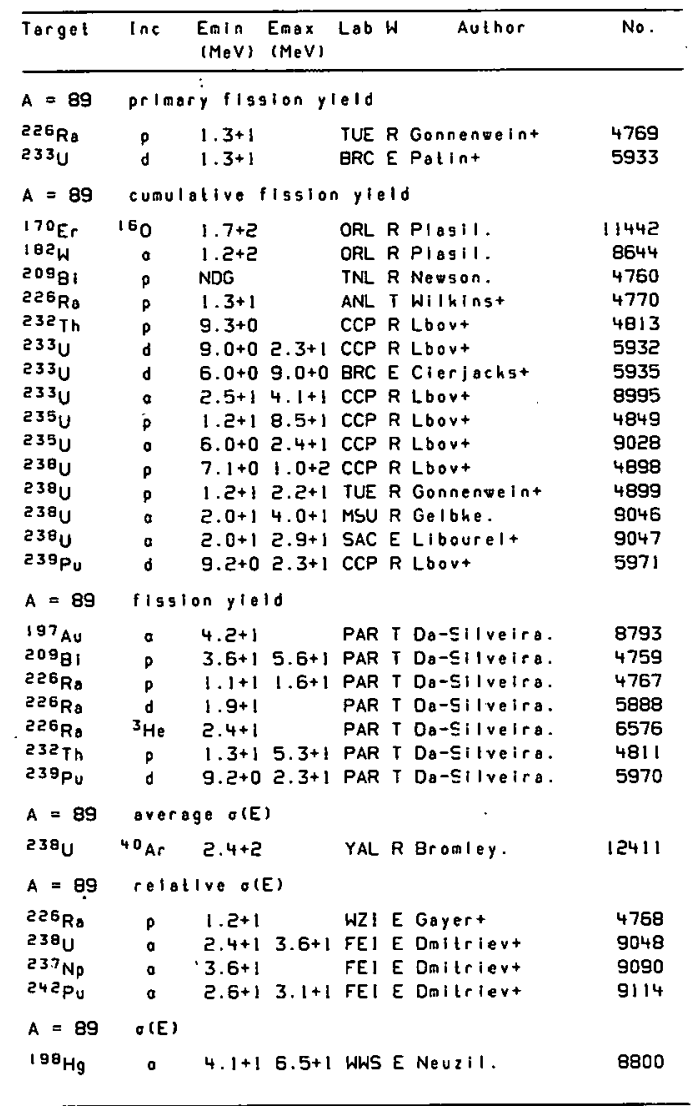




\begin{tabular}{|c|c|c|c|c|c|c|}
\hline Torget & $\ln c$ & $\begin{array}{l}\varepsilon_{m} \mid n \\
\left(M_{e} V\right)\end{array}$ & $\begin{array}{l}E_{\max } \\
(\operatorname{MeV})\end{array}$ & Lab & Author & No. \\
\hline$A=89$ & $O(E)$ & & & & & \\
\hline 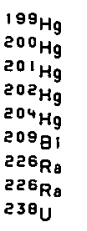 & $\begin{array}{c}a \\
a \\
a \\
a \\
a \\
a \\
t \\
3_{\mathrm{He}} \\
12_{\mathrm{C}}\end{array}$ & $\begin{array}{l}4.1+1 \\
4.1+1 \\
4.1+1 \\
4.1+1 \\
4.1+1 \\
\text { NDG } \\
2.3+1 \\
\text { NDG } \\
\text { NDG }\end{array}$ & $6.5+1$ & $\begin{array}{l}\text { WWS } \\
\text { WWS } \\
\text { WWS } \\
\text { WWS } \\
\text { WWS } \\
\text { GER } \\
\text { LAS } \\
\text { GER } \\
\text { OUB }\end{array}$ & $\begin{array}{l}\text { E Nouzil. } \\
\text { E Neuzil. } \\
\text { E Nouzil. } \\
\text { E Nouzil. } \\
\text { E Neuzil. } \\
R \text { Mosel. } \\
\text { E Webert } \\
R \text { Mosel. } \\
\text { R Flerov. }\end{array}$ & $\begin{array}{r}8805 \\
8810 \\
8814 \\
8821 \\
8828 \\
8927 \\
6068 \\
6577 \\
10157\end{array}$ \\
\hline$A=90$ & \multicolumn{6}{|c|}{ primary figsion yield } \\
\hline $\begin{array}{l}20981 \\
226 R_{B} \\
233 \mathrm{U}\end{array}$ & $\begin{array}{l}p \\
p \\
d\end{array}$ & $\begin{array}{l}3 \cdot 2+1 \\
1 \cdot 3+1 \\
1 \cdot 3+1\end{array}$ & & $\begin{array}{l}\text { TUE } \\
\text { TUE } \\
\text { BRC }\end{array}$ & $\begin{array}{l}\text { R Gonnenveint } \\
\text { R Gonnenweint } \\
\text { E Potint }\end{array}$ & $\begin{array}{l}4761 \\
4769 \\
5933\end{array}$ \\
\hline$A=90$ & \multicolumn{6}{|c|}{ cumulative fisgion yield } \\
\hline 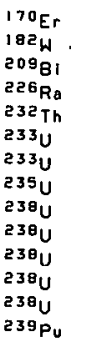 & $\begin{array}{r}160 \\
0 \\
p \\
p \\
p \\
d \\
q \\
q \\
p \\
p \\
o \\
q \\
q \\
d\end{array}$ & $\begin{array}{l}1.7+2 \\
1.2+2 \\
\text { NDG } \\
1.3+1 \\
9.3+0 \\
6.0+0 \\
2.5+1 \\
6.0+0 \\
7.1+0 \\
1.2+1 \\
2.0+1 \\
2.0+1 \\
5.0+1 \\
9.2+0\end{array}$ & $\begin{array}{l} \\
9.0+0 \\
4.1+1 \\
2.4+1 \\
1.0+2 \\
2.2+1 \\
4.0+1 \\
2.9+1 \\
2.3+1\end{array}$ & $\begin{array}{l}\text { ORL } \\
\text { ORL } \\
\text { TNL } \\
\text { ANL } \\
\text { CCP } \\
\text { BRC } \\
\text { CCP } \\
\text { CCP } \\
\text { CCP } \\
\text { TUE } \\
\text { MSU } \\
\text { SAC } \\
\text { BON } \\
\text { CCP }\end{array}$ & $\begin{array}{l}R \text { Piosil. } \\
R \text { Plosil. } \\
R \text { Newson. } \\
T \text { Wilkinst } \\
R \text { Lbovt } \\
\text { E Clerlachst } \\
R \text { Lbovt } \\
R \text { Lbovt } \\
R \text { Lbovt } \\
R \text { Gonnengelnt } \\
R \text { Gelbke. } \\
E \text { Lloourelt } \\
\text { E Davidt } \\
R \text { Lbovt }\end{array}$ & $\begin{array}{r}11442 \\
8644 \\
4760 \\
4770 \\
4813 \\
5935 \\
8995 \\
9028 \\
4898 \\
4899 \\
9046 \\
9047 \\
9049 \\
597 !\end{array}$ \\
\hline$A=90$ & \multicolumn{6}{|c|}{ fissten yield } \\
\hline $\begin{array}{l}197 \mathrm{Au} \\
209 \mathrm{BI} \\
226 \mathrm{Ra} \\
226 \mathrm{Ra} \\
225 \mathrm{Ra} \\
232 \mathrm{Th} \\
239 \mathrm{Pu}\end{array}$ & $\begin{array}{c}0 \\
p \\
0 \\
d \\
3^{\mathrm{He}} \\
p \\
0 \\
0\end{array}$ & $\begin{array}{l}4.2+1 \\
3 \cdot 6+1 \\
1.1+1 \\
1.9+1 \\
2 \cdot 4+1 \\
1.3+1 \\
9 \cdot 2+0\end{array}$ & $\begin{array}{l}5.6+1 \\
1.6+1 \\
5.3+1 \\
2.3+1\end{array}$ & $\begin{array}{l}\text { PAR } \\
\text { PAR } \\
\text { PAR } \\
\text { PAR } \\
\text { PAR } \\
\text { PAR } \\
\text { PAR }\end{array}$ & $\begin{array}{l}\text { T Da-Silveira. } \\
\text { T Da-Silvaira. } \\
\text { T Da-Silveira. } \\
\text { T Da-Silveira. } \\
\text { T Da-Silvaira. } \\
\text { T Da-Silvelra. } \\
\text { T Da-Silvelra. }\end{array}$ & $\begin{array}{l}8793 \\
4759 \\
4767 \\
5888 \\
6576 \\
4811 \\
5970\end{array}$ \\
\hline
\end{tabular}

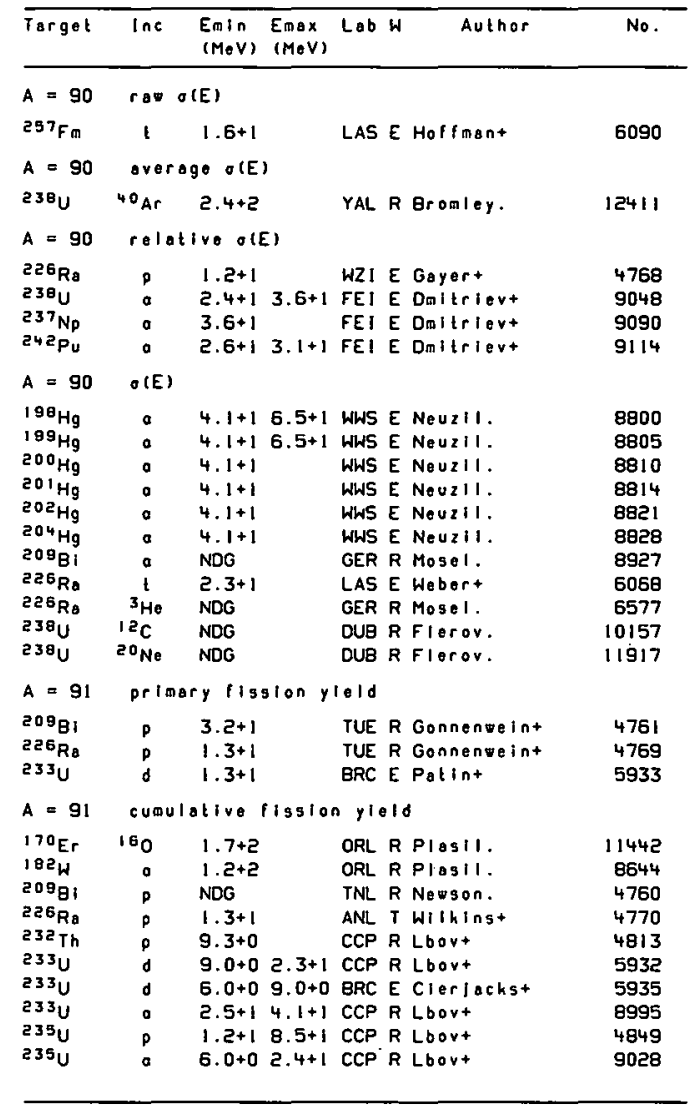




\begin{tabular}{|c|c|c|c|c|c|c|}
\hline Torget & Inc & $\begin{array}{l}\text { Emin } \\
(M e V)\end{array}$ & $\begin{array}{l}\text { Emax } \\
(\operatorname{MeV})\end{array}$ & Lob & Author & No. \\
\hline$A=91$ & \multicolumn{6}{|c|}{ cumulative fission yleld } \\
\hline $\begin{array}{l}238 \mathrm{U} \\
238 \mathrm{U} \\
238_{\mathrm{U}} \\
23 \mathrm{~B}_{\mathrm{U}} \\
23 \mathrm{Pu}^{\mathrm{Pu}}\end{array}$ & $\begin{array}{l}p \\
p \\
a \\
a \\
d\end{array}$ & 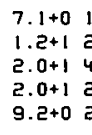 & $\begin{array}{l}1.0+2 \\
2.2+1 \\
4.0+1 \\
2.9+1 \\
2.3+1\end{array}$ & $\begin{array}{l}\text { CCP F } \\
\text { TUE F } \\
\text { MSU F } \\
\text { SAC E } \\
\text { CCP F }\end{array}$ & $\begin{array}{l}\text { R Lbovt } \\
R \text { Gonnenweint } \\
R \text { Gelbke. } \\
\text { E Libourelt } \\
R \text { Lbovt }\end{array}$ & $\begin{array}{l}4898 \\
4899 \\
9046 \\
9047 \\
5971\end{array}$ \\
\hline$A=91$ & \multicolumn{6}{|c|}{ fisston yleld } \\
\hline $\begin{array}{l}197 \mathrm{Au} \\
200 \mathrm{BI} \\
226 \mathrm{Ra} \\
226 \mathrm{Ra} \\
226 \mathrm{Ra} \\
232 \mathrm{Th} \\
239 \mathrm{Pu}\end{array}$ & $\begin{array}{c}a \\
p \\
\rho \\
d \\
y_{\mathrm{He}} \\
p \\
0 \\
0\end{array}$ & $\begin{array}{l}4 \cdot 2+1 \\
3 \cdot 6+15 \\
1.1+1 \quad 1 \\
1.9+1 \\
2.4+1 \\
1.3+15 \\
9 \cdot 2+0\end{array}$ & $\begin{array}{l}5.6+1 \\
1.6+1 \\
5.3+1 \\
2.3+1\end{array}$ & $\begin{array}{l}\text { PAR } \\
\text { PAR } \\
\text { PAR } \\
\text { PAR } \\
\text { PAR } \\
\text { PAR } \\
\text { PAR }\end{array}$ & $\begin{array}{l}\text { T Da-Silvelra. } \\
\text { T Da-Silveira. } \\
\text { T Da-Silveira. } \\
\text { T Da-Silvelra. } \\
\text { T Da-Silveira. } \\
\text { T Da-Silveira. } \\
\text { T Da-Silveira. }\end{array}$ & $\begin{array}{l}8793 \\
4759 \\
4767 \\
5888 \\
6576 \\
4811 \\
5970\end{array}$ \\
\hline$A=91$ & \multicolumn{6}{|c|}{ average $O(E)$} \\
\hline $\begin{array}{l}{ }^{230} U \\
A=91\end{array}$ & \multicolumn{3}{|c|}{ rolative o(E) } & \multicolumn{3}{|c|}{ 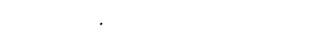 } \\
\hline $\begin{array}{l}226 \mathrm{Ra} \\
238^{8} \mathrm{U} \\
23{ }^{3} \mathrm{NP} \\
24{ }^{2} \mathrm{Pu} \\
24{ }^{3} \mathrm{Am} .\end{array}$ & $\begin{array}{l}p \\
: \\
a \\
0 \\
a\end{array}$ & $\begin{array}{l}1 \cdot 2+1 \\
2 \cdot 4+1= \\
3 \cdot 6+1 \\
2 \cdot 6+1 \\
2 \cdot 4+1\end{array}$ & $\begin{array}{l}3.6+1 \\
3.1+15 \\
3.6+1\end{array}$ & $\begin{array}{l}\text { WZI } \\
\text { FEI } \\
\text { FEI } \\
\text { FEI } \\
\text { FEI }\end{array}$ & $\begin{array}{l}\text { E Gayert } \\
\text { E Omitrievt } \\
\text { E Daitrievt } \\
\text { E Dmilrievt } \\
\text { E Daltrievt }\end{array}$ & $\begin{array}{l}4768 \\
9048 \\
9090 \\
9114 \\
9116\end{array}$ \\
\hline$A=91$ & \multicolumn{6}{|l|}{$\sigma(E)$} \\
\hline $\begin{array}{l}19 \theta_{\mathrm{Hg}} \\
199_{\mathrm{Hg}}\end{array}$ & $a$ & $\begin{array}{l}4.1+1 E \\
4.1+1 E\end{array}$ & $\begin{array}{l}6.5+1 \\
6.5+1\end{array}$ & $\begin{array}{l}\text { WWS E } \\
\text { WWS E }\end{array}$ & $\begin{array}{l}\text { E Neuz }: 1 \text {. } \\
\text { E Neuz:I. }\end{array}$ & $\begin{array}{l}8800 \\
8805\end{array}$ \\
\hline $\begin{array}{l}200 \mathrm{Hg} \\
201 \mathrm{Hg}\end{array}$ & $a$ & $\begin{array}{l}4.1+1 \\
4.1+1\end{array}$ & & $\begin{array}{l}\text { WWS } \\
\text { WWS }\end{array}$ & $\begin{array}{l}\text { E Neuzil. } \\
\text { E Neuzili. }\end{array}$ & $\begin{array}{l}8810 \\
8814\end{array}$ \\
\hline $20 \mathrm{ZHg}_{\mathrm{Hg}}$ & a & $4.1+1$ & & WW5 & E Nevail. & 8821 \\
\hline $204 \mathrm{Hg}$ & a & $4.1+1$ & & WWS & E Neuzil. & 8828 \\
\hline $209_{8 i}$ & $a$ & NDG & & GER $F$ & R Mosel. & 8927 \\
\hline $226 \mathrm{Ro}$ & $t$ & $2.3+1$ & & LAS E & E Webert & 6068 \\
\hline $226_{\mathrm{Ra}}$ & ${ }^{3} \mathrm{He}$ & NDG & & GER $F$ & R Mosel. & 6577 \\
\hline $23 \theta_{U}$ & $12 \mathrm{C}$ & NDG & & DUB $F$ & R Flerov. & 10157 \\
\hline $239_{0}$ & $20 \mathrm{Ne}$ & NDG & & DUP F & R Fleroy. & 11917 \\
\hline$A=92$ & \multicolumn{6}{|c|}{ primary fission yield fission fragments } \\
\hline $233 \mathrm{U}$ & d & $1.4+1$ & & BRC E & EPalint & 5934 \\
\hline
\end{tabular}

\begin{tabular}{|c|c|c|c|c|c|c|}
\hline Torget & $\ln c$ & $\begin{array}{l}\text { Emin } \\
(\mathrm{MeV})\end{array}$ & $\begin{array}{l}\text { Emax } \\
(\operatorname{MaV})\end{array}$ & Lob W & Author & No. \\
\hline$A=92$ & \multicolumn{6}{|c|}{ prlary fission yield } \\
\hline $\begin{array}{l}{ }^{209} \mathrm{Bi}_{\mathrm{Bi}} \\
22 \mathrm{~B}_{\mathrm{Ra}} \\
233_{\mathrm{U}}\end{array}$ & $\begin{array}{l}p \\
p \\
d\end{array}$ & $\begin{array}{l}3 \cdot 2+1 \\
1 \cdot 3+1 \\
1.3+1\end{array}$ & & $\begin{array}{l}\text { TUE R } \\
\text { TUE R } \\
\text { BRC E }\end{array}$ & $\begin{array}{l}\text { Gonnenwelrt } \\
\text { Gonnenweint } \\
\text { Patint }\end{array}$ & $\begin{array}{l}4761 \\
4769 \\
5933\end{array}$ \\
\hline$A=92$ & \multicolumn{6}{|c|}{ cumulative fission yleld } \\
\hline 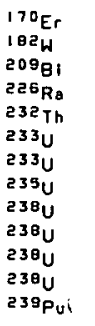 & $\begin{array}{r}160 \\
a \\
p \\
p \\
p \\
d \\
a \\
a \\
p \\
p \\
a \\
a \\
d\end{array}$ & $\begin{array}{l}1.7+2 \\
1.2+2 \\
\text { NDG } \\
1.3+1 \\
9.3+0 \\
6.0+0 \\
2.5+1 \\
6.0+0 \\
7.1+0 \\
1.2+1 \\
2.0+1 \\
2.0+1 \\
9.2+0\end{array}$ & $\begin{array}{rr} & 0 \\
& \\
& \\
& \\
& \\
9.0+0 & \\
4.1+1 & 0 \\
2.4+1 & 0 \\
1.0+2 & 0 \\
2.2+1 & 1 \\
4.0+1 & \\
2.9+1 & 5 \\
2.3+1 & 0\end{array}$ & 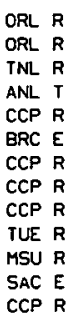 & 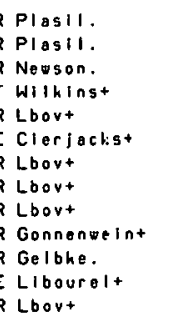 & $\begin{array}{r}11442 \\
8644 \\
4760 \\
4770 \\
4813 \\
5935 \\
8995 \\
9028 \\
4898 \\
4899 \\
5046 \\
9047 \\
5971\end{array}$ \\
\hline$A=92$ & \multicolumn{4}{|c|}{ flasion yield } & : & \\
\hline $\begin{array}{l}197 \mathrm{Au} \\
209 \mathrm{Bi} \\
226 \mathrm{Ra} \\
226 \mathrm{RB} \\
226 \mathrm{Ra} \\
232 \mathrm{Th} \\
239 \mathrm{Pu}\end{array}$ & $\begin{array}{c}a \\
p \\
p \\
d \\
{ }^{3} \mathrm{He} \\
p \\
d \\
d\end{array}$ & $\begin{array}{l}4 \cdot 2+1 \\
3 \cdot 6+1 \\
1.1+1 \\
1.9+1 \\
2 \cdot 4+1 \\
1 \cdot 3+1 \\
9 \cdot 2+0\end{array}$ & $\begin{array}{r}5.6+1 F \\
1.6+1 F \\
. F \\
5.3+1 F \\
2.3+1 F\end{array}$ & $\begin{array}{l}\text { PAR } T \\
\text { PAR } \\
\text { PAR } \\
\text { PAR } \\
\text { PAR } \\
\text { PAR } \\
\text { PAR }\end{array}$ & $\begin{array}{l}\text { Da-Silveira. } \\
\text { T Da-Silveira. } \\
\text { T Da-Silvelra. } \\
\text { T Da-Silveira. } \\
\text { T Da-Silveira. } \\
\text { T Da-Silveira. } \\
\text { T Da-Silveira. }\end{array}$ & $\begin{array}{l}8793 \\
4759 \\
4767 \\
5888 \\
6576 \\
4811 \\
5970\end{array}$ \\
\hline$A=92$ & \multicolumn{6}{|c|}{ overage $O(E)$} \\
\hline $\begin{array}{l}238_{U} \\
A=92\end{array}$ & \multicolumn{6}{|c|}{ relalive o(E) } \\
\hline $\begin{array}{l}256 \mathrm{Ra} \\
238_{U} \\
237 \mathrm{~Np} \\
242 \mathrm{Pu} \\
243_{\mathrm{Am}}\end{array}$ & $\begin{array}{l}p \\
a \\
a \\
a \\
0\end{array}$ & $\begin{array}{l}1.2+1 \\
2.4+1= \\
3.6+1 \\
2.6+1 \\
2.4+1\end{array}$ & $\begin{array}{l}3.6+1 \\
F \\
3.1+1\end{array}$ & $\begin{array}{l}\text { WZI E } \\
\text { FEI E } \\
\text { FEI E } \\
\text { FEI E } \\
\text { FEI E }\end{array}$ & 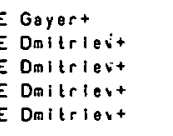 & $\begin{array}{l}4768 \\
9048 \\
9090 \\
9114 \\
9116\end{array}$ \\
\hline$A=92$ & $\sigma(E)$ & & & & & \\
\hline $198 \mathrm{Hg}$ & $a$ & $4.1+1=$ & $6.5+1 \mathrm{r}$ & WWS E & E Neuzil. & 8800 \\
\hline
\end{tabular}




\begin{tabular}{|c|c|c|c|c|c|c|}
\hline Target & $\operatorname{lnc}$ & $\begin{array}{l}\mathrm{Em} \text { in } \\
(\mathrm{MeV})\end{array}$ & $\begin{array}{l}\max \\
(\mathrm{MeV})\end{array}$ & Lab & Author & No. \\
\hline$A=92$ & $\sigma(E)$ & & & & & \\
\hline $1999_{\mathrm{Hg}}$ & a & $4 \cdot 1+1$ & $6.5+1$ & WWS & E Neuzil. & 8805 \\
\hline $200 \mathrm{Hg}$ & $a$ & $4.1+1$ & & WWS & E Neuzil. & 8810 \\
\hline $20: \mathrm{Hg}$ & a & $4,1+1$ & & WWS & E Neuzii. & 8814 \\
\hline $202 \mathrm{Hg}$ & $a$ & $4,1+i$ & & WWS & E Neuzil. & 8821 \\
\hline $204 \mathrm{Hg}$ & $a$ & $4.1+1$ & & WWS & E Neuzil. & 8828 \\
\hline 2098 & $a$ & NDG & & GER & R Mosel. & 8927 \\
\hline $226 \mathrm{Ra}$ & i & $2.3+1$ & & LAS & E Webert & 6068 \\
\hline $226 \mathrm{Ra}$ & ${ }^{3} \mathrm{He}$ & NOG & & GER F & R Mosel. & 6577 \\
\hline $238_{\mathrm{U}}$ & $12^{2} \mathrm{C}$ & NDG & & OUB F & R Flerov. & 10157 \\
\hline $238^{4}$ & $20 \mathrm{Ne}$ & NOG & & DUB F & R Fierov. & 11917 \\
\hline$A=93$ & \multicolumn{6}{|c|}{ petmary fission yield fission frogments } \\
\hline $233 \mathrm{U}$ & d & $1.4+1$ & & BRC & E Patint & 5934 \\
\hline$A=93$ & \multicolumn{6}{|c|}{ primary fission yield } \\
\hline $2 \log _{B 1}$ & 。 & $3.2+1$ & & TUE & R Gonnenueint & 4761 \\
\hline $556^{R a}$ & 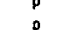 & $1.3+1$ & & TUE & R Gonnenweint & 4769 \\
\hline $233 \mathrm{U}$ & d & $1.3+1$ & & BRC & E Patint & 5933 \\
\hline$A=93$ & \multicolumn{6}{|c|}{ cumulative fission yield } \\
\hline $170 \mathrm{Er}$ & 160 & $1.7+2$ & & ORL. F & R Plasil. & 11442 \\
\hline $182 \mathrm{~W}$ & 0 & $1.5+5$ & & ORL & R Plasil. & 8644 \\
\hline $200^{81}$ & p & NDG & & TNL & $R$ Newson. & 4760 \\
\hline $225_{\mathrm{Ra}}$ & p & $1.3+1$ & & ANL. & TWilkingt & 4770 \\
\hline $232 T h$ & p & $9.3+0$ & & CCP & $R$ Lbort & 4813 \\
\hline $233 \mathrm{U}$ & d & $6.0+0$ & $9.0+0$ & BRC & E Clerjachs & 5935 \\
\hline $233 \mathrm{U}$ & 0 & $2.5+1$ & $4.1+1$ & CCP & R Lbovt & B995 \\
\hline $235 \mathrm{u}$ & p & $1.2+1$ & $8.5+1$ & CCP & R Lbout & 4849 \\
\hline $235 \mathrm{U}$ & $\begin{array}{l}p \\
a\end{array}$ & $6.0+0$ & $2.4+1$ & CCP & R Lbovt & 9028 \\
\hline $238_{U}$ & p & $7.1+0$ & $1.0+5$ & CCP & R Lbovt & 4898 \\
\hline $238_{U}$ & p & $1.5+1$ & $2.2+1$ & TUE $F$ & R Gonnenweint & 4899 \\
\hline $238_{U}$ & $a$ & $2.0+1$ & $4.0+1$ & MSU F & R Gelbke. & 9046 \\
\hline $23 \theta_{U}$ & 0 & $2.0+1$ & $2.9+1$ & SAC & E Libourelt & 9047 \\
\hline $239 \mathrm{Pu}$ & d & $9.2+0$ & $2.3+1$ & CCP & R Lbout & 5971 \\
\hline$A=93$ & \multicolumn{6}{|c|}{ fission yleld } \\
\hline $197 \mathrm{AU}$ & a & $4.2+1$ & & PAR & T Da-Silveira. & 8793 \\
\hline $209^{\circ i}$ & 0 & $3.6+1$ & $5.6+1$ & PAR & T Da-silvelra. & 4759 \\
\hline $226_{R_{B}}$ & p & $1.1+1$ & $1.6+1$ & PAR & T Da-Silveira. & 4767 \\
\hline $256_{\mathrm{Ra}}$ & $\begin{array}{l}9 \\
0\end{array}$ & $.9+1$ & & PAR & T Da-silveira. & 5888 \\
\hline
\end{tabular}

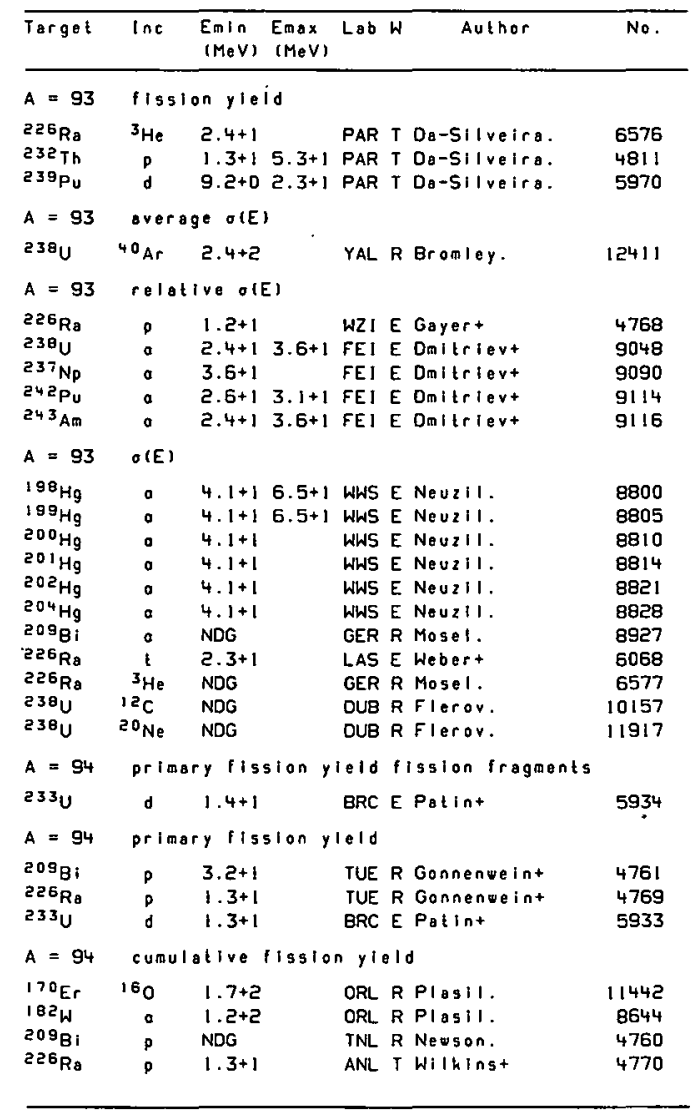




\begin{tabular}{|c|c|c|c|c|c|c|}
\hline Torget & $\ln c$ & $\begin{array}{l}\text { Emln } \\
(\mathrm{MeV})\end{array}$ & $\begin{array}{l}\text { Emax } \\
\text { (MeVi }\end{array}$ & Lab W & Author & No. \\
\hline$A=94$ & cumu & atlye $f$ & fission & $n y 101$ & & \\
\hline $\begin{array}{l}232 \mathrm{Th} \\
233 \mathrm{U} \\
233 \mathrm{U} \\
235 \mathrm{U} \\
238_{U} \\
238_{U} \\
23 \theta_{U} \\
23 \theta_{U} \\
239 \mathrm{pu}\end{array}$ & $\begin{array}{l}p \\
d \\
q \\
a \\
p \\
p \\
q \\
q \\
d\end{array}$ & $\begin{array}{l}9 \cdot 3+0 \\
6 \cdot 0+0 \\
2 \cdot 5+1 \\
6.0+0 \\
7.1+0 \\
1.2+1 \\
2.0+1 \\
2.0+1 \\
9.2+0\end{array}$ & $\begin{array}{l}9.0+0 \\
4 \cdot 1+1 \\
2.4+1 \\
1.0+2 \\
2 \cdot 2+1 \\
4.0+1 \\
2.9+1 \\
2.3+1\end{array}$ & $\begin{array}{ll}\text { CCP } & R \\
\text { GRC } & E \\
\text { CCP } & R \\
\text { CCP } & R \\
\text { CCP } & R \\
\text { TUE } & R \\
\text { MSU } & R \\
\text { SAC } & E \\
\text { CCP } & R\end{array}$ & $\begin{array}{l}\text { R Loovt } \\
\text { Clarjachst } \\
\text { R Lbovt } \\
\text { R Lbovt } \\
\text { R Lbovt } \\
\text { R Gonnenuelnt } \\
\text { R Gelbhe. } \\
\text { E Libourelt } \\
\text { R Lbovt }\end{array}$ & $\begin{array}{l}4813 \\
5935 \\
8995 \\
9028 \\
4898 \\
4899 \\
9046 \\
9047 \\
5971\end{array}$ \\
\hline$A=94$ & fiss & ion yiel & & & & \\
\hline $\begin{array}{l}197 \mathrm{Au} \\
209 \mathrm{Bi} \\
226 \mathrm{Ra} \\
226 \mathrm{Ra} \\
226 \mathrm{RB} \\
232 \mathrm{Th} \\
239 \mathrm{Pu}\end{array}$ & $\begin{array}{c}0 \\
p \\
p \\
\delta \\
{ }^{3} \mathrm{He} \\
p \\
d\end{array}$ & $\begin{array}{l}4.2+1 \\
3 \cdot 6+1 \\
1.1+1 \\
1.9+1 \\
2 \cdot 4+1 \\
1.3+1 \\
9.2+0\end{array}$ & $\begin{array}{l}5.6+1 \\
1.6+1 \\
5.3+! \\
2.3+1\end{array}$ & $\begin{array}{l}\text { PAR T } \\
\text { PAR T } \\
\text { PAR T } \\
\text { PAR T } \\
\text { PAR T } \\
\text { PAR T } \\
\text { PAR T }\end{array}$ & $\begin{array}{l}\text { T Da-Silveira. } \\
\text { T Da-Silveira. } \\
\text { T Da-SIlveira. } \\
\text { T Da-Silveira. } \\
\text { T Da-Silveira. } \\
\text { T Da-Silveira. } \\
\text { T Da-SIlveira. }\end{array}$ & $\begin{array}{l}8793 \\
4759 \\
4767 \\
5888 \\
6576 \\
48 ! 1 \\
5970\end{array}$ \\
\hline$A=94$ & aver & $g e \quad \sigma(E)$ & & & & \\
\hline $\begin{array}{l}238 U \\
A=94\end{array}$ & $\begin{array}{l}40_{\mathrm{Ar}} \\
\mathrm{rela}\end{array}$ & $\begin{array}{l}2.4+2 \\
\text { ive olE }\end{array}$ & & YAL $R$ & R Bromley. & 12411 \\
\hline $\begin{array}{l}226 \mathrm{Ra} \\
23 \mathrm{~B}_{\mathrm{U}} \\
23{ }^{3} \mathrm{~Np} \\
242 \mathrm{PU} \\
24{ }^{2} \mathrm{Am}\end{array}$ & $\begin{array}{l}p \\
a \\
a \\
a \\
a\end{array}$ & $\begin{array}{l}1 \cdot 2+1 \\
2 \cdot 4+1 \\
3 \cdot 6+1 \\
2 \cdot 6+1 \\
2 \cdot 4+1\end{array}$ & $\begin{array}{l}3.6+1 \\
3.1+1 \\
3.6+1\end{array}$ & $\begin{array}{ll}\text { WZI } & E \\
\text { FEI } & E \\
\text { FEI } & E \\
\text { FEI } & E \\
F E I & E\end{array}$ & $\begin{array}{l}\text { E Goyert } \\
\text { Emitrievt } \\
\text { Emitrlevt } \\
\text { E Omitrievt } \\
\text { E Omitrlevt }\end{array}$ & $\begin{array}{l}4768 \\
9048 \\
9090 \\
9114 \\
9116\end{array}$ \\
\hline$A=94$ & $O(E)$ & & & & & \\
\hline $\begin{array}{l}198_{\mathrm{Hg}} \\
199 \mathrm{Hg} \\
200 \mathrm{Hg} \\
201 \mathrm{Hg} \\
202^{\mathrm{Hg}} \\
204 \mathrm{Hg} \\
209 \mathrm{Bi} \\
226 \mathrm{Ra} \\
226 \mathrm{Ra} \\
238 \mathrm{U}\end{array}$ & $\begin{array}{c}a \\
a \\
a \\
a \\
0 \\
0 \\
a \\
i \\
3^{3} \mathrm{He} \\
1{ }^{2} \mathrm{C}\end{array}$ & $\begin{array}{l}4.1+1 \\
4.1+1 \\
4.1+1 \\
4.1+1 \\
4.1+1 \\
4.1+1 \\
\text { NDG } \\
2.3+1 \\
\text { NDG. } \\
\text { NDG. }\end{array}$ & $\begin{array}{c}6.5+5 \\
6.5+5 \\
\end{array}$ & $\begin{array}{l}\text { WWS E } \\
\text { WWS E } \\
\text { WWS E } \\
\text { WWS E } \\
\text { WWS E } \\
\text { WWS E } \\
\text { GER R } \\
\text { LAS E } \\
\text { GER R } \\
\text { DUB R }\end{array}$ & $\begin{array}{l}\text { E Neuzil. } \\
\text { E Neuzil. } \\
\text { E Neuzil. } \\
\text { E Neuzil. } \\
\text { E Neuzil. } \\
\text { E Neuzil. } \\
\text { R Mosel. } \\
\text { E Webert } \\
\text { R Mosel. } \\
\text { R Flerov. }\end{array}$ & $\begin{array}{r}8800 \\
8805 \\
8810 \\
8814 \\
8821 \\
8828 \\
8927 \\
6058 \\
6577 \\
10157\end{array}$ \\
\hline
\end{tabular}

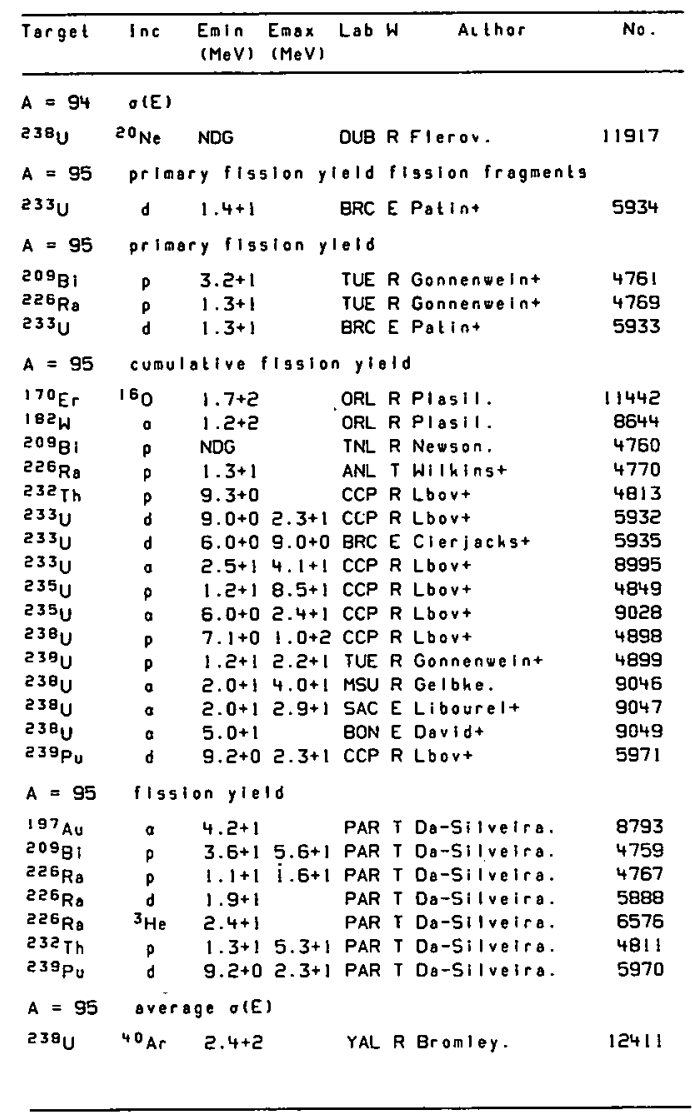




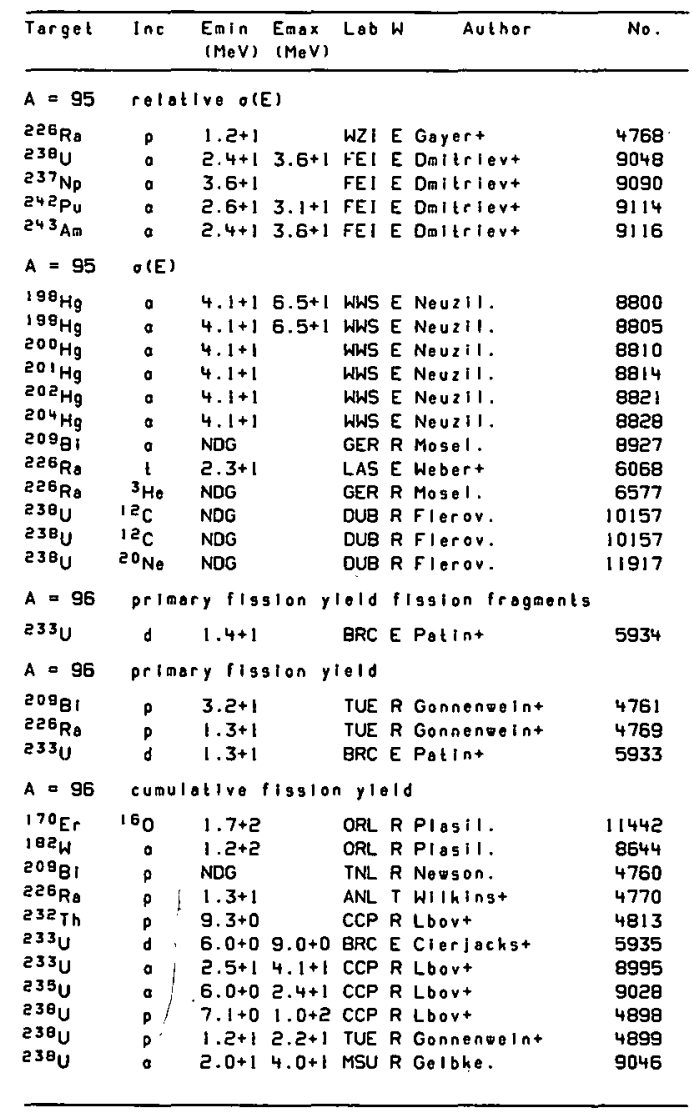

\begin{tabular}{lllll}
\hline Target Ine & $\begin{array}{l}\text { Emin } \\
(\mathrm{MeV})\end{array}$ & $\begin{array}{l}\text { Emax } \\
(\mathrm{MeV})\end{array}$ & Lab $W$ Author \\
\hline
\end{tabular}

$A=95$ cunulative fission yield

239u $2.0+12.9+1$ SAC E Llbourelt 9047

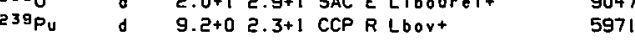

$A=96$ figgion yield

197AU a 4.2+1 PART Da-silveira. 8793

$208^{8} \mathrm{~B} \quad \mathrm{P} .6+15.6+1$ PAR T Da-SIIveira. 4759

$226 R_{A} \rho \quad 1.1+11.6+1$ PAR T Da-SIIvelra. 4767

${ }^{226} \mathrm{Ra} d \mathrm{~d}$ 1.9+1 PART Da-SIIvelra. 5888

${ }_{225} \mathrm{Re} \quad{ }^{3} \mathrm{He} \quad 2.4+1 \quad$ PAR T Da-Silveira. 6576

$232 \mathrm{Th} P \quad 1.3+15.3+1$ PAR T Da-Silveira. 4811

$239 \mathrm{Pu} d \mathrm{9.2+0} 2.3+1$ PAR T Da-sllvelra. 5970

$A=98$ overage $O(E)$

238U ${ }^{40} \mathrm{Ar} 2.4+2 \quad$ YAL R Bromley, 12411

$A=96$ relative o(E)

$226 \mathrm{Ra} \quad 1.2+1$ Hil E Gayert

$\begin{array}{lllll}228 \mathrm{Ra} & \rho & 1.2+1 & \text { WLI E Gayert } & 4768 \\ 23 \theta_{\mathrm{U}} & 0 & 2.4+1 & 3.6+1 \text { FEI E Dmitrievt } & 9048 \\ 237 \mathrm{~Np} & 0 & 3.6+1 & \text { FEI E Dmilrievt } & 9090\end{array}$

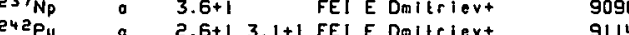

$\begin{array}{lllll}243 \mathrm{Pu} & 0 & 2.6+13.1+1 \text { FEI E Dmltrievt } & 9114 \\ 243 \mathrm{Am} & 0 & 2.4+13.6+1 \text { FEI E Dnitrievt } & 9116\end{array}$

$A=S Q \quad O(E)$

$198 \mathrm{Hg} \quad 4 \quad 4.1+16.5+1$ WWS E NeUzil. 8800

$199 \mathrm{Hg} O 4.1+16.5+1$ WWS E NeUz11.

$200 \mathrm{Hg} \quad 4.1+1$ WWS E Neuz11.

$201 \mathrm{Hg} \quad 4 \quad 4.1+1$ WWS E Neuz1l.

$202 \mathrm{Hg} 04.1+1$ WWS E NeVzII.

$204 \mathrm{Hg} \quad 4.1+1$ HWS E NeUzil.

O NDG GER R Mosel. 8927

${ }^{226} R_{a} \quad l \quad 2.3+1 \quad$ LAS E Hebert

${ }^{226} \mathrm{Ra}_{\mathrm{a}}{ }^{3} \mathrm{He}$ NDG $\quad$ GER R Mosel. 6577

$230 \mathrm{U} 12 \mathrm{C}$ NDG $\quad 10157$

$238^{2}{ }^{2} \mathrm{Ne}$ NDG DUB Rlerov. 11917

$A=97$ primory figsion yield fission fragments

$233 \mathrm{U} d \mathrm{~d} 1.4+1 \quad$ BRC E Potint 5934

$A=97$ primary fission yleld

${ }^{209} 8 i$ P $3.2+1$ TUE R Gonnenweint 4761 


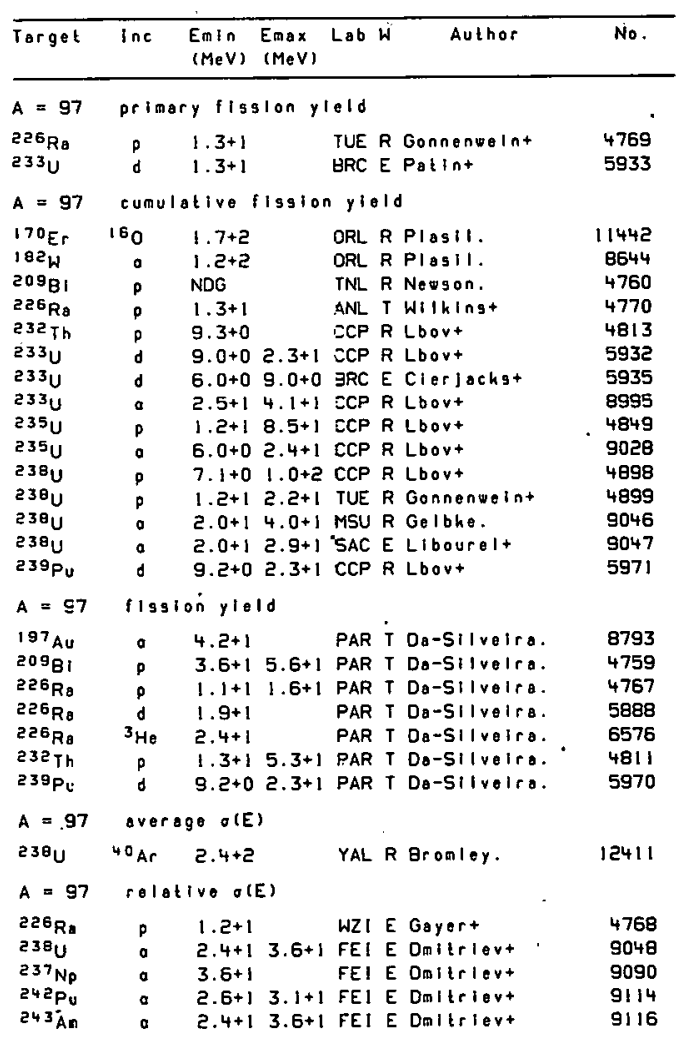

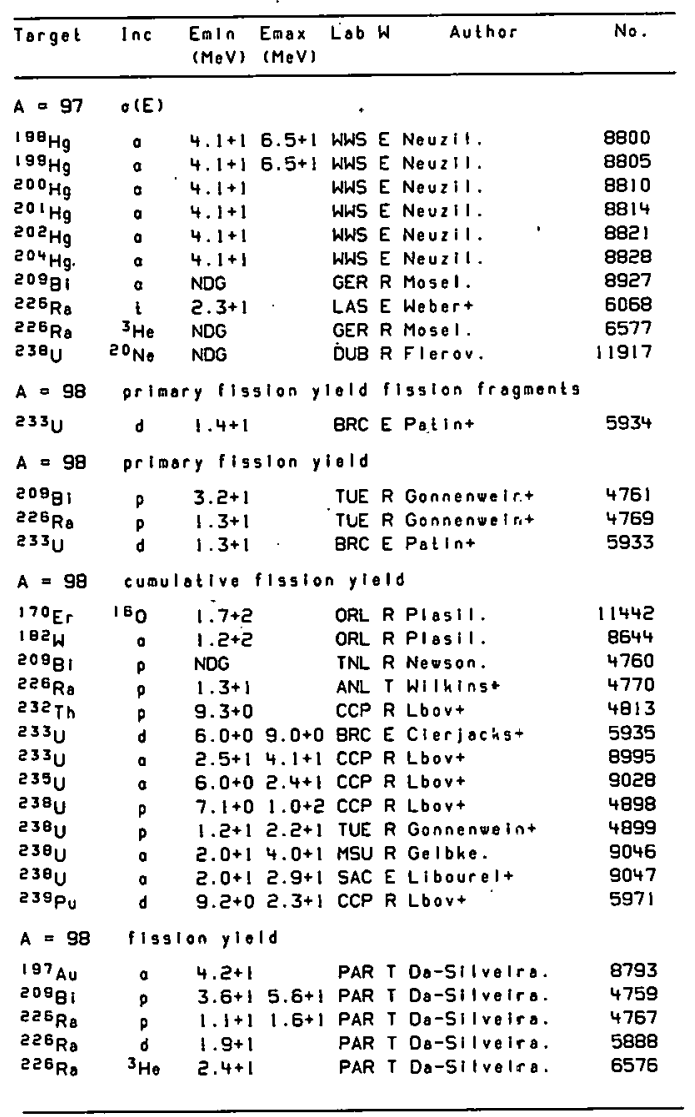




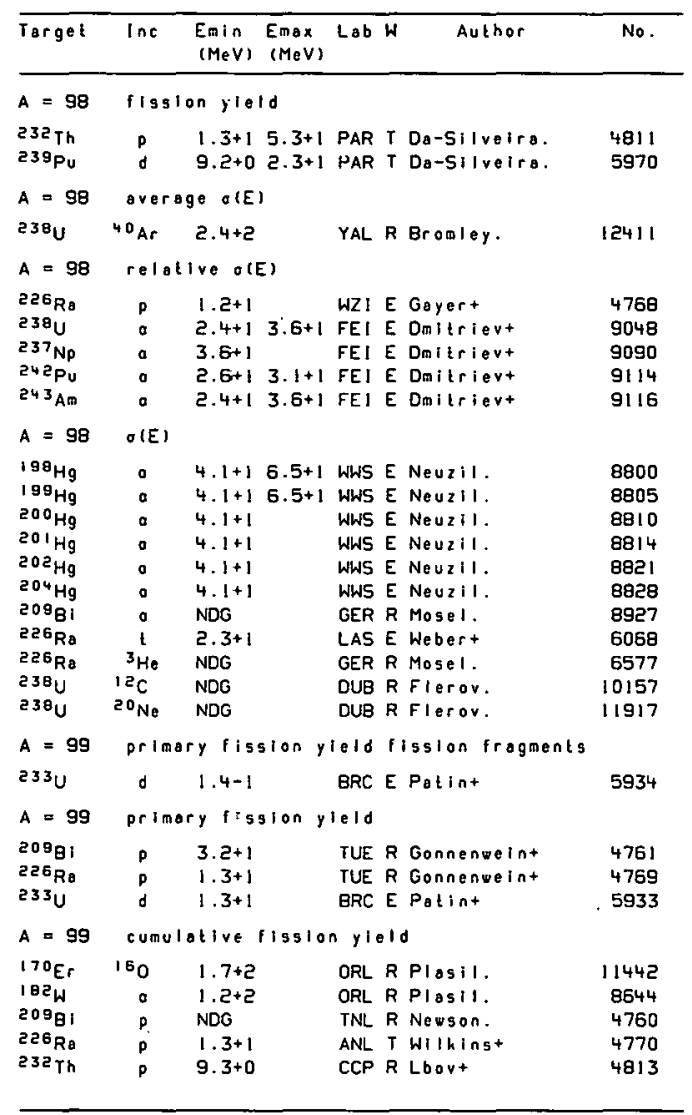

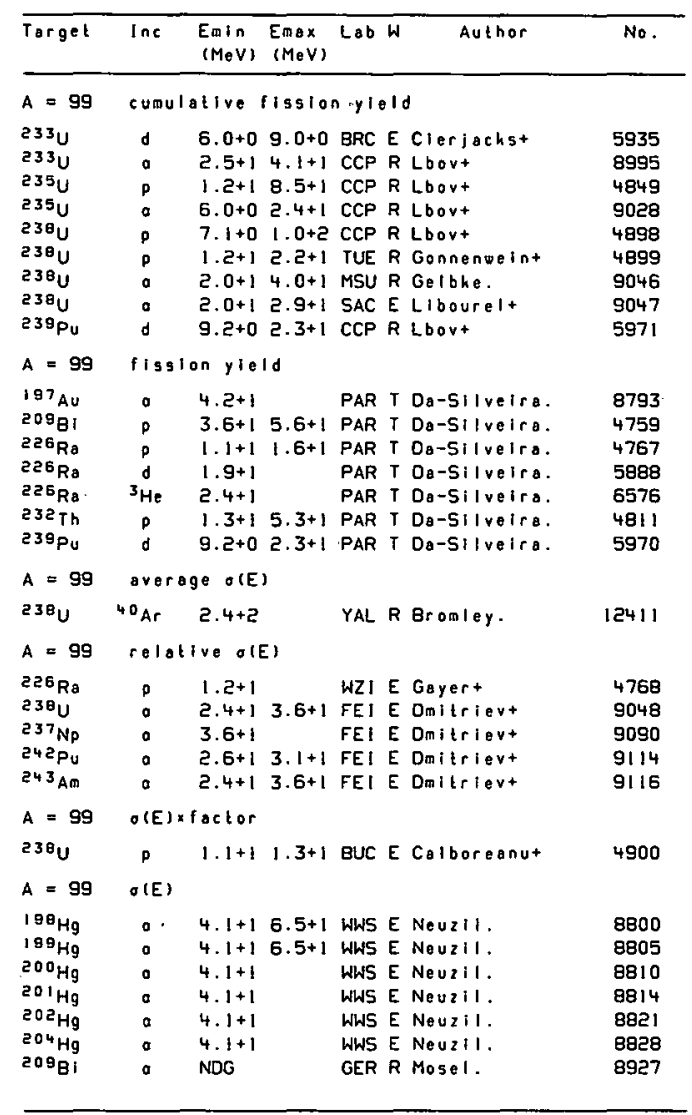




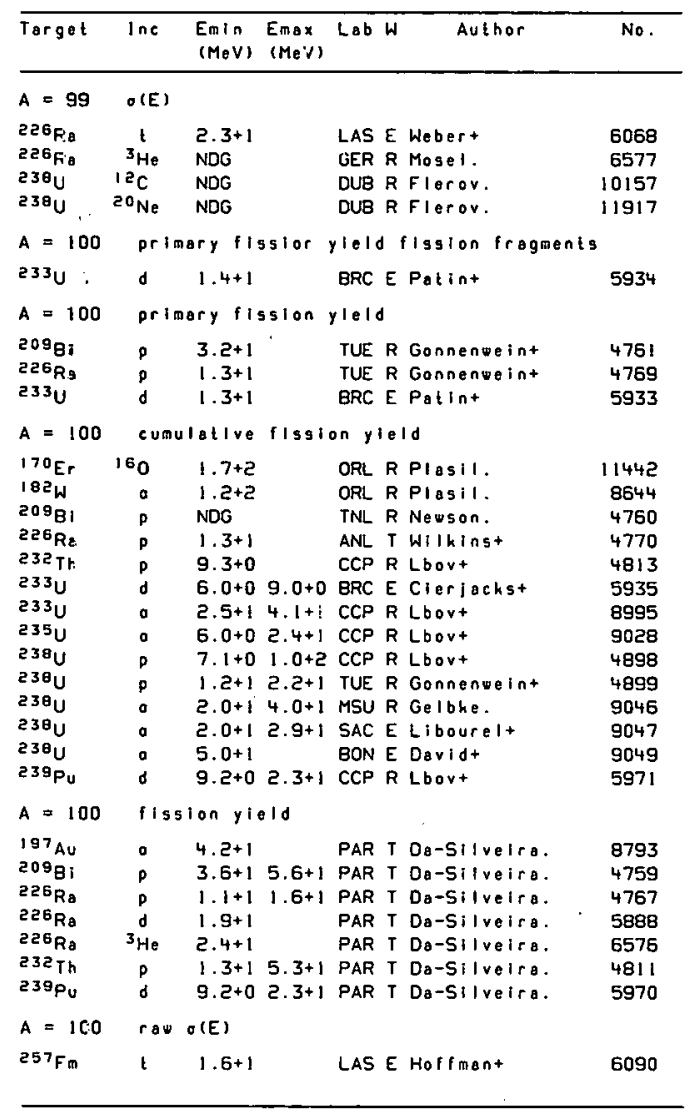

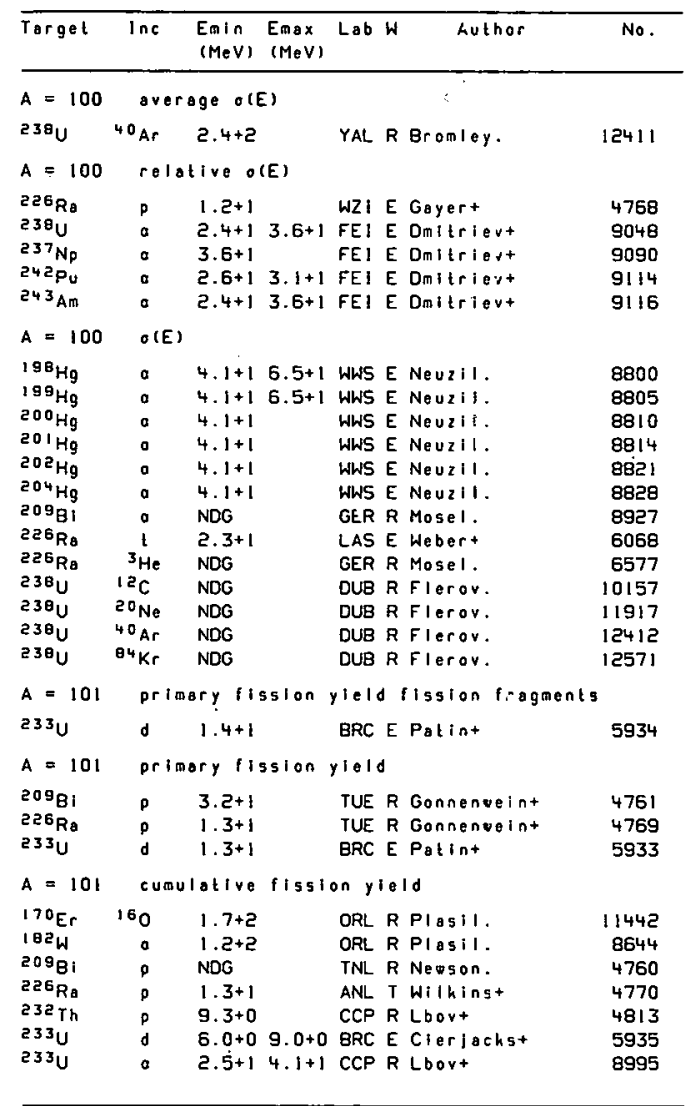




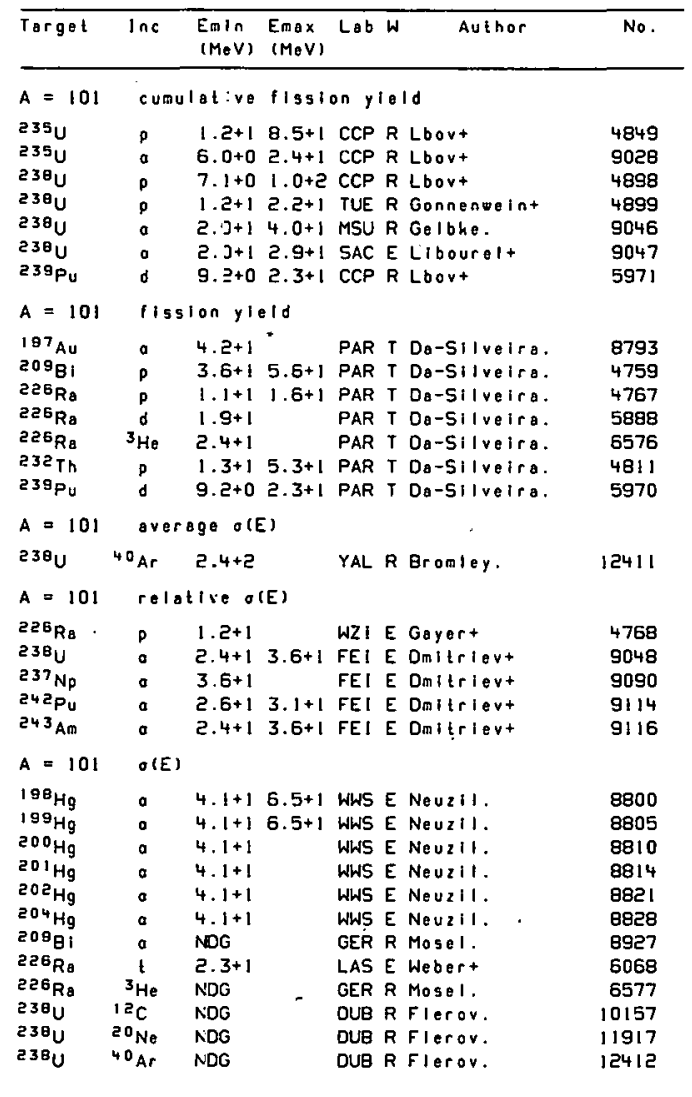

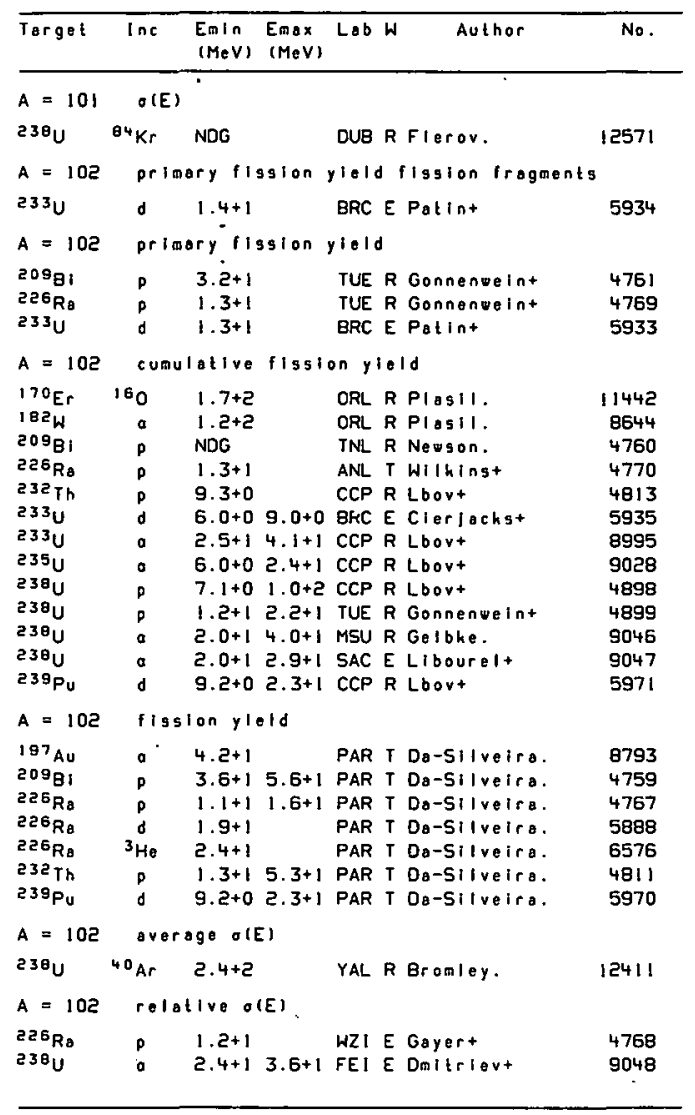




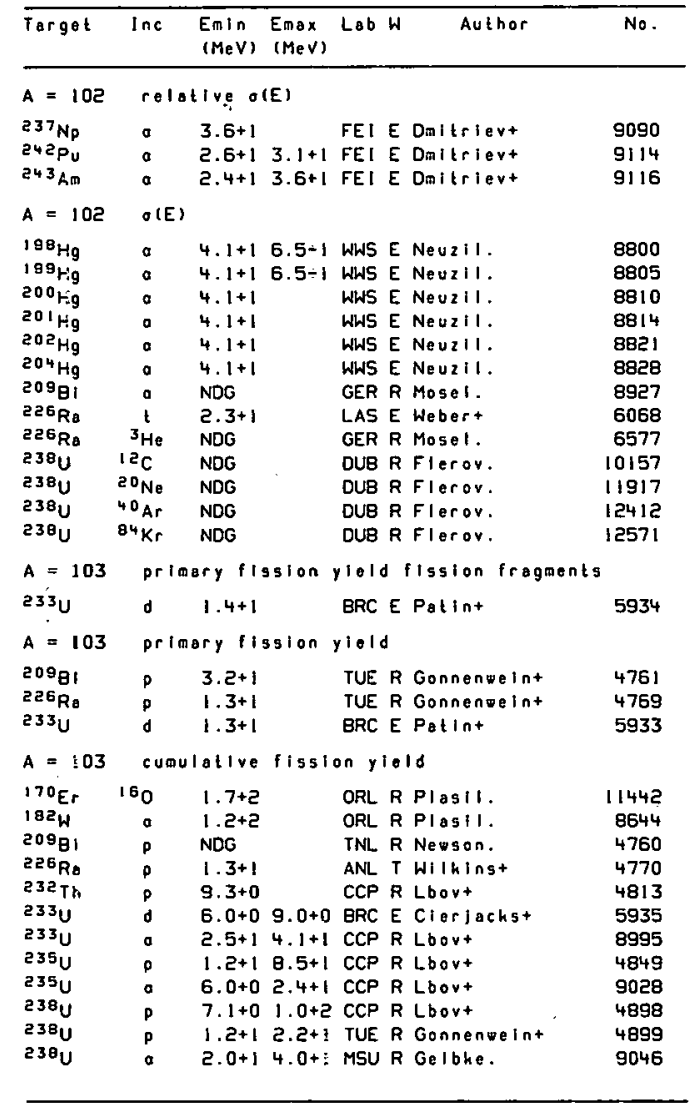

\begin{tabular}{|c|c|c|c|c|c|c|}
\hline Target & $\ln c$ & $\begin{array}{l}\text { Emin } \\
(\mathrm{MeV})\end{array}$ & $\begin{array}{l}E \max \\
(\mathrm{MeV})\end{array}$ & Lab W & W Author & No. \\
\hline$A=103$ & \multicolumn{6}{|c|}{ cumulative } \\
\hline $\begin{array}{l}238 \mathrm{U} \\
239 \mathrm{Pu}\end{array}$ & d & $\begin{array}{l}2.0+1 \\
9.2+0\end{array}$ & $\begin{array}{l}2.9+1 \\
2.3+1\end{array}$ & $\begin{array}{l}\text { SAC E } \\
\text { CCP R }\end{array}$ & $\begin{array}{l}\text { E Libolrelt } \\
R \text { Lbovt }\end{array}$ & $\begin{array}{l}9047 \\
5971\end{array}$ \\
\hline$A=103$ & \multicolumn{6}{|c|}{ fisston yield } \\
\hline $\begin{array}{l}197 \mathrm{Au} \\
209 \mathrm{BI}\end{array}$ & a & $\begin{array}{l}4 \cdot 2+1 \\
3 \cdot 6+1\end{array}$ & $5.6+1$ & $\begin{array}{l}\text { PAR T } \\
\text { PAR T }\end{array}$ & $\begin{array}{l}\text { T Da-Silveira. } \\
\text { T Da-Silveira. }\end{array}$ & $\begin{array}{l}8793 \\
4759\end{array}$ \\
\hline $226 \mathrm{Ro}$ & & - $1.1+1$ & $1.6+1$ & PAR T & T Da-Silveira. & 4767 \\
\hline $226 \mathrm{Ra}$ & d & $1.9+1$ & & PAR T & T Da-5ilveira. & 5888 \\
\hline $226 \mathrm{Ra}$ & ${ }^{3} \mathrm{He}$ & $2.4+1$ & & PAR T & T Da-silveira & 6576 \\
\hline $232 \mathrm{Th}$ & $p$ & $1.3+1$ & $5.3+1$ & PAR T & T Da-Silvaira. & 4811 \\
\hline${ }^{239} p_{u}$ & $d$ & $9.5+0$ & $2.3+1$ & PAR T & T Da-Silveira. & 5970 \\
\hline
\end{tabular}

$A=103$ everage $O(E)$

$238 \mathrm{40}$ Ar $2.4+2 \quad$ YAL R Bromley. 12411

$A=103$ relative o(E)

$\begin{array}{lllll} & & & \\ 23 \theta_{U} & P & 1.2+1 & \text { WLI E Gayert } & 4768 \\ & 0 & 2.4+1 & 3.6+1 \text { FEI E Daitrievt. } & 9048\end{array}$

$237^{7} \mathrm{~Np} a \quad 3.6+1$ FEI E Daitriert 9090

$242 \mathrm{Pu}$ a $2.6+13.1+1$ FEI E Dmitrlert 9114

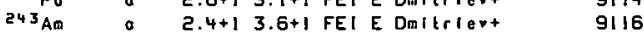

$A=103 \quad \sigma(E)$

$198_{\mathrm{Hg}}$ व $4.1+16.5+1$ WWS E Neuz11. 8800

$199 \mathrm{Hg}^{\circ}: 4.1+16.5+1$ WWS E Neuz11.

$200 \mathrm{Hg} \quad 4.1+1$ WWS E NeUz1!.

$201 \mathrm{HO} \quad 4.1+1 \quad$ WWS E Nev211.

202 a $4.1+1$ WWS $\mathrm{E}$ NeUz11.

$204 \mathrm{Hg}$ a $4.1+1$ WWS E NeV211.

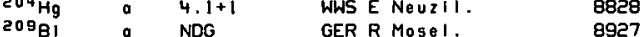

$256 \mathrm{Ra} t \quad 2.3+1$ LAS E Webert

$25 \mathrm{RB}^{3} \mathrm{He}$ NDG 6068

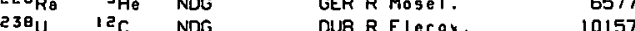

$230^{2} 20 \mathrm{NO}$ NDO 10157

$230^{\circ} 40 \mathrm{Ar} N D \mathrm{NO} \quad 119$

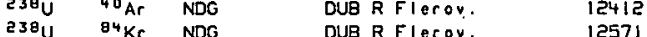

$A=104$ primary flssion yleld flagion fragments

$233 \mathrm{U} d 1.4+1 \quad$ BRC E PBlint; 5934 


\begin{tabular}{|c|c|c|c|c|c|c|}
\hline Target & $\operatorname{lnc}$ & $\begin{array}{l}\text { Emin } \\
\text { (Mev) }\end{array}$ & $\begin{array}{l}\text { Emax } \\
(\operatorname{MeV})\end{array}$ & Lob & Author & No. \\
\hline$A=104$ & \multicolumn{6}{|c|}{ primary fission yleld } \\
\hline $\begin{array}{l}209_{\mathrm{Bi}} \\
226_{\mathrm{Ra}} \\
233_{\mathrm{U}}\end{array}$ & $\begin{array}{l}p \\
p \\
d\end{array}$ & $\begin{array}{l}3.2+1 \\
1.3+1 \\
1.3+1\end{array}$ & & $\begin{array}{l}\text { TUE F } \\
\text { TUE F } \\
\text { ERC E }\end{array}$ & $\begin{array}{l}\text { R Gonnenweint } \\
\text { R Gonnenweint } \\
\text { E Patint }\end{array}$ & $\begin{array}{l}4761 \\
4769 \\
5933\end{array}$ \\
\hline$A=104$ & \multicolumn{6}{|c|}{ cumulative fission yleld } \\
\hline 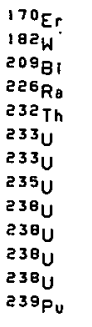 & $\begin{array}{r}160 \\
a \\
p \\
p \\
p \\
d \\
a \\
a \\
p \\
p \\
0 \\
a \\
d\end{array}$ & $\begin{array}{l}1.7+2 \\
1.2+2 \\
\text { NDG } \\
1.3+1 \\
9.3+0 \\
6.0+0 \\
2.5+1 \\
6.0+0 \\
7 \cdot 1+0 \\
1.2+1 \\
2 \cdot 0+1 \\
2.0+1 \\
9.2+0\end{array}$ & $\begin{array}{l} \\
\\
9.0+0 \\
4.1+1 \\
2.4+1 \\
1.0+2 \\
2.2+1 \\
4.0+1 \\
2.9+1 \\
2.3+1\end{array}$ & $\begin{array}{l}\text { ORL F } \\
\text { ORL F } \\
\text { TNL F } \\
\text { ANL } \\
\text { CCP F } \\
\text { ERC E } \\
\text { CCP F } \\
\text { CCP F } \\
\text { CCP F } \\
\text { TUE F } \\
\text { MSU F } \\
\text { SAC E } \\
\text { CCP F }\end{array}$ & $\begin{array}{l}\text { R Plasil. } \\
\text { R Plasil. } \\
\text { R Newson. } \\
\text { T Wilkinst } \\
\text { R Lbovt } \\
\text { E Clerjachst } \\
\text { R Lbovt } \\
\text { R Lbovt } \\
\text { R Lbovt } \\
\text { R Gonnenweint } \\
\text { R Gelohe. } \\
\text { E Libourelt } \\
\text { R Lbovt }\end{array}$ & $\begin{array}{r}11442 \\
8644 \\
4760 \\
4770 \\
4813 \\
5935 \\
8995 \\
9028 \\
4898 \\
4899 \\
9046 \\
9047 \\
5971\end{array}$ \\
\hline$A=104$ & \multicolumn{6}{|c|}{ fission ylelo } \\
\hline $\begin{array}{l}197 \mathrm{Au} \\
209 \mathrm{Bi} \\
226 \mathrm{Ra} \\
226 \mathrm{Ra} \\
226 \mathrm{Ro} \\
232 \mathrm{Th} \\
239 \mathrm{Pu}\end{array}$ & $\begin{array}{c}a \\
p \\
p \\
d \\
{ }^{3} \mathrm{He} \\
p \\
d\end{array}$ & $\begin{array}{l}4 \cdot 2+1 \\
3 \cdot 6+1 \\
1 \cdot 1+1 \\
1.9+1 \\
2 \cdot 4+1 \\
1 \cdot 3+1 \\
9 \cdot 2+0\end{array}$ & $\begin{array}{l}5.5+1 \\
1.6+1 \\
\\
5.3+1 \\
2.3+1\end{array}$ & $\begin{array}{l}\text { PAR } \\
\text { PAR T } \\
\text { PAR } 1 \\
\text { PAR } \\
\text { PAR } \\
\text { PAR } \\
\text { PAR }\end{array}$ & $\begin{array}{l}\text { T Da-Silveira. } \\
\text { T Da-Silveira. } \\
\text { T Da-Silveira. } \\
\text { T Da-Silveira. } \\
\text { T Da-Silveira. } \\
\text { T Da-Silveira. } \\
\text { T Da-Silveira. }\end{array}$ & $\begin{array}{l}8793 \\
4759 \\
4767 \\
5888 \\
6576 \\
4811 \\
5970\end{array}$ \\
\hline$A=104$ & \multicolumn{6}{|c|}{ average o(E) } \\
\hline $\begin{array}{l}238 \mathrm{U} \\
A=104\end{array}$ & $\begin{array}{l}{ }^{40} \mathrm{Ar} \\
\mathrm{rel}\end{array}$ & $\begin{array}{l}2.4+2 \\
\text { t1 }\end{array}$ & (E) & YAL $F$ & R Bromley. & 12411 \\
\hline $\begin{array}{l}226 \mathrm{Ra} \\
238_{\mathrm{U}} \\
23{ }^{7} \mathrm{~Np} \\
242 \mathrm{Pu} \\
24{ }^{3} \mathrm{Am}\end{array}$ & $\begin{array}{l}p \\
a \\
a \\
a \\
a\end{array}$ & $\begin{array}{l}1.2+1 \\
2.4+1 \\
3 \cdot 6+1 \\
2.6+1 \\
2 \cdot 4+1\end{array}$ & $\begin{array}{l}3.6+1 \\
3.1+1 \\
3.6+1\end{array}$ & $\begin{array}{l}\text { WZ: } \\
\text { FEI } \\
\text { FEI } \\
\text { FEI E } \\
\text { FEI }\end{array}$ & $\begin{array}{l}\text { E Gayert } \\
\text { E Omitrievt } \\
\text { E Dmitrievt } \\
\text { E Dmitrieyt } \\
\text { E Dolitrieyt }\end{array}$ & $\begin{array}{l}4768 \\
9048 \\
9090 \\
9114 \\
9116\end{array}$ \\
\hline$A=104$ & $o l \varepsilon$ & & & & & \\
\hline${ }^{198} \mathrm{Hg}_{9}$ & a & $4.1+1$ & $6.5+1$ & WWS E & E Neuzil. & 8800 \\
\hline
\end{tabular}

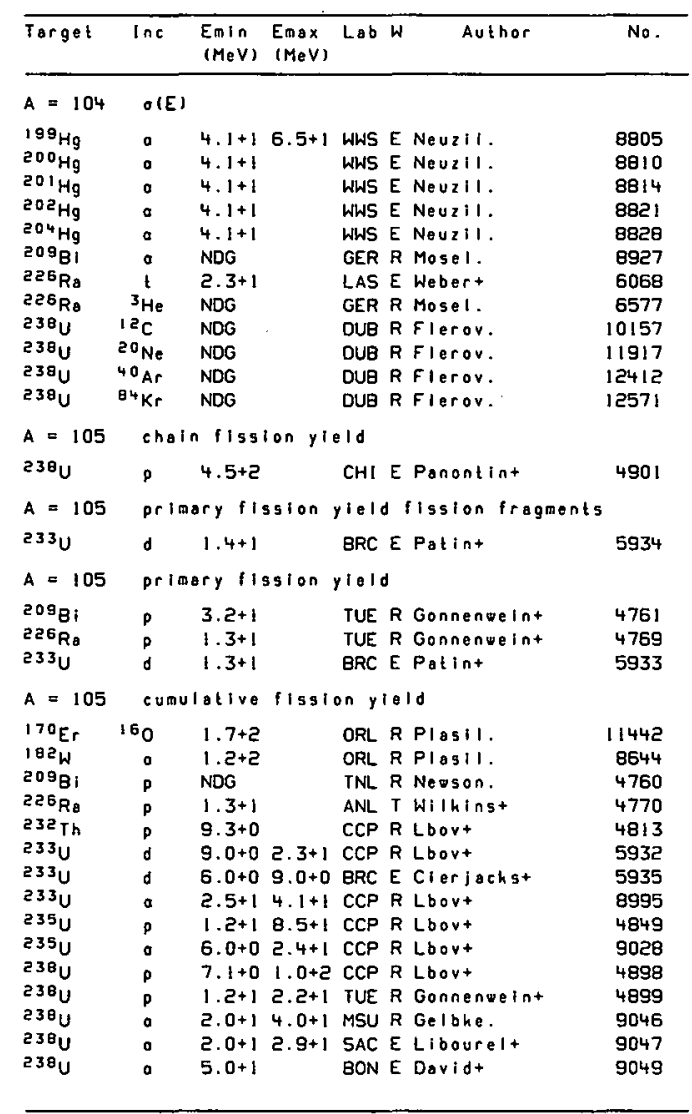




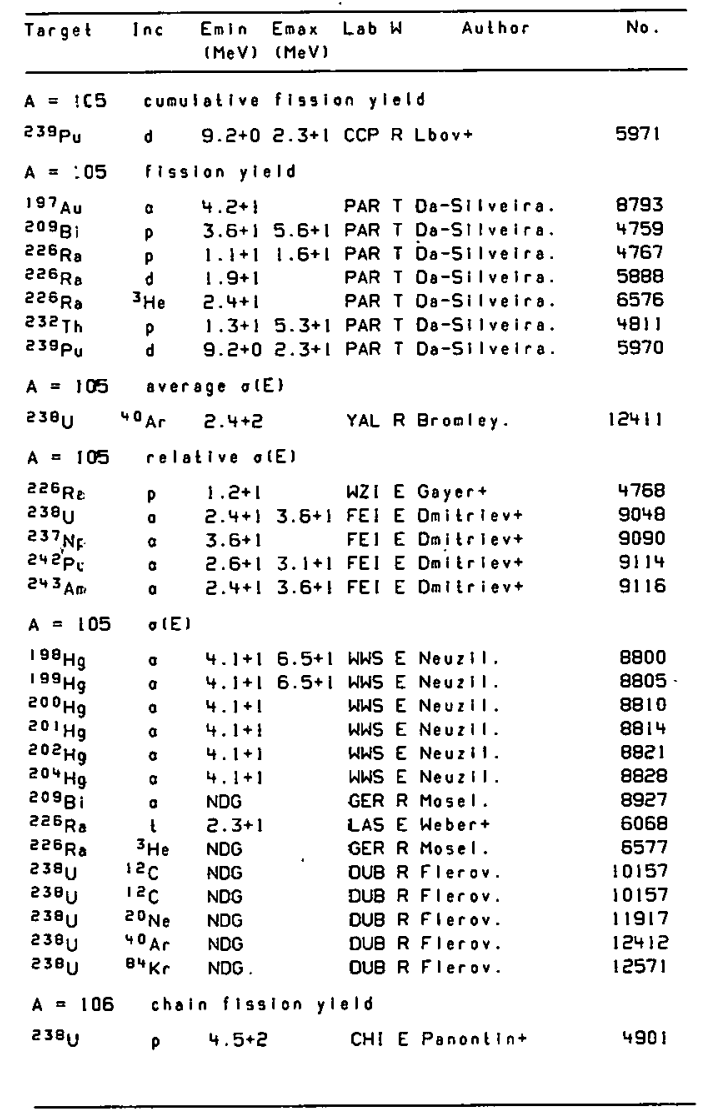

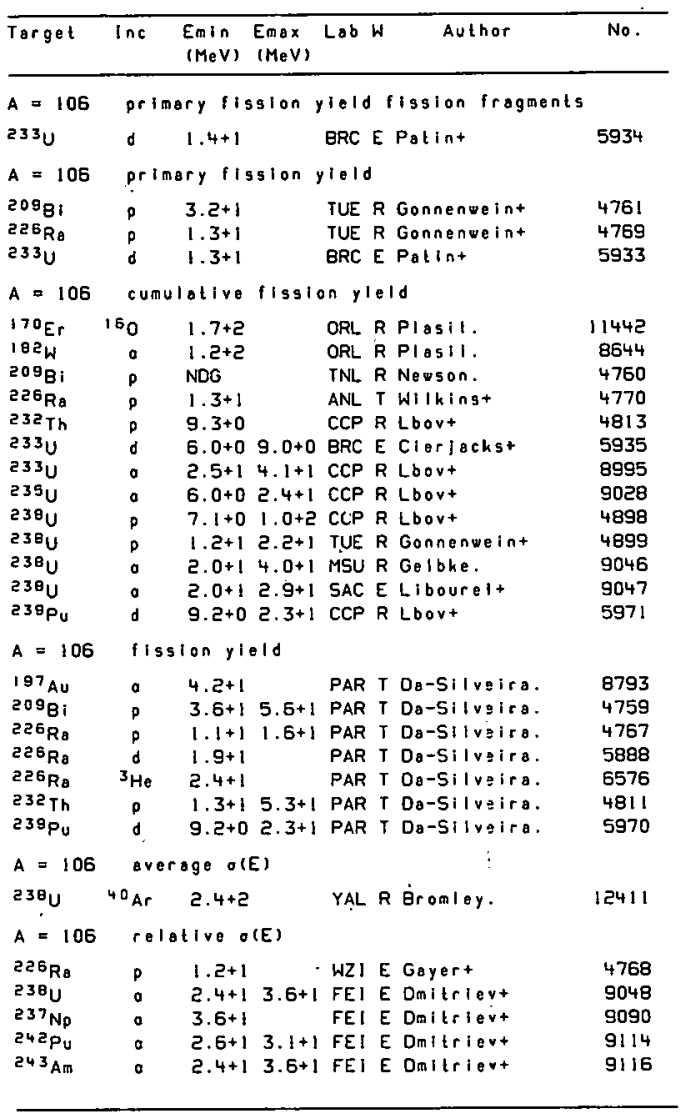




\begin{tabular}{lllll}
\hline Target $\operatorname{lnc}$ & $\begin{array}{c}\text { Emin } \\
\text { (MeV) }\end{array}$ & Eas Lov \\
& Nob $W$ & Author & No. \\
\hline
\end{tabular}

\section{$A=106$ o(E)}

$198_{\mathrm{Hg}} \quad 4 j+16.5+1$ HWS E Nevz11.

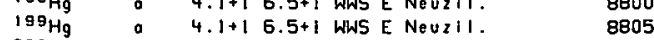

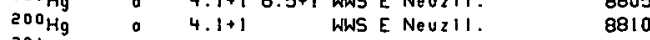

$201 H_{9} \circ 4.1+1$ WWS E NeVz11.

$202_{\mathrm{Hg}} \mathrm{O} 4.1+1$ WWS E NeUzil.

$204 \mathrm{Hg} 4.1+1$ WWS E NeUzil.

$209^{\mathrm{Bi}}$ a NDG GER R Mosel. 8927

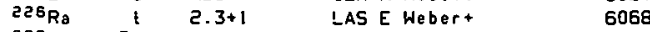

$256_{\mathrm{Ro}}{ }^{3} \mathrm{He}$ NDG GER R Mosel. 6577

$23 \theta^{2}{ }^{12} \mathrm{C}$ NDG DUB R Flerov.

$238 \mathrm{U} 20 \mathrm{Ne}$ NDG DUB R Flerov.

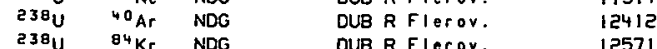

$A=107$ choln lission rield

$230_{U} P$ 4.5-2 CHI E Panontint 4901

息 $\quad A=107$ primory fisgion yleld fisgion fragments

233U d $1.4-1$ BRC E Patint

$A=107$ primory fission yiold

$\begin{array}{lllll}20 \theta_{B i} & p & 3.2+1 & \text { TUE R Gonnenueint } & 4761 \\ 226_{\mathrm{Ra}} & P & 1.3+1 & \text { TUE R Gonnenvelnt } & 4769\end{array}$

$\begin{array}{lllll}253_{0} & P & 1.3+1 & \text { TUE R Gonnenuelnt } & 4769 \\ 233^{\prime} & d & 1.3+1 & \text { BRC E Patint } & 5933\end{array}$

$A=107$ cumulatice fission yield

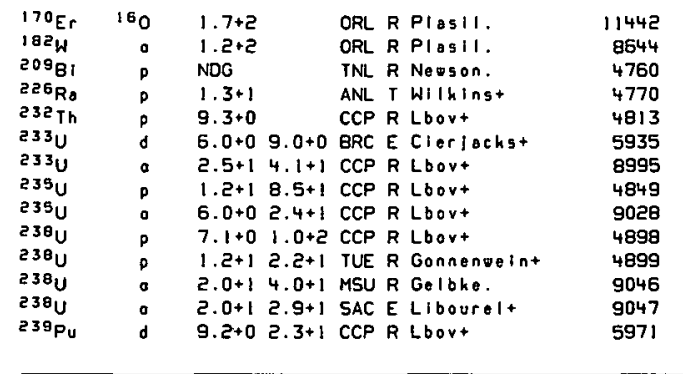

\begin{tabular}{llllll}
\hline Target Inc & $\begin{array}{l}\text { EaIn } \\
(M e V)\end{array}$ & $\begin{array}{l}\text { Emax Lab } \\
\text { (MeV) }\end{array}$ & Author & No. \\
\hline
\end{tabular}

$A=107$ fission yield

197 Au $\quad 4.2+1 \quad$ PART Da-Silvelra. 8793

${ }^{209} \mathrm{gi} \quad \mathrm{P} \quad 3.6+15.6+1$ PAR T Da-Silveira. 4759

$226_{R a} P \quad 1.1+1$ 1.6+1 PAR T Da-silvelra. 4767

$25 \mathrm{Ra}^{2} \mathrm{O} \quad 1.9+1 \quad$ PAR T Da-SIlvelra. 5888

$226 \mathrm{Ra}{ }^{3} \mathrm{He} 2.4+1 \quad$ PAR T Do-silveira. 6576

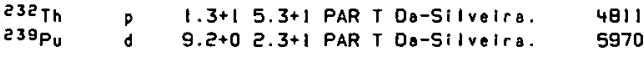

$A=107$ overage $O(E)$

$238_{\mathrm{U}} 40 \mathrm{Ar} 2.4+2$ YAL R Bromley.

$A=107$ rolotive $O(E)$

$226 \mathrm{Ra} P \quad 1.2+1$ WZI E Goyert 4768

$230_{U} \quad 2.4+13.6+1 \mathrm{FE}$ ! E Onitriovt 9048

$237 \mathrm{~Np} a 3.6+1 \quad$ FEI E Oritrievt 9090

$\begin{array}{llll}242 P_{u} & a & 2.6+13.1+1 \text { FEI E Ombirlevt } & 9114 \\ 243 \mathrm{Am} & 0 & 2.4+13.6+1 \text { FEI E Ombirievt } & 9116\end{array}$

$A=107 \quad O(E)$

${ }^{198} \mathrm{Hg}$ a $4.1+16.5+1$ WWS E NeUz11. 8800

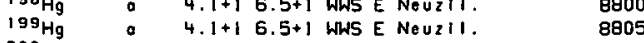

$200 \mathrm{Hg} 20.4 .1+1.6 .5+1$

8810

$202 \mathrm{Hg}^{2} \mathrm{O} \quad 4.1+1$ (1) 8814

$202 \mathrm{Hg}_{9} \mathrm{a} \quad 4.1+1$ WWS E Nouzil.

${ }^{204} \mathrm{Hg} 0 \mathrm{H} \quad 4.1+1$ WWS E NoUzil.

${ }^{209} \mathrm{Bi}$ N NDG GER R Mosel.

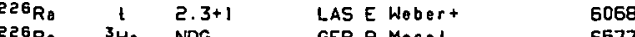

${ }^{226} \mathrm{Ra}{ }^{3} \mathrm{He}$ NDG GER R Mosel. 6577

$230^{2} 20 \mathrm{Ne}$ NDG OUB R Flerov. 11917

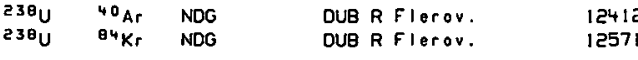

$A=108$ choln lisgion yiold

${ }^{238} U$ P $4.5+2 \quad C H I$ E Ponontint 4901

$A=108$ primory flssion yield flsgion frogments

$233_{\mathrm{J}} \mathrm{d} 1.4+1 \quad$ BRC E Patint 5934

$A=100$ primory fission yleld

$209_{B i} P \quad 3.2+1$ TUE R Gonnendeint 4761 


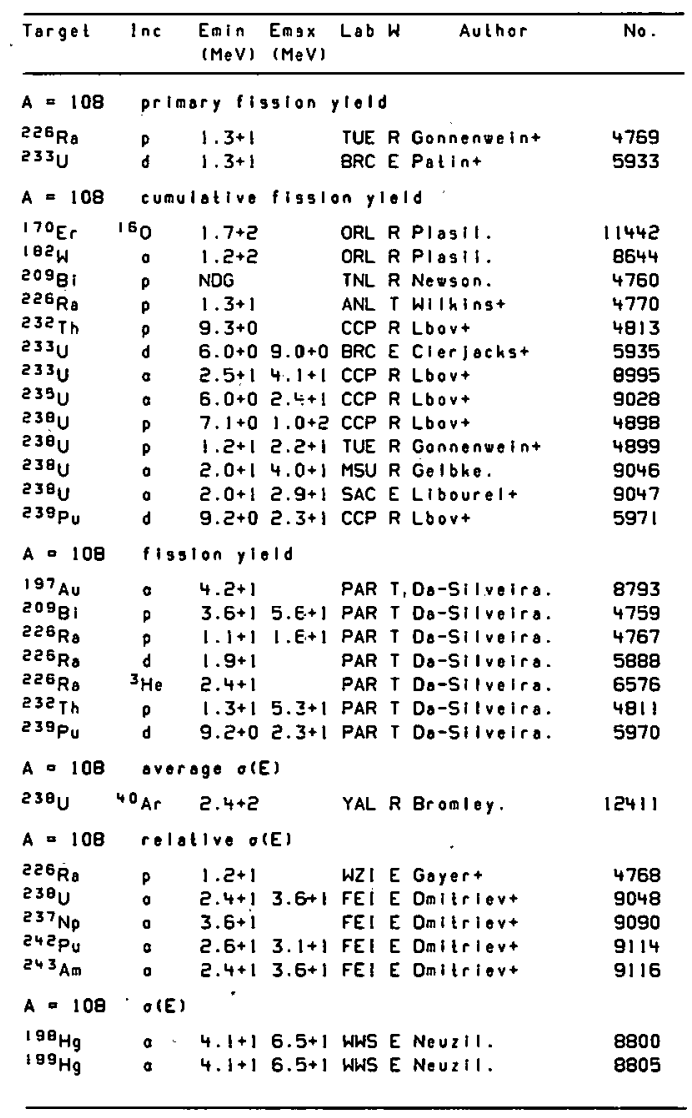

\begin{tabular}{|c|c|c|c|c|c|c|}
\hline Target & $\operatorname{lnc}$ & $\begin{array}{l}\text { Emin } \\
(M \in V)\end{array}$ & $\begin{array}{l}\operatorname{Emax} \\
(M e V)\end{array}$ & Lab $W$ & Author & No. \\
\hline$A=100$ & $O(E)$ & & & & & \\
\hline $200 \mathrm{Hg}$ & a & $4.1+1$ & & WWS E & Neu2il. & 8810 \\
\hline $201 \mathrm{Hg}$ & a & $4.1+1$ & & WWS E & Neuzil. & $89: 4$ \\
\hline $202 \mathrm{Hg}$ & 0 & $4.1+1$ & & WWS $\mathrm{E}$ & Neuzil. & 8821 \\
\hline $204 \mathrm{Hg}$ & a & $4.1+1$ & & WWS $\mathrm{E}$ & Neuzal. & 8828 \\
\hline $200_{\mathrm{BI}}$ & a & NDG & & GER R & Mosel. & 8927 \\
\hline $225_{\mathrm{Ra}}$ & $i$ & $2.3+1$ & & LAS E & Webert & 6068 \\
\hline $226 \mathrm{Ro}_{0}$ & ${ }^{3} \mathrm{He}$ & NDG & & GER $R$ & Mosel. & 6577 \\
\hline $238_{U}$ & ${ }^{12} \mathrm{C}$ & NDG & & DUB $R$ & Fierov. & 10157 \\
\hline $238 \mathrm{U}$ & $20_{\mathrm{Ne}}$ & NDG & & DUB $R$ & Flerov. & 11917 \\
\hline $238 \mathrm{U}$ & ${ }^{40} \mathrm{Ar}$ & NDG & & DUB $R$ & Flerov. & 12412 \\
\hline 2384 & ${ }^{84} \mathrm{Kr}$ & NOG & & DUB $R$ & Flerov. & 12571 \\
\hline
\end{tabular}

$A=109$ chointission yleld

${ }^{238} \mathrm{U} P \quad 4.5+2$ CHI E Panointint 4901

$A=109$ prtary flssion yiold fission irogments

$233 \mathrm{U} d 1.4+1 \quad$ BRC E PBlint 5934

$A$ - 109 prisory fission yield

$209_{\mathrm{BI}} P \quad 3.2+1$ TUE R Gonnenveint 4761

$\begin{array}{lllll}209^{\circ I} & p & 3.2+1 & \text { TUE R Gonnenvelnt } & 4761 \\ 226 \mathrm{Ro} & \mathrm{p} & 1.3+1 & \text { TUE R Gonnengelnt } & 4769\end{array}$

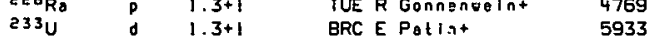

$A=109$ cuaviative flission yleld

\begin{tabular}{|c|c|c|c|c|c|c|}
\hline $\begin{array}{l}170 \mathrm{Er} \\
182_{\mathrm{W}}\end{array}$ & 160 & $\begin{array}{r}1.7+2 \\
1.2+5\end{array}$ & & ORL & R Plasil. & $\begin{array}{r}11442 \\
8644\end{array}$ \\
\hline $209^{81}$ & 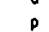 & NDG & & TNL & $R$ Newgon. & 4760 \\
\hline $226^{\circ} \mathrm{s}$ & p & $1.3+1$ & & ANL & I Wilhinst & 4770 \\
\hline${ }^{232} \mathrm{Th}$ & p & $9.3+0$ & & CCP & R Lbovt & 4813 \\
\hline $233_{\mathrm{U}} \mathrm{U}$ & d & $9.0+0$ & $2.3+1$ & CCP & $R$ Lbov- & 5932 \\
\hline $233 \mathrm{U}$ & d & $6.0+0$ & $9.0+0$ & BRC & E cleriachst & 5935 \\
\hline $233 \mathrm{U}$ & a & $2.5+1$ & $4.1+1$ & CCP & $R$ Lbov:- & 8995 \\
\hline${ }^{235} \mathrm{U}$ & p & $1.2+1$ & $8.5+1$ & CCP & R Lbov- & 4849 \\
\hline $235 \mathrm{u}$ & a & $6.0+0$ & $2.4+1$ & CCP & R Lbove & 9028 \\
\hline $238 \mathrm{U}$ & p & $7.1+0$ & $1.0+2$ & $\mathrm{CCP}$ & R Lbovt & 4898 \\
\hline $238 \mathrm{U}$ & $p$ & $1.2+1$ & $2.2+1$ & TUE & R Gonnenuelnt & 4899 \\
\hline $23 B_{u}$ & a & $2.0+1$ & $4.0+1$ & MSU & R.Gelble & 9046 \\
\hline $238 \mathrm{Y}$ & 。 & $2.0+1$ & $2.9+1$ & SAC & E Libourel+ & 9047 \\
\hline $239 \mathrm{p}$ & d & $9.2+0$ & $2.3+1$ & $\mathrm{CCP}$ & R Lbovt & 5971 \\
\hline
\end{tabular}




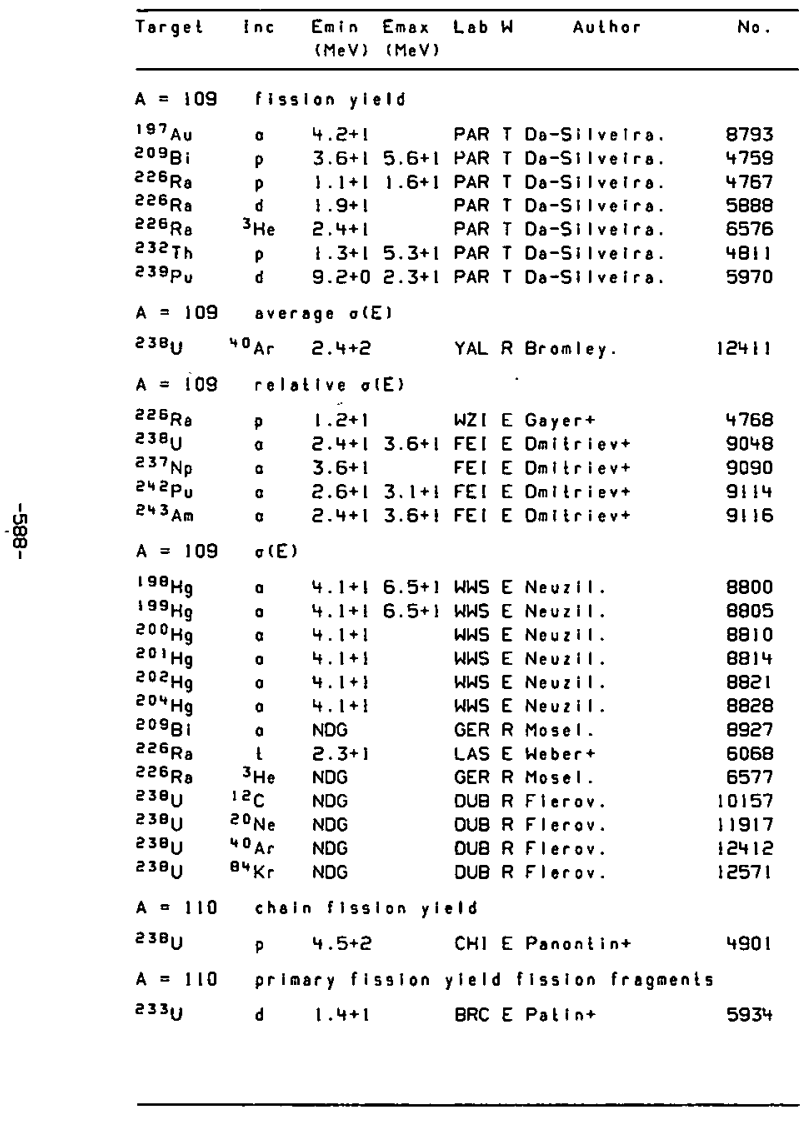

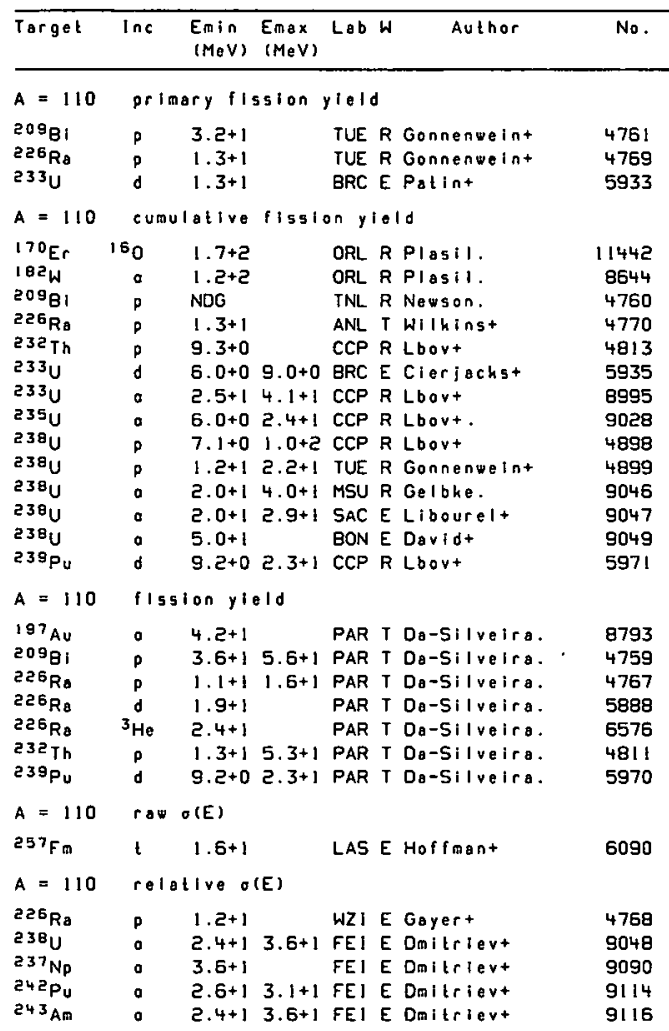




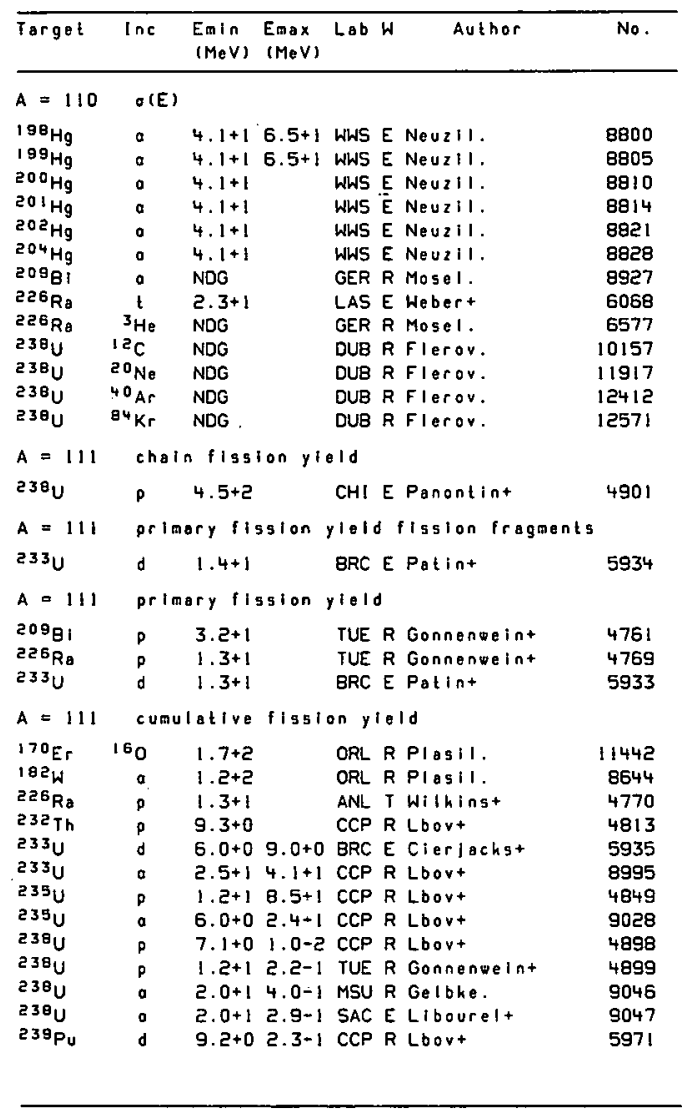

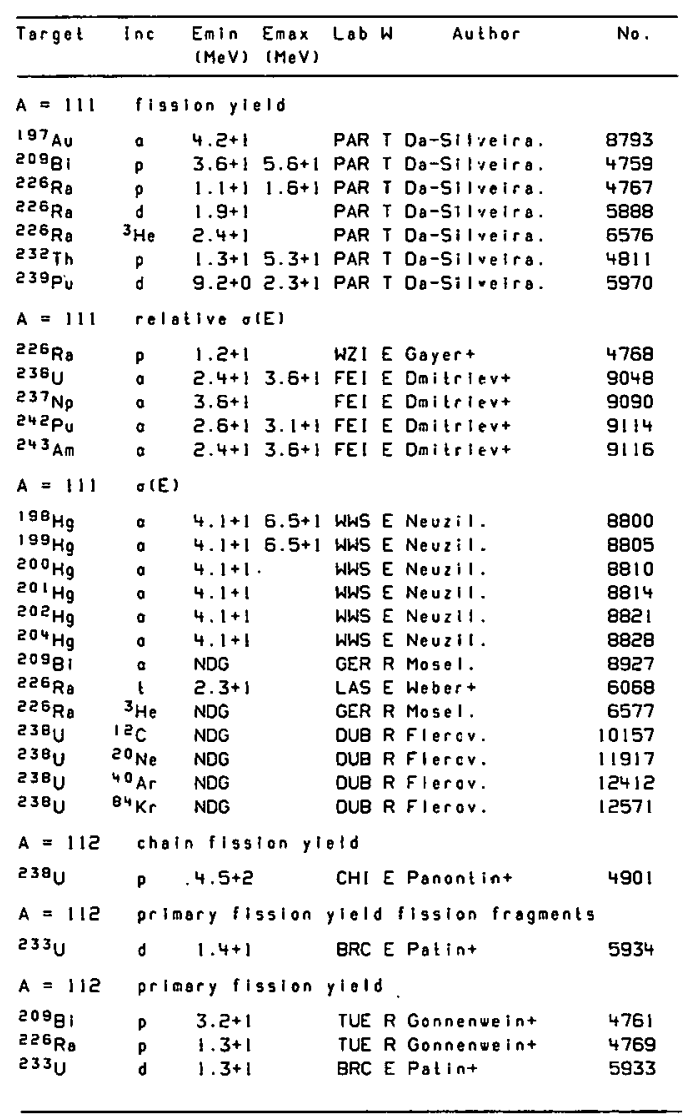




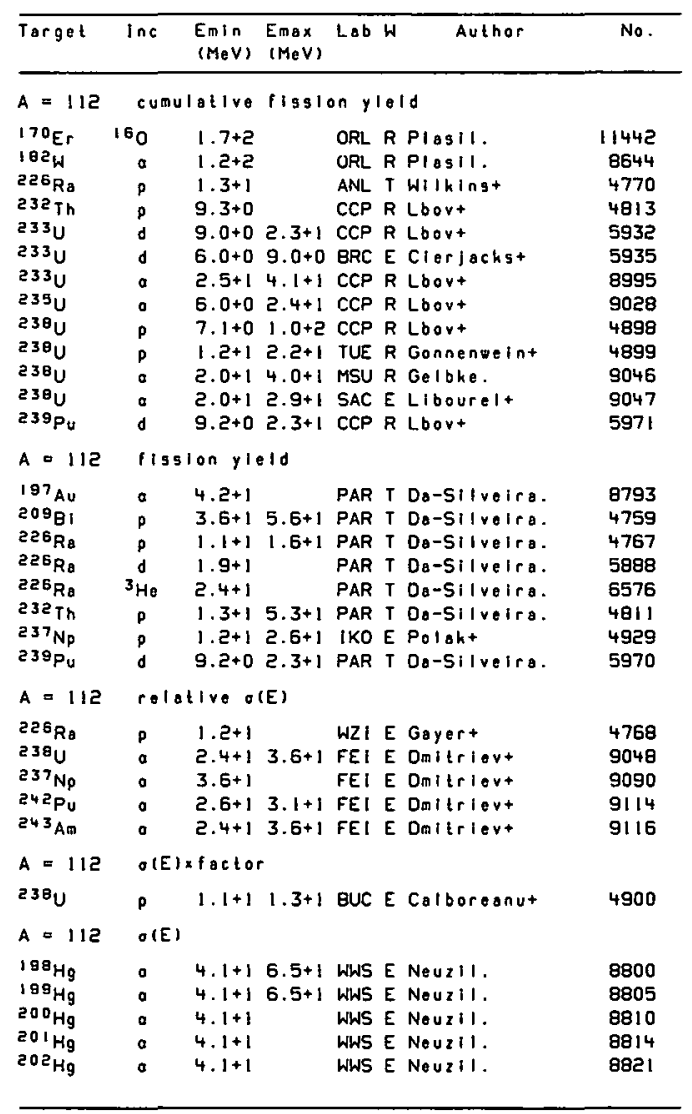

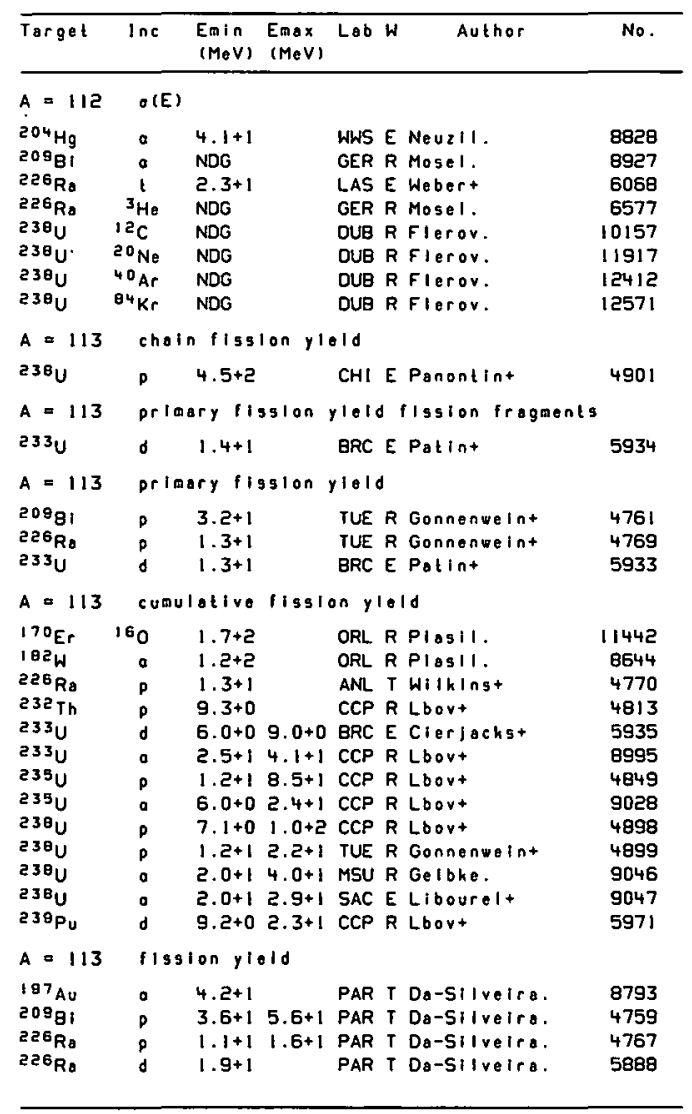




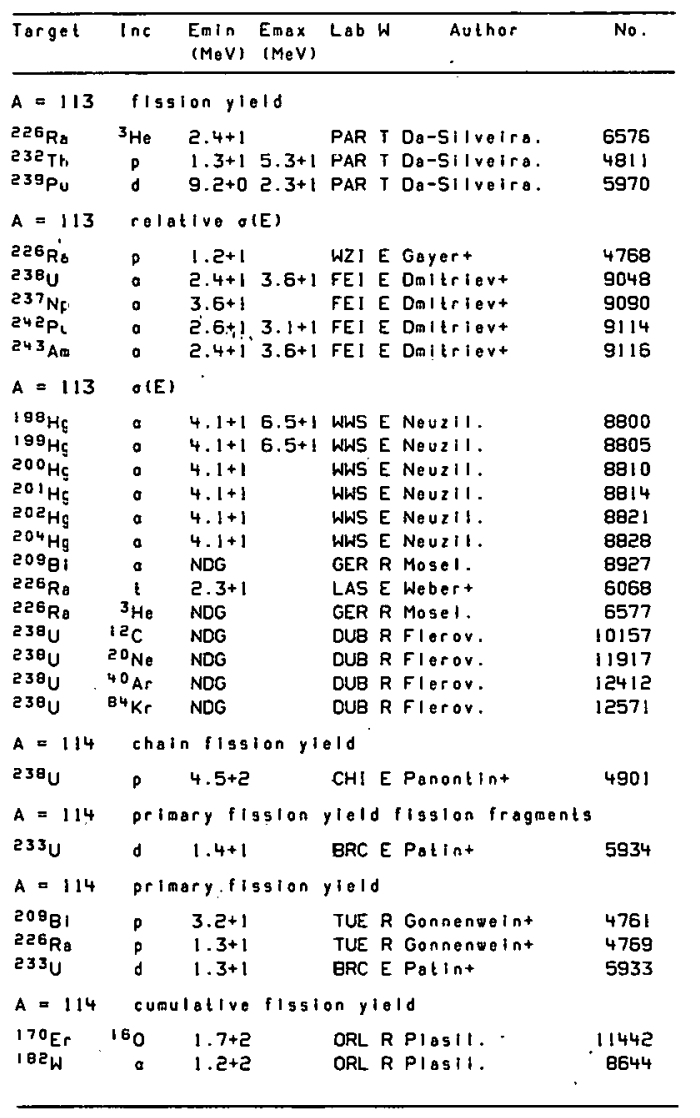

\begin{tabular}{|c|c|c|c|c|c|c|}
\hline Target & $\operatorname{lnc}$ & $\begin{array}{l}\text { Emln } \\
(M \circ V)\end{array}$ & $\begin{array}{l}\text { Emox } \\
\left(M_{\theta} V\right)\end{array}$ & Lob $W$ & Author & No. \\
\hline
\end{tabular}

$A=114$ cumulative flagion yleld

${ }^{226} \mathrm{Ra} \quad p \quad 1.3+1 \quad$ ANL T WIIhIng+ 4770

$232 \mathrm{Th} P \mathrm{P} \quad 9.3+0 \quad$ CCP R Lbovt 4813

$233 \mathrm{U} d 6.0+09.0+0$ BRC E Cierjackst 5935

$233 \mathrm{U}$ a $2.5+14.1+1$ CCP R Lbovt 8995

$235 \mathrm{U} \quad 0.0+02.4+1$ CCP R Lbovt 9028

$\begin{array}{lllll}230 & P & 7+1+0 & 1.0+2 \text { CCP R Lbovt } & 4898\end{array}$

$230 \mathrm{U} P$ P. $1.2+12.2+1$ TUE R Gonnenvelint 4899

230U $02.0+14.0+1$ MSU R Gelbke. 9046

$23 \theta_{U} 0$ 2.0+1 $2.9+1$ SAC E Llbourelt 9047

${ }^{239} \mathrm{Pu} d \quad 9.2+02.3+1$ CCP R Lbovt 597

$A=114 \quad f 135100 y+10$

$197 \mathrm{Au} \quad 4.2+1 \quad$ PART Da-Silveira. 8793

20981 3.6+15.6+1 PAR T Da-Silvelra. 4759

$226 R_{a} \quad P \quad 1.1+1 \quad 1.6+1$ PAR T Da-Silveira. 4767

${ }^{226} R_{a} d \quad 1.9+1 \quad$ PAR T Da-Silveira. 5888

$226 \mathrm{R}_{0}{ }^{3} \mathrm{He} \quad 2.4+1 \quad$ PAR T Da-Silveira. 6576

23eTh $P$ Th $1.3+15.3+1$ PAR T Da-Silveira. 4811

$239 \mathrm{Pu}$ d $9.2+02.3+1$ PAR T Da-5ilveira. 5970

$A=114$ Pelalive $O(E)$

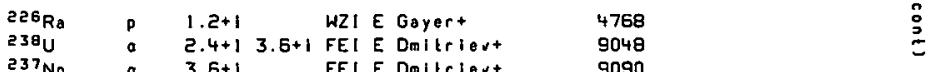

$237 \mathrm{~Np} \quad 3.6+1 \quad$ FEI E Dmilriert 9090

$242 P_{2} \quad 2.6+13.1+1$ FEI E Dmililert 9114

$A=114 \quad$ (E)

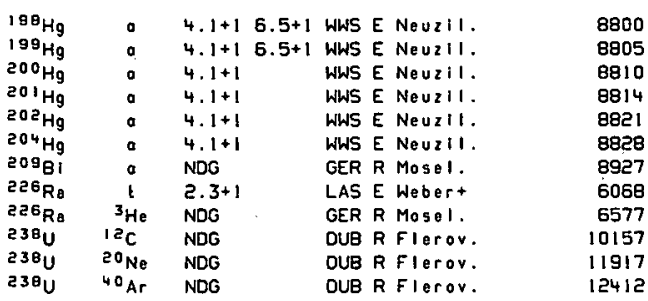




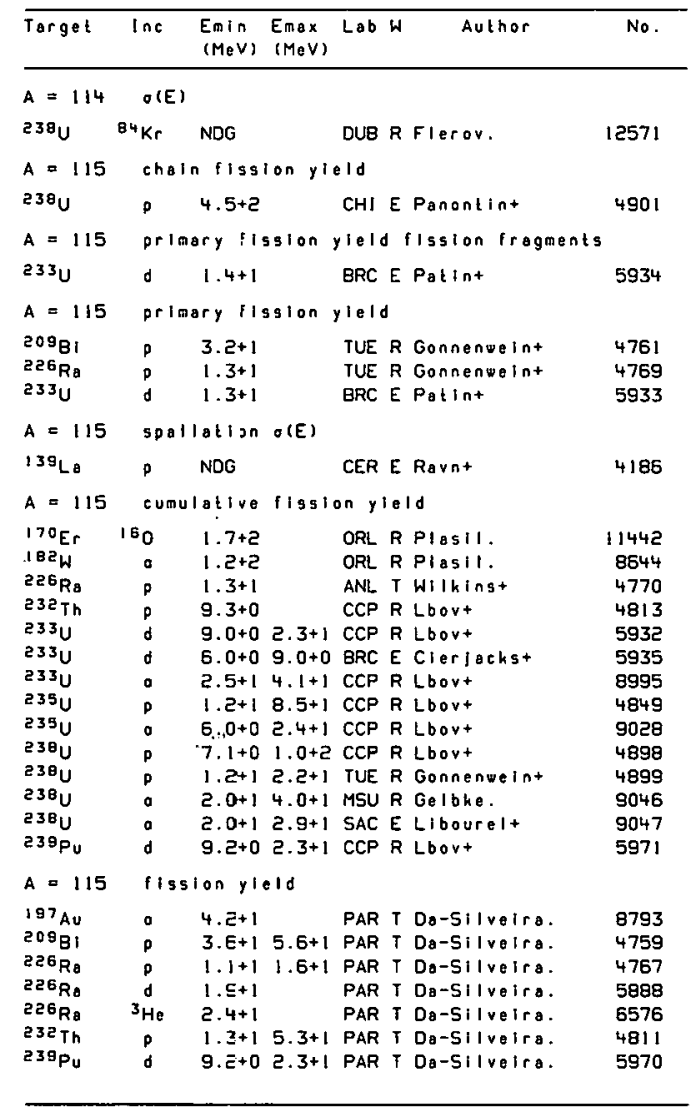

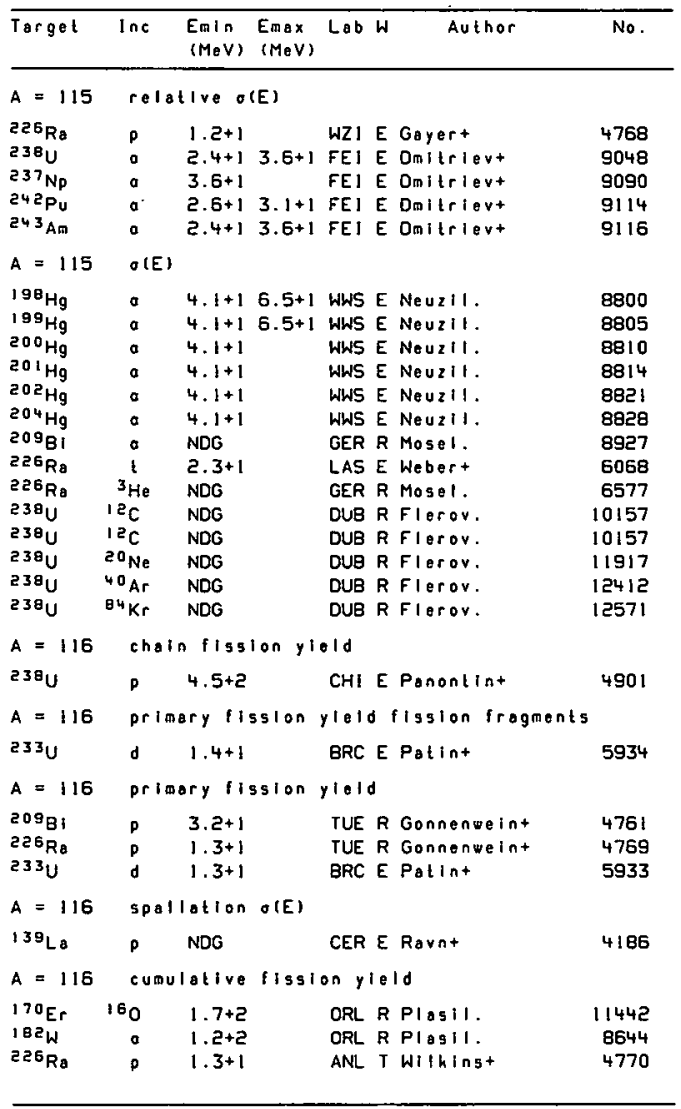




\begin{tabular}{|c|c|c|c|c|c|c|}
\hline Target & $\ln c$ & $\begin{array}{l}\text { Emin } \\
(M e V)\end{array}$ & $\begin{array}{l}\varepsilon_{\max } \\
(\operatorname{MeV})\end{array}$ & Lab $W$ & Author & No. \\
\hline
\end{tabular}

$A=116$ cumulative fission yield

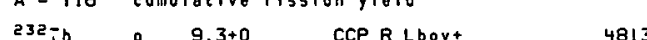

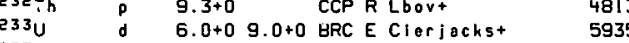

$233 \mathrm{U}$ a $2.5+14.1+1$ CCP R Lbovt 8995

$235 \mathrm{U}$ a $6.0+02.4+1$ CCP R Lbovt 9028

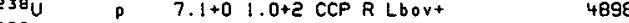

$230^{2} \quad P \quad 1.2+12.2+1$ TUE R Gonnenweint 4899

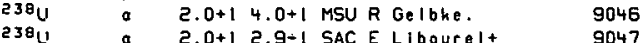

239Fu d $2.0+1$ 2.9-1 SAC E Libourelt 5

$A=116$ fission yleld

${ }^{209} \mathrm{~B}_{1} P \quad 3.6+15.6+1$ PAR T $D_{B}-5$ ilveifo. 4759

226 Ro $P$ P $1+11.6+1$ PAR T Da-5ilveira. 4757

$25 R_{R} \quad 1.9+1$ PAR T De-5ilvelre 5988

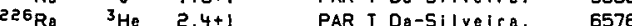

$232 \mathrm{Th} \quad 1.3+15.3+1$ PAR T Da-5ilveira. 6576

4811
5970

$A=116$ relative o(E)

$226 R_{B} P \quad 1.2+1 \quad$ WZI E Goyert 4768

$238^{2}$ a $2.4+13.6+1$ FEI E Omitrlevt 9048

$237 \mathrm{No}$ a $3.6+1$ FEl E Dmitrlevt 9090

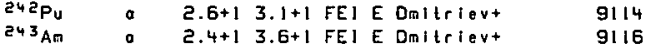

$A=: 16 \quad \sigma(E)$

${ }^{65} \mathrm{Cu} \quad{ }^{63} \mathrm{Cu} \quad 5.0+1 \quad 1.1+2$ PAR M Girard+ 12478

$20{ }^{0} \mathrm{BI} a$ NDG GER R Mosel. 1957

$255_{0} R_{0} \quad 2.3+1$ LASE Webert 6068

${ }^{226} \mathrm{Re}{ }^{3} \mathrm{He}$ NDG GER R Mosel. 6577

$23 \theta^{2}{ }^{32} \mathrm{C}$ NDG DUB R Flerov. 10157

$230 \mathrm{U} 20 \mathrm{Ne}$ NDG DUB R Flerov. 11917

$238 \mathrm{U} 40 \mathrm{Ar}$ NDG DUB R Flerov. 12412

$23 \mathrm{BU}^{84} \mathrm{Kr}$ NDG DUB R Flerov.

$A=117$ chain ission $y: e 1 d$

$230_{U} \quad 4.5+2 \quad C H I$ E Panontint 4901

$A=117$ primaryfission yleld fission fregments

$233 \mathrm{U} d 1.4+1 \quad$ ERC E Palint 5934

\begin{tabular}{|c|c|c|c|c|c|c|}
\hline Target & Inc & $\begin{array}{l}\text { Emin } \\
(\mathrm{MeV})\end{array}$ & $\begin{array}{l}E \max \\
(\operatorname{MaV})\end{array}$ & Lab & Aulhor & No... \\
\hline
\end{tabular}

$A=117$ primary fission yield

$209 \mathrm{Bi} P 3.2+1$ TUE R Gonnenweint 4761

$226 R_{a} \quad p \quad 1.3+1 \quad$ TUE R Gonnenweint 4769

$233 \mathrm{U} d \mathrm{~d} 1.3+1 \quad$ BRC E Palitit 5933

$A=117$ spallalion $O(E)$

139LO $P$ NDG CER E Ravnt

4186

$A=117$ cumulative ission yield

$\begin{array}{lrrrr}170 \mathrm{Er} & 160 & 1.7+2 & \text { ORLR Plasil. } & 11442 \\ 182 \mathrm{~W} & \mathrm{a} & 1.2+2 & \text { ORL RPIBSil. } & 8544\end{array}$

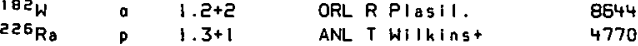

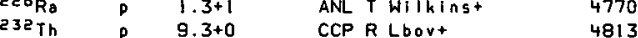

$233 \mathrm{U}$ o $9.0+02.3+1$ CCP R Loovt 4813

$233 \mathrm{U} d \quad 6.0+0$ g. $0+0$ BRC E Clerlacist 5935

$233 \mathrm{U} \quad 2.5+14.1+1$ CCP R Lbort

$235 \mathrm{U} P \quad 1.2+18.5+1$ CCP R Lbovt 4849

$235 \mathrm{U} \quad 6.0+02.4+1$ CCPR Lbovt

$23 \theta_{U} P \quad 7.1+01.0+2$ CCP R Lbovt

$23 \theta_{U} P \quad 1.2+13.2+1$ TUE R Gonnenveint 4898

$23 \theta_{4} \quad 1.2+12.2+1$ TSE R Gonnenveint 4899

$230^{2} \quad 2.0+14.0+1$ MSU R Geloke. 9046

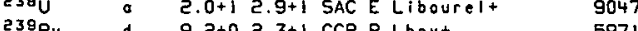

d $9.2+02.3+1$ CCP R Lbout 5971

$A=117$ fission yield

$209_{B i} P \quad 3.6+15.6+1$ PAR T Da-Silveira, 4759

${ }^{226_{R a}} \quad P \quad 1.1+11.6+1$ PAR T Da-Silveira. 4767

$25 R_{A} d \quad 1.9+1$ PAR T Da-silvelra. 5888

${ }^{226 \mathrm{Ra}}{ }^{3} \mathrm{He} \quad 2.4+1 \quad$ PAR T Da-Silverra. 6576

$232 \mathrm{Th} \quad \mathrm{O} \quad 1.3+15.3+1$ PAR T Da-Silveira. 4811

9.2+0 $2.3+1$ PAR I De-Silveira. 5970

$A=117$ relative $O(E)$

$226 \mathrm{Ra} D 1.2+1$ D 21 E Gayert 4768

$23 \theta_{U}$ a $2.4+13.6+1$ FEI E Dmitrievt 9048

$237 \mathrm{~Np}$ a 3.6+1 FEI E Daitrievt 9090

${ }^{242} \mathrm{Pu} \quad$ a $2.6+13.1+1 \mathrm{FEI} \mathrm{E}$ Dmitriev+ 9114

$243 \mathrm{Am}$ a $2.4+13.6+1$ FEI E Dmitrlevt 9116

$A=117 \quad \sigma(E)$

${ }^{65} \mathrm{Cu}{ }^{63} \mathrm{Cu} \quad 5.0+11.1+2$ PAR M GIrard+ 12478 


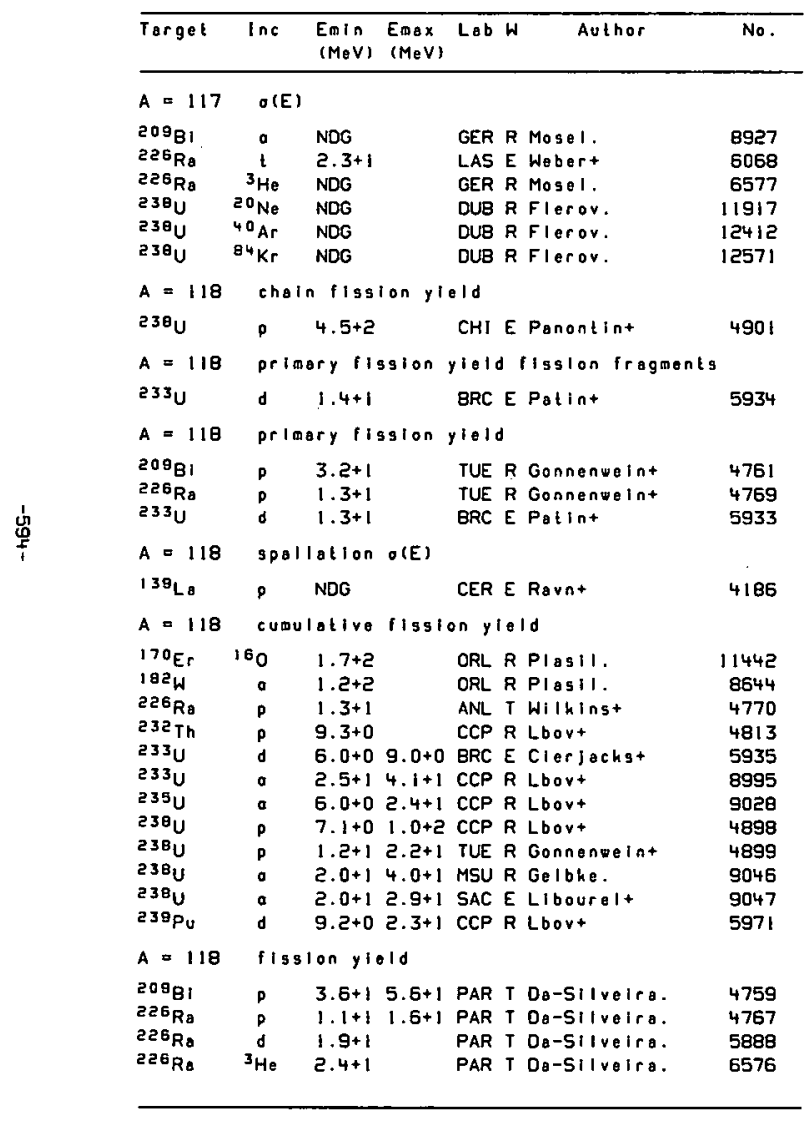

\begin{tabular}{|c|c|c|c|c|c|c|}
\hline Target & $\operatorname{Inc}$ & $\begin{array}{l}\text { Enin } \\
(M e V)\end{array}$ & $\begin{array}{l}\text { Emax } \\
(M \mathrm{M} V)\end{array}$ & Lab W & Author & No. \\
\hline
\end{tabular}

$232 \mathrm{Th} P \quad 1.3+15.3+1$ PAR T Da-Silveira. 4811

239Pu d $9.2+02.3+1$ PAR T Da-Silveira. 5970

$A=118$ rolalive $\sigma(E)$

$226_{\mathrm{Ra}} P \quad 1.2+1 \quad$ WZI E Gayert 4768

$238 \mathrm{U}$ a $2.4+13.6+1$ FEI E Dmitriert 9048

$237 \mathrm{Ng}$ a $3.6+1 \quad$ FEI E Daltrlevt 9090

$242 P_{0}$ a $2.6+13.1+1$ FEI E Dilirievt 9114

$243 \mathrm{Am}$ a $2.4+13.6+1$ FEI E Dmilerievt 9116

$A=11 \theta \quad \sigma(E)$

${ }^{65} \mathrm{Cu}{ }^{63} \mathrm{Cu} 5.0+1 \quad 1.1+2$ PAR M Girardt 12478

${ }^{209} \mathrm{BI}$ a NDG GER R Mosel.

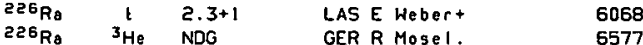

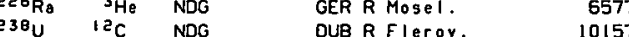

$38^{2} 20^{N}$ NDG OLBR Fleroy.

$238 \mathrm{U} 4 \mathrm{NOA}^{\mathrm{Ar}} \mathrm{NDG} \quad$ DUBR Flerov.

$238 \mathrm{U}{ }^{84} \mathrm{Kr}$ NDG $\quad$ OUBR Flerov. 12571

$A=119$ chain flgsion yield

$238 \mathrm{U} P \mathrm{P} 4.5+2$ CHi E Panontint 4901

$A=119$ priary lission yield figsion fragaents

$233 \mathrm{U}$ o $1.4+1 \quad$ BRC E Palint 5934

$A=119$ primary fission yleld

${ }^{209} \mathrm{gr} P \quad 3.2+1$ TUE R Gonnenweint 4761

$\begin{array}{lllll}226 \mathrm{Ra} & 0 & 1.3+1 & \text { TUE R Gonnenweint } & 4769 \\ 233^{2} \mathrm{U} & d & 1.3+1 & \text { BRC E Palint } & 5933\end{array}$

$A=119$ spallation $O(E)$

139 La D NDG CER E Ravnt 4186

$A=119$ cumulative flission yield

$\begin{array}{lrlll}170 \mathrm{Er} & 160 & 1.7+2 & \text { ORL R Plasil. } & 11442 \\ 182 \mathrm{~W} & \mathrm{a} & 1.2+2 & \text { ORL R PIBsil. } & 8644 \\ 226 \mathrm{Ra} & \rho & 1.3+1 & \text { ANL T WIIhinst } & 4770 \\ 232 \mathrm{Th} & \mathrm{p} & 9.3+0 & \text { CCP R Lbovt } & 4813 \\ 233 \mathrm{U} & d & 6.0+09.0+0 & \text { BRC E Cleriochst } & 5935\end{array}$




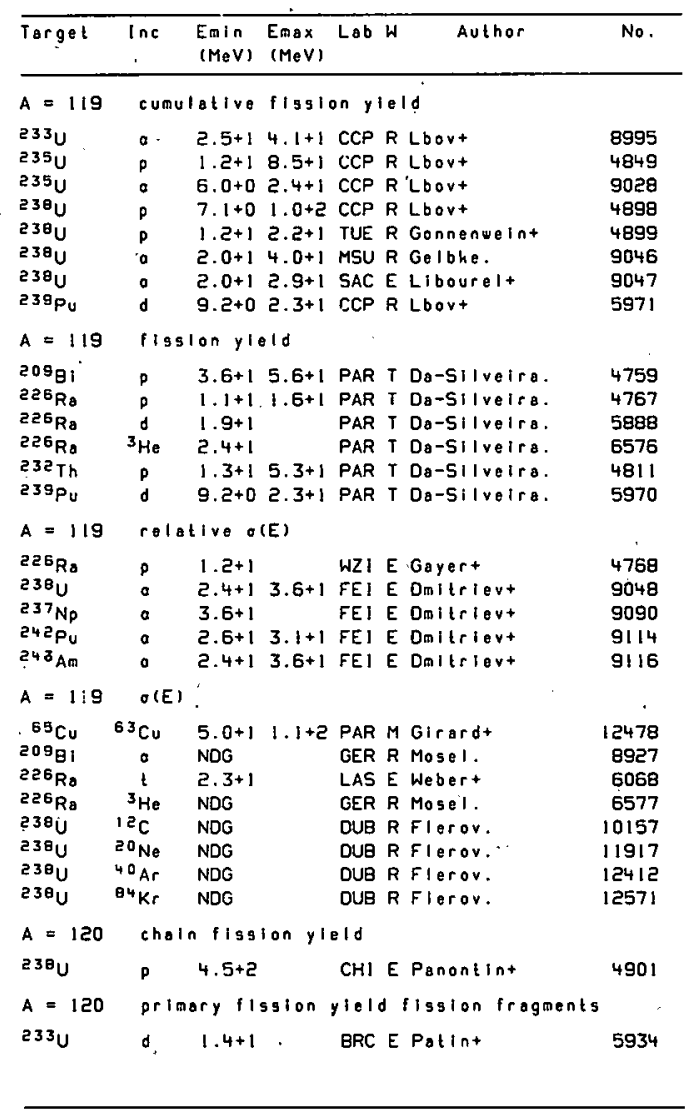

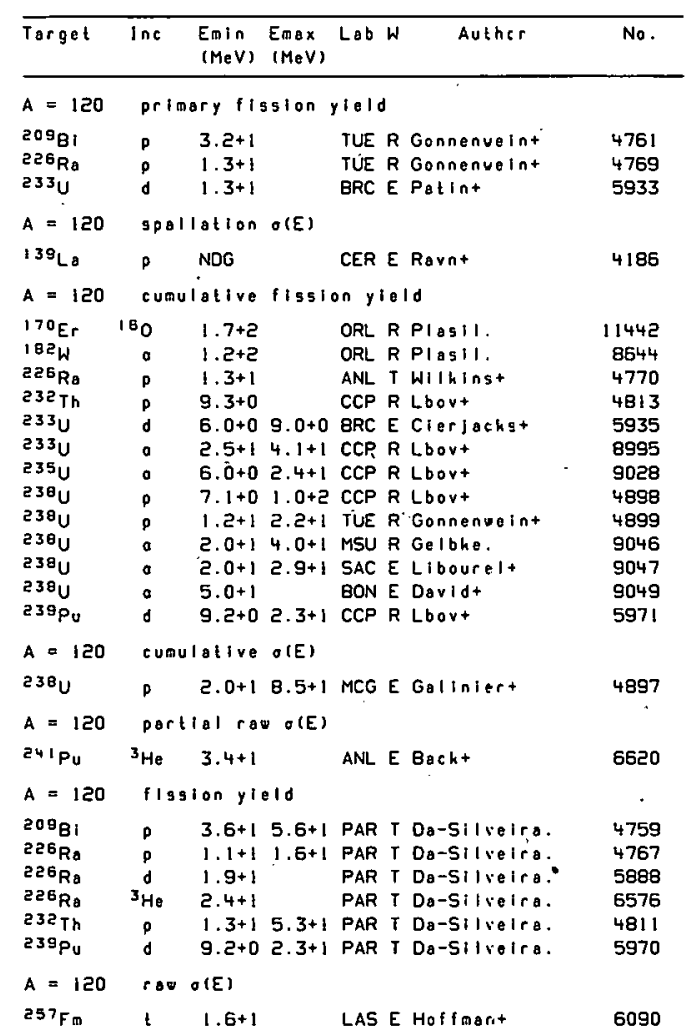




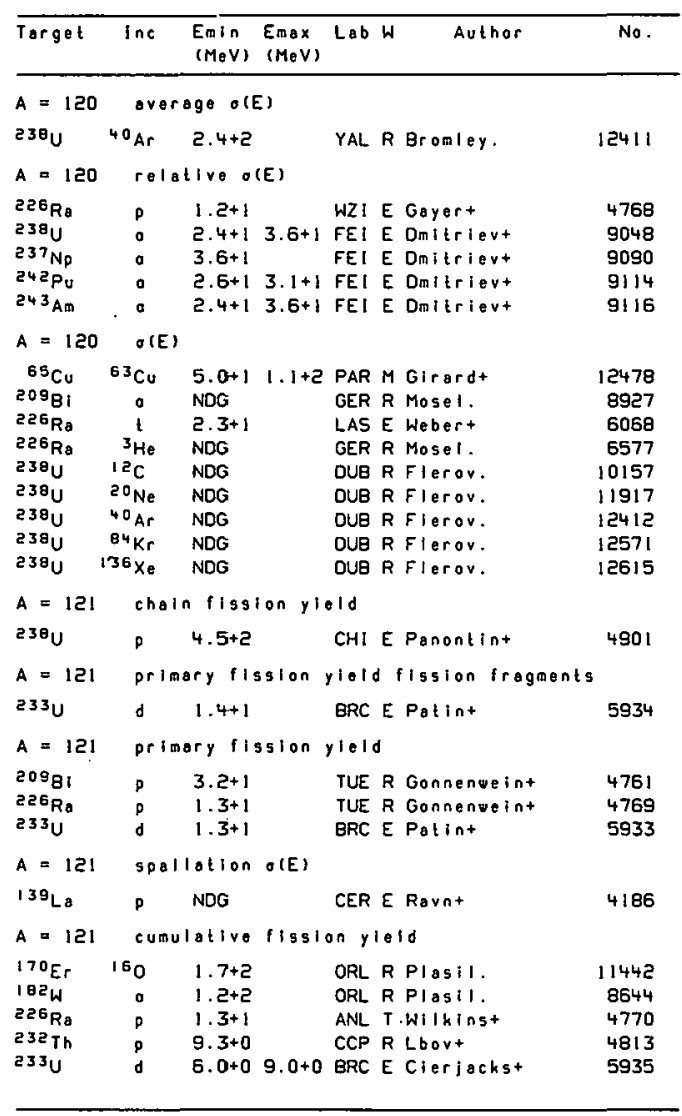

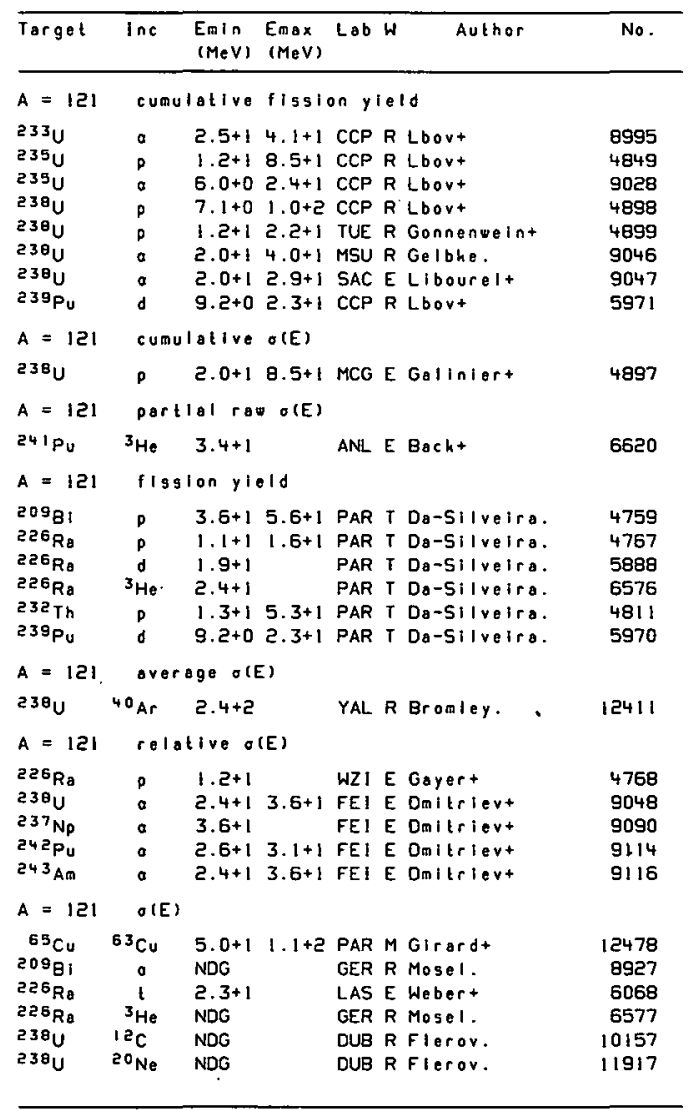




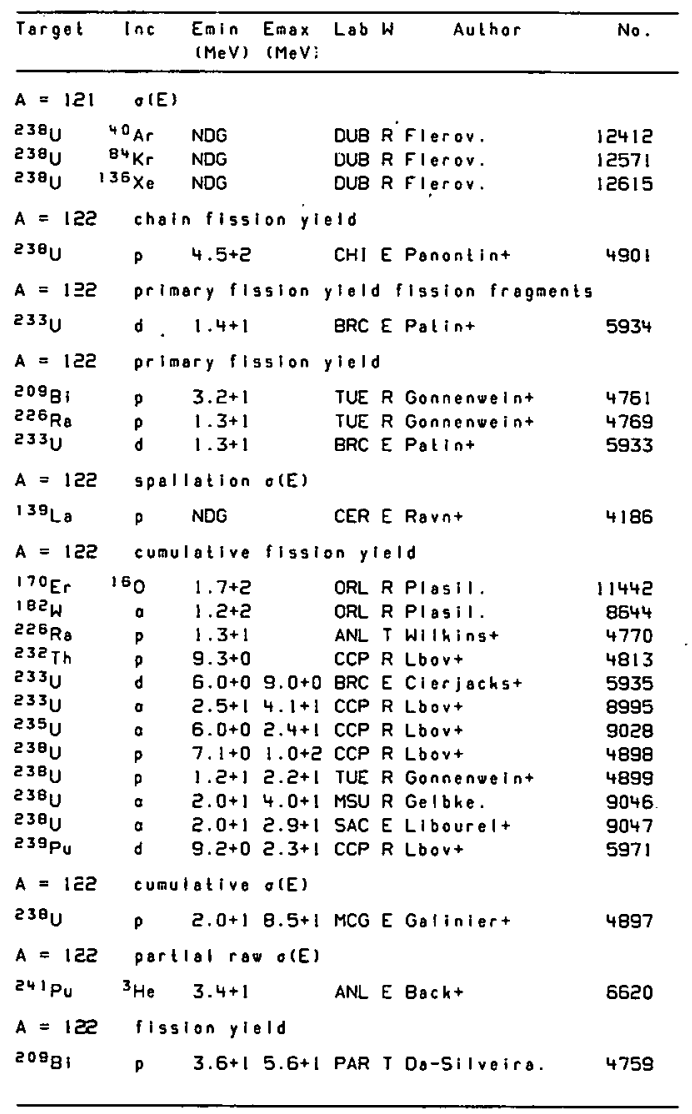

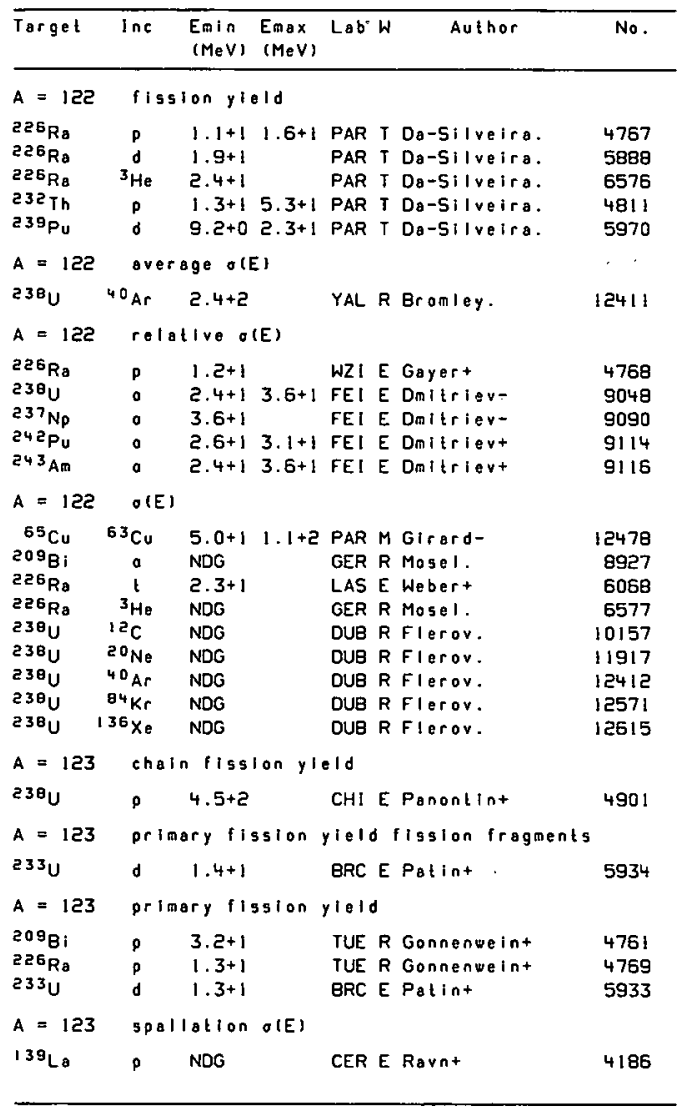




\begin{tabular}{|c|c|c|c|c|c|c|}
\hline Target & $\ln c$ & $\begin{array}{l}E_{m} / n \\
(\mathrm{MeV})\end{array}$ & $\begin{array}{l}\text { Emax } \\
(\operatorname{MeV})\end{array}$ & Lab & Author & No. \\
\hline$A=123$ & \multicolumn{6}{|c|}{3 cumulative lission yiald } \\
\hline 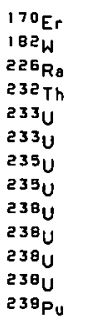 & $\begin{array}{c}160 \\
0 \\
p \\
p \\
d \\
a \\
p \\
0 \\
p \\
p \\
0 \\
0 \\
0\end{array}$ & $\begin{array}{l}1.7+2 \\
1.2+2 \\
1.3+1 \\
9.3+0 \\
6.0+0 \\
2.5+1 \\
1.2+1 \\
6.0+0 \\
7.1+0 \\
1.2+1 \\
2.0+1 \\
2.0+1 \\
9.2+0\end{array}$ & $\begin{array}{l} \\
9.0+0 \\
4.1+1 \\
8.5+1 \\
2.4+1 \\
1.0+2 \\
2.2+1 \\
4.0+1 \\
2.9+1 \\
2.3+1\end{array}$ & $\begin{array}{l}\text { ORL } \\
\text { URL } \\
\text { ANL } \\
\text { CCP } \\
\text { BRC } \\
\text { CCP } \\
\text { CCP } \\
\text { CCP } \\
\text { CCP } \\
\text { TUE } \\
\text { MSU } \\
\text { SAC } \\
\text { CCP }\end{array}$ & $\begin{array}{l}\text { R Plasil. } \\
R \text { Plasil. } \\
T \text { Wilkinst } \\
R \text { Lbovt } \\
\text { E Cleriachst } \\
R \text { Lbovt } \\
R \text { Lbovt } \\
R \text { Lbovt } \\
R \text { Lbovt } \\
R \text { Gonnenweint } \\
R \text { Gelbhe. } \\
\text { E Libourelt } \\
R \text { Lbovt }\end{array}$ & $\begin{array}{r}11442 \\
8644 \\
4770 \\
48 ! 3 \\
5935 \\
8995 \\
4849 \\
9028 \\
4898 \\
4899 \\
9046 \\
9047 \\
5971\end{array}$ \\
\hline$A=123$ & \multicolumn{6}{|c|}{ C Cumulatlve $O(E)$} \\
\hline $\begin{array}{l}{ }^{238} U \\
A=123\end{array}$ & \multicolumn{5}{|c|}{3 partlal row o(E). } & 4897 \\
\hline $241 P_{u}$ & ${ }^{3} \mathrm{He}$ & $3.4+1$ & & ANL & E Back+ & 6620 \\
\hline$A=123$ & \multicolumn{6}{|c|}{ fission yield } \\
\hline $\begin{array}{l}\log _{\mathrm{Bl}} \\
226 \mathrm{Ra} \\
226 \mathrm{Ra} \\
226 \mathrm{Ra} \\
232 \mathrm{Th} \\
23 \mathrm{Pu}^{2}\end{array}$ & $\begin{array}{c}\rho \\
\rho \\
d \\
3_{\mathrm{He}} \\
\rho \\
d\end{array}$ & $\begin{array}{l}3.6+1 \\
1.1+1 \\
1.9+1 \\
2.4+1 \\
1.3+1 \\
9.2+0\end{array}$ & $\begin{array}{l}5.6+1 \\
1.6+1 \\
\\
5.3+1 \\
2.3+1\end{array}$ & $\begin{array}{l}\text { PAR } \\
\text { PAR } \\
\text { PAR } \\
\text { PAR } \\
\text { PAR } \\
\text { PAR }\end{array}$ & $\begin{array}{ll}\text { T } & \text { Da-SIIVeira. } \\
\text { T Da-SIIVeira. } \\
\text { T Da-Silveira. } \\
\text { T Da-SIIvelra. } \\
\text { T Da-Silveira. } \\
\text { T Da-Silveira. }\end{array}$ & $\begin{array}{l}4759 \\
4767 \\
5888 \\
6576 \\
4811 \\
5970\end{array}$ \\
\hline$A=123$ & \multicolumn{6}{|c|}{ average $O(E)$} \\
\hline $\begin{array}{l}230 \mathrm{U} \\
A=123\end{array}$ & \multicolumn{5}{|c|}{ relotive o(E) } & 12411 \\
\hline $\begin{array}{l}226 \mathrm{Ra} \\
23 \theta_{\mathrm{U}} \\
237_{\mathrm{Np}} \\
242 \mathrm{Pu} \\
24{ }^{\mathrm{Am}}\end{array}$ & $\begin{array}{l}p \\
a \\
a \\
a\end{array}$ & $\begin{array}{l}1.2+1 \\
2.4+1 \\
3.6+1 \\
2.6+1 \\
2.4+1\end{array}$ & $\begin{array}{l}3.6+1 \\
3.1+1 \\
3.6+1\end{array}$ & $\begin{array}{l}\text { WZI } \\
\text { FEI } \\
\text { FEI } \\
\text { FEI } \\
\text { FEI }\end{array}$ & $\begin{array}{l}\text { E Gayert } \\
\text { E Ditrlevt } \\
\text { E Dmitrievt } \\
\text { E Dmitrievt } \\
\text { E Dmitrievt }\end{array}$ & $\begin{array}{l}4768 \\
9048 \\
9090 \\
9114 \\
9116\end{array}$ \\
\hline$A=123$ & \multicolumn{6}{|c|}{$O(E)$} \\
\hline${ }^{65} \mathrm{Cu}$ & ${ }^{63} \mathrm{Cu}$ & $5.0+1$ & $1.1+2$ & PAR & MGirard+ & 12478 \\
\hline
\end{tabular}

\begin{tabular}{|c|c|c|c|c|c|c|}
\hline Torget & $\ln c$ & $\begin{array}{l}\text { Emin } \\
(M e V)\end{array}$ & $\begin{array}{l}\text { Emax } \\
(M e V)\end{array}$ & Lab $W$ & Author & No. \\
\hline$A=123$ & $O(E)$ & & & & & \\
\hline $\begin{array}{l}209 g_{1} \\
22 R_{0}\end{array}$ & $i$ & $\begin{array}{l}\text { NDG } \\
2.3+1\end{array}$ & & $\begin{array}{l}\text { GER R } \\
\text { LAS E }\end{array}$ & $\begin{array}{l}\text { Mosel. } \\
\text { Webert }\end{array}$ & $\begin{array}{l}8927 \\
6068\end{array}$ \\
\hline $22{ }^{6} R_{a}$ & ${ }^{3} \mathrm{He}$ & NDG & & GER $R$ & Mosel. & 6577 \\
\hline 2380 & $12 c$ & NDG & & DUB $R$ & Flerov. & 10157 \\
\hline $238 \mathrm{U}$ & $20 \mathrm{Ne}$ & NDG & & DUB $\mathrm{R}$ & Flerov. & 11917 \\
\hline $238 \mathrm{U}$ & $40 \mathrm{Ar}$ & NDG & & DUQ $R$ & Flerov. & 12412 \\
\hline $238 \mathrm{U}$ & ${ }^{84} \mathrm{Kr}$ & NDG & & DUB $R$ & Flerov. & 12571 \\
\hline $238 \mathrm{U}$ & $136 \times e$ & NDG & & DUB $R$ & Flerov. & 12615 \\
\hline
\end{tabular}

$238 \mathrm{U} P \quad 4.5+2 \quad \mathrm{CHI}$ E Panontint 4901

$A=124$ pelmary figsion yiold fission fragments

$233 \mathrm{U} d 1.4+1 \quad$ BRC E Patint 5934

$A=124$ primory figsion yield

$209_{B i} \quad 3.2+1$ TUE R Gonnenteint 4761

$\begin{array}{lllll}206_{\text {Ro }} & p & 3.2+1 & & \\ 23 & 1.3+1 & \text { TUE R Gonnenweint } & 4769\end{array}$

$233 \mathrm{U} O \mathrm{O} 1.3+1 \quad$ BRC E Patint 5933

$A=124$ spollation $O(E)$

139 LO $P$ NDS CERERBVnt 4186

$A=124$ cumulative fission yleld

$170 \mathrm{Er} 160 \quad 1.7+2 \quad$ ORL RPlOSil. 11442

$182 \mathrm{~W} O \quad 1.2+2$ ORL R PIASIl. 8544

$\begin{array}{lllll}225 \mathrm{RB} & \mathrm{p} & 1.3+1 & \text { ANL T Wilkinst } & 4770 \\ 232 \mathrm{Th} & \mathrm{p} & 9.3+0 & \text { CCP R Lbovt } & 4813\end{array}$

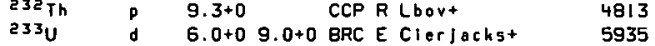

$233 \mathrm{U} \quad$ a $2.5+14.1+1$ CCP R Lbovt 8995

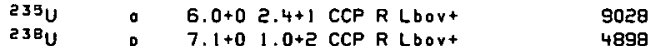

$23 \theta_{U} p \quad 1.2+12.2+1$ TUE R Gonnenweint 4899

$238 \mathrm{U} a \mathrm{2} .0+14.0+1$ MSU R Gelbhe. 5046

$\begin{array}{lll}238 \mathrm{U} & 2.0+12.9+1 \text { SAC E Libourel+ } & 9047\end{array}$

$239 \mathrm{Pu}_{4}$ d $9.2+02.3+1$ CCP R Lbovt 5971

$A=124$ cumulative $O(E)$

$238 U$ Q $2.0+18.5+1$ MCG E GBliniert 4897 


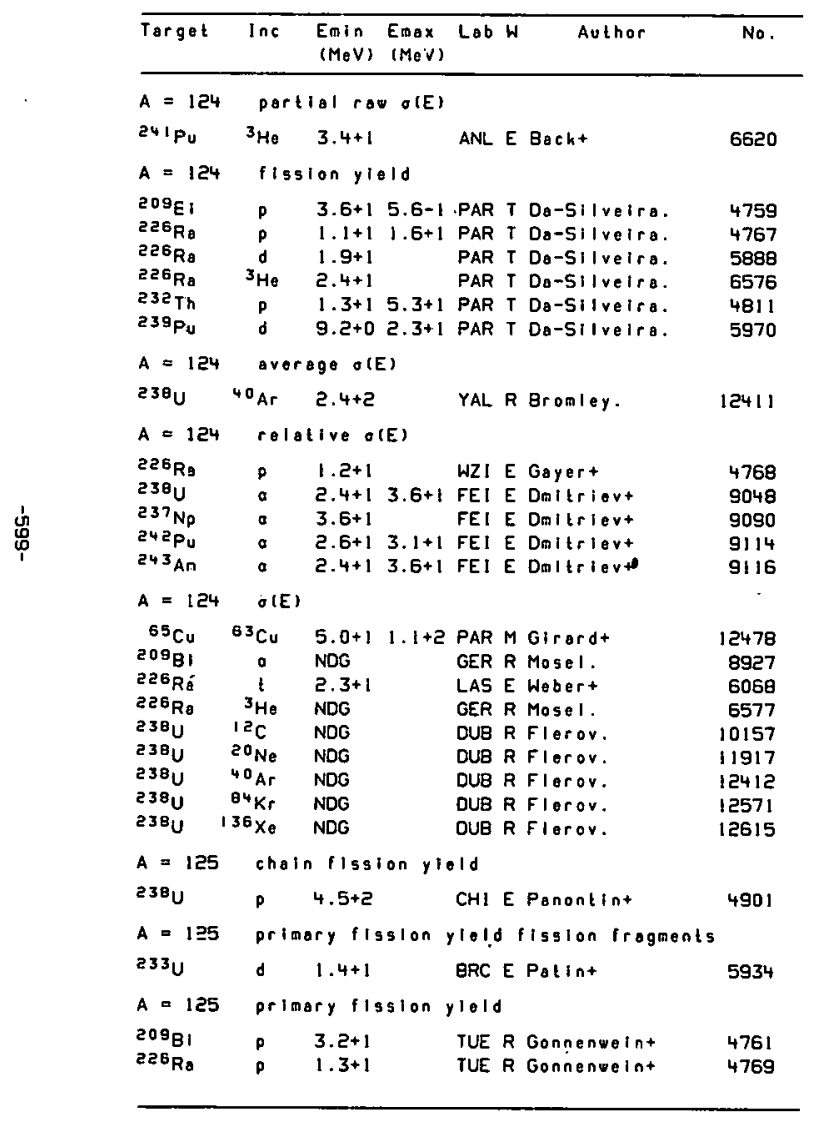

\begin{tabular}{|c|c|c|c|c|c|c|}
\hline Target & $\ln c$ & $\begin{array}{l}\text { Eain } \\
\text { (MeV) }\end{array}$ & $\begin{array}{l}\text { Emax } \\
(\operatorname{Mev})\end{array}$ & Lab W & Guthor & No. \\
\hline$A=125$ & \multicolumn{6}{|c|}{ primary fission yield } \\
\hline $233 \mathrm{U}$ & d & $1.3+1$ & & BRC E & E Potint & 5933 \\
\hline$A=125$ & \multicolumn{3}{|c|}{ spallation o(E) } & & \\
\hline $139 \mathrm{La}$ & $\rho$ & NDG & & CER E & E Roynt & 4185 \\
\hline$A=125$ & \multicolumn{2}{|c|}{ cumulative } & \multicolumn{3}{|c|}{ fission yield } & \\
\hline $170 \mathrm{Er}$ & ${ }^{160} 0$ & $1.7+2$ & & \multirow{2}{*}{\multicolumn{2}{|c|}{ ORL R PIasil. }} & 11442 \\
\hline $182_{W}$ & $\circ$ & \multirow{2}{*}{$\begin{array}{l}1.2+2 \\
1.3+1\end{array}$} & & \multirow{2}{*}{\multicolumn{2}{|c|}{$\begin{array}{l}\text { ORL R PIasil. } \\
\text { ANL T Wilkinst, }\end{array}$}} & 8644 \\
\hline${ }^{256} \mathrm{Ra}$ & $p$ & & & & & 4770 \\
\hline $232 \mathrm{Th}$ & p & $9.3+0$ & & & 4813 \\
\hline $233 \mathrm{U}$ & d & $6.0+0$ & $9.0+0$ & $\begin{aligned} \text { CCP R } \\
.0 \text { BRC E }\end{aligned}$ & E Clerjachst & 5935 \\
\hline $233 \mathrm{U}$ & - & $2.5+1$ & $4.1+1$ & CCP R & $R$ Lbovt & 8995 \\
\hline $235 \mathrm{U}$ & $\rho$ & $1.2+1$ & $8.5+1$ & CCP R & R Lbovt & 4849 \\
\hline $235 \mathrm{U}$ & o & $6.0+0$ & $2.4+1$ & CCP R & R Loovt: & 9028 \\
\hline $53 \theta^{2} u$ & p & $7.1+0$ & $1.0+2$ & CCP R & $R$ Lbovt & 4898 \\
\hline $238 \mathrm{U}$ & p & $1.2+1$ & $5.2+1$ & TUE $R$ & R Gonnenwelnt & 4899 \\
\hline $23 \theta^{2}$ & - & $2.0+1$ & $4.0+1$ & MSU $R$ & R Gelbke. & 9046 \\
\hline $23 \theta^{2}$ & a & $2.0+1$ & $2.9+1$ & SAC E & E Libourel+ & 9047 \\
\hline $238 \mathrm{U}$ & 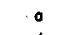 & $5 \cdot 0+1$ & & BON E & E Davidt & 9049 \\
\hline $230 \mathrm{Pu}$ & $d$ & $9.2+0$ & $2.3+1$ & $\mathrm{CCP} R$ & R Lbovt & 5971 \\
\hline$A=125$ & eum & ulative & $O(E)$ & & & \\
\hline $238_{U}$ & p & $2.0+1$ & $8.5+1$ & MCG E & E Goliniert & 4897 \\
\hline$A=125$ & par & tal raw & - $\sigma(E)$ & & & \\
\hline $241 \mathrm{Pu}$ & ${ }^{3} \mathrm{He}$ & $3.4+1$ & & ANL E & Eacht & 6620 \\
\hline$A=125$ & $\mathrm{fts}$ & ion yio & Q1d & & & \\
\hline $\begin{array}{l}209 \mathrm{Bi} \\
226 \mathrm{~PB}\end{array}$ & p & $3.6+1=$ & $5.6+1$ & PAR T & To-Silveira. & 4759 \\
\hline $\begin{array}{l}226 \mathrm{Ro} \\
226 \mathrm{R}\end{array}$ & $p$ & $1.1+1$ & $1.6+1$ & PAR T & T $D_{a}-S 11$ veira. & 4767 \\
\hline $\begin{array}{l}256 \mathrm{Ra} \\
25 \mathrm{R}\end{array}$ & ${ }_{3}^{6} \mathrm{He}$ & $1.9+1$ & & PAR I & I Da-Silivelra. & 5888 \\
\hline $\begin{aligned} 535 \\
230\end{aligned}$ & & $2.4+1$ & & PAR T & T Da-Silveira. & 6576 \\
\hline $\begin{array}{l}239 \mathrm{Pu} \\
29\end{array}$ & $p$ & $1.3+15$ & $5.3+1$ & PAR T & I Da-Silveira. & 4811 \\
\hline & & $9.2+0=$ & $2.3+1$ & PAR J & T Da-Silve?ra. & 5970 \\
\hline$A=125$ & ave & age olE & & & & \\
\hline $238_{U}$ & ${ }^{40} \mathrm{Ar}$ & $2.4+2$ & & YAL R & Bromlez. & 12411 \\
\hline$A=125$ & rel & alive olE & (E) & & & \\
\hline $22 \mathrm{R}_{\theta}$ & p & $1.2+1$ & & WZI E & Eayort & 4768 \\
\hline
\end{tabular}




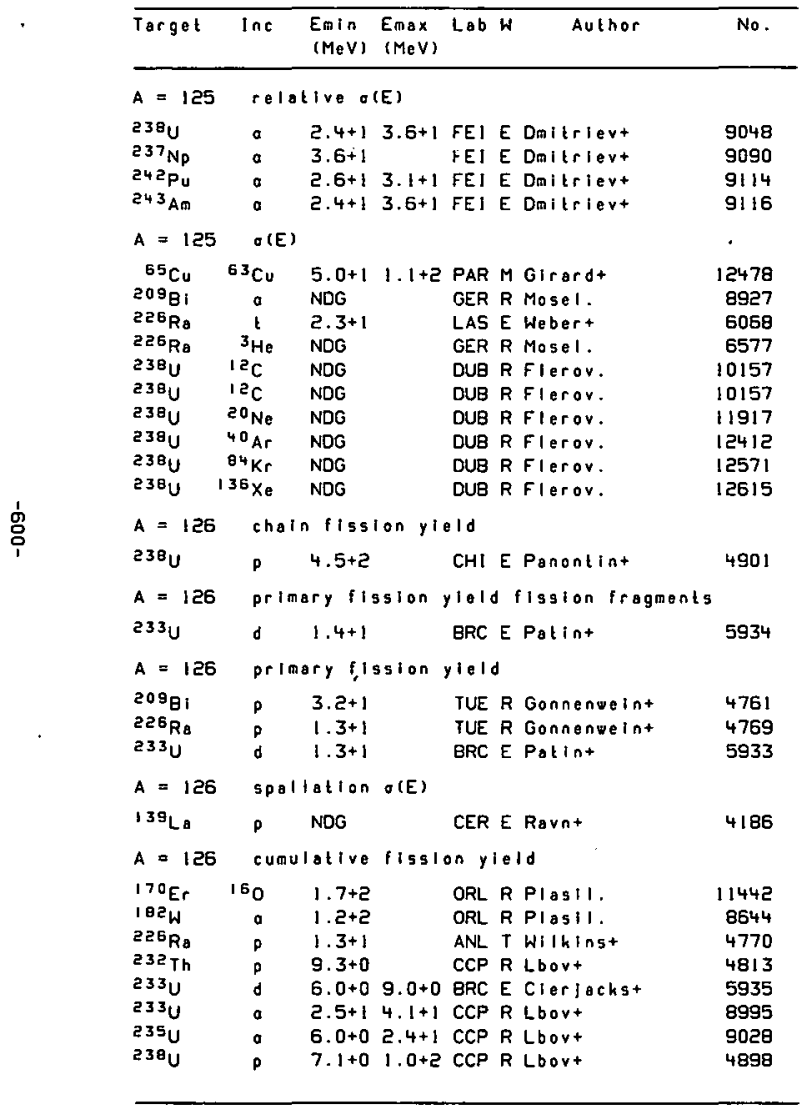

\begin{tabular}{|c|c|c|c|c|c|c|}
\hline Target & inc & $\begin{array}{l}\text { Emin } \\
(\mathrm{MeV})\end{array}$ & $\begin{array}{l}\text { Emax } \\
(M a V)\end{array}$ & Lab & Author & No. \\
\hline$A=126$ & \multicolumn{6}{|c|}{ cumulative fission yield } \\
\hline 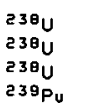 & $\begin{array}{l}p \\
a \\
a \\
d\end{array}$ & $\begin{array}{l}1.2+1 \\
2.0+1 \\
2.0+1 \\
9.2+0\end{array}$ & $\begin{array}{l}2.2+1 \\
4.0+1 \\
2.9+1 \\
2.3+1\end{array}$ & $\begin{array}{l}\text { TUE } \\
\text { MSU } \\
\text { SAC } \\
\text { CCP }\end{array}$ & $\begin{array}{l}\text { R Gonnenweint } \\
R \text { Gelbke. } \\
\text { E Libourelt } \\
R \text { Lbovt }\end{array}$ & $\begin{array}{l}4899 \\
9046 \\
9047 \\
5971\end{array}$ \\
\hline$A=126$ & \multicolumn{6}{|c|}{ cumulative o(E) } \\
\hline $\begin{array}{l}238 \mathrm{U} \\
A=126\end{array}$ & $\begin{array}{l}\text { p } \\
\text { par }\end{array}$ & $\begin{array}{l}2.0+1 \\
181 \mathrm{ras}\end{array}$ & $\begin{array}{r}8.5+1 \\
O(E)\end{array}$ & MCG & E Galiniert & 4897 \\
\hline $241 P u_{u}$ & $3_{\mathrm{He}}$ & $3.4+1$ & & ANL & E Bacht & 6620 \\
\hline$A=126$ & \multicolumn{6}{|c|}{ fission yield } \\
\hline $\begin{array}{l}209 \mathrm{Bi} \\
22 \mathrm{R}_{\mathrm{B}}\end{array}$ & $\begin{array}{l}p \\
p\end{array}$ & $\begin{array}{l}3.6+1 \\
1.1+1\end{array}$ & $\begin{array}{l}5.6+1 \\
1.6+1\end{array}$ & $\begin{array}{l}\text { PAR } \\
\text { PAR }\end{array}$ & $\begin{array}{l}\text { T Da-Silvelra. } \\
\text { T Da-Siliveira. }\end{array}$ & $\begin{array}{l}4759 \\
4767\end{array}$ \\
\hline $\begin{array}{l}256 \mathrm{Ra} \\
25 \mathrm{P}_{\mathrm{B}}\end{array}$ & $\begin{array}{l}0 \\
3 \\
3\end{array}$ & $1.9+1$ & & PAR & TDa-Silveira. & 5888 \\
\hline $\begin{array}{l}25 R_{\theta} \\
535 T h\end{array}$ & she & $\begin{array}{l}2.4+1 \\
1.3+1\end{array}$ & $5 \cdot 3+1$ & $\begin{array}{l}\text { PAR } \\
\text { PAR }\end{array}$ & $\begin{array}{l}\text { T Da-Silveira. } \\
\text { T Da-silveira. }\end{array}$ & $\begin{array}{l}6576 \\
4811\end{array}$ \\
\hline $239 \mathrm{Pu}$ & d & $9.2+0$ & $2.3+1$ & PAR & T Da-silveira. & 5970 \\
\hline$A=126$ & \multicolumn{6}{|c|}{ average $\sigma(E)$} \\
\hline $23 \theta^{2}$ & $40 \mathrm{Ar}$ & $2.4+2$ & & YAL & R Bromley. & 12411 \\
\hline$A=126$ & \multicolumn{6}{|c|}{ relative o(E) } \\
\hline $\begin{array}{l}226 \mathrm{Ra} \\
23 \theta_{\mathrm{U}} \\
237 \mathrm{~Np} \\
242 \mathrm{Pu} \\
24{ }^{2} \mathrm{Am}\end{array}$ & $\begin{array}{l}p \\
a \\
a \\
a \\
a \\
a\end{array}$ & $\begin{array}{l}1.2+1 \\
2.4+1 \\
3.6+1 \\
2.6+1 \\
2.4+1\end{array}$ & $\begin{array}{l}3.6+1 \\
3.1+1 \\
3.6+1\end{array}$ & $\begin{array}{l}\text { WZI } \\
\text { FEI } \\
\text { FEI } \\
\text { FEI } \\
\text { FEI }\end{array}$ & $\begin{array}{l}\text { E Gayert } \\
\text { E Dmitrievt } \\
\text { E Omitrievt } \\
\text { E Omitrievt } \\
\text { E Dmitrievt }\end{array}$ & $\begin{array}{l}4768 \\
9048 \\
9090 \\
9114 \\
9116\end{array}$ \\
\hline$A=126$ & \multicolumn{6}{|c|}{$O(E)$} \\
\hline $\begin{array}{r}65 \mathrm{Cu} \\
20{ }^{60 \mathrm{Bi}}\end{array}$ & $\begin{array}{c}63 \mathrm{Cu} \\
0\end{array}$ & $\begin{array}{l}5.0+1 \\
\text { NDG }\end{array}$ & $1.1+2$ & $\begin{array}{l}\text { PAR } \\
\text { GER }\end{array}$ & $\begin{array}{l}\text { M Girardt } \\
\text { R Mosel. }\end{array}$ & $\begin{array}{r}12478 \\
8927\end{array}$ \\
\hline $225_{\mathrm{Ra}}$ & $i$ & $2 \cdot 3+1$ & & LAS & E Webert & 6068 \\
\hline $225 \mathrm{Ra}$ & ${ }^{3} \mathrm{He}$ & NDG & & GER & R Mosel. & 6577 \\
\hline $238_{U}$ & $12 \mathrm{C}$ & NDG & & DUB & R Flerov. & 10157 \\
\hline $238 \mathrm{U}$ & ${ }^{20} \mathrm{Ne}$ & NDG & & DUB & R Fleroy. & 11917 \\
\hline $238 \mathrm{u}$ & $40 \mathrm{Ar}$ & NDG & & DUB & RFlerov. & 12412 \\
\hline $238 \mathrm{U}$ & ${ }^{84} \mathrm{Kr}$ & NDG & & DUB & Rfflerov. & 12571 \\
\hline $238 U$ & $136 \mathrm{Xe}_{\mathrm{e}}$ & NDG & & DUB & R Flerov. & 12615 \\
\hline
\end{tabular}




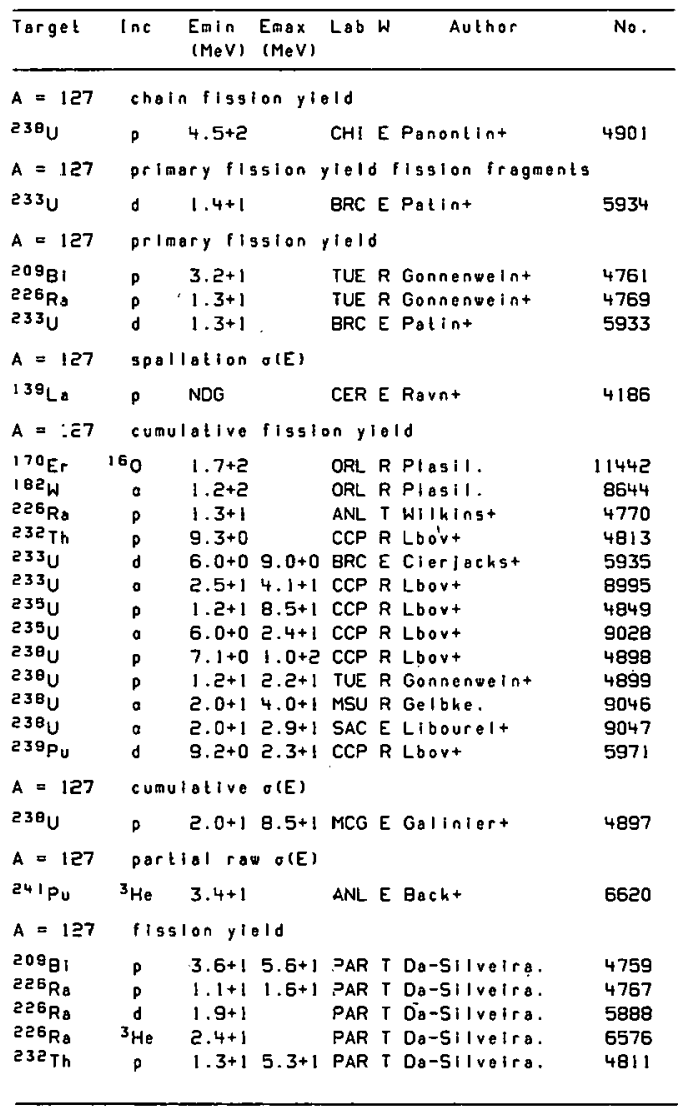

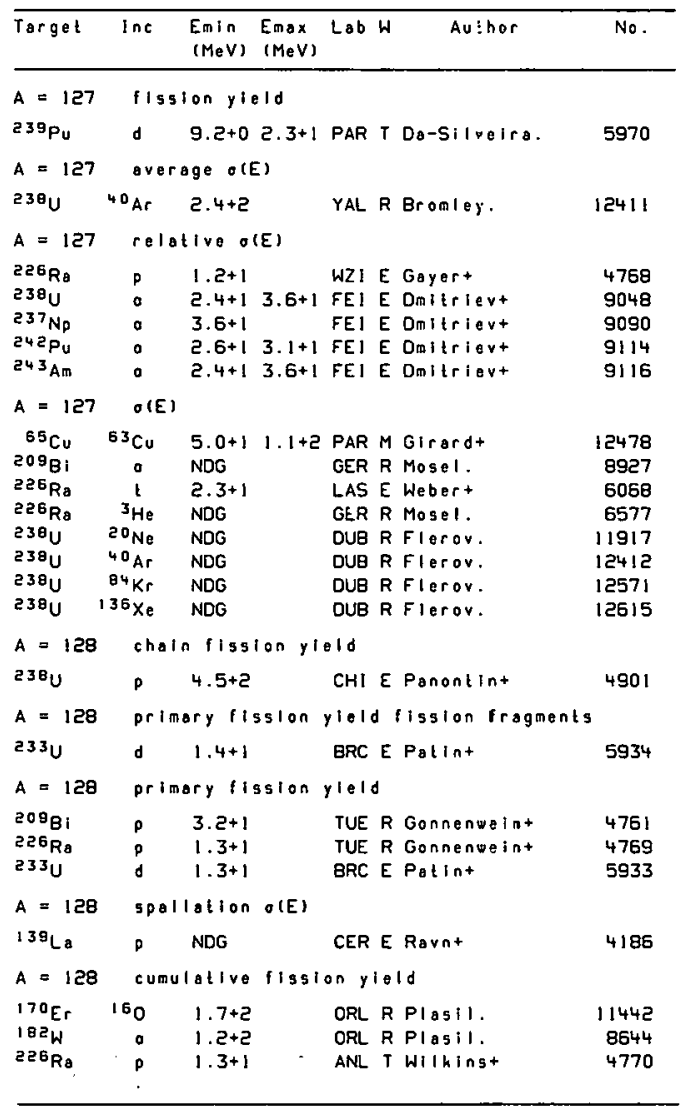




\begin{tabular}{|c|c|c|c|c|c|c|}
\hline Target & $\ln c$ & $\begin{array}{l}\text { Emin } \\
(\mathrm{MeV})\end{array}$ & $\begin{array}{l}\operatorname{Emax} \\
(\mathrm{MeV})\end{array}$ & Lab & Author & No. \\
\hline$A=128$ & \multicolumn{6}{|c|}{ cumulative figsion yiold } \\
\hline $\begin{array}{l}232 \mathrm{Th} \\
233_{\mathrm{U}} \\
233 \mathrm{U} \\
233_{\mathrm{U}} \\
238_{\mathrm{U}} \\
238_{\mathrm{U}} \\
238_{\mathrm{U}} \\
238_{\mathrm{U}} \\
239_{\mathrm{Pu}}\end{array}$ & $\begin{array}{l}\text { p } \\
\text { d } \\
\text { a } \\
o \\
p \\
p \\
\text { p } \\
0 \\
d\end{array}$ & $\begin{array}{l}9 \cdot 3+0 \\
6 \cdot 0+0 \\
2 \cdot 5+1 \\
6 \cdot 0+0 \\
7 \cdot 1+0 \\
1.2+1 \\
2 \cdot 0+1 \\
2 \cdot 0+1 \\
9.2+0\end{array}$ & $\begin{array}{l}9 \cdot 0+0 \\
4.1+1 \\
2 \cdot 4+1 \\
1.0+2 \\
2.2+1 \\
4 \cdot 0+1 \\
2.9+1 \\
2.3+1\end{array}$ & $\begin{array}{l}\text { CCP } \\
\text { BRC } \\
\text { CCP } \\
\text { CCP } \\
\text { CCP } \\
\text { TUE } \\
\text { MSU } \\
\text { SAC } \\
\text { CCP }\end{array}$ & $\begin{array}{l}\text { R Lbovt } \\
\text { E Clerjockst } \\
R \text { Lbovt } \\
R \text { Lbovt } \\
R \text { Lbovt } \\
R \text { Gonnenweint } \\
R \text { Gelbke. } \\
\text { E Libourelt } \\
\text { R Lbovt }\end{array}$ & $\begin{array}{l}4813 \\
5935 \\
8995 \\
9028 \\
4898 \\
4899 \\
9048 \\
9047 \\
5971\end{array}$ \\
\hline$A=128$ & \multicolumn{6}{|c|}{ cunulative o(E) } \\
\hline $\begin{array}{l}{ }^{230} U \\
A=128\end{array}$ & \multicolumn{6}{|c|}{ Dortlol roo o(E) } \\
\hline $241 \mathrm{Pu}$ & ${ }^{3} \mathrm{He}$ & $3.4+1$ & & ANL & E Bocht & 6620 \\
\hline$A=128$ & \multicolumn{6}{|c|}{ flisgion yield } \\
\hline $\begin{array}{l}209 \mathrm{Bl} \\
226 \mathrm{Ra} \\
226 \mathrm{Ra} \\
226 \mathrm{Ra} \\
232 \mathrm{Th} \\
239 \mathrm{Pu}\end{array}$ & $\begin{array}{c}p \\
p \\
d \\
{ }^{d} \mathrm{He} \\
0 \\
0\end{array}$ & $\begin{array}{l}3.6+1 \\
1.1+1 \\
1.9+1 \\
2.4+1 \\
1.3+1 \\
9.2+0\end{array}$ & $\begin{array}{l}5.6+1 \\
1.6+1 \\
\\
5.3+1 \\
2.3+1\end{array}$ & $\begin{array}{l}\text { PAR } \\
\text { PAR } \\
\text { PAR } \\
\text { PAR } \\
\text { PAR } \\
\text { PAR }\end{array}$ & $\begin{array}{l}\text { T Da-Silveira. } \\
\text { T Da-silveira. } \\
\text { T Do-Silveira. } \\
\text { T Da-SIIveira. } \\
\text { T Do-SIIveira. } \\
\text { T Da-Silveira. }\end{array}$ & $\begin{array}{l}4759 \\
4767 \\
5888 \\
6576 \\
4811 \\
5970\end{array}$ \\
\hline $\begin{array}{l}A=128 \\
230 \mathrm{U}\end{array}$ & $\begin{array}{l}\text { avo } \\
{ }^{40} \mathrm{Ar}\end{array}$ & $\begin{array}{l}.90 .1 E \\
2.4+2\end{array}$ & & YAL & R Bromley. & 12411 \\
\hline$A=128$ & \multicolumn{6}{|c|}{ relotive o(E) } \\
\hline $\begin{array}{l}226 \mathrm{Ra} \\
23 \theta_{\mathrm{U}} \\
237 \mathrm{~Np} \\
242 \mathrm{Pu} \\
243_{\mathrm{Am}}\end{array}$ & $\begin{array}{l}p \\
0 \\
0 \\
0 \\
0\end{array}$ & $\begin{array}{l}1.2+1 \\
2.4+1 \\
3.6+1 \\
2.5+1 \\
2.4+1\end{array}$ & $\begin{array}{l}3.6+1 \\
3.1+1 \\
3.6+1\end{array}$ & $\begin{array}{l}\text { WZI } \\
\text { FEI } \\
\text { FEI } \\
\text { FEI } \\
\text { FEI }\end{array}$ & $\begin{array}{l}\text { E Gayert } \\
\text { E Dmilrievt } \\
\text { E Dnilrievt } \\
\text { E Dmilrlevt } \\
\text { E Dmbirleyt }\end{array}$ & $\begin{array}{l}4768 \\
9048 \\
9090 \\
9114 \\
9116\end{array}$ \\
\hline$A=128$ & \multicolumn{6}{|c|}{$O(E)$} \\
\hline $\begin{array}{l}509 \theta_{i} \\
256 R_{s} \\
25 R_{a} \\
23 \theta_{U} \\
53 \theta_{U}\end{array}$ & $\begin{array}{c}a \\
k \\
3 \mathrm{He} \\
12 \mathrm{C} \\
20 \mathrm{Ne}\end{array}$ & $\begin{array}{l}\text { NOG } \\
2.3+1 \\
\text { NDG } \\
\text { NOG } \\
\text { NDG }\end{array}$ & & $\begin{array}{l}\text { GER } \\
\text { LAS } \\
\text { GER } \\
\text { DUB } \\
\text { DUB }\end{array}$ & $\begin{array}{l}\text { R Mosel. } \\
E \text { Webert } \\
R \text { Mosel. } \\
R \text { Flerov. } \\
R \text { Flerov. }\end{array}$ & $\begin{array}{r}8927 \\
6068 \\
6577 \\
10157 \\
11917\end{array}$ \\
\hline
\end{tabular}

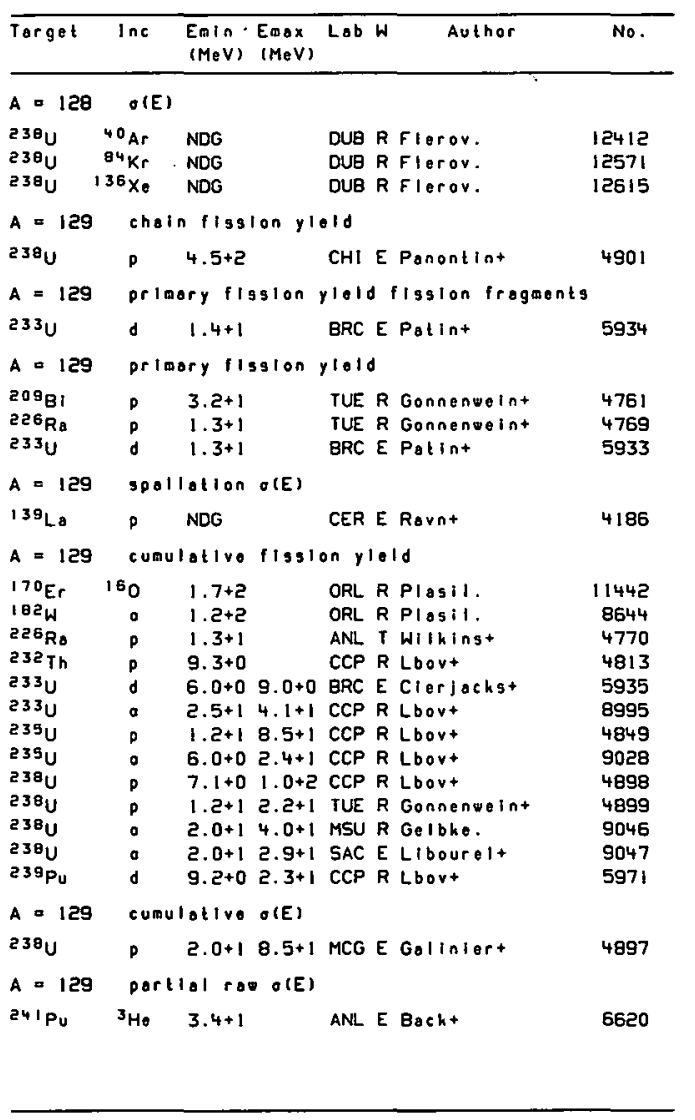




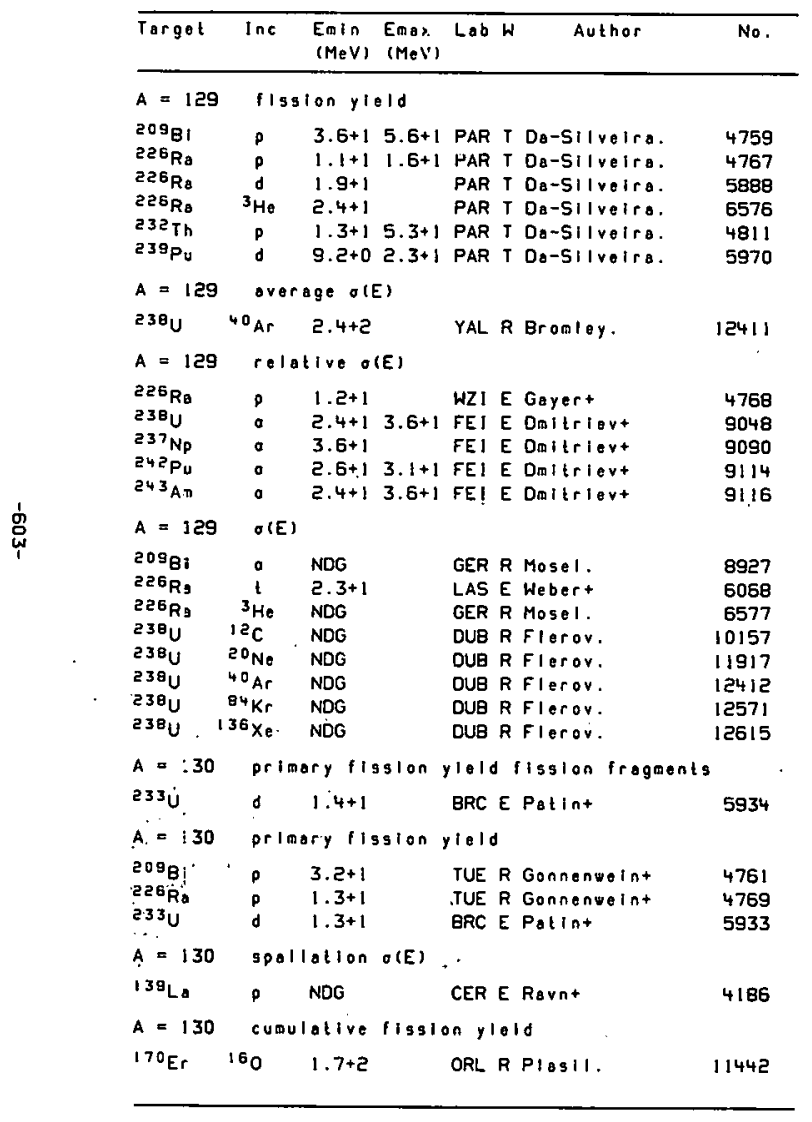

\begin{tabular}{|c|c|c|c|c|c|c|}
\hline Torget & Inc & $\begin{array}{l}E_{q} \mid n \\
\left(M_{0} \in\right)\end{array}$ & $\begin{array}{l}\varepsilon_{\mathrm{qax}} \\
(\mathrm{MQV})\end{array}$ & Lab W & Author & No. \\
\hline
\end{tabular}

$A=130$ cumulalive lission yield

$102 \mathrm{~W} \quad 1.2+2$ ORL R Plasil.

$22 \mathrm{R}_{\theta} D \quad 1.3+1 \quad$ ANL T Wilkins- 4770

$232 T h$ P $9.3+0 \quad$ CCP R Lbovt 4813

$233 \mathrm{U} d \quad 6.0+09.0+0$ BRC E Cierlachst 5935

$233 \mathrm{U} O 2.5+14.1+1$ CCP R Loovt 8995

${ }^{233} \mathrm{U} \quad 0 \quad 6.0+02.4+1$ CCP R Lbovt 9028

$\begin{array}{lllll}238 \mathrm{U} & \rho^{\prime} & 7.1+0 & 1.0+2 \text { CCP R Lbovt } & 4899 \\ 230 \mathrm{U} & P^{2} & 1.2+1 & 2.2+1 \text { TUE R Gonnenwelnt } & 4899\end{array}$

$23 \mathrm{U}^{2} \quad 2.0+14.0+1$ MSU R Golbho. 9046

$23 \mathrm{U}^{2} \mathrm{O} 2.0+12.9+1$ SAC $E$ Liboure 1+ 9047

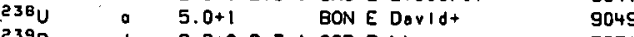

$239 \mathrm{Pu} 9.2+02.3+1$ CCP R Lbovt 5971

$A=130$ cumulativo $O(E)$

$238 \mathrm{U} P \quad 2.0+18.5+1$ MCGE Golin-ort 4997

$A=130$ parllal rovo(E)

$24^{1} \mathrm{Pu} \quad{ }^{3} \mathrm{He} \quad 3.4+1 \quad$ ANL E Bacht 6620

$A=130$ fission yleld

209日i P 3.6+1 5.6+1 PAR T Do-sIlvelra. 4759

$226 \mathrm{Ra} P \mathrm{P} \quad 1.1+1$ 1.6+1 PAR T Do-Silvelra. 4767

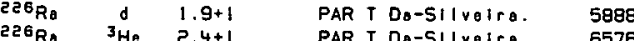

$232 \mathrm{Th}$ He $2.4+1$ PAR T Do-SIlvelira. 657

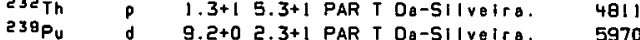

$A=130$ row ote

$257^{7} \mathrm{Fm} \quad t \quad 1.6+1$ LAS E Hoffment 6090

$A=130$ average o(E)

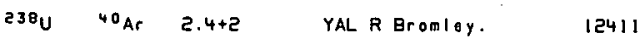

$A=130$ relatlve o(E)

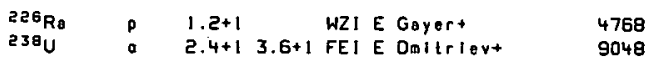

$\begin{array}{lllll}238_{U} & \text { a } & 2.4+1 & 3.6+1 \text { FEI E ODItrlevt } & 9048 \\ 237 \mathrm{~Np} & \text { a } & 3.6+1 & \text { FEI E Omitrievt } & 9090\end{array}$

$\begin{array}{llll}242 \mathrm{Pu} & 9 & 9090 \\ 243+13.1+1 \text { FEI E Datriavt } & 9114\end{array}$

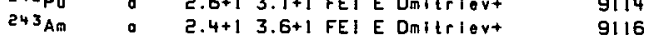




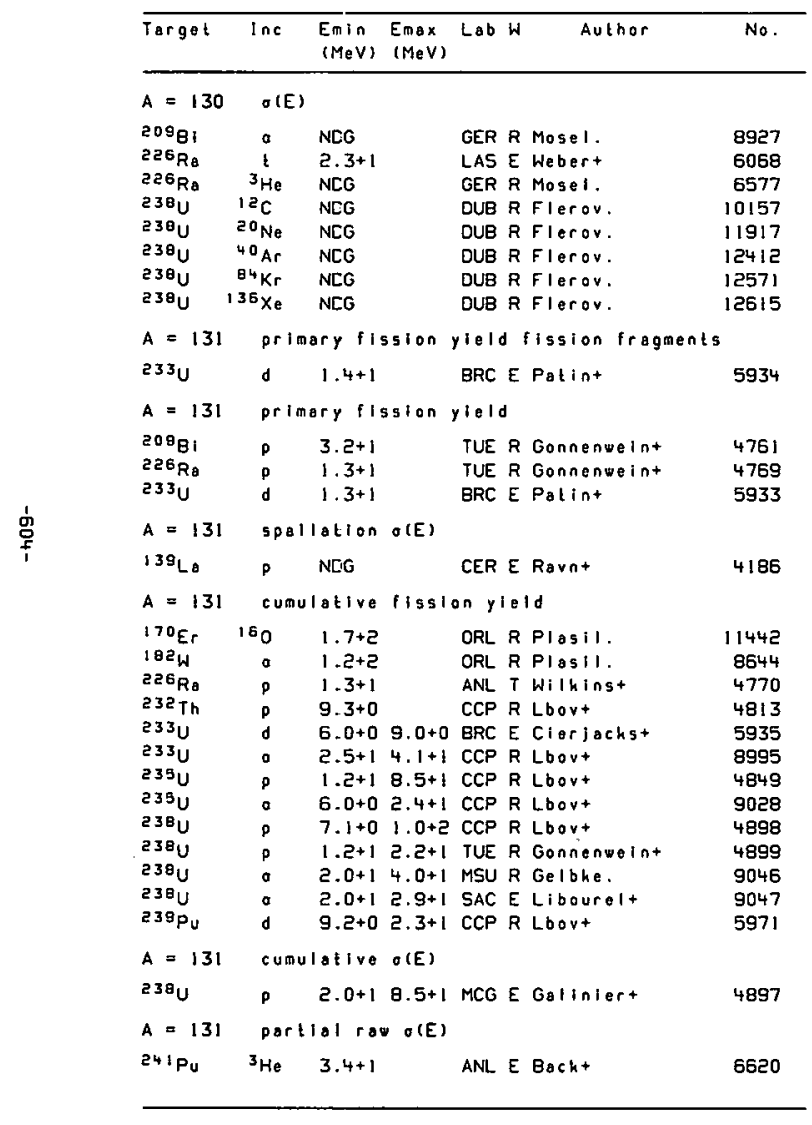

\begin{tabular}{|c|c|c|c|c|c|c|c|}
\hline et & $\operatorname{lnc}$ & $\begin{array}{l}\text { Emin } \\
(\mathrm{MeV})\end{array}$ & $\begin{array}{l}\text { Enax } \\
(M e V)\end{array}$ & Lab & & Author & \\
\hline
\end{tabular}

$A=131$ fission yield

$226_{R a} P \quad 1.1+11.6+1$ PAR T Da-5ilveirs. 4767

$226 R_{a} d \quad 1.9+1 \quad$ PAR T Da-5ilveira. 5888

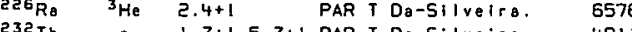

$232 \mathrm{Th} \quad \mathrm{P} \quad 1.3+15.3+1$ PAR T Da-5ilveira, 4811

239Pu d 9.2+0 2.3+1 PAR T Da-Silveira. 5970

$A=131$ average $\sigma(E)$

$238_{\mathrm{U}} \quad{ }^{40 \mathrm{Ar}} 2.4+2$ YAL R Bromiey. 12411

$A=131$ relalive $\sigma(E)$

$226_{R a} D \quad 1.2+1 \quad$ WZI E Gayert 4768

$238_{U} \quad 0 \quad 2.4+13.6+1$ FEI E Deitrievt 9048

$237 \mathrm{~Np} a \quad 3.6+1$ FEI E Dmitrievt 9090

$242 \mathrm{Pu} O 2.6+13.1+1$ FEI E Ditrievt 9114

$\begin{array}{llll}243 \mathrm{Am} & 0 & 2.4+13.6+1 \text { FEI E Dmitrievt } & 9116\end{array}$

$209_{B 1}{ }^{2}$ NDG GER R MOSel.

$\begin{array}{llll}256 \mathrm{Ra} & \mathrm{k} & 2.3+1 & \text { LAS E Webert }\end{array}$

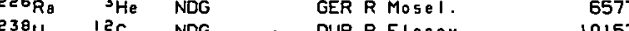

$238^{2} 20$ NDG DU 10157

$238_{4} 40^{\circ}$ NDG 11917

$23 \theta_{14} 84 \mathrm{Kr} N \mathrm{NOG} 12412$

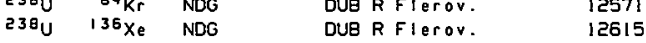

$A=132$ priary fission ylelo fission frogments

$233 \mathrm{y}$ o $1.4+1 \quad$ BRC E Patint 5934

$A=132$ primary fission ylelo

$\operatorname{209}_{B 1} P \quad 3.2+1$ TUE R Gonnenveint 4.761

$226 R a$ TUE R Gonnenweint $\quad P \quad 1.3+1 \quad 4759$

$233 \mathrm{U} d \mathrm{~d} 1.3+1 \quad$ BRCEPatint 5933

$A=132$ spallation o(E)

139 La $P$ NDG CER E Ravnt 4186

$A=132$ cumulative fission yleld

$\begin{array}{lrrrr}170 E_{r} & 150 & 1.7+2 & \text { ORL R PIAS11. } & 11442 \\ 102 \mathrm{~W} & 0 & 1.2+2 & \text { ORL R PIOSI1. } & 8644\end{array}$ 


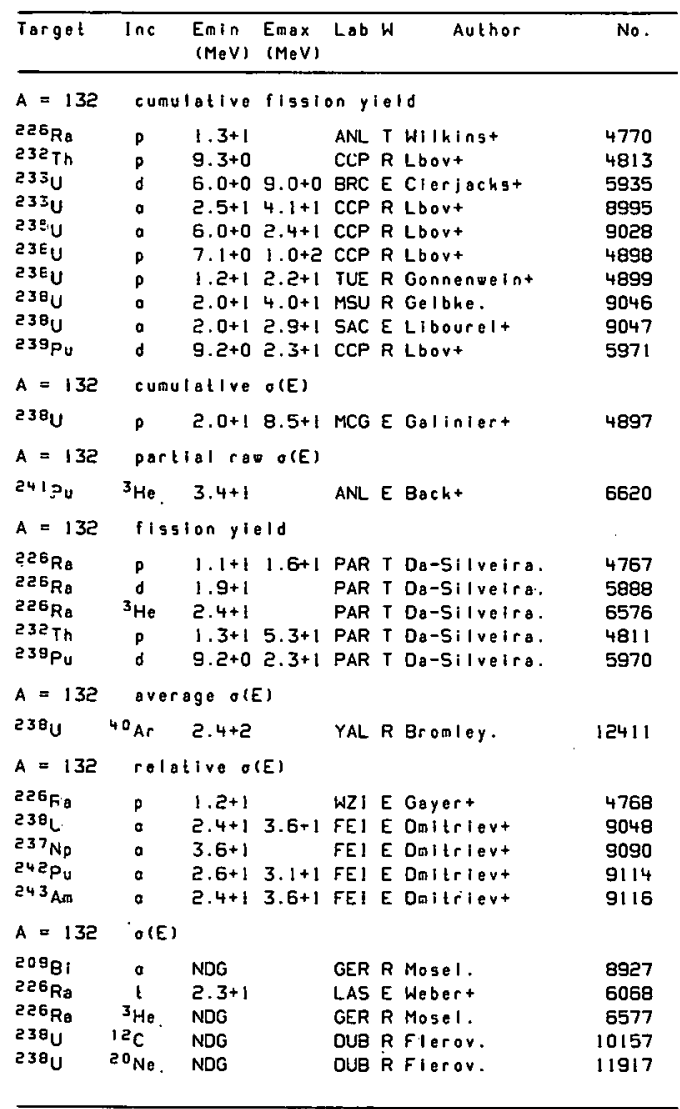

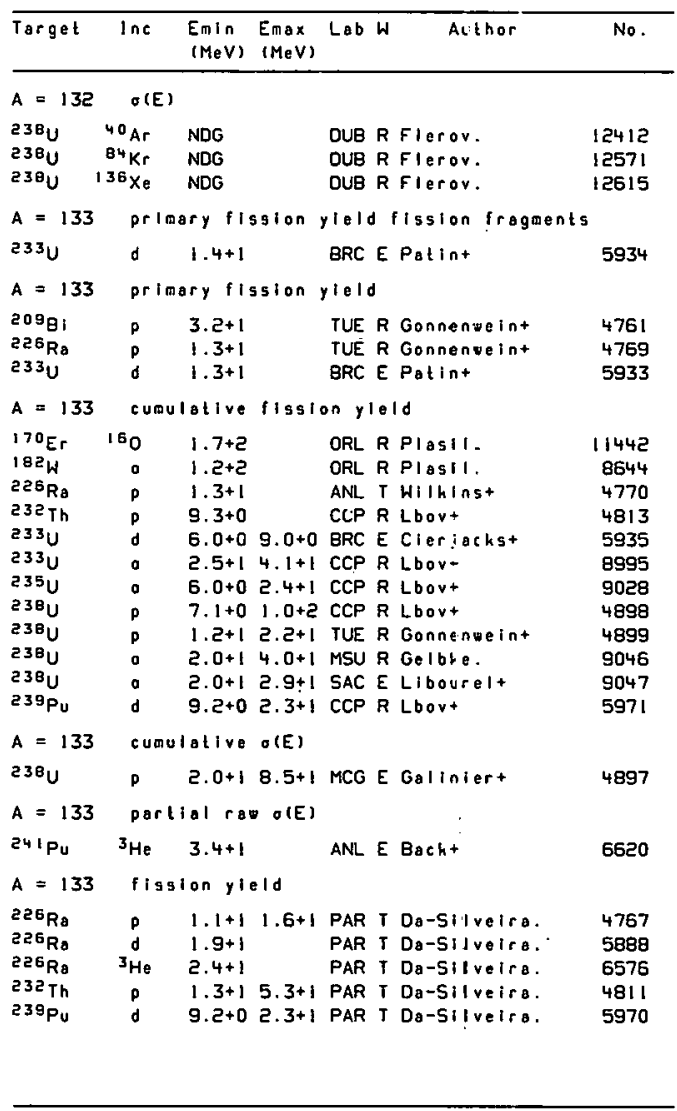




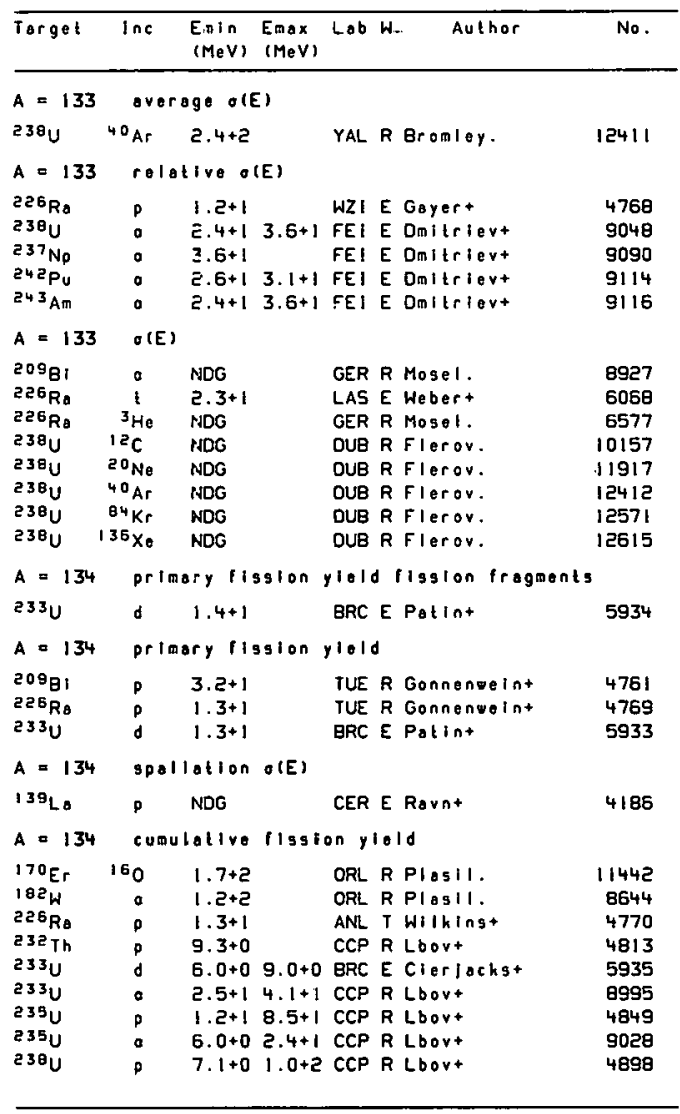

\begin{tabular}{llll}
\hline Target Inc & $\begin{array}{l}\text { Emin Emax Lab } \\
(M e V) \text { Author }\end{array}$ & Nov) \\
\hline
\end{tabular}

$A=134$ cunulative fisgion yleld

$23 \theta_{U}$ P $1.2+12.2+1$ TUE R Gonnenveint 4899

230 U a $2.0+14.0+1$ MSU R Golbhe. 9046

$238^{2} \mathrm{O} \quad 2.0+12.9+1$ SAC E Libourel+ 9047

$239 \mathrm{Pu}$ d 9.2+0 $2.3+1$ CCP R Lbov+ 5971

$A=134$ cumulative $\theta(E)$

$230 \mathrm{U} P \quad 2.0+18.5+1$ MCG E Gallniert 4897

$A=134$ partlal ravo(E)

${ }^{24} 1 \mathrm{Pu} \quad{ }^{3} \mathrm{He} \quad 3.4+1 \quad$ ANL E Backt 6620

$A=134$ fisglon yiolo

$226_{\mathrm{Ra}} \rho \quad 1.1+1 \quad 1.6+1$ PAR T Da-Silveira. 4767

256 Ra d $1.9+1$ PAR T Da-SIIveira. 5888

${ }^{226 \mathrm{Ra}}{ }^{3} \mathrm{He} \quad 2.4+1 \quad$ PAR T Da-SIIveira. 6576

$232 \mathrm{Th} p \mathrm{p} 1.3+15.3+1$ PAR T Da-Silveira. 4811

$A=134$ overogo o(E).

$230^{4} 4{ }^{\circ} \mathrm{Ar} 2.4+2$ YAL R Bromloy. 12411

$A=134$ rolative o(E)

$256_{R} \quad p \quad 1.2+1 \quad$ WZI E Gayert 4768

$238_{\mathrm{U}} a \quad 2.4+13.6+1$ FEI E Dmitrievt 9048

$237 \mathrm{~Np}$ a $3.6+1$ FEI E Dnitrlevt 9090

$\begin{array}{llll}242 \mathrm{Pu} & 0 & 2.6+13.1+1 \mathrm{FEI} \text { E Onitrlevt } & 9114 \\ 243 \mathrm{Am} & 0 & 2.4+13.6+1 \mathrm{FEI} \text { E Onitrievt } & 9116\end{array}$

$A=134$ O(E)

$209^{\circ}:$ O NDG GER R Mosel. 9927

$256_{\mathrm{Ra}} \mathrm{t} 2.3+1$ LASE Webert

$226 \mathrm{Re}^{3}{ }^{3} \mathrm{He}$ NDG $\quad$ GER R Mosel.

$238_{U}{ }^{3} \mathrm{C}$ NDG DUB R Flerov. 10157

$238^{2}{ }^{20} \mathrm{Ne}$ NDO $\quad$ OUB RFIerov. 11917

$238 \mathrm{U}{ }^{40} \mathrm{Ar}$ NDG DUB R Flerov.

$23 \theta^{84} \mathrm{Kr}$ NDG DUB R Flerov. 1257

$230^{3} 136 \times$ NDG DUB R Flerov. 12615

$A=135$ primery flission yleld flssion fragents

$233 \mathrm{U}$ d $1.4+1 \quad$ BRC E Potint - 5934 


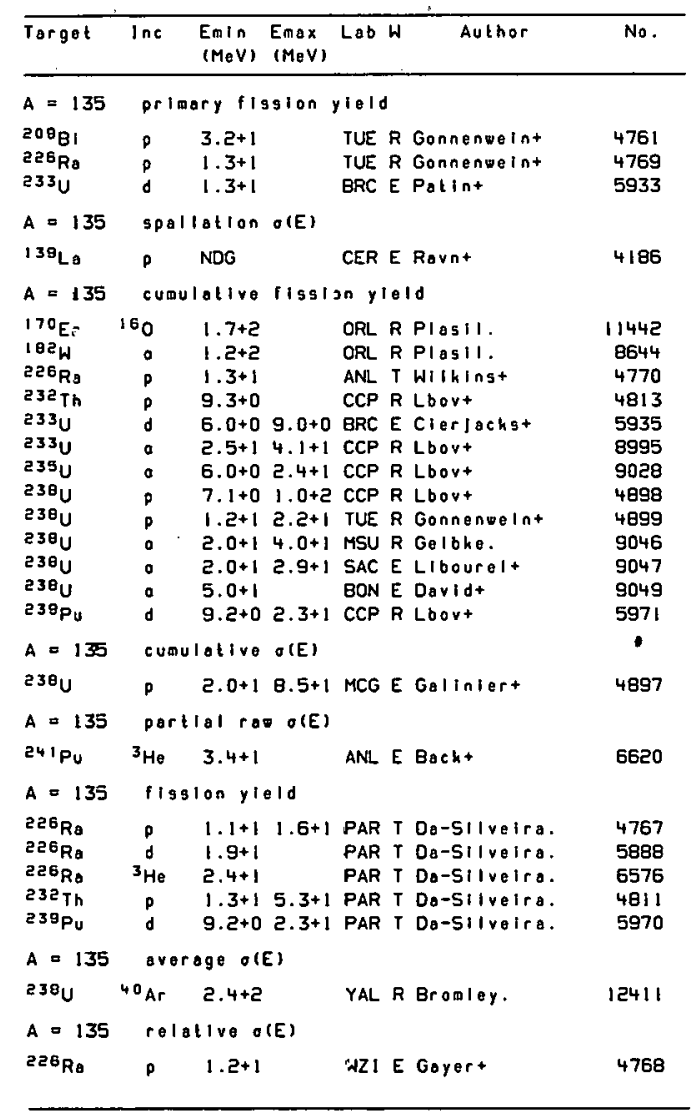

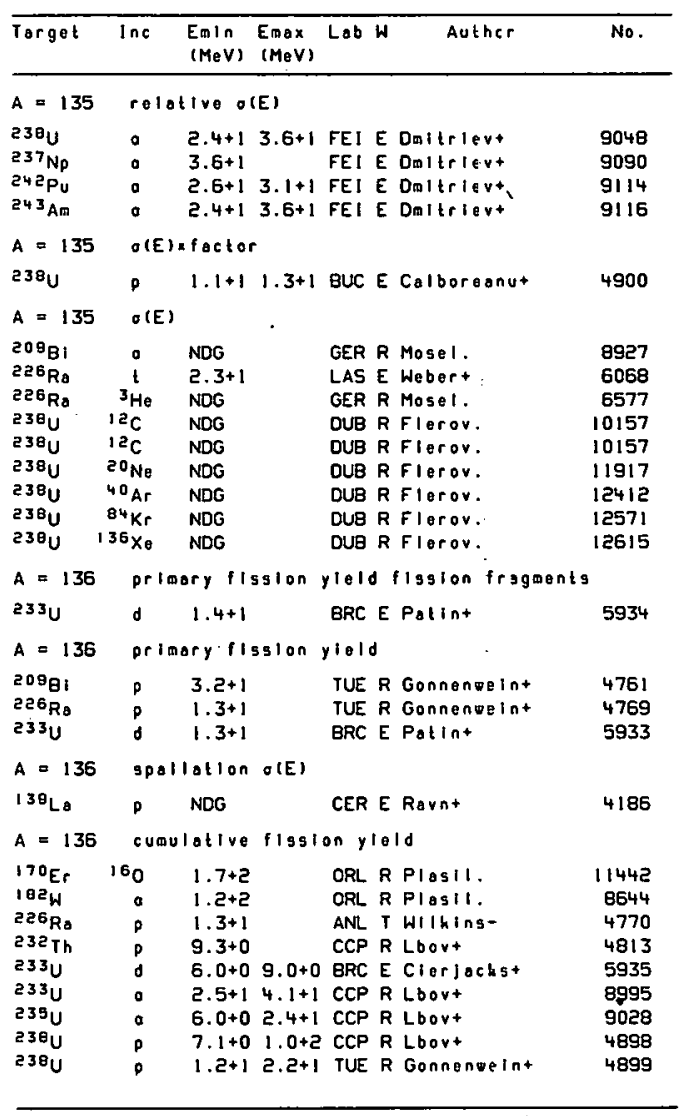




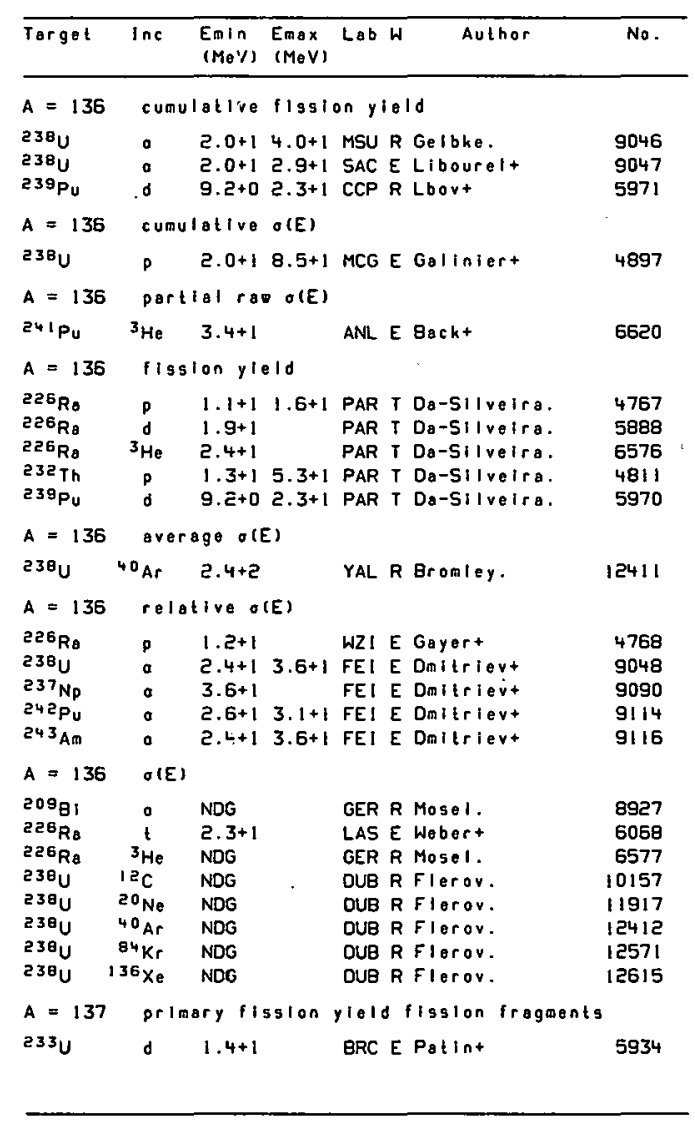

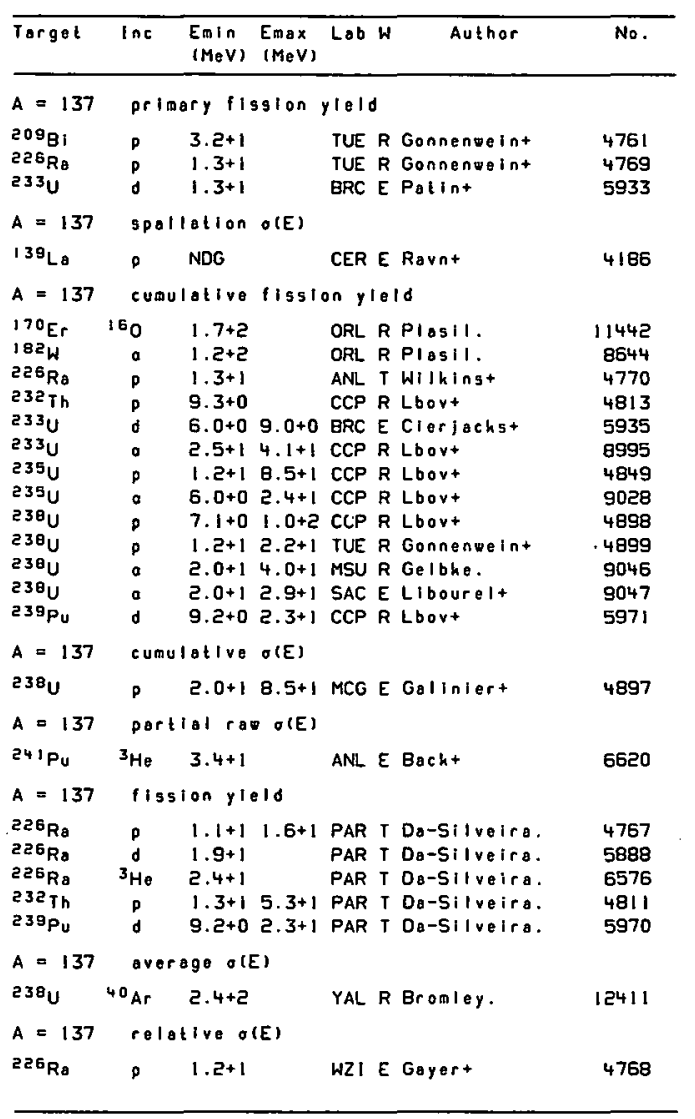




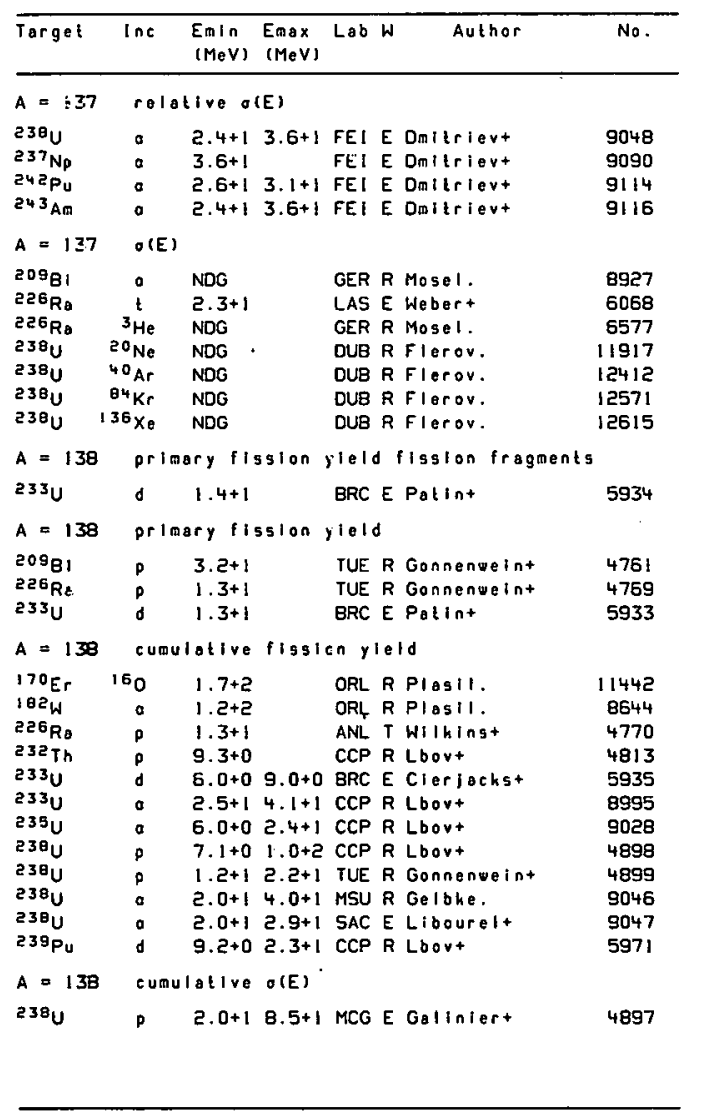

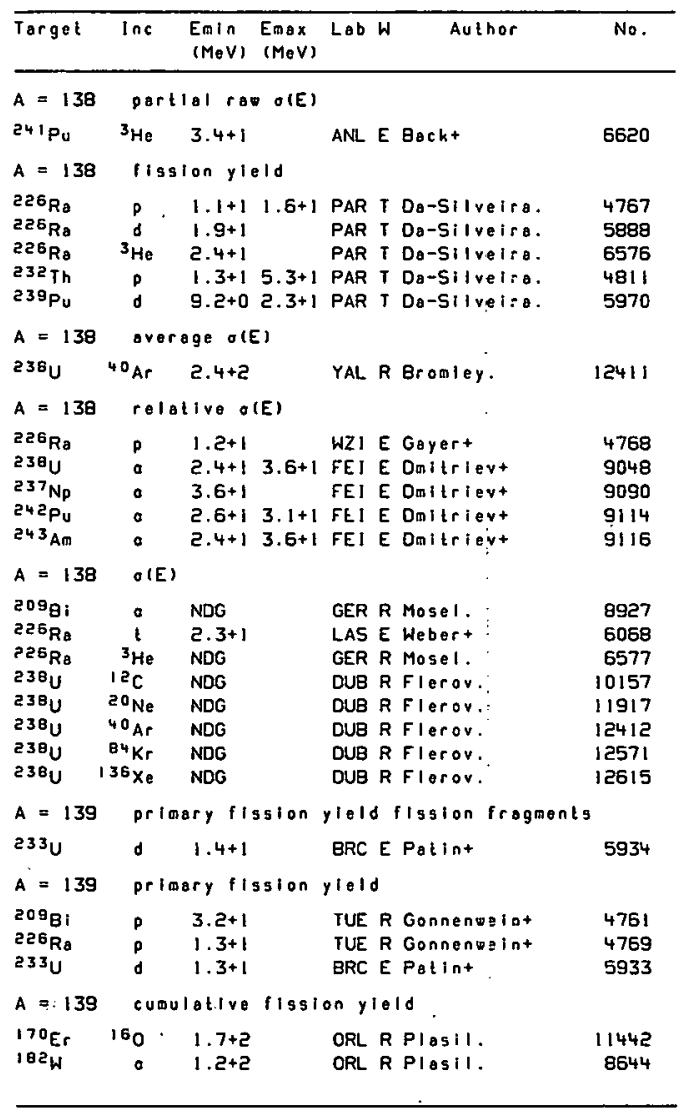




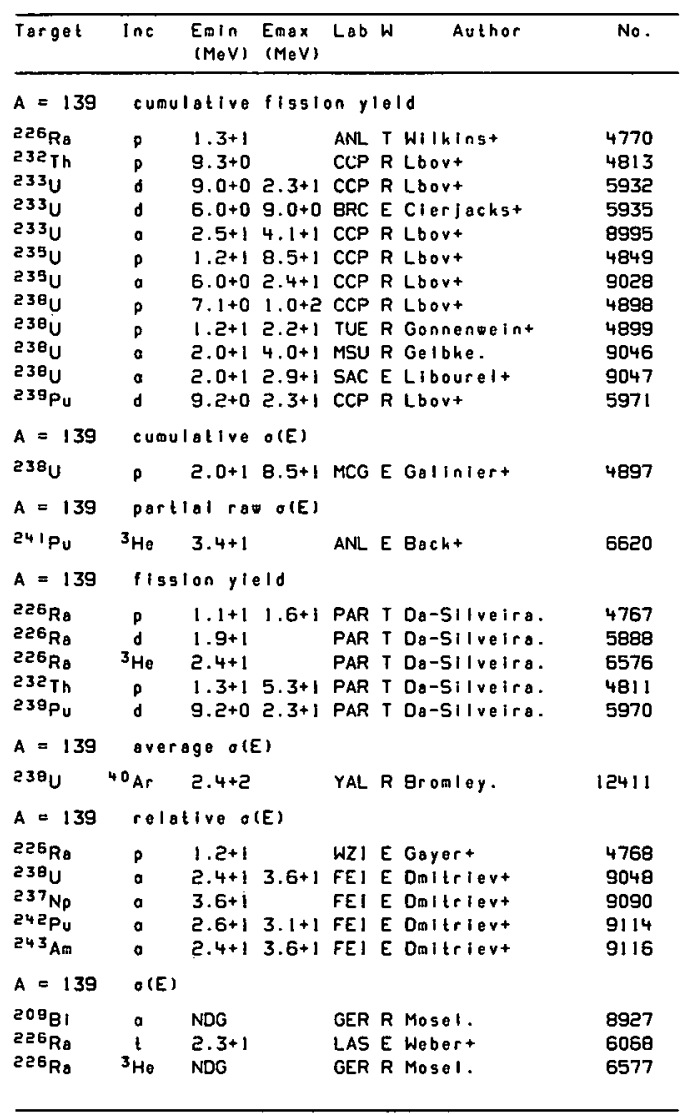

\begin{tabular}{|c|c|c|c|c|c|c|}
\hline Targel & $\ln c$ & $\begin{array}{l}\text { Emin } \\
(M e V\end{array}$ & $\begin{array}{l}\text { Emax } \\
(\mathrm{MeV})\end{array}$ & Lab W & Author & No. \\
\hline$A=139$ & OIE & & & & & \\
\hline 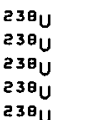 & $\begin{array}{l}12 \mathrm{C} \\
20 \mathrm{Ne} \\
{ }^{40} \mathrm{Ar} \\
84 \mathrm{Kr} \\
136 \mathrm{xe}\end{array}$ & $\begin{array}{l}\text { NDG } \\
\text { NDG } \\
\text { NDG } \\
\text { NDG }\end{array}$ & & $\begin{array}{l}\text { DUB R } \\
\text { OUB } R \\
\text { OUB R } \\
\text { OUB R }\end{array}$ & $\begin{array}{l}\text { R Flerov. } \\
\text { R Flerov. } \\
\text { R Flerov. } \\
\text { R Flerov. }\end{array}$ & $\begin{array}{l}10157 \\
11917 \\
12412 \\
12571 \\
12615\end{array}$ \\
\hline
\end{tabular}

$A=140$ primary fission yield fission fragnents

$233 \mathrm{U} d 1.4+1 \quad$ BRC E Patint 5934

$A=140$ primory fission yield

$209^{B i} \quad P \quad 3.2+1 \quad$ TUE R Gonnenvelnt

$\begin{array}{llll}225 \mathrm{Ra} & P & 1.3+1 \text { - TUE R Gonnenueint } & 4761 \\ & & \end{array}$

${ }^{233} \mathrm{U} d \mathrm{~B} 1.3+1$ BRC E Patint

$A=140$ cumulative fission yleld

$170 \mathrm{Er} \quad 16 \mathrm{O} \quad 1.7+2 \quad$ ORL R Plasil. 1144

$182 \mathrm{~W} a \mathrm{l}$ OKL R PIasil. 8644

$225 \mathrm{Ra} P \quad 1.3+1 \quad$ ANL T Wilkinst 4770

232 Th $P \quad 9.3+0 \quad$ CCP R Lbovt 4813

$233 \mathrm{U} d \quad 9.0+02.3+1$ CCP R Lbovt 593

$233 \mathrm{U} d \quad 6.0+09.0+0$ BRC E Clerlackst 5935

$235 \mathrm{U} a \mathrm{a} 2.5+14.1+1$ CCP R Lbovt 289

$23 \theta^{\prime} \mathrm{P} \quad 7.1+01.0+2$ CCP R Lbovt 4898

230U $P \quad 1.2+12.2+1$ TUE R Gonnenueint 4899

$230 \mathrm{U} 2.0+14.0+1$ MSU R Gelbke.

$238 \mathrm{U} \quad 2.0+12.9+1$ SAC E Libourelt 9047

$230^{2} \mathrm{U} \quad 5.0+1 \quad$ BON E David+ 9049

$2{ }^{39} \mathrm{Pu} d \quad 9.2+02.3+1$ CCP R Lbovt 597

$A=140$ cumulative $O(E)$

$23 \theta^{U} P \quad 2.0+18.5+1$ MCG E Galiniert 4897

$A=140$ partiol rav $O(E)$

$241 \mathrm{Pu} \quad{ }^{3} \mathrm{He} \quad 3.4+1 \quad$ ANl. E Bacht 6620

$A=140$ fIssion yield

$226 \mathrm{Ra} \quad 0 \quad 1.1+1$ 1.6+1 PAR T Do-Silvelra. 4767

$226_{\mathrm{Ra} a} \mathrm{~d} \quad 1.9+1$ PAR T Da-Silveira. 5898

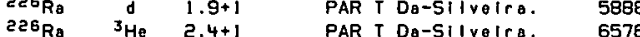




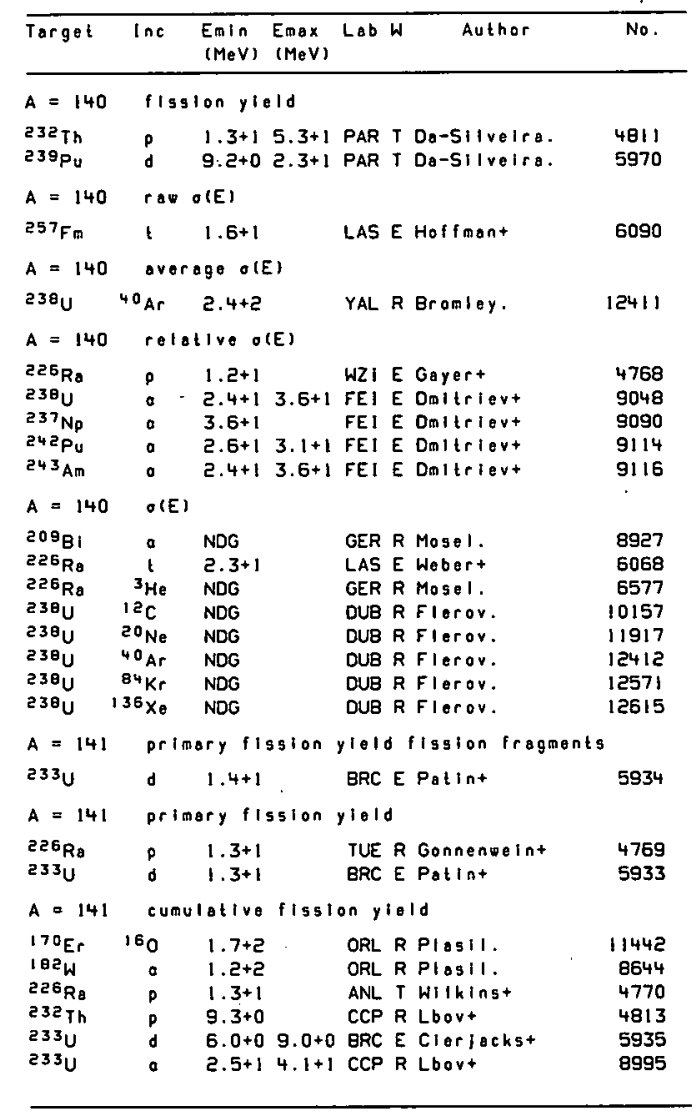

\begin{tabular}{|c|c|c|c|c|c|c|}
\hline Target & $\ln c$ & $\begin{array}{l}\text { Emin } \\
(\mathrm{MeV})\end{array}$ & $\begin{array}{l}E_{\max } \\
(\mathrm{MeV})\end{array}$ & Lab W & Author & No. \\
\hline
\end{tabular}

$235 \mathrm{U} P \quad 1.2+1$ B.5+1 CCP R Lbout

$235 \mathrm{U}(1.0+02.4+1$ CCP R Lbovt 4849

$238 \mathrm{O} 9.0+0.4+1$ CCP R Lbovt 9028

$238 \mathrm{U} \quad 7.1+01.0+2$ CCP R Lbovt 4898

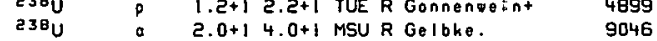

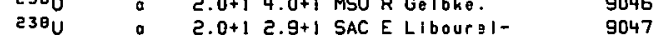

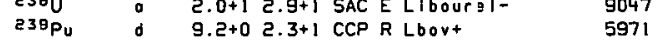

$A=141$ cumulatlve $\sigma(E)$

${ }^{230} U$ P $2.0+18.5+1$ MCG E Galinier- 4897

$A=141$ partlal rov o(E)

24IPu $3 \mathrm{He} 3.4+1 \quad$ ANL E Backt 6620

$A=141$ fission yleld

${ }^{256} \mathrm{R}_{\theta} \quad p \quad 1.1+1 \quad 1.6+1$ PAR T Da-511.ye:ra. 4767

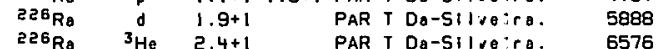

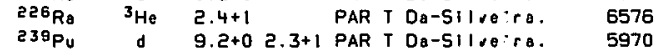

$A=141$ average $O(E)$

$A=141$ average $O(E)$
$238 \mathrm{U} \quad 40 \mathrm{Ar} 2.4+2 \quad$ YAL $R$ Bromley.

$A=141$ relative o(E)

$256 \mathrm{Re}^{2} \quad 1.2+1 \quad$ WZI E Gayert 4768

$230_{U} \quad a \quad 2.4+13.6+1$ FEI E Dmitriev- 9048

$237 \mathrm{~Np} a$ a $3.6+1$ FE1 E Dmitriev- 9090

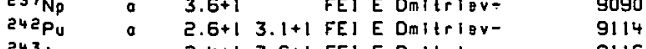

$243 \mathrm{Am} a \quad 2.4+13.6+1$ FEI E Doltrlev- 9116

$A=141 \quad O(E)$

$236_{\mathrm{Ra}} t \quad 2.3+1$ LAS E Webert 6068

${ }^{256} \mathrm{Ro}^{3} \mathrm{He}$ NDG GER R Mosel. 6577

$23 \mathrm{Q}_{\mathrm{U}}{ }^{12 \mathrm{C}}$ NDG $\quad$ DUA R Flerov. 10157

$230^{20} \mathrm{Ne}$ NDG DUB R Flerov. 11917

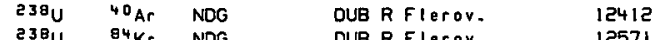

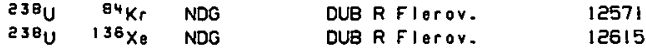




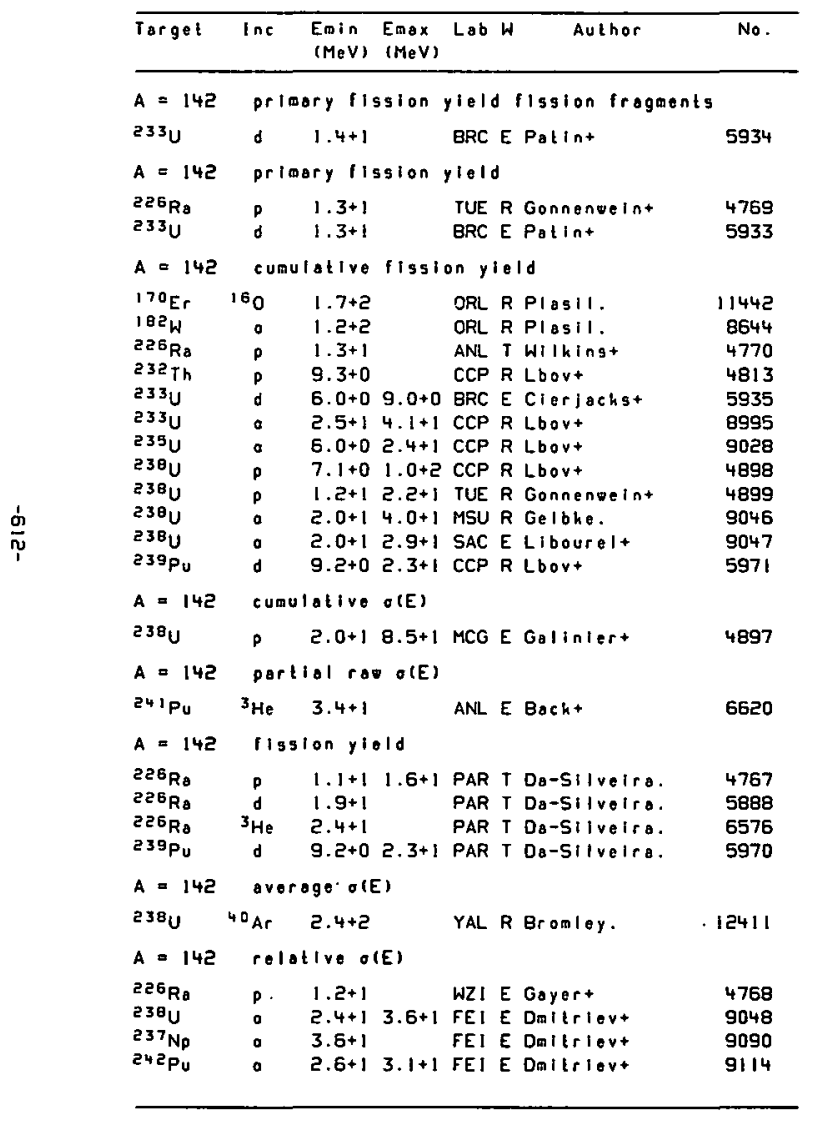

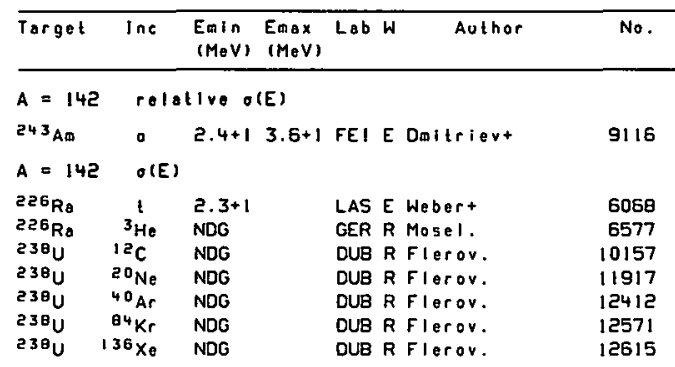

$A=143$ primary fission yield fission fragments

$233 \mathrm{U}$ d $1.4+1 \quad$ BRC E Patint 5934

$A=143$ primery lission yield

${ }^{226} \mathrm{Ra} \quad \mathrm{P} \quad 1.3+1 \quad$ TUE R Gonnengeint 4769

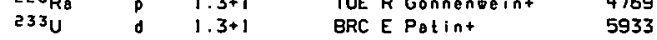

$A=143$ cumulotive figsion yield

$170 \mathrm{Er} \quad 160 \quad 17+2 \quad 08 \mathrm{R}$ Plasil.

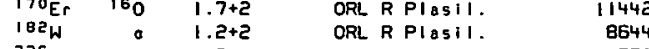

$225_{R a} P \quad 1.3+1 \quad$ ANL T Wilkinst 4770

$232 \mathrm{Th} P \quad 9.3+0 \quad$ CCP R Lbovt 4813

$233 \mathrm{U} d \quad 6.0+09.0+0$ BRC E Cleriachst 5935

$233 \mathrm{U} a 2.5+14.1+1$ CCP R Lbovt 8995

$235 \mathrm{U} P \quad 1.2+18.5+1$ CCP R Lbovt 4849

$235 \mathrm{U} 06.0+02.4+1$ CCP R Lbovt 9028

$238 \mathrm{U} P \quad 7.1+01.0+2$ CCP R Lbout 4898

$\begin{array}{lllll}23 \theta_{U} & 0 & 1.2+12.2+1 & \text { TUE R Gonnenveint } & 4899 \\ 238_{U} & 0 & 2.0+1 & 4.0+1 \text { MSU R Golbhe. } & 9046\end{array}$

$\begin{array}{lll}239 & 104 & 9046\end{array}$

$239 \mathrm{Pu} d \quad 9.2+02.3+1$ CCP R Lbovt 5971

$A=143$ cumulative $O(E)$

${ }^{230} U$ P $2.0+18.5+1$ MCG E Galiniert 4897

$A=143$ partial row o(E)

$241 \mathrm{Pu} \quad{ }^{3} \mathrm{He} \quad 3.4+1 \quad$ ANL E Bacht 6620 


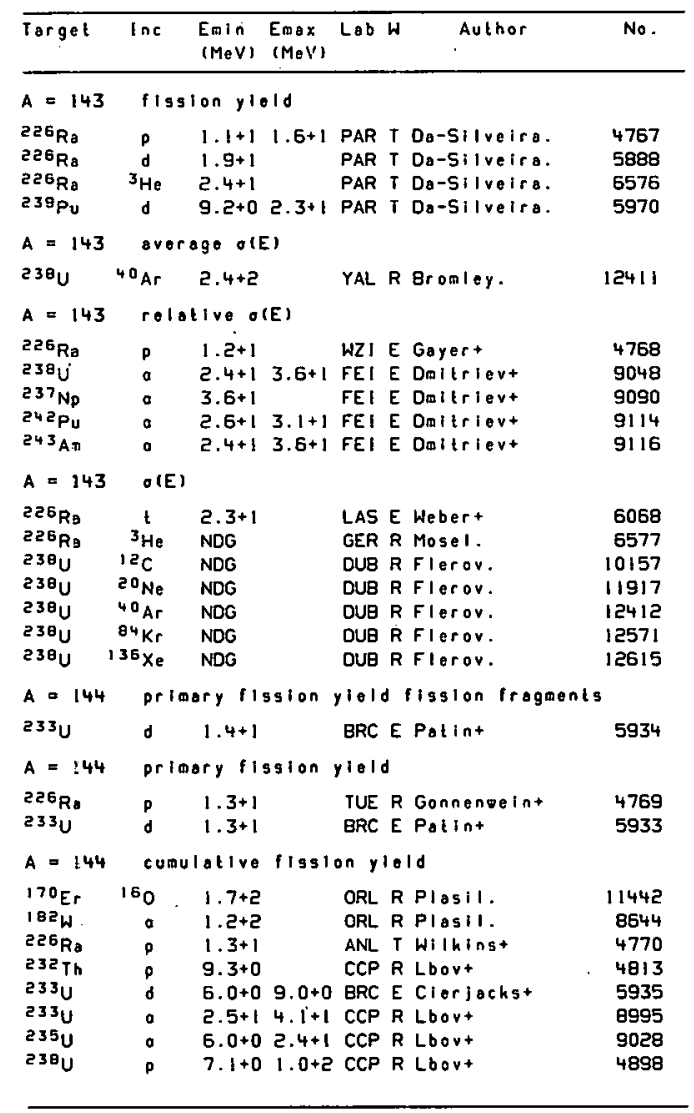

\begin{tabular}{|c|c|c|c|c|c|c|}
\hline Targot & $\ln c$ & $\begin{array}{l}\text { Emin } \\
(\mathrm{MeV})\end{array}$ & $\begin{array}{l}E \max \\
(\mathrm{MeV})\end{array}$ & Lab $h$ & Author & No. \\
\hline$A=144$ & \multicolumn{6}{|c|}{ cumulative lission yield } \\
\hline $\begin{array}{l}238 \mathrm{U} \\
238 \mathrm{U} \\
23 \theta^{2} \mathrm{U} \\
239 \mathrm{Pu}\end{array}$ & $\begin{array}{l}p \\
0 \\
d \\
d\end{array}$ & $\begin{array}{l}1.2+1 \\
2.0+1 \\
2.0+1 \\
9.2+0\end{array}$ & $\begin{array}{l}2 \cdot 2+1 \\
4 \cdot 0+1 \\
2 \cdot 9+1 \\
2 \cdot 3+1\end{array}$ & $\begin{array}{l}\text { TUE F } \\
\text { MSU F } \\
\text { SAC E } \\
\text { CCP F }\end{array}$ & $\begin{array}{l}\text { R Gonnenueint } \\
R \text { Gelbke. } \\
\text { E Libourelt } \\
\text { R Lbovt }\end{array}$ & $\begin{array}{l}4899 \\
9046 \\
9047 \\
5971\end{array}$ \\
\hline$A=144$ & \multicolumn{6}{|c|}{ cumulative o(E) } \\
\hline $\begin{array}{l}238 U \\
A=144\end{array}$ & $\begin{array}{l}\text { D } \\
\text { por }\end{array}$ & \multicolumn{4}{|c|}{ Dortial rav O(E) } & 4897 \\
\hline $241 p_{0}$ & ${ }^{3} \mathrm{He}$ & $3.4+1$ & & ANL E & E Bacht & 6620 \\
\hline$A=144$ & \multicolumn{6}{|c|}{ fission yield } \\
\hline $\begin{array}{l}256_{\mathrm{Ro}} \\
256_{\mathrm{Ra}} \\
226_{\mathrm{Ro}} \\
239_{\mathrm{Pu}}\end{array}$ & 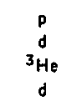 & $\begin{array}{l}1.1+1 \\
1.9+1 \\
2.4+1 \\
9.2+0\end{array}$ & $\begin{array}{l}1.6+1 \\
2.3+1\end{array}$ & $\begin{array}{l}\text { PAR } \\
\text { PAR } \\
\text { PAR } \\
\text { PAR }\end{array}$ & $\begin{array}{l}\text { T Da-Silveira. } \\
\text { T Da-Silveira. } \\
\text { T Da-Silveira. } \\
\text { T Da-Silveira. }\end{array}$ & $\begin{array}{l}4767 \\
5888 \\
6576 \\
5970\end{array}$ \\
\hline$A=144$ & \multicolumn{6}{|c|}{ overage o(E) } \\
\hline $238^{2}$ & ${ }^{40} \mathrm{Ar}$ & $2.4+2$ & & YAL F & R Bromley. & 12411 \\
\hline$A=144$ & \multicolumn{6}{|c|}{ relative o(E) } \\
\hline $\begin{array}{l}226_{\mathrm{RB}} \\
23 \mathrm{~B}_{\mathrm{U}} \\
23 . \mathrm{Np} \\
24 \mathrm{Pu}^{\mathrm{Pu}} \\
243_{\mathrm{Am}}\end{array}$ & $\begin{array}{l}p \\
: \\
: \\
:\end{array}$ & $\begin{array}{l}1.2+1 \\
2 \cdot 4+1 \\
3 \cdot 6+1 \\
2 \cdot 6+1 \\
2.4+1\end{array}$ & $\begin{array}{l}3.6+1 \\
3.1+1 \\
3.6+1\end{array}$ & $\begin{array}{l}\text { WZI } \\
\text { FEI } \\
\text { FEI } \\
\text { FEI } \\
\text { FEI }\end{array}$ & $\begin{array}{l}\text { E Gayer- } \\
\text { E Daitrient } \\
\text { E Daltriert } \\
\text { E Dallriert } \\
\text { E Dmitriert }\end{array}$ & $\begin{array}{l}4768 \\
9048 \\
9090 \\
9114 \\
9116\end{array}$ \\
\hline$A=144$ & \multicolumn{6}{|c|}{$\theta(E)$} \\
\hline $148_{\mathrm{Nd}}$ & 160 & $7.2+1$ & & NBI & E Oeschlert & 11400 \\
\hline $14 \mathrm{BNO}^{2}$ & 180 & $7.2+1$ & & NBI & E Oeschlert & 11710 \\
\hline $225_{\mathrm{RB}}$ & \multirow{2}{*}{${ }^{3} \mathrm{t}$} & $2.3+1$ & & LAS & E Webert & 6068 \\
\hline $226 \mathrm{Ra}$ & & NDG & & GER F & R Mosel. & 6577 \\
\hline $238 \mathrm{U}$. & & NDG & & DUB $\mathrm{f}$ & R Flerov. & 10157 \\
\hline $23 \theta_{U}$ & & NDG & & DUB: & R flerov. & $119 ! 7$ \\
\hline $238^{\circ}$ & $20 \mathrm{Ne}$ & NDG & & DUB: & R flecou. & 12412 \\
\hline $238 \mathrm{u}$ & $84 \mathrm{Kr}$ & NDG & & DUB & R Flerou. & 12571 \\
\hline $238_{U}$ & $\begin{array}{l}136 \times 8 \\
136\end{array}$ & NDG & & DUB & R Flerov. & 12615 \\
\hline$A=145$ & \multicolumn{6}{|c|}{ priaary fission yield fission frogments } \\
\hline $233 \mathrm{U}$ & d & $1.4+1$ & & BRC & E Patint & 5934 \\
\hline
\end{tabular}




\begin{tabular}{|c|c|c|c|c|c|c|}
\hline Target & $\ln c$ & $\begin{array}{l}\text { Emin } \\
(M e V)\end{array}$ & $\begin{array}{l}\text { Emax } \\
(\operatorname{MeV})\end{array}$ & Lab W & Author & No. \\
\hline$A=145$ & \multicolumn{6}{|c|}{ primary fisgion yleld } \\
\hline $\begin{array}{l}2266_{R} \\
233_{U}\end{array}$ & $\begin{array}{l}d \\
p \\
p\end{array}$ & $\begin{array}{l}1.3+1 \\
1.3+1\end{array}$ & & $\begin{array}{l}\text { TUE } R \\
\text { BRC E }\end{array}$ & $\begin{array}{l}\text { R Gonnenweint } \\
\text { E Patint }\end{array}$ & $\begin{array}{l}4769 \\
5933\end{array}$ \\
\hline$A=145$ & \multicolumn{6}{|c|}{ cumulative fisglon yleld } \\
\hline $\begin{array}{l}170 \mathrm{Er} \\
182 \mathrm{~W} \\
226 \mathrm{Ra} \\
232 \mathrm{Th} \\
233_{\mathrm{U}} \\
233 \mathrm{U} \\
235 \mathrm{U} \\
235 \mathrm{U} \\
238_{\mathrm{U}} \\
23 \mathrm{~B}_{\mathrm{U}} \\
238_{\mathrm{U}} \\
238_{\mathrm{U}} \\
238_{\mathrm{U}} \\
239_{\mathrm{PU}}\end{array}$ & $\begin{array}{r}160 \\
a \\
p \\
p \\
d \\
a \\
p \\
a \\
p \\
p \\
a \\
a \\
a \\
d\end{array}$ & $\begin{array}{l}1 \cdot 7+2 \\
1.2+2 \\
1.3+1 \\
9 \cdot 3+0 \\
6 \cdot 0+0 \\
2 \cdot 5+1 \\
1.2+1 \\
6 \cdot 0+0 \\
7 \cdot 1+0 \\
1 \cdot 2+1 \\
2 \cdot 0+1 \\
2 \cdot 0+1 \\
5 \cdot 0+1 \\
9 \cdot 2+0\end{array}$ & $\begin{array}{l}1 \\
9.0+0 \\
4 \cdot 1+1 \\
8.5+1 \\
2.4+1 \\
1.0+2 \\
2.2+1 \\
4 \cdot 0+1 \\
2.9+1 \\
2.3+1\end{array}$ & $\begin{array}{ll}\text { ORL } & R \\
\text { ORL } & R \\
\text { ANL } & T \\
\text { CCP } & R \\
\text { BRC } & E \\
\text { CCP } & R \\
\text { CCP } & R \\
\text { CCP } & R \\
\text { CCP } & R \\
\text { TUE } & R \\
\text { MSU } & R \\
\text { SAC } & E \\
\text { BON } & E \\
\text { CCP } & R\end{array}$ & $\begin{array}{l}R \text { Plasli. } \\
R \text { Plaslil. } \\
T \text { WlIklingt } \\
R \text { Lbovt } \\
\text { E Cierlachst } \\
R \text { Lbovt } \\
R \text { Lbovt } \\
R \text { Lbovt } \\
R \text { Lbovt } \\
R \text { Gonnengelnt } \\
R \text { Gelbke. } \\
\text { E Libourelt } \\
\text { E Davidt } \\
R \text { Lbovt }\end{array}$ & $\begin{array}{r}11442 \\
8644 \\
4770 \\
4813 \\
5935 \\
8995 \\
4849 \\
9028 \\
4898 \\
4899 \\
9046 \\
9047 \\
9049 \\
5971\end{array}$ \\
\hline$A=145$ & \multicolumn{6}{|c|}{ cumulative o(E) } \\
\hline $\begin{array}{l}238_{U} \\
A=145\end{array}$ & par & $\begin{array}{l}2.0+1 \\
1.1 \mathrm{rov}\end{array}$ & $\begin{array}{r}8.5+1 \\
\text { O(E) }\end{array}$ & MCG E & E Galinlert & 4897 \\
\hline $241 \mathrm{Pu}$ & ${ }^{3} \mathrm{He}$ & $3.4+1$ & & ANL $E$ & E Bacht & 6620 \\
\hline$A=145$ & \multicolumn{6}{|c|}{ flssion yleld } \\
\hline $\begin{array}{l}256 \mathrm{Ra} \\
226 \mathrm{Ro} \\
226 \mathrm{Ra} \\
239 \mathrm{Pu}\end{array}$ & $\underset{\substack{p \\
d \\
3^{3} \mathrm{He} \\
d}}{0}$ & $\begin{array}{l}1 \cdot 1+1 \\
1.9+1 \\
2 \cdot 4+1 \\
9.2+0\end{array}$ & $\begin{array}{l}1.6+1 \\
2.3+1\end{array}$ & $\begin{array}{l}\text { PAR T } \\
\text { PAR T } \\
\text { PAR T } \\
\text { PAR T }\end{array}$ & $\begin{array}{ll}\text { T Da-Silveira. } \\
\text { T Da-Silveira. } \\
\text { T Da-Silveira. } \\
\text { T Da-Silveira. }\end{array}$ & $\begin{array}{l}4767 \\
5888 \\
6576 \\
5970\end{array}$ \\
\hline$A=145$ & \multicolumn{6}{|c|}{ avoroge $O(E)$} \\
\hline $23 \theta_{U}$ & ${ }^{40 \mathrm{Ar}}$ & $2.4+2$ & & YAL $R$ & R Bronley. & 12411 \\
\hline$A=145$ & \multicolumn{6}{|c|}{ relative o(E) } \\
\hline $\begin{array}{l}226 \mathrm{Ro} \\
238_{\mathrm{U}} \\
237 \mathrm{~Np} \\
242 \mathrm{Pu} \\
243_{\mathrm{Am}}\end{array}$ & $\begin{array}{l}p \\
a \\
a \\
a \\
a\end{array}$ & $\begin{array}{l}1.2+1 \\
2 \cdot 4+1 \\
3 \cdot 6+1 \\
2 \cdot 6+1 \\
2.4+1\end{array}$ & $\begin{array}{l}3.6+1 \\
3.1+1 \\
3.6+1\end{array}$ & $\begin{array}{l}\text { WZI } \\
\text { FEI } \\
\text { FEI } \\
\text { FEI } \\
\text { FEI } \\
\text { FEI }\end{array}$ & 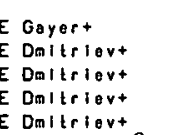 & $\begin{array}{l}4768 \\
9048 \\
9090 \\
9114 \\
9116\end{array}$ \\
\hline
\end{tabular}

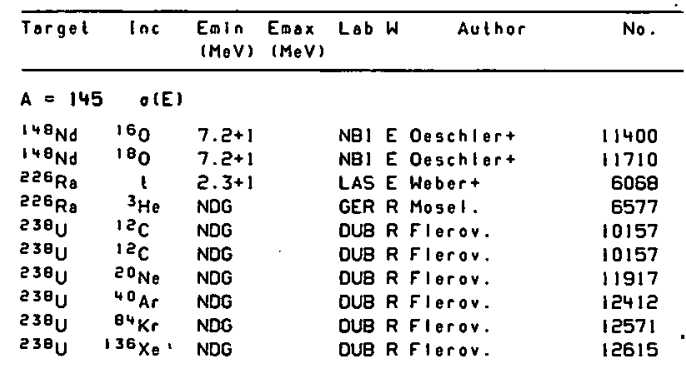

$A=146$ primary fligion yleld flagton frogments

${ }^{233} \mathrm{U}$ d $1.4+1 \quad$ BRC E Patint 5934

$A=146$ primary flsgion ylold

$\begin{array}{lllll}226 \mathrm{Ra} & P & 1.3+1 & \text { TUE R Gonnenvolnt } & 4769 \\ 233 \mathrm{U} & d & 1.3+1 & \text { BRC E Patint } & 5933\end{array}$

$A=146$ cuaulative flasion yleld

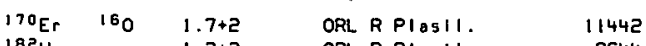

$182 \mathrm{~W} O 1.2+2$ ORL R Plosil. 8644

$\begin{array}{llll} & 1.3+1 & \text { ANL T Wilklnst } & 4770\end{array}$

$33 \mathrm{U} O \mathrm{U}$

$233 \mathrm{U} \quad 6.0+09.0+0$ BRC E Clerjochst 5935

$335 \mathrm{U} \quad 0995$

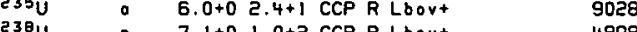

$230 \mathrm{U} \quad P \quad 7.1+01.0+2$ CCP R L6ovt 4898

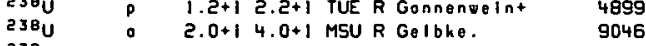

$\begin{array}{lll}238 U & 2.0+12.9+1 \text { SAC E Llbourelt } 9047\end{array}$

${ }^{239} \mathrm{Pu} d \quad 9.2+02.3+1$ CCP R Lbovt 5971

$A=146$ cumulative $\sigma(E)$

$238_{U} \quad P \quad 2.0+18.5+1$ MCG E Galiniort 4897

$A=146$ portlol ravo(E)

241Pu ${ }^{3} \mathrm{He} \quad 3.4+1 \quad$ ANL $E$ Backt $\quad 6620$

$A=146$ IIssion ylold

${ }^{226} 6_{R} \quad P \quad 1.1+1 \quad 1.6+1$ PAR T Da-Silvaira. 4767 


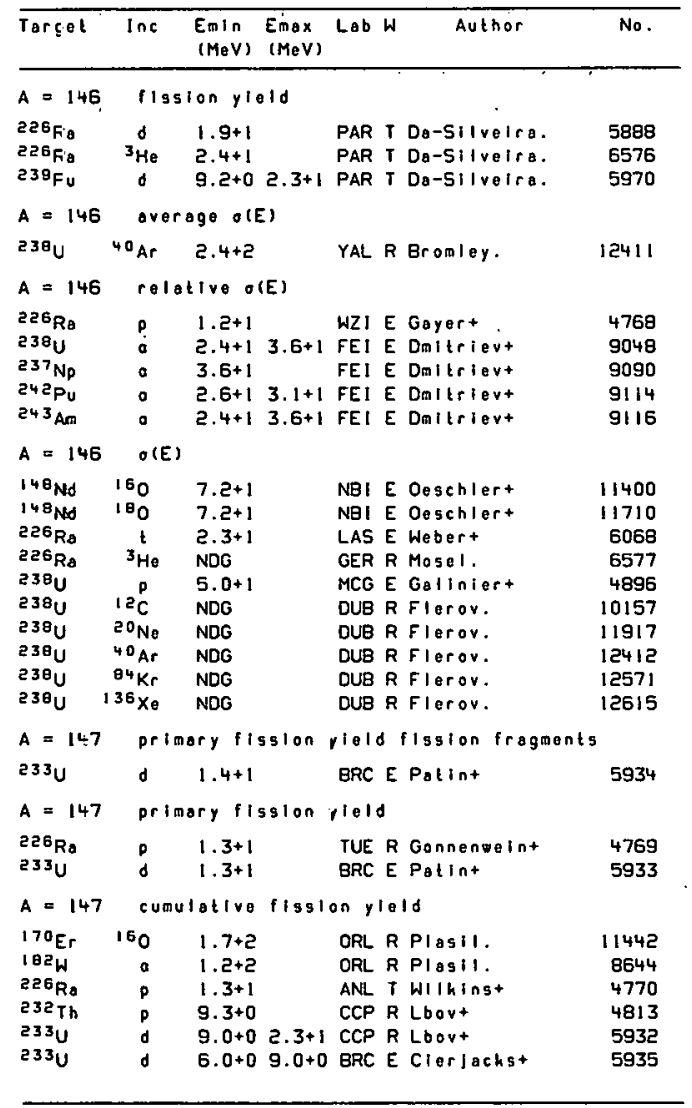

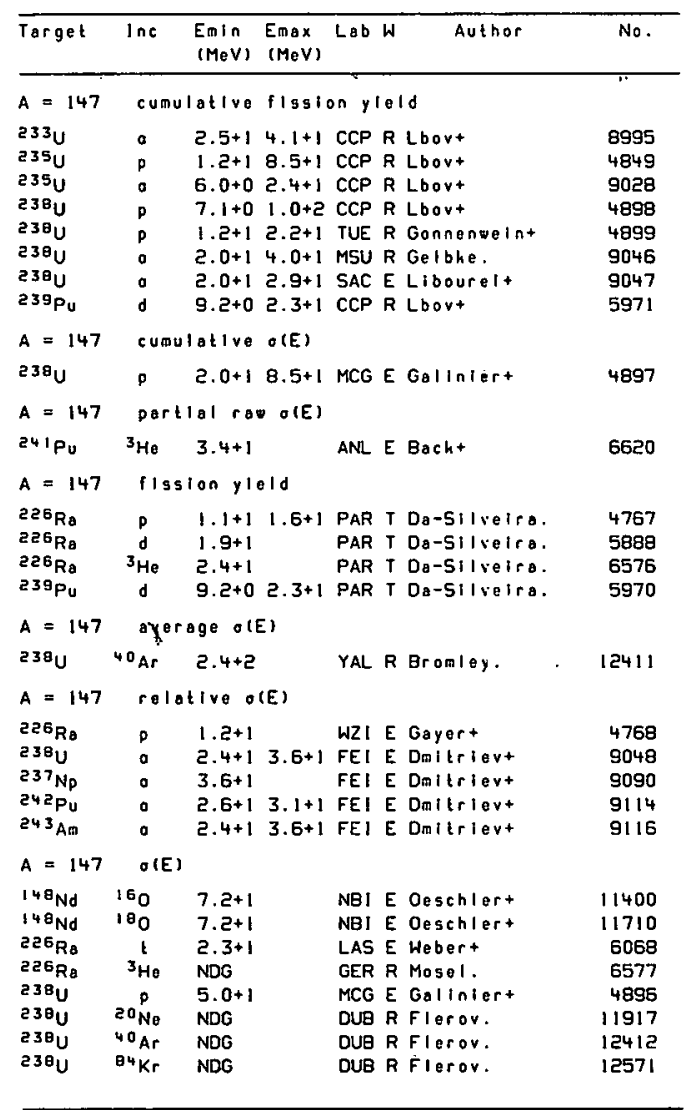




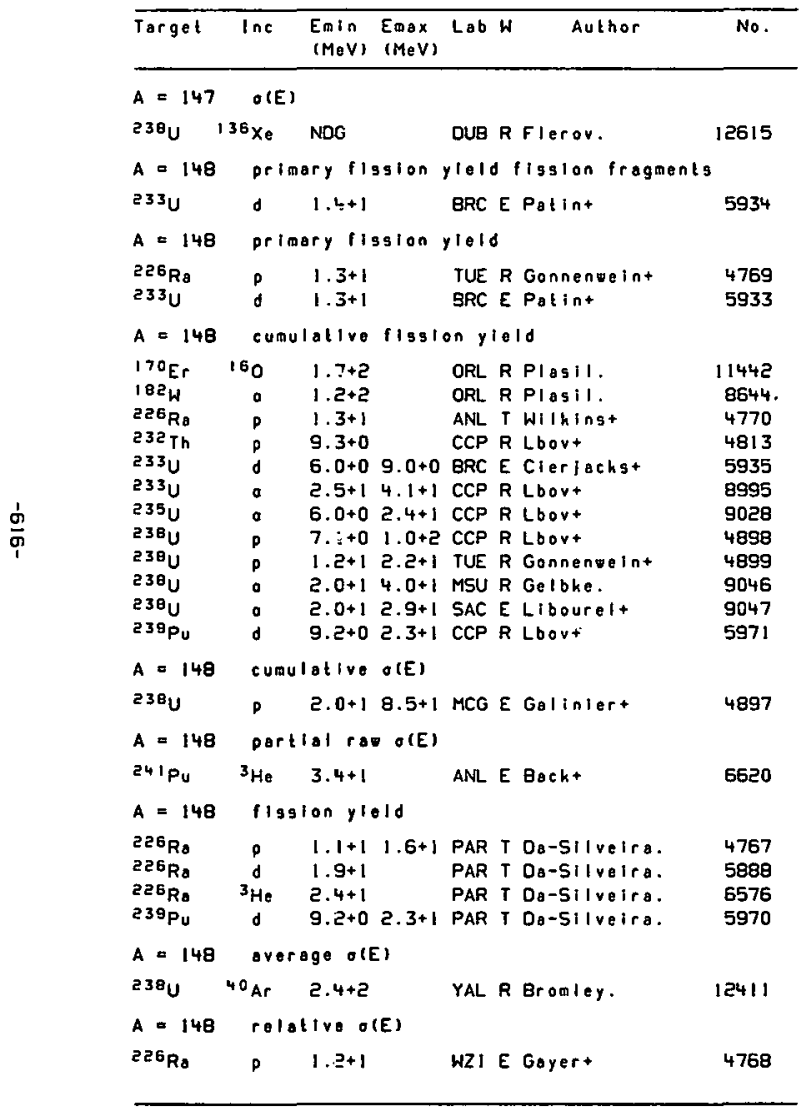

\begin{tabular}{lccccc}
\hline Target Inc & $\begin{array}{c}\text { Emla } \\
(\mathrm{MeV})\end{array}$ & Emsx Lab $(\mathrm{MeV})$ & Author & No. \\
\hline
\end{tabular}

$A=14 E$ relotive o(E)

$23 \mathrm{O}^{2} \quad$ o $2.4+13.6+1$ FEI E Doitriert 9048

$237_{\mathrm{Np}} a$ 3.6+1 $3.6+1$ FEI E DoItrievt 9090

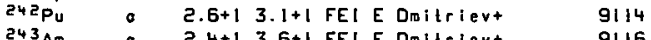

$A=148 \quad \sigma(E)$

$148 \mathrm{Nd} 160 \quad 7.2+1 \quad$ NBI E Oeschlort

$\begin{array}{llll}148_{\text {Nd }} 180 & 7.2+1 & \text { NBIE Deschlert } & 11710\end{array}$

${ }^{226} \mathrm{Re}_{\mathrm{B}} \mathrm{t} 2.3+1$ LASE Webert 6068

$226 \mathrm{Ra}^{3}{ }^{\mathrm{He}}$ NDG GER R Mosel. 6577

$\begin{array}{llllr}238_{\mathrm{U}} & { }^{\mathrm{p}} & 5.0+1 & \text { MCG E Galiniert } & 4896 \\ 238_{\mathrm{U}} & 12 \mathrm{C} & \text { NDG } & \text { DUB R Flerov. } & 10157\end{array}$

$230^{20}{ }^{20}$ NOG DUB R Flerov. 11917

$230 \mathrm{U} 4{ }^{\circ} \mathrm{Ar}$ NDG DUB R Flerov. 12412

${ }^{230}{ }^{84} \mathrm{Kr} N D G \quad$ DUB R Flerov. 12571

${ }^{238} \mathrm{U}{ }^{136} \mathrm{Xe}$ NDG DUB R Flerov. 12615

$A=149$ prlaary flasion ylold flasion fragaents

$233 \mathrm{U} d \quad 1.4+1 \quad$ BRC E Palint 5934

$A=149$ primory flasion yleld

$226 \mathrm{Ra} \quad P \quad 1.3+1 \quad$ TUE R Ganaendeint 4769

$233 \mathrm{U}$ d $1.3+1$ BRC E Palint 5933

$A=149$ cumulative fission yleld

170 Er ${ }^{160} \quad 1.7+2 \quad$ ORL R PIOSII. 11442

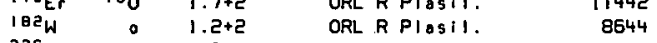

$\begin{array}{lllll}226 \mathrm{Ra} & 0 & 1.2+2 & \text { ORL R Plosil. } & 8644 \\ 20 & 1.3+1 & \text { ANL T Wilhinst } & 4770\end{array}$

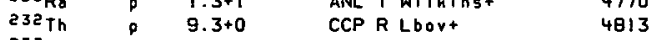

$233 \mathrm{U} d$ 6.0+0 9.0+0 BRC E Cleriackst 5935

$233 \mathrm{U} \quad 0 \quad 2.5+14.1+1$ CCP R Lbovt 8995

$235 \mathrm{U} P \quad 1.2+18.5+1$ CCP R Lbovt 4849

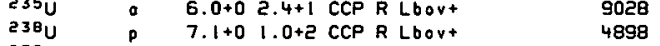

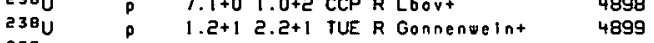

$23 \theta_{U} \quad 0 \quad 2.0+14.0+1$ MSU R Gelbke. 9046

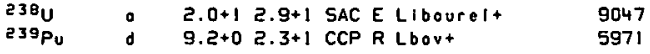




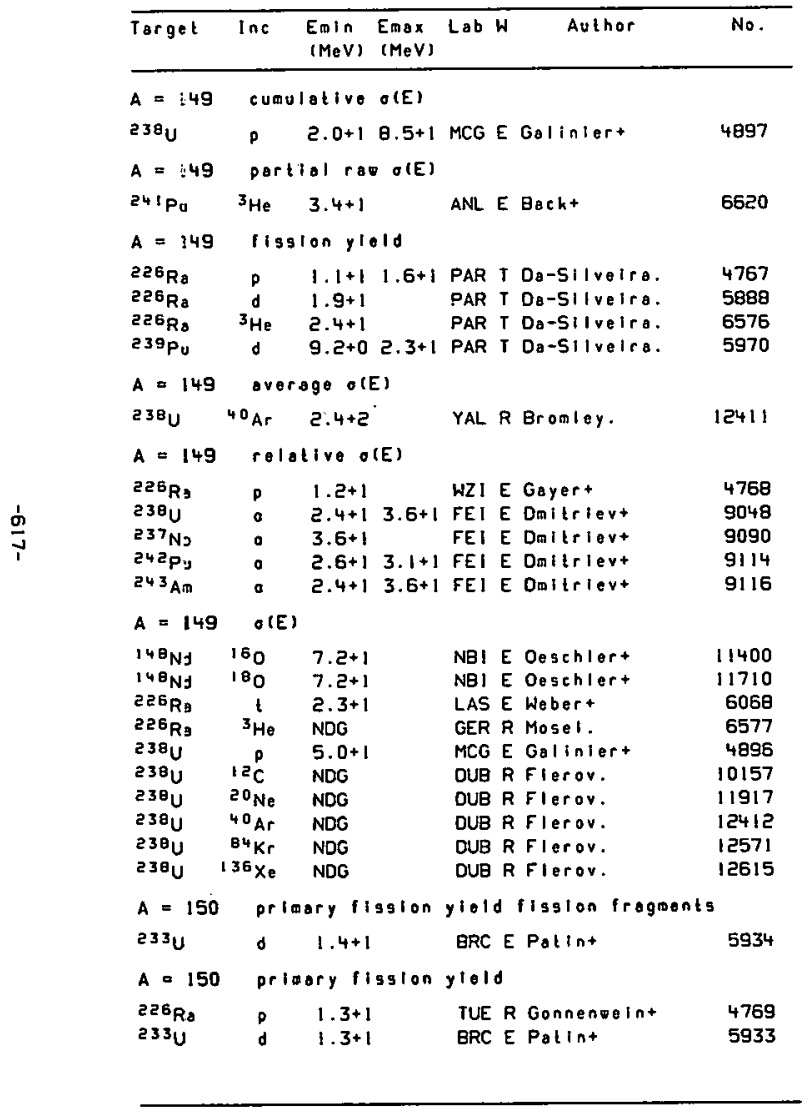

\begin{tabular}{|c|c|c|c|c|c|c|}
\hline Target & $\operatorname{Inc}$ & $\begin{array}{l}E_{m} \mid n \\
(M e V)\end{array}$ & $\begin{array}{l}\text { Emax } \\
(\operatorname{MeV})\end{array}$ & Lab W & Autho: & No. \\
\hline
\end{tabular}

$A=150$ cumulative tission yleld

$170 \mathrm{Er} \quad 160 \quad 1.7+2 \quad$ ORL R Plosil. 11442

$102_{\mathrm{W}}$ a $1.2+2$ ORL R Plasil. 8644

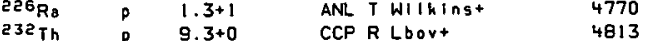

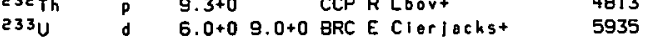

$233 \mathrm{U} \quad 2.5+14.1+1$ CCP R Lbovt

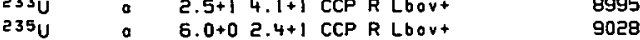

$238 \mathrm{U} P \mathrm{p} \quad 7.1+01.0+2$ CCP R Lbovt 4898

$23 \theta_{U} \mathrm{U} \quad 1.2+12.2+1$ TUE R Gonnenweint 4899

$230^{2} \mathrm{U} \quad 2.0+14.0+1$ MSU R Gelbhe. 9046

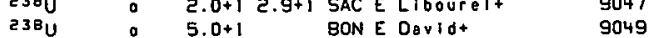

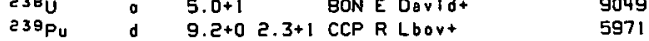

$A=150$ cumulative $\theta(E)$

$238 U$ P $2.0+1$ B.5+1 MCG E Galinlert 4897

$A=150$ porllol ravole

$241 \mathrm{Pu}^{3} \mathrm{He} \quad 3.4+1 \quad$ ANI. E Bacht 6620

$A=150$ 11ssion yielo

${ }^{226} \mathrm{Ra}_{a} \quad \mathrm{O} \quad 1.1+11.6+1$ PAR T Da-5llvelra. 4767

$226 R_{0} d \quad 1.9+1$ PART Da-5llvelra. 5888

$226_{\mathrm{Ra}}{ }^{3} \mathrm{He} \quad 2.4+1 \quad$ PAR T Da-Silvelra. 6576

$23{ }^{9} \mathrm{Pu}$ d $9.2+0$ 2.3+1 PAR T Da-Silveira. 5970

$A=150 \quad r a D O(E)$

$25{ }^{7} \mathrm{Fm} \mid 1.6+1$ LAS E Horfmort 6090

$A=150$ averogo $\sigma(E)$

$230^{4}{ }^{40} \mathrm{Ar} 2.4+2$ YAL R Bromles. 12411

$A=150$ Colative o(E)

${ }^{226} \mathrm{Ra} P$ P $1.2+1 \quad$ WZ! E Gayert 4768

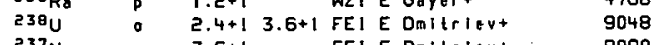

$237 \mathrm{~Np} \quad 0 \quad 3.6+1 \quad$ FEI E Omitrlevt 9090

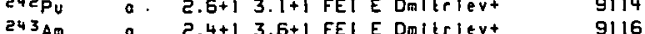




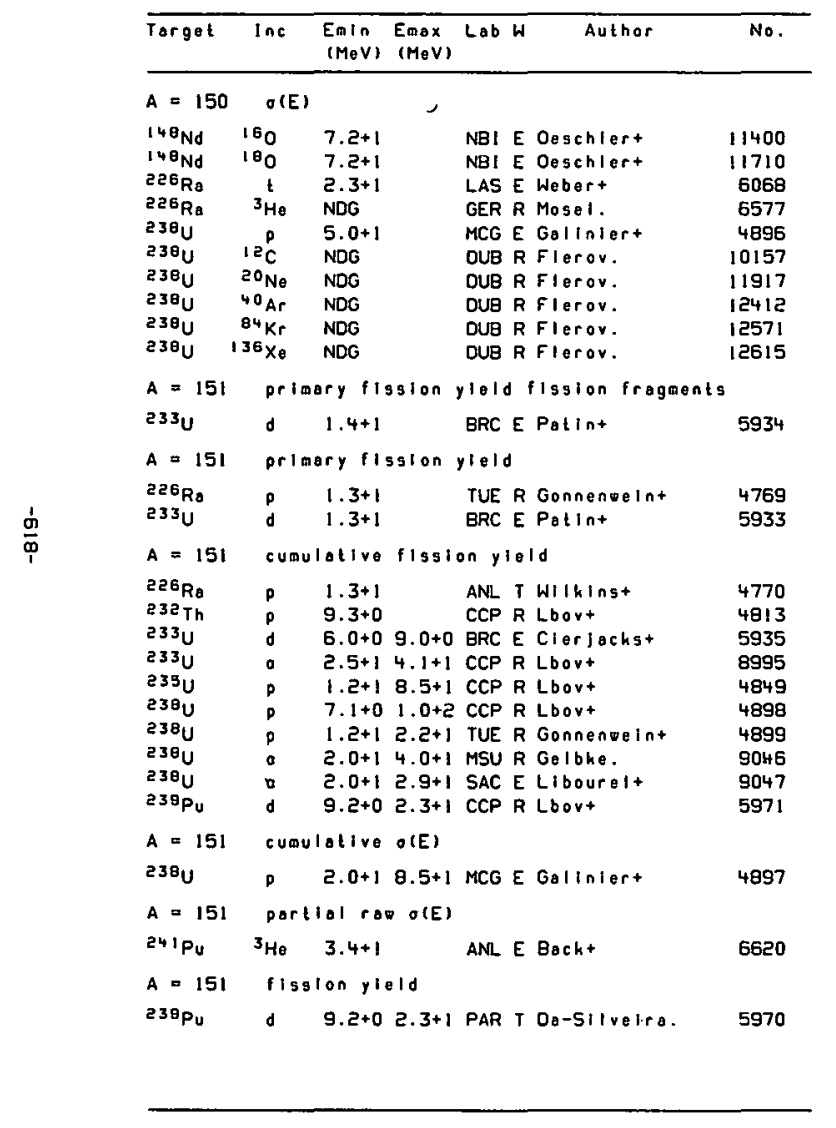

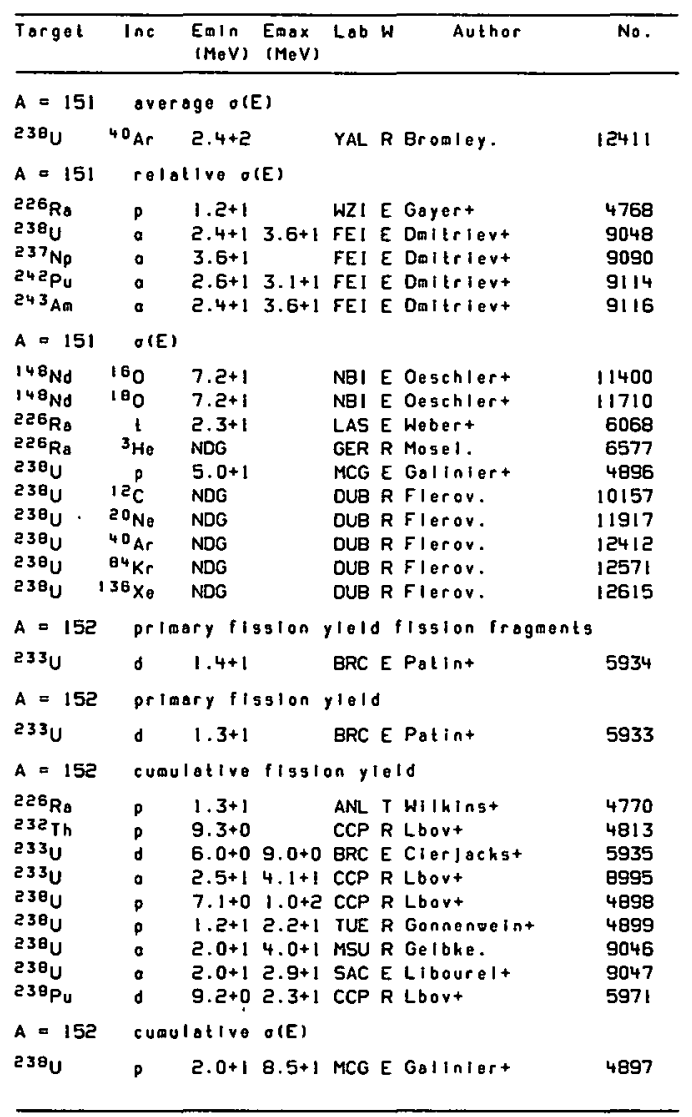




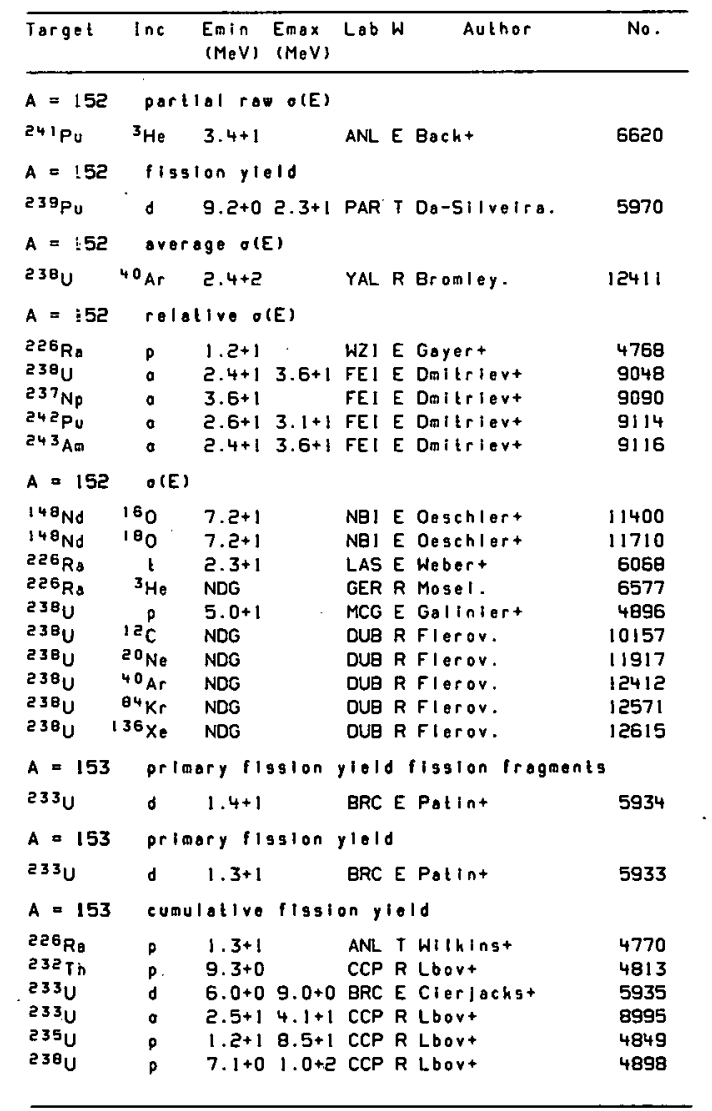

\begin{tabular}{lllll}
\hline Target Inc & $\begin{array}{l}\text { Emin Emax Lab } \\
\text { (MeV) }\end{array}$ & Author & No. \\
\hline
\end{tabular}

$A=153$ cumulative flisgion yleld

$238 \mathrm{U} P \quad 1.2+12.2+1$ TUE R Gonnenieint 4899

$238 \mathrm{U} \quad 2.0+14.0+1$ MSU R Gelbhe. 9046

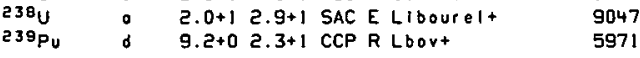

$A=153$ cumulative of(E)

$23 \theta U$ P $2.0+18.5+1$ MCG E Galiniert 4897

$A=153$ parllal rao o(E)

$241 \mathrm{Pu} \quad{ }^{3} \mathrm{He} 3.4+1 \quad$ ANL E Bacht 6620

$A=153$ ligsion yleld

$239 \mathrm{Pu} \quad \mathrm{d} 9.2+02.3+1$ PAR T Da-Silveira.
$A=153$ overoge O(E)

$238 \mathrm{U} \quad{ }^{40} \mathrm{Ar} 2.4+2$ YAL R Bromloy.

$A=153$ relotive of(E)

$226 \mathrm{Re} \quad \mathrm{P} \quad 1.2+1 \quad$ WZI E Gayert 4768

$23 \mathrm{U}^{\circ} \quad 2.4+13.6+1$ FEI E Dmltriert 9048

$23{ }^{37} \mathrm{~Np} a \quad 3.6+1$ FEI E Doltrieut 9090

$242 \mathrm{Pu} a$ a $2.6+13.1+1 \mathrm{FEI} E$ Dnitrievt 9114

243 Am a $2.4+13.6+1$ FEI E Dmiteiert 9116

$\begin{array}{lllll}148_{\text {No }} & 160 & 7.2+1 & \text { NEI E Oeschiert } & 11400 \\ 148_{\text {Nd }} & 10_{0} & 7.2+1 & \text { NEI E Oeschiert } & 11710\end{array}$

$\begin{array}{llll}256 \mathrm{Ra} & t & 2.3+1 & \text { LAS E Webert }\end{array}$

${ }^{226} \mathrm{Re} \quad{ }^{3} \mathrm{He} \quad$ NDG $\quad$ GER R Mosel.

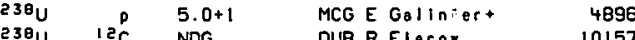

$23 \theta^{2} \mathrm{I} C \mathrm{C}$ NDG DUB R Flerov. 10157

$230 \mathrm{U} 20 \mathrm{Ne}$ NDG $204 \mathrm{~B}$ R Flerov. 11917

$230^{4}{ }^{40} \mathrm{Ar}$ NDG DUB R Flerov.

${ }^{238} \mathrm{U}{ }^{84} \mathrm{Kr}$ NDG DUB R Flerov. 1257

${ }^{230} \mathrm{U}{ }^{136} \mathrm{Xe}$ NDG DUB R Flerov. 12615

$A=154$ primary fission yield fisglon tragments

$233 \mathrm{U}$ o $1.4+1 \quad$ BRC E Patin- 5934 


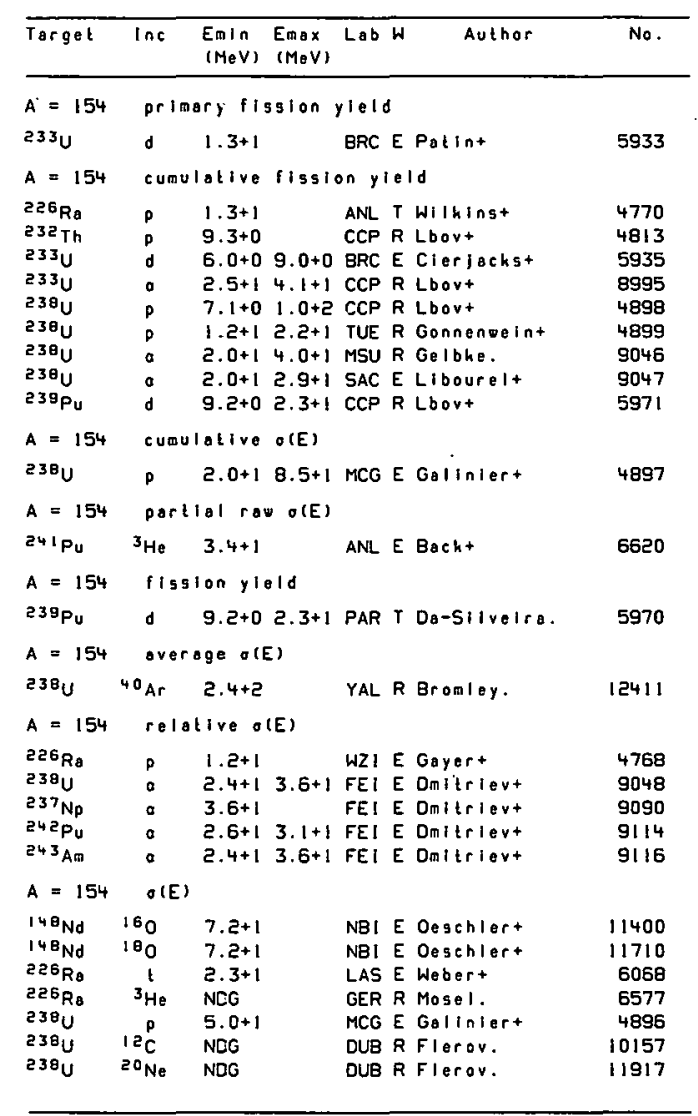

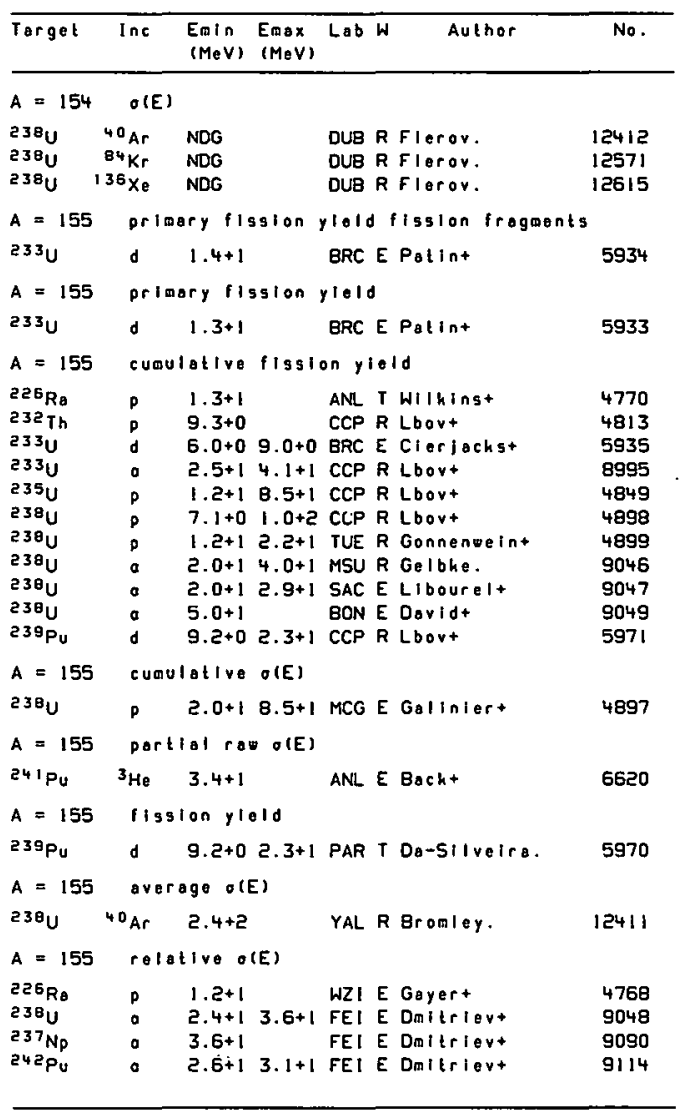




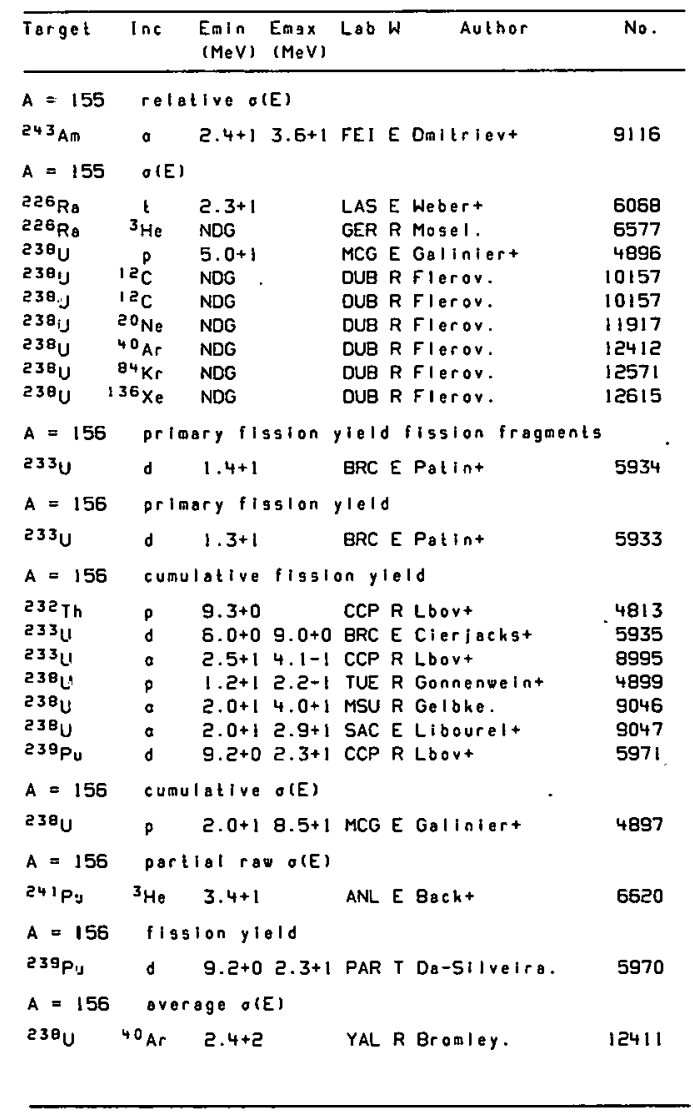

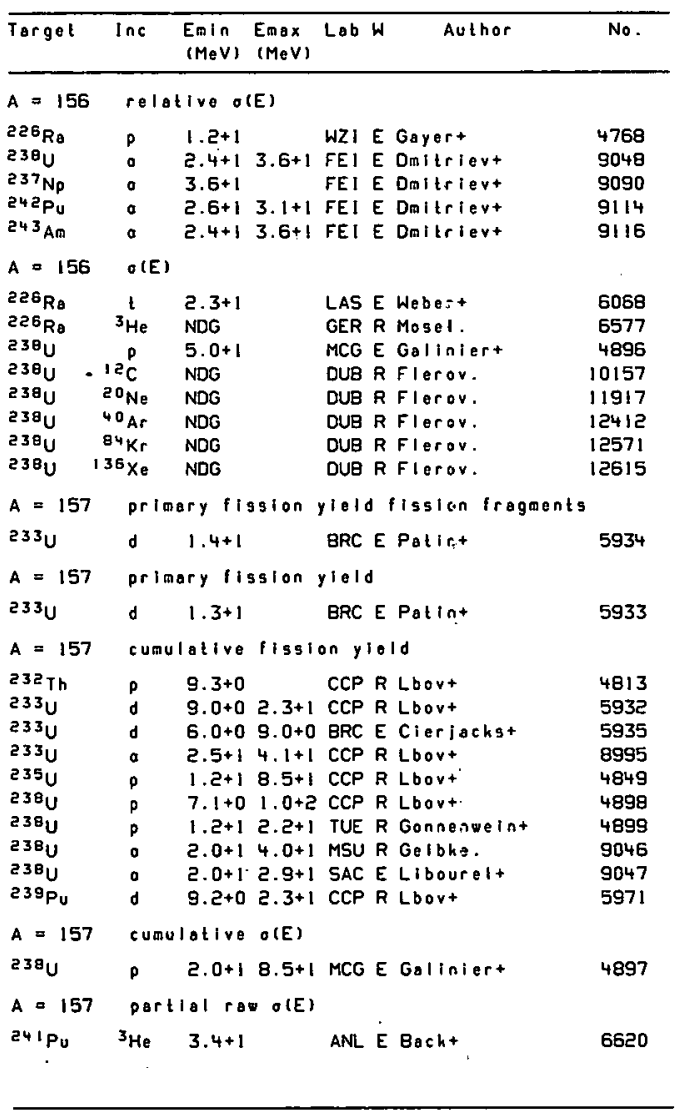




\begin{tabular}{|c|c|c|c|c|c|c|}
\hline Target & $\ln c$ & $\begin{array}{l}E n \ln \\
(M e V)\end{array}$ & $\begin{array}{l}\text { Emax } \\
\text { (MeV) }\end{array}$ & Lab & Author & No. \\
\hline$A=157$ & \multicolumn{6}{|c|}{ fisglon yleld } \\
\hline $\begin{array}{l}239 P_{U} \\
A=157\end{array}$ & d & \multicolumn{4}{|c|}{ average o(E) } & 5970 \\
\hline $\begin{array}{l}238 \mathrm{U} \\
A=157\end{array}$ & $\begin{array}{r}{ }^{40} \mathrm{Ar} \\
\mathrm{rO}\end{array}$ & \multicolumn{5}{|c|}{ relalive o(E) } \\
\hline $\begin{array}{l}226_{\mathrm{Ra}} \\
23 \theta_{\mathrm{U}} \\
237_{\mathrm{Np}} \\
242 \mathrm{Pu} \\
243_{\mathrm{Am}}\end{array}$ & $\begin{array}{l}p \\
a \\
a \\
a \\
a\end{array}$ & $\begin{array}{l}1.2+1 \\
2.4+1 \\
z .6+1 \\
\bar{z} \cdot 6+1 \\
2.4+1\end{array}$ & $\begin{array}{l}3.6+1 \\
3.1+1 \\
3.6+1\end{array}$ & $\begin{array}{l}\text { WZI E } \\
\text { FEI E } \\
\text { FEI E } \\
\text { FEI E } \\
\text { FEI E }\end{array}$ & $\begin{array}{l}\text { E Gayert } \\
\text { E Omitrievt } \\
\text { E Omitrievt } \\
\text { E Omitrievt } \\
\text { E Omitrievt }\end{array}$ & $\begin{array}{l}4768 \\
9048 \\
9090 \\
9114 \\
9116\end{array}$ \\
\hline$A=157$ & \multicolumn{6}{|c|}{$\sigma(E)$} \\
\hline $\begin{array}{l}228_{R s} \\
226 R_{B} \\
238_{U} \\
238_{U} \\
238_{U} \\
238_{U} \\
238_{U}\end{array}$ & 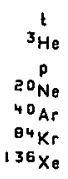 & $\begin{array}{l}2.3+1 \\
\text { NDG } \\
5.0+1 \\
\text { NDG } \\
\text { NDG } \\
\text { NDG } \\
\text { NDG }\end{array}$ & & $\begin{array}{l}\text { LAS } \\
\text { GER F } \\
\text { MCG E } \\
\text { DUE F } \\
\text { OUE F } \\
\text { OU日 F } \\
\text { OU日 F }\end{array}$ & $\begin{array}{l}\text { E Webert } \\
\text { R Mosel. } \\
\text { E Galiniert } \\
\text { R Flerov. } \\
\text { R Flerov. } \\
\text { R Flerov. } \\
\text { R Flerov. }\end{array}$ & $\begin{array}{r}6068 \\
6577 \\
4896 \\
11917 \\
12412 \\
12571 \\
12615\end{array}$ \\
\hline \multicolumn{4}{|c|}{ primary fission } & yleld & digsion frogme & \\
\hline $233 \mathrm{U}$ & d & $1.4+1$ & & BRC & E Patint & 5934 \\
\hline \multicolumn{4}{|c|}{ primary fission } & $y 1010$ & & \\
\hline $233 \mathrm{U}$ & $d$ & $1.3+1$ & & BRC E & E Pat In+ & 5933 \\
\hline$A=158$ & \multicolumn{2}{|c|}{ cumulative } & fisslo & on $y 10$ & 1010 & \\
\hline $\begin{array}{l}232 \mathrm{Th} \\
233 \mathrm{U} \\
233_{\mathrm{U}} \\
238_{\mathrm{U}} \\
238_{\mathrm{U}} \\
238_{\mathrm{U}} \\
23 \mathrm{P}_{\mathrm{Pu}}\end{array}$ & $\begin{array}{l}p \\
d \\
0 \\
p \\
0 \\
0 \\
d\end{array}$ & $\begin{array}{l}9 \cdot 3+0 \\
6 \cdot 0+0 \\
2 \cdot 5+1 \\
1.2+1 \\
2 \cdot 0+1 \\
2 \cdot 0+1 \\
9 \cdot 2+0\end{array}$ & $\begin{array}{l}9.0+0 \\
4.1+1 \\
2.2+1 \\
4.0+1 \\
2.9+1 \\
2.3+1\end{array}$ & $\begin{array}{l}\text { CCP F } \\
\text { BRC } \\
\text { CCP F } \\
\text { TUE F } \\
\text { MSU F } \\
\text { SAC } \\
\text { CCP F }\end{array}$ & $\begin{array}{l}\text { R Lbovt } \\
\text { E Cierjackst } \\
R \text { Lbovt } \\
R \text { Gonnenwelnt } \\
R \text { Gelbhe. } \\
\text { E Libourelt } \\
\text { R Lbovt }\end{array}$ & $\begin{array}{l}4813 \\
5935 \\
8995 \\
4899 \\
9046 \\
9047 \\
5971\end{array}$ \\
\hline \multirow{2}{*}{$\begin{array}{l}A=158 \\
238 \mathrm{U}\end{array}$} & \multicolumn{2}{|c|}{ cumulative } & $\sigma(E)$ & & & \\
\hline & p & $2.0+1$ & $8.5+1$ & MCG & E Golintert & 4897 \\
\hline
\end{tabular}

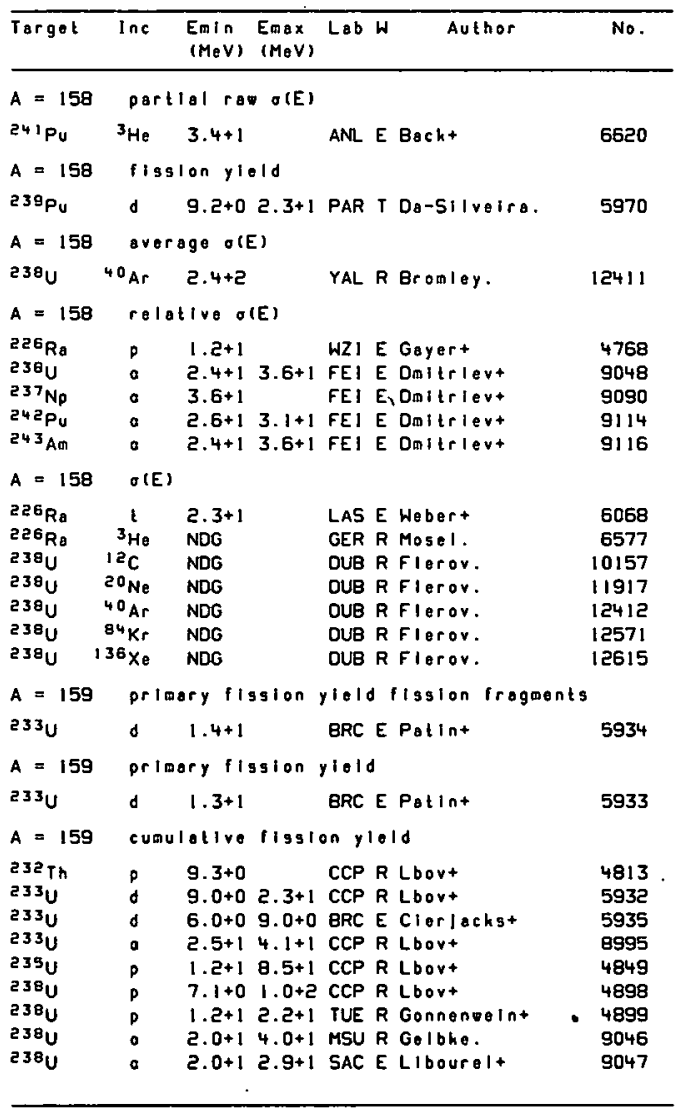




\begin{tabular}{|c|c|c|c|c|c|c|}
\hline Target & Inc & $\begin{array}{l}\text { Emin } \\
(\mathrm{MeV})\end{array}$ & $\begin{array}{l}\text { Emax } \\
(\operatorname{MeV} V)\end{array}$ & Lob & Author & No. \\
\hline$A=159$ & \multicolumn{6}{|c|}{ cumulative flgsion yleld } \\
\hline $\begin{array}{l}{ }^{239} \mathrm{Pu} \\
A=159\end{array}$ & $\begin{array}{l}\text { d } \\
\text { cum }\end{array}$ & $\begin{array}{l}9.2+0 \\
\text { lattve }\end{array}$ & $\begin{array}{l}2.3+1 \\
\sigma(E)\end{array}$ & CCP F & R Lbovt & 5971 \\
\hline $238 \mathrm{U}$ & $p$ & $2.0+1$ & $8.5+1$ & MCO E & E Galinlert & 4897 \\
\hline$A=159$ & \multicolumn{6}{|c|}{ figsion yiold } \\
\hline $\begin{array}{l}{ }^{23}{ }^{2} P_{4} \\
A=159\end{array}$ & d & \multicolumn{4}{|c|}{ average o(E) } & 5970 \\
\hline $\begin{array}{l}{ }^{238} U \\
A=159\end{array}$ & $\begin{array}{l}40 \mathrm{Ar} \\
\mathrm{r} \in \mathrm{I}\end{array}$ & \multicolumn{4}{|c|}{ relotive $\sigma(E)$} & 12411 \\
\hline $\begin{array}{l}23 \theta^{2} \mathrm{U} \\
237_{\mathrm{Np}} \\
242^{2} \mathrm{Pu} \\
243_{\mathrm{Am}}\end{array}$ & $\begin{array}{l}a \\
a \\
a \\
a\end{array}$ & $\begin{array}{l}2.4+1 \\
3.6+1 \\
2.6+1 \\
2.4+1\end{array}$ & $\begin{array}{l}3.6+1 \\
3.1+1 \\
3.6+1\end{array}$ & $\begin{array}{l}\text { FEI } \\
\text { FEI } \\
\text { FEI } \\
\text { FEI }\end{array}$ & $\begin{array}{l}\text { E Doilrlevt } \\
\text { E Doitrlevt } \\
\text { E Doitrleyt } \\
\text { E Daltrlevt }\end{array}$ & $\begin{array}{l}9048 \\
9090 \\
9114 \\
9116\end{array}$ \\
\hline$A=159$ & \multicolumn{6}{|c|}{$\sigma(E)$} \\
\hline $\begin{array}{l}226 R \Delta \\
226 R B \\
238 U \\
238 U \\
238 U \\
238 U \\
238 U\end{array}$ & $\begin{array}{c}t \\
3 \mathrm{He} \\
12 \mathrm{C} \\
20 \mathrm{Ne} \\
40 \mathrm{Ar} \\
84 \mathrm{Kr} \\
136 \times \mathrm{Xo}\end{array}$ & $\begin{array}{l}2.3+1 \\
\text { NDG } \\
\text { NDG } \\
\text { NDG } \\
\text { NDG } \\
\text { NDG } \\
\text { NDG }\end{array}$ & & $\begin{array}{l}\text { LAS } \\
\text { GER } \\
\text { DUB } \\
\text { DUB } \\
\text { DUA } \\
\text { DUB } \\
\text { DUA }\end{array}$ & $\begin{array}{l}\text { E Webert } \\
\text { R Mosel. } \\
R \text { Flerov. } \\
R \text { Flerov. } \\
R \text { Flerov. } \\
\text { R Flerov. } \\
\text { R Flerov. }\end{array}$ & $\begin{array}{r}6068 \\
6577 \\
10157 \\
11917 \\
12412 \\
12571 \\
12615\end{array}$ \\
\hline$A=160$ & \multicolumn{2}{|c|}{ cumulative } & \multicolumn{3}{|c|}{ fission yield } & \\
\hline 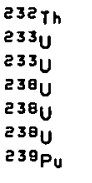 & $\begin{array}{l}p \\
d \\
a \\
p \\
a \\
a \\
d\end{array}$ & $\begin{array}{l}9.3+0 \\
6.0+0 \\
2.5+1 \\
1.2+1 \\
2.0+1 \\
2.0+1 \\
9.2+0\end{array}$ & $\begin{array}{l}9.0+0 \\
4 \cdot 1+1 \\
2.2+1 \\
4.0+1 \\
2.9+1 \\
2.3+1\end{array}$ & $\begin{array}{l}\text { CCP } \\
\text { BRC } \\
\text { CCP } \\
\text { TUE } \\
\text { MSU } \\
\text { SAC } \\
\text { CCP }\end{array}$ & $\begin{array}{l}\text { R Lbovt } \\
\text { E Clarjackst } \\
R \text { Lbovt } \\
\text { R Gonnenwelnt } \\
R \text { Gelbke. } \\
\text { E Llbourelt } \\
\text { R Lbovt }\end{array}$ & $\begin{array}{l}4813 \\
5935 \\
8995 \\
4899 \\
9046 \\
9047 \\
5971\end{array}$ \\
\hline$A=160$ & cum & lotive & $O(E)$ & & & \\
\hline $\begin{array}{l}230^{2} U \\
A=160\end{array}$ & p & $\begin{array}{l}2.0+1 \\
\operatorname{lon} y i \theta\end{array}$ & $\begin{array}{l}8.5+1 \\
010\end{array}$ & MCG & E Galinlert & 4897 \\
\hline $238 \mathrm{Pu}$ & 0 & $9.2+0$ & $2.3+1$ & PAR & T Da-Silvelra. & 5970 \\
\hline
\end{tabular}

\begin{tabular}{|c|c|c|c|c|c|c|}
\hline Torget & $\ln c$ & $\begin{array}{l}\text { Eoln } \\
\left(M_{\theta} V\right)\end{array}$ & $\begin{array}{l}\text { Emax } \\
(\mathrm{MeV})\end{array}$ & Lob & Author & No. \\
\hline$A=160$ & \multicolumn{6}{|c|}{$\operatorname{ros} \theta(E)$} \\
\hline $257_{\mathrm{Fm}}$ & t & $1.6+1$ & & LAS & E Hoffinont & 6090 \\
\hline$A=160$ & \multicolumn{6}{|c|}{ average $O(E)$} \\
\hline $\begin{array}{l}230 \mathrm{U} \\
A=160\end{array}$ & $\begin{array}{l}40 \mathrm{Ar} \\
\mathrm{reI}\end{array}$ & $\begin{array}{l}2.4+2 \\
\text { tive of }\end{array}$ & (E) & YAL & R Gromley. & 12411 \\
\hline $\begin{array}{l}230 \mathrm{U} \\
237_{\mathrm{Np}} \\
24 \mathrm{Pu}^{2} \\
243_{\mathrm{Am}}\end{array}$ & $\begin{array}{l}0 \\
\vdots \\
0 \\
\circ\end{array}$ & $\begin{array}{l}2.4+1 \\
3.6+1 \\
2.6+1 \\
2.4+1\end{array}$ & $\begin{array}{l}3.6+1 \\
3 \cdot 1+1 \\
3.6+1\end{array}$ & $\begin{array}{l}\text { FEI } \\
\text { FEI } \\
\text { FEI } \\
\text { FEI }\end{array}$ & $\begin{array}{l}\text { E Dmltrient } \\
\text { E Dmilriovt } \\
\text { E Dallrievt } \\
\text { E Dollriovt }\end{array}$ & $\begin{array}{l}9048 \\
9090 \\
9114 \\
9116\end{array}$ \\
\hline$A=160$ & \multicolumn{6}{|c|}{$O(E)$} \\
\hline 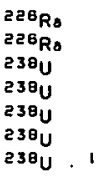 & $\begin{array}{c}3^{\prime} \\
3_{\mathrm{He}} \\
12 \mathrm{C} \\
20 \mathrm{Ne} \\
40 \mathrm{Ar} \\
84 \mathrm{Kr} \\
136 \mathrm{Xe}\end{array}$ & $\begin{array}{l}2.3+1 \\
\text { NDG } \\
\text { NDG } \\
\text { NDG } \\
\text { NDG } \\
\text { NDG } \\
\text { NDG }\end{array}$ & & $\begin{array}{l}\text { LAS } \\
\text { GER } \\
\text { DUB } \\
\text { DUB } \\
\text { DUB } \\
\text { DUB } \\
\text { OUB }\end{array}$ & $\begin{array}{l}\text { E Webert } \\
\text { R Mosel. } \\
\text { R Flerov. } \\
\text { R Flerov. } \\
\text { R Flerov. } \\
\text { R Flerov. } \\
\text { R Flerov. }\end{array}$ & $\begin{array}{r}6068 \\
6577 \\
10157 \\
11917 \\
12412 \\
12571 \\
12615\end{array}$ \\
\hline \multicolumn{3}{|l|}{$A=161$} & fissto & on $y$ & $101 d$ & \\
\hline $\begin{array}{l}238_{U} \\
238 \mathrm{U} \\
23 \mathrm{Pu}^{2}\end{array}$ & $\begin{array}{l}p \\
p \\
d\end{array}$ & $\begin{array}{l}7.1+0 \\
1.2+1 \\
9.2+0\end{array}$ & $\begin{array}{l}1.0+2 \\
2.2+1 \\
2.3+1\end{array}$ & $\begin{array}{l}\text { CCP } \\
\text { TUE } \\
\text { CCP }\end{array}$ & $\begin{array}{l}R \text { Lbovt } \\
R \text { Gonnenwelnt } \\
R \text { Lbovt }\end{array}$ & $\begin{array}{l}4898 \\
4899 \\
5971\end{array}$ \\
\hline$A=161$ & cum & olotive & $\sigma(E)$ & & & \\
\hline $\begin{array}{l}23 \theta_{U} \\
A=161\end{array}$ & $\begin{array}{l}\text { D } \\
\text { ove }\end{array}$ & $\begin{array}{l}2.0+1 \\
.90 \quad 0 / E\end{array}$ & $8.5+1$ & MCG & E Goliniert. & 4897 \\
\hline${ }^{230} \mathrm{U}$ & ${ }^{40} \mathrm{Ar}$ & $2.4+2$ & & YAL & R Bromler. & 12411 \\
\hline$A=161$ & rol & ative ol & (E) & & & . \\
\hline $\begin{array}{l}23 \theta_{U} \\
237 \mathrm{~Np} \\
24 P_{u} \\
243_{A \infty}\end{array}$ & $\begin{array}{l}a \\
a \\
a \\
a\end{array}$ & $\begin{array}{l}2.4+1 \\
3.6+1 \\
2.6+1 \\
2.4+1\end{array}$ & $\begin{array}{l}3.6+1 \\
3.1+1 \\
3.6+1\end{array}$ & $\begin{array}{l}\text { FEI } \\
\text { FEI } \\
\text { FEI } \\
\text { FEI }\end{array}$ & $\begin{array}{l}\text { E Omitrievt } \\
\text { E Dol trievt } \\
\text { E Dmitrievt } \\
\text { E Dmitrievt }\end{array}$ & $\begin{array}{l}9048 \\
9090 \\
9114 \\
9116\end{array}$ \\
\hline$A=161$ & OtE & & & & & \\
\hline $\begin{array}{l}23 \theta_{U} \\
23 \theta_{U}\end{array}$ & $\begin{array}{l}{ }_{12} \mathrm{C} \\
20 \mathrm{No}\end{array}$ & $\begin{array}{l}\text { NDG } \\
\text { NDG }\end{array}$ & & $\begin{array}{l}\text { DUB } \\
\text { DUB }\end{array}$ & $\begin{array}{l}\text { R Fleror. } \\
\text { R Flerov. }\end{array}$ & $\begin{array}{l}10157 \\
11917\end{array}$ \\
\hline
\end{tabular}




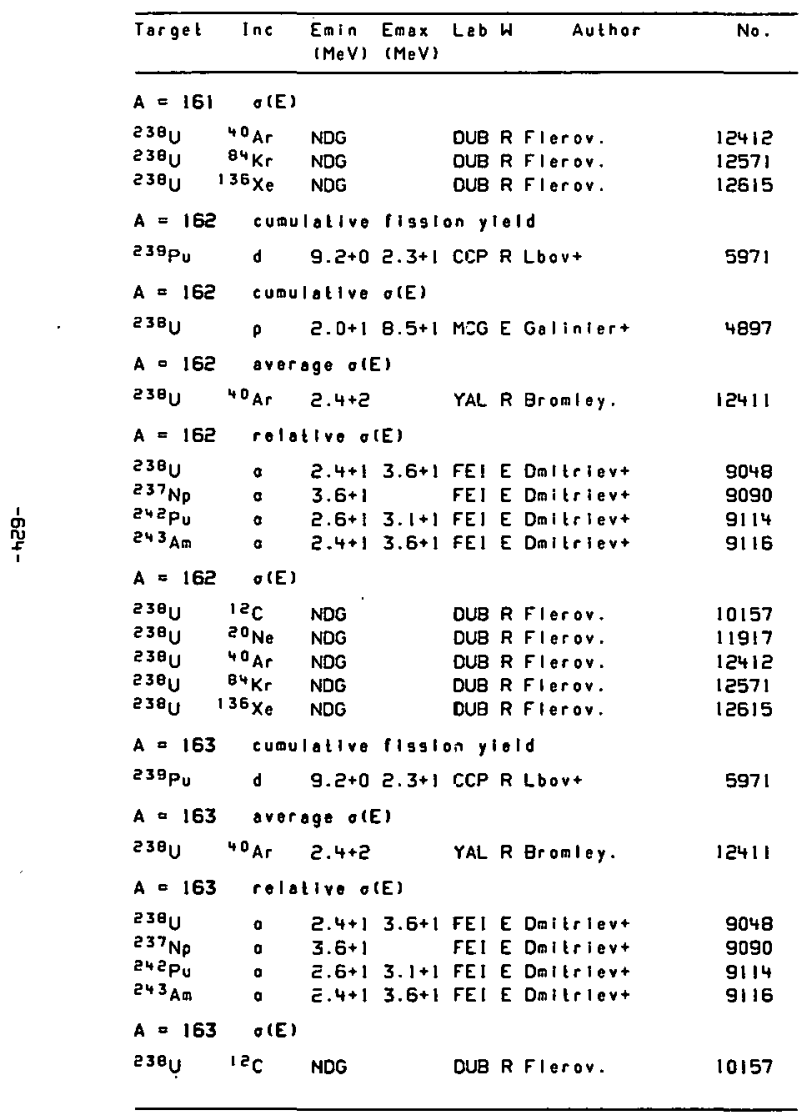

\begin{tabular}{|c|c|c|c|c|c|c|}
\hline Target & $\operatorname{lnc}$ & $\begin{array}{l}\text { Emin } \\
\text { (MeV) }\end{array}$ & $\begin{array}{l}\text { Emax } \\
(\operatorname{MeV})\end{array}$ & Lab W & Author & No. \\
\hline$A=163$ & OIE & & & & & \\
\hline $\begin{array}{l}23 \theta_{U} \\
23 \theta_{U} \\
23 \theta_{U} \\
23 \theta_{U}\end{array}$ & $\begin{array}{l}20 \mathrm{Ne} \\
{ }^{40} \mathrm{Ar} \\
{ }^{84} \mathrm{Kr} \\
136 \mathrm{Xe}\end{array}$ & $\begin{array}{l}\text { NDG } \\
\text { NDG } \\
\text { NDG } \\
\text { NDG }\end{array}$ & & $\begin{array}{l}\text { OUB R } \\
\text { DUB R } \\
\text { DUB R } \\
\text { DUB R }\end{array}$ & $\begin{array}{l}\text { R Flerov. } \\
R \text { Flerov. } \\
R \text { Flerov. } \\
\text { R Flerov. }\end{array}$ & $\begin{array}{l}11917 \\
12412 \\
12571 \\
126 ! 15\end{array}$ \\
\hline
\end{tabular}

$A=164$ comulative fission yiold

239Pu $9.2+02.3+1$ CCP R Lbovt 597

$A=164$ overage $\sigma(E)$

$238 \mathrm{U} \quad{ }^{40} \mathrm{Ar} 2.4+2$ YAL R Bromley. 12411

$A=164$ relotive o(E)

$238 \mathrm{U} O 2.4+13.6+1 \mathrm{FEI}$ E Dmltrlev+ 9048

$237 \mathrm{~Np} O 3.6+1$. FEI E Omitrlevt 9090

$242 P_{0} \circ 2.6+13.1+1$ FEI E Dmiteievt 9114

243Am O $2.4+13.6+1$ FEI E Dmitrievt

$A=164$ O(E)

$23 \theta^{\circ} \quad 12 \mathrm{C}$ NDG DUB R FIerov.

$\begin{array}{lll}230 \mathrm{O} 20_{\mathrm{Ne}} \text { NDG } & \text { DUB R Flerov. } & 10157 \\ & & 11917\end{array}$

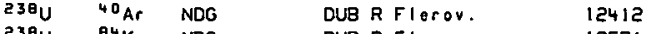

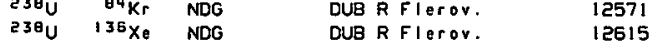

$23{ }^{9} \mathrm{Pu}$ o $9.2+02.3+1 \mathrm{CCP} R$ Lbovt 5971

$A=165$ overoge o(E)

$238 \mathrm{U}{ }^{40} \mathrm{Ar} 2.4+2 \quad$ YAL R Bromley. 12411

$A=165$ relative o(E)

$\begin{array}{lllll}238 \mathrm{U} & 0 & 2.4+13.6+1 \text { FEI E Dmlfrlevt } & 9048\end{array}$

$\begin{array}{lllll}237 \mathrm{~Np} & a & 3.6+1 & \text { FEI E Dmilrievt } & 9090 \\ 242 \mathrm{Pu} & a & 2.6+13.1+1 \text { FEI E Dolirlovt } & 9114\end{array}$

243 Am O $2.4+13.6+1$ FEI E Dmlirlevt $9 ! 16$

$A=165$ O(E)

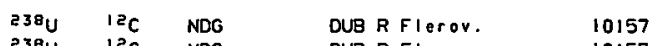

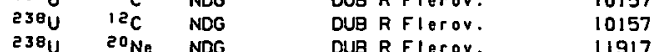






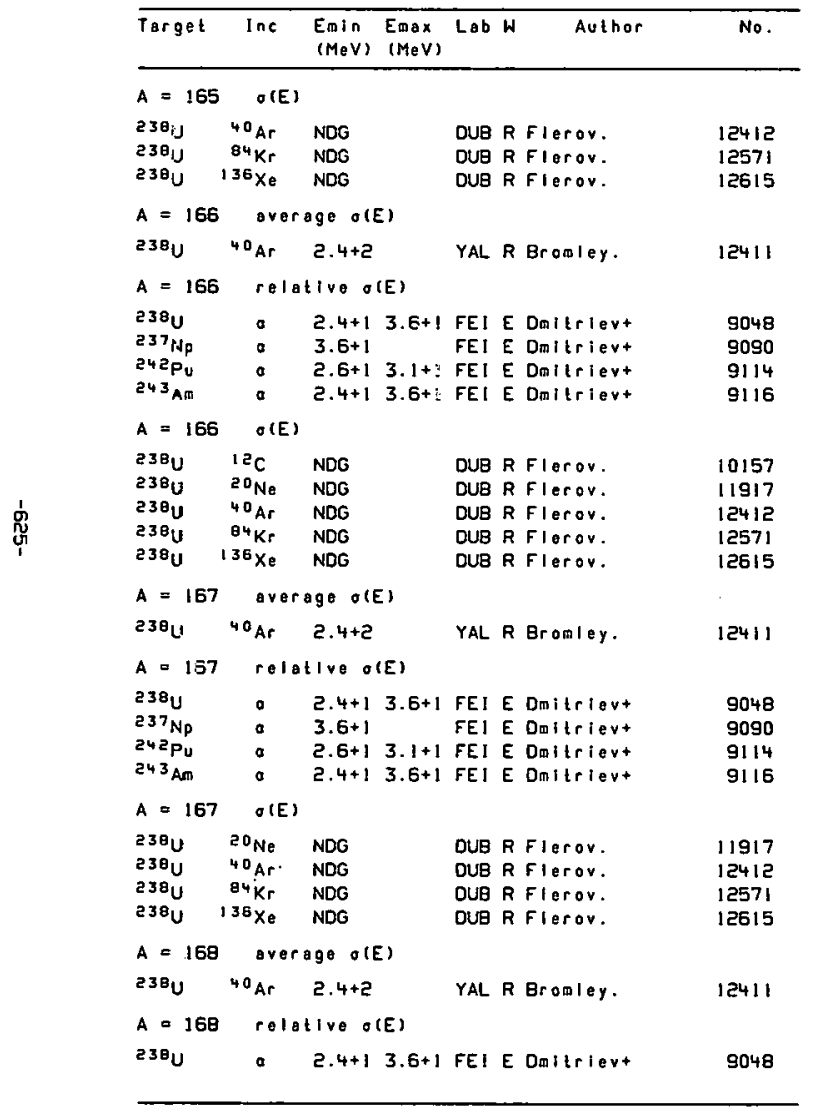

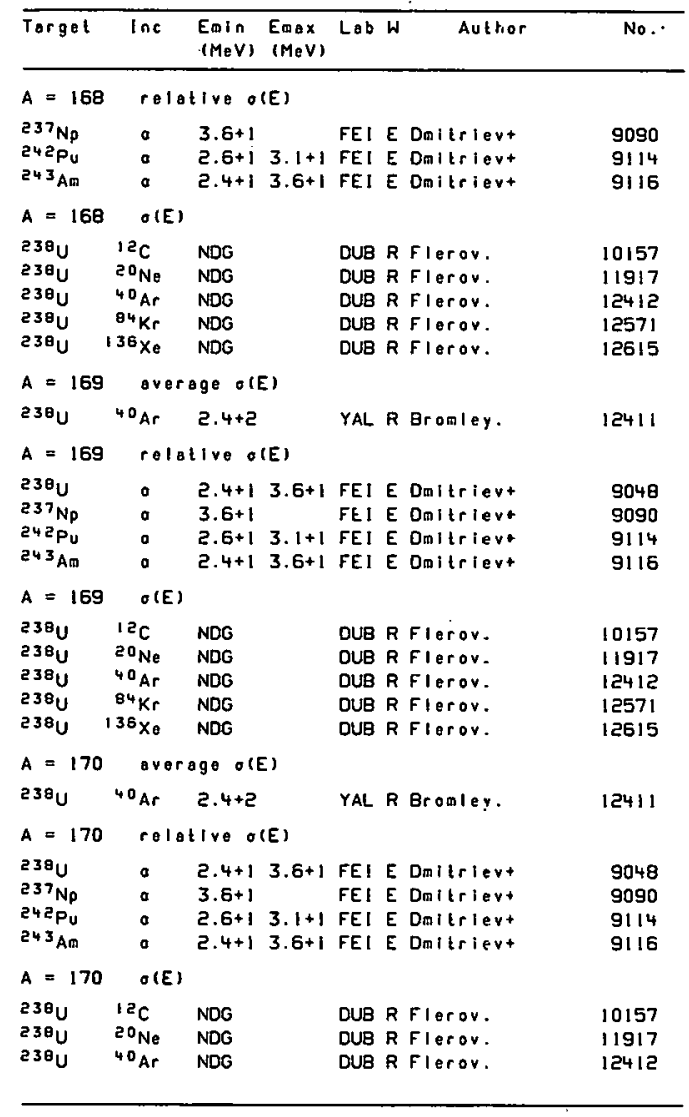




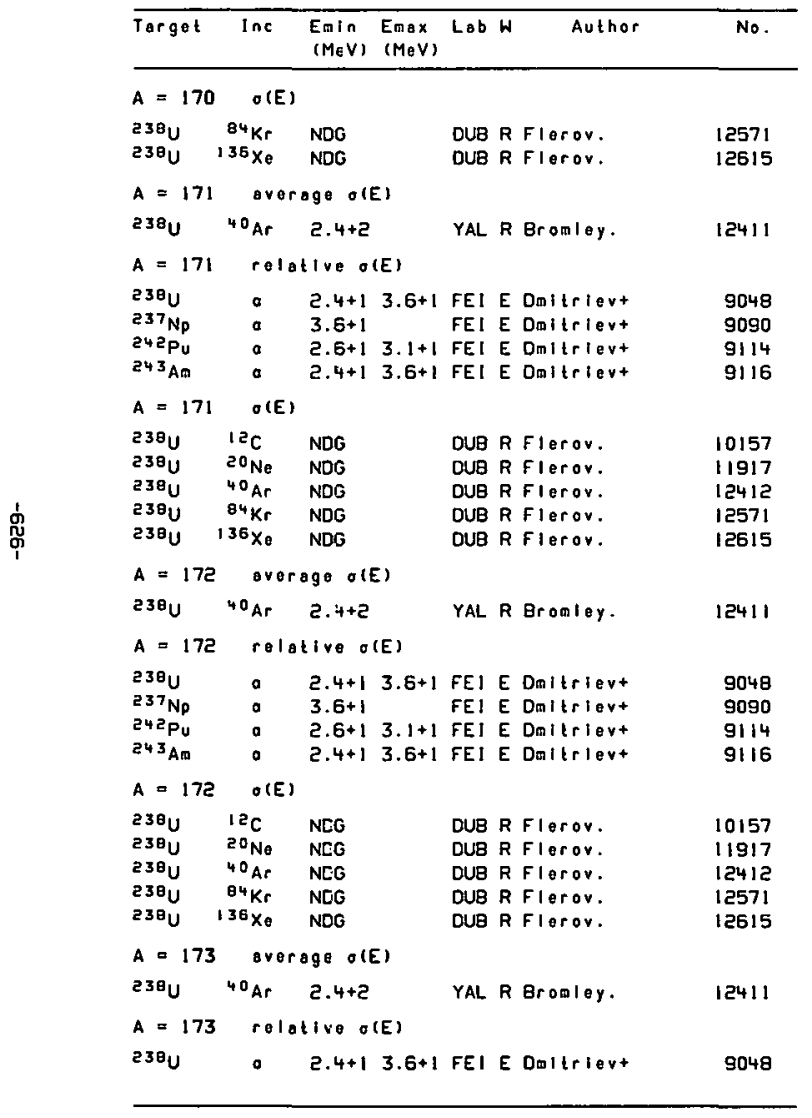

\begin{tabular}{|c|c|c|c|c|c|c|}
\hline Targel & $\ln e$ & $\begin{array}{l}\text { Enin } \\
\text { (MeV) }\end{array}$ & $\begin{array}{l}\text { Emax } \\
(M \circ V)\end{array}$ & Lab & Author & No. \\
\hline$A=173$ & \multicolumn{6}{|c|}{ reletive o(E) } \\
\hline $\begin{array}{l}237 \mathrm{~Np} \\
242 \mathrm{Pu} \\
243_{\mathrm{Am}}\end{array}$ & $\begin{array}{l}0 \\
0 \\
0\end{array}$ & $\begin{array}{l}3.6+1 \\
2.6+1 \\
2.4+1\end{array}$ & $\begin{array}{l}3.1+1 \\
3.6+1\end{array}$ & $\begin{array}{l}\text { FEI } \\
\text { FEI } \\
\text { FEI }\end{array}$ & $\begin{array}{l}\text { E Dmilirievt } \\
\text { E Dallrievt } \\
\text { E Daitrievt }\end{array}$ & $\begin{array}{l}9090 \\
9114 \\
9116\end{array}$ \\
\hline$A=173$ & \multicolumn{6}{|c|}{$O(E)$} \\
\hline 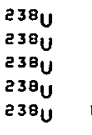 & $\begin{array}{l}{ }_{12} \mathrm{C} \\
20 \mathrm{Ne} \\
{ }^{4} \mathrm{Ne} \\
{ }^{84} \mathrm{Ar} \\
13 \mathrm{Kr} \\
1{ }^{36} \times \mathrm{Xe}\end{array}$ & $\begin{array}{l}\text { NDG } \\
\text { NDG } \\
\text { NDG } \\
\text { NDG } \\
\text { NDG }\end{array}$ & & $\begin{array}{l}\text { DUB } \\
\text { DUE } \\
\text { DUE } \\
\text { DUE } \\
\text { DUE }\end{array}$ & $\begin{array}{l}\text { R Flerov. } \\
\text { R Flerov. } \\
\text { R Flerov. } \\
R \text { Flerov. } \\
\text { R Flerov. }\end{array}$ & $\begin{array}{l}10157 \\
11917 \\
12412 \\
12571 \\
12615\end{array}$ \\
\hline \multicolumn{7}{|c|}{$A=174$ average $0(E)$} \\
\hline \multicolumn{5}{|c|}{$A=174$ reletive o(E) } & & \\
\hline $\begin{array}{l}23 \theta_{\mathrm{U}} \\
237 \mathrm{~Np} \\
242 \mathrm{Pu} \\
243 \mathrm{Am}\end{array}$ & $\begin{array}{l}a \\
a \\
a \\
a\end{array}$ & \multirow{2}{*}{\multicolumn{2}{|c|}{$\begin{array}{ll}2.4+1 & 3.6+1 \\
3.6+1 & \\
2.6+1 & 3.1+1 \\
2.4+1 & 3.6+1\end{array}$}} & $\begin{array}{l}\text { FEI } \\
\text { FEI } \\
\text { FEI } \\
\text { FEI }\end{array}$ & $\begin{array}{l}\text { E Omitrievt } \\
\text { E Omitrievt } \\
\text { E Omitrievt } \\
\text { E Omitrievt }\end{array}$ & $\begin{array}{l}9048 \\
9090 \\
9114 \\
9116\end{array}$ \\
\hline \multicolumn{6}{|c|}{$A=174 \quad O(E)$} & \\
\hline $\begin{array}{l}538^{4} \\
538^{4} \\
53 \theta^{4} \\
53 \theta^{4} \\
53 \theta^{4}\end{array}$ & $\begin{array}{l}12 \mathrm{C} \\
20 \mathrm{Ne} \\
40 \mathrm{Ar} \\
84 \mathrm{Kr} \\
138 \mathrm{Xe}\end{array}$ & $\begin{array}{l}\text { NDG } \\
\text { NDG } \\
\text { NDG } \\
\text { NDG } \\
\text { NDG }\end{array}$ & & $\begin{array}{l}\text { DUB } \\
\text { DUB } \\
\text { DUB } \\
\text { DUB } \\
\text { DUB }\end{array}$ & $\begin{array}{l}\text { R Flerov. } \\
\text { R Flerov. } \\
\text { R Flerov. } \\
\text { R Flerov. } \\
\text { R Flerov. }\end{array}$ & $\begin{array}{l}10157 \\
11917 \\
12412 \\
12571 \\
12615\end{array}$ \\
\hline \multicolumn{7}{|c|}{$A=175$ average $O(E)$} \\
\hline $\begin{array}{l}238 U \\
A=175\end{array}$ & $\begin{array}{l}{ }^{40} \mathrm{Ar} \\
5 \quad \mathrm{rel}\end{array}$ & \multicolumn{2}{|l|}{$2.4+2$} & YAL & R Bromiey. & 12411 \\
\hline $\begin{array}{l}238 \mathrm{U} \\
237 \mathrm{~Np} \\
242 \mathrm{Pu} \\
243_{\mathrm{Am}}\end{array}$ & $\begin{array}{l}0 \\
0 \\
a \\
0\end{array}$ & $\begin{array}{l}2.4+1 \\
3 \cdot 6+1 \\
2.6+1 \\
2.4+1\end{array}$ & $\begin{array}{l}3 \cdot 6+1 \\
3 \cdot 1+1 \\
3 \cdot 6+1\end{array}$ & $\begin{array}{l}\text { FEI } \\
\text { FEI } \\
\text { FEI } \\
\text { FEI }\end{array}$ & $\begin{array}{l}\text { E Dolirlert } \\
\text { E Daitrlert } \\
\text { E Doitrlevt } \\
\text { E Dnitriout }\end{array}$ & $\begin{array}{l}9048 \\
9090 \\
9114 \\
9116\end{array}$ \\
\hline \multicolumn{7}{|c|}{$A=175 \quad O(E)$} \\
\hline 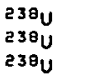 & $\begin{array}{l}12 \mathrm{C} \\
20 \mathrm{No} \\
40 \mathrm{Ar}\end{array}$ & $\begin{array}{l}\text { NDG } \\
\text { NDG } \\
\text { NDG }\end{array}$ & & $\begin{array}{l}\text { DUB } \\
\text { DUB } \\
\text { DUB }\end{array}$ & $\begin{array}{l}\text { R Flerov. } \\
\text { R Flerov. } \\
\text { R flerov. }\end{array}$ & $\begin{array}{l}10157 \\
11917 \\
12412\end{array}$ \\
\hline
\end{tabular}




\begin{tabular}{|c|c|c|c|c|c|c|}
\hline Target & Inc & $\begin{array}{l}\text { Emin } \\
(\operatorname{MeV})\end{array}$ & $\begin{array}{l}\text { Emos) } \\
\text { (MeVi) }\end{array}$ & Lab & Author & No. \\
\hline$A=175$ & S OLE & & & & & \\
\hline $\begin{array}{l}23 \theta_{U} \\
238_{U}^{U}\end{array}$ & $\begin{array}{r}{ }^{84} \mathrm{Kr} \\
1{ }^{36} \mathrm{Xe}\end{array}$ & $\begin{array}{l}\text { NDG } \\
\text { NDG }\end{array}$ & & $\begin{array}{l}\text { DUB } \\
\text { DUE }\end{array}$ & $\begin{array}{l}\text { R Flerov. } \\
\text { R Flerov. }\end{array}$ & $\begin{array}{l}12571 \\
12615\end{array}$ \\
\hline \multicolumn{7}{|c|}{$A=176$ overoge $O(E)$} \\
\hline $\begin{array}{l}238_{U} \\
A=176\end{array}$ & $\begin{array}{l}\text { "OAr } \\
\quad r \in 1\end{array}$ & $2.4+2$ & & YAL & R Bromley. & 12411 \\
\hline $\begin{array}{l}238 \mathrm{U} \\
2337 \mathrm{~Np} \\
242 \mathrm{Pu} \\
243 \mathrm{Am} .\end{array}$ & $\begin{array}{l}a \\
a \\
a \\
a\end{array}$ & $\begin{array}{l}2.4+1 \\
3.6+1 \\
2.6+1 \\
2.4+1\end{array}$ & $\begin{array}{l}3.6+1 \\
3.1+1 \\
3.6+1\end{array}$ & $\begin{array}{l}\text { FEI } \\
\text { FEI } \\
\text { FEI } \\
\text { FEI }\end{array}$ & $\begin{array}{l}\text { E Dalitrievt } \\
\text { E Daitrievt } \\
\text { E Dalirievt } \\
\text { E Dnitrievt }\end{array}$ & $\begin{array}{l}9048 \\
9090 \\
9114 \\
9116\end{array}$ \\
\hline$A=176$ & OLE & & & & & \\
\hline $\begin{array}{l}238_{U} \\
238_{U} \\
238_{U} \\
238_{U}\end{array}$ & $\begin{array}{l}20 \mathrm{Ne} \\
40 \mathrm{Ar} \\
84 \mathrm{Kr} \\
136 \mathrm{Xe}\end{array}$ & $\begin{array}{l}\text { NDG } \\
\text { NDG } \\
\text { NDG } \\
\text { NDG }\end{array}$ & & $\begin{array}{l}\text { DUE } \\
\text { OUB } \\
\text { DUB } \\
\text { DUB }\end{array}$ & $\begin{array}{l}\text { R Flerov. } \\
\text { R Flerov. } \\
\text { R Flerov. } \\
\text { R Flerov. }\end{array}$ & $\begin{array}{l}11917 \\
12412 \\
12571 \\
12615\end{array}$ \\
\hline \multicolumn{7}{|c|}{$A=177$ overege $O(E)$} \\
\hline $23 \theta^{2} \mathrm{U}$ & ${ }^{40} \mathrm{Ar}$ & $2.4+2$ & & YAL & R Bromley. & 12411 \\
\hline$A=177$ & $r e 1$ & tive o & (E) & & & \\
\hline $\begin{array}{l}238_{\mathrm{U}} \\
237_{\mathrm{NO}} \\
24 \mathrm{P}_{\mathrm{P}} \\
243_{\mathrm{An}}\end{array}$ & $\begin{array}{l}\dot{0} \\
\dot{0} \\
\dot{0}\end{array}$ & $\begin{array}{l}2.4+1 \\
3.6+1 \\
2.6+1 \\
2.4+1\end{array}$ & $\begin{array}{l}3.6+1 \\
3.1+1 \\
3.6+1\end{array}$ & $\begin{array}{l}\text { FEI } \\
\text { FEI } \\
\text { FEI } \\
\text { FEI }\end{array}$ & $\begin{array}{l}\text { E Dmilriert } \\
\text { E Dallriert } \\
\text { E Dmilriert } \\
\text { E Doitriert }\end{array}$ & $\begin{array}{l}9048 \\
9090 \\
9114 \\
9116\end{array}$ \\
\hline$A=177$ & 7 OIE & & & & & \\
\hline $\begin{array}{l}530 u \\
530 u \\
538 u \\
530 u\end{array}$ & $\begin{array}{r}20 \mathrm{Ne} \\
{ }^{40} \mathrm{Ar} \\
84 \mathrm{Kr} \\
136 \mathrm{Xe}\end{array}$ & $\begin{array}{l}\text { NDG } \\
\text { NDG } \\
\text { NDG } \\
\text { NDG }\end{array}$ & & $\begin{array}{l}\text { OUB } \\
\text { DUB } \\
\text { OUB } \\
\text { DUB }\end{array}$ & $\begin{array}{l}\text { R Flerov. } \\
\text { R Flerov. } \\
\text { R Flerov. } \\
\text { R Flerov. }\end{array}$ & $\begin{array}{l}11917 \\
12412 \\
12571 \\
12615\end{array}$ \\
\hline$A=178$ & o ovo & ogo olE & & & & \\
\hline $\begin{array}{l}230^{2} U \\
A=178\end{array}$ & $\begin{array}{l}40 \mathrm{Ar} \\
\quad \mathrm{POI}\end{array}$ & $\begin{array}{l}2.4+2 \\
1100\end{array}$ & & YAL & R Bromley. & 12411 \\
\hline $\begin{array}{l}23 \theta_{U} \\
237 \mathrm{~Np} \\
242 \mathrm{Pu}\end{array}$ & a & $\begin{array}{l}2.4+1 \\
3.6+1 \\
2.6+1\end{array}$ & $\begin{array}{l}3.6+1 \\
3.1+1\end{array}$ & $\begin{array}{l}\text { FEI } \\
\text { FEI } \\
\text { FEI }\end{array}$ & $\begin{array}{l}\text { E Dmitrlevt } \\
\text { E Dmitrlevt } \\
\text { E Omitrievt }\end{array}$ & $\begin{array}{l}9048 \\
9090 \\
9114\end{array}$ \\
\hline
\end{tabular}

\begin{tabular}{|c|c|c|c|c|c|c|}
\hline Target & $\ln c$ & $\begin{array}{l}\text { Emln } \\
(M 0 V)\end{array}$ & $\begin{array}{l}\text { Emax } \\
(M 0 V)\end{array}$ & Lab $W$ & Author & No. \\
\hline
\end{tabular}

$A=178$ relotive o(E)

$243_{\mathrm{Am}}$ O $2.4+13.6+1$ FEI E Dmltriert 9116

$A=178 \quad \sigma(E)$

$538 \mathrm{U} 20_{\mathrm{Ne}} \mathrm{NDG}$ DUB R Fleror.

$238 \mathrm{U} 40 \mathrm{Ar}$ NDG DUB R Fleror. 12412

$23 B^{94} \mathrm{Kr}$ NDG DUB R Fleros. 12571

$230_{U} 136 X_{e}$ NDG DUBRFlerod.

$A=179$ overago $\sigma(E)$

$230^{\circ} \quad 40 \mathrm{Ar} \quad 2.4+2$

YAL R BromIsy. $\quad 12411$

$A=179$ rololivo $\theta(E)$

$23 \theta_{\text {U }}$ a $2.4+13.6+1$ FEl E Dmltrlevt 9048

$237 \mathrm{~Np} a \quad 3.6+1 \quad$ FEI E Doritelert 9090

$24 \mathrm{Pu}^{2} \quad 2.6+13.1+1 \mathrm{FEI} E$ Daltrlert 9114

243 Aar a $2.4+13.6+1$ FEI E Daltrlert 9116

$A=179 \quad O(E)$

$238 \mathrm{U} 20 \mathrm{Ne}$ NDG DUB R Flerov.

$238^{4}{ }^{40} \mathrm{Ar}$ NDG DUB R Fleror. 12412

$23 B_{U}{ }^{84} \mathrm{Kr}$ NDG DUB R FIeror. 12571

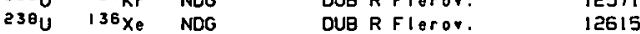

$A=180$ averoge olE

$23 \mathrm{BU}^{2} \quad{ }^{40} \mathrm{Ar} \quad 2.4+2$

YAL R Bromley. 12411

$A=180$ rolotive otE

$238_{U}$ a $2.4+13.6+1$ FEI E Dmitriert 9048

$237 \mathrm{~Np} a 3.6+1$ FEI E DmItrievt 9090

24 $2 \mathrm{Pu} a 2.6+13.1+1$ FEl E Dmltrlevt 9114

$243 \mathrm{Am} \quad 2.4+13.6+1$ FEI E Dmlkrievt 9116

$A=180 \quad O(E)$

$230^{2} \mathrm{U} 0_{\mathrm{Ne}} \mathrm{NDG}$ DUB R FleroY. 11917

$230^{40} \mathrm{Ar}$ NDG DUB R FleroN. 12412

${ }^{23 \theta}{ }^{84} \mathrm{Kr}$ NDG DUB R Fleror. 12571

${ }^{230} \mathrm{U} 136 x_{e}$ NDG DUB R Fleror.

$A=181$ overoge o(E)

$238 \mathrm{U}{ }^{40} \mathrm{Ar} 2.4+2$ YAL R Bromley. 12411 


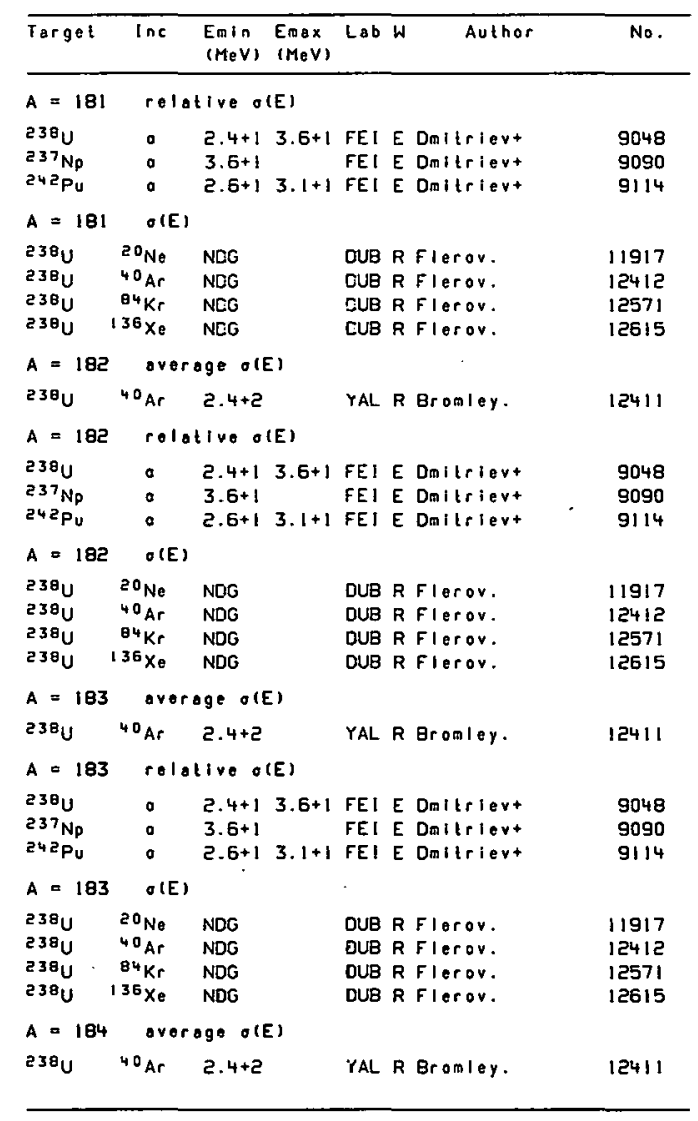

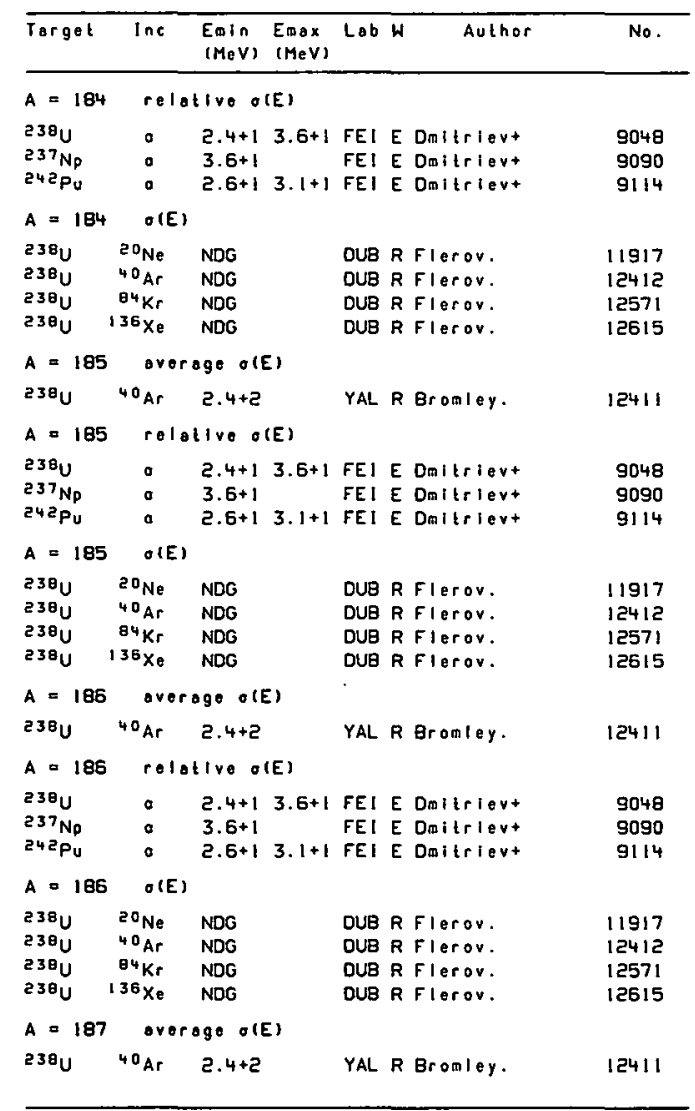




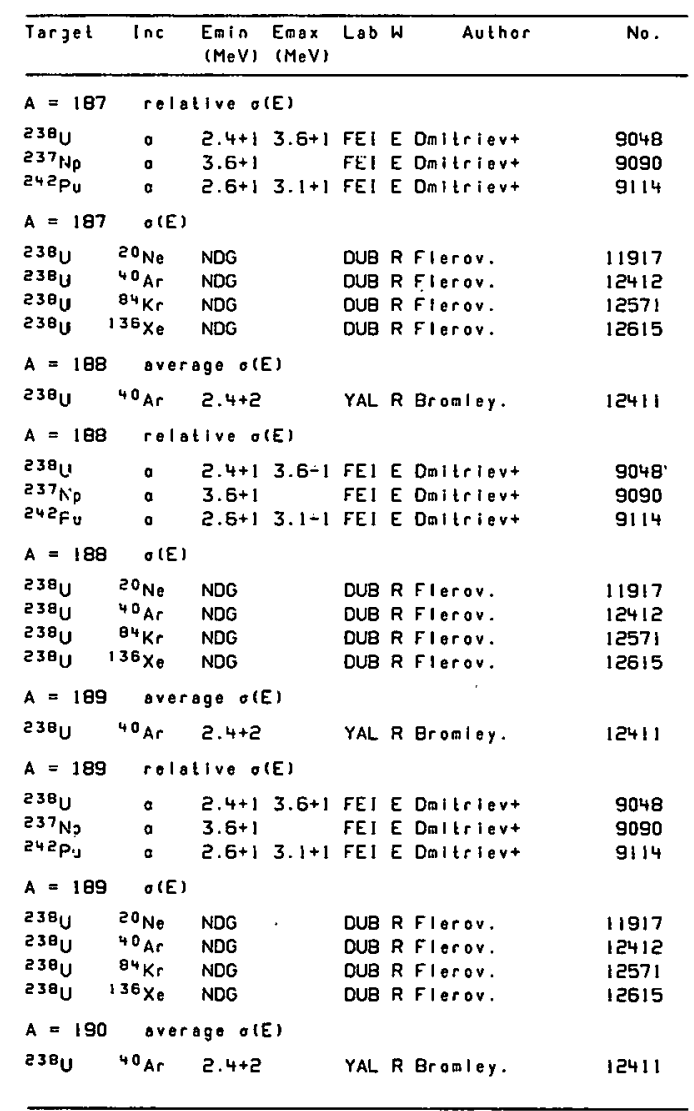

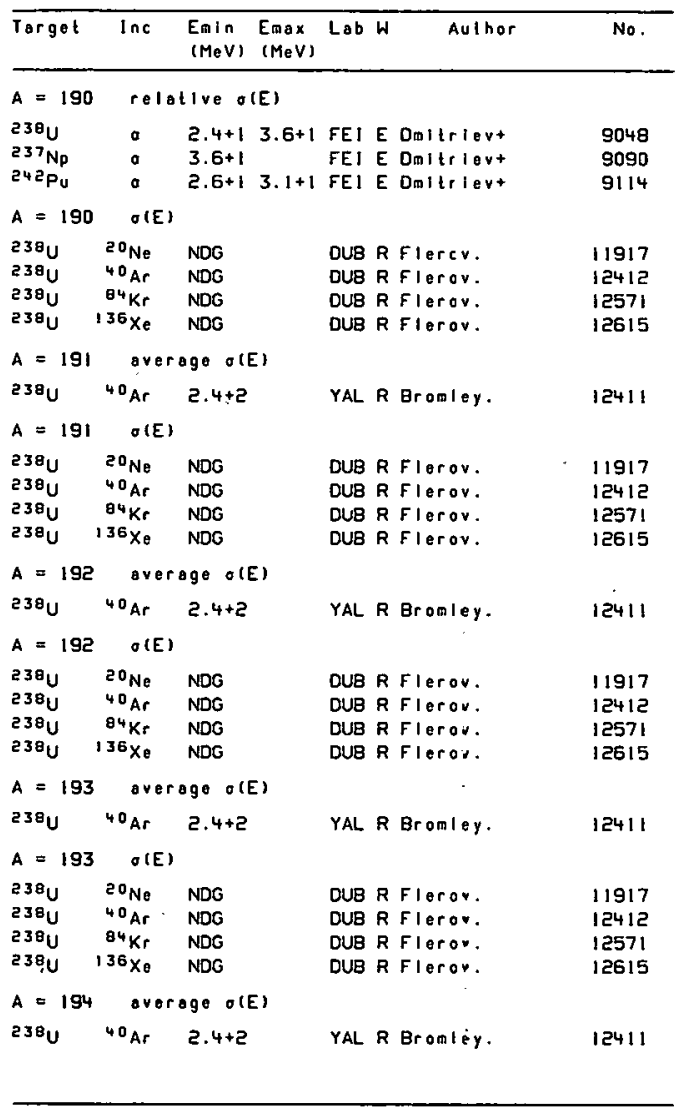




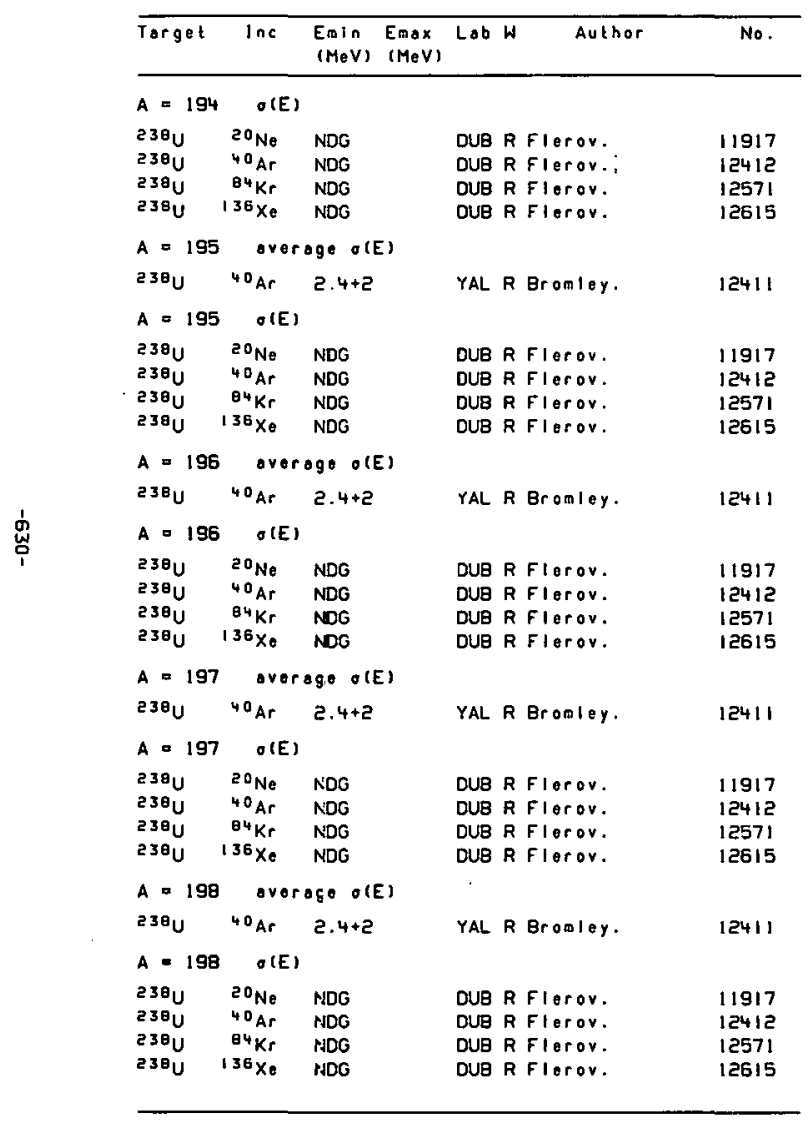

\begin{tabular}{|c|c|c|c|c|c|}
\hline Torget & $\ln c$ & $\begin{array}{ll}\text { Emin } & \text { Emax } \\
(M e V) & (M e V)\end{array}$ & Lob & Author & No. \\
\hline \multicolumn{6}{|c|}{$A=199$ overago $O(E)$} \\
\hline $\begin{array}{l}238 U \\
A=199\end{array}$ & $\begin{array}{l}{ }^{40} \mathrm{Ar} \\
O(E)\end{array}$ & $2.4+2$ & YAL & R Broaloy. & 12411 \\
\hline $\begin{array}{l}238 u \\
238 u \\
238 u \\
238 u\end{array}$ & $\begin{array}{l}20 \mathrm{Ne} \\
{ }^{40} \mathrm{Ar} \\
8{ }^{84} \mathrm{Kr} \\
136 \mathrm{Xe}\end{array}$ & $\begin{array}{l}\text { NDG } \\
\text { NDG } \\
\text { NDG } \\
\text { NDG }\end{array}$ & $\begin{array}{l}\text { DUB } \\
\text { DUB } \\
\text { OUB } \\
\text { DUB }\end{array}$ & $\begin{array}{l}\text { R Flerov. } \\
\text { R Flerov. } \\
\text { R flerov. } \\
\text { R flerov. }\end{array}$ & $\begin{array}{l}11917 \\
12412 \\
12571 \\
12615\end{array}$ \\
\hline \multicolumn{6}{|c|}{$A=200$ overoge $O(E)$} \\
\hline $\begin{array}{l}2{ }^{3} \mathrm{~B}_{\mathrm{U}} \\
\mathrm{A}=200\end{array}$ & $\begin{array}{l}40 \mathrm{Ar} \\
\quad \sigma(E)\end{array}$ & $2.4+2$ & YAL & R Gromley. & 12411 \\
\hline $\begin{array}{l}23 \theta_{U} \\
23 \theta_{U} \\
23 \theta_{U} \\
23 \theta_{U}\end{array}$ & $\begin{array}{l}20 \mathrm{Ne} \\
{ }^{20} \mathrm{Ar} \\
84 \mathrm{Kr} \\
136 \mathrm{Xe}\end{array}$ & $\begin{array}{l}\text { NDG } \\
\text { NDG } \\
\text { NDG } \\
\text { NDG }\end{array}$ & $\begin{array}{l}\text { DUB } \\
\text { DUB } \\
\text { DUB } \\
\text { DUB }\end{array}$ & $\begin{array}{l}\text { R Fleroy. } \\
\text { R fleroy. } \\
\text { R Flerov. } \\
\text { R flerov. }\end{array}$ & $\begin{array}{l}11917 \\
12412 \\
12571 \\
12615\end{array}$ \\
\hline \multicolumn{6}{|c|}{$A=201$ overage o(E) } \\
\hline $\begin{array}{l}230 \mathrm{U} \\
A=201\end{array}$ & $\begin{array}{l}40_{A r} \\
O(E)\end{array}$ & $2.4+2$ & YAL & R Bromley. & 12411 \\
\hline $\begin{array}{l}238 \mathrm{U} \\
238 \mathrm{U} \\
238 \mathrm{U} \\
238 \mathrm{U}\end{array}$ & $\begin{array}{l}20 \mathrm{Ne} \\
{ }^{40} \mathrm{Ar} \\
{ }^{64} \mathrm{Kr} \\
1{ }^{36} \mathrm{Xe}\end{array}$ & $\begin{array}{l}\text { NDG } \\
\text { NDG } \\
\text { NDG } \\
\text { NDG }\end{array}$ & $\begin{array}{l}\text { DUE } \\
\text { DUB } \\
\text { DUA } \\
\text { OUB }\end{array}$ & $\begin{array}{l}\text { R Flerov. } \\
R \text { Flerov. } \\
R \text { Flerov. } \\
R \text { Flerov. }\end{array}$ & $\begin{array}{l}11917 \\
12412 \\
12571 \\
12615\end{array}$ \\
\hline \multicolumn{6}{|c|}{$A=202$ average $O(E)$} \\
\hline $\begin{array}{l}23 \theta^{2} \\
A=202\end{array}$ & $\begin{array}{l}40_{\mathrm{Ar}} \\
\quad O(E)\end{array}$ & $2.4+2$ & YAL & R Bromley. & 12411 \\
\hline $\begin{array}{l}23 \theta^{\prime} \\
23 \theta^{2} \\
23 \theta^{\prime} \\
23 \theta^{2}\end{array}$ & $\begin{array}{l}20 \mathrm{Ne} \\
40 \mathrm{Ar} \\
{ }^{94} \mathrm{Kr} \\
1{ }^{36} \times \mathrm{Xe}\end{array}$ & $\begin{array}{l}\text { NDG } \\
\text { NDG } \\
\text { NDG } \\
\text { NDG }\end{array}$ & $\begin{array}{l}\text { DUB } \\
\text { DUB } \\
\text { DUB } \\
\text { DUB }\end{array}$ & $\begin{array}{l}\text { R Flerov. } \\
\text { R Flerov. } \\
\text { R flerov. } \\
\text { R Flerov. }\end{array}$ & $\begin{array}{l}11917 \\
12412 \\
12571 \\
12615\end{array}$ \\
\hline$A=203$ & 3 over & oge o(E) & & & \\
\hline $\begin{array}{l}{ }^{238} U \\
A=203\end{array}$ & $\begin{array}{l}40 \mathrm{Ar} \\
\mathrm{O}(\mathrm{E})\end{array}$ & $2.4+2$ & YAL & R Bromloy. & 12411 \\
\hline $23 \theta_{U}$ & $20 \mathrm{Ne}$ & NDG & DUB & R Fleroy. & 11917 \\
\hline
\end{tabular}




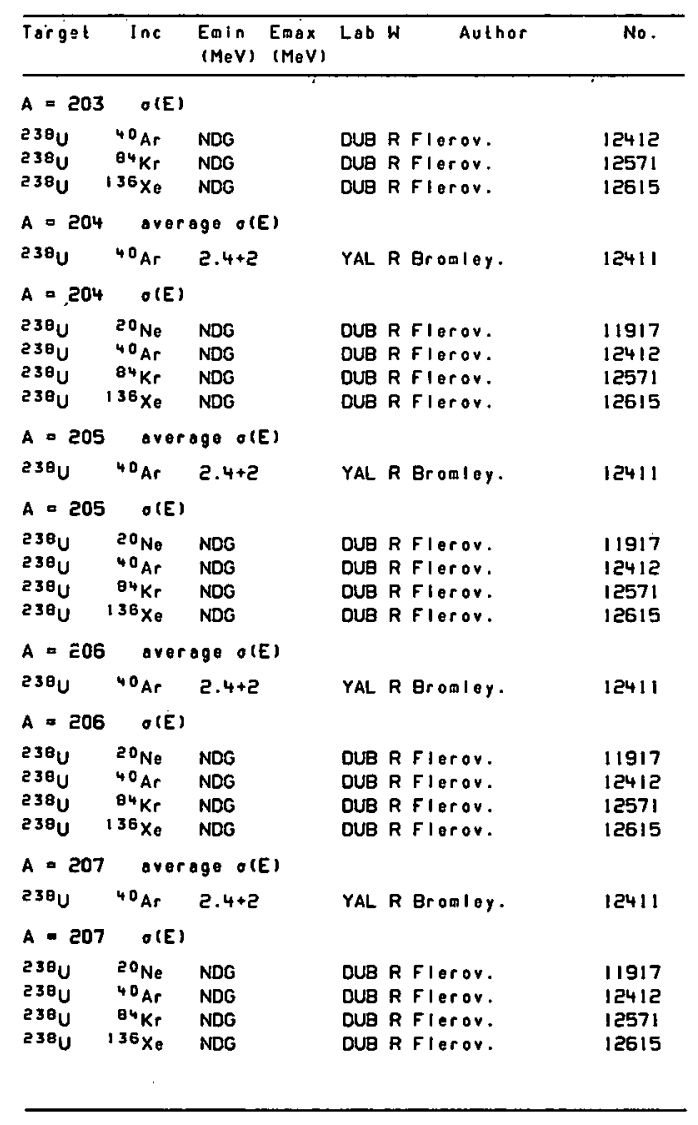

\begin{tabular}{|c|c|c|c|c|c|c|}
\hline Target & Ine & $\begin{array}{l}\text { Enin } \\
(\mathrm{MeV})\end{array}$ & $\begin{array}{l}\text { Emax } \\
(\operatorname{MeV})\end{array}$ & Lob & Author & No. \\
\hline \multicolumn{7}{|c|}{$A=200$ overoge $\sigma(E)$} \\
\hline $\begin{array}{l}230 \mathrm{U} \\
A=208\end{array}$ & $\begin{array}{l}40 \mathrm{Ar} \\
\quad \mathrm{OCE}\end{array}$ & $2.4+2$ & & YAL & R Bromley. & 12411 \\
\hline $\begin{array}{l}23 \theta_{U} \\
23 \theta_{U} \\
23 \theta_{U} \\
23 \theta_{U}\end{array}$ & $\begin{array}{l}20 \mathrm{Ne} \\
40 \mathrm{Ar} \\
84 \mathrm{Ke} \\
136 \times \mathrm{Xe}\end{array}$ & $\begin{array}{l}\text { NDG } \\
\text { NDG } \\
\text { NDG } \\
\text { NDG }\end{array}$ & & $\begin{array}{l}\text { DUB } \\
\text { DUE } \\
\text { DUA } \\
\text { DUA }\end{array}$ & $\begin{array}{l}\text { R Fleror. } \\
\text { R Fleror. } \\
\text { R Fleror. } \\
\text { R Fleror. }\end{array}$ & $\begin{array}{l}11917 \\
12412 \\
12571 \\
12615\end{array}$ \\
\hline \multicolumn{7}{|c|}{$A=209$ averaga $O(E)$} \\
\hline $\begin{array}{l}23 \theta_{U} \\
A=205\end{array}$ & $\begin{array}{l}40 \mathrm{Ar} \\
9 \text { OIE }\end{array}$ & $2.4+2$ & & YAL & R Bromiey. & 12411 \\
\hline $\begin{array}{l}238_{U} \\
23 \theta_{U} \\
23 \theta_{U} \\
238_{U}\end{array}$ & $\begin{array}{l}20 \mathrm{Ne} \\
{ }^{40} \mathrm{Ar} \\
8{ }^{4} \mathrm{Kr} \\
136 \mathrm{Xe}\end{array}$ & $\begin{array}{l}\text { NDG } \\
\text { NDG } \\
\text { NDG } \\
\text { NDG }\end{array}$ & & $\begin{array}{l}\text { DUB } \\
\text { DUB } \\
\text { DUB } \\
\text { DUB }\end{array}$ & $\begin{array}{l}\text { R Flerov. } \\
\text { R Fleroy. } \\
\text { R Flerov. } \\
\text { R fleron. }\end{array}$ & $\begin{array}{l}11917 \\
12412 \\
12571 \\
12615\end{array}$ \\
\hline \multicolumn{7}{|c|}{$A=210$ overage $O(E)$} \\
\hline \multicolumn{7}{|c|}{$A=210 \quad O(E)$} \\
\hline $205 \mathrm{TI}$ & $19_{F}$ & $1.8+2$ & & PAR & E Gordest & 11817 \\
\hline $209_{B i}$ & ${ }^{12} \mathrm{c}$ & $6.8+1$ & B. $7+1$ & PAR & E Gerdest & 10151 \\
\hline $209_{B i}$ & $14 \mathrm{~N}$ & $6.1+i$ & $1.6+2$ & PAR & E Gardest & 10510 \\
\hline $200_{B 1}$ & 180 & $7.7+1$ & $9.4+1$ & PAR & E Gordest & 11551 \\
\hline $209_{\mathrm{BI}}$ & $19 \mathrm{~F}$ & $6.8+1$ & $1.9+2$ & PAR & E Gordest & 11829 \\
\hline $209 \mathrm{Bi}$ & $20_{\mathrm{Ne}}$ & $9.7+1$ & $1.9+2$ & PAR & E Gardest & 11912 \\
\hline $200_{\mathrm{BI}}$ & $40 \mathrm{Ar}$ & $1.8+2$ & $2.9+2$ & PAR & E Gardest & 12390 \\
\hline & ${ }^{40} \mathrm{Ca}$ & $1.8+2$ & $2.8+2$ & PAR & E Gardest & 12445 \\
\hline $209^{8}$ & ${ }^{96} \mathrm{Fe}$ & $2.7+2$ & $4.0+2$ & PAR & E Gardegt & 12473 \\
\hline 2098 & ${ }^{63} \mathrm{Cu}$ & $2.9+2$ & $4.1+2$ & PAR & E Gerdest & 12494 \\
\hline $238 \mathrm{u}$ & $20_{\mathrm{Ne}}$ & NDG & & DUB & R Flerov. & 11917 \\
\hline & ${ }^{40} \mathrm{Ar}$ & NDG & & DUB & R Flerov. & 12412 \\
\hline $238_{U}$ & ${ }^{84} \mathrm{Kr}$ & NDG & & DUE & R Flerov. & 12571 \\
\hline $23 \theta_{u}$ & ${ }^{136} \times e$ & NOG & & DUB & R Flerov. & 12615 \\
\hline \multicolumn{7}{|c|}{$A=211$ overoge o(E) } \\
\hline $23 \theta_{U}$ & ${ }^{40} \mathrm{Ar}$ & $2.4+2$ & & YAL & R Brooler. & 12411 \\
\hline
\end{tabular}




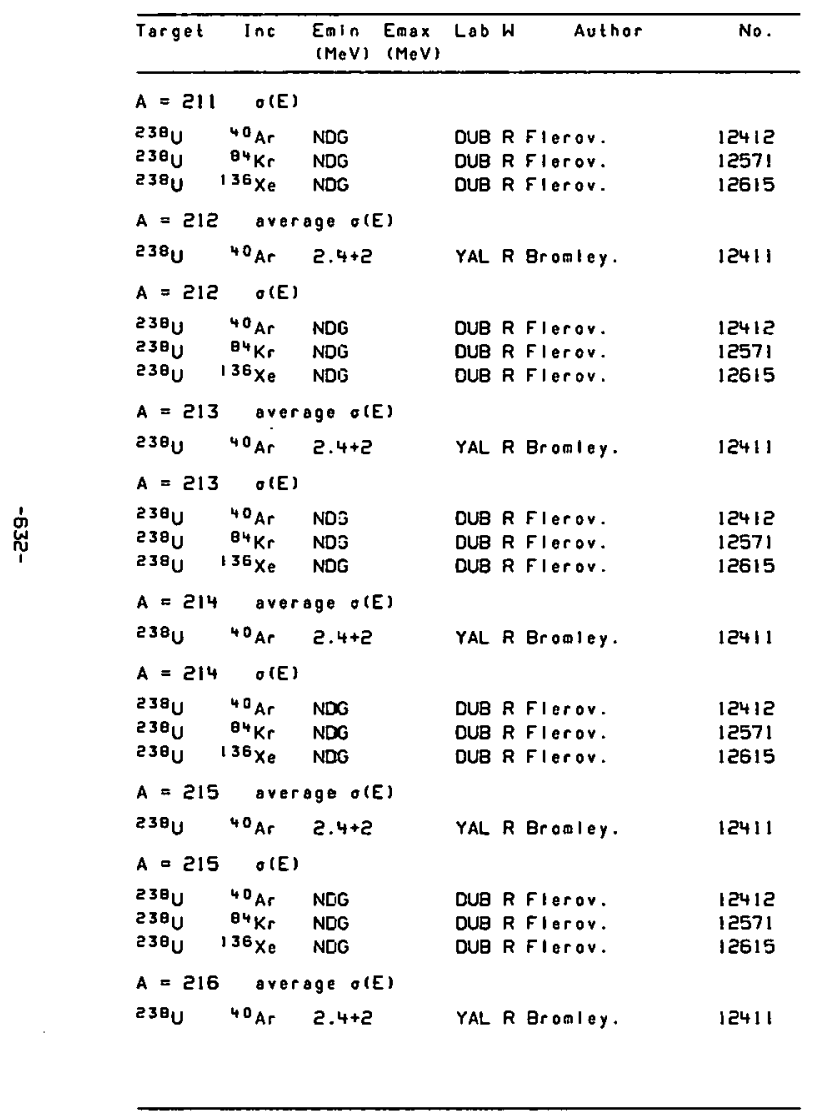

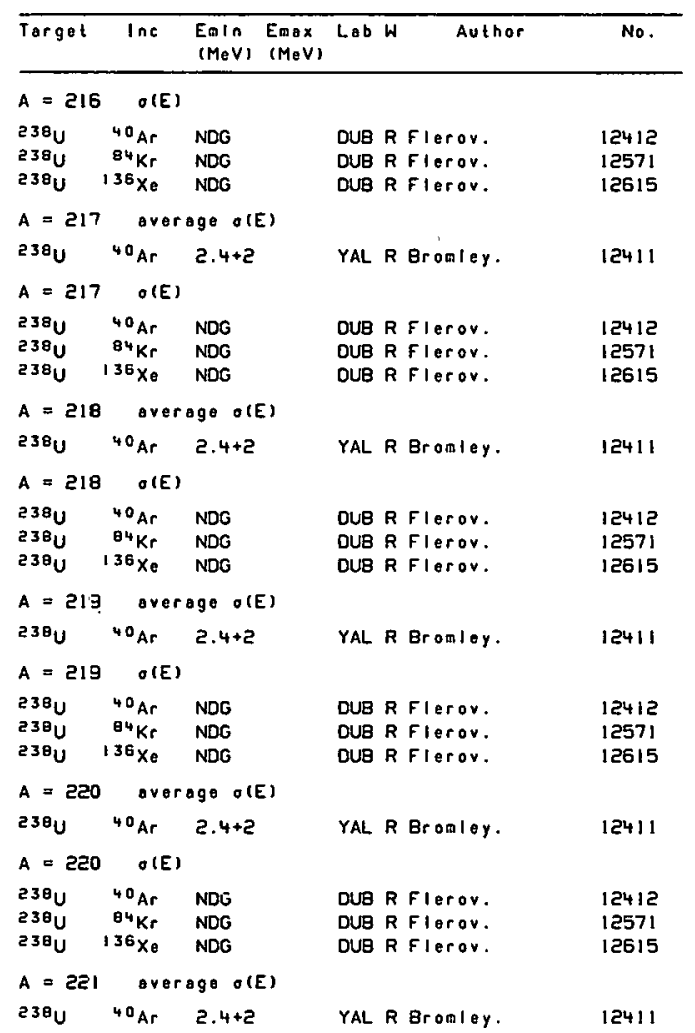




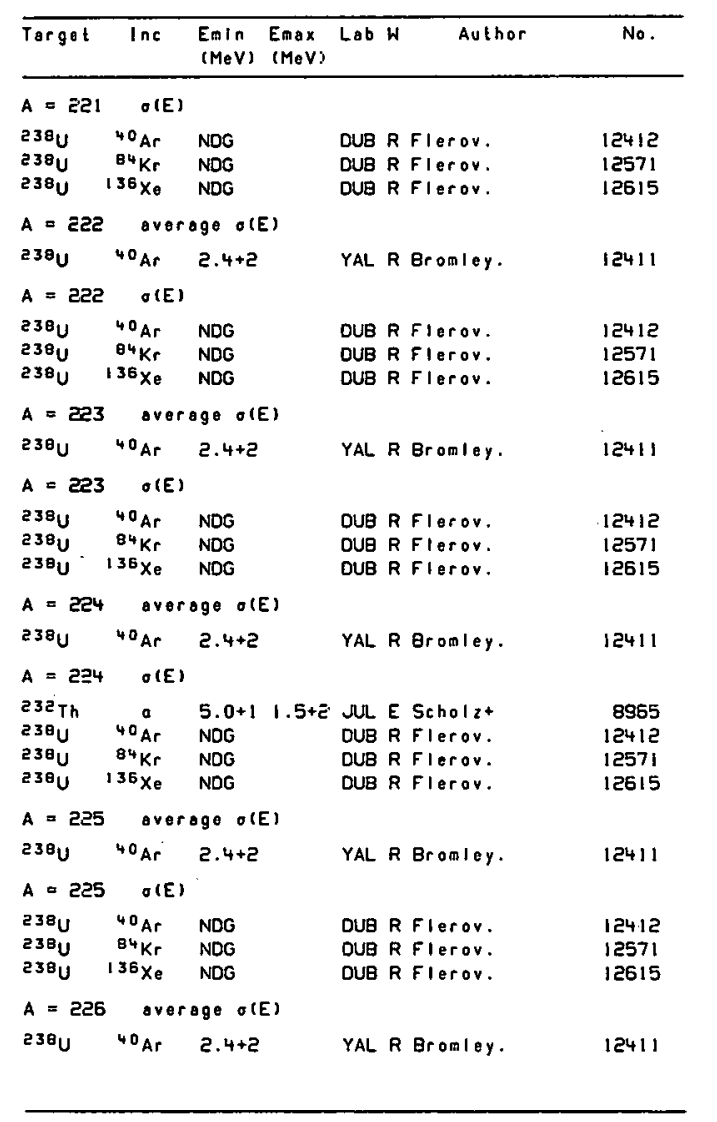

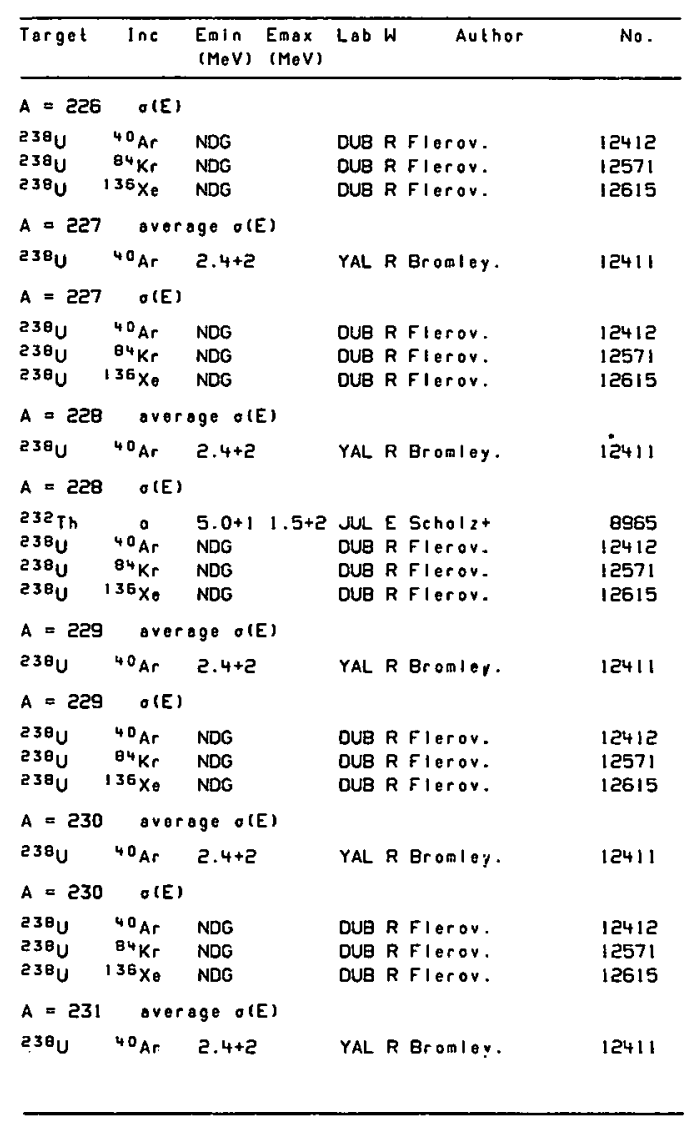




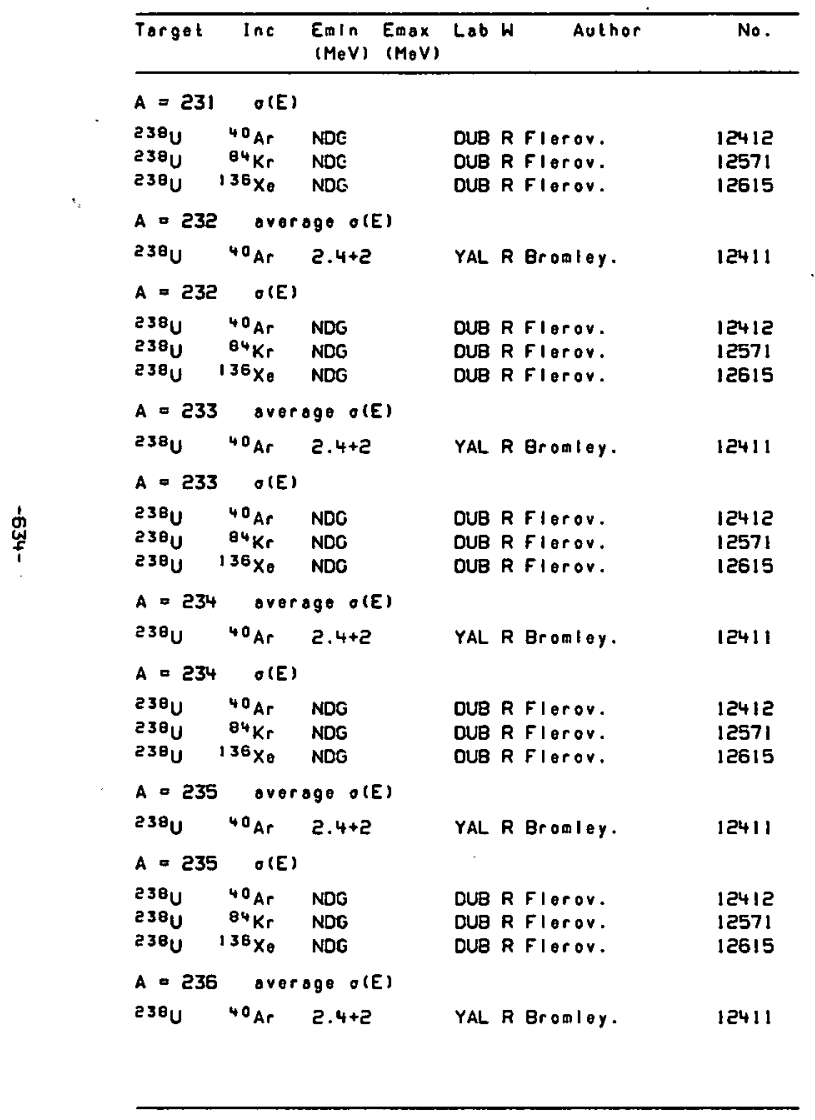

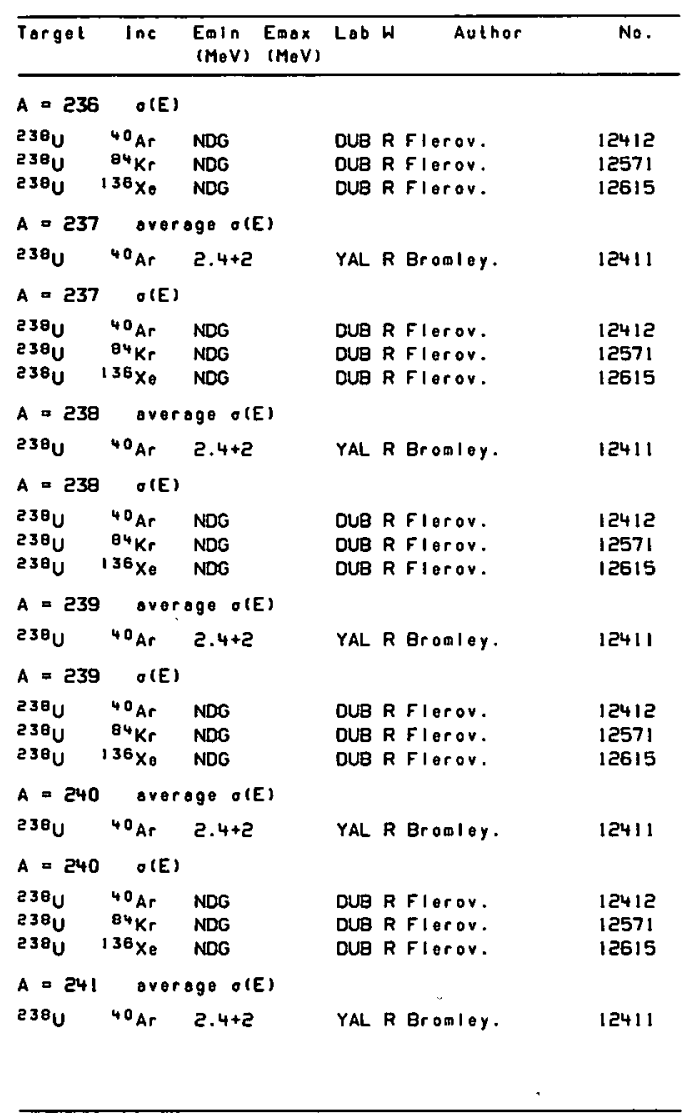




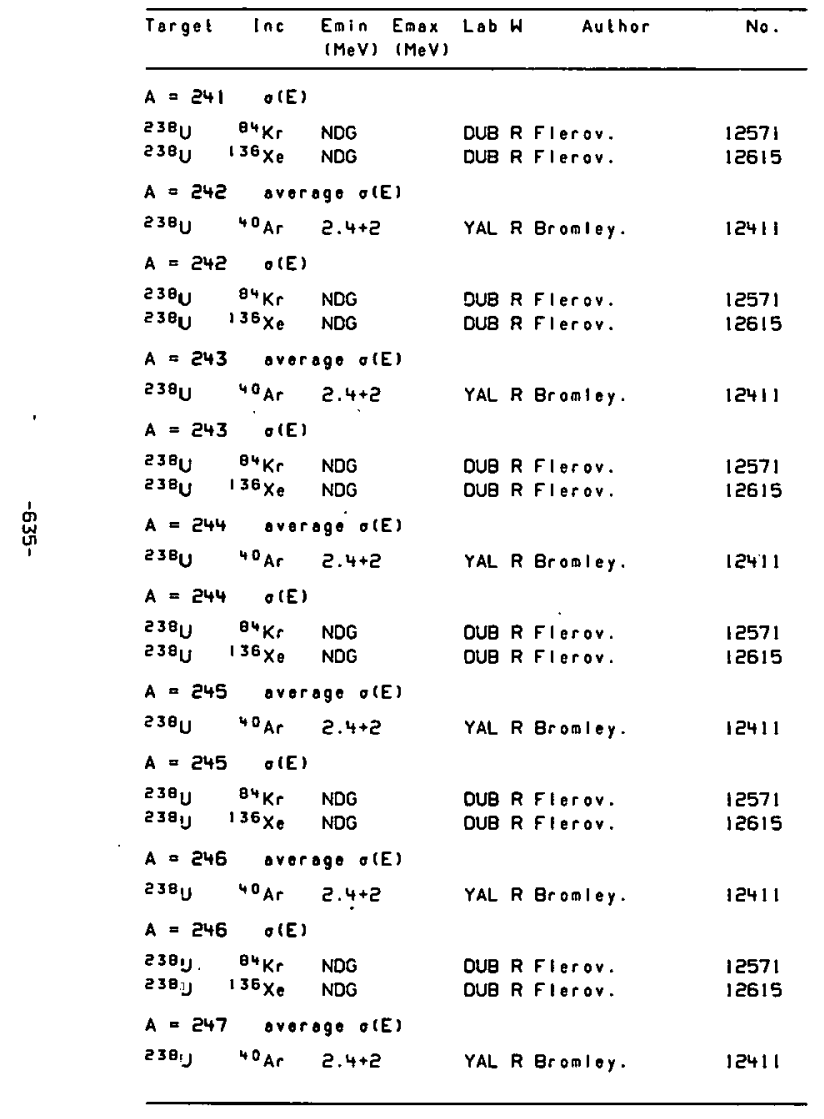

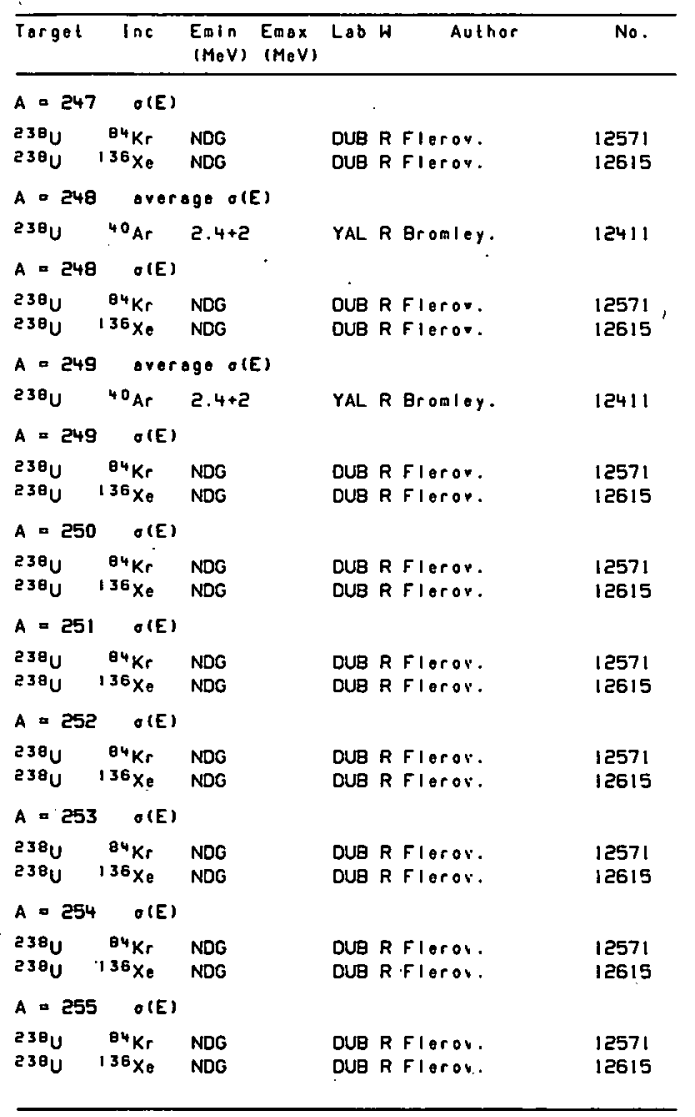




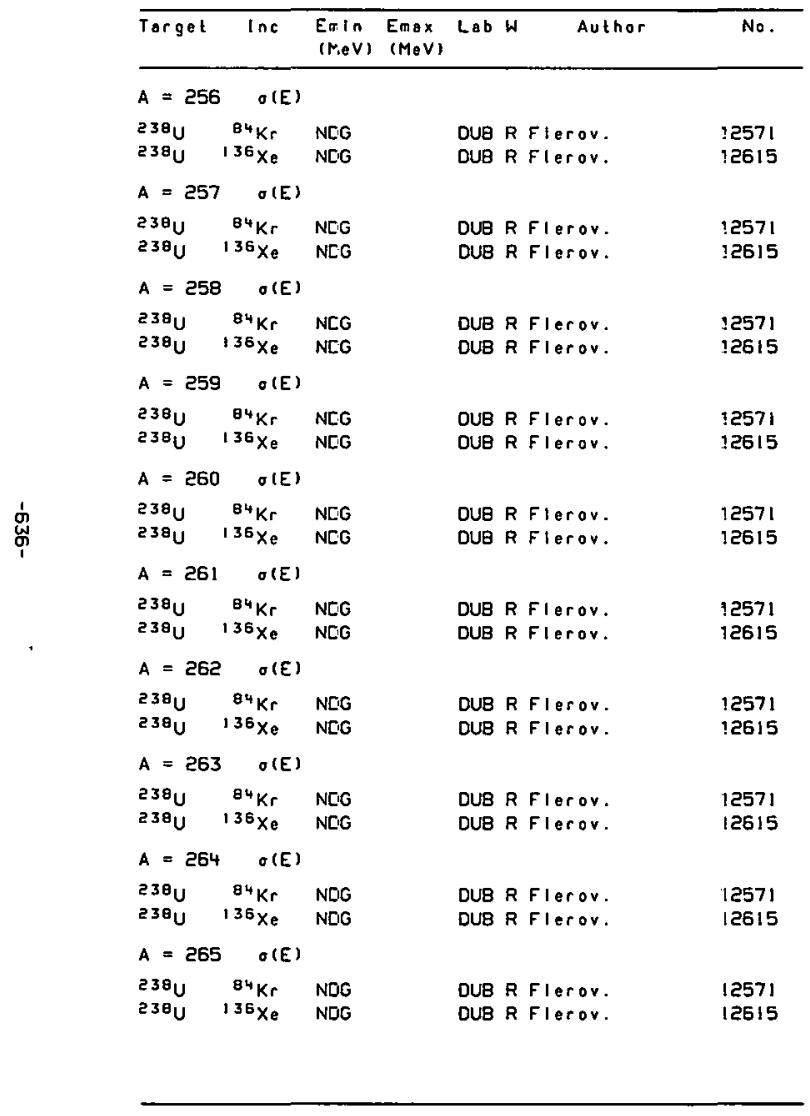

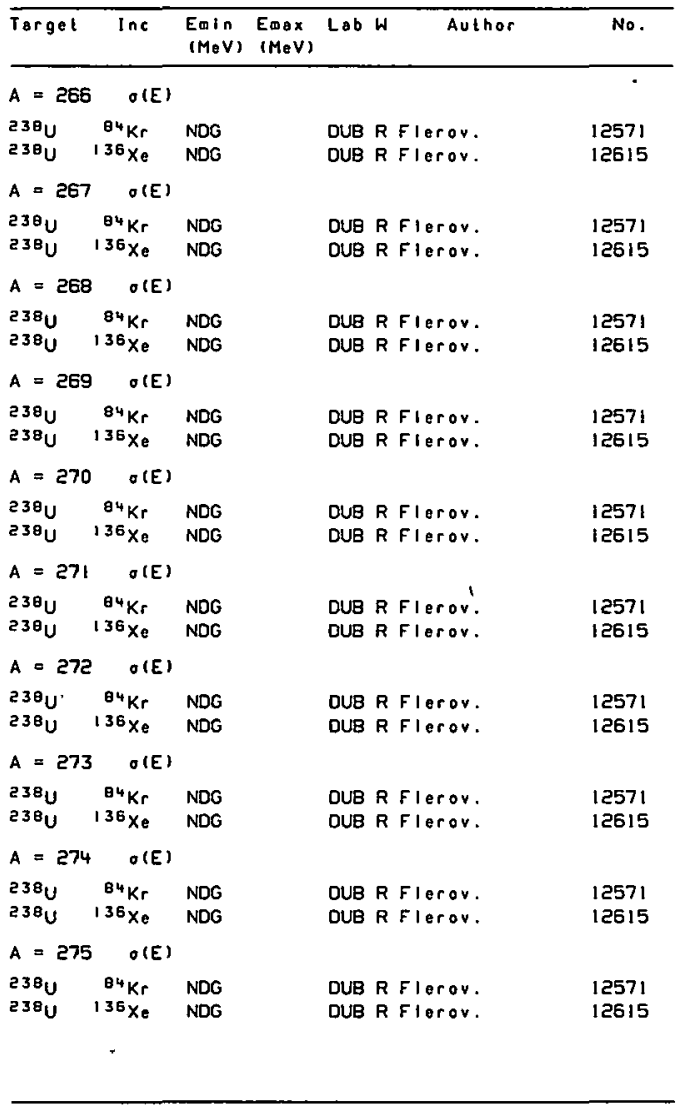




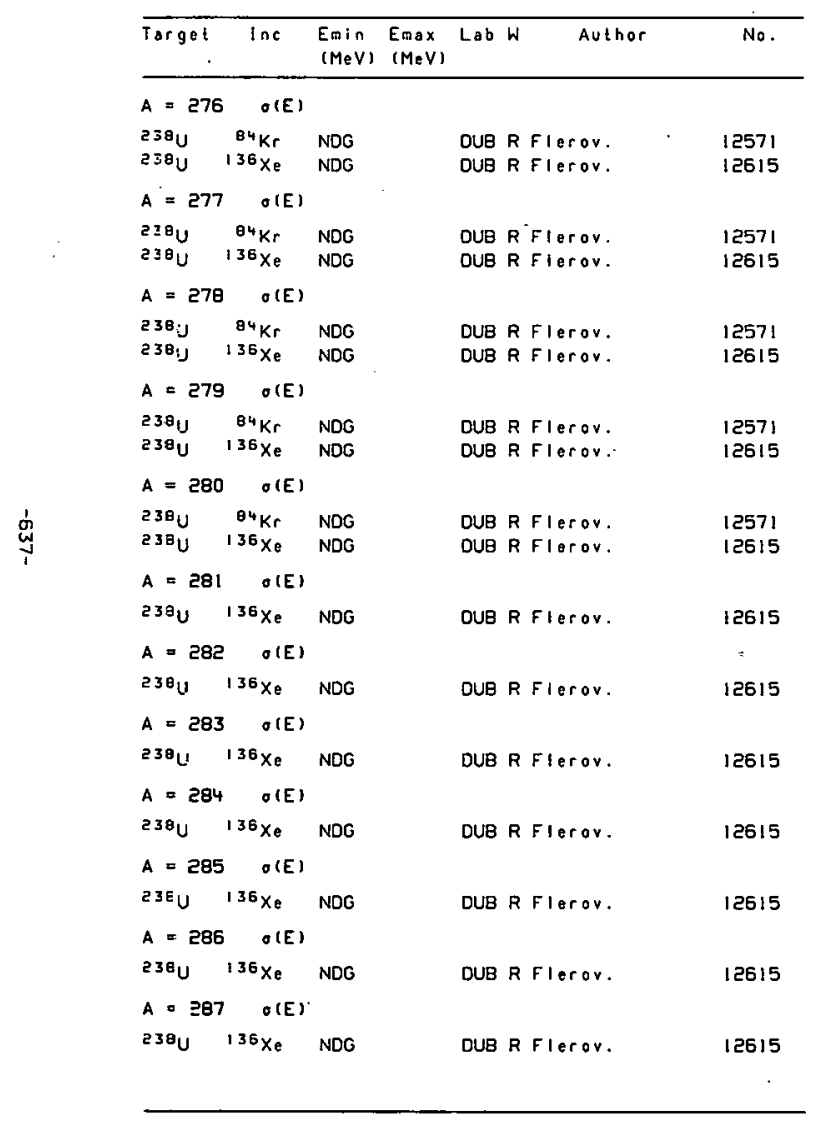

\begin{tabular}{|c|c|c|c|c|c|}
\hline Target lnc & $\begin{array}{l}\text { Emin } \\
(\mathrm{MeV})\end{array}$ & $\begin{array}{l}\max \\
(\operatorname{MeV})\end{array}$ & $\mathrm{Lab} \mathrm{W}$ & Author & No. \\
\hline \multicolumn{6}{|l|}{$A=28 \theta \quad 0(E)$} \\
\hline $238 \mathrm{U} \quad{ }^{136} \mathrm{Xe}$ & NDO & & DUB $R$ & R Flerov. & 12615 \\
\hline \multicolumn{6}{|l|}{$A=289 \quad O(E)$} \\
\hline $23 \theta_{u} \quad{ }^{36} \times \mathrm{Xe}$ & NDG & & DUB $R$ & R Flerov. & 12615 \\
\hline \multicolumn{6}{|l|}{$A=290 \quad O(E)$} \\
\hline $238 \mathrm{u} \quad 136 \times \mathrm{xe}$ & NDG & ' & DUB $R$ & R flerov. & 12615 \\
\hline \multicolumn{6}{|l|}{$A=291 \quad O(E)$} \\
\hline $238 \mathrm{U} \quad{ }^{136} x_{e}$ & NDG & & DUB R & R Flerov. & 12615 \\
\hline \multicolumn{6}{|l|}{$A=292 \quad O(E)$} \\
\hline $238 \mathrm{U} \quad{ }^{136} \mathrm{Xe}$ & NDG & & DUB R & R Flerov. & 12615 \\
\hline \multicolumn{6}{|l|}{$A=293 \quad O(E)$} \\
\hline${ }^{238} \mathrm{U} \quad{ }^{336} \mathrm{Xe}$ & NDG & & DUB $R$ & R Flerov. & 12615 \\
\hline \multicolumn{6}{|l|}{$A=.294 \quad O(E)$} \\
\hline${ }^{238} \mathrm{U} \quad{ }^{136} \times \mathrm{x}$ & NOG & & DUP $R$ & R flerer. & 12615 \\
\hline \multicolumn{6}{|l|}{$A=295 \quad \sigma(E)$} \\
\hline $238 u \quad 136 x_{0}$ & NDG & & DUA $\mathrm{R}$ & R Flerov. & 12615 \\
\hline \multicolumn{6}{|l|}{$A=296 \quad \sigma(E)$} \\
\hline $238 \mathrm{U} \quad 136 \mathrm{xe}$ & NDG & & OUE $R$ & R Flerov. & 12615 \\
\hline \multicolumn{6}{|l|}{$A=297 \quad O(E)$} \\
\hline $238 u \quad 136 x e$ & NDG & & DUB R & R Flerov. & 12615 \\
\hline \multicolumn{6}{|l|}{$A=298 \quad \sigma(E)$} \\
\hline${ }^{238} U \quad{ }^{136} x e$ & NOG & & DUB R & R Flerov. & 12615 \\
\hline \multicolumn{6}{|l|}{$A=299 \quad O(E)$} \\
\hline${ }^{230} \mathrm{U} \quad 136 \mathrm{Xe}_{0}$ & NDG & & DUB $R$ & R Flerow. & 12615 \\
\hline \multicolumn{6}{|l|}{$A=300 \quad \sigma(E)$} \\
\hline $238 \mathrm{U} \cdot 1{ }^{36} \mathrm{X}_{0}$ & NDG & & DUB $R$ & R Fleroy. & 12615 \\
\hline \multicolumn{6}{|l|}{$A=301 \quad O(E)$} \\
\hline $2380 \quad 136 x$ & NDG & & DUB $R$ & R Flerov. & 12615 \\
\hline
\end{tabular}




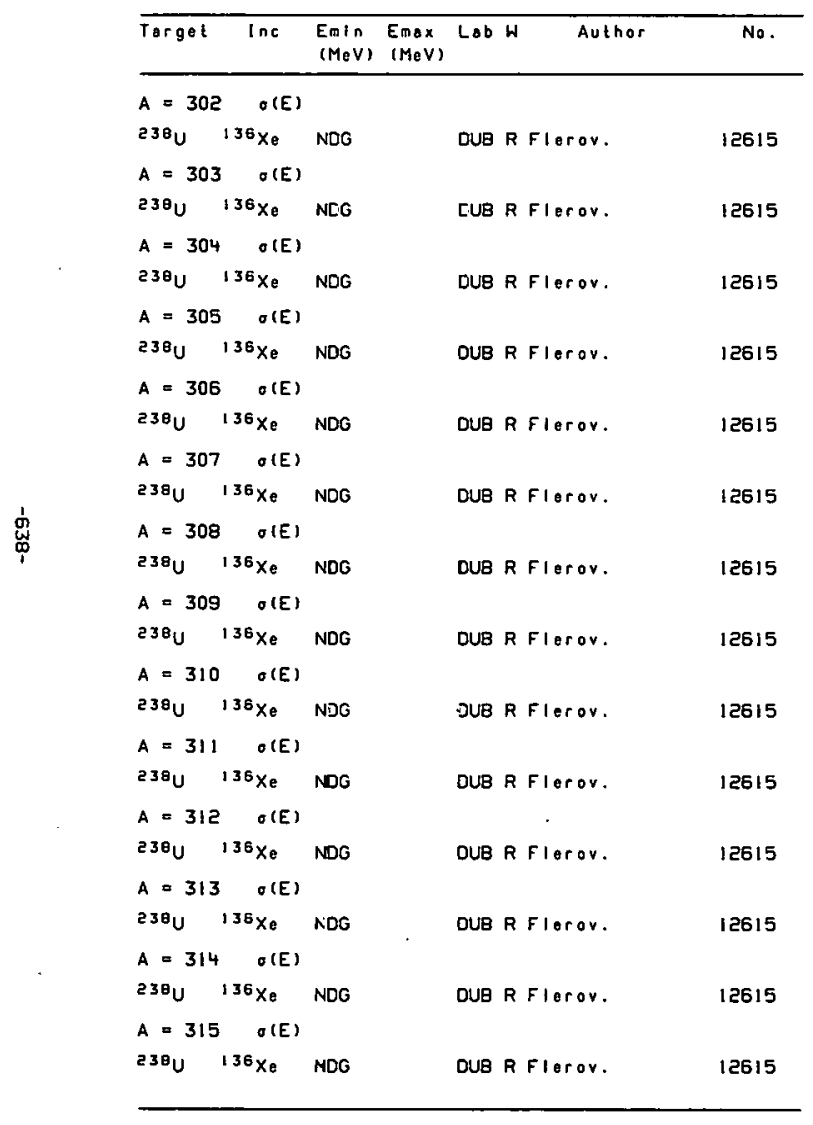

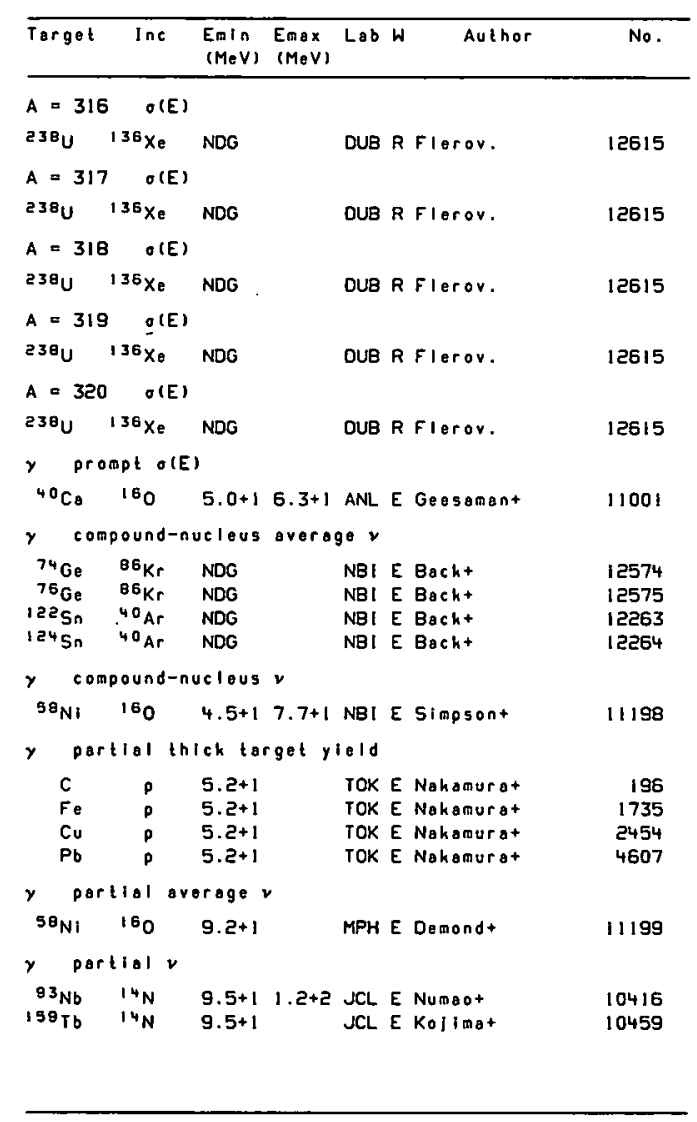




\begin{tabular}{|c|c|c|c|c|c|c|}
\hline Target & Inc & $\begin{array}{l}\text { Emin } \\
(\mathrm{MeV})\end{array}$ & $\begin{array}{l}\text { Emax } \\
(\operatorname{MeV})\end{array}$ & Lab & Author & No. \\
\hline \multicolumn{7}{|c|}{$\gamma$ partlal relative o(E) } \\
\hline $\begin{array}{l}12 \mathrm{C} \\
40 \mathrm{Ca} \\
{ }^{40} \mathrm{Cg} \\
72 \mathrm{Ge} \\
140 \mathrm{Ce} \\
140 \mathrm{Ce} \\
101 \mathrm{Ta}\end{array}$ & $\begin{array}{r}160 \\
160 \\
118 \\
108 \\
0 \\
0 \\
160\end{array}$ & $\begin{array}{l}2.8+1 \\
4 \cdot 5+1 \\
2 \cdot 5+1 \\
3 \cdot 1+1 \\
2.8+1 \\
4.5+1 \\
7.6+0\end{array}$ & $\begin{array}{l}3 \cdot 5+1 \\
6 \cdot 3+1 \\
5 \cdot 0+1 \\
4 \cdot 0+1 \\
4 \cdot 0+1 \\
5 \cdot 9+1 \\
3 \cdot 4+1\end{array}$ & $\begin{array}{l}\text { MON } \\
\text { ANL } \\
\text { BNL } \\
\text { FSU } \\
\text { IPS } \\
\text { IKO E } \\
\text { WAU }\end{array}$ & $\begin{array}{l}\text { E Tarast } \\
\text { E Geesamant } \\
\text { E Nothant } \\
\text { E Clementst } \\
\text { E Ludziejeusklt } \\
\text { E Ludzieleashit } \\
\text { E Chont }\end{array}$ & $\begin{array}{r}10624 \\
11002 \\
9571 \\
9526 \\
8405 \\
8406 \\
11456\end{array}$ \\
\hline \multicolumn{7}{|c|}{$y$ portial ofE } \\
\hline $\begin{array}{l}12 c \\
1{ }^{12} \mathrm{C} \\
13 \mathrm{C} \\
160 \\
160 \\
160\end{array}$ & $\begin{array}{l}180 \\
24 \mathrm{Mg} \\
160 \\
p \\
a \\
28 \mathrm{SI}\end{array}$ & $\begin{array}{l}2.2+1 \\
5 \cdot 0+1 \\
1.6+1 \\
8.0+0 \\
1.5+1 \\
6.0+1\end{array}$ & $\begin{array}{l}6.2: \\
7 \cdot 0+1 \\
4.6+1 \\
8.0+1 \\
1.0+3 \\
9.0+1\end{array}$ & $\begin{array}{l}\text { WAU } \\
\text { STR } \\
\text { WAU } \\
\text { GSF } \\
\text { GSF } \\
\text { STR }\end{array}$ & $\begin{array}{l}\text { E Chant } \\
\text { E Freamant } \\
\text { E Chant } \\
\text { D Ramalyt } \\
\text { O Ramaly+ } \\
\text { E Freemant }\end{array}$ & $\begin{array}{r}11607 \\
11936 \\
10725 \\
482 \\
6834 \\
11955\end{array}$ \\
\hline \multicolumn{7}{|c|}{$\gamma$ relative I, } \\
\hline${ }^{12} \mathrm{C}$ & $\begin{array}{l}{ }^{2} \mathrm{C} \\
\text { produce }\end{array}$ & \multicolumn{5}{|c|}{$y$ roo product yield } \\
\hline$C \bullet P_{F e}$ & a & $5.0+0$ & & SUN & E Giholat & 7586 \\
\hline \multicolumn{7}{|c|}{ r ralative produci yield } \\
\hline $\operatorname{copfe}$ & a & $5.0+0$ & & SUN & E Gihualat & 7587 \\
\hline \multicolumn{7}{|c|}{$y$ pioduct yleld } \\
\hline Ir & P & $3.4+1$ & $4.8+1$ & TOK & E Numoot & 4435 \\
\hline$y \quad r s$ & thich & $\operatorname{target}$ & yeld & & & \\
\hline $\begin{array}{c}{ }^{9} \mathrm{Be} \\
\mathrm{B}\end{array}$ & $p$ & $\begin{array}{l}2.0+0 \\
2.0+0\end{array}$ & $\begin{array}{l}2.5+0 \\
2.5+0\end{array}$ & $\begin{array}{l}\text { AUA } \\
\text { AUA }\end{array}$ & $\begin{array}{l}\text { E Birdt } \\
\text { E Birdt }\end{array}$ & $\begin{array}{l}131 \\
145\end{array}$ \\
\hline $12 \mathrm{c}$ & a & $5.0+0$ & & SUN & E Gilest & 6745 \\
\hline $23^{2} \mathrm{Na}$ & p & $2.0+0$ & $2.5+0$ & $A \cup A$ & E Birdt & 677 \\
\hline$\underset{27}{M g}$ & p & $\begin{array}{l}2 \cdot 0+0 \\
2 \cdot 0+0\end{array}$ & $\begin{array}{l}2.5+0 \\
2.5+0\end{array}$ & AUA & E Birdt & $\begin{array}{l}715 \\
809\end{array}$ \\
\hline Si & $?$ & $\begin{array}{l}5.0+0 \\
2.0+0\end{array}$ & $\begin{array}{l}2.5+0 \\
2.5+0\end{array}$ & AUA & E Birdt & 955 \\
\hline $31 p$ & $\beta$ & $2.0+0$ & $2.5+0$ & AUA & E Blrdt & 1018 \\
\hline$s$ & 0 & $5.0+0$ & & SUN & E Gllest & 7153 \\
\hline $\begin{array}{r}\mathrm{C}_{\theta} \\
{ }^{9{ }^{9}} \mathrm{Co}_{0}\end{array}$ & $a$ & $\begin{array}{l}5.0+0 \\
5.0+0\end{array}$ & $\cdots$ & $\begin{array}{l}\text { SUN } \\
\text { SUN }\end{array}$ & $\begin{array}{l}\text { E Gilest } \\
\text { E Gilest }\end{array}$ & $\begin{array}{l}7272 \\
7602\end{array}$ \\
\hline
\end{tabular}

\begin{tabular}{lllll}
\hline Target Inc & $\begin{array}{c}\text { Emln } \\
(M e V)\end{array}$ & $\begin{array}{l}\text { Emax } \\
(M e V)\end{array}$ & Lob $W$ & Author \\
\hline
\end{tabular}

$\gamma$ raw thich torget yleld

\begin{tabular}{|c|c|c|c|c|}
\hline Go & a & $5.0+0$ & SUN E Gllest & 7850 \\
\hline & a & $5 \cdot 0+0$ & SUN E Gllest & 8029 \\
\hline${ }^{89}{ }_{Y}$ & $a$ & $5.0+0$ & SUN E Gilest & 8042 \\
\hline $93^{\mathrm{Nb}}$ & 0 & $5.0+0$ & SUN E Gilest & 8082 \\
\hline $\ln$ & $a$ & $5.0+0$ & SUN E Gllest & 8287 \\
\hline $5 n$ & 0 & $5.0+0$ & SUN E Gllest & ө299 \\
\hline $\mathrm{Ba}$ & $a$ & $5.0+0$ & SUN E Gllest & 8390 \\
\hline$\theta_{\text {La }}$ & $a$ & $5.0+0$ & SUN E Giliest & 8399 \\
\hline $\mathrm{Ce}_{\mathrm{e}}$ & a & $5.0+0$ & SUN E Gillest & 8404 \\
\hline $141 \mathrm{Pr}$ & 0 & $5.0+0$ & SUN E Gilest & 8415 \\
\hline Nd & 0 & $5.0+0$ & SUN $\bar{E}$ Gllest & 8419 \\
\hline $\mathrm{Pb}$. & 0 & $5.0+0$ & SUN E Gllest & 8843 \\
\hline $209_{\mathrm{BI}}$ & 0 & $5.0+0$ & SUN E Gllest & 8902 \\
\hline
\end{tabular}

$y$ thich target yield

\begin{tabular}{|c|c|c|c|c|c|c|}
\hline c & $p$ & $1.2+1$ & $.3+1$ & CCP & Motusericht & 195 \\
\hline c & a & $4.6+1$ & & CCP & Matuserich* & 6734 \\
\hline $\mathrm{Mg}$ & p & $1.2+1$ & $2.3+1$ & $\mathrm{CCP}$ & Matusericht & 715 \\
\hline Mg & 0 & $4.6+1$ & & $\mathrm{CCP}$ & Eatusericht & 6965 \\
\hline $27 \mathrm{AI}$ & $\rho$ & $1.2+1$ & $2.3+1$ & CCP & Maluserlcht & 800 \\
\hline $27 \mathrm{Al}$ & o & $4.6+1$ & & CCP & Matusericht & 7021 \\
\hline $\mathrm{Ca}_{\mathrm{B}}$ & $p$ & $1.0+0$ & $2.7+1$ & CLU & E Intratort & 1240 \\
\hline II & p & $1.2+1$ & $2.3+1$ & CCP & Eatusericht & 1395 \\
\hline$T_{1}$ & a & $4.6+1$ & & $\mathrm{CCP}$ & Matuseylcht & 7367 \\
\hline $51 \mathrm{~V}$ & 160 & $3.6+1$ & $4.6+1$ & ORL & E Wellst & 11135 \\
\hline$F_{\theta}$ & p & $1.2+1$ & $2.3+1$ & CCP & E Malusericht & 1734 \\
\hline$F_{0}$ & a & $4.6+1$ & & CCP & E Matusevicht & 7531 \\
\hline Ni & p & $1.2+1$ & $2.3+1$ & CCP & E Matusericht & 2138 \\
\hline NI & a & $4.6+1$ & & $\mathrm{CCP}$ & E Matusericht & 7630 \\
\hline Cu & $\mathrm{p}$ & $1.0+0$ & $2.7+1$ & CLU & E Intratort+ & 2452 \\
\hline Cu & $p$ & $1.2+1$ & $2.3+1$ & CCP & E Maluserlcht & 2453 \\
\hline Cu & 0 & $4.6+1$ & & $\mathrm{CCP}$ & E Malusericht & 7739 \\
\hline $2 n$ & p & $1.0+0$ & $2.7+1$ & $\mathrm{CLU}$ & E Intratort & 2727 \\
\hline $9^{93} \mathrm{Nb}$ & p & $1.2+1$ & $2.3+1$ & $\mathrm{CCP}$ & E Motusericht & 3508 \\
\hline${ }^{93} \mathrm{Nb}$ & a & $4.6+1$ & & ' $\mathrm{CCP}$ & E Motusericht & 8083 \\
\hline $\mathrm{Cd}$ & p & $1.2+1$ & $2.3+1$ & $\mathrm{CCP}$ & E Motusevicht & 3724 \\
\hline Co & $a$ & $4.6+1$ & & $\mathrm{CCP}$ & E Matusericht & 8249 \\
\hline 'To & p & $1.2+1$ & $2.3+1$ & $\mathrm{CCP}$ & E Matusericht & 4381 \\
\hline ' Ta & a & $4.6+1$ & & CCP & E Matusericht & 8624 \\
\hline W & D & $1.2+1$ & $2 \cdot 3+1$ & CCP & E Metusericht & 4412 \\
\hline
\end{tabular}




\begin{tabular}{|c|c|c|c|c|c|c|c|}
\hline Targed & $\ln c$ & $\begin{array}{l}\text { Emin } \\
(M e v)\end{array}$ & $\begin{array}{l}E \max \\
(\mathrm{MeV})\end{array}$ & Lab & $w$ & Author & No. \\
\hline \multicolumn{8}{|c|}{$\gamma$ thich target yiold } \\
\hline w & a & $4.6+1$ & & $\mathrm{CCP}$ & E 1 & Matusevicht & 8636 \\
\hline $\mathrm{Pb}$ & p & $1.2+1$ & $2.3+1$ & $\mathrm{CCP}$ & EI & Matusevicht & 4606 \\
\hline $\mathrm{Pb}$ & $a$ & $4.6+1$ & & CCP & E & Matusevicht & 8844 \\
\hline${ }^{23 \theta} \mathrm{U}$ & $p$ & $1.2+1$ & $2.3+1$ & $\mathrm{CCP}$ & E & Matusevicht & 4867 \\
\hline $238 \mathrm{U}$ & a & $4.6+1$ & & CCP & E & Matusevicht & 9039 \\
\hline Many & $p$ & $1.2+1$ & $2.3+1$ & $\mathrm{CCP}$ & E & Matusevicht & 5000 \\
\hline Many & 0 & $4.6+1$ & & CCP & E & Matusericht & 9157 \\
\hline \multicolumn{8}{|l|}{$\gamma \quad \nu$} \\
\hline $107 \mathrm{Ag}$ & $86_{\mathrm{Kr}}$ & NDG & & BRK & E & Wozniont & 12592 \\
\hline $\log _{\mathrm{Ag}}$ & ${ }^{86} \mathrm{Kr}$ & NDG & . & BRK & E & Woznight & 12594 \\
\hline $165 \mathrm{Ho}$ & $88_{\mathrm{Kr}}$ & NDG & . & BRK & E & Wozniaht & 12595 \\
\hline${ }^{197} \mathrm{Au}$ & ${ }^{86} \mathrm{Kr}$ & NDS & & BRK & E & Woznlaht & 12596 \\
\hline \multicolumn{8}{|c|}{$y$ relative o(E) } \\
\hline CDPH & p & $7.0+1$ & $1.1+4$ & $\mathrm{HRV}$ & $T$ & Epstein. & 53 \\
\hline $\operatorname{CAPH}$ & & $2.8+2$ & $4.4+4$ & HRV & $T$ & Epstein. & 6663 \\
\hline${ }^{12} \mathrm{C}$ & $12 \mathrm{c}$ & $9.0+0$ & $2.8+1$ & SAC & R & Papineau. & 9677 \\
\hline 160 & 160 & $1.4+1$ & $3.3+1$ & YAL & $\mathbf{R}$ & Bromley. & 10773 \\
\hline \multicolumn{8}{|c|}{$y$ o(E) x factor } \\
\hline${ }^{12} \mathrm{C}$ & $12 \mathrm{C}$ & $6.0+0$ & $4.8+1$ & YAL & $T$ & Erbt & 9678 \\
\hline $1 a_{c}$ & $12 \mathrm{C}$ & $6.2+0$ & $1.6+1$ & MIT & $\mathrm{R}$ & Feshbach. & 9679 \\
\hline $1 a_{c}$ & ${ }^{12} \mathrm{C}$ & $6.4+0$ & $1.5+1$ & BRC & R & Cindro. & 9680 \\
\hline $12 \mathrm{c}$ & ${ }^{12} \mathrm{c}$ & $6.4+0$ & $1.6+1$ & KTO & $\mathbf{R}$ & Abe. & 9681 \\
\hline $12 \mathrm{c}$ & ${ }^{13} \mathrm{C}$ & $1.5+1$ & $1.6+1$ & YAL & E & Willett & $10 ! 72$ \\
\hline 160 & $12 \mathrm{c}$ & $1 . \subseteq+1$ & $4.8+1$ & NBI & $\bar{E}$ & Suftkousklt & 9826 \\
\hline 160 & $10_{0}$ & $1.4+1$ & $2.3+1$ & YAL & $R$ & Bromley. & 10774 \\
\hline 160 & $15_{0}$ & $z . E+1$ & $4.6+1$ & MIT & R & Feshbach. & 10775 \\
\hline $18 e^{2} W$ & ${ }^{12} \mathrm{C}$ & $7.0+1$ & $1.3+2$ & $B O R$ & $T$ & Delagranget & 10071 \\
\hline \multicolumn{8}{|c|}{$y \quad O(E)$} \\
\hline $10 \mathrm{~B}$ & ${ }^{14} \mathrm{~N}$ & $8.9+0$ & $1.8+1$ & CAL & $E$ & Wut & 10238 \\
\hline c & $p$ & TR & $2.4+1$ & WAU & $E$ & Bodanshyt & 194 \\
\hline c & & TR & $2.7+1$ & WAU & $E$ & Bodanskyt & 6735 \\
\hline $12 \mathrm{c}$ & $12 \mathrm{C}$ & $1.3+1$ & $1.6+1$ & YAL & E & Erb+ & 9683 \\
\hline $12 \mathrm{c}$ & $14 \mathrm{~N}$ & $5.9+1$ & $7.0+1$ & GRE & $E$ & Colet & 10265 \\
\hline $14 \mathrm{~N}$ & p & TR & $2.4+1$ & WAU & $E$ & Bodanskyt & 366 \\
\hline $14 \mathrm{~N}$ & & TR & $2.7+1$ & WAU & $\vec{E}$ & Bodonskyt & $679 !$ \\
\hline${ }^{14} \mathrm{~N}$ & $10_{\mathrm{B}}$ & $5.0+0$ & $1.3+1$ & CAL & $M$ & Wut & 9511 \\
\hline
\end{tabular}

\begin{tabular}{lllll}
\hline Targat inc & $\begin{array}{c}\text { Earno } \\
(M e V)\end{array}$ & Emax Lab $W$ & Author & No. \\
& & & \\
\hline
\end{tabular}

\begin{tabular}{|c|c|c|c|c|c|c|}
\hline \multicolumn{7}{|c|}{$y \quad O(E)$} \\
\hline 160 & P & TR & $2.4+1$ & WAU E & E Bodanshyt & 483 \\
\hline 160 & 0 & $T R$ & $2.7+1$ & WAU E & E Bodanshy+ & 6835 \\
\hline${ }^{20} \mathrm{Ne}$ & p & TR & $2.4+1$ & WAU E & E Bodanshyt & 644 \\
\hline${ }^{20} \mathrm{Ne}$ & 8 & $4.0+0$ & $4.0+2$ & GSF D & D Ramaty+ & 645 \\
\hline${ }^{20} \mathrm{Ne}$ & - & $\mathrm{TR}$ & $2.7+1$ & WAU E & E Bodanshy+ & 6922 \\
\hline${ }^{20} \mathrm{Ne}$ & a & $8.0+0$ & $1.2+3$ & GSF D & D Ramaty+ & 6923 \\
\hline $24 \mathrm{Mg}$ & $p$ & $T R$ & $2.4+1$ & WAU E & E Bodanshyt & 736 \\
\hline $24 \mathrm{Mg}$ & $p$ & $5.0+0$ & $6.0+2$ & GSF D & D Ramaty+ & 737 \\
\hline${ }^{24} \mathrm{Mg}$ & 。 & TR & $2.7+1$ & WAU E & E Bodanshy+ & 6976 \\
\hline${ }^{24} \mathrm{Mg}$ & 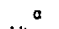 & $8.0+0$ & $2.4+3$ & GSF D & D Ramaty+ & 6977 \\
\hline $27 \mathrm{AI}$ & ${ }^{14} \mathrm{~N}$ & $5.9+1$ & & GRE E & E colet & 10349 \\
\hline 2051 & $p$ & TR & $2.4+1$ & WAU E & E Bodanshyt & 973 \\
\hline 2851 & $p$ & $6.0+0$ & $1.0+3$ & GSF D & D Ramaty+ & 974 \\
\hline${ }^{2 \theta} 51$ & : & TR & $2.7+1$ & WAU E & E Bodanshyt & 7092 \\
\hline $28 \mathrm{SI}$ & 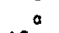 & $0.0+0$ & $4.0+3$ & GSF D & D Ramaty+ & 7093 \\
\hline${ }^{20} 51$ & ${ }^{16} 0$ & $5.0+1$ & $6.5+1$ & YAL E & E Bettst & 10980 \\
\hline${ }^{2 \theta} \mathrm{SI}$ & ${ }^{28} \mathrm{~S} 1$ & $6.5+1$ & $9.0+1$ & FSU E & E Medskert & $1 ! 970$ \\
\hline${ }^{30} \mathrm{SI}$ & ${ }^{14} \mathrm{~N}$ & $5.9+1$ & $7.0+1$ & GRE E & E Colet & 10360 \\
\hline $\mathrm{Ca}_{\mathrm{a}}$ & 0 & $9.0+1$ & & OSA E & Efirit & 7273 \\
\hline${ }^{56} \mathrm{Fe}$ & p & $T R$ & $2.4+1$ & WAU E & E Bodanshy+ & 1885 \\
\hline${ }^{56} \mathrm{Fe}$ & p p p la & $4.0+0$ & $8.0+2$ & GSF D & D Ramaty+ & 1886 \\
\hline${ }^{56} \mathrm{Fe}$ & 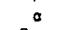 & TR & $2.7+1$ & WAU E & E Bodonshyt & 7551 \\
\hline${ }^{56} \mathrm{Fe}$ & ${ }^{6} \mathrm{LI}$ & $6.6+1$ & $9.1+1$ & INU E & E Karwowshit & 9192 \\
\hline${ }^{5 \theta_{\mathrm{Ni}}}$ & & $2.0+2$ & & LAS $E$ & E Jachsont & 2229 \\
\hline${ }^{50} \mathrm{NI}$ & ${ }^{6} \mathrm{Li}$ & $6.6+1$ & $9.1+1$ & INUE & E Karwowshit & 9209 \\
\hline${ }^{60} \mathrm{NI}$ & $p$ & $2.0+2$ & & LAS $E$ & E Jacksont & 2330 \\
\hline${ }^{80} \mathrm{Sr}$ & $p$ & $3.0+0$ & +1 & LRL E & E detricht & 3255 \\
\hline${ }^{89} Y$ & $p$ & $3.0+0$ & +1 & LRL $E$ & E Dretrient & 3313 \\
\hline${ }^{90} \mathrm{Zr}$ & p & $3.0+0$ & +1 & LRL E & E oletrleht & 3400 \\
\hline Meny & $p$ & $3.5+0$ & $1.0+3$ & GSF D & D Ramaty ${ }^{+}$ & 5007 \\
\hline Many & a & $\theta .0+0$ & $4.0+3$ & GSF D & D Ramoty+ & 9156 \\
\hline
\end{tabular}

$n$ prompt $v$

\begin{tabular}{|c|c|c|c|c|}
\hline $19 \theta_{\mathrm{Hg}}$ & 。 & $4.1+16.5+1$ & WWS E Neuzil. & 8798 \\
\hline $199_{\mathrm{Hg}}$ & 0 & $4.1+16.5+1$ & WWS E Neuzil. & 8803 \\
\hline $200 \mathrm{Hg}$ & 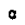 & $4.1+1$ & WWS E Neuzil. & 8808 \\
\hline $201 \mathrm{Hg}$ & 。 & $4.1+1$ & WWS E Neuzil. & 8813 \\
\hline $202 \mathrm{Hg}$ & c & $4.1+1$ & WWS E Neuzll. & 8817 \\
\hline${ }^{204} \mathrm{Hg}$ & o & $4.1+1$ & WWS E Neuzil. & 8824 \\
\hline${ }^{226} \mathrm{Ra}$ & $p$ & $\mid .2+1$ & WZI E Gayert & 4756 \\
\hline
\end{tabular}




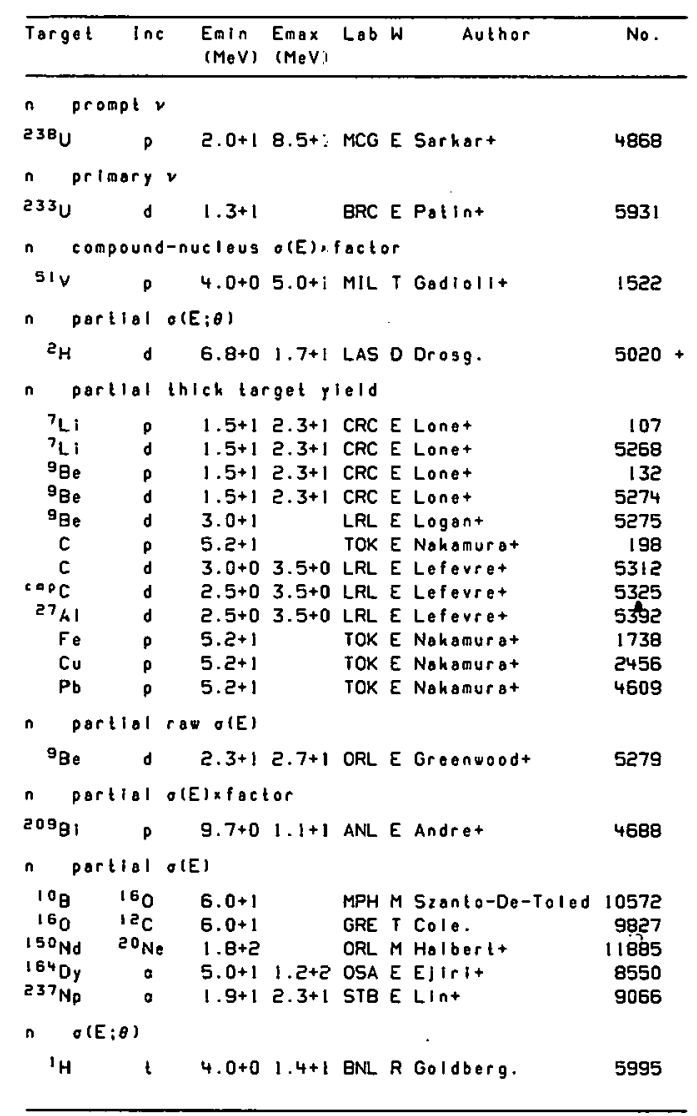

\begin{tabular}{lllll}
\hline Target Ine & $\begin{array}{c}\text { Eminn } \\
(\operatorname{MeV})\end{array}$ & $\begin{array}{c}\text { (Mav) Lab } \\
\text { Mev }\end{array}$ & Author & No. \\
\hline
\end{tabular}

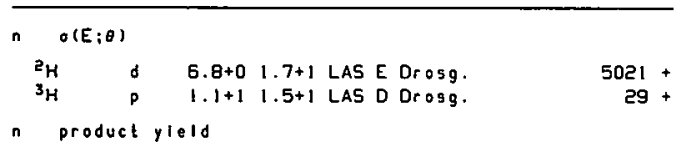

$n$ product yield

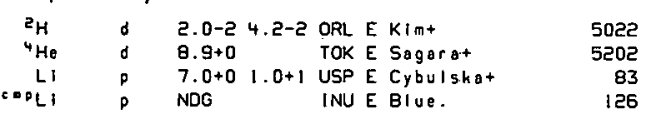

n thich target yleldxfactor

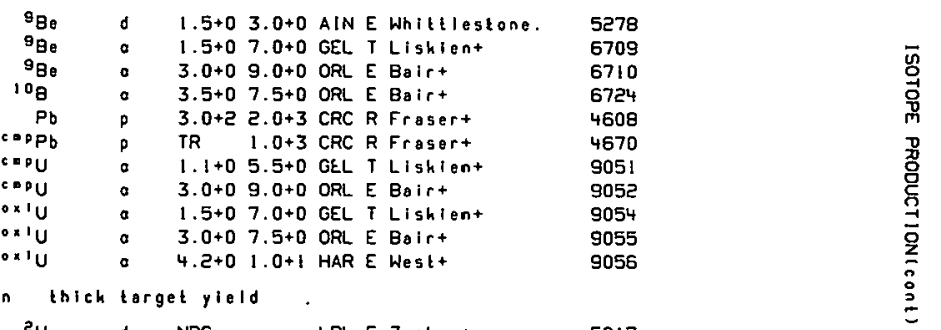

\begin{tabular}{|c|c|c|c|c|c|c|}
\hline${ }^{2} \mathrm{H}$ & d & NDG & & LRL E & Zuchert & 5017 \\
\hline${ }^{2} H$ & d & NDG & & VNV E & Bernardt & 5018 \\
\hline${ }^{a_{H}}$ & d & $2.5-2$ & $3.0-1$ & ORL E & Kim. & 5019 \\
\hline${ }^{2} \mathrm{H}$ & $t$ & NDG & & LASE & Armstrongt & 5999 \\
\hline${ }^{2} \mathrm{H}$ & $i$ & $2.5-2$ & $3.0-1$ & ORL $\bar{E}$ & Kim. & 6000 \\
\hline${ }^{2} \mathrm{H}$ & $a$ & +0 & $7.2+0$ & HAR E & West+ & 6654 \\
\hline${ }^{3} \mathrm{H}$ & d & $2.5-2$ & $3.0-1$ & ORL E & $\mathrm{Kim}$. & 5101 \\
\hline${ }^{3} \mathrm{H}$ & d & $1.6-1$ & & ITL E & Hourst+ & 5102 \\
\hline${ }^{3} \mathrm{H}$ & d & $2.1-1$ & & WISE & Chenever :- & 5103 \\
\hline${ }^{3} \mathrm{H}$ & d & $4.0-1$ & & LRL E & Bootht & 5104 \\
\hline${ }^{3} \mathrm{H}$ & d & $5.0-i$ & & RDI E & cleland. & 5105 \\
\hline LI & p & TR & $3.0+0$ & BIRE & Campbell+ & 84 \\
\hline LI & $p$ & +1 & & TNL E & Nelsont & 85 \\
\hline LI & d & +0 & & TOH E & Miyagit & 5211 \\
\hline LI & d & $6.0+0$ & $3.2+1$ & ANL $R$ & Persianit & 5212 \\
\hline LI & d & +1 & & TNL E & Nelsont & 5213 \\
\hline LI & 0 & $3.5+0$ & $9.0+0$ & ORL E & Bairt & 6699 \\
\hline
\end{tabular}




\begin{tabular}{|c|c|c|c|c|c|c|}
\hline Target & $\operatorname{Inc}$ & $\begin{array}{l}\text { Emin } \\
\text { (Mev) }\end{array}$ & $\begin{array}{l}\text { Emax } \\
(\operatorname{MeV})\end{array}$ & Lab & Author & No. \\
\hline thic & \multicolumn{6}{|c|}{ target yield } \\
\hline LI & a & $4.4+0$ & $7.0+0$ & GEL & T Liskient & 6700 \\
\hline Li & $a$ & $7.5+0$ & & HAR & E West t & 6701 \\
\hline$\sigma_{L I}$ & $a$ & $6.0+0$ & $9.0+0$ & ORL & E Bairt & 6702 \\
\hline${ }^{7} \mathrm{LI}$ & a & $3.5+0$ & $9.0+0$ & ORL & E Bairt & 6705 \\
\hline${ }^{9} \mathrm{Be}$ & p & TR & $3.0+0$ & BIR & E Campbell+ & 133 \\
\hline${ }^{9} \mathrm{Be}$ & d & $6.0+0$ & $4.0+1$ & ANL & R Persianit & 5276 \\
\hline $\mathbf{9}_{\mathrm{Be}}$ & d & $4.0+1$ & & ORL & E Soltmarsht & 5277 \\
\hline$B$ & 0 & $3.0-1$ & $5.2+0$ & GEL & T Lishient & 6721 \\
\hline $\mathrm{B}$ & a & $3.5+0$ & $7.5+0$ & ORL & E Bairt & 6722 \\
\hline a & $a$ & $7.5+0$ & & HAR & E West + & 6723 \\
\hline $11 B$ & $a$ & $3.5+0$ & $7.5+0$ & ORL & E Balrt & $672 \theta$ \\
\hline c & $a$ & $1.0+0$ & $5.4+0$ & ORL & E Boirt & 6736 \\
\hline c & $a$ & $1.1+0$ & $7.0+0$ & GEL & T Lishient & 6737 \\
\hline c & a & $2.0+0$ & $1.0+1$ & ORL & E Machlin. & 6738 \\
\hline c & a & $3 \cdot 0+0$ & $1.0+1$ & HAR & E West + & 6739 \\
\hline c & $a$ & $5.4+0$ & $9.0+0$ & ORL & E Bair. & 6740 \\
\hline $12 \mathrm{c}$ & 0 & $3.6+0$ & $1.0+1$ & HAR & E West+ & 6746 \\
\hline $13 \mathrm{c}$ & o & $1.0+0$ & $1.0+0$ & ORL & E Boirt & 6782 \\
\hline $\mathrm{N}$ & a & $6.1+0$ & $7.0+0$ & GEL & Tliskient & 6790 \\
\hline 0 & 0 & $1.5+0$ & $7.0+0$ & GEL & TLishient & 6830 \\
\hline 0 & a & $3.5+0$ & $8.0+0$ & ORL & E Balrt & 6931 \\
\hline$\because P O$ & o & $3 \cdot 0+0$ & B. $0+0$ & ORL & E Boirt & 6896 \\
\hline CApo & $a$ & $4.0+0$ & $5 \cdot 0+0$ & HAR & T Hootent & 6897 \\
\hline $19^{F}$ & $a$ & $3.5+0$ & $7.5+0$ & ORL & E Bairt & 6902 \\
\hline $18_{F}$ & $a$ & $7.5+0$ & & HAR & E West + & 6903 \\
\hline $\mathrm{Ne}$ & 。 & $1.9+0$ & $5.1+0$ & GEL & T Lishlent & 6919 \\
\hline Mg & $a$ & $3.0+0$ & $5.0+0$ & GEL & t Lishiont & 6966 \\
\hline $\mathrm{Mg}$ & a & $3.6+0$ & $1.0+1$ & HAR & E West+ & 6967 \\
\hline $\mathrm{Mg}$ & 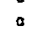 & $4.0+0$ & $9.0+0$ & ORL & E Balrt & 6968 \\
\hline $27 \mathrm{Al}$ & $a$ & $3.3+0$ & $7.0+0$ & GEL & T Lisklent & 7022 \\
\hline $27 \mathrm{Al}$ & $a$ & $3.5+0$ & $9.0+0$ & ORL & E Bairt & 7023 \\
\hline $27 \mathrm{Al}$ & a & $3.8+0$ & $1.0+1$ & HAR & $\varepsilon$ West+ & 7024 \\
\hline si & $a$ & $2.4+0$ & $4.7+0$ & GEL & T Lishient & 7089 \\
\hline Si & $a$ & $3.8+0$ & $1.0+1$ & HAR & E Westt & 7090 \\
\hline Si & $a$ & $4.5+0$ & $9.0+0$ & ORL & E Bairt & 7091 \\
\hline $30 \mathrm{si}$ & a & +0 & $7.2+0$ & HAR & E West + & 7114 \\
\hline $0 \times 151$ & a & $5.0+0$ & $8.0+0$ & ORL & E Boirt & 7130 \\
\hline$C_{0}$ & p & $1.0+0$ & $2.7+1$ & CLU & E Intratort & 1241 \\
\hline$F_{e}$ & a & $5.1+0$ & $1.0+1$ & HAR & E West+ & 7532 \\
\hline $56 \mathrm{Fe}$ & $a$ & $2.0+0$ & $7.5+0$ & HAR & E West+ & 7552 \\
\hline
\end{tabular}

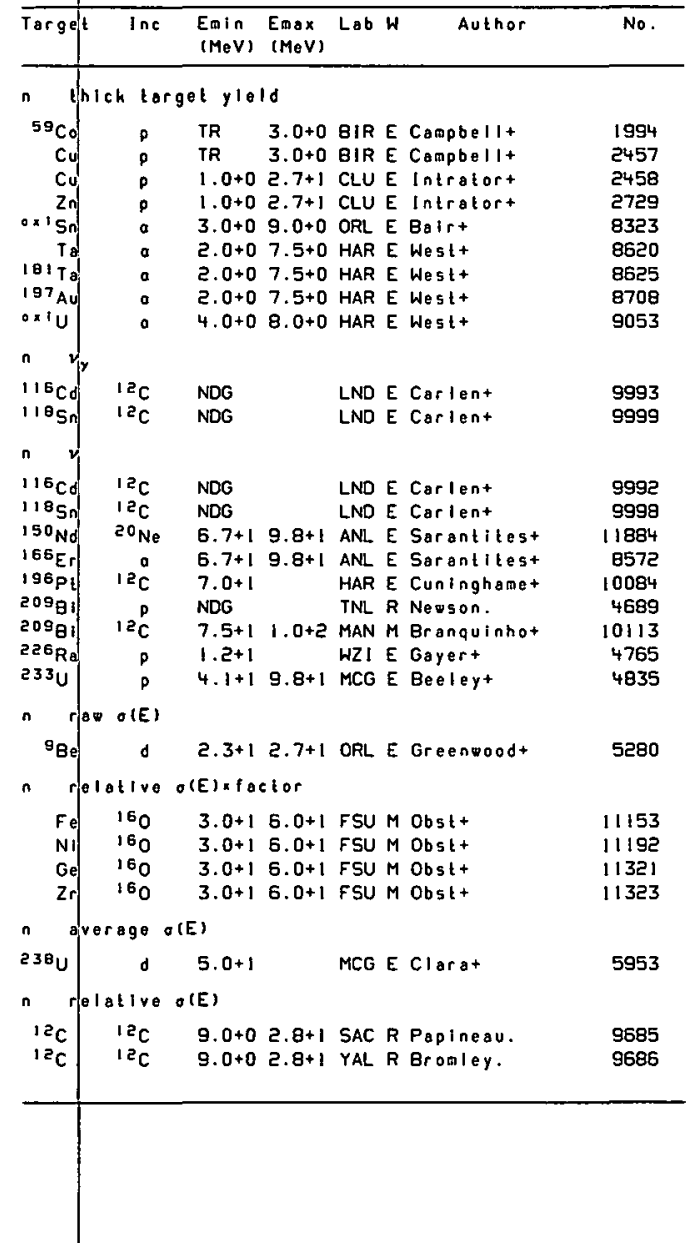




\begin{tabular}{|c|c|c|c|c|c|c|}
\hline Target & inc & $\begin{array}{l}\text { Emin } \\
(\mathrm{MeV})\end{array}$ & $\begin{array}{l}E_{\max } \\
(M \in V)\end{array}$ & Lab & Author & No. \\
\hline \multicolumn{7}{|c|}{$n \quad \sigma(E) \times f$ actor } \\
\hline $3_{\mathrm{H}}$ & 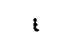 & $3.4-2$ & $1.6-1$ & CCP & E Serout & 6019 \\
\hline${ }^{6} \mathrm{Li}$ & d & $2.0-1$ & $7.7-1$ & ANL & E Hollandt & 5221 \\
\hline Cd & $p$ & $2.5+0$ & $5.8+0$ & ORL & E Johnsont & 3726 \\
\hline $\ln$ & $p$ & $2.5+0$ & $7.0+0$ & ORL & M Johnsont & 3825 \\
\hline & $p$ & $2.6+0$ & $5.7+0$ & ORL & E Johnsont & 3939 \\
\hline $197 \mathrm{Au}$ & $7_{L i}^{r}$ & $3.4+1$ & & LAS & E Goldstonet & 9460 \\
\hline $232 \mathrm{Th}$ & ${ }^{7} \mathrm{LI}$ & $3.4+1$ & & LAS & E Goldstonet & 9473 \\
\hline $233 \mathrm{u}$ & $a$ & $2.0+1$ & $5.0+1$ & STB & E Delagranget & 8988 \\
\hline $234 \mathrm{U}$ & $a$ & $2.0+1$ & $5.0+i$ & STB & E Delagranget & 9002 \\
\hline $235^{u}$ & a & $2.0+1$ & $5.0+1$ & STB & E Delagranget & 9018 \\
\hline $236 u$ & ${ }^{7} \mathrm{Li}$ & $3.4+1$ & & LAS & E Goldstonet & 9478 \\
\hline \multicolumn{7}{|c|}{$n \quad O(E)$} \\
\hline$e_{H}$ & d & NDG & & LRL & E Zuchert & 5016 \\
\hline${ }^{2} \mathrm{H}$ & $d$ & $5.0+0$ & $1.9+2$ & NOT & T Fonsecat & 5023 \\
\hline $2_{H}$ & $\mathrm{t}$ & $0.0+0$ & $6.0-1$ & LAS & E Armstrongt & 6001 \\
\hline${ }^{3} \mathrm{H}$ & d & $0.0+0$ & $4.0-1$ & LAS & E Arostrongt & 5106 \\
\hline${ }^{4} \mathrm{He}$ & p & $3.0+1$ & $2.0+2$ & NMX & R Ahluealia. & 67 \\
\hline${ }^{6} \mathrm{~L} ;$ & d & $2.0-1$ & $7.8-1$ & ANL & E Hollandt & 5220 \\
\hline${ }^{9} \mathrm{Be}$ & $a$ & $1.5+0$ & $7.9+0$ & OTC & D Gelgert & $6711+$ \\
\hline${ }^{10}{ }_{B}$ & 150 & $6.0+1$ & & MPH & MSzanto-De-Toled & 10573 \\
\hline c & p & $3 \cdot 0+1$ & $2.0+2$ & NMX & R Ahluvalia. & 197 \\
\hline c & $14 \mathrm{~N}$ & $1.6+1$ & $7.5+1$ & FSU & T Holubt & 10244 \\
\hline c & 150 & $1.6+1$ & $7.5+1$ & FSU & T Holubt & 10590 \\
\hline${ }^{12} \mathrm{C}$ & ${ }^{12} \mathrm{c}$ & NDG & & ORL & E Bairt & 9684 \\
\hline${ }^{12} \mathrm{C}$ & ${ }^{13} \mathrm{C}$ & $7.0+0$ & $3.0+1$ & ORL & E Bolrt & 10173 \\
\hline${ }^{12} \mathrm{C}$ & 160 & NDG & & ORL & E Balrt & 10625 \\
\hline $12 \mathrm{C}$ & 160 & $4.1+1$ & $6.5+1$ & STR & $R$ Hoast & 10626 \\
\hline$=12 \mathrm{C}$ & $10_{0}$ & NDG & & ORL & E Bairt & 11608 \\
\hline $12_{c} \mathrm{C}$ & $10_{0}$ & NDG & & ORL & E Bairt & 11509 \\
\hline $13 \mathrm{C}$ & ${ }^{12} \mathrm{c}$ & $7.0+0$ & $4.0+1$ & ORL & E Balrt & 9785 \\
\hline${ }^{13} \mathrm{C}$ & ${ }^{13} \mathrm{C}$ & NOG & & ORL & E Boirt & 10188 \\
\hline${ }^{14} \mathrm{~N}$ & p & $7.0+0$ & $2.0+2$ & NMX & R Ahluwalia. & 367 \\
\hline 0 & a & $3.0+0$ & $8.0+0$ & ORL & E Bairt & 6829 \\
\hline 160 & & $1.8+1$ & $2.0+2$ & NMX & R Ahluoglla. & 484 \\
\hline 160 & $12 \mathrm{c}$ & NDG & & ORL & E Balrt & 9828 \\
\hline 160 & ${ }^{13} \mathrm{C}$ & NDG & & ORL & E Balrt & 10193 \\
\hline 160 & 160 & NOG & & ORL & E Bairt & 10776 \\
\hline 150 & 160 & TR & $5.5+1$ & ORL & E Balrt & 10777 \\
\hline 160 & 150 & $2.5+1$ & & STF & E Chengt & 10778 \\
\hline
\end{tabular}

\begin{tabular}{|c|c|c|c|c|c|c|}
\hline Target & $\ln c$ & $\begin{array}{l}\text { EmIn } \\
(M e V)\end{array}$ & $\begin{array}{l}\text { Emax } \\
(M e v)\end{array}$ & Lab & Au $\vdots$ hor & No. \\
\hline \multicolumn{7}{|c|}{$n \quad O(E)$} \\
\hline${ }^{16} 0$ & 160 & $2.5+1$ & & ISTR & E Kolatat & 10779 \\
\hline 160 & 180 & NDG & & ORL & E Bairt & 11643 \\
\hline 160 & 180 & TR & $5.5+1$ & ORL & E Bairt & 11644 \\
\hline 170 & $a$ & $1.0+0$ & $1.9+0$ & ORL & E Balrt & 6877. \\
\hline 180 & & $1.0+0$ & $1.9+0$ & ORL & E Bairt & 6882 \\
\hline 180 & $12 \mathrm{c}$ & NDG & & ORL & E Bairt & 9853 \\
\hline 180 & ${ }^{13} \mathrm{C}$ & NDG & & ORL & E Bairt & 10196 \\
\hline 180 & 160 & NDG & & ORL & E Bairt & 10862 \\
\hline $10_{0}$ & 160 & TR & $5.5+1$ & ORL & E Bairt & 10863 \\
\hline 180 & 180 & NDG & & ORL & E Bairt & 11645 \\
\hline 180 & 180 & TR & $5.5+1$ & ORL & E Bairt & 11646 \\
\hline $\mathrm{Mg}$ & 160 & $1.6+1$ & $7.5+1$ & FSU & T Holubt & 10868 \\
\hline $27 \mathrm{Al}$ & 160 & $1.6+1$ & $7.5+1$ & FSU & T Holub+ & 10920 \\
\hline Si & $14 \mathrm{~N}$ & $1.6+1$ & $7.5+1$ & FSU & T Holub+ & 10352 \\
\hline $\mathrm{Cr}$ & p & $4.0+0$ & $5.5+0$ & LRL & E Albert. & 1588 \\
\hline $\mathrm{Fe}_{\mathrm{e}}$ & p & $3.5+0$ & $4.0+2$ & NMX & R Ahlusalis. & 1736 \\
\hline $\mathrm{Fe}_{\mathrm{O}}$ & p & $4.0+0$ & $5.5+0$ & LKL & E Albert. & 1737 \\
\hline $\mathrm{Ni}$ & 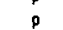 & $7.0+0$ & & LRL & E Albert. & 2139 \\
\hline${ }^{5 \theta_{\mathrm{NI}}}$ & 160 & $3.6+1$ & $5.5+1$ & ORL & M Robinsont & 11200 \\
\hline $5 \theta_{\mathrm{NI}}$ & 160 & $3.8+1$ & $5.5+1$ & YAL & R Bromley. & 11201 \\
\hline $58_{\mathrm{Ni}}$ & 180 & $3.6+1$ & $5.5+1$ & ORL & M Robinsont & 11667 \\
\hline $60 \mathrm{Ni}$ & 160 & $3.6+1$ & $5.5+1$ & ORL & M Robinsont & 11225 \\
\hline $60 \mathrm{Ni}$ & 160 & $3.8+i$ & $5.5+1$ & YAL & R Bromley. & 11226 \\
\hline $60 \mathrm{NI}$ & 180 & $3.6+1$ & $5.5+1$ & ORL & M Robinsont & 11669 \\
\hline${ }^{61} \mathrm{NI}$ & 160 & $3.6+1$ & $5.5+1$ & ORL & M Robinsont & 11228 \\
\hline${ }^{6} I_{N I}$ & 160 & $3.8+1$ & $5.5+1$ & YAL & R Bromley. & 11229 \\
\hline GINI & 180 & $3.6+1$ & $5.5+1$ & ORL & M Robinsont & 11670 \\
\hline $62^{N I}$ & 160 & $3.6+1$ & $5.5+1$ & ORL & M Robinsont & 11258 \\
\hline $62_{\mathrm{Ni}}$ & 160 & $3.8+1$ & $5.5+1$ & YAL & R Bromley. & 11269 \\
\hline $62_{\mathrm{Ni}}$ & 180 & $3.6+1$ & $5.5+1$ & ORL & M Robinsont & $1167 !$ \\
\hline${ }^{64} \mathrm{NI}$ & 160 & $3.6+1$ & $5.5+1$ & ORL & M Robinsont & 11270 \\
\hline $64 \mathrm{NI}$ & 160 & $3.8+1$ & $5.5+1$ & YAL & R Bromley. & $1127 !$ \\
\hline${ }^{64} \mathrm{NI}$ & 180 & $3.6+1$ & $5.5+1$ & ORL & $M$ Robinsont & 11672 \\
\hline Cu & & $4.0+0$ & $5.5+0$ & LRL & E Albert: & 2455 \\
\hline${ }^{63} \mathrm{Cu}$ & 160 & $3 \cdot 6+1$ & $5.5+1$ & ORL & M Robinsont & 11275 \\
\hline${ }^{63} \mathrm{Cu}$ & 180 & $3.6+1$ & $5.5+1$ & ORL & M Robinsont & 11675 \\
\hline${ }^{65} \mathrm{Cu}$ & 160 & $3.6+1$ & $5 \cdot 5+1$ & ORL & MRobinsont & 11296 \\
\hline${ }^{85} \mathrm{Cu}$ & 100 & $3.6+1$ & $5.5+1$ & ORL & M Robinsont & 11676 \\
\hline $2 n$ & & $4.0+0$ & $5.5+0$ & LRL & E Albert: & 2728 \\
\hline 7 & & $3.6+1$ & $5.5+1$ & ORL & $M$ Robinsont & 11314 \\
\hline
\end{tabular}




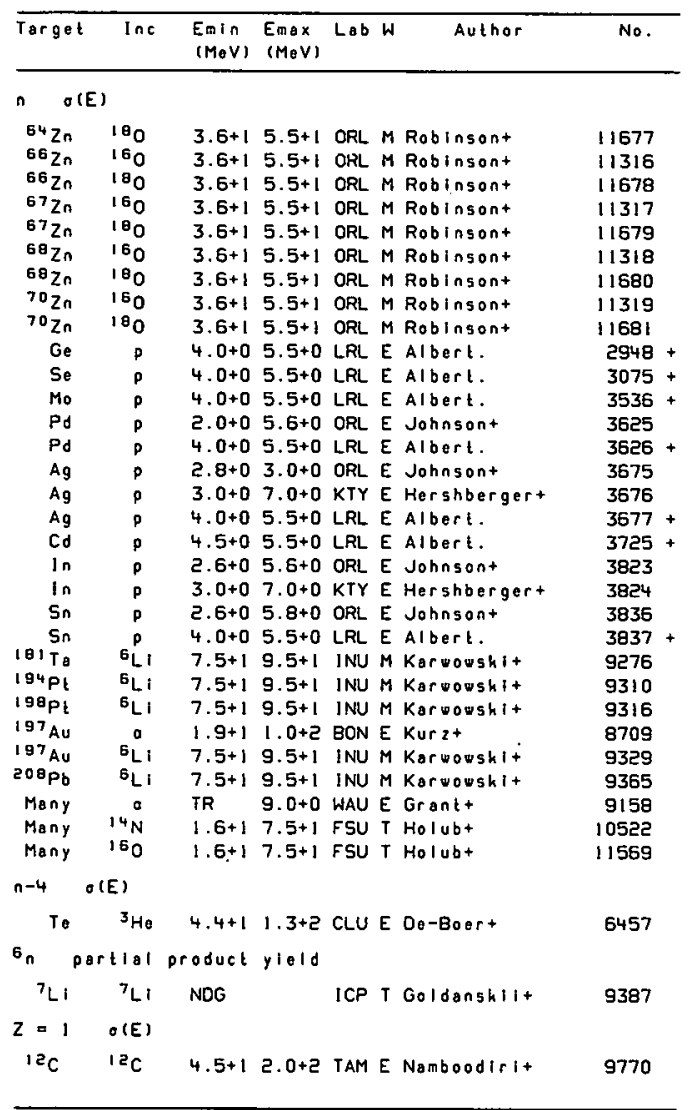

\begin{tabular}{|c|c|c|c|c|c|c|}
\hline Targett & $\ln c$ & $\begin{array}{l}E_{m i n} \\
(\mathrm{MeV})\end{array}$ & $\begin{array}{l}\text { Emax } \\
(M e V)\end{array}$ & Lab & Author & No. \\
\hline $\begin{array}{l}Z=1 \\
27_{\mathrm{AI}}\end{array}$ & $\begin{array}{l}O(E) \\
1{ }^{14} \mathrm{~N}\end{array}$ & $1.0+2$ & & LYO & E Billereyt & 10351 \\
\hline $\begin{array}{c}{ }^{1} H \\
27_{A 1} \\
58_{N i} \\
90 \\
{ }^{20}{ }_{3} \\
{ }^{8}{ }_{B i}\end{array}$ & $\begin{array}{c}\text { compound } \\
p \\
p \\
p \\
p\end{array}$ & $\begin{array}{c}\text { d-nueleus } \\
9.0+1 \\
9.0+1 \\
9.0+1 \\
9.0+1\end{array}$ & semiss & $\begin{array}{l}\text { gion } \\
\text { MRY } \\
\text { MRY } \\
\text { MRY } \\
\text { MRY }\end{array}$ & $\begin{array}{l}o(E) \\
E \text { Wut } \\
E \text { Wut } \\
E \text { Wut } \\
E \text { Wut }\end{array}$ & $\begin{array}{r}813 \\
2233 \\
3404 \\
4693\end{array}$ \\
\hline $\begin{array}{c}1 \mathrm{H} \\
27_{\mathrm{Al}} \\
58_{\mathrm{Ni}} \\
90^{2} \mathrm{Zr} \\
20{ }^{\circ} \mathrm{Bi}\end{array}$ & $\begin{array}{c}p \\
p \\
p \\
p\end{array}$ & $\begin{array}{c}\text { enis } 5 \text { ion } \\
9.0+1 \\
9.0+1 \\
9.0+1 \\
9.0+1\end{array}$ & $n \circ(E)$ & $\begin{array}{l}\text { MRY } \\
\text { MRY } \\
\text { MRY } \\
\text { MRY }\end{array}$ & $\begin{array}{l}\text { E } \text { Hut }^{+} \\
\text {E } \mathrm{Hut}^{+} \\
\text {E } \mathrm{Wut} \\
\text { E } \mathrm{Wut}^{-}\end{array}$ & $\begin{array}{r}811 \\
2231 \\
3402 \\
4691\end{array}$ \\
\hline $\begin{array}{c}{ }^{1} \mathrm{H} \\
27_{\mathrm{Al}} \\
58_{\mathrm{Ni}} \\
90 \\
209_{\mathrm{Zi}}\end{array}$ & $\begin{array}{c}\text { anission } \\
p \\
p \\
p \\
p\end{array}$ & $\begin{array}{c}o(E) \times 10 \\
9.0+1 \\
9.0+1 \\
9.0+1 \\
9.0+1\end{array}$ & $\begin{array}{l}\text { actor } \\
1.0+2\end{array}$ & $\begin{array}{l}\text { MRY } \\
\text { MFY } \\
\text { MRY } \\
\text { MRY }\end{array}$ & $\begin{array}{l}\text { E } \text { Wut } \\
\text { E } \text { Wut } \\
\text { E } \text { Wut } \\
\text { E } \text { Wut }^{+}\end{array}$ & $\begin{array}{r}812 \\
2232 \\
3403 \\
4692\end{array}$ \\
\hline${ }^{1} H$ & oission & n $O(E)$ & & & & \\
\hline $\begin{array}{r}27 \\
58 \mathrm{Al} \\
90 \mathrm{Ni} \\
20 \mathrm{Zr} \\
209_{\mathrm{Bi}}\end{array}$ & $\begin{array}{l}p \\
p \\
p \\
p\end{array}$ & $\begin{array}{l}9.0+1 \\
9.0+1 \\
9.0+1 \\
9.0+1\end{array}$ & $1.0+2$ & $\begin{array}{l}\text { MRY } \\
\text { MRY } \\
\text { MRY } \\
\text { MRY }\end{array}$ & $\begin{array}{l}\text { E } \text { Wut }^{+} \\
\text {E Wut } \\
E \text { Wut } \\
\text { E } \text { Wut }^{-}\end{array}$ & $\begin{array}{r}810 \\
2230 \\
3401 \\
4690\end{array}$ \\
\hline${ }^{\prime} H$ & : onpound & $d$-nucleus & s $O(E) \times$ & $\therefore \mathrm{facl}$ & tor & \\
\hline $\begin{array}{l}5 I_{V} \\
{ }^{H} \mathrm{H}\end{array}$ & $\begin{array}{c}P \\
\text { :ompound }\end{array}$ & $\begin{array}{r}4.0+0 \\
\text {-nucleus }\end{array}$ & $\begin{array}{l}5.0+1 \\
5 \quad O(E)\end{array}$ & MIL & T Godiolit & 1523 \\
\hline $\begin{array}{c}27 \mathrm{Al} \\
5 \theta_{\mathrm{NI}} \\
90 \mathrm{Zr} \\
197 \mathrm{Au} \\
208 \mathrm{~Pb} \\
208 \mathrm{~Pb} \\
232 \mathrm{Th}\end{array}$ & $\begin{array}{c}d \\
d \\
d \\
e_{L} \\
d \\
\sigma_{L} \\
d\end{array}$ & $\begin{array}{l}8 \cdot 0+1 \\
8 \cdot 0+1 \\
8 \cdot 0+1 \\
7 \cdot 5+1 \\
7 \cdot 0+1 \\
7 \cdot 5+1 \\
7 \cdot 0+1\end{array}$ & $\begin{array}{l}9.5+1 \\
9.5+1\end{array}$ & $\begin{array}{l}\text { MRY } \\
\text { MRY } \\
\text { MRY } \\
\text { INU } \\
\text { MRY } \\
\text { INU } \\
\text { MRY }\end{array}$ & $\begin{array}{l}\text { E Wut } \\
\text { E Wut } \\
\text { E Wut } \\
\text { E Vigdort } \\
\text { E Wut } \\
\text { E Vigdort } \\
\text { E Wut }\end{array}$ & $\begin{array}{l}5397 \\
5473 \\
5587 \\
9330 \\
5862 \\
9366 \\
5901\end{array}$ \\
\hline${ }^{\prime} H$ & $\theta=k i a \mid$ & $\sigma(E) \times f a c$ & ctor & & & \\
\hline${ }^{1} H$ & $p$ & $0.0+0$ & $2.0+1$ & MUN & T Albrechit & 17 \\
\hline
\end{tabular}




\begin{tabular}{lcccc}
\hline Target Inc & $\begin{array}{c}\text { Emin } \\
\text { (MeV) }\end{array}$ Emax \\
(MeV)
\end{tabular}

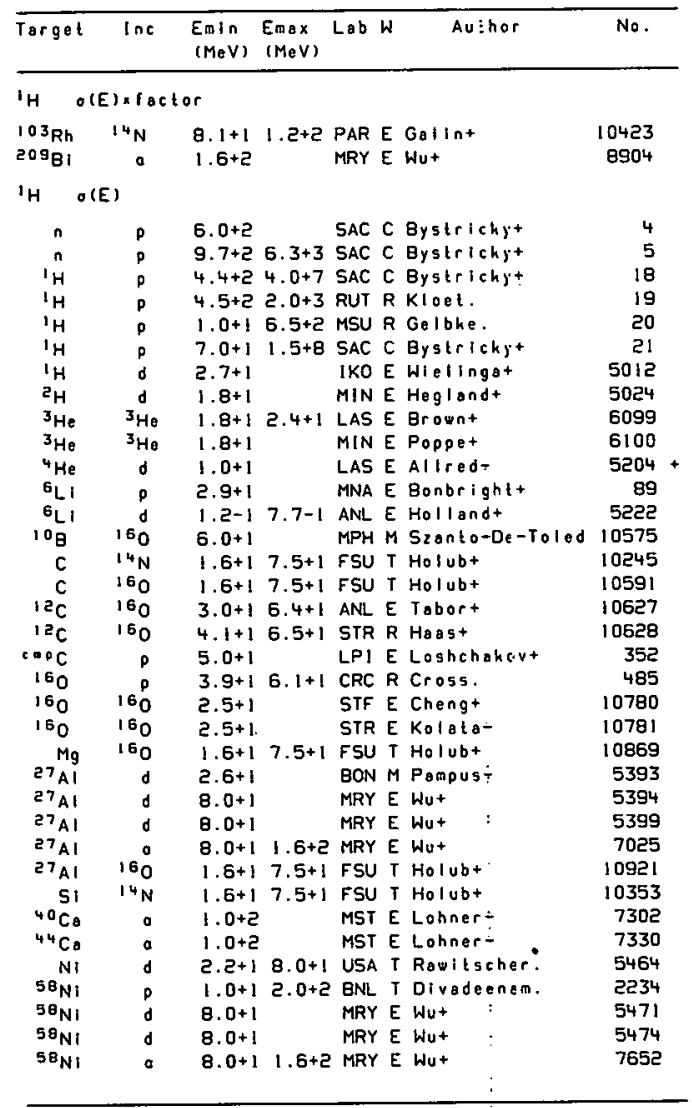




\begin{tabular}{|c|c|c|c|c|c|c|}
\hline Target & $\ln c$ & $\begin{array}{l}\text { Emin } \\
(\mathrm{MeV})\end{array}$ & $\begin{array}{l}\text { Emax } \\
(\operatorname{MeV})\end{array}$ & Lab & Author & No. \\
\hline \multicolumn{7}{|c|}{ 'H $H(E)$} \\
\hline $62^{N i}$ & d & $2.6+1$ & & BON $\mathrm{r}$ & $M$ Pampust & 5496 \\
\hline${ }^{64} \mathrm{Zn}$ & $p$ & $1.3+1$ & & PUR E & E Lux. & 2748 \\
\hline${ }^{66} \mathrm{Zn}$ & p & $1.3+1$ & & PUR E & E Lux. & 2782 \\
\hline $68 \mathrm{Zn}$ & p & $1.3+1$ & & PUR E & E Lux. & 2815 \\
\hline $70 \mathrm{Zn}$ & & $1.3+1$ & & PUR E & E Lux. & 2861 \\
\hline Se & $40^{\circ} \mathrm{Ar}$ & $2.0+5$ & & PAR E & E Golint & 12162 \\
\hline 7750 & $40_{\mathrm{Ar}}$ & $1.5+2$ & $2.0+2$ & PAR & E Golint & 12173 \\
\hline $90 \mathrm{Zr}$ & d & $8.0+1$ & & MRY E & E Wut & 5585 \\
\hline $90 \mathrm{Zr}$ & 9 & $8 \cdot 0+1$ & & MRY & E Wut & 5588 \\
\hline${ }^{90} \mathrm{Zr}$ & a & $8.0+1$ & $1.6+2$ & MRY E & E Wut & 8052 \\
\hline $103 \mathrm{Rh}$ & $14 \mathrm{~N}$ & $8.1+1$ & $1.5+5$ & PAR E & E Golint & 10422 \\
\hline $118 \mathrm{sn}$ & ${ }^{40} \mathrm{Ar}$ & $2.2+2$ & $3.4+2$ & BRK E & E Delagranget & 12232 \\
\hline $119 \mathrm{Sn}$ & d & $2.6+1$ & & BON $\mathrm{N}$ & M Pampus & 5716 \\
\hline $1545 \mathrm{~m}$ & ${ }^{40} \mathrm{Ar}$ & $2.2+2$ & $3.4+2$ & BRK E & E Delagranget & 12335 \\
\hline $164 D_{y}$ & $40 \mathrm{Ar}$ & $2.2+2$ & $3.4+2$ & BRK E & E Delagranget & 12342 \\
\hline $181 \mathrm{Tg}$ & d & $2.6+1$ & & BON $\mathrm{T}$ & M Pampust & 5788 \\
\hline $181 \mathrm{To}$ & ${ }^{6} \mathrm{LI}$ & $7.5+1$ & $9.5+1$ & INU $\mathrm{r}$ & M Korwooshlt & 9277 \\
\hline $194 \mathrm{Pt}$ & $\mathrm{G}_{\mathrm{LI}}$ & $7.5+1$ & $9.5+1$ & INU & M Karwouskit & 9311 \\
\hline $19 \theta_{\mathrm{Pt}}$ & $\sigma_{\mathrm{Li}}$ & $7.5+1$ & $9.5+1$ & INU $\mathrm{r}$ & M Karwowshit & 9317 \\
\hline $197 \mathrm{Au}$ & ${ }^{6} L_{1}$ & $7.5+1$ & $9.5+1$ & INU $\mathrm{r}$ & M Korwoosk 1+ & 9331 \\
\hline${ }^{197} \mathrm{Au}$ & ${ }^{40} \mathrm{Ar}$ & $2.2+2$ & $3.4+2$ & BRK E & E Delagranget & 12364 \\
\hline $208 \mathrm{~Pb}$ & d & $7-0+1$ & & MRY E & E Wut & 5860 \\
\hline $20{ }^{2} \mathrm{~Pb}$ & d & $7.0+1$ & & MRY E & E Wut & 5863 \\
\hline $200 \mathrm{~Pb}$ & ${ }^{6} \mathrm{LI}$ & $7.5+1$ & $9.5+1$ & INU & M Korwooshit & 9367 \\
\hline $2098 i$ & $a$ & $1.6+2$ & & MRY E & E Wut & 8903 \\
\hline $232 \mathrm{Th}$ & d & $7.0+1$ & & MRY & E Hot & 5899 \\
\hline $23 \mathrm{Thh}^{2}$ & d & $7.0+1$ & & MRY & E Wu+ & 5902 \\
\hline Mony & $14 \mathrm{~N}$ & $1.6+1$ & $7.5+1$ & FSU & I Holub+ & 10523 \\
\hline Many & 160 & $1.6+1$ & $7.5+1$ & FSU & T Holubt & 11570 \\
\hline \multicolumn{7}{|c|}{${ }^{2} H \quad$ compound-nucleus ea 1} \\
\hline${ }^{27} \mathrm{Al}$ & d & $8 \cdot 0+1$ & & MRY E & E Wut & 5402 \\
\hline${ }^{58} \mathrm{Ni}$ & g & 8. $0+1$ & & MRY E & E Wut & 5477 \\
\hline${ }^{90} \mathrm{zr}$ & d & $8.0+1$ & & MRY E & E Wut & 5591 \\
\hline $20 \theta^{2} \mathrm{~Pb}$ & d & $7.0+1$ & & MRY E & E Wut & 5866 \\
\hline $232 \mathrm{Th}$ & d & $7 \cdot 0+1$ & & MRY E & E Wut & 5905 \\
\hline \multicolumn{7}{|c|}{$e_{H}$ portlol onlsglon $O(E)$} \\
\hline $\begin{array}{l}27 \mathrm{Al}^{2} \\
{ }^{5 \theta_{\mathrm{NI}}}\end{array}$ & $\begin{array}{l}\text { d } \\
d\end{array}$ & $\begin{array}{l}8 \cdot 0+1 \\
8 \cdot 0+1\end{array}$ & & $\begin{array}{l}\text { MRY } \\
\text { MRY }\end{array}$ & $\begin{array}{l}\text { E Wut } \\
\text { E Wut }\end{array}$ & $\begin{array}{l}5401 \\
5476\end{array}$ \\
\hline
\end{tabular}

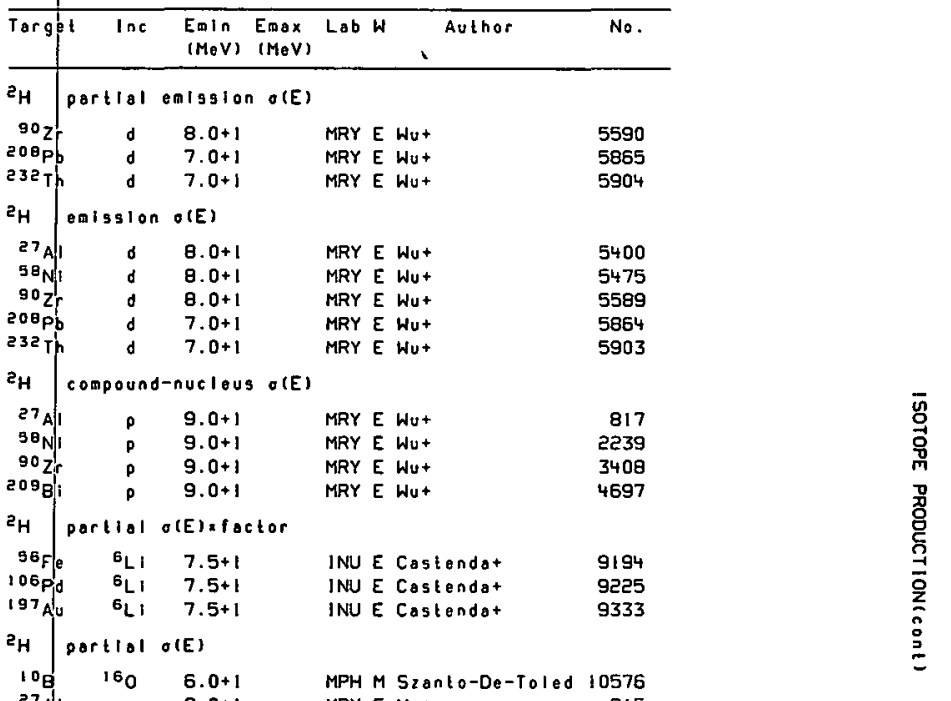




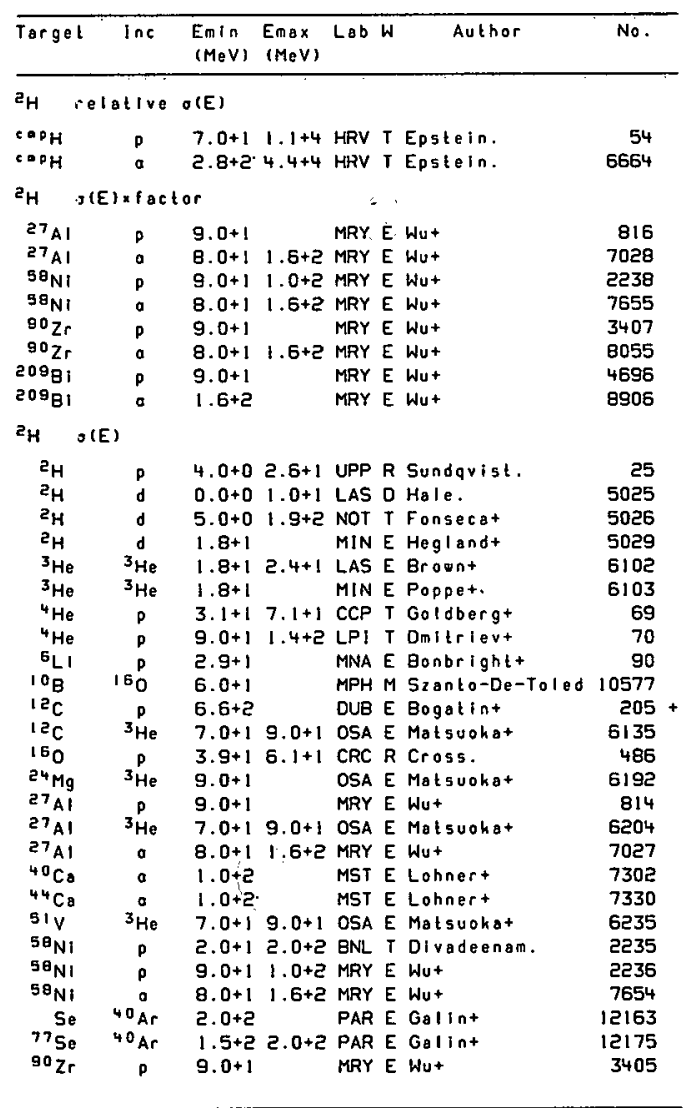

\begin{tabular}{|c|c|c|c|c|c|c|}
\hline Target & $\ln s$ & $\begin{array}{l}\text { Emin } \\
(M e V)\end{array}$ & $\begin{array}{l}\text { Emax } \\
(M e V)\end{array}$ & Lab & $A u=h e r$ & No. \\
\hline \multicolumn{7}{|c|}{${ }^{2} H \quad O(E)$} \\
\hline${ }^{90} \mathrm{Zr}$ & ${ }^{3} \mathrm{He}$ & $7.0+1$ & $9.0+1$ & OSA & E Matsuohat & 6422 \\
\hline $90 \mathrm{Zr}$ & & a. $0+1$ & $1.6+2$ & MRY & E Wut & 8054 \\
\hline $103 \mathrm{Rh}$ & 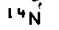 & $8.1+1$ & $1.2+2$ & PAR & E Galint & 10424 \\
\hline $1185 n$ & ${ }^{3} \mathrm{He}$ & $7.0+1$ & $9.0+1$ & OSA & E Matsuohat & 6449 \\
\hline $159 \mathrm{~Tb}$ & ${ }^{3} \mathrm{He}$ & $7.0+1$ & $9.0+1$ & OSA & E Matsuokat & 6490 \\
\hline $181 \mathrm{Ta}$ & $3_{\mathrm{He}}$ & $9.0+1$ & & OSA & E Matsuokat & 6506 \\
\hline $197 \mathrm{Au}$ & $3_{\mathrm{He}}$ & $9.0+1$ & & OSA & E Matsuokat & 6521 \\
\hline $209_{B 1}$ & & $9.0+1$ & & MRY & E Wu+ & 4694 \\
\hline 20981 & ${ }^{3} \mathrm{He}$ & $7.0+1$ & $9.0+1$ & OSA & E Matsuokat & 6571 \\
\hline $2098 i$ & a & $1.6+2$ & & MRY & E Wut & 8905 \\
\hline
\end{tabular}

${ }^{3} \mathrm{H}$ compound-nucleus o(E)

\begin{tabular}{|c|c|c|c|c|c|}
\hline $27 \mathrm{Al}$ & p & $9.0+1$ & MRY E & Wut & 822 \\
\hline $27 \mathrm{Al}$ & d & $8.0+1$ & MRY E & Wut & 5405 \\
\hline${ }^{59} \mathrm{NI}$ & 0 & $9.0+1$ & MRY E & Wut & 2244 \\
\hline${ }^{58} \mathrm{Ni}$ & d & $8.0+1$ & MRY E & Hut & 5480 \\
\hline${ }^{90} \mathrm{Zr}$ & $D$ & $9.0+1$ & MFY E & Wut & 3412 \\
\hline $90 \mathrm{Zr}$ & $\delta$ & $8.0+1$ & MRY E & Wut & 5594 \\
\hline $200 \mathrm{pb}$ & d & $7.0+1$ & MRY E & Wut & 5869 \\
\hline $200^{B i}$ & p & $9.0+1$ & MRY E & Wut & 4701 \\
\hline $232 \mathrm{Th}$ & d & $7.0+1$ & MRY E & Wut & 5908 \\
\hline \multicolumn{6}{|c|}{${ }^{3} \mathrm{H}$ partlal o(E) afactor } \\
\hline $\begin{array}{r}56 \mathrm{Fe} \\
106 \mathrm{Pd}\end{array}$ & 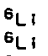 & $\begin{array}{l}7.5+1 \\
7.5+1\end{array}$ & $\begin{array}{l}\text { INU E } \\
\text { INU E }\end{array}$ & $\begin{array}{l}\text { Castendat } \\
\text { Castendat }\end{array}$ & $\begin{array}{l}9196 \\
9227\end{array}$ \\
\hline $199 \mathrm{AU}$ & $\sigma_{L i}$ & $7.5+1$ & INU E & Castendat & 9335 \\
\hline \multicolumn{6}{|c|}{${ }^{3} H$ partial of(E) } \\
\hline $27 \mathrm{AI}$ & p & $9.0+1$ & MRY E & Wut & 820 \\
\hline $27 \mathrm{Al}$ & d & $8.0+1$ & MRY E & Wut & 5405 \\
\hline${ }^{56} \mathrm{Fe}$ & ${ }^{6} L_{1}$ & $7.5+1$ & INUE & Castendat & 9195 \\
\hline${ }^{58} \mathrm{Ni}$ & p & $9.0+1$ & MRY E & Wut & 2242 \\
\hline${ }^{5} \theta_{\mathrm{NI}}$ & d & $8.0+1$ & MRY E & Wut & 5479 \\
\hline${ }^{90} \mathrm{Zr}$ & p & $9.0+1$ & MRY E & Wu+ & 3410 \\
\hline${ }^{90} \mathrm{Zr}$ & g & $8.0+1$ & MRY E & $\mathrm{Wu}^{+}$ & 5593 \\
\hline${ }^{106} \mathrm{Pd}$ & ${ }^{6} \mathrm{Li}$ & $7.5+1$ & INU E & Castense + & 9228 \\
\hline $197 \mathrm{Au}$ & ${ }^{6} L i$ & $7.5+1$ & INU E & Castendat & 9334 \\
\hline $200 \mathrm{~Pb}$ & 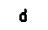 & $7.0+1$ & MRY E & Wut & 5868 \\
\hline $20{ }^{\circ} \mathrm{BI}$ & p & $9.0+1$ & MRY E & Wut & 4699 \\
\hline $232 \mathrm{Th}$ & 9 & $7.0+1$ & MRY E & Wut & 5907 \\
\hline
\end{tabular}




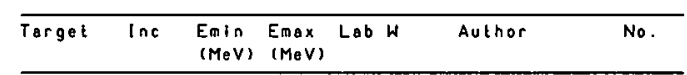

${ }^{3} H \quad \sigma(E ; \theta)_{d}$

$3_{H}$ d $9.6-13.2+0$ MIN E Strattont $5110+$

${ }^{3} \mathrm{H} d$ LAS E Allred+ $1.0+1$ 5:11.

${ }^{3} H \quad O(E ; \theta)$,

$\Sigma_{H}$ d 2.0+0 6.2+0 KTYE Schulte+ $5051+$

$3_{H} O(E ; \theta)^{\circ} \times$ foctor

$2_{H}$ d 3.9-2 8.7-2 OXF E Bootht $5056+$

${ }^{3} H \quad O(E ; \theta)$,

${ }^{2} H$ o 3.9-2 8.7-2 OXF E Bootht 5052

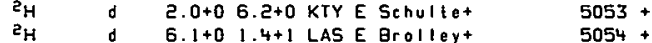

${ }^{3} H \quad O(E ; \theta)_{n}$

$3^{3} \mathrm{H} d \quad 2.3+03.7+0$ LAS E Henkel+ $5112+$

官 $\quad 3^{3} H \quad O(E ; \theta) \times k^{2}$

${ }^{3} \mathrm{H}$ o 9.6-1 3.2+0 MIN E Strattont $5109+$

${ }^{3} H \quad O(E ; \theta)$ cosine coefficlent exponsion

$3_{H}$ d $1.0+1$ LAS E Allred+

$5108+$

${ }^{3} H \quad O(E ; \theta)$ cosine coefficlent expansionxo(E:0*)

$2_{H}$ d 5.2-2 8.7-2 OXF E Bootht 5050 +

${ }^{3} H \quad \sigma(E ; \theta)$ Legendre coefficient expansion

${ }^{2} H$ d $2.0+05.2+0$ KTY E Schultet $5048+$

$2_{H} \mathrm{~d} 6.1+01.4+1$ LAS E Brolley+ $5049+$

${ }^{3} H \quad O(E ; \theta) \times$ factor

$2_{H}$ d 1.3-2 1.1-1 LAS E Arnoldt 5046 +

${ }^{2_{H}}$ d 3.9-2 8.7-2 OXF E Bootht 5047 +

$3_{H} \quad \sigma(E ; \theta)$

$2_{H}$ d 3.9-2 8.7-2 OXF E Bootht 5055

$3_{\mathrm{H}} \mathrm{i} 1.8+01.9+0$ LAS E Allent $6021+$

${ }^{3} \mathrm{H}$ produce yleld

Mg a $2.4+1 \quad$ KFK C Muenzelt 6971

\begin{tabular}{|c|c|c|c|c|c|c|c|}
\hline Target & $\ln c$ & $\begin{array}{l}\text { Emin } \\
(\mathrm{MeV})\end{array}$ & $\begin{array}{l}\text { Emax } \\
\text { (MoV) }\end{array}$ & Lab & $w$ & Author & No. \\
\hline H & roduct & yleid & & & & & \\
\hline & a & $4.8+1$ & & BRK & $\varepsilon$ & Gonzalez-Vidalt & 6972 \\
\hline $27 \mathrm{Al}$ & $\mathbf{p}$ & & $2.5+1$ & KFK & & Merkelt & 825 \\
\hline $27 \mathrm{Al}$ & p & $3.2+1$ & & BRK & E & Gonzalez-V & 826 \\
\hline $27 \mathrm{Al}$ & & $3.2+1$ & & KFK & c & Muenzel+ & 827 \\
\hline $27 \mathrm{~A} / \mathrm{I}$ & d & $1.2+1$ & $5.1+1$ & KFK & E & Merkel+ & 5409 \\
\hline $27 \mathrm{Al}$ & D & $2.4+1$ & & BRK & $\mathrm{E}$ & Gonzalez-vidalt & 5410 \\
\hline $27 \mathrm{All}$ & d & $2.4+1$ & & KFK & c & Muenzelt & 5411 \\
\hline $27 \mathrm{Al}$ & 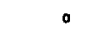 & $2.0+1$ & $4.8+1$ & KFK & E & Merkelt & 7032 \\
\hline $27 \mathrm{~A}$ & @ & $4.8+1$ & & BRK & E & Gonzalez-Vidalt & 7033 \\
\hline 27Ali & a & $4.8+1$ & & KFK & c & Muenzel+ & 7034 \\
\hline $\mathrm{T}$ & a & $4.8+1$ & & BRK & E & Gonzalez-VI & 7370 \\
\hline & 0 & $4.8+1$ & & KFK & c & Muenzel+ & 7371 \\
\hline$v$ & $p$ & TR & $2.5+1$ & KFK & E & Merkel+ & 1524 \\
\hline $51 \mathrm{v}$ & d & $1.2+1$ & $5.0+1$ & KFK & E & Merkel+ & 5448 \\
\hline $51 \mathrm{v}$ & - & $2.0+1$ & $5.0+1$ & KFK & E & Merkel+ & 7431 \\
\hline $\mathbf{F}$ & व & $4.8+1$ & & BRK & E & Gonzalez-vidalt & 7535 \\
\hline & a & $4.8+1$ & & KEK & c & Muenze $1+$ & 7536 \\
\hline${ }^{59} \mathrm{co}$ & 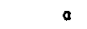 & $4.8+1$ & & BRK & E & Gonzalez-Vidalt & 7605 \\
\hline${ }^{59} \mathrm{co}$ & & $4.8+1$ & & KFK & c & Muenze1+ & 7606 \\
\hline$N /$ & & $4.8+1$ & & BRK & E & Gonzalez-vidalt & 7633 \\
\hline $\mathrm{Ni}$ & a & $4.8+1$ & & KFK & c & Muenzel+ & 7634 \\
\hline cu & 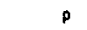 & $3.2+1$ & & BRK & $E$ & Gonzalez-VIdale & 2461 \\
\hline ch & p & $3.2+1$ & & KFK & c & Muenzel+ & 2462 \\
\hline ch & d & $2.4+1$ & & BRK & E & Gonzalez-vidalt & 5506 \\
\hline ch & & $2.4+1$ & & KFK & c & Muenzel+ & 5507 \\
\hline cu & & $4.8+1$ & & BRK & E & Gonzolez-Vidalt & 7742 \\
\hline ch & a & $4.8+1$ & & KFK & c & Muenzel+ & 7743 \\
\hline$z h$ & a & $3.2+1$ & & BRK & E & Gonzalez-vic & 2732 \\
\hline $2 h$ & 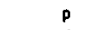 & $3.2+1$ & & KFK & c & Muenzel+ & 2733 \\
\hline $2 n$ & $d$ & $2.4+1$ & & BRK & E & Gonzalez-VI & 5526 \\
\hline$z h$ & 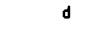 & $2.4+1$ & & KFK & c & Muenzelt & 5527 \\
\hline$z h$ & & $4.8+1$ & & BRK & E & Gonzolez-Vidslt & 7792 \\
\hline $2 h$ & - & $4.8+1$ & & KFK & c & Muenzelt & 7793 \\
\hline${ }^{3} \mathrm{Nb}$ & D & TR & $2.5+1$ & KFK & E & Merkel+ & 3510 \\
\hline${ }^{93} \mathrm{Nb}$ & d & $1.2+1$ & $5.1+1$ & KFK & E & Merkelt & 5607 \\
\hline $93 \mathrm{Nb}$ & a & $2.0+1$ & $5.0+1$ & KFK & & Merkelt & 8085 \\
\hline Ab & $\mathbf{p}$ & $3.2+1$ & & BRK & ( & Gonzalez-vidalt & 3680 \\
\hline$A g$ & 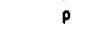 & $3.2+1$ & & KFK & & Muenzel+ & 3681 \\
\hline$A_{9}^{\prime}$ & d & $2.4+1$ & & BRK & ( & Gonzalez-vi & 5649 \\
\hline$A_{B}^{\prime}$ & d & $2.4+1$ & & UJV & & Muenzel+ & 5650 \\
\hline
\end{tabular}




\begin{tabular}{|c|c|c|c|c|c|c|c|}
\hline Target & $\ln c$ & $\begin{array}{l}\text { Enin } \\
(\mathrm{MeV})\end{array}$ & $\begin{array}{l}\text { Emax } \\
(\operatorname{Me} v)\end{array}$ & Lab & $w$ & Author & No. \\
\hline${ }^{3} \mathrm{H}$ & product & yleld & & & & & \\
\hline $\mathrm{Ag}$ & a & $4,8+1$ & & BRK & & Gonzelez-VIdalt+ & 8231 \\
\hline$A g$ & a & $4.8+1$ & & KFK & & Muenzelt & 8232 \\
\hline Co & $\mathrm{p}$ & $3.2+1$ & & BRK & & Gonzalez-Vidal+ & 3729 \\
\hline Cd & $p$ & $3.2+1$ & & KFK & & Muenzel+ & 3730 \\
\hline $\mathrm{Cd}$ & d & $2.4+1$ & & BRK & EC C & Gonzalez-Vidalt & 5659 \\
\hline $\mathrm{Cd}$ & d & $2.4+1$ & & KFK & & Muenzelt & 5660 \\
\hline Cd & a & $4.8+1$ & & BRK & E & Gonzalez-Vidalt & 8252 \\
\hline Cd & a & $4.8+1$ & & KFK & $\mathrm{Cr}$ & Muenzelt & 8253 \\
\hline $\operatorname{In}$ & $a$ & $4.8+1$ & & BRK & E C & Gonzalez-Vidalt+ & 8290 \\
\hline $\ln$ & a & $4.8+1$ & & KFK & $\mathrm{cr}-\mathrm{r}-\mathrm{r}$ & Muenzelt & 8291 \\
\hline sn & d & $2.4+1$ & & BRK & EC & Gonzolez-Vidolt & 5710 \\
\hline$S_{n}$ & d & $2.4+1$ & & KFK & cr & Muenzelt & 5711 \\
\hline$S_{n}$ & a & $4.8+1$ & & BRK & EC & Gonzalez-Vidalt & 8302 \\
\hline Sn & o & $4.8+1$ & & KFK & $\mathrm{cr}-\mathrm{r}-\mathrm{r}$ & Muenzelt & 8303 \\
\hline Sb & a & $4 . \theta+1$ & & BRK & $E$ & Gonzalez-Vidalt & 8327 \\
\hline Sb & a & $4 \cdot 8+1$ & & KFK & c 1 & Muenzeft & 8328 \\
\hline $\mathbf{P i}$ & a & $4.8+1$ & & BRK & E & Gonzalez-vidolt & 8684 \\
\hline & a & $4.8+1$ & & KFK & C 1 & Muenzel+ & 8685 \\
\hline $197 \mathrm{Au}$ & p & TR & $2.5+1$ & KFK & E & Merkelt & 4462 \\
\hline $197 \mathrm{Au}$ & p & $3.2+1$ & & BRK & E & Gonzalez-vidalt & 4463 \\
\hline $197 \mathrm{Au}$ & p & $3.2+1$ & & KFK & cr & Muenzelt & 4464 \\
\hline $197 \mathrm{Au}$ & d & $1.5+1$ & $5.1+1$ & KFK & E & Merkelt & 5821 \\
\hline $197 \mathrm{Au}$ & d & $2.4+1$ & & BRK & $E$ & Gonzalez-VIdalt & 5822 \\
\hline $197 \mathrm{Au}$ & d & $2.4+1$ & & KFK & C 1 & Muenzelt & 5823 \\
\hline $197 \mathrm{AU}$ & 0 & $2 \cdot 0+1$ & $5.0+1$ & KFK & E & Merkelt & 8712 \\
\hline $197 \mathrm{Au}$ & 0 & $4.8+1$ & & BRK & E & Gonzalez-vidale & 8713 \\
\hline $197 \mathrm{Au}$ & 0 & $4.8+1$ & & KFK & C 1 & Muenzel+ & 8714 \\
\hline $\mathrm{Pb}$ & p & $3 \cdot 2+1$ & & BRK & $E$ & Gonzalez-vidalt & 4612 \\
\hline $\mathrm{Pb}$ & o & $3.2+1$ & & KFK & cr & Muenzelt+ & 4613 \\
\hline Pb & d & $2.4+1$ & & BRK & E & Gonzalez-VIdalt & 5854 \\
\hline $\mathrm{Pb}$ & $d$ & $2.4+1$ & & KFK & c & Muenzelt & 5855 \\
\hline $\mathrm{Pb}$ & $a$ & $4.8+1$ & & BRK & $E$ & Gonzalez-vidalt & 8847 \\
\hline $\mathrm{Pb}$ & 0 & $4.8+1$ & & KFK & C & Muenzelt & 8848 \\
\hline $232 \mathrm{Th}$ & p & $3.2+1$ & & BRK & $E$ & Gonzalez-Vioalt & 4781 \\
\hline $232 \mathrm{Th}$ & p & $3.2+1$ & & KFK & $\mathrm{C}$ & Muenzel+ & 4782 \\
\hline $232 \mathrm{Th}$ & d & $2.4+1$ & & BRK & $\mathrm{E}$ & Gonzalez-Vidalt & 5911 \\
\hline $232 \mathrm{Th}$ & d & $2.4+1$ & & KFK & $\mathrm{c}$ & Muenze1+ & 5912 \\
\hline $232 \mathrm{Th}$ & $a$ & $4.8+1$ & & BRK & $\varepsilon$ & Gonzalez-vidal+ & 8935 \\
\hline $232 \mathrm{Th}$ & 0 & $4.8+1$ & & KFK & & Muenzelt & 8936 \\
\hline 2380 & p & $3.2+1$ & & BRK & $E$ & Gonzalez-Vidalt & 4871 \\
\hline
\end{tabular}

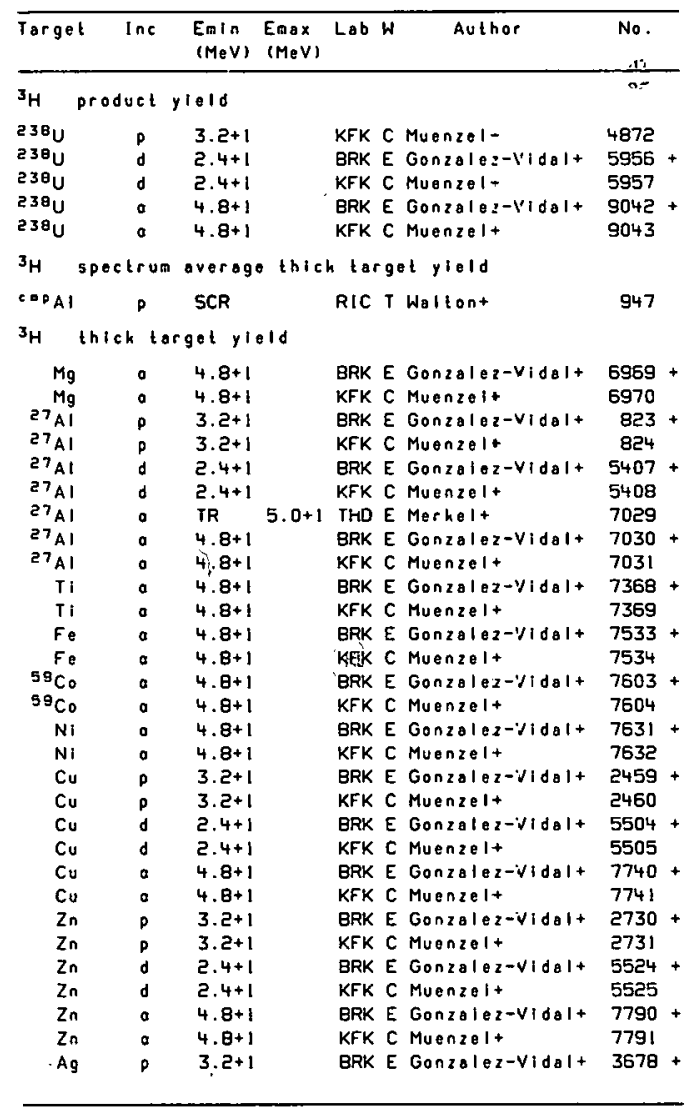




\begin{tabular}{|c|c|c|c|c|c|c|}
\hline Target & $\operatorname{lnc}$ & $\begin{array}{l}\text { Emln } \\
(M e V)\end{array}$ & $\begin{array}{l}\text { Emax } \\
(\operatorname{MeV})\end{array}$ & Lab & Author & No. \\
\hline \multicolumn{7}{|c|}{${ }^{3} \mathrm{H}$ thick target yleld } \\
\hline Ag & $p$ & $3.2+1$ & & KFK & C Muenzelt & 3679 \\
\hline $\mathrm{Ag}$ & d & $2.4+1$ & & BRK & E Gonzalez-Vidale & 5647 \\
\hline & d & $2.4+1$ & & KFK & C Muenzel+ & 5648 \\
\hline $\mathrm{Ag}$ & a & $4.8+1$ & & BRK & E Gonzalez-Vidalt & 8229 \\
\hline $\mathrm{Ag}$ & a & $4.8+1$ & & KFK & C Muenzel+ & 8230 \\
\hline$c d$ & $p$ & $3.2+1$ & & BRK & E Gonzalez-Vidalt & 3727 \\
\hline Cd & $p$ & $3.2+1$ & & KFK & C Muenzelt & 3728 \\
\hline \multirow{14}{*}{$\begin{array}{l}\mathrm{C} \\
\mathrm{C} \\
\mathrm{C} \\
\mathrm{C} \\
\mathrm{I} \\
\mathrm{I} \\
\mathrm{S} \\
\mathrm{S} \\
\mathrm{S} \\
\mathrm{S} \\
\mathrm{S} \\
\mathrm{P} \\
\mathrm{P}\end{array}$} & d & $2.4+1$ & & BRK & E Gonzalez-Vidalt & $5657+$ \\
\hline & d & $2.4+1$ & & KFK & C Muenzel+ & 5658 \\
\hline & $a$ & $4.8+1$ & & BRK & E Gonzalez-Vidalt & 8250 \\
\hline & $a$ & $4.8+1$ & & KFK & C Muenzel+ & 8251 \\
\hline & a & $4.8+1$ & & BRK & E Gonzalez-Vidalt & $8288+$ \\
\hline & $a$ & $4.8+1$ & & KFK & C Muenzelt & 8289 \\
\hline & d & $2.4+1$ & & BRK & E Gonzalez-Vidalt & 5700 \\
\hline & d & $2.4+1$ & & KFK & C Muenzelt & 5709 \\
\hline & $a$ & $4.8+1$ & & BRK & E Gonzalez-Vidalt & $8300+$ \\
\hline & $a$ & $4.8+1$ & & KFK & C Muenzelt & 8301 \\
\hline & a & $4.8+1$ & & ERK & E Gonzalez-Vidalt & $8325+$ \\
\hline & $a$ & $4.8+1$ & & KFK & C Muenzelt & 8326 \\
\hline & $a$ & $4.8+1$ & & ERK & E Gonzalez-VIdalt & $8682+$ \\
\hline & $a$ & $4.8+1$ & & KFK & C Muenzal + & 8683 \\
\hline $197 \mathrm{Au}$ & p & $3.2+1$ & & ERK & E Gonzalez-Vidalt & 4460 \\
\hline $197 \mathrm{Au}$ & $p$ & $3.2+1$ & & KFK & C Muenzel+ & 4461 \\
\hline $197 \mathrm{Au}$ & d & $2.4+1$ & & BRK & E Gonzalez-Vidalt & $5819+$ \\
\hline $197 \mathrm{Au}$ & d & $2.4+1$ & & KFK & C Muenzelt & 5820 \\
\hline $197 \mathrm{Au}$ & $a$ & $4.8+1$ & & BRK & E Gonzalez-VIdalt+ & $8710+$ \\
\hline $197 \mathrm{Au}$ & $a$ & $4.8+1$ & & KFK & C Muenzelt & 8711 \\
\hline $\mathrm{Pb}$ & p & $3.2+1$ & & BRK & E Gonzalez-VIdal+ & $4610+$ \\
\hline $\mathrm{Pb}$ & p & $3.2+1$ & & KFK & C Muenzelt & 4611 \\
\hline $\mathrm{Pb}$ & o & $2.4+1$ & & BRK & E Gonzalez-vidolt & $5852+$ \\
\hline $\mathrm{Pb}$ & $\delta$ & $2.4+1$ & & $\mathrm{KFK}$ & C Muenze1+ & 5853 \\
\hline $\mathrm{Pb}$ & $a$ & $4,8+1$ & & BRK & E Gonzalez-Vidalt & $8845+$ \\
\hline $\mathrm{Pb}$ & $a$ & $4.8+1$ & & KFK & C Muenzel+ & 8846 \\
\hline $232 \mathrm{Th}$ & $p$ & $3.2+1$ & & BRK & E Gonzalez-Vidalt & $4779+$ \\
\hline $232 \mathrm{Th}$ & p & $3.2+1$ & & KFK & C Muenzelt & 4780 \\
\hline $232 \mathrm{Th}$ & d & $2.4+1$ & & BRK & E Gonzalez-Vidalt & 5909 \\
\hline $232 \mathrm{Th}$ & d & $2.4+1$ & & KFK & C Muenzelt & 5910 \\
\hline $232 \mathrm{Th}$ & a & $4.8+1$ & & BRK & E Gonzalez-vidolt & 8933 \\
\hline $232 \mathrm{Th}$ & $a$ & $4.8+1$ & & KFK & C Muenzel+ & 8934 \\
\hline $238^{U}$ & p & $3.2+1$ & & BRK & E Gonzalez-Vidal+ & 4869 \\
\hline
\end{tabular}

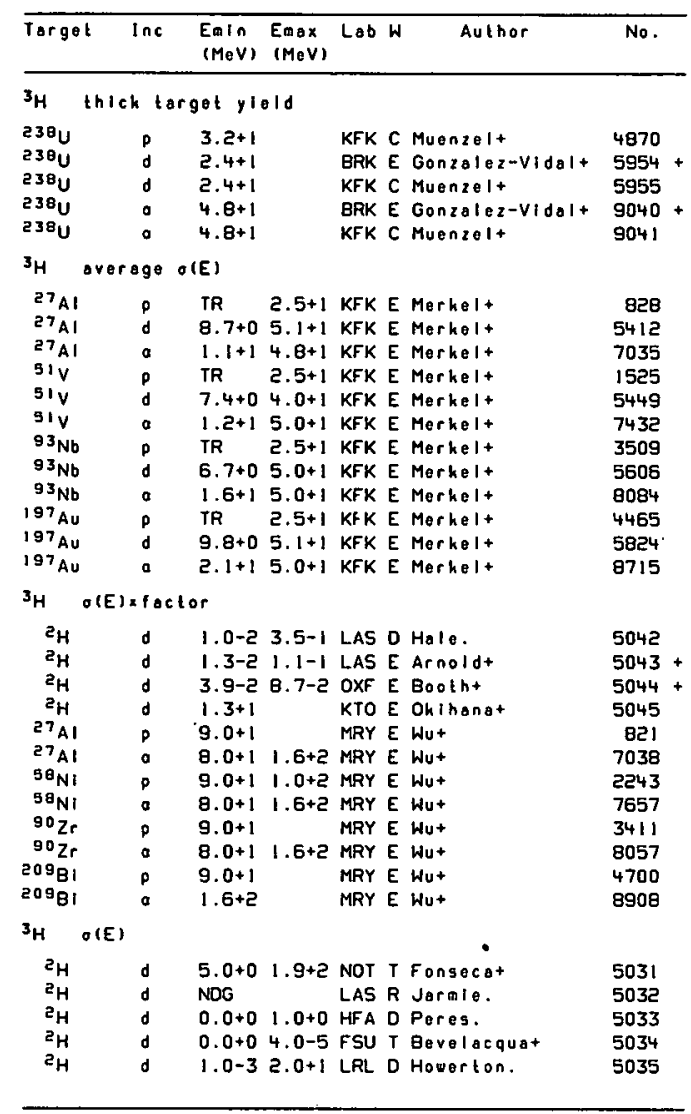




\begin{tabular}{|c|c|c|c|c|c|c|}
\hline Target & $\ln c$ & $\begin{array}{l}\text { Emin } \\
(\mathrm{MeV})\end{array}$ & $\begin{array}{l}E \max \\
(\mathrm{MeV})\end{array}$ & Lab & Author & No. \\
\hline \multicolumn{7}{|c|}{$3_{H} \quad \sigma(E)$} \\
\hline$e_{H}$ & $\sigma$ & $1.0-2$ & $5.0-1$ & LAS & D Hale. & 5036 \\
\hline$e_{\mathrm{H}}$ & $d$ & $1.3-2$ & $1.1-1$ & LAS & E Arnoldt & $5037+$ \\
\hline${ }^{2} \mathrm{H}$ & d & $5.2-2$ & 8.7-2 & OXF & E Bootht & $5038+$ \\
\hline$\varepsilon_{\mathrm{H}}$ & $d$ & $2.0-1$ & $1.0+5$ & Ui & T Miloyt & 5039 \\
\hline$e_{H}$ & d & $6.1+0$ & $1.4+1$ & LAS & E Brolleyt & $5040+$ \\
\hline$a_{\mathrm{H}}$ & 0 & $1.8+1$ & & MIN & E Heglandt & 5041 \\
\hline${ }^{3} \mathrm{H}$ & p & NDG & & LAS & O Halet & 30 \\
\hline $3^{3} \mathrm{H}$ & d & $0.0+0$ & $8.0+0$ & LAS & D Hale. & 5107 \\
\hline${ }^{3} \mathrm{H}$ & $i$ & $2.0-2$ & $2.0+0$ & LAS & $0 \mathrm{Halet}$ & 6020 \\
\hline${ }^{3} \mathrm{He}$ & ${ }^{3} \mathrm{He}$ & $1.8+1$ & $2.4+1$ & LAS & E Brownt & 6104 \\
\hline${ }^{3} \mathrm{He}$ & ${ }^{3} \mathrm{He}$ & $1 . \theta+1$ & & MIN & E Poppet & 6105 \\
\hline${ }^{4} \mathrm{He}$ & $p$ & $3.1+1$ & $7.1+1$ & $\mathrm{CCP}$ & T Goldbergt & 71 \\
\hline${ }^{4} \mathrm{He}$ & p & $9.0+1$ & $1.4+2$ & LPI & T Dolterievt & 72 \\
\hline${ }^{5} \mathrm{LI}$ & d & $1.0-1$ & $9.0-1$ & ANL & E Hollandt & 5224 \\
\hline $12 \mathrm{c}$ & p & $6.6+2$ & & DUB & E Bogatint & $206+$ \\
\hline 160 & p & $3.9+1$ & $6.1+1$ & CRC & R Cross. & 487 \\
\hline 160 & p & $1.6+2$ & & LPI & T Doltrient & 498 \\
\hline $27 \mathrm{Al}$ & p & $9.0+1$ & & MRY & E Wut & B18 \\
\hline $27 \mathrm{Al}$ & p & $2.5+3$ & & CER & E Goebelt & 819+ \\
\hline $27 \mathrm{AI}$ & d & $1.0+1$ & $5.0+1$ & KFK & E Merkel- & 5403 \\
\hline $27 \mathrm{Al}$ & g & $8.0+1$ & & MRY & E Wut & 5404 \\
\hline $27_{A 1}$ & a & $1.2+1$ & $5.0+1$ & KFK & E Merkelt+ & 7036 \\
\hline $27 \mathrm{Al}$ & $a$ & $8.0+1$ & $1.6+2$ & MRY & E Wut & 7037 \\
\hline${ }^{40} \mathrm{Ca}$ & a. & $1.0+5$ & & MST & E Lohner* & 7302 \\
\hline${ }^{44} \mathrm{Ca}$ & 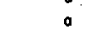 & $1.0+2$ & & MST & E Lohnert & 7330 \\
\hline $\operatorname{siv}$ & d & $1.0+1$ & $5.0+1$ & KFK & E Merkelt & 5450 \\
\hline $51 v$ & 0 & $1.5+1$ & $5.0+1$ & KFK & E Merhelt & 7433 \\
\hline $\mathrm{Fe}_{\mathrm{\theta}}$ & p & $2.5+3$ & & CER & E Goebel- & $1739+$ \\
\hline $5 \theta_{\mathrm{Ni}}$ & 8 & $1.0+1$ & $2.0+2$ & BNL & T Divadeenam. & 2240 \\
\hline${ }^{5 \theta_{\mathrm{NI}}}$ & : & $9.0+1$ & $1.0+2$ & MRY & E Wut & 2241 \\
\hline $5 \theta_{\mathrm{NI}}$ & d & $8.0+1$ & & MRY & E Wut & 5478 \\
\hline $5 \theta_{\mathrm{NI}}$ & a & $0.0+1$ & $1.6+2$ & MRY & E Wut & 7656 \\
\hline $\mathrm{Cu}$ & D & $2.5+3$ & & CER & E Goebel+ & $2463+$ \\
\hline${ }^{63} \mathrm{Cu}$ & ${ }^{3} \mathrm{He}$ & $6.0+0$ & $7.0+1$ & ANL & E Golchertt & 6294 \\
\hline${ }^{65} \mathrm{Cu}$ & ${ }^{3} \mathrm{He}$ & $6.0+0$ & $7.0+1$ & ANL & E Golehertt & 6335 \\
\hline Se & ${ }^{40} \mathrm{Ar}$ & $2.0+2$ & & PAR & E Golint & 12164 \\
\hline${ }^{77} \mathrm{se}$ & $40 \mathrm{Ar}$ & $1.5+2$ & $2.0+2$ & PAR & E Galint & 12176 \\
\hline $90 \mathrm{Zr}$ & p & $9.0+1$ & & MRY & E Wut & 3409 \\
\hline $90 \mathrm{Zr}$ & d & $8.0+1$ & & MRY & E Wut & 5592 \\
\hline $90 \mathrm{Zr}$ & 0 & $8.0+1$ & $1.6+2$ & MRY & E Wut & 1056 \\
\hline
\end{tabular}

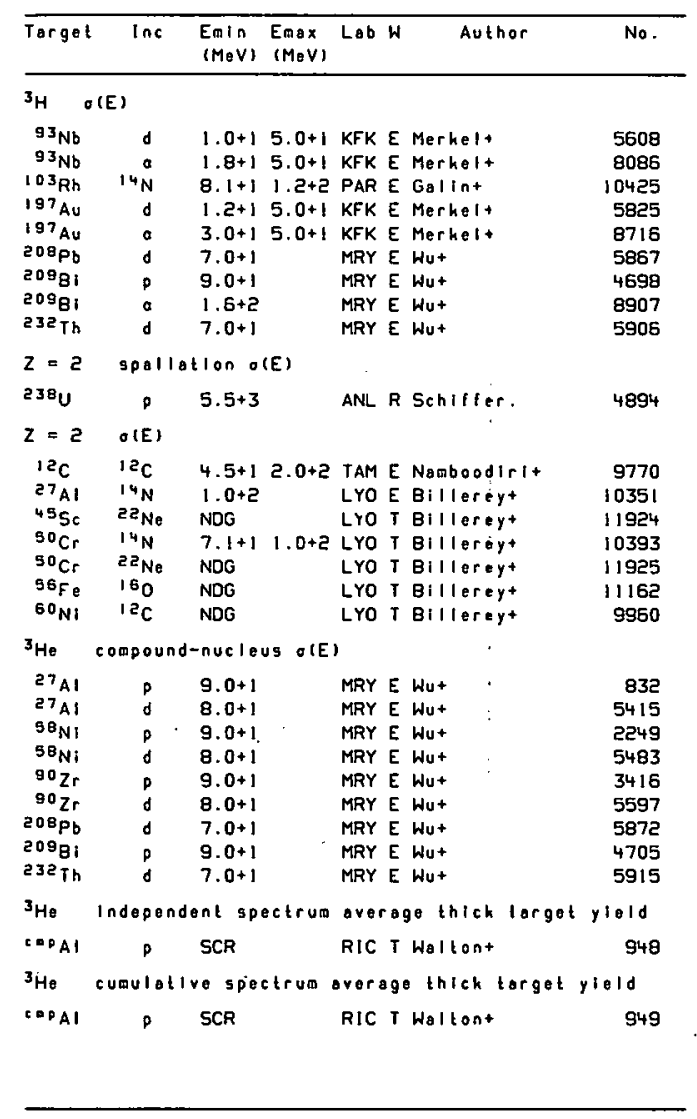




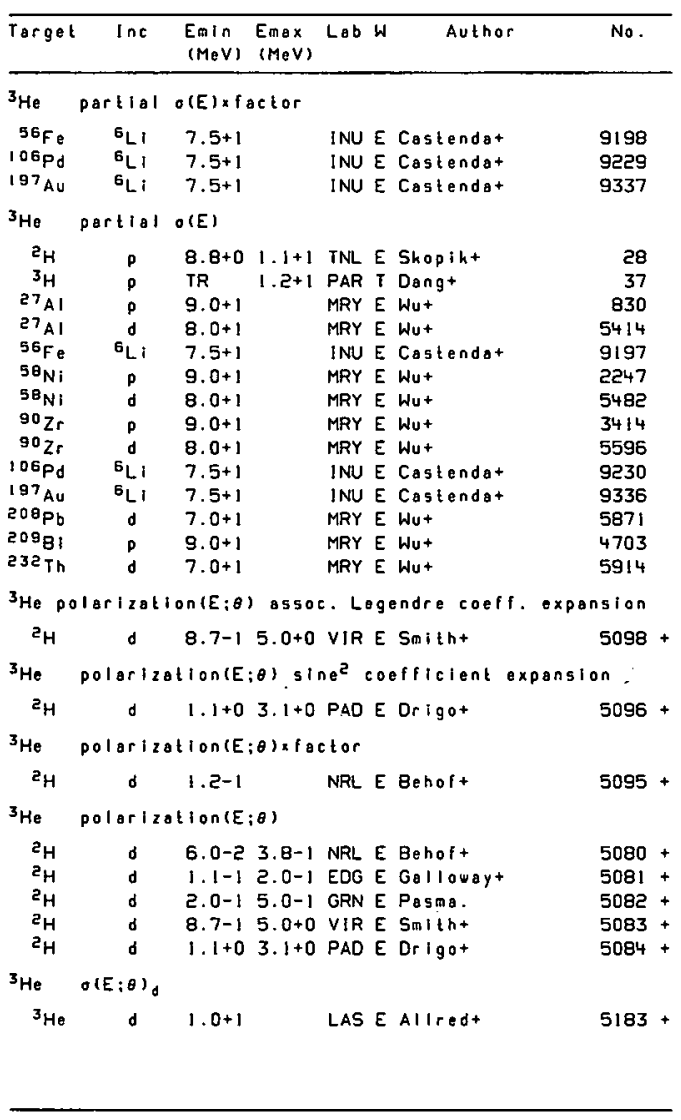

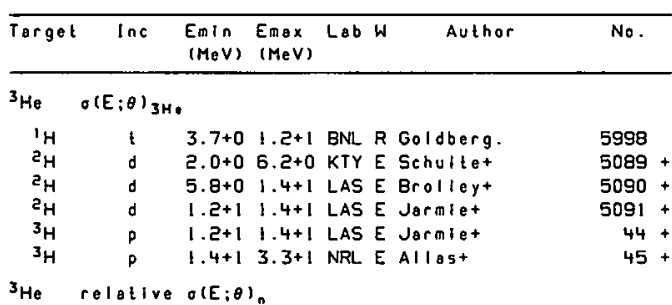

$a_{H} d 5.0+01.2+1$ LRL E Goldbergt $5099+$ ${ }^{3}$ He $\sigma(E ; \theta)_{n}$ ef actor

$a_{H}$ d 3.9-2 8.7-2 OXF E Bootht $5100+$

${ }^{3} \mathrm{He}_{\mathrm{H}} \quad O(E ; \theta)_{\mathrm{n}}$

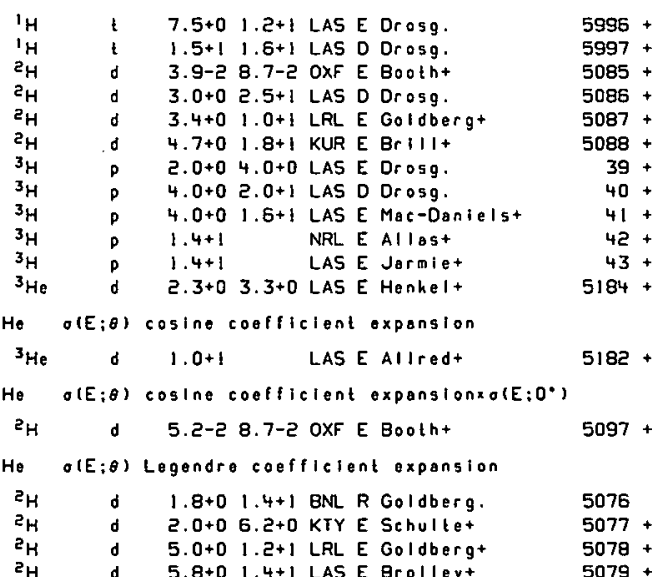




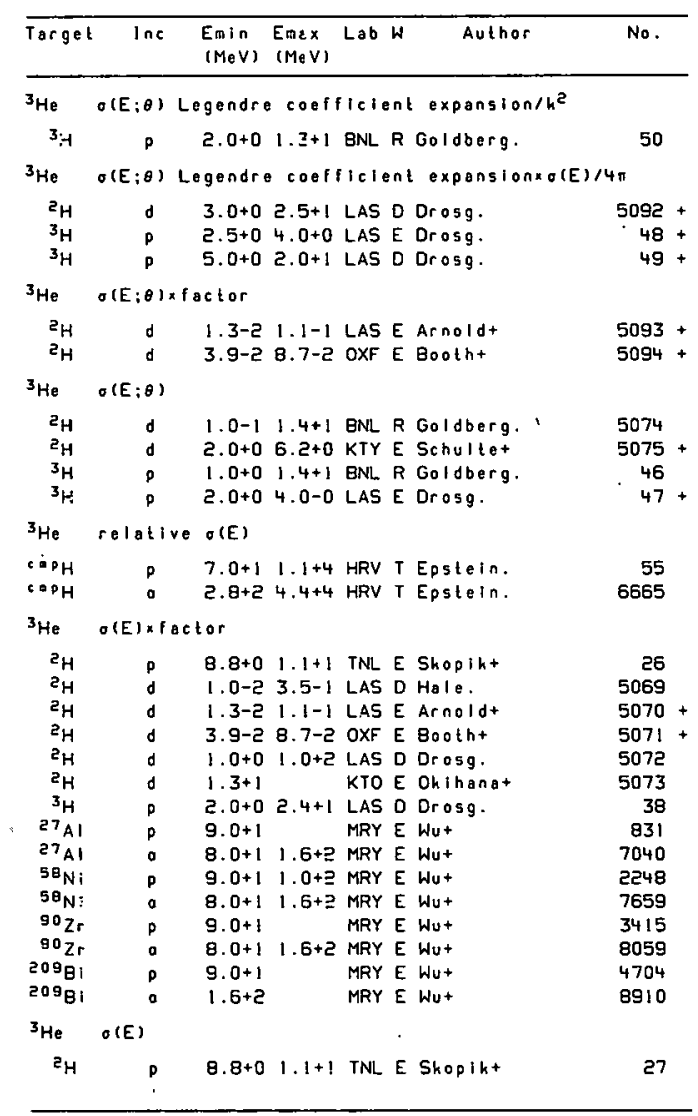

\begin{tabular}{|c|c|c|c|c|c|c|}
\hline Target & $\ln c$ & $\begin{array}{l}\text { Emin } \\
(\mathrm{MeV})\end{array}$ & $\begin{array}{l}E \max \\
(\mathrm{MeV})\end{array}$ & Lab & Author & No. \\
\hline${ }^{3} \mathrm{He}$ & $O(E)$ & & & & & \\
\hline${ }^{2} \mathrm{H}$ & d & $0.0+0$ & $1.0+0$ & HFA & D Peres. & 5057 \\
\hline $2_{H}$ & d & $1.0-3$ & $9.5+1$ & UI & T Mile,t & 5058 \\
\hline$e_{H}$ & d & i. $0-3$ & $2.0+1$ & LRL & D Howerton. & 5059 \\
\hline$e_{H}$ & d & $5 \cdot 0-3$ & $2.0+1$ & LRL. & D Howerton. & 5060 \\
\hline $2_{H}$ & d & $1.0-2$ & $5.0-1$ & LAS & D Hale. & 5051 \\
\hline$e_{\mathrm{H}}$ & d & $1.3-2$ & $1.1-1$ & LAS & E Arno.d+ & $5062+$ \\
\hline$e_{H}$ & d & $5.0-2$ & $1.2-1$ & LAS & E Jarmiet & 5063 \\
\hline $2_{H}$ & d & $5.2-2$ & $8.7-2$ & OXF & E Bootht & $5064+$ \\
\hline${ }^{2} \mathrm{H}$ & d & $2.0+0$ & $6.2+0$ & KTY & E Schultet & $5065+$ \\
\hline$a_{H}$ & d & $3.0+0$ & $2.5+1$ & LAS & D Drose. & $5056+$ \\
\hline${ }^{2} H$ & d & $5.0+0$ & $1.2+1$ & LRL & E Goldterg+ & $5067+$ \\
\hline $2_{H}$ & d & $1.8+1$ & & MIN & E Heglandt & 5068 \\
\hline${ }^{3} \mathrm{H}$ & $\mathbf{p}$ & NDG & & LAS & D Hale: & 31 \\
\hline${ }^{3} \mathrm{H}$ & p & $1.0+0$ & $1.2+1$ & BNL & R Goldberg. & 32 \\
\hline${ }^{3} \mathrm{H}$ & p & $1.0+0$ & $1.3+1$ & FSU & I Heldersont & 33 \\
\hline${ }^{3} \mathrm{H}$ & p & $1.0+0$ & $2.0+1$ & LRL & 0 Howertion. & 34 \\
\hline $3_{\mathrm{H}}$ & p & $2.5+0$ & $4.0+0$ & LAS & E Drosg. & $35+$ \\
\hline${ }^{3} \mathrm{H}$ & p & $5.0+0$ & $2.0+1$ & LAS & O Drosg. & $36+$ \\
\hline${ }^{3} \mathrm{He}$ & p & $0.0+0$ & $2.0+1$ & LAS & D Hale. & 60 \\
\hline $3_{\mathrm{He}}$ & g & $0.0+0$ & $1.0+1$ & LAS & D Hale. & 5181 \\
\hline $3_{\mathrm{He}}$ & ${ }^{3} \mathrm{He}$ & $1.8+1$ & $2.4+1$ & LAS & E Brownt & 6106 \\
\hline${ }^{4} \mathrm{He}$ & p & $3.1+1$ & $7.1+1$ & CCP & T Goldbergt & 73 \\
\hline${ }^{4} \mathrm{He}$ & p & $9.0+1$ & $1.4+2$ & LPI & T Dmitriegt & 74 \\
\hline${ }^{4} \mathrm{He}$ & p & $2.5+0$ & $7.1+1$ & CCP & T Goldbergt & 75 \\
\hline${ }^{4} \mathrm{He}$ & p & $9.0+1$ & $1.4+2$ & LPI & T Doitrieyt & 76 \\
\hline${ }^{6} \mathrm{LI}$ & g & $1.0-1$ & $9.0-1$ & ANL & E Holland- & 5225 \\
\hline $12 \mathrm{c}$ & p & $6.6+2$ & & DUB & E Bogalint & $207+$ \\
\hline 160 & p & $6.1+1$ & & CRC & R Cross. & 489 \\
\hline$M_{g}$ & p & $1.2+1$ & $4.5+1$ & RIC & E Wallont & 717 \\
\hline${ }^{27} \mathrm{Ai}$ & p & $9.0+1$ & & MRY & E Wut & 829 \\
\hline $27{ }_{A 1}$ & $d$ & $8.0+1$ & & MRY & $\mathrm{E} \mathrm{Hut}$ & 5413 \\
\hline $27 \mathrm{Al}$ & a & $8.0+1$ & $1.6+2$ & MRY & E Wu+ & 7039 \\
\hline $5 i$ & p & $1.8+1$ & $4,4+1$ & RIC & E Waltorit & 957 \\
\hline${ }^{40} \mathrm{Ca}$ & 0 & $1.0+2$ & & MST & E Lohnert & 7302 \\
\hline${ }^{44} \mathrm{Ca}$ & 0 & $1.0+2$ & & MST & E Lohnert & 7330 \\
\hline$\because P \mathrm{Ca}_{\mathrm{a}}$ & p & $1.9+1$ & $4.4+1$ & RIC & E Wallort & 1325 \\
\hline $\mathrm{Fe}_{\mathrm{\theta}}$ & $p$ & $2.5+3$ & & CER & E Goobelt & 1843 \\
\hline${ }^{58} \mathrm{NI}$ & p & $3.5+1$ & $2.0+2$ & $\mathrm{BNL}$ & T Divadeenam. & 2245 \\
\hline $5 \theta^{N i}$ & $p$ & $9.0+1$ & $1.0+5$ & MRY & E Wu+ & 2246 \\
\hline${ }^{S E} \mathrm{Ni}$ & do & $8.0+1$ & & MRY & E Wut & 5481 \\
\hline
\end{tabular}




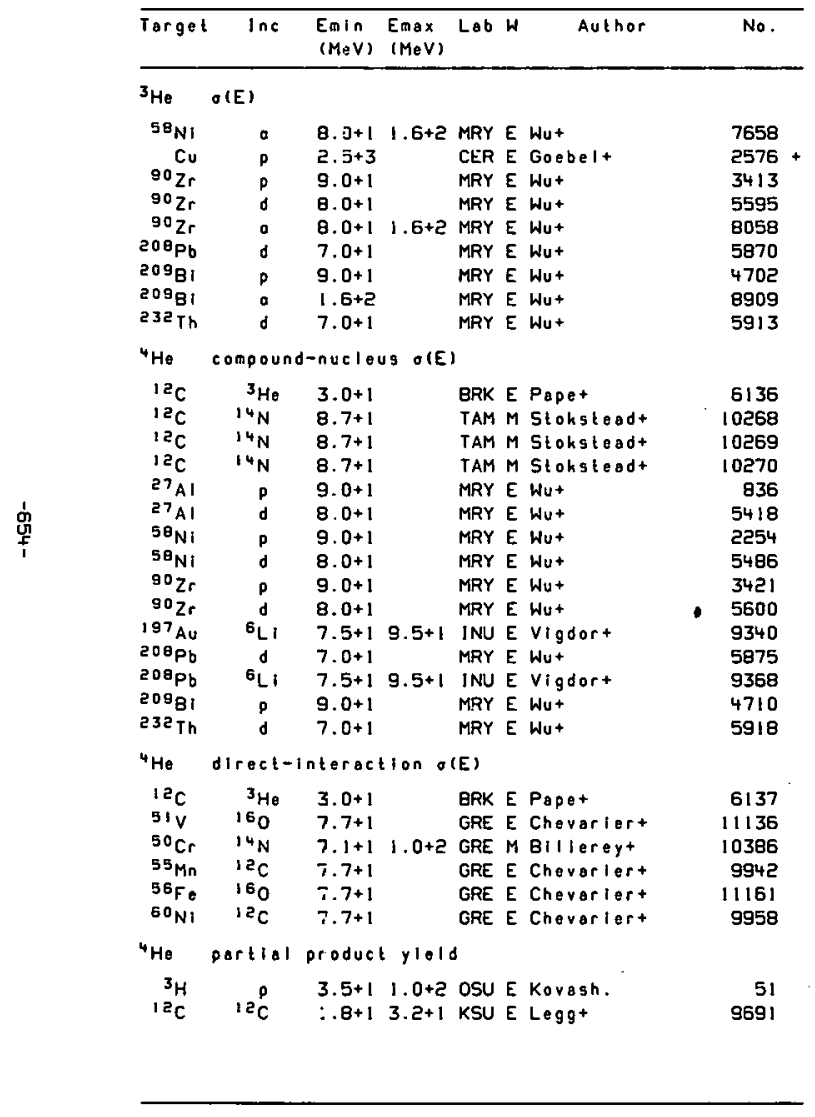

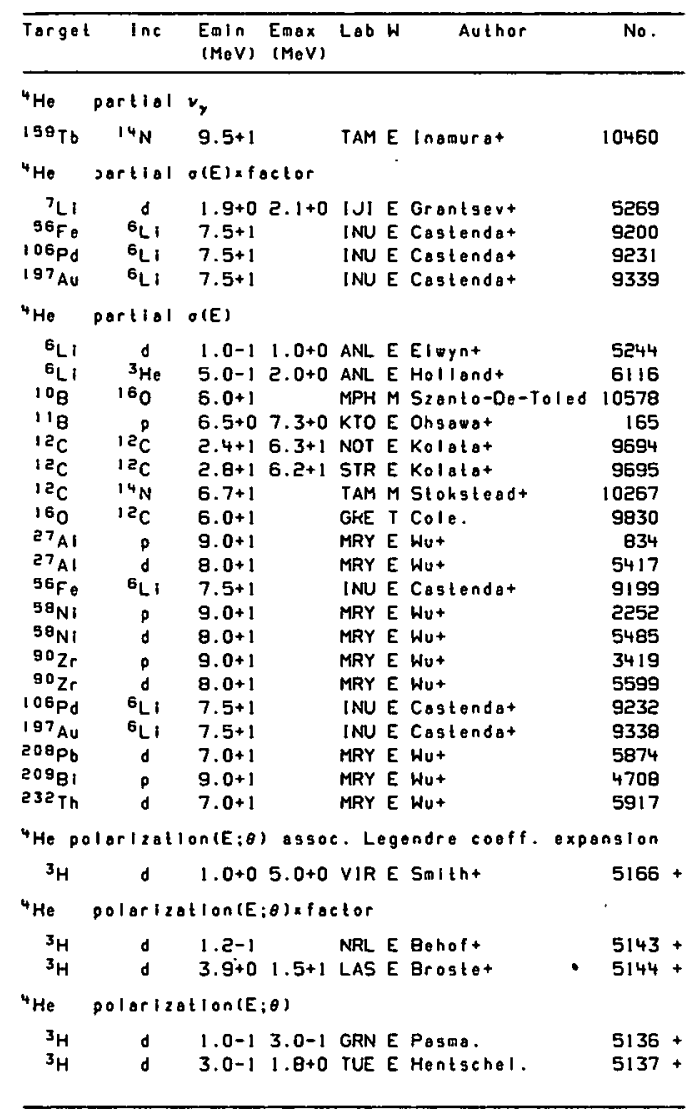




\begin{tabular}{|c|c|c|c|c|c|c|}
\hline Target & Inc & $\begin{array}{l}\text { Emin } \\
(M e V)\end{array}$ & $\begin{array}{l}\text { Emax } \\
(M e V)\end{array}$ & Lab & Author & No. \\
\hline${ }^{4} \mathrm{He}$ & polarizat & $\operatorname{tion}(E: C$ & & & & \\
\hline $\begin{array}{l}3_{H} \\
3_{H} \\
3_{H} \\
3_{H} \\
3_{H}\end{array}$ & $\begin{array}{l}\text { d } \\
d \\
d \\
d \\
d\end{array}$ & $\begin{array}{l}6.0-1 \\
1.0+0 \\
2.1+0 \\
3.9+0 \\
8.7+0\end{array}$ & $\begin{array}{l}1.2+0 \\
5 \cdot 0+0 \\
5.8+0 \\
1.5+1 \\
1.9+2\end{array}$ & $\begin{array}{l}\text { BKB } \\
\text { VIR } \\
\text { BER } \\
\text { LAS } \\
\text { CCP }\end{array}$ & $\begin{array}{l}\text { E Borelit } \\
\text { E Smitht } \\
\text { E Busset } \\
\text { E Brostet } \\
\text { E Alekseovt }\end{array}$ & $\begin{array}{l}5138+ \\
5139: \\
5140 \div \\
5141+ \\
5142+\end{array}$ \\
\hline${ }^{4} \mathrm{He}$ & $\theta(E ; \theta)_{0} \times 4$ & $4 n / 0(E)$ & & & & \\
\hline${ }_{4}^{3} \mathrm{He}$ & $\begin{array}{c}t \\
\text { relotive }\end{array}$ & $\begin{array}{l}1.0-1 \\
0(E ; \theta)\end{array}$ & $\begin{array}{l}2.5-i: \\
\text { ?. }\end{array}$ & LAS & D Stevartt & $6032+$ \\
\hline $\begin{array}{l}z_{H} \\
2_{H} \\
3_{H}\end{array}$ & $\begin{array}{l}\mathrm{k} \\
\mathrm{k} \\
\mathrm{t}\end{array}$ & $\begin{array}{l}7.5-2 \\
1.2-1 \\
1.0-1\end{array}$ & $\begin{array}{l}2.0-1 \\
2.5-1\end{array}$ & $\begin{array}{l}\text { LAS } \\
\text { HAR } \\
\text { LAS }\end{array}$ & $\begin{array}{l}\text { E Brelschert } \\
\text { E Allant } \\
\text { D Stegart+ }\end{array}$ & $\begin{array}{l}6017+ \\
6018+ \\
6030+\end{array}$ \\
\hline${ }^{4} \mathrm{He}$ & $\sigma(E ; \theta)_{0} \times f$ & foctor & & & & \\
\hline $\begin{array}{l}{ }^{2} \mathrm{H} \\
{ }^{4} \mathrm{He}\end{array}$ & $\begin{array}{c}t \\
-(E ; \theta)\end{array}$ & $1.3+1$ & & LAS & E Mc-Danlelst & $6016+$ \\
\hline $\begin{array}{l}2_{H} \\
2_{H} \\
2_{H} \\
2_{H} \\
3_{H} \\
3_{H} \\
{ }^{3} H\end{array}$ & $\begin{array}{l}1 \\
\vdots \\
1 \\
t \\
d \\
0 \\
0\end{array}$ & $\begin{array}{l}1.2-1 \\
1.5+0 \\
1.5+0 \\
2.0+1 \\
1.0-2 \\
2.2+0 \\
1.0+1\end{array}$ & $\begin{array}{l}1.6+0 \\
1.9+0 \\
1.7+c\end{array}$ & $\begin{array}{l}\text { DUB } \\
\text { LAS } \\
\text { LAS } \\
\text { LAS } \\
\text { RIC } \\
\text { MIN } \\
\text { LAS }\end{array}$ & $\begin{array}{l}\text { E Kobzevt } \\
\text { E Heanendingert } \\
\text { E Allent } \\
\text { E Jarmlet } \\
\text { E Connert } \\
\text { E Stroltont } \\
\text { E Brolleyt }\end{array}$ & $\begin{array}{l}6012+ \\
6013+ \\
6014+ \\
6015+ \\
5167+ \\
5168+ \\
5169+\end{array}$ \\
\hline${ }^{4} \mathrm{He}$ & $\sigma(E ; \theta)_{0}$ & & & & & \\
\hline${ }^{4} \mathrm{He}$ & $\begin{array}{c}d \\
0(E ; \theta)\end{array}$ & $1.0+1$ & & LAS & E Allredt & $5207+$ \\
\hline $\begin{array}{l}{ }^{4} \mathrm{He} \\
{ }^{4} \mathrm{Ho}\end{array}$ & $\begin{array}{c}1 \\
O(E: \theta)_{0}\end{array}$ & $1.7+0$ & & LAS & E Allent & $6035+$ \\
\hline${ }^{3} \mathrm{He}$ & $\begin{array}{c}d \\
\theta(E ; \theta)_{n} \times 4\end{array}$ & $\begin{array}{l}5.9+0 \\
4 \pi / \sigma(E)\end{array}$ & $1.4+1$ & LAS E & E Stewart+ & $5194+$ \\
\hline${ }^{{ }^{3} \mathrm{H}}$ & pelotive & $\begin{array}{l}1.0-1 \\
\sigma(E ; \theta)\end{array}$ & $2.5-1$ & LAS & D Slewarlt & $6031+$ \\
\hline${ }^{3} \mathrm{H}$ & d & $5.0-1$ & $7.0+0$ & LAS E & E Bame + & $5170+$ \\
\hline
\end{tabular}

\begin{tabular}{|c|c|c|c|c|c|c|}
\hline Terget & $\operatorname{Inc}$ & $\begin{array}{l}\text { EmIn } \\
(M e V)\end{array}$ & $\begin{array}{l}\text { Emox } \\
\text { (MeV) }\end{array}$ & Lab h & Author & No. \\
\hline " $\mathrm{He}$ & relolive & $\sigma(\varepsilon ; \theta)$ & & & & \\
\hline $\begin{array}{l}3^{3} \mathrm{H} \\
{ }^{3} \mathrm{H}\end{array}$ & $d$ & $\begin{array}{l}6.2+0 \\
1.0-1\end{array}$ & $\begin{array}{l}1.1+1 \\
2.5-1\end{array}$ & LRL & $\begin{array}{l}\text { E Goldberg+ } \\
\text { O Stevart+ }\end{array}$ & $\begin{array}{l}5171+ \\
6029+\end{array}$ \\
\hline${ }^{4} \mathrm{He}$ & $\sigma(E ; \theta)_{n}$ & & & & & \\
\hline $\begin{array}{l}{ }^{2} \mathrm{H} \\
{ }^{2} \mathrm{H} \\
{ }^{3} \mathrm{H} \\
{ }^{3} \mathrm{H} \\
{ }^{3} \mathrm{H} \\
{ }^{3} \mathrm{H} \\
{ }^{3} \mathrm{H} \\
{ }^{3} \mathrm{H} \\
{ }^{3} \mathrm{H} \\
{ }^{3} \mathrm{H} \\
{ }^{3} \mathrm{H} \\
{ }^{4} \mathrm{He}\end{array}$ & $\begin{array}{l}b \\
b \\
d \\
d \\
d \\
d \\
d \\
d \\
d \\
d \\
d \\
d\end{array}$ & $\begin{array}{l}8.0-2 \\
1.0+1 \\
5.0-1 \\
1.1+0 \\
4.0+0 \\
5.0+0 \\
5.0+0 \\
6.1+0 \\
6.4+0 \\
7.0+0 \\
1.0+1 \\
2.7+0\end{array}$ & $\begin{array}{l}4.0-1 \\
1.5+1 \\
7.0+0 \\
5.0+0 \\
1.9+1 \\
1.9+1 \\
1.1+1 \\
1.4+1 \\
1.1+1 \\
1.9+1 \\
3.6+0\end{array}$ & $\begin{array}{l}\text { LAS } \\
\text { LAS } \\
\text { LASE } \\
\text { ORL } \\
\text { KUR } \\
\text { LAS } \\
\text { LASE } \\
\text { LAS } \\
\text { LRL } \\
\text { LAS } \\
\text { LAS } \\
\text { LAS }\end{array}$ & $\begin{array}{l}\text { E Argot } \\
\text { D Orosg. } \\
\text { E Bamet } \\
\text { E Galonsky+ } \\
\text { E Brillt } \\
\text { D Drosg. } \\
\text { E Mac-Danlelst } \\
\text { E Steqarl+ } \\
\text { E Goldberg+ } \\
\text { E Simaonst } \\
\text { E Brolley+ } \\
\text { E Henkel+ }\end{array}$ & $\begin{array}{l}6010+ \\
6011+ \\
5150+ \\
5151 \\
5152+ \\
5153+ \\
5154 \\
5155 \\
5156+ \\
5157 \\
5158 \\
5210\end{array}$ \\
\hline${ }^{4} \mathrm{He}$ & rolotive & - $O(E ; \theta)$ & ) Legen & ndre & coefficlent expon & ion \\
\hline${ }^{3} \mathrm{H}$ & 0 & $6.2+0$ & $1.1+1$ & LRL & E Goldberg* & 5135 \\
\hline${ }^{4} \mathrm{He}$ & $\sigma(E ; \theta)$ Lo & - gendre & - coeff & ficler & ont expansion & \\
\hline $\begin{array}{l}{ }^{3} \mathrm{H} \\
3^{3} \mathrm{H} \\
{ }^{3} \mathrm{H} \\
{ }^{3} \mathrm{He}\end{array}$ & $\begin{array}{l}9 \\
9 \\
9 \\
d\end{array}$ & $\begin{array}{l}5.0-1 \\
1.0+0 \\
1.8+0 \\
5.9+0\end{array}$ & $\begin{array}{l}7.0+0 \\
5.0+0 \\
1.2+1 \\
1.4+1.1\end{array}$ & $\begin{array}{l}\text { LAS } \\
\text { VIR } \\
\text { BNL } \\
\text { LAS }\end{array}$ & $\begin{array}{l}\text { E Bamet } \\
\text { E Smilht } \\
\text { R Goldberg. } \\
\text { E Stevartt }\end{array}$ & $\begin{array}{l}5147 \\
5148 \\
5149 \\
5192\end{array}$ \\
\hline "Ho & $\theta(E ; \theta)$ Le & -egendre & $\theta \cos f$ & flaclor & ont expansion/he & \\
\hline${ }^{3} \mathrm{H}$ & d & $5.0+0$ & $1.6+1$ & LAS & E Mc-Danielst & 5160 \\
\hline${ }^{4} \mathrm{He}$ & $\theta(E ; \theta)$ Le & -egendre & ecoofil & flele & ont exponglonxo(E) & $4 \pi$ \\
\hline${ }^{3} \mathrm{H}$ & d & $5.0+0$ & $1.9+1$ & LAS & D Drosg. & $5159+$ \\
\hline${ }^{4} \mathrm{H}_{\mathrm{O}}$ & relstive & $\sigma(E ; \theta)$ & & & & \\
\hline${ }^{3} \mathrm{H}$ & d & $5.0+0$ & $1.6+1$ & LAS E & E Mc-Danlelst & 5134 \\
\hline${ }^{4} \mathrm{He}$ & $O(E ; \theta) \times f \theta$ & foctor & & & & \\
\hline $\begin{array}{l}{ }_{3}^{3} \mathrm{H} \\
3_{H} \\
3_{H}\end{array}$ & $\begin{array}{l}d \\
d\end{array}$ & $\begin{array}{l}4.7-1 \\
3.9+0 \\
1.3+1\end{array}$ & $1.5+1$ & $\begin{array}{l}\text { LAS } \\
\text { LAS } \\
\text { LAS }\end{array}$ & $\begin{array}{l}\text { E Coont: } \\
\text { E Broglet } \\
\text { E Mc-Danielgt }\end{array}$ & $\begin{array}{l}5161 \\
5162 \\
5163\end{array}$ \\
\hline
\end{tabular}




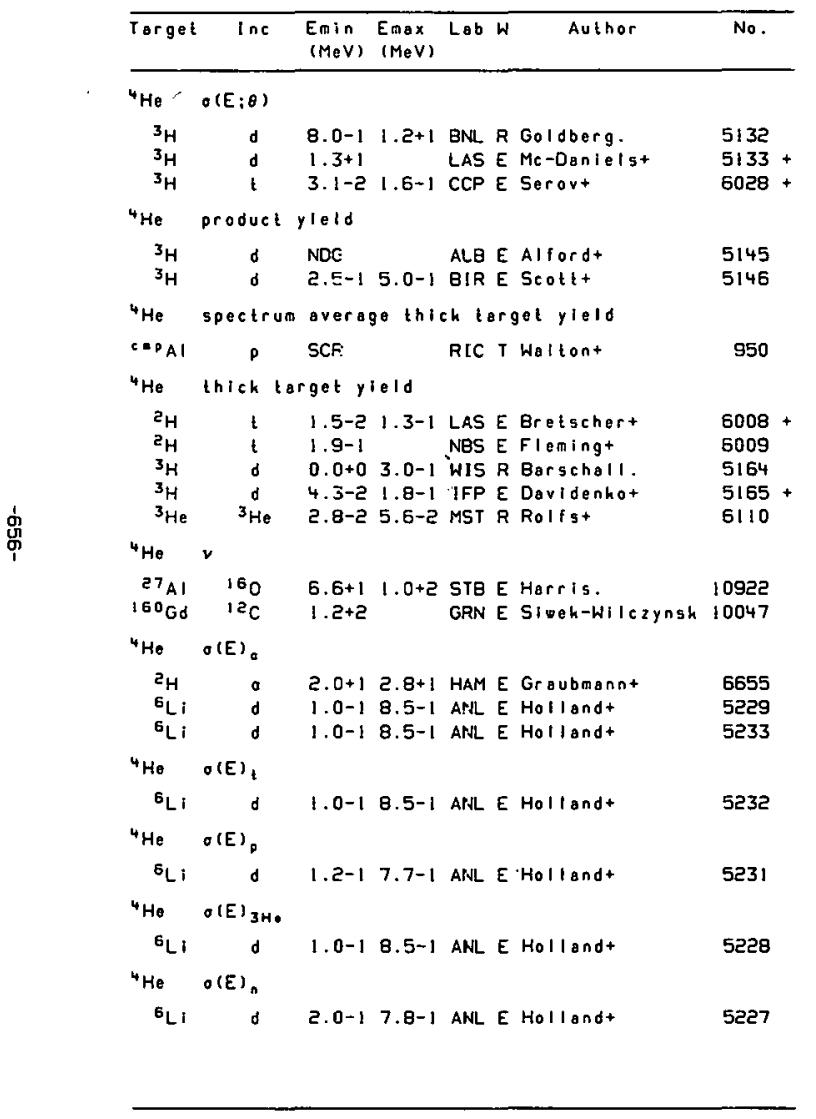

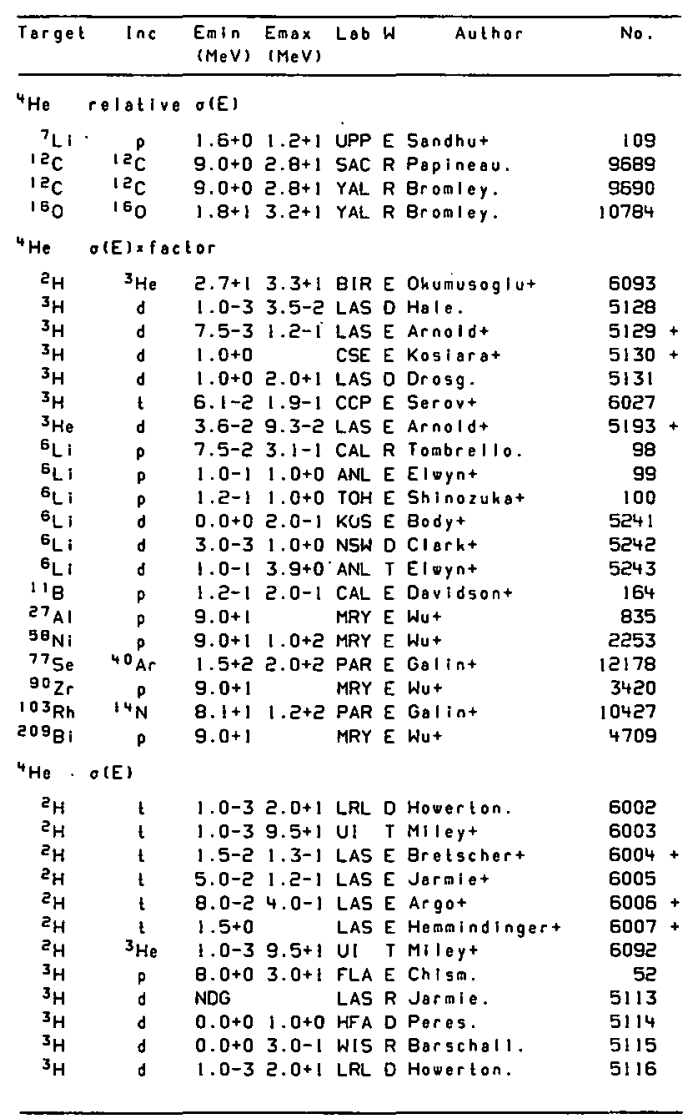




\begin{tabular}{|c|c|c|c|c|c|c|c|}
\hline Target & $\ln c$ & $\begin{array}{l}\text { Emin } \\
(\mathrm{MeV})\end{array}$ & $\begin{array}{l}\max \\
(\operatorname{MeV})\end{array}$ & Lab & W & Author & No. \\
\hline${ }^{4} \mathrm{He}$ & $\sigma(E)$ & & & & & & \\
\hline${ }^{3} \mathrm{H}$ & d & $7.5-3$ & $1.2-1$ & LAS & $E$ & Arnold + & $5117+$ \\
\hline${ }^{3} \mathrm{H}$ & d & $1.0-2$ & $8.0+0$ & LAS & 0 & Hale. & $5118+$ \\
\hline${ }^{3} \mathrm{H}$ & d & $5.0-2$ & $1.7-1$ & IFP & $E$ & Davidentiot & $5119+$ \\
\hline${ }^{3} \mathrm{H}$ & d & $1.1-1$ & & RIC & $E$ & Connert & $5120+$ \\
\hline${ }^{3} \mathrm{H}$ & d & $2.0-1$ & $1.0+2$ & UI & $T$ & Mileyt & 5121 \\
\hline${ }^{3} \mathrm{H}$ & $\delta$ & 2.5-1 & $5.0-1$ & BIR & E & Walther. & 5122 \\
\hline${ }^{3} \mathrm{H}$ & d & $5.0-1$ & $7.0+0$ & LAS & $E$ & Bamet & $5123+$ \\
\hline${ }^{3} \mathrm{H}$ & d & $1.5+0$ & $5.8+0$ & ORL & $E$ & Galonsky+ & $5124+$ \\
\hline${ }^{3} \mathrm{H}$ & d & $5.0+0$ & $1.9+1$ & LAS & D & Drosg. & $5125+$ \\
\hline${ }^{3} \mathrm{H}$ & $d$ & $6.2+0$ & $1.1+1$ & LRL & E & Goldbergt & $5126+$ \\
\hline${ }^{3} \mathrm{H}$ & g & $1.0+1$ & & LAS & E & Brolley+ & $5127+$ \\
\hline${ }^{3} \mathrm{H}$ & $t$ & NOG & & LAS & $\mathbf{R}$ & Jormie. & 6022 \\
\hline${ }^{3} \mathrm{H}$ & $t$ & $0.0+0$ & $1.0+0$ & HFA & D & Peres. & 6023 \\
\hline${ }^{3} \mathrm{H}$ & t & $1.0-2$ & $3.5+0$ & LAS & D & Hale. & 6024 \\
\hline${ }^{3} \mathrm{H}$ & t & $3.1-2$ & 1.6-1 & $\mathrm{CCP}$ & E & Seravt & $6025+$ \\
\hline${ }^{3} \mathrm{H}$ & $t$ & $1.4-1$ & $1.0+2$ & UI & $T$ & Mileyt & 6026 \\
\hline${ }^{3} \mathrm{H}$ & ${ }^{3} \mathrm{He}$ & $4.5-1$ & $1.1+0$ & MNA & $T$ & Mc-Keet & 6096 \\
\hline${ }^{3} \mathrm{He}$ & $d$ & $0.0+0$ & $1.0+0$ & HFA & D & Peres. & 5185 \\
\hline${ }^{3} \mathrm{He}$ & d & $5.0-3$ & $2.0+1$ & LRL & D & Howerton. & 5186 \\
\hline $3^{3} \mathrm{He}$ & d & $1.0-2$ & $8.0+0$ & LAS & D & Hole. & 5187 \\
\hline${ }^{3} \mathrm{He}$ & 0 & $3.6-2$ & 9. $3-2$ & LAS & E & Arnoldt & $5188+$ \\
\hline${ }^{3} \mathrm{He}$ & d & $1.0-1$ & $6.0-1$ & ALG & $M$ & Allab+ & 5189 \\
\hline${ }^{3} \mathrm{Ha}$ & g & $4.0-1$ & $1.0+2$ & ui & $\mathrm{T}$ & Mileyt & 5190 \\
\hline${ }^{3} \mathrm{He}$ & d & $5.9+0$ & $1.4+1$ & LAS & E & Stevartt & $5191+$ \\
\hline${ }^{3} \mathrm{He}$ & ${ }^{3} \mathrm{He}$ & $1.8+1$ & $2.4+1$ & LAS & E & Brownt & 6107 \\
\hline${ }^{3} \mathrm{He}$ & ${ }^{3} \mathrm{He}$ & $6.0-2$ & & MST & $\mathbf{R}$ & Rolfst & 6108 \\
\hline${ }^{3} \mathrm{He}$ & ${ }^{3} \mathrm{He}$ & $1.8+1$ & $2.4+1$ & LAS & E & Brount & 6109 \\
\hline${ }^{4} \mathrm{He}$ & p & $0.0+0$ & $3.0+1$ & LAS & D & Hale. & 77 \\
\hline${ }^{4} \mathrm{He}$ & p & $2.5+1$ & $4.2+1$ & NI! & $T$ & Kanadat & 78 \\
\hline${ }^{4} \mathrm{He}$ & $p$ & $3.0+1$ & $6.5+1$ & OSA & $T$ & Saito. & 79 \\
\hline${ }^{4} \mathrm{He}$ & p & $9.0+1$ & $1.4+2$ & LPI & $T$ & Dmitrieut & 80 \\
\hline${ }^{4} \mathrm{He}$ & d & $0.0+0$ & $1.5+1$ & LAS & D & Hale. & 5205 \\
\hline${ }^{4} \mathrm{He}$ & d & $1.0+1$ & & LAS & E & Allredt & $5206+$ \\
\hline${ }^{4} \mathrm{He}$ & 9 & $4.0+0$ & $3 \cdot 0+1$ & ITY & $\bar{T}$ & Osman. & 5208 \\
\hline${ }^{4} \mathrm{He}$ & $\delta$ & $7.8+0$ & & кTO & E & Kambarat & 5209 \\
\hline${ }^{4} \mathrm{He}$ & t & $0.0+0$ & $1.4+1$ & LAS & 0 & Hale. & 6034 \\
\hline${ }^{4} \mathrm{He}$ & ${ }^{3} \mathrm{He}$ & $0.0+0$ & $1.1+1$ & LAS & D & Hahe. & 6112 \\
\hline${ }^{6} L i$ & p & $1.0-4$ & $2.5+0$ & LAS & D & Hate. & 91 \\
\hline${ }^{E_{L}} \mathrm{i}$ & p & -2 & +0 & BRK & $T$ & Ruby+ & 92 \\
\hline${ }^{6} \mathrm{Li}$ & $p$ & $1.0-1$ & $1.8-1$ & DEB & $E$ & Sonogy $1+$ & 93 \\
\hline
\end{tabular}

\begin{tabular}{|c|c|c|c|c|c|c|}
\hline Target & $\ln c$ & $\begin{array}{l}\text { Emin } \\
(\operatorname{MeV})\end{array}$ & $\begin{array}{l}\text { Emax } \\
(\operatorname{MeV})\end{array}$ & Lab & Author & No. \\
\hline${ }^{4} \mathrm{He}$ & $O(E)$ & & & & & \\
\hline $6_{L i}$ & $p$ & $1.2-1$ & $1.0+0$ & $\mathrm{rOH}$ & E Shinozukat & 94 \\
\hline$\sigma_{L i}$ & p & $1.4-1$ & $3.0+0$ & ANL & E Eloynt & 95 \\
\hline$\sigma_{L I}$ & p & $1.0+0$ & $2.6+0$ & $\mathrm{SHI}$ & E Chia-shout & 96 \\
\hline $6_{\mathrm{Li}}$ & p & $3.0+0$ & $1.2+1$ & TNL & E Gould+ & 97 \\
\hline $6 \mathrm{Li}^{-}$ & d & $1.0-1$ & $1.0+0$ & ANL & E Eloyn- & 5226 \\
\hline$\sigma_{L i}$ & d & $1.2-1$ & 0.0-1 & ANL & E Hollandt & 5230 \\
\hline${ }^{6} \mathrm{Li}$ & d & $1.0-3$ & $2.0+1$ & LRL & o Howertor. & 5234 \\
\hline$B_{L i}$ & d & $1.5-1$ & $8.0-1$ & ANL & E Hollandt & 5235 \\
\hline$b_{L i}$ & d & $1.0-3$ & $2.0+1$ & LRL & D Howerlar. & 5236 \\
\hline $6_{\mathrm{Li}}$ & d & $3.0-3$ & $1.0+0$ & NSW & O Clarkt & 5237 \\
\hline${ }^{6}{ }_{L 1}$ & d & $1.0-1$ & $1.0+0$ & ANL & E Elaynt & 5238 \\
\hline $6_{\mathrm{Li}}$ & d & $1.0-i$ & $1.8-1$ & KOS & E Bodyt & 5239 \\
\hline$G_{L i}$ & d & $2.3+0$ & $6.0+0$ & TNL & E Gouldt & 5240 \\
\hline $6_{\mathrm{Li}}$ & ${ }^{3} \mathrm{He}$ & $5.0-1$ & $2.0+0$ & ANL & E Hollandt & 6114 \\
\hline${ }^{6} L_{1}$ & ${ }^{3} \mathrm{He}$ & $3.0+0$ & $6.0+0$ & TNL & E Gouldt & 6115 \\
\hline $6_{L i}$ & ${ }^{B} \mathrm{Li}$ & NDG & & BRK & T Ruby+ & 9161 \\
\hline${ }^{6} L_{i}$ & ${ }^{6} \mathrm{Li}$ & $1.0+0$ & $5.5+0$ & sul & E Norbeck. & 9162 \\
\hline $7_{\mathrm{Li}}$ & $p$ & $1.0-1$ & $1.8-1$ & DEB & E Somogyit & 108 \\
\hline $7_{L I}$ & t & $5.0+0$ & $6.0+0$ & CCP & E Abramovich & 6039 \\
\hline $10_{B}$ & p & $4.8-1$ & $2.0+1$ & LRL & OHovertion. & 148 \\
\hline $10_{B}$ & d & $1.0-3$ & $2.0+1$ & LRL & 0 Howerton. & 5290 \\
\hline $10_{B}$ & 150 & $6.0+1$ & & $\mathrm{MPH}$ & MSzanta-De-Toled & 10579 \\
\hline 118 & p & $5.0-4$ & $3.0+0$ & LAS & o Hale. & 160 \\
\hline $1_{\mathrm{B}}$ & p & $6.8-2$ & $2.0+1$ & LRL & D Hovertion. & 161 \\
\hline 118 & p & $2.1-1$ & $3.3-1$ & CAL & E Davidsont & 162 \\
\hline 118 & p & $3.0-i$ & $1.0+2$ & UI & T MIley+ & 163 \\
\hline 118 & d & $3.8+0$ & & $\mathrm{IJI}$ & E Granlsevt & 5304 \\
\hline c & $14 \mathrm{~N}$ & $1.6+1$ & $7.5+1$ & FSU & T Holubt & 10246 \\
\hline$c$ & 150 & $1.6+i$ & $7.5+1$ & FSU & T Holubt & 10592 \\
\hline $12 c$ & p & $6.6+2$ & & DUB & E Bogalint & 208 \\
\hline $12 \mathrm{c}$ & p & $9.0+1$ & & LPI & Tomitrievat & 209 \\
\hline $12^{2} \mathrm{C}$ & & $9.0+1$ & & LPI & T Dmilirievt & 510 \\
\hline $12 \mathrm{c}$ & م & $9.0+1$ & & LPI & T Dmilitievt & 211 \\
\hline $12 \mathrm{c}$ & & $9.0+1$ & & LPI & T Dofitrieyt & 212 \\
\hline $12 \mathrm{c}$ & g & $9.0+1$ & & LPI & T Datirievt & 213 \\
\hline $12 \mathrm{C}$ & p & $9.0+1$ & & LPI & T Dmitrievt & 214 \\
\hline $12 \mathrm{C}$ & 0 & $9.0+1$ & & LPI & T Dritrievt & 215 \\
\hline $12 \mathrm{c}$ & p & $9.0+1$ & & LPI & T Dmitrlevt & 216 \\
\hline $12 \mathrm{C}$ & $p$ & $9.0+1$ & & LPI & T Dmitrievt & 217 \\
\hline $1 a_{c}$ & p & $5.0+1$ & & LPI & E Vdovint & 218 \\
\hline
\end{tabular}




\begin{tabular}{|c|c|c|c|c|c|c|}
\hline Target & Inc & $\begin{array}{l}\text { Emin } \\
(M e V)\end{array}$ & $\begin{array}{l}\text { Emax } \\
(M e V)\end{array}$ & Lab & Author & No. \\
\hline${ }^{4} \mathrm{He}$ & $O(E)$ & & & & & \\
\hline${ }^{12} \mathrm{C}$ & $\mathbf{p}$ & $9.0+1$ & & LPI & T Dofitrlevt & 219 \\
\hline${ }^{12} \mathrm{c}$ & p & $5.0+1$ & & LPI & E Voovint & 220 \\
\hline${ }^{12} \mathrm{C}$ & p & $9.0+1$ & & LPI & T Datitrievt & 221 \\
\hline${ }^{12} \mathrm{C}$ & p & $5 \cdot 0+1$ & & LPI & E vdovint & 222 \\
\hline $12 \mathrm{C}$ & p & $9.0+1$ & & LPI & T Onitrievt & 223 \\
\hline${ }^{12} \mathrm{C}$ & p & $5.0+1$ & & LPI & E Vdovint & 224 \\
\hline $12 \mathrm{C}$ & p & $9.0+1$ & & LPI & I Denftrlovt & 225 \\
\hline${ }^{12} \mathrm{C}$ & p & $9.0+1$ & & LPI & T Dmitrievt & 226 \\
\hline${ }^{12} \mathrm{C}$ & p & $5.0+1$ & & LPI & E voovint & 227 \\
\hline${ }^{12} \mathrm{C}$ & p & $9.0+1$ & & LPI & T Omlitient & 228 \\
\hline${ }^{12} \mathrm{C}$ & $12 \mathrm{C}$ & $2.4+1$ & $6.3+1$ & NOT & E Kolatat & 9692 \\
\hline${ }^{12} \mathrm{C}$ & ${ }^{12} \mathrm{c}$ & $2.8+1$ & $6.2+1$ & SIR & E Kolatat & 9693 \\
\hline${ }^{12} \mathrm{C}$ & ${ }^{12} \mathrm{C}$ & $2.4+1$ & $6 \cdot 3+1$ & NOT & E Kolata+ & 9696 \\
\hline${ }^{12} \mathrm{C}$ & $12 \mathrm{C}$ & $2.8+1$ & $6.2+1$ & STR & E Kolatat & 9697 \\
\hline${ }^{12} \mathrm{c}$ & 160 & $3.0+1$ & $6.4+1$ & ANL & E Tabort & 10629 \\
\hline $1^{2} c$ & 160 & $4.0+1$ & $6.5+1$ & SIR & M Koletat & 10630 \\
\hline $14 N$ & $p$ & $5 \cdot 0+1$ & & LPI & E vdovint & 368 \\
\hline${ }^{14} \mathrm{~N}$ & p & $5.0+1$ & & LPI & E vdovint & 369 \\
\hline${ }^{14} \mathrm{~N}$ & $p$ & $5.0+1$ & & LPI & E vdovint & 370 \\
\hline $14 \mathrm{~N}$ & p & $5.0+1$ & & LPI & E Vdovint & 371 \\
\hline 180 & $p$ & $3.9+1$ & $6.1+1$ & CRC & R Cross. & 490 \\
\hline 150 & $p$ & $5.0+1$ & & LPI & E Vdovint & 491 \\
\hline 160 & & $5.0+1$ & & LPI & E Vdovint & 492 \\
\hline 180 & 160 & $2.5+1$ & & STF & E Chengt & 10782 \\
\hline 160 & 160 & $2.5+1$ & & STR & E Kolata+ & 10783 \\
\hline $\mathrm{Mg}$ & $\mathbf{p}$ & $1.2+1$ & $4.5+1$ & RIC & E Wallont & 718 \\
\hline $\mathrm{Mg}$ & 160 & $1.6+1$ & $7.5+1$ & FSU & I Holub+ & 10870 \\
\hline${ }^{27} \mathrm{AI}$ & p & $9.0+1$ & & MRY & E Wut & 833 \\
\hline${ }^{27} \mathrm{Al}$ & d & $8.0+1$ & & MRY & E Wu+ & 5416 \\
\hline${ }^{27} \mathrm{Al}$ & 160 & $1.6+1$ & $7.5+1$ & FSU & T Holubt & 10923 \\
\hline si & 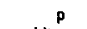 & $1.7+1$ & $4.5+1$ & RIC & E Waltont & 958 \\
\hline $5 i$ & $14 N$ & $1.6+1$ & $7.5+1$ & FSU & I T Holubt & 10354 \\
\hline${ }^{40} \mathrm{CB}$ & a & $1.0+2$ & & MST & E Lohnert & 7302 \\
\hline${ }^{44} \mathrm{CB}_{\mathrm{B}}$ & a & $1.0+2$ & & MST & E Lohnert & 7330 \\
\hline$\because P C_{\theta}$ & p & $1.9+1$ & $4.4+1$ & RIC & E Waltont & 1326 \\
\hline $\mathrm{Fe}$ & p & $2.5+3$ & & CER & E Goebelt & 1843 \\
\hline${ }^{5 \theta_{N i}}$ & & $3.0+1$ & $2.0+2$ & $\mathrm{BNL}$ & T Olvadeenam. & 2250 \\
\hline${ }^{30} \theta_{\mathrm{Ni}}$ & $p$ & $9.0+1$ & $1.0+5$ & MRY & E Wut & 2251 \\
\hline $58_{\mathrm{N} 1}$ & d & 8. $0+i$ & & MRY & E Wot & 5484 \\
\hline Cu & p & $2.5+3$ & & CER & E Goebelt & 2576 \\
\hline
\end{tabular}

\begin{tabular}{|c|c|c|c|c|c|c|}
\hline Target & $\operatorname{Inc}$ & $\begin{array}{l}\text { Emin } \\
(\mathrm{MeV})\end{array}$ & $\begin{array}{l}\text { Emax } \\
(\operatorname{MeV})\end{array}$ & Lab & Author & No. \\
\hline${ }^{4} \mathrm{He}$ & \multicolumn{6}{|l|}{$\sigma(E)$} \\
\hline${ }^{54} \mathrm{Zn}$ & p & $1.8+1$ & & PUR & E Lux. & 2749 \\
\hline $662 n$ & p & $1.8+1$ & & PUR & $E$ Lux. & 2783 \\
\hline${ }^{68} \mathrm{Zn}$ & p & $1.8+1$ & & PUR & E Lux. & 2816 \\
\hline $70 \mathrm{Zn}$ & م & $1.8+1$ & & PUR & E Lux. & 2862 \\
\hline & ${ }^{40} \mathrm{Ar}$ & $2.0+2$ & & PAR & E Golint & 12165 \\
\hline${ }^{77} \mathrm{Se}$ & ${ }^{40} \mathrm{Ar}$ & $1.5+2$ & $2.0+2$ & PAR & E Galint & 12177 \\
\hline $90 \mathrm{Zr}$ & p & $2.0+1$ & $8.0+1$ & MCG & E Kanlelot & 3417 \\
\hline${ }^{90} \mathrm{Zr}$ & p & $9.0+1$ & & MRY & E Wut & 3418 \\
\hline $90 \mathrm{Zr}$ & g & $8.0+1$ & & MRY & E Wut & 5598 \\
\hline $10^{3} \mathrm{Rh}$ & $14 \mathrm{~N}$ & $8.1+1$ & $1.2+2$ & PAR & E Gallnt & 10426 \\
\hline $11 \mathrm{~S}_{\mathrm{Sn}}$ & $40 \mathrm{Ar}$ & $5.5+5$ & $3.4+2$ & BRK & E Delagranget & 12233 \\
\hline $1545 \mathrm{~m}$ & ${ }^{40} \mathrm{Ar}$ & $5.5+5$ & $3.4+2$ & BRK & E Delagranget & 12336 \\
\hline $160^{\circ} \mathrm{Gd}$ & $12 \mathrm{c}$ & $9.0+1$ & $2.0+2$ & GRN & E siveh-Wilczynsh & 10046 \\
\hline $160 \mathrm{Gd}$ & ${ }^{12} \mathrm{C}$ & $9.0+1$ & $1.2+2$ & GRN & E Slveh-WIIczynsh & 10048 \\
\hline $160 \mathrm{Gd}$ & $12 \mathrm{c}$ & 9. $0+1$ & $1.5+5$ & GRN & E Siweh-WIlczynsh & 10049 \\
\hline $164 \mathrm{OY}_{\mathrm{Y}}$ & ${ }^{40} \mathrm{Ar}$ & $2.2+2$ & $3.4+2$ & BRK & E Delagranget & 12343 \\
\hline $181 \mathrm{Ta}$ & $p$ & $2.0+1$ & $8.5+1$ & MIL & T Gadiall+ & 4382 \\
\hline $181 \mathrm{Ta}$ & ${ }_{6}^{6} L_{1}$ & $7.5+1$ & $9.5+1$ & INU & M Karqoushit & 9278 \\
\hline $194 \mathrm{Pz}$ & $\mathrm{s}_{\mathrm{Li}}$ & $7.5+1$ & $9.5+1$ & INU & M Karwoushit & 9312 \\
\hline $198 \mathrm{pl}$ & $\sigma_{L i}$ & $7.5+1$ & $9.5+1$ & INU & M Karwowshit & 9318 \\
\hline $197 \mathrm{Au}$ & p & $2.0+1$ & $8.5+1$ & MIL & T Gadialit & 4466 \\
\hline $197 \mathrm{AU}$ & ${ }^{6} \mathrm{~L} 1$ & $7.5+1$ & $9.5+1$ & INU & M Karwowshit & 9341 \\
\hline $197 \mathrm{AU}$ & $40 \mathrm{Ar}$ & $5.5+5$ & $3.4+2$ & BRK & E Delagranget & 12365 \\
\hline $202 \mathrm{Hg}$ & p & $2.0+1$ & $8.0+1$ & MCG & E Kantelot & 4515 \\
\hline $202 \mathrm{Hg}$ & p & $2.0+1$ & $8.5+1$ & MIL & T Gadiolit & 4516 \\
\hline $206 \mathrm{pb}$ & p & $2.0+1$ & $8.5+1$ & MIL & T Gadialit & 4633 \\
\hline $207 \mathrm{~Pb}$ & p & $2.0+1$ & $8.5+1$ & MIL & T Gadiolit & $464 !$ \\
\hline $208 \mathrm{~Pb}$ & d & $7.0+1$ & & MRY & E Wut & 5873 \\
\hline $208 \mathrm{pb}$ & ${ }^{6} \mathrm{Li}$ & $7.5+1$ & $9.5+1$ & INU & M Karwowshl+ & 9369 \\
\hline $209 \mathrm{Bi}$ & p & $2.0+1$ & $8.5+1$ & MiL & T Gadialit & 4706 \\
\hline $200^{8 i}$ & & $9.0+1$ & & MRY & E Wut & 4707 \\
\hline $209 \mathrm{Bi}$ & $14 N$ & $8.5+1$ & $9.5+1$ & $\mathrm{JCL}$ & E Nomurat & 10497 \\
\hline $232 \mathrm{Th}$ & d & $7.0+1$ & & MRY & E Wut & 5916 \\
\hline Meny & ${ }^{14} \mathrm{~N}$ & $1.6+1$ & $7.5+1$ & FSU & T Holubt & 10524 \\
\hline Many & 160 & $1.6+1$ & $7.5+1$ & FSU & T Holubt & 11571 \\
\hline${ }^{5} \mathrm{He}$ & pou & nucl & us rol & ative & e $\sigma(E)$ & \\
\hline${ }^{3} \mathrm{H}$ & d & $1.5-1$ & $1.3+0$ & MPH & E guss+ & 517 \\
\hline
\end{tabular}




\begin{tabular}{|c|c|c|c|c|c|c|}
\hline Target & $\operatorname{Inc}$ & $\begin{array}{l}\text { Emin } \\
(M e V)\end{array}$ & $\begin{array}{l}E_{\max } \\
(\operatorname{Me} y)\end{array}$ & Lab & Auther & No. \\
\hline${ }^{5} \mathrm{He}$ & direct-in & nteract & tion re & elati & Ive $\theta(E)$ & \\
\hline${ }^{3} \mathrm{H}$ & $d$ & $1.5-1$ & $1.3+0$ & MPH & E Busst & $5177+$ \\
\hline${ }^{5} \mathrm{He}$ & partialo & $\sigma(E)$ & & & & \\
\hline${ }^{7} \mathrm{Li}$ & $d$ & $2.5-1$ & $1.2-0$ & LIE & E Robaye+ & 5270 \\
\hline${ }^{3} \mathrm{He}$ & cosonance & $e v i d t h$ & & & & \\
\hline $3_{\mathrm{Fi}}$ & d & $1.0+0$ & & CSE E & E Kogiarat & $5174+$ \\
\hline${ }^{5} \mathrm{He}$ & $\sigma(E ; \theta)$, & & & & & \\
\hline $\begin{array}{l}{ }^{3} \mathrm{H} \\
{ }^{3} \mathrm{H}\end{array}$ & $d$ & $\begin{array}{l}4.7-1 \\
1.0+0\end{array}$ & & $\begin{array}{l}\text { LAS } \\
\text { CSE }\end{array}$ & $\begin{array}{l}\text { E Coont } \\
\text { E Kosiarat }\end{array}$ & $\begin{array}{l}5179+ \\
5180+\end{array}$ \\
\hline${ }^{5} \mathrm{He}$ & rolative & thick & tarcet & t yie & eld & \\
\hline${ }^{3} \mathrm{H}$ & $d$ & $1.5-1$ & $1.3+0$ & $\mathrm{MPH}$ & E Busst & $5175+$ \\
\hline${ }^{5} \mathrm{He}$ & relative & $O(E)$ & & & & \\
\hline${ }^{3} \mathrm{H}$ & d & $1.5-1$ & $1.3+0$ & $\mathrm{MPH}$ & E Busst & $5176+$ \\
\hline${ }^{5} \mathrm{He}$ & $\sigma(E) \times f a c t$ & tor & & & & \\
\hline${ }^{3} \mathrm{H}$ & $d$ & $1.0+0$ & & CSE & E Kosiarat & $5173+$ \\
\hline${ }^{5} \mathrm{He}$ & $\sigma(E)$ & & & & & \\
\hline $3 \mathrm{H}$ & d & $1.0+0$ & & CSE & E kosiarat & $5172+$ \\
\hline${ }^{6} \mathrm{He}$ & $O(E)$ & & & & & \\
\hline $\begin{array}{l}{ }^{4} \mathrm{He} \\
7 \mathrm{Li} \\
12 \mathrm{C} \\
14 \mathrm{~N} \\
160\end{array}$ & $\begin{array}{l}a \\
t \\
p \\
a \\
a\end{array}$ & $\begin{array}{l}6 \cdot 0+1 \\
5 \cdot 0+0 \\
5 \cdot 0+1 \\
3 \cdot 3+1 \\
3 \cdot 3+1\end{array}$ & $\begin{array}{l}1.6+2 \\
6.0+0 \\
5.1+1 \\
5.1+1\end{array}$ & $\begin{array}{l}\text { MRY } \\
\text { CCP } \\
\text { LPI } \\
\text { KFK } \\
\text { KFK }\end{array}$ & $\begin{array}{l}\text { E Glagola. } \\
\text { E Abramovicht } \\
\text { E Vdovint } \\
\text { E Vidal-Quadrast } \\
\text { E Vidal-Quadrast }\end{array}$ & $\begin{array}{r}6673 \\
6039 \\
229 \\
6820 \\
6876\end{array}$ \\
\hline$z=3$ & binory & $O(\varepsilon)$ & & & & \\
\hline 160 & $\mathbf{p}$ & $2.0+1$ & $1.0+2$ & INU & T Compani-Tabrizi & 565 \\
\hline$z=3$ & spallat & tion olf & & & & \\
\hline${ }^{12} \mathrm{C}$ & $p$ & $4.5+1$ & $1.0+2$ & MRY & E Mathewst & 309 \\
\hline$z=3$ & $\sigma(E) \times f \theta$ & octor & & & & \\
\hline $\begin{array}{l}200 \mathrm{Pt} \\
200 \mathrm{Pt}\end{array}$ & $\begin{array}{l}160 \\
160\end{array}$ & $\begin{array}{l}1.4+2 \\
1.4+2\end{array}$ & $\begin{array}{l}3.2+2 \\
3.2+2\end{array}$ & $\begin{array}{l}\text { BRK } \\
\text { MSU }\end{array}$ & $\begin{array}{l}\text { E Gelbke+ } \\
\text { R Gelbke. }\end{array}$ & $\begin{array}{l}11538 \\
11539\end{array}$ \\
\hline
\end{tabular}

\begin{tabular}{|c|c|c|c|c|c|c|c|}
\hline Torget & $\ln c$ & $\begin{array}{l}\text { Emin } \\
(\mathrm{MeV})\end{array}$ & $\begin{array}{l}\text { Emax } \\
(\mathrm{MeV})\end{array}$ & Lab & $W$ & Author & No. \\
\hline$z=3$ & $\sigma(E)$ & & & & & & \\
\hline${ }^{12} \mathrm{C}$ & $p$ & $9.0+1$ & & LP I & & Doitelert & 310 \\
\hline $12 \mathrm{c}$ & p & $9.0+1$ & & LPI & $T$ & Omitrievt & 315 \\
\hline${ }^{12} \mathrm{c}$ & p & $9.0+1$ & & LPl & $T$ & Dmitrievt & 316 \\
\hline${ }^{12} \mathrm{C}$ & p & $9.0+1$ & & LPI & $\mathrm{T}$ & Dofitrievt & 317 \\
\hline $12 \mathrm{c}$ & p & $9.0+1$ & & [P] & $\mathrm{T}$ & Doitrlert & 318 \\
\hline $12 \mathrm{c}$ & g & $9.0+1$ & & LPI & $T$ & Datrleva & 319 \\
\hline $12 \mathrm{C}$ & g & $9.0+1$ & & LPI & $\mathbf{T}$ & Dmitrlevt & 320 \\
\hline $12 \mathrm{c}$ & p & $9.0+i$ & & [P] & $\mathrm{T}$ & Dmitrievt & 324 \\
\hline${ }^{12} \mathrm{C}$ & p & $9.0+1$ & & LP] & & Datitrieva & 325 \\
\hline $12 \mathrm{c}$ & p & $9.0+i$ & & [P] & $\mathrm{T}$ & Omitrievt & 326 \\
\hline${ }^{12} \mathrm{C}$ & $p$ & $9.0+i$ & & LPI & & Omitrievt & 327 \\
\hline${ }^{12} \mathrm{C}$ &.$p$ & $9.0+1$ & & LPI & & Oafitiont & 329 \\
\hline $12 \mathrm{C}$ & $p$ & $9.0+1$ & & [P] & & Dnitrieva & 330 \\
\hline${ }^{12} \mathrm{C}$ & ${ }^{12} \mathrm{C}$ & $4.5+1$ & $2.0+2$ & TAM & $E$ & Namboodirlt & 9770 \\
\hline $12 \mathrm{C}$ & $14 \mathrm{~N}$ & $8.6+1$ & $1.8+2$ & ORL & & Del-Campot & 10319 \\
\hline${ }^{48} \mathrm{Ca}$ & 160 & $5.6+1$ & & ANL & 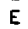 & Kovort & 11107 \\
\hline${ }^{45} \mathrm{Sc}$ & $22_{\mathrm{Ne}}$ & NDS & & Cro & & Billereyt & 11924 \\
\hline $51 \mathrm{v}$ & 160 & $7.7+1$ & & GRE & & Chevartert & 11142 \\
\hline${ }^{50} \mathrm{Cr}$ & $14 \mathrm{~N}$ & $7.1+1$ & $1.0+2$ & LYO & & Billereyt & 10393 \\
\hline${ }^{50} \mathrm{Cr}$ & $22_{\mathrm{Ne}}$ & NDG & & EYo & & Billereyt & 11925 \\
\hline${ }^{55} \mathrm{Mn}$ & $12 \mathrm{C}$ & $7.7+1$ & & GRE & & Cheveriert & 9944 \\
\hline $56_{\mathrm{Fe}}$ & 160 & NDG. & & LYO & $\mathrm{T}$ & Blilereyt & 11162 \\
\hline $56 \mathrm{Fe}$ & 150 & $7.7+1$ & & GRE & & Chevarlert & 11163 \\
\hline${ }^{60} \mathrm{NI}$ & ${ }^{12} \mathrm{C}$ & NDG & & LYo & $T$ & Biltereyt & 9960 \\
\hline $60^{N I}$ & ${ }^{12} \mathrm{c}$ & $7.7+1$ & & GRE & & Chevarlert & 9961 \\
\hline Se & $40 \mathrm{Ar}$ & $2.0+2$ & & PAR & $\mathrm{E}$ & Golint & 12166 \\
\hline $77 \mathrm{se}$ & $40 \mathrm{Ar}$ & $1.5+2$ & $2.0+2$ & PAR & & Galint & 12179 \\
\hline $197 \mathrm{Au}$ & ${ }^{12} \mathrm{c}$ & $1.3+2$ & & KFK & & Muenzel+ & 10095 \\
\hline $197 \mathrm{AU}$ & 160 & $1.4+2$ & $1.7+2$ & KFK & & Muenzeit+ & 11481 \\
\hline${ }^{4} \mathrm{LI}$ & $\sigma(E)$ & & & & & & \\
\hline${ }^{4} \mathrm{He}$ & p & $3.1+1$ & $7.1+1$ & $\mathrm{CCP}$ & & Goldberg+ & 81 \\
\hline${ }^{5} \mathrm{LI}$ & portiol & $\sigma(E ; \theta)$ & $\cos \ln \theta$ & $10 \mathrm{co}$ & & flelent expansion & \\
\hline${ }^{3} \mathrm{He}$ & d & $4.8-1$ & $1.0+0$ & $M P H$ & & Busst & $5198+$ \\
\hline${ }^{5} \mathrm{LI}$ & partlal & rolotis & Ve OlE : & $: \theta\}$ & & & \\
\hline${ }^{3} \mathrm{He}$ & d & $4.8-1$ & $1.0+0$ & $\mathrm{MPH}$ & & Busst & $5197+$ \\
\hline
\end{tabular}




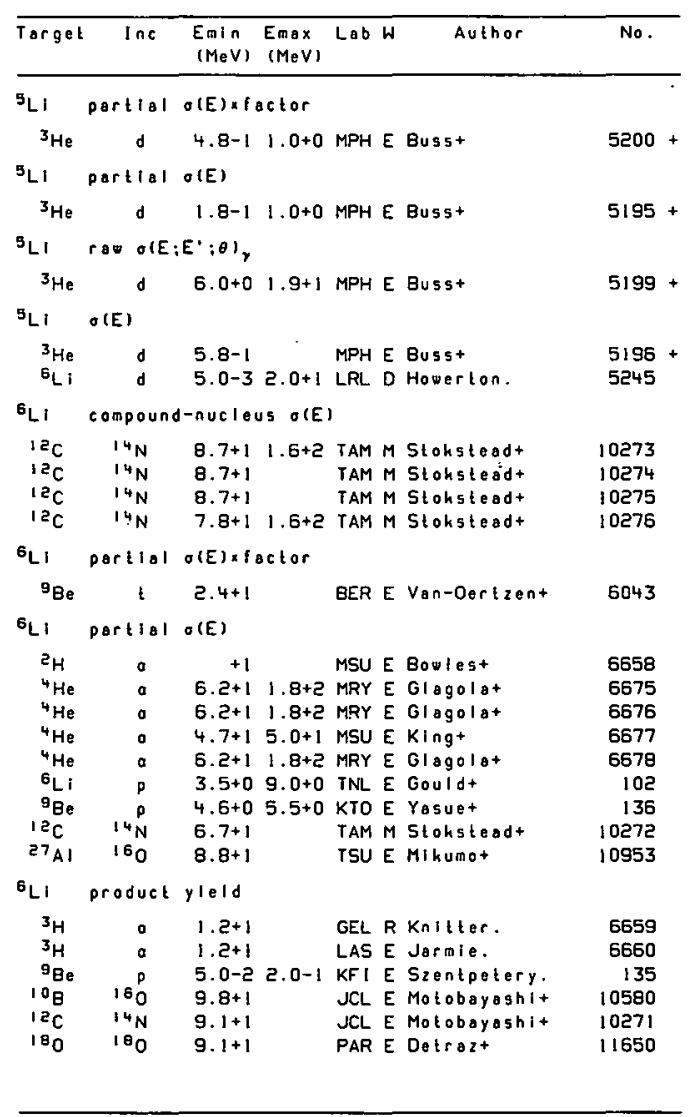

\begin{tabular}{|c|c|c|c|c|c|c|}
\hline Ta:get & $\operatorname{lnc}$ & $\begin{array}{l}\text { Emin } \\
(\mathrm{MeV})\end{array}$ & $\begin{array}{l}\text { Emax } \\
(\mathrm{MeV})\end{array}$ & Lob & Author & No. \\
\hline${ }^{6} L_{1}$ & spectrum & averas & ge OlE & $1 \times f a c$ & ictor & \\
\hline $\cos 0$ & p & $0.0+0$ & $9.9+9$ & MRY & M Rochet & 602 \\
\hline${ }^{6} L_{1}$ & relative & $O(E)$ & & & & \\
\hline $\begin{array}{l}C \sim P \mathrm{He} \\
1 \geq \mathrm{C} \\
\therefore \triangle \mathrm{C}\end{array}$ & $\begin{array}{l}q \\
\rho\end{array}$ & $\begin{array}{l}2.8+2 \\
1.0+3 \\
1.0+3\end{array}$ & $4.4+4$ & $\begin{array}{l}\text { HRV } \\
\text { PAR } \\
\text { PAR }\end{array}$ & $\begin{array}{l}\text { T Epstein. } \\
\text { M Fontes. } \\
M \text { Fontes. }\end{array}$ & $\begin{array}{r}6697 \\
230 \\
354\end{array}$ \\
\hline${ }^{6} \mathrm{LI}_{1}$ & $O(E) \times f a c t$ & tor & & & & \\
\hline $\begin{array}{r}{ }^{9} \mathrm{Be} \\
{ }^{50} \mathrm{Cr} \\
20 \mathrm{~Pb} \\
20 \mathrm{~Pb}\end{array}$ & $\begin{array}{l}14 p \\
14 \mathrm{~N} \\
160 \\
160\end{array}$ & $\begin{array}{l}7.5-2 \\
7.1+1 \\
1.4+2 \\
1.4+2\end{array}$ & $\begin{array}{l}1.9-1 \\
1.0+2 \\
3.2+2 \\
3.2+2\end{array}$ & $\begin{array}{l}\text { KFI } \\
\text { LYO } \\
\text { BRK } \\
\text { MSU }\end{array}$ & $\begin{array}{l}\text { E Szentpetery. } \\
\text { T Blllereyt } \\
\text { E Gelbke+ } \\
\text { R Gelbke. }\end{array}$ & $\begin{array}{r}134 \\
10387 \\
11541 \\
11542\end{array}$ \\
\hline${ }^{6} \mathrm{LI}$ & $O(\varepsilon)$ & & & & & \\
\hline $\begin{array}{l}z_{H} \\
2_{H}\end{array}$ & : & $\begin{array}{l}8.8+0 \\
2.3+1\end{array}$ & $\begin{array}{l}2.6+1 \\
3.3+1\end{array}$ & $\begin{array}{l}\text { ANL } \\
\text { MSU }\end{array}$ & $\begin{array}{l}\text { E Bowlest } \\
\text { E Bowlest }\end{array}$ & $\begin{array}{l}6656 \\
6657\end{array}$ \\
\hline $3_{\mathrm{H}}$ & $a$ & $1.2+1$ & 1. $.6+1$ & UFP & E Andersont & 6661 \\
\hline${ }^{4} \mathrm{He}$ & $\mathrm{t}$ & $0.0+0$ & $1.4+1$ & LAS & D Hale. & 6036 \\
\hline${ }^{4} \mathrm{He}$ & $a$ & $6.0+1$ & $1.6+2$ & MRY & E Glagola. & 6674 \\
\hline${ }^{B_{L I}}$ & p & $0.0+0$ & $2.5+0$ & LAS & D Hale. & 101 \\
\hline${ }^{6} \mathrm{Li}$ & $p$ & & $2.0+0$ & ANL & E Eloynt & 103 \\
\hline$B_{L i}$ & d & $2.3+0$ & $6.0+0$ & TNL. & E Gouldt & 5246 \\
\hline${ }^{8 L i}$ & d & -1 & $2.0+0$ & ANL & E Elwynt & 5247 \\
\hline${ }^{5} \mathrm{Li}$ & $a$ & -1 & $2.0+0$ & ANL & E Eloynt & 6703 \\
\hline $12 \mathrm{C}$ & $p$ & $5.0+1$ & & LPI & Evdovint & 231 \\
\hline $12 \mathrm{c}$ & $p$ & $5.0+1$ & & LPI & E Vovint & 232 \\
\hline${ }^{12} \mathrm{c}$ & p & $5.0+1$ & & LPI & E voovint & 233 \\
\hline${ }^{12} \mathrm{C}$ & 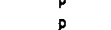 & $5.0+1$ & & LPI & $E$ & 234 \\
\hline $12 \mathrm{C}$ & p & $5.0+1$ & & LPI & E Vdovint & 235 \\
\hline $12 \mathrm{c}$ & $a$ & $4.3+1$ & $5.1+1$ & KFK & E vidal-Quadrast & 6747 \\
\hline $12^{2} \mathrm{C}$ & $a$ & $5.1+1$ & & KFK & Evidal-Quadrast & 6748 \\
\hline $12 c$ & 0 & $3.3+1$ & $5.1+1$ & KFK & E vidal-Quadrast & 6781 \\
\hline $14 \mathrm{~N}$ & & $5.0+1$ & & LPI & E Vouvint & 372 \\
\hline $14 N$ & p & $5.0+1$ & & LPI & E voovint & 373 \\
\hline $1+N$ & p & $5.0+1$ & & LPI & Evoovint & 374 \\
\hline $14 \mathrm{~N}$ & p & $5.0+1$ & & LPI & Evoovint & 375 \\
\hline $14 \mathrm{~N}$ & p & $5.0+1$ & & LPi & Evoovint & 376 \\
\hline $14 \mathrm{~N}$ & p & $5.0+1$ & & LPI & E voovint & 377 \\
\hline${ }^{14} \mathrm{~N}$ & p & $5.0+1$ & & LPI & E vdovint & 378 \\
\hline $14 \mathrm{~N}$ & $a$ & $3.3+1$ & $5 \cdot 1+1$ & KFK & E vidal-Quadrast & 6792 \\
\hline
\end{tabular}




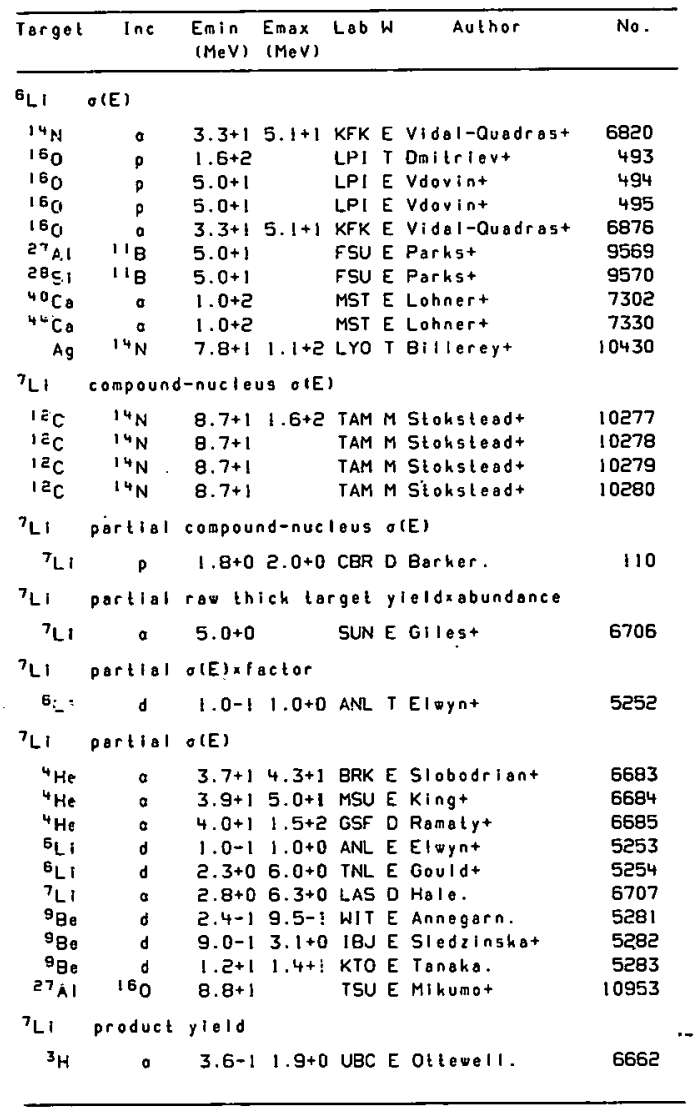

\begin{tabular}{llll}
\hline Target Ine & $\begin{array}{l}\text { Emin Emax Lab } W \text { Author } \\
(\mathrm{MeV})(\mathrm{MeV})\end{array}$ & No. \\
\hline
\end{tabular}

iLi product yield

$10_{0}{ }^{180} 9.1+1 \quad$ PARE Detraz+ 11650

? $L 1$ spectrum average ofElxfactor

COPO D $0.0+09.9+9$ MRY M Rochet 603

'L1 relative $\theta(E)$

$\because$ OPe O $2.8+24.4+4$ HRV T Epstein. 6698

$\begin{array}{rllll}{ }^{12} \mathrm{C} & p & 1.0+3 & \text { PAR M Fontes. } & 236 \\ \therefore \circ \mathrm{P} & \mathrm{P} & 1.0+3 & \text { PAR M Fontes. } & 355\end{array}$

TL1 O(E) xfactor

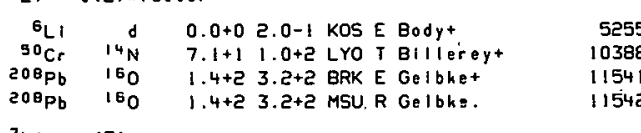

7 LI $\quad O(E)$

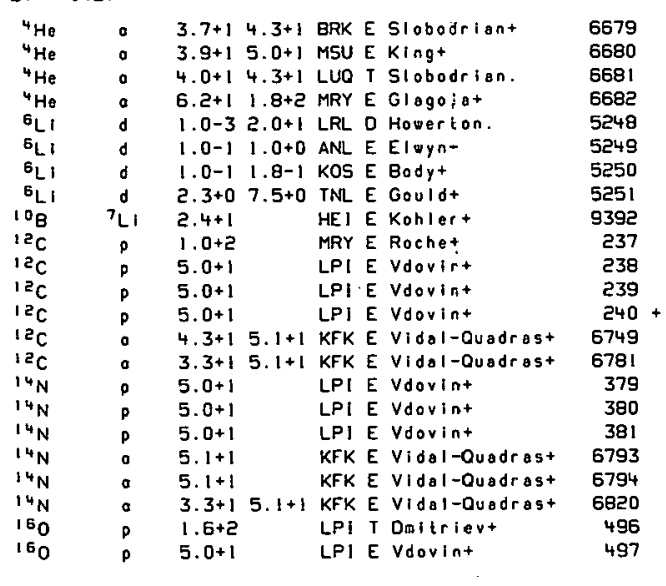




\begin{tabular}{|c|c|c|c|c|c|c|}
\hline Target & $\operatorname{lnc}$ & $\begin{array}{l}E m \mid n \\
{[M e V)}\end{array}$ & $\begin{array}{l}\text { Emax } \\
(\mathrm{MeV})\end{array}$ & Lab W & Author & No. \\
\hline${ }^{7} \mathrm{LI}$ & $\theta(E)$ & & & & & \\
\hline $\begin{array}{l}160 \\
160 \\
27 \mathrm{Al} \\
20 \mathrm{Si} \\
40 \mathrm{Ca} \\
{ }^{4} \mathrm{CO} \\
\mathrm{Ag}\end{array}$ & $\begin{array}{r}p \\
118 \\
118 \\
0 \\
14 N \\
14\end{array}$ & $\begin{array}{l}9 \cdot 0+1 \\
3 \cdot 3+1 \\
5 \cdot 0+1 \\
5 \cdot 0+1 \\
1 \cdot 0+2 \\
1 \cdot 0+2 \\
7 \cdot 8+1\end{array}$ & $5.1+1$ & $\begin{array}{l}\text { MRY E F } \\
\text { KFK E V } \\
\text { FSU E F } \\
\text { FSU E F } \\
\text { MST E S } \\
\text { MST E L } \\
\text { LYO T E }\end{array}$ & $\begin{array}{l}\text { Moylet } \\
\text { Vidal-Quadrast } \\
\text { Porhst } \\
\text { Porkst } \\
\text { Lohnert } \\
\text { Lohnert } \\
\text { Billereyt }\end{array}$ & $\begin{array}{r}567 \\
6876 \\
9569 \\
9570 \\
7302 \\
7330 \\
10430\end{array}$ \\
\hline${ }^{8} \mathbf{L I}_{1}$ & product & pleid & & & & \\
\hline 180 & $1 \theta_{0}$ & $9.1+1$ & & PAR E C & Detraz+ & 11650 \\
\hline${ }^{B_{\mathbf{L}}}$ & relative & thick & target & $t$ yield & & \\
\hline${ }^{10} \mathrm{Be}$ & $\mathfrak{\imath}$ & $1.2+1$ & & PEN E & Alburgert & 6047 \\
\hline${ }^{8} \mathrm{LI}$ & $O(E)$ & & & & & \\
\hline $\begin{array}{l}{ }^{7} \mathrm{LI} \\
{ }^{4}{ }^{0} \mathrm{Cg} \\
{ }^{4}{ }^{4} \mathrm{Cg}\end{array}$ & $\begin{array}{l}\text { d } \\
\text { a } \\
0\end{array}$ & $\begin{array}{l}6.1-1 \\
1.0+2 \\
1.0+2\end{array}$ & $2.0+0$ & $\begin{array}{l}\text { BYU E S } \\
\text { MST E L } \\
\text { MST E L }\end{array}$ & $\begin{array}{l}\text { Schillingt } \\
\text { Lohnert } \\
\text { Lohnert }\end{array}$ & $\begin{array}{l}5271 \\
7302 \\
7330\end{array}$ \\
\hline${ }^{9} \mathrm{LI}$ & pertlal & $\theta(E)$ & & & & \\
\hline${ }^{3} \mathrm{LI}$ & $\mathrm{t}$ & $5.0+0$ & $6.0+0$ & CCP E & Abromovicht & 6041 \\
\hline${ }^{8} \mathrm{LI}$ & relotive & thlck & torget & $t$ yield & & \\
\hline${ }^{10} \mathrm{Be}$ & $\mathbf{t}$ & $1.2+1$ & & PEN E & Alburgert & 6048 \\
\hline${ }^{9} \mathrm{~L} 1$ & $\theta(E)$ & & & & & \\
\hline $\begin{array}{l}{ }^{3} H \\
{ }^{7} \mathrm{LI}\end{array}$ & $\begin{array}{l}{ }^{7} L i \\
1\end{array}$ & $\begin{array}{l}3.0+1 \\
5.0+0\end{array}$ & $6.0+0$ & $\begin{array}{l}\text { FSU E } \\
\text { CCP E }\end{array}$ & $\begin{array}{l}\text { Delaune. } \\
\text { Abramovicht }\end{array}$ & $\begin{array}{l}9386 \\
6040\end{array}$ \\
\hline$z=4$ & binars & $\sigma(E)$ & & & & \\
\hline 160 & $p$ & $2.0+1$ & $1.0+2$ & INU T & Compani-Tabrizi & 565 \\
\hline$z=4$ & spalla & ation of & (E) & & & \\
\hline $12 \mathrm{C}$ & p & $4: 5+1$ & $1.0+21$ & MRY E F & Matheost & 309 \\
\hline$Z=4$ & $\sigma(E) \times 5$ & sector & & & & \\
\hline $\begin{array}{l}200 \mathrm{~Pb} \\
200 \mathrm{~Pb}\end{array}$ & $\begin{array}{l}160 \\
160\end{array}$ & $\begin{array}{l}1.4+2 \\
1.4+2\end{array}$ & $\begin{array}{l}3.2+2 \\
3.2+2\end{array}$ & $\begin{array}{l}\text { BRK E } \\
\text { MSU R }\end{array}$ & $\begin{array}{l}\text { Geloket } \\
\text { Gelbke. }\end{array}$ & $\begin{array}{l}11538 \\
11539\end{array}$ \\
\hline
\end{tabular}

\begin{tabular}{|c|c|c|c|c|c|c|}
\hline Target & $\operatorname{lnc}$ & $\begin{array}{l}E_{m} \mid n \\
(M e V)\end{array}$ & $\begin{array}{l}\text { Emax } \\
\text { (MeV) }\end{array}$ & Lab & Author & No. \\
\hline \multicolumn{7}{|l|}{$z=4$} \\
\hline $12 \mathrm{c}$ & $p$ & $9.0+1$ & & LPI & T Dolteleyt & 312 \\
\hline $12 \mathrm{c}$ & p & $9.0+1$ & & LPI & T Daltrlevt & 313 \\
\hline $12 \mathrm{c}$ & $p$ & $9.0+1$ & & LPI & T Onitrievt & 314 \\
\hline $12 \mathrm{c}$ & p & $9.0+1$ & & LPI & T Daitriert & 322 \\
\hline $12_{c}$ & $b^{0}$ & $9.0+1$ & & LPI & T Dotitriert & 323 \\
\hline $12 c$ & p & $3.0+1$ & & LPI & T Dafierievt & 331 \\
\hline $12_{C}$ & $\operatorname{la}_{C}^{p}$ & $4.5+1$ & $2.0+2$ & TAM & E Namboodieit & 9770 \\
\hline $1 e_{c}$ & $14 \mathrm{~N}$ & $8.6+1$ & $1.8+20$ & ORL & E Del-Campot & 10319 \\
\hline $12 c$ & 160 & $4.0+1$ & $6.6+11$ & NOT & E Malaint & 10722 \\
\hline${ }^{48} \mathrm{co}$ & 150 & $5.6+1$ & & ANL. & E Kovort & 11107 \\
\hline${ }^{45} \mathrm{Sc}$ & $22_{\mathrm{Ne}}$ & NDG & & LYO & T Blllereyt & 11924 \\
\hline $2 I^{2}$ & 160 & $7.7+1$ & & GRE & E Chevartert & 11142 \\
\hline $50 \mathrm{cr}$ & $14 \mathrm{~N}$ & $7.1+1$ & $1.0+2 L$ & LYO & T Billereyt & 10393 \\
\hline $\mathrm{So}^{\circ} \mathrm{Cr}$ & $23_{\mathrm{Ne}}$ & NDG & & LYO & T Billereyt & 11925 \\
\hline${ }^{53} \mathrm{Mn}$ & $12 \mathrm{C}$ & $7.7+1$ & & GRE & E Chevarlert & 9944 \\
\hline${ }^{56} \mathrm{Fe}$ & 160 & NDG & & LYO & T Billereyt & 11162 \\
\hline${ }^{56} \mathrm{Fe}$ & 160 & $7.7+1$ & & GFE & E Chevarlert & 11163 \\
\hline $60^{\mathrm{Ni}}$ & $12 \mathrm{c}$ & NDG & & LYO & T Billereyt & 9960 \\
\hline $80^{8 i}$ & $1 a_{c}$ & $7.7+1$ & & GRE & E Chevarlert & 9961 \\
\hline $197_{A U}$ & $12_{c}$ & $1.3+2$ & & KFK & C Muenzelt & 10095 \\
\hline $197 \mathrm{Au}$ & 160 & $1.4+2$ & $1.7+2 x$ & KFK & C Muenzolt & 11481 \\
\hline \multicolumn{7}{|c|}{ compound-nucleus $O(E) \times f a c t$ or } \\
\hline${ }^{7} \mathrm{LI}$ & p & $1.8+0$ & $2.0+0$ & CBR & D Barker. & 121 \\
\hline \multicolumn{7}{|c|}{ ompound-nucleus otE). } \\
\hline $12 \mathrm{c}$ & $14 \mathrm{~N}$ & $8.7+1$ & $1.6+2$ & TAM & M Stoksteadt & 10282 \\
\hline $12 c$ & $14 n$ & $\begin{array}{l}8.7+1 \\
8.7+1\end{array}$ & $1.0+5$ & TAM I & M Stoksteadt & $\begin{array}{l}10205 \\
10283\end{array}$ \\
\hline $12 \mathrm{c}$ & $14 \mathrm{~N}$ & $8.7+1$ & & TAM : & M Stohsteadt & 10284 \\
\hline $12 c$ & ${ }^{14} \mathrm{~N}$ & $8.7+1$ & & TAM I & M Stoksteadt & 10285 \\
\hline \multicolumn{7}{|c|}{${ }^{7} \mathrm{Bg} \quad \sigma(E)$} \\
\hline${ }^{6} L_{1}$ & $\sigma_{\mathrm{LI}}$ & $3.0+0$ & $1.5+1$ & BRK & E Wong. & 9164 \\
\hline${ }^{7} \mathrm{Be}$ & portiol & $\theta(E ; \theta)$ & & & & \\
\hline${ }^{2} L_{1}$ & $\rho$ & TR & +0 & BNL & R Goldberg. & 123 \\
\hline \multicolumn{7}{|c|}{ partial rapo(E) } \\
\hline$z_{L I}$ & p & $3.1+0$ & $4.9+0 \mathrm{r}$ & KTY & E Brandenbergert & 122 \\
\hline
\end{tabular}




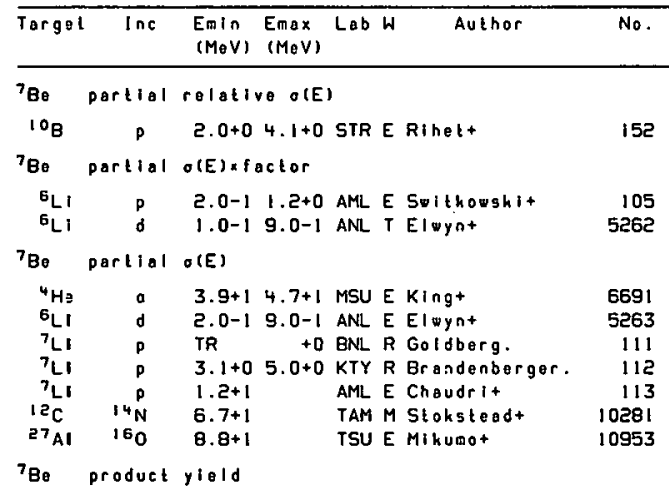

$180 \quad 180 \quad 9.1+1 \quad$ PARE Detrazt 11650

'Be thich target yleldafictor

$\begin{array}{clllll}\text { C } & 14 \mathrm{~N} & 3.5+1 & 5.8+1 & \text { FSU E Holub+ } & 10247 \\ 27 & 16 \mathrm{O} & 3.5+1 & 5.8+1 \text { FSU E Holubt } & 10924\end{array}$

$\begin{array}{llll}\text { S1 } 1{ }^{4} \mathrm{~N} & 3.8+15.8+1 \text { FSU E Holubt } & 10924 \\ & & \end{array}$

7Be thick target yiold

\begin{tabular}{|c|c|c|c|c|c|c|}
\hline LI & p & $2.8+0$ & $2.2+1$ & FEI & E Dmitrievt & 86 \\
\hline Li & d & $7.5+0$ & $2.2+1$ & FEI & E Datriert & 5214 \\
\hline${ }^{6} \mathrm{LI}$ & d & $1.0-1$ & $1.8-1$ & KOS & M Szabot & 5264 \\
\hline${ }^{9} \mathrm{Be}$ & p & $2.2+1$ & & FEI & E Datirievt & 138 \\
\hline${ }^{9} \mathrm{Be}$ & d & $2.2+1$ & & FEI & E Dmlirievt & 5284 \\
\hline${ }^{9} B_{\theta}$ & - & $4 \cdot 3+1$ & & FEI & E Doltrient & 6712 \\
\hline $\mathbf{\theta}$ & p & $2.8+0$ & $2.2+1$ & FEI & E Daftrleyt & 146 \\
\hline$\theta$ & d & $7.0+0$ & $2.2+1$ & FEI & E Dmitrievt & 5289 \\
\hline 100 & & $7.6-2$ & $2.9+0$ & CLU & M Roughtont & 153 \\
\hline${ }^{12} \mathrm{C}$ & $19 \%$ & $2.5+1$ & $7.5+1$ & FSU & M Zellert & 11745 \\
\hline${ }^{13} \mathrm{C}$ & ${ }^{19} \mathrm{~F}$ & $2.5+1$ & $7.5+1$ & FSU & M Zellert & 11773 \\
\hline $27 \mathrm{Al}$ & a & $3.8+1$ & $1.6+2$ & JUL & E Probstt & 7042 \\
\hline
\end{tabular}

${ }^{3} \theta_{0}$ raw o(E)

ANL M Meadows.

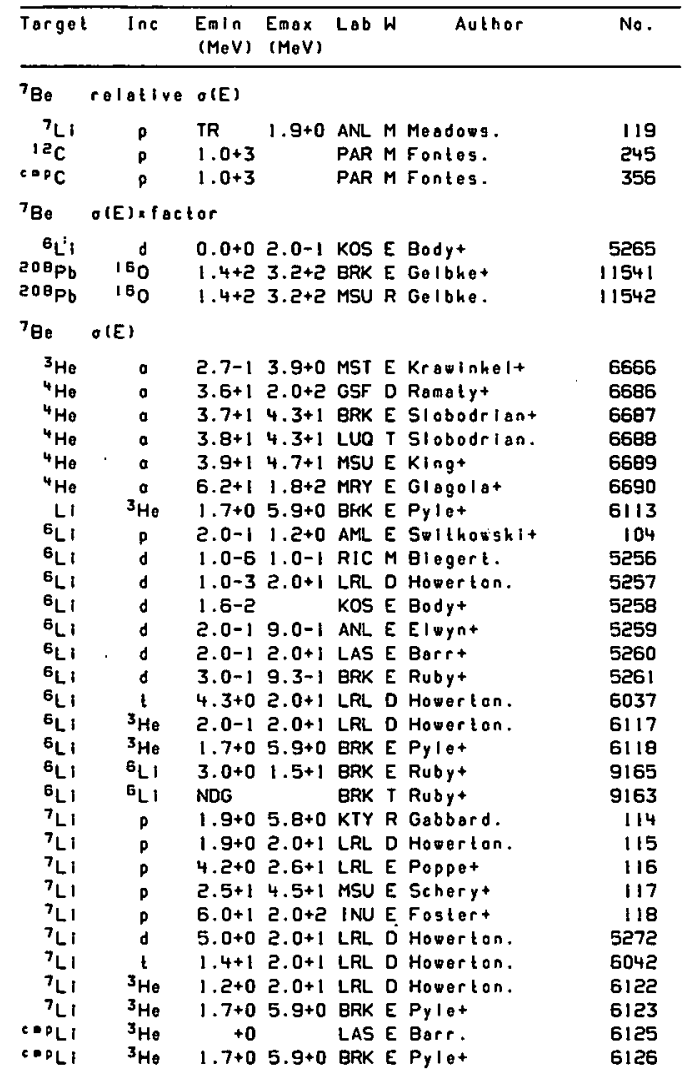




\begin{tabular}{|c|c|c|c|c|c|c|c|}
\hline Target & $\ln c$ & $\begin{array}{l}\text { Emin } \\
(M e V)\end{array}$ & $\begin{array}{l}\text { Emax } \\
(M e V)\end{array}$ & Lab & $W$ & Author & No. \\
\hline \multicolumn{8}{|c|}{$\sigma(E)$} \\
\hline$C O P L i$ & $6_{L i}$ & $2.3+0$ & $1.5+1$ & BRK & E & Rubyt & 9170 \\
\hline${ }^{9} \mathrm{Be}$ & p & $1.5+1$ & $5.2+1$ & TOK & & Inovet & 137 \\
\hline & p & $4.0+0$ & $5.2+1$ & TOK & E & Inouet & 147 \\
\hline $10 \mathrm{~B}$ & p & $7.6-2$ & $2.9+0$ & $\mathrm{CLU}$ & EF & Roughtont & 149 \\
\hline $10_{B}^{\circ}$ & p & $1.0-1$ & $2.0+1$ & LRL & D & Hoverton. & 150 \\
\hline $10_{\mathrm{B}}$ & p & 8.0-1 & $2.5+0$ & FLA & $E C$ & Griggs. & $15 !$ \\
\hline $12 \mathrm{C}$ & $p$ & $2.5+1$ & $1.6+2$ & BNL & E & Arminit & 241 \\
\hline $12_{\mathrm{C}}$ & p & 5. $0+1$ & $2.8+4$ & BNL & D & Cumming. & 242 \\
\hline $12 \mathrm{C}$ & p & $5 \cdot 0+1$ & $2.8+4$ & KFK & c & Muenzelt & 243 \\
\hline $1 \mathrm{ac}_{\mathrm{c}}$ & p & $1.0+2$ & & MRY & E & Rochet & 244 \\
\hline $1{ }^{2} \mathrm{C}$ & p & $5.0+1$ & & LPI & E & Voovint & 246 \\
\hline${ }^{12} \mathrm{c}$ & p & 5. $0+1$ & & LPI & $\bar{E}$ & Vdovint & 247 \\
\hline${ }^{12} \mathrm{C}$ & $p$ & $5.0+1$ & & LPI & $\vec{E}$ & Vdovint & 248 \\
\hline $12_{C}$ & ${ }^{3} \mathrm{He}$ & $0.0+0$ & $3.1+1$ & BRK & E & Papet & 6138 \\
\hline${ }^{12} \mathrm{C}$ & $a$ & $9.2+2$ & & LPI & T & Dmitrient & 6750 \\
\hline${ }^{12} \mathrm{C}$ & a & $3.3+1$ & $5.1+1$ & $\mathrm{KFX}$ & E & vidal-Ouadrast & 6781 \\
\hline $\mathrm{N}$ & p & $1.1+1$ & $5.2+1$ & TOK & $\bar{E}$ & Inouet & 361 \\
\hline${ }^{14} \mathrm{~N}$ & p & $5.0+1$ & & LPI & $E$ & Vdovint & 382 \\
\hline $14 \mathrm{~N}$ & p & $5.0+1$ & & LPI & E & Vdovint & 383 \\
\hline $14 \mathrm{~N}$ & p & $5.0+1$ & & LPI & $\vec{E}$ & Vdovint & 384 \\
\hline $14 \mathrm{~N}$ & p & $5.0+1$ & & LPI & $E$ & Voovint & 385 \\
\hline $14 \mathrm{~N}$ & p & $5.0+1$ & & LPI & $E$ & & 386 \\
\hline $14 \mathrm{~N}$ & p & $5.0+1$ & & LPI & E & Vdovint & 387 \\
\hline $14 \mathrm{~N}$ & p & $5.0+1$ & & LPI & $E$ & & 388 \\
\hline $14 \mathrm{~N}$ & p & $5.0+1$ & & LPI & E & voovint & 389 \\
\hline $14 \mathrm{~N}$ & a & $5.1+1$ & & KFK & $\varepsilon$ & vidal-Ouadrast & 6795 \\
\hline $14 \mathrm{~N}$ & a & $4 \cdot 3+1$ & $5 \cdot 1+1$ & KFK & $E$ & vidal-Quadrast & 6796 \\
\hline $14 \mathrm{~N}$ & $a$ & $3.3+1$ & $5.1+1$ & KFK & E & vidal-Quadrast & 6820 \\
\hline 0 & p & $2.8+1$ & $.5 .2+1$ & TOK & E & Inouet & 472 \\
\hline 150 & $p$ & $1.6+2$ & & LPI & 1 & Dafilitieyt & 498 \\
\hline 150 & p & 5. $0+1$ & & LPI & $E$ & Vdovint & 499 \\
\hline 160 & p & $5.0+1$ & & LPI & $\vec{E}$ & Vourint & 500 \\
\hline 150 & p & $9.0+1$ & & MRY & $E$ & Moylet & 567 \\
\hline${ }^{16} 0$ & $\alpha$ & $3 \cdot 3+1$ & $5.1+1$ & KFK & $E$ & vidal-Quadrast & 6876 \\
\hline $\operatorname{COPF}$ & $G_{L 1}$ & $2.3+0$ & $1.5+1$ & BRK & $\vec{E}$ & Rubyt & 9176 \\
\hline $27_{A 1}$ & & $6.1+1$ & & KFK & $\mathrm{c}$ & Muenzelt & 837 \\
\hline $27 \mathrm{Al}$ & p & $6.1+1$ & & ORL & E & WIIllomst & 938 \\
\hline $27_{\mathrm{Al}}$ & p & 8. $2+1$ & $8.0+2$ & PUR & $E$ & Heydeggort & 839 \\
\hline $27_{A I}$ & p & $6.0+2$ & & MTR & & Clinet & 840 \\
\hline $27 \mathrm{Al}$ & a & $5.0+1$ & $1.6+2$ & JUL & $R$ & Stocklint & 7041 \\
\hline
\end{tabular}

\begin{tabular}{|c|c|c|c|c|c|c|}
\hline Terget & $\ln c$ & $\begin{array}{l}E \mathrm{~m} / \mathrm{n} \\
(\mathrm{MeV})\end{array}$ & $\begin{array}{l}\text { Emax } \\
(\mathrm{MeV})\end{array}$ & Lab & Author & No. \\
\hline${ }^{7} \mathrm{Be}$ & $\sigma(E)^{\circ}$ & & & & & \\
\hline 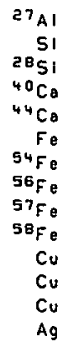 & 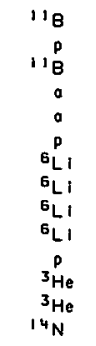 & $\begin{array}{l}5 \cdot 0+1 \\
4 \cdot \theta+1 \\
5 \cdot 0+1 \\
1 \cdot 0+2 \\
1 \cdot 0+2 \\
5 \cdot 9+2 \\
9 \cdot 8+0 \\
6 \cdot 5+1 \\
6 \cdot 5+1 \\
6 \cdot 5+1 \\
5 \cdot 9+2 \\
3 \cdot 9+1 \\
4 \cdot 0+1 \\
7 \cdot 8+1\end{array}$ & $\begin{array}{r}9.4+1 \\
8.5+1 \\
8.5+1 \\
8.5+1 \\
6.9+1 \\
7.0+1 \\
1.1+2\end{array}$ & $\begin{array}{l}\text { FSU } \\
\text { UPP } \\
\text { FSU } \\
\text { MST } \\
\text { MST } \\
\text { LAS E } \\
\text { INU E } \\
\text { INU E } \\
\text { INU E } \\
\text { INU E } \\
\text { LAS E } \\
\text { KFK O } \\
\text { ANL E } \\
\text { LYO }\end{array}$ & $\begin{array}{l}\text { E Parhst } \\
\text { E Lundavist+ } \\
\text { E Parhst } \\
\text { E Lohnert } \\
\text { E Lohnert } \\
\text { E Ortht } \\
\text { E Jastrzebshit } \\
\text { E Jastrzebshit } \\
\text { E Singht } \\
\text { E Josirzebshit } \\
\text { E Ortht } \\
\text { C Muenzel+ } \\
\text { E Golcherlt } \\
\text { T Billereyt }\end{array}$ & $\begin{array}{c}9569 \\
959 \\
9570 \\
7302 \\
7330 \\
1740+ \\
9185 \\
9204 \\
9206 \\
9207 \\
2464+ \\
6287 \\
6280+ \\
10430\end{array}$ \\
\hline${ }^{8} \mathrm{Be}$ & compound & d-nucleus & US $\sigma(E)$ & & & \\
\hline $\begin{array}{l}10 \mathrm{~B} \\
1{ }^{12} \mathrm{C}\end{array}$ & $\begin{array}{l}7_{\mathrm{T}}^{7} \mathrm{I} \\
{ }^{3} \mathrm{He}\end{array}$ & $\begin{array}{l}2.4+1 \\
3.0+1\end{array}$ & & $\begin{array}{l}\text { ME }] ~ \\
\text { BRK E }\end{array}$ & $\begin{array}{l}\text { M Kohlert } \\
\text { E Popet }\end{array}$ & $\begin{array}{l}9394 \\
6140\end{array}$ \\
\hline${ }^{8} \mathrm{Be}$ & partial & compoun & nd-nucle & leus & $\sigma(E)$ & \\
\hline${ }^{1 / B}$ & $\frac{P}{\text { olrect- }}$ & $\begin{array}{l}3.0+0 \\
\text { Interact }\end{array}$ & $\begin{array}{l}8.0+0 E \\
\text { tion ote }\end{array}$ & $\begin{array}{l}B O C \\
\text { (E) }\end{array}$ & T Borcherst & 169 \\
\hline $\begin{array}{l}10 \mathrm{~B} \\
1{ }^{12} \mathrm{C}\end{array}$ & $\begin{array}{l}{ }^{3} \mathrm{LI} \\
{ }^{3} \mathrm{He}\end{array}$ & $\begin{array}{l}2.4+1 \\
3.0+1\end{array}$ & & $\begin{array}{l}\text { HEI } \\
\text { BRK E }\end{array}$ & $\begin{array}{l}\text { M Kohlert } \\
\text { E Popet }\end{array}$ & $\begin{array}{l}9393 \\
5139\end{array}$ \\
\hline${ }^{8} \mathrm{Be}_{0}$ & portiol & product & $1 y \operatorname{lold}$ & & & \\
\hline $\begin{array}{l}{ }^{7} \mathrm{LI} \\
{ }^{8} \mathrm{Be}\end{array}$ & $\begin{array}{c}p \\
\text { portial }\end{array}$ & $\begin{array}{c}2.2+1 \\
\text { Co }\end{array}$ & & TNLE & E Blott+ & 125 \\
\hline $\begin{array}{l}{ }^{4} \mathrm{He} \\
{ }^{6} \mathrm{Li}\end{array}$ & d & $\begin{array}{l}3.3+1 \\
6.8+0\end{array}$ & $\begin{array}{l}3.5+15 \\
7.1+0\end{array}$ & $\begin{array}{l}\text { HEI E } \\
\text { STB E }\end{array}$ & $\begin{array}{l}\text { E Peul+ } \\
\text { E Noet }\end{array}$ & $\begin{array}{l}6693 \\
5266\end{array}$ \\
\hline${ }^{8} \mathrm{Be}$ & portial & relotive & ve o(E) & & & \\
\hline $\begin{array}{l}{ }^{4} \mathrm{He} \\
{ }^{7} \mathrm{Li} \\
{ }^{7} \mathrm{LI} \\
{ }^{\circ}{ }^{\circ} \mathrm{B}\end{array}$ & $\underset{d}{a} \underset{j}{a}$ & $\begin{array}{l}1.6+1 \\
1.6+0 \\
1.6+0 \\
1.6+0\end{array}$ & $\begin{array}{l}1.7+1 \\
2.2+0 \\
2.2+0 \\
2.2+0\end{array}$ & $\begin{array}{l}\text { YAL } \\
\text { CBR D } \\
\text { CBR D } \\
\text { CBR D }\end{array}$ & $\begin{array}{l}\text { E Longt } \\
\text { D Borker. } \\
\text { D Borker. } \\
\text { D Berker. }\end{array}$ & $\begin{array}{r}6692 \\
124 \\
6124 \\
5291\end{array}$ \\
\hline
\end{tabular}

25

693

692

6124

531 


\begin{tabular}{llll}
\hline Target Inc & $\begin{array}{c}\text { Emin } \\
\text { (MeV) }\end{array}$ & Emax Lab $(\mathrm{MeV}:$ & Author \\
\hline
\end{tabular}

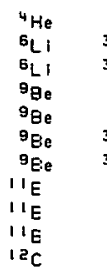

3.2+1 3.6+1 PTN $E$ Bovlest

He 4.0-1 2.0+0 ANL E Hollandt

3.0+0 6.0+0 TNL E Gould+

2.4-1 9.5-1 WIT E Annegarn.

d. $1.2+1 \quad 1.4+1$ KTO $E$ Tanaha.

5. $0+0 \quad 1.0+1$ STR E Bilwest

5. $0-15 \cdot 0+1$ CUL E Bucht

$2.0+15.0+1$ MIL T Gadiolit

$1.7+1 \quad 3.3+1$ STR E Brocherd

${ }^{8} B$ e $O\left(E_{i} E^{\prime}\right)$.

${ }^{9} \mathrm{ge} \quad$ a $4.3+0 \quad 7.4+0$ OTC D Gelgert

6120

615

5286

6127

6128

166
167

167

6751

$6713+$

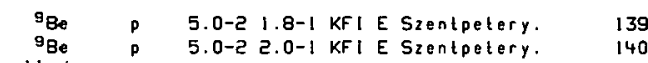

${ }^{8} \mathrm{Be}$ partial o(E)

$1.8+03.0+C$ KTO E Sahamoto

Be $\sigma(E) \times f a c t o r$

\begin{tabular}{|c|c|c|c|c|c|c|}
\hline Target & $\ln c$ & $\begin{array}{l}\text { Emin } \\
(\mathrm{MeV})\end{array}$ & $\begin{array}{l}E_{m B x} \\
(\operatorname{MeV})\end{array}$ & Lab W & Author & No. \\
\hline
\end{tabular}

\begin{tabular}{|c|c|c|c|}
\hline $12_{C} C$ & & & \\
\hline$\because \bar{C}$ & $p$ & $1.0+3$ & PAR M Fontes. \\
\hline
\end{tabular}

${ }^{8} \mathrm{Be}$ o(E) x factor

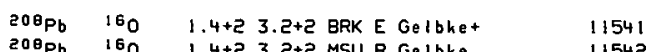

日a $\sigma(E) \quad 1.4+23.2+2$ MSU R Gelbke.

${ }^{10} \mathrm{~B} \quad 7 \mathrm{~L} \quad 2.4+1 \quad H E I M$ Kohlert 9395

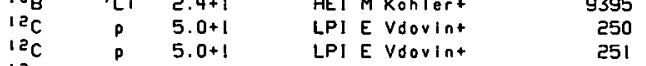

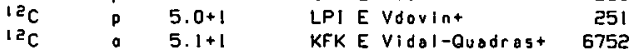

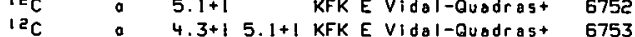

$12 \mathrm{C} a \mathrm{C} .3+15.1+1$ KFK E Vidal-Quedrast 6781

118 P 5.0-2 2.0-1 KFIE Szentpetery. 170

${ }^{B} \mathrm{Be} \quad \sigma(E)$

$6 \mathrm{Li}{ }^{3} \mathrm{He} \quad-1$ to ANL E Elwynt 6119

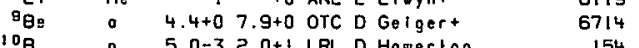

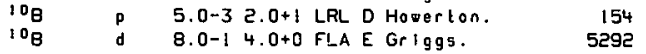

${ }^{9} B e$ compound-nucleus olE

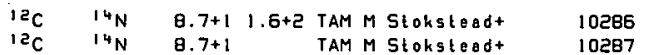

$\begin{array}{lllll}12 \mathrm{C} & 14 \mathrm{~N} & 8.7+1 & \text { TAM M Stokstead+ } & 10287 \\ 12 \mathrm{C} & 14 \mathrm{~N} & 8.7+1 & \text { TAM M Stokstead+ } & 10288\end{array}$

$\begin{array}{llll}{ }^{12} \mathrm{C} & 14 \mathrm{~N} & 8.7+1 & \text { TAM M Stoksteadt } 10289\end{array}$

${ }^{9} \mathrm{Be}$ partial o(E)xfoctor

${ }^{9}$ Be p $4.0+06.0+0$ kTO E Yasuet

${ }^{\theta}$ Be partial o(E)

27Al ${ }^{160}$ 8.8+1 TSU E Mikumo+ 10953

${ }^{3} \mathrm{Be}$ product yield

${ }^{180} \quad{ }^{180} \quad 9.1+1 \quad$ PAR E Detraz+ 11650 


\begin{tabular}{|c|c|c|c|c|c|c|c|}
\hline & Target & $\ln c$ & $\begin{array}{l}\text { Emin } \\
(M e V)\end{array}$ & $\begin{array}{l}E_{\operatorname{mox}} \\
(\operatorname{Mov})\end{array}$ & Lob & Author & No. \\
\hline \multirow[t]{10}{*}{ - } & ${ }^{10} \mathrm{Bg}$ & direct-it & interac & ction. & $\sigma(E)$ & & \\
\hline & ${ }^{10} \mathrm{~B}$ & ${ }^{3} \mathrm{LI}$ & $2.4+1$ & & HEI & M Kohlert & 9396 \\
\hline & 1000 & partlal & $\theta(E)$ & & & & \\
\hline & ${ }^{9} \mathrm{Be}$ & $ه$ & $1.0+0$ & & WIT E & E Selischopt & 5287 \\
\hline & $10 \mathrm{Bg}$ & product & yield & & & & \\
\hline & $10_{0}$ & 180 & $9.1+1$ & & PAR & E Detrazt & 11650 \\
\hline & ${ }^{10} \mathrm{B \theta}_{0}$ & relative & $\sigma(E)$ & & & & \\
\hline & $\begin{array}{l}12 C \\
c=0 C\end{array}$ & $\begin{array}{l}\text { p } \\
\text { p }\end{array}$ & $\begin{array}{l}1.0+3 \\
1.0+3\end{array}$ & & $\begin{array}{l}\text { PAR } \\
\text { PAR }\end{array}$ & $\begin{array}{l}M \text { Fontes. } \\
\text { M Fontes. }\end{array}$ & $\begin{array}{l}252 \\
358\end{array}$ \\
\hline & ${ }^{10} \mathrm{Be}$ & $O(E) \times 10 C$ & ctor & & & & \\
\hline & $\begin{array}{l}20 \theta^{2} \mathrm{~Pb} \\
200_{\mathrm{Pb}}\end{array}$ & $\begin{array}{l}160 \\
160\end{array}$ & $\begin{array}{l}1.7+2 \\
1.4+2\end{array}$ & $\begin{array}{l}3.2+2 \\
3.2+2\end{array}$ & $\begin{array}{l}\text { BRK } \\
\text { MSU F }\end{array}$ & $\begin{array}{l}\text { E Gelbhet } \\
\text { R Gelbke. }\end{array}$ & $\begin{array}{l}11541 \\
11542\end{array}$ \\
\hline & ${ }^{10} \mathrm{Be}$ & $O(E)$ & & & & & \\
\hline & $10_{B}$ & ${ }^{7} \mathrm{LI}$ & $2.4+1$ & & HEI $\mathrm{N}$ & M Kohlert & 9398 \\
\hline & $12 \mathrm{C}$ & $p$ & $1.0+2$ & & MRY & E Rochet & 253 \\
\hline & $12 \mathrm{C}$ & p & $5.0+1$ & & LPI E & E Vdovint & 254 \\
\hline & $12 \mathrm{c}$ & a & $4.3+1$ & $5.1+1$ & $\mathrm{KFK}$ & E Vidol-Ouadrast & 6754 \\
\hline & $12 \mathrm{c}$ & a & $3 \cdot 3+1$ & $5.1+1$ & KFK & Evidal-auadrast & 6781 \\
\hline & $14 \mathrm{~N}$ & $p$ & $5.0+1$ & & LPI E & E vdovint & 395 \\
\hline & $14 N$ & $p$ & $5.0+1$ & & LPI E & Evdovint & 396 \\
\hline & ${ }^{14} \mathrm{~N}$ & a & $4,3+1$ & $5.1+1$ & $\mathrm{KFK}$ & E Vidal-Ouadrast & 6799 \\
\hline & $14 \mathrm{~N}$ & a & $5.1+i$ & & KFK & E vidal-0uadrast & 6800 \\
\hline & ${ }^{14} \mathrm{~N}$ & a & $3 \cdot 3+1$ & $5.1+1$ & KFK E & E videl-Quadrast & 6820 \\
\hline & 150 & $p$ & $1.6+2$ & sent & LPI & T Denitrlevt & 506 \\
\hline & 160 & p & $5.0+1$ & & LPI & E Vdovint & 507 \\
\hline & 150 & $p$ & $9.0+1$ & & MRY E & E Moylet & 567 \\
\hline & 160 & a & $5.1+1$ & & KFK E & E vidal-Ouadrast & 6838 \\
\hline - & 180 & $\circ$ & $3.3+1$ & $5.1+1$ & KFK E & E vidal-Quadrast & 6876 \\
\hline & ${ }^{27} \mathrm{Al}$ & 118 & $5 \cdot 0+1$ & & FSU E & E Parkst & 9569 \\
\hline & ${ }^{28} \mathrm{SI}$ & "в & $5.0+1$ & & FSU E & E Porkst & 9570 \\
\hline & ${ }^{40} \mathrm{Co}$ & a & $1.0+2$ & & MST E & E Lohnert & 7302 \\
\hline & ${ }^{44} \mathrm{Co}$ & a & $1.0+2$ & & MST E & E Lohnert & 7330 \\
\hline & $\mathrm{Ag}$ & $14 \mathrm{~N}$ & $7.8+1$ & $1.1+2$ & LYO & T Billerey+ & 10430 \\
\hline & "Bo & product & ylold & & & & \\
\hline • & 180 & 180 & $9.1+1$ & & PAR E & E Detraz- & 11650 \\
\hline
\end{tabular}

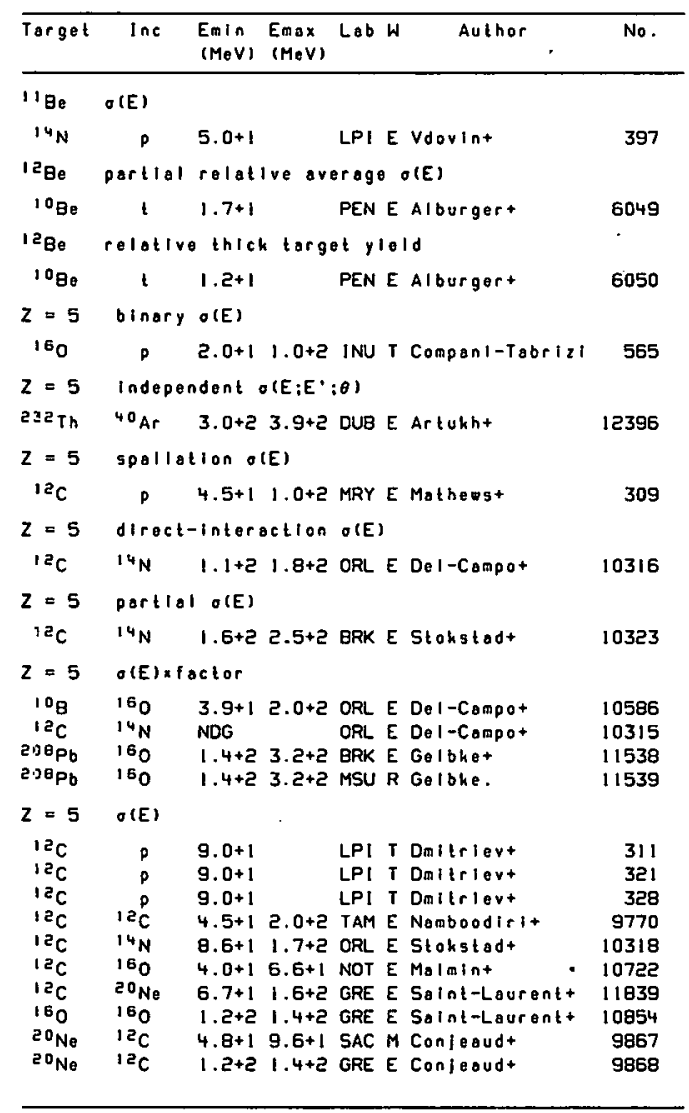




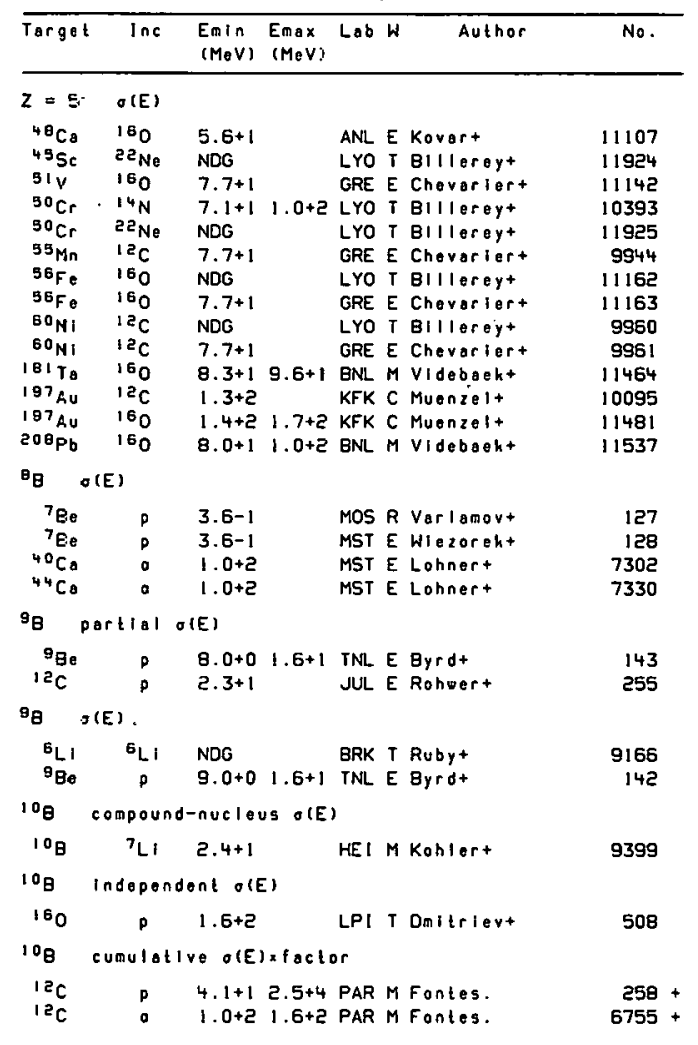

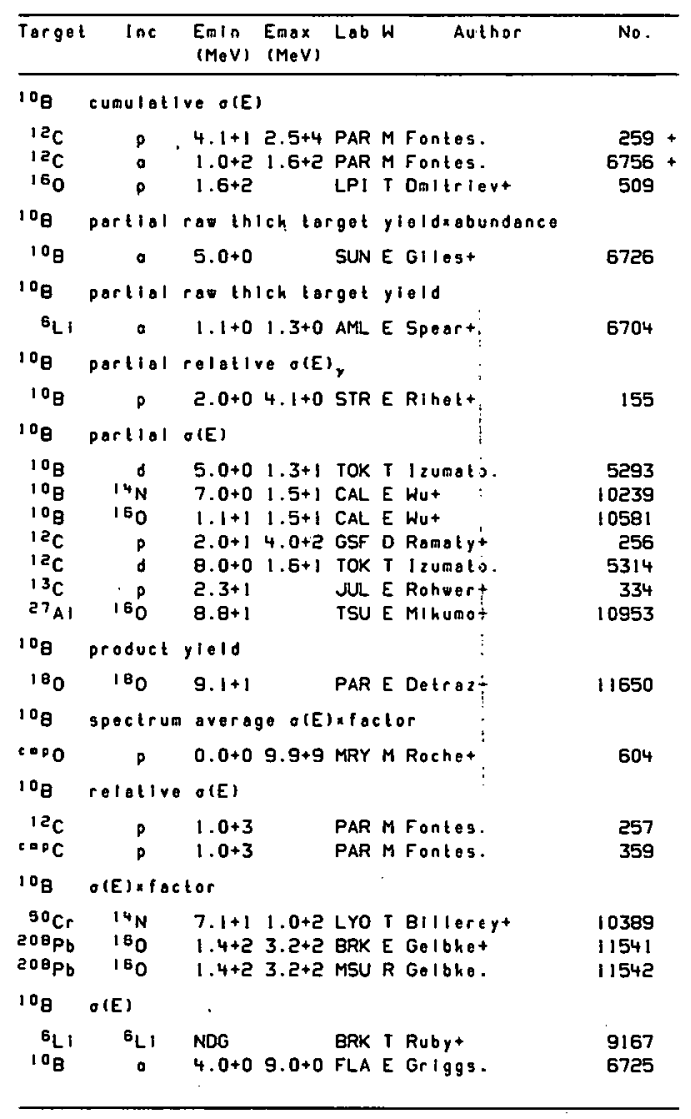




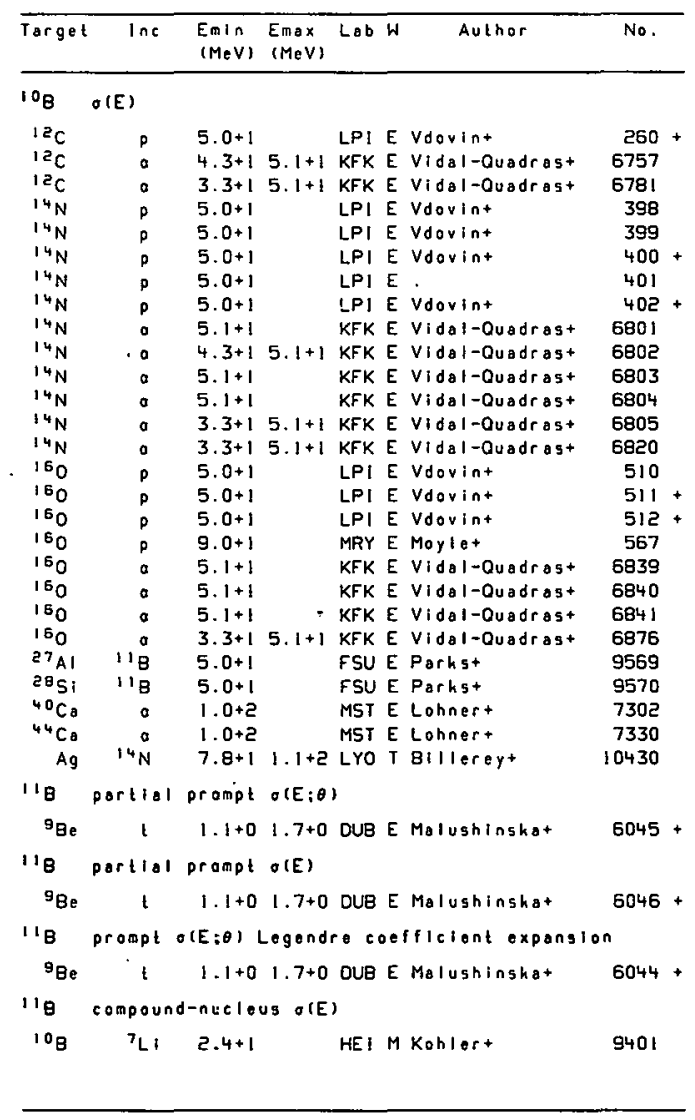

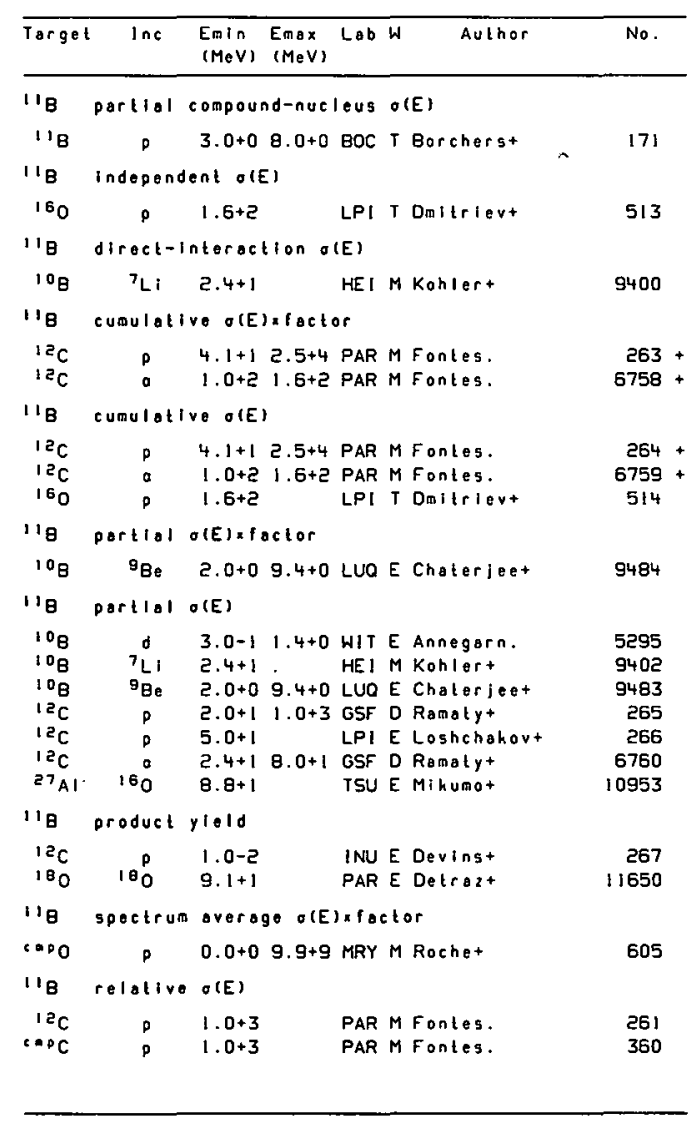




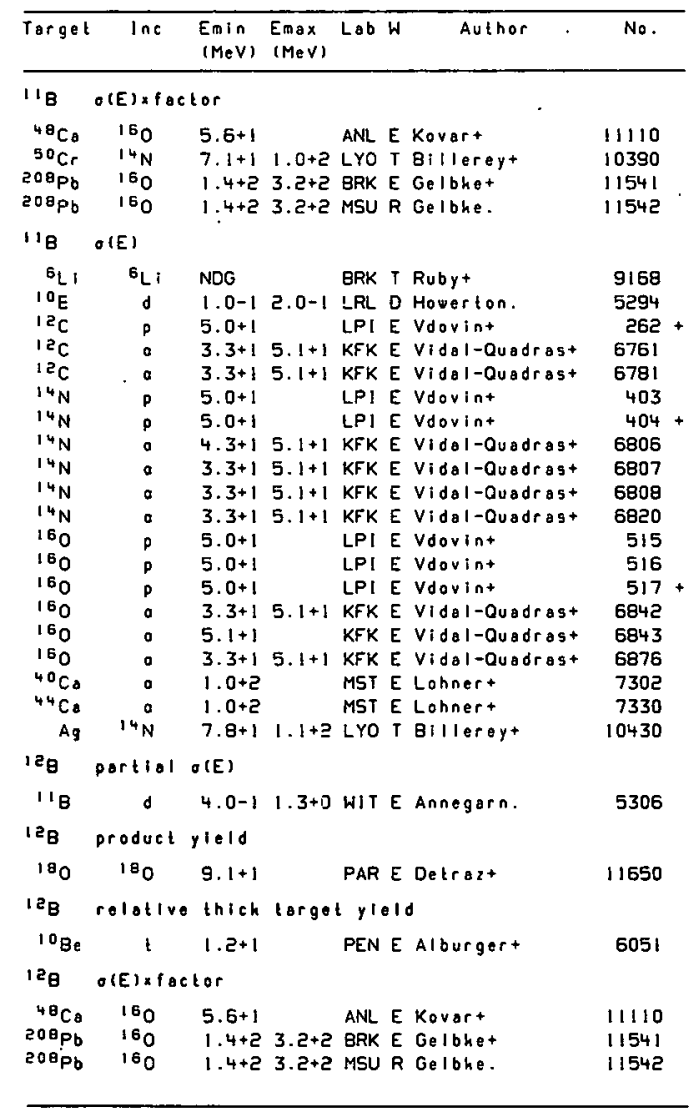

\begin{tabular}{|c|c|c|c|c|c|c|}
\hline Torget & $\ln c$ & $\begin{array}{l}\text { Emin } \\
(M e V)\end{array}$ & $\begin{array}{l}\text { Emax } \\
(\operatorname{MeV})\end{array}$ & Lab W & Luthor & No. \\
\hline 128 & $O(E)$ & & & & & \\
\hline $\begin{array}{l}118 \\
14 \mathrm{~N} \\
160 \\
160 \\
\text { Ag }\end{array}$ & $\begin{array}{r}d \\
p \\
p \\
0 \\
14 \mathrm{~N}\end{array}$ & $\begin{array}{l}1 \cdot 0-1 \\
5 \cdot 0+1 \\
5 \cdot 0+1 \\
5 \cdot 1+1 \\
7 \cdot 8+1\end{array}$ & $2.0+1$ & $\begin{array}{l}\text { LRL } \\
\text { LPI } \\
\text { LPI E } \\
\text { KFK E } \\
\text { LYO }\end{array}$ & $\begin{array}{l}\text { D Howerton. } \\
\text { E Vdovint } \\
\text { E Vdovint } \\
\text { E Vidal-ouadrast } \\
\text { T Billereyt }\end{array}$ & $\begin{array}{r}5305 \\
405 \\
518 \\
6844 \\
10430\end{array}$ \\
\hline${ }^{13} \mathrm{~B}$ & product & yleld & & & & \\
\hline 180 & 180 & $9.1+1$ & & PAR E & E Detrazt & 11650 \\
\hline${ }^{13} B_{B}$ & $O(E) \times f O C$ & etor & & & & \\
\hline $\begin{array}{c}{ }^{48} \mathrm{Ca} \\
{ }^{13} \mathrm{~g}\end{array}$ & $\begin{array}{l}160 \\
\sigma(E)\end{array}$ & $5.6+1$ & & ANL E & E kovart & 11110 \\
\hline TLI & ${ }^{7} \mathrm{Li}$ & $2.6+1$ & & FSU E & E Delaune. & 9388 \\
\hline $14 \mathrm{~B}$ & product & $y / 01 d$ & & & & \\
\hline 180 & 180 & $9.1+1$ & & PAR E & E Detrazt & 11650 \\
\hline$z=6$ & binary & $y O(E)$ & & & & \\
\hline 160 & $p$ & $2.0+1$ & $1.0+2$ & INU T & T Compani-Tabrizi & 565 \\
\hline$z=6$ & indepe & ondont. & OLE;E': & ;(1) & & \\
\hline $232 \mathrm{Th}$ & ${ }^{40} \mathrm{Ar}$ & $3.0+2$ & $3.9+2$ & OUB E & E Artuhtat & 12398 \\
\hline$z=6$ & direct & t-intero & oction & $O(E)$ & & \\
\hline $\begin{array}{l}12 \mathrm{C} \\
20 \mathrm{Ne}\end{array}$ & $\begin{array}{l}14 \mathrm{~N} \\
{ }^{12} \mathrm{C}\end{array}$ & $\begin{array}{l}1.5+5 \\
1.1+5\end{array}$ & $\begin{array}{l}1.8+2 \\
1.4+2\end{array}$ & $\begin{array}{l}\text { ORL E } \\
\text { GRE E }\end{array}$ & $\begin{array}{l}\text { E Del-Campot } \\
\text { E Conjerudt }\end{array}$ & $\begin{array}{r}10316 \\
9869\end{array}$ \\
\hline$z=6$ & portlo & $.1 \sigma(E)$ & & & & \\
\hline${ }^{12} \mathrm{c}$ & $14 \mathrm{~N}$ & $1.6+2$ & $2.5+2$ & BRK E & E Stokstadt & 10323 \\
\hline$z=6$ & produc & ct yiold & & & & \\
\hline $\begin{array}{l}10 \mathrm{~g} \\
12 \mathrm{c}\end{array}$ & $\begin{array}{l}160 \\
14 \mathrm{~N}\end{array}$ & $\begin{array}{l}9.8+1 \\
9.1+1\end{array}$ & & $\begin{array}{l}\text { JCL E } \\
\text { JCL E }\end{array}$ & $\begin{array}{l}\text { E Motobayashit } \\
\text { E Molobayashit }\end{array}$ & $\begin{array}{l}10587 \\
10320\end{array}$ \\
\hline$z=6$ & row ol & (E) & & & & \\
\hline${ }^{12} \mathrm{c}$ & ${ }^{12} \mathrm{C}$ & $4.0+1$ & & MUN E & E Everst & 9773 \\
\hline$z=6$ & $\sigma(E)=f$ & factor & & & & \\
\hline $10 \mathrm{~B}$ & 180 & $3.9+1$ & $2.0+2$ & ORL E & E Del-Compot & 10586 \\
\hline
\end{tabular}




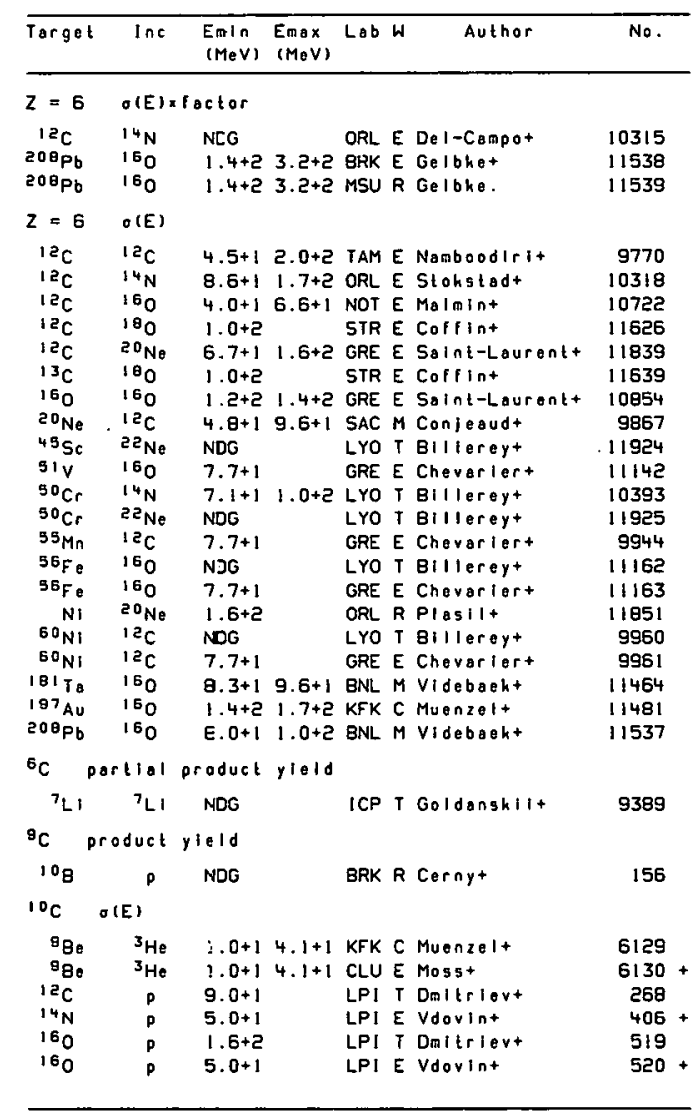

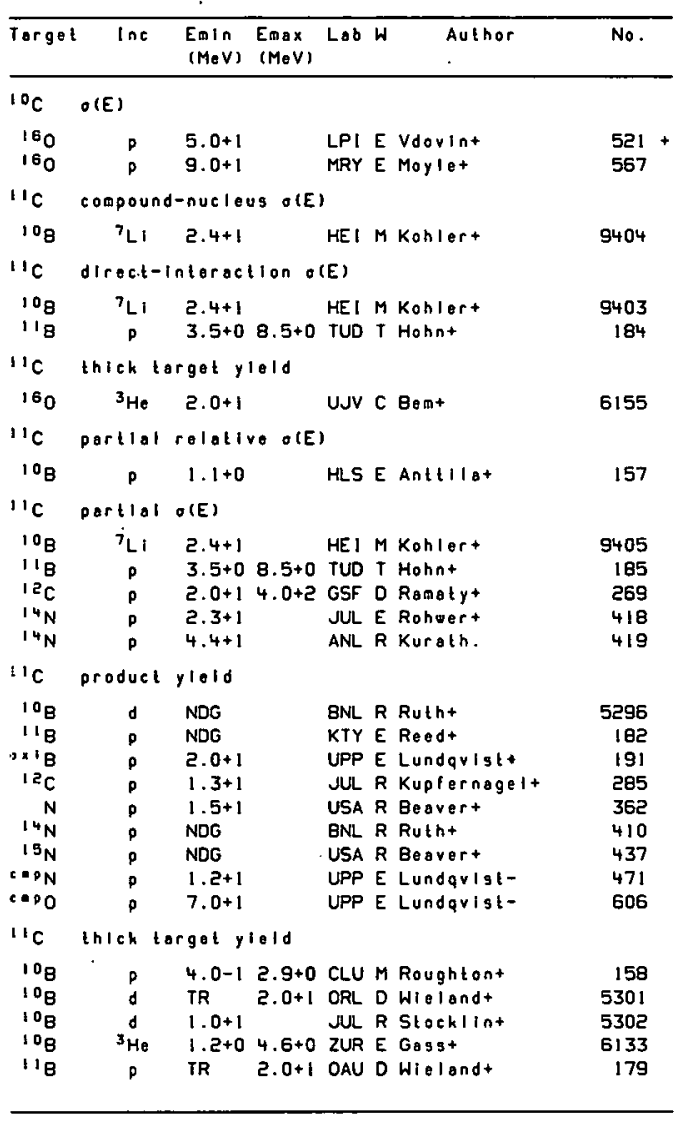




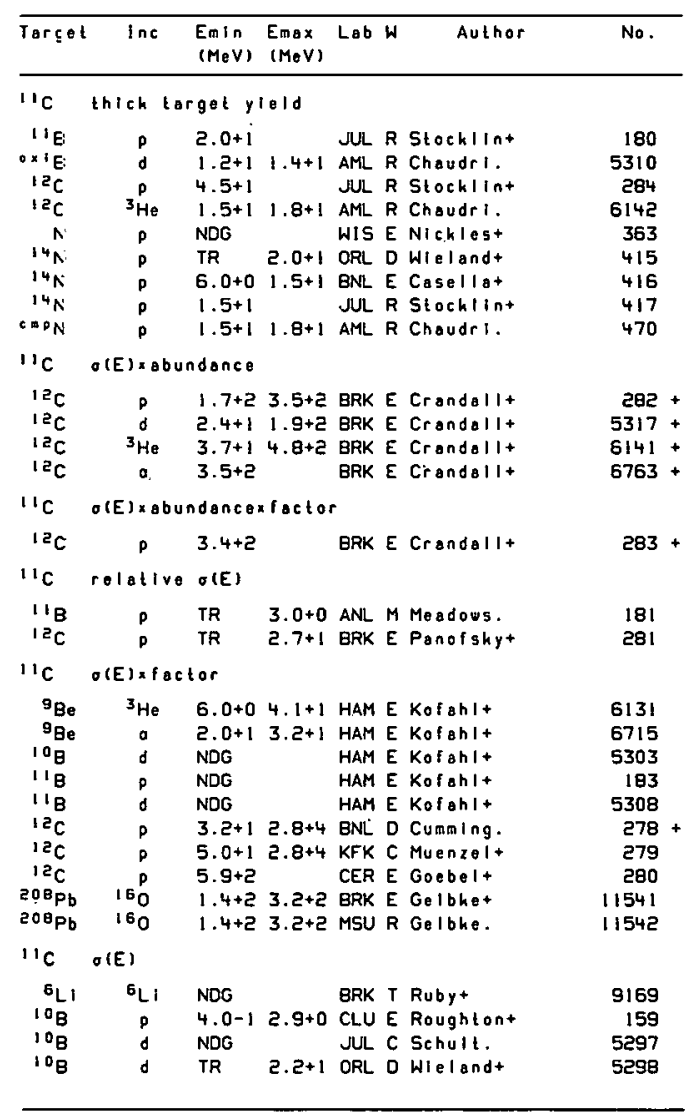

\begin{tabular}{|c|c|c|c|c|c|c|c|c|}
\hline Target & Inc & $\begin{array}{l}\text { EnIn } \\
(M e V)\end{array}$ & $\begin{array}{l}\text { Emax } \\
\text { (MeV) }\end{array}$ & Lab & W & & ther & No. \\
\hline $11 \mathrm{C}$ & $O(E)$ & , & & & & & & \\
\hline${ }^{10} \mathrm{~B}$ & d & $1.0-3$ & $2.0+1$ & LRL & D H & Howert? & on. & 5299 \\
\hline $10_{B}^{\circ}$ & d & $7.5-2$ & $1.7-1$ & USA & $E$ L & Lent & & 5300 \\
\hline $10 \mathrm{~B}$ & ${ }^{3} \mathrm{He}$ & $1.2+0$ & $4.6+0$ & ZUR & E C & Gasst & & 6132 \\
\hline$U^{\prime}$ & p & $\mathrm{TR}$ & $2.2+1$ & OAU & D h & Wielend & $d+$ & 172 \\
\hline " & p & $2.0-1$ & $9.9+1$ & HRV & E r & $\mathrm{HIn} \mathrm{l}_{2}+$ & & 173 \\
\hline$U_{B}$ & p & $2.0-1$ & $9.8+1$ & KFK & C r & Muenzel & $1+$ & 174 \\
\hline 118 & p & $3.0+0$ & $2.0+1$ & LRL & D & Howerto & on. & 175 \\
\hline$I_{B}$ & p & $3.5+0$ & $8.5+0$ & TUD & $\mathrm{TH}$ & Hohnt & & 176 \\
\hline$" 1 B$ & p & $3.0+1$ & $1.1+2$ & MIL & $T$ & Gadiol & $1+$ & 177 \\
\hline "'B & p & $3.6+1$ & $1.5+2$ & PAR & E & Albouyt & & 178 \\
\hline 118 & d & NDG & & JUL & $c$ & Sehult! & & 5307 \\
\hline${ }^{12} \mathrm{C}$ & $p$ & TR & $2.7+1$ & BRK & E & Aamodt & & 272 \\
\hline${ }^{12} \mathrm{C}$ & $p$ & $8.0+0$ & $3.4+2$ & KFK & C & Muenzel & & 273 \\
\hline $12 \mathrm{C}$ & $p$ & $8.5+0$ & $1.0+2$ & $\mathrm{MRV}$ & E & $\operatorname{Hin} t z+$ & & 274 \\
\hline${ }^{12} \mathrm{c}$ & p & $2.0+1$ & $5.0+1$ & ENL & $E$ & Cumming & & 275 \\
\hline${ }^{12} \mathrm{c}$ & $p$ & $3.2+1$ & $2.8+4$ & BNL & 0 & Cumming & & 276 \\
\hline $12_{\mathrm{C}}$ & $p$ & $5.9+2$ & & CER & $E$ & Goobelt & & 277 \\
\hline $12_{C}$ & $p$ & $9.0+1$ & & LPI & $\tau$ & Daterie & evt & 270 \\
\hline $12_{c}$ & p & $0.0+0$ & $4 \cdot 2+1$ & JUL & $R$ & Stocki! & Int & 271 \\
\hline $12 \mathrm{c}$ & o & $2.3+3$ & & KFK & c & Muenze & $1+$ & 5315 \\
\hline${ }^{12} \mathrm{c}$ & d & $2.3+3$ & & SAC & $E$ & Bonolg & $15+$ & 5316 \\
\hline $12 \mathrm{C}$ & 0 & $9.2+2$ & & LPI & $T$ & Dmitrie & eqt & 6762 \\
\hline $12 \mathrm{c}$ & $a$ & $5.1+1$ & & KFK & E & Vidal-o & Quzodrast. & 6764 \\
\hline $12 \mathrm{c}$ & $a$ & $3.3+1$ & $5.1+1$ & KFK & E & vidal-ó & Que orost & 6781 \\
\hline $14 \mathrm{~N}$ & $\mathbf{p}$ & $5.0+1$ & & LPI & $E$ & Voovint & & 407 \\
\hline${ }^{14} \mathrm{~N}$ & p & $5.0+1$ & & LPI & $E$ & . & & 408 \\
\hline${ }^{14} \mathrm{~N}$ & p & $5.0+1$ & & LPI & E & Voovint & & 409 \\
\hline $14 \mathrm{~N}$ & $p$ & TR & $2.2+1$ & ORL & 0 & Wheland & $\frac{1}{d+}+$ & 411 \\
\hline $14 N$ & p & $3.8+0$ & $6.5+0$ & CAL & $E$ & Ingall & $g+$ & 412 \\
\hline $14 \mathrm{~N}$ & p & $4.0+0$ & $1.5+1$ & JUL & $\mathbf{R}$ & Stochli & $i_{n+}$ & 413 \\
\hline${ }^{14} \mathrm{~N}$ & $p$ & $4.7+0$ & $1.5+1$ & ENL & $E$ & Casoll! & $b^{2}$ & 414 \\
\hline $14 \mathrm{~N}$ & $a$ & $4.3+1$ & $5.1+1$ & KFK & $E$ & VIdaI-c & Quadrast & 6809 \\
\hline $14 \mathrm{~N}$ & a & $3 \cdot 3+1$ & $5.1+1$ & KFK & $E$ & $v \mid d a l=0$ & OQuadrast & 6810 \\
\hline $14 \mathrm{~N}$ & $a$ & $3.3+1$ & $5.1+1$ & KFK & $E$ & vidal-o & Quadrast & 6820 \\
\hline 160 & p & $1.6+2$ & & LPI & $\mathrm{T}$ & Dmitrie & évt & 522 \\
\hline $15_{0}$ & $p$ & $5.0+1$ & & LPI & $E$ & Voovint & & 523 \\
\hline 160 & p & $5.0+1$ & & LPI & $E$ & Vdovint & & 524 \\
\hline 160 & 0 & $2.5+5$ & & {$[P]$} & $T$ & Dofitris & $\frac{1}{g} v+$ & 6845 \\
\hline 180 & 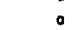 & $3.3+1$ & $5.1+1$ & KFK & $E$ & vidol-c & Quadrast & 6876 \\
\hline Ag & $14 \mathrm{~N}$ & $7.8+1$ & $1.1+2$ & LYO & T & Billere & êt & 10430 \\
\hline
\end{tabular}




\begin{tabular}{|c|c|c|c|c|c|c|}
\hline iget & $\operatorname{lng}$ & $\begin{array}{l}E_{m I n} \\
(\mathrm{MeV})\end{array}$ & $\begin{array}{l}E \max \\
(\operatorname{MeV})\end{array}$ & Lab W & Author & No. \\
\hline c & $O(E)$ & & & & & \\
\hline Many & d & NDG & & \multicolumn{2}{|c|}{ TNL E Nelsont } & 5993 \\
\hline${ }^{12} \mathrm{C}$ & \multicolumn{5}{|c|}{ compound-nuclous o(E) x foctor } & \\
\hline${ }^{12} \mathrm{C}$ & $12_{C}$ & $5.0+1$ & $7.4+1$ & STB E & Cormlert & 9714 \\
\hline $12_{c}$ & \multicolumn{3}{|c|}{ partlal compound-nuc } & lous re & - lotlve o(E) & \\
\hline${ }^{12} \mathrm{C}$ & 160 & $5.7+1$ & $6.8+1$ & TSU E & Furunot & 10644 \\
\hline${ }^{12} \mathrm{C}$ & \multicolumn{3}{|c|}{ partl.ald } & oction & o(E) & \\
\hline $12 c$ & $12 \mathrm{C}$ & $2.8+1$ & $6.2+1$ & STR E & Kolatat & 9690 \\
\hline $1 \mathrm{CC}_{\mathrm{C}}$ & portlal. & $\sigma(E ; \theta) \mathrm{L}$ & Logondr & \multicolumn{3}{|c|}{ re cooff. exponsionao(E;0.) } \\
\hline${ }^{9} \mathrm{Be}_{0}$ & a & $6.1-1$ & $9.9+0$ & OTC D & Geigert & 6719 \\
\hline${ }^{12} \mathrm{C}$ & portial & $O(E ; \theta)$ & & & & \\
\hline${ }^{9} \mathrm{Be}$ & a & $1.4+0$ & $8.0+0$ & OTC D & Gelgert & 6718 \\
\hline${ }^{12} \mathrm{c}$ & portial & product & $t y i e 10$ & & & \\
\hline $\begin{array}{l}I_{B} \\
I_{C}\end{array}$ & $160_{0}^{p}$ & $\begin{array}{l}3.5+1 \\
1.0+2\end{array}$ & $1.0+2$ & $\begin{array}{l}\text { OSU E } \\
\text { ROC E }\end{array}$ & $\begin{array}{l}\text { Kovash. } \\
\text { Cormlert }\end{array}$ & $\begin{array}{r}189 \\
10643\end{array}$ \\
\hline${ }^{12} \mathrm{c}$ & \multicolumn{2}{|c|}{ portial ofEld } & & & & \\
\hline${ }^{12} \mathrm{C}$ & $3^{3} \mathrm{He}$ & $7.0+1$ & $9.0+1$ & OSA E & Motsuokot & 6144 \\
\hline${ }^{12} \mathrm{C}$ & portial & $O(E)_{y} \times f$ & factor & & & \\
\hline $\begin{array}{l}12 c \\
12 c\end{array}$ & $12^{p}$ & $\begin{array}{l}1.8+1 \\
2.1+1\end{array}$ & $\begin{array}{l}2.3+1 \\
4.1+1\end{array}$ & $\begin{array}{l}\text { UBC E } \\
\text { TEX E }\end{array}$ & $\begin{array}{l}\text { Berghofert } \\
\text { Bralthooltet }\end{array}$ & $\begin{array}{r}288 \\
9715\end{array}$ \\
\hline${ }^{12} \mathrm{C}$ & \multicolumn{2}{|c|}{ portial o(E), } & & & & \\
\hline $\begin{array}{l}12 c \\
12 c \\
12 c \\
12 c \\
12 c \\
12 c\end{array}$ & $\begin{array}{r}p \\
a d \\
12 c \\
12 c \\
12 c \\
160\end{array}$ & $\begin{array}{l}5 \cdot 0+0 \\
8 \cdot 0+0 \\
2.0+1 \\
3 \cdot 0+1 \\
3 \cdot 0+1 \\
4.7+1\end{array}$ & $\begin{array}{l}1.1+2 \\
1.2+2 \\
5 \cdot 2+1 \\
7.0+1 \\
9 \cdot 0+1 \\
5 \cdot 6+1\end{array}$ & $\begin{array}{l}\text { GSF D } \\
\text { GSF D } \\
\text { STR E } \\
\text { BNL E } \\
\text { STB E } \\
\text { WAU E }\end{array}$ & $\begin{array}{l}\text { Romalyt } \\
\text { Ronalyt } \\
\text { Kolatat } \\
\text { Jocheinshit } \\
\text { Cormlert } \\
\text { Ehangt }\end{array}$ & $\begin{array}{r}286 \\
6765 \\
9711 \\
9712 \\
9713 \\
10541\end{array}$ \\
\hline${ }^{2} \mathrm{c}$ & portlat & $O(E) \times f$ & octor. & ot roso & onance & \\
\hline${ }^{12} \mathrm{C}$ & 160 & $4.6+1$ & $.3+1$ & STB E & OMolonint & 10540 \\
\hline
\end{tabular}

\begin{tabular}{|c|c|c|c|c|c|c|}
\hline$\overline{\text { Torget }}$ & $\ln e$ & $\begin{array}{l}E m \mid n \\
(M \circ V)\end{array}$ & $\begin{array}{l}\text { Emax } \\
(M e V)\end{array}$ & Lab & Author & No. \\
\hline${ }^{12} \mathrm{C}$ & partlol & $O(E)$. & treso & nance & & \\
\hline $\begin{array}{l}12 \mathrm{C} \\
{ }^{12} \mathrm{C}\end{array}$ & $\begin{array}{l}12 \mathrm{c} \\
160\end{array}$ & $\begin{array}{l}6.2+1 \\
4.6+1\end{array}$ & $5.6+1$ & $\begin{array}{l}\text { ROC } \\
\text { STQ }\end{array}$ & $\begin{array}{l}\text { E Clovert } \\
\text { E Malolnt }\end{array}$ & $\begin{array}{r}9710 \\
10639\end{array}$ \\
\hline${ }^{12} \mathrm{C}$ & partial & colati & ve olE & & & \\
\hline $\begin{array}{l}12 \mathrm{C} \\
12 \mathrm{C} \\
12 \mathrm{C} \\
4 \mathrm{C}^{1} 1\end{array}$ & $\begin{array}{l}160 \\
2851 \\
2851 \\
160\end{array}$ & $\begin{array}{l}2.4+1 \\
5 \cdot 8+1 \\
5 \cdot 8+1 \\
9.6+1\end{array}$ & $\begin{array}{l}4.6+1 \\
1.1+2 \\
1.1+2\end{array}$ & $\begin{array}{l}\text { STB } \\
\text { ROC } \\
\text { ROC } \\
\text { MUN }\end{array}$ & $\begin{array}{l}\text { E Cormlert } \\
\text { E Clovert } \\
\text { E Clovert } \\
\text { M Heringt }\end{array}$ & $\begin{array}{l}10638 \\
11952 \\
11953 \\
11115\end{array}$ \\
\hline${ }^{12} \mathrm{C}$ & pertlal & $O(E) \times f$ & octor & & & \\
\hline $\begin{array}{l}12 \mathrm{C} \\
15_{\mathrm{N}}\end{array}$ & p & $\begin{array}{l}3.0+0 \\
9.3-2\end{array}$ & $\begin{array}{l}6.1+1 \\
4.2-1\end{array}$ & $\begin{array}{l}\text { MiL } \\
\text { CAL }\end{array}$ & $\begin{array}{l}\text { T Bonottit } \\
\text { E Zyshindt }\end{array}$ & $\begin{array}{l}289 \\
445\end{array}$ \\
\hline${ }^{1} a_{C}$ & portial & $O(E)$ & & & & \\
\hline $\begin{array}{l}{ }^{9} \mathrm{Be} \\
1_{\mathrm{B}}\end{array}$ & a & $\begin{array}{l}1.5+0 \\
\text { NDG }\end{array}$ & $7.9+0$ & $\begin{array}{l}\text { OrC } \\
\text { ORL }\end{array}$ & $\begin{array}{l}\text { D Geigert } \\
\text { T Ramavatramt }\end{array}$ & $\begin{array}{r}6720 \\
186\end{array}$ \\
\hline$I_{B}$ & p & $6.0+0$ & $2.3+1$ & WAU & E Snovert & 187 \\
\hline $12 c$ & p & $1.9+1$ & $2.5+1$ & GRE & E Galllardt & 287 \\
\hline $12 \mathrm{C}$ & $12 \mathrm{C}$ & $1.5+1$ & $4.5+1$ & NAG & T Kondot & 9701 \\
\hline${ }^{12} \mathrm{C}$ & $12 \mathrm{c}$ & $2.0+1$ & $8.0+1$ & YAL & E Kondot & 9702 \\
\hline $12 \mathrm{c}$ & $12 \mathrm{c}$ & $2.2+1$ & $8.0+1$ & W1S & T Friedmant & 9703 \\
\hline $12 \mathrm{C}$ & $12 \mathrm{c}$ & $2.4+1$ & $8.8+i$ & SAC & E Harar. & 9704 \\
\hline $12_{\mathrm{C}}$ & $12 \mathrm{c}$ & $2.4+1$ & $9.0+1$ & STB & R Cormiert & 9705 \\
\hline $1 a_{c}$ & $12 \mathrm{c}$ & $2.9+1$ & $6.4+1$ & PEN & E Cannelit & 9705 \\
\hline $12 \mathrm{C}$ & $12 \mathrm{c}$ & $3.0+1$ & $9.0+1$ & BNL & E Cormlert & 9707 \\
\hline $12 \mathrm{c}$ & $12 \mathrm{c}$ & $3.2+1$ & $0.0+1$ & TOK & T Tanimurat & 9708 \\
\hline${ }^{12} \mathrm{c}$ & $12 \mathrm{c}$ & $4.4+1$ & B. $0+1$ & ROC & E Fultont & 9709 \\
\hline $12 \mathrm{C}$ & $12 \mathrm{C}$ & $4.0+1$ & $8.8+1$ & ENL & T fuller. & 9718 \\
\hline $\operatorname{lac} C$ & $1 e_{C}$ & $3.4+1$ & $6.6+1$ & YAL & E Erb+ & 9719 \\
\hline${ }^{12} \mathrm{c}$ & 160 & $1.4+1$ & $3.2+1$ & TOK & T Tanimurat & 10632 \\
\hline $12 \mathrm{C}$ & 160 & $3.2+1$ & $4 \cdot 3+1$ & CBR & E Branforot & 10633 \\
\hline $12 \mathrm{C}$ & 160 & $3 \cdot 3+1$ & $5.4+1$ & ST日 & E Malmint & 10634 \\
\hline $12 \mathrm{C}$ & 160 & $3.4+1$ & $7.2+1$ & SUB & E Jacholnshit & 10635 \\
\hline $12 \mathrm{c}$ & 160 & $3.6+1$ & $4.7+1$ & STR & R Hast & 10636 \\
\hline${ }^{12} \mathrm{C}$ & 160 & $5.0+1$ & $7.0+1$ & ROC & E Shopirat & 10637 \\
\hline${ }^{12} \mathrm{C}$ & 160 & $4.0+1$ & $6.5+1$ & SAC & M Bertault+ & 10645 \\
\hline${ }^{12} \mathrm{C}$ & 170 & $3.1+1$ & $3.4+1$ & WZI & E Chechikt & 11577 \\
\hline${ }^{12} \mathrm{c}$ & 180 & $3.2+1$ & $3.5+1$ & WZI & E Chechikt & 11611 \\
\hline $15_{N}$ & $p$ & $9.3-2$ & $4.2-1$ & $\mathrm{CAL}$ & E Zyshindt & 438 \\
\hline $15 \mathrm{~N}$ & p & $1.8-1$ & $2.5+0$ & CAL & E Rolfgt & 439 \\
\hline
\end{tabular}




\begin{tabular}{|c|c|c|c|c|c|c|}
\hline Torget & Inc & $\begin{array}{l}\text { Emin } \\
\text { (Mev) }\end{array}$ & $\begin{array}{l}E_{\max } \\
(\operatorname{MeV})\end{array}$ & Lab & Author & No. \\
\hline${ }^{12} \mathrm{C}$ & partial & $\sigma(E)$ & & & & \\
\hline $\begin{array}{l}15_{\mathrm{N}} \\
15_{\mathrm{N}} \\
15_{\mathrm{N}} \\
15_{\mathrm{N}} \\
15_{\mathrm{N}} \\
16_{0} \\
15_{0} \\
20_{\mathrm{Ne}} \\
27_{\mathrm{A}} !\end{array}$ & $\begin{array}{r}p \\
p \\
p \\
p \\
p \\
p \\
: \\
16 \\
\end{array}$ & $\begin{array}{r}9.0-1 \\
+0 \\
2.9+0 \\
2.0+1 \\
4.4+1 \\
1.8+1 \\
1.6+1 \\
1.3+1 \\
8.8+1\end{array}$ & $\begin{array}{l}1.3+0 \\
3.5+0 \\
5.0+1 \\
1.0+3 \\
8.0+1 \\
2.1+1\end{array}$ & $\begin{array}{l}\text { CBR } \\
\text { SAC } \\
\text { CBR } \\
\text { MIL } \\
\text { ANL } \\
\text { GSF } \\
\text { GSF } \\
\text { WIS } \\
\text { TSU }\end{array}$ & $\begin{array}{l}\text { E Bray+ } \\
\text { R Engelaann+ } \\
\text { E Frawloy+ } \\
\text { T Gadiali+ } \\
\text { R Kurath. } \\
\text { D Ramaty+ } \\
\text { D Ramaty+ } \\
\text { E Oavis. } \\
\text { E Mikumo+ }\end{array}$ & $\begin{array}{r}440 \\
441 \\
442 \\
443 \\
444 \\
525 \\
6846 \\
6924 \\
10953\end{array}$ \\
\hline${ }^{12} \mathrm{c}$ & relative & $I_{\gamma}$ & & & & \\
\hline $\begin{array}{l}{ }^{11 B} B \\
{ }^{12} \mathrm{C}\end{array}$ & $\begin{array}{c}\text { P } \\
\text { producl }\end{array}$ & $\begin{array}{l}4.0+1 \\
y i e l d\end{array}$ & & osu & T Arnold. & 188 \\
\hline $\begin{array}{l}12 \mathrm{C} \\
12 \mathrm{C} \\
18_{0}\end{array}$ & $\begin{array}{l}13 c \\
180 \\
180\end{array}$ & $\begin{array}{l}1.8+1 \\
4.0+1 \\
9.1+1\end{array}$ & $\begin{array}{l}3 \cdot 2+1 \\
6 \cdot 0+1\end{array}$ & $\begin{array}{l}\text { KSU } \\
\text { NOT } \\
\text { PAR }\end{array}$ & $\begin{array}{l}\text { E Legg+ } \\
\text { E Oavist } \\
\text { E Detraz+ }\end{array}$ & $\begin{array}{l}10174 \\
11610 \\
11650\end{array}$ \\
\hline $\begin{array}{c}{ }^{12} \mathrm{C} \\
{ }^{12} \mathrm{C}\end{array}$ & $\begin{array}{r}\sigma(E), \\
l^{2} \mathrm{C}\end{array}$ & $2.0+1$ & $9.0+1$ & MBG & R Fich. & 9717 \\
\hline${ }^{12} \mathrm{c}$ & $\sigma(E)$ at & resonan & nce & & & \\
\hline${ }^{12} \mathrm{c}$ & ${ }^{12} \mathrm{C}$ & $6.2+1$ & & ROC & E Clovert & 9699 \\
\hline${ }^{12} \mathrm{C}$ & reletive & $\sigma(E)$ & & & & \\
\hline${ }^{12} \mathrm{C}$ & 180 & $2.9+1$ & $3.4+1$ & MON & E Tarast & 10631 \\
\hline${ }^{12} \mathrm{C}$ & $\sigma(E) \times f \theta C$ & tor & & & & \\
\hline $\begin{array}{l}{ }^{40} \mathrm{Co} \\
500 \mathrm{Cr} \\
2008 \mathrm{~Eb} \\
200 \overline{P b}\end{array}$ & $\begin{array}{l}160 \\
14 \mathrm{~N} \\
160 \\
160\end{array}$ & $\begin{array}{l}5.6+1 \\
7.1+1 \\
1.4+2 \\
1.4+2\end{array}$ & $\begin{array}{l}1.0+2 \\
3.2+5 \\
3.5+2\end{array}$ & $\begin{array}{l}\text { ANR } \\
\text { LYO } \\
\text { BRK } \\
\text { MSU }\end{array}$ & $\begin{array}{l}\text { E Kovart } \\
\text { T Gillereyt } \\
\text { E Gelbhet } \\
\text { R Gelbke. }\end{array}$ & $\begin{array}{l}11110 \\
10391 \\
11541 \\
11542\end{array}$ \\
\hline${ }^{12} \mathrm{c} \quad 0$ & $\sigma(E)$ & & & & & \\
\hline $\begin{array}{l}98 \mathrm{Be} \\
9 \mathrm{Be} \\
918 \\
118 \\
12 \mathrm{C} \\
12 \mathrm{C}\end{array}$ & $\begin{array}{l}a \\
a \\
p \\
d \\
\rho \\
d\end{array}$ & $\begin{array}{l}\text { NDG } \\
4.4-1 \\
\text { TR } \\
1.0-3 \\
4.9+1 \\
1.4+1\end{array}$ & $\begin{array}{l}8.0-1 \\
3.5+1 \\
2.0+1 \\
1.6+2 \\
2.6+1\end{array}$ & $\begin{array}{l}\text { BNL } \\
\text { IPS } \\
\text { INU } \\
\text { LRL } \\
\text { SUR } \\
\text { TPI }\end{array}$ & $\begin{array}{l}\text { R Goldberg. } \\
\text { E Ramstroemt } \\
\text { E Blatt+ } \\
\text { O Howerton. } \\
\text { T Abdul Jaililt } \\
\text { T Motusevicht }\end{array}$ & $\begin{array}{r}6716 \\
6717 \\
190 \\
5309 \\
290 \\
5318\end{array}$ \\
\hline
\end{tabular}

\begin{tabular}{|c|c|c|c|c|c|c|}
\hline Target & $\ln \mathrm{s}$ & $\begin{array}{l}\text { Emin } \\
(\mathrm{MeV})\end{array}$ & $\begin{array}{l}\text { Emax } \\
(\operatorname{MeV})\end{array}$ & Lab & Author & No. \\
\hline${ }^{12} \mathrm{C}$ & $\sigma(E)$ & & & & & \\
\hline $1 e_{C}$ & ${ }^{3} \mathrm{He}$ & $1.0+1$ & $2.0+1$ & TPI & T Makuserlcht & 5143 \\
\hline${ }^{12} \mathrm{c}$ & 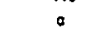 & $8.8+0$ & $1.7+1$ & TPi & T Matusericht & 6766 \\
\hline${ }^{12} \mathrm{c}$ & $12 \mathrm{C}$ & NOG & & ROC & R Cormier. & 9700 \\
\hline $1 z_{c}$ & $12 \mathrm{C}$ & $3.4+1$ & $6.6+1$ & YAL & E Erbt & 9716 \\
\hline $12 c$ & 150 & $3.3+1$ & $5.4+1$ & STB & E Malmint & 10642 \\
\hline $12 \mathrm{c}$ & 170 & $3.1+1$ & $3.4+1$ & WZI & E Chechikt & 11578 \\
\hline $12 \mathrm{C}$ & $18_{0}$ & $3.2+1$ & $3.5+1$ & WZI & E Chechikt & 11612 \\
\hline${ }^{12} \mathrm{C}$ & $20 \mathrm{Ne}$ & $5.4+1$ & $8.0+1$ & ORL & E Shapirat & 11838 \\
\hline $14 \mathrm{~N}$ & p & $5.0+1$ & & LPI & E Vdovint & $420+$ \\
\hline 180 & p & $5.0+1$ & & LPI & E Vdovint. & 526 \\
\hline 160 & p & $5.0+1$ & & LP! & E Vdovint & 527 \\
\hline 180 & p & $5.0+1$ & & LPI & E Vdovint & $528+$ \\
\hline 160 & $\begin{array}{l}p \\
p\end{array}$ & $\begin{array}{l}5.0+1 \\
5.0+1\end{array}$ & & LPi & E Vdovint & $529+$ \\
\hline 160 & $a$ & $5.1+1$ & & KFK & Evidal-Cuadrast & 6847 \\
\hline 160 & 0 & $3.3+1$ & $5.1+1$ & $\mathrm{KFK}$ & E vidal-Guadrast & 6848 \\
\hline 180 & & $4.3+1$ & $5.1+1$ & KFK & E vidal-Quadrast & 6849 \\
\hline $27 \mathrm{Al}$ & $1{ }_{B}$ & $5.0+1$ & & FSU & E Parkst & 9569 \\
\hline $2 \theta_{5 i}$ & $W_{B}$ & $5 \cdot 0+1$ & & FSU & E Parkst & 9570 \\
\hline Ag & $14 \mathrm{~N}$ & $7.8+1$ & $1.1+2$ & LYO & T Billereyt & 10430 \\
\hline${ }^{13} \mathrm{C}$ & compound & d-nucleu & ug $O(E)$ & & $!$ & \\
\hline $10 \mathrm{~B}$ & ${ }^{7} L I$ & $2.4+1$ & & MEI & M Kohlert. & 9406 \\
\hline${ }^{13} \mathrm{C}$ & portiol & roothi & ick tor & irget & ylelorobundonce & \\
\hline $10_{B}$ & 0 & $5.0+0$ & & SUN & E Gilest & 6727 \\
\hline${ }^{13} \mathrm{C}$ & partlol & relativ & ve $O(E)$ & & 1 & \\
\hline${ }^{48} \mathrm{Ti}$ & 160 & $9.6+1$ & & MUN & M Her Ingt: & 11116 \\
\hline${ }^{13} \mathrm{C}$ & partlal & $O(E) \times 18$ & actor & & & \\
\hline $\begin{array}{l}{ }^{9} \mathrm{Be} \\
{ }^{9} \mathrm{Be}\end{array}$ & $\begin{array}{l}1{ }^{2} \mathrm{C} \\
1{ }^{2} \mathrm{C}\end{array}$ & $\begin{array}{l}2.3+0 \\
3.5+0\end{array}$ & $\begin{array}{l}1.4+1 \\
9.5+0\end{array}$ & $\begin{array}{l}\text { LUQ } \\
\text { CAL }\end{array}$ & $\begin{array}{l}\text { E Cheung } \\
\text { M Switkooghlt }\end{array}$ & $\begin{array}{l}9601 \\
9602\end{array}$ \\
\hline${ }^{13} \mathrm{C}$ & portlol & $O(E)$ & & & & \\
\hline${ }^{9} \mathrm{Be}$ & $12 \mathrm{C}$ & $2.3+0$ & $1.4+1$ & LUO & E Cheungt & 9603 \\
\hline $10_{\mathrm{B}}$ & $T_{L I}$ & $2.4+1$ & & HEI & M kohlert & 9407 \\
\hline $12 \mathrm{C}$ & d & $2.5-1$ & $5.0-1$ & WIT & E Annegarn. & 5319 \\
\hline $12 c$ & ${ }^{12} \mathrm{C}$ & $9.4+i$ & & ORL & E Fulmert & 9721 \\
\hline $13 c$ & $12 \mathrm{c}$ & $1.8+i$ & $4 \cdot 0+1$ & STR & $\mathrm{R} \mathrm{Has}+$ & 9786 \\
\hline $13 \mathrm{c}$ & 170 & $3.0+1$ & $3.2+1$ & WZI & E Chechikt & 11582 \\
\hline
\end{tabular}




\begin{tabular}{|c|c|c|c|c|c|c|}
\hline Target & $\operatorname{lnc}$ & $\begin{array}{l}\text { Emin } \\
(M e V)\end{array}$ & $\begin{array}{l}\text { Emax } \\
(\mathrm{MeV})\end{array}$ & Lab & Author & No. \\
\hline${ }^{13} \mathrm{C}$ & Dartial & $\sigma(E)$ & & & , & \\
\hline $\begin{array}{l}13 \mathrm{C} \\
14 \mathrm{~N} \\
16 \mathrm{O} \\
27 \mathrm{Al}\end{array}$ & $\begin{array}{r}10_{0} \\
p \\
p \\
100\end{array}$ & $\begin{array}{l}3 \cdot 1+1 \\
5 \cdot 0+1 \\
2 \cdot 1+1 \\
8 \cdot 8+1\end{array}$ & $\begin{array}{l}3.3+1 \\
4.0+2\end{array}$ & $\begin{array}{l}\text { WZI } \\
\text { LPI } \\
\text { GSF } \\
\text { ISU }\end{array}$ & $\begin{array}{l}\text { E Chechlkt } \\
\text { E Loshchohort } \\
\text { D Ramaly+ } \\
\text { E Mihumo+ }\end{array}$ & $\begin{array}{r}11631 \\
422 \\
530 \\
10953\end{array}$ \\
\hline${ }^{13} \mathrm{C}$ & product & yield & & & & \\
\hline $\begin{array}{l}12 \mathrm{c} \\
180\end{array}$ & $\begin{array}{l}12 \mathrm{C} \\
180\end{array}$ & $\begin{array}{l}7.0+1 \\
9.1+1\end{array}$ & & $\begin{array}{l}\text { ROC } \\
\text { PAR }\end{array}$ & $\begin{array}{l}\text { E Hermant } \\
\text { E Detrazt }\end{array}$ & $\begin{array}{r}9720 \\
11650\end{array}$ \\
\hline${ }^{13} \mathrm{C}$ & thick t & target y & $101 d$ & & & \\
\hline${ }^{9} \mathrm{Be}$ & ${ }^{12} \mathrm{C}$ & $2.1+0$ & $5.2+0$ & $\mathrm{BOC}$ & MUhlhornt & 9604 \\
\hline${ }^{13} \mathrm{C}$ & $O(E) \times f O$ & actor & & & & \\
\hline $\begin{array}{r}40 \mathrm{Ca} \\
50 \mathrm{Cr} \\
200 \mathrm{~Pb} \\
200 \mathrm{~Pb}\end{array}$ & $\begin{array}{l}150 \\
14 \mathrm{~N} \\
160 \\
160\end{array}$ & $\begin{array}{l}5.6+1 \\
7.1+1 \\
1.4+2 \\
1.4+2\end{array}$ & $\begin{array}{l}1.0+2 \\
3.2+2 \\
3.2+2\end{array}$ & $\begin{array}{l}\text { ANL } \\
\text { LYO } \\
\text { BRK } \\
\text { MSU }\end{array}$ & $\begin{array}{l}\text { E Kovart } \\
\text { T Billereyt } \\
\text { E Gelbke+ } \\
\text { R Gelbke. }\end{array}$ & $\begin{array}{l}11110 \\
10392 \\
11541 \\
11542\end{array}$ \\
\hline${ }^{13} \mathrm{C}$ & $\sigma(E)$ & & & & & \\
\hline $\begin{array}{l}9 \mathrm{ge} \\
13 \mathrm{C} \\
13 \mathrm{C}\end{array}$ & $\begin{array}{l}12 \mathrm{C} \\
170 \\
180\end{array}$ & $\begin{array}{l}2.1+0 \\
3 \cdot 0+1\end{array}$ & $\begin{array}{l}5.2+0 \\
3.2+1\end{array}$ & $\begin{array}{l}\text { BOC } \\
\text { WZI }\end{array}$ & $\begin{array}{l}\text { M Uhlhornt } \\
\text { E Chechiht }\end{array}$ & $\begin{array}{r}9605 \\
11583\end{array}$ \\
\hline${ }_{14 \mathrm{~N}}^{13 \mathrm{C}}$ & $\begin{array}{r}180 \\
p\end{array}$ & $\begin{array}{l}3 \cdot 1+1 \\
5 \cdot 0+1\end{array}$ & $3.3+1$ & $\begin{array}{l}\text { WZI } \\
\text { LPI }\end{array}$ & $\begin{array}{l}\text { E Chechiht } \\
\text { E Vdovint }\end{array}$ & $42532+$ \\
\hline 160 & p & $5.0+1$ & & LPI & E Vdovint & 531 \\
\hline 160 & p & $5 \cdot 0+1$ & & LPI & E Vdovint & $532+$ \\
\hline 160 & $a$ & $4.3+1$ & $5.1+1$ & KFK & E Vidal-Ouadrast & 6850 \\
\hline Ag & ${ }^{14} \mathrm{~N}$ & $7.8+1$ & $1.1+2$ & LYO & T Billerey+ & 10430 \\
\hline${ }^{14} \mathrm{C}$ & parllal & $\sigma(E)$ & & & & \\
\hline I'B & - & $4.4+0$ & $6.7+0$ & TAI & E Hout & 6730 \\
\hline${ }^{14} \mathrm{C}$ & product & yield & & & & \\
\hline $\begin{array}{l}12_{C} \\
10_{0}\end{array}$ & $\begin{array}{l}180 \\
180\end{array}$ & $\begin{array}{l}4.0+1 \\
9.1+1\end{array}$ & $6.0+1$ & $\begin{array}{l}\text { NOT } \\
\text { PAR }\end{array}$ & $\begin{array}{l}\text { E Oavist } \\
\text { E Detraz+ }\end{array}$ & $\begin{array}{l}11613 \\
11650\end{array}$ \\
\hline${ }^{14} \mathrm{C}$ & $O(E) \times f \circ$ & etor & & & & \\
\hline $\begin{array}{r}4 \theta_{\mathrm{Cb}} \\
208 \mathrm{~Pb} \\
208 \mathrm{~Pb}\end{array}$ & $\begin{array}{l}160 \\
160 \\
160\end{array}$ & $\begin{array}{l}5.6+1 \\
1.4+2 \\
1.4+2\end{array}$ & $\begin{array}{l}3.2+2 \\
3.2+2\end{array}$ & $\begin{array}{l}\text { ANL } \\
\text { BRK } \\
\text { MSU }\end{array}$ & $\begin{array}{l}\text { E Kovort } \\
\text { E Gelbket } \\
\text { R Gelbke. }\end{array}$ & $\begin{array}{l}11110 \\
11541 \\
11542\end{array}$ \\
\hline
\end{tabular}

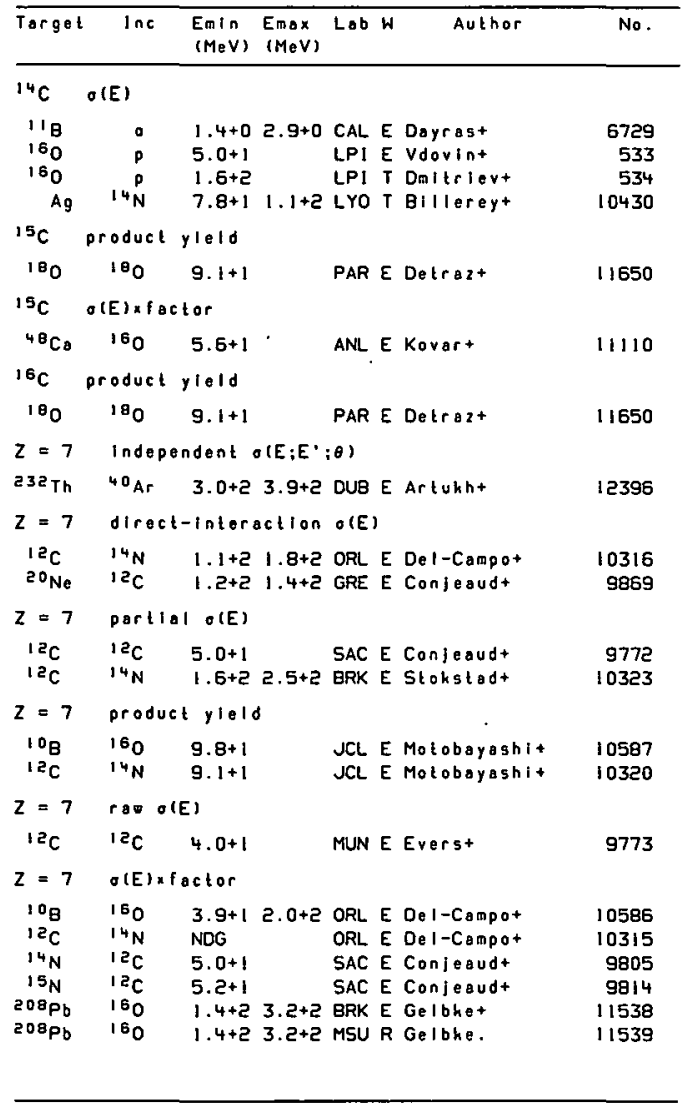




\begin{tabular}{|c|c|c|c|c|c|c|}
\hline Target & $\ln c$ & $\begin{array}{l}\text { Emin } \\
(\mathrm{MeV})\end{array}$ & $\begin{array}{l}\text { Emax } \\
\text { (MeV) }\end{array}$ & Lab $\mathrm{W}$ & Author & No. \\
\hline$z=7$ & $O(E)$ & & & & & \\
\hline $12 \mathrm{C}$ & $\operatorname{lac}$ & $1.5+1$ & $6.2+1$ & $A N L E$ & E Kovart & 9768 \\
\hline${ }^{12} \mathrm{C}$ & ${ }^{12} \mathrm{c}$ & $4.5+1$ & $2.0+2$ & TAME & E Nomboodirit & 9770 \\
\hline${ }^{12} \mathrm{c}$ & ${ }^{13} \mathrm{c}$ & $1.6+1$ & $5.2+1$ & ANL $E$ & E Kovart & 10185 \\
\hline${ }^{12} \mathrm{C}$ & ${ }^{14 N}$ & $8.6+1$ & $1.7+2$ & ORL E & E Stohstodt & 10318 \\
\hline $12 \mathrm{C}$ & 160 & $4.0+1$ & $6.6+1$ & NOT E & E Molmint & 10722 \\
\hline${ }^{12} \mathrm{C}$ & 180 & $1.0+2$ & & STR E & E Coffint & 11626 \\
\hline${ }^{12} \mathrm{C}$ & $20 \mathrm{Ne}$ & $6.7+1$ & $1.6+2$ & GRE $E$ & E Solnt-Lourent+ & 11839 \\
\hline${ }^{13} \mathrm{C}$ & 180 & $1.0+2$ & & STRE & E Coffint & 11639 \\
\hline $14 \mathrm{n}$ & ${ }^{13} \mathrm{C}$ & $4.4+1$ & $5.4+1$ & SAC E & E Conleaudt & 10192 \\
\hline $15_{N}$ & ${ }^{12} \mathrm{c}$ & $3.0+1$ & $5.0+1$ & $\operatorname{SAC} \varepsilon$ & E Conleaudt & 9815 \\
\hline 160 & 160 & $1.2+2$ & $1.4+2$ & GRE E & E Sạnt-Leurent+ & 10854 \\
\hline${ }^{20} \mathrm{Ne}$ & ${ }^{12} \mathrm{C}$ & $4.8+1$ & $9.6+1$ & SAC $M$ & M Conjeaudt & 9867 \\
\hline${ }^{45} \mathrm{Se}$ & ${ }^{22} \mathrm{Ne}$ & NDG & & LYO T & I Blliereyt & 11924 \\
\hline $\operatorname{siv}$ & 160 & $7.7+1$ & & GRE $\varepsilon$ & E Chevariert & 11142 \\
\hline${ }^{50} \mathrm{Cr}$ & ${ }^{14} \mathrm{~N}$ & $7.1+1$ & $1.0+2$ & LYO T & T Billereyt & 10393 \\
\hline${ }^{50} \mathrm{Cr}$ & ${ }^{22} \mathrm{Ne}$ & NDG & & LYO T & I Billereyt & 11925 \\
\hline${ }^{55} \mathrm{Mn}$ & ${ }^{12} \mathrm{c}$ & $7.7+1$ & & GRE E & E Chevarlert & 5944 \\
\hline${ }^{56} \mathrm{Fe}$ & 160 & NDG & & LYO T & T Elllereyt & 11162 \\
\hline${ }^{56} \mathrm{Fe}$ & 160 & $7.7+1$ & & GRE E & E Chevariert & 11163 \\
\hline i & ${ }^{20} \mathrm{Ne}$ & $1.6+2$ & & ORL R & R Plosilt+ & 11851 \\
\hline${ }^{60} \mathrm{NI}$ & ${ }^{12} \mathrm{c}$ & NOG & & LYO T & T Bullereyt & 9960 \\
\hline${ }^{60} \mathrm{NI}$ & ${ }^{12} \mathrm{C}$ & $7.7+1$ & & GRE E & E Chevertert & 9961 \\
\hline $181 \mathrm{~T}$ & 160 & $8.3+1$ & $9.6+1$ & BNL $M$ & M Vidobaght & 11464 \\
\hline $197 \mathrm{Au}$ & 160 & $1.4+2$ & $1.7+2$ & $\mathrm{KFK} \mathrm{C}$ & C Muenzel+ & 11481 \\
\hline $20{ }^{\circ} \mathrm{Pb}$ & 160 & $8.0+1$ & $1.0+2$ & ENL $M$ & M Videbaeh+ & 11537 \\
\hline $1 a_{N}$ & (E) & & & & & \\
\hline 160 & p & $5.0+1$ & & LPIE & E vdovint & $535+$ \\
\hline $13 \mathrm{~N}$ & thich to & arget y 1 & $1.1 d$ & & & \\
\hline 150 & ${ }^{3} \mathrm{He}$ & NDG & & uJv $\mathrm{C}$ & $C \mathrm{Bem}+$ & 6156 \\
\hline $13_{N}$ & portlat & product & $1 y 1010$ & & & \\
\hline $\begin{array}{ll}12 \\
12\end{array}$ & $\rho$ & $1.4+1$ & $1.4+1$ & $B I R E$ & E Khant & 293 \\
\hline${ }^{12} \mathrm{C}$ & p & $1.4+1$ & $1.4+1$ & MOS R & R Vorlomovt & 294 \\
\hline${ }^{12} \mathrm{c}$ & D & $3.5+1$ & $1.0+2$ & OSU E & E Kovash. & 295 \\
\hline${ }^{13} \mathrm{~N}$ & oartlal & ROO OLE & & & & \\
\hline${ }^{12} \mathrm{C}$ & p & $1.2+0$ & $1.6+0$ & DUB E & E voltovit & 308 \\
\hline
\end{tabular}

\begin{tabular}{|c|c|c|c|c|c|c|}
\hline Targot & Inc & $\begin{array}{l}\text { Emin } \\
(\mathrm{MeV})\end{array}$ & $\begin{array}{l}\text { Emax } \\
(\mathrm{MeV})\end{array}$ & Lob & Author & No. \\
\hline${ }^{13} \mathrm{~N}$ & portlal & $\sigma(E) \times f \circ$ & octor & & & \\
\hline $\begin{array}{l}1{ }^{2} c \\
1{ }^{2} c\end{array}$ & p & $\begin{array}{l}9.0+0 \\
1.4+1\end{array}$ & $\begin{array}{l}2.4+1 \\
1.4+1\end{array}$ & $\begin{array}{l}\text { UBC } \\
\text { WAU }\end{array}$ & $\begin{array}{l}\text { E Berghofert } \\
\text { M Mars- }\end{array}$ & $\begin{array}{l}301 \\
302\end{array}$ \\
\hline $13_{N}$ & portiol & $\sigma(E)$ & & & & \\
\hline $\begin{array}{l}15 c \\
15 c \\
15 c \\
15 c \\
15 c \\
15 c \\
13 c \\
134 \\
1100\end{array}$ & $\begin{array}{c}p \\
p \\
p \\
12{ }_{C}^{p} \\
3^{p} \mathrm{He} \\
p\end{array}$ & $\begin{array}{r}3.9-1 \\
+0 \\
1.2+0 \\
9.0+0 \\
9.4+1 \\
\text { NDG } \\
3.5-1 \\
2.3+1\end{array}$ & $\begin{array}{l}5.5-1 \\
1.6+0 \\
1.6+1 \\
6.4-1\end{array}$ & $\begin{array}{l}\text { WAU } \\
\text { SAC } \\
\text { DUB } \\
\text { TNL } \\
\text { ORL } \\
\text { TNL } \\
\text { DEB } \\
\text { JUL }\end{array}$ & $\begin{array}{l}\text { M Marst } \\
R \text { Engelmannt } \\
\text { E Voltert } \\
\text { E Turnert } \\
\text { E Fuldert } \\
\text { T Byrdt } \\
\text { E Somogyit } \\
\text { E Rohwert }\end{array}$ & $\begin{array}{r}296 \\
297 \\
298 \\
299 \\
9723 \\
342 \\
6151 \\
546\end{array}$ \\
\hline $13 \mathrm{~N}$ & relative & 12 & & & & \\
\hline${ }^{12} \mathrm{c}$ & p & $4.0+1$ & & osu & T Arnold. & 300 \\
\hline $13 \mathrm{~N}$ & product & $y|e| d$ & & & & \\
\hline $\begin{array}{l}12 \mathrm{C} \\
12 \mathrm{C} \\
12 \mathrm{C} \\
13 \mathrm{C} \\
13 \mathrm{C} \\
14 \mathrm{~N} \\
18 \mathrm{O} \\
180 \\
600\end{array}$ & $\begin{array}{r}p \\
p \\
12 C \\
d \\
p \\
p \\
p \\
p \\
p\end{array}$ & $\begin{array}{l}-1 \\
7.7-1 \\
\text { NOG } \\
7.0+1 \\
\text { NDG } \\
\text { NDG } \\
1.4+1 \\
2.7+1 \\
7.0+1\end{array}$ & & $\begin{array}{l}\text { AML } \\
\text { MOS } \\
\text { BNL } \\
\text { ROC } \\
\text { BNL } \\
\text { KTY } \\
\text { DAV } \\
\text { IKO } \\
\text { UPP }\end{array}$ & $\begin{array}{l}\text { E Hegglet } \\
\text { R Vorlamout } \\
\text { R Rutht } \\
\text { E Hormant } \\
\text { R Ruth+ } \\
\text { E Roodt } \\
\text { E Logunas-Solert } \\
\text { E Brinknant } \\
\text { E Lundagist+ }\end{array}$ & $\begin{array}{r}291 \\
292 \\
5320 \\
9722 \\
335 \\
423 \\
548 \\
549 \\
607\end{array}$ \\
\hline${ }^{13} \mathrm{~N}$ & thich to & arget $y^{\prime}$ & teld 100 & ector & & \\
\hline $\begin{array}{l}{ }^{13} \mathrm{C} \\
{ }^{13} \mathrm{~N}\end{array}$ & thick to & $\begin{array}{l}1.1+1 \\
\text { orget y } 1\end{array}$ & leld & WIS & E Nichlas- & 341 \\
\hline $\begin{array}{l}12 \mathrm{C} \\
12 \mathrm{C} \\
12 \mathrm{C} \\
12 \mathrm{C} \\
12 \mathrm{C} \\
13 \mathrm{C} \\
14 \mathrm{~N} \\
18 \mathrm{O} \\
160\end{array}$ & $\begin{array}{l}p \\
p \\
d \\
d \\
d \\
d \\
3_{\mathrm{He}}^{p} \\
p \\
p\end{array}$ & $\begin{array}{l}3.5-1 \\
1.6+0 \\
T R \\
5.8+0 \\
1.4+1 \\
T R \\
3.0+1 \\
T R \\
1.8+1\end{array}$ & $\begin{array}{l}2.4+0 \\
1.8+0 \\
1.8+1 \\
2.0+1 \\
1.8+1\end{array}$ & $\begin{array}{l}\text { CLU } \\
\text { OHO } \\
\text { ORL } \\
\text { JUL } \\
\text { AML } \\
\text { OAU } \\
\text { AML } \\
\text { ORL } \\
\text { AML }\end{array}$ & $\begin{array}{l}\text { M Rough:ont } \\
\text { E Tsin. } \\
\text { D Wlelandt } \\
\text { R Stoch leit } \\
\text { R Chaudri. } \\
\text { D Wlolandt } \\
\text { R Chaudel. } \\
\text { D Wiolandt } \\
\text { R Choudr } 1 .\end{array}$ & $\begin{array}{r}303 \\
304 \\
5322 \\
5323 \\
5324 \\
340 \\
6150 \\
542 \\
543\end{array}$ \\
\hline
\end{tabular}




\begin{tabular}{lllll}
\hline Target Inc & $\begin{array}{l}\text { EmIn } \\
\text { Mev) }\end{array}$ & $\begin{array}{l}\text { Emax Lab } \\
\text { (MeV) }\end{array}$ & Author & No. \\
\hline
\end{tabular}

$13_{N}$ thick larget yield

$\begin{array}{lllll}160 & 0 & 1.9+1 & \text { DAVE Porkst } & 544 \\ 160 & p & 1.9+1 & \text { JUL R Slochlint } & 545\end{array}$

$\begin{array}{lllll}160 & p & 1.9+1 & \text { JUL R Stochlint } & 545 \\ & p & 1.0+1 & \text { WISENichlest } & 608\end{array}$

$13 \mathrm{~N}$ spectrum average o(E)

${ }^{160}$ P $1.0+11.2+1$ JAP E Suzuki+ 547

$13 \mathrm{~N}$ relotive o(E) $\times$ factor

${ }^{16} 0$ P $6.7+09.2+0$ KLN E Grunlet 550

$1{ }^{3} \mathrm{~N} \quad \sigma(E) \times$ factor

${ }^{48} \mathrm{Ca} \quad 160 \quad 5.6+1 \quad$ ANL E Kovart 11110

$200 \mathrm{~Pb} \quad 160 \quad 1.4+23.2+2$ BRK E Gelbhet 11541

$1.4+23.2+2$ MSU R Gelbhe. 11542

$13^{3} \mathrm{~N} \quad O(E)$

\begin{tabular}{|c|c|c|c|c|c|c|}
\hline Target & $\operatorname{lnc}$ & $\begin{array}{l}\text { Emin } \\
(M e V)\end{array}$ & $\begin{array}{c}\text { Emax } \\
(\operatorname{MeV})\end{array}$ & Lab & Author & No. \\
\hline${ }^{14} \mathrm{~N}$ & direct- & Interact & lion ol & (E) & & \\
\hline${ }^{10} \mathrm{~B}$ & ${ }^{7} \mathrm{LI}$ & $2.4+1$ & & HEI & M Kohlert & $\cdot 9408$ \\
\hline${ }^{14} \mathrm{~N}$. & portlat & rasthi & lek tor & irget & yleldxabundanco & \\
\hline $1 ' B$ & a & $5.0+0$ & & SUN & E Gilest & 6731 \\
\hline${ }^{14} \mathrm{~N}$ & partlal & $\sigma(E)$, & & & & \\
\hline $\begin{array}{l}14 \mathrm{~N} \\
14 \mathrm{~N}\end{array}$ & p & $\begin{array}{l}2.4+1 \\
3.0+0\end{array}$ & $\begin{array}{l}8.0+1 \\
1.1+2\end{array}$ & GSF & $\begin{array}{l}\text { D Ramoty+ } \\
\text { D Ramaty+ }\end{array}$ & $\begin{array}{r}424 \\
6811\end{array}$ \\
\hline${ }^{14} \mathrm{~N}$ & partiol & $\operatorname{ros} \theta \cos$ & & & & \\
\hline $\begin{array}{l}13 \mathrm{C} \\
{ }^{13} \mathrm{C}\end{array}$ & $\begin{array}{l}p \\
p\end{array}$ & $\begin{array}{l}1.7+0 \\
3.8+0\end{array}$ & $\begin{array}{l}1.7+0 \\
3.8+0\end{array}$ & $\begin{array}{l}\text { HLS } \\
\text { LUO }\end{array}$ & $\begin{array}{l}\text { E Keinonent } \\
\text { E Rangacharyulut }\end{array}$ & $\begin{array}{l}346 \\
347\end{array}$ \\
\hline${ }^{14} \mathrm{~N}$ & Dor 11.01 & rolatio & vo $O(E)$ & & & \\
\hline $\begin{array}{l}12 \mathrm{C} \\
170 \\
170 \\
4011\end{array}$ & $\begin{array}{r}12 c \\
p \\
160\end{array}$ & $\begin{array}{l}9.4+1 \\
1.0-1 \\
6.7-1 \\
9.6+1\end{array}$ & $\begin{array}{l}3.0+0 \\
6.8-1\end{array}$ & $\begin{array}{l}\text { ORL } \\
\text { STR } \\
\text { TUR } \\
\text { MUN }\end{array}$ & $\begin{array}{l}\text { E Fulmert } \\
\text { E Senst } \\
\text { E Kiesert } \\
M \text { Heringt }\end{array}$ & $\begin{array}{r}9727 \\
569 \\
570 \\
11117\end{array}$ \\
\hline$: 4 \mathrm{~N}$ & partial & $o(E) \times f o$ & octor & & & \\
\hline $\begin{array}{l}{ }^{13} \mathrm{C} \\
{ }^{14} \mathrm{~N}\end{array}$ & $\stackrel{p}{\text { partial }}$ & $\begin{array}{l}1.0+0 \\
O(E)\end{array}$ & $1.7+0$ & HLS & E Keinanent & 348 \\
\hline $\begin{array}{l}{ }^{4} \mathrm{He} \\
10_{\mathrm{B}}\end{array}$ & $\begin{array}{l}{ }^{14} \mathrm{~N} \\
{ }^{7} \mathrm{Li}\end{array}$ & $\begin{array}{l}\text { NDG } \\
2.4+1\end{array}$ & 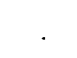 & $\begin{array}{l}\text { TOR } \\
\text { HEI }\end{array}$ & $\begin{array}{l}\text { E kioser. } \\
\text { M Kohlert }\end{array}$ & $\begin{array}{r}10234 \\
9410\end{array}$ \\
\hline $10 \mathrm{~B}$ & ${ }^{9} \mathrm{Be}$ & $4.0+0$ & $9.4+0$ & LUO & E Choterjeet & 9485 \\
\hline $1^{2} \mathrm{C}$ & ${ }^{12} \mathrm{c}$ & $4.4+1$ & $8.0+1$ & ROC & E Clovert & 9724 \\
\hline${ }^{12} \mathrm{c}$ & ${ }^{12} \mathrm{C}$ & $9.4+1$ & & ORL & E Fulmort & 9725 \\
\hline${ }^{13} \mathrm{C}$ & $p$ & & & SAC & R Engelmannt & 343 \\
\hline${ }^{13} \mathrm{C}$ & p & $6.2+0$ & $1.7+1$ & TNL & E Turner. & 344 \\
\hline${ }^{13} \mathrm{C}$ & $p$ & $2.5+1$ & & INL & E Blattt & 345 \\
\hline $14 \mathrm{~N}$ & d & $6.0+0$ & $1.8+1$ & TOK & T Izumato. & 5329 \\
\hline $14 \mathrm{~N}$ & d & & $1.8+1$ & OSA & E Aohlt & 5330 \\
\hline 160 & p & $2.0+1$ & $1.0+3$ & GSF & D Ramoty ${ }^{+}$ & 551 \\
\hline 160 & d & $6.7+0$ & $1.2+1$ & WIS & E Riedhausert & 5345 \\
\hline 160 & a & $2.4+1$ & $0.0+1$ & GSF & O Ramaty+ & 6853 \\
\hline $\begin{array}{l}170 \\
27{ }_{A 1}\end{array}$ & $160^{p}$ & $\begin{array}{l}4.0-1 \\
8 . \theta+1\end{array}$ & $1.4+0$ & $\begin{array}{l}\text { TOR } \\
\text { TSU }\end{array}$ & $\begin{array}{l}\text { E Kieser. } \\
\text { E Mihumot }\end{array}$ & $\begin{array}{r}574 \\
10953\end{array}$ \\
\hline
\end{tabular}

.




\begin{tabular}{|c|c|c|c|c|c|c|}
\hline Targel & $\ln c$ & $\begin{array}{l}\text { Emin } \\
(M e V)\end{array}$ & $\begin{array}{l}\text { Emax } \\
(\text { Me'f) }\end{array}$ & Lab $h$ & Author & No. \\
\hline $14 \mathrm{~N}$ & product & yield & & & & \\
\hline $\begin{array}{l}14 \mathrm{~N} \\
180\end{array}$ & $18_{0}^{\circ}$ & $\begin{array}{l}5.6+0 \\
9.1+1\end{array}$ & $5.6+0 \mathrm{~K}$ & $\begin{array}{l}\text { KOU E } \\
\text { PAR E }\end{array}$ & $\begin{array}{l}\text { E Mah+ } \\
\text { E Dairaz+ }\end{array}$ & $\begin{array}{r}6812 \\
11650\end{array}$ \\
\hline${ }^{14} \mathrm{~N}$ & $O(E)$ ot & resonon & & & & \\
\hline${ }^{12} \mathrm{C}$ & ${ }^{12} \mathrm{C}$ & $6.2+1$ & & ROC E & E Clovert & 9726 \\
\hline $14 \mathrm{~N}$ & row o(E) & & & & & \\
\hline $\begin{array}{l}17 \mathrm{C} \\
170\end{array}$ & p & $\begin{array}{l}4.6-1 \\
7.4-1\end{array}$ & $\begin{array}{l}9.6-1 \\
1.5+0\end{array}$ & $\begin{array}{l}\text { TOR E } \\
\text { MCM E }\end{array}$ & $\begin{array}{l}\text { E Kiesert } \\
\text { E Kiesert }\end{array}$ & $\begin{array}{l}575 \\
576\end{array}$ \\
\hline $14 \mathrm{~N}$ & O(E) XfOE & tor & & & & \\
\hline $\begin{array}{l}170 \\
170 \\
170 \\
40 \mathrm{Cs} \\
200 \mathrm{~Pb} \\
200 \mathrm{~Pb}\end{array}$ & $\begin{array}{r}p \\
p \\
16 p \\
160 \\
160\end{array}$ & $\begin{array}{l}0.0+0 \\
0.0+0 \\
1.0-2 \\
5.6+1 \\
1.4+2 \\
1.4+2\end{array}$ & $\begin{array}{l}1.5-1 \\
1.5-1 \\
1.4-1 \\
3.2+2 \\
3.2+2\end{array}$ & $\begin{array}{l}\text { CAL E } \\
\text { MST F } \\
\text { TOR E } \\
\text { ANL E } \\
\text { BRK E } \\
\text { MSU F }\end{array}$ & $\begin{array}{l}\text { E Rolfgt } \\
\text { R Rolfgt } \\
\text { E Klesert } \\
\text { E Kovart } \\
\text { E Golbhet } \\
\text { R Gelbhe. }\end{array}$ & $\begin{array}{r}571 \\
572 \\
573 \\
11110 \\
11541 \\
11542\end{array}$ \\
\hline $14 \mathrm{~N}$ & O(E) & & & & & \\
\hline $\begin{array}{l}11 \mathrm{C} \\
12 \mathrm{C} \\
160 \\
150 \\
170 \\
A 9\end{array}$ & $\begin{array}{r}12 a \\
12 C \\
p \\
d \\
0 \\
1{ }^{2} N\end{array}$ & $\begin{array}{l}\text { NDG } \\
7.0+1 \\
5.0+1 \\
6.5-1 \\
4.0-1 \\
7.8+1\end{array}$ & $\begin{aligned} 1.1+0 k \\
1.4+0 \\
1.1+2\end{aligned}$ & $\begin{array}{l}\text { CAL } \\
\text { ROC } \\
\text { LPI } \\
\text { KOU E } \\
\text { TOR } \\
\text { LYO }\end{array}$ & $\begin{array}{l}\text { M Ingallst } \\
\text { E Hermont } \\
\text { E Voovint } \\
\text { E Moc-Arthurt } \\
\text { M Kiesert } \\
\text { T Billereyt }\end{array}$ & $\begin{array}{r}6741 \\
9728 \\
552+ \\
5346 \\
568 \\
10430\end{array}$ \\
\hline $15_{N}$ & compound & S-nuclou & us $\sigma(E)$ & & & \\
\hline 108 & $T_{L i}$ & $2.4+1$ & & HEI $\mathrm{r}$ & M Kohlert & 9411 \\
\hline $15_{N}$ & portlol & row ofE & & & & \\
\hline${ }^{15} \mathrm{~N}$ & $\alpha$ & $7.9+0$ & $8.4+0$ & OXF E & E Symonst & 6822 \\
\hline $15 \mathrm{~N}$ & porl1.01 & o(E) of & tresond & nonce & & \\
\hline${ }^{13} \mathrm{C}$ & $3_{\mathrm{He}}$ & $4.6+0$ & & OXF E & E Cheost & 6148 \\
\hline${ }^{15} \mathrm{~N}$ & partlal & relotiv & vo o(E) & & & \\
\hline $\begin{array}{l}12 \mathrm{C} \\
13 \mathrm{C} \\
48 \mathrm{Ti}\end{array}$ & $\begin{array}{c}a_{0}^{a} \\
{ }_{160}^{3} \mathrm{He} \\
10^{2}\end{array}$ & $\begin{array}{l}1.8+1 \\
2.0+1 \\
9.6+1\end{array}$ & $1.9+1$ & $\begin{array}{l}\text { KLN } \\
\text { NBI E } \\
\text { MUN }\end{array}$ & $\begin{array}{l}\text { E Moebiust } \\
\text { E Pedersent } \\
\text { M Heringt }\end{array}$ & $\begin{array}{r}6768 \\
6147 \\
11118\end{array}$ \\
\hline
\end{tabular}

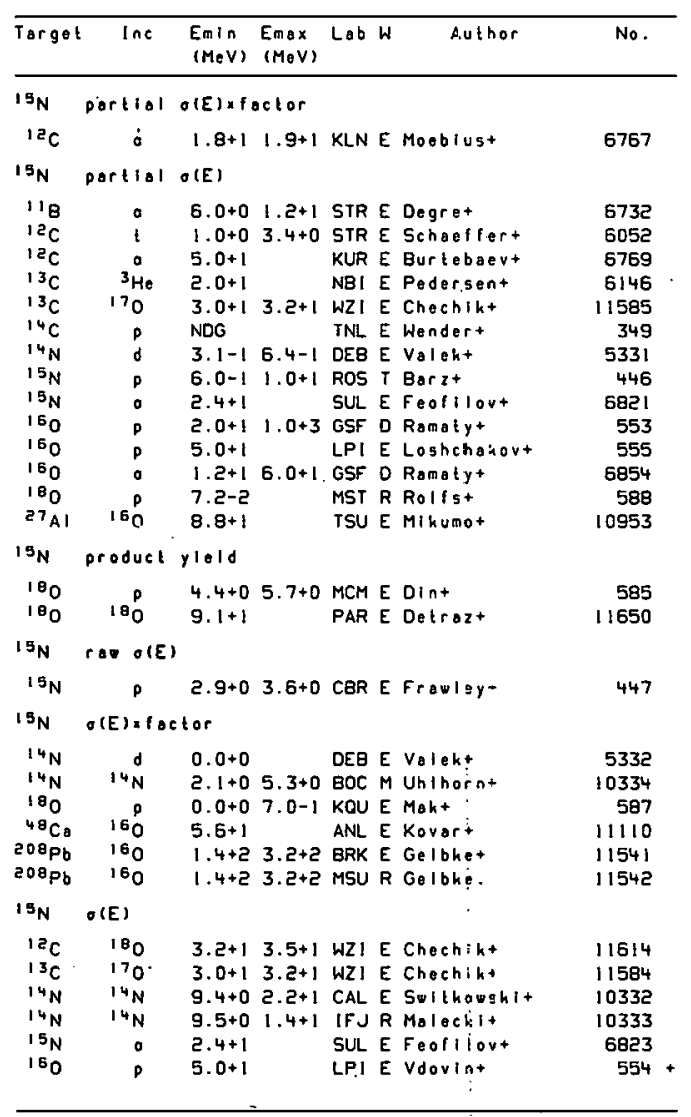




\begin{tabular}{|c|c|c|c|c|c|c|}
\hline Target & $\ln c$ & $\begin{array}{l}E_{m i n} \\
\left(\mathrm{YeV}_{\mathrm{e}}\right)\end{array}$ & $\begin{array}{l}\text { Emax } \\
(\operatorname{MeV})\end{array}$ & Lab h & Author & No. \\
\hline $15_{N}$ & $O(E)$ & & & & & \\
\hline $\begin{array}{l}180 \\
19 F \\
27 \mathrm{AI} \\
28 \mathrm{Si} \\
\mathrm{Ag}\end{array}$ & $\begin{array}{l}3^{\mathrm{p}} \\
3 \mathrm{He} \\
11 \mathrm{~B} \\
11 \mathrm{~B} \\
14 \mathrm{~N}\end{array}$ & $\begin{array}{l}2.4-1 \\
\text { IR } \\
5.0+1 \\
5.0+1 \\
7.8+1\end{array}$ & $\begin{array}{l}7.0-1 \\
5.9+0 \\
1.1+2\end{array}$ & $\begin{array}{l}\text { KOU E } \\
\text { BRK E } \\
\text { FSU E } \\
\text { FSU E } \\
\text { LYO T }\end{array}$ & $\begin{array}{l}\text { E Maht } \\
\text { E Pylat } \\
\text { E Parkst } \\
\text { E Parkst } \\
\text { T Billereyt }\end{array}$ & $\begin{array}{r}586 \\
6174 \\
9569 \\
9570 \\
10430\end{array}$ \\
\hline $16_{N}$ & compound & $y$-nucleu & US O(E) & & & \\
\hline${ }^{10} \mathrm{~B}$ & ${ }^{7} L i$ & $2.4+1$ & & HEI $M$ & M Kohlert & 9412 \\
\hline $16 \mathrm{~N}$ & portiol & direct- & - Intero & oction & n O(E) xfactor & \\
\hline $14 \mathrm{~N}$ & 1 & $1.5+1$ & & PEN E & E Fortunet & 6054 \\
\hline${ }^{16} \mathrm{~N}$ & portlal & $O(E) \times f a$ & actor & & & \\
\hline${ }^{14} \mathrm{~N}$ & 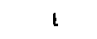 & $1.5+1$ & & PEN E & E Fortunet & 6053 \\
\hline${ }^{10} \mathrm{~N}$ & product & yield & & & & \\
\hline 180 & $10_{0}$ & $9.1+1$ & & PAR E & E Detraz+ & 11650 \\
\hline $16 \mathrm{~N}$ & ofE) afoc & eter & & & & \\
\hline${ }^{48} \mathrm{Co}$ & 160 & $5.6+1$ & & ANL E & E Kovart & 11110 \\
\hline $18_{N}$ & $O(E)$ & & & & & \\
\hline Ag & ${ }^{14} \mathrm{~N}$ & $\therefore .8+1$ & $1,1+2$ & LYO 1 & I Bullereyt & 10430 \\
\hline $17_{N}$ & portiol & OIEIAfO & octor & & & \\
\hline${ }^{15} \mathrm{~N}$ & 1 & $1.5+1$ & & PEN E & E Mooret & 6056 \\
\hline $17_{N}$ & product & yiold & & & & \\
\hline 180 & 180 & $9.1+1$ & & PAR E & E Detrozt & 11650 \\
\hline $17_{N}$ & $O(E) \times f \Delta C$ & ctor & & & & \\
\hline $\begin{array}{l}24 \mathrm{Mg} \\
25 \mathrm{Mg} \\
26 \mathrm{Mg} \\
325 \\
345 \\
{ }^{34} 5 \\
{ }^{40} \mathrm{Co} \\
{ }^{4} \mathrm{Cg} \\
{ }^{4} \mathrm{C}^{\mathrm{C} 8}\end{array}$ & $\begin{array}{r}p \\
p \\
p \\
p \\
p \\
p \\
160\end{array}$ & $\begin{array}{l}1.0+3 \\
1.0+3 \\
1.0+3 \\
1.0+3 \\
1.0+3 \\
1.0+3 \\
1.0+3 \\
5.6+1\end{array}$ & & $\begin{array}{l}\text { LIN E } \\
\text { LINE } \\
\text { LIN E } \\
\text { LIN E } \\
\text { LIN E } \\
\text { LIN E } \\
\text { LIN E } \\
\text { ANL E }\end{array}$ & $\begin{array}{l}\text { E Batist+ } \\
\text { E Batist+ } \\
\text { E Batist+ } \\
\text { E Batist+ } \\
\text { E Batist+ } \\
\text { E Batist+ } \\
\text { E Batist+ } \\
\text { E Kovart }\end{array}$ & $\begin{array}{r}739+ \\
755+ \\
769+ \\
1060+ \\
1070+ \\
1255+ \\
1291+ \\
11110\end{array}$ \\
\hline
\end{tabular}

\begin{tabular}{|c|c|c|c|c|c|c|}
\hline Torget & $\ln c$ & $\begin{array}{l}\text { Emin } \\
(M \circ V)\end{array}$ & $\begin{array}{l}\text { Emax } \\
(M \circ V)\end{array}$ & Lab h & Author & No. \\
\hline $17 \mathrm{~N}$ & \multicolumn{6}{|l|}{$O(E)$} \\
\hline 18 & ${ }^{7} L i$ & $3.5+1$ & & FSU E & E Delaune. & 9414 \\
\hline${ }^{24} \mathrm{Mg}$ & p & $1.0+3$ & & LINE & E Batist+ & 738 \\
\hline${ }^{25} \mathrm{Mg}$ & p & $1.0+3$ & & LIN & $E-B a t i s t+$ & 754 \\
\hline $26 \mathrm{Mg}$ & p & $1.0+3$ & & LIN & E Batistt & 768 \\
\hline 325 & $p$ & $1.0+3$ & & LINE & E Batistt & 1059 \\
\hline${ }^{34} \mathrm{~S}$ & $p$ & $1.0+3$ & & LINE & E Batistt & 1069 \\
\hline${ }^{40} \mathrm{Ca}$ & p & $1.0+3$ & & LINE & E Batist+ & 1254 \\
\hline${ }^{44} \mathrm{Ca}$ & $p$ & $1.0+3$ & & LINE & E Batlistt & 1290 \\
\hline${ }^{5 \theta^{N}} \mathrm{Ni}$ & $p$ & $1.0+3$ & & LINE & E Batist+ & 2255 \\
\hline${ }^{62} \mathrm{Ni}$ & p & $1.0+3$ & & LINE & E Batistt & 2385 \\
\hline${ }^{64} \mathrm{Ni}$ & $p$ & $1.0+3$ & & LIN E & E Batist+ & 2420 \\
\hline $1225 n$ & $p$ & $1.0+3$ & & LIN $E$ & E Batrstt & 3844 \\
\hline $1165 n$ & p & $1.0+3$ & & LIN $E$ & E Batista & 3851 \\
\hline $119 \mathrm{Sn}$ & p & $1.0+3$ & & LIN & E Batist+ & 3882 \\
\hline $120 \mathrm{Sn}$ & p & $1.0+3$ & & LIN E & E Botistt & 3894 \\
\hline $122 \mathrm{sn}$ & $p$ & $1.0+3$ & & LIN E & E Batista & 3904 \\
\hline $1245 n$ & p & $1.0+3$ & & LIN & E Batist+ & 3911 \\
\hline $1 \theta_{N}$ & \multicolumn{6}{|c|}{ product ylold } \\
\hline 180 & $18_{0}$ & $9.1+1$ & & PAR E & E Detrazt & 11650 \\
\hline $2=8$ & \multicolumn{6}{|c|}{ Independont $O\left(E_{;} ; E^{\prime} ; \theta\right)$} \\
\hline $232 \mathrm{Th}$ & $40_{\mathrm{Ar}}$ & $3.0+2$ & $3.9+2$ & DUA E & E Artukht & 12396 \\
\hline$z=\theta$ & \multicolumn{6}{|c|}{ Sosllation o(E) } \\
\hline $238^{2}$ & p & $5.5+3$ & & ANL $F$ & Rethiffer. & 4894 \\
\hline$z=\theta$ & \multicolumn{6}{|c|}{ direct-interoction $O(E)$} \\
\hline $12 c$ & $14 N$ & $1.1+2$ & $1.8+2$ & ORL E & E Del-Campot & 10316 \\
\hline 160 & 160 & $6.7+1$ & $1.6+2$ & GRE E & E Conjeaudt & 10855 \\
\hline $20 \mathrm{Ne}$ & ${ }^{12} \mathrm{C}$ & $1.2+2$ & $1.4+2$ & GRE E & E Conjesudt & 9869 \\
\hline$z=\theta$ & \multicolumn{6}{|c|}{ portial o(E) } \\
\hline${ }^{12} \mathrm{C}$ & ${ }^{12} \mathrm{C}$ & $5.0+1$ & & SAC E & E Conjeaudt & 9772 \\
\hline $12 \mathrm{C}$ & ${ }^{14} \mathrm{~N}$ & $1.6+2$ & $5+2$ & BRK E & E Stokstadt & 10323 \\
\hline $2 \cdot \theta$ & \multicolumn{6}{|c|}{ product yleld } \\
\hline $10 \mathrm{~B}$ & 160 & $9.8+1$ & & JCL $\dot{E}$ & E Motobayaghit & 10587 \\
\hline $12 \mathrm{C}$ & ${ }^{14} \mathrm{~N}$ & $9.1+1$ & & JCL E & E Motobayashit & 10320 \\
\hline
\end{tabular}




\begin{tabular}{|c|c|c|c|c|c|c|c|}
\hline Target & $\operatorname{lnc}$ & $\begin{array}{l}\text { Emin } \\
(\mathrm{MeV})\end{array}$ & $\begin{array}{l}\text { Emax } \\
(\operatorname{Me} V \bar{V})\end{array}$ & Lab & $W$ & Author & No. \\
\hline$z=\theta$ & raso & & & & & & \\
\hline${ }^{12} \mathrm{c}$ & ${ }^{12} \mathrm{C}$ & $4.0+1$ & & MUN & E & Everst & 9773 \\
\hline$z=8$ & $\theta(E) \times$ & octor & & & & & \\
\hline $\begin{array}{l}10^{10} B \\
{ }^{12} \mathrm{C} \\
1{ }^{4} \mathrm{~N}\end{array}$ & $\begin{array}{l}160 \\
14 \mathrm{~N} \\
12 \mathrm{C}\end{array}$ & $\begin{array}{l}3.9+1 \\
\text { NDG } \\
5.0+1\end{array}$ & $2.0+2$ & $\begin{array}{l}\text { ORL } \\
\text { ORL } \\
\text { SAC }\end{array}$ & $\begin{array}{l}\text { E } \\
\text { E } \\
\text { E }\end{array}$ & $\begin{array}{l}\text { Del-Campot } \\
\text { Del-Campot } \\
\text { Conjesudt }\end{array}$ & $\begin{array}{r}10586 \\
10315 \\
9805\end{array}$ \\
\hline$z=8$ & $\sigma(E)$ & & & & & & \\
\hline $\begin{array}{l}1{ }^{12} \mathrm{c} \\
1{ }^{2} \mathrm{c}\end{array}$ & $\begin{array}{l}12 \mathrm{C} \\
12 \mathrm{C}\end{array}$ & $\begin{array}{l}1.5+1 \\
2.8+1\end{array}$ & $\begin{array}{l}6.2+1 \\
5.4+1\end{array}$ & ANL & & $\begin{array}{l}\text { Kovart } \\
\text { Confeaudt }\end{array}$ & $\begin{array}{l}9768 \\
9769\end{array}$ \\
\hline $12 \mathrm{C}$ & $12 \mathrm{C}$ & $4.5+1$ & $2.0+2$ & TAM & E & Namboodirit & 9770 \\
\hline${ }^{12} \mathrm{c}$ & ${ }^{13} \mathrm{c}$ & $1.6+1$ & $5.2+1$ & ANL & E & Kovart & 10185 \\
\hline${ }^{12} \mathrm{c}$ & $14 \mathrm{~N}$ & $3 \cdot 3+1$ & $5.2+1$ & ANL & E & Kovart & 10317 \\
\hline $1{ }^{2} \mathrm{c}$ & $14 \mathrm{~N}$ & $8.6+1$ & $1.7+2$ & ORL & E & Stohstaot & 10318 \\
\hline $12 c$ & $15 \mathrm{~N}$ & $2.0+1$ & $6.0+1$ & ANL & E & Kovart & 10528 \\
\hline$i{ }^{2} c$ & 160 & $4.0+1$ & $6.6+1$ & NOT & E I & Molmint & 10722 \\
\hline $12 c$ & $10_{0}$ & $1.0+2$ & & STR & $E$ & Coffint & 11626 \\
\hline $1{ }^{2} \mathrm{c}$ & $20 \mathrm{Ne}$ & $6.7+1$ & $1.6+2$ & GRE & E & Saint-Laurent+ & 11839 \\
\hline${ }^{13} \mathrm{C}$ & $10_{0}$ & $1.0+2$ & & STR & E & Coffint & 11639 \\
\hline $14 \mathrm{~N}$ & $12 c$ & $3.4+1$ & $5.0+1$ & SAC & E & Conjeaud & 9806 \\
\hline $14 \mathrm{~N}$ & ${ }^{13} \mathrm{C}$ & $4.4+1$ & $5.4+1$ & SAC & E & Conjeaudt & 10192 \\
\hline $15 \mathrm{~N}$ & $12_{C}$ & $3.0+1$ & $5.0+1$ & SAC & E & Conjeauot & 9815 \\
\hline 160 & 160 & $1.2+2$ & $1.4+2$ & GRE & $E$ & Saint-Leurentt & 10854 \\
\hline 170 & $12 \mathrm{C}$ & $3.5+1$ & $4.9+1$ & SAC & E & Conjeaud & 9845 \\
\hline 170 & ${ }^{13} \mathrm{C}$ & $3.8+1$ & $5.3+1$ & SAC & E & Conjeaudt & 10195 \\
\hline $20 \mathrm{Ne}$ & $12 \mathrm{C}$ & $4.8+1$ & $9.6+1$ & SAC I & $M$ & Conjeaust & 9867 \\
\hline${ }^{45} 5 \mathrm{c}$ & ${ }^{22} \mathrm{Ne}$ & NOG & & LYO & $T$ & Billereyt & 11924 \\
\hline 514 & 160 & $7.7+1$ & & GRE & E & Chevariert & 11142 \\
\hline${ }^{50} \mathrm{Cr}$ & $14 \mathrm{~N}$ & $7.1+1$ & $1.0+2$ & LYO & $\mathrm{T}$ & Billereyt & 10393 \\
\hline${ }^{50} \mathrm{Cr}$ & $22_{\mathrm{Ne}}$ & NOG & & LYO & $T E$ & Billereyt & 11925 \\
\hline${ }^{55} \mathrm{Mn}$ & ${ }^{12} \mathrm{C}$ & $7.7+1$ & & GRE & E & Chevariert & 9944 \\
\hline${ }^{56} \mathrm{Fe}, \mathrm{e}$ & 160 & NDG & & LYO & $\mathrm{T}$ & Billereyt & 11162 \\
\hline${ }^{56} \mathrm{Fe}$ & 160 & $7.7+1$ & & GRE & E & Chevarlert & 11163 \\
\hline & $20 \mathrm{Ne}$ & $1.6+2$ & & ORL & R F & Plesilt+ & 11851 \\
\hline${ }^{60} \mathrm{NI}$ & ${ }^{12} \mathrm{C}$ & NDG & & LYO & $T$ & Billereyt & 9960 \\
\hline $60 \mathrm{Ni}$ & $12 \mathrm{C}$ & $7.7+1$ & & GRE & E & Chevarlert & 9961 \\
\hline $181 \mathrm{Tg}$ & 160 & $8.3+1$ & $9.6+1$ & BNL 1 & MI & videboent & 11464 \\
\hline $200 \mathrm{~Pb}$ & 160 & $8.0+1$ & $1.0+2$ & BNL 1 & M & Videboekt & 11537 \\
\hline $232 \mathrm{Th}$ & ${ }^{40} \mathrm{Ar}$ & $7.2+4$ & & BRK & $\mathbf{R}=$ & Scott. & 12399 \\
\hline
\end{tabular}

\begin{tabular}{|c|c|c|c|c|c|c|}
\hline Target & $\ln e$ & $\begin{array}{l}\text { Emin } \\
(M e V)\end{array}$ & $\begin{array}{l}\text { Emax } \\
(\operatorname{MeV})\end{array}$ & Lab & Author & No: \\
\hline 130 & product, & $y 1010$ & & & & \\
\hline $14 N$ & $p$ & NDG & & BRK & R Cernyt & 425 \\
\hline${ }^{14} 0$ & rOD O(E) & & & & & \\
\hline${ }^{14} \mathrm{~N}$ & $p$ & $6.3+0$ & $6.4+0$ & NZA & E Whilet & 427 \\
\hline 140 & $\sigma(E)$ & & & & & \\
\hline $\begin{array}{l}14 \mathrm{~N} \\
160\end{array}$ & p & $\begin{array}{l}6.0+0 \\
1.6+2\end{array}$ & $1.5+1$ & $\begin{array}{l}\text { JCL } \\
\text { LPI }\end{array}$ & $\begin{array}{l}\text { E Nosakit } \\
\text { T Dolitievt }\end{array}$ & $\begin{array}{l}426 \\
556\end{array}$ \\
\hline 150 & diroct-in & interact & tion ot & (E) & & \\
\hline $15 \mathrm{~N}$ & $p$ & $1.0+0$ & $1.0+1$ & ROS & T Bar 2+ & 460 \\
\hline 150 & thich tor & orgot $y^{\prime}$ & 1018 & & i & \\
\hline 160 & $p$ & $2.7+1$ & & AML & R Chaudri. & 560 \\
\hline 190 & partlal & O(E) of & trason & nonce & & \\
\hline $15_{N}$ & 0 & $8.9+0$ & $1.8+1$ & BIR & E Cheyt & 459 \\
\hline 150 & portlol. & relativ & ve $O(E)$ & & & \\
\hline${ }^{12} \mathrm{C}$ & ${ }^{12} \mathrm{C}$ & $3.0+1$ & $1.9+2$ & BRK & R Horvey: & 9729 \\
\hline 150 & portiolo & $O(E)$ & & & . & \\
\hline $14 N$ & $p$ & NDG & & INL & E Wendert & 428 \\
\hline $14 N$ & p & +0 & & SAC & R Engelmarn- & 429 \\
\hline${ }^{15} \mathrm{~N}$ & p & NDG & & TNL & T Byrd+ & 455 \\
\hline $15_{N}$ & $p$ & $1.0+0$ & $1.0+1$ & ROS & T Barz+ & 456 \\
\hline $15_{N}$ & p & $5.0+0$ & $1.6+1$ & MUN & T Ardit . & 457 \\
\hline $15 \mathrm{~N}$ & $p$ & $5.8+0$ & $8.6+0$ & BIR & E Chewt & 458 \\
\hline 160 & p & $2.0+1$ & $1.0+3$ & GSF & D Ramaty+ & 557 \\
\hline 190 & rolativo & $l_{y}$ & & & & \\
\hline $14 \mathrm{~N}$ & $p$ & $1.7+0$ & $1.8+0$ & LYO & E Deaint & 431 \\
\hline 150 & product & $y 1010$ & & & & \\
\hline$N$ & d & NDG & & USA & R Beavert & 5328 \\
\hline $14 \mathrm{~N}$ & p & $7.0+0$ & & USA & R Beavert & 430 \\
\hline $14 \mathrm{~N}$ & d & NDG & & BNL & R Ruth+ & 5333 \\
\hline${ }^{15} \mathrm{~N}$ & p & NDG & & BNL & R Rutht & 448 \\
\hline $15 \mathrm{~N}$ & p & +1 & & USA & R Beavert & 449 \\
\hline 0 & p & $2.7+1$ & & USA & R Beavert & 473 \\
\hline
\end{tabular}




\begin{tabular}{|c|c|c|c|c|c|c|}
\hline Torget & $\operatorname{lnc}$ & $\begin{array}{l}\text { Emin } \\
(\mathrm{MeV})\end{array}$ & $\begin{array}{l}\text { Emax } \\
(\mathrm{MeV})\end{array}$ & Lob & Author & No. \\
\hline 150 & product & $y \in=10$ & & & & \\
\hline $\begin{array}{r}160 \\
180 \\
C A P\end{array}$ & $\begin{array}{r}18 p \\
p \\
p\end{array}$ & $\begin{array}{l}2.6-1 \\
9.1-1 \\
4.5-1\end{array}$ & 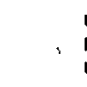 & $\begin{array}{l}\text { USA } \\
\text { PAR } \\
\text { UPP }\end{array}$ & $\begin{array}{l}\text { R Geovert } \\
\text { E Detrazt } \\
\text { E Lundquist+ }\end{array}$ & $\begin{array}{r}559 \\
11650 \\
609\end{array}$ \\
\hline 150 & thick to & arget $y^{1}$ & 1018 & & & \\
\hline $\begin{array}{l}N \\
14 N \\
14 N \\
14 N \\
14 N \\
14 N \\
14 N \\
15 N\end{array}$ & $\begin{array}{l}d \\
p \\
d \\
d \\
d \\
d \\
3^{\mathrm{He}} \\
\rho\end{array}$ & $\begin{array}{l}\text { NDG } \\
3.8-1 \\
\text { NDG } \\
\text { TR } \\
5.0+0 \\
6.0+0 \\
1.7+1 \\
\text { TR }\end{array}$ & $\begin{array}{l}6.8+0 \\
6.0+0 \\
1.2+1\end{array}$ & $\begin{array}{l}\text { WIS } \\
\text { CLU } \\
\text { TNL } \\
\text { ORL } \\
\text { AML } \\
\text { JUL } \\
\text { AML } \\
\text { OAU }\end{array}$ & $\begin{array}{l}\text { E Nicklest } \\
\text { M Roughiont } \\
\text { E Nelson. } \\
\text { D Wielandt } \\
\text { R Chaudri. } \\
\text { R Stochlint } \\
\text { R Chaudri. } \\
\text { D Wielandt }\end{array}$ & $\begin{array}{r}5327 \\
434 \\
5336 \\
5337 \\
5338 \\
5339 \\
6152 \\
454\end{array}$ \\
\hline 150 & relative & - OIEI & & & & \\
\hline $14 \mathrm{~N}$ & d & $3.0+0$ & $1.0+1$ & TNL & E Nelsont & 5340 \\
\hline 150 & $O(E) \times f a c$ & ctor. & & & & \\
\hline $\begin{array}{l}{ }^{48} \mathrm{C} . \\
{ }^{15} \mathrm{O}^{\circ}\end{array}$ & ${ }^{15} 0$ & $5.6+1$ & & ANL & E Kovart & 11110 \\
\hline $12 c$ & 。 & $T R$ & $2.6+1$ & KLN & M Gruhlet & 6770 \\
\hline $14 \mathrm{~N}$ & $p$ & $0.0+0$ & $4.2+1$ & INU & T Compani-Tabrizi & 432 \\
\hline $14 \mathrm{~N}$ & p & $2.0-1$ & $2.6+0$ & YAL & E Troutvettert & 433 \\
\hline${ }^{14} \mathrm{~N}$ & d & TR & $5.0+0$ & ORL & D Wielandt & 5334 \\
\hline $14 \mathrm{~N}$ & d & $6.0-1$ & $2.8+0$ & 8NL & E Ruiz+ & 5335 \\
\hline $15_{N}$ & p & $T R$ & $1.2+1$ & OAU & D Wlel andt & 450 \\
\hline $15 \mathrm{~N}$ & p & $1.0+0$ & $1.0+1$ & ROS & T Bor zt & 451 \\
\hline $15_{N}$ & p & $4.0+0$ & $1.4+1$ & MUN & T Ardit & 452 \\
\hline $15 \mathrm{~N}$ & p & $9.0+0$ & $1.6+1$ & TNL & E Byrdt & 453 \\
\hline 160 & p & $1.6+2$ & & LPI & T Daltrievt & 558 \\
\hline 160 & 0 & $9.2+5$ & & LPI & T Dmitrievt & 6855 \\
\hline Ag & $14 \mathrm{~N}$ & $7.8+1$ & $1.1+2$ & LYO & T Billereyt & 10430 \\
\hline 160 & compound & $d$-nucles & US $O(E)$ & & & \\
\hline $10 \mathrm{~B}$ & $7_{L I}$ & $2.4+1$ & & HEI & M Kohlert & $94: 3$ \\
\hline 160 & Dartiol & compoun & ind-nucl & leus & $O(E)$ & \\
\hline${ }^{180} 0$ & p & $2.0+1$ & $4.4+1$ & TAM & T Kubot & 590 \\
\hline
\end{tabular}

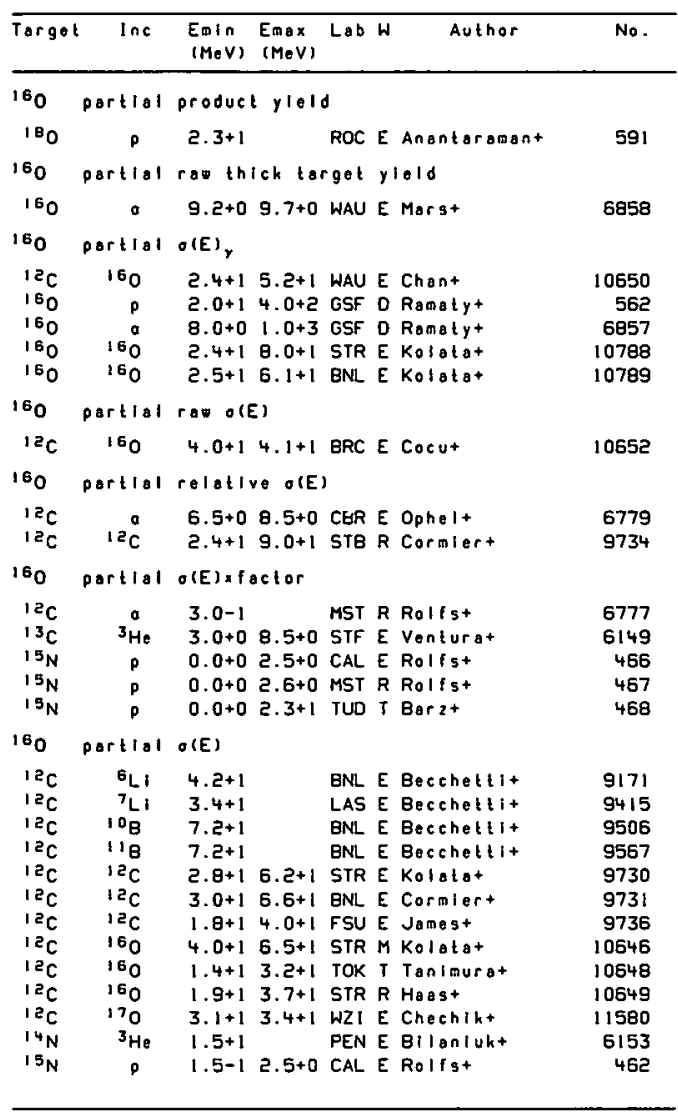




\begin{tabular}{|c|c|c|c|c|c|c|}
\hline Target & $\ln c$ & $\begin{array}{l}\text { Emin } \\
(\mathrm{MeV})\end{array}$ & $\begin{array}{l}\text { Emax } \\
\text { (MeV: }\end{array}$ & Lab & Author & No. \\
\hline 160 & partlal o & $\sigma(E)$ & & & & \\
\hline 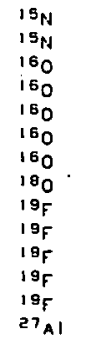 & $\begin{array}{r}p \\
p \\
p \\
a \\
q \\
160 \\
160 \\
p \\
p \\
p \\
p \\
p \\
p \\
p \\
160\end{array}$ & $\begin{array}{l}6.0+0 \\
8 \cdot 0+0 \\
2.0+1 \\
1.8+1 \\
1.5+1 \\
\text { NDG } \\
3.6+1 \\
2.0+1 \\
1.5+0 \\
1.2+1 \\
1 \cdot 4+1 \\
2 \cdot 0+1 \\
2 \cdot 3+1 \\
8.8+1\end{array}$ & $\begin{array}{l}2.2+1 \\
1.3+1 \\
1.5+2 \\
2.0+1 \\
2.0+1 \\
0.4+1 \\
4.4+1 \\
2.0+0 \\
1.0+2 \\
5.0+1\end{array}$ & $\begin{array}{l}\text { OXF E } \\
\text { SIF } \\
\text { JUL } \\
\text { WIS E } \\
\text { WIS E } \\
\text { WIS } \\
\text { YAL } \\
\text { TAM } \\
\text { PAD } \\
\text { HEI } \\
\text { TUE } \\
\text { MIL } \\
\text { JUL } \\
\text { TSU }\end{array}$ & $\begin{array}{l}\text { E Chewt } \\
\text { M Calarcot } \\
\text { T Wambacht } \\
\text { E Blllen. } \\
\text { E Billent } \\
\text { T Friedment } \\
\text { T Phllilipst } \\
\text { T Kubot } \\
\text { M Oe-Rosot } \\
\text { E Stablert } \\
\text { M Rohwert } \\
\text { T Gadiolit } \\
\text { E Rohwert } \\
\text { E Mikumot }\end{array}$ & $\begin{array}{r}463 \\
464 \\
561 \\
6856 \\
6859 \\
10786 \\
10787 \\
589 \\
614 \\
615 \\
616 \\
617 \\
618 \\
10953\end{array}$ \\
\hline 160 & product y & yield & & & & \\
\hline $\begin{array}{l}12 c \\
180\end{array}$ & $\begin{array}{l}12 \mathrm{C} \\
180\end{array}$ & $\begin{array}{l}7.0+1 \\
9.1+1\end{array}$ & & $\begin{array}{l}\text { ROC } \\
\text { PAR }\end{array}$ & $\begin{array}{l}\text { E Hermant } \\
\text { E Detraz+ }\end{array}$ & $\begin{array}{r}9735 \\
11650\end{array}$ \\
\hline $\begin{array}{l}16_{0} \\
19_{F}\end{array}$ & $\begin{array}{c}\text { rolative } \\
\text { p }\end{array}$ & $\begin{array}{l}\text { thlch } \\
8.4-1\end{array}$ & $\begin{array}{l}\text { target } \\
9.6-t\end{array}$ & & $\begin{array}{l}\text { eld } \\
\text { E Crumptont. }\end{array}$ & 619 \\
\hline${ }^{160} 0$ & thick tor & rget $y 1$ & teld & & & \\
\hline $\begin{array}{l}13 \mathrm{C} \\
13 \mathrm{C}\end{array}$ & व & $\begin{array}{l}6.0-1 \\
3.0+0\end{array}$ & $\begin{array}{l}1.2+0 \\
9.0+0\end{array}$ & $\begin{array}{l}\text { AE } \\
\text { ORL }\end{array}$ & $\begin{array}{l}\text { M Ramgtront } \\
\text { E Bairt }\end{array}$ & $\begin{array}{l}6786 \\
6787\end{array}$ \\
\hline 160 & O(E) at $r$ & resonanc & nee & & & \\
\hline $\begin{array}{l}12 \mathrm{C} \\
15 \mathrm{~A}\end{array}$ & a & $\begin{array}{l}1.4+0 \\
8.6+0\end{array}$ & $1.8+!$ & $\begin{array}{l}\text { MST } \\
\text { STF }\end{array}$ & $\begin{array}{l}\text { R Rolfgt } \\
\text { E O-Connell+ }\end{array}$ & $\begin{array}{r}6776 \\
465\end{array}$ \\
\hline 160 & relative & $\sigma(E)$ & & & & \\
\hline $\begin{array}{l}1 z_{C} \\
15^{k} \\
19^{2}\end{array}$ & $\begin{array}{l}\text { a } \\
\dot{p} \\
\mathbf{p}\end{array}$ & $\begin{array}{l}6.5+0 \\
6.0+0 \\
3.0-1\end{array}$ & $\begin{array}{l}8.5+0 \\
9.0+0 \\
1.1+0\end{array}$ & $\begin{array}{l}\text { CBR } \\
\text { WAU } \\
\text { BKB }\end{array}$ & $\begin{array}{l}\text { E Ophelt } \\
\text { E Snovert } \\
\text { E Subolict }\end{array}$ & $\begin{array}{r}6778 \\
469 \\
620\end{array}$ \\
\hline 160 & $O(E) \times f o c t$ & tor & & & & \\
\hline $\begin{array}{l}1{ }^{12} c \\
1{ }^{2} c \\
1{ }^{2} c \\
1{ }^{2} c\end{array}$ & $\begin{array}{r}: \\
12 a \\
c\end{array}$ & $\begin{array}{l}1.3-1 \\
4.0-1 \\
4.0-1 \\
6.0+0\end{array}$ & $\begin{array}{l}4.7+0 \\
4.1+0 \\
1.4+1\end{array}$ & $\begin{array}{l}\text { MST } \\
\text { CAL } \\
\text { LAS } \\
\text { NCS }\end{array}$ & $\begin{array}{l}\text { R Rolfst } \\
\text { T Humblett } \\
\text { E Bocchettlt } \\
\text { M Porkt }\end{array}$ & $\begin{array}{l}6771 \\
6772 \\
6773 \\
9732\end{array}$ \\
\hline
\end{tabular}

\begin{tabular}{|c|c|c|c|c|c|c|c|}
\hline Target & $\ln c$ & $\begin{array}{l}\text { Emin } \\
(\mathrm{MeV})\end{array}$ & $\begin{array}{l}\text { Emax } \\
(\operatorname{MeV})\end{array}$ & Lab & w & Author & No. \\
\hline 160 & \multicolumn{7}{|c|}{ olEl) foctor } \\
\hline $\begin{array}{l}13 \mathrm{C} \\
16 \mathrm{O} \\
{ }^{48} \mathrm{CO}\end{array}$ & $\begin{array}{l}10^{\circ} \\
160\end{array}$ & $\begin{array}{l}7.0-1 \\
1.6+1 \\
5.6+1\end{array}$ & $\begin{array}{l}1.0+0 \\
2.9+1\end{array}$ & $\begin{array}{l}\text { AE } \\
\text { NBI } \\
\text { ANL }\end{array}$ & & $\begin{array}{l}\text { Ramstromt } \\
\text { Chrigtensont } \\
\text { Kovart }\end{array}$ & $\begin{array}{r}6788 \\
10791 \\
11110\end{array}$ \\
\hline 160 & \multicolumn{7}{|c|}{$O(E)$} \\
\hline $\begin{array}{l}{ }^{10} \mathrm{~B} \\
10_{\mathrm{B}}\end{array}$ & $\begin{array}{l}10^{8} \\
14^{N}\end{array}$ & $\begin{array}{l}2.0+1 \\
6.9+0\end{array}$ & $1.8+1$ & $\begin{array}{l}\text { PEN E } \\
\text { CAL E }\end{array}$ & $\begin{array}{l}\varepsilon \\
E\end{array}$ & $\begin{array}{l}\text { Ajzenberg-Selov } \\
\text { Wut }\end{array}$ & $\begin{array}{r}9500 \\
10243\end{array}$ \\
\hline $12 \mathrm{C}$ & $a$ & NDG & & WAU & $M$ & Ebisaua. & 6774 \\
\hline${ }^{12} \mathrm{C}$ & $a$ & $T R$ & $3.0+0$ & MST F & $R$ & Rolfst & 6775 \\
\hline $12 c$ & $12 \mathrm{c}$ & $3.0+1$ & $6.6+1$ & MBG F & $\mathrm{R}$ & Fick. & 9733 \\
\hline${ }^{12} \mathrm{C}$ & ${ }^{12} \mathrm{C}$ & $2.8+1$ & $6.2+1$ & STR & E 1 & Kolatat & 9775 \\
\hline $12 \mathrm{C}$ & 160 & $4.0+1$ & $6.5+1$ & STR & MI & Kolalot & 10647 \\
\hline $.12 \mathrm{C}$ & 160 & $3.5+1$ & $6.2+1$ & ANL & E & Tabort & 10651 \\
\hline${ }^{12} \mathrm{C}$ & ${ }^{150}$ & $6.0+1$ & & MUN E & E & Weidingert & 10723 \\
\hline${ }^{12} \mathrm{C}$ & 170 & $3.1+1$ & $3-4+1$ & WZI & E & Chechiht & 11579 \\
\hline $12_{C}$ & 180 & $3.2+1$ & $3.5+1$ & WZ1 & $E$ & Chechiht & 11615 \\
\hline${ }^{13} \mathrm{C}$ & a & $7.0-1$ & $1.0+0$ & AE $i$ & $M$ & Ramstront & 6783 \\
\hline${ }^{13} \mathrm{C}$ & 0 & $1.0+0$ & $5.4+0$ & ORL & $E$ & Bairt & 6784 \\
\hline${ }^{13} \mathrm{c}$ & $a$ & $3.0+0$ & $9.0+0$ & ORL & E & Bairt & 6785 \\
\hline${ }^{13} \mathrm{C}$ & 170 & $3.0+1$ & $3.2+1$ & WZ! & E & Chechilt & 11586 \\
\hline $13_{N}$ & p & $2.0+1$ & $2.4+1$ & MOS & $\mathbf{R}$ & Varlaciout & 461 \\
\hline${ }^{15} \mathrm{~N}$ & d & NOG & & BNL & $\mathbf{R}$ & Goldberg. & 5341 \\
\hline 160 & ${ }^{12} \mathrm{C}$ & $6.0+1$ & & GRE & $T$ & Cole. & 9842 \\
\hline 160 & 160 & $2.4+1$ & $6.0+1$ & STR & $\mathbf{R}$ & Has s+ & 10785 \\
\hline 160 & 160 & $5.6+1$ & & NBI & E & Fernandez+ & 10790 \\
\hline 160 & 160 & $2.0+1$ & $6.0+1$ & BNL & T & Fuller. & 10792 \\
\hline $19_{F}$ & D & NDG & & CAL & T & Woosleyt & 612 \\
\hline${ }^{19} \mathrm{~F}$ & p & $1.2+1$ & $1.8+1$ & HEI & E & Stablert & 613 \\
\hline${ }^{27} \mathrm{Al}$ & 1'B & $5.0+1$ & & FSU & E & Parkst & 9569 \\
\hline${ }^{28} \mathrm{Si}$ & 18 & $5.0+1$ & & FSU & $E$ & Parkst & 9570 \\
\hline $\mathrm{Ag}$ & $14 \mathrm{~N}$ & $7 \cdot \theta+1$ & $1.1+2$ & LYO & T & Billereyt & 10430 \\
\hline 1690 & $\sigma(E)$ & & & & & & \\
\hline${ }^{12} \mathrm{C}$ & ${ }^{12} \mathrm{C}$ & $3.5+1$ & $6.9+1$ & MUU & E & Weidingert & 9737 \\
\hline \multicolumn{8}{|c|}{ cumulative? o(E) } \\
\hline $1{ }^{2} \mathrm{C}$ & ${ }^{13} \mathrm{C}$ & $8.8+0$ & $2.5+1$ & ORL & $R$ & Stakstead. & 10175 \\
\hline${ }^{12} \mathrm{C}$ & ${ }^{13} \mathrm{C}$ & $1.0+1$ & $3.3+1$ & CAL & & Dayrast & 10176 \\
\hline & & & & & & . & \\
\hline
\end{tabular}




\begin{tabular}{|c|c|c|c|c|c|c|}
\hline Target & $\ln c$ & $\begin{array}{l}E(m) n \\
(M \leq V)\end{array}$ & $\begin{array}{l}\text { Emax } \\
(\mathrm{MeV})\end{array}$ & Lab & Author & No. \\
\hline 170 & partlal & ravthi & ich tor & rget & yield & \\
\hline $14 \mathrm{~N}$ & 。 & $5.17+0$ & & SUN & E Gilest & 6813 \\
\hline $\begin{array}{l}170 \\
170\end{array}$ & $\begin{array}{c}\text { partlat } \\
p\end{array}$ & $\begin{array}{c}\text { relatir } \\
1 . J-1\end{array}$ & $\begin{array}{l}\text { ve } O(E) \\
3.0+0\end{array}$ & $\begin{array}{l}\text { 'y } \\
\text { STR }\end{array}$ & E Senst & 577 \\
\hline 170 & partlal & relatir & ve $O(E)$ & )$\times 8 a c$ & ctor & \\
\hline${ }^{9} \mathrm{Be}$ & 160 & $8.9+0$ & $2.0+1$ & $\mathrm{CTH}$ & E Christianssont & 10550 \\
\hline 170 & parllal & rolativ & ve $O(E)$ & & & \\
\hline${ }^{9} \mathrm{Be}$ & 160 & $8.9+0$ & $2.0+1$ & $\mathrm{CTH}$ & E Christianssont & 10549 \\
\hline 170 & partial & $\sigma(E) \times f o$ & actor & & & \\
\hline $\begin{array}{l}98 \mathrm{Be} \\
13 \mathrm{C} \\
160\end{array}$ & $\begin{array}{l}150 \\
160 \\
100\end{array}$ & $\begin{array}{l}5.0+0 \\
1.3+1 \\
\text { NDS }\end{array}$ & $\begin{array}{l}1.5+1 \\
1.5+1\end{array}$ & $\begin{array}{l}\text { CAL } \\
\text { LVP } \\
\text { CAL }\end{array}$ & $\begin{array}{l}\text { M Sultkougkit } \\
\text { M Durellt } \\
\text { M Svitkouskit }\end{array}$ & $\begin{array}{r}10552 \\
10726 \\
9516\end{array}$ \\
\hline 170 & portlol & $O(E)$ & & & & \\
\hline $\begin{array}{l}9 \mathrm{Be} \\
10_{\mathrm{B}} \\
13 \mathrm{C} \\
14 \mathrm{~N} \\
16 \mathrm{O} \\
19_{\mathrm{F}}\end{array}$ & $\begin{array}{r}10_{0} \\
10_{0} \\
10_{0} \\
t \\
d \\
d\end{array}$ & $\begin{array}{l}5 \cdot 0+0 \\
4 \cdot 3+0 \\
3 \cdot 1+1 \\
8 \cdot 0-1 \\
5 \cdot 0-1 \\
3 \cdot 0-1\end{array}$ & $\begin{array}{l}1.5+1 \\
8.6+0 \\
3.3+1 \\
3.3+0 \\
1.2+0 \\
4.6-1\end{array}$ & $\begin{array}{l}\text { CAL } \\
\text { NBI } \\
\text { WZI } \\
\text { STR } \\
\text { WIT } \\
\text { DEB }\end{array}$ & $\begin{array}{l}\text { M Solthouskit } \\
\text { D Brogliat } \\
\text { E Chechint } \\
\text { E Lincht } \\
\text { E Annegarn. } \\
\text { E Somogyit }\end{array}$ & $\begin{array}{r}10551 \\
11600 \\
11634 \\
6055 \\
5347 \\
5352\end{array}$ \\
\hline 170 & product & yield & & & & \\
\hline $\begin{array}{l}12 \mathrm{C} \\
180\end{array}$ & $\begin{array}{l}180 \\
180\end{array}$ & $\begin{array}{l}4.0+1 \\
9.1+1\end{array}$ & $6.0+1$ & $\begin{array}{l}\text { NOT } \\
\text { PAR }\end{array}$ & $\begin{array}{l}\text { E Oavist } \\
\text { E Detraz+ }\end{array}$ & $\begin{array}{l}11617 \\
11650\end{array}$ \\
\hline 170 & relative & e o(E) & & & & \\
\hline $\begin{array}{l}24 \mathrm{Mg} \\
26 \mathrm{Mg}\end{array}$ & $\begin{array}{l}{ }^{3} \mathrm{He} \\
{ }^{3} \mathrm{He}\end{array}$ & $\begin{array}{l}8 \cdot 0+1 \\
8 \cdot 0+1\end{array}$ & & $\begin{array}{l}\text { NRL } \\
\text { NRL }\end{array}$ & $\begin{array}{l}E \operatorname{singht} \\
E \operatorname{singht}\end{array}$ & $\begin{array}{l}6200 \\
6202\end{array}$ \\
\hline 170 & $\sigma(E) \times f \Delta C$ & ctor & & & & \\
\hline${ }^{4} \mathrm{C}_{\mathrm{Ca}}$ & 150 & $5.6+1$ & & ANL & E Kovart & 11110 \\
\hline 170 & $O(E)$ & & & & & \\
\hline $\begin{array}{l}12 \mathrm{C} \\
13 \mathrm{C} \\
13 \mathrm{C} \\
13 \mathrm{C}\end{array}$ & $\begin{array}{l}180 \\
160 \\
160 \\
180\end{array}$ & $\begin{array}{l}3.2+1 \\
\text { NDG } \\
1.3+1 \\
3.1+1\end{array}$ & $\begin{array}{l}3.5+1 \\
1.5+1 \\
3.3+1\end{array}$ & $\begin{array}{l}\text { WZI } \\
\text { NCS } \\
\text { LVP } \\
\text { WZI }\end{array}$ & $\begin{array}{l}\text { E Chechikt } \\
\text { T Porkt } \\
\text { M Ourell+ } \\
\text { E Chechikt }\end{array}$ & $\begin{array}{l}11616 \\
10727 \\
10728 \\
11633\end{array}$ \\
\hline
\end{tabular}

\begin{tabular}{|c|c|c|c|c|c|c|}
\hline Target & $\ln c$ & $\begin{array}{l}\text { Emin } \\
(\mathrm{MeV})\end{array}$ & $\begin{array}{l}\text { Egax } \\
(\operatorname{MeV} V)\end{array}$ & Lab & Author & No. \\
\hline 170 & $O(E)$ & & & & & \\
\hline${ }^{2{ }^{20} \mathrm{~F}}$ & $14 \mathrm{p}$ & $\begin{array}{l}\text { NDG } \\
7.8+1\end{array}$ & $1.1+2$ & $\begin{array}{l}\text { CAL } \\
\text { LYO }\end{array}$ & $\begin{array}{l}\text { T Woosleyt } \\
\text { T Billereyt }\end{array}$ & $\begin{array}{r}631 \\
10430\end{array}$ \\
\hline 180 & port1.01 & $O(E)$, & & & & \\
\hline $\begin{array}{l}12 c \\
12 c\end{array}$ & $\begin{array}{l}180 \\
180\end{array}$ & $\begin{array}{l}2.3+1 \\
2.5+1\end{array}$ & $\begin{array}{l}6.8+1 \\
5.9+1\end{array}$ & $\begin{array}{l}\text { WAU E } \\
\text { STR E }\end{array}$ & $\begin{array}{l}\text { E Chant } \\
\text { E Freenont }\end{array}$ & $\begin{array}{l}11618 \\
11619\end{array}$ \\
\hline $18_{0}$ & jortial & overage & e $\sigma(E)$. & a facto & or & \\
\hline${ }^{12} \mathrm{C}$ & ${ }^{7} L I$ & $1.6+1$ & $1.8+1$ & PEN E & E Fortunet & 9418 \\
\hline $1 \varepsilon_{0}$ & jortlol & row ole & & & & \\
\hline${ }^{15} \mathrm{~N}$ & 。 & $7.9+0$ & $8.4+0$ & OXF E & E Symonst & 6824 \\
\hline $1 E_{0}$ & partlal & average & o $O(E)$ & & & \\
\hline${ }^{12} \mathrm{C}$ & 'Li & $1.6+1$ & $1.8+1$ & PEN 8 & E Fortunet & 9419 \\
\hline $1 \varepsilon_{0}$ & portlal & relativ & ve $\sigma(E)$ & & & \\
\hline${ }^{40} \mathrm{TI}$ & 160 & $9.6+1$ & & MUN & $M$ Heringt & 11119 \\
\hline $10_{0}$ & partial & ofEIafa & actor & & & \\
\hline $\begin{array}{l}12 \mathrm{c} \\
160\end{array}$ & $\stackrel{7 L I}{t}$ & $\begin{array}{l}1.6+1 \\
1.5+1\end{array}$ & $1.8+1$ & $\begin{array}{l}\text { PEN } \\
\text { PEN E }\end{array}$ & $\begin{array}{l}\text { E Fortunet } \\
\text { E Mooret }\end{array}$ & $\begin{array}{l}5417 \\
6057\end{array}$ \\
\hline 180 & partiol & $G(E)$ & & & & \\
\hline $\begin{array}{l}12 \mathrm{C} \\
13 \mathrm{C} \\
180 \\
120 \mathrm{sn}\end{array}$ & $\begin{array}{l}7 \mathrm{LI} \\
170 \\
12 \mathrm{C} \\
10_{0}\end{array}$ & $\begin{array}{l}1.6+1 \\
3.0+1 \\
1.7+1 \\
7.2+1\end{array}$ & $\begin{array}{l}1.0+1 \\
3.2+1 \\
4.0+1\end{array}$ & $\begin{array}{l}\text { PEN E } \\
\text { WZ1 E } \\
\text { STR F } \\
\text { MUU E }\end{array}$ & $\begin{array}{l}\text { E Fortunet } \\
\text { E Chechikt } \\
\text { R Hast } \\
\text { E Spielert }\end{array}$ & $\begin{array}{r}9416 \\
11589 \\
9854 \\
11683\end{array}$ \\
\hline 180 & product, & yleloy & & & & \\
\hline 180 & $p$ & $4.4+0$ & $5.7+0$ & MCM E & E Dint & 593 \\
\hline 180 & product & $y 1010$ & & & & \\
\hline 180 & 180 & $9.1+1$ & & PAR & E Delraz+ & 11650 \\
\hline 180 & $r O D O(E)$, & , ot $r e$ & esonanc & & & \\
\hline$i \theta_{0}$ & $p$ & $3.0+0$ & $3.5+0$ & CBR & E Din. & 592 \\
\hline 180 & relative & $\sigma(E)$ & & & & \\
\hline${ }^{24} \mathrm{Mg}$ & ${ }^{3} \mathrm{Ho}$ & $8.0+1$ & & NRL & E singht & 6200 \\
\hline
\end{tabular}




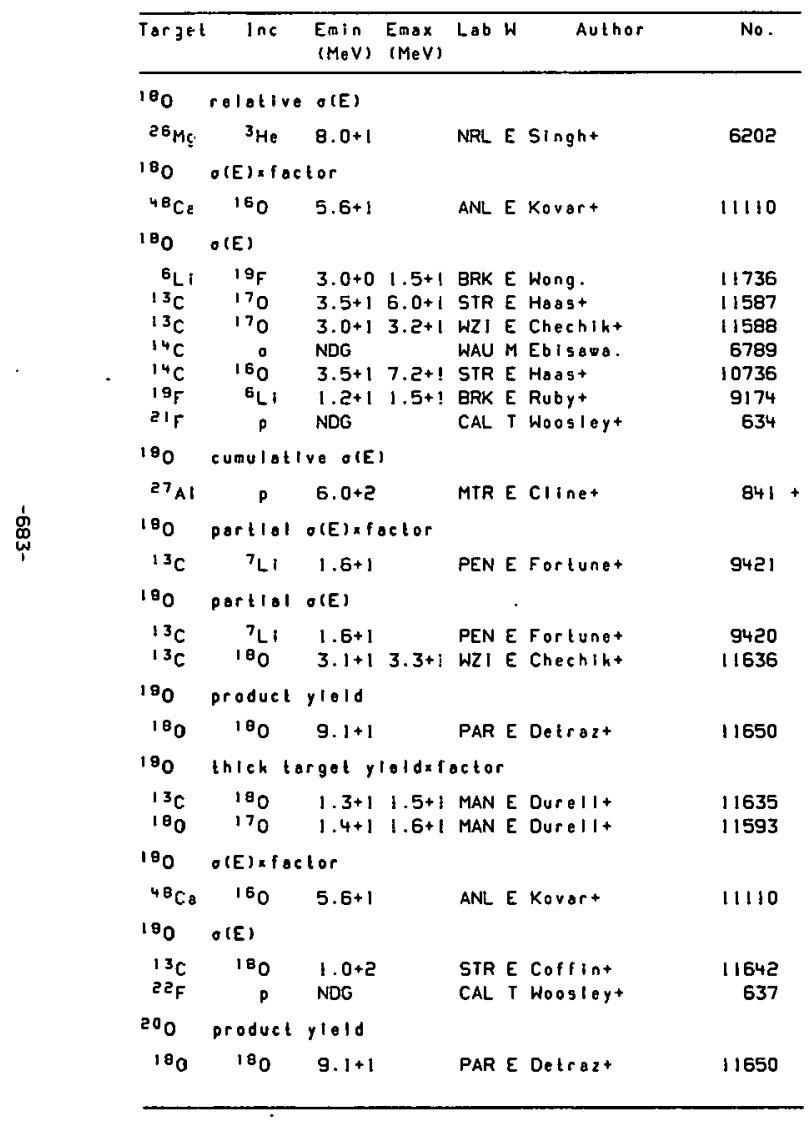

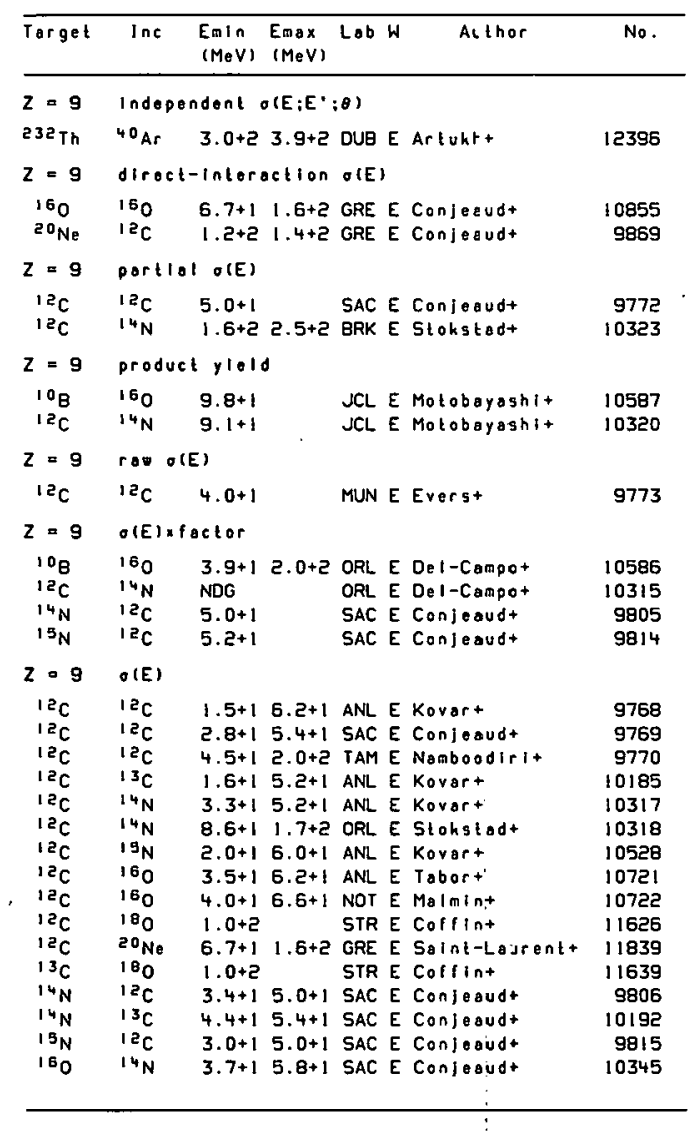




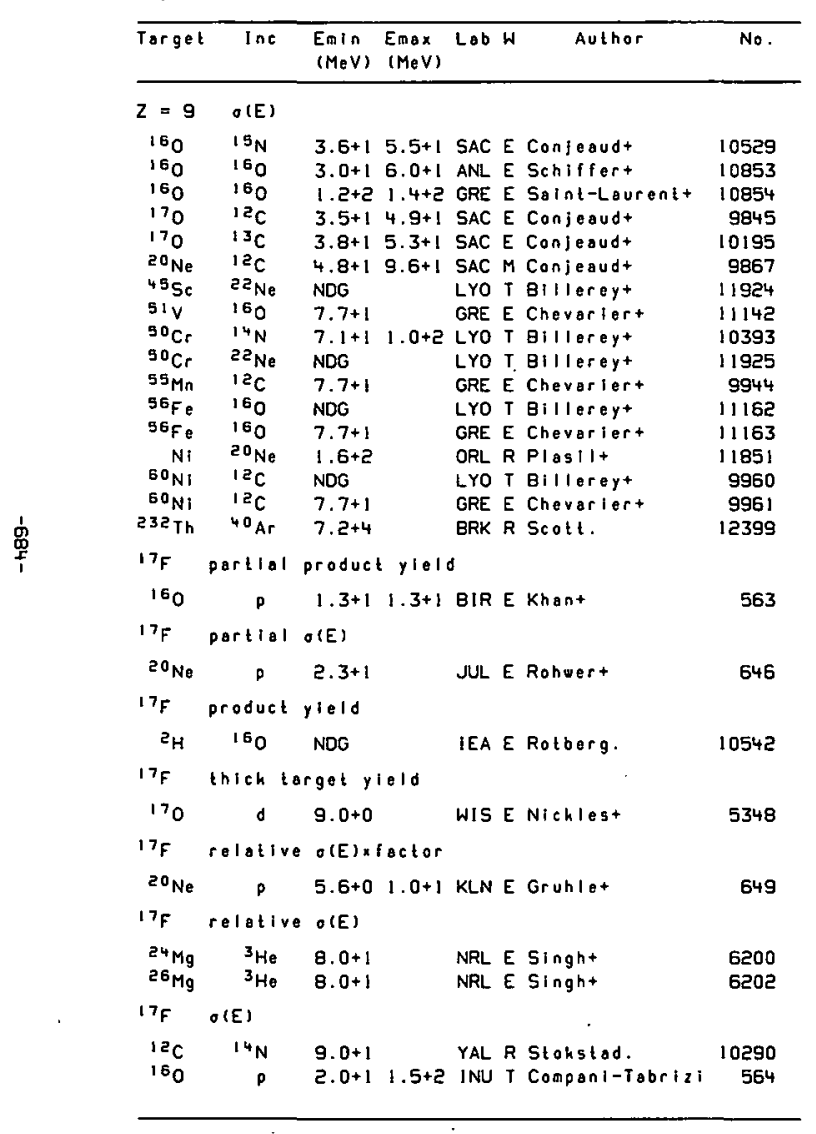

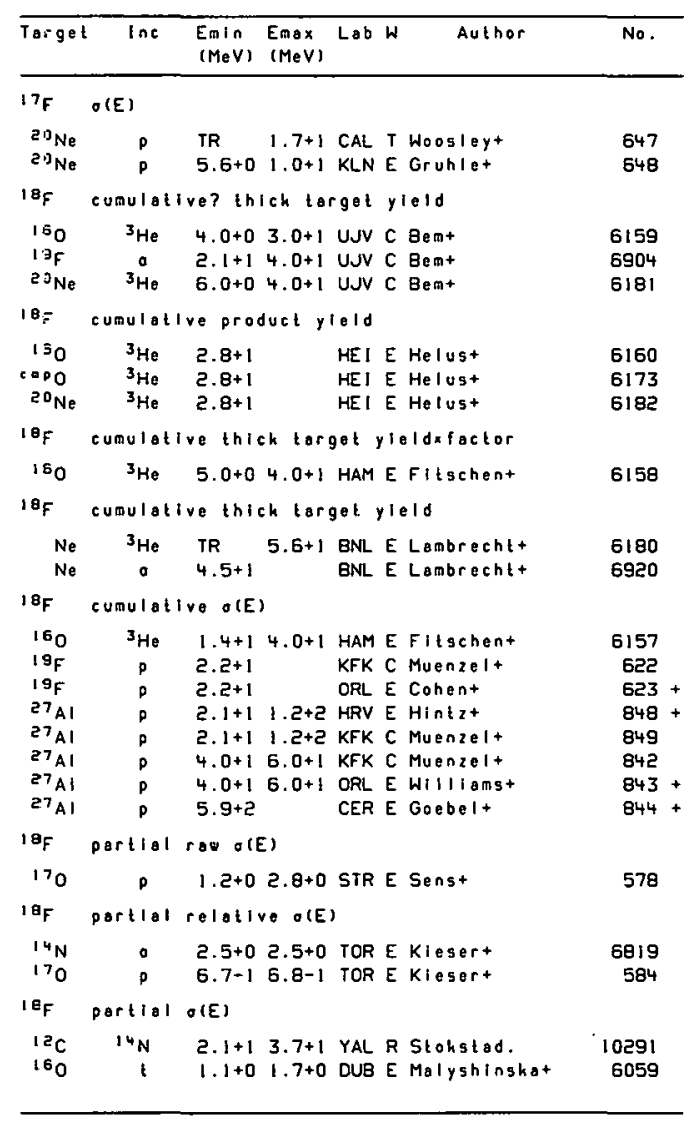




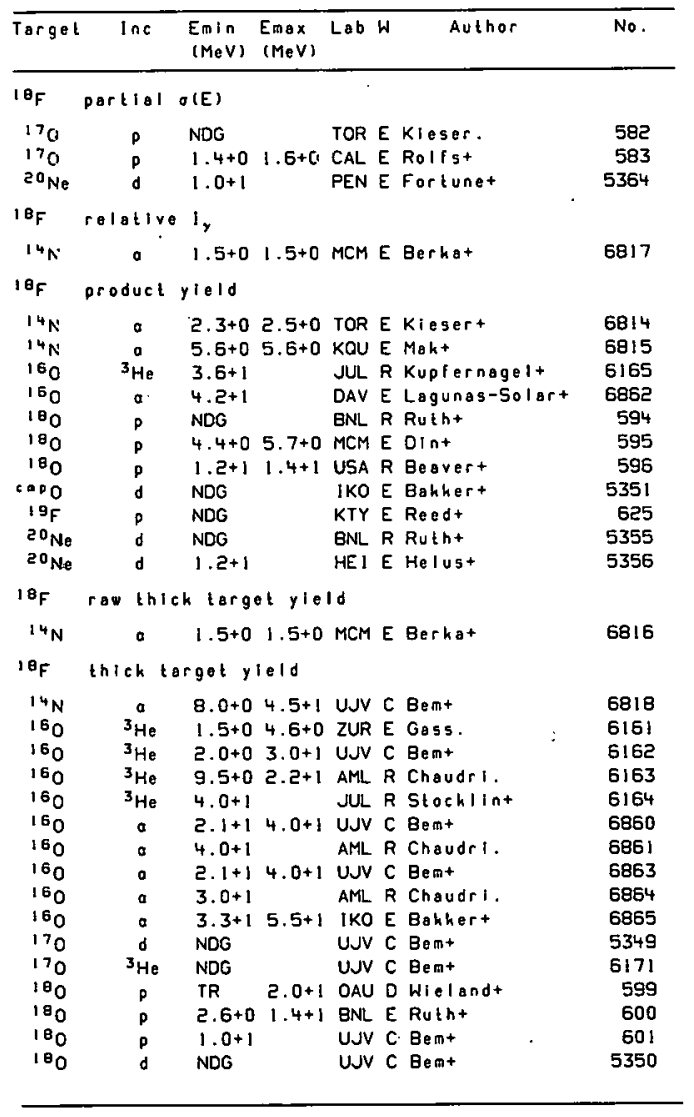

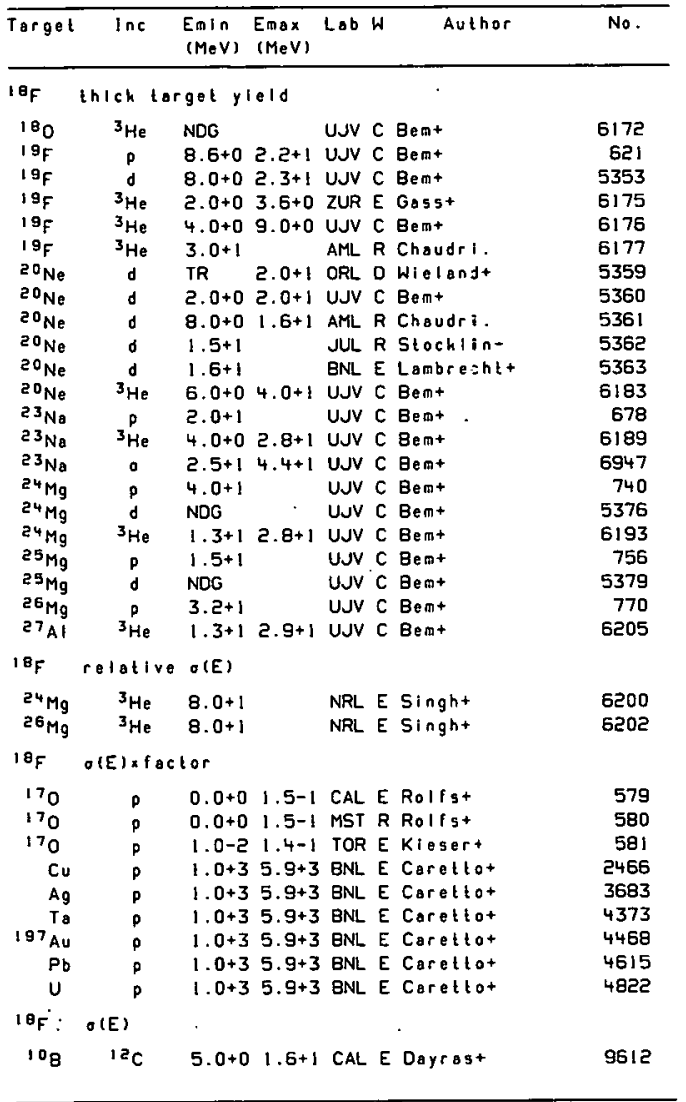




\begin{tabular}{cccccc}
\hline Target & Inc & $\begin{array}{c}\text { Emln } \\
\text { (MeV) }\end{array}$ (MaV) \\
(Mab
\end{tabular}

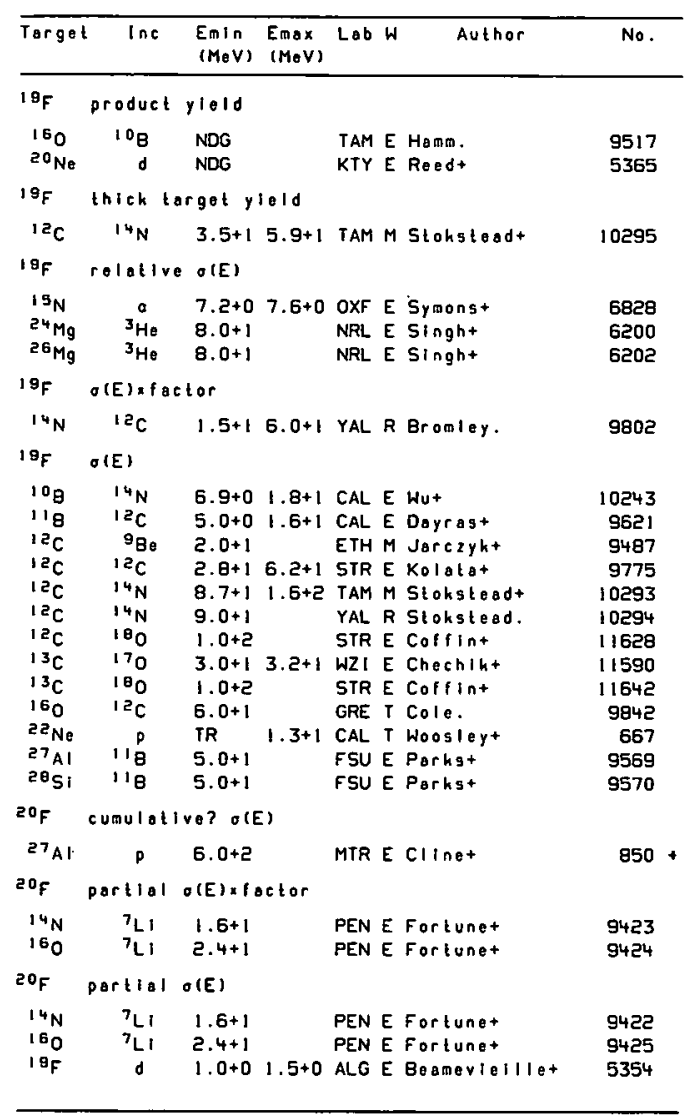




\begin{tabular}{|c|c|c|c|c|c|c|}
\hline Target & $\ln c$ & $\begin{array}{l}\text { Emin } \\
(\mathrm{MeV})\end{array}$ & $\begin{array}{l}\text { Emax } \\
(\operatorname{MeV})\end{array}$ & Lob W & Author & No. \\
\hline $20_{F}$ & relative & $O(E)$ & & & & \\
\hline $\begin{array}{l}24 \mathrm{Mg} \\
26 \mathrm{Mg}\end{array}$ & $\begin{array}{l}{ }^{3} \mathrm{He} \\
{ }^{3} \mathrm{He}\end{array}$ & $\begin{array}{l}8.0+1 \\
8.0+1\end{array}$ & $\cdot i$ & $\begin{array}{l}\text { NRL E } \\
\text { NRL E }\end{array}$ & $\begin{array}{l}\text { Singht } \\
\text { Singht }\end{array}$ & $\begin{array}{l}6200 \\
6202\end{array}$ \\
\hline \multicolumn{7}{|c|}{$20_{F} \quad O(E) \times$ factor } \\
\hline${ }^{9} \mathrm{Ba}$ & $19_{F}$ & $7.0+0$ & $1.6+1$ & CAL $M$ & Swithouskit & 11737 \\
\hline $20_{F}$ & $o(E)$ & & & & & \\
\hline $\begin{array}{l}12 c \\
12 c \\
12 c \\
13 c\end{array}$ & $\begin{array}{l}9 \mathrm{Be} \\
14 \mathrm{~N} \\
10_{0} \\
18_{0}\end{array}$ & $\begin{array}{l}2.0+1 \\
8 \cdot 7+1 \\
1.0+2 \\
1.0+2\end{array}$ & $1.6+2$ & $\begin{array}{l}\text { ETH M } \\
\text { TAM M } \\
\text { STR E } \\
\text { STR E }\end{array}$ & $\begin{array}{l}\text { Jarczykt } \\
\text { Stohsteadt } \\
\text { Coffint } \\
\text { Coffint }\end{array}$ & $\begin{array}{r}9488 \\
10296 \\
11628 \\
11642\end{array}$ \\
\hline $21_{F}$ & $O(E)$ & & & & & \\
\hline $\begin{array}{l}{ }_{12} \mathrm{C} \\
{ }^{3} \mathrm{C}\end{array}$ & $\begin{array}{l}180 \\
180\end{array}$ & $\begin{array}{l}1.0+2 \\
1.0+2\end{array}$ & & $\begin{array}{l}\text { STR E } \\
\text { STR E }\end{array}$ & $\begin{array}{l}\text { Corfint } \\
\text { Coffint }\end{array}$ & $\begin{array}{l}11628 \\
11642\end{array}$ \\
\hline $22_{F}$ & $\sigma(E)$ & & & & & \\
\hline${ }^{13} \mathrm{c}$ & $10_{0}$ & $1.0+2$ & & STR E & Coffint & 11642 \\
\hline$z=10$ & indep & endent & OIE;E' & $\because ; \theta)$ & & \\
\hline $232 \mathrm{Th}$ & ${ }^{40} \mathrm{Ar}$ & $3.0+2$ & $3.9+2$ & DUB E & Artukht & 12396 \\
\hline$z=10$ & direc & t-inter & raction & $n O(E)$ & & \\
\hline $20 \mathrm{He}$ & ${ }^{12} \mathrm{C}$ & $1.2+2$ & $1.4+2$ & GRE E & Conleaudt & 9869 \\
\hline$z=10$ & part 1 & al o(E) & & & & \\
\hline $\begin{array}{l}12 c c \\
12 c\end{array}$ & $\begin{array}{l}12 \mathrm{C} \\
14_{N}\end{array}$ & $\begin{array}{l}5.0+1 \\
1.6+2\end{array}$ & $2.5+2$ & $\begin{array}{l}\text { SAC E } \\
\text { BRK E }\end{array}$ & $\begin{array}{l}\text { Conjeaudt } \\
\text { Stokstadt }\end{array}$ & $\begin{array}{r}9772 \\
10323\end{array}$ \\
\hline$z=10$ & produ & ct ylol & & & & \\
\hline $\begin{array}{l}100 \\
12 c\end{array}$ & $\begin{array}{l}160 \\
14 \mathrm{~N}\end{array}$ & $\begin{array}{l}9.8+1 \\
9.1+1\end{array}$ & & $\begin{array}{l}\text { JCL E } \\
\text { JCL E }\end{array}$ & $\begin{array}{l}\text { Motobayoshit } \\
\text { Motobayoshit }\end{array}$ & $\begin{array}{l}10587 \\
10320\end{array}$ \\
\hline$z=10$ & $v_{n}$ & & & & & \\
\hline hg & ${ }^{40} \mathrm{Ar}$ & $3.4+2$ & & PAR R & Lefortt & 12202 \\
\hline$z=10$ & $\cos 0$ & $(E)$ & & & & \\
\hline${ }^{12} \mathrm{C}$ & ${ }^{12} \mathrm{c}$ & $4.0+1$ & 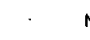 & MUN E & Everst & 9773 \\
\hline
\end{tabular}

\begin{tabular}{|c|c|c|c|c|c|c|}
\hline$\overline{\text { Target }}$ & inc & $\begin{array}{l}\text { EnIn } \\
\text { (MeV) }\end{array}$ & $\begin{array}{l}\text { Emax } \\
(\mathrm{MeV})\end{array}$ & Lab & Author & No. \\
\hline$z=10$ & \multicolumn{2}{|c|}{ O(E) xfactor } & & & & \\
\hline $10_{B}$ & 160 & $3.9+1$ & $2.0+20$ & ORL E & E Del-Compot & 10586 \\
\hline $12 \mathrm{c}$ & $14 \mathrm{~N}$ & NDG & & ORL E & E Del-Campot & 10315 \\
\hline $14 \mathrm{~N}$ & ${ }^{12} \mathrm{C}$ & $5.0+1$ & & SAC E & E Conjesudt & 9805 \\
\hline $15_{N}$ & $1 z_{c}$ & $5.2+1$ & & SAC E & E Conjeoudt & 9814 \\
\hline $235 \mathrm{Th}$ & ${ }^{40} \mathrm{Ar}$ & $3.9+2$ & & OXF $F$ & R Hodgson. & 12398 \\
\hline$z=10$ & $\theta(E)$ & & & & & . \\
\hline${ }^{12} \mathrm{C}$ & $12 \mathrm{c}$ & $1.5+1$ & $6.2+1$ & ANL E & E Kovart & 9768 \\
\hline $12^{2} \mathrm{c}$ & $12_{c}$ & $2.8+1$ & $5.4+1=$ & SAC E & E Conjesudt & 9769 \\
\hline $12^{2} \mathrm{C}$ & $12 \mathrm{c}$ & $4.5+1$ & $2.0+2$ & TAM E & E Nambocdirit & 9770 \\
\hline${ }^{12} \mathrm{c}$ & $13 \mathrm{c}$ & $1.6+1$ & $5.2+1$ & ANL E & E Kovart & 10185 \\
\hline${ }^{12} \mathrm{C}$ & $14 \mathrm{~N}$ & $3.3+1$ & $5.2+1$ & ANL E & E Kovart & 10317 \\
\hline${ }^{12} \mathrm{c}$ & $14 \mathrm{~N}$ & $8.6+1$ & $1.7+20$ & ORL E & E Stokstaot & 10310 \\
\hline $12 \mathrm{c}$ & $15 \mathrm{~N}$ & $2.0+1$ & $6.0+1$ & ANL E & E Kovart & 10528 \\
\hline $12 \mathrm{C}$ & 160 & $3.5+1$ & $6.2+1$ & ANL $E$ & E Tabort & $1072 !$ \\
\hline $12 \mathrm{C}$ & 160 & $4.0+1$ & $6.6+11$ & NOT E & E Malmint & 10722 \\
\hline $12 \mathrm{c}$ & 170 & $3.4+1$ & $8.0+1 k$ & MUN E & E Hertza & 11581 \\
\hline $12 \mathrm{C}$ & $10_{0}$ & $1.0+5$ & & STR E & E Colfint & 11626 \\
\hline${ }^{12} \mathrm{C}$ & $20 \mathrm{Ne}$ & $6.7+1$ & $1.6+2$ & GRE E & E Saint-Laurent+ & 11839 \\
\hline${ }^{13} \mathrm{c}$ & 100 & $1.0+2$ & & STR E & E Colfint & 11639 \\
\hline $14 \mathrm{~N}$ & $12 \mathrm{c}$ & $3.4+1$ & $5.0+1$ & SAC E & E Conjeaudt & 9806 \\
\hline $14 \mathrm{~N}$ & ${ }^{13} \mathrm{C}$ & $4.4+1$ & $5 \cdot 4+1 \leq$ & SAC E & E Conjeaudt & 10192 \\
\hline $15 \mathrm{~N}$ & 120 & $3.0+1$ & $5 \cdot 0+1$ & SAC E & E Conjeaud+ & 9815 \\
\hline 180 & $14 \mathrm{~N}$ & $3.7+1$ & $5.8+1=$ & SAC E & E Conjeaudt & 10345 \\
\hline 160 & $15 \mathrm{~N}$ & $3.6+1$ & $5.5+1$ & SAC E & E Conjeaudt & 10529 \\
\hline 150 & 160 & $3.0+1$ & $6.0+1$ & ANL E & E Schiffert & 10853 \\
\hline 160 & 160 & $1.2+2$ & $1.4+2$ & GRE E & E Saint-Lourent+ & 10854 \\
\hline 170 & $12 \mathrm{c}$ & $3.5+1$ & $4.9+15$ & SAC E & E Conjeaudt & 9845 \\
\hline 170 & ${ }^{13} \mathrm{C}$ & $3.8+1$ & $5.3+15$ & SAC E & E Conjeaudt & 10195 \\
\hline $20_{\mathrm{Ne}}$ & $12 \mathrm{c}$ & $4.8+1$ & $9.6+1$ & SAC & M Conjeaudt & 9867 \\
\hline${ }^{45} \mathrm{Se}$ & $2 z_{\mathrm{Ne}}$ & NDG & & LYO & T Billereyt & 11924 \\
\hline${ }^{50} \mathrm{Cr}$ & $14 \mathrm{~N}$ & $7.1+1$ & $1.0+2$ & LYO & I Billereyt & 10393 \\
\hline $\mathrm{so}^{\mathrm{Cr}}$ & $22 \mathrm{Ne}$ & NDG & & LYo & T Billereyt & 11925 \\
\hline $566_{\mathrm{Fe}}$ & 160 & NDG & & LYO & T Blllerey+ & 11162 \\
\hline $\mathrm{N} 1$ & $20 \mathrm{Ne}$ & $1.6+2$ & & ORL F & R Plasilt & 11851 \\
\hline 50 & $\operatorname{lig}^{2} \mathrm{c}$ & NDG & & LYO & T Billereyt & 9960 \\
\hline $232 \mathrm{Th}$ & $40 \mathrm{Ar}$ & $7.2+4$ & & BRK F & R Scott. & 12399 \\
\hline $17 \mathrm{Ne}$ & product & $y \mid e l d$ & & & & \\
\hline 160 & ${ }^{3} \mathrm{He}$ & NDG & & BRK F & R Cernyt & 6168 \\
\hline
\end{tabular}




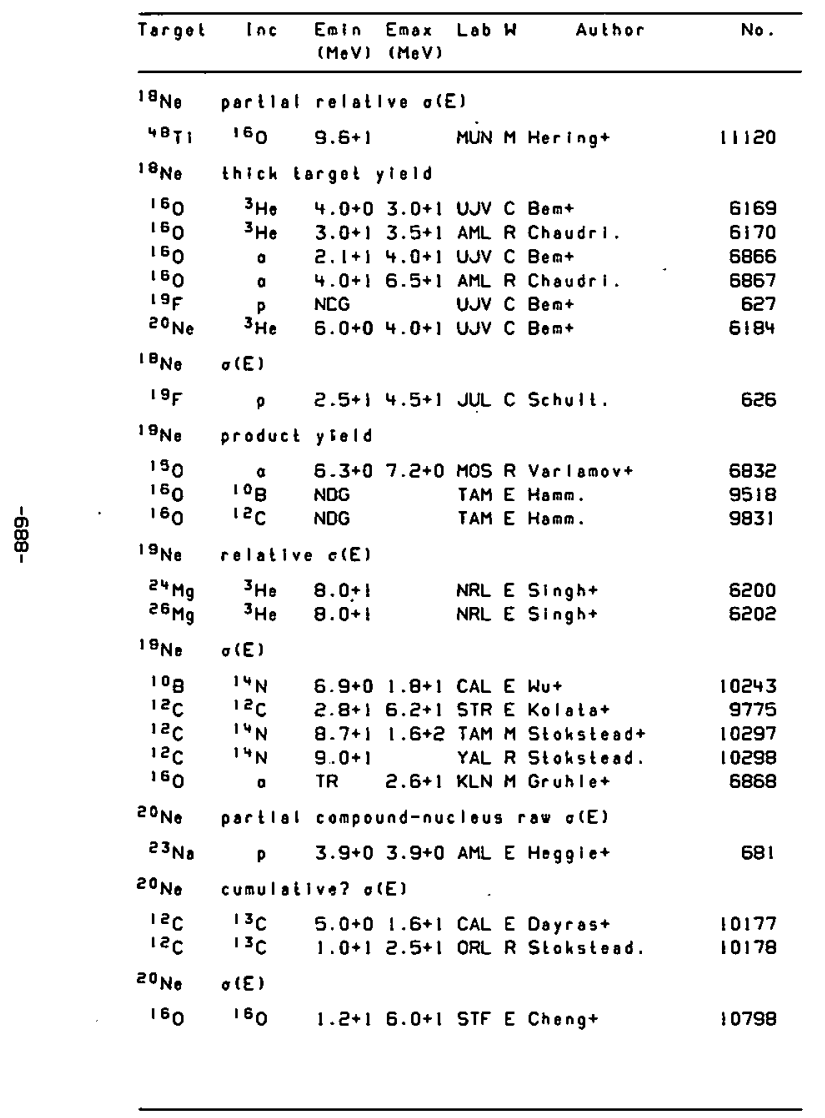

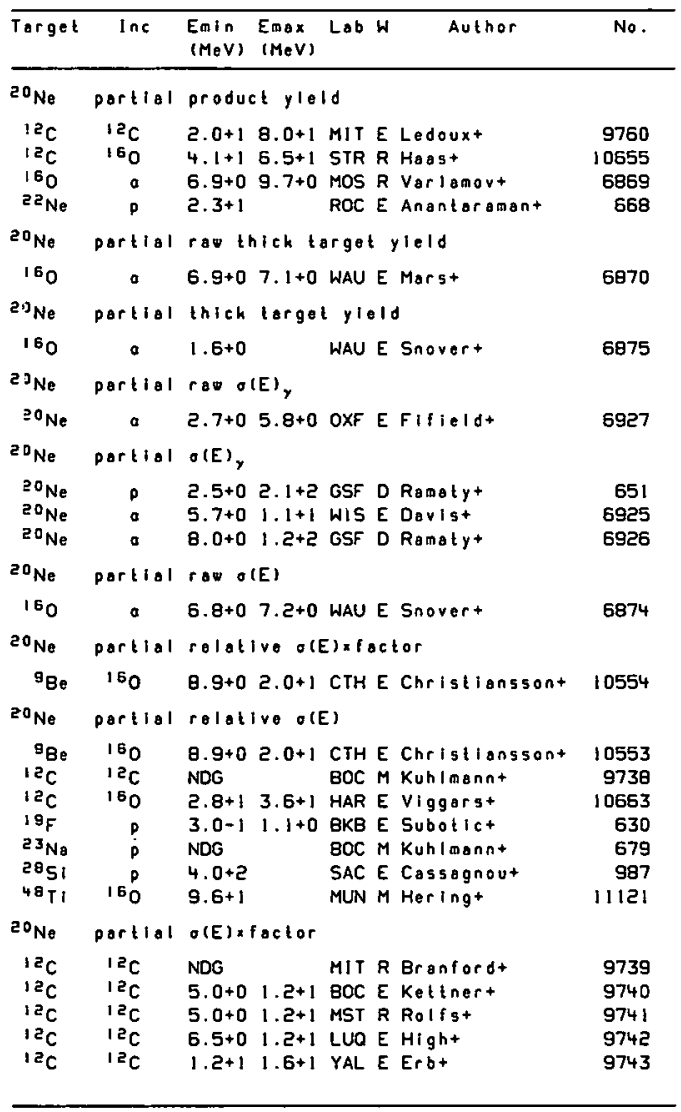




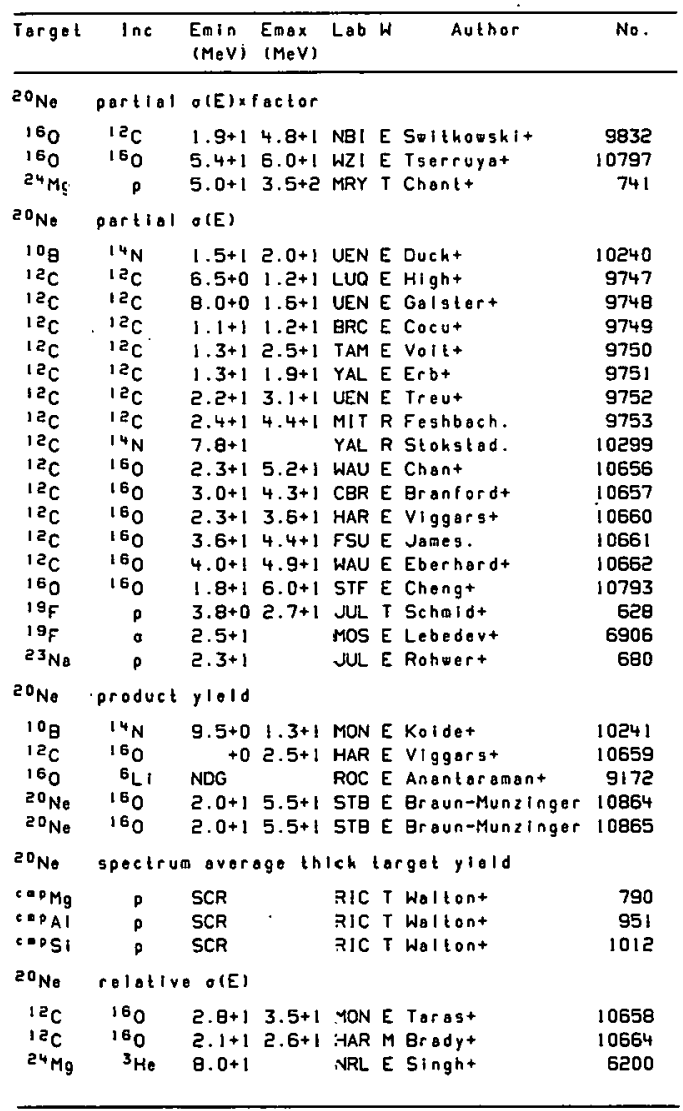

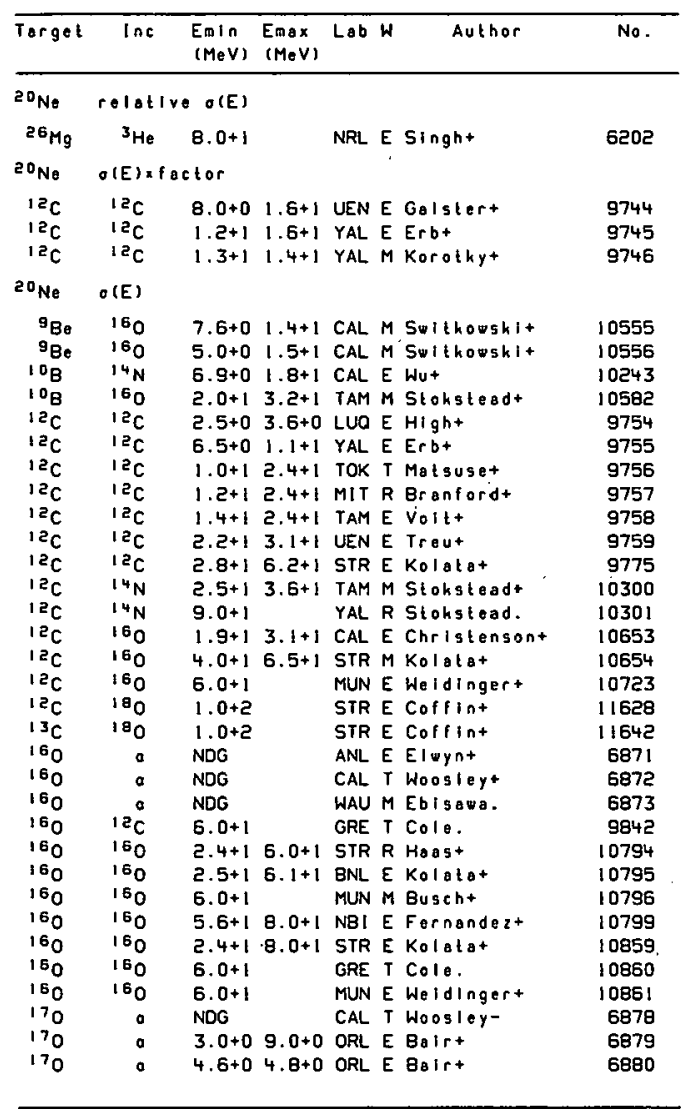




\begin{tabular}{|c|c|c|c|c|c|c|}
\hline Target & $\operatorname{lnc}$ & $\begin{array}{l}\text { Emin } \\
(M e V)\end{array}$ & $\begin{array}{l}E \max \\
(M \otimes V)\end{array}$ & Lab & Author & No. \\
\hline $20 \mathrm{Ne}$ & $O(E)$ & & & & & \\
\hline $17 \mathrm{~F}$ & a & NDG & & CAL & T Woosleyt & 6898 \\
\hline $19^{F}$ & $p$ & NDG & & CAL & I Woosleyt & 629 \\
\hline $20 \mathrm{~F}$ & p & NDG & & CAL & T Woosleyt & 632 \\
\hline $20 \mathrm{Ne}$ & p & $2.4+1$ & & oxF & T Mac-Kintosht & 650 \\
\hline $20^{\circ} \mathrm{Ne}$ & $12 \mathrm{c}$ & $2.0+1$ & $5.4+1$ & NOT & E De-Youngt & 9870 \\
\hline${ }^{20} \mathrm{Ne}$ & ${ }^{12} \mathrm{C}$ & $6.7+1$ & & GRE & T Cole. & 9871 \\
\hline $2{ }^{2} \mathrm{No}$ & p & NDG & & CAL & T Woosleyt & 682 \\
\hline Mg & $\rho$ & $1.2+1$ & $4.5+1$ & RIC & E Waltont & 719 \\
\hline $27 \mathrm{AI}$ & p & $1.6+1$ & $4.2+1$ & RIC & E Waltont & 851 \\
\hline $27 \mathrm{Al}$ & p & TR & $8.0+1$ & NRL & T Peterson. & 852 \\
\hline si & p & $2.9+1$ & $4.4+1$ & RIC & E Waltont & 960 \\
\hline $2{ }^{1} \mathrm{Ne}$ & partial & raw th & hlch to & orgel & l yleldxabundance & \\
\hline 180 & a & $5.0+0$ & & SUN & E Gllest & 6883 \\
\hline${ }^{21} \mathrm{Ne}$ & partiol & raw ol & (E), & & & \\
\hline $21 \mathrm{Ne}$ & p & $7.0-1$ & $2.0+0$ & HLS & E Keinonent & 660 \\
\hline $21 \mathrm{Ne}$ & partial & relati & ive ole & EIxfo & actor & \\
\hline${ }^{9} \theta e$ & 180 & $0.9+0$ & $2.0+1$ & $\mathrm{CTH}$ & E Christionssont & 10558 \\
\hline${ }^{21} \mathrm{Ne}$ & partial & relati & ive ole & & & \\
\hline 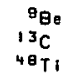 & $\begin{array}{l}160 \\
160 \\
160\end{array}$ & $\begin{array}{l}8.9+0 \\
2.5+1 \\
9.6+1\end{array}$ & $\begin{array}{l}2.0+1 \\
5 \cdot 3+1\end{array}$ & $\begin{array}{l}\text { CTH } \\
\text { WAU } \\
\text { MUN }\end{array}$ & $\begin{array}{l}\text { E Chrigtianssont } \\
\text { E Bhangt } \\
\text { M Heringt }\end{array}$ & $\begin{array}{l}10557 \\
10729 \\
11122\end{array}$ \\
\hline $2 I_{\mathrm{Ne}}$ & partial & $\sigma(E)$ & & & & \\
\hline $\begin{array}{l}12 \mathrm{C} \\
13 \mathrm{C} \\
10 \mathrm{O} \\
20 \mathrm{Ne}\end{array}$ & $\begin{array}{r}14 \mathrm{~N} \\
170 \\
a \\
0\end{array}$ & $\begin{array}{l}1 \cdot 3+1 \\
3 \cdot 0+1 \\
\text { TR } \\
5 \cdot 0-1\end{array}$ & $\begin{array}{l}3 \cdot 7+1 \\
3 \cdot 2+1 \\
6 \cdot 5+0 \\
6 \cdot 4-1\end{array}$ & $\begin{array}{l}\text { YAL } \\
\text { WZ1 } \\
\text { AML } \\
\text { DEB }\end{array}$ & $\begin{array}{l}\text { R Stohstod. } \\
\text { E Chechikt } \\
\text { E Switkowshit } \\
\text { E Valek. }\end{array}$ & $\begin{array}{r}10302 \\
11592 \\
6888 \\
5366\end{array}$ \\
\hline$e^{\prime}{ }^{n}$ & $\mathrm{I}_{y}$ & & & & & \\
\hline $\begin{array}{l}180 \\
20 \mathrm{Ne} \\
21 \mathrm{Ne}\end{array}$ & a & $\begin{array}{l}2.7+0 \\
2.7+0 \\
2 \cdot 7+0\end{array}$ & $\begin{array}{l}6.0+0 \\
6.0+0 \\
6.0+0\end{array}$ & $\begin{array}{l}\text { CRC } \\
\text { CRC } \\
\text { CRC }\end{array}$ & $\begin{array}{l}\text { E Mc-Donaldt } \\
\text { E Mc-Donsidt+ } \\
\text { E Mc-Donoldt }\end{array}$ & $\begin{array}{r}6889 \\
5367 \\
661\end{array}$ \\
\hline$a^{\prime} \mathrm{Ne}$ & spectrum & a avero & age thi & ick t & torgot yiold & \\
\hline $\begin{array}{l}\because A M g \\
\because P A I\end{array}$ & $p$ & $\begin{array}{l}\text { SCR } \\
\text { SCR }\end{array}$ & & $\begin{array}{l}\text { RIC } \\
\text { RIC }\end{array}$ & $\begin{array}{l}\text { T Waltont } \\
\text { T Waltont }\end{array}$ & $\begin{array}{l}791 \\
952\end{array}$ \\
\hline
\end{tabular}

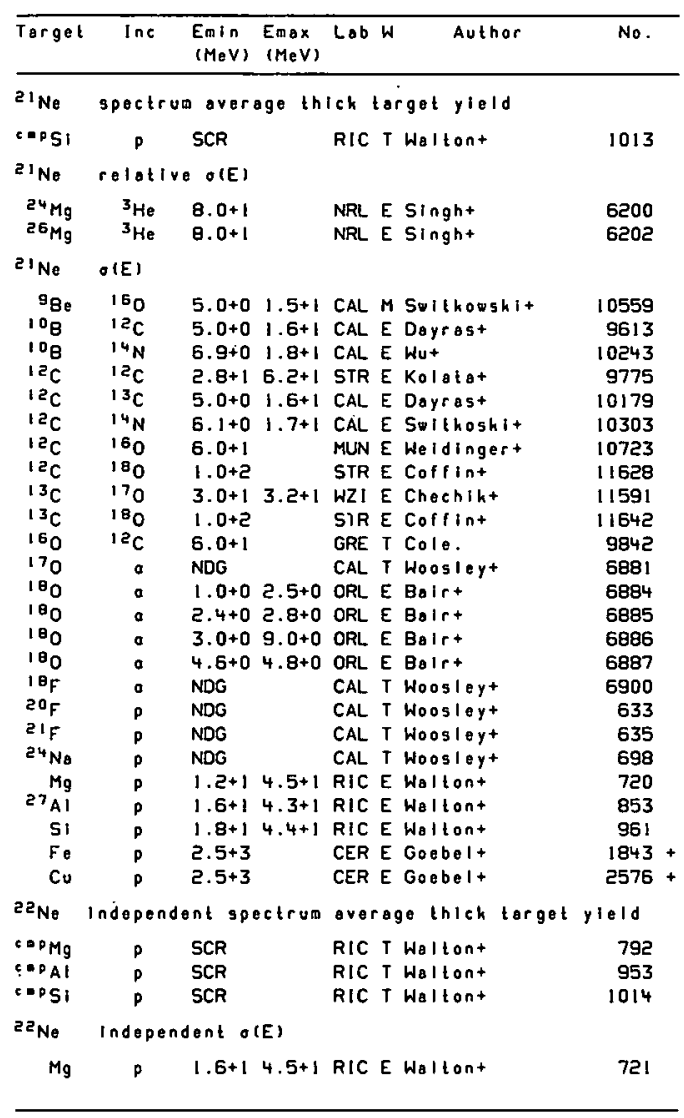




\begin{tabular}{|c|c|c|c|c|c|c|}
\hline Target & $\ln e$ & $\begin{array}{l}\text { Emin } \\
(\mathrm{MeV})\end{array}$ & $\begin{array}{l}E \max \\
(M=V)\end{array}$ & Lab & Author & No. \\
\hline $22_{\mathrm{Ne}}$ & independe & dent ol & (E) & & & \\
\hline $\begin{array}{r}27 \mathrm{Al} \\
\mathrm{Si}\end{array}$ & $p$ & $\begin{array}{l}1.6+1 \\
3.4+1\end{array}$ & $\begin{array}{l}4.2+1 \\
4.5+1\end{array}$ & $\begin{array}{l}\text { RIC E } \\
\text { RIC E }\end{array}$ & $\begin{array}{l}\text { E Wallont } \\
\text { E Wallont }\end{array}$ & $\begin{array}{l}854 \\
962\end{array}$ \\
\hline $22_{\mathrm{Ne}}$ & cumulati & Ive spe & ectrum & avero & age thich target & yield \\
\hline $\begin{array}{l}\qquad \triangle P M g \\
\operatorname{CPPAI} \\
\operatorname{CPPSI}\end{array}$ & $\begin{array}{l}p \\
p \\
p\end{array}$ & $\begin{array}{l}\text { SCR } \\
\text { SCR } \\
\text { SCR }\end{array}$ & & $\begin{array}{l}\text { RIC T } \\
\text { RIC T } \\
\text { RIC T }\end{array}$ & $\begin{array}{l}\text { T Waltiont } \\
\text { T Waltiont } \\
\text { T Waltont }\end{array}$ & $\begin{array}{r}793 \\
954 \\
1015\end{array}$ \\
\hline $2 e_{\mathrm{Ne}}$ & cunulotis & Ive otE & & & & \\
\hline $\begin{array}{l}M g \\
\text { Si }\end{array}$ & $\begin{array}{l}p \\
p\end{array}$ & $\begin{array}{l}1.2+1 \\
2.9+1\end{array}$ & $\begin{array}{l}4.5+1 \\
4.5+1\end{array}$ & $\begin{array}{l}\text { RIC E } \\
\text { RIC E }\end{array}$ & $\begin{array}{l}\text { E Waltiont } \\
\text { E Waltont }\end{array}$ & $\begin{array}{l}722 \\
963\end{array}$ \\
\hline $22 \mathrm{Ne}$ & partlal & rov th & hict to & $\operatorname{argot}$ & yleldxabundance & \\
\hline $19_{F}$ & 。 & $5.0+0$ & & SUN E & E Gilest & 6907 \\
\hline $22_{\mathrm{Ne}}$ & porttol r & ras oll & (E) & & & \\
\hline 180 & a & $6.0-1$ & $2.2+0$ & IFSE & E Trautveltert & 6890 \\
\hline $2 e_{\mathrm{Ne}}$ & portial? & relati & IVO OLE & E) & & \\
\hline $\begin{array}{l}198 \\
2851 \\
40_{11}\end{array}$ & 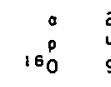 & $\begin{array}{l}2.3+0 \\
4.0+2 \\
9.6+1\end{array}$ & $4.7+0$. & $\begin{array}{l}\text { OTC E } \\
\text { SAC E } \\
\text { MUN } M\end{array}$ & $\begin{array}{l}\text { E Van-Der-Zuant } \\
\text { E Cassagnout } \\
\text { M Heringt }\end{array}$ & $\begin{array}{r}6908 \\
987 \\
11123\end{array}$ \\
\hline $2 a_{\mathrm{Ne}}$ & portial & $\theta(E)$ & & & & \\
\hline $\begin{array}{l}12 c \\
1 \geq c \\
13 c\end{array}$ & $\begin{array}{l}160 \\
180 \\
180\end{array}$ & $\begin{array}{l}3 \cdot 1+1 \\
2 \cdot 5+1 \\
3 \cdot 1+1\end{array}$ & $\begin{array}{l}5 \cdot 2+1 \\
5 \cdot 9+1 \\
3 \cdot 3+1\end{array}$ & $\begin{array}{l}\text { WAU E } \\
\text { STR E } \\
\text { WZI E }\end{array}$ & $\begin{array}{l}\text { E Chant } \\
\text { E Freenant } \\
\text { E Chechiht }\end{array}$ & $\begin{array}{l}10665 \\
11620 \\
11638\end{array}$ \\
\hline $22_{\text {Ne }}$ & product, & yield & & & & \\
\hline 180 & ${ }^{6} \mathrm{Li}$ & NDG & & ROC E & E Anantaramant & 9173 \\
\hline${ }^{22} \mathrm{Ne}_{\mathrm{N}}$ & relotive & $\sigma(E)$ & & & & \\
\hline $\begin{array}{l}26 \mathrm{Mg} \\
26 \mathrm{Mg}\end{array}$ & $\begin{array}{l}{ }^{3} \mathrm{He} \\
{ }^{3} \mathrm{He}\end{array}$ & $\begin{array}{l}8.0+1 \\
8.0+1\end{array}$ & & $\begin{array}{l}\text { NRL : } \\
\text { NRL E }\end{array}$ & $\begin{array}{l}\text { E singht } \\
\text { E Singht }\end{array}$ & $\begin{array}{l}6200 \\
6202\end{array}$ \\
\hline $2 e_{W_{0}}$ & OIEI) foct & tor & & & & \\
\hline $\begin{array}{l}180 \\
180\end{array}$ & 。 & $\begin{array}{l}0.0+0 \\
0.0+0\end{array}$ & $\begin{array}{l}2.6-0 \\
2.5-0\end{array}$ & $\begin{array}{l}\text { IFS E } \\
\text { MSI F }\end{array}$ & $\begin{array}{l}\text { E Trautvellort } \\
\text { R Rolfgt }\end{array}$ & $\begin{array}{l}6891 \\
6892\end{array}$ \\
\hline
\end{tabular}

\begin{tabular}{|c|c|c|c|c|c|c|}
\hline Target & $\ln c$ & $\begin{array}{l}E_{m / n} \\
\left(M_{\theta} V\right)\end{array}$ & $\begin{array}{l}\text { Emax } \\
(M e V)\end{array}$ & Lab & Author & No. \\
\hline $2 e_{\mathrm{Ne}}$ & $O(E)$ & & & & & . \\
\hline $10 \mathrm{~B}$ & $14 \mathrm{~N}$ & $6.9+0$ & $1.8+1$ & CAL & E Wut & 10243 \\
\hline 118 & 120 & $5.0+0$ & $1.6+1$ & CAL & E Dayras+ & 9622 \\
\hline${ }^{12} \mathrm{c}$ & $12 \mathrm{c}$ & $2.8+1$ & $6.2+1$ & SiR & E Kolata+ & $\mathbf{9 7 7 5}$ \\
\hline${ }^{12} \mathrm{C}$ & 160 & $6.0+1$ & & MUN & E Weldingert & 10723 \\
\hline $12 \mathrm{C}$ & 180 & $1.0+5$ & & STR & E Coffint & 11628 \\
\hline${ }^{13} \mathrm{C}$ & 180 & $3.1+1$ & $3.3+1$ & WZ1 & E Chechint & 11637 \\
\hline${ }^{13} \mathrm{c}$ & 180 & $1.0+5$ & & STR & E Coffint & 11642 \\
\hline 160 & $12 c$ & $6.0+1$ & & GRE & T cole. & 9842 \\
\hline 180 & o & NDG & & CAL & T Woosleyt & 6893 \\
\hline 190 & a & NDG & & CAL & T Woosleyt & 6894 \\
\hline $19 \mathrm{~F}$ & 0 & NDG & & CAL & T Woosleyt & 6909 \\
\hline $2 I_{F}$ & p & NDG & & CAL & T Woosleyt & 636 \\
\hline $22_{F}$ & p & NDG & & CAL & T Woosieyt & 638 \\
\hline${ }^{25} \mathrm{Na}$ & p & NDG & & $\mathrm{CAL}$ & T Woosleyt & 701 \\
\hline Fe & p & $2.5+3$ & & CER & E Goebelt & 1843 \\
\hline $\mathrm{Cu}$ & p & $2.5+3$ & & CER & E Goebe1+ & 2576 \\
\hline${ }^{23} \mathrm{Ne}$ & \multicolumn{6}{|c|}{ cumulative $O(E)$} \\
\hline${ }^{27} \mathrm{Al}$ & p & $6.0+2$ & & MIR & E Clinet & 855 \\
\hline $23_{\mathrm{No}}$ & \multicolumn{6}{|l|}{$O(E)$} \\
\hline${ }^{12} \mathrm{C}$ & 180 & $1.0+2$ & & STR & E Coffint & 11628 \\
\hline${ }^{13} \mathrm{c}$ & 180 & $1.0+2$ & & STR & E Coff: $n^{+}$ & 11642 \\
\hline 190 & a & NDG & & CAL & T Woosleyt & 6895 \\
\hline $20^{\circ}$ & a & NDG & & CAL & T Woosleyt & 6914 \\
\hline $22_{F}$ & $p$ & NDG & & CAL & I Woosleyt & 639 \\
\hline $26_{\mathrm{No}}$ & p & NDG & & CAL & I Woosleyt & 704 \\
\hline${ }^{24} \mathrm{No}$ & \multicolumn{6}{|c|}{ cumulative o(E)xlactor } \\
\hline Cu & D & $1.0+3$ & $3.0+3$ & BNL & E Hudis+ & 2469 \\
\hline Cu & $p$ & $1.0+3$ & $3.0+3$ & KFK & C Muenzelt & 2470 \\
\hline Ag & $p$ & $1.0+3$ & $3.0+3$ & $\mathrm{BNL}$ & E Hudist & 3686 \\
\hline $\mathrm{Ag}$ & p & $1.0+3$ & $3.0+3$ & KFK & C Muenzelt & 3687 \\
\hline $197 \mathrm{Au}$ & p & $1.0+3$ & $3.0+3$ & ENL & E Hudist & 4471 \\
\hline $197 \mathrm{Au}$ & $p$ & $1.0+3$ & $3 \cdot 0+3$ & KFK & C Muenzelt & 4472 \\
\hline$u$ & $p$ & $7.0+2$ & $3.0+3$ & BNL & E Hudist & 4825 \\
\hline u & $p$ & $7.0+2$ & $3.0+3$ & KFK & C Muenzelt & 4826 \\
\hline${ }^{24} \mathrm{Ne}$ & \multicolumn{6}{|c|}{ cuavlative o(E) } \\
\hline $27_{\mathrm{Al}}$ & $p$ & $6.0+2$ & & MTR & E Clinet & 85 \\
\hline
\end{tabular}




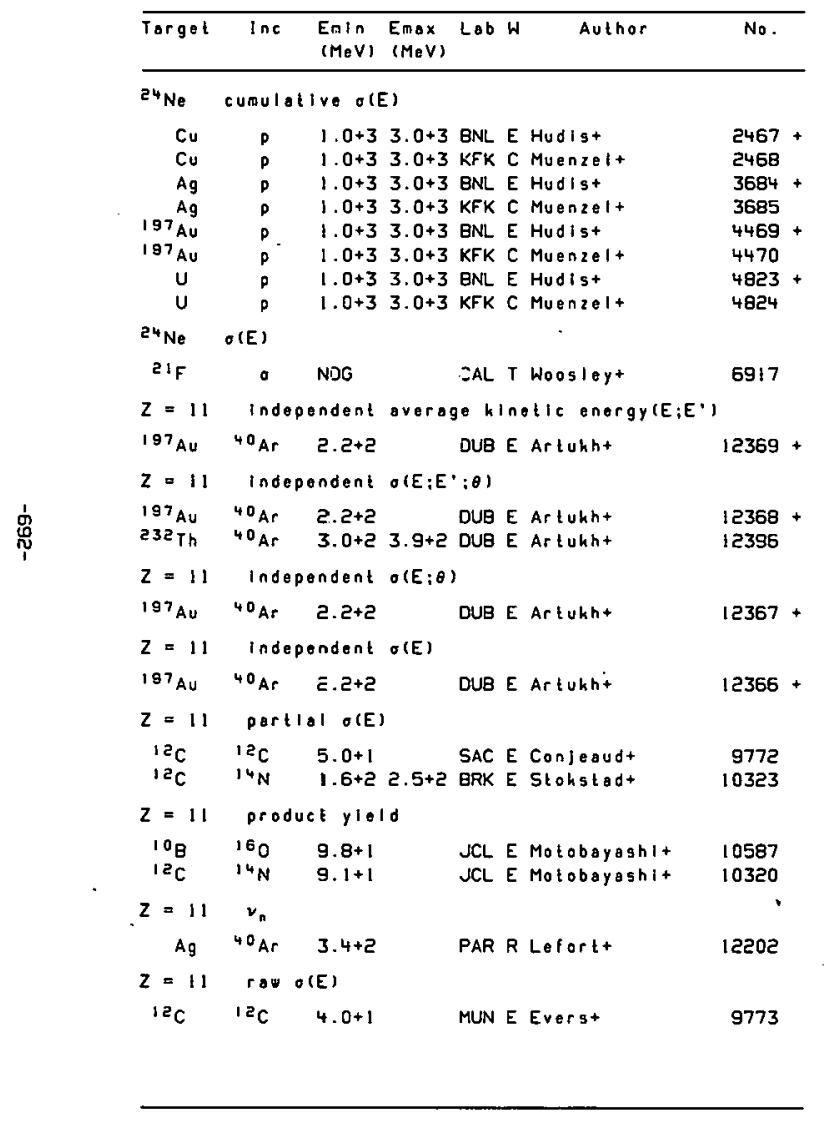

\begin{tabular}{|c|c|c|c|c|c|c|}
\hline$\overline{T a r g e t}$ & $\ln c$ & $\begin{array}{l}E_{m} \mid n \\
(M e V)\end{array}$ & $\begin{array}{l}E_{\max } \\
(\mathrm{MeV})\end{array}$ & Lab & Author & No. \\
\hline$z=11$ & \multicolumn{2}{|c|}{ o(E)xfactor } & & & & \\
\hline${ }^{10} \mathrm{~B}$ & 160 & $3.9+1$ & $2.0+2$ & ORL & E Del-Campot & 10586 \\
\hline $12 \mathrm{C}$ & $14 \mathrm{~N}$ & NDG & & ORL & E Del-Campot & 10315 \\
\hline $14 \mathrm{~N}$ & $12 \mathrm{c}$ & $5.0+1$ & & SAC & E Conjeaudt & 9805 \\
\hline $15_{N}$ & ${ }^{12} \mathrm{c}$ & $5.2+1$ & & SAC & EConjeaudt & 9814 \\
\hline $232 \mathrm{Th}$ & ${ }^{40} \mathrm{Ar}$ & $3.9+2$ & & OXF & R Hodgson. & 12398 \\
\hline$z=11$ & \multicolumn{2}{|c|}{$\sigma(E)$} & & & & \\
\hline${ }^{12} \mathrm{C}$ & $\mathrm{I}^{2} \mathrm{C}$ & $1.5+1$ & $6.2+1$ & ANL & E Kovart & 9768 \\
\hline $12 \mathrm{C}$ & $12 \mathrm{c}$ & $2.8+1$ & $5.4+1$ & SAC E & E Conjeaudt & 9769 \\
\hline $1 z_{c}$ & $12 \mathrm{c}$ & $4.5+1$ & $2.0+2$ & TAM E & E Namboodirit & 9770 \\
\hline${ }^{12} \mathrm{C}$ & ${ }^{13} \mathrm{c}$ & $1.5+1$ & $5.2+1$ & ANL & E Kovart & 10185 \\
\hline${ }^{12} \mathrm{C}$ & $14 \mathrm{~N}$ & $3 \cdot 3+1$ & $5.2+1$ & ANL. & E Kovort & 10317 \\
\hline $1 z_{\mathrm{c}}$ & $14 \mathrm{~N}$ & $8.6+1$ & $1.7+2$ & ORL & E Stohstodt & 10318 \\
\hline $12 \mathrm{C}$ & $15 \mathrm{~N}$ & $2.0+1$ & $6.0+1$ & ANL & E Kovart & 10528 \\
\hline${ }^{12} \mathrm{c}$ & 160 & $3.5+1$ & $6.2+1$ & ANL & E Tabort & 10721 \\
\hline $12 \mathrm{C}$ & 150 & $4.0+1$ & $6.5+1$ & NOT $E$ & E Malmint & 10722 \\
\hline $12 \mathrm{C}$ & 170 & $3.4+1$ & $8.0+1$ & MUN E & E Hertz+ & $1158 \mid$ \\
\hline $12 \mathrm{C}$ & 180 & $1.0+2$ & & STR E & E Coffint & 11626 \\
\hline $12 \mathrm{C}$ & $20 \mathrm{No}$ & $5 \cdot 7+1$ & $1.6+2$ & GRE & E Soint-Laurentt & 11839 \\
\hline${ }^{13} \mathrm{C}$ & 100 & $1.0+2$ & & STR E & E Coffint & 11639 \\
\hline $14 \mathrm{~N}$ & $12 \mathrm{c}$ & $3.4+1$ & $5.0+1$ & SAC E & E Conjeaudt & 9806 \\
\hline $14 N$ & $13 \mathrm{c}$ & $4.4+1$ & $5.4+1$ & SAC & E Conjeaudt & 10192 \\
\hline $15_{N}$ & $12 \mathrm{c}$ & $3 \cdot 0+1$ & $5.0+i$ & SAC E & E Conjeaudt & 9815 \\
\hline 160 & $14 \mathrm{~N}$ & $3.7+1$ & $5.8+i$ & SAC E & E Conjeaudt & 10345 \\
\hline 160 & $15_{\mathrm{N}}$ & $3.6+1$ & $5.5+i$ & SAC E & E Conjeaudt & 10529 \\
\hline 160 & 160 & $3.0+1$ & $6.0+1$ & ANL & E Schiffert & 10853 \\
\hline 160 & 160 & $1.2+2$ & $1.4+2$ & GRE & E Saint-Laurentt & 10854 \\
\hline 170 & $12 \mathrm{c}$ & $3.5+1$ & $4.9+1$ & SAC E & E Conjeaudt & 9845 \\
\hline 170 & $13 \mathrm{c}$ & $3.8+1$ & $5.3+1$ & SAC E & E Conjeaudt & 10195 \\
\hline $20 \mathrm{Ne}$ & $12 \mathrm{c}$ & $4.8+1$ & $9.6+1$ & SAC N & $M$ Conjeaudt & 9867 \\
\hline $20 \mathrm{Ne}$ & $12 \mathrm{c}$ & $1.5+5$ & $1.4+2$ & GRE & E Conjeaudt & 9868 \\
\hline${ }^{45} \mathrm{Sc}$ & $22_{\mathrm{Ne}}$ & NDG & & LYo & T Billereyt & 11924 \\
\hline${ }^{50} \mathrm{Cr}$ & $14 \mathrm{~N}$ & $7.1+1$ & $1.0+2$ & LYO & T Billereyt & 10393 \\
\hline${ }^{50} \mathrm{Cr}$ & $25^{n}$ & NOG & & LYO & T Billereyt & 11925 \\
\hline${ }^{56} \mathrm{Fe}$ & 160 & NDG & & LYO & T Billereyt & 11162 \\
\hline & $20 \mathrm{No}$ & $1.6+2$ & & ORL F & RPlasilt & 11851 \\
\hline$a^{n}$ & ${ }^{12} \mathrm{C}$ & NOC & & LYO & Billereyt & 9960 \\
\hline $232 \mathrm{Ib}$ & $40 \mathrm{Ar}$ & $7.2+$ & & BRK & & 12399 \\
\hline
\end{tabular}




\begin{tabular}{|c|c|c|c|c|c|c|}
\hline Targat & $\ln c$ & $\begin{array}{l}\text { Emin } \\
(M e V)\end{array}$ & $\begin{array}{l}E \operatorname{Egx} \\
(M \in V)\end{array}$ & Lab & Author & No. \\
\hline $20 \mathrm{Na}$ & $O(E)$ & & & & & \\
\hline $20 \mathrm{Ne}$ & $p$ & $T R$ & $1.7+1$ & CAL & T Woosleyt & 652 \\
\hline $2{ }^{1} \mathrm{Na}$ & cumulat & ive pro & oduct y & yield & & \\
\hline $20 \mathrm{Ne}$ & ${ }^{3} \mathrm{He}$ & TR & +1 & HEI & E Helust & 6185 \\
\hline${ }^{2}{ }^{\mathrm{Na}}$ & partial & rovol & $(E)$ & & & \\
\hline${ }^{20} \mathrm{Ne}$ & p & $1.2+0$ & $1.3+0$ & HLS & E Keinonent & 654 \\
\hline $2^{\prime} \mathrm{Na}$ & partial & relati & IVe ofE & & & \\
\hline $28 \mathrm{SI}$ & $p$ & $4.0+2$ & & SAC & E Cassagnout & 987 \\
\hline $2 I_{\mathrm{Na}}$ & partial & $O(E)$ & & & & \\
\hline $\begin{array}{l}12 \mathrm{C} \\
24 \mathrm{Mg}\end{array}$ & ${ }_{\mathrm{P}}^{14 \mathrm{~N}}$ & $\begin{array}{l}2.1+1 \\
2.3+1\end{array}$ & $3.7+1$ & $\begin{array}{l}\text { YAL } \\
\text { JUL }\end{array}$ & $\begin{array}{l}\text { R Stohstad. } \\
\text { E Rohwert }\end{array}$ & $\begin{array}{r}10304 \\
742\end{array}$ \\
\hline $2{ }^{\prime} \mathrm{Na}$ & product & $y 1018$ & & & & \\
\hline $\begin{array}{l}20 \mathrm{Ne} \\
20 \mathrm{Ne}\end{array}$ & $\begin{array}{l}\text { d } \\
\text { p }\end{array}$ & $\begin{array}{l}1.2+0 \\
T R\end{array}$ & -5 & MOS & $\begin{array}{l}\text { R Varlamovt } \\
\text { E Helust }\end{array}$ & $\begin{array}{r}653 \\
5368\end{array}$ \\
\hline $2 I_{\mathrm{Na}}$ & relotive & e $\sigma(E) \times$ & $\times$ factor & & & \\
\hline $24 \mathrm{Mg}$ & P & $9.2+0$ & $1.4+1$ & KLN & E Gruhle+ & 745 \\
\hline $2 I_{\mathrm{Na}}$ & relative & - $O(E)$ & & & & \\
\hline $\begin{array}{l}24 \mathrm{Mg} \\
26 \mathrm{Mg}\end{array}$ & $\begin{array}{l}{ }^{3} \mathrm{He} \\
{ }^{3} \mathrm{He}\end{array}$ & $\begin{array}{l}8.0+1 \\
8.0+1\end{array}$ & & $\begin{array}{l}\text { NRL } \\
\text { NRL }\end{array}$ & $\begin{array}{l}\text { E singht } \\
\text { E singht }\end{array}$ & $\begin{array}{l}6200 \\
6202\end{array}$ \\
\hline $21 \mathrm{Na}$ & $O(E) \times f a C$ & ctor & & & & \\
\hline $\begin{array}{l}20 \mathrm{Ne} \\
{ }^{20} \mathrm{Ne}\end{array}$ & $p$ & $\begin{array}{l}0.0+0 \\
3.8-1\end{array}$ & $\begin{array}{l}1.8 \div 0 \\
1.5+0\end{array}$ & MST & $\begin{array}{l}\text { R Rolfst } \\
\text { E Rolfst }\end{array}$ & $\begin{array}{l}657 \\
658\end{array}$ \\
\hline $2 I_{\mathrm{Na}}$ & $O(E)$ & & & & & \\
\hline $\begin{array}{l}10 \mathrm{~B} \\
10_{0}\end{array}$ & $\begin{array}{l}12 \mathrm{C} \\
160\end{array}$ & $\begin{array}{l}5 \cdot 0+0 \\
6 \cdot 0+1\end{array}$ & $1.6+1$ & CAL & $\begin{array}{l}\text { E Dayrast } \\
\text { T Cole. }\end{array}$ & $\begin{array}{r}9614 \\
10860\end{array}$ \\
\hline $17 \mathrm{~F}$ & a & NDG & & CAL & T Woosleyt & 6899 \\
\hline $20_{\mathrm{Ne}}$ & p & NDG & & CAL & T Woogleyt & 655 \\
\hline $20 \mathrm{Ne}$ & p & $0.0+0$ & $1.1+2$ & INU & T Componi-Tabrizi & 656 \\
\hline $21 \mathrm{Ne}$ & p & TR & $1.3+1$ & CAL & T Woosleyt & 662 \\
\hline Mg & p & $1.5+1$ & $2.7+i$ & CLU E & E Wilsont & 723 \\
\hline $24 \mathrm{Mg}$ & p & & $1.7+1$ & CAL & T Woosloyt & 743 \\
\hline $24 \mathrm{Mg}$ & p & $9.2+0$ & $1.4+1$ & $\mathrm{KLN}$ & E Gruhlet & 744 \\
\hline
\end{tabular}

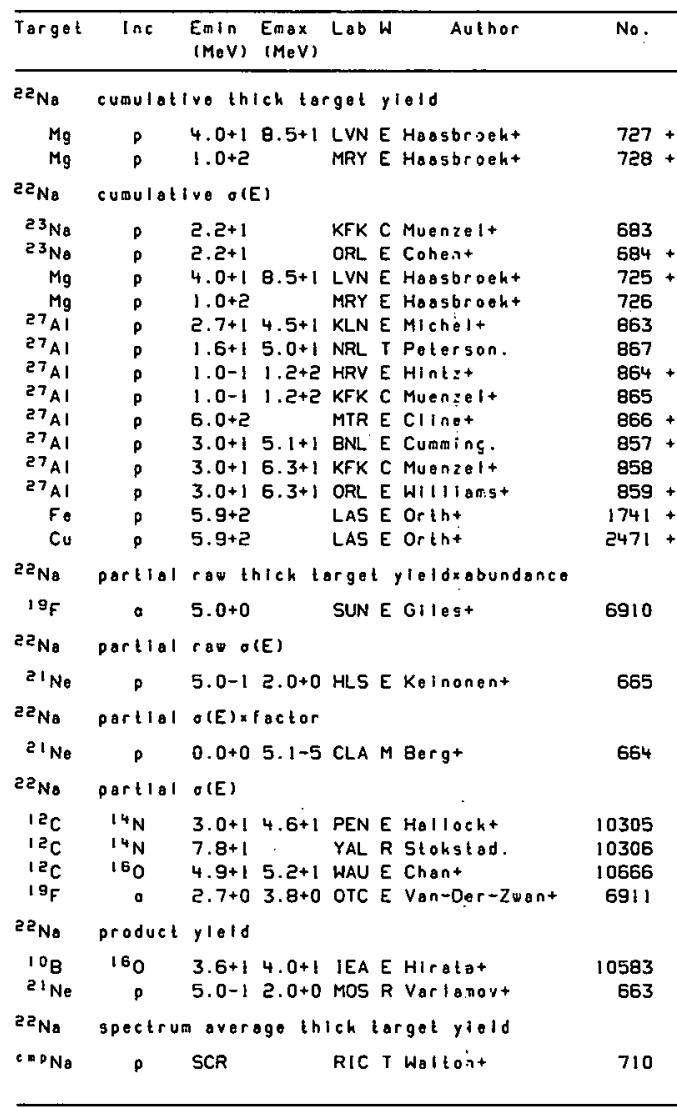




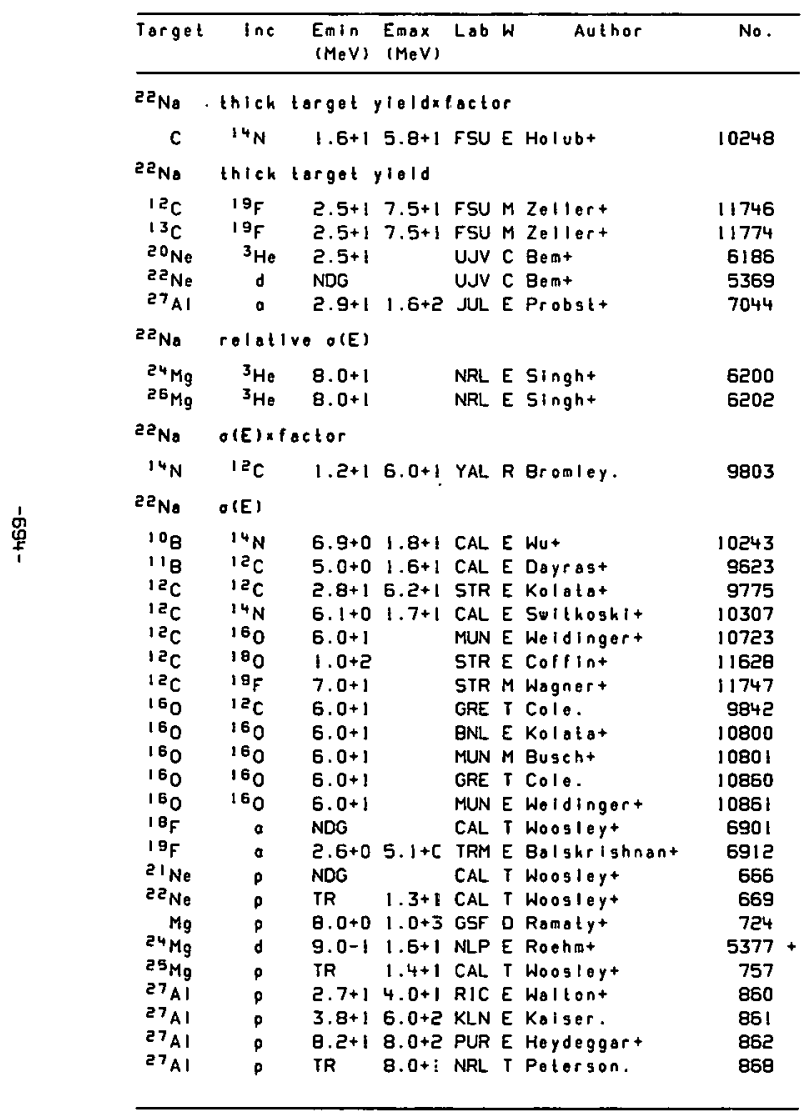

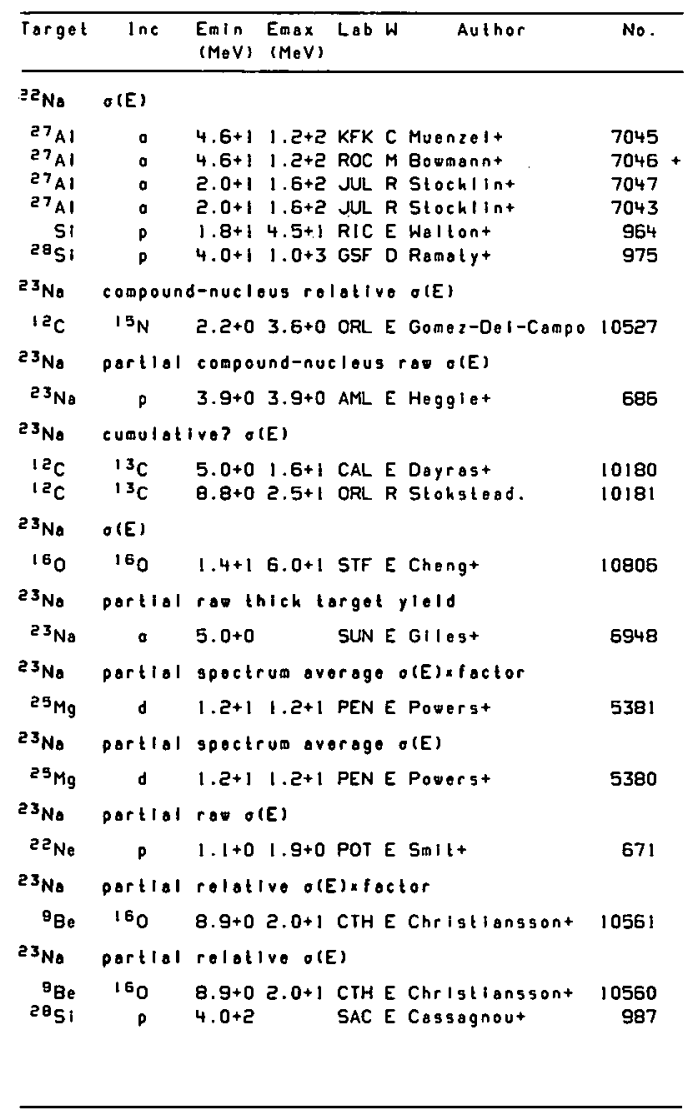




\begin{tabular}{|c|c|c|c|c|c|c|}
\hline Target & $\ln c$ & $\begin{array}{l}\text { Emin } \\
(M e V)\end{array}$ & $\begin{array}{l}\text { Emax } \\
(\operatorname{MeV})\end{array}$ & Lab & Author & No. \\
\hline $23^{2} \mathrm{~g}$ & partlol & $O(E) \times f$ & factor & & & \\
\hline $\begin{array}{l}12 c \\
12 c \\
12 c \\
160 \\
160\end{array}$ & $\begin{array}{l}12 c \mathrm{c} \\
12 \mathrm{C} \\
12 \mathrm{C} \\
12 \mathrm{c} \\
160\end{array}$ & $\begin{array}{l}5 \cdot 0+0 \\
5 \cdot 0+0 \\
6 \cdot 5+0 \\
1 \cdot 9+1 \\
5 \cdot 4+1\end{array}$ & $\begin{array}{l}1.2+1 \\
1.2+1 \\
1.2+1 \\
4.8+1 \\
6 \cdot 0+1\end{array}$ & $\begin{array}{l}\text { BOC } \\
\text { MST } \\
\text { LUO } \\
\text { NB1 } \\
\text { WZ1 }\end{array}$ & $\begin{array}{l}\text { E Kelknert } \\
\text { R Rolfgt } \\
\text { E Hight } \\
\text { E Soltkowshit } \\
\text { E Tserruyat }\end{array}$ & $\begin{array}{r}9761 \\
9762 \\
9763 \\
9833 \\
10805\end{array}$ \\
\hline $23^{3} \mathrm{Na}$ & partlal & $\sigma(E)$ & & & & \\
\hline $\begin{array}{l}12 \mathrm{C} \\
12 \mathrm{C} \\
160 \\
26 \mathrm{Mg}\end{array}$ & $\begin{array}{r}160 \\
160 \\
160 \\
p\end{array}$ & $\begin{array}{l}1.8+1 \\
3 \cdot 0+1 \\
1.8+1 \\
2 \cdot 3+1\end{array}$ & $\begin{array}{l}5 \cdot 2+1 \\
4 \cdot 3+1 \\
6 \cdot 0+1\end{array}$ & $\begin{array}{l}\text { WAU } \\
\text { CBR } \\
\text { STF } \\
\text { JUL }\end{array}$ & $\begin{array}{l}\text { E Chont } \\
\text { E Branfordt } \\
\text { E Chengt } \\
\text { E Rohert }\end{array}$ & $\begin{array}{r}10669 \\
10670 \\
10802 \\
771\end{array}$ \\
\hline $23 \mathrm{Na}$ & product & yield & & & & \\
\hline $\begin{array}{l}10 \mathrm{~B} \\
22^{\mathrm{Ne}}\end{array}$ & ${ }^{14 N}$ & $\begin{array}{l}9.5+0 \\
1.3+0\end{array}$ & $1.3+1$ & MON & $\begin{array}{l}\text { E Koidet } \\
\text { R Varlamovt }\end{array}$ & $\begin{array}{r}10242 \\
670\end{array}$ \\
\hline $23^{2} \mathrm{No}$ & relative & o $O(E) \times$ & f factor & & & \\
\hline $\begin{array}{r}1{ }^{12} \mathrm{C} \\
23^{\mathrm{Na}}\end{array}$ & $\begin{array}{l}160 \\
\text { relative }\end{array}$ & $\begin{array}{l}7.9+0 \\
. \quad O(E)\end{array}$ & $1.5+1$ & SAC & R Popineau. & 10672 \\
\hline $\begin{array}{l}12 \mathrm{C} \\
24 \mathrm{Mg} \\
26 \mathrm{Mg}\end{array}$ & $\begin{array}{l}{ }^{16} 0_{0} \\
{ }^{3} \mathrm{He} \\
{ }^{3} \mathrm{He}\end{array}$ & $\begin{array}{l}2.8+1 \\
8.0+1 \\
8.0+1\end{array}$ & $3.5+1$ & $\begin{array}{l}\text { MON } \\
\text { NRL } \\
\text { NRL }\end{array}$ & $\begin{array}{l}\text { E Trast, } \\
\text { E Singht } \\
\text { E Singht }\end{array}$ & $\begin{array}{r}10671 \\
6200 \\
6202\end{array}$ \\
\hline $23^{30}$ & $\sigma(E)$ & & & & & \\
\hline $\begin{array}{rl}9 & \mathrm{Be} \\
{ }^{9} \mathrm{Be}\end{array}$ & $\begin{array}{l}160 \\
160\end{array}$ & $\begin{array}{l}7.6+0 \\
5.0+0\end{array}$ & $\begin{array}{l}1.4+1 \\
1.5+1\end{array}$ & CAL & $\begin{array}{l}\text { MSorthowshit } \\
\text { M Swithowshit }\end{array}$ & $\begin{array}{l}10562 \\
10563\end{array}$ \\
\hline $\begin{array}{l}10 \mathrm{~B} \\
12 \mathrm{C}\end{array}$ & $\begin{array}{l}14 \mathrm{~N} \\
15^{\mathrm{C}} \mathrm{C}\end{array}$ & $\begin{array}{l}6.9+0 \\
2.5+0\end{array}$ & $\begin{array}{l}1.8+1 \\
3.6+0\end{array}$ & $\begin{array}{l}\text { CAL } \\
\text { LUO }\end{array}$ & $\begin{array}{l}\text { E Wut } \\
\text { E Hight }\end{array}$ & $\begin{array}{r}10243 \\
9764\end{array}$ \\
\hline $12 \mathrm{C}$ & $12 \mathrm{c}$ & $2.8+1$ & $6.2+1$ & STR & E Kolata+ & 9775 \\
\hline${ }^{12} \mathrm{C}$ & 160 & $1.7+1$ & $3.1+1$ & CAL & E Christensont & 10667 \\
\hline $1 \mathrm{C}$ & 160 & $4.0+1$ & $6.5+1$ & STR & E Kolatat & 10668 \\
\hline${ }^{12} \mathrm{C}$ & 160 & $6.0+1$ & & MUN & $E$ Weldingert & 10723 \\
\hline $12 \mathrm{C}$ & 180 & $1.0+2$ & & STR & E Colfint & 11628 \\
\hline $12 \mathrm{c}$ & $19 \mathrm{~F}$ & $5.0+1$ & $7.0+1$ & STR I & M Wagnert & 11748 \\
\hline${ }^{13} \mathrm{C}$ & 180 & $1.0+2$ & & STR E & E Coffint & 11642 \\
\hline 160 & ${ }^{12} \mathrm{C}$ & $6.0+1$ & & GRE & T Cole. & 9842 \\
\hline 160 & 160 & $6.0+1$ & & 8NL & E Kolalat & 10803 \\
\hline 160 & 160 & $6.0+1$ & & MUN & M Buscht & 10804 \\
\hline 160 & 160 & $2.4+1$ & $8.0+1$ & STR E & E Kolatat & 10859 \\
\hline
\end{tabular}

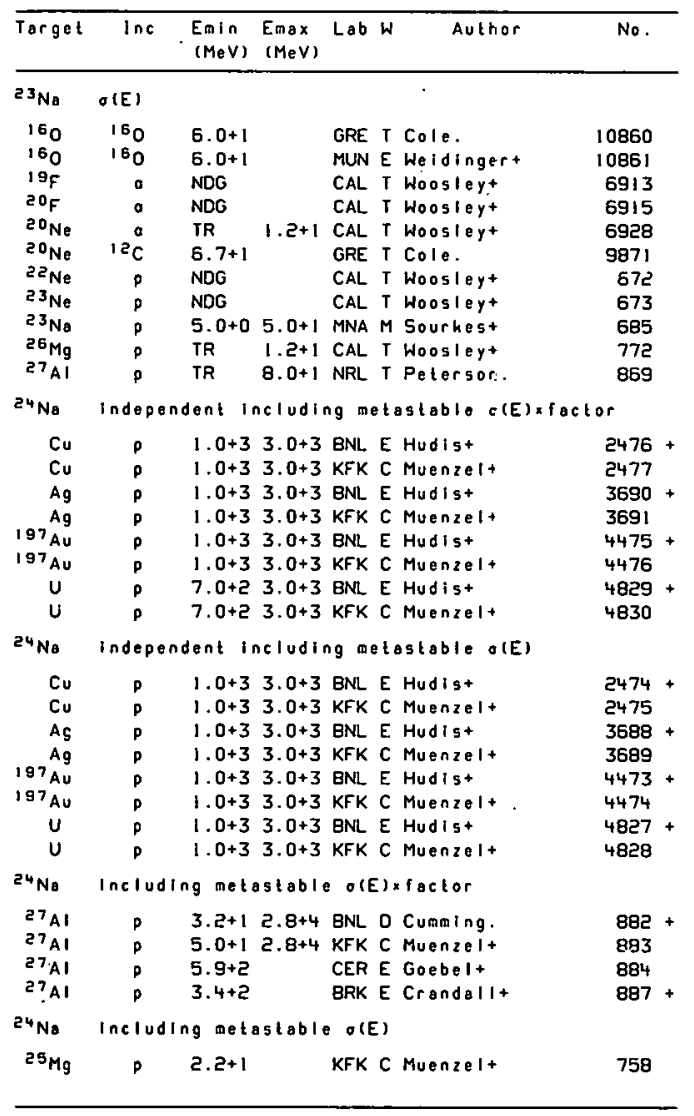




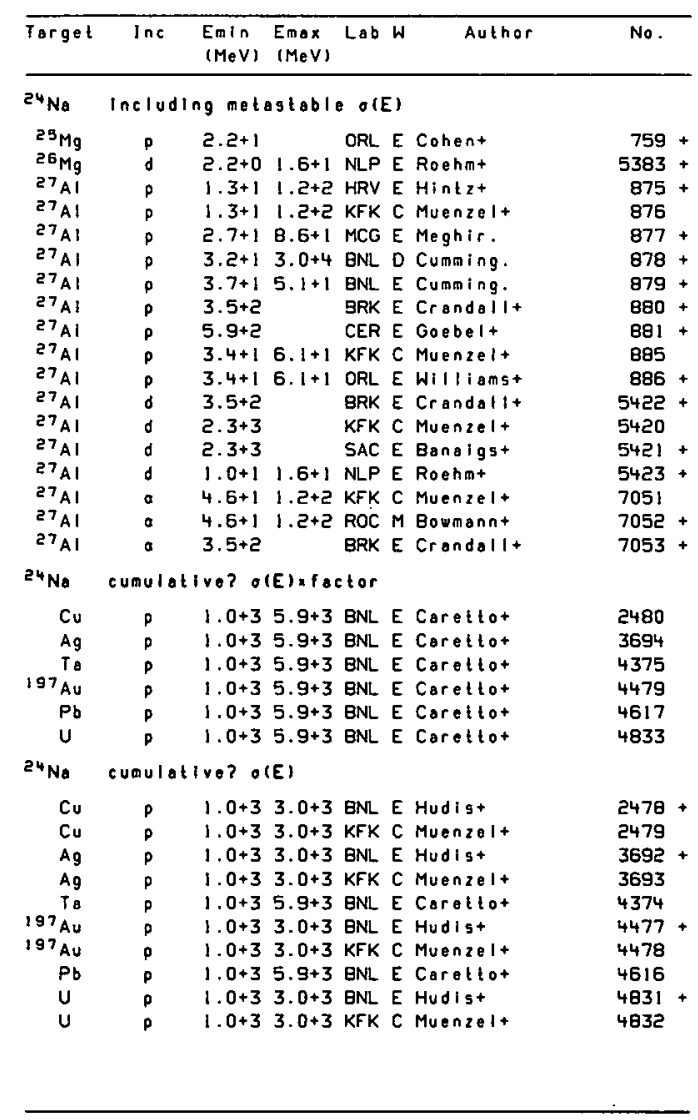

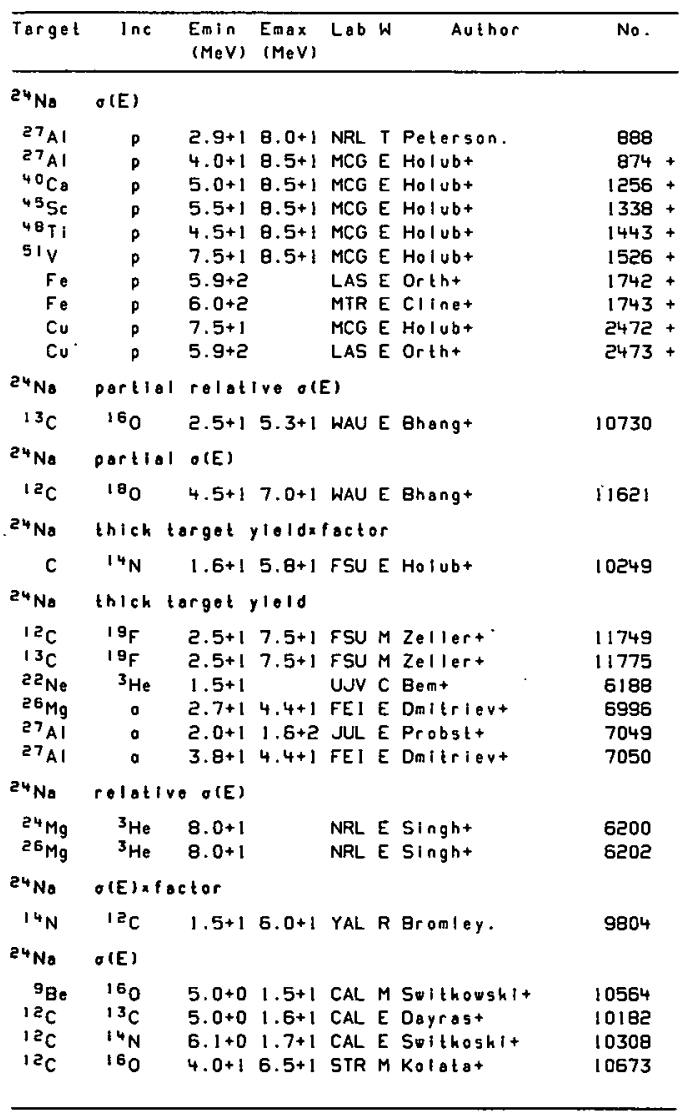




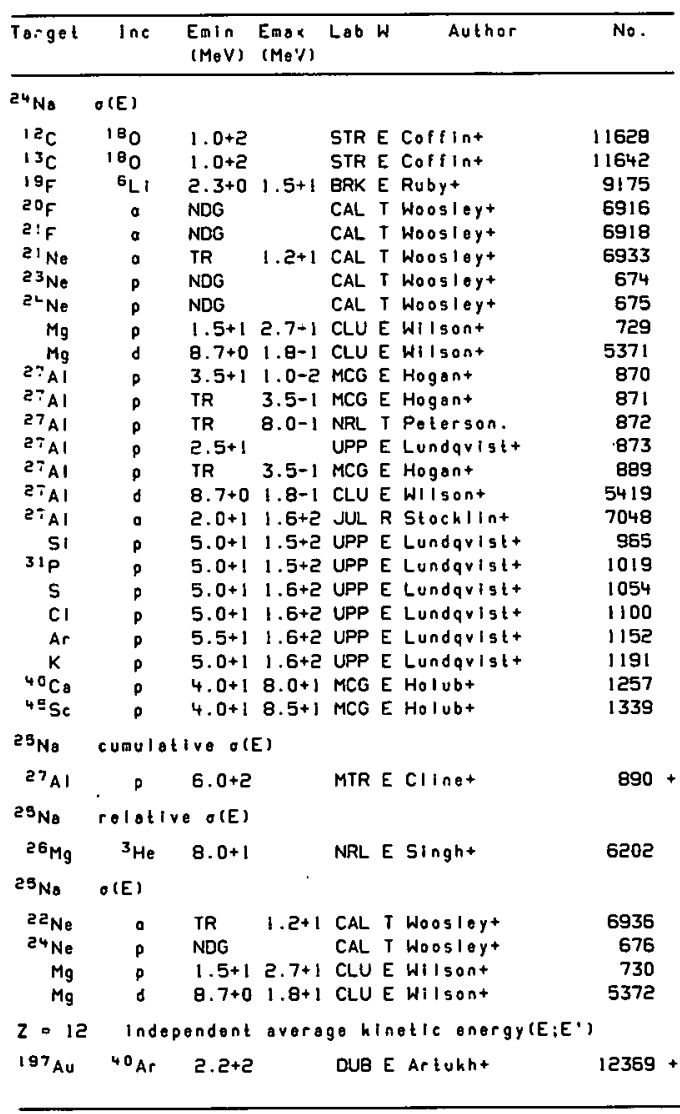

\begin{tabular}{|c|c|c|c|c|c|c|}
\hline Target & $\ln c$ & $\begin{array}{l}E_{\square(n)} \\
(M e V)\end{array}$ & $\begin{array}{l}\text { Emax } \\
(\operatorname{MeV})\end{array}$ & Lab. & Author & No. \\
\hline$z=12$ & Inder & ondent & $O(E ; E$. & $\because(\theta)$ & & \\
\hline $\begin{array}{l}187 \mathrm{Au} \\
232 \mathrm{Th}\end{array}$ & $\begin{array}{l}40 \mathrm{Ar} \\
40 \mathrm{Ar}\end{array}$ & $\begin{array}{l}2.2+2 \\
3.0+2\end{array}$ & $3.9+2$ & $\begin{array}{l}\text { DUB } \\
\text { DUP }\end{array}$ & $\begin{array}{l}\text { E Artuhht } \\
\text { E Arlukht }\end{array}$ & $\begin{array}{l}12368+ \\
12396\end{array}$ \\
\hline$z=12$ & inder & endent & $\theta(E ; \theta)$ & & & \\
\hline $\begin{array}{l}197 \mathrm{Au} \\
Z=12\end{array}$ & $\begin{array}{l}{ }^{40} \mathrm{Ar} \\
\text { Indor }\end{array}$ & $\begin{array}{r}2.2+2 \\
\text { endent }\end{array}$ & $O(E)$ & OUB & E Artukht & $12367+$ \\
\hline $\begin{array}{l}197 \mathrm{AU} \\
Z=12\end{array}$ & $\begin{array}{c}40_{A r} \\
v_{n}\end{array}$ & $2.2+2$ & & DUB & E Artukht & $12366+$ \\
\hline $\mathrm{Ag}$ & ${ }^{40} \mathrm{Ar}$ & $3.4+2$ & & PAR & R Lefortt & 12202 \\
\hline$z=12$ & row & (E) & & & & \\
\hline${ }^{12} \mathrm{C}$ & ${ }^{12} \mathrm{C}$ & $4.0+1$ & & MUN & E Everst & 9773 \\
\hline$z=12$ & $\sigma(E)$ & foctor & & & & \\
\hline $\begin{array}{r}10 \mathrm{~B} \\
1 \mathrm{C}_{\mathrm{C}} \\
14 \mathrm{~N} \\
15_{\mathrm{N}} \\
232 \mathrm{Th}\end{array}$ & $\begin{array}{l}16 \mathrm{O} \\
14 \mathrm{~N} \\
12 \mathrm{C} \\
12 \mathrm{C} \\
40_{\mathrm{Ar}}\end{array}$ & $\begin{array}{l}3.9+1 \\
\text { NDG } \\
5.0+1 \\
5.2+1 \\
3.9+2\end{array}$ & $2.0+2$ & $\begin{array}{l}\text { OHL } \\
\text { ORL } \\
\text { SAC } \\
\text { SAC } \\
\text { OXF }\end{array}$ & $\begin{array}{l}\text { E Del-Campot } \\
\text { E Del-Compot } \\
\text { E Conjeaudt } \\
\text { E Conjesudt } \\
\text { R Hodgson. }\end{array}$ & $\begin{array}{r}10586 \\
10315 \\
9805 \\
9814 \\
12398\end{array}$ \\
\hline$z=12$ & $\theta(\varepsilon)$ & & & & & \\
\hline $\begin{array}{l}12 c \\
12 c\end{array}$ & $\begin{array}{l}1 e_{c} \\
1{ }^{2} c\end{array}$ & $\begin{array}{l}2.8+1 \\
4.5+1\end{array}$ & $\begin{array}{l}5.4+1 \\
2.0+2\end{array}$ & $\begin{array}{l}\text { SAC } \\
\text { TAM }\end{array}$ & $\begin{array}{l}\text { E Conjoaudt } \\
\text { E Namboodirit }\end{array}$ & $\begin{array}{l}9769 \\
9770\end{array}$ \\
\hline $12 c$ & $13 \mathrm{C}$ & $1.6+1$ & $2.2+1$ & ANL. & E Kovart & $10 ! 85$ \\
\hline $12 c$ & $14 \mathrm{~N}$ & $3.3+1$ & $5.2+1$ & ANL & E Kovart & 0317 \\
\hline${ }^{12} \mathrm{c}$ & $14 N$ & $8.6+1$ & $1.7+2$ & ORL & E Stohstadt & 10318 \\
\hline $12 \mathrm{c}$ & $15_{N}$ & $2.0+1$ & $6.0+1$ & ANL & E Kovert & 10528 \\
\hline $1{ }^{2} c$ & 180 & $3.5+1$ & $6 \cdot 2+1$ & ANL & E Tabort & 10721 \\
\hline $12 \mathrm{C}$ & 160 & $4.0+1$ & $6.6+1$ & NOT & E MelnInt & 10722 \\
\hline $12 \mathrm{c}$ & 170 & $3.4+1$ & $8.0+1$ & MUN & E Hertz+ & 11581 \\
\hline $12 \mathrm{c}$ & $1 \theta_{0}$ & $1.0+2$ & & STR & E coffint & 11626 \\
\hline $12^{2}$ & $20 \mathrm{Ne}$ & $6.7+1$ & $1.6+2$ & GRE & E Saint-Laurent+ & 11839 \\
\hline${ }^{13} \mathrm{C}$ & 180 & $1.0+2$ & & STR & E Coffint & 11639 \\
\hline $14 \mathrm{~N}$ & $12 c$ & $3.4+1$ & $5 \cdot 0+1$ & SAC & E Conjeaudt & 9806 \\
\hline $14 \mathrm{~N}$ & $13 \mathrm{c}$ & $4.4+1$ & $5.4+1$ & SAC & E Conjeaudt & 10192 \\
\hline 150 & $14 \mathrm{~N}$ & $3.7+1$ & $5.8+1$ & SAC & E Conjeaudt & 10345 \\
\hline 160 & $19_{N}$ & $3.6+1$ & $5.5+1$ & SAC & E Conjeaudt & 10529 \\
\hline 160 & 160 & $3.0+1$ & $6 \cdot 0+1$ & ANL & E Schiffert & 10853 \\
\hline
\end{tabular}




\begin{tabular}{|c|c|c|c|c|c|c|}
\hline Target & $\operatorname{lnc}$ & $\begin{array}{l}\text { Emin } \\
(\text { MeV) }\end{array}$ & $\begin{array}{l}\max \\
(\operatorname{MeV} V)\end{array}$ & Lob & Author & No. \\
\hline$z=12$ & $\sigma(E)$ & & & & & \\
\hline 160 & 160 & $1.2+2$ & $1.4+2$ & GRE & E Salnt-Laurent+ & 10854 \\
\hline 170 & ${ }^{12} \mathrm{c}$ & $3.5+1$ & $4.9+1$ & SAC & E Conjeaudt & 9845 \\
\hline 170 & $13 \mathrm{C}$ & $3.8+1$ & $5.3+1$ & SAC & E Conjeaudt & 10195 \\
\hline${ }^{20} \mathrm{Ne}$ & ${ }^{12} \mathrm{c}$ & $4.8+1$ & $9.6+1$ & SAC & M Conioaudt & 9867 \\
\hline $20_{\mathrm{Ne}}$ & $12 \mathrm{c}$ & $1.2+2$ & $1.4+2$ & GRE & E Conjeaudt & 9868 \\
\hline${ }^{45} \mathrm{Sc}$ & ${ }^{2}{ }^{\mathrm{Ne}}$ & NOG & & LYO & T Billereyt & 11924 \\
\hline${ }^{50} \mathrm{Cr}$ & ${ }^{14} \mathrm{~N}$ & $7.1+1$ & $1.0+2$ & LYO & T Billereyt & 10393 \\
\hline${ }^{50} \mathrm{Cr}$ & ${ }^{22} \mathrm{Ne}$ & NDG & & LYO & T Blllereyt & 11925 \\
\hline $56_{\mathrm{Fe}}$ & 160 & NOG & & LYO & T Billereyt & 11162 \\
\hline Ni & ${ }^{20} \mathrm{Ne}$ & $1.6+2$ & & ORL & RPlasilt & $|185|$ \\
\hline${ }^{60} \mathrm{Ni}$ & ${ }^{12} \mathrm{C}$ & NDG & & LYO & T Billereyt & 9960 \\
\hline $232 \mathrm{Th}$ & ${ }^{40} \mathrm{Ar}$ & $7.2+4$ & & BRK & R Scolt. & 12399 \\
\hline $20 \mathrm{Mg}$ & row $O(E)$ & & & & & \\
\hline${ }^{12} \mathrm{C}$ & $12 \mathrm{C}$ & $4.0+1$ & & MUN & E Everst & 9777 \\
\hline $2 \mathbf{I M g}_{\mathrm{Mg}}$ & $r a \theta^{\prime}(E)$ & & & & & \\
\hline${ }^{12} \mathrm{C}$ & $12 \mathrm{C}$ & $4.0+1$ & & MUN & E Everst & 9777 \\
\hline $21 M g$ & $\sigma(E)$ & & & & & \\
\hline${ }^{20} \mathrm{Ne}$ & ${ }^{3} \mathrm{He}$ & NDG & & BRK & R Cernyt & 6187 \\
\hline $22_{M g}$ & ravo(E) & & & & & \\
\hline${ }^{12} \mathrm{C}$ & ${ }^{12} \mathrm{c}$ & $4.0+1$ & & MUN & E Everst & 9777 \\
\hline $22_{\mathrm{Mg}}$ & relotive & - $O(E)$ & & & & \\
\hline $\begin{array}{l}24 \mathrm{Mg} \\
26 \mathrm{Mg}\end{array}$ & $\begin{array}{l}{ }^{3} \mathrm{Me} \\
{ }^{3} \mathrm{He}\end{array}$ & $\begin{array}{l}8 \cdot 0+1 \\
8 \cdot 0+1\end{array}$ & & $\begin{array}{l}\text { NRL } \\
\text { NRL }\end{array}$ & $\begin{array}{l}\text { E singht } \\
\text { E Singht }\end{array}$ & $\begin{array}{l}6200 \\
6202\end{array}$ \\
\hline $22 \mathrm{Mg}$ & $O(E)$ & & & & & \\
\hline $\begin{array}{r}\mathrm{Mg} \\
232 \mathrm{Th}\end{array}$ & $40_{\mathrm{Ar}}^{\mathrm{P}}$ & $\begin{array}{l}1.5+1 \\
2 \cdot 0+2\end{array}$ & $\begin{array}{l}2.7+1 \\
1.0+3\end{array}$ & $\begin{array}{l}\text { CLU } \\
\text { BRK }\end{array}$ & $\begin{array}{l}\text { E Wilsont } \\
\text { R Scott. }\end{array}$ & $\begin{array}{r}731 \\
12401\end{array}$ \\
\hline $23 \mathrm{Mg}$ & partial & $\sigma(E) \times f$ & factor & & & \\
\hline 160 & ${ }^{12} \mathrm{C}$ & $1.9+1$ & $4.8+1$ & NBI & E Switkowskit & 9834 \\
\hline $23 \mathrm{Mg}$ & partlal & $O(E)$ & & & & \\
\hline $\begin{array}{l}{ }_{12} \mathrm{C} \\
{ }^{23} \mathrm{Na}\end{array}$ & $\begin{array}{r}160 \\
p\end{array}$ & $\begin{array}{l}2.6+1 \\
4.8+0\end{array}$ & $\begin{array}{l}5.2+1 \\
7.5+0\end{array}$ & $\begin{array}{l}\text { WAU } \\
\text { KTY }\end{array}$ & $\begin{array}{l}\text { E Chan+ } \\
\text { M Flyant }\end{array}$ & $\begin{array}{r}10575 \\
690\end{array}$ \\
\hline
\end{tabular}

\begin{tabular}{|c|c|c|c|c|c|c|}
\hline Target & $\ln e$ & $\begin{array}{l}\text { Emin } \\
(M e V)\end{array}$ & $\begin{array}{l}\text { Emax } \\
(\operatorname{MeV})\end{array}$ & Lab & Author & No. \\
\hline $23 \mathrm{Mg}$ & $r o v \sigma(E)$ & & & & & \\
\hline${ }^{12} \mathrm{C}$ & $12 c$ & $4.0+1$ & & MUN & E Everst & 9777 \\
\hline $23 \mathrm{Mg}$ & rolative & - $O(E)$ & & & & \\
\hline $\begin{array}{l}24 \mathrm{Mg} \\
26 \mathrm{Mg}\end{array}$ & $\begin{array}{l}{ }^{3} \mathrm{He} \\
{ }^{3} \mathrm{He}\end{array}$ & $\begin{array}{l}9.0+1 \\
8 \cdot 0+1\end{array}$ & & $\begin{array}{l}\text { NRL } \\
\text { NRL }\end{array}$ & $\begin{array}{l}\text { E singht } \\
\text { E singht }\end{array}$ & $\begin{array}{l}6200 \\
6202\end{array}$ \\
\hline $23 \mathrm{Mg}$ & $O(E)$ & & & & & \\
\hline $10_{B}$ & $14 \mathrm{~N}$ & $6.9+0$ & $1.8+1$ & CAL & E Wut & 10243 \\
\hline $12 \mathrm{c}$ & 160 & $4.0+1$ & $6.5+1$ & STR & M Kolatat & 10674 \\
\hline $1 z_{C}$ & 160 & $6.0+1$ & & MUN & E Weidingert & 10723 \\
\hline $1 a_{c}$ & 180 & $1.0+2$ & & STR & E Coffint & 11628 \\
\hline 160 & ${ }^{12} \mathrm{c}$ & $6.0+1$ & & GRE & T cole. & 9842 \\
\hline $15_{0}$ & 160 & $6 . c+1$ & & GRE & TCole. & 10860 \\
\hline $20_{\mathrm{Ne}}$ & $a$ & TR & $1.2+1$ & CAL & T Woosley+ & 6929 \\
\hline $20 \mathrm{Ne}$ & & TR & $3.1+1$ & KLN & MGruhlet & 6930 \\
\hline $20 \mathrm{Ne}$ & ${ }^{12} \mathrm{c}$ & $6.7+1$ & & GRE & T Cole. & 9871 \\
\hline $23_{\mathrm{No}}$ & p & TR & $8.1+0$ & CAL & T Woosleyt & 687 \\
\hline $23^{\mathrm{Na}}$ & p & $5.0+0$ & $5.8+0$ & $\mathrm{KTY}$ & E Gabbardt & 688 \\
\hline${ }^{23} \mathrm{Ne}$ & $p$ & $5.1+0$ & $1.2+1$ & CAL & E Mannt & 689 \\
\hline $\mathrm{Mg}$ & p & $1.5+1$ & $2.7+1$ & CLU & E Wilisont & 732 \\
\hline${ }^{27} \mathrm{AI}$ & p & $1.5+1$ & $2.7+1$ & CLU & E Wilsont & 891 \\
\hline $\begin{array}{l}27 \mathrm{Al} \\
232 \mathrm{Th}\end{array}$ & ${ }^{4} \mathrm{O}_{\mathrm{Ar}}^{\mathrm{P}}$ & $\begin{array}{l}\text { TR } \\
2.0+2\end{array}$ & $\begin{array}{l}8.0+1 \\
1.0+3\end{array}$ & $\begin{array}{l}\text { NRL } \\
\text { BRK }\end{array}$ & $\begin{array}{l}\text { T Pelerson. } \\
\text { R Scolt. }\end{array}$ & $\begin{array}{r}892 \\
12401\end{array}$ \\
\hline $24 \mathrm{Mg}$ & $O(E)$ & & & & & \\
\hline 160 & 160 & $1.8+1$ & $6.0+1$ & STF & E Cheng* & 10812 \\
\hline${ }^{4} \mathrm{Mg}$ & partial & produc & ct $y|\in|$ & & & \\
\hline $26 \mathrm{Mg}$ & p & $2.3+1$ & & ROC & E Anantaramant & 773 \\
\hline${ }^{24} \mathrm{Mg}$ & partiol & $O(\varepsilon)_{d}$ & & & & \\
\hline $24 \mathrm{Mg}$ & ${ }^{3} \mathrm{He}$ & $7.0+1$ & $9.0+1$ & OSA & E Motsuokat & 6194 \\
\hline$=4 \mathrm{Mg}$ & partlal & rav or & (E), & & & \\
\hline${ }^{24} \mathrm{Mg}$ & a & $3.2+0$ & $3.8+0$ & UTR & E Meast & 6979 \\
\hline $24 \mathrm{Mg}_{9}$ & portlal & $\sigma(E)_{y}$ & & & & \\
\hline $\begin{array}{l}24 \mathrm{Mg} \\
24 \mathrm{Mg}\end{array}$ & p & $\begin{array}{l}3 \cdot 5+0 \\
8 \cdot 0+0\end{array}$ & $\begin{array}{l}2.0+2 \\
4.0+1\end{array}$ & $\begin{array}{l}\text { GSF } \\
\text { GSF }\end{array}$ & $\begin{array}{l}\text { D Ramaty } \\
\text { D Romaly+ }\end{array}$ & $\begin{array}{r}747 \\
6978\end{array}$ \\
\hline
\end{tabular}




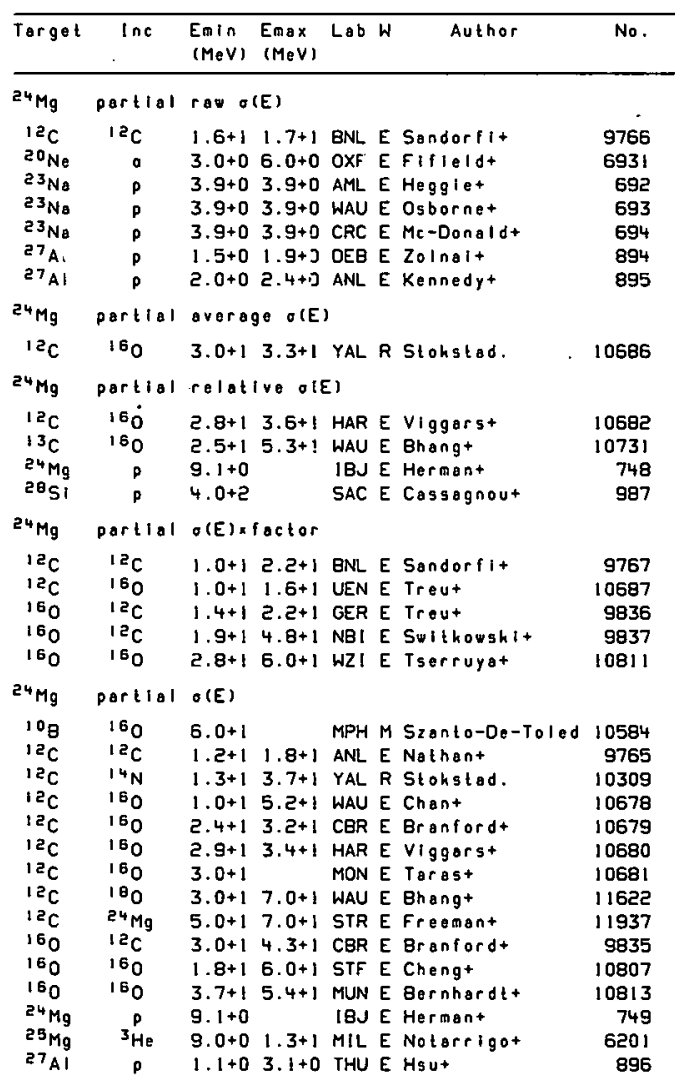

\begin{tabular}{|c|c|c|c|c|c|c|}
\hline$\overline{\text { Target }}$ & $\ln c$ & $\begin{array}{l}\text { Emin } \\
(M e v)\end{array}$ & $\begin{array}{l}\text { Emax } \\
(\operatorname{MeV})\end{array}$ & Lab & Author & No. \\
\hline $24 \mathrm{Mg}$ & partiol & $O(E)$ & & & & \\
\hline $\begin{array}{l}27_{A 1} \\
27_{A 1}\end{array}$ & $p$ & $\begin{array}{l}1.5+0 \\
2.3+1\end{array}$ & $-1.9+00$ & $\begin{array}{l}\text { DEB E } \\
\text { JUL E }\end{array}$ & $\begin{array}{l}\text { E Somogrit } \\
\text { E Roherert }\end{array}$ & $\begin{array}{l}997 \\
898\end{array}$ \\
\hline${ }^{24} \mathrm{Mg}$ & jroduct, & yield & & & & \\
\hline $\begin{array}{l}20 \mathrm{Ne} \\
20 \mathrm{Ne} \\
23 \mathrm{Na} \\
27 \mathrm{Al}\end{array}$ & $\begin{array}{c}6 \mathrm{E} / \\
160 \\
p \\
p\end{array}$ & $\begin{array}{l}\text { NDG } \\
4.1+1 \\
3.9+0 \\
1.4+0\end{array}$ & $\begin{array}{l}6.3+1 \quad 8 \\
3.9+0 \\
2.2+0\end{array}$ & $\begin{array}{l}\text { ROC E } \\
\text { BNL E } \\
\text { MOS R } \\
\text { DEB E }\end{array}$ & $\begin{array}{l}\text { E Ananteranant } \\
\text { E Barrette+ } \\
R \text { Vorlarove } \\
\text { E Zolnat+ }\end{array}$ & $\begin{array}{r}9177 \\
10866 \\
691 \\
904\end{array}$ \\
\hline${ }^{24} \mathrm{Mg}$ & row o(E) & & & & & \\
\hline $\begin{array}{l}12 \mathrm{C} \\
23_{\mathrm{Na}}\end{array}$ & $\begin{array}{r}12 \mathrm{c} \\
\mathrm{p}\end{array}$ & $\begin{array}{l}4 \cdot 0+1 \\
5 \cdot 1-1\end{array}$ & 5.2-1 & $\begin{array}{l}\text { MUN E } \\
\text { AML E }\end{array}$ & $\begin{array}{l}\text { E Everst } \\
\text { E Painet }\end{array}$ & $\begin{array}{r}9777 \\
697\end{array}$ \\
\hline $24 \mathrm{Mg}$ & relallive & $O(E)=$ & - factor & & & \\
\hline${ }^{12} \mathrm{c}$ & 160 & $7.9+0$ & $1.5+15$ & SAC R & R Popinesu. & 10690 \\
\hline $24 \mathrm{Mg}$ & relative & $O(E)$ & & & & \\
\hline $\begin{array}{l}12 \mathrm{C} \\
12 \mathrm{C} \\
12 \mathrm{C} \\
24 \mathrm{Mg} \\
24 \mathrm{Mg} \\
26 \mathrm{Mg}\end{array}$ & $\begin{array}{l}160 \\
160 \\
150 \\
p \\
3^{3} \mathrm{He} \\
{ }^{3} \mathrm{He}\end{array}$ & $\begin{array}{l}6 \cdot 3+0 \\
2 \cdot 1+1 \\
2 \cdot 8+1 \\
9.1+0 \\
8 \cdot 0+1 \\
8 \cdot 0+1\end{array}$ & $\begin{array}{r}3.1+10 \\
2.6+1 \\
3.5+1 \\
n\end{array}$ & $\begin{array}{l}\text { CAL E } \\
\text { HAR } \\
\text { MON E } \\
\text { IBJ E } \\
\text { NRL E } \\
\text { NRL E }\end{array}$ & $\begin{array}{l}\text { E Christensont } \\
\text { M Brady+ } \\
\text { E Torast } \\
\text { E Hermont } \\
\text { E Singht } \\
\text { E Singht }\end{array}$ & $\begin{array}{r}10683 \\
10684 \\
10685 \\
750 \\
6200 \\
6202\end{array}$ \\
\hline $24 \mathrm{Mg}$ & $O(E) \times f \circ C$ & etor & & & & \\
\hline${ }^{13} \mathrm{C}$ & ${ }^{13} \mathrm{C}$ & $6.3+0$ & $1.4+15$ & LUDE & E Chatteritet & 10190 \\
\hline${ }^{4} \mathrm{Mg}_{\mathrm{g}}$ & $O(\varepsilon)$ & & & & & \\
\hline $\begin{array}{l}9 \mathrm{Be} \\
12^{2} \mathrm{C} \\
12^{2} \mathrm{C}\end{array}$ & $\begin{array}{l}150 \\
13 \mathrm{C} \\
13 \mathrm{c}\end{array}$ & $\begin{array}{l}5.0+0 \\
5.0+0 \\
1.6+1\end{array}$ & $\begin{array}{r}1.5+10 \\
1.5+10\end{array}$ & $\begin{array}{l}\text { CAL } \\
\text { CAL E } \\
\text { PEN E }\end{array}$ & $\begin{array}{l}\text { M Solthoughit } \\
\text { E Doyrast } \\
\text { E Gellert }\end{array}$ & $\begin{array}{l}10565 \\
10183 \\
10184\end{array}$ \\
\hline $12 \mathrm{c}$ & $14 N$ & $6.1+0$ & $1.7+10$ & CAL E & E Solthoskit & 10310 \\
\hline $12 c$ & 150 & 6. $3+0$ & $3.1+10$ & CAL E & Ehristensont & 10676 \\
\hline${ }^{12} \mathrm{C}$ & 180 & $4.0+1$ & $6.5+15$ & STR E & E Kolalat & 10677 \\
\hline $12 \mathrm{c}$ & 180 & $1.1+1$ & $2.3+10$ & CAL & E Swlthoush1+ & 10688 \\
\hline $12 c$ & 160 & $2.0+1$ & $1.0+5 r$ & MIT E & E Youngt & 10689 \\
\hline $1 a_{c}$ & 150 & $6.0+1$ & & MUN E & E Weidingert & 10723 \\
\hline $12 \mathrm{c}$ & $1 \theta_{0}$ & $1.0+2$ & & STR E & E Corfint & 11628 \\
\hline $13 \mathrm{c}$ & $13 \mathrm{C}$ & $6.3+0$ & $1.4+1 \mathrm{~L}$ & LUOE & E Chattorjoat & 10189 \\
\hline $13 \mathrm{C}$ & 180 & $1.0+2$ & & STR E & E Coffint & 11642 \\
\hline
\end{tabular}




\begin{tabular}{|c|c|c|c|c|c|c|}
\hline Target & $\operatorname{Inc}$ & $\begin{array}{l}E_{\mathrm{m} I n} \\
(\mathrm{MeV})\end{array}$ & $\begin{array}{l}\text { Emax } \\
(\operatorname{MeV})\end{array}$ & Lab & Author & No. \\
\hline $24 \mathrm{Mg}$ & $\theta(E)$ & & & & & \\
\hline 160 & ${ }^{12} \mathrm{C}$ & $6.0+1$ & & GRE & T Cole. & 9842 \\
\hline 160 & 160 & $2.4+1$ & $6.0+1$ & STR F & R HaAs+ & 10808 \\
\hline 160 & 160 & $2.5+1$ & $6.1+1$ & BNL & E Kolalot & 10809 \\
\hline 160 & 160 & $6.0+1$ & & MUN & M Buscht & 10810 \\
\hline 160 & 160 & $2.4+1$ & 8. $0+1$ & STR & E Kolatat & 10859 \\
\hline 150 & 160 & $6.0+1$ & & GRE & T Colo. & 10860 \\
\hline 180 & 160 & $6.0+1$ & & MUN E & E Weldingert & 10881 \\
\hline $20 \mathrm{Ne}$ & 0 & NDG & & CAL & T Woosleyt & 6932 \\
\hline${ }^{20} \mathrm{Ne}$ & ${ }^{12} \mathrm{C}$ & $2.0+1$ & $5.4+1$ & NOT & E De-Youngt & $\mathbf{8 8 7 0}$ \\
\hline $20 \mathrm{Ne}$ & $12 \mathrm{C}$ & $6.7+1$ & & GRE & T Cole. & 9871 \\
\hline $21 \mathrm{Ne}$ & : & NDG & & CAL & T Woosleyt & 6934 \\
\hline${ }^{2} 1_{\mathrm{Na}}$ & $\therefore$ & NDG & & CAL & T Woosleyt & 6943 \\
\hline${ }^{23} \mathrm{No}_{0}$ & 0 & NDG & & CAL & T Woosleyt & 695 \\
\hline${ }^{23} \mathrm{Na}$ & p & $5.0-1$ & $2.5+0$ & USA E & E No Author-Lab. & 696 \\
\hline${ }^{24} \mathrm{Na}$ & ? & NDG & & CAL & T Woosleyt & 699 \\
\hline $24 \mathrm{Mg}$ & p & $1.8+1$ & $5.0+1$ & OXF & T Mac-Kintosht & 746 \\
\hline${ }^{24} \mathrm{Mg}$ & ${ }^{12} \mathrm{C}$ & $2.2+1$ & $3.5+i$ & STR F & R Haost & 9881 \\
\hline $24 \mathrm{Mg}$ & ${ }^{12} \mathrm{C}$ & $4.2+1$ & $8.4+!$ & HEI & E Novotny+ & 9882 \\
\hline $27_{\mathrm{AI}}$ & D & TR & $0.0+1$ & NRL & T Peterson. & 893 \\
\hline $27 A 1$ & p & NDG & & CAL & T Woosleyt & 899 \\
\hline $27 \mathrm{Al}$ & $p$ & & $0.0+1$ & NRL & $T$ Peterson. & 900 \\
\hline${ }^{27} \mathrm{Al}$ & p & +0 & & DEB E & E Hunyadit & 901 \\
\hline $27_{A I}$ & p. & $1.6+1$ & $4.0+1$ & RIC E & E Walliont & 902 \\
\hline $27_{\mathrm{AI}}$ & p & $3.7+1$ & & MIL E & E Collit & 903 \\
\hline $232 \mathrm{Th}$ & $40 \mathrm{Ar}$ & $2.0+2$ & $1.0+3$ & BRK F & R seoti. & 12401 \\
\hline${ }^{25} \mathrm{Mg}$ & partlal & row th & hick ta & argot & yloldxabundance & \\
\hline $29 \mathrm{Mg}$ & 。 & $5.0+0$ & & SUN E & E Gllegt & 6988 \\
\hline${ }^{25} \mathrm{Mg}$ & pertial & reloti & IVO OCE & & & \\
\hline $\begin{array}{l}25 \mathrm{Mg} \\
28 \mathrm{Si}\end{array}$ & p & $\begin{array}{l}9.1+0 \\
4.0+2\end{array}$ & & $\begin{array}{l}\text { IBJ } \\
\text { SAC }\end{array}$ & $\begin{array}{l}\text { E Hermant } \\
\text { E Cossagnout }\end{array}$ & $\begin{array}{l}750 \\
987\end{array}$ \\
\hline $25 \mathrm{Mg}$ & portial & $O(E) \times f$ & factor & & & \\
\hline $24 \mathrm{Mg}$ & d & $1.4+1$ & & IJIE & E Toharevshilit & 5378 \\
\hline $25 \mathrm{Mg}$ & portlal & $O(E)$ & & & & \\
\hline $\begin{array}{l}12 c c \\
12 c\end{array}$ & $\begin{array}{l}14 \mathrm{~N} \\
160\end{array}$ & $\begin{array}{l}7.8+1 \\
3.5+1\end{array}$ & $5.2+1$ & $\begin{array}{l}\text { YAL F } \\
\text { WAU E }\end{array}$ & $\begin{array}{l}\text { R Stohstod. } \\
\text { E Chont }\end{array}$ & $\begin{array}{l}10311 \\
10692\end{array}$ \\
\hline $12 \mathrm{c}$ & 180 & $\begin{array}{l}3.5+1 \\
2.5+1\end{array}$ & $\begin{array}{l}5.2+1 \\
5.9+1\end{array}$ & STR E & E Freement & 11652 \\
\hline
\end{tabular}

\begin{tabular}{|c|c|c|c|c|c|c|}
\hline Torge! & Inc & $\begin{array}{l}\text { Enin } \\
(M \otimes V)\end{array}$ & $\begin{array}{l}E_{\max } \\
\left(M_{\theta} V\right)\end{array}$ & Lob $\mathrm{K}$ & Author & No. \\
\hline $29_{\mathrm{Mg}}$ & portiol & $\sigma(E)$ & & & & \\
\hline $\begin{array}{l}{ }^{12} \mathrm{C} \\
{ }^{13} \mathrm{C} \\
{ }^{27} \mathrm{Al}\end{array}$ & $\begin{array}{r}180 \\
160 \\
d\end{array}$ & $\begin{array}{l}2.5+1 \\
1.6+1 \\
5 \cdot 4-1\end{array}$ & $\begin{array}{l}7 \cdot 0+1 \\
5 \cdot 6+1 \\
6 \cdot 5-1\end{array}$ & $\begin{array}{l}\text { WAU E } \\
\text { WAU E } \\
\text { DEB E }\end{array}$ & $\begin{array}{l}\text { E Ghangt } \\
\text { E Chant } \\
\text { E Sonogyit }\end{array}$ & $\begin{array}{r}11624 \\
10732 \\
5424\end{array}$ \\
\hline $29_{\mathrm{Mg}}$ & row o(E) & & & & & \\
\hline${ }^{12} \mathrm{C}$ & ${ }^{12} \mathrm{C}$ & $4.0+1$ & & MUN E & E Everst & 9777 \\
\hline $25 \mathrm{Mg}$ & celative & O OIE) & & & & \\
\hline $\begin{array}{l}24 M g \\
25 M_{g} \\
2 B_{M g}\end{array}$ & $\begin{array}{l}{ }^{3} \mathrm{He} \\
\mathbf{p}^{\mathbf{p}} \\
{ }^{\mathrm{He}}\end{array}$ & $\begin{array}{l}8 \cdot 0+1 \\
9 \cdot 1+0 \\
8 \cdot 0+1\end{array}$ & & $\begin{array}{l}\text { NRL E } \\
\text { IBJ E } \\
\text { NRL E }\end{array}$ & $\begin{array}{l}\text { E Singht } \\
\text { E Hermant } \\
\text { E Singht }\end{array}$ & $\begin{array}{r}6200 \\
761 \\
6202\end{array}$ \\
\hline $29 \mathrm{Mg}$ & $\sigma(E)$ & & & & & \\
\hline $1{ }^{2} \mathrm{C}$ & $14 N$ & $6.1+0$ & $1.7+1$ & CAL E & E Solthogkit & 10312 \\
\hline${ }^{12} \mathrm{C}$ & 180 & $4.0+1$ & $6.5+i$ & STR M & M Kolotat & 10691 \\
\hline${ }^{12} \mathrm{C}$ & 160 & $6.0+1$ & & MUN E & E Weidingert & 10723 \\
\hline $12 \mathrm{C}$ & 180 & $1.0+2$ & & SIR E & E Coffint & 11628 \\
\hline $1{ }^{12} \mathrm{C}$ & $19_{F}$ & $5.0+1$ & $7.0+1$ & STR M & M Wagnert & 11750 \\
\hline${ }^{13} \mathrm{C}$ & 180 & $1.0+2$ & & STR E & E Coffint & 11642 \\
\hline 160 & $12 \mathrm{c}$ & $6.0+1$ & & GRE I & T Cole. & 9842 \\
\hline 150 & $14 \mathrm{~N}$ & $1.2+1$ & $2.7+1$ & CAL E & E Solitkouski+ & 10342 \\
\hline 160 & 160 & $6.0+1$ & & GRE T & T Cole. & 10860 \\
\hline $20 \mathrm{Ne}$ & $12 c$ & $6.7+1$ & & GRE $T$ & i cole. & 9871 \\
\hline $21 \mathrm{Ne}$ & a & NDG & & CAL T & T Woosleyt & 6935 \\
\hline $22_{\mathrm{Ne}}$ & a & TR & $1.2+1$ & CAL T & T Woosleyt & 6937 \\
\hline $2{ }^{2} \mathrm{No}$ & 0 & NDG & & CAL $T$ & T Woosley+ & 6945 \\
\hline${ }^{24} \mathrm{Na}$ & p & NDG & & CAL T & T Woosley+ & 700 \\
\hline${ }^{25} \mathrm{Na}$ & p & NDG & & CAL T & T Woosley+ & 702 \\
\hline${ }^{27} \mathrm{Al}$ & p & $\mathrm{TR}$ & $8.0+1$ & NRL T & T Peterson. & 905 \\
\hline $27 \mathrm{Al}$ & p & $1.6+1$ & $4 \cdot 3+1$ & RIC E & E Waltont & 906 \\
\hline $2 \theta_{A l}$ & p & NDG & & CAL T & I Woosleyt & 935 \\
\hline${ }^{48} \mathrm{TI}$ & & $4.0+1$ & $9.0+1$ & MCG E & E Holub+ & 1444 \\
\hline $232 \mathrm{Th}$ & Ar & $2.0+2$ & $1.0+3$ & ERK $R$ & R scott. & 12401 \\
\hline${ }^{28} \mathrm{Mg}$ & partiol & row th & hlch to & orget & $y \in e l d$ & \\
\hline${ }^{23} \mathrm{Na}$ & a & $5.0+0$ & & SUN E & E Gllest & 6949 \\
\hline $26 \mathrm{Mg}$ & partiol & reloti & ive otE & & & \\
\hline $\begin{array}{l}26 \mathrm{Mg} \\
28 \mathrm{si}\end{array}$ & $\begin{array}{l}p \\
p\end{array}$ & $\begin{array}{l}9.1+0 \\
4.0+2\end{array}$ & & $\begin{array}{l}\text { IBJ E } \\
\text { SAC E }\end{array}$ & $\begin{array}{l}\text { E Hermant } \\
\text { E Cassagnout }\end{array}$ & $\begin{array}{l}774 \\
987\end{array}$ \\
\hline
\end{tabular}




\begin{tabular}{|c|c|c|c|c|c|c|}
\hline Target & $\ln c$ & $\begin{array}{l}\text { Emin } \\
(\mathrm{MeV})\end{array}$ & $\begin{array}{l}\text { Emax } \\
(M e V)\end{array}$ & Lab W & Author & No. \\
\hline $26 \mathrm{Mg}$ & partial & $\sigma(E) \times f$ & factor & & & \\
\hline $\begin{array}{l}160 \\
25 \mathrm{Mg}\end{array}$ & ${ }_{0}^{12} \mathrm{C}$ & $\begin{array}{l}1.9+1 \\
1.4+1\end{array}$ & $4.8+1$ & $\begin{array}{l}\text { NBI E } \\
\text { IJIE }\end{array}$ & $\begin{array}{l}\text { E Swithowshlt } \\
\text { E Toharevsklit }\end{array}$ & $\begin{array}{l}9838 \\
5382\end{array}$ \\
\hline $26 \mathrm{Mg}$ & partial & $O(E)$ & & & & \\
\hline $\begin{array}{l}12 c \\
12 c \\
12 c \\
12 c\end{array}$ & $\begin{array}{l}160 \\
160 \\
160 \\
180\end{array}$ & $\begin{array}{l}1.2+1 \\
1 \cdot 6+1 \\
3 \cdot 0+1 \\
2.2+1\end{array}$ & $\begin{array}{l}5.2+1 \\
7.0+1 \\
4.3+1 \\
4.8+1\end{array}$ & $\begin{array}{l}\text { WAU E } \\
\text { WAU E } \\
\text { CBR E } \\
\text { WAU E }\end{array}$ & $\begin{array}{l}\text { E Chant } \\
\text { E Chant } \\
\text { E Branfordt } \\
\text { E Chant }\end{array}$ & $\begin{array}{l}10695 \\
10696 \\
10697 \\
11625\end{array}$ \\
\hline $26 \mathrm{Mg}$ & product & yield & & & & \\
\hline $22_{\mathrm{Ne}}$ & ${ }^{6} L_{1} \cdot$ & NDG & & ROC E & E Anantaramant & 9178 \\
\hline${ }^{26} \mathrm{Mg}$ & rav o(E) & & & & & \\
\hline${ }^{12} \mathrm{c}$ & ${ }^{12} \mathrm{c}$ & $4.0+1$ & & MUN E & E Everst & 9777 \\
\hline $26 \mathrm{Mg}$ & relative & e $O(E)$ & & & . & \\
\hline $\begin{array}{l}12 \mathrm{C} \\
26 \mathrm{Mc} \\
26 \mathrm{Mc}\end{array}$ & $\begin{array}{l}100 \\
3_{\mathrm{He}}\end{array}$ & $\begin{array}{l}2.8+1 \\
9.1+0 \\
8.0+1\end{array}$ & $3.5+1$ & $\begin{array}{l}\text { MON E } \\
\text { IBJ E } \\
\text { NRL E }\end{array}$ & $\begin{array}{l}\text { E Tarast } \\
\text { E Hermant } \\
\text { E Singht }\end{array}$ & $\begin{array}{r}10698 \\
775 \\
6202\end{array}$ \\
\hline $25 \mathrm{Mg}$ & $\sigma(E)$ & & & & & \\
\hline${ }^{12} \mathrm{C}$ & 160 & $1.3+1$ & $3.1+1$ & CAL E & E Christensont & 10593 \\
\hline${ }^{12} \mathrm{C}$ & 150 & $4.0+1$ & $6.5+:$ & STR $M$ & M Kolatat & 10694 \\
\hline $12 \mathrm{C}$ & $1 \epsilon_{0}$ & $6.0+1$ & & MUN E & E Weldingert & 10723 \\
\hline $1{ }^{2} \mathrm{C}$ & 180 & $1.0+2$ & & STR E & E Coffint & 11628 \\
\hline $12 \mathrm{C}$ & $19 \mathrm{~F}$ & $5 \cdot 0+1$ & $7.0+:$ & STR M & M Wagnert & 11751 \\
\hline $13 \mathrm{C}$ & 180 & $1.0+2$ & & STR E & E Coffint & 11642 \\
\hline 150 & $12 \mathrm{C}$ & $5.0+1$ & & GRE $T$ & T Cole. & 9842 \\
\hline $15_{0}$ & 160 & $6.0+1$ & & BNLE & E Kolalat & 10814 \\
\hline 180 & 160 & $5.0+1$ & & MUN M & M Bugcht & 10815 \\
\hline 160 & 160 & $2.4+1$ & $8.0+1$ & STR $E$ & E Kolatat & 10859 \\
\hline 160 & 160 & E. $0+1$ & & GRE T & T Cole. & 10860 \\
\hline 160 & 160 & $6.0+1$ & & MUN E & E Weldingert & 10861 \\
\hline${ }^{25} \mathrm{Ne}$ & o & NDG & & CAL T & T Woosleyt & 6938 \\
\hline${ }^{23} \mathrm{Ne}$ & a & NDG & & CAL $T$ & T Woosleyt & 6939 \\
\hline $233_{\mathrm{Na}}$ & a & NDG & & CAL $T$ & T Woosley+ & 6950 \\
\hline $25 \mathrm{Na}$ & p & NDG & & CAL T & T Woosley+ & 703 \\
\hline${ }^{26} \mathrm{No}$ & p & NDG & & CAL T & T Woosleyt & 705 \\
\hline $27 \mathrm{Al}$ & p & $T R$ & $8.0+1$ & NRL T & T Peterson. & 907 \\
\hline $29_{\mathrm{Al}}$ & p & NDG & & CAL $T$ & T Woosley+ & 938 \\
\hline
\end{tabular}

\begin{tabular}{|c|c|c|c|c|c|c|}
\hline Target & $\ln c$ & $\begin{array}{l}\text { Emin } \\
(\mathrm{MeV})\end{array}$ & $\begin{array}{l}\text { Emax } \\
(\mathrm{MeV})\end{array}$ & Lab W & Althor & No. \\
\hline $26 \mathrm{Mg}$ & $\sigma(E)$ & & & & & \\
\hline $232 \mathrm{Th}$ & ${ }^{40} \mathrm{Ar}$ & $2.0+2$ & $1.0+3$ & BRK $R$ & Rcott. & 12401 \\
\hline $27 \mathrm{Mg}$ & thich & arget $y$ & yield & & & \\
\hline $\begin{array}{l}12 \mathrm{c} \\
13 \mathrm{c}\end{array}$ & $\begin{array}{l}19_{F} \\
1 g_{F}\end{array}$ & $\begin{array}{l}2.5+1 \\
2.5+1\end{array}$ & $\begin{array}{l}7.5+1 \\
7.5+1\end{array}$ & $\begin{array}{l}\text { FSU M } \\
\text { FSU M }\end{array}$ & $\begin{array}{l}\text { M Zellert } \\
\text { M Zellert }\end{array}$ & $\begin{array}{l}11752 \\
11776\end{array}$ \\
\hline $27 \mathrm{Mg}$ & raw OCE & & & & & \\
\hline${ }^{12} \mathrm{C}$ & ${ }^{12} \mathrm{C}$ & $4.0+1$ & & MUN E & E Everst & 9777 \\
\hline $27_{\mathrm{Mg}}$ & relatir & e $O(E)$ & & & & \\
\hline $25 \mathrm{Mg}$ & ${ }^{3} \mathrm{He}$ & $8.0+1$ & & NFL E & E singht & 6202 \\
\hline $27 \mathrm{Mg}$ & $\sigma(E)$ & & & & & \\
\hline${ }^{12} \mathrm{C}$ & 180 & $1.0+2$ & & STR E & E Coffint & 11628 \\
\hline${ }^{13} \mathrm{C}$ & 180 & $1.0+2$ & & STR E & E Coffint & 11642 \\
\hline 180 & ${ }^{14} \mathrm{C}$ & $1.3+1=$ & $3.9+1$ & ETH E & E Eyal+ & 10223 \\
\hline 180 & 180 & $2.0+1$ & $4.0+1$ & EIHE & E Eyalt & 11647 \\
\hline${ }^{23} \mathrm{Ne}$ & a & NDG & & CAL $T$ & T Woosleyt & 6940 \\
\hline${ }^{24} \mathrm{Ne}$ & 0 & NDG & & CAL T & T Woosleyt & 6941 \\
\hline${ }^{24} \mathrm{Na}$ & a & NDG & & CAL $T$ & I Woogleyt & 6954 \\
\hline${ }^{26} \mathrm{Na}$ & p & NDG & & CAL T & I Woosleyt & 706 \\
\hline $27 \mathrm{No}$ & p & NDG & & CAL T & I Woosleyt & 707 \\
\hline $\mathrm{Mg}$ & d & $8.7+0$ & $1.8+1$ & CLU E & E Wilsont & 5373 \\
\hline $27_{\mathrm{Al}}$ & d & $8.7+0$ & $1.8+1$ & CLUE & E WIIsont & 5425 \\
\hline $27 \mathrm{Al}$ & a & $5 \cdot 0+1$ & $1.5+2$ & JUL E & E OQim. & 7054 \\
\hline $\mathbf{3 0}_{\mathrm{Al}}$ & $p$ & NDG & & CAL T & T Woosleyt & 941 \\
\hline $232 \mathrm{Th}$ & ${ }^{40} \mathrm{Ar}$ & $2.0+2$ & $1.0+3$ & BRK $R$ & R Scott. & 12401 \\
\hline $28_{\mathrm{Mg}}$ & cumulat & líe olE & & & & \\
\hline $\begin{array}{l}\mathrm{Fe} \\
\mathrm{Cu}\end{array}$ & $p$ & $\begin{array}{l}5 \cdot 9+2 \\
5 \cdot 9+2\end{array}$ & & $\begin{array}{l}\text { LAS E } \\
\text { LAS E }\end{array}$ & $\begin{array}{l}\text { E Ortht } \\
\text { E Ortht }\end{array}$ & $\begin{array}{l}1744+ \\
2481+\end{array}$ \\
\hline $2 \mathrm{~B}_{\mathrm{Mg}}$ & product & $y \mid 010$ & & & & \\
\hline$=D P N A$ & $p$ & $1.0+2$ & & UPP E & E Lundquist+ & 711 \\
\hline 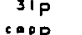 & p & $1.0+2$ & & UPP E & E Lundquist+ & 1023 \\
\hline capp & p & $1.0+2$ & & UPP E & E Lundavisl+ & 1052 \\
\hline $\operatorname{cop} \mathrm{Cl}$ & p & $1.0+2$ & & UPP E & E Lundavigtt & 1150 \\
\hline $\operatorname{cop} \mathrm{p}$ & p & $1.0+2$ & & UPP $\varepsilon$ & $\varepsilon$ Lundquist+ & 1236 \\
\hline
\end{tabular}




\begin{tabular}{|c|c|c|c|c|c|c|}
\hline Target & $\ln c$ & $\begin{array}{l}\text { Emin } \\
(\mathrm{MeV})\end{array}$ & $\begin{array}{l}\text { Emax } \\
(M e V)\end{array}$ & Lab & Author & No. \\
\hline $2 \theta_{M g}$ & \multicolumn{6}{|c|}{ thick torget yieloxfactor } \\
\hline $27_{\mathrm{Al}}^{\mathrm{M}_{9}}$ & a & $\begin{array}{l}2.7+1 \\
3.8+1\end{array}$ & $\begin{array}{l}4.4+1 \\
4.4+1\end{array}$ & $\begin{array}{l}\mathrm{FEI} \\
\mathrm{FEI}\end{array}$ & $\begin{array}{l}\text { E Dmitrievt } \\
\text { E Dmitrievt }\end{array}$ & $\begin{array}{l}6973 \\
7059\end{array}$ \\
\hline $2 \theta_{M g}$ & \multicolumn{6}{|c|}{ thlch target yleld } \\
\hline $\begin{array}{l}{ }^{12} \mathrm{C} \\
13 \mathrm{C}\end{array}$ & $\begin{array}{l}199_{F} \\
199_{F}\end{array}$ & $\begin{array}{l}2.5+1 \\
2.5+1\end{array}$ & $\begin{array}{l}7.5+1 \\
7.5+1\end{array}$ & $\begin{array}{l}\text { FSU } \\
\text { FSU }\end{array}$ & $\begin{array}{l}M \text { Zellert } \\
M \text { Zellert }\end{array}$ & $\begin{array}{l}11753 \\
11777\end{array}$ \\
\hline$M g$ & a & $4.4+1$ & & JUL & $R$ Weinreleht & 6974 \\
\hline $26 \mathrm{Mg}$ & $i$ & $3.5+0$ & $4.0+0$ & JUL & R Weinrelcht & 6060 \\
\hline $26 \mathrm{Mg}$ & $a$ & NOG & & JUL & c Schult. & 6998 \\
\hline $26 \mathrm{Mg}$ & 0 & $2.7+1$ & $4.4+1$ & FEJ & E Dmitrievt & 6999 \\
\hline $27 \mathrm{AI}$ & $\mathrm{t}$ & $2.6+1$ & & JUL & E Probst+ & 6062 \\
\hline $27_{A 1}$ & a & $3.8+1$ & $4.4+1$ & FEI & E Doribrieva & 7056 \\
\hline $27 \mathrm{Al}$ & 0 & $4.5+1$ & $1.4+2$ & AML & R Choudri. & 7057 \\
\hline $27_{A 1}$ & $a$ & $1.4+2$ & & JUL & C Sehult. & 7058 \\
\hline $31 \hat{p}$ & p & $6.2+1$ & $1.5+2$ & UPP & E Lundquist+ & 1021 \\
\hline 315 & $p$ & $1.5+2$ & & JuL & E Probst+ & 1022 \\
\hline s & p & $8.8+1$ & $1.6+2$ & UPP & E Malmborgt & 1056 \\
\hline $\mathrm{Cl}$ & $p$ & $7.5+1$ & & UPP & E Lundavista & 1102 \\
\hline $\mathrm{Cl}$ & $p$ & $1.6+2$ & & JUL & R Weinreicht & 1103 \\
\hline $\mathrm{Ar}$ & p & $7.8+1$ & & UPP & E Lundquist+ & 1154 \\
\hline$k$ & 0 & $1.2+2$ & & UPP & E Lundquist+ & 1193 \\
\hline $2 a_{M g}$ & \multicolumn{6}{|c|}{ raw o(E) } \\
\hline${ }^{12} \mathrm{C}$ & $12 \mathrm{C}$ & $4.0+!$ & & MUN & E Everst & 9777 \\
\hline $2 \theta_{M g}$ & \multicolumn{6}{|l|}{$\sigma(E)$} \\
\hline${ }^{24} \mathrm{Ne}$ & a & NDG & & CAL & T Woosleyt & 6942 \\
\hline $25_{\mathrm{Na}}$ & $a$ & NDG & & CAL & T Woosleyt & 6957 \\
\hline $27^{\mathrm{Na}}$ & $p$ & NDG & & CAL & T Woosleyt & 708 \\
\hline$\because \mathrm{Na}^{-1}$ & $p$ & NDG & & UPP & E Lundquist+ & 712 \\
\hline $26 \mathrm{Mg}$ & $\mathrm{i}$ & NDG & & JUL & C Schult. & 6051 \\
\hline $26 \mathrm{Mg}$ & : & $2.0+1$ & $1.6+2$ & JUL & R Stocklint & 6997 \\
\hline $27_{A I}$ & $a$ & $3.0+1$ & $1.6+2$ & JUL & R Stocklint & 7055 \\
\hline $31 \mathrm{AI}$ & p & NDG & & CAL & T Woosleyt & 944 \\
\hline & & $5.5+1$ & $1.5+2$ & UPP & E Lundquist+ & 956 \\
\hline 3051 & p & $1.3+2$ & & UPP & E Lundarist+ & 997 \\
\hline $31 p$ & & $5.0+1$ & $1.5+2$ & UPP & E Lundavist+ & 1020 \\
\hline$\triangle A P P$ & p & NDG & & UPP & E Lundarist+ & 1053 \\
\hline 5 & $p$ & $5.0+1$ & $1.5+2$ & UPP & E Lundquigt+ & 1055 \\
\hline $\mathrm{cl}$ & p & $5.0+1$ & $1.5+2$ & UPP & E Lundquist+ & 1101 \\
\hline
\end{tabular}

\begin{tabular}{|c|c|c|c|c|c|c|}
\hline Torget & $\operatorname{Inc}$ & $\begin{array}{l}\text { Enin } \\
(M \circ V)\end{array}$ & $\begin{array}{l}\text { Emex } \\
(M e V)\end{array}$ & Lab & Author & No. \\
\hline $28 \mathrm{Mg}$ & $O(E)$ & & & & & \\
\hline 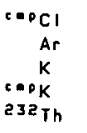 & $\begin{array}{r}p \\
p \\
p \\
40 \\
40_{A r}\end{array}$ & $\begin{array}{l}\text { NDG } \\
5.5+1 \\
8.0+1 \\
\text { NDG } \\
2.0+2\end{array}$ & $\begin{array}{l}1.7+2 \\
1.5+2 \\
1.0+3\end{array}$ & $\begin{array}{l}\text { UPP } \\
\text { UPP } \\
\text { UPP } \\
\text { UPP } \\
\text { BRK }\end{array}$ & $\begin{array}{l}\text { E Lundarist } \\
\text { E Lundaristt } \\
\text { E Lundaristt } \\
\text { E Lundaristt } \\
R \text { Scoti. }\end{array}$ & $\begin{array}{r}1151 \\
1153 \\
1192 \\
1237 \\
12401\end{array}$ \\
\hline $28 \mathrm{Mg}$ & rav ole & & & & & \\
\hline${ }^{12} \mathrm{C}$ & $12 c$ & $4.0+1$ & & MUN & E Everst & 9777 \\
\hline $29 \mathrm{Mg}$ & $O(E)$ & & & & & \\
\hline $\begin{array}{r}26 \mathrm{Na} \\
232 \mathrm{Th}\end{array}$ & ${ }_{40}^{\circ} \mathrm{Ar}$ & $\begin{array}{l}\text { NDG } \\
2.0+2\end{array}$ & $1.0+3$ & $\begin{array}{l}\text { CAL } \\
\text { BRK }\end{array}$ & $\begin{array}{l}\text { T Woosleyt } \\
\text { R Scott. }\end{array}$ & $\begin{array}{r}6950 \\
12401\end{array}$ \\
\hline $30_{\mathrm{Mg}}$ & row ole & & & & & \\
\hline${ }^{12} \mathrm{C}$ & $12 c$ & $4.0+1$ & & MUN & E Everst & 9777 \\
\hline $30 \mathrm{Mg}$ & $O(E)$ & & & & & \\
\hline $232 \mathrm{Th}$ & ${ }^{40} \mathrm{Ar}$ & $2.0+2$ & $1.0+3$ & BRK & R Scott. & 12401 \\
\hline$z=13$ & Indep & endent & everag & $g \circ k 1$ & Inetlc energy & \\
\hline $197 \mathrm{Au}$ & ${ }^{40} \mathrm{Ar}$ & $2.2+2$ & & DuB & E Artukht & $12369+$ \\
\hline$Z=13$ & Indep & ondent & $\sigma\left(E ; E^{\prime}\right.$ & $\cdot(\theta)$ & & \\
\hline $\begin{array}{l}187 \mathrm{Au} \\
232 \mathrm{Th}\end{array}$ & $\begin{array}{l}40 \mathrm{Ar} \\
40 \mathrm{Ar}\end{array}$ & $\begin{array}{l}2.2+2 \\
3.0+2\end{array}$ & $3.9+2$ & $\begin{array}{l}\text { DUB } \\
\text { DUB }\end{array}$ & $\begin{array}{l}\text { E Artukht } \\
\text { E Artukht }\end{array}$ & $\begin{array}{l}12368+ \\
12396\end{array}+$ \\
\hline$z=13$ & indep & endent & $\theta(E ; \theta)$ & & & \\
\hline $187 \mathrm{AU}$ & $40 \mathrm{Ar}$ & $2.2+2$ & & DUB & E Artukht & $12367+$ \\
\hline$z=13$ & indep & endent & $O(E)$ & & & \\
\hline $\begin{array}{l}157 \mathrm{AU} \\
z=13\end{array}$ & $\begin{array}{c}40 \mathrm{Ar} \\
v_{n}\end{array}$ & $2.2+2$ & & DUB & E Artukht & $12366+$ \\
\hline Ag & $40 \mathrm{Ar}$ & $3.4+2$ & & PAR & R Lefort+ & 12202 \\
\hline$z=13$ & $\operatorname{ros} 0$ & (E) & & & & \\
\hline${ }^{12} \mathrm{c}$ & ${ }^{12} \mathrm{C}$ & $4.0+1$ & & MUN & E Everst & 9773 \\
\hline$z=13$ & $O(E) x$ & factor & & & & \\
\hline $232 \mathrm{Th}$ & $40 \mathrm{Ar}$ & $3.9+2$ & & OXF & R Hodgson. & 12398 \\
\hline
\end{tabular}




\begin{tabular}{|c|c|c|c|c|c|c|}
\hline Target & $\ln c$ & $\begin{array}{l}\text { Emin } \\
(\operatorname{MeV})\end{array}$ & $\begin{array}{l}\text { Emax } \\
(\mathrm{MeV})\end{array}$ & Lab & Author & No. \\
\hline$z=13$ & o(E) & & & & & \\
\hline $1 a_{C}$ & ${ }^{12} \mathrm{c}$ & $2.8+1$ & $5.4+1 \subseteq$ & SAC & E Conjeaudt & 9769 \\
\hline $12 \mathrm{c}$ & $14 \mathrm{~N}$ & $3.3+1$ & $5.2+1$ & ANL & E Kovart & 10317 \\
\hline $12 \mathrm{C}$ & $15 \mathrm{~N}$ & $2.0+1$ & $6.0+1$ & ANL $E$ & E kovort & 10528 \\
\hline $12 \mathrm{C}$ & 150 & $3.5+1$ & $6.2+1$ & ANL & E Tabort & 10721 \\
\hline $12 \mathrm{c}$ & 170 & $3.4+1$ & $8.0+11$ & MUN E & E Hertz+ & $1158 i$ \\
\hline${ }^{12} \mathrm{C}$ & 180 & $1.0+2$ & & STR & E Coffint & 11626 \\
\hline $12 \mathrm{c}$ & $20 \mathrm{Ne}$ & $6.7+1$ & $1.6+2$ & GRE & E Saint-Laurentt & 11839 \\
\hline $13 \mathrm{C}$ & $10_{0}$ & $1.0+2$ & & STR & E Colfint & 11639 \\
\hline $14 N$ & ${ }^{13} \mathrm{C}$ & $4.4+1$ & $5.4+1$ & SAC & E Conleaudt & 10192 \\
\hline 160 & $14 \mathrm{~N}$ & $3.7+1$ & $5.8+1$ & SAC E & E Conjeaudt & 10345 \\
\hline 160 & $15_{N}$ & $3.6+1$ & $5.5+1$ & SAC E & E Confeoudt & 10529 \\
\hline 160 & 160 & $3.0+1$ & $6.0+1$ & ANL & E Sehiffert & 10853 \\
\hline 160 & $16_{0}$ & $1.2+2$ & $1.4+2$ & GRE & E Saint-Laurentt & 10854 \\
\hline 170 & $12^{2} \mathrm{C}$ & $3.5+1$ & $4.9+1$ & SAC & E Conjeaudt & 9845 \\
\hline 170 & $13 \mathrm{C}$ & $3.8+1$. & $5.3+1$ & SAC & E Conjeaudt & 10195 \\
\hline $20 \mathrm{Ne}$ & $12 \mathrm{C}$ & $4.8+1$ & $9.6+1$ & SAC 1 & M Conjeaudt & 9867 \\
\hline $20 \mathrm{Ne}$ & $12 \mathrm{C}$ & $1.2+5$ & $1,4+2$ & GRE & E Conjeaudt & 9868 \\
\hline $\mathrm{Ni}$ & $20 \mathrm{Ne}$ & $1.6+2$ & & ORL & R plasilt & 11851 \\
\hline $232 \mathrm{Th}$ & $40 \mathrm{Ar}$ & $7.2+4$ & & BRK & R Scott. & 12399 \\
\hline $23_{\mathrm{Al}}$ & product & $y=18$ & & & & \\
\hline $24 \mathrm{Mg}$ & D & NDG & & BRK & R Cernyt & 751 \\
\hline${ }^{24} \mathrm{Al}$ & $\theta(E)$ & & & & & \\
\hline $24 \mathrm{Mg}$ & p & $\begin{array}{l}1.5+1 \\
\text { IR }\end{array}$ & $\begin{array}{l}2.7+1 \\
1.7+1\end{array}$ & CLU & E Wilsont & 733 \\
\hline $25 \mathrm{Al}$ & portial & reloti & IVe ole & & & \\
\hline & & & & & & \\
\hline${ }^{2 \theta} \mathrm{Si}$ & p & $4.0+2$ & & SAC & E Cossagnout & 987 \\
\hline $25_{A 1}$ & portio. & $\cdot(E)$ & & & & \\
\hline $25_{M g}$ & $p$ & IR & +0 & CAL & E Oovidsont & 762 \\
\hline $25_{\mathrm{Al}}$ & polotive & C. $O(E)$ & & & & \\
\hline $\begin{array}{l}24 \mathrm{Mg} \\
26 \mathrm{Mg}\end{array}$ & $\begin{array}{l}{ }_{3}^{3} \mathrm{He} \\
{ }_{3}^{3} \mathrm{He}\end{array}$ & $\begin{array}{l}8 \cdot 0+1 \\
8 \cdot 0+1\end{array}$ & & $\begin{array}{l}\text { NRL } \\
\text { NRL }\end{array}$ & $\begin{array}{l}\text { E singht } \\
\text { E singht }\end{array}$ & $\begin{array}{l}6200 \\
6202\end{array}$ \\
\hline $25_{A I}$ & $O(E)$ & & & & & \\
\hline $\begin{array}{l}12 \mathrm{C} \\
12 \mathrm{C}\end{array}$ & $\begin{array}{l}14 \mathrm{~N} \\
19 \mathrm{~F}\end{array}$ & $\begin{array}{l}6.1+0 \\
7.0+1\end{array}$ & $1.7+1$ & $\begin{array}{l}\text { CAL } \\
\text { STR }\end{array}$ & $\begin{array}{l}\text { E Sollkashit } \\
\text { M Wagnert }\end{array}$ & $\begin{array}{l}10313 \\
11754\end{array}$ \\
\hline
\end{tabular}

\begin{tabular}{|c|c|c|c|c|c|c|}
\hline Target & $\ln c$ & $\begin{array}{l}\text { Emin } \\
(\mathrm{MeV})\end{array}$ & $\begin{array}{l}\text { Emax } \\
(M e V)\end{array}$ & Lab & Author & No. \\
\hline${ }^{25} \mathrm{Al}$ & $\theta(E)$ & & & & & \\
\hline $2 I_{\mathrm{Ne}}$ & 0 & NDG & & CAL & T Woosleyt & 6944 \\
\hline$M_{9}$ & d & $8.7+0$ & $1.8+1$ & $\mathrm{CLU}$ & E Wilsont & 5374 \\
\hline${ }^{24} \mathrm{Mg}$ & $\mathbf{p}$ & NDG & & CAL & T Woosley+ & 753 \\
\hline $24 \mathrm{Mg}$ & ${ }^{3} \mathrm{He}$ & $7.0+1$ & $9.0+1$ & OSA & M Malsuotat & 6195 \\
\hline $25 \mathrm{Mg}$ & p & IR & $1.4+1$ & CAL & T Woosleyt & 763 \\
\hline $27_{A I}$ & $p$ & TR & $3.2+1$ & BRK & E Gonzolez-VIdolt & 908 \\
\hline $27_{\mathrm{Al}}$ & $p$ & $1.6+1$ & $4.5+1$ & RIC & E Waltont & 909 \\
\hline si & $p$ & $1.5+1$ & $2.7+1$ & CLU & E Wilsont & 967 \\
\hline$\theta_{51}$ & p & & $1.8+1$ & CAL & T Wooslest & 976 \\
\hline${ }^{20} \mathrm{Si}$ & $p$ & $3.7+1$ & & MIL & E Collit & 977 \\
\hline
\end{tabular}

26 Al Spollotion o(E)

$\begin{array}{llll}F e & 1.5+2 & 3.7+2 \text { NRL R Silbertergt } 1844\end{array}$

$26_{A 1} O(E)$

$160160 \quad 1.5+16.0+1$ STF E Cheng+ 10820

26 Al portial product yield

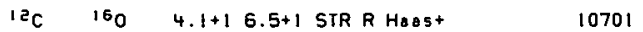

${ }^{26}$ Al portial roo thlck target ylold

${ }^{23} \mathrm{Na} \quad 5.0+0$ SUNE Gilest 6951

$2{ }^{2}$ Al portlal rolative O(E)

${ }^{25} \mathrm{Mg}$ P 8.0-2 3.5-1 MST E ElIX+ 765

${ }^{28} \mathrm{Si} P$ P.0+2 SAC E Cassagrout 987

${ }^{26} A 1$ portial OIE) afactor

160 12 C $1.9+14.8+1$ NBI E Sulthoushit 9840

$160150 \quad 5.4+16.0+1$ WZI E Tserruyat 10819

2EAI portiol O(E)

$12 \mathrm{C} 160 \quad 1.4+15.2+1$ WAU E Chant 10702

$12 \mathrm{C} \quad 160 \quad 3.0+14.3+1$ CBR E Branfordt 10703

160 lE C $2.3+13.2+1$ CBR E Bronfordt 9839

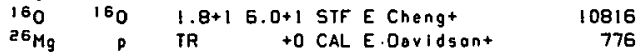

${ }^{28} \mathrm{Al} \mathrm{I}_{\mathrm{Y}}$ at resonanco

$25_{M g}$ P 3.0-1 3.2-1 MST E EIIX+ 


\begin{tabular}{lcccr}
\hline Target & InC & $\begin{array}{c}\text { Emin } \\
\text { (MGQV) }\end{array}$ Emax \\
(MeV)
\end{tabular}

\begin{tabular}{|c|c|c|c|c|c|c|}
\hline Target & Inc & $\begin{array}{l}\text { EnIn } \\
(M e V)\end{array}$ & $\begin{array}{l}\text { Emax } \\
(\operatorname{MeV})\end{array}$ & Lab & Author & No. \\
\hline${ }^{27} \mathrm{Al}$ & 30011011 & $\operatorname{lon} \theta(E$ & & & & \\
\hline $\mathrm{Fe}$ & P & $1.5+2$ & $3 \cdot 7+2$ & NRL F & R Silberberg+ & 1844 \\
\hline $27 \mathrm{Al}$ & $\sigma(E)$ & & & & & \\
\hline 160 & 160 & $1.8+1$ & $6.0+1$ & STF & E Cheng* & 10826 \\
\hline $27 \mathrm{Al}$ & portlat & raoth & hich to & arget & tyloldxabundance & \\
\hline $24 \mathrm{Mg}$ & 。 & $5.0+0$ & & SUN & E Gilest & 6980 \\
\hline $27 \mathrm{Al}$ & portlat & raeth & hich to & arget & $y 1010$ & \\
\hline $2 ?_{A I}$ & 。 & $5.0+0$ & & SUN & E Gilest & 7061 \\
\hline $27_{A 1}$ & portiol & $O(E)^{d}$ & & & & \\
\hline${ }^{23} \mathrm{Al}_{\mathrm{I}}$ & ${ }^{3} \mathrm{He}$ & $7.0+1$ & $9.0+1$ & OSA & E Malsuahat & 6206 \\
\hline${ }^{27} \mathrm{Al}$ & Dortial & rav of & (E), & & & \\
\hline $27 \mathrm{Al}$ & p & $2.0+0$ & $2.4+0$ & ANL & E Kennedy+ & 912 \\
\hline${ }^{27} \mathrm{Al}$ & portiol & spectr & rum oye & geroge & - O(E) & \\
\hline${ }^{27} \mathrm{Al}$ & - & $2.3+1$ & $2.7+1$ & MUU & E Eberhard+ & 7060 \\
\hline $27 \mathrm{Al}$ & partiol & raw ol & (E) & & & \\
\hline $\begin{array}{l}24 \mathrm{Mg} \\
26 \mathrm{Mg}\end{array}$ & : & $\begin{array}{l}3.2+0 \\
7 \cdot 0-1\end{array}$ & $\begin{array}{l}3.8+0 \\
1.0+0\end{array}$ & $\begin{array}{l}\text { UTR } \\
\text { UTR }\end{array}$ & $\begin{array}{l}\text { E Masast } \\
\text { E Meast }\end{array}$ & $\begin{array}{r}6981 \\
781\end{array}$ \\
\hline${ }^{27} \mathrm{Al}$ & partial & rolati & IVe olE & (E) & & \\
\hline $\begin{array}{l}{ }^{13} \mathrm{C} \\
{ }^{3} \mathrm{Si}\end{array}$ & ${ }^{160}$ & $\begin{array}{l}2.5+1 \\
4.0+2\end{array}$ & $5 \cdot 3+1$ & WAU & $\begin{array}{l}\text { E Bhang+ } \\
\text { E Cossagnout }\end{array}$ & $\begin{array}{r}10733 \\
987\end{array}$ \\
\hline $27_{A 1}$. & partial & $O(E) \times f$ & factor & & & \\
\hline 160 & 160 & $2.8+1$ & $6.0+1$ & WZI & E Tsereruyat & 10825 \\
\hline${ }^{27} \mathrm{Al}$ & portiol & $O(E)$ & & & & \\
\hline $\begin{array}{l}12 \mathrm{C} \\
12 \mathrm{C} \\
160 \\
27 \mathrm{Al} \\
30 \mathrm{SI}\end{array}$ & $\begin{array}{r}160 \\
160 \\
160 \\
120 \\
p\end{array}$ & $\begin{array}{l}1.0+1 \\
3 \cdot 0+1 \\
1 \cdot 8+1 \\
8 \cdot 2+1 \\
4 \cdot 1+1\end{array}$ & $\begin{array}{l}2 \cdot 6+1 \\
4 \cdot 3+1 \\
6 \cdot 0+1\end{array}$ & $\begin{array}{l}\text { WAU } \\
\text { CBR } \\
\text { STF } \\
\text { YAL } \\
\text { MIL }\end{array}$ & $\begin{array}{l}\text { E Chant } \\
\text { E Branfordt } \\
\text { E Chengt } \\
\text { E Betlgt } \\
\text { E Pellegrinit }\end{array}$ & $\begin{array}{r}10712 \\
10713 \\
10821 \\
9899 \\
998\end{array}$ \\
\hline $277_{A 1}$ & product & yelo & & & & \\
\hline${ }^{12} \mathrm{C}$ & 160 & NDG & & HAR & E Walgont & 10714 \\
\hline
\end{tabular}




\begin{tabular}{|c|c|c|c|c|c|c|}
\hline Torgek & Inc & $\begin{array}{l}\text { Emin } \\
(\mathrm{MeV})\end{array}$ & $\begin{array}{l}E_{\max } \\
(\operatorname{MeV})\end{array}$ & Lab h & Author & No. \\
\hline $27_{A 1}$ & \multicolumn{6}{|c|}{ COW O(E) } \\
\hline $26 \mathrm{Mg}_{9}$ & $p$ & $5.0-1$ & $2.0+0 A$ & AML & E Poinet & 783 \\
\hline $2 ?_{A I}$ & \multicolumn{6}{|c|}{ relative $O(E) \times f a c t o r$} \\
\hline${ }^{12} \mathrm{C}$ & 160 & $7.9+0$ & $1.5+1 \mathrm{~s}$ & SAC F & R Popinazu. & 10715 \\
\hline $27_{\mathrm{Al}}$ & \multicolumn{6}{|c|}{ relative o(E) } \\
\hline $\begin{array}{l}12 \mathrm{C} \\
12 \mathrm{C} \\
2 \mathrm{E}_{\mathrm{Mg}}\end{array}$ & $\begin{array}{l}160 \\
160 \\
3 \mathrm{He}\end{array}$ & $\begin{array}{l}6.3+0 \\
2.9+1 \\
8 \cdot 0+1\end{array}$ & $\begin{array}{l}3.1+10 \\
3.4+19\end{array}$ & $\begin{array}{l}\text { CAL } \\
\text { MON } \\
\text { NRL }\end{array}$ & $\begin{array}{l}\text { E Christensont } \\
\text { E Toragt } \\
\text { E Singht }\end{array}$ & $\begin{array}{r}10707 \\
10708 \\
62002\end{array}$ \\
\hline $27 \mathrm{Al}$ & \multicolumn{6}{|l|}{$\sigma(E)$} \\
\hline${ }^{12} \mathrm{C}$ & 160 & $4.0+1$ & $6.5+1 s$ & STR & M Kolata+ & 10706 \\
\hline${ }^{12} \mathrm{c}$ & 160 & $6.3+0$ & $3.1+10$ & CAL & E Christensont & 10709 \\
\hline${ }^{12} \mathrm{C}$ & 160 & $1.1+1$ & $2.3+10$ & CAL & E Swithowghit & 10710 \\
\hline${ }^{12} \mathrm{C}$ & 160 & $3.3+1$ & $5.4+1 \subseteq$ & STB & E Molmint & 10711 \\
\hline${ }^{12} \mathrm{c}$ & 160 & $6.0+1$ & & MUN E & E Weldingert & 10723 \\
\hline${ }^{12} \mathrm{C}$ & 180 & $1.0+2$ & & STR & E Coffint & 11628 \\
\hline${ }^{12} \mathrm{C}$ & $19 \mathrm{~F}$ & $5.0+1$ & $7.0+15$ & STR & M Wagnert & 11756 \\
\hline${ }^{13} \mathrm{C}$ & 180 & $1.0+2$ & & STR & E Coffint & 11642 \\
\hline 160 & $12 \mathrm{c}$ & $6.0+1$ & & GRE $T$ & T Cole. & 9842 \\
\hline 160 & 160 & $1.8+1$ & $6.0+15$ & STF & E Chengt & 10822 \\
\hline 180 & 160 & $6.0+1$ & & BNL & E Kolatat & 10823 \\
\hline 180 & 160 & $6.0+1$ & & MUN $\mathrm{N}$ & M Buscht & 10824 \\
\hline 160 & 160 & $2.4+1$ & $8.0+1$ & STR & E Kolatat & 10859 \\
\hline 160 & 180 & $6.0+1$ & & GRE & T Cole. & 10860 \\
\hline 160 & 160 & $6.0+1$ & & MUN E & E Weloingert & 10861 \\
\hline $20 \mathrm{Ne}$ & ${ }^{12} \mathrm{c}$ & $2.0+1$ & $5.4+1 i$ & NOT & E Do-Youngt & 9870 \\
\hline $20 \mathrm{~N}=$ & ${ }^{12} \mathrm{c}$ & $6.7+1$ & & GRE $T$ & T Cole. & 9871 \\
\hline${ }^{2}{ }^{3} \mathrm{No}$ & 0 & NDG & & CAL & T Woosleyt & 6953 \\
\hline${ }^{24} \mathrm{Ng}$ & a & NDG & & CAL & T Woosleyt & 6955 \\
\hline $24 \mathrm{Mg}$ & a & TR & $1.2+1$ & CAL & T Woosley+ & 6982 \\
\hline${ }^{26} \mathrm{Mg}$ & p & NDG & & CAL & T Woosleyt & 782 \\
\hline $27 \mathrm{Mg}$ & p & NDG & & CAL & T Woosleyt & 784 \\
\hline $27 \mathrm{AI}$ & p & $5.2+1$ & $8.0+11$ & NRL & T Peterson. & 913 \\
\hline 27 AI & d & $2.6+1$ & & BON $N$ & M Pampust & 5427 \\
\hline${ }^{30} \mathrm{si}$ & p & $T R$ & $1.3+1$ & CAL & T Woosleyt & 999 \\
\hline $2 \theta_{\mathrm{Al}}$ & \multicolumn{6}{|c|}{ gpallation o(E) } \\
\hline$F=$ & $p$ & $1.5+2$ & $3.7+2$ & NRL F & R silberbergt & 1844 \\
\hline
\end{tabular}

\begin{tabular}{|c|c|c|c|c|c|c|}
\hline Target & $\ln c$ & $\begin{array}{l}\text { Emin } \\
(M e V)\end{array}$ & $\begin{array}{l}\text { Emax } \\
(\mathrm{MeV})\end{array}$ & Lab W & Author & No. \\
\hline
\end{tabular}

Fe $\quad 6.0+2$ MTRE Clinet $1745+$

29) thick torget yiold

${ }^{12} \mathrm{C} \quad{ }^{19} \mathrm{~F} \quad 2.5+17.5+1$ FSU M Zellert $\quad 11757$

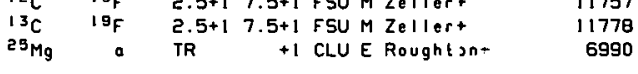

$2 \theta_{A 1} O(E) \times$ foctor

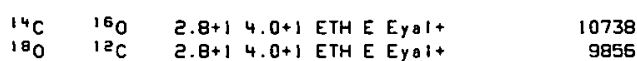

$2 \theta_{\mathrm{Al}}$ O(E) $2.8+14.0+1$ ETH E Eyalt

${ }^{2} \mathrm{C} \quad 19 \mathrm{~F} \quad 5.0+17.0+1$ STR M Wagnor- 1175

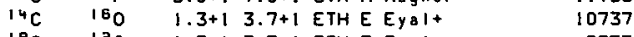

$18_{0} \quad{ }^{2} \mathrm{C} \quad 1.3+13.7+1$ ETH E Eyal+ 9855

${ }^{24} \mathrm{Na}$ a NDG CAL T HoOSlayt 6956

${ }^{25} \mathrm{Na} \quad$ a NDG CAL T Woosle?+ $\quad 6950$

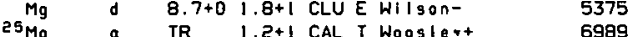

$27 \mathrm{Mg} P$ NDG CAL T WOOSla $:^{+} \quad 785$

$28 \mathrm{Mg} P$ NDG CAL T Woosle:+

$27 \mathrm{Al} d \quad 8.7+01.8+1$ CLUE Wilson- 5428

$27 \mathrm{Al} \quad 3.0+15.0+1$ JUL E Probst- 7062

$\begin{array}{rrrr}27 \text { AI } & 0.0+1 & 1.6+2 \text { MRY E Wut } & 7053 \\ 51 & 1.5+12.7+1 & \text { CLUE Wilson- } & 968\end{array}$

51 d $8.7+01.8+1$ CLUE Wilson- 5430

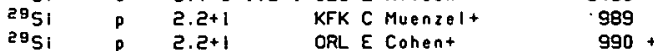

$\begin{array}{llllr}{ }^{29} \mathrm{Si} & p & 2.2+1 & \text { ORL E Cohent } & 990+ \\ \text { Siv } & p & 6.5+18.5+1 & \text { MCG } E \text { Holubt } & 1527\end{array}$

$2 \theta_{A 1}$ cumulative o(E)

Fe $0.0+2$ MTRE clinet $1746+$

${ }^{29} \mathrm{Al}$ thick torget yield

$\begin{array}{lllll}12 \mathrm{C} & 19 \mathrm{~F} & 2.5+1 & 7.5+1 \text { FSU M Zellert } & 11759\end{array}$

$\begin{array}{lrlrr}13 \mathrm{C} & 19_{\mathrm{F}} & 2.5+17.5+1 \text { FSU M Zellert } & 11779 \\ 25 \mathrm{Mg} & \text { a } & \text { TR } & +1 \text { CLU E Roughtent } & 7002\end{array}$

$29_{A 1}$ O(E)xfoctor

${ }^{14} \mathrm{C} \quad 160 \quad 2.8+14.0+1$ ETH E Eyolt 10740 


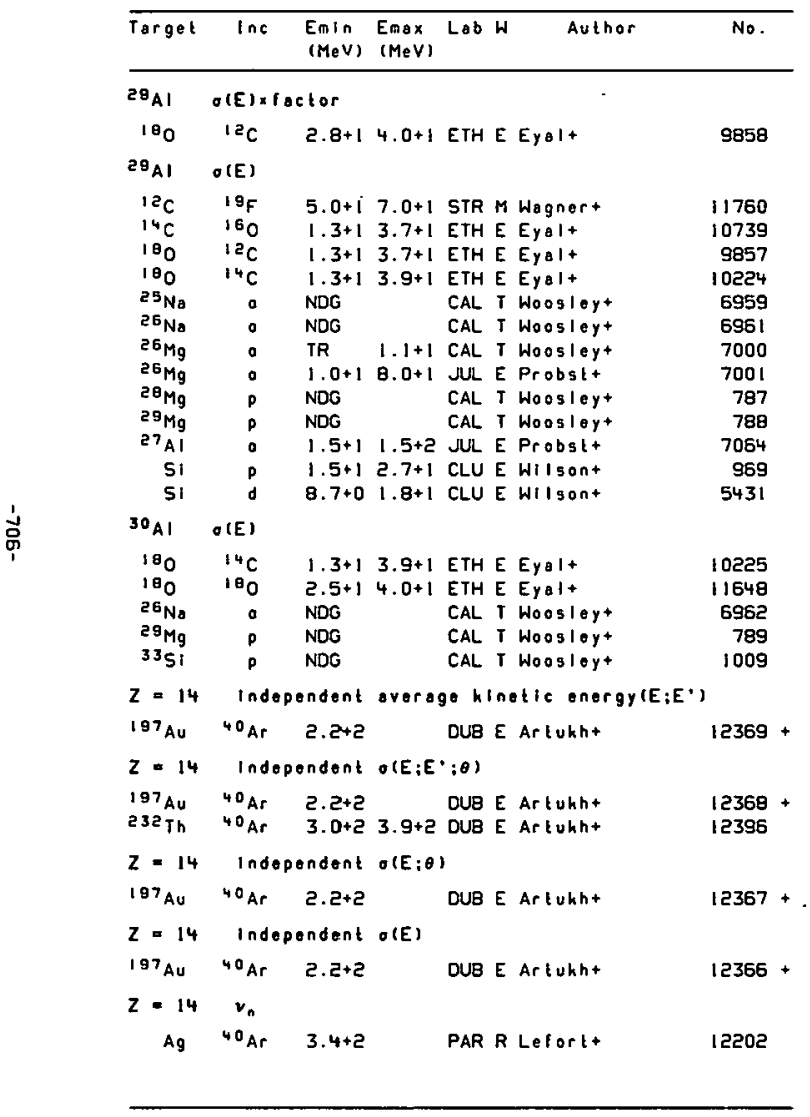

\begin{tabular}{|c|c|c|c|c|c|c|}
\hline Target & $\operatorname{lnc}$ & $\begin{array}{l}\text { Eoln } \\
(\mathrm{MeV})\end{array}$ & $\begin{array}{l}\text { Emax } \\
(M e V)\end{array}$ & Lab $h$ & Author & No. \\
\hline$z=14$ & \multicolumn{2}{|c|}{ o(E)xfactor } & & \multirow{3}{*}{ OXF $P$} & \multirow{3}{*}{ R Hodgson. } & \multirow{3}{*}{12398} \\
\hline 23гTh & $40 \mathrm{Ar}$ & $3.9+2$ & & & & \\
\hline$z=14$ & $\theta(E)$ & & & & & \\
\hline $\begin{array}{l}12 \mathrm{c} \\
12 \mathrm{C}\end{array}$ & $\begin{array}{l}160 \\
20 \mathrm{Ne}\end{array}$ & $\begin{array}{l}3.5+1 \\
6.7+1\end{array}$ & $\begin{array}{l}6.2+1 \\
1.6+2\end{array}$ & $\begin{array}{l}\text { ANL E } \\
\text { GRE E }\end{array}$ & $\begin{array}{l}\text { E Tobart } \\
\text { E Soint-Laurentt }\end{array}$ & $\begin{array}{l}10721 \\
11839\end{array}$ \\
\hline 160 & $14 \mathrm{~N}$ & $3.7+1$ & $5.8+15$ & SAC E & E Conjeaudt & 10345 \\
\hline 160 & $13 \mathrm{~N}$ & $3.6+1$ & $5.5+15$ & SAC E & E Conleaudt & 10529 \\
\hline 150 & ${ }^{16} 0$ & $3.0+1$ & $6.0+1$ & ANL E & E Schiffert & 10853 \\
\hline 160 & 160 & $1.2+2$ & $1.4+20$ & GRE E & E Soint-Laurent+ & 10854 \\
\hline $20 \mathrm{Ne}$ & ${ }^{12} \mathrm{C}$ & $4.8+1$ & $9.6+15$ & SAC M & M Conjeaudt & 9867 \\
\hline $20 \mathrm{Ne}$ & ${ }^{12} \mathrm{c}$ & $1.2+2$ & $1.4+2$ & GRE E & E Conjeaudt & 9868 \\
\hline $\mathrm{NI}$ & ${ }^{20} \mathrm{Ne}$ & $1.6+2$ & & ORL' $R$ & RPIasila & 11851 \\
\hline $232 \mathrm{Th}$ & ${ }^{40} \mathrm{Ar}$ & $7.2+4$ & & & R Scott. & 12399 \\
\hline 2451 & parllal & produc & ct yiald & & & \\
\hline $24 \mathrm{Mg}$ & $3^{3} \mathrm{He}$ & NDG & & MSU E & E Bowlest & 6196 \\
\hline${ }^{24} \mathrm{SI}$ & product & $y=1 d_{0}$ & & & & \\
\hline $\mathrm{Mg}$ & ${ }^{3} \mathrm{He}$ & $5.7+1$ & & PIN E & E Robertgont & 6191 \\
\hline${ }^{25} \mathbf{S}_{1}$ & product & yield & & & & \\
\hline${ }^{24} \mathrm{Mg}$ & ${ }^{3} \mathrm{He}$ & NDG & & BRK $R$ & R Cernyt & 6197 \\
\hline${ }^{25} 51$ & $\theta(E)$ & & & & & \\
\hline $\begin{array}{l}2^{4} \mathrm{Mg} \\
24 \mathrm{Mg}\end{array}$ & $\begin{array}{l}{ }^{3} \mathrm{He} \\
{ }^{3} \mathrm{He}\end{array}$ & $\begin{array}{l}2.1+1 \\
2.5+1\end{array}$ & $\begin{array}{l}4.3+1 \quad \\
4.3+1 k\end{array}$ & $\begin{array}{l}\text { CLU E } \\
\text { KFK C }\end{array}$ & $\begin{array}{l}\text { E Mosst } \\
\text { C Muenzelt }\end{array}$ & $\begin{array}{l}6198+ \\
6199\end{array}$ \\
\hline${ }^{26} \mathrm{~S} 1$ & $\theta(E)$ & & & & & \\
\hline${ }^{27} \mathrm{Al}$ & $p$ & $1.5+1$ & $2.7+1$ & CLUE & E Wilsont & 914 \\
\hline $27 \mathrm{Si}$ & portiol & cos ol & (E) & & & \\
\hline $27_{A I}$ & p & $5.8+0$ & $5.8+0 \mathrm{r}$ & MON E & E Azuelost & 921 \\
\hline 2751 & portiol & relate & IVe ole & & & \\
\hline${ }^{28} \mathrm{Si}$ & $p$ & $4.0+2$ & & SAC E & E Cossegnout & 987 \\
\hline${ }^{27} \mathrm{SI}$. & portiol & $\sigma(E)$ & & & & \\
\hline${ }^{12} \mathrm{C}$ & 160 & $1.2+1$ & $2.6+1 h$ & WAU E & E Chant & 10720 \\
\hline
\end{tabular}




\begin{tabular}{|c|c|c|c|c|c|c|}
\hline Target & $\ln c$ & $\begin{array}{l}\text { Emin } \\
(\mathrm{MeV})\end{array}$ & $\begin{array}{l}\text { Emax } \\
(\operatorname{MeV})\end{array}$ & Lab $W$ & Author & No. \\
\hline${ }^{7} \mathrm{SI}$ & product & yield & & & & \\
\hline${ }^{24} \mathrm{Mg}$ & ${ }^{6} \mathrm{Li}$ & TR & $2.4+0 \mathrm{r}$ & WITE & Eelischopt & 9179 \\
\hline $27 \mathrm{si}$ & raw thict & in torg & get y 101 & 19 & & \\
\hline $27_{A 1}$ & p & $T R$ & +01 & NZA E & Naylort & 920 \\
\hline 2751 & $r \Delta O(E)$ & & & & & 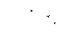 \\
\hline $27 \mathrm{Al}$ & $p$ & $T R$ & +o & WMU M & 1 Bernsteln. & 919 \\
\hline $27 \mathrm{si}$ & $O(E)$ & & & & & \\
\hline $12 c$ & 160 & $1.1+1$ & $2.3+10$ & CAL E & Switkoughit & 10716 \\
\hline $12 \mathrm{c}$ & 160 & $2.9+1$ & $3.4+1 i$ & MON E & Earast & 10717 \\
\hline $12 c$ & 160 & $3.3+1$ & $5.4+15$ & STB E & Malmint & 10718 \\
\hline${ }^{12} \mathrm{c}$ & 160 & $4.0+1$ & $6.5+1=$ & STR M & 4 Kolatat & 10719 \\
\hline 160 & 150 & $6.0+1$ & & BNL E & Kolata+ & 10827 \\
\hline 160 & 160 & $6.0+1$ & & MUN M & 4 Buscht & 10828 \\
\hline 160 & 160 & $6.0+1$ & & GRE $T$ & T Cole. & 10860 \\
\hline 160 & 160 & $6.0+1$ & & MUN $\varepsilon$ & Eeidingert & 10861 \\
\hline $20 \mathrm{Ne}$ & $12 \mathrm{C}$ & $6.7+1$ & & GRE $T$ & T cole. & 9871 \\
\hline${ }^{24} \mathrm{Mg}$ & a & TR & $1.2+1$ & CAL T & T Woosleyt & 6983 \\
\hline $24 \mathrm{Mg}$ & o & IR & $2.7+1 k$ & KLN M & 4 Gruhlet & 6984 \\
\hline $24 \mathrm{Mg}$ & 0 & $8.4+0$ & $1.3+1=$ & BIRE & Blyth+ & 6985 \\
\hline $26_{A}:$ & p & NDG & & CAL T & T Woosleyt & 795 \\
\hline${ }^{27} \mathrm{~A}:$ & p & TR & $0.3+0$ & CAL T & I Woosley+ & 915 \\
\hline${ }^{27} \mathrm{Al}$ & p & IR & $8.0+11$ & NRL $\tau$ & Teterson. & 916 \\
\hline $27 \mathrm{AI}$ & p & $5.8+0$ & $6.4+9$ r & KTY E & Elynnt & 917 \\
\hline $27 \mathrm{AI}$ & $p$ & $1.5+1$ & $2.7+1$ & CLU E & Wilsont & 918 \\
\hline $27 \mathrm{Al}$ & 8 & $8.7+0$ & $1.8+1$ & CLUE & E Wilsont & 5429 \\
\hline $5 i$ & $p$ & $1.5+1$ & $2.7+10$ & CLU E & Wilsont & 970 \\
\hline${ }^{20} \mathrm{si}$ & $\sigma(E)$ & & & & & \\
\hline 160 & 160 & $1.8+1$ & $6.0+15$ & STF E & E Chengt & 10833 \\
\hline${ }^{20} \mathrm{SI}$ & portial & produc & ct $y 1.10$ & & & \\
\hline $\begin{array}{l}24 \mathrm{MC} \\
27 \mathrm{AI} \\
30 \mathrm{Si}\end{array}$ & $\begin{array}{r}14 \mathrm{~N} \\
\mathrm{P} \\
\mathrm{P}\end{array}$ & $\begin{array}{l}7 \cdot 0+1 \\
3 \cdot 5+1 \\
2 \cdot 3+1\end{array}$ & $1.0+2$ & $\begin{array}{l}\text { CER E } \\
\text { OSU E } \\
\text { ROC E }\end{array}$ & $\begin{array}{l}\text { Zellert } \\
\text { Kovash. } \\
\text { Anantaramant }\end{array}$ & $\begin{array}{r}10346 \\
923 \\
1000\end{array}$ \\
\hline${ }^{2 \theta} \mathrm{SI}$ & portlal & rooth & hlck :or & orget & yleldxobundence & \\
\hline $\begin{array}{l}25 \mathrm{Mg} \\
28 \mathrm{Si}\end{array}$ & o & $\begin{array}{l}5.0+0 \\
5.0+0\end{array}$ & & $\begin{array}{l}\text { SUN E } \\
\text { SUN E }\end{array}$ & $\begin{array}{l}\text { Gilest } \\
\text { Erlest }\end{array}$ & $\begin{array}{l}6991 \\
7094\end{array}$ \\
\hline
\end{tabular}

\begin{tabular}{|c|c|c|c|c|c|}
\hline Target & $\ln c$ & $\begin{array}{l}\text { Emin } \\
(\mathrm{MeV})\end{array}$ & $\begin{array}{l}\text { Emax } \\
(\mathrm{MeV})\end{array}$ & Lab $\mathrm{K}$ & Author \\
\hline
\end{tabular}

20SI portial overoge o(E),

${ }^{20} 51$ o $1.0+02.4+1$ WAU.E Bodonshy+ S8!

est partiol o(E),

28S1 $3.0+0$ 2.0+2 GSF D Ramaly+ 979

$285101.4+14.0+1 \mathrm{MILE} \mathrm{De}-\mathrm{LeO}_{0+}$

$28_{51} \quad 8.0+01.0+5$ GSF D Ramalyt 7095

2BSI parllol rOU O(E)

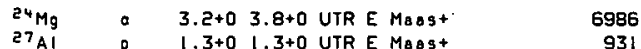

$27 \mathrm{Al} \quad 3.7+03.7+0$ WAUE Osboriet

20Si partlal relative o(E).

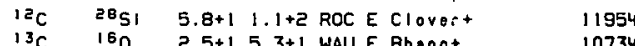

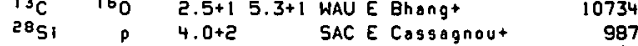

28Si partlal o(E)

$12 \mathrm{C} \quad 24 \mathrm{Mg} 5.0+17.0+1$ STR E Freemant 11938

$160 \quad 160 \quad 1.8+16.0+1$ STF E Cheng: 10829

160 SB $6.0+19.0+1$ STR E Freemant 11956

a $2.5+1 \quad 7067$

28 Si rov I,

27 AI P 2.0+0 5.1+0 ANL E Kennedyt 957

20: relotive $]^{2}$

$27 \mathrm{Al} \quad \mathrm{P} 7.6-11.8+0$ HLS E Ant111a+ 924

27Al $P$ 9.9-1 GEO E Scottt

27Al $P$ 2.140 $2.5+0$ ANL E Kennedy+ 926

2051 product y101d

$160160 \quad 1.7+13.2+1$ ATH E Foxt, 10834

${ }^{24} \mathrm{Mg}{ }^{6} \mathrm{LI}$ TR $2.4+0$ WIT E Selischopt 9180

${ }^{24} \mathrm{Mg}{ }^{7} \mathrm{LI}$ TR $2.4+0 \mathrm{WIT}$ E Selischopt 9426

$24 \mathrm{Mg} 180 \mathrm{O} 4.0+16.1+1$ BNL E Barrettet 11653

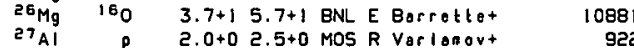




\begin{tabular}{|c|c|c|c|c|c|c|}
\hline Target & $\ln c$ & $\begin{array}{l}\text { Emin } \\
(\mathrm{MeV})\end{array}$ & $\begin{array}{l}E \max \\
(\operatorname{MeV})\end{array}$ & Lab & Author & No. \\
\hline${ }^{2 \theta} \mathbf{S I}$ & thich & terget & yield & & & \\
\hline 2851 & d & $6.8+0$ & & TNL & T Eastgatet & 5433 \\
\hline${ }^{20} \mathrm{si}$ & \multicolumn{6}{|c|}{ rov $O(E)$ of resonance } \\
\hline${ }^{27} \mathrm{Al}$ & $p$ & $6.3-1$ & $6.5-1$ & AML & E Poine* & 934 \\
\hline${ }^{28} \mathrm{Si}$ & \multicolumn{6}{|c|}{ raw o(E) } \\
\hline $\begin{array}{l}27 \mathrm{Al} \\
27 \mathrm{Al} \\
27_{\mathrm{Al}}\end{array}$ & $\begin{array}{l}p \\
p \\
p\end{array}$ & $\begin{array}{l}5.0-1 \\
9.9-1 \\
1.5+0\end{array}$ & $\begin{array}{l}2.0+0 \\
2.2+0\end{array}$ & $\begin{array}{l}\text { AML } \\
\text { HLS } \\
\text { OEB }\end{array}$ & $\begin{array}{l}\text { E Painet } \\
\text { E Anlillat } \\
\text { E Zolnait }\end{array}$ & $\begin{array}{l}928 \\
929 \\
930\end{array}$ \\
\hline${ }^{28} \mathrm{si}$ & \multicolumn{6}{|c|}{ relollve o(E) } \\
\hline $26 \mathrm{Mg}$ & ${ }^{3} \mathrm{He}$ & $8.0+1$ & & NRL & E singht & 6202 \\
\hline${ }^{20} \mathrm{si}$ & \multicolumn{6}{|l|}{$\theta(E)$} \\
\hline${ }^{12} \mathrm{C}$ & $19 \mathrm{~F}$ & $5.0+1$ & $7.0+1$ & STR & M Wognert & 11761 \\
\hline 150 & $14 \mathrm{~N}$ & $1.2+1$ & $2.7+1$ & CAL & E Soltkowshit & 10344 \\
\hline 160 & 160 & $2.4+1$ & $5.0+1$ & STR & $\mathrm{R}$ Hast & 10830 \\
\hline 160 & 180 & $2.5+1$ & $5.1+1$ & BNL & E Kolatat & 10831 \\
\hline 150 & 160 & $6.0+1$ & & MUN & M Buseht & 10932 \\
\hline 160 & 160 & $2.4+1$ & $8.0+1$ & STR & E Kolatat & 10859 \\
\hline 160 & 160 & $6 \cdot 0+1$ & & GRE & I Cole. & 10860 \\
\hline $16_{0}$ & 150 & $6 \cdot 0+1$ & & MUN & E Weidingert & 10861 \\
\hline $20 \mathrm{Ne}$ & $12 \mathrm{c}$ & $6.7+1$ & & GRE & T Cole. & 9871 \\
\hline $24 \mathrm{Mg}$ & a & NDG & & CAL & T Woosleyt & 6987 \\
\hline $24 \mathrm{Mg}$ & $12 \mathrm{c}$ & $2.2+1$ & $3.5+1$ & SIR & $\mathrm{RHOast}^{+}$ & 9883 \\
\hline $25 \mathrm{Mg}$ & $a$ & NDG & & CAL & T Woosleyt & 6992 \\
\hline $25 \mathrm{Mg}$ & $a$ & NDG & & KTY & E Flynnt & 6993 \\
\hline $25 \mathrm{Mg}$ & 0 & TR & $5.5+0$ & LAS & T Fostert & 6994 \\
\hline $26 \mathrm{Mg}$ & 160 & $4.1+1$ & $5.3+1$ & ANL & E Henningt & 10882 \\
\hline $255_{A 1}$ & a & NDG & & $\mathrm{CAL}$ & T Woosleyt & 7012 \\
\hline $27 \mathrm{Al}$ & & NDO & & $\mathrm{CAL}$ & T Woosley+ & 933 \\
\hline $27 \mathrm{Al}$ & ${ }^{3} \mathrm{He}$ & $7.0+1$ & $9.0+1$ & OSA & M Matsuohat & 6207 \\
\hline $27 \mathrm{Al}$ & a & TR & $4.8+1$ & BRK & E Gonzalez-Vidalt & 7065 \\
\hline $27_{\mathrm{Al}}$ & a & $8.0+1$ & $1.6+2$ & MRY & $\mathrm{E} \mathrm{Wu}^{+}$ & 7066 \\
\hline $2 \theta_{\mathrm{Al}}$ & p & NDG & & $\mathrm{CAL}$ & T Woosley+ & 936 \\
\hline $31 p$ & p & NDG & & CAL & I Woosleyt & 1024 \\
\hline 2851 & \multicolumn{6}{|c|}{ spollatlon $\theta(E)$} \\
\hline $\mathrm{Fe}$ & p & $1.5+2$ & $3.7+2$ & NRL & R silberbergt & 1844 \\
\hline
\end{tabular}

\begin{tabular}{|c|c|c|c|c|c|c|}
\hline$\overline{\text { Target }}$ & $\ln c$ & $\begin{array}{l}\text { Emin } \\
(M e V)\end{array}$ & $\begin{array}{l}\text { Emax } \\
(M e V)\end{array}$ & Lob $h$ & Author & No. \\
\hline $28 \mathrm{SI}$ & $\sigma(E)$ & & & & & \\
\hline 160 & 160 & $1.8+1$ & $5.0+1$ & STF E & E Chengt & 10839 \\
\hline 2851 & partlol & row in & hich to & argot & yloldyeabundance & \\
\hline${ }^{29} \mathrm{Si}$ & 。 & $5.0+0$ & & SUN E & E Gilest & 7105 \\
\hline $29 \mathrm{si}$ & portiol & row th & hlek to & orgot & yloldrabundance & \\
\hline $26 \mathrm{Mg}$ & a & $5.0+0$ & & SUN E & E Gllest & 7003 \\
\hline $295 i$ & porttol & relati & ive spe & ectrua & overoge o(E) & \\
\hline $31 p$ & d & $2.0+0$ & $3.0+0$ & THU E & E Huang+ & 5435 \\
\hline 2951 & partial & spectr & rum ave & erago & of $(E) \times$ foclor & \\
\hline $31 p$ & d & $2.0+0$ & $3.0+0$ & THU E & E Huangt & 5436 \\
\hline 2951 & partlat & $O(E)$ & & & & \\
\hline 160 & 160 & $1.8+1$ & $6.0+1$ & STF E & E Chengt & 10835 \\
\hline${ }^{29} \mathrm{SI}$ & produet & yiold & & & & \\
\hline${ }^{24} \mathrm{Mg}$ & ${ }^{\prime} L_{1}$ & TR & $2.4+0$ & WITE & E Selischopt & 9427 \\
\hline $29_{5 i}$ & $O(E)$ & & & & & \\
\hline $12 \mathrm{C}$ & $19 \mathrm{~F}$ & $5.0+1$ & $7.0+1$ & STR $\mathrm{M}$ & M Wagnert & 11762 \\
\hline 180 & 160 & $1.8+1$ & $6.0+1$ & STF $E$ & E Chengt & 10836 \\
\hline 160 & 160 & $6.0+1$ & & BNL E & E Kolstat & 10837 \\
\hline 150 & 160 & $6.0+1$ & & MUN $M$ & M Buscht & 10838 \\
\hline 160 & 160 & $2.4+1$ & $8.0+1$ & STR E & E kolatat & 10859 \\
\hline 160 & 180 & $6.0+1$ & & GRE $T$ & T Cole. & 10860 \\
\hline 160 & 160 & $6.0+1$ & & MUN E & E Weldingert & 10851 \\
\hline $20_{\mathrm{Ne}}$ & ${ }^{12} \mathrm{c}$ & $2.0+1$ & $5.4+1$ & NOT E & E De-Youngt & 9870 \\
\hline $20 \mathrm{Ne}$ & ${ }^{12} \mathrm{c}$ & $6.7+1$ & & GRE T & T cole. & 9871 \\
\hline $25 \mathrm{Mg}$ & : & NDG & & CAL I & I Woosleyt & 6995 \\
\hline $26 \mathrm{Mg}$ & a & NDG & & CAL I & T Woosley+ & 7004 \\
\hline $26 \mathrm{Mg}$ & $a$ & NDG & & KTYE & E Flynnt & 7005 \\
\hline $26 \mathrm{Mg}$ & a & TR & $5.5+0$ & LAS T & T fostert & 7005 \\
\hline $26 \mathrm{Mg}$ & $a$ & $3.1+0$ & $5.4+0$ & ORL & E Bairt & 7007 \\
\hline $26_{\mathrm{Al}}$ & $a$ & NOG & & CAL $\mathrm{T}$ & T Woosleyt & 7014 \\
\hline $27 \mathrm{Al}$ & 0 & $8.0+1$ & $1.6+2$ & MRY E & $\mathrm{E} \mathrm{Wut}$ & 7068 \\
\hline $28 \mathrm{Al}$ & p & NDG & & CAL $T$ & T Woosleyt & 937 \\
\hline $29_{A 1}$ & p & NDG & & CAL & T Woosleyt & 939 \\
\hline $32_{p}$ & p & NDG & & CAL I & I Woosley+ & 1035 \\
\hline
\end{tabular}




\begin{tabular}{|c|c|c|c|c|c|c|}
\hline Target & $\ln c$ & $\begin{array}{l}\text { Emin } \\
(\mathrm{MeV})\end{array}$ & $\begin{array}{l}\text { Emex } \\
(M e V)\end{array}$ & Lab & Author & No. \\
\hline${ }^{30} 5 i$ & spalloti & IOn ole & & & & \\
\hline $\mathrm{Fe}$ & p & $1.5+2=$ & $3.7+2$ & NRL & R Sllberberg+ & 1844 \\
\hline 3051 & partial & produc & $|y| 0 \mid$ & & & \\
\hline $26 \mathrm{Mg}$ & ${ }^{14} \mathrm{~N}$ & $7.0+1$ & & $\mathrm{COR}$ & E. Zellert & 10347 \\
\hline${ }^{30} 5 i$ & partlal & raw th & ick ta & arget & tyleldabundance & \\
\hline${ }^{30} \mathrm{SI}$ & a & $5.0+0$ & & SUN & E Gilest & 7115 \\
\hline $30_{51}$ & partiol & rasth & ich to & orget & $y|y| 010$ & \\
\hline $27 \mathrm{Al}$ & a & $5.0+0$ & & SUN & E Gliest & 7069 \\
\hline 3051 & partial & $O(E) \times f$ & factor & & & \\
\hline 150 & 160 & $2.8+1$ & $6.0+1$ & WZI & E Tserruyat & 10840 \\
\hline $30 \mathrm{si}$ & partial & $O(E)$ & & & & \\
\hline 180 & 160 & $1.8+1$ & $6.0+:$ & STF & E Chengt & 10841 \\
\hline $30 \mathrm{si}$ & product & yield & & & & \\
\hline${ }^{26} \mathrm{Mg}$ & 150 & $3.7+1=$ & $5.7+:$ & BNL & E Barretlet & 10883 \\
\hline 3051 & $O(E)$ & & & & & \\
\hline $\begin{array}{l}180 \\
180\end{array}$ & $\begin{array}{l}150 \\
160\end{array}$ & $\begin{array}{l}1.8+1 \\
2.4+1\end{array}$ & $\begin{array}{l}6.0+1 \\
8.0+1\end{array}$ & $\begin{array}{l}\text { STF } \\
\text { STR }\end{array}$ & $\begin{array}{l}\text { E Chengt } \\
\text { E Kolated }\end{array}$ & $\begin{array}{l}10842 \\
10859\end{array}$ \\
\hline 150 & 150 & $\begin{array}{l}2.4+1 \\
6.0+1\end{array}$ & & GRE & $\begin{array}{l}\text { E Kolatat } \\
\text { T Cole. }\end{array}$ & $\begin{array}{l}10859 \\
10860\end{array}$ \\
\hline $20 \mathrm{Ne}$ & ${ }^{12} \mathrm{C}$ & $6.7+1$ & & GRE & T Cole. & 9871 \\
\hline $26 \mathrm{Mg}$ & 0 & NDG & & CAL & T Woosleyt & 7008 \\
\hline $26 \mathrm{Mg}$ & 150 & NDG & & ANL & E Henningt & 10884 \\
\hline $27 \mathrm{Mg}$ & $a$ & NDG & & CAL & T Woosley+ & 7009 \\
\hline $27 \mathrm{Al}$ & $a$ & NDG & & CAL & T Woosleyt & 7070 \\
\hline $27 \mathrm{Al}$ & 0 & $0.0+11$ & $1.6+2$ & MRY & E Wut & $707 !$ \\
\hline $28_{\mathrm{Al}}$ & p & NOG & & CAL & T Woosleyt & 940 \\
\hline${ }^{30} 0_{A 1}$ & p & NDG & & CAL & T Woosleyt & 942 \\
\hline 330 . & $p$ & NDG & & CAL & T Woosleyt & 1038 \\
\hline $315 i$ & spollatl & Ion $O(E)$ & & & & \\
\hline Fo & $p$ & $1.5+2=$ & $3.7+\hat{\varepsilon}$ & NFL F & R Slliberbefgt & 1844 \\
\hline $315 i$ & $O(E)$ & & & & & . \\
\hline $\begin{array}{l}27 \mathrm{Mg} \\
28 \mathrm{Al}\end{array}$ & a & $\begin{array}{l}\text { NDG } \\
\text { NDG }\end{array}$ & . & $\begin{array}{l}\text { CAL } \\
\text { CAL }\end{array}$ & $\begin{array}{l}\text { T Woosleyt } \\
\text { T Woosleyt }\end{array}$ & $\begin{array}{l}7010 \\
7080\end{array}$ \\
\hline
\end{tabular}

\begin{tabular}{|c|c|c|c|c|c|c|}
\hline Target & Inc & $\begin{array}{l}\text { EmIn } \\
(M e V)\end{array}$ & $\begin{array}{l}\text { Emax } \\
(M e V)\end{array}$ & Lab & Autror & No. \\
\hline 3151 & $\dot{\theta}(E)$ & & & & & \\
\hline $\begin{array}{l}30 \mathrm{Al} \\
311_{\mathrm{Al}} \\
3{ }^{34} \mathrm{P}\end{array}$ & $\begin{array}{l}p \\
p \\
p\end{array}$ & $\begin{array}{l}\text { NDG } \\
\text { NDG } \\
\text { NDG }\end{array}$ & & $\begin{array}{l}\text { CAL } \\
\text { CAL } \\
\text { CAL }\end{array}$ & $\begin{array}{l}\text { T Woosleyt } \\
\text { T Woosleyt } \\
\text { T Woosleyt }\end{array}$ & $\begin{array}{r}943 \\
945 \\
1040\end{array}$ \\
\hline $32 \mathrm{si}$ & spollat & $\operatorname{lon} \sigma(E)$ & & & & \\
\hline $\begin{array}{c}\mathrm{Fe} \\
{ }^{32} \mathrm{SI}\end{array}$ & O(E) & $1.5+2=$ & $3.7+21$ & NRL F & R Silberberg+ & 1844 \\
\hline $\begin{array}{l}28_{M g} \\
299_{A 1} \\
311_{A 1} \\
35 \mathrm{P}\end{array}$ & $\begin{array}{l}a \\
\dot{\alpha} \\
p \\
p\end{array}$ & $\begin{array}{l}\text { NDG } \\
\text { NDG } \\
\text { NDG } \\
\text { NDG }\end{array}$ & & $\begin{array}{l}\text { CAL } \\
\text { CAL } \\
\text { CAL } \\
\text { CAL }\end{array}$ & $\begin{array}{l}\text { T Woosley+ } \\
\text { T Woosley+ } \\
\text { T Woosley+ } \\
\text { T Woosley+ }\end{array}$ & $\begin{array}{r}7011 \\
7083 \\
946 \\
1043\end{array}$ \\
\hline 3351 & $\theta(E)$ & & & & & \\
\hline $\begin{array}{l}36 \mathrm{p} \\
3{ }^{4} \mathrm{si}\end{array}$ & $\begin{array}{r}p \\
O(E)\end{array}$ & NDG & & CAL & I Woosley+ & 1046 \\
\hline${ }^{37} \mathrm{P}$. & $p$ & NDG & . & CAL & T Woosley+ & 1049 \\
\hline$z=15$ & $\operatorname{lndep}$ & endent & average & ge kir & inetic onergy $\left(E_{i} E^{\prime}\right)$ & \\
\hline $187 \mathrm{Au}$ & ${ }^{\circ} \mathrm{Ar}$ & $2.2+2$ & & OUB & E Artuhht & $12369+$ \\
\hline$z=15$ & Indep & endent & $O(E: E)$ & $\because \theta 1$ & & \\
\hline $\begin{array}{l}197 \mathrm{Au} \\
232 \mathrm{Th}\end{array}$ & $\begin{array}{l}{ }^{40} \mathrm{Ar} \\
{ }^{40} \mathrm{Ar}\end{array}$ & $\begin{array}{l}2.2+2 \\
3.0+2\end{array}$ & $3.9+2$ & $\begin{array}{l}\text { DUB } \\
\text { DUB }\end{array}$ & $\begin{array}{l}\text { E Artuhht } \\
\text { E Artuhht }\end{array}$ & $\begin{array}{l}12368+ \\
12396\end{array}$ \\
\hline $\begin{array}{l}Z=15 \\
197\end{array}$ & $\begin{array}{l}\text { Indep } \\
{ }^{40} \mathrm{Ar}\end{array}$ & endent & $\theta(\varepsilon ; \theta)$ & & & \\
\hline$z=15$ & indep & $\begin{array}{l}2.2+2 \\
\text { endent }\end{array}$ & $a(E)$ & DUQ & E Arlukh+ & $12367+$ \\
\hline $\begin{array}{l}197_{A v} \\
z=15\end{array}$ & $\begin{array}{c}40 \mathrm{Ar} \\
v_{n}\end{array}$ & $2.2+5$ & & DUB E & E Artukh+ & $12366+$ \\
\hline Ag & $40 \mathrm{Ar}$ & $3.4+2$ & & PAR F & R Lefortt & 12202 \\
\hline$z=15$ & $\sigma(E) \times$ & foctor & & & & \\
\hline $\begin{array}{l}232 \mathrm{Th} \\
z=15\end{array}$ & $\begin{array}{l}{ }^{40} \mathrm{Ar} \\
\text { O(E) }\end{array}$ & $3.9+2$ & & oxf $F$ & R Hodgson. & 12398 \\
\hline${ }^{12} \mathrm{C}$ & ${ }^{20} \mathrm{Ne}$ & $6.7+1$ & $1.6+2$ & GRE E & E Saint-Laurentt & 11839 \\
\hline
\end{tabular}




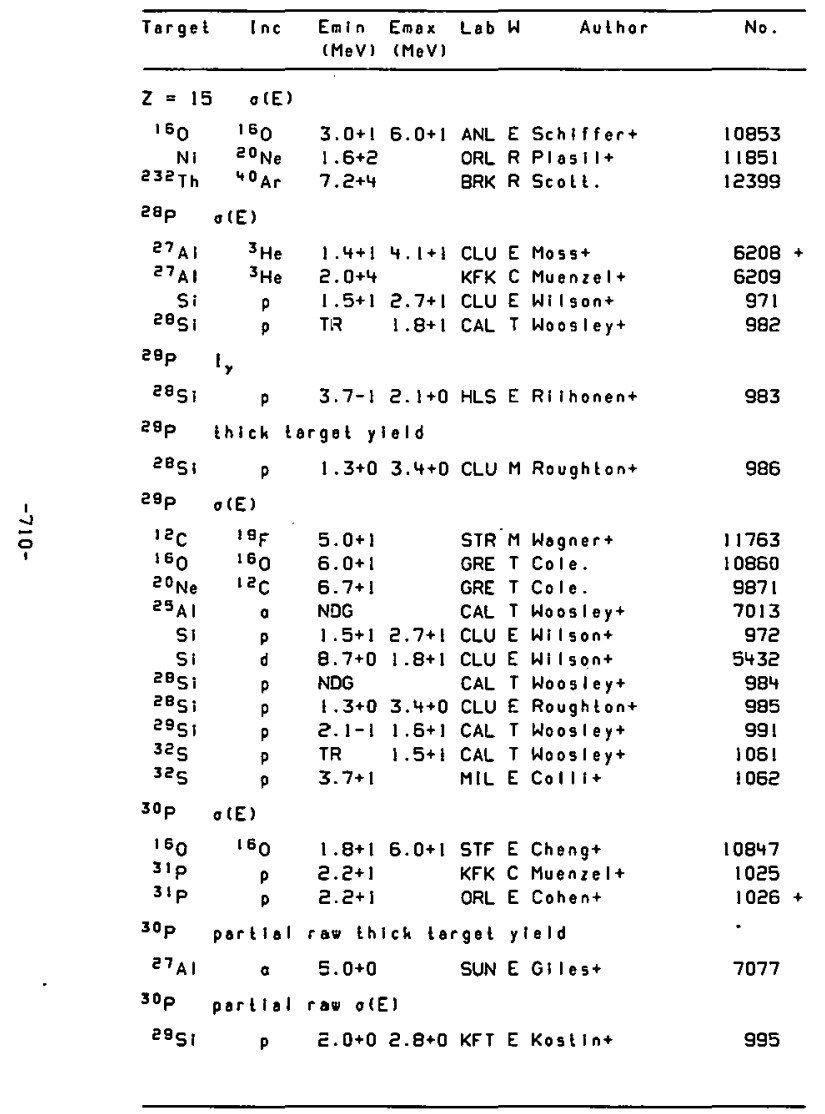

\begin{tabular}{|c|c|c|c|c|c|c|}
\hline Target & $\ln c$ & $\begin{array}{l}E_{\mathrm{Noln}} \\
(\mathrm{MeV})\end{array}$ & $\begin{array}{l}\text { Emax } \\
\text { (MeV) }\end{array}$ & Lab & Author & No. \\
\hline${ }^{30} \mathrm{P}$ & partiol & $O(E) \times f$ & actor & & & \\
\hline 160 & 160 & $2.8+1$ & $6.0+1$ & WZI & E Tserruyat & 10846 \\
\hline${ }^{30} \mathrm{P}$ & partiel & $O(E)$ & & & & \\
\hline 160 & 160 & $1.8+1$ & $6.0+1$ & SIF & E.Chengt & 10843 \\
\hline${ }^{30} \mathrm{p}$ & $I_{y}$ & & & & & \\
\hline${ }^{29} \mathrm{Si}$ & p & $4.2-1$ & & HLS & E Rilihonent & 992 \\
\hline $30 p$ & thick to & arget $y 1$ & 1010 & & & \\
\hline $27 \mathrm{Al}$ & a & $2.8+1$ & & JUL & R Stochlint & 7075 \\
\hline${ }^{27} \mathrm{Al}$ & a & $3.5+1$ & & JUL $\varepsilon$ & E Sahahundut & 7076 \\
\hline${ }^{29} \mathrm{Si}_{\mathrm{i}}$ & $p$ & $3.7-1$ & $3.5+0$ & CLU & M Roughiont & 996 \\
\hline${ }^{3: P} \mathrm{P}$ & p & $2.8+1$ & & JUL & E Sahahundu+ & 1027 \\
\hline $30 \mathrm{p}$ & $\theta(E)$ & & & & & \\
\hline 160 & 160 & $1.8+1$ & $6.0+1$ & SIF & E Chengt & 10844 \\
\hline 160 & 150 & $6.0+i$ & & BNL & E Kolatat & 10845 \\
\hline 160 & 150 & $2.4+1$ & $8.0+1$ & STR & E Kolatat & 10859 \\
\hline 160 & 160 & $6.0+1$ & & GRE & T Cole. & 10860 \\
\hline $20 \mathrm{Ne}$ & $12 \mathrm{C}$ & $6.7+1$ & & GRE & r cole. & 9871 \\
\hline $26_{\mathrm{Al}}$ & a & NDG & & CAL. & T Woosleyt & 7015 \\
\hline $27 \mathrm{AI}$ & a & TR & $1.1+1$ & CAL & T Woosleyt & 7072 \\
\hline${ }^{27} \mathrm{Al}$ & 0 & $3.1+0$ & $6.1+0 \mathrm{~K}$ & KTY & E Flynnt & 7073 \\
\hline $27 \mathrm{Al}$ & 。 & $1.0+1$ & $2.8+1$ & JuL & E Sahokundut & 7074 \\
\hline${ }^{29} \mathrm{Si}$ & p & NDG & & CAL & T Woosleyt & 993 \\
\hline${ }^{29} \mathrm{si}$ & p & $3.7-1$ & $3.5+0$ & CLU & E Roughtiont & 994 \\
\hline${ }^{30} \mathrm{Si}$ & p & $2.1-1$ & $1.3+1$ & CAL & T Woosleyt & 1001 \\
\hline $31 p$ & p & $1.9+1$ & $3.5+1$ & JUL E & E Sohahundut & 1028 \\
\hline $33 \mathrm{~s}$ & p & $2.1-1$ & $1.1+1$ & CAL & T Woosley+ & 1065 \\
\hline $31 p$ & compound & d-nuclou & US $O(E)$ & & & \\
\hline${ }^{12} \mathrm{C}$ & ${ }^{19} \mathrm{~F}$ & $5 \cdot 0+1$ & $7.6+1$ & THD & T Puhlhofer. & 11764 \\
\hline $31 P$ & spollat & $\operatorname{lon} O(E)$ & & & & \\
\hline $\mathrm{Fe}$ & $\rho$ & $1.5+2$ & $3.7+2$ & NRL & R Silberbergt & 1844 \\
\hline $31 \mathrm{p}$ & Dartlal & row thi & Ick tor & get & yieldxabundance & \\
\hline${ }^{28} \mathbf{S i}_{1}$ & a & $5.0+0$ & & SUN & E Gilest & 7096 \\
\hline
\end{tabular}




\begin{tabular}{|c|c|c|c|c|c|c|}
\hline Target & $\ln c$ & $\begin{array}{l}\text { Emin } \\
(\mathrm{MeV})\end{array}$ & $\begin{array}{l}E_{\max } \\
(\mathrm{MeV})\end{array}$ & Lab & Author & No. \\
\hline $31_{P}$ & partiol & $\operatorname{raw} t h 1$ & ich lor & rget & yielo & \\
\hline $31 p$ & a & $5.0+0$ & & SUN & E Gilest & 7135 \\
\hline $31 p$ & partiol & $\sigma(E)$ & & & & \\
\hline $\begin{array}{l}12 \mathrm{C} \\
160 \\
30 \mathrm{si} \\
34 \mathrm{~s}\end{array}$ & $\begin{array}{c}24 \mathrm{Mg} \\
180 \\
\rho \\
p\end{array}$ & $\begin{array}{l}5.0+1 \\
1.8+1 \\
5.0+0 \\
3.6+1\end{array}$ & $\begin{array}{l}7.0+1 \\
6.0+1 \\
2.8+1\end{array}$ & $\begin{array}{l}\text { STR } \\
\text { STF } \\
\text { TNL. } \\
\text { MIL }\end{array}$ & $\begin{array}{l}\text { E Freomant } \\
\text { E Chengt } \\
\text { E Cameront } \\
\text { E Pellegrinlt }\end{array}$ & $\begin{array}{r}11939 \\
10849 \\
1005 \\
107 !\end{array}$ \\
\hline $31 p$ & $\mathrm{I}_{\gamma}$ & & & & & \\
\hline $30 \mathrm{Si}$ & p & $5.0-1$ & $8.5-1$ & HLS & E Rithonent & 1002 \\
\hline $31 p$ & raw o(E) & & & & & \\
\hline${ }^{30} 51$ & $p$ & $5.0-1$ & $2.0+0$ & AML & E Painet & 1004 \\
\hline $31 p$ & $O(E)$ & & & & & \\
\hline 160 & 160 & $6.0+1$ & & $\mathrm{BNL}$ & E Kolata+ & 10848 \\
\hline 160 & 150 & $1.8+1$ & $6.0+1$ & STF & E Chengt & 10850 \\
\hline 160 & 150 & $6.0+1$ & & BNL & E Kolatat & 10851 \\
\hline 160 & 150 & $2.4+1$ & $8.0+1$ & STR & E Kolatat & 10859 \\
\hline $20 \mathrm{Ne}$ & $12 \mathrm{c}$ & $6.7+1$ & & GRE & T Cole. & 9871 \\
\hline $24 \mathrm{Mg}$ & ${ }^{12} \mathrm{C}$ & $2.2+1$ & $3.5+1$ & STR & 8 Hast & 9884 \\
\hline $27 \mathrm{Al}$ & a & NDG & & CAL & T Woosleyt & 7078 \\
\hline $27 \mathrm{Al}$ & 160 & $5.0+1$ & $6.0+1$ & NBI & M Kuang-Shit & 10925 \\
\hline $28 \mathrm{Al}$ & $a$ & NOG & & CAL & T Woosleyt & 7081 \\
\hline 2851 & a & TR & $1.1+1$ & CAL & T Woosley+ & 7097 \\
\hline $305 i$ & p & NDG & & CAL & T Woosleyt & 1003 \\
\hline $315 i$ & p & NOG & . & CAL. & T Woosley+ & 1006 \\
\hline $3^{34} \mathrm{~S}$ & p & TR & $1.1+1$ & CAL & T Woosleyt & 1072 \\
\hline $32_{p}$ & spallati & $\operatorname{lon} \sigma(E)$ & & & & \\
\hline $\mathrm{Fe}$ & $\mathbf{p}$ & $1.5+2$ & $3.7+2$ & NRL & R silberbergt & 1844 \\
\hline $3 e_{p}$ & portiol & rawthi & lick tar & rget & yieldrabundance & \\
\hline $2{ }^{2} \mathrm{si}$ & 0 & $5.0+0$ & & SUN & E Gllest & 7106 \\
\hline${ }^{32} \mathrm{P}$ & product & yleld & & & & \\
\hline${ }^{40} \mathrm{Ar}$ & p & $5 \cdot 2+1$ & & iko & E Kaspersent & 1164 \\
\hline${ }^{32} \mathrm{P}$ & $G(E) \times 1 a C$ & etor & & & & \\
\hline${ }^{40} \mathrm{Ar}$ & $p$ & $5.2+1$ & & IKO & E Kospersent & 1165 \\
\hline
\end{tabular}

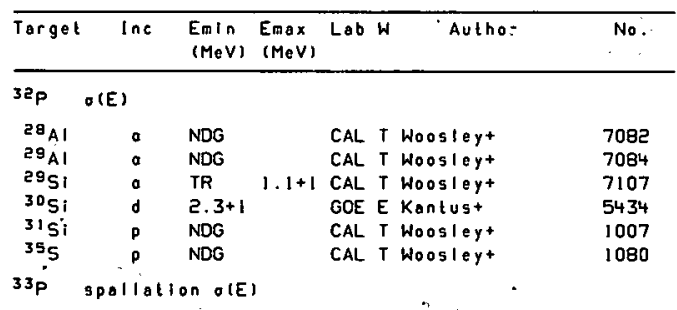

$\mathrm{Fe} P \quad 1.5+23.7+2 \mathrm{NRL}$ R Silberberg+ 1844 ${ }_{33} \mathrm{p}$ produci rleld

${ }^{40} \mathrm{Ar} P 5.2+1$ IKO E Kaspersen- 1166

${ }^{33} \mathrm{P} \quad \sigma(E) \times f a c t o r$

$40 \mathrm{Ar} P$ P.2+1 IKO E Kaspersen- 1167

$33 \mathrm{P}$ O(E)

$29_{\text {Al }}$ a NDG CAL T Woosleyt 7085

30 Al a NDG $\quad$ CAL T Woosleyt 7086

$325 i \quad P \quad$ NDG $1.1+1$ CAL T Hoosley+ 7116

$335 i \quad P$ NDG $\quad 1008$

365 NDG CAL T Woosleyt

${ }^{34} \mathrm{P}$ Spollation o(E)

$\mathrm{Fe} P \quad 1.5+23.7+2 \mathrm{NRL} R$ Silberbergt 1844 ${ }^{34} P \quad O(E)$

$1001001.6+14.0+1$ ETH E Eya1+ 11649

$\begin{array}{lll}30 & \text { Al a NDG } & \\ 3351 & \text { CAL T Woosley- } & 7087\end{array}$

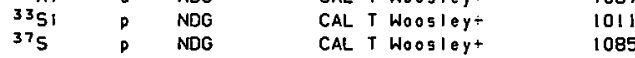

${ }^{35} p$ spollotion $O(E)$

Fe $P \quad 1.5+23.7+2$ NRL R Silberbergt 1844

$35^{P} \quad \sigma(E)$

$38_{\text {S }} D$ NDG CAL T Woosleyt 


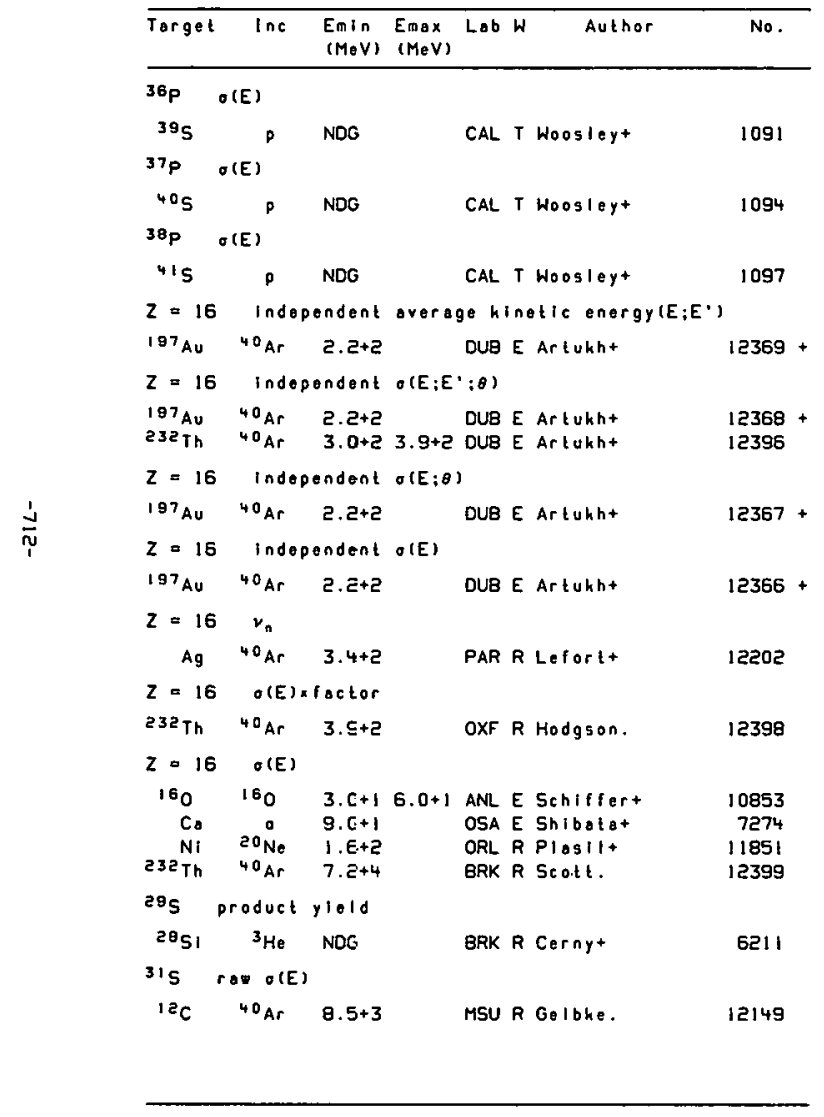

\begin{tabular}{|c|c|c|c|c|c|}
\hline Target & $t \ln c$ & $\begin{array}{l}\text { En In } \\
(M \bullet V)\end{array}$ & $\begin{array}{l}\text { Emax } \\
(\operatorname{MoV})\end{array}$ & Author & No. \\
\hline \multicolumn{6}{|c|}{$315 \quad O(E)$} \\
\hline $\begin{array}{l}160 \\
2051 \\
30 \mathrm{p} \\
31 \mathrm{p}\end{array}$ & $\begin{array}{r}160 \\
0 \\
p \\
p\end{array}$ & $\begin{array}{l}2.5+1 \\
T R \\
\text { NDG } \\
2.1-1\end{array}$ & $\begin{array}{l}1.1+1 \\
8.3+0\end{array}$ & $\begin{array}{l}\text { STF E Chengt } \\
\text { CAL T Woogleyt } \\
\text { CAL T Woosleyt } \\
\text { CAL T Woosleyt }\end{array}$ & $\begin{array}{r}10852 \\
7098 \\
1017 \\
1029\end{array}$ \\
\hline \multicolumn{6}{|c|}{325 Spallation o(E) } \\
\hline${ }_{325}^{\mathrm{Fe}}$ & $\begin{array}{c}D \\
\text { partial }\end{array}$ & $\begin{array}{l}1.5+2 \\
\text { product }\end{array}$ & $\begin{array}{l}3.7+2 \\
t y 101 d\end{array}$ & NRL R SIlberbergt & 1844 \\
\hline $\begin{array}{l}28 \mathrm{SI} \\
34 \mathrm{~S}\end{array}$ & $\dot{p}$ & $\begin{array}{l}\text { NDG } \\
2.3+1\end{array}$ & & $\begin{array}{l}\text { TNL T Lafferty+ } \\
\text { ROC E Anantaramant }\end{array}$ & $\begin{array}{l}7104 \\
1073\end{array}$ \\
\hline 325 & portial & overoge & - $O(E)$ & & \\
\hline $\begin{array}{ll}2 \theta_{51} \\
{ }^{32} 5\end{array}$ & $\begin{array}{c}a \\
\text { partial }\end{array}$ & $\begin{array}{l}1.2+1 \\
0(E)\end{array}$ & $2.0+1$ & STF E Kuhlmannt & 7103 \\
\hline $\begin{array}{l}12 \mathrm{c} \\
2851 \\
2851\end{array}$ & $\begin{array}{l}24 \mathrm{Mg} \\
180^{\circ}\end{array}$ & $\begin{array}{l}5.0+1 \\
5.0+0 \\
4.6+1\end{array}$ & $\begin{array}{l}7.0+1 \\
1.6+1 \\
5.2+1\end{array}$ & $\begin{array}{l}\text { SIR E Freemant } \\
\text { STF E Kuhlmannt } \\
\text { PUP E Pengt }\end{array}$ & $\begin{array}{r}11940 \\
7100 \\
10951\end{array}$ \\
\hline${ }^{32} \mathrm{~s}$ & produck & $y 1010$ & & & \\
\hline $\begin{array}{l}2851 \\
2951 \\
31 \mathrm{p}\end{array}$ & $\vdots$ & $\begin{array}{l}1.4+0 \\
1.8+0 \\
1.4+0\end{array}$ & $\begin{array}{l}3.8+0 \\
2.5+0 \\
1.5+0\end{array}$ & $\begin{array}{l}\text { MOS R Varlamovt } \\
\text { TRM E Bolshrishnont } \\
\text { MOS R Varlamovt }\end{array}$ & $\begin{array}{l}7099 \\
7111 \\
1030\end{array}$ \\
\hline $32 \mathrm{~s}$ & row thic & $=k$ targo & of yiel & & \\
\hline $\begin{array}{l}28 \mathrm{Si} \\
32_{\mathrm{S}}\end{array}$ & 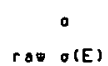 & $1.4+0$ & $3.8+0$ & OTC E Rogerst & 7101 \\
\hline $\begin{array}{l}12 c \\
2951 \\
31 p \\
31 p \\
{ }^{40} \mathrm{Ca}\end{array}$ & $\begin{array}{c}40 \mathrm{Ar} \\
0 \\
{ }_{0} \\
{ }^{40} \mathrm{Ar}\end{array}$ & $\begin{array}{l}0.5+3 \\
2.0+0 \\
8.1-1 \\
1.9+0 \\
2.4+2\end{array}$ & $\begin{array}{l}4.3+0 \\
8.2-1 \\
3.0+0\end{array}$ & $\begin{array}{l}\text { MSU R Golbhe. } \\
\text { ALE E Ohont } \\
\text { AML E Painet } \\
\text { CPC E Kostint } \\
\text { MSU R Gelbhe. }\end{array}$ & $\begin{array}{r}12149 \\
7110 \\
1033 \\
1034 \\
12151\end{array}$ \\
\hline $32 \mathrm{~s}$ & $\sigma(E)$ & & & & \\
\hline $\begin{array}{l}2 \theta_{51} \\
29_{51} \\
29_{51} \\
29 \mathrm{p}\end{array}$ & $\begin{array}{l}\circ \\
\vdots \\
a\end{array}$ & $\begin{array}{l}\text { NDG } \\
\text { IR } \\
3.0+0 \\
\text { NDG }\end{array}$ & $\begin{array}{l}1.1+1 \\
6.8+0\end{array}$ & $\begin{array}{l}\text { CAL T Woosleyt } \\
\text { CAL T Woosleyt } \\
\text { KTY E Gabbordt } \\
\text { CAL T Woosleyt }\end{array}$ & $\begin{array}{l}7102 \\
7100 \\
7109 \\
7131\end{array}$ \\
\hline
\end{tabular}




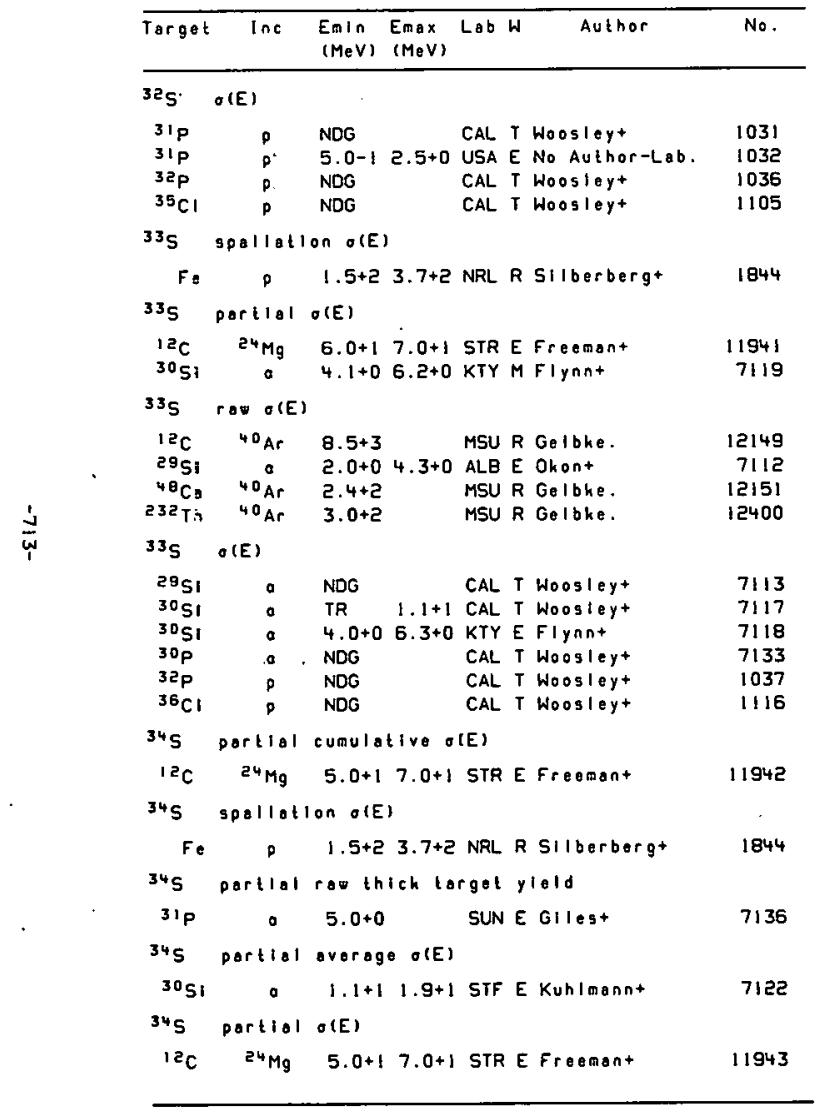

\begin{tabular}{|c|c|c|c|c|c|c|}
\hline Target & $\ln c$ & $\begin{array}{l}\text { Emin } \\
(M e V)\end{array}$ & $\begin{array}{l}\text { Emax } \\
(\operatorname{MeV})\end{array}$ & Lob h & Author & No. \\
\hline 345 & portlal & $\sigma(E)$ & & & & \\
\hline${ }^{30} \mathrm{s!}$ & $\circ$ & $4.0+0$ & $1.5+1$ & STF E & E Kuhlagonnt & 7120 \\
\hline${ }^{34} \mathrm{~s}$ & $r a w o(E)$ & & & & & \\
\hline $\begin{array}{c}12 \mathrm{C} \\
4 \mathrm{Ca} \\
232 \mathrm{Th}\end{array}$ & $\begin{array}{l}{ }^{40} \mathrm{Ar} \\
{ }^{40} \mathrm{Ar} \\
40 \mathrm{Ar}\end{array}$ & $\begin{array}{l}8.5+3 \\
2.4+2 \\
3.0+2\end{array}$ & & $\begin{array}{l}\text { MSU R } \\
\text { MSU R } \\
\text { MSU R }\end{array}$ & $\begin{array}{l}\text { R Gelbke. } \\
R \text { Gelbke. } \\
\text { R Gelbke. }\end{array}$ & $\begin{array}{l}12149 \\
12151 \\
12400\end{array}$ \\
\hline 345 & $O(E)$ & & & & & \\
\hline $\begin{array}{l}24 \mathrm{Mg} \\
27_{\mathrm{Al}} \\
27_{\mathrm{Al}} \\
27_{\mathrm{Al}} \\
30 \mathrm{SI} \\
3151 \\
31 \mathrm{P} \\
33^{\mathrm{P}} \\
34 \mathrm{P} \\
37^{7} \mathrm{Cl}\end{array}$ & $\begin{array}{r}12 c \\
160 \\
160 \\
160 \\
a \\
a \\
a \\
p \\
p \\
p\end{array}$ & $\begin{array}{l}2.2+1 \\
5.0+1 \\
5.0+1 \\
5.0+1 \\
\text { NDG } \\
\text { NDG } \\
\text { NDG } \\
\text { NDG } \\
\text { NDG } \\
\text { NDG }\end{array}$ & $\begin{array}{r}3.5+1 \\
6.0+1 \\
6.0+1 \\
6.0+1\end{array}$ & $\begin{array}{l}\text { STR } \\
\text { NBI } \\
\text { NBI } \\
\text { NBI } \\
\text { CAL } \\
\text { CAL } \\
\text { CAL } \\
\text { CAL } \\
\text { CAL } \\
\text { CAL T }\end{array}$ & $\begin{array}{l}\text { R Haost } \\
\text { M Kuang-Shit } \\
\text { M Kuang-Shit } \\
\text { M Kuang-Shit } \\
\text { T Woosleyt } \\
\text { T Woosleyt } \\
\text { T Woogleyt } \\
\text { T Woogleyt } \\
\text { T Woogleyt } \\
\text { T Woosleyt }\end{array}$ & $\begin{array}{r}9885 \\
10927 \\
10928 \\
10926 \\
7121 \\
7123 \\
7137^{\circ} \\
1039 \\
1041 \\
1118\end{array}$ \\
\hline${ }^{35} \mathrm{~s}$ & spolletlo & $\operatorname{lon} \sigma(E)$ & & & & \\
\hline${ }^{35} \mathrm{Se}$ & product & $\begin{array}{l}1.5+2 \\
y 1.10\end{array}$ & $3.7+21$ & NRL $R$ & R Silberterg+ & 1844 \\
\hline${ }^{40} \mathrm{Ar}$ & p & $5.2+1$ & & IKO E & E Kaspersent & 1168 \\
\hline${ }^{35} \mathrm{~s}$ & $r a \Phi \theta(E)$ & & & & & \\
\hline $\begin{aligned} 12 \mathrm{C} \\
48 \mathrm{Ca} \\
232 \mathrm{Th}\end{aligned}$ & $\begin{array}{l}40 \mathrm{Ar} \\
{ }^{40} \mathrm{Ar} \\
40 \mathrm{Ar}\end{array}$ & $\begin{array}{l}8.5+3 \\
2.4+2 \\
3.0+2\end{array}$ & & $\begin{array}{l}\text { MSU F } \\
\text { MSU F } \\
\text { MSU F }\end{array}$ & $\begin{array}{l}\text { R Gelbke. } \\
R \text { Gelbhe. } \\
\text { R Gelbke. }\end{array}$ & $\begin{array}{l}12149 \\
1215 ! \\
12400\end{array}$ \\
\hline${ }^{35} \mathrm{~s}$ & of(E)afact & tor & & & & \\
\hline $\begin{array}{l}{ }^{40} \mathrm{Ar} \\
{ }^{35} 5\end{array}$ & $\sigma(E)^{p}$ & $5.2+1$ & & IKO E & E Kasporsent & 1169 \\
\hline $\begin{array}{l}315 i \\
34 p \\
33 p \\
38 \mathrm{Cl}\end{array}$ & $\begin{array}{l}p \\
p \\
p \\
p\end{array}$ & $\begin{array}{l}\text { NDG } \\
\text { NDG } \\
\text { NDG } \\
\text { NDG }\end{array}$ & & $\begin{array}{l}\text { CAL } \\
\text { CAL } \\
\text { CAL } \\
\text { CAL T }\end{array}$ & $\begin{array}{l}\text { T Woosley+ } \\
\text { T Woosley+ } \\
\text { T Woosley+ } \\
\text { T Woosleyt }\end{array}$ & $\begin{array}{l}7124 \\
1042 \\
1044 \\
1126\end{array}$ \\
\hline$\cdot$ & & & & & & \\
\hline
\end{tabular}




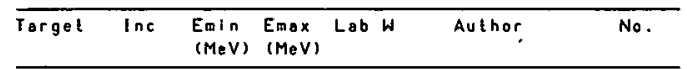

$36 \mathrm{~S}$ spallation o(E)

Fo $P \quad 1.5+23.7+2$ NRL R Silberbergt 1844 $36 S \quad r Q W O(E)$

$\begin{array}{lllll}12 \mathrm{C} & 40 \mathrm{Ar} & 8.5+3 & \text { MSU R Gelbke. } & 12149 \\ 48_{\mathrm{C}} & 40 \mathrm{Ar} & 2.4+2 & \text { MSU R Gelbke. } & 12151\end{array}$

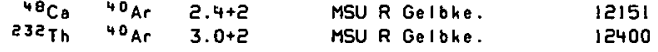

$36_{S}$ o(E)

\begin{tabular}{|c|c|c|c|c|}
\hline 3251 & a & NOG & CAL T Woogleyt & 7125 \\
\hline${ }^{3} 5 i$ & 。 & NDG & CAL T Woosloyt & 7126 \\
\hline $35 p$ & p & NOG & CAL T Woosleyt & 1045 \\
\hline${ }^{36 p}$ & p & NOG & CAL T Woosleyt & 1047 \\
\hline${ }^{39} \mathrm{Cl}$ & $p$ & NDIG & CAL T Woosleyt & 1129 \\
\hline
\end{tabular}

37s spollation o(E)

$\mathrm{Fe} P \quad 1.5+23.7+2$ NRL R SIIberbergt 1844

375 ran $0(E)$

$\begin{array}{llll}12 \mathrm{C} & 40 \mathrm{Ar} & 0.5+3 & \mathrm{ASU} R \text { Gelbke. }\end{array}$

235 40 Ar 40 Ar $3 .++2$ Melbke. 12151

375 o(E)

\begin{tabular}{|c|c|c|c|c|}
\hline${ }^{33} \mathrm{si}$ & a & NOG & CAL T Woosleyt & 7127 \\
\hline 3451 & a & NOG & CAL $T$ Woosleyt & 7128 \\
\hline${ }^{36} \mathrm{p}$ & $p$ & NDG & CAL T Woosley+ & 1048 \\
\hline $37_{p}$ & p & NDG & CAL T Woosleyt & 1050 \\
\hline${ }^{\circ} \mathrm{Cl}$ & $p$ & NDG & CAL T Woosleyt & 1132 \\
\hline
\end{tabular}

${ }^{30} \mathrm{~S}$ thick torget yleld

$\begin{array}{lllll}{ }^{40} \mathrm{Ar} & P & 5.0+1 & \text { IKOE Leurst } & 1172 \\ { }^{40} \mathrm{Ar} & P & 5.0+1 & \text { JUL ROBint } & 1173\end{array}$

$30^{2}$ raw o(E)

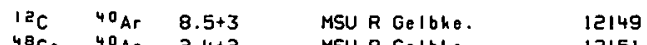

$48 \mathrm{Ca} 40 \mathrm{Ar} 2.4+2$ MSU R Gelbke.

$232 \mathrm{Th} 40^{\mathrm{Ar}} 3.0+2 \quad$ MSU R Gelbke. 12400

30s o(E) afactor

${ }^{40} \mathrm{Ar} \quad 5.2+1$ iKo E Kaspersent 1174

3

174

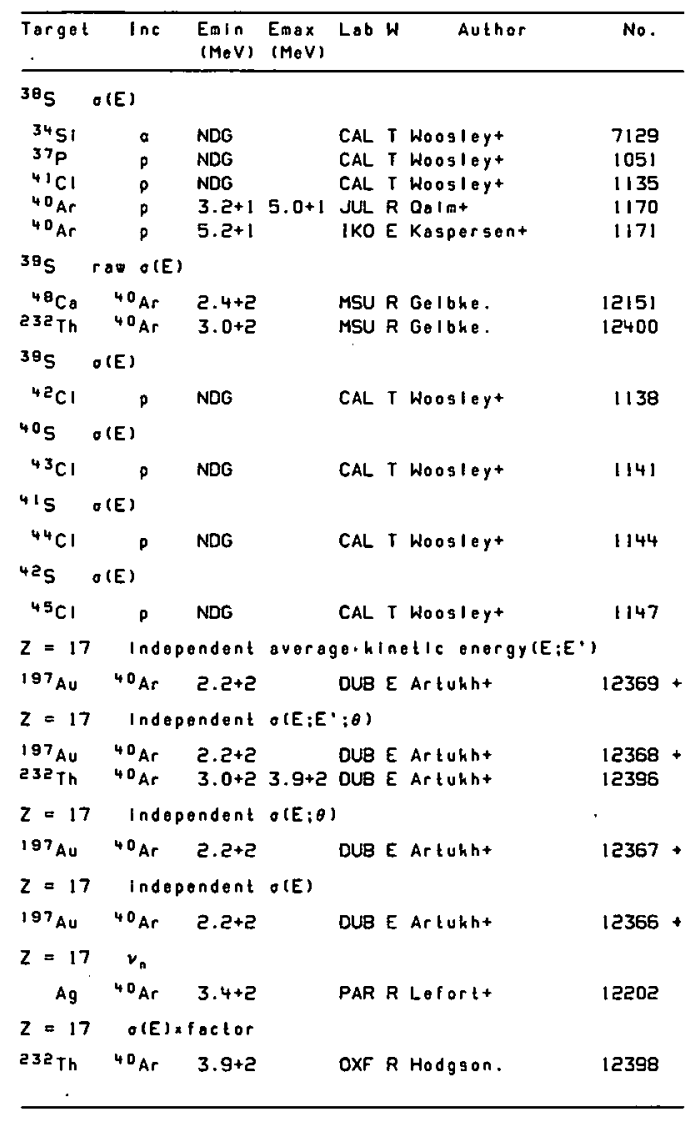




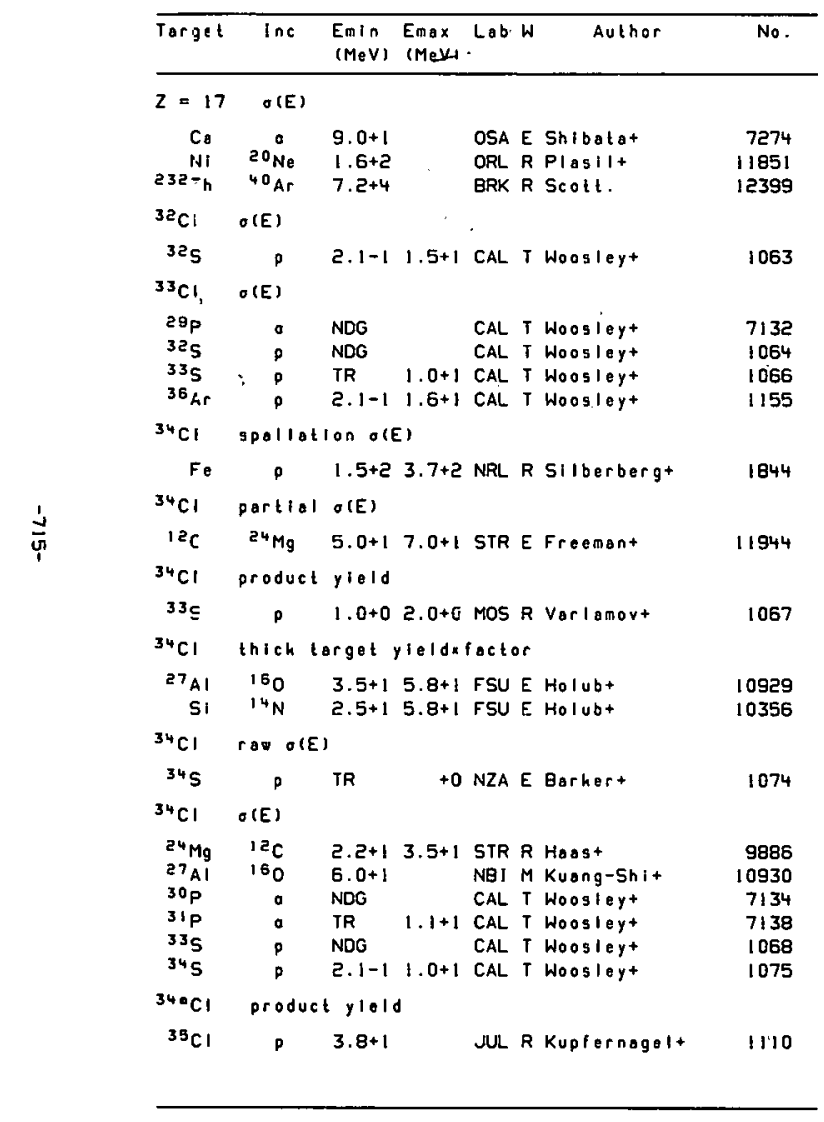

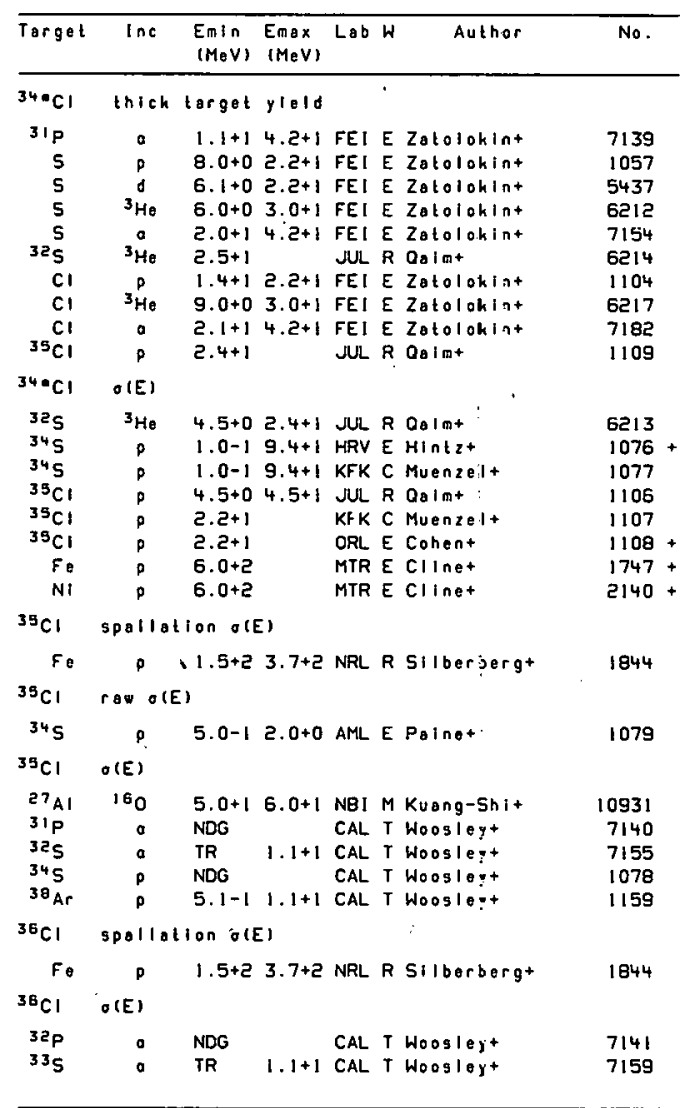




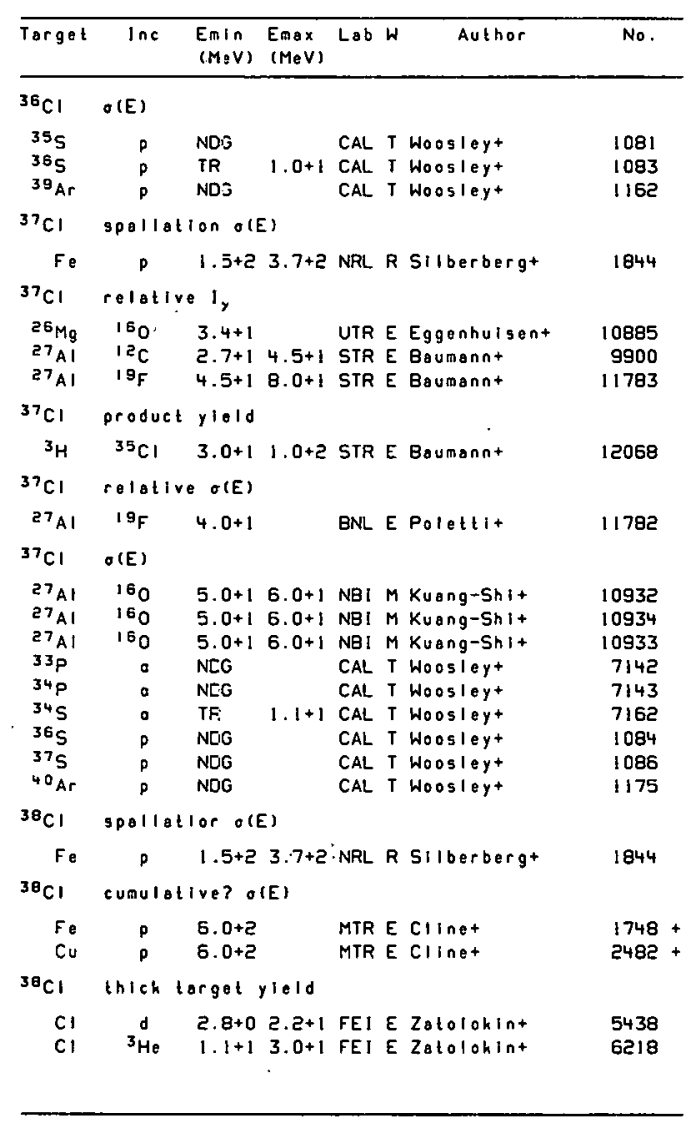

\begin{tabular}{|c|c|c|c|c|c|c|}
\hline Target & $\ln c$ & $\begin{array}{l}\text { Emin } \\
(M e V)\end{array}$ & $\begin{array}{l}\text { Emax } \\
(\mathrm{MeV})\end{array}$ & Lab & Author & No. \\
\hline${ }^{38} \mathrm{Cl}$ & $\sigma(E)$ & & & & & \\
\hline $\begin{array}{l}34 \mathrm{p} \\
375 \\
385 \\
41 \mathrm{Ar}\end{array}$ & $\begin{array}{l}a \\
p \\
p \\
p\end{array}$ & $\begin{array}{l}\text { NDG } \\
\text { NDG } \\
\text { NDG } \\
\text { NDG }\end{array}$ & & $\begin{array}{l}\text { CAL } \\
\text { CAL } \\
\text { CAL } \\
\text { CAL }\end{array}$ & $\begin{array}{l}\text { I Woosleyt } \\
\text { I Woosleyt } \\
\text { T Woosleyt } \\
\text { T Woosleyt }\end{array}$ & $\begin{array}{l}7144 \\
1087 \\
1089 \\
1179\end{array}$ \\
\hline${ }^{39} \mathrm{Cl}$ & Spatlo & Ion OlE & & & & \\
\hline $\begin{array}{c}\mathrm{Fe}_{\mathrm{e}} \\
{ }^{39} \mathrm{Cl}\end{array}$ & $\begin{array}{c}P \\
\text { cumule }\end{array}$ & $\begin{array}{l}\text { 1.5+2 } \\
\text { HVe OlE }\end{array}$ & E) $3.7+2$ & NRL & R Silberberg ${ }^{+}$ & 1844 \\
\hline $\begin{array}{l}\mathrm{Fe} \\
\mathrm{Cu}\end{array}$ & p & $\begin{array}{l}6.0+2 \\
6.0+2\end{array}$ & & $\begin{array}{l}\text { MTR } \\
\text { MTR }\end{array}$ & $\begin{array}{l}\text { E Clinet } \\
\text { E Clinet }\end{array}$ & $\begin{array}{l}1749+ \\
2483+\end{array}$ \\
\hline${ }^{39} \mathrm{Cl}$ & $O(E)$ & ' & & & & \\
\hline $\begin{array}{l}35 P \\
36 P \\
36 S \\
38 S \\
39 S \\
42 \mathrm{Ar}\end{array}$ & $\begin{array}{l}a \\
a \\
a \\
p \\
p \\
p\end{array}$ & $\begin{array}{l}\text { NDG } \\
\text { NDG } \\
\text { IR } \\
\text { NDG } \\
\text { NDG } \\
\text { NDG }\end{array}$ & $1.2+1$ & $\begin{array}{l}\text { CAL } \\
\text { CAL } \\
\text { CAL } \\
\text { CAL } \\
\text { CAL } \\
\text { CAL }\end{array}$ & $\begin{array}{l}\text { T Woosley+ } \\
\text { I Woosley+ } \\
\text { I Woogley+ } \\
\text { T Woosley+ } \\
\text { T Woosley+ } \\
\text { T Woosley+ }\end{array}$ & $\begin{array}{l}7145 \\
7146 \\
7167 \\
1090 \\
1092 \\
1182\end{array}$ \\
\hline${ }^{40} \mathrm{Cl}$ & cuaula & Ire olE & & & & \\
\hline $\begin{array}{l}\mathrm{Cu} \\
{ }^{40} \mathrm{Cl}\end{array}$ & $\begin{array}{r}P \\
\sigma(E)\end{array}$ & $6.0+2$ & & MTR & E Clinet & $2484+$ \\
\hline $36 p$ & a & NDG & & CAL & T Woogley+ & 7147 \\
\hline${ }^{37 p} p$ & - & NDG & & CAL & T Woosley+ & 7148 \\
\hline${ }^{38} \mathrm{~s}$ & p & NDG & & CAL & T Woosleyt & 1093 \\
\hline $40 \mathrm{~s}$ & p & NDG & & CAL & I Woogleyt & 1095 \\
\hline${ }^{43} \mathrm{Ar}$ & p & NDG & & CAL & T Woosleyt & 1184 \\
\hline $41 \mathrm{Cl}$ & $O(E)$ & & & & & \\
\hline $37 p$ & - & NDG & & CAL & T Woosleyt & 7149 \\
\hline $3 \theta^{2}$ & a & NDG & & CAL & T Woosleyt & 7150 \\
\hline${ }^{405}$ & p & NDG & & CAL & I Woosley+ & 1085 \\
\hline 415 & p & NDG & & CAL & T Woosleyt & 1098 \\
\hline${ }^{4} \mathrm{C} \mathrm{Cl}$ & $\sigma(E)$ & & & $\cdot$ & & \\
\hline $38 p$ & a & NDG & & CAL & I Woosleyt & 7151 \\
\hline 415 & p & NDG & & CAL & T Woosley+ & 1099 \\
\hline
\end{tabular}




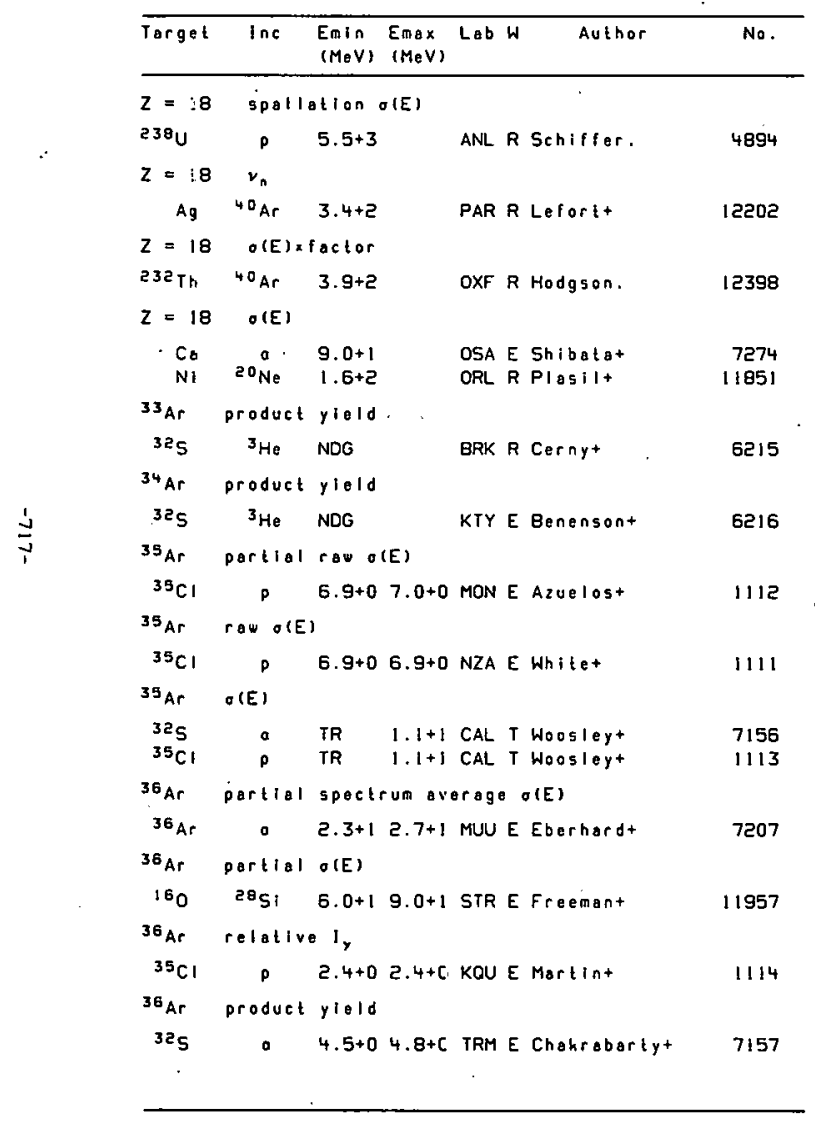

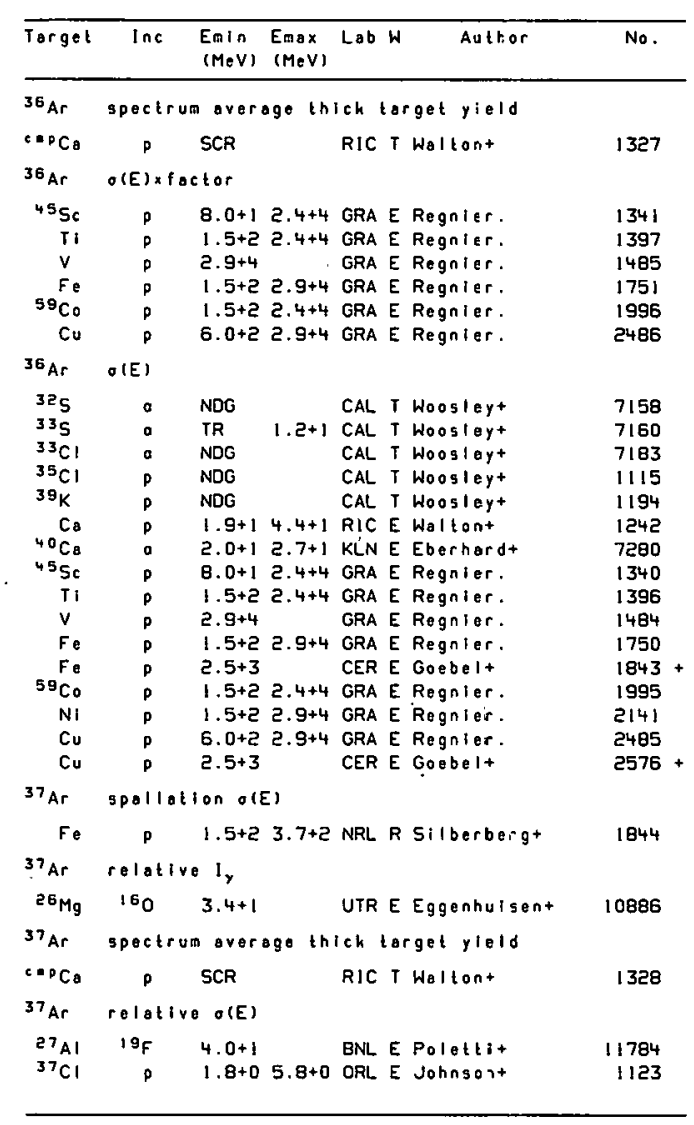




\begin{tabular}{|c|c|c|c|c|c|c|}
\hline Target & Inc & $\begin{array}{l}\text { Emin } \\
(\mathrm{MeV})\end{array}$ & $\begin{array}{l}\text { Emax } \\
(\mathrm{MeV})\end{array}$ & Lob & Author & No. \\
\hline${ }^{37} \mathrm{Ar}$ & $\sigma(E)$ & & & & & \\
\hline $27 \mathrm{Al}$ & 160 & $6 \cdot 01+1$ & & NEI & M Kuang-Shit & 10935 \\
\hline $27 \mathrm{Al}$ & 160 & $6.0+1$ & & NEI & M Kuang-Shit & 10937 \\
\hline $27_{A 1}$ & 160 & $5: 0+1$ & $6.0+1$ & $\mathrm{NBI}$ & M Kuang-Shit & 10936 \\
\hline${ }^{33} \mathrm{~s}$ & a & NDC & & CAL & T Woosleyt & 7161 \\
\hline 345 & a & $T R$ & $1.2+1$ & CAL. & T Woosleyt & 7163 \\
\hline${ }^{34} \mathrm{Cl}$ & a & NDO & & CAL & T Woosléyt & 7185 \\
\hline${ }^{36} \mathrm{Cl}$ & p & NOC & & CAL & T Woogleyt & 1117 \\
\hline${ }^{37} \mathrm{Cl}$ & p & TR & $1.0+1$ & CAL & T Woosleyt & 1119 \\
\hline${ }^{37} \mathrm{Cl}$ & p & TR & $5.0+0$ & ORL & E Johnsont & $1120+$ \\
\hline${ }^{37} \mathrm{Cl}$ & p & $1.6+0$ & $5.0+0$ & $\mathrm{KFK}$ & C Muenzelt & 1121 \\
\hline${ }^{37} \mathrm{Cl}$ & p & $2.5+0$ & $5.2+0$ & ORL & E Johnsont & 1122 \\
\hline${ }^{40} \mathrm{~K}$ & $p$ & NDO & & CAL & T Woosley+ & 1202 \\
\hline Co & p & $1.9+1$ & $4.4+1$ & RIC & E Waltont & 1243 \\
\hline $\mathrm{Fe}$ & $p$ & $2.5+3$ & & CER & E Goebelt & $1752+$ \\
\hline $\mathrm{Fe}$ & $p$ & $2.5+3$ & & CER & E Goebel+ & $1843+$ \\
\hline $\mathrm{Cu}$ & p & $2.5+3$ & & CER & E Goobelt & $2487+$ \\
\hline $\mathrm{Cu}$ & p & $2.5+3$ & & CER & E Goebelt & $2576+$ \\
\hline${ }^{38} \mathrm{Ar}$ & partial & cunula & attue & $\sigma(E)$ & & \\
\hline 150 & $28_{51}$ & $6.0+1$ & $9.0+1$ & STR & E Fremant & 11959 \\
\hline $38_{\text {Ar }}$ & spallati & ion $O(E$ & & & & v \\
\hline $\mathrm{Fe}$ & $p$ & $1.5+2$ & $3.7+2$ & NRL & R silberbergt & 1844 \\
\hline${ }^{38} \mathrm{Ar}$ & partiol & rovth & hick te & arget & $t$ yieldxabundance & \\
\hline${ }^{35} \mathrm{Cl}$ & a & $5.0+0$ & & SUN & E Gilest & 7187 \\
\hline${ }^{30} \mathrm{Ar}$ & partiol & ravor & & & & \\
\hline $2 \theta_{S i}$ & 160 & $5.0+1$ & $6.5+1$ & YAL & E Bettst & 10962 \\
\hline${ }^{30} \mathrm{Ar}$ & portlal & relati & ive ole & (E) & & \\
\hline $24 \mathrm{Mg}$ & 160 & $3 \cdot 3+1$ & $4.8+1$ & UTR & E Aortst & 10875 \\
\hline${ }^{3 \theta_{\mathrm{Ar}}}$ & portiol & $\theta(E)$ & & & & \\
\hline 150 & $285 i$ & $6.0+1$ & $9.0+1$ & STR & E Froemant & 11958 \\
\hline 38 Ar & spectrua & an overa & age th & ilck & target yleId & \\
\hline $\operatorname{COPMg}$ & $\mathrm{p}$ & $\mathrm{SCR}$ & & RIC & I Woltont & 794 \\
\hline CADAI & $p$ & SCR & & RIC & T Weltont & 955 \\
\hline$c=051$ & p & SCR & & RIC & I Wallont & 1016 \\
\hline
\end{tabular}

\begin{tabular}{lcccc}
\hline Target Inc Emin Emax Lab W Author & No. \\
& (MeV) $(M e V)$ & & \\
\hline
\end{tabular}

$38^{A r}$ raw OIE)

${ }^{37} \mathrm{Cl} P$ 5.0-1 $2.0+0$ AML E Paingt 1125

$38_{\text {Ar relative o(E) }}$

$27 \mathrm{Al}{ }^{19} \mathrm{~F} \quad 4.0+1 \quad$ BNLE Polett1+ 11785

$38_{\text {Ar }}$ oletafactor

${ }^{45} \mathrm{Sc} P \quad 8.0+12.4+4$ GRA E Regnier. 1343

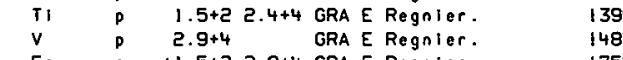

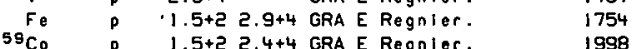

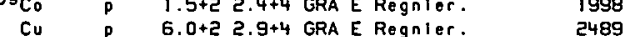

${ }^{30} \mathrm{Ar} O(E)$

$27 \mathrm{Al} 160 \mathrm{O} 5.0+16.0+1 \mathrm{NBI} M$ Kuang-Shit 10939

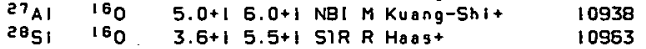

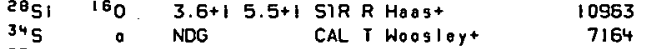

$\begin{array}{lllll}34 \mathrm{~S} & \text { a } & \text { NDG } & \text { CAL T Woosleyt } & 7164 \\ 35 \mathrm{~S} & 0 & \text { NDG } & \text { CAL T Woogleyt } & 7165\end{array}$

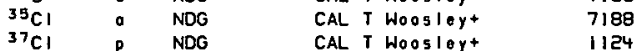

$\begin{array}{lllll}{ }^{37} \mathrm{Cl} & \text { D } & \text { NDG } & \text { CAL T Woosleyt } & 1124 \\ { }^{38} \mathrm{Cl} & \mathrm{p} & \text { NDG } & \text { CAL W Woosloyt } & 1127\end{array}$

$\begin{array}{lllll}{ }^{38} \mathrm{Cl} & \text { P } & \text { NDG } & \text { CAL T Woosleyt } & 1127 \\ 41 \mathrm{~K} & \mathrm{P} & \text { NDG } & \text { CAL T Woosleyt } & 1205\end{array}$

$\begin{array}{rllll}C_{B} & p & 1.9+1 & 4.4+1 \text { RIC E Waltont } & 1244 \\ { }^{45} \mathrm{Sc} & p & 8.0+12.4+4 \text { GRA E Regnier. } & 1342\end{array}$

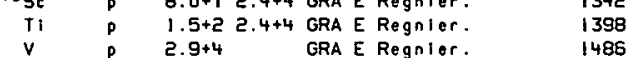

$\begin{array}{lllll}V & p & 2.9+4 & \text { GRA E Regnier. } & 1486 \\ \mathrm{Fe} & p & 1.5+2 & 2.9+4 \text { GRA E Regnier. } & 1753\end{array}$

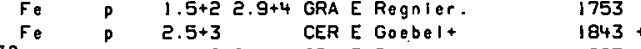

${ }^{39} \mathrm{Co} P$ P $1.5+22.4+4$ GRA E Regnier. 1997

Cu p $6.0+22.9+4$ GRA R Regnier.

Cu p $2.5+3$ CER E Goebel+ 2576 +

${ }^{39^{A}}$ spollation ole

Fe p $1.5+23.7+2$ NRL R Sllberberg+ 1844 ${ }^{39}$ ar spectrum average thlch target yield

OPTI P SCR RIC T WBltont 1482 


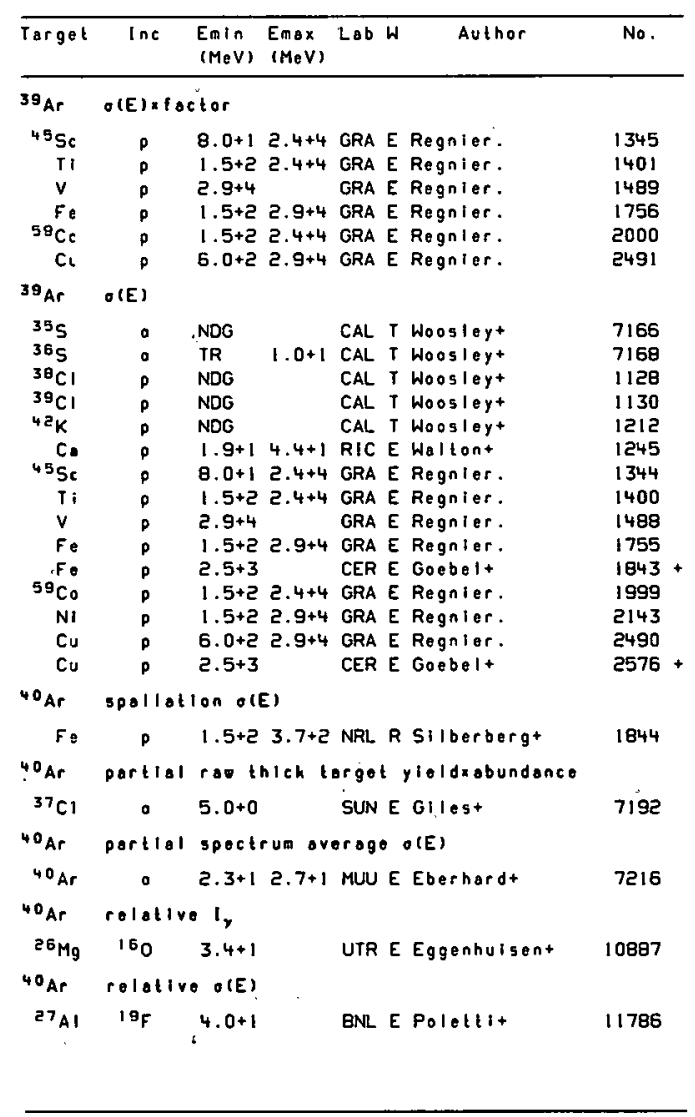

\begin{tabular}{|c|c|c|c|c|c|c|}
\hline Target & $\ln c$ & $\begin{array}{l}\text { Emin } \\
(\mathrm{MeV})\end{array}$ & $\begin{array}{l}\text { Emax } \\
(M e V)\end{array}$ & Lab W & Author & No. \\
\hline . ${ }^{A} \mathrm{ar}$ & $\sigma(E)$ & & & & & \\
\hline $\begin{array}{l}27 \mathrm{Al} \\
36 \mathrm{~S} \\
37 \mathrm{~S} \\
37 \mathrm{Cl} \\
39 \mathrm{Cl} \\
{ }^{40} \mathrm{Cl} \\
40_{\mathrm{Ar}} \\
43_{\mathrm{K}}\end{array}$ & $\begin{array}{r}160 \\
0 \\
a \\
0 \\
p \\
p \\
p \\
p\end{array}$ & $\begin{array}{l}5.0+1 \\
\text { NDG } \\
\text { NDG } \\
\text { TR } \\
\text { NDG } \\
\text { NDG } \\
2.4+1 \\
\text { NDG }\end{array}$ & $6.0+1$ & $\begin{array}{ll}\text { NBI } & M \\
\text { CAL } & T \\
\text { CAL } & T \\
\text { CAL } & T \\
\text { CAL } & T \\
\text { CAL } & T \\
\text { HAM T } \\
\text { CAL T }\end{array}$ & $\begin{array}{l}\text { Kuang-Shit } \\
\text { Woosleyt } \\
\text { Woogleyt } \\
\text { Woosleyt } \\
\text { Woosley+ } \\
\text { Woosleyt } \\
\text { Fitz. } \\
\text { Woosleyt }\end{array}$ & $\begin{array}{r}10940 \\
7169 \\
7170 \\
7193 \\
1131 \\
1133 \\
7217 \\
1215\end{array}$ \\
\hline "iar & cumula & ve spe & ollot & on $O(E)$ & & \\
\hline Cu & $p$ & $2.0+2$ & $5.9+2$ & LASE & ortht & 2577 \\
\hline
\end{tabular}

P $2.0+25.9+2$ LAS E Ortht

2577

- 'Aa spaliotion o(E)

Fe p $1.5+23.7+2$ NRL R Silberbergt 1844

41 Ar cumulallive ole

\begin{tabular}{|c|c|c|c|c|c|}
\hline $\mathrm{Fe}$ & $\dot{p}$ & $6.0+2$ & & MTR E Cline+ & $1757+$ \\
\hline "Ar & $\sigma(E)$ & & & & \\
\hline $37 \mathrm{~s}$ & 。 & NDG & & CAL T Woosley+ & 7171 \\
\hline $3 \theta_{s}$ & $a$ & NDG & & CAL T Woosles+ & 7172 \\
\hline${ }^{40} \mathrm{Cl}$ & p & NDG & & CAL T Hooslej+ & 1134 \\
\hline $41 \mathrm{Cl}$ & p & NDG & & CAL T Woosley+ & 1136 \\
\hline${ }^{44} \mathrm{~K}$ & $p$ & NDG & & CAL T Woosley+ & 1218 \\
\hline $\operatorname{siv}$ & d & TR & $9.0+1$ & JUL E Probst+ & 5452 \\
\hline
\end{tabular}

$42 \mathrm{Ar}$ gDallallion o(E)

Fe $D \quad 1.5+23.7+2$ NRL R S1Iberbergt 1844

$42 \mathrm{Ar} O(E) \times f a c t o r$

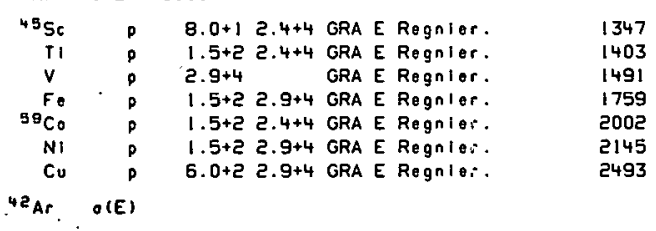

$\begin{array}{lll}{ }^{30} \mathrm{~S}^{\circ} \text { a NDG CAL T Woostert } & 7173\end{array}$ 


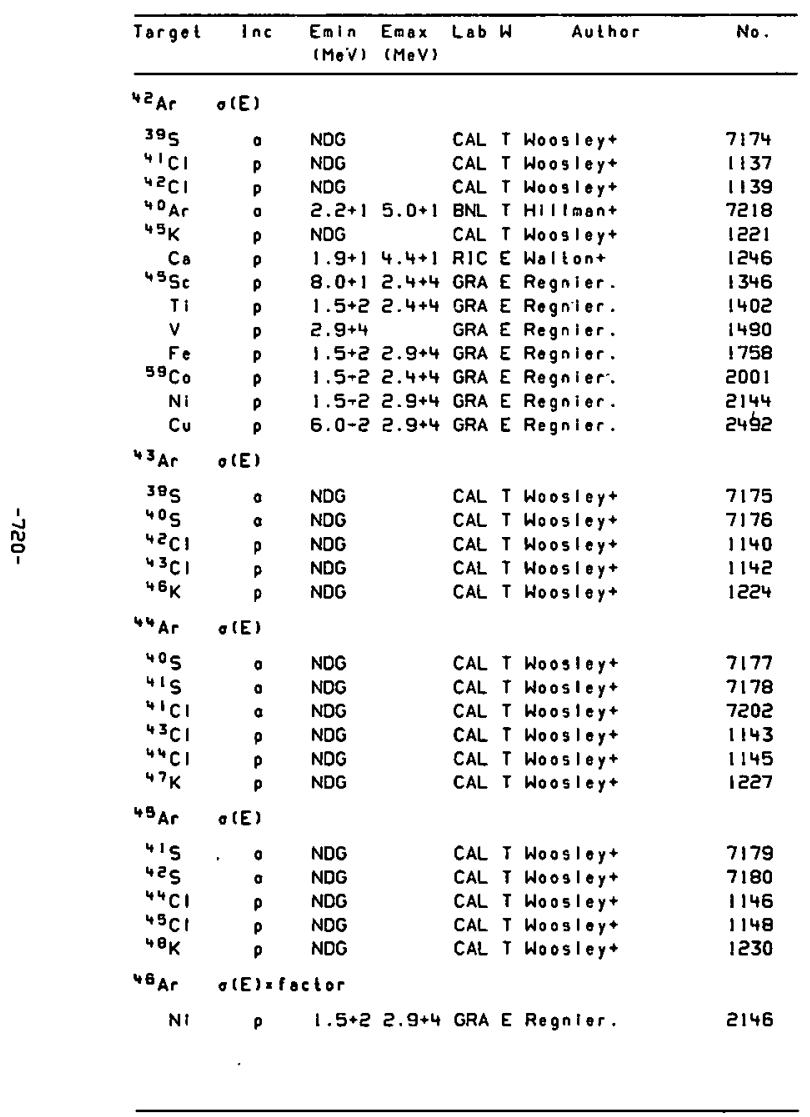

\begin{tabular}{|c|c|c|c|c|c|c|}
\hline Target & $\ln c$ & $\begin{array}{l}\text { Emin } \\
(\mathrm{MeV})\end{array}$ & $\begin{array}{l}\text { Enax } \\
(M e V)\end{array}$ & Lab & Author & No. \\
\hline${ }^{46} \mathrm{Ar}$ & $g(E)$ & & & & & \\
\hline $\begin{array}{l}425 \\
45 \mathrm{Cl} \\
49 \mathrm{~K}\end{array}$ & $\begin{array}{l}q \\
p \\
p\end{array}$ & $\begin{array}{l}\text { NDG } \\
\text { NDG } \\
\text { NDG }\end{array}$ & & $\begin{array}{l}\text { CAL } \\
\text { CAL } \\
\text { CAL }\end{array}$ & $\begin{array}{l}\text { T Woosleyt } \\
\text { T Woosleyt } \\
\text { T Woosleyt }\end{array}$ & $\begin{array}{l}718 ! \\
1149 \\
1233\end{array}$ \\
\hline $4 \mathrm{APr}$ & $g(E)=f$ & ctor & & & & \\
\hline${ }_{4 \mathrm{Ar}}^{\mathrm{NI}}$ & $\begin{array}{c}P \\
O(E) \times f\end{array}$ & $\begin{array}{l}1.5+2 \\
\text { ctor }\end{array}$ & $2.9+4$ & GRA & E Regnier. & 2147 \\
\hline $2=19$ & inde & $\begin{array}{l}1.5+2 \\
\text { endent }\end{array}$ & $\begin{array}{l}2.9+4 \\
\text { ovoros }\end{array}$ & $\begin{array}{l}\text { GRA } \\
\text { ge } k \text { i }\end{array}$ & $\begin{array}{l}\text { E Regnier. } \\
\text { inetic energyt }\end{array}$ & 2148 \\
\hline $\begin{array}{l}197 A U \\
Z=19\end{array}$ & $\begin{array}{l}40 \mathrm{Ar} \\
\text { inde }\end{array}$ & $\begin{array}{r}2.2+2 \\
\text { endont }\end{array}$ & $O(E ; E$ & $\begin{array}{l}\text { DUB } \\
\cdot ; \theta\}\end{array}$ & E Artukht & 12369 \\
\hline $\begin{array}{l}197 \mathrm{Au} \\
232 \mathrm{Th}\end{array}$ & $\begin{array}{l}40 \mathrm{Ar} \\
40_{\mathrm{Ar}}\end{array}$ & $\begin{array}{l}2.2+5 \\
3.0+5\end{array}$ & $3.9+2$ & $\begin{array}{l}\text { DUB } \\
\text { DUB }\end{array}$ & $\begin{array}{l}\text { E Artuhht } \\
\text { E Artukht }\end{array}$ & $\begin{array}{l}12368 \\
12396\end{array}$ \\
\hline$z=19$ & inde & endont & $\sigma(E ; \theta)$ & & & \\
\hline $\begin{array}{l}197_{A U} \\
Z=19\end{array}$ & $\begin{array}{l}40 \mathrm{Ar} \\
\text { inde }\end{array}$ & $\begin{array}{r}2.2+2 \\
\text { endent }\end{array}$ & $\sigma(E)$ & DUB & E Artukht & 12367 \\
\hline $\begin{array}{l}197 \mathrm{AU} \\
2=19\end{array}$ & $\begin{array}{c}{ }^{40} \mathrm{Ar} \\
v_{n}\end{array}$ & $2.2+2$ & & DUB & E Arlukht & 12366 \\
\hline$A g$ & ${ }^{40} \mathrm{Ar}$ & $3.4+2$ & & PAR & R Lefort+ & 12202 \\
\hline $2=19$ & $O(E)$ & foctor & & & & \\
\hline $\begin{array}{l}232 \mathrm{Th} \\
2=19\end{array}$ & $\begin{array}{l}4 O_{A r} \\
O(E)\end{array}$ & $3.9+2$ & & oxf & $R$ Hodgson. & 12398 \\
\hline $\begin{array}{l}\mathrm{C}_{B} \\
\mathrm{Ni}\end{array}$ & $20^{\circ} \mathrm{Ne}$ & $\begin{array}{l}9.0+1 \\
1.6+2\end{array}$ & & $\begin{array}{l}\text { OSA } \\
\text { ORL }\end{array}$ & $\begin{array}{l}\text { E Shibatat } \\
\text { R Plasllt }\end{array}$ & $\begin{array}{r}7274 \\
11851\end{array}$ \\
\hline $36 \mathrm{k}$ & $O(E)$ & & & & & \\
\hline${ }^{36_{\mathrm{Ar}}}$ & $O(E)^{D}$ & TR & $1.0+1$ & CAL & T Woogleyt & 1156 \\
\hline $\begin{array}{l}{ }^{33} \mathrm{Cl} \\
{ }^{36} \mathrm{Ar} \\
{ }^{40} \mathrm{Co}\end{array}$ & $:$ & $\begin{array}{l}\text { NDG } \\
\text { NDG } \\
\theta .2-1\end{array}$ & $1.6+1$ & $\begin{array}{l}\text { CAL } \\
\text { CAL } \\
\text { CAL }\end{array}$ & $\begin{array}{l}\text { I Woogley+ } \\
\text { T Woosley+ } \\
\text { T Woogley+ }\end{array}$ & $\begin{array}{l}7184 \\
1157 \\
1258\end{array}$ \\
\hline
\end{tabular}




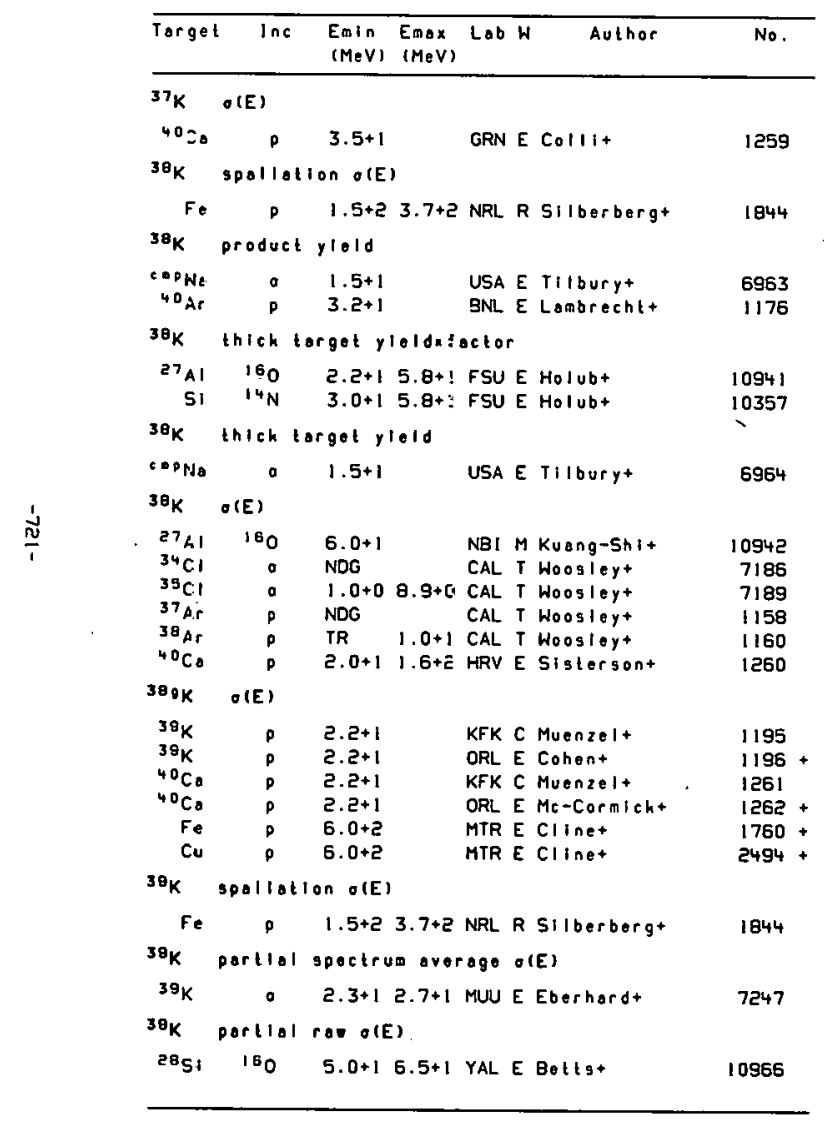

\begin{tabular}{|c|c|c|c|c|c|c|}
\hline Target & $\ln c$ & $\begin{array}{l}\text { Emin } \\
(\operatorname{MeV})\end{array}$ & $\begin{array}{l}\text { EqBx } \\
(M e V)\end{array}$ & Lob & Adthor & No. \\
\hline${ }^{39_{k}}$ & partlolo & $o(E)$ & & & & \\
\hline 160 & ${ }^{2 \theta} \mathrm{si}$ & $6.0+1$ & $9.0+1$ & STR E & E Freemant & 11960 \\
\hline${ }^{39} \mathrm{~K} \quad r$ & relative & $1 y$ & & & & \\
\hline $285 i$ & 180 & $4.5+1$ & & UTR E & E Eggenhuisent & 10965 \\
\hline${ }^{3 \theta_{K}}$ & $O(E)$ & & & & & \\
\hline $39_{K}$ & $p$ & $5.7+0$ & $6.0+0$ & SUN E & E Standert & 1197 \\
\hline $38_{k}$ & $O(E)$ & & & & & \\
\hline $\begin{array}{l}28 \mathrm{SI} \\
35 \mathrm{Cl} \\
36 \mathrm{Ar} \\
38_{\mathrm{Ar}} \\
{ }^{40} \mathrm{Co} \\
{ }^{42} \mathrm{Co}\end{array}$ & $\begin{array}{r}160 \\
a \\
a \\
p \\
a \\
p\end{array}$ & $\begin{array}{l}3.1+1 \\
\text { NDG } \\
\text { TR } \\
\text { NDG } \\
2.0+1 \\
\text { NDG }\end{array}$ & $\begin{array}{l}5.5+1 \\
1.1+1 \\
2.7+1\end{array}$ & $\begin{array}{l}\text { STR R } \\
\text { CAL T } \\
\text { CAL T } \\
\text { CAL T } \\
\text { KLN E } \\
\text { CAL T }\end{array}$ & $\begin{array}{l}\text { R Haast } \\
\text { I Woogleyt } \\
\text { T Woosleyt } \\
\text { T Woosley+ } \\
\text { E Eberhard+ } \\
\text { T Woosleyt }\end{array}$ & $\begin{array}{r}10964 \\
7190 \\
7208 \\
1161 \\
7281 \\
1275\end{array}$ \\
\hline${ }^{40} \mathrm{~K}$ & gpollatlo & on $O(E)$ & & & & \\
\hline${ }^{40} \mathrm{Fe}$ & $\begin{array}{c}\text { polative } \\
\text { relot }\end{array}$ & $\begin{array}{l}1.5+2 \\
1,\end{array}$ & $3.7+2$ & NRL R & R Silberberg+ & 1844 \\
\hline${ }^{26}{ }^{26} \mathrm{Kg}$ & $\begin{array}{c}160 \\
\text { relalive }\end{array}$ & $\begin{array}{l}2.6+1 \\
\sigma(E)\end{array}$ & $4.9+1$ & UTR E & E Eggonhuisent & 10888 \\
\hline${ }^{27} \mathrm{~K} \mathrm{~K}$. & $\begin{array}{l}19 \mathrm{~F} \\
O(E)\end{array}$ & $+.0+1$ & & BNL E & E Polettit & 11787 \\
\hline $\begin{array}{l}27 \mathrm{Al} \\
27 \mathrm{Al} \\
3 \mathrm{C}^{6} \mathrm{Cl} \\
37 \mathrm{Cl} \\
39_{\mathrm{AP}} \\
{ }^{40} \mathrm{Ar} \\
{ }^{4} \mathrm{CB}\end{array}$ & $\begin{array}{r}160 \\
180 \\
0 \\
0 \\
p \\
p \\
0\end{array}$ & $\begin{array}{l}6.0+1 \\
5.0+1 \\
\text { NDG } \\
\text { B.9-1 } \\
\text { NDG } \\
\text { TR } \\
\text { NDG }\end{array}$ & $\begin{array}{l}6.0+1 \\
8.6+0 \\
1.0+1\end{array}$ & $\begin{array}{ll}\text { NBI } & M \\
\text { NBI } & M \\
\text { CAL } & T \\
\text { CAL } & T \\
\text { CAL } & T \\
\text { CAL } & \text { T } \\
\text { CAL } & \text { T }\end{array}$ & $\begin{array}{l}\text { M Kuang-jhit } \\
\text { M Kuang-5hit } \\
\text { T Woosley+ } \\
\text { T Woosley+ } \\
\text { T Wooslert } \\
\text { T Wooslegt+ } \\
\text { T Wooslegt+ }\end{array}$ & $\begin{array}{r}10944 \\
10943 \\
7191 \\
7194 \\
1163 \\
1177 \\
1284\end{array}$ \\
\hline $41 \mathrm{~K} \quad 9$ & spallatlo & on $O(E)$ & & & & \\
\hline${ }^{4} \mathrm{~K}^{\mathrm{Ke}}$ & $\begin{array}{c}p \\
\text { partial o }\end{array}$ & $\begin{array}{l}1.5+2= \\
o(E),\end{array}$ & $3.7+2$ & NRL $R$ & R Silberbergt & 1844 \\
\hline $41 k$ & 160 & $2.2+1=$ & $2.4+1$ & LVP E & E Lister- & 10982 \\
\hline
\end{tabular}




\begin{tabular}{|c|c|c|c|c|c|c|}
\hline Target & $\operatorname{Inc}$ & $\begin{array}{l}\text { Emin } \\
\text { (Meiv) }\end{array}$ & $\begin{array}{l}E_{\max } \\
(\mathrm{MeV})\end{array}$ & Lab W & Author & No. \\
\hline $41_{k}$ & relativa & $\mathrm{I}_{y}$ & & & & \\
\hline $26 \mathrm{Mg}$ & 180 & $3.4+1$ & & UTR E & Eggenhulsent & 11655 \\
\hline$n^{\prime} k$ & $O(E)$ & & & & & \\
\hline $4 / K$ & p & $5.7+0$ & $6.0+0$ & SUN E & E Standert & 1206 \\
\hline${ }^{41} k$ & relative & $O(E)$ & & & & \\
\hline $27 \mathrm{Al}$ & $19_{F}$ & $4.0+1$ & & BNL E & E Poletti+ & 11788 \\
\hline $4{ }^{1} k$ & $O(E)$ & & & & & \\
\hline $27_{A 1}$ & 160 & $5.0+1$ & $6.0+1$ & NBI $M$ & M Kueng-Shit & 10945 \\
\hline${ }^{37} \mathrm{Cl}$ & $a$ & NDE & & CAL $T$ & T Woosleyt & 7195 \\
\hline${ }^{37} \mathrm{Cl}$ & a & $2.5+0$ & $5.4+0$ & CAL E & E Zyskindt & 7196 \\
\hline${ }^{38} \mathrm{Cl}$ & a & NDE & & CAL T & T Woosleyt & 7197 \\
\hline${ }^{38} \mathrm{Ar}$ & a & $T R$ & $1.1+1$ & CAL $T$ & T Woosleyt & 7212 \\
\hline $40 \mathrm{Ar}$ & $p$ & NDG & & CAL T & T Woosleyt & 1178 \\
\hline${ }^{41} \mathrm{Ar}$ & $p$ & NDG & & CAL T & T Woosleyt & 1180 \\
\hline${ }^{44} \mathrm{Cg}$ & p & $5 \cdot 1-1$ & $1.1+1$ & CAL $T$ & T Woosleyt & 1292 \\
\hline \multicolumn{7}{|c|}{ cunulative spallation o(E) } \\
\hline Cu & D & $2.0+2$ & $5.9+2$ & LAS E & E ortht & 2577 \\
\hline \multicolumn{7}{|c|}{ spallation o(E) } \\
\hline $\mathrm{Fe}$ & p & $1.5+2$ & $3.7+2$ & NRL R & R silberbergt & 1844 \\
\hline \multicolumn{7}{|c|}{ cumulative? o(E) } \\
\hline $\begin{array}{l}\mathrm{Fo} \\
\mathrm{Cu}\end{array}$ & p & $\begin{array}{l}5.9+2 \\
5.9+2\end{array}$ & & $\begin{array}{l}\text { LAS E } \\
\text { LAS E }\end{array}$ & $\begin{array}{l}\text { E Ortht } \\
\text { E Ortht }\end{array}$ & $\begin{array}{l}1762+ \\
2496+\end{array}$ \\
\hline \multicolumn{7}{|c|}{ thick torget yieldxfactor } \\
\hline si & $14 \mathrm{~N}$ & $2.5+1$ & $5.8+1$ & FSU E & E Holubt & 10358 \\
\hline \multicolumn{7}{|c|}{ thich target yield } \\
\hline $40 \mathrm{Ar}$ & $a$ & $1.7+1$ & $2.8+1$ & usv c & $C$ Bent & 7219 \\
\hline${ }^{4} 2_{K}$ & relative & $\sigma(E)$ & & & & . \\
\hline $27 \mathrm{Al}$ & $19 \mathrm{~F}$ & $4.0+1$ & & BNLE & E Poleltl+ & 11789 \\
\hline $4 a_{k}$ & $O(E)$ & & & & & \\
\hline${ }^{36} \mathrm{Cl}$ & a & NDG & & CAL T & T Woosley+ & 7198 \\
\hline${ }^{40} \mathrm{Ar}$ & a & $2.4+1$ & & HAM T & $T F t i 2$. & 7220 \\
\hline
\end{tabular}

\begin{tabular}{|c|c|c|c|c|c|c|}
\hline Target & $\ln c$ & $\begin{array}{l}\text { Emin } \\
(M e V)\end{array}$ & $\begin{array}{l}\text { Emax } \\
(\mathrm{MeV})\end{array}$ & Lab & Author & No. \\
\hline $4 e_{K}$ & \multicolumn{6}{|c|}{$\theta(E)$} \\
\hline $41 \mathrm{Ar}$ & p & NDG & & CAL & T Woosleyt & 1181 \\
\hline${ }^{4} 3 \mathrm{Ca}$ & p & $2.2+1$ & & KFK & C Muenzel+ & 1285 \\
\hline${ }^{43} \mathrm{Ca}$ & p & $2.2+1$ & & ORL & E Cohent & $1286+$ \\
\hline${ }^{4} \mathrm{Ca}$ & p & $2.2+1$ & & KFK & C Muenzelt & 1293 \\
\hline${ }^{4} 4 \mathrm{Co}$ & $p$ & $2.2+1$ & & ORL & E Mc-Cormicht & 1294 \\
\hline${ }^{45} \mathrm{Se}$ & p & $1.0+1$ & $8.5+1$ & MCG & E Mc-Geet & 1348 \\
\hline${ }^{45} \mathrm{Sc}$ & p & $4.0+1$ & $8.5+1$ & KFK & C Muenzelt & 1349 \\
\hline & 0 & NDG & & KLN & E Herrt & 1404 \\
\hline $31 \mathrm{~V}$ & d & $\mathrm{TR}$ & $9.0+1$ & JUL & EProbst+ & 5452 \\
\hline $\mathrm{Fe}$ & p & $6.0+2$ & & MTR & E Clinet & 1761 \\
\hline $\mathrm{Ni}$ & p & $6.0+2$ & & MTR & E Clinet & $2149+$ \\
\hline $\mathrm{Cu}$ & p & $6.0+2$ & & MTR & E Clinet & 2495 \\
\hline
\end{tabular}

$43 \mathrm{~K}$ cumulative spollation o(E)

Cu $P \quad 2.0+25.9+2$ LAS E Ortht 2577

$43 \mathrm{~K}$ spallotion o(E)

Fe $P \quad 1.5+23.7+2$ NRL R Stlberberg+ 1844 $43 \mathrm{~K}$ cumulative? $O(E)$

Tr $P$ S $3.5+14.5+1$ KLN E Michelt 1406 +

$43_{\mathrm{K}}$ cunulative o(E)

$\begin{array}{lllll}\mathrm{Fe} & \mathrm{p} & 5.9+2 & \text { LASE Oriht } & 1763+ \\ \mathrm{Fe} & \mathrm{p} & 6.0+2 & \text { MTRE Clinet } & 1764+ \\ \mathrm{Ni} & \mathrm{p} & 6.0+2 & \text { MTRE Cllnet } & 2150+ \\ \mathrm{Cu} & \mathrm{p} & 5.9+2 & \text { LASE Oriht } & 2497+ \\ \mathrm{Cu} & \mathrm{P} & 6.0+2 & \text { MTRE Clinet } & 2498+\end{array}$

$4{ }^{3} \mathrm{~K}$ thich target yleldxabundance

${ }^{40} \mathrm{Ar}$ a $6.0+11.0+2 \mathrm{KFKE} \mathrm{Helus+} 7226$

${ }^{43} \mathrm{~K}$ thick target yleld

${ }_{40}^{40} \mathrm{Ar} \quad 1.1+12.6+1$ UJV C Bemt $\quad 7221$

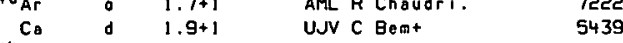

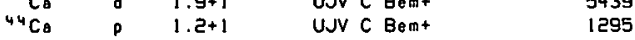

${ }^{43} \mathrm{~K}$ O(E)

$\begin{array}{llll}{ }^{39} \mathrm{CI} \text { a NDG CAL T Woosleyt } & 7199\end{array}$ 


\begin{tabular}{|c|c|c|c|c|c|c|}
\hline Torget & $\ln c$ & $\begin{array}{l}\text { EmIn } \\
\text { (MeV) }\end{array}$ & $\begin{array}{l}\text { Emex } \\
(\operatorname{MeV})\end{array}$ & Lab & Author & No. \\
\hline${ }^{43} \mathrm{~K}$ & \multicolumn{6}{|l|}{$\sigma(E)$} \\
\hline${ }^{40} \mathrm{Cl}$ & a & NDG & & CAL & T Woosleyt & 7200 \\
\hline $40 \mathrm{Ar}$ & $a$ & TR & $1,1+1$ & CAL & I Woosleyt & 7223 \\
\hline${ }^{40} \mathrm{Ar}$ & a & $1.2+1$ & $4 \cdot c+1$ & JUL' & $R$ Qaimt & $722^{4}$ \\
\hline${ }^{40} \mathrm{Ar}$ & a & $2.4+1$ & & HAM & T Fitz. & 7225 \\
\hline $42_{\mathrm{Ar}}$ & p & NDG & & CAL & T Woosleyt & 1183 \\
\hline${ }^{43} \mathrm{Ar}$ & p & NDG & & CAL & T Woosleyt & 1185 \\
\hline${ }^{44} \mathrm{CB}_{\mathrm{B}}$ & p & $2.5+1$ & & KFK & C Muenzelt & 1296 \\
\hline${ }^{44} \mathrm{Co}$ & p & $2.2+1$ & & ORL & E Cohent & $1297+$ \\
\hline${ }^{44} \mathrm{Ca}$ & a & $2.0+1$ & $2.7+1$ & KLN & E Eberhardt & 7317 \\
\hline${ }^{46} \mathrm{CO}$ & p & $5.1-1$ & $1.2+1$ & CAL & T Woosleyt & 1303 \\
\hline${ }^{45} \mathrm{Se}$ & p & $1.0+1$ & $8.5+1$ & MCG & E Mc-Gee+ & $1350+$ \\
\hline${ }^{45} \mathrm{Sc}$ & p & $4.0+1$ & $8.5+1$ & KFK & C Muenzelt & $135 \mid$ \\
\hline Ti & p & NDG & & KLN & E Herrt & 1405 \\
\hline$T_{1}^{T}$ & d & $5.0+1$ & $8.5+1$ & JUL & R Oaimt & 5444 \\
\hline $51 \mathrm{v}$ & d & TR & $9.0+1$ & JUL & E Probst+ & 5452 \\
\hline \multicolumn{7}{|c|}{${ }^{44} \mathrm{~K} \quad O(E)$} \\
\hline${ }^{40} \mathrm{Cr}$ & a & NDG & & CAL & T Woosleyt & 7201 \\
\hline${ }^{41} \mathrm{Cl}$ & 0 & NDG & & CAL & T Woosleyt & 7203 \\
\hline${ }^{43} \mathrm{Ar}$ & p & NDG & & CAL & T Woosleyt & 1186 \\
\hline${ }^{44} \mathrm{Ar}$ & p & NDG & & CAL & T Woosleyt & 1187 \\
\hline \multicolumn{7}{|c|}{${ }^{49} K \quad O(E)$} \\
\hline $41 \mathrm{Cl}$ & $a$ & NDG & & CAL & T Woosleyt & 7204 \\
\hline${ }^{42} \mathrm{Cl}$ & 0 & NDG & & CAL & T Woosleyt & 7205 \\
\hline${ }^{44} \mathrm{Ar}$ & p & NDG & & CAL & T Woosleyt & 1188 \\
\hline${ }^{45} \mathrm{Ar}$ & $p$ & NDG & & CAL & T Woosley+ & 1189 \\
\hline${ }^{48} \mathrm{Co}$ & p & $5.1-1$ & $1 \cdot 3+1$ & CAL & T Woosleyt & 1309 \\
\hline \multicolumn{7}{|l|}{$46_{K}$} \\
\hline${ }^{42} \mathrm{Cl}$ & 。 & NDG & & CAL & T Woosleyt & 7206 \\
\hline Ar & p & NOG & & CAL & T Woosleyt & 1190 \\
\hline \multicolumn{7}{|c|}{ portiol o(E) } \\
\hline${ }^{48} \mathrm{Co}$ & 160 & $5.6+1$ & & ANL & E Kovart & 11078 \\
\hline$z=20$ & Indep & enden & avera & $g \circ k i$ & Inetlc energ & \\
\hline $197 \mathrm{Au}$ & $40 \mathrm{Ar}$ & $2.2+2$ & & DUB & E Artuhht & 12369 \\
\hline
\end{tabular}

\begin{tabular}{|c|c|c|c|c|c|c|}
\hline Target & $\ln c$ & $\begin{array}{l}\text { Emin } \\
(M e V)\end{array}$ & $\begin{array}{l}\text { Emax } \\
(\operatorname{MeV})\end{array}$ & Lab W & 4u!hor & No. \\
\hline$z=20$ & Indepen & indent & $O(E ; E)$ & $\because 81$ & & . \\
\hline $\begin{array}{l}197 \mathrm{Au} \\
232 \mathrm{Th}\end{array}$ & $\begin{array}{l}{ }^{40} \mathrm{Ar} \\
{ }^{40} \mathrm{Ar}\end{array}$ & $\begin{array}{l}2.2+2 \\
3.0+2\end{array}$ & $3.9+2$ & $\begin{array}{l}\text { DUB E } \\
\text { DUB E }\end{array}$ & $\begin{array}{l}\text { E Artuah- } \\
\text { E Artuah- }\end{array}$ & $\begin{array}{l}12368 \\
12396\end{array}$ \\
\hline$z=20$ & indepen & endent & $O(E ; \theta)$ & & & \\
\hline $197_{\mathrm{AU}}$ & ${ }^{40} \mathrm{Ar}$ & $2.2+2$ & & DUB E & E Artusht & 12367 \\
\hline$z=20$ & Indepen & endent & $O(E)$ & & & \\
\hline $197 \mathrm{AU}$ & ${ }^{40} \mathrm{Ar}$ & $2.2+2$ & & OUB E & E Artukht & 12366 \\
\hline$z=20$ & $v_{n}$ & & & & & \\
\hline Ag & ${ }^{40} \mathrm{Ar}$ & $3.4+2$ & & PAR $R$ & R Lefort+ & 12202 \\
\hline$z=20$ & $\sigma(E) \times f_{0}$ & factor & & & & \\
\hline $232 \mathrm{Th}$ & ${ }^{40} \mathrm{Ar}$ & $3.9+2$ & & OXF R & R Hodgsor. & 12398 \\
\hline$z=20$ & $\theta(\varepsilon)$ & & & & & \\
\hline $\begin{array}{l}\mathrm{Ca} \\
\mathrm{Ni}\end{array}$ & $20_{\mathrm{Ne}}^{\circ}$ & $\begin{array}{l}9.0+1 \\
1.6+2\end{array}$ & & $\begin{array}{l}\text { OSA E } \\
\text { ORL R }\end{array}$ & $\begin{array}{l}\text { E Shibata+ } \\
\text { R Plasile }\end{array}$ & $\begin{array}{r}7274 \\
11851\end{array}$ \\
\hline${ }^{37} \mathrm{Co}$ & product $y$ & $y / 01 d$ & 、 & & & \\
\hline${ }^{36} \mathrm{Ar}$ & ${ }^{3} \mathrm{He}$ & NDG & & ERK $R$ & R Cerny+ & 6219 \\
\hline${ }^{39} \mathrm{Co}$ & row $O(E)$ & & & & & \\
\hline${ }^{39} \mathrm{~K}$ & $p$ & $7.5+0$ & $7.5+0$ & MON E & E RoO+: & 1198 \\
\hline${ }^{39} \mathrm{Cs}$ & $O(E)$ & & & & & , \\
\hline $\begin{array}{l}36 \mathrm{Ar} \\
39_{\mathrm{K}}\end{array}$ & $\dot{p}$ & $\begin{array}{l}\text { TR } \\
\text { TR }\end{array}$ & $\begin{array}{l}1.7+1 \\
1.0+1\end{array}$ & $\begin{array}{l}\text { CAL T } \\
\text { CAL T }\end{array}$ & $\begin{array}{l}\text { T Woosleyt } \\
\text { T Woos liey+ }\end{array}$ & $\begin{array}{l}7209 \\
1199\end{array}$ \\
\hline${ }^{40} \mathrm{Co}$ & compound & d-nucle & eus pro & oduet & $y / e 1 d$ & \\
\hline $\begin{array}{l}{ }^{40} \mathrm{CO} \\
{ }^{4}{ }^{0} \mathrm{Ca}\end{array}$ & $\begin{array}{l}p \\
p\end{array}$ & $\begin{array}{l}7.2+0 \\
7.2+0\end{array}$ & & $\begin{array}{l}\text { OSU E } \\
\text { OSU E }\end{array}$ & $\begin{array}{l}\text { E Hausmant } \\
\text { E Housmant }\end{array}$ & $\begin{array}{l}1265 \\
1266\end{array}$ \\
\hline${ }^{4}{ }^{\circ} \mathrm{Co}$ & portllol & compou & und-nue & cleus & $O(E)$ & \\
\hline${ }^{28} 51$ & 160 & $2.4+1$ & $4.6+11$ & MUN E & E Appe I+ & 10967 \\
\hline${ }^{40} \mathrm{Ca}$ & spallatlo & Ion of & & & & \\
\hline
\end{tabular}

197AU ${ }^{40} \mathrm{Ar} 2.2+2$ DUB E Artuhht $12369+$

$\mathrm{Fe} \quad \mathrm{P} \quad 1.5+23.7+2 \mathrm{NRL} R$ silberbergt

1844 


\begin{tabular}{|c|c|c|c|c|c|c|}
\hline Target & $\operatorname{lnc}$ & $\begin{array}{l}\text { Emin } \\
(\mathrm{MeV})\end{array}$ & $\begin{array}{l}\text { Emax } \\
(\operatorname{MeV})\end{array}$ & Lab & Author & No. \\
\hline${ }^{40} \mathrm{Ca}$ & portial & spectr & rum ave & erage & $O(E)$ & \\
\hline${ }^{40} \mathrm{Ca}$ & - & $2 \cdot 3+1$ & $2.7+1$ & MuU & E Eberhardt & 7282 \\
\hline $4^{\circ} \mathrm{Co}$ & partlol & overag & $g \circ O(E)$ & & & \\
\hline${ }^{40} \mathrm{Ca}$ & $\circ$ & $2.0+1$ & $6.5+1$ & UBC & T Brinht & 7283 \\
\hline${ }^{40} \mathrm{Co}$ & portlol & $\sigma(E) \times f$ & factor & & & \\
\hline${ }^{46} \mathrm{TI}$ & $p$ & $4.2+1$ & & FIN & E Freedmant & 1419 \\
\hline${ }^{40} \mathrm{Co}$ & parllol & $O(\varepsilon)$ & & & & \\
\hline $\begin{array}{l}{ }^{40} \mathrm{Cs} \\
{ }^{40} \mathrm{Co} \\
{ }^{40} \mathrm{Co} \\
{ }^{40} \mathrm{Co}\end{array}$ & $\begin{array}{r}p \\
p \\
130 \\
13 C\end{array}$ & $\begin{array}{l}5 \cdot 5+0 \\
2 \cdot 0+1 \\
1.0+1 \\
4.0+1\end{array}$ & $\begin{array}{l}1 \cdot 1+1 \\
1 \cdot 5+2 \\
2 \cdot 7+1 \\
7.0+1\end{array}$ & $\begin{array}{l}\text { FIC } \\
\text { SUL } \\
\text { KLN } \\
\text { ENL }\end{array}$ & $\begin{array}{l}\text { I Brown. } \\
\text { I Wombacht } \\
\text { E Eberhardt } \\
\text { T Fuller. }\end{array}$ & $\begin{array}{r}1263 \\
1264 \\
7285 \\
10199\end{array}$ \\
\hline${ }^{40} \mathrm{Cs}$ & relative & - $r_{j}$ & & & & \\
\hline${ }^{40} \mathrm{Ca}$ & $\circ$ & $1.4+1$ & $2.4+1$ & ANL & E Meyer-Schutzmei & 7286 \\
\hline${ }^{40} \mathrm{Co}$ & product & $y=\theta$ id & & & . & \\
\hline${ }^{36} \mathrm{Ar}$ & ${ }^{6} L_{1}$ & NOG & & P.OC & E Anontarament & 9181 \\
\hline${ }^{40} \mathrm{Co}$ & rop O(E) & & & & & \\
\hline $39_{k}$ & $p$ & $5.0-1$ & $2.0+0$ & AML & E Paine+ & 1201 \\
\hline${ }^{40} \mathrm{Co}$ & overage & $O|E|$ & & & & \\
\hline${ }^{40} \mathrm{Co}$ & a & $2.0+1$ & $2.6+1$ & WAU & E Eberhardt & 7284 \\
\hline${ }^{40} \mathrm{Co}$ & relotive & - $O(E)$ & & & & \\
\hline $\begin{array}{l}27 \mathrm{Al} \\
{ }^{40} \mathrm{CA}\end{array}$ & $\begin{array}{r}19 \\
F\end{array}$ & $\begin{array}{l}4.0+1 \\
1.4+1\end{array}$ & $2.4+1$ & $\begin{array}{l}\text { ENL } \\
\text { ANL }\end{array}$ & $\begin{array}{l}\text { E Polettit } \\
\text { E Meyer-5chutzmei }\end{array}$ & $\begin{array}{r}11790 \\
7280\end{array}$ \\
\hline${ }^{40} \mathrm{Co}$ & $\sigma(E)$ & & & & & \\
\hline $208 i$ & 160 & $2.5+1$ & $4.5+1$ & MUN & E singht & 10968 \\
\hline${ }^{36} \mathrm{Ar}$ & a & TR & $1.7+1$ & CAL & T Woosley+ & 7210 \\
\hline $37 \mathrm{~K}$ & a & NOG & & CAL & T Woosleyt & 7243 \\
\hline $39 \mathrm{~K}$ & p & NDG & & CAL & I Hoosleyt & 1200 \\
\hline${ }^{40} \mathrm{~K}$ & p & NDG & & CAL & T Woosleyt & 1203 \\
\hline${ }^{40} \mathrm{CO}$ & $a$ & $2.4+1$ & & HAM & T Fitz. & 7287 \\
\hline${ }^{40} \mathrm{Co}$ & 160 & $5.6+1$ & & ANL & E vigdort & 110031 \\
\hline${ }^{40} \mathrm{Co}$ & ${ }^{16} 0$ & $5.6+1$ & & HEI & R zeidanan. & 11004 \\
\hline${ }^{43} \mathrm{5c}$ & $p$ & NDG & & CAL & T Woosleyt & 1331 \\
\hline
\end{tabular}

\begin{tabular}{|c|c|c|c|c|c|c|}
\hline Target & $\ln e$ & $\begin{array}{l}\text { Emin } \\
(\mathrm{MeV})\end{array}$ & $\begin{array}{l}\text { Emax } \\
(\mathrm{MeV})\end{array}$ & Lab W & Author & No. \\
\hline${ }^{41} \mathrm{C}_{0}$ & \multicolumn{6}{|c|}{ spotlation o(E) } \\
\hline $\mathrm{Fe}$ & $p$ & $1.5+2$ & $3.7+2$ & NRL $R$ & R silberbergt & 1844 \\
\hline${ }^{41} \mathrm{Co}$ & portiol & row oll & (E) & & & \\
\hline $28_{5 i}$ & 150 & $5.0+1$ & $6.5+1$ & YAL E & E Betist & 10970 \\
\hline${ }^{41} \mathrm{Co}$ & portiol & relatil & I ve OLE & & & \\
\hline $27 \mathrm{Al}$ & 160 & $2.5+1=$ & $5 \cdot 0+1$ & KLN T & T Hellmeistert & 10947 \\
\hline${ }^{4} \mathrm{CO}$ & portlot & $\theta(E)$ & & & & \\
\hline 160 & ${ }^{28} \mathrm{SI}$ & $6.0+1$ & $9.0+1$ & STR E & E Freemont & 11961 \\
\hline${ }^{41} \mathrm{Co}$ & \multicolumn{6}{|c|}{ relative $I_{\text {, }}$} \\
\hline $26 \mathrm{Mg}$ & $10_{0}$ & $3.4+1$ & & UTR E & E Eggenhulsent & 11656 \\
\hline${ }^{41} \mathrm{Co}$ & average & $O(E)$ & & & & \\
\hline $41 x$ & $p$ & $1.2+0$ & $1.3+0$ & ORL E & E Johnsont & 1210 \\
\hline${ }^{4} \mathrm{CO}$ & \multicolumn{3}{|c|}{ reletive o(E) } & \multicolumn{3}{|c|}{ 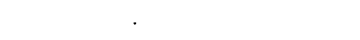 } \\
\hline $27 \mathrm{AI}$ & ${ }^{19} \mathrm{~F}$ & $4.0+1$ & & BNL. E & E Polettit & 11791 \\
\hline${ }^{41} \mathrm{co}_{0}$ & \multicolumn{6}{|l|}{$\sigma(E)$} \\
\hline $\begin{array}{l}27 \mathrm{Al} \\
27 \mathrm{Al}\end{array}$ & $\begin{array}{l}14 \mathrm{~N} \\
160\end{array}$ & $\begin{array}{l}5.9+1 \\
3.5+1\end{array}$ & $7.0+1$ & $\begin{array}{l}\text { GRE E } \\
\text { KLN }\end{array}$ & $\begin{array}{l}\text { E Vianot } \\
\text { T Hellmeigtert }\end{array}$ & $\begin{array}{l}10350 \\
10946\end{array}$ \\
\hline $285 i$ & 160 & $3.6+15$ & $5.5+1$ & STR R & R Mast & 10969 \\
\hline${ }^{37} \mathrm{Ar}$ & 0 & NDG & & CAL $T$ & T Woosleyt & 7211 \\
\hline${ }^{3 \theta_{A r}}$ & 。 & TR & $1.2+1$ & CAL $\mathrm{T}$ & I Woosleyt & 7213 \\
\hline${ }^{3 \theta_{\mathrm{K}}}$ & 0 & NDG & & CAL $T$ & T Woosleyt & 7245 \\
\hline $40 \mathrm{~K}$ & $p$ & NDG & & CAL T & I Woosleyt & 1204 \\
\hline $41 k$ & p & $T R$ & $1.0+1$ & CAL $T$ & T Woosleyt & 1207 \\
\hline${ }^{41} k$ & $p$ & $1.1+0$ & $6.0+0$ & KFK C & C Muenzelt & 1208 \\
\hline $41 \mathrm{k}$ & $p$ & $1.1+0 E$ & $6.0+0$ & ORL E & E Johnsont & 1209 \\
\hline${ }^{40} \mathrm{CB}$ & $14 \mathrm{~N}$ & $1.9+1=$ & $2.8+1$ & IFJ $R$ & R Malechl+ & 10367 \\
\hline${ }^{44} \mathrm{Sc}$ & $p$ & NDG & & CAL $T$ & T Woostey+ & 1333 \\
\hline${ }^{42} \mathrm{Co}$ & portial & \multicolumn{5}{|c|}{ Cumulative $O(E)$} \\
\hline 160 & 285 & $6.0+15$ & $9.0+1$ & STR E & E Freemant & 11962 \\
\hline${ }^{42} \mathrm{Ca}$ & \multicolumn{6}{|c|}{ spollation o(E) } \\
\hline $\mathrm{Fe}$ & P & $1.5+2=$ & $3.7+2$ & NRL $R$ & R Silberberg+ & 1844 \\
\hline
\end{tabular}




\begin{tabular}{|c|c|c|c|c|c|c|}
\hline Target & $\ln c$ & $\begin{array}{l}\text { Emin } \\
\text { (MeV) }\end{array}$ & $\begin{array}{l}\text { Emax } \\
\text { CMeVI }\end{array}$ & Lab $k$ & Author & No. \\
\hline${ }^{42} \mathrm{Co}$ & cumulativ & ive? ol & (E) & & & \\
\hline $2 \theta_{5}$ & 160 & $3 \cdot 1+1$ & $5.5+15$ & STR F & R Hast & 10971 \\
\hline${ }^{42} \mathrm{CB}_{8}$ & partial & row th & hick :ar & $\operatorname{arget}$ & yieldxabundance & \\
\hline${ }^{39} \mathrm{~K}$ & a & $5.0+0$ & & SUN E & E Gilest & 7248 \\
\hline${ }^{42} \mathrm{Co}$ & partial & $O(E), x$ & lactor & & & \\
\hline $\begin{array}{l}{ }_{2 \theta^{N I}} \\
2_{2 \theta^{N I}} \\
20^{2}\end{array}$ & $\begin{array}{l}p \\
p \\
p\end{array}$ & $\begin{array}{l}1.0+3 \\
1.0+3 \\
1.0+3\end{array}$ & & $\begin{array}{l}\text { LIN } \\
\text { LIN } \\
\text { LIN }\end{array}$ & $\begin{array}{l}\text { E Volnint } \\
\text { E Volnint } \\
\text { E Volnint }\end{array}$ & $\begin{array}{l}2316+ \\
2320+ \\
2321+\end{array}$ \\
\hline${ }^{42} \mathrm{Co}$ & gartial = & spectr & rum aver & erage & $O(E)$ & \\
\hline${ }^{42} \mathrm{Ca}$ & a & $2.3+1$ & $2.7+1 M$ & MUU E & E Eberhard+ & 7304 \\
\hline${ }^{42} \mathrm{CO}_{0}$ & partial & $\cos \theta$ & (E) & & & \\
\hline${ }^{28} \mathrm{Si}$ & 160 & $5.0+1$ & $6.5+: Y$ & YAL E & E Bettst & 10974 \\
\hline${ }^{42} \mathrm{Cs}$ & partial & coloti & ive o(E) & & & \\
\hline${ }^{40} \mathrm{Ca}$ & $\circ$ & $1.4+1$ & $2.4+\vdots A$ & ANL & E Meyer-Sehutzmei & 7289 \\
\hline${ }^{42} \mathrm{Co}$ & portlal & $O(E)$ & & & & \\
\hline 160 & ${ }^{28} \mathrm{Si}$ & $6.0+1$ & $9.0+15$ & STR E & E Freemant & 11963 \\
\hline${ }^{42} \mathrm{Ca}$ & relative & $e_{y}$ & & & & \\
\hline${ }^{28} \mathrm{Si}$ & 160 & $4.5+1$ & & UTR E & E Eggentuisent & 10973 \\
\hline${ }^{42} \mathrm{Cs}$ & relative & $\theta \sigma(E)_{Y}$ & & & & \\
\hline${ }^{4}{ }^{2} \mathrm{Ca}$ & $p$ & $2.6+0$ & $2.7+0 \mathrm{~s}$ & STR E & E Dint & 1276 \\
\hline${ }^{42} \mathrm{Co}$ & relative & $\sigma(E)$ & & & & \\
\hline $27 \mathrm{Al}$ & ${ }^{19} \mathrm{~F}$ & $4.0+1$ & & BNLE & E Poleltit & 11792 \\
\hline${ }^{42} \mathrm{Cs}$ & $O(E)$ & & & & & \\
\hline 2851 & 160 & $3.1+1$ & $5.5+15$ & STR R & R Hoast & 10972 \\
\hline $\begin{array}{l}{ }^{38} 8_{\mathrm{Ar}} \\
39_{\mathrm{K}}\end{array}$ & a & $\begin{array}{l}\text { NDG } \\
\text { TR }\end{array}$ & $1.1+10$ & $\begin{array}{l}\text { CAL T } \\
\text { CAL T }\end{array}$ & $\begin{array}{l}\text { T Woosleyt } \\
\text { T Woosleyt }\end{array}$ & $\begin{array}{l}7214 \\
7249\end{array}$ \\
\hline $41 k$ & $p$ & NDG & & CAL T & T Woosleyt & 1211 \\
\hline $4 z_{K}$ & & NOG & & CAL $T$ & T Woosleyt & 1213 \\
\hline${ }^{42} \mathrm{Co}$ & 160 & $5.6+1$ & & ANL $E$ & E Vigdort & 11043 \\
\hline${ }^{42} C_{0}$ & 160 & $5 \cdot 6+1$ & & HEI F & R Zeidman. & 11044 \\
\hline${ }^{44} \mathrm{Ca}$ & - & $2.0+1$ & $2.7+1 \mathrm{~K}$ & KLN E & E Eberhardt & 7318 \\
\hline
\end{tabular}

\begin{tabular}{|c|c|c|c|c|c|c|}
\hline Target & $\operatorname{lnc}$ & $\begin{array}{l}\text { Enln } \\
(\mathrm{MeV})\end{array}$ & $\begin{array}{l}\text { Emax } \\
\text { (MoV) }\end{array}$ & Lab & Althor & No. \\
\hline${ }^{42} \mathrm{Ca}$ & $\theta(E)$ & & & & & \\
\hline $\begin{array}{l}{ }^{45} \mathrm{Sc} \\
5 \theta_{\mathrm{Ni}} \\
60 \mathrm{Ni}\end{array}$ & $p$ & $\begin{array}{l}\text { NDG } \\
2.0+2 \\
2.0+5\end{array}$ & & $\begin{array}{l}\text { CAL } \\
\text { ANL } \\
\text { ANL }\end{array}$ & $\begin{array}{l}\text { T Woosleyt } \\
\text { E Schiffer. } \\
\text { E Schiffer. }\end{array}$ & $\begin{array}{l}1352 \\
2319 \\
2363\end{array}$ \\
\hline${ }^{43} \mathrm{Ca}$ & spollat & IOn $O(E$ & & & & \\
\hline $\begin{array}{r}F_{e} \\
{ }^{43} \mathrm{Ca}\end{array}$ & $\begin{array}{c}P \\
\text { partlat }\end{array}$ & $\begin{array}{l}1.5+2 \\
\text { produc }\end{array}$ & $\begin{array}{l}3.7+2 \\
\text { ct } y i e l\end{array}$ & $\begin{array}{l}\text { NRL } \\
\text { Id }\end{array}$ & R Silberbergt & 1844 \\
\hline $\begin{array}{l}{ }^{40} \mathrm{Ar} \\
{ }^{43} \mathrm{Co}\end{array}$ & ${ }_{\text {relative }}^{a}$ & $\begin{array}{r}5.5+0 \\
. \quad \sigma(E)\end{array}$ & $1.9+1$ & LVP & E Bahbehanit & 7227 \\
\hline $\begin{array}{l}{ }^{27} \mathrm{Al} \\
{ }^{43} \mathrm{Ca}\end{array}$ & $\begin{array}{l}19_{F} \\
O(E)\end{array}$ & $4.0+1$ & & BNL & E Poletlit & 11793 \\
\hline $\begin{array}{l}{ }^{39} \mathrm{Ar} \\
{ }^{40} \mathrm{Ar} \\
40 \mathrm{Ar} \\
40 \mathrm{~K} \\
4 \mathrm{~K}^{4} \\
4 \mathrm{~K}^{4} \\
4 \mathrm{~K}^{4} \mathrm{CB} \\
46 \mathrm{SC}\end{array}$ & $\begin{array}{l}a \\
a \\
a \\
a \\
p \\
p \\
a \\
p\end{array}$ & $\begin{array}{l}\text { NDG } \\
\text { TR } \\
2.4+1 \\
\text { TR } \\
\text { NDG } \\
\text { NDG } \\
2.0+1 \\
\text { NDG }\end{array}$ & $\begin{array}{l}9.0+0 \\
1.1+1 \\
2.7+1\end{array}$ & $\begin{array}{l}\text { CAL } \\
\text { CAL } \\
\text { HAM } \\
\text { CAL } \\
\text { CAL } \\
\text { CAL } \\
\text { KLN } \\
\text { CAL }\end{array}$ & $\begin{array}{l}\text { T Woosleyt } \\
\text { T Woosleyt } \\
\text { T Fitz- } \\
\text { T Woosleyt } \\
T \text { Woosleyt } \\
T \text { Woosleyt } \\
\text { E Eberhardt } \\
\text { T Woogleyt }\end{array}$ & $\begin{array}{l}7215 \\
7228 \\
7229 \\
7252 \\
1214 \\
1216 \\
7319 \\
1376\end{array}$ \\
\hline${ }^{44} \mathrm{Ca}$ & spollot & ion oCE & & & & \\
\hline $\begin{array}{r}\mathrm{Fe} \\
{ }^{4{ }^{4}} \mathrm{Cg}\end{array}$ & $\begin{array}{c}p \\
\text { partiol }\end{array}$ & $\begin{array}{l}1.5+2 \\
O(E)_{y}\end{array}$ & $\begin{array}{r}3.7+2 \\
\text { a factor }\end{array}$ & NRL & R Silberbergt & 1844 \\
\hline $\begin{array}{l}5 \theta_{\mathrm{Ni}} \\
5 \theta_{\mathrm{Ni}} \\
5 \theta_{\mathrm{Ni}}\end{array}$ & $p$ & $\begin{array}{l}1.0+3 \\
1.0+3 \\
1.0+3\end{array}$ & & $\begin{array}{l}\text { LIN } \\
\text { LIN } \\
\text { LIN }\end{array}$ & $\begin{array}{l}\text { E Volnint } \\
E \text { Volnint } \\
\text { E Volnolnt }\end{array}$ & $\begin{array}{l}2316+ \\
2320+ \\
2321+\end{array}$ \\
\hline${ }^{44} \mathrm{CB}$ & portial & averos & $g e$ ofE & & & \\
\hline $\begin{array}{l}{ }^{44} \mathrm{Ca} \\
{ }_{4}^{4} \mathrm{Ca}\end{array}$ & partial & $\begin{array}{l}2.0+1 \\
O(E)\end{array}$ & $2.6+1$ & WAU & E Eberhardt & 7320 \\
\hline $\begin{array}{l}{ }^{44} \mathrm{Ca} \\
{ }^{44} \mathrm{Co}\end{array}$ & $\underset{\text { relative }}{0}$ & $\begin{array}{l}1.0+1 \\
0(\varepsilon)\end{array}$ & 2. $7+1$ & KLN & E Eberhardt & 7321 \\
\hline${ }^{27} \mathrm{Al}$ & $19_{F}$ & $4.0+1$ & & BNL & E Polettit & 11794 \\
\hline
\end{tabular}




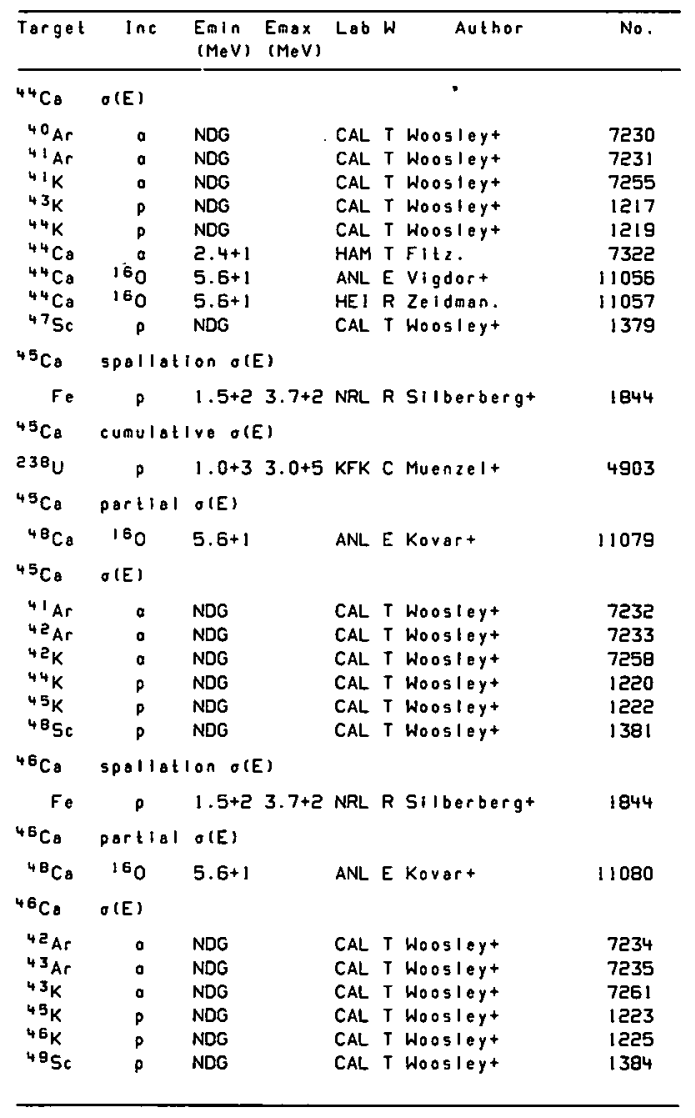

\begin{tabular}{|c|c|c|c|c|c|c|}
\hline Target & $\ln c$ & $\begin{array}{l}\text { Em in } \\
(\mathrm{MeV})\end{array}$ & $\begin{array}{l}\text { Emax } \\
(\mathrm{MeV})\end{array}$ & Lab & Author & No. \\
\hline${ }^{47} \mathrm{Co}$ & spaltati & ION OIE & & & & \\
\hline $\begin{array}{l}\mathrm{Fe} \\
{ }^{47} \mathrm{Ce}\end{array}$ & $\begin{array}{c}p \\
\text { cumulat } ~\end{array}$ & $\begin{array}{l}1.5+2 \\
\text { ivo ole }\end{array}$ & $\begin{array}{l}3.7+21 \\
\text { E) }\end{array}$ & NRL & R silberbergt & 1844 \\
\hline $238^{u}$ & $p$ & $1.0+3$ & $3.0+5$ & KFK & C Muenzel+ & 4903 \\
\hline${ }^{47} \mathrm{Co}$ & portiol & relati & I ve olE & & & \\
\hline $\begin{array}{l}{ }^{48} \mathrm{Ca} \\
{ }^{47} \mathrm{Ca}\end{array}$ & $\begin{array}{c}\text { "1B } \\
\text { portlal }\end{array}$ & $\begin{array}{l}2.5+1 \\
O(E)\end{array}$ & $5.0+1$ & BNL & E Nathant & 9572 \\
\hline${ }^{48} \mathrm{Co}$ & 100 & $5.6+1$ & & ANL & E Kovert & 11081 \\
\hline${ }^{47} \mathrm{Co}$ & thlck to & orgety & $y=10$ & & & \\
\hline$T$ & d & $9.0+1$ & & JUL & E Probst. & 5446 \\
\hline${ }^{47} \mathrm{CB}$ & $O(E)$ & & & & & \\
\hline${ }^{43} \mathrm{Ar}$ & . & NDG & & CAL & T Woosleyt & 7236 \\
\hline${ }^{44} \mathrm{Ar}$ & a & NDG & & CAL & T Woosloy+ & 7237 \\
\hline${ }^{44} \mathrm{~K}$ & a & NDG & & CAL & T Woosleyt & 7263 \\
\hline${ }^{46} \mathrm{~K}$ & p & NDG & & CAL & T Woosleyt & 1228 \\
\hline $47 k$ & p & NDG & & CAL & T Woosleyt & 1228 \\
\hline${ }^{\circ} \mathrm{Sc}$ & p & NDG & & CAL & T Woosloy+ & 1387 \\
\hline$T i$ & d & TR & $9: 0+1$ & JUL E & E Probst. & 5445 \\
\hline $51 \mathrm{~V}$ & d & $T R$ & $9.0+1$ & JUL & E Probst+ & 5452 \\
\hline${ }^{48} \mathrm{Co}$ & portial & spectr & rua ave & oroge & e O(E) & \\
\hline${ }^{4} \theta_{\mathrm{C}}$ & - & $2.3+1$ & $2.7+1$ & MUU & E Eberhardt & 7338 \\
\hline${ }^{48} \mathrm{Co}$ & portlal & $\theta(E)$ & & & & \\
\hline $\begin{array}{l}{ }^{48} \mathrm{Ca} \\
{ }^{48} \mathrm{Ca} \\
{ }^{48} \mathrm{Co} \\
{ }^{48} \mathrm{Ca}\end{array}$ & $\begin{array}{l}160 \\
160 \\
160 \\
160\end{array}$ & $\begin{array}{l}5 \cdot 6+1 \\
5 \cdot 6+1 \\
5 \cdot 6+1 \\
5 \cdot 6+1\end{array}$ & & $\begin{array}{l}\text { ANL } \\
\text { SAC } \\
\text { NB! } \\
\text { SAC }\end{array}$ & $\begin{array}{l}\text { E Kovart } \\
R \text { Low. } \\
R \text { Hansont } \\
R \text { Low. }\end{array}$ & $\begin{array}{l}11084 \\
11085 \\
11086 \\
11087\end{array}$ \\
\hline${ }^{4 \theta} \mathrm{Ca}_{\mathrm{B}}$ & $\theta(E)$ & & & & & \\
\hline $44 \mathrm{Ar}$ & a & NDG & & CAL & T Woosloyt & 7238 \\
\hline${ }^{45} \mathrm{Ar}$ & a & NDG & & CAL & T Woosleyt & 7239 \\
\hline $45 k$ & a & NDG & & CAL & I Woogloyt & 7266 \\
\hline $47 x$ & & NDG & & CAL & T Woosleyt & 1229 \\
\hline${ }^{48} \mathrm{~K}$ & $p$ & NDG & & CAL & T Woosloyt & 1231 \\
\hline${ }^{48} \mathrm{Co}$ & 160 & $5.6+1$ & & ANL & E Vigdort & 11082 \\
\hline
\end{tabular}




\begin{tabular}{|c|c|c|c|c|c|c|}
\hline Target & $\ln c$ & $\begin{array}{l}\text { Emin } \\
(\operatorname{MeV})\end{array}$ & $\begin{array}{l}\text { Emax } \\
(\operatorname{MeV})\end{array}$ & Lab & Author & No. \\
\hline${ }^{48} \mathrm{C}_{\theta}$ & $O(E)$ & & & & & \\
\hline $\begin{array}{l}48 \mathrm{Ca} \\
515 \mathrm{c}\end{array}$ & $\begin{array}{r}160 \\
p\end{array}$ & $\begin{array}{l}5.6+1 \\
\text { NDG }\end{array}$ & & $\begin{array}{l}\text { HEI } \\
\text { CAL }\end{array}$ & $\begin{array}{l}\text { R Zeldman. } \\
\text { T Woosleyt }\end{array}$ & $\begin{array}{r}11083 \\
1390\end{array}$ \\
\hline${ }^{48} \mathrm{CO}$ & pertial & lhlck & targot & $t y 10$ & oldafoctor & \\
\hline $\begin{array}{l}{ }^{48} \mathrm{Ca} \\
{ }^{49} \mathrm{Co}\end{array}$ & $\begin{array}{c}! \\
\text { portiol }\end{array}$ & $\begin{array}{l}2.5+0 \\
\theta(E)\end{array}$ & $3.5+0 \mathrm{r}$ & MAN & $M$ Hylandt & 6063 \\
\hline $\begin{array}{l}48 \mathrm{Ca} \\
48 \mathrm{Ca} \\
4 \mathrm{C}^{\mathrm{Ca}}\end{array}$ & $\begin{array}{l}18 \\
170 \\
170\end{array}$ & $\begin{array}{l}2 \cdot 5+0 \\
5 \cdot 6+1 \\
3 \cdot 0+1\end{array}$ & $3.5+0$ & $\begin{array}{l}\text { MAN } \\
\text { ANL } \\
\text { MAN }\end{array}$ & $\begin{array}{l}\text { M Hylandt } \\
\text { E Kovart } \\
\text { R Goldifarb. }\end{array}$ & $\begin{array}{r}6064 \\
11088 \\
11598\end{array}$ \\
\hline${ }^{48} \mathrm{Co}_{0}$ & $O(E)$ & & & & & \\
\hline $\begin{array}{l}49_{\mathrm{Ar}} \\
46^{\mathrm{Ar}} \\
46 \mathrm{~K} \\
48_{\mathrm{K}} \\
49_{\mathrm{K}}\end{array}$ & $\begin{array}{l}a \\
a \\
a \\
p \\
p\end{array}$ & $\begin{array}{l}\text { NDG } \\
\text { NDG } \\
\text { NDG } \\
\text { NDG } \\
\text { NDG }\end{array}$ & c & $\begin{array}{l}\text { CAL } \\
\text { CAL } \\
\text { CAL } \\
\text { CAL } \\
\text { CAL }\end{array}$ & $\begin{array}{l}\text { T Woosley+ } \\
\text { T Woosley+ } \\
\text { T Woosley+ } \\
\text { T Woosley+ } \\
\text { T Woosley+ }\end{array}$ & $\begin{array}{l}7240 \\
7241 \\
7269 \\
1232 \\
1234\end{array}$ \\
\hline${ }^{50} \mathrm{Co}$ & $\sigma(E)$ & & & & . & \\
\hline $\begin{array}{l}46 \mathrm{Ar} \\
{ }^{49} \mathrm{~K}\end{array}$ & a & $\begin{array}{l}\text { NDG } \\
\text { NDG }\end{array}$ & & $\begin{array}{l}\text { CAL } \\
\text { CAL }\end{array}$ & $\begin{array}{l}T \text { Woosleyt } \\
\text { T Woosleyt }\end{array}$ & $\begin{array}{l}7242 \\
1235\end{array}$ \\
\hline$z=21$ & indepe & endent & overoge & go his & inotlc onergy & \\
\hline $197 \mathrm{AU}$ & ${ }^{40} \mathrm{Ar}$ & $2.2+2$ & & DUB & E Artukh: & $12369+$ \\
\hline$z=21$ & Indepe & ondent & OLE:E': & $:(\theta)$ & & \\
\hline $\begin{array}{l}197 \mathrm{Au} \\
232 \mathrm{Th}\end{array}$ & $\begin{array}{l}{ }^{40} \mathrm{Ar} \\
40 \mathrm{Ar}\end{array}$ & $\begin{array}{l}2.2+2 \\
3 \cdot 0+2\end{array}$ & $3.9+2$ & $\begin{array}{l}\text { DUB } \\
\text { DUB }\end{array}$ & $\begin{array}{l}\text { E Artukht } \\
\text { E Artukht }\end{array}$ & $\begin{array}{l}12368+ \\
12396\end{array}$ \\
\hline$z=21$ & Indepe & endent & $\theta(E ; \theta)$ & & & \\
\hline $\begin{array}{l}197 \mathrm{Au} \\
Z=21\end{array}$ & $\begin{array}{l}{ }^{40} \mathrm{Ar} \\
\text { Indepe }\end{array}$ & $\begin{array}{l}2.2+2 \\
\text { ondent }\end{array}$ &.$(E)$ & DUB & E Artuhht & $12367+$ \\
\hline $\begin{array}{l}197 \mathrm{Au} \\
Z=21\end{array}$ & $\begin{array}{c}{ }^{40} \mathrm{Ar} \\
v_{n}\end{array}$ & $2.2+2$ & & DUP & E Artukht & $12366+$ \\
\hline Ag & $40 \mathrm{Ar}$ & $3.4+2$ & & PAR F & R Lefortt & 12202 \\
\hline$z=21$ & $\sigma(E) \times 1$ & foctor & & & & \\
\hline $232 \mathrm{Th}$ & ${ }^{40} \mathrm{Ar}$ & $3.9+2$ & & OXF & $\mathrm{R}$ Hodgson. & 12398 \\
\hline
\end{tabular}

\begin{tabular}{|c|c|c|c|c|c|c|}
\hline Target & $\operatorname{lnc}$ & $\begin{array}{l}\text { EmIn } \\
(M e V)\end{array}$ & $\begin{array}{l}\text { Emax } \\
(\operatorname{MeV})\end{array}$ & Lab & $\begin{array}{l}\text { Author } \\
\text {. }\end{array}$ & No. \\
\hline$z=21$ & $O(E)$ & & & & & \\
\hline $\begin{array}{l}\mathrm{Co}_{0} \\
\mathrm{Ni}\end{array}$ & $200^{\circ} \mathrm{Ne}$ & $\begin{array}{l}9.0+1 \\
1.6+2\end{array}$ & & $\begin{array}{l}\text { OSA } \\
\text { ORL }\end{array}$ & $\begin{array}{l}\text { E Shibata+ } \\
\text { R Plagilat }\end{array}$ & $\begin{array}{r}7274 \\
11851\end{array}$ \\
\hline${ }^{40} \mathrm{se}$ & o(E) & & & & & \\
\hline $\begin{array}{l}{ }^{40} \mathrm{Ca} \\
{ }^{41 \mathrm{SC}}\end{array}$ & relative & $\begin{array}{l}\text { TR } \\
I_{r}\end{array}$ & $1.0+1$ & CAL & T Woosleyt & 1267 \\
\hline $\begin{array}{l}{ }^{40} \mathrm{Ca} \\
{ }^{415 e}\end{array}$ & $\stackrel{p}{\text { product }}$ & $\begin{array}{l}1.8+0 \\
y 1010\end{array}$ & $1.9+0$ & KOU & E Kozubt & 1269 \\
\hline $\begin{array}{l}{ }^{4{ }^{\circ}} \mathrm{Ca} \\
41 \mathrm{Se}\end{array}$ & thich to & $\begin{array}{l}1.8+0 \\
\text { orget y }\end{array}$ & $\begin{array}{l}4.1+0 \\
y 1010\end{array}$ & MOS $\mathrm{F}$ & R Verlanort & 1268 \\
\hline $\begin{array}{l}{ }^{40} \mathrm{Ca} \\
+15 \mathrm{se}\end{array}$ & $\begin{array}{c}p \\
r a g\end{array}$ & $8.5-1$ & $4.7+0$ & CLU & M Roughtont & 1273 \\
\hline $\begin{array}{l}{ }^{40} \mathrm{Ca} \\
{ }^{4} \mathrm{Sc}\end{array}$ & $O(E) \times f O C$ & $\begin{aligned} & 5.0-1 \\
& \text { etor }\end{aligned}$ & $2.0+0$ & AML & E Painet & 1272 \\
\hline $\begin{array}{l}.516 \\
4150\end{array}$ & $\begin{array}{r}p \\
o(E)\end{array}$ & $9.0+1$ & & BNL & T Divadeonan. & 1573 \\
\hline $\begin{array}{l}37 \mathrm{~K} \\
{ }^{40} \mathrm{Co} \\
{ }^{40} \mathrm{Co} \\
31 \mathrm{~V}\end{array}$ & $\begin{array}{l}\text { p } \\
p \\
p\end{array}$ & $\begin{array}{l}\text { NDG } \\
\text { NDG } \\
8.5-1 \\
9.0+1\end{array}$ & $4.7+0$ & $\begin{array}{l}\text { CAL } \\
\text { CAL } \\
\text { CLU } \\
\text { BNL }\end{array}$ & $\begin{array}{l}\text { T Woogleyt } \\
\text { T Woosleyt } \\
\text { E Roughtont } \\
\text { T Divadoenan. }\end{array}$ & $\begin{array}{l}7244 \\
1270 \\
1271 \\
1572\end{array}$ \\
\hline${ }^{4} \mathrm{sc}$ & spallati & Ion olE & & & & \\
\hline $\begin{array}{r}F e \\
425 e\end{array}$ & $\frac{p}{\text { partlat }}$ & $\begin{array}{l}1.5+2 \\
0(E)\end{array}$ & $3.7+2$ & NRL F & R Silberbergt & 1844 \\
\hline${ }^{40} \mathrm{Co}$ & a & $4.0+1$ & & MSU E & E Nonnt & 7290 \\
\hline${ }^{42} \mathrm{Sc}$ & $O(E) \times f a c$ & elor & & & & \\
\hline $\begin{array}{r}31 \mathrm{~V} \\
42 \mathrm{Se}\end{array}$ & $\begin{array}{r}p \\
O(E)\end{array}$ & $9.0+1$ & & BNL & T Divadeenan. & 1573 \\
\hline $\begin{array}{l}38_{K} \\
39_{K} \\
{ }^{40} \mathrm{C}_{8}\end{array}$ & : & $\begin{array}{l}\text { NDG } \\
\text { TR } \\
2.4+1\end{array}$ & $9.9+0$ & $\begin{array}{l}\text { CAL } \\
\text { CAL } \\
\text { HAM }\end{array}$ & $\begin{array}{l}\text { T Woosleyt } \\
\text { T Woosleyt } \\
\text { T Fllz. }\end{array}$ & $\begin{array}{l}7246 \\
7250 \\
7291\end{array}$ \\
\hline
\end{tabular}




\begin{tabular}{|c|c|c|c|c|c|c|}
\hline Target & $\ln c$ & $\begin{array}{l}\text { Emin } \\
(\mathrm{MeV})\end{array}$ & $\begin{array}{l}\text { Emax } \\
(\mathrm{MeV})\end{array}$ & Lab W & Author & No. \\
\hline${ }^{42} \mathrm{sc}$ & $\theta(E)$ & & & & & \\
\hline $\begin{array}{l}40 \mathrm{Ca} \\
{ }^{41} \mathrm{Ca} \\
4 \mathrm{Ca}^{\mathrm{C} a} \\
4 \mathrm{Ca}^{4} \mathrm{Ca} \\
46 \mathrm{~T} \\
51 \mathrm{~V}\end{array}$ & $\begin{array}{r}180 \\
p \\
p \\
p \\
p \\
p\end{array}$ & $\begin{array}{l}5.6+1 \\
\text { NDG } \\
\text { TR } \\
\because .3+0 \\
2.2+1 \\
9.0+1\end{array}$ & $\begin{array}{l} \\
1.2+1 \\
1.0+1 \\
3.4+1\end{array}$ & $\begin{array}{l}\text { ANL. } \\
\text { CAL } \\
\text { CAL T } \\
\text { CRC E } \\
\text { MSU } \\
\text { ENL } \\
\text { EN T }\end{array}$ & $\begin{array}{l}\text { R Vigdor. } \\
T \text { Woosleyt } \\
T \text { Woosleyt } \\
\text { E Jocksont } \\
M \text { Edmistont } \\
T \text { Olvadeenam. }\end{array}$ & $\begin{array}{r}11005 \\
1274 \\
1277 \\
1278 \\
1420 \\
1572\end{array}$ \\
\hline wensc & cumulat & live oll & (E) & & & \\
\hline $\begin{array}{r}\mathrm{Cu} \\
42=5 c\end{array}$ & $\begin{array}{c}\text { p } \\
\text { product }\end{array}$ & $\begin{array}{l}6.0+2 \\
t \quad y / e 10\end{array}$ & d. & MTR E & E clinet & $2499+$ \\
\hline $\begin{array}{l}40 \mathrm{Ca} \\
{ }^{43} \mathrm{St}\end{array}$ & ${ }^{14} \mathrm{~N}$ & $\begin{array}{l}2.9+1 \\
d-\text { nucle }\end{array}$ & $\begin{array}{l}5.5+1 \\
\text { eUs } \sigma(E\end{array}$ & $\begin{array}{l}\text { STB E } \\
\text { E) }\end{array}$ & E Geesamant & 10368 \\
\hline $\begin{array}{l}{ }^{27} \mathrm{Al} \\
{ }^{4} \mathbf{S} \mathrm{SC}\end{array}$ & $\begin{array}{l}160 \\
\text { spalloti }\end{array}$ & $\begin{array}{l}3.0+1 \\
\text { Ion } \sigma(E\end{array}$ & E) & THD T & T Puhlhofer. & 10948 \\
\hline $\begin{array}{r}\mathrm{Fe} \\
{ }^{43} \mathrm{Sc}\end{array}$ & cumulati & $\begin{array}{l}1.5+2 \\
\text { IVe olE }\end{array}$ & E) & NRL R & R Silberberg+ & 1844 \\
\hline $\begin{array}{l}\mathrm{Tl}^{\prime} \\
\mathrm{Fe} \\
\mathrm{Cu}\end{array}$ & $\begin{array}{l}p \\
p \\
p\end{array}$ & $\begin{array}{l}1 \cdot 3+1 \\
6 \cdot 0+2 \\
6 \cdot 0+2\end{array}$ & $4.5+1$ & $\begin{array}{l}\text { KLN E } \\
\text { MTR E } \\
\text { MTR E }\end{array}$ & $\begin{array}{l}\text { E Mlchelt } \\
\text { E Clinet } \\
\text { E Clinet }\end{array}$ & $\begin{array}{l}1400+ \\
1765+ \\
2500+\end{array}$ \\
\hline $4^{3} 56$ & partial & relatt & Ive OLE & & & \\
\hline $\begin{array}{l}{ }^{40} \mathrm{C}_{\mathrm{a}} \\
{ }^{4} \mathrm{C}_{\mathrm{B}}\end{array}$ & a & $\begin{array}{l}1.4+1 \\
2.6+0\end{array}$ & $2.4+1$ & $\begin{array}{l}\text { ANL E } \\
\text { STR E }\end{array}$ & $\begin{array}{l}\text { E. Meyer-Schulzmel } \\
\text { E.Dint }\end{array}$ & $\begin{array}{l}7292 \\
1283\end{array}$ \\
\hline $4^{3} 5 c$ & partial & $\sigma(E) \times f$ & fàctor & & & \\
\hline $\begin{array}{l}{ }^{46} \mathrm{Tt} \\
{ }^{43} \mathrm{5c}\end{array}$ & $\begin{array}{c}p \\
\text { partial }\end{array}$ & $\begin{array}{l}4.2+1 \\
\sigma(E)\end{array}$ & & PTN E & E Freedmant & 1421 \\
\hline $\begin{array}{l}{ }^{40} \mathrm{Ca} \\
{ }^{43} \mathrm{Sc}\end{array}$ & $\begin{array}{c}a \\
\text { praduct }\end{array}$ & $\begin{array}{l}1.0+1 \\
y \in e \mid d\end{array}$ & $2.7+1$ & KLN E & E Appel+ & 7293 \\
\hline $\begin{array}{l}{ }^{42} \mathrm{Ca} \\
{ }^{43} \mathrm{Sc}\end{array}$ & $\frac{p}{t h \mid c k \text { to }}$ & $\begin{array}{l}1.8+0 \\
\text { orget y }\end{array}$ & $\begin{array}{l}4.1+0 \\
y 1010\end{array}$ & $\operatorname{MOS} R$ & R Varlamovt & 1279 \\
\hline 3051 & $14 \mathrm{~N}$ & $4.0+1$ & $6.2+1$ & FSU M & M Zellert & 10361 \\
\hline
\end{tabular}

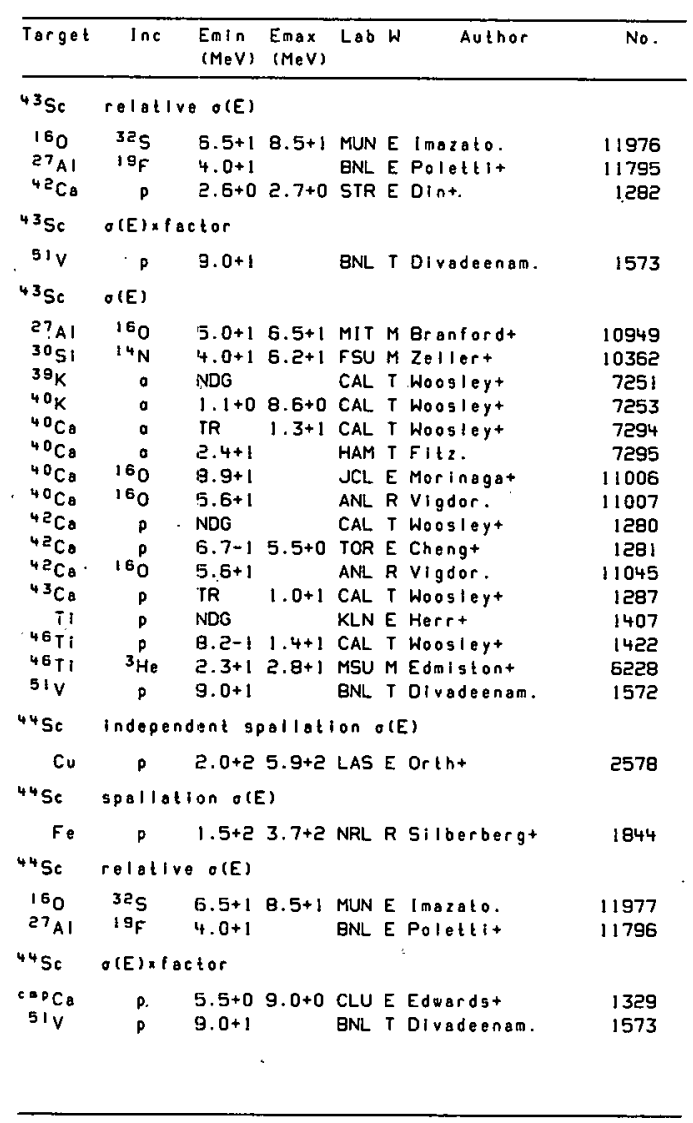




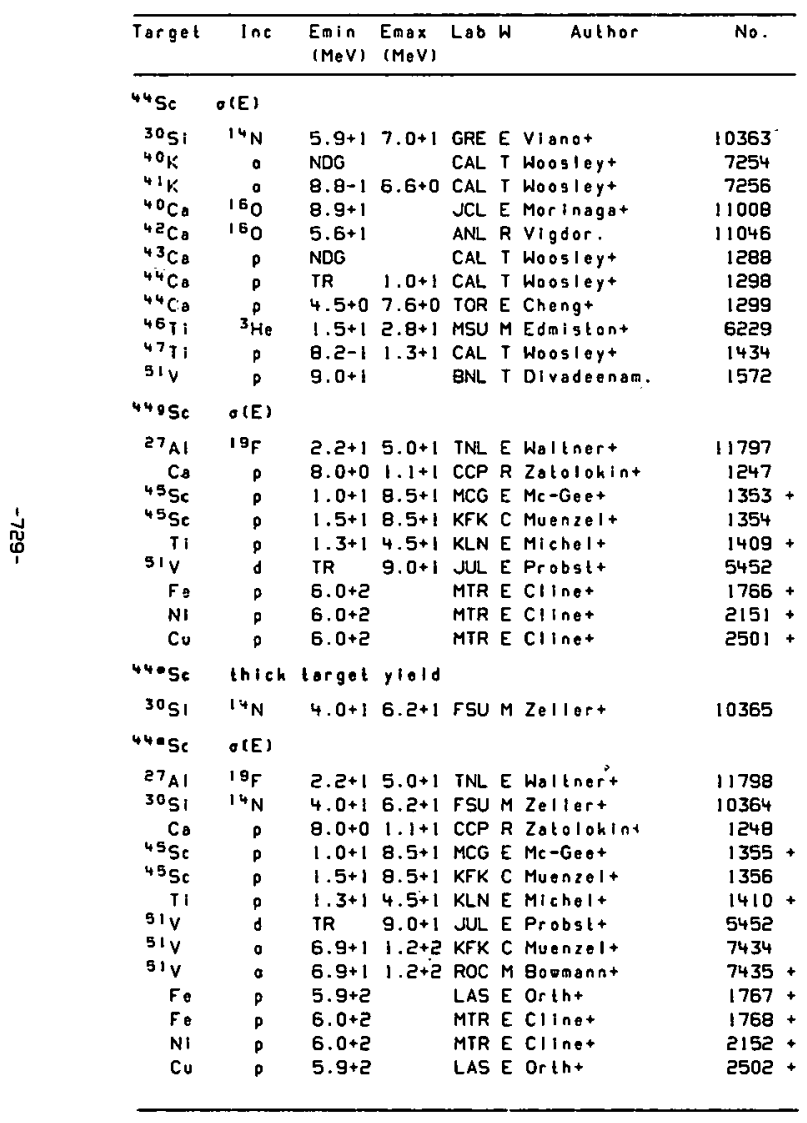

\begin{tabular}{|c|c|c|c|c|c|c|}
\hline Target & $\ln s$ & $\begin{array}{l}E m 1 n \\
(M e V)\end{array}$ & $\begin{array}{l}E_{\max } \\
(\mathrm{MeV})\end{array}$ & Lab W & Aushor & No. \\
\hline $44.5 \mathrm{C}$ & $\theta(E)$ & & & & & \\
\hline $\mathrm{Cu}$ & $p$ & $6.0+2$ & & MTR E & E clinet & $2503+$ \\
\hline $44 a \cdot 95 c$ & SUDAO & ationo & $O(E)$ & & & \\
\hline $\begin{array}{l}45 \mathrm{Sc} \\
4^{45} \mathrm{Sc}\end{array}$ & $\begin{array}{l}p \\
p\end{array}$ & $\begin{array}{l}1.0+1 \\
1.5+1\end{array}$ & $\begin{array}{l}8.5+1 k \\
8.5+1 k\end{array}$ & $\begin{array}{l}\text { MCG E } \\
\text { KFK C }\end{array}$ & $\begin{array}{l}\text { E Me-Geet } \\
\text { C Muenzelt }\end{array}$ & $\begin{array}{l}1357+ \\
1358\end{array}$ \\
\hline 44.1 .58 & ratio & $O(E)$ & & & & \\
\hline $\begin{array}{l}27 \mathrm{Al} \\
{ }^{45} \mathrm{SC}\end{array}$ & $\begin{array}{l}19 \mathrm{~F} \\
\text { spallati }\end{array}$ & $\begin{array}{l}2.2+1 \\
\text { ion otE }\end{array}$ & $5.0+1$ & TNL E & E Walinert & 11799 \\
\hline $\mathrm{Fe}$ & $p$ & $1.5+2$ & $3.7+2 \mathrm{~N}$ & NRL R & R SIlberbergt & 1844 \\
\hline${ }^{45} \mathrm{Sc}$ & portlol & row th & hlch tor & orget & $y 1010$ & \\
\hline${ }^{49} \mathrm{Sc}$ & $\circ$ & $5.0+0$ & & SUN E & E Glitest & 7349 \\
\hline${ }^{45} \mathrm{Sc}$ & Dortial & $O(E)$ & & & & \\
\hline${ }^{44} \mathrm{Ca}$ & ${ }^{7} \mathrm{LH}$ & $1.0+1$ & $3.5+1 r$ & MUN E & E Fortunat & 9429 \\
\hline${ }^{45} \mathrm{Sc}$ & $I_{y}$ & & & & & \\
\hline${ }^{49} 5 c$ & $p$ & $2.0+0$ & $4.0+00$ & DEB E & E Dongt & 1359 \\
\hline${ }^{45} \mathrm{Se}$ & relotive & $O(E)$ & & & & \\
\hline $\begin{array}{l}27 \mathrm{Al} \\
44 \mathrm{Ca} \\
56 \mathrm{Fo} \\
58_{\mathrm{Ni}} \\
64 \mathrm{Zn}\end{array}$ & $\begin{array}{l}{ }^{19} \mathrm{~F} \\
{ }^{\mathrm{p}} \\
{ }^{3} \mathrm{He} \\
{ }^{3} \mathrm{He} \\
{ }^{3} \mathrm{He}\end{array}$ & $\begin{array}{l}4.0+1 \\
1.6+0 \\
8 \cdot 0+1 \\
8 \cdot 0+1 \\
8 \cdot 0+1\end{array}$ & $\begin{array}{r}1.7+0 \\
i \\
i\end{array}$ & $\begin{array}{l}\text { BNL E } \\
\text { CER E } \\
\text { NRL E } \\
\text { NRL E } \\
\text { NRL E }\end{array}$ & $\begin{array}{l}\text { E Polettit } \\
\text { E Awglt } \\
\text { E Singht } \\
\text { E Singht } \\
\text { E Singht }\end{array}$ & $\begin{array}{r}11800 \\
1301 \\
6263 \\
6278 \\
6371\end{array}$ \\
\hline${ }^{45} \mathrm{se}$ & $O(E)=f O C$ & etor & & & & \\
\hline $\begin{array}{r}51 \mathrm{~V} \\
{ }_{45 \mathrm{Sc}}\end{array}$ & $\begin{array}{r}p \\
O(E)\end{array}$ & $9.0+1$ & & BNL T & T Olvadeenam. & 1573 \\
\hline $41 k$ & a & NDG & & CAL I & I Woosleyt & 7257 \\
\hline $42_{K}$ & - & NDG & & CAL T & T Woosleyt & 7259 \\
\hline $\begin{array}{l}{ }^{4}{ }^{2} C_{8} \\
{ }^{4}{ }^{2} C_{8}\end{array}$ & $160_{0}^{\circ}$ & $\begin{array}{l}\text { IR } \\
5.6+1\end{array}$ & $1.1+1$ & $\begin{array}{l}\text { CAL T } \\
\text { ANL } 8\end{array}$ & $\begin{array}{l}\text { T Woosleyt } \\
\text { R Vigdor. }\end{array}$ & $\begin{array}{r}7305 \\
11047\end{array}$ \\
\hline${ }^{4} \mathrm{CO}$ & $p$ & NDG & & CAL T & T Woosteyt & 1300 \\
\hline${ }^{44} \mathrm{C}_{8}$ & 160 & $5.6+1$ & & ANL $F$ & R vigdor. & 11058 \\
\hline $\begin{array}{l}{ }^{48} \mathrm{Co} \\
{ }^{4} \mathrm{C}^{\circ} \mathrm{TI}\end{array}$ & $p$ & $\begin{array}{l}4.0+2 \\
5.1-1\end{array}$ & $1.3+1$ & $\begin{array}{l}\text { NRL } \\
\text { CAL }\end{array}$ & $\begin{array}{l}\text { I Silberjerg* } \\
\text { T Wooslert }\end{array}$ & $\begin{array}{l}1322 \\
1445\end{array}$ \\
\hline
\end{tabular}




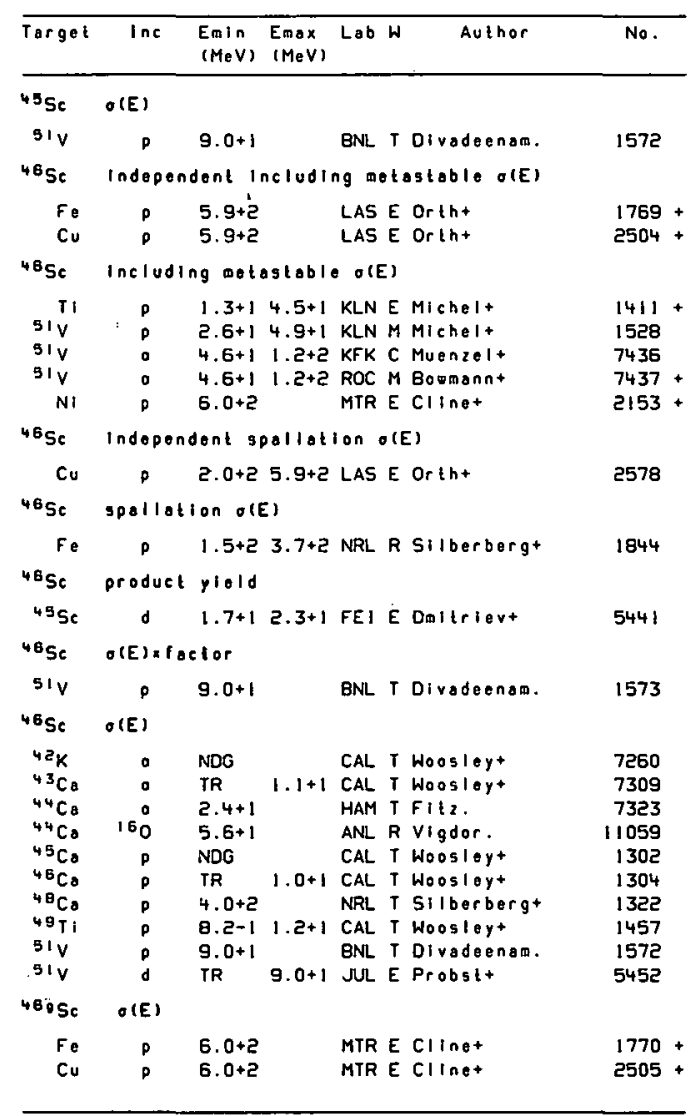

\begin{tabular}{|c|c|c|c|c|c|c|}
\hline Targat & $\ln c$ & $\begin{array}{l}E m i n \\
(M \circ V)\end{array}$ & $\begin{array}{l}\text { Emax } \\
(\operatorname{MeV})\end{array}$ & Lab & Author & No. \\
\hline $48 \cdot 5 c$ & $O(E)$ & & & & & \\
\hline $\begin{array}{l}\mathrm{Fe}_{\mathrm{e}} \\
\mathrm{Cu}\end{array}$ & p & $\begin{array}{l}6.0+2 \\
6.0+2\end{array}$ & & $\begin{array}{l}\text { MTR } \\
\text { MTR }\end{array}$ & $\begin{array}{l}\text { E Cllnet } \\
\text { E Cllnet }\end{array}$ & $\begin{array}{l}1771+ \\
2506+\end{array}$ \\
\hline $48 \cdot+95$ & sua & iatlon & $O(E)$ & & & \\
\hline $50 v$ & p & $\begin{array}{l}1 . \theta+1 \\
1 . \theta+1\end{array}$ & $\begin{array}{l}4.9+1 \\
4.9+1\end{array}$ & $\begin{array}{l}\text { KLN } \\
\text { KLN }\end{array}$ & $\begin{array}{l}\text { M Michel, } \\
\text { Mithel. }\end{array}$ & $\begin{array}{l}1492 \\
1504\end{array}$ \\
\hline${ }^{47} \mathrm{Sc}$ & cumula & lve $s p$ & 00110110 & on of & (E) & \\
\hline Cu & 9 & $2.0+2$ & $25.9+2$ & LAS & E Ortht & 2577 \\
\hline${ }^{47} \mathrm{Se}$ & spollo & ion $O E$ & & & & \\
\hline $\begin{array}{r}\mathrm{Fe} \\
{ }^{47} \mathrm{Se}\end{array}$ & $\begin{array}{c}P \\
\text { cumula }\end{array}$ & $\begin{array}{l}1.5+2 \\
\text { ive? }\end{array}$ & $\begin{array}{l}3.7+2 \\
\text { (E) }\end{array}$ & NRL & R Silberbergt & 1844 \\
\hline $\begin{array}{l}\mathrm{Fe} \\
\mathrm{NI} \\
\mathrm{Cu}\end{array}$ & $\begin{array}{l}p \\
p \\
p\end{array}$ & $\begin{array}{l}6.0+2 \\
6.0+2 \\
6.0+2\end{array}$ & & $\begin{array}{l}\text { MTR } \\
\text { MTR } \\
\text { MTR }\end{array}$ & $\begin{array}{l}\text { E Clinet } \\
\text { E Clinet } \\
\text { E Clinet }\end{array}$ & $\begin{array}{l}1772+ \\
2154+ \\
2507+\end{array}$ \\
\hline${ }^{47} \mathrm{Se}$ & cumula & ive ole & & & & \\
\hline $\begin{array}{l}\mathrm{Fe} \\
\mathrm{Cu}\end{array}$ & P & $\begin{array}{l}5.9+2 \\
5.9+2\end{array}$ & & $\begin{array}{l}\text { LAS } \\
\text { LAS }\end{array}$ & $\begin{array}{l}\text { E Orlht } \\
\text { E Orlht }\end{array}$ & $\begin{array}{l}1773+ \\
2508+\end{array}$ \\
\hline${ }^{47} \mathrm{Sc}$ & $O(E) \times f$ & ictor & & & & \\
\hline 5 IV & $p$ & $9.0+1$ & & BNL & T Divadeenam. & 1573 \\
\hline${ }^{47} \mathrm{Sc}$ & $O(E)$ & & & & & \\
\hline${ }^{43} \mathrm{~K}$ & 。 & NDG & & CAL & T Woosleyt & 7262 \\
\hline${ }^{44} \mathrm{~K}$ & a & NDG & & CAL & T Woosleyt & 7264 \\
\hline$c_{0}$ & p & $1.4+1$ & $4.1+1$ & RIC & E Waltont & 1249 \\
\hline${ }^{44} \mathrm{Cs}$ & a & IR & $1.1+1$ & CAL & I Woosleyt & 7324 \\
\hline${ }^{44} \mathrm{Ca}$ & $\therefore$ & $2.4+1$ & & HAM & 1 T Fill. & 7325 \\
\hline${ }^{44} \mathrm{Ca}$ & 160 & $5.6+1$ & & ANL & R vigdor. & 11060 \\
\hline${ }^{46} \mathrm{Ca}$ & p & NDO & & CAL & T Woosloyt & 1305 \\
\hline${ }^{47} \mathrm{Ca}$ & D & NDG & & CAL & T Woosleyt & 1306 \\
\hline${ }^{48} \mathrm{Co}$ & $p$ & $4.0+2$ & & NRL & I silberbergt & 1322 \\
\hline$T I$ & $p$ & $1.3+1$ & $14.5+1$ & KLN & I E Michel+ & $1412+$ \\
\hline${ }^{\circ} \mathrm{TH}$ & D & $5.1-1$ & $1.3+1$ & CAL & T Woogleyt & 1465 \\
\hline & p & $1.8+1$ & $14.9+1$ & KLN & Michelt & 1493 \\
\hline 516 & $p$ & NDG & & KLN & 1 E Herrt & 1529 \\
\hline Siv & D & $2.0+1$ & $11.0+2$ & BNL & T Divadeonam. & 1530 \\
\hline
\end{tabular}




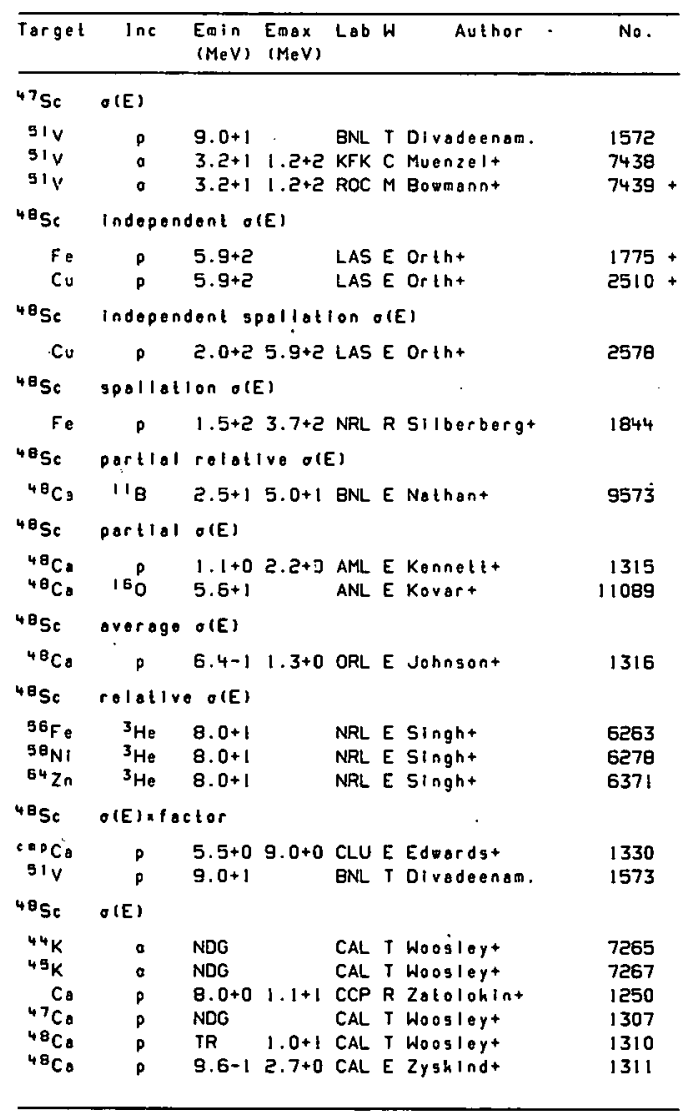

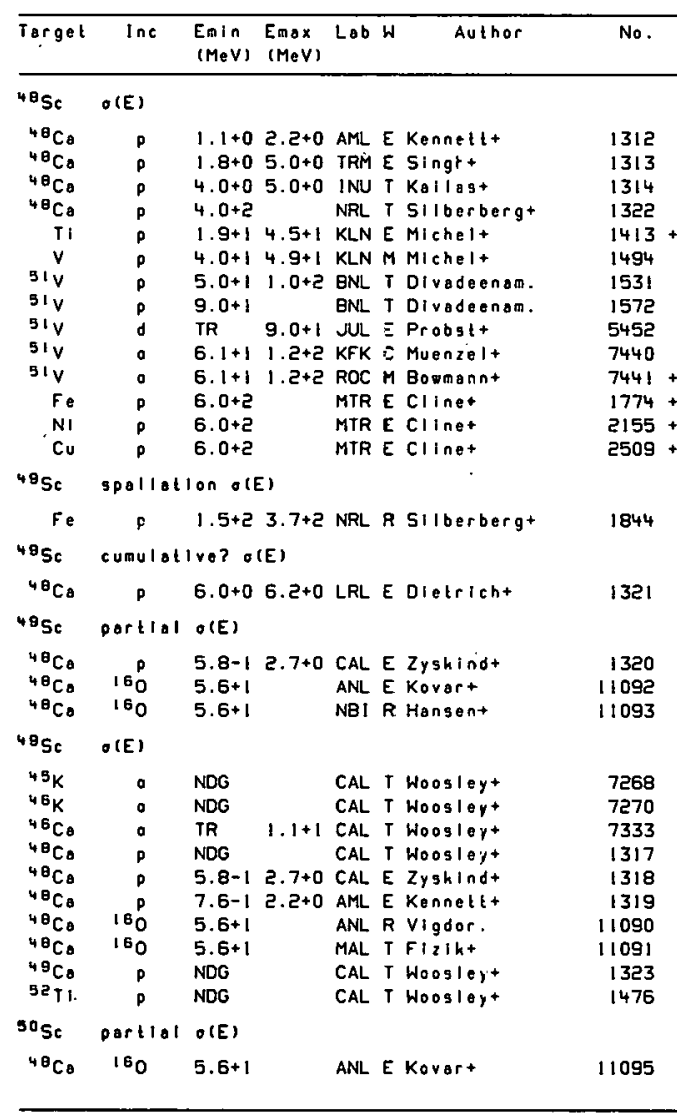




\begin{tabular}{|c|c|c|c|c|c|c|}
\hline Target & $\ln c$ & $\begin{array}{l}\text { Emin } \\
(M e V)\end{array}$ & $\begin{array}{l}E_{\max } \\
(\mathrm{MeV})\end{array}$ & Lab $h$ & Author & No. \\
\hline${ }^{50} \mathrm{Sc}$ & $\theta(E)$ & & & & & \\
\hline $\begin{array}{l}46 \mathrm{~K} \\
{ }^{48} \mathrm{Ca} \\
{ }^{49} \mathrm{Ca} \\
53 \mathrm{Tl}\end{array}$ & $\begin{array}{r}10_{0}^{\circ} \\
p \\
p\end{array}$ & $\begin{array}{l}\text { NOG } \\
5.6+1 \\
\text { NDG } \\
\text { NOG }\end{array}$ & & $\begin{array}{l}\text { CAL } \\
\text { ANL } \\
\text { CAL } \\
\text { CAL }\end{array}$ & $\begin{array}{l}T \text { Woosleyt } \\
\text { R Vigdor. } \\
T \text { Woosleyt } \\
T \text { Woosleyt }\end{array}$ & $\begin{array}{r}7271 \\
11094 \\
1324 \\
1479\end{array}$ \\
\hline $5005 c$ & $\sigma(\varepsilon)$ & & & & & \\
\hline${ }^{48} \mathrm{C}_{9}$ & 160 & $5.6+1$ & & ANL $E$ & E Kovart & 11096 \\
\hline $51 \mathrm{Sc}$ & $\sigma(E)$ & & & & & \\
\hline $\begin{array}{l}{ }^{48} \mathrm{Ca} \\
{ }^{4 \theta} \mathrm{Ca}\end{array}$ & $160^{\circ}$ & $\begin{array}{l}\text { TR } \\
5.6+1\end{array}$ & $1.2+1$ & $\begin{array}{l}\text { CAL } \\
\text { ANL } F\end{array}$ & $\begin{array}{l}\text { T Woosleyt } \\
\text { R Vigdor. }\end{array}$ & $\begin{array}{r}7339 \\
11097\end{array}$ \\
\hline$z=22$ & inde & endent & averog & $g e k 1 n$ & netic energyce & \\
\hline $\begin{array}{l}197 \mathrm{Au} \\
Z=22\end{array}$ & $\begin{array}{l}{ }^{40} \mathrm{Ar} \\
\text { inde }\end{array}$ & $\begin{array}{l}2.2+2 \\
\text { endent }\end{array}$ & OIE;E. & $\begin{array}{l}\text { OUB E } \\
\cdot ; \theta 1\end{array}$ & E Artukh+ & $12369+$ \\
\hline $\begin{array}{l}197 \mathrm{Au} \\
232 \mathrm{Th}\end{array}$ & $\begin{array}{l}40 \mathrm{Ar} \\
40 \mathrm{Ar}\end{array}$ & $\begin{array}{l}z .2+2 \\
3.0+5\end{array}$ & $3.9+2$ & $\begin{array}{l}\text { OUB E } \\
\text { DUB E }\end{array}$ & $\begin{array}{l}\text { E Arlukht } \\
\text { E Artukht }\end{array}$ & $\begin{array}{l}12368+ \\
12396\end{array}$ \\
\hline$z=22$ & inde & erdent & $\theta(E: \theta)$ & & & \\
\hline $\begin{array}{l}197 \mathrm{Au} \\
z=22\end{array}$ & $\begin{array}{l}{ }^{40} \mathrm{Ar} \\
\text { inde }\end{array}$ & $\begin{array}{r}2.2+2 \\
\text { endent }\end{array}$ & $\sigma(E)$ & DUB E & E Arlukht & $12367+$ \\
\hline $\begin{array}{l}197 \mathrm{AU} \\
Z=22\end{array}$ & $\begin{array}{c}40 \mathrm{Ar} \\
\nu_{\mathrm{n}}\end{array}$ & $2.2+2$ & & DUB E & E Artuhht & $12366+$ \\
\hline$A g$ & $40^{\mathrm{Ar}}$ & $3.4+2$ & ' & PAR F & R Lefortt & 12202 \\
\hline$z=22$ & $O(E)$ & foctor & & & & \\
\hline $\begin{array}{l}232 \mathrm{Th} \\
z=22\end{array}$ & $\begin{array}{l}40 \mathrm{Ar} \\
0\{E\}\end{array}$ & $3.9+2$ & & OXF F & $R$ Hodgion. & 12398 \\
\hline${ }^{4 I} \mathrm{NI}$ & $\begin{array}{l}20 \mathrm{Ne} \\
\sigma(E)\end{array}$ & $1.6+2$ & & ORL F & RPlasilt & 11851 \\
\hline${ }^{4} \mathrm{Co}$ & ${ }^{3} \mathrm{He}$ & NDG & & BRK $F$ & R Cernyt & 6221 \\
\hline${ }^{43} \mathrm{TI}$ & spalla & fion $O(E$ & & & & \\
\hline $\mathrm{Fe}$ & p & $1.5+2$ & $3.7+2$ & NRL $F$ & R Silberbergt & 1844 \\
\hline
\end{tabular}

\begin{tabular}{|c|c|c|c|c|c|c|c|}
\hline$\overline{\text { Target }}$ & $\ln c$ & $\begin{array}{l}\text { Emin } \\
(M e V)\end{array}$ & $\begin{array}{l}E_{\max } \\
(\operatorname{MeV})\end{array}$ & Lab W & Author & No. & \\
\hline $43 T 1$ & partlat & relat & ive ore & & & & \\
\hline${ }^{40} \mathrm{Co}$ & $a$ & $1.4+1$ & $2.4+1$ & ANL E & E Meyer-Schutzmei & 7296 & \\
\hline $43 \mathrm{TI}$ & $\sigma(E)$ & & & & & & \\
\hline $\begin{array}{l}{ }^{40} \mathrm{Ca} \\
{ }^{40} \mathrm{Ca}\end{array}$ & a & $\begin{array}{l}\text { TR } \\
2.4+1\end{array}$ & $1 . \theta+1$ & $\begin{array}{l}\text { CAL T } \\
\text { HAM T }\end{array}$ & $\begin{array}{l}\text { T Woosleyt } \\
\text { T Fitz. }\end{array}$ & $\begin{array}{l}7297 \\
7299\end{array}$ & \\
\hline${ }^{44} \mathrm{TI}$ & Independ & dent $t$ & hick tor & arget & $t y(e) d y$ & & \\
\hline $\begin{array}{l}{ }^{45} \mathrm{Sc} \\
{ }^{45} \mathrm{Sc}\end{array}$ & $\begin{array}{l}\text { p } \\
\text { d }\end{array}$ & $\begin{array}{l}1.4+1 \\
1.7+1\end{array}$ & $\begin{array}{l}2.2+1 \\
2 \cdot 3+1\end{array}$ & $\begin{array}{l}\text { FEI E } \\
\text { FEI E }\end{array}$ & $\begin{array}{l}\text { E Daitrievt } \\
\text { E Daitriest }\end{array}$ & $\begin{array}{l}1360+ \\
5442+\end{array}$ & \\
\hline${ }^{44} \mathrm{Ti}$ & spollati & ion $\sigma(E$ & & & & & \\
\hline $\mathrm{Fe}$ & $\rho$ & $1.5+2$ & $3.7+2$ & NRL $R$ & R Silberbergt & 1844 & \\
\hline${ }^{44} \mathrm{Ti}$ & partial & raw of & & & & & \\
\hline${ }^{40} \mathrm{Ca}$ & $a$ & $3.5+0$ & $4.0+0$ & CAL E & E Coopermant & 7299 & \\
\hline${ }^{44} \mathrm{TI}$ & partial & $\sigma(E) \times$ & factor & & & & \\
\hline${ }^{46} \mathrm{TI}$ & p & $4.2+1$ & & PIN E & E Freedmant & 1424 & \\
\hline $44 \mathrm{TI}$ & rolative & e $O(E)$ & & & & & \\
\hline${ }^{27} \mathrm{Al}$ & $19 \mathrm{~F}$ & $4.0+1$ & & BNLE & EPolettit & 11801 & \\
\hline${ }^{44} \mathrm{TI}$ & $O(E)$ & & & & & & \\
\hline${ }^{40} \mathrm{Ca}$ & a & NDG & & CAL I & I Woosleyt & 7300 & \\
\hline
\end{tabular}

$\begin{array}{llll}{ }^{40} \mathrm{CB} & \text { a NDG } & \text { CAL T WoOSley+ } & 7300\end{array}$

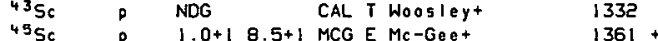

${ }_{45 \mathrm{Sc}}^{4} \mathrm{SP}^{43 \mathrm{Sc}} 1.0+18.5+1$ MCG E Mc-Geet $1361+$

${ }^{45 \mathrm{SC} P} \quad 1.5+18.5+1 \mathrm{KFK} C$ Muenzelt 1362

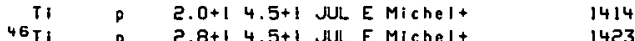

${ }_{47}^{46} \mathrm{II} \quad \mathrm{P} 2.8+14.5+1 \mathrm{JUL}$ E Michelt 1423

$\begin{array}{lllll}47 \mathrm{r} & \text { P } & 4.0+14.5+1 & \text { JUL E Michelt } & 1435\end{array}$

45 Ti gpallation o(E)

Fe p $1.5+23.7+2$ NRL R SIlberbergt 1844 $45 T$ partlal o(E)

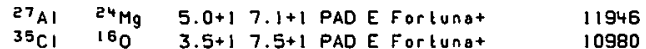




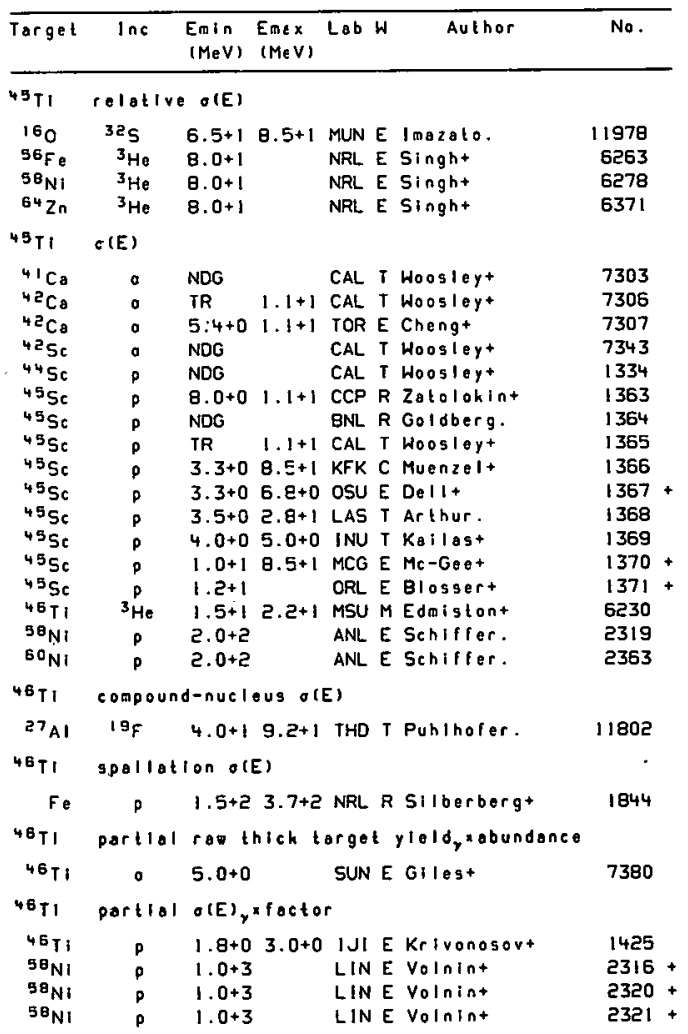

\begin{tabular}{|c|c|c|c|c|c|c|}
\hline Target & Ine & $\begin{array}{l}\text { Emin } \\
(\mathrm{MeV})\end{array}$ & $\begin{array}{l}E \operatorname{Eax} L \\
(\operatorname{MeV})\end{array}$ & Lab W & Author & No. \\
\hline${ }^{46} \mathrm{TI}$ & partlal & $O(E) \times f o$ & foctor & & & \\
\hline${ }^{45} \mathrm{Sc}$ & a & $2.7+1$ & & IJIE & E Tohareushitit & 7351 \\
\hline${ }^{46} \mathrm{TI}$ & portlolo & $\sigma(E)$ & & & $\cdot$ & \\
\hline $\begin{array}{l}40 \mathrm{Co} \\
{ }^{45} \mathrm{Sc}\end{array}$ & ${ }_{a}^{12} c$ & $\begin{array}{l}3 \cdot 4+1 \\
2 \cdot 7+1\end{array}$ & $\begin{array}{r}6.2+1 \mathrm{M} \\
\mathrm{I}\end{array}$ & $\begin{array}{l}\text { MUN E } \\
\text { IJIE }\end{array}$ & $\begin{array}{l}\text { E Fortungt } \\
\text { E Tokareaskita }\end{array}$ & $\begin{array}{l}9905 \\
7350\end{array}$ \\
\hline${ }^{46} \mathrm{Tl}$ & relotive & $I_{r}$ & & & & \\
\hline${ }^{43} \mathrm{Co}$ & a & $8.0+0$ & $1.5+1 \mathrm{c}$ & CBR E & E Dracouligt & 7310 \\
\hline ( & $t_{y}$ & & & & & \\
\hline${ }^{43} \mathrm{Ca}$ & 。 & NDG & & CBR E & E Radford+ & 7311 \\
\hline $45 \pi$ & product & y1010 & & & & \\
\hline${ }^{45} \mathrm{Se}$ & 9 & $1.2+0$ & $3.0+0 \mathrm{R}$ & RAM E & E Rahman- & 1375 \\
\hline${ }^{46} \mathrm{TI}$ & $r o o o(E)$ & & & & & \\
\hline${ }^{45} 5 c$ & p & $1.2+0$ & $1.5+0 \mathrm{~A}$ & AML E & E Solonont & 1372 \\
\hline${ }^{46} \mathrm{Tt}$ & relalive & $\sigma(E)$ & & & & \\
\hline $\begin{array}{l}{ }^{40} \mathrm{Ca} \\
48 \mathrm{TI}\end{array}$ & $\begin{array}{r}12 \mathrm{C} \\
0\end{array}$ & $\begin{array}{l}3.3+1 \\
2.1+1\end{array}$ & $\begin{array}{l}4.8+1 \mathrm{M} \\
3.5+1 \mathrm{C}\end{array}$ & $\begin{array}{l}\text { MUN E } \\
\text { GRE E }\end{array}$ & $\begin{array}{l}\text { E linazito. } \\
\text { E Giorit }\end{array}$ & $\begin{array}{l}9906 \\
7390\end{array}$ \\
\hline${ }^{46} \mathrm{Tl}_{1}$ & $\sigma(E)$ & & & & & \\
\hline $\begin{array}{l}{ }^{4} \mathrm{C}_{\mathrm{B}} \\
{ }^{43} \mathrm{C}_{\theta}\end{array}$ & 0 & $\begin{array}{l}\text { NDG } \\
\text { NDG }\end{array}$ & & $\begin{array}{l}\text { CAL T } \\
\text { CAL T }\end{array}$ & $\begin{array}{l}\text { T Wooglept } \\
\text { T Wooglept }\end{array}$ & $\begin{array}{l}7308 \\
7312\end{array}$ \\
\hline $\begin{array}{l}{ }^{43} \mathrm{CO} \\
{ }^{4} \mathrm{~S}_{\mathrm{Sc}}\end{array}$ & a & $\begin{array}{l}\text { NDG } \\
\text { NDG }\end{array}$ & & $\begin{array}{l}\text { CAL T } \\
\text { CAL T }\end{array}$ & T Wooslept & 7345 \\
\hline${ }^{45} \mathrm{Sc}$ & $p$ & NDG & & CAL I & I Woosleyt & 1373 \\
\hline${ }^{45} \mathrm{Sc}$ & D & $7.0-1$ & $4.2+0 \mathrm{~A}$ & AML E & E Solonont & 1374 \\
\hline $455 c$ & p & NDG & & CAL 1 & T Wooslert & 1377 \\
\hline${ }^{49} \mathrm{~V}$ & p & NDG & & CAL $T$ & T Woogley+ & 1502 \\
\hline${ }^{50} \mathrm{Ni}$ & p & $8.0+1$ & $1.6+21$ & INUE & E Sadlert & 2317 \\
\hline $58_{N i}$ & p & $8.0+1$ & $1.4+2 M$ & MIL I & T Gadiolit & 2310 \\
\hline $5 B_{N 1}$ & p & $2.0+2$ & & ANL E & E Sehiffer. & 2319 \\
\hline${ }^{60} \mathrm{Ni}$ & p & $2.0+2$ & & ANL E & E Schififer. & 2363 \\
\hline 47 & spollatio & IOA OCE & & & & \\
\hline $\mathrm{Fe}$ & $p$ & $1.5+2$ & $3.7+21$ & NRL R & R Silberterg+ & 1844 \\
\hline${ }^{47} \mathrm{TI}$ & portlol & roth & hick tor & orget & yleld, acbundance & \\
\hline $47 \mathrm{Ti}$ & $\cdot$ & $5.0+0$ & & SUN E & E Gilest & 7385 \\
\hline
\end{tabular}




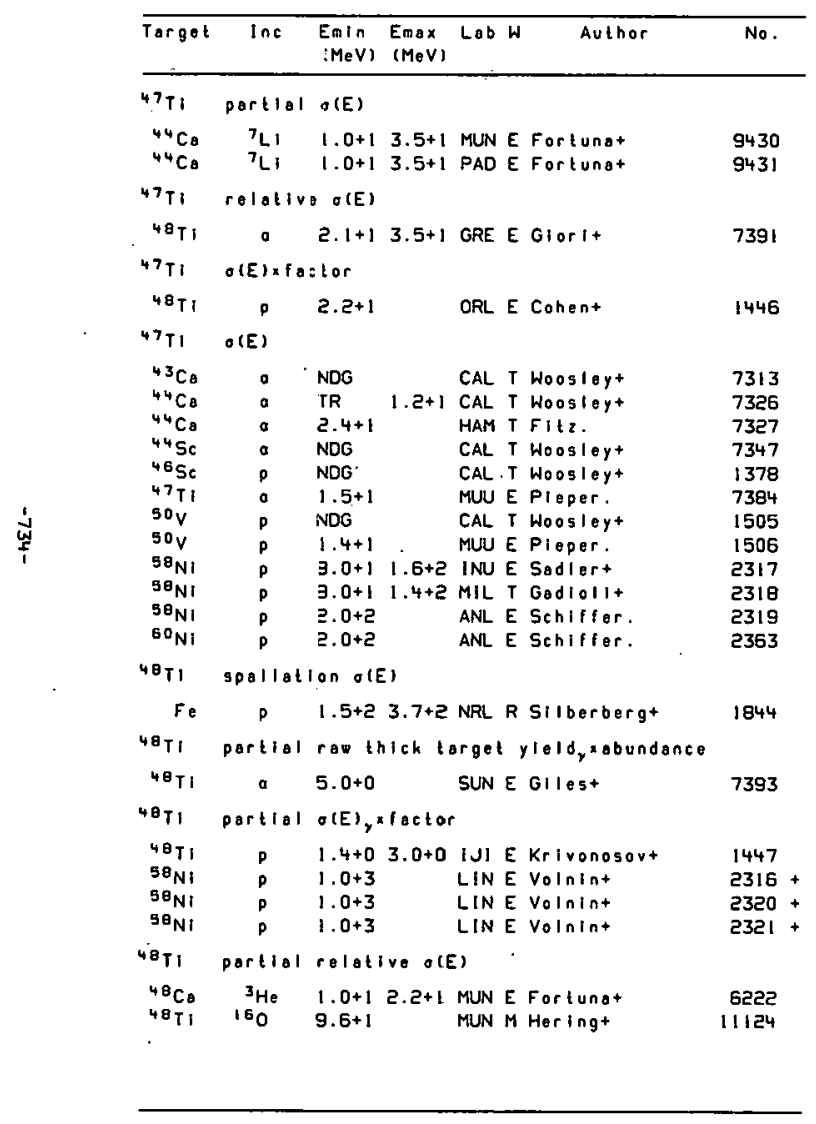

\begin{tabular}{|c|c|c|c|c|c|c|}
\hline Target & $\ln c$ & $\begin{array}{l}\text { Emin } \\
\left(M_{\theta} V\right)\end{array}$ & $\begin{array}{l}E_{\max } \\
(\operatorname{MeV})\end{array}$ & Lab & Author & No. \\
\hline${ }^{48} \mathrm{Ti}$ & portlat & $O(E)$ & & & & \\
\hline $\begin{array}{l}{ }^{40} \mathrm{CB} \\
{ }^{44} \mathrm{CB} \\
50 \mathrm{TI}\end{array}$ & $\begin{array}{c}{ }^{12 C} C \\
7_{L i} \\
p\end{array}$ & $\begin{array}{l}5.5+1 \\
1.0+1 \\
4.0+1\end{array}$ & $\begin{array}{l}6.2+1 \\
3.5+1\end{array}$ & $\begin{array}{l}\text { MUN } \\
\text { MUN } \\
\text { MSU }\end{array}$ & $\begin{array}{l}\text { E Fortunat } \\
\text { E Fortunat } \\
\text { M Brownt }\end{array}$ & $\begin{array}{l}9907 \\
9432 \\
1466\end{array}$ \\
\hline${ }^{40} \mathrm{TI}$ & relative & $\sigma(E)$ & & & & \\
\hline $\begin{array}{l}4 \theta_{\mathrm{TI}} \\
56 \mathrm{Fe} \\
5 \theta_{\mathrm{Ni}} \\
6{ }^{4} \mathrm{Zn}\end{array}$ & $\begin{array}{l}{ }^{0} \mathrm{He} \\
{ }^{3} \mathrm{He} \\
{ }^{3} \mathrm{He}\end{array}$ & $\begin{array}{l}2.1+1 \\
8.0+1 \\
8.0+1 \\
8.0+1\end{array}$ & $3.5+1$ & $\begin{array}{l}\text { GRE } \\
\text { NRL } \\
\text { NRL } \\
\text { NRL }\end{array}$ & $\begin{array}{l}\text { E Glorlt } \\
\text { E Singht } \\
\text { E Singht } \\
\text { E Singht }\end{array}$ & $\begin{array}{l}7392 \\
6263 \\
6278 \\
6371\end{array}$ \\
\hline${ }^{40} \mathrm{Ti}$ & $\sigma(E)$ & & & & & \\
\hline $\begin{array}{l}44 \mathrm{Ca} \\
45 \mathrm{Ca} \\
45 \mathrm{SC} \\
475 \mathrm{C} \\
48 \mathrm{Sc} \\
51 \mathrm{~V} \\
58_{\mathrm{NI}} \\
58_{\mathrm{NI}} \\
58_{\mathrm{NI}} \\
60 \mathrm{NI} \\
62_{\mathrm{NI}}\end{array}$ & $\begin{array}{l}0 \\
q \\
q \\
p \\
p \\
p \\
p \\
p \\
p \\
p \\
p\end{array}$ & $\begin{array}{l}\text { NDG } \\
\text { NDG } \\
\text { NDG } \\
\text { NDG } \\
\text { NDG } \\
\text { NDG } \\
8.0+1 \\
8.0+1 \\
2.0+2 \\
2.0+2 \\
1.4+2\end{array}$ & $\begin{array}{l}1.6+2 \\
1.4+2\end{array}$ & $\begin{array}{l}\text { CAL } \\
\text { CAL } \\
\text { CAL } \\
\text { CAL } \\
\text { CAL } \\
\text { CAL } \\
\text { INU } \\
\text { MIL } \\
\text { ANL } \\
\text { ANL } \\
\text { MIL }\end{array}$ & $\begin{array}{l}\text { T Woosleyt } \\
\text { T Woosley+ } \\
\text { T Woosleyt } \\
\text { T Woosleyt } \\
\text { T Woosley+ } \\
\text { T Woosleyt } \\
\text { E Sadlert } \\
\text { T Gadialit } \\
\text { E Schiffer. } \\
\text { E Schlffer. } \\
\text { T Gadialit }\end{array}$ & $\begin{array}{l}7328 \\
7331 \\
7352 \\
1380 \\
1382 \\
1532 \\
2317 \\
2318 \\
2319 \\
2363 \\
2412\end{array}$ \\
\hline${ }^{49} \mathrm{TI}_{1}$ & spollati & IOO OIE & & & & \\
\hline $\begin{array}{r}\mathrm{Fe} \\
4971\end{array}$ & $p$ & $\begin{array}{l}1.5+2 \\
o(E)\end{array}$ & $3.7+2$ & NRL & R Silberberg* & 1844 \\
\hline${ }^{48} \mathrm{Ca}$ & 150 & $5.6+1$ & & ANL & E Kovart & 11098 \\
\hline${ }^{49} \mathrm{TI}$ & relative & $O(E)$ & & & : & \\
\hline $\begin{array}{l}56 \mathrm{Fe} \\
50_{\mathrm{NI}} \\
54 \mathrm{Zn}\end{array}$ & $\begin{array}{l}{ }^{3} \mathrm{He} \\
{ }^{3} \mathrm{He} \\
{ }^{3} \mathrm{He}\end{array}$ & $\begin{array}{l}8.0+1 \\
8 \cdot 0+1 \\
8 \cdot 0+1\end{array}$ & & $\begin{array}{l}\text { NRL } \\
\text { NRL } \\
\text { NRL }\end{array}$ & $\begin{array}{l}\text { E Singht } \\
\text { E Singht } \\
E \text { Singht }\end{array}$ & $\begin{array}{l}6263 \\
6278 \\
6371\end{array}$ \\
\hline${ }^{48} \mathrm{TI}$ & $O(E)$ & & & & . & \\
\hline $\begin{array}{l}{ }^{45} \mathrm{Ca} \\
{ }^{45} \mathrm{Cb} \\
{ }^{46} \mathrm{Sc} \\
{ }^{48} \mathrm{Sc} \\
{ }^{49} \mathrm{Sc}\end{array}$ & $\begin{array}{l}0 \\
0 \\
a \\
p \\
p\end{array}$ & $\begin{array}{l}\text { NDG } \\
\text { TR } \\
\text { NDG } \\
\text { NDG } \\
\text { NDG }\end{array}$ & $1.2+1$ & $\begin{array}{l}\text { CAL } \\
\text { CAL } \\
\text { CAL } \\
\text { CAL } \\
\text { CAL }\end{array}$ & $\begin{array}{l}\text { T Woosley+ } \\
\text { T Woosley+ } \\
\text { T Woosleyt } \\
\text { T Woosley+ } \\
\text { T Woosley+ }\end{array}$ & $\begin{array}{l}7332 \\
7334 \\
7355 \\
1383 \\
1385\end{array}$ \\
\hline
\end{tabular}




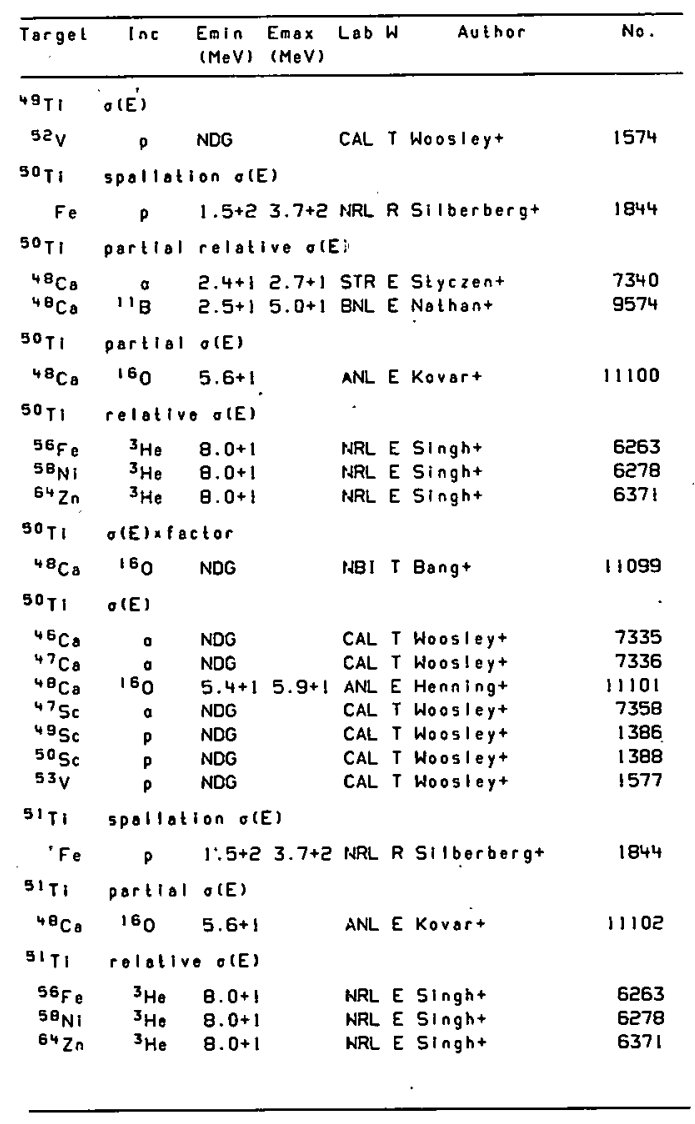

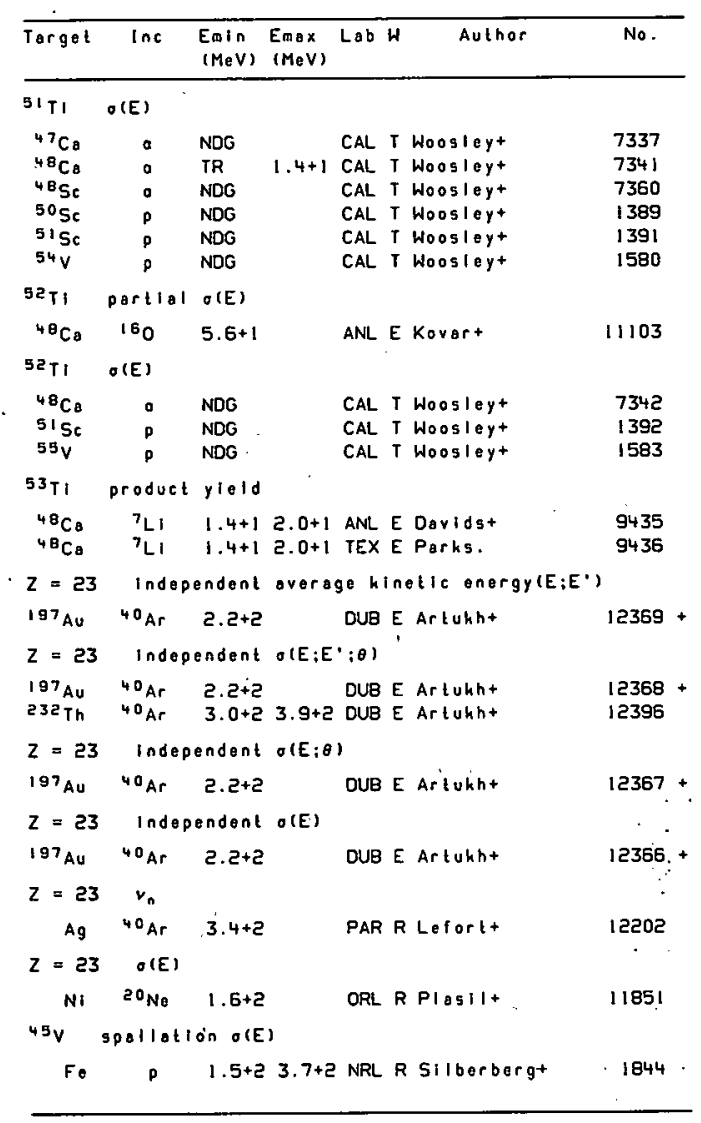




\begin{tabular}{|c|c|c|c|c|c|c|}
\hline Target & $\operatorname{lnc}$ & $\begin{array}{l}\text { Emin } \\
(\mathrm{MeV})\end{array}$ & $\begin{array}{l}\text { Emax } \\
(\operatorname{MeV})\end{array}$ & Lab $r$ & Author & No. \\
\hline \multicolumn{7}{|c|}{ tial product yleld } \\
\hline $\begin{array}{l}{ }^{40} \mathrm{Ca} \\
{ }^{40} \mathrm{Ca}\end{array}$ & $\begin{array}{l}\sigma_{L L} \\
7_{L I}\end{array}$ & $\begin{array}{l}\text { NDG } \\
\text { NDG }\end{array}$ & & $\begin{array}{l}\text { ANL } \\
\text { ANL }\end{array}$ & $\begin{array}{l}\text { E Gronmeyert } \\
\text { E Gronmeyert }\end{array}$ & $\begin{array}{l}9182 \\
942 \theta\end{array}$ \\
\hline \multicolumn{7}{|c|}{$45 \mathrm{~V} \quad O(E)$} \\
\hline${ }^{46} \mathrm{TI}$ & $p$ & $2.2+1$ & $3.4+1$ & MSU & M Edmistont & 1426 \\
\hline \multicolumn{7}{|c|}{ spollation o(E) } \\
\hline $\mathrm{Fe}$ & P & $1.5+2$ & $3.7+2$ & NRL F & R Silberberg+ & 1844 \\
\hline \multicolumn{7}{|c|}{ rtial o(E) } \\
\hline${ }^{40} \mathrm{Ca}$ & $12 \mathrm{C}$ & $4.8+1$ & $6.2+1$ & MUN E & E Fortunat & 9908 \\
\hline \multicolumn{7}{|c|}{$46 \mathrm{~V}$ O(E) } \\
\hline $425 c$ & o & NDG & & CAL 1 & T Woosleyt & 7344 \\
\hline${ }^{45} \mathrm{Ti}$ & $p$ & NDG & & CAL $T$ & T Woosleyt & 1418 \\
\hline${ }^{46} \mathrm{Ti}$ & $p$ & NDG & & KTY E & E Flynnt & 1427 \\
\hline${ }^{46} \mathrm{TI}$ & 9 & $T R$ & $1.1+1$ & CAL T & T Woosleyt & 1428 \\
\hline${ }^{46} \mathrm{TI}$ & i & $7.3+0$ & $1.0+1$ & CRC E & E Jachiont & 1429 \\
\hline $46 \pi$ & p & $2.0+1$ & $3.4+1$ & MSU $\mathrm{F}$ & MEdmistiont & 1430 \\
\hline${ }^{46} \mathrm{TI}$ & $3_{\mathrm{He}}$ & $2.0+1$ & $2.8+1$ & MSU $\mathrm{r}$ & MEdmistont & 6231 \\
\hline \multicolumn{7}{|c|}{ ollation o(E) } \\
\hline $\mathrm{Fe}$ & $p$ & $1.5+2$ & $3.7+2$ & NRL $F$ & R silberbergt & 1844 \\
\hline \multicolumn{7}{|c|}{$47 \mathrm{~V}$ portiol o(E) } \\
\hline${ }^{40} \mathrm{Co}$ & ${ }^{12} \mathrm{C}$ & $2.7+1$ & $6.2+1$ & MUN E & E Fortunat & 9909 \\
\hline \multicolumn{7}{|c|}{ ck torget yleld } \\
\hline${ }^{46} \mathrm{TI}$ & 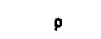 & $7.0-1$ & $3.5+0$ & CLU $\mathrm{r}$ & M Roughlont & 1433 \\
\hline \multicolumn{7}{|c|}{ relative o(E) } \\
\hline${ }^{40} \mathrm{Ca}$ & ${ }^{12} \mathrm{c}$ & $3.3+1$ & $4.8+1$ & MUN E & E Imazalo. & s910 \\
\hline \multicolumn{7}{|c|}{$47 \mathrm{~V}$ O(E) x lactor } \\
\hline $48 \mathrm{Ti}$ & p & $2.2+1$ & & ORL E & E Cohent & 1450 \\
\hline \multicolumn{7}{|c|}{$47 V \quad \sigma(E)$} \\
\hline${ }^{40} \mathrm{Co}$ & 150 & $7.0+1$ & & NBI $\mathrm{r}$ & M Kuang-ShIt & 11010 \\
\hline${ }^{40} \mathrm{Co}$ & 160 & $7.0+1$ & & NBI & M Kuong-Shlt & 11011 \\
\hline${ }^{40} \mathrm{Co}$ & 160 & $7.0+1$ & & NBI $\mathrm{M}$ & M Kuang-Sh1+ & 11009 \\
\hline
\end{tabular}

\begin{tabular}{|c|c|c|c|c|c|c|}
\hline Target & $\ln c$ & $\begin{array}{l}E m_{1} \mid n \\
\left(M_{\theta} V\right)\end{array}$ & $\begin{array}{l}\text { Enox } \\
(\mathrm{MeV})\end{array}$ & Lab $\mathrm{W}$ & Author & No. \\
\hline
\end{tabular}

\begin{tabular}{|c|c|c|c|c|c|c|}
\hline $47 v$ & $o(E)$ & & & & & \\
\hline${ }^{43} \mathrm{Sc}$ & a & NDG & & CAL $T$ & T Woosleyt & 7346 \\
\hline${ }^{46} \mathrm{Ti}$ & p & NDG & & CAL $T$ & Thosleyt & 1431 \\
\hline${ }^{46} \mathrm{TI}$ & p & $7.0-1$ & $3.5+0$ & CLUE & Roughtont & 1432 \\
\hline${ }^{46} \mathrm{Ti}$ & ${ }^{3} \mathrm{He}$ & $1.5+i$ & $2.8+1$ & MSU M & 4 Edmistont & 6232 \\
\hline $47 \mathrm{TI}$ & $p$ & TR & $1.1+1$ & CAL $T$ & Thosleyt & 1436 \\
\hline${ }^{47} \mathrm{TI}$ & $p$ & $4.2+0$ & $1.4+1$ & KFK C & Cuenzelt+ & 1437 \\
\hline $47 \mathrm{TH}$ & p & $4.2+0$ & $1.4+1$ & TOK E & Tonahat & $1438+$ \\
\hline $47 \mathrm{Ti}$ & p & $4.9+0$ & $6.7+0$ & ROC E & Taketanit & $1439+$ \\
\hline${ }^{4} \theta_{\mathrm{TI}}$ & p & $2.2+1$ & & KFK C & Muenzalt & 1448 \\
\hline $48 \mathrm{TI}$ & p & $2.2+1$ & & ORL E & Cohent & $1449+$ \\
\hline $50 \mathrm{Cr}$ & p & $9.2-1$ & $1.5+1$ & CAL $T$ & T Woosleyt & 1534 \\
\hline${ }^{5 \theta_{N 1}}$ & p & $8.0+1$ & $1.6+2$ & INU E & Sadlert & 2317 \\
\hline${ }^{58} \mathrm{~N}_{1}$ & p & $8.0+1$ & $1.4+2$ & MIL T & T Gadiolit & 2318 \\
\hline
\end{tabular}

$4 \theta_{V}$ cumulallve spollotion $\sigma(E)$

Cu p 2.0+2 5.9+2 LAS E Ortht 2577

${ }^{4 \theta} \mathrm{V}$ spollation o(E)

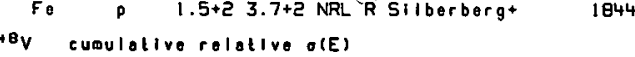

$160325 \quad 6.5+18.5+1$ MUN E Inozato. 11979

$48 \mathrm{~V}$ curulative o(E)

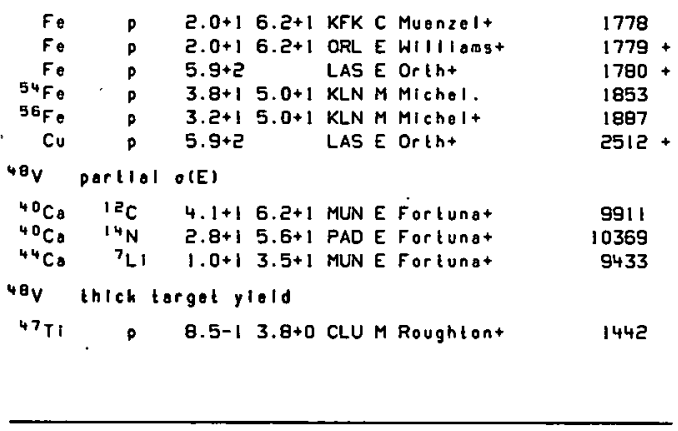




\begin{tabular}{|c|c|c|c|c|c|c|}
\hline Target & $\operatorname{lnc}$ & $\begin{array}{l}\text { Emin } \\
(M e V)\end{array}$ & $\begin{array}{l}E \max \\
(M \in V)\end{array}$ & Lab & Author & No. \\
\hline $48 \mathrm{~V}$ & \multicolumn{6}{|l|}{$\sigma(E)$} \\
\hline${ }^{44} \mathrm{Sc}$ & $a$ & NOG & & CAL & T Woogleyt & 7348 \\
\hline${ }^{45} \mathrm{sc}$ & 0 & IR & $8.7+0$ & CAL & T Woosleyt & 7353 \\
\hline $\mathrm{Ti}$ & p & $8.0+0$ & $1.1+1$ & CCP & R Zotolokint & 1415 \\
\hline TI & p & $1.3+14$ & $4.5+1$ & KLN & E Michelt & $1416+$ \\
\hline $\mathrm{TI}$ & p & $1.7+1$ & $4.3+1$ & RIC & E Waltont & 1417 \\
\hline${ }^{45} \mathrm{Ti}$ & 0 & NDG & & CAL & T Hoosleyt & 7378 \\
\hline $47 \mathrm{TI}$ & p & NDG & & CAL & $T$ Woosleyt & 1440 \\
\hline${ }^{47} \mathrm{TI}$ & p & $8.5-1=$ & $3.8+0$ & CLU & E Roughtont & 1441 \\
\hline${ }^{40} \mathrm{TI}$ & p & TR & $1.1+1$ & CAL & T Woosleyt & 1451 \\
\hline${ }^{48} \mathrm{TI}$ & p & $5.1+0$ & $1.4+1$ & KFK & C Muenzelt & 1452 \\
\hline $48 T$ & p & $5.1+0$ & $1.4+1$ & TOK & E Tonohat & $1453+$ \\
\hline$v$ & p & $1.7+1$ & $4.5+1$ & KLN & M Michelt & 1495 \\
\hline $50 \mathrm{v}$ & $p$ & $2.3+1=$ & $5 \cdot 0+1$ & $\mathrm{KLN}$ & M Michel. & 1507 \\
\hline $51 \mathrm{~V}$ & p & NDG & & KLN & E Herrt & 1533 \\
\hline $51 \mathrm{~V}$ & $p$ & $3.4+15$ & $5.0+1$ & KLN & M Michelt & 1534 \\
\hline $51 \mathrm{~V}$ & p & $5.0+1$ & $1.0+2$ & $\mathrm{BNL}$ & T Divadeenam. & 1535 \\
\hline $51 \mathrm{~V}$ & d & $T R$ & $9.0+1$ & JUL & E Probstt & 5452 \\
\hline $51 \mathrm{~V}$ & a & $5.2+1$ & $1.2-2$ & KFK & C Muenzel+ & 7442 \\
\hline $51 \mathrm{~V}$ & a & $5.2+1$ & $1.2-2$ & ROC & M Bowmannt & $7443+$ \\
\hline $\mathrm{Fe}_{\mathrm{e}}$ & p & $3.9+1$ & $4.4-1$ & KLN & Michelt & 1776 \\
\hline $\mathrm{Fe}$ & $p$ & $5.0+1$ & $6.3+1$ & DAV & E Schoent & 1777 \\
\hline${ }^{54} \mathrm{Fe}$ & ${ }^{6} L_{1}$ & $9.8+0$ & $9.4+1$ & INU & E Jestrzebskit & 9185 \\
\hline $57 \mathrm{Fe}$ & p & $4.7+1$ & $5 \cdot 0+i$ & KLN & M Michelst & 1944 \\
\hline $\mathrm{Ni}$ & p & $6.0+2$ & & MTR & E clinet & $2156+$ \\
\hline${ }^{5 \theta_{N i}}$ & p & $8.0+1$ & $1.6+2$ & INU & E Sadlert & 2317 \\
\hline${ }^{50} \mathrm{NI}$ & p & $8.0+1$ & $1.4+2$ & MIL & T Godiolit+ & 2318 \\
\hline $60 \mathrm{NI}$ & p & $1.4+2$ & & INU & E Sadlert & 2361 \\
\hline $60_{\mathrm{Ni}}$ & p & $1.4+2$ & & MIL & T Gadialit & 2362 \\
\hline $62_{\mathrm{NI}}$ & p & $1.4+2$ & & MIL & T Gadiolit & 2412 \\
\hline $\mathrm{Cu}$ & p & $6.0+2$ & & MTR & E clinet & $2511+$ \\
\hline \multicolumn{7}{|l|}{$49 v$} \\
\hline $\mathrm{Fe}$ & $p$ & $1.5+2$ & $3.7+2$ & NRL & R silberberg+ & 1844 \\
\hline $49 \mathrm{~V}$ & partial & $\theta(E)$ & & & & \\
\hline $\begin{array}{l}{ }^{40} \mathrm{Ca} \\
{ }^{40} \mathrm{Ca} \\
4{ }^{4} \mathrm{Ca} \\
51 \mathrm{~V}\end{array}$ & $\begin{array}{c}12 C \\
12 C \\
7 L 1 \\
{ }^{7} \mathrm{C} \\
p\end{array}$ & $\begin{array}{l}2.7+1 \\
3.4+1 \\
1.0+1 \\
4.0+1\end{array}$ & $\begin{array}{l}6.2+1 \\
6.2+1 \\
3.5+1\end{array}$ & $\begin{array}{l}\text { PAD } \\
\text { MUN } \\
\text { MUN } \\
\text { MSU }\end{array}$ & $\begin{array}{l}\text { E Forlunat } \\
\text { E Forlunat } \\
\text { E Forlunat } \\
\text { M Brount }\end{array}$ & $\begin{array}{l}9912 \\
9913 \\
9434 \\
1536\end{array}$ \\
\hline
\end{tabular}

\begin{tabular}{lllll}
\hline Target Inc Eain Eaax Lab $W$ & $\begin{array}{c}\text { Euthor } \\
(\mathrm{MeV})\end{array}$ & No. \\
\hline $49 \mathrm{VeV})$ &
\end{tabular}

\section{$49 \mathrm{~V}$ average o(E)}

$49 \mathrm{Ti} P \quad 1.4+01.5+0$ ORL E Johnsont i

$49 \mathrm{~V}$ rolotive $O(E)$

${ }^{40} \mathrm{Ca} \quad{ }^{12} \mathrm{C} \quad 3.3+14.8+1$ MUN E Imazato. 9914

${ }^{48} \mathrm{TI}$ a $2.7+13.5+1$ GRE E Glorit $\quad 7394$

$4 \mathrm{~g}^{\mathrm{V}}$ o(E)

${ }^{45} \mathrm{Sc} \quad \mathrm{NC}$ NDG CAL T Woosleyt .7354

${ }_{46 \mathrm{Sc}}^{45}$ NDG CAL T Woos eyt 7356

$\begin{array}{llll}45 T 1 & 0 & \text { IR } 1.2+1 \text { CAL T Woosleyt } & 739 !\end{array}$

$\begin{array}{lllll}48 \mathrm{Ti} & \mathrm{P} & \text { NDG } & \text { CAL T Hoosleyt } & 1454 \\ 49 \mathrm{Ti} & \rho & \text { TR } & 1.1+1 \text { CAL T Hoosleyt } & 1458\end{array}$

${ }_{49}^{4} \mathrm{Tl} P \quad 1.4+05.8+0 \mathrm{KFKCM}$ Muenzel+ 1459

${ }^{49} \mathrm{Tl} P \quad 1.4+05.8+0$ ORL $E$ Johnsont 1460 .

${ }^{52} \mathrm{Cr} \quad$ 8.2-1 $1.4+1$ CAL T Woosleyt 1611

${ }_{50} 8_{N i} \rho 8.0+11.6+2$ INU E Sadtart 2317

${ }^{98} \mathrm{NI} P \quad 8.0+11.4+2$ MIL T Gadiollt 2318

$58 \mathrm{NI}$. $2.0+2$ ANL E Schiffer.

$60_{\mathrm{NI}} \mathrm{P} 1.4+2$ INU E Sadiert

$60 \mathrm{N1} P \quad 1.4+2$ MIL T Gadiolit 2362

$60 \mathrm{NI} P$ P $2.0+2$ ANL E Sehiffer.

$65_{N 1} \mathrm{~N} 1.4+2$ MIL T Gadiolit

SOV spallation $\sigma(E)$

Fe $\quad 1.5+23.7+2$ NRL R Silberbergt 1844

Sov portlal o(E) at resonance

SOTi P TR TO ORE E Leferget 1467

SOV partial row $O(E)$

$50 T_{1} P$ TR TO ORE E Lefeuret 1468

$20 \mathrm{~V}$ relative $\mathrm{I}^{4}$

${ }^{4 \theta_{\mathrm{Tl}}}$ a 3.2+1 GRE E GIori- 7398

Sov relotive o(E)

$\begin{array}{llllll}{ }^{40} \mathrm{TI} & \text { a } & 2.1+1 & 3.5+1 & \text { GRE E Glorit } & 7395 \\ { }^{56} \mathrm{Fe} & { }^{3} \mathrm{He} & 8.0+1 & \text { NRL E Singht } & 6263\end{array}$

$\begin{array}{lllll}{ }^{58} \mathbf{F i}_{\mathrm{Ni}} & { }^{3} \mathrm{He} . & 8.0+1 & \text { NRL E Singht } & 6263 \\ { }_{64} \mathrm{He} & 8.0+1 & \text { NRL E Singht } & 6278\end{array}$

$\begin{array}{lllll}{ }^{64} \mathrm{Zn} & { }^{3} \mathrm{He} & 8.0+1 & \text { NRL E Singht } & 6278 \\ & & & & \end{array}$ 


\begin{tabular}{|c|c|c|c|c|c|c|}
\hline Target & $\ln c$ & $\begin{array}{l}\text { Emin } \\
(M e V)\end{array}$ & $\begin{array}{l}\text { Emax } \\
(\mathrm{MeV})\end{array}$ & Lab & Author & No. \\
\hline${ }^{50} \mathrm{v}$ & \multicolumn{6}{|l|}{$\sigma(E)$} \\
\hline $\begin{array}{l}46 \mathrm{Sc} \\
47 \mathrm{Ti} \\
47 \mathrm{Ti} \\
49 \mathrm{Ti} \\
50 \mathrm{Ti} \\
50 \mathrm{Ti} \\
50 \mathrm{Ti} \\
50 \mathrm{~V} \\
53 \mathrm{Cr} \\
58_{\mathrm{Ni}} \\
58_{\mathrm{Ni}} \\
50 \mathrm{Ni} \\
60_{\mathrm{Ni}} \\
62_{\mathrm{Ni}}\end{array}$ & $\begin{array}{l}a \\
a \\
a \\
p \\
p \\
p \\
p \\
p \\
p \\
p \\
p \\
p \\
p \\
p\end{array}$ & $\begin{array}{l}\text { NDG } \\
\text { TR } \\
1.5+1 \\
\text { NDG } \\
\text { NDG } \\
\text { NDG } \\
\text { TR } \\
1.4+1 \\
8.2-1 \\
8.0+1 \\
8.0+1 \\
1.4+2 \\
1.4+2 \\
1.4+2\end{array}$ & $\begin{array}{c}1.2+1 \\
1.1+1 \\
1.2+1 \\
1.6+2 \\
1.4+2\end{array}$ & $\begin{array}{l}\text { CAL } \\
\text { CAL } \\
\text { MUU } \\
\text { CAL } \\
\text { KTY } \\
\text { IRM E } \\
\text { CAL } \\
\text { MUU } \\
\text { CAL } \\
\text { INU } \\
\text { MIL } \\
\text { INU } \\
\text { MIL } \\
\text { MIL }\end{array}$ & $\begin{array}{l}\text { T Woosley+ } \\
\text { T Woosley+ } \\
\text { E Pieper. } \\
\text { T Woosleyt } \\
\text { E Flynnt } \\
\text { E Kailast } \\
\text { T Woosleyt } \\
\text { E Pieper. } \\
\text { T Woosley+ } \\
\text { E Sadlert } \\
\text { T Gadialit } \\
\text { E Sadlert } \\
\text { T Gadiolit } \\
\text { T Gadiolit }\end{array}$ & $\begin{array}{l}7357 \\
7386 \\
7387 \\
1462 \\
1469 \\
1470 \\
1471 \\
1508 \\
1648 \\
2317 \\
2318 \\
2361 \\
2362 \\
2412\end{array}$ \\
\hline 312 & \multicolumn{6}{|c|}{ Spallation $\sigma(E)$} \\
\hline Fe & $p_{\text {partial }}^{p}$ & $\begin{array}{l}1.5+2 \\
\text { roothi }\end{array}$ & $\begin{array}{l}3.7+2 \text { o } \\
\text { ich tors }\end{array}$ & $\begin{array}{l}\text { NRL F } \\
\text { rgot }\end{array}$ & $\begin{array}{l}\text { R Silberbergt } \\
\text { yleld }\end{array}$ & 1844 \\
\hline 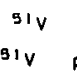 & $\begin{array}{c}a \\
\text { partial }\end{array}$ & $\begin{array}{l}5.0+0 \\
O(E)_{d}\end{array}$ & & SUN E & E Gilest & 7445 \\
\hline $\begin{array}{l}51 \mathrm{~V} \\
51 \mathrm{~V}\end{array}$ & $\begin{array}{c}{ }^{3} \mathrm{He} \\
\text { partlel }\end{array}$ & $\begin{array}{l}7.0+1 \\
0(E)_{y} \times f\end{array}$ & $\begin{array}{l}9.0+1 \\
\text { factor }\end{array}$ & OSA & E Matsuokat & 6236 \\
\hline $\operatorname{siv}$ & $\begin{array}{c}\text { p } \\
\text { partiol }=\end{array}$ & $\begin{array}{l}1.2+0 \\
\text { spoctro }\end{array}$ & $\begin{array}{l}3.0+0 \\
\text { un over }\end{array}$ & $\begin{aligned} \text { IJI E } \\
\text { rage }\end{aligned}$ & $\begin{array}{l}\text { E Krivonosovt } \\
\text { o(E) }\end{array}$ & 1537 \\
\hline $\begin{array}{l}51 \mathrm{~V} \\
51 \mathrm{~V}\end{array}$ & $\begin{array}{c}a \\
\text { portial }\end{array}$ & $\begin{array}{l}2.3+1 \\
\text { relotiv }\end{array}$ & $\begin{array}{l}2.7+1 \\
\text { ve } O(E)\end{array}$ & MUU $=$ & E Eberhardt & 7444 \\
\hline $\begin{array}{l}{ }^{48} \mathrm{Ca} \\
50 \mathrm{Ti}\end{array}$ & $\begin{array}{r}1_{B} \\
p\end{array}$ & $\begin{array}{l}2.5+1 \\
2.1+0\end{array}$ & $\begin{array}{l}5 \cdot 0+1= \\
3 \cdot 1+0 \mathrm{C}\end{array}$ & BNL & $\begin{array}{l}\text { E Nathant } \\
\text { E Erlondssont }\end{array}$ & $\begin{array}{l}9575 \\
1473\end{array}$ \\
\hline $51 \mathrm{~V}$, & partial & $O(E)$ & & & & \\
\hline $\begin{array}{l}{ }^{48} \mathrm{Ca} \\
51 \mathrm{~V}\end{array}$ & $\begin{array}{l}160 \\
\text { relotive }\end{array}$ & $\begin{array}{l}5.6+1 \\
O(E)\end{array}$ & & ANL & E Kovort & 11104 \\
\hline $\begin{array}{l}48 \mathrm{TI} \\
56 \mathrm{Fe}\end{array}$ & $\underset{3_{\mathrm{He}}}{a}$ & $\begin{array}{l}2 \cdot 1+1 \\
8 \cdot 0+1\end{array}$ & $3.5+10$ & $\begin{array}{l}\text { GRE } \\
\text { NRL E }\end{array}$ & $\begin{array}{l}\text { E Giorit } \\
\text { E Singht }\end{array}$ & $\begin{array}{l}7397 \\
6263\end{array}$ \\
\hline
\end{tabular}

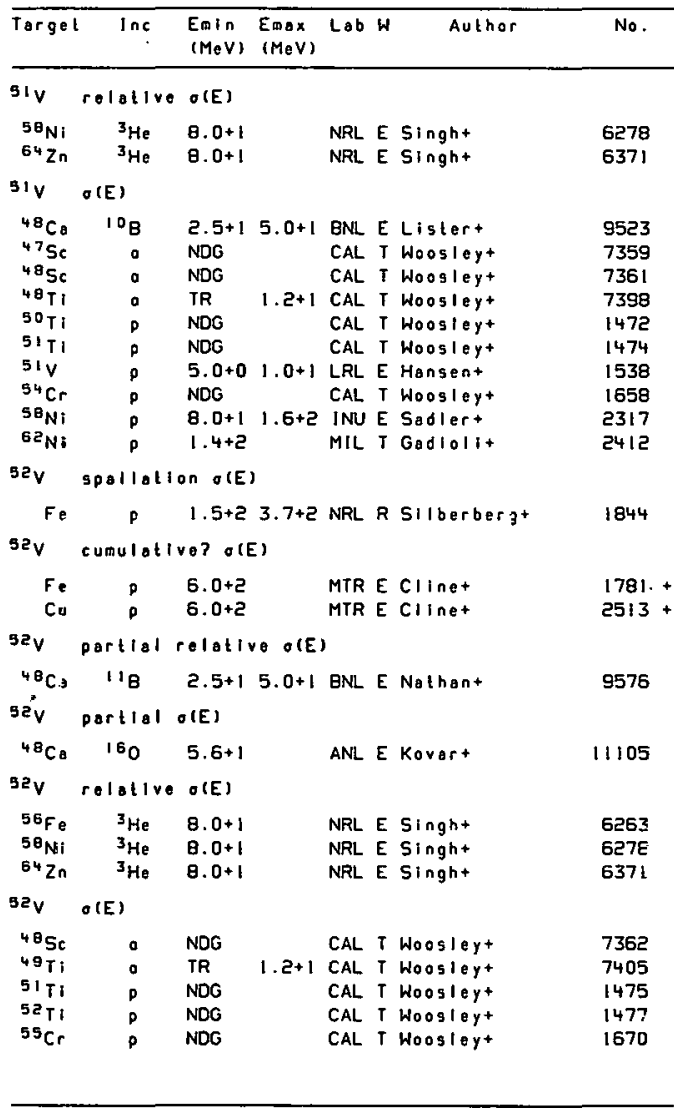




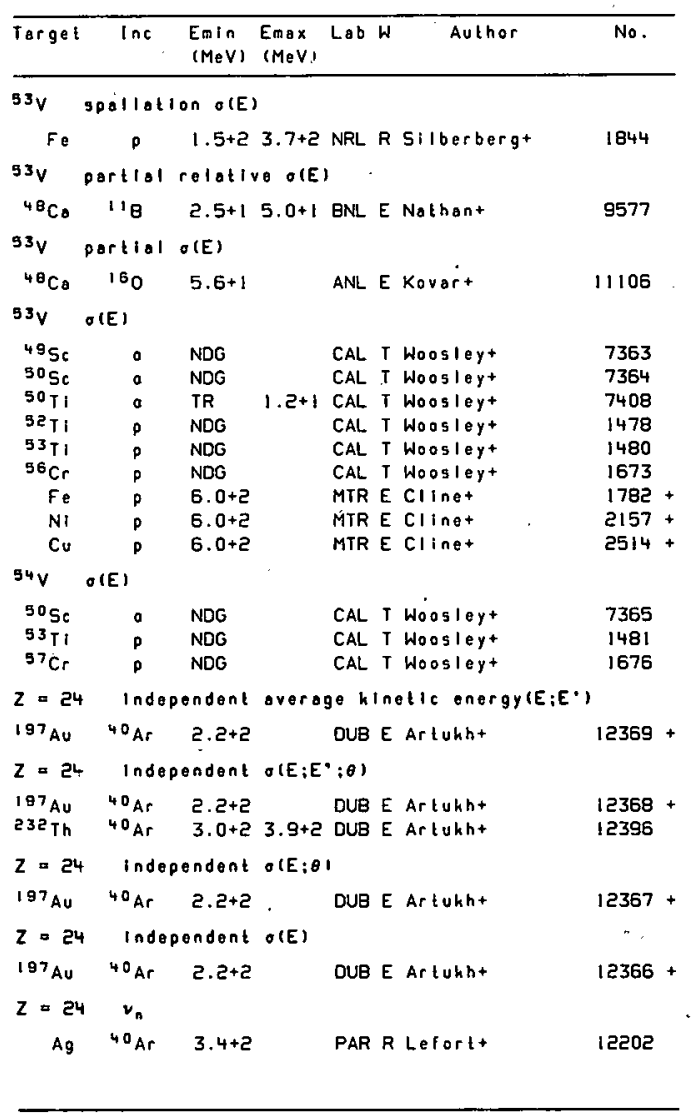

\begin{tabular}{|c|c|c|c|c|c|c|}
\hline Target & $\operatorname{lnc}$ & $\begin{array}{l}\text { Emln } \\
(\mathrm{MeV})\end{array}$ & $\begin{array}{l}\text { Emax } \\
(M e V)\end{array}$ & Lab W & Author & No. \\
\hline$z=24$ & $O(E)$ & & & & & \\
\hline $\mathrm{Ni}$ & $20 \mathrm{Ne}$ & $1.6+2$ & & \multirow{2}{*}{\multicolumn{2}{|c|}{ ORL R PIasil+ }} & \multirow[t]{2}{*}{11851} \\
\hline${ }^{45} \mathrm{Cr}$ & produet & yiold & & & & \\
\hline $32 \mathrm{~s}$ & 160 & NDG & & BRK $R$ & R Cerny & 10977 \\
\hline${ }^{49} \mathrm{Cr}$ & $O(E)$ & & & & & \\
\hline${ }^{32} \mathrm{~s}$ & 160 & NDG & & BRK $R$ & R Cerny. & 10978 \\
\hline${ }^{47} \mathrm{Cr}$ & \multicolumn{5}{|c|}{ Spollotlon o(E) } & \multirow{3}{*}{1844} \\
\hline $\mathrm{Fe}$ & $p$ & \multirow{2}{*}{\multicolumn{2}{|c|}{$1.5+2 \quad 3.7+2$}} & \multirow{2}{*}{\multicolumn{2}{|c|}{ NRL R stlberjergt }} & \\
\hline${ }^{47} \mathrm{Cr}$ & $O(E)$ & & & & & \\
\hline $\begin{array}{l}46 \mathrm{TI} \\
51 \mathrm{~V}\end{array}$ & $\stackrel{3}{3} \mathrm{He}$ & $\begin{array}{l}1.5+1 \\
9.0+1\end{array}$ & $2.8+1$ & $\begin{array}{l}\text { MSU M } \\
\text { BNL T }\end{array}$ & $\begin{array}{l}\text { MEdmistion- } \\
\text { T Divadeanam. }\end{array}$ & $\begin{array}{l}6233 \\
1571\end{array}$ \\
\hline${ }^{4 \theta} \mathrm{Cr}$ & \multicolumn{5}{|c|}{ cumulatlve spollation o(E) } & \\
\hline $\mathrm{Cu}$ & $p$ & $2.0+2$ & $5.9+2$ & LAS E & E Ortht & 2577 \\
\hline${ }^{40} \mathrm{Cr}$ & \multicolumn{3}{|c|}{ spollation o(E) } & & & $\ddot{\ddot{\theta}}$ \\
\hline $\mathrm{Fe}$ & $p$ & $1.5+2$ & $3.7+2$ & NRL $R$ & R Silberberg+ & 1844 \\
\hline${ }^{48} \mathrm{Cr}$ & \multicolumn{3}{|c|}{ cumulative thich tar } & rgety & $y \mid 010$ & \\
\hline$T_{11}$ & ${ }^{3} \mathrm{He}$ & $\begin{array}{l}1.0+1 \\
1.0+1\end{array}$ & $\begin{array}{l}1.4+1 \\
1.4+1\end{array}$ & $\begin{array}{l}\text { JUL E } \\
\text { JUL E }\end{array}$ & $\begin{array}{l}\text { E Probst- } \\
\text { E Probsit }\end{array}$ & $\begin{array}{l}6225 \\
7374\end{array}$ \\
\hline${ }_{48} \mathrm{Cr}$ & \multicolumn{5}{|c|}{ portial rolative o(E) } & \\
\hline $\begin{array}{l}34 \mathrm{~S} \\
{ }^{40} \mathrm{CB}\end{array}$ & $\begin{array}{l}160 \\
108\end{array}$ & $\begin{array}{l}3.4+1 \\
1.4+1\end{array}$ & $3.6+1$ & $\begin{array}{l}\text { AAU E } \\
\text { AAU E }\end{array}$ & $\begin{array}{l}\text { E Hansent } \\
\text { E Hansent }\end{array}$ & $\begin{array}{r}10979 \\
9522\end{array}$ \\
\hline${ }^{40} \mathrm{Cr}$ & \multicolumn{5}{|c|}{ portial o(E) } & \\
\hline${ }^{40} \mathrm{Ca}$ & $14 \mathrm{~N}$ & $2.8+1$ & $5.6+1$ & PAD E & E Fortunat & 10370 \\
\hline${ }^{48} \mathrm{Cr}$ & \multicolumn{5}{|c|}{ thlch torget yield } & \\
\hline 11 & ${ }^{3} \mathrm{He}$ & $3.6+1$ & & JUL E & E Weinrlacht & 6226 \\
\hline${ }^{48} \mathrm{Cr}$ & \multicolumn{3}{|c|}{ relative o(E) } & & & \\
\hline $\begin{array}{l}160 \\
{ }^{40} \mathrm{Ca} \\
56 \mathrm{Fe} \\
58 \mathrm{Ni}\end{array}$ & $\begin{array}{l}3{ }^{3} \mathrm{~s} \\
1^{2} \mathrm{C} \\
3^{3} \mathrm{He} \\
{ }^{3} \mathrm{He}\end{array}$ & $\begin{array}{l}6 \cdot 5+1 \\
3 \cdot 3+1 \\
8 \cdot 0+1 \\
8 \cdot 0+1\end{array}$ & $\begin{array}{l}8.5+1 \\
4.8+1\end{array}$ & $\begin{array}{l}\text { MUN E } \\
\text { MUN E } \\
\text { NRL E } \\
\text { NRL E }\end{array}$ & $\begin{array}{l}E \text { Inazato. } \\
E \text { lmazato. } \\
E \text { Singht } \\
E \text { Singht }\end{array}$ & $\begin{array}{r}11980 \\
9915 \\
6263 \\
6278\end{array}$ \\
\hline
\end{tabular}




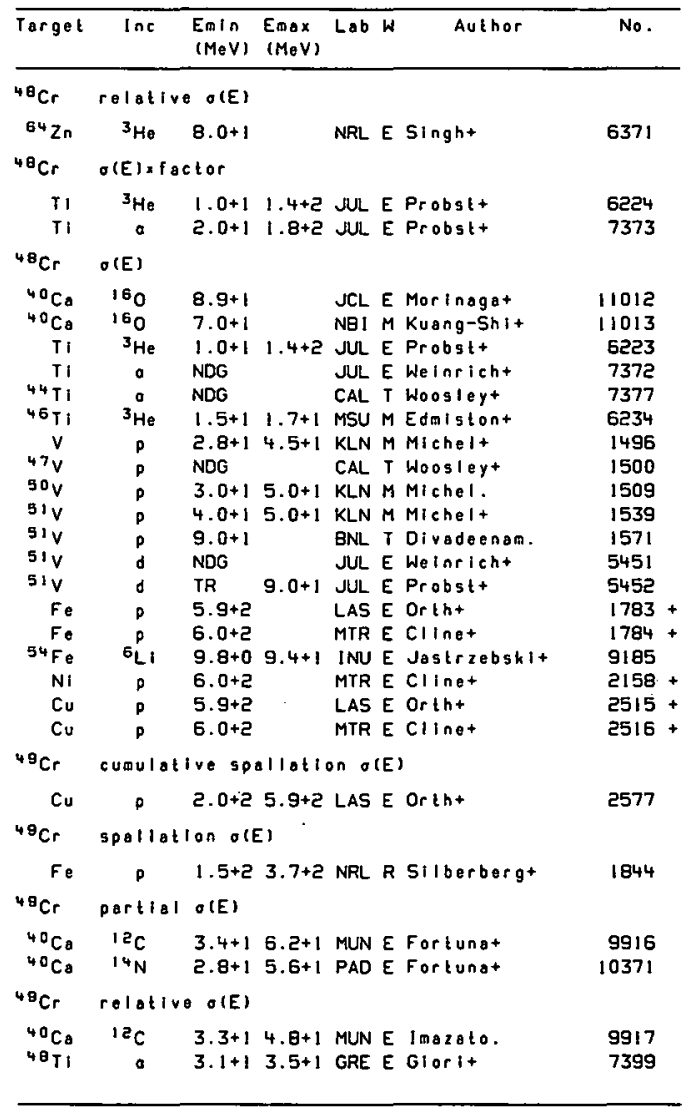

\begin{tabular}{|c|c|c|c|c|c|c|}
\hline Target & Inc & $\begin{array}{l}\text { Emin } \\
(M e V)\end{array}$ & $\begin{array}{l}E \max \\
(\operatorname{Me} v)\end{array}$ & Lab W & Author & No. \\
\hline${ }^{8} \mathrm{Cr}$ & relative & e OIE) & & & & \\
\hline $\begin{array}{l}56_{\mathrm{Fe}} \\
58_{\mathrm{Ni}} \\
64 \mathrm{ZB}\end{array}$ & $\begin{array}{l}{ }^{3} \mathrm{He} \\
{ }^{3} \mathrm{He} \\
{ }^{3} \mathrm{He}\end{array}$ & $\begin{array}{l}8 \cdot 0+1 \\
8 \cdot 0+1 \\
8 \cdot 0+1\end{array}$ & & $\begin{array}{l}\text { NRL E } \\
\text { NRL E } \\
\text { NRL E }\end{array}$ & $\begin{array}{l}\text { E Singht } \\
\text { Singht } \\
\text { E singht }\end{array}$ & $\begin{array}{l}6263 \\
6278 \\
6371\end{array}$ \\
\hline${ }^{4}{ }^{8} \mathrm{Cr}$ & $\sigma(E)$ & & & & & \\
\hline $\begin{array}{l}40 \mathrm{Ca} \\
45 \mathrm{TI}\end{array}$ & $\begin{array}{r}160 \\
0\end{array}$ & $\begin{array}{l}8.9+1 \\
\text { NDG }\end{array}$ & & $\begin{array}{l}J C L E \\
\text { CAL T }\end{array}$ & $\begin{array}{l}\text { E Morinagat } \\
\text { T Woosley+ }\end{array}$ & $\begin{array}{r}11014 \\
7379\end{array}$ \\
\hline${ }^{46} \mathrm{TI}$ & $a$ & TR 1 & $1.5+1$ & CAL T & $T$ Woosleyt & 7382 \\
\hline$v$ & p & $2.6+14$ & $4.5+1$ & KLN M & M Michelt & 1497 \\
\hline $6 \mathrm{v}$ & $a$ & NDG & & CAL T & T Hoosleyt & 7418 \\
\hline${ }^{48} \mathrm{~V}$ & p & NDC & & CAL T & T Woosleyt & 1501 \\
\hline $50 \mathrm{v}$ & p & $1.8+15$ & $5.0+1$ & KLN M & Michelt & 1510 \\
\hline $51 \mathrm{~V}$ & p & NDG & & KLLN E & E Herrt & 1540 \\
\hline $51 \mathrm{~V}$ & p & $2.5+1$ & $1.0+2$ & BNL T & T Divadeenam. & 1541 \\
\hline $51 \mathrm{~V}$ & p & $9.0+1$ & & BNL T & T Divadeenam. & 1571 \\
\hline $51 \mathrm{~V}$ & d & $\mathrm{TR} S$ & $9.0+1$ & JUL E & E Probst+ & 5452 \\
\hline $\mathrm{Fe}_{54 \mathrm{e}}$ & $\sigma_{1}^{p}$ & $6.0+5$ & & MIR E & E Clinet & $1785+$ \\
\hline${ }^{4} \mathrm{Fe}$ & ${ }^{6} L_{1}$ & $9.8+0 s$ & $9.4+1$ & INU E & E Jostrzebshit & 9185 \\
\hline Ine & p & $6.0+2$ & & MTR E & E Clinet & $2159+$ \\
\hline${ }^{5 \theta_{N i}}$ & $p$ & $8.0+1 \quad 1$ & $1.6+2$ & INU E & E Sadlert & 2317 \\
\hline $23^{3} \mathrm{NI}$ & $p$ & $8.0+1 \quad 1$ & $1.4+2$ & MIL T & T Gadiolit & 2318 \\
\hline${ }^{60} \mathrm{NI}$ & p & $1.4+2$ & & MIL T & T Gadialit & 2362 \\
\hline Cu & o & $6.0+2$ & & MTR E & E Clinet & $2517+$ \\
\hline${ }^{50} \mathrm{Cr}$ & spallati & $\operatorname{lon} O(E)$ & & & & \\
\hline $\mathrm{Fe}$ & $\rho$ & $1.5+23$ & $3.7+2$ & NRL $R$ & R Sllberbergt & 1644 \\
\hline${ }^{50} \mathrm{Cr}$ & partiol & product & $=t$ ylel & & & \\
\hline${ }^{40} \mathrm{Co}$ & ${ }^{14} \mathrm{~N}$ & $2.9+1=$ & $3.8+1$ & STB E & E Geesamant & 10372 \\
\hline${ }^{50} \mathrm{Ce}$ & partlal & row thi & hick to & $\operatorname{argot}$ & yleldxabundance & \\
\hline${ }^{50} \mathrm{Cr}$ & 0 & $5.0+0$ & & SUN E & E Gilest & 7473 \\
\hline${ }^{50} \mathrm{Cr}$ & partiol & $O(E)_{p}$ & & & & \\
\hline${ }^{50} \mathrm{Cr}$ & p & $6.0+0 \mathrm{E}$ & $6.2+0$ & MOS E & E Romanovisilit & 1597 \\
\hline${ }^{30} \mathrm{Cr}$ & portlal & $\theta(E)^{2} \times t^{\prime}$ & f actor & & & \\
\hline $\begin{array}{l}{ }^{0}{ }^{\circ} \mathrm{Cr} \\
{ }^{20} \mathrm{Ni}\end{array}$ & p & $\begin{array}{l}1.6+0 \\
1.0+3\end{array}$ & $3.0+0$ & $\begin{array}{l}\text { IJIE E } \\
\text { LIN E }\end{array}$ & $\begin{array}{l}\text { E Krivonosovt } \\
\text { E Volnint }\end{array}$ & $\begin{array}{l}1595 \\
2316+\end{array}$ \\
\hline${ }^{28^{N I}}$ & $p$ & $1.0+3$ & & LIN E & E volnint & $2320+$ \\
\hline
\end{tabular}




\begin{tabular}{|c|c|c|c|c|c|c|}
\hline Target & $\operatorname{Inc}$ & $\begin{array}{l}\text { Emin } \\
(\mathrm{MeV})\end{array}$ & $\begin{array}{l}E_{\max } \\
\text { (MeV: }\end{array}$ & Lab h & Author & No. \\
\hline${ }^{50} \mathrm{Cr}$ & partiol & $\theta(E), n$ & "factor & & & \\
\hline${ }^{5 \theta_{N i}}$ & $p$ & $1.0+3$ & & LINE & E Volnint & $2321+$ \\
\hline${ }^{50} \mathrm{Cr}$ & partial & relati & IVe oIE & & & \\
\hline $\begin{array}{l}40 \mathrm{CO} \\
48 \mathrm{Ti}\end{array}$ & $\begin{array}{l}160 \\
160\end{array}$ & $\begin{array}{l}4.5+1 \\
9.6+1\end{array}$ & $6.3+1$ & $\begin{array}{l}\text { ANL E } \\
\text { MUN } r\end{array}$ & $\begin{array}{l}\text { E Geesamant } \\
M \text { Heringt }\end{array}$ & $\begin{array}{l}11015 \\
11125\end{array}$ \\
\hline${ }^{30} \mathrm{Cr}$ & partial & $O(E) \times f$ & factor & & & \\
\hline${ }^{48} \mathrm{Ti}$ & 160 & $9.6+1$ & & MUU E & E Puchlat & 11126 \\
\hline${ }^{50} \mathrm{Cr}$ & portial & $\sigma(E)$ & & & & \\
\hline $\begin{array}{l}{ }^{40} \mathrm{Ca} \\
{ }^{40} \mathrm{Ca}\end{array}$ & $\begin{array}{l}12 \mathrm{C} \\
14 \mathrm{~N}\end{array}$ & $\begin{array}{l}3.4+1 \\
2.8+1\end{array}$ & $\begin{array}{l}6.2+1 \\
5.6+1\end{array}$ & MUN E & $\begin{array}{l}\text { E Fortunat } \\
\text { E Fortunat }\end{array}$ & $\begin{array}{r}9918 \\
10373\end{array}$ \\
\hline${ }^{50} \mathrm{Cr}$ & $\sigma(E)_{p}$ & & & & & \\
\hline $\begin{array}{l}{ }^{50} \mathrm{Cr} \\
{ }^{50} \mathrm{Cr}\end{array}$ & $e_{\text {rololive }}^{p}$ & $\begin{array}{r}6.0+0 \\
. \quad \sigma(E)\end{array}$ & $6.2+0 \mathrm{~N}$ & MOS E & E Romanorshisit & 1596 \\
\hline 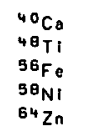 & $\begin{array}{c}{ }^{12} \mathrm{C} \\
0 \\
3^{0} \mathrm{He} \\
3^{3} \mathrm{He} \\
{ }^{3} \mathrm{He}\end{array}$ & $\begin{array}{l}3 \cdot 3+1 \\
2 \cdot 1+1 \\
8 \cdot 0+1 \\
8 \cdot 0+1 \\
8 \cdot 0+1\end{array}$ & $\begin{array}{r}4.8+1 \\
3.5+1\end{array}$ & $\begin{array}{l}\text { MUN } \\
\text { GRE } \\
\text { NRL } \\
\text { NRL } \\
\text { NRL }\end{array}$ & $\begin{array}{l}\text { E l mazalo. } \\
\text { E Glorit } \\
\text { E Singht } \\
\text { E Singht } \\
\text { E Singht }\end{array}$ & $\begin{array}{l}9919 \\
7400 \\
6263 \\
6278 \\
6371\end{array}$ \\
\hline${ }^{50} \mathrm{Cr}$ & $\sigma(E)$ & & & . & & \\
\hline $\begin{array}{l}{ }^{40} \mathrm{Cg} \\
{ }^{4} \mathrm{C}\end{array}$ & $\begin{array}{l}160 \\
160\end{array}$ & $\begin{array}{l}7.0+1 \\
7.0+1\end{array}$ & & NBI & $\begin{array}{l}\text { M Kuang-Sh 1+ } \\
\text { M Kuang-Shit }\end{array}$ & $\begin{array}{l}11016 \\
11018\end{array}$ \\
\hline${ }^{40} \mathrm{Ce}$ & 160 & $7.0+1$ & & NQ1 & MKuang-Shit & 11017 \\
\hline${ }^{46} \mathrm{TI}$ & a & NDG & & CAL & I Woosley+ & 7383 \\
\hline $47 \pi$ & a & TR & $1.4+1$ & CAL & T Woosleyt & 7388 \\
\hline${ }^{47} \mathrm{~V}$ & a & NDG & & CAL & I Woosleyt & 7420 \\
\hline $49 v$ & $p$ & NDG & & CAL & T Woosleyt & 1503 \\
\hline $50 \mathrm{~V}$ & p & NDG & & CAL & T Woosleyt & 1511 \\
\hline $51 \mathrm{~V}$ & p & $9.0+1$ & & BNL & T Divadeenam. & 1571 \\
\hline $53 \mathrm{Mn}$ & D & NOG & & CAL & T Woosleyt & 1681 \\
\hline${ }^{5 \theta^{N}} \mathrm{Ni}$ & p & $8.0+1$ & $1.6+2$ & INU & E Sadlert & 2317 \\
\hline${ }^{5 \theta} \mathrm{Ni}$ & $\rho$ & $8.0+1$ & $1.4+21$ & MIL & T Gadiolit & 2318 \\
\hline${ }^{58_{\mathrm{Ni}}}$ & p & $2.0+2$ & & ANL & E Schlffer. & 2319 \\
\hline $60 \mathrm{Ni}$ & p & $1.4+2$ & & INU & E Sadert & 2361 \\
\hline${ }^{60} \mathrm{Ni}$ & $\rho$ & $1.4+2$ & & MIL & T Gadialit+ & 2362 \\
\hline${ }^{60} \mathrm{Ni}$ & $p$ & $2.0+2$ & & ANL & ESchiffer. & 2363 \\
\hline
\end{tabular}

\begin{tabular}{|c|c|c|c|c|c|c|}
\hline Target & $\ln c$ & $\begin{array}{l}\text { Emin } \\
(M e V)\end{array}$ & $\begin{array}{l}\text { Emax } \\
\text { (MeV) }\end{array}$ & LaO W & Author & No. \\
\hline${ }^{50} \mathrm{Cr}$ & $O(E)$ & & & & & \\
\hline $62_{\mathrm{NI}}$ & g & $1.4+2$ & & \multicolumn{2}{|c|}{ MIL T Gadiolit } & 2412 \\
\hline${ }^{5} \mathrm{Cr}$ & \multicolumn{5}{|c|}{ cuaulative spallation o(E) } & \\
\hline $\mathrm{Cu}$ & p & $2.0+2$ & $5.9+2$ & LAS E & Or th+ & 2577 \\
\hline
\end{tabular}

${ }^{51} \mathrm{Cr}$ spallation $O(E)$

Fe $\quad 1.5+23.7+2$ NRL R Silberbergt 1844

Sic: cumulative o(E)

\begin{tabular}{|c|c|c|c|c|c|c|c|c|}
\hline$F_{\theta}$ & p & $1.0+1$ & $5.0+1$ & I KLN M & $M$ Michelt & 1786 & & \\
\hline $\mathrm{Fe}$ & $p$ & $3.2+1$ & $6.3+1$ & KFK C & C Muenzel+ & 1787 & & \\
\hline $\mathrm{Fe}$ & $P$ & $3.2+1$ & $6.3+1$ & I ORL E & E Wllllomst & 1788 & & $\bar{n}$ \\
\hline$F_{\mathbf{e}}$ & ó & $5.9+2$ & & LAS E & E ortht & 1789 & + & U. \\
\hline $\begin{array}{r}F_{e} \\
54 F^{2}\end{array}$ & $p$ & $6.0+2$ & & MTR E & Eclinot & 1790 & + & 욤 \\
\hline $\begin{array}{l}{ }^{54} \mathrm{Fe} \\
{ }^{56} \mathrm{Fe}_{\mathrm{e}}\end{array}$ & $p+4$ & $3.4+1$ & $5.0+1$ & $1 \mathrm{KLN} M$ & Michel. & 1854 & & $m$ \\
\hline $\begin{array}{l}{ }^{56} \mathrm{Fe}_{\mathrm{e}} \\
\mathrm{Ni}\end{array}$ & p & $: 2.9+1$ & $5.0+1$ & $1 \mathrm{KLN} M$ & Michel+ & 1888 & & 몸 \\
\hline $\mathrm{NI}$ & D & $6.0+2$ & & MTR E & E Clinet & 2160 & + & 9 \\
\hline$C_{v}$ & p & $5.9+2$ & & LAS E & E Ortht & 2518 & + & ह \\
\hline $\mathrm{Cu}_{u}$ & $p$ & $6.0+2$ & & MTR E & E Clinet & 2519 & & \\
\hline${ }^{3} \mathrm{Cr}$ & partiol & proou & set $y 10$ & old & & & & \\
\hline${ }^{40} \mathrm{Co}$ & ${ }^{14} \mathrm{~N}$ & $2.9+1$ & $3.8+1$ & 1 STB & Geesamant & 10375 & & כ \\
\hline
\end{tabular}

$48 \mathrm{ri}$ - 5.0+0 SUN E Gilest 7401

${ }^{51} \mathrm{Cr}$ partial o(E)

${ }^{40} \mathrm{Co} 14 \mathrm{~N} 2.8+15.6+1$ PAD E Fortunat 10374

$51 \mathrm{~V} P \quad 4.0+04.4+0$ KTY E Sekhoran. 1565

${ }^{3} \mathrm{Cr} \mathrm{I}_{y}$

siv P $4.0+04.6+0$ TOK E Kasagit 1564

${ }^{51} \mathrm{Cr}$ product yield

siv $P$ NDG CAL E Zyshingt 1553

s $\mathrm{Cr}$ o(E) zabundance

$51 \mathrm{~V} P 1.3+14.5+1 \mathrm{KLN}$ M Michel+ 1562 


\begin{tabular}{|c|c|c|c|c|c|c|}
\hline Targot & $\ln c$ & $\begin{array}{l}\text { Emin } \\
(M e V)\end{array}$ & $\begin{array}{l}\text { Emox } \\
(\mathrm{MeV})\end{array}$ & $206 \mathrm{~W}$ & Author & No. \\
\hline${ }^{51} \mathrm{Cr}$ & average & $\theta(E)$ & & & & . \\
\hline $51 \mathrm{~V}$ & p & $1.6+0$ & $1.6+0$ & ORL E & E Johnsont & 1566 \\
\hline $51 \mathrm{Cr}$ & relative & $\theta \theta(E)$ & & & & \\
\hline $\begin{array}{l}{ }^{48} \mathrm{Ti} \\
56 \mathrm{Fe} \\
50_{\mathrm{Ni}} \\
64 \mathrm{Zn}\end{array}$ & $\begin{array}{l}3^{a} \\
{ }^{3} \mathrm{He} \\
3^{3} \mathrm{He} \\
{ }^{3} \mathrm{He}\end{array}$ & $\begin{array}{l}2.1+1 \\
8 \cdot 0+1 \\
8 \cdot 0+1 \\
8.0+1\end{array}$ & $3.5+1$ & $\begin{array}{l}\text { GRE E } \\
\text { NRL E } \\
\text { NRL E } \\
\text { NRL E }\end{array}$ & $\begin{array}{l}\text { E Giorit } \\
\text { E Singht } \\
\text { E Singht } \\
\text { E Singht }\end{array}$ & $\begin{array}{l}7402 \\
6263 \\
6278 \\
6371\end{array}$ \\
\hline${ }^{31} \mathrm{Cr}$ & olEl $\times f$ ac & etor & & & & \\
\hline $\begin{array}{r}\mathrm{Ir} \\
\mathrm{T}^{\prime} \\
{ }^{2} \mathrm{Cr}\end{array}$ & $\begin{array}{c}{ }^{3} \mathrm{He} \\
a \\
p\end{array}$ & $\begin{array}{l}1.0+1 \\
2 \cdot 0+1 \\
2 \cdot 2+1\end{array}$ & $\begin{array}{l}1.4+2 \\
1.8+2\end{array}$ & $\begin{array}{l}\text { JUL E } \\
\text { JUL E } \\
\text { ORL E }\end{array}$ & $\begin{array}{l}\text { E Probstt } \\
\text { E Probstt } \\
\text { E Cohent }\end{array}$ & $\begin{array}{l}6227 \\
7375 \\
1614\end{array}$ \\
\hline sicr & $O(E)$ & & & & & \\
\hline${ }^{47} \mathrm{TI}$ & a & NDG & & CAL T & T Woosleyt & 7389 \\
\hline${ }^{48} \mathrm{TI}$ & a & TR & $1.4+1$ & CAL T & T Woosleyt & 7403 \\
\hline $\mathrm{v}$ & p & $2.0+0$ & $5.0+1$ & KLN M & M Mlchel+ & 1498 \\
\hline $48 \mathrm{v}$ & o & NDG & & CAL $\mathrm{T}$ & I Woosleyt & 7422 \\
\hline $50 \mathrm{~V}$ & p & NDG & & CAL $T$ & T Woosley+ & 1512 \\
\hline $51 \mathrm{~V}$ & p & NDG & & KTY E & E Sekhorant & 1542 \\
\hline $51 \mathrm{~V}$ & p & TR & $1.1+1$ & CAL I & I Woogleyt & 1543 \\
\hline $51 \mathrm{~V}$ & p & TR & $5.7+0$ & ORL E & E Johnsont & $1544+$ \\
\hline $51 \mathrm{~V}$ & $p$ & $1.6+0$ & $1.3+1$ & KFK C & C Muenzelt & 1545 \\
\hline $5 \mathrm{IV}$ & $p$ & $1.6+0$ & $2.3+0$ & BNL $\mathrm{R}$ & R Goldberg. & 1546 \\
\hline $51 \mathrm{~V}$ & p & $1.6+0$ & $4.5+0$ & CAL E & E Zyskind.. & 1547 \\
\hline $5 ! \mathrm{V}$ & p & $2.0+0$ & $3.0+0$ & KFT E & E Krivonosort & 1548 \\
\hline $5 i v$ & $p$ & $3.0+0$ & $5.0+0$ & INU T & T Kailast & 1549 \\
\hline $51 \mathrm{~V}$ & p & $3.1+0$ & $1.0+1$ & ANL $E$ & E Wingt & $1550+$ \\
\hline $51 \mathrm{~V}$ & p & $3.7+0$ & $6.7+0$ & OSU E & E Delit & $1551+$ \\
\hline $51 \mathrm{v}$ & p & $4.0+0$ & $5.0+1$ & KLN & M Michelt & 1552 \\
\hline $51 \mathrm{~V}$ & p & $4.0+0$ & $5.5+0$ & LRL E & E Albert. & $1553+$ \\
\hline $51 \mathrm{~V}$ & p & $4.5+0$ & $6.7+0$ & ROC E & E Takelanl+ & $1554+$ \\
\hline $51 \mathrm{~V}$ & p & $5.0+0$ & $1.1+1$ & LRL E & E Hensent & $1555+$ \\
\hline $51 \mathrm{~V}$ & $p$ & $6.7+0$ & $1.3+1$ & TOK E & E Tanakat & $1556+$ \\
\hline $51 \mathrm{~V}$ & p & $7.5+0$ & & MIT E & E Shore+ & $1557+$ \\
\hline $5 ! v$ & $p$ & $8.0+0$ & $1.1+1$ & CCP R & R Zotolokint & 1558 \\
\hline $51 \mathrm{~V}$ & p & $1.0+1$ & $1.2+2$ & BNL I & T DIvadeenam. & 1559 \\
\hline $51 \mathrm{v}$ & p & $1.1+1$ & $4.5+1$ & MIL E & E Godiolit & $1560+$ \\
\hline $51 \mathrm{~V}$ & p & $3.2+1$ & $1.5+2$ & PAR E & E Albouyt & $1561+$ \\
\hline $51 \mathrm{~V}$ & p & $9.0+1$ & & BNL $T$ & T Divadeensm. & 1571 \\
\hline
\end{tabular}

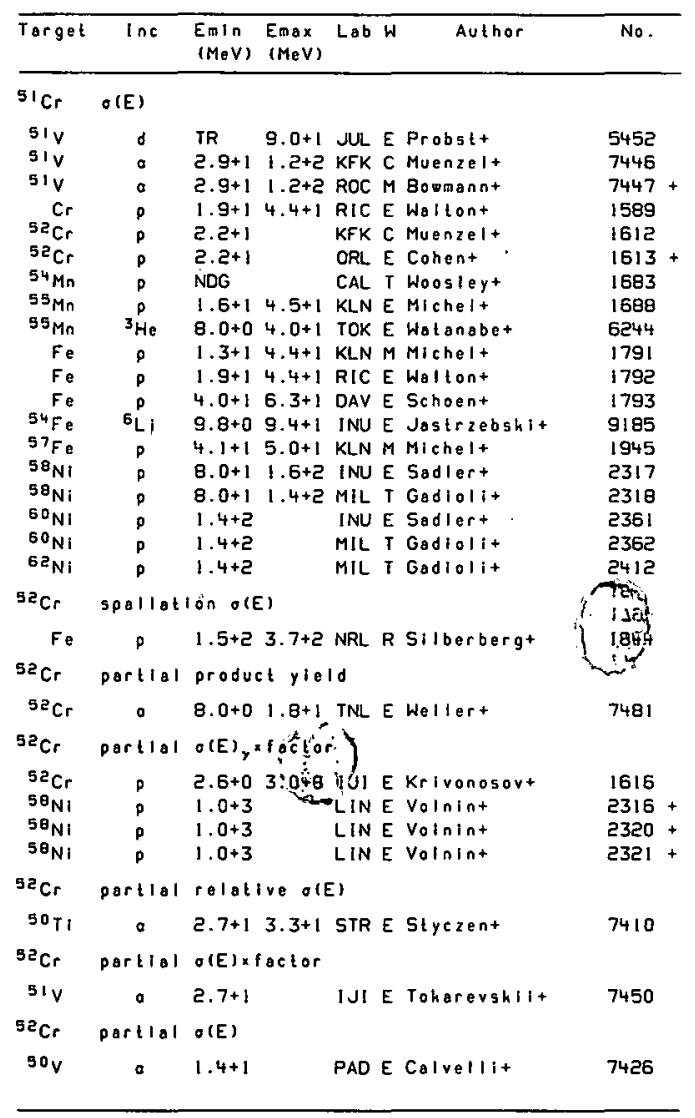




\begin{tabular}{|c|c|c|c|c|c|c|}
\hline Target & $\ln c$ & $\begin{array}{l}\text { Emin } \\
(\mathrm{MeV})\end{array}$ & $\begin{array}{l}\text { Emax } \\
(\operatorname{MeV})\end{array}$ & Lab & Author & No. \\
\hline${ }^{s 2} \mathrm{Cr}$ & partial & $O(E)$ & & & & \\
\hline $\begin{array}{l}51 v \\
51 \mathrm{~V}\end{array}$ & 0 & $\begin{array}{l}2.5+1 \\
2.7+1\end{array}$ & & $\begin{array}{l}\text { MOS } \\
\text { IJI }\end{array}$ & $\begin{array}{l}\text { E Lebedert } \\
\text { E Toharerghit }\end{array}$ & $\begin{array}{l}7448 \\
7449\end{array}$ \\
\hline${ }^{s e} \mathrm{Cr}$ & relolive & - $1_{\gamma}$ & & & & \\
\hline $\begin{array}{l}{ }^{50} \mathrm{Ti} \\
{ }^{52} \mathrm{Cr}\end{array}$ & of(E) & $1.8+1$ & $2.5+1$ & Buc & E Berindet & 7409 \\
\hline $\begin{array}{l}{ }^{52} \mathrm{Cr} \\
{ }^{32} \mathrm{Cr}\end{array}$ & $\begin{array}{c}D \\
\text { relotive }\end{array}$ & $\begin{array}{r}6.0+0 \\
\text { CO(E) }\end{array}$ & $6.2+0$ & MOS & E Romenovghist & 1617 \\
\hline${ }^{52} \mathrm{Cr}$ & $\theta(E)^{\gamma}$ & $4.1+0$ & $4.2+0$ & KF I & E Fodort & 1615 \\
\hline${ }^{32} \mathrm{Cr}$ & $p$ & $4.1+0$ & $4.3+0$ & $\mathrm{KF} \mathbf{i}$ & E Fodort & 1618 \\
\hline${ }^{32} \mathrm{Cr}$ & $\cos \theta(E)$ & & & & & \\
\hline $\begin{array}{l}52 \mathrm{Cr} \\
52 \mathrm{Cr}\end{array}$ & $\begin{array}{c}P \\
\text { celotive }\end{array}$ & $\begin{array}{r}4.1+0 \\
\text { e } O(E)\end{array}$ & $4.2+0$ & $\mathrm{KF} !$ & E Lovas Fodort & 1619 \\
\hline $\begin{array}{l}56 \mathrm{Fz} \\
5 \theta_{\mathrm{Ni}} \\
54 \mathrm{Zn}\end{array}$ & $\begin{array}{l}{ }^{3} \mathrm{He} \\
{ }^{3} \mathrm{He} \\
{ }^{3} \mathrm{He}\end{array}$ & $\begin{array}{l}8.0+1 \\
8.0+1 \\
8.0+1\end{array}$ & & $\begin{array}{l}\text { NRL } \\
\text { NRL } \\
\text { NRL }\end{array}$ & $\begin{array}{l}\text { E singht } \\
\text { E Singht } \\
\text { E Singht }\end{array}$ & $\begin{array}{l}6263 \\
6278 \\
6371\end{array}$ \\
\hline${ }^{52} \mathrm{Cr}$ & $\theta(E) \times f \circ C$ & ctor & & & & \\
\hline SIV & p & NDG & & CAL & E Zyshindt & 1567 \\
\hline${ }^{s 2} \mathrm{Cr}$ & $O(E)$ & & & & & \\
\hline${ }^{48} \mathrm{Ti}$ & 。 & NDG & & CAL & T Woosleyt & 7404 \\
\hline${ }^{49} \mathrm{Ti}$ & a & NDG & & CAL & T Woosleyt & 7406 \\
\hline $49 \mathrm{~V}$ & 0 & NDG & & CAL & T Woosleyt & 7424 \\
\hline $51 \mathrm{~V}$ & p & NDG & & CAL & T Woosleyt & 1568 \\
\hline $51 \mathrm{v}$ & $p$ & $9.3-1$ & $4.5+0$ & CAL & E Zyshind. & 1569 \\
\hline $31 \mathrm{~V}$ & p & $9.0+1$ & & BNL & T Divadeenam. & 1571 \\
\hline $51 \mathrm{~V}$ & ${ }^{3} \mathrm{He}$ & $7.0+1$ & $9.0+1$ & OSA & M Molsuokot & 6237 \\
\hline 254 & $p$ & NDG & & CAL & T Woosleyt & 1575 \\
\hline $55 \mathrm{Mn}$ & p & NDG & & $\mathrm{CAL}$ & T Woosleyt & 1689 \\
\hline${ }^{38} \mathrm{Ni}$ & p & $8.0+1$ & $1.6+2$ & INU & E Sadiert & 2317 \\
\hline${ }^{58} \mathrm{Ni}$ & p & $8.0+1$ & $1.4+2$ & MIL & T Gadiolit & 2318 \\
\hline${ }^{5 \theta^{N} \mathrm{i}}$ & p & $2.0+2$ & & ANL & E Sehiffer. & 2319 \\
\hline $60 \mathrm{Ni}$ & p & $1.4+2$ & & INU & E Sodlert & $236 !$ \\
\hline
\end{tabular}

\begin{tabular}{|c|c|c|c|c|c|c|}
\hline Target & $\ln c$ & $\begin{array}{l}\text { Enin } \\
(\mathrm{MeV})\end{array}$ & $\begin{array}{l}\text { Emax } \\
(\mathrm{MeV})\end{array}$ & Lab W & Author & No. \\
\hline${ }^{52} \mathrm{Cr}$ & $O(E)$ & & & & & \\
\hline $\begin{array}{l}60 \mathrm{Ni} \\
60 \mathrm{Ni} \\
62^{6 i} \\
64 \mathrm{Ni} \\
64 \mathrm{Ni}\end{array}$ & $\begin{array}{l}p \\
p \\
p \\
p \\
p\end{array}$ & $\begin{array}{l}1.4+2 \\
2.0+2 \\
1.4+2 \\
1.4+2 \\
1.4+2\end{array}$ & & $\begin{array}{l}\text { MIL T } \\
\text { ANL E } \\
\text { MIL T } \\
\text { INU E } \\
\text { MIL T }\end{array}$ & $\begin{array}{l}\text { Godiolit } \\
\text { Schiffer. } \\
\text { Gadiolit } \\
\text { Sadlert } \\
\text { Gadialit }\end{array}$ & $\begin{array}{l}2362 \\
2363 \\
2412 \\
2434 \\
2435\end{array}$ \\
\hline${ }^{53} \mathrm{Cr}$ & spallati & ion OIE & & & & \\
\hline $\begin{array}{l}\mathrm{Fe} \\
{ }^{53} \mathrm{Cr}\end{array}$ & $\begin{array}{c}p \\
\text { portl.al }\end{array}$ & $\begin{array}{l}1.5+2 \\
\ln 1 \mathrm{ch}\end{array}$ & $\begin{array}{l}3.7+2 \\
\text { target }\end{array}$ & $\begin{array}{l}\text { NRTL R } \\
1 y 1010\end{array}$ & $\begin{array}{l}\text { Silberbergt } \\
d_{y} \text { afaclor }\end{array}$ & 1844 \\
\hline $\begin{array}{l}53 \mathrm{Cr} \\
53 \mathrm{Cr} \\
53 \mathrm{Cr} \\
53 \mathrm{Cr}\end{array}$ & $\begin{array}{l}p \\
p \\
0 \\
0\end{array}$ & $\begin{array}{l}2.5+0 \\
2.5+0 \\
4.0+0 \\
4.0+0\end{array}$ & $\begin{array}{l}3.5+0 \\
3.5+0 \\
7.0+0 \\
7.0+0\end{array}$ & $\begin{array}{l}\text { TRM E } \\
\text { VIP E } \\
\text { TRM E } \\
\text { VIP E }\end{array}$ & $\begin{array}{l}\text { Patrawale+ } \\
\text { Patrawale+ } \\
\text { Patraoble+ } \\
\text { Patraoale+ }\end{array}$ & $\begin{array}{l}1650 \\
1651 \\
7488 \\
7489\end{array}$ \\
\hline${ }^{53} \mathrm{Cr}$ & partlal & rav th & hich ta & argot $y$ & yloldxabundance & \\
\hline${ }^{53} \mathrm{Cr}$ & 。 & $5.0+0$ & & SUN E & Gllest & 7487 \\
\hline${ }^{53} \mathrm{Cr}$ & portlal & $O(E)$, & - factor & & & \\
\hline${ }^{53} \mathrm{Cr}$ & $\begin{array}{c}p \\
\text { relative }\end{array}$ & $\begin{array}{r}1.8+0 \\
. \quad \sigma(E)\end{array}$ & $3.0+0$ & IJIE & Krivonosort & 1649 \\
\hline $\begin{array}{l}{ }^{56} \mathrm{Fe} \\
{ }^{5} \theta_{\mathrm{Ni}} \\
{ }^{64} \mathrm{Zn}\end{array}$ & $\begin{array}{l}{ }^{3} \mathrm{He} \\
{ }^{3} \mathrm{He} \\
{ }^{3} \mathrm{He}\end{array}$ & $\begin{array}{l}8 \cdot 0+1 \\
8 \cdot 0+1 \\
8 \cdot 0+1\end{array}$ & & $\begin{array}{l}\text { NRL E } \\
\text { NRL E } \\
\text { NRL E }\end{array}$ & $\begin{array}{l}\operatorname{singh*} \\
\operatorname{singh*} \\
\operatorname{singh.}\end{array}$ & $\begin{array}{l}6263 \\
6278 \\
6371\end{array}$ \\
\hline${ }^{53} \mathrm{Cr}$ & $\theta(E)$ & & & & & \\
\hline $\begin{array}{l}49 T i \\
50 T i\end{array}$ & a & $\begin{array}{l}\text { NDG } \\
\text { TR }\end{array}$ & $1.4+1$ & $\begin{array}{l}\text { CAL } ' T \\
\text { CAL } T\end{array}$ & $\begin{array}{l}\text { Woosleyt } \\
\text { Woosleyt }\end{array}$ & $\begin{array}{l}7407 \\
7411\end{array}$ \\
\hline $50 \mathrm{~V}$ & 0 & NDG & & CAL T & Woosity+ & 7427 \\
\hline $25 v$ & $p$ & NDG & & CAL $T$ & Woosley+ & 1576 \\
\hline $53 \mathrm{~V}$ & p & NDG & & CAL $T$ & Waosleyt & 1578 \\
\hline${ }^{56} \mathrm{Mn}$ & p & NDG & & CAL $T$ & Woosleyt & 1712 \\
\hline${ }^{60} \mathrm{Ni}$ & 0 & $1.4+2$ & & INU E & Sodlert & 2361 \\
\hline $60^{\mathrm{NI}}$ & p & $1.4+2$ & & MIL T & Gadio:it & 2362 \\
\hline${ }^{62} \mathrm{Ni}$ & p & $1.4+2$ & & MIL T & Gadio:it & 2412 \\
\hline${ }^{64} \mathrm{NI}$ & $p$ & $1.4+2$ & & INU E & Sadlert & 2434 \\
\hline${ }^{64} \mathrm{NI}$ & $p$ & $1.4+2$ & & MIL T & Gadioit & 2435 \\
\hline
\end{tabular}




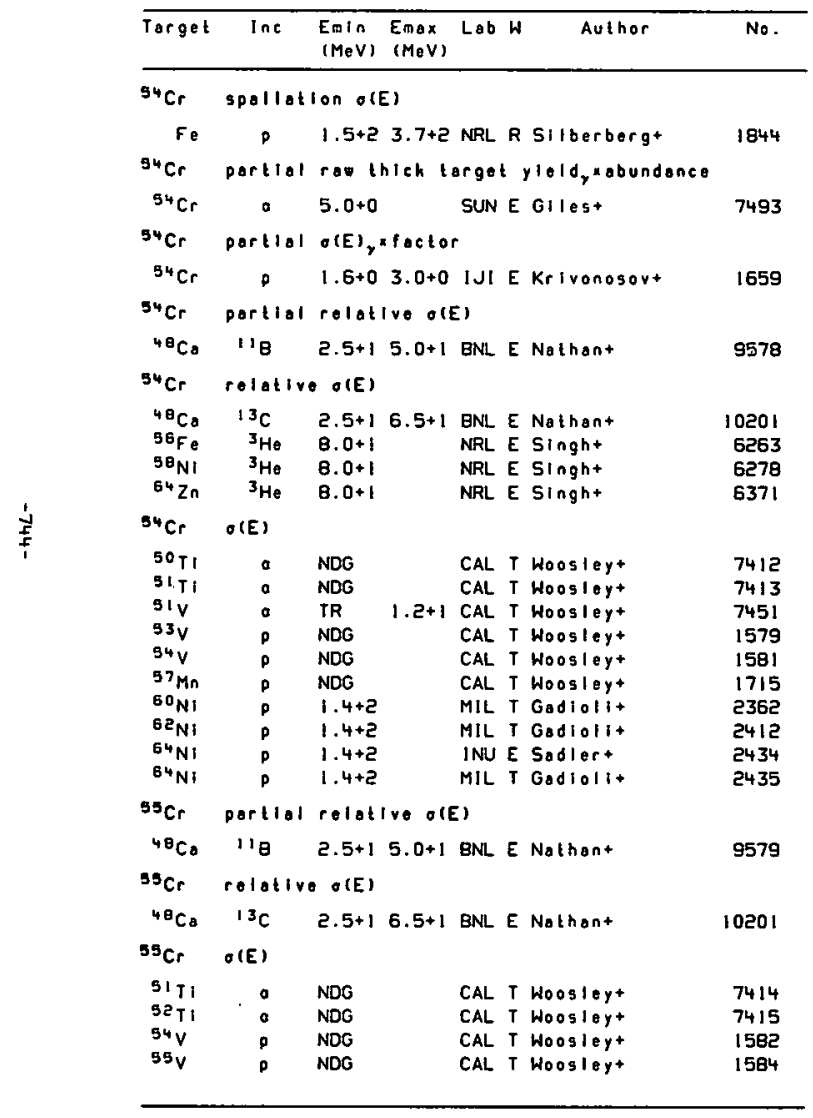

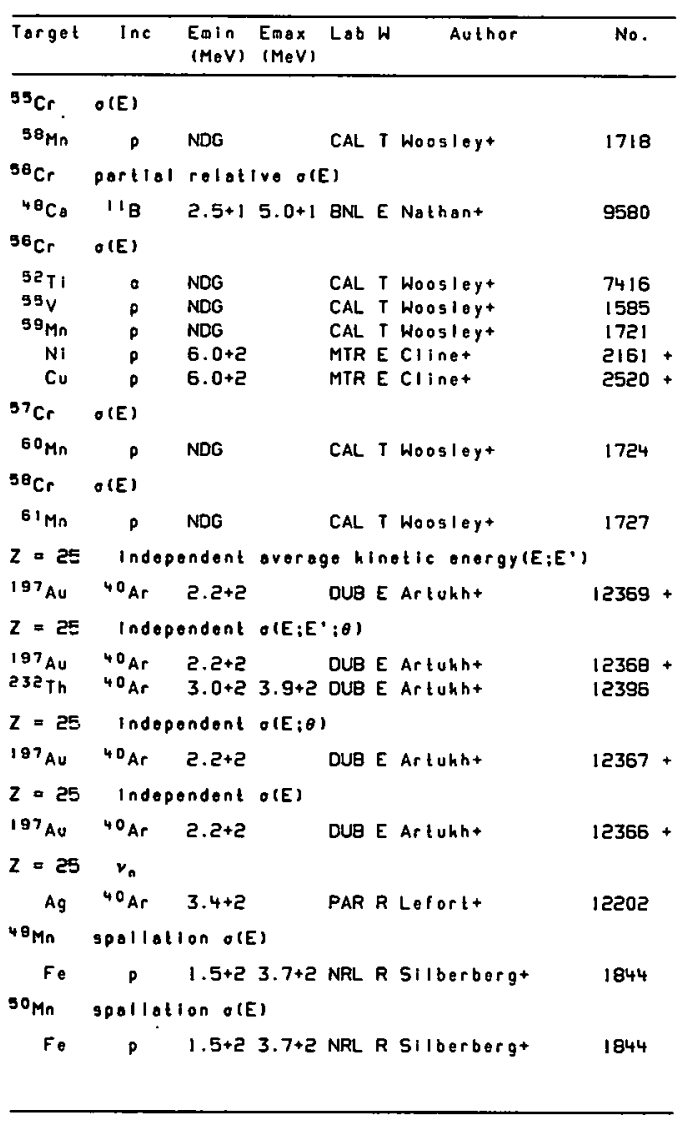




\begin{tabular}{|c|c|c|c|c|c|c|}
\hline Targe: & $\ln c$ & $\begin{array}{l}E_{m 1 n} \\
(M e V)\end{array}$ & $\begin{array}{l}\text { Emax } L \\
(\operatorname{MeV} V)\end{array}$ & Lob W & Author & No. \\
\hline${ }^{50} \mathrm{Mn}$ & relative & - $O(E)$ & & & & \\
\hline $\begin{array}{l}{ }^{56} \mathrm{Fe} \\
5^{5} \mathrm{Bi} \\
6{ }^{4} \mathrm{Zn}\end{array}$ & $\begin{array}{l}{ }_{3}^{3} \mathrm{He} \\
{ }^{3} \mathrm{He} \\
{ }^{3} \mathrm{He}\end{array}$ & $\begin{array}{l}8 \cdot 0+1 \\
8 \cdot 0+1 \\
8 \cdot 0+1\end{array}$ & & $\begin{array}{l}\text { NRL E } \\
\text { NRL E } \\
\text { NRL E }\end{array}$ & $\begin{array}{l}\operatorname{singht} \\
\operatorname{singht} \\
\operatorname{singht}\end{array}$ & $\begin{array}{l}6263 \\
6278 \\
6371\end{array}$ \\
\hline $50 \mathrm{Mn}$ & $O(E)$ & & & & & \\
\hline $\begin{array}{l}{ }^{40} \mathrm{Co} \\
{ }^{40} \mathrm{Co} \\
{ }^{40} \mathrm{Co} \\
4 \mathrm{CV}^{4} \\
{ }^{49} \mathrm{Cr} \\
50 \mathrm{Cr} \\
50_{\mathrm{N} 1} \\
{ }^{50} \mathrm{NI}\end{array}$ & $\begin{aligned} 12 c \\
160 \\
160 \\
0 \\
p \\
p \\
p \\
p\end{aligned}$ & $\begin{array}{l}3.3+1 \\
7.0+1 \\
7.0+1 \\
\text { NDG } \\
\text { NDG } \\
\text { TR } \\
8.0+1 \\
B \cdot 0+1\end{array}$ & $\begin{array}{r}4.8+1 M \\
N \\
N \\
C \\
C \\
1.1+1 \quad C \\
1.6+2 ~ \\
1.4+2\end{array}$ & $\begin{array}{l}\text { MUN E } \\
\text { NBI } M \\
\text { NBI } \\
\text { CAL } \\
\text { CAL } \\
\text { CAL } \\
\text { INU } \\
\text { MIL } \\
\text { MIL }\end{array}$ & $\begin{array}{l}\text { lmazolo. } \\
\text { Kuang-Shit } \\
\text { Kuang-Shit } \\
\text { Woosleyt } \\
\text { Woosleyt } \\
\text { Woosleyt } \\
\text { Sadlert } \\
\text { Gadiolit }\end{array}$ & $\begin{array}{r}9920 \\
11019 \\
11020 \\
7419 \\
1593 \\
1598 \\
2317 \\
2318\end{array}$ \\
\hline $509 \mathrm{Mn}$ & $\sigma(E)$ & & & & & \\
\hline $\begin{array}{l}50 \mathrm{Cr} \\
{ }^{50} \mathrm{Cr}\end{array}$ & P & $\begin{array}{l}8.5+0 \\
8.6+0\end{array}$ & $\begin{array}{l}8.6+0 F \\
8.6+0 \mathrm{~K}\end{array}$ & $\begin{array}{l}\text { FSU E } \\
\text { KFK C }\end{array}$ & $\begin{array}{l}\text { Me-Kennot } \\
\text { Muenzel+ }\end{array}$ & $\begin{array}{l}1599+ \\
1600\end{array}$ \\
\hline $50 \cdot M n$ & product & $t y \mid e 10$ & & & & \\
\hline $\begin{array}{l}{ }^{40} \mathrm{Co} \\
50 \cdot \mathrm{Mn}\end{array}$ & $\begin{array}{l}-14 \mathrm{~N} \\
0(E)\end{array}$ & $2.9+1$ & $5.5+15$ & STB E & Geesamant & 10376 \\
\hline $\begin{array}{l}{ }^{50} \mathrm{Cr} \\
{ }^{50} \mathrm{Cr} \\
\mathrm{Fe} \\
\mathrm{Cu}\end{array}$ & $\begin{array}{l}p \\
p \\
p \\
p\end{array}$ & $\begin{array}{l}8.6+0 \\
8.6+0 \\
6.0+2 \\
6.0+2\end{array}$ & $\begin{array}{r}9.0+0 k \\
9.0+0\end{array}$ & $\begin{array}{l}\text { KFK C } \\
\text { FSU E } \\
\text { MTR E } \\
\text { MTR E }\end{array}$ & $\begin{array}{l}\text { Muenzel+ } \\
\text { Me-Kennat } \\
\text { Clinget } \\
\text { Clinet }\end{array}$ & $\begin{array}{l}1601 \\
1602+ \\
1794+ \\
2521+\end{array}$ \\
\hline $51 M n$ & spalloti & I on $O(E)$ & & & & \\
\hline $\begin{array}{c}\mathrm{Fe} \\
\mathrm{SIMn}\end{array}$ & $\sigma(E)$ & $1.5+2=$ & $3.7+2 \mathrm{~N}$ & NRL $R$ & SIlberberg* & 1844 \\
\hline $\begin{array}{l}{ }^{54} \mathrm{Fe} \\
{ }^{56} \mathrm{Fe}\end{array}$ & $\begin{array}{l}p \\
p\end{array}$ & $\begin{array}{l}1.0+1= \\
3 \cdot 2+1=\end{array}$ & $\begin{array}{l}5.0+1 k \\
5.0+1 k\end{array}$ & $\begin{array}{l}K L N \text { M } \\
K L N M\end{array}$ & $\begin{array}{l}1 \text { Michel. } \\
1 \text { Michelt }\end{array}$ & $\begin{array}{l}1855 \\
1889\end{array}$ \\
\hline $31 \mathrm{Mn}$ & portiol & produe & et yield & & & \\
\hline $\begin{array}{l}{ }^{40} \mathrm{Co} \\
51 \mathrm{Mn}\end{array}$ & $\begin{array}{l}14 \mathrm{~N} \\
\text { portlel }\end{array}$ & $\begin{array}{l}2.9+1= \\
O(E)\end{array}$ & $3.8+15$ & STB E & Geesamant & 10377 \\
\hline $\begin{array}{l}{ }^{40} \mathrm{Cs} \\
{ }^{4}{ }^{\circ} \mathrm{Co}\end{array}$ & $\begin{array}{l}14 \mathrm{~N} \\
14 \mathrm{~N}\end{array}$ & $\begin{array}{l}2.8+1 \\
2.8+1\end{array}$ & $\begin{array}{l}5.6+1 F \\
5.6+1 F\end{array}$ & $\begin{array}{l}\text { PAD E } \\
\text { PAD E }\end{array}$ & $\begin{array}{l}\text { Fortunat } \\
\text { Fortunat }\end{array}$ & $\begin{array}{l}10378 \\
10380\end{array}$ \\
\hline
\end{tabular}

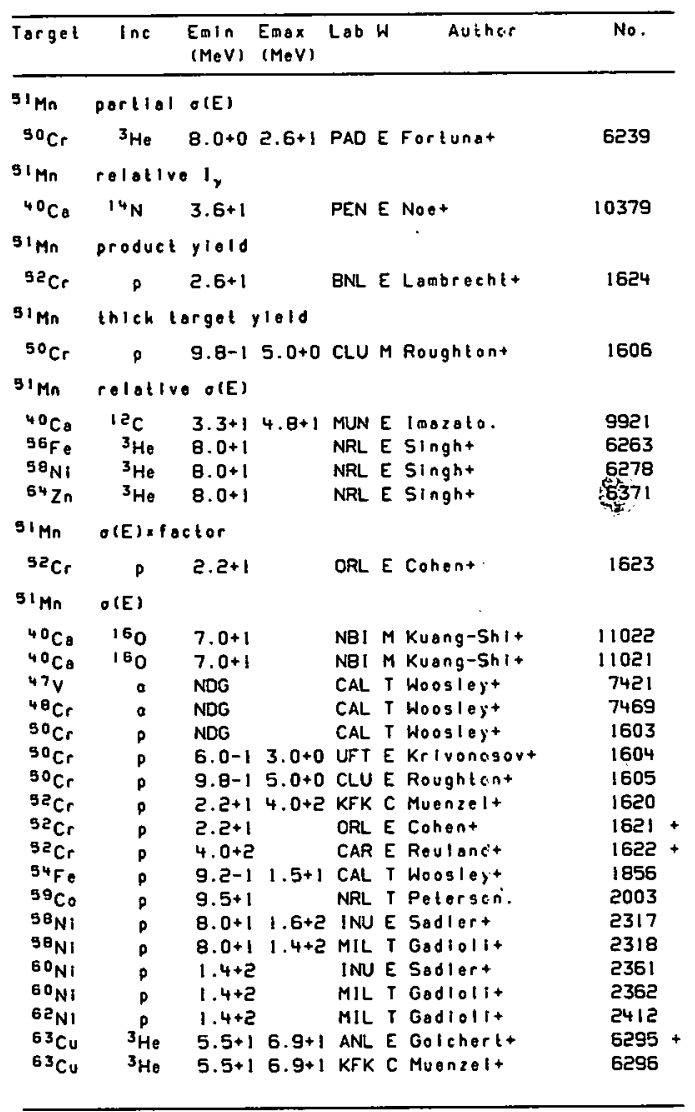




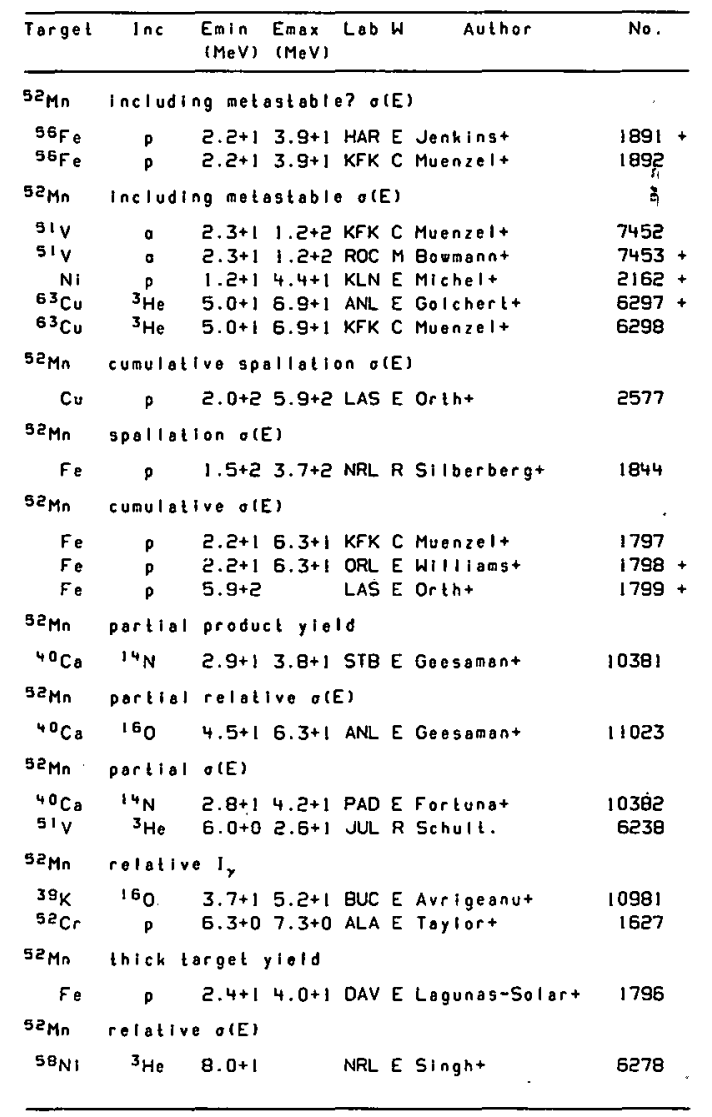

\begin{tabular}{|c|c|c|c|c|c|c|}
\hline Target & Inc & $\begin{array}{l}\text { Emin } \\
(\mathrm{MeV})\end{array}$ & $\begin{array}{l}\text { Emax } \\
(\operatorname{MeV})\end{array}$ & Lob & Author & No. \\
\hline $52 \mathrm{Mn}$ & \multicolumn{6}{|c|}{ relative $\sigma(E)$} \\
\hline${ }^{64} \mathrm{Zn}$ & ${ }^{3} \mathrm{He}$ & $8.0+1$ & & NRL & E singht & 6371 \\
\hline $52 M n$ & \multicolumn{6}{|c|}{$g(E) \times$ factor } \\
\hline $\begin{array}{l}{ }_{92} \mathrm{Cr}^{2} \\
\mathrm{Fe}\end{array}$ & $p$ & $\begin{array}{l}4.0+2 \\
1.5+1\end{array}$ & $4.0+1$ & $\begin{array}{l}\text { CAR } \\
\text { DAV }\end{array}$ & $\begin{array}{l}\text { E Reulandt } \\
\text { E Lagunas-Solart }\end{array}$ & $\begin{array}{l}1626 \\
1795\end{array}$ \\
\hline $52 \mathrm{Mn}$ & \multicolumn{6}{|l|}{$g(E)$} \\
\hline $\begin{array}{l}24 \mathrm{Mg} \\
48 \mathrm{~V}\end{array}$ & $\begin{array}{r}325 \\
32\end{array}$ & $\begin{array}{l}7.0+1 \\
\text { NDG }\end{array}$ & $i .2+2$ & $\begin{array}{l}J U L \\
\text { CAL }\end{array}$ & $\begin{array}{l}\text { R Schult. } \\
\text { T Woosleyt }\end{array}$ & $\begin{array}{r}11986 \\
7423\end{array}$ \\
\hline $\mathrm{Cr}_{r}$ & p & $8.0+0$ & $1.1+1$ & $\mathrm{CCP}$ & R Zatolohint & 1590 \\
\hline$C_{r}$ & D & $1.9+!$ & & RIC & E Waltonr & 1591 \\
\hline${ }^{49} \mathrm{Cr}$ & a & NDG & & CAL & T Woosleyt & 7471 \\
\hline $91 \mathrm{Cr}$ & p & NDG & & CAL & T Woosleyt & 1607 \\
\hline${ }^{52} \mathrm{Cr}$ & $p$ & TR & $1.2+1$ & CAL & T Woosleyt & 1625 \\
\hline $55 \mathrm{Mn}$ & ${ }^{3} \mathrm{He}$ & $8.0+0$ & $4.0+1$ & TOK & E Watanabet & 6245 \\
\hline${ }^{56} \mathrm{Fe}$ & p & $2.0+1$ & $1.0+3$ & GSF & D Ramaty + & 1890 \\
\hline${ }^{56} \mathrm{Fe}$ & $p$ & $2.0+1$ & $7.0+1$ & BNL & T Divadeenam. & 1893 \\
\hline${ }^{59} \mathrm{C}_{0}$ & p & $7.1+1$ & & NRL & T Pelerson. & 2004 \\
\hline $58_{\mathrm{Ni}}$ & p & $4.0+1$ & $5.5+1$ & NRL & T Peterson. & 2256 \\
\hline${ }^{58_{N i}}$ & $p$ & $8.0+1$ & $1.6+2$ & INU & E Sadlert & 2317 \\
\hline${ }^{98} \mathrm{Ni}$ & p & $8.0+1$ & $1.4+2$ & MIL & T Godiolit & 2318 \\
\hline $60^{\circ} \mathrm{Ni}$ & p & $1.4+2$ & & INU & E Sadlert & 2361 \\
\hline $60^{N I}$ & $p$ & $1.4+2$ & & MIL & T Gadiolit & 2362 \\
\hline $62_{\mathrm{Ni}}$ & p & $1.4+2$ & & MIL & T Gadiolit & 2412 \\
\hline S20Mn & \multicolumn{6}{|c|}{ Including metastable? o(E) } \\
\hline $\begin{array}{l}51 y \\
\operatorname{siv}\end{array}$ & : & $\begin{array}{l}2.3+1 \\
2.3+1\end{array}$ & $\begin{array}{l}1.5+5 \\
1.5+5\end{array}$ & $\begin{array}{l}\text { KFK } \\
\text { ROC }\end{array}$ & $\begin{array}{l}\text { C Muenzelt } \\
\text { M Bowmannt }\end{array}$ & $\begin{array}{l}7454 \\
7455\end{array}$ \\
\hline $529 M n$ & \multicolumn{6}{|c|}{ cumulative $\sigma(E)$} \\
\hline $56 \mathrm{Fe}$ & $p$ & $5.9+2$ & & LAS & E Orint & 1894 \\
\hline $528 \mathrm{Mn}$ & \multicolumn{6}{|l|}{$o(E)$} \\
\hline${ }^{40} \mathrm{C} \theta$ & 160 & $8.9+1$ & & JCL & E Morinagat & 11024 \\
\hline $52 \mathrm{Cr}$ & p & $5.8+0$ & $4.0+2$ & KFK & C Muenzel+ & 1628 \\
\hline $5{ }^{2} \mathrm{Cr}$ & p & $5.8+0$ & $1.0+1$ & ANL & E Wingt & 1629 \\
\hline${ }^{52} \mathrm{Cr}$ & p & $1.2+1$ & & ORL & E Blossert & 1630 \\
\hline $52 \mathrm{Cr}$ & p & $4.0+2$ & & CAR & E Reulandt & 1631 \\
\hline $\mathrm{Fe}$ & p & $6.0+2$ & & MTR & E clinet & 1800 \\
\hline${ }^{54} \mathrm{Fe}$ & $\sigma_{L i}$ & $9.8+0$ & $9.4+1$ & INU & E Jastrzebshit & 9185 \\
\hline
\end{tabular}




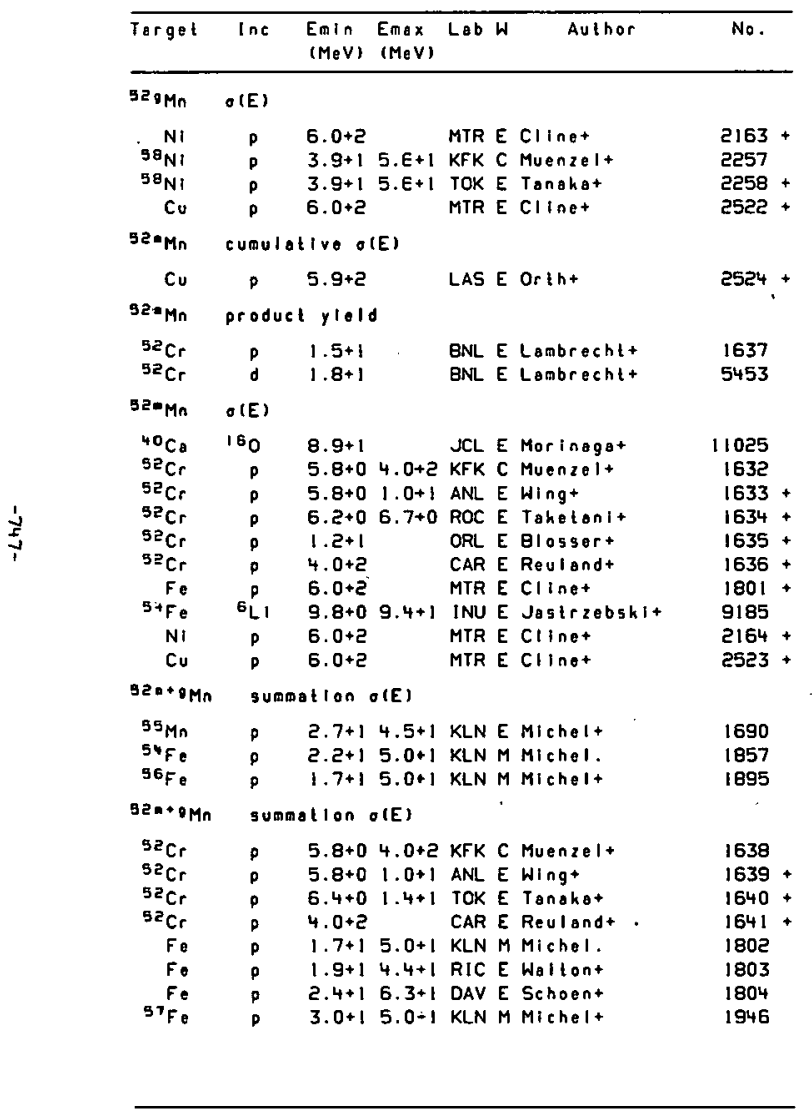

\begin{tabular}{|c|c|c|c|c|c|c|}
\hline Target & $\operatorname{lnc}$ & $\begin{array}{l}\text { Emin } \\
(\mathrm{MeV})\end{array}$ & $\begin{array}{l}\text { Emax } \\
(M e V)\end{array}$ & Lab & Author & No. \\
\hline $52 \cdot+9 M n$ & $O(E) \times$ & f actor & & & & \\
\hline${ }^{52} \mathrm{Cr}$ & $\mathbf{p}$ & $4.0+2$ & & CAR & E Reulanot & 1642 \\
\hline $53 \mathrm{Mn}$ & spollell & ion olE & & & & \\
\hline $\mathrm{Fe}$ & p & $1.5+2$ & $3 \cdot 7+2$ & NRL & R Silberberg+ & 1844 \\
\hline $93 \mathrm{Mn}$ & partlol & ras ol & (E) & & & \\
\hline${ }^{52} \mathrm{Cr}$ & $p$ & $1.0+0$ & $1.0+0$ & AML & E Soltrowshit & 1644 \\
\hline${ }^{33} \mathrm{Mn}$ & portiol & relati & IVe OlE & E). & & \\
\hline $\begin{array}{l}{ }^{40} \mathrm{Ca} \\
{ }^{52} \mathrm{Cr}\end{array}$ & $\begin{array}{r}160 \\
p\end{array}$ & $\begin{array}{l}4.5+1 \\
4.1+0\end{array}$ & $\begin{array}{l}6.3+1 \\
4.3+0\end{array}$ & $\begin{array}{l}\text { ANL } \\
\text { KF! }\end{array}$ & $\begin{array}{l}\text { E Geessmant } \\
\text { E Fodort }\end{array}$ & $\begin{array}{r}11027 \\
1645\end{array}$ \\
\hline $53 M_{n}$ & partlal & $O(E) \times f$ & factor & & & \\
\hline${ }^{50} \mathrm{Cr}$ & $\circ$ & $2.6+1$ & & $\mathrm{CBR}$ & E Aniol* & 7474 \\
\hline $93 M_{n}$ & portlal & $O(E)$ & & & & \\
\hline${ }^{52} \mathrm{Cr}$ & $p$ & $4.1+0$ & $4.3+0$ & $\mathrm{KFI}$ & E Fodort & 1647 \\
\hline $93 \mathrm{mn}$ & row o(E) & & & & & \\
\hline${ }^{52} \mathrm{Cr}$ & $\rho$ & $1.0+0$ & $1.0+0$ & AML & E Switlowshit & 1643 \\
\hline $53_{\mathrm{Mn}}$ & relative & $\theta \sigma(E)$ & & & & \\
\hline $\begin{array}{l}56 \mathrm{Fe} \\
5 \theta_{\mathrm{Ni}} \\
64 \mathrm{Zn}\end{array}$ & $\begin{array}{l}{ }^{3} \mathrm{He} \\
{ }^{3} \mathrm{He} \\
{ }^{3} \mathrm{He}\end{array}$ & $\begin{array}{l}8 \cdot 0+1 \\
8 \cdot 0+1 \\
8 \cdot 0+1\end{array}$ & & $\begin{array}{l}\text { NRL } \\
\text { NRL } \\
\text { NRL }\end{array}$ & $\begin{array}{l}\text { E Singht } \\
\text { E Singht } \\
\text { E Singht }\end{array}$ & $\begin{array}{l}6263 \\
6278 \\
6371\end{array}$ \\
\hline $53^{3} \mathrm{Mn}$ & $O(E) \times f O C$ & ctor & & & & \\
\hline $\begin{array}{l}{ }^{52} \mathrm{Cr} \\
{ }^{53} \mathrm{Mn}\end{array}$ & $\begin{array}{l}14 N \\
O(E)\end{array}$ & $7.1+1$ & $1.0+2$ & LYO & T Billereyt & 10395 \\
\hline $\begin{array}{l}40 \mathrm{Ca} \\
48 \mathrm{~s}\end{array}$ & $\begin{array}{l}150 \\
100\end{array}$ & $7.0+1$ & & $\mathrm{NBI}$ & M Kuang-Shit & 11026 \\
\hline $\begin{array}{l}{ }^{48} \mathrm{Co} \\
4{ }^{4} \mathrm{~V}\end{array}$ & $\begin{array}{r}{ }^{10} \theta \\
0\end{array}$ & $\begin{array}{l}2.5+1 \\
\text { NDG }\end{array}$ & $5.0+1$ & $\begin{array}{l}\text { BNL } \\
\text { CAL }\end{array}$ & $\begin{array}{l}\text { E Listert } \\
\text { T Woosleyt }\end{array}$ & $\begin{array}{l}9524 \\
7425\end{array}$ \\
\hline $50 \mathrm{~V}$ & 0 & $4.2+0$ & $1.1+1$ & CAL & T Woosley+ & 7428 \\
\hline${ }^{50} \mathrm{Cr}$ & 0 & TR & $1.2+1$ & CAL & T Woosleyt & 7475 \\
\hline${ }^{52} \mathrm{Cr}$ & p & NDG & & CAL & T Woosley+ & 1646 \\
\hline${ }^{53} \mathrm{Cr}$ & p & $T R$ & $1.2+1$ & CAL & T Woosleyt & 1652 \\
\hline${ }^{53} \mathrm{Cr}$ & p & $T R$ & $5.9+0$ & ORL & E Johnsont & $1653+$ \\
\hline${ }^{53} \mathrm{Cr}$ & p & $1.4+0$ & $5.9+0$ & KFK & C Muenzo1+ & 1654 \\
\hline${ }^{33} \mathrm{Cr}$ & p & $2.0+0$ & $3.0+0$ & $\mathrm{KFT}$ & E Krivanosovt & 1655 \\
\hline
\end{tabular}




\begin{tabular}{|c|c|c|c|c|c|c|}
\hline Target & $\ln c$ & $\begin{array}{l}\text { Enin } \\
(\mathrm{MeV})\end{array}$ & $\begin{array}{l}\text { Emax } \\
(\operatorname{MeV})\end{array}$ & Lab & Author & No. \\
\hline${ }^{53} \mathrm{Mn}$ & $\sigma(E)$ & & & & & \\
\hline $\begin{array}{l}5{ }^{3} \mathrm{Cr} \\
56 \mathrm{Fe} \\
59 \mathrm{CO} \\
58_{\mathrm{Ni}} \\
58_{\mathrm{Ni}} \\
60 \mathrm{Ni} \\
60 \mathrm{Ni} \\
62 \mathrm{Ni} \\
64 \mathrm{Ni} \\
6{ }^{4} \mathrm{Ni}\end{array}$ & $\begin{array}{l}p \\
p \\
p \\
p \\
p \\
p \\
p \\
p \\
p \\
p\end{array}$ & $\begin{array}{l}2.8+0 \\
9.2-1 \\
5 \cdot 7+1 \\
8.0+1 \\
8.0+1 \\
1.4+2 \\
1.4+2 \\
1.4+2 \\
1.4+2 \\
1.4+2\end{array}$ & $\begin{array}{l}3.9+0 \\
1.3+1 \\
1.6+2 \\
1.4+2\end{array}$ & $\begin{array}{l}\text { IJI } \\
\text { CAL } \\
\text { NRL } \\
\text { INU } \\
\text { MIL } \\
\text { INU } \\
\text { MIL } \\
\text { MIL } \\
\text { INU } \\
\text { MIL }\end{array}$ & $\begin{array}{l}\text { E Dryapaghenkot } \\
\text { T Woosleyt } \\
\text { T Pelerson. } \\
\text { E Sadlert } \\
\text { T Godiolit } \\
\text { E Sadlert } \\
\text { T Gadiolit } \\
\text { T Gadiolit } \\
\text { E Sodiert } \\
\text { T Godiolit }\end{array}$ & $\begin{array}{l}1656 \\
1896 \\
2005 \\
2317 \\
2318 \\
2361 \\
2362 \\
2412 \\
2434 \\
2435\end{array}$ \\
\hline${ }^{54} \mathrm{Mn}$ & indepen & dent ol & & & & \\
\hline $\begin{array}{l}\mathrm{Fe} \\
\mathrm{Cu}\end{array}$ & $\begin{array}{l}p \\
p\end{array}$ & $\begin{array}{l}5.9+2 \\
5.9+2\end{array}$ & & $\begin{array}{l}\text { LAS } \\
\text { LAS }\end{array}$ & $\begin{array}{l}\text { E Orlht } \\
\text { E Orlht }\end{array}$ & $\begin{array}{l}1810+ \\
2526+\end{array}$ \\
\hline $54 \mathrm{Mn}$ & indepen & dent sp & pollati & ion. & $\sigma(E)$ & \\
\hline $\begin{array}{l}\mathrm{Cu} \\
54 \mathrm{Mn}\end{array}$ & $\begin{array}{c}p \\
\text { spallat }\end{array}$ & $\begin{array}{l}2.0+2 \\
\text { ion } 0(E\end{array}$ & $\begin{array}{l}5.9+2 \\
\text { E) }\end{array}$ & LAS & E Ortht & 2578 \\
\hline $\begin{array}{l}\mathrm{Fe} \\
{ }_{54} \mathrm{Mn}\end{array}$ & $O(E)$ & $1.5+2$ & $3.7+2$ & NRL & R Sllberberg+ & 1844 \\
\hline $\begin{array}{l}55 \mathrm{Mn} \\
{ }^{56} \mathrm{Fe} \\
{ }^{59} \mathrm{Co}\end{array}$ & $\begin{array}{l}p \\
p \\
p\end{array}$ & $\begin{array}{l}9.3+0 \\
8.0+0 \\
2.6+1\end{array}$ & $\begin{array}{l}4.5+1 \\
5 \cdot 0+1 \\
5 \cdot 0+1\end{array}$ & $\begin{array}{l}\text { KLN } \\
\text { KLN } \\
\text { KLN }\end{array}$ & $\begin{array}{l}\text { E Michel+ } \\
\text { Michel+ } \\
\text { Michel+ }\end{array}$ & $\begin{array}{l}1691 \\
1898 \\
2007\end{array}$ \\
\hline $\begin{array}{l}54 \mathrm{Mn} \\
51 \mathrm{~V}\end{array}$ & partial & $\begin{array}{l}\mathrm{cos} \text { th } \\
5.0+0\end{array}$ & hlck ta & $\begin{array}{l}\text { orget } \\
\text { SUN }\end{array}$ & t yleld & 7456 \\
\hline $54 \mathrm{mn}$ & partiol & relots & ive ole & & & \\
\hline $\begin{array}{l}48 i_{\theta} \\
\operatorname{siv}\end{array}$ & $\begin{array}{r}i_{B} \\
a\end{array}$ & $\begin{array}{l}\tilde{c} .5+1 \\
E .8+0\end{array}$ & $\begin{array}{l}5.0+1 \\
1.5+1\end{array}$ & $\begin{array}{l}\mathrm{BNL} \\
\mathrm{CCP}\end{array}$ & $\begin{array}{l}\text { E Nathant } \\
\text { E Kydojarovt }\end{array}$ & $\begin{array}{l}9581 \\
7461\end{array}$ \\
\hline $54 \mathrm{Mn}$ & thick $t$ & $\operatorname{arget} y$ & yield & & & \\
\hline $\begin{array}{l}51 \mathrm{~V} \\
54 \mathrm{Mn}\end{array}$ & $\begin{array}{c}a \\
\sigma(E) \times f o\end{array}$ & $\begin{array}{l}\text { TR } \\
\text { ctor }\end{array}$ & +1 & $\mathrm{CLu}$ & E Roughtont & 7460 \\
\hline $\begin{array}{l}52 \mathrm{Cr} \\
5 \mathrm{Mro}\end{array}$ & ${ }_{P}^{14} N$ & $\begin{array}{l}7.1+1 \\
2.2+1\end{array}$ & $1.0+2$ & $\begin{array}{l}\text { LYO } \\
\text { ORL }\end{array}$ & $\begin{array}{l}\text { I Billereyt } \\
\text { E Cohent }\end{array}$ & $\begin{array}{r}10396 \\
1694\end{array}$ \\
\hline
\end{tabular}

\begin{tabular}{|c|c|c|c|c|c|c|c|}
\hline Target & Inc & $\begin{array}{l}\text { EmIn } \\
(M e V)\end{array}$ & $\begin{array}{l}\text { Emax } \\
(M e V)\end{array}$ & Lab & $w$ & Author & No. \\
\hline${ }^{4} \mathrm{Mn}$ & $\sigma(E)$ & & & & & & \\
\hline $50 \mathrm{~V}$ & a & NDG & & CAL & $\mathbf{T}$ & Woosleyt & 7429 \\
\hline $51 \mathrm{v}$ & a & TR & $1.0+1$ & CAL & & Woosleyt & 7457 \\
\hline $91 \mathrm{~V}$ & 0 & $6.6+0$ & $8.5+1$ & KFK & & Muenzelt & 7458 \\
\hline $51 \mathrm{~V}$ & a & $6.6+0$ & $8.5+1$ & ROC & M & Boovennt & $7459+$ \\
\hline $\mathrm{Cr}$ & p & 8. $0+0$ & $1.1+1$ & CCP & $R$ & Zalolohint & 1592 \\
\hline${ }^{3} \mathrm{Cr}$ & p & NDG & & CAL & & Woosleyt & 1657 \\
\hline${ }^{54} \mathrm{Cr}$ & p & TR & $5.5+0$ & ORL & E & Johnsont & 1660 \\
\hline${ }^{4} \mathrm{Cr}$ & p & TR & $1.1+1$ & CAL & & Woosleyt & 1661 \\
\hline${ }^{4} \mathrm{Cr}$ & p & 8.3-1 & $3.6+0$ & CAL & E & Zyskindt & 1662 \\
\hline${ }^{4} \mathrm{Cr}$ & p & $2.3+0$ & $5.5+0$ & KFK & $\mathrm{c}$ & Muenzelt & 1663 \\
\hline${ }^{4} \mathrm{Cr}$ & p & $3.0+0$ & $5.0+0$ & INU & $T$ & Kailast & 1664 \\
\hline $5 \mathrm{mn}$ & p & $2.2+1$ & & KFK & c & Muenzel+ & 1692 \\
\hline${ }^{55} \mathrm{Mn}$ & & $2.2+1$ & & ORL & E & Cohent & $1693+$ \\
\hline $55 \mathrm{Mn}$ & ${ }^{3} \mathrm{He}$ & $8.0+0$ & $4.0+1$ & TOK & $\vec{E}$ & Watanabet & 6246 \\
\hline $\mathrm{Fe}$ & $\mathbf{p}$ & $1.7+1$ & $4.4+1$ & KLN & M & Michel+ & 1805 \\
\hline $\mathrm{Fe}$ & p & $2.6+1$ & $6.3+1$ & DAV & $E$ & Schoent & 1806 \\
\hline $\mathrm{Fe}$ & p & $3 \cdot 1+1$ & $6.2+1$ & $\mathrm{KF} \mathrm{K}$ & c & Muenzelt & 1807 \\
\hline $\mathrm{Fe}$ & p & $3 \cdot 1+1$ & $6.2+1$ & ORL & E & Williamst & 1808 \\
\hline $\mathrm{Fe}$ & & $6.0+2$ & & MTR & E & Clinet & 1809 \\
\hline${ }^{4} \mathrm{Fe}$ & ${ }^{6} \mathrm{Li}$ & $9.8+0$ & $9.4+1$ & INU & E & Jestrzebshit & 9185 \\
\hline${ }^{55} \mathrm{Fe}$ & p & $2.5+1$ & $3.9+1$ & HAR & $E$ & Jenkingt & $1899+$ \\
\hline${ }^{56} \mathrm{Fe}$ & p & $2.5+1$ & $3.9+1$ & KFK & c & Muenzelt & 1900 \\
\hline${ }^{55} \mathrm{Fe}$ & p & $3.0+1$ & $1.0+3$ & GSF & 0 & Ramaty+ & 1897 \\
\hline${ }^{36} \mathrm{Fe}$ & p & $3.0+i$ & $7.0+1$ & BNL & T & Oivadeenam. & 1901 \\
\hline${ }^{37} \mathrm{Fe}$ & p & $3.5+1$ & $5 \cdot 0+1$ & KLN & M & Michelt & 1947 \\
\hline${ }^{57} \mathrm{Fe}$ & p & NDG & & CAL & T & Woosleyt & 1948 \\
\hline${ }^{5 \theta_{F e}}$ & p & $1.8+1$ & $5.0+1$ & KLN & M & Michelt & 1963 \\
\hline${ }^{59} \mathrm{Co}$ & p & NDG & & $\mathrm{KLN}$ & E & Michelt & 2008 \\
\hline${ }^{59} \mathrm{Co}$ & p & $3.2+1$ & $6.3+1$ & DAV & E & Schoent & 2009 \\
\hline${ }^{39} \mathrm{Co}$ & p & $2.7+1$ & $4.5+1$ & KLN & M & Michelt & 2006 \\
\hline${ }^{59} \mathrm{Co}_{0}$ & p & $8 \cdot 1+1$ & & NRL & $\mathbf{T}$ & Peterson. & 2010 \\
\hline${ }^{59} \mathrm{Co}_{0}$ & p & $4.5+1$ & & NRL & $\mathbf{T}$ & Peterson. & 2011 \\
\hline${ }^{59} \mathrm{Co}$ & p & $4.0+1$ & & NRL & $T$ & Peterson. & 2012 \\
\hline $\mathrm{Ni}$ & p & $6.0+2$ & & MTR & E & Clinet & $2165+$ \\
\hline${ }^{58} \mathrm{Ni}$ & & $8.0+1$ & $1.6+2$ & INU & E & Sodlert & 2317 \\
\hline${ }^{58} \mathrm{Ni}$ & p & $8.0+1$ & $1.4+2$ & MIL & & Gadlolit & 2318 \\
\hline${ }^{60} \mathrm{Ni}$ & p & $4.2+1$ & $5.6+1$ & KFK & $c$ & Muenzel+ & 2331 \\
\hline${ }^{60} \mathrm{Ni}$ & p & $4.2+1$ & $5.6+1$ & TOK & $E$ & Tanakat & 2332 \\
\hline${ }^{50} \mathrm{Ni}$ & p & $1.4+2$ & & INU & $\bar{E}$ & Sadiert & 2361 \\
\hline${ }^{60} \mathrm{Ni}$ & p & $1.4+2$ & & MIL & $\mathbf{T}$ & Gadiolit & 2362 \\
\hline
\end{tabular}




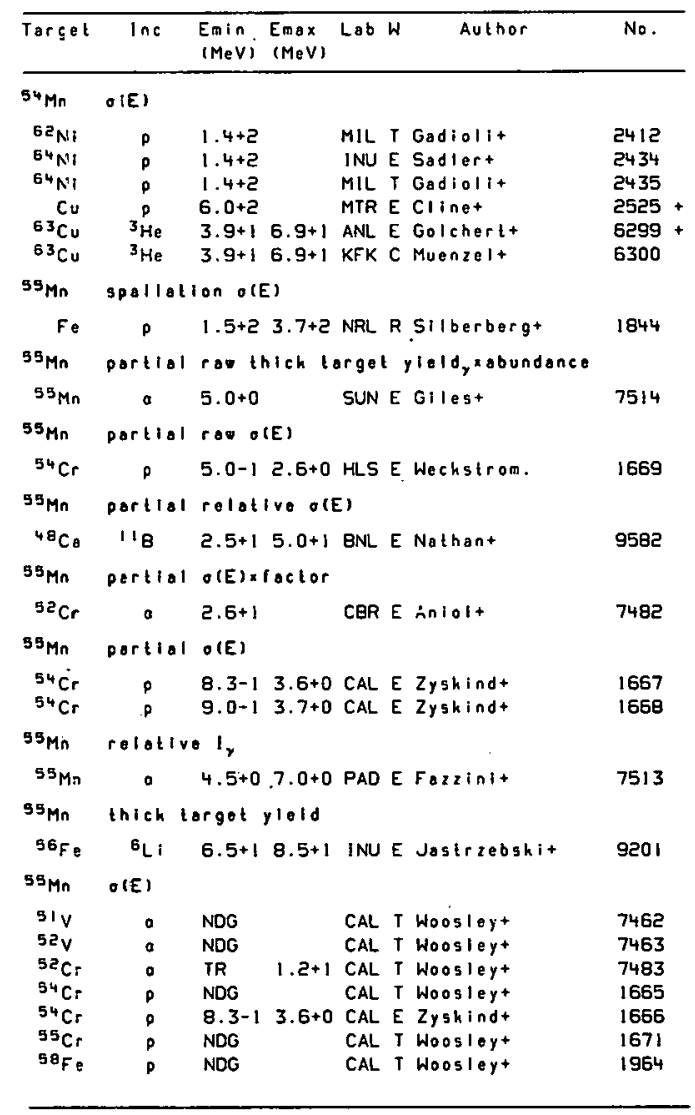

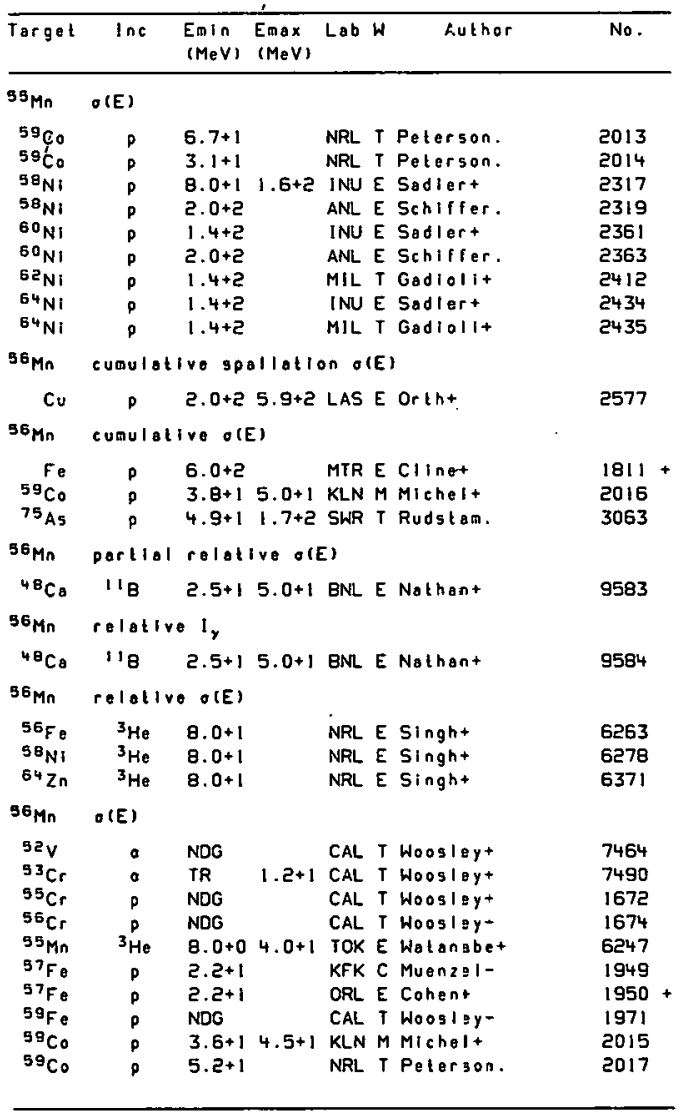




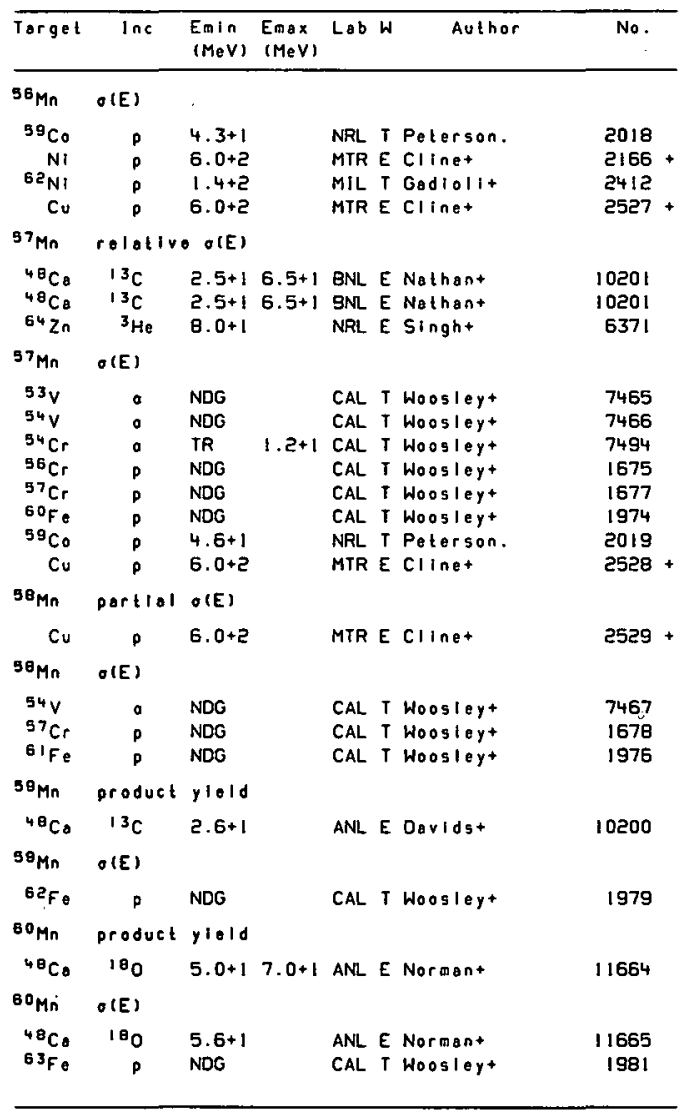

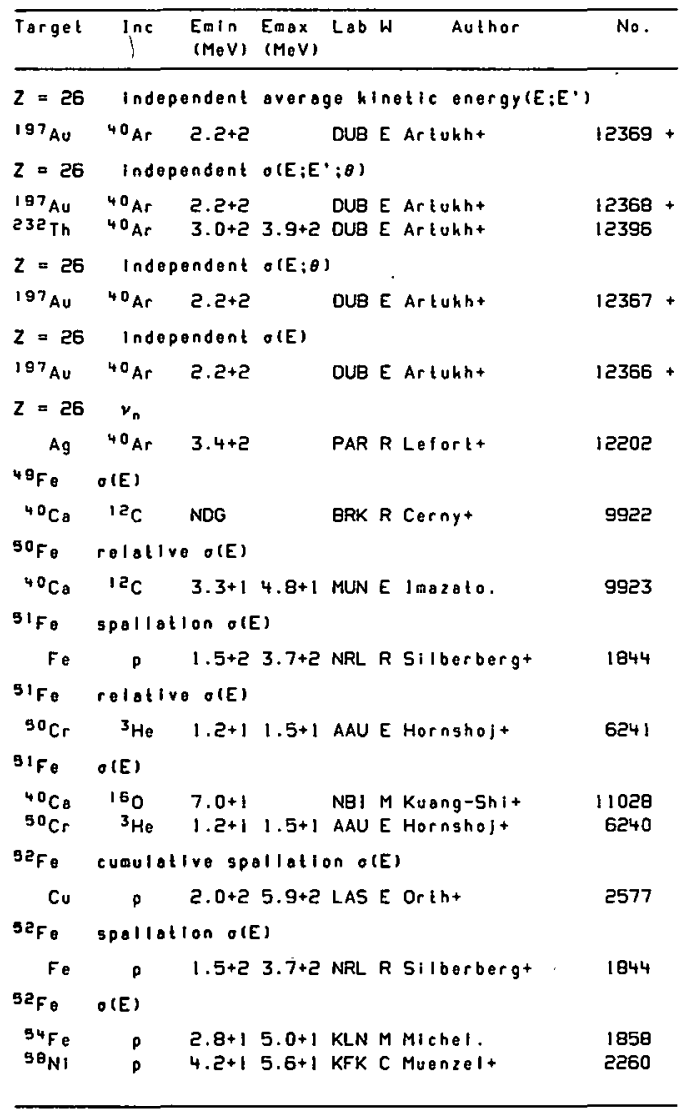




\begin{tabular}{|c|c|c|c|c|c|c|}
\hline Target & $\ln c$ & $\begin{array}{l}\text { Emin } \\
(\mathrm{MeV})\end{array}$ & $\begin{array}{l}\text { Emax } \\
(M e V)\end{array}$ & Lab & Author & No. \\
\hline $\mathrm{se}_{\mathrm{Fo}}$ & \multicolumn{6}{|c|}{ cumulative $O(E)$} \\
\hline${ }^{5 \theta_{N i}}$ & $p$ & $4.2+1$ & $5.6+1$ & TOK & E Tanathat & $2261+$ \\
\hline $52 \mathrm{Fe}$ & portial & produc & et yield & & & \\
\hline${ }^{40} \mathrm{Ca}$ & $14 \mathrm{~N}$ & $2.9+1$ & $3.8+15$ & STB & E Geesamant & 10383 \\
\hline${ }^{52} \mathrm{Fe}$ & partial & relati & ive $\sigma(E)$ & & & \\
\hline $40 \mathrm{Ca}$ & ${ }^{12} \mathrm{c}$ & $3 \cdot 3+1$ & $4.8+11$ & MUU & E Imazatat & 9924 \\
\hline $52 \mathrm{Fe}$ & portial & $\sigma(E) \times f$ & factor & & & \\
\hline${ }^{54} \mathrm{Fe}$ & $p$ & $4.5+1$ & & MSU & E Decooshit & 1859 \\
\hline${ }^{52} \mathrm{Fe}$ & partial & $\theta(E)$ & & & & \\
\hline${ }^{40} \mathrm{Ca}$ & $14 \mathrm{~N}$ & $2.8+1$ & $4.2+1 F$ & PAD & E Fortunat & 10384 \\
\hline $52 \mathrm{Fe}$ & colative & o $I_{r}$. & & & & \\
\hline${ }^{90} \mathrm{Cr}$ & ${ }^{3} \mathrm{He}$ & $8.0+0$ & & TOK & E Iritanit & 6242 \\
\hline $52 \mathrm{Fe}$ & product & yield & & & & \\
\hline${ }^{55} \mathrm{Mr}$ & $\rho$ & $5.0+1$ & & UPP & E' Lundquistt & 1696 \\
\hline${ }^{2} \mathrm{Fo}$ & thlck to & arget $y$ & $y=10 \times 0$ & abund & sence & \\
\hline $\begin{array}{l}{ }^{50} \mathrm{Cr} \\
{ }^{52} \mathrm{Cr}\end{array}$ & $\stackrel{a}{3_{\mathrm{He}}}$ & $\begin{array}{l}3 \cdot 0+1 \\
4 \cdot 6+1\end{array}$ & & $\begin{array}{l}\text { AML } \\
\text { AML }\end{array}$ & $\begin{array}{l}\text { R Chaudri. } \\
\text { R Chaudri. }\end{array}$ & $\begin{array}{l}7476 \\
6243\end{array}$ \\
\hline $32 \mathrm{Fo}_{0}$ & thick ta & arget $y$ & yield & & & \\
\hline $\begin{array}{l}55 \mathrm{Mr} \\
5 \theta_{\mathrm{NI}}\end{array}$ & $p$ & $\begin{array}{l}2.3+1 \\
5 \cdot 5+1\end{array}$ & $6.5+1$ & $\begin{array}{l}\text { AML } \\
\text { IKO }\end{array}$ & $\begin{array}{l}\text { R Chaudri. } \\
\text { E Lourst }\end{array}$ & $\begin{array}{l}1695 \\
2259\end{array}$ \\
\hline${ }^{52} \mathrm{Fo}$ & relative & $\sigma(E)$ & & & & \\
\hline${ }^{40} \mathrm{C}_{8}$ & ${ }^{12} \mathrm{c}$ & $3 \cdot 3+1$ & $4.8+11$ & MUN & E Imazato. & 9925 \\
\hline $52 \mathrm{Fe}_{\mathrm{e}}$ & $\theta(E)$ & & & & & \\
\hline $\begin{array}{l}{ }^{48} \mathrm{Cr} \\
{ }^{50} \mathrm{Cr}\end{array}$ & $a$ & $\begin{array}{l}\text { NDG } \\
\text { TR }\end{array}$ & $2.8+1$ & $\begin{array}{l}\text { CAL } \\
\text { UK }\end{array}$ & $\begin{array}{l}\text { T Woosleyt } \\
\text { E Watergt }\end{array}$ & $\begin{array}{l}7470 \\
7477\end{array}$ \\
\hline $48 \mathrm{Mn}$ & a & NDG & & CAL & T Woosleyt & 7504 \\
\hline $51 \mathrm{Mn}$ & p & NDG & & CAL & T Woosley+ & 1679 \\
\hline${ }^{55} \mathrm{Mn}$ & P & $T R$ & $1.6+2$ & HAR & E Symet & 1697 \\
\hline$t \oplus p M n$ & p & $T R$ & $5.5+1$ & IKO & E Leurst & 1730 \\
\hline $\mathrm{Fo}$ & p & $3.3+1$ & $4.4+1$ & KLN & M Michol+ & 1812 \\
\hline $\mathrm{Fe}$ & p & $3.4+1$ & $6.1+1$ & KFK & C Muenzel+ & 1813 \\
\hline
\end{tabular}

\begin{tabular}{|c|c|c|c|c|c|c|}
\hline Target & $\ln c$ & $\begin{array}{l}\text { Enin } \\
(\mathrm{MeV})\end{array}$ & $\begin{array}{l}\text { Emax } \\
(\mathrm{MeV})\end{array}$ & Lab & Author & No. \\
\hline${ }^{52} \mathrm{Fe}$ & $\sigma(E)$ & & & & & \\
\hline $\mathrm{Fe}$ & D & $3.4+1$ & $6.1+1$ & ORL E & E Willianst & $1814+$ \\
\hline $\mathrm{Fe}$ & p & $5.9+2$ & 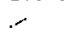 & LAS E & Ortht & $1815+$ \\
\hline $\mathrm{Fe}$ & $p$ & $6.0+2$ & & MTR E & E Clinet & $1816+$ \\
\hline${ }^{54} \mathrm{Fe}$ & ${ }^{6} \mathrm{~L} 1$ & $9.8+0$ & $9.4+1$ & INU E & E Jastrzebshit & 9185 \\
\hline${ }^{59} \mathrm{Co}$ & $p$ & $9.5+1$ & & NRL & T Petersan. & 2020 \\
\hline${ }^{59} \mathrm{Co}$ & p & $9.1+1$ & & NRL & T Peterson: & 2021 \\
\hline${ }^{59} \mathrm{Co}$ & p & $8.2+1$ & & NRL T & T Petersan. & 2022 \\
\hline NI & p & $6.0+2$ & & MTR E & E Clinet & $2167+$ \\
\hline${ }^{62} \mathrm{NI}$ & p & $1.4+2$ & & MIL T & T Gadiolit & 2412 \\
\hline Cu & p & $5.9+2$ & & LAS E & E Ortht & $2530+$ \\
\hline $\mathrm{Cu}$ & $p$ & $6.0+2$ & & MTR E & E Clinet & $253 ! 1+$ \\
\hline $52 \bullet_{\mathrm{Fe}}$ & produ & $t$ yiold & & & & \\
\hline${ }^{40} \mathrm{CB}$ & ${ }^{14} \mathrm{~N}$ & $2.9+1$ & $5.5+1$ & STB E & E Geesamant & 10385 \\
\hline $53_{\mathrm{FO}}$ & cumula & ive lnc & cluoding & 9 melo & ostable? o(E) & \\
\hline $\begin{array}{l}5 \theta_{N 1} \\
5 \theta_{N 1}\end{array}$ & $\begin{array}{l}p \\
p\end{array}$ & $\begin{array}{l}3 \cdot 3+1 \\
3 \cdot 3+1\end{array}$ & $\begin{array}{l}5 \cdot 6+1 \\
5 \cdot 6+1\end{array}$ & $\begin{array}{l}\text { KFK O } \\
\text { TOK E }\end{array}$ & $\begin{array}{l}\text { C Muenzel+ } \\
\text { E Tanaho+ }\end{array}$ & $\begin{array}{l}2262 \\
2253+\end{array}$ \\
\hline $53 \mathrm{Fe}$ & lnclud & ng meta & astable & e? OLE & & \\
\hline $\begin{array}{r}F e \\
54 \mathrm{Fe} \\
54 \mathrm{Fe} \\
\mathrm{Cu}\end{array}$ & $\begin{array}{l}p \\
p \\
p \\
p\end{array}$ & $\begin{array}{l}6.0+2 \\
2.2+1 \\
2.2+1 \\
6.0+2\end{array}$ & & $\begin{array}{l}\text { MTR E } \\
\text { KFK C } \\
\text { ORL E } \\
\text { MTR E }\end{array}$ & $\begin{array}{l}\text { E Clinet } \\
\text { C Muenzel+ } \\
\text { E Cohent } \\
\text { E Cline+ }\end{array}$ & $\begin{array}{l}1817+ \\
1860 \\
1861+ \\
2532+\end{array}$ \\
\hline $5^{3} \mathrm{Fo}$ & spalla & IOn OCE & & & & \\
\hline $\mathrm{Fe}$ & p & $1.5+2$ & $3.7+2$ & NRL F & R Silberberg+ & 1844 \\
\hline${ }^{33} \mathrm{Fo}$ & rolati & $O O(E)$ & & & & \\
\hline $\begin{array}{l}{ }^{56} \mathrm{Fe} \\
50_{\mathrm{Ni}} \\
64 \mathrm{Zn}\end{array}$ & $\begin{array}{l}{ }^{3} \mathrm{He} \\
{ }^{3} \mathrm{He} \\
{ }^{3} \mathrm{He}\end{array}$ & $\begin{array}{l}8 \cdot 0+1 \\
8 \cdot 0+1 \\
8 \cdot 0+1\end{array}$ & & $\begin{array}{l}\text { NRL E } \\
\text { NRL E } \\
\text { NRL E }\end{array}$ & $\begin{array}{l}E \operatorname{singht} \\
E \operatorname{singht} \\
\text { E singht }\end{array}$ & $\begin{array}{l}6263 \\
6278 \\
6371\end{array}$ \\
\hline $5^{53} \mathrm{Fe}$ & $\sigma(E)$ & & & & & \\
\hline${ }^{40} \mathrm{Ca}$ & 180 & $7.0+1$ & & NBI $M$ & M Kuang-Sil- & 11029 \\
\hline${ }^{49} \mathrm{Cr}$ & a & NDG & & CAL & I Woosioyt & 7472 \\
\hline${ }^{50} \mathrm{Cr}$ & 0 & TR & $1.6+1$ & CAL I & I Woosleyt & 7478 \\
\hline $50 \mathrm{Mn}$ & a & NDG & & CAL $T$ & T Woosleyt & 7505 \\
\hline $52 \mathrm{Mn}$ & p & NDG & & CAL $T$ & T Woosleyt & 1680 \\
\hline${ }^{59} \mathrm{Co}_{0}$ & p & $8.4+1$ & & NRL T & T Peterson. & 2023 \\
\hline
\end{tabular}




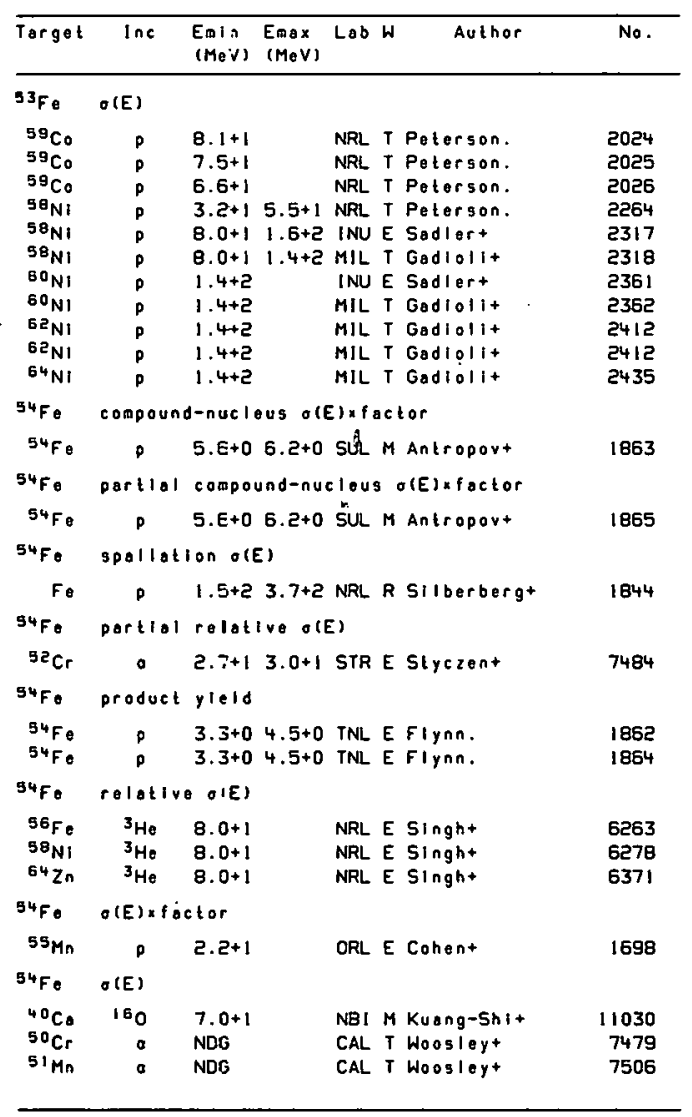

\begin{tabular}{|c|c|c|c|c|c|c|}
\hline Target & $\ln c$ & $\begin{array}{l}\text { Emin } \\
\text { (MeV) }\end{array}$ & $\begin{array}{l}\text { Emox } \\
(M e V)\end{array}$ & Lob $W$ & Author & No. \\
\hline${ }^{54} \mathrm{Fe}$ & $O(E)$ & & & & & \\
\hline 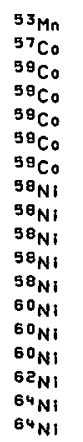 & $\begin{array}{l}p \\
p \\
p \\
p \\
p \\
p \\
p \\
p \\
p \\
p \\
p \\
p \\
p \\
p \\
p \\
p \\
p \\
p\end{array}$ & $\begin{array}{l}\text { NDG } \\
\text { NDG } \\
7.0+1 \\
6.5+1 \\
6.2+1 \\
4.2+1 \\
8.1+1 \\
T R \\
T R \\
6.0+1 \\
8.0+1 \\
2.0+2 \\
1.4+2 \\
1.4+2 \\
2.0+2 \\
1.4+2 \\
1.4+2 \\
1.4+2\end{array}$ & $\begin{array}{l}8 \\
8.0+1 \\
8.0+1 \\
1.6+2 \\
1.4+2 \\
\\
\\
3 \\
3\end{array}$ & $\begin{array}{ll}\text { CAL } & T \\
\text { CAL } & T \\
\text { NRL } & T \\
\text { NRL } & T \\
\text { NRL } & T \\
\text { NRL } & T \\
\text { NRL } & T \\
\text { NRL } & T \\
\text { NRL } & T \\
\text { INU E } \\
\text { MIL } & T \\
\text { ANL } & E \\
\text { INU } & E \\
\text { MIL } & T \\
\text { ANR } & \text { E } \\
\text { MIL } & T \\
\text { INU } & E \\
\text { MIL } & T\end{array}$ & $\begin{array}{l}\text { T Woosleyt } \\
\text { T Woosleyt } \\
\text { T Peterson. } \\
\text { T Peterson. } \\
\text { T Peterson. } \\
\text { T Peterson. } \\
\text { T Peterson. } \\
\text { T Peterson. } \\
\text { T Potergon. } \\
\text { E Sadlert } \\
T \text { Gadiolit } \\
\text { E Schiffer. } \\
\text { E Sadiert } \\
\text { T Gadiolit } \\
\text { E Schiffer. } \\
\text { T Gadiallt } \\
\text { E Sadiert } \\
\text { T Gadialit }\end{array}$ & $\begin{array}{l}1682 \\
1987 \\
2027 \\
2028 \\
2029 \\
2030 \\
2031 \\
2265 \\
2266 \\
2317 \\
2318 \\
2319 \\
2361 \\
2362 \\
2363 \\
2412 \\
2434 \\
2435\end{array}$ \\
\hline${ }^{55} \mathrm{Fe}_{\mathrm{e}}$ & spollat & IOn OLE & & & & \\
\hline $\begin{array}{l}\mathrm{Fe}_{\mathrm{e}} \\
\mathrm{Ss}_{\mathrm{Fo}}\end{array}$ & $\begin{array}{c}p \\
\text { relative }\end{array}$ & $\begin{array}{l}1.5+2 \\
.1 y\end{array}$ & $3.7+2$ & NRL $R$ & R sllberbergt & 1844 \\
\hline $\begin{array}{l}59_{\mathrm{Mn}} \\
55_{\mathrm{Fo}}\end{array}$ & $\begin{array}{c}\text { p } \\
\text { product }\end{array}$ & $\begin{array}{l}2.8+0 \\
y 1010\end{array}$ & $3.6+0$ & KaU E & E Robertsont & 1708 \\
\hline $\begin{array}{l}{ }^{55} \mathrm{Mn} \\
{ }^{55} \mathrm{Fo}\end{array}$ & $\stackrel{p}{\text { thick to }}$ & $\begin{array}{l}\text { NDG } \\
\text { orget } y\end{array}$ & yiold & RoS E & E Bockerlt & 1707 \\
\hline $\begin{array}{l}{ }^{59} \mathrm{Co}_{0} \\
{ }^{55} \mathrm{~F}_{0}\end{array}$ & $\begin{array}{c}D \\
\text { overoge }\end{array}$ & $\begin{array}{l}2.5+1 \\
O(E)\end{array}$ & $8.5+1$ & LVN E & E Hassbroght & $2037+$ \\
\hline $\begin{array}{l}55 \mathrm{Mn} \\
{ }^{55} \mathrm{~F} 。\end{array}$ & $\begin{array}{c}p \\
\text { relotire }\end{array}$ & $\begin{array}{r}1.0+0 \\
. \quad O(E)\end{array}$ & $1.2+0$ & ORL E & E Johnsont & 1709 \\
\hline $\begin{array}{l}4 \theta_{\mathrm{TI}} \\
32 \mathrm{Cr} \\
56_{\mathrm{Fe}} \\
58_{\mathrm{Ni}} \\
58_{\mathrm{Ni}}\end{array}$ & $\begin{array}{c}160 \\
12^{2} \mathrm{C} \\
3_{\mathrm{He}} \\
3_{\mathrm{He}} \\
0\end{array}$ & $\begin{array}{l}5.8+1 \\
5 \cdot 6+1 \\
8.0+1 \\
8.0+1 \\
6.2+1\end{array}$ & & $\begin{array}{l}\text { SAC E } \\
\text { SAC E } \\
\text { NRL E } \\
\text { NRL E } \\
\text { TOK E }\end{array}$ & $\begin{array}{l}\text { E Nomurat } \\
\text { E Nomurat } \\
\text { E Singht } \\
\text { E Singht } \\
\text { E Hamagahit }\end{array}$ & $\begin{array}{r}11129 \\
9935 \\
6263 \\
6278 \\
7660\end{array}$ \\
\hline
\end{tabular}




\begin{tabular}{|c|c|c|c|c|c|c|}
\hline Target & $\ln e$ & $\begin{array}{l}\text { Enin } \\
(M e V)\end{array}$ & $\begin{array}{l}\text { Emax } \\
(\mathrm{MeV})\end{array}$ & Lab W & Author & No. \\
\hline${ }^{95} \mathrm{Fe}$ & \multicolumn{2}{|c|}{ relative ofE } & & & \multirow[b]{2}{*}{ E singht } & \\
\hline${ }^{64} \mathrm{Zn}$ & $3_{\mathrm{He}}$ & $8.0+1$ & & NRL E & & 6371 \\
\hline${ }^{55} \mathrm{Fe}$ & \multicolumn{2}{|c|}{ OIE) X foctor } & & & & \\
\hline $\begin{array}{l}48 \mathrm{Tr} \\
4 \mathrm{Tl}^{2} \mathrm{Cr} \\
53 \mathrm{Cr} \\
56 \mathrm{Fg}\end{array}$ & $\begin{array}{l}6 \\
160 \\
12 \mathrm{C} \\
1{ }^{2} \mathrm{~N} \\
0\end{array}$ & $\begin{array}{l}5.8+1 \\
5.6+1 \\
7.1+1 \\
2.2+1\end{array}$ & $1.0+2$ & $\begin{array}{l}\text { SAC E } \\
\text { SAC E } \\
\text { LYO T } \\
\text { ORL E }\end{array}$ & $\begin{array}{l}\text { E Nomurat } \\
\text { E Nomurat } \\
\text { T Billereyt } \\
\text { E Cohent }\end{array}$ & $\begin{array}{r}11128 \\
9934 \\
10397 \\
1906\end{array}$ \\
\hline${ }^{55} \mathrm{Fo}$ & \multicolumn{2}{|l|}{$O(E)$} & & & & \\
\hline $\begin{array}{l}48 \mathrm{TI} \\
51 \mathrm{Cr}\end{array}$ & $\begin{array}{r}160 \\
0\end{array}$ & $\begin{array}{l}5.8+1 \\
\text { NDG }\end{array}$ & & $\begin{array}{l}\text { SAC } \varepsilon \\
\text { CAL T }\end{array}$ & $\begin{array}{l}\text { E Nomurat } \\
\text { T Woosleyt }\end{array}$ & $\begin{array}{r}11130 \\
7480\end{array}$ \\
\hline $25 \mathrm{Cr}$ & 0 & TR & $1.5+1$ & CAL T & T Woosleyt & 7485 \\
\hline $52 \mathrm{Cr}$ & $12_{C}$ & $5.6+1$ & & SAC E & E Nomurat & 9936 \\
\hline $52 \mathrm{Mn}$ & 0 & NDG & & CAL T & T Woosleyt & 7508 \\
\hline $54 \mathrm{Mn}$ & p & NDG & & CAL $T$ & T Woosleyt & 1684 \\
\hline $55 \mathrm{Mn}$ & p & TR & $1.2+1$ & CAL T & T Woosleyt & 1699 \\
\hline $55 \mathrm{Mn}$ & $p$ & $1.0+0$ & $6.8+0$ & KFK C & C Muenzel+ & 1700 \\
\hline${ }^{55} \mathrm{Mn}$ & p & $1.4+0=$ & $5.4+0$ & TRM E & E Viyogit & 1701 \\
\hline $55 \mathrm{Mn}$ & p & $1.4+0=$ & $5.4+0$ & TRM E & E Kallast & 1702 \\
\hline $55 \mathrm{Mn}$ & $p$ & $2.0+0$ & $5.0+0$ & INU $\mathrm{T}$ & T Kollagt & 1703 \\
\hline $55 \mathrm{Mn}$ & p & $2.1+0$ & $5.8+0$ & ORL E & E Johnsont & $1704+$ \\
\hline $55 \mathrm{Mn}$ & p & $3.5+0$ & $6.7+0$ & OSU E & E Dellt & 1705 \\
\hline $55 \mathrm{Mn}$ & D & $4.0+0$ & $5.5+0$ & LRLE & E Albert. & 1706 \\
\hline${ }^{56} \mathrm{Fe}$ & p & $1.5+1$ & $3.3+1$ & HAR E & E Jenkingt & $1902+$ \\
\hline${ }^{56} \mathrm{Fe}$ & p & $1.5+1$ & $3.3+1$ & KFK C & C Muenzel+ & 1903 \\
\hline $56_{\mathrm{Fe}}$ & p & $2.2+1$ & & ORL E & E Cohent & 1904 \\
\hline $56 \mathrm{Fe}$ & $p$ & $1.0+1$ & $7.0+1$ & BNL T & T Olyadeenam. & 1905 \\
\hline${ }^{58} \mathrm{Co}$ & p & NDG & & CAL $T$ & T Woosleyt & 1989 \\
\hline${ }^{59} \mathrm{Co}$ & $p$ & $2.4+1$ & $7.6+1$ & LVN E & E Hoasbroeht & $2036+$ \\
\hline${ }^{39} \mathrm{Co}$ & p & $5.9+1$ & & NRL $T$ & T Peterson. & 2032 \\
\hline${ }^{59} \mathrm{Co}$ & p & $5 \cdot 3+1$ & & NRL $T$ & T Peterson. & 2033 \\
\hline${ }^{59} \mathrm{Co}_{0}$ & p & $5.2+1$ & & NRL T & T Pelerson. & 2034 \\
\hline${ }^{59} \mathrm{Co}$ & p & $6.5+1$ & & NRL $T$ & T Potergon. & 2035 \\
\hline${ }^{59} \mathrm{Co}$. & p & $2.8+1$ & & NRL & T Pelerson. & 2038 \\
\hline $58_{\mathrm{Ni}}$ & p & TR & $8.0+1$ & NRL T & T Poterson. & 2267 \\
\hline $5 \theta_{N I}$ & p & 8.0+1 & $1.6+2$ & INUE & E Sadlert & 2317 \\
\hline $5 \theta_{\mathrm{Ni}}$ & p & $8.0+1$ & $1.4+2$ & MIL T & T Godiolit+ & 2318 \\
\hline${ }^{50} \mathrm{Ni}$ & p & $2.0+2$ & & ANL $E$ & E Schiffer. & 2319 \\
\hline $60_{\mathrm{NI}}$ & p & $1.4+2$ & & INUE & E. Sodlert & 2361 \\
\hline $60 \mathrm{NI}$ & p & $1.4+2$ & & MIL T & T Gadiolit & 2362 \\
\hline
\end{tabular}

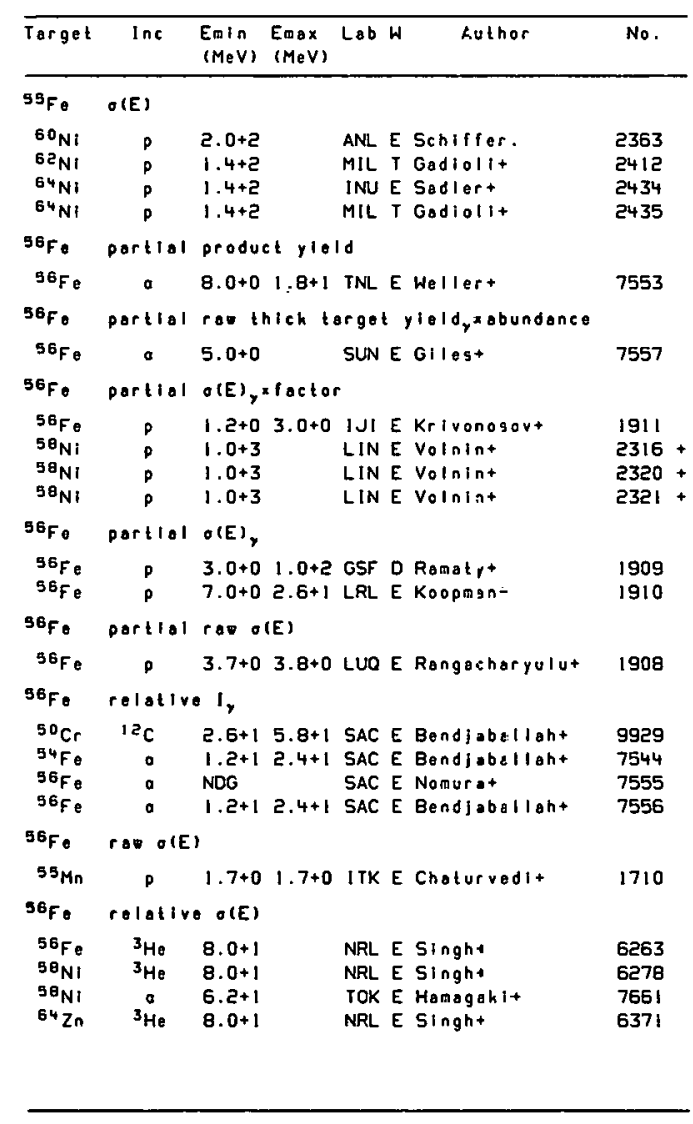




\begin{tabular}{|c|c|c|c|c|c|c|}
\hline Target & $\operatorname{lnc}$ & $\begin{array}{l}\text { Eain } \\
(\mathrm{MeV})\end{array}$ & $\begin{array}{l}E \max \\
(\mathrm{MeV})\end{array}$ & Lab & Author & No. \\
\hline${ }^{56} \mathrm{Fe}$ & \multicolumn{6}{|c|}{ O(E) x factor } \\
\hline $\begin{array}{l}{ }^{52} \mathrm{Cr} \\
56 \mathrm{Fe} \\
59 \mathrm{Co}\end{array}$ & $\begin{array}{r}14 \mathrm{~N} \\
0 \\
p\end{array}$ & $\begin{array}{l}7.1+1 \\
7.0+0 \\
7.0+0\end{array}$ & $\begin{array}{l}1.0+2 \\
1.2+1 \\
1.2+1\end{array}$ & $\begin{array}{l}\text { LYO } \\
\text { COL } \\
\text { COL }\end{array}$ & $\begin{array}{l}\text { T Billereyt } \\
\text { R Miller. } \\
\text { R Miller. }\end{array}$ & $\begin{array}{r}10398 \\
7554 \\
2043\end{array}$ \\
\hline${ }^{56} \mathrm{Fe}$ & \multicolumn{6}{|l|}{$\theta(E)$} \\
\hline${ }^{52} \mathrm{Cr}$ & a & NDG & & CAL & T Woosleyt & 7486 \\
\hline${ }^{53} \mathrm{Cr}$ & a & TR & $1.3+1$ & CAL & I Woosleyt & 7491 \\
\hline $53 \mathrm{Mn}$ & $a$ & NDG & & CAL & I Woosleyt & 7510 \\
\hline $55 \mathrm{Mn}$ & p & NDG & & CAL & T Woosleyt & 1711 \\
\hline${ }^{56} \mathrm{Mn}$ & p & NDG & & CAL & T Woosleyt & 1713 \\
\hline${ }^{56} \mathrm{Fe}$ & p & $7.0+0$ & $2.6+1$ & LRL & E Koopmant & 1907 \\
\hline${ }^{56} \mathrm{Fe}$ & $p$ & $7.0+0$ & $2.6+1$ & LRL & E Koopmant & 1912 \\
\hline${ }^{59} \mathrm{Co}$ & $p$ & $4.7+1$ & & NRL & T Peterson. & 2039 \\
\hline${ }^{59} \mathrm{C}_{0}$ & p & $3.8+1$ & & NRL & TPeterson. & 2040 \\
\hline${ }^{59} \mathrm{Co}_{0}$ & $p$ & $5.1+1$ & & NRL & T Peterson. & 2041 \\
\hline${ }^{59} \mathrm{Co}$ & $p$ & $3.7+1$ & & NRL & T Peterson. & 2042 \\
\hline${ }^{59} \mathrm{Co}$ & $p$ & NDG & & CAL & T Woosleyt & 2044 \\
\hline${ }^{59} \mathrm{Co}$ & $p$ & $1.7+1$ & & NRL & T Peterson. & 2045 \\
\hline${ }^{5 \theta_{N I}}$ & $p$ & TR & $8.0+1$ & NRL & T Peterson. & 2268 \\
\hline $5 \mathbf{\theta}_{\mathrm{NI}}$ & p & $8.0+1$ & $1.6+2$ & INU & E Sadlert & 2317 \\
\hline $5 \theta_{\mathrm{Ni}}$ & p & $8.0+1$ & $1.4+2$ & MIL & T Gadiolit & 2318 \\
\hline $5 \theta^{N 1}$ & $p$ & $2.0+2$ & & ANL & E Sehiffer. & 2319 \\
\hline $60_{\mathrm{NI}}$ & p & $1.4+2$ & & INU & E Sadlert & 2361 \\
\hline${ }^{60} \mathrm{NI}$ & p & $1.4+2$ & & MIL & T Gadiolit & 2362 \\
\hline $60_{N I}$ & $p$ & $2.0+2$ & & ANL & E Sehiffer. & 2363 \\
\hline${ }^{64} \mathrm{NI}$ & $p$ & $1.4+2$ & & INU & E Sadlert & 2434 \\
\hline $57 \mathrm{Fo}$ & \multicolumn{6}{|c|}{ partial rov thick target yieldyabundance } \\
\hline${ }^{57} \mathrm{Fe}$ & $\circ$ & $5.0+0$ & & SUN E & E Gilest & 7569 \\
\hline $57 \mathrm{Fo}$ & \multicolumn{6}{|c|}{ partial o(E), afactor } \\
\hline${ }^{57} \mathrm{Fe}$ & $p$ & $1.2+0$ & $3.0+0$ & $|u|$ & E Krivonosort & 1951 \\
\hline $57_{\mathrm{Fe}}$ & \multicolumn{6}{|c|}{ relotive o(E) } \\
\hline $\begin{array}{l}{ }^{4}{ }^{8} \mathrm{Ca} \\
5 \mathrm{Ca}^{5} \\
50_{\mathrm{NI}} \\
{ }^{6} \mathrm{CO}\end{array}$ & $\begin{array}{l}{ }^{13} \mathrm{C} \\
{ }^{3} \mathrm{He} \\
{ }^{3} \mathrm{He} \\
{ }^{3} \mathrm{He}\end{array}$ & $\begin{array}{l}E .5+1 \\
B .0+1 \\
8.0+1 \\
8.0+1\end{array}$ & $6.5+1$ & $\begin{array}{l}\text { BNL } \\
\text { NRL E } \\
\text { NRL E } \\
\text { NRL }\end{array}$ & $\begin{array}{l}\text { E Nathant } \\
\text { E Singht } \\
\text { E Singht } \\
\text { E Singht }\end{array}$ & $\begin{array}{r}10201 \\
6263 \\
6278 \\
6371\end{array}$ \\
\hline
\end{tabular}

\begin{tabular}{|c|c|c|c|c|c|c|}
\hline Target & $\ln e$ & $\begin{array}{l}E \min \\
(M e V)\end{array}$ & $\begin{array}{l}\text { Emax } \\
(\operatorname{MeV})\end{array}$ & Lab & Author & No. \\
\hline${ }^{57} \mathrm{Fe}$ & $\sigma(E)$ & & & & & \\
\hline $\begin{array}{l}53 \mathrm{Cr} \\
{ }^{54} \mathrm{Cr} \\
58 \mathrm{Mn} \\
57 \mathrm{Mn} \\
59 \mathrm{Co} \\
{ }^{59} \mathrm{Co} \\
60 \mathrm{Co} \\
60 \mathrm{Ni} \\
60 \mathrm{Ni} \\
62_{\mathrm{Ni}} \\
64 \mathrm{Ni} \\
64 \mathrm{Ni}\end{array}$ & $\begin{array}{l}a \\
a \\
p \\
p \\
p \\
p \\
p \\
p \\
p \\
p \\
p \\
p\end{array}$ & $\begin{array}{l}\text { NDG } \\
\text { TR } \\
\text { NDG } \\
\text { NDG } \\
3.9+1 \\
3.3+1 \\
\text { NDG } \\
1.4+2 \\
1.4+2 \\
1.4+2 \\
1.4+2 \\
1.4+2\end{array}$ & $1.2+1$ & $\begin{array}{l}\text { CAL } \\
\text { CAL } \\
\text { CAL } \\
\text { CAL } \\
\text { NRL } \\
\text { NRL } \\
\text { CAL } \\
\text { INU } \\
\text { MIL } \\
\text { MIL } \\
\text { INU } \\
\text { MIL }\end{array}$ & $\begin{array}{l}\text { T Woosleyt } \\
\text { T Woosleyt } \\
\text { T Woosleyt } \\
\text { T Woosleyt } \\
\text { T Pelerson, } \\
\text { T Peterson. } \\
\text { T Woosleyt } \\
\text { E Sadiert } \\
\text { I Gadiolit } \\
\text { T Gadiolit } \\
\text { E Sadiert } \\
\text { T Gadiolit }\end{array}$ & $\begin{array}{l}7492 \\
7495 \\
1714 \\
1716 \\
2046 \\
2047 \\
2116 \\
2361 \\
2362 \\
2412 \\
2434 \\
2435\end{array}$ \\
\hline${ }^{50} \mathrm{Fe}$ & partial & produc & cl y lel & 18 & & \\
\hline $\begin{array}{l}{ }^{58} \mathrm{Fe} \\
{ }^{58} \mathrm{Fe}\end{array}$ & $\begin{array}{c}a \\
\text { port|al }\end{array}$ & $\begin{array}{l}8.0+0 \\
r a 0 t h\end{array}$ & $\begin{array}{l}1.8+1 \\
\text { hick to }\end{array}$ & TNL & $\begin{array}{l}\text { E Wellert } \\
\text { yleld, abundance }\end{array}$ & 7574 \\
\hline${ }^{50} \mathrm{Fe}$ & $a$ & $5.0+0$ & & SUN E & E Gilest & 7575 \\
\hline${ }^{58} \mathrm{Fe}$ & portial & $O(E)_{Y} \times$ & x factor & & & \\
\hline $\begin{array}{l}{ }^{58} \mathrm{Fe}_{\mathrm{e}} \\
{ }^{58} \mathrm{Fe}\end{array}$ & $t^{p}$ & $1.6+0$ & $3.0+0$ & $|\lambda|$ & E Krivonasovt & 1965 \\
\hline $\begin{array}{l}{ }^{55} \mathrm{Mn} \\
{ }^{50} \mathrm{Fe}\end{array}$ & $\begin{array}{c}0 \\
\text { relotive }\end{array}$ & $\begin{array}{l}1.2+1 \\
-O(E)\end{array}$ & $2.4+1$ & SAC & E Cavallarot & 7515 \\
\hline $\begin{array}{l}{ }^{48} \mathrm{Ca} \\
{ }^{64} \mathrm{Zn}\end{array}$ & ${ }^{13} \mathrm{C}$ & $\begin{array}{l}2.5+1 \\
8.0+1\end{array}$ & $6.5+1$ & $\begin{array}{l}\text { BNL } \\
\text { NRL }\end{array}$ & $\begin{array}{l}\text { E Nalhant } \\
\text { E Singht }\end{array}$ & $\begin{array}{r}10201 \\
6371\end{array}$ \\
\hline${ }^{50} \mathrm{Fe}$ & $\sigma(E)$ & & & & & \\
\hline $\begin{array}{l}{ }^{54} \mathrm{Cr} \\
{ }_{55} \mathrm{Cr}\end{array}$ & $\ddot{a}$ & $\begin{array}{l}\text { NDG } \\
\text { NDG }\end{array}$ & & $\begin{array}{l}\text { CAL } \\
\text { CAL }\end{array}$ & $\begin{array}{l}\text { T Woosleyt } \\
\text { T Woosleyt }\end{array}$ & $\begin{array}{l}7496 \\
7497\end{array}$ \\
\hline $55 \mathrm{Mn}$ & $a$ & TR & $1.2+1$ & CAL & T Woosleyt & 7516 \\
\hline $\begin{array}{l}57 \mathrm{Mn} \\
5 \theta_{\mathrm{Mn}}\end{array}$ & $p$ & $\begin{array}{l}\text { NDG } \\
\text { NDG }\end{array}$ & & CAL & $\begin{array}{l}\text { T Woosleyt } \\
\text { T Woosleyt }\end{array}$ & $\begin{array}{l}1717 \\
1719\end{array}$ \\
\hline${ }^{59} \mathrm{Co}$ & p & $2.6+1$ & & NRL & T Peterson. & 2048 \\
\hline 6 I $C_{0}$ & $p$ & NDG & & CAL 1 & T Woosleyt & 2119 \\
\hline${ }^{60} \mathrm{Ni}$ & p & $1.4+2$ & & INU E & E Sadtert & 2361 \\
\hline $\begin{array}{l}60^{6 \mathrm{Ni}} \\
64 \mathrm{Ni}\end{array}$ & p & $1.4+2$ & & MIL & T Gadiolit & 2362 \\
\hline${ }^{64} \mathrm{NI}$ & $p$ & $1.4+2$ & & INU E & E Sadlert & 2434 \\
\hline
\end{tabular}




\begin{tabular}{lllll}
\hline Target Inc & $\begin{array}{l}\text { Emin } \\
\text { (MeV) }\end{array}$ & Emax Lab & Lab $W$ & Author
\end{tabular}

${ }^{59} \mathrm{Fe}$ cumulative spallation o(E)

$C_{u} P \quad 2.0+25.9+2$ LAS E Orlht 2577

${ }^{59} \mathrm{Fe}$ cumulative $O(E)$

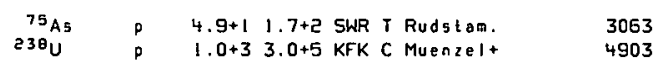

${ }^{59} \mathrm{FO}_{\mathrm{O}} \mathrm{O}(\mathrm{E})$

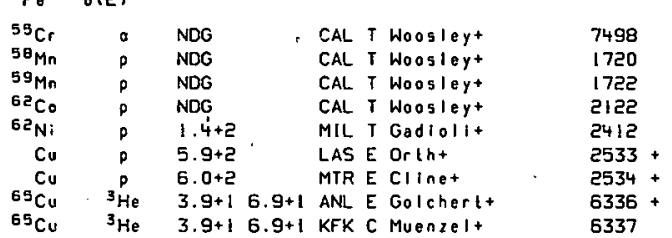

\begin{tabular}{|c|c|c|c|c|c|}
\hline${ }^{56} \mathrm{Cr}$ & a & NDG & CAL $T$ & Woosleyt & 7499 \\
\hline${ }^{57} \mathrm{Cr}$ & 。 & NDG & CAL T & Woosleyt & 7500 \\
\hline $59 \mathrm{Mn}$ & p & NDG & CAL T & I Woosleyt & 1723 \\
\hline${ }^{60} \mathrm{Mn}$ & p & NDG & CAL $T$ & Woosleyt & 1725 \\
\hline${ }^{63} \mathrm{Co}$ & p & NDG & CAL $T$ & Woosleyt & 2125 \\
\hline${ }^{61} \mathrm{Fe}$ & $\theta(E)$ & & & & \\
\hline${ }^{57} \mathrm{Cr}$ & a & NDG & CAL $T$ & Woosleyt & 7501 \\
\hline${ }^{5 \theta} \mathrm{Cr}$ & 。 & NDG & CAL T & I Woosleyt & 7502 \\
\hline${ }^{60} \mathrm{Mn}$ & $p$ & NDG & CAL T & Woosley+ & 1726 \\
\hline $51 \mathrm{Mn}$ & p & NDG & CAL T & Woosley+ & 1728 \\
\hline${ }^{54} \mathrm{Co}$ & $\rho$ & NDG & CAL $\mathrm{T}$ & r Woosieyt & 2128 \\
\hline${ }^{62} \mathrm{Fe}$ & $O(E)$ & & & & \\
\hline${ }^{58} \mathrm{Cr}$ & a & NDG & CAL $T$ & r Woosleyt & 7503 \\
\hline $61 \mathrm{Mn}$ & $\mathrm{p}$ & NOG & CAL $T$ & I Woosleyt & 1729 \\
\hline${ }^{65} \mathrm{C}_{0}$ & p & NDG & CAL $T$ & Woosleyt & 2131 \\
\hline
\end{tabular}

$Z=27$ Independent overoge kinetic energy(E;E',

$197 \mathrm{Au} \quad{ }^{40} \mathrm{Ar} \quad 2.2+2$ DUB E Artuhht

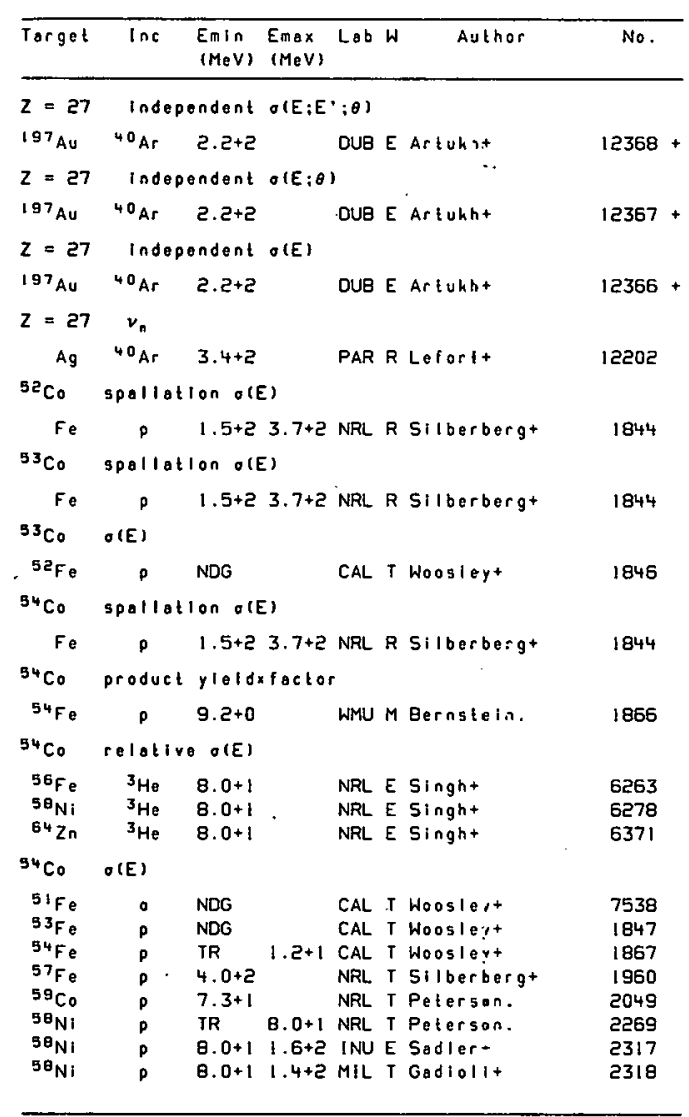




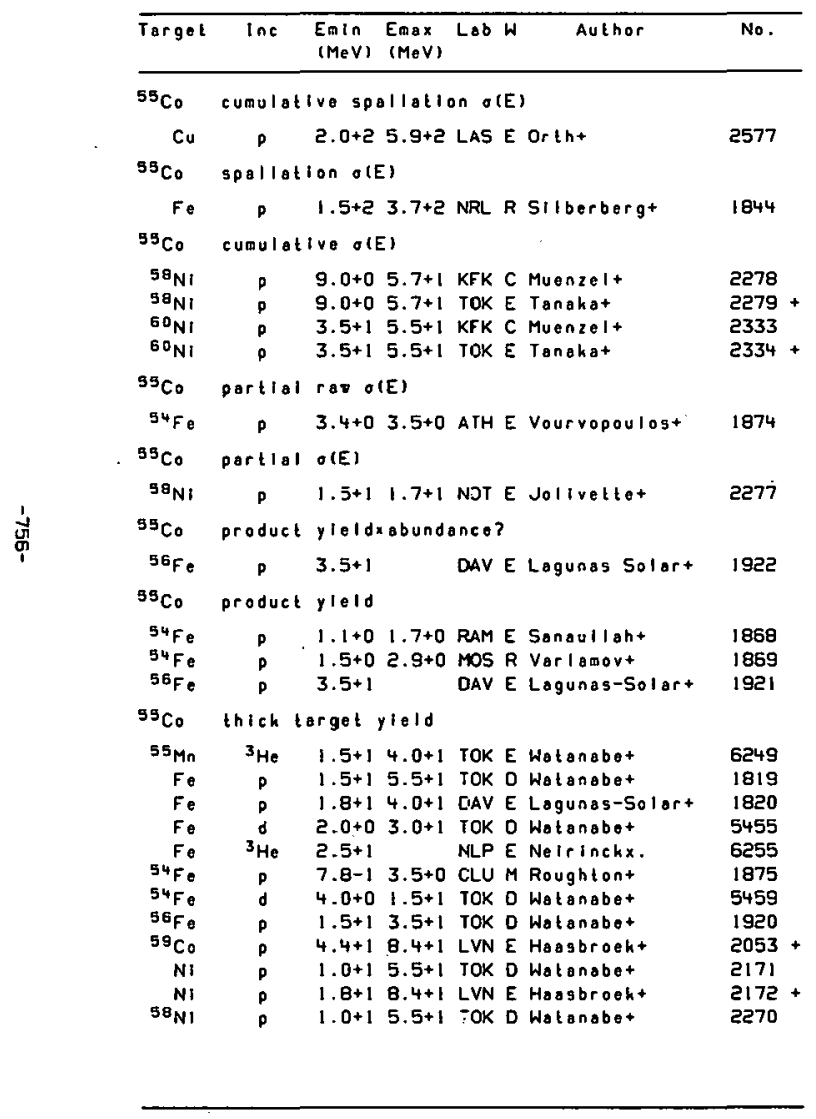

\begin{tabular}{|c|c|c|c|c|c|c|}
\hline$\overline{\text { Target }}$ & $\ln c$ & $\begin{array}{l}\text { Emin } \\
(M e V)\end{array}$ & $\begin{array}{l}\text { Emax } \\
(M e v)\end{array}$ & Leb & Author & No. \\
\hline${ }^{59} \mathrm{Co}_{0}$ & \multicolumn{6}{|c|}{ relative $O(E)$} \\
\hline $\begin{array}{l}56 \mathrm{~F}_{\mathrm{e}} \\
58 \mathrm{NI} \\
64 \mathrm{Zn}\end{array}$ & $\begin{array}{l}{ }^{3} \mathrm{He} \\
3^{3} \mathrm{He} \\
{ }^{3} \mathrm{He}\end{array}$ & $\begin{array}{l}8.0+1 \\
8 \cdot 0+1 \\
8.0+1\end{array}$ & & $\begin{array}{l}\text { NRL } \\
\text { NRL } \\
\text { NRL }\end{array}$ & $\begin{array}{l}\text { E singht } \\
\text { E singht } \\
\text { E singht }\end{array}$ & $\begin{array}{l}6263 \\
6278 \\
6371\end{array}$ \\
\hline${ }^{55} \mathrm{C}_{0}$ & \multicolumn{6}{|c|}{$\sigma(E)$ a factor } \\
\hline $\begin{aligned} F_{e} \\
55_{\mathrm{Fe}}\end{aligned}$ & $\begin{array}{l}p \\
p\end{array}$ & $\begin{array}{l}1.5+1 \\
2.2+1\end{array}$ & $4.0+1$ & $\begin{array}{l}\text { DAV } \\
\text { ORL }\end{array}$ & $\begin{array}{l}\text { E Lagunas-Solart } \\
\text { E Cohent }\end{array}$ & $\begin{array}{l}1818 \\
1919\end{array}$ \\
\hline${ }^{55} \mathrm{Co}$ & $\sigma(E)$ & . & & & & \\
\hline${ }^{40} \mathrm{Ca}$ & 180 & 9.9+1 & & $J C L$ & E Morinag & 11031 \\
\hline $51 M n$ & & NDG & & CAL & T Woosley+ & 7507 \\
\hline $55 \mathrm{Mn}$ & ${ }^{3} \mathrm{He}$ & 8. $0+0$ & $4.0+1$ & TOK & E Watanabet & 6248 \\
\hline $55 \mathrm{Mn}$ & 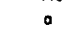 & +1 & $4.0+1$ & TOK & E Watanabot & 7517 \\
\hline $\mathrm{Fe}_{\mathrm{e}}$ & p & $1.3+i$ & $6.0+1$ & KFK & C Muenzel- & 1821 \\
\hline$F_{e}$ & p & $1.3+1$ & $4.4+1$ & KLN & M Michelt & 1822 \\
\hline $\mathrm{Fe}_{\mathrm{e}}$ & p & $1.3+1$ & $6 \cdot 0+1$ & ORL & E Willianst & 1823 \\
\hline$F_{\mathbf{e}}$ & p & $5.9+2$ & & LAS & E Ortht & 1824 \\
\hline $52_{\mathrm{Fe}}$ & : & NDG & & CAL & T Woosleyt & 7540 \\
\hline${ }^{54} \mathrm{Fe}$ & p & NDG & & CAL & T Woosloyt & 1870 \\
\hline${ }^{54} \mathrm{Fe}$ & $p$ & $7.8-1$ & $3.5+0$ & CLU & E Roughtont & 1871 \\
\hline${ }^{54} \mathrm{Fe}$ & p & $1.1+1$ & & KFK & C Muenzel+ & 1872 \\
\hline${ }^{54} \mathrm{Fe}$ & 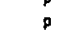 & $1.1+i$ & & ORL & E Cohen. & 1873 \\
\hline${ }^{54} \mathrm{Fe}$ & ${ }^{6} \mathrm{Li}$ & $9.8+0$ & $9.4+1$ & INU & E Jagtrzebskit & 9185 \\
\hline${ }^{56} \mathrm{Fe}$ & $p$ & $1.2+1$ & $3.0+2$ & GSF & O Ramaty+ & 1913 \\
\hline${ }^{56} \mathrm{Fe}_{\mathrm{e}}$ & p & $1.6+1$ & $4.0+1$ & DAV & $E$ Lagunas-Solar & 1914 \\
\hline${ }^{56} \mathrm{Fe}$ & p & $1.6+1$ & $3.9+1$ & HAR & E Jenkinst & 1915 \\
\hline${ }^{36} \mathrm{Fe}$ & p & $1.6+1$ & $3.9+1$ & KFK & C Muenzelt & 1918 \\
\hline${ }^{56} \mathrm{Fo}$ & p & $1.8+1$ & $5.0+1$ & KLN & M Michel+ & 1917 \\
\hline${ }^{56} \mathrm{Fe}$ & p & $2.2+1$ & & ORL & E Cotient & 1918 \\
\hline${ }^{57} \mathrm{Fe}$ & p & $4.0+2$ & & NRL & T Silberbergt & 1960 \\
\hline${ }^{50} \mathrm{Fe}$ & p & $4.0+2$ & & NRL & T Silberbergt & 1970 \\
\hline${ }^{59} \mathrm{Co}$ & p & $4.4+1$ & $8.4+1$ & LVN & E Hassbroekt & 2052 \\
\hline${ }^{59} \mathrm{Co}$ & p & $5.8+1$ & & NRL & $T$ Paterson. & 2050 \\
\hline${ }^{59} \mathrm{Co}_{0}$ & p & $4.9+1$ & & NPL & T Peterson. & 2051 \\
\hline${ }^{59} \mathrm{Co}$ & p & $5.8+1$ & & NRL & T Peterson. & 2054 \\
\hline $\mathrm{Ni}$ & p & $1.2+1$ & $4.4+1$ & KLN & E Mlchelt & 2168 \\
\hline NI & p & $1.8+1$ & $8.4+1$ & LVN & E Hasbroel & 2169 \\
\hline $\mathrm{Ni}$ & p & $6.0+2$ & & MTR & E Clinet & 2170 \\
\hline Ni & d & $2.5+1$ & & IKO & $E$ Brinkmant & 5465 \\
\hline $\mathrm{Ni}$ & p & $4.0+1$ & $5.5+1$ & NRL & T Peterson. & 2271 \\
\hline
\end{tabular}




\begin{tabular}{|c|c|c|c|c|c|c|}
\hline Target & $\ln 5$ & $\begin{array}{l}E \operatorname{lin} \\
(\mathrm{MeV})\end{array}$ & $\begin{array}{l}\text { Emax } \\
\text { (MeV) }\end{array}$ & Lob $W$ & Author & No. \\
\hline${ }^{55} \mathrm{C}_{0}$ & $O(E)$ & & & & & \\
\hline $\begin{array}{l}5 \theta_{\mathrm{Ni}} \\
5 \theta_{\mathrm{Ni}} \\
5 \theta_{\mathrm{Ni}} \\
5 \theta_{\mathrm{Ni}} \\
5 \theta_{\mathrm{Ni}} \\
5 \theta_{\mathrm{Ni}} \\
60_{\mathrm{Ni}} \\
60_{\mathrm{Ni}} \\
62_{\mathrm{Ni}} \\
\mathrm{Cu} \\
C_{U}\end{array}$ & $\begin{array}{l}p \\
p \\
p \\
p \\
p \\
p \\
p \\
p \\
p \\
p \\
p\end{array}$ & $\begin{array}{l}T R \\
1.0+0 \\
7.4+0 \\
7.4+0 \\
3.7+1 \\
8.0+1 \\
1.4+2 \\
1.4+2 \\
1.4+2 \\
5.9+2 \\
6.0+2\end{array}$ & $\begin{array}{l}8.0+1 \\
1.4+1 \\
5.6+1 \\
1.8+1 \\
1.4+2\end{array}$ & $\begin{array}{ll}\text { NRL } & \text { T } \\
\text { CAL } & T \\
\text { KFK } & C \\
\text { PIN } & \text { E } \\
\text { MIL } & \text { E } \\
\text { MIL } & T \\
\text { INU } & \text { E } \\
\text { MIL } & \text { T } \\
\text { MIL } & \text { T } \\
\text { LAS } & \text { E } \\
\text { MTR E }\end{array}$ & 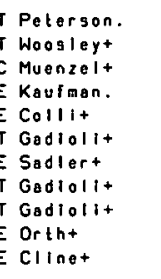 & $\begin{array}{l}2272 \\
2273 \\
2274 \\
2275+ \\
2275 \\
2318 \\
2361 \\
2362 \\
2412 \\
2535+ \\
2536+\end{array}$ \\
\hline $\begin{array}{l}{ }^{56} \mathrm{Co} \\
\mathrm{Cu}_{0}\end{array}$ & $\begin{array}{c}\text { cumulo } \\
p\end{array}$ & $\begin{array}{l}\text { ive spa } \\
2.0+2\end{array}$ & $\begin{array}{l}5.9+2 \\
0.110 t i 0\end{array}$ & $\begin{array}{l}\text { ON OIE } \\
\text { LAS E }\end{array}$ & $=$ Ortht & 2577 \\
\hline $\begin{array}{l}{ }^{56} \mathrm{Co}_{0} \\
\mathrm{Fe} \\
{ }^{56} \mathrm{C}_{0}\end{array}$ & $\begin{array}{c}\text { spalla } \\
\text { cumula }\end{array}$ & $\begin{array}{l}\text { lon ole } \\
1.5+5 \\
\text { ive? oll }\end{array}$ & $\begin{array}{l}\text { E) } \\
3.7+2 \\
\text { (E) }\end{array}$ & NRL $R$ & Siltberberg+ & 1844 \\
\hline $\begin{array}{l}\mathrm{Fe} \\
{ }^{86} \mathrm{Co}\end{array}$ & $\begin{array}{c}P \\
\text { cunula }\end{array}$ & $\begin{array}{l}6.0+2 \\
\text { ive thi }\end{array}$ & ick tar & $\begin{array}{l}\text { MTR E } \\
\text { rget y }\end{array}$ & $\begin{array}{l}=\text { clinet } \\
\text { yield }\end{array}$ & $1828+$ \\
\hline $\begin{array}{r}{ }^{59} \mathrm{Co} \\
\mathrm{Ni} \\
\mathrm{Ni}\end{array}$ & $\begin{array}{l}p \\
p \\
p\end{array}$ & $\begin{array}{l}4.4+1 \\
2.5+1 \\
1.0+2\end{array}$ & $\begin{array}{l}8.4+1 \\
8.5+1\end{array}$ & $\begin{array}{l}\text { LVN E } \\
\text { LVN E } \\
\text { MRY E }\end{array}$ & $\begin{array}{l}\text { Hasbroekt } \\
\text { Haasbroekt } \\
\text { Haosbroekt }\end{array}$ & $\begin{array}{l}2061+ \\
2176+ \\
2177+\end{array}$ \\
\hline${ }^{55} \mathrm{Co}$ & $O(E)$ & . & . & & & \\
\hline 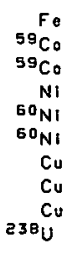 & $\begin{array}{l}\text { PLI } \\
p \\
p \\
p \\
p \\
p \\
p \\
p \\
p \\
p\end{array}$ & $\begin{array}{l}1.0+1 \\
3.4+1 \\
4.4+1 \\
2.5+1 \\
2.7+1 \\
2.7+1 \\
5 \cdot 2+1 \\
5.2+1 \\
5.9+2 \\
1.0+3\end{array}$ & $\begin{array}{l}2.4+1 \\
5 \cdot 0+1 \\
8 \cdot 4+1 \\
8 \cdot 5+1 \\
5 \cdot 5+1 \\
5 \cdot 5+1 \\
5 \cdot 8+1 \\
5 \cdot 8+1 \\
\\
3 \cdot 0+5\end{array}$ & $\begin{array}{l}\text { HAR E } \\
\text { KLN M } \\
\text { LVN E } \\
\text { LVN E } \\
\text { KFK C } \\
\text { TOK E } \\
\text { KFK C } \\
\text { ORL E } \\
\text { LAS E } \\
\text { KFK C }\end{array}$ & $\begin{array}{l}\text { Conlont } \\
\text { M Mlchelt } \\
\text { E Hasbroekt } \\
\text { Haasbraekt } \\
\text { C Muenzel+ } \\
\text { E Tonahat } \\
\text { C Muenzelt } \\
\text { E Willianst } \\
\text { Ortht } \\
\text { C Muenzelt }\end{array}$ & $\begin{array}{l}9437 \\
2058 \\
2050+ \\
2175+ \\
2335 \\
2336+ \\
2538 \\
2539+ \\
2540+ \\
4903\end{array}$ \\
\hline${ }^{56} \mathrm{Co}$ & rolotis & o thick & $k \operatorname{targe}$ & et $y 10$ & 18 & \\
\hline${ }^{55} \mathrm{Mn}$ & ${ }^{3} \mathrm{He}$ & $3.7+1$ & $4.0+1$ & TOK E & Watonabe+ & 6252 \\
\hline
\end{tabular}

\begin{tabular}{|c|c|c|c|c|c|c|}
\hline Target & $\ln c$ & $\begin{array}{l}\text { Enin } \\
\text { (MeV) }\end{array}$ & $\begin{array}{l}E m B x \\
(M e V)\end{array}$ & Lab & Autior & No. \\
\hline${ }^{56} \mathrm{Co}$ & \multicolumn{6}{|c|}{ relative thlck target yleld } \\
\hline $\begin{array}{r}\mathrm{Fe} \\
{ }^{5{ }^{8}} \mathrm{Co}\end{array}$ & $\begin{array}{c}\text { P } \\
\text { thlck }\end{array}$ & $\begin{array}{c}\text { TR } \\
\text { target }\end{array}$ & $\begin{array}{l}6.3+0 \\
\text { yold }\end{array}$ & TNL & E Nillert & 1836 \\
\hline 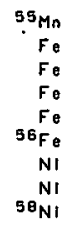 & $\begin{array}{c}{ }_{3}^{3} \mathrm{He} \\
p \\
p \\
0 \\
{ }^{3} \mathrm{He} \\
p \\
p \\
0 \\
p\end{array}$ & $\begin{array}{l}9 \cdot 0+0 \\
9 \cdot 0+0 \\
1 \cdot 7+1 \\
9 \cdot 0+0 \\
2 \cdot 5+1 \\
5 \cdot 0+0 \\
2 \cdot 8+1 \\
1.0+1 \\
3 \cdot 0+1\end{array}$ & $\begin{array}{l}4 \cdot 0+1 \\
5 \cdot 5+1 \\
4 \cdot 0+1 \\
3 \cdot 5+1 \\
5 \cdot 5+1 \\
4 \cdot 0+1 \\
5 \cdot 5+1\end{array}$ & $\begin{array}{l}\text { TOK } \\
\text { TOK } \\
\text { DAV } \\
\text { TOK } \\
\text { NLP } \\
\text { TOK } \\
\text { TOK } \\
\text { TOK } \\
\text { TOK }\end{array}$ & $\begin{array}{l}\text { E Watanabet } \\
\text { O Watanabet } \\
\text { E Logunas-Solart } \\
\text { O Watanabet } \\
\text { E Neirinchx. } \\
\text { D Watanabet } \\
\text { D Watanabet } \\
\text { E Muramatisut } \\
\text { D Watanabet }\end{array}$ & $\begin{array}{l}6251 \\
1826 \\
1827 \\
5456 \\
6256 \\
1933 \\
2178 \\
7638 \\
2280\end{array}$ \\
\hline${ }^{56} \mathrm{Co}$ & \multicolumn{6}{|c|}{ rolative o(E) } \\
\hline $\begin{array}{l}\mathrm{Fe} \\
56 \mathrm{Fe}^{2} \\
58_{\mathrm{Ni}} \\
58_{\mathrm{Ni}} \\
6{ }^{4} \mathrm{Zn}\end{array}$ & $\begin{array}{l}{ }_{3}^{p} \\
{ }^{3} \mathrm{He} \\
{ }^{3} \mathrm{He} \\
{ }^{0} \\
{ }^{0} \mathrm{He}\end{array}$ & $\begin{array}{l}\text { TR } \\
8 \cdot 0+1 \\
8 \cdot 0+1 \\
6 \cdot 2+1 \\
8 \cdot 0+1\end{array}$ & $6.3+0$ & $\begin{array}{l}\text { TNL } \\
\text { NRL } \\
\text { NKL } \\
\text { TOK } \\
\text { NRL }\end{array}$ & $\begin{array}{l}\text { E Nillort } \\
\text { E Singht } \\
\text { E Singht } \\
\text { E Homagakit } \\
\text { E Singht }\end{array}$ & $\begin{array}{l}1835 \\
6263 \\
6278 \\
7662 \\
6371\end{array}$ \\
\hline${ }^{56} \mathrm{Co}_{0}$ & \multicolumn{6}{|c|}{ atelafactor. } \\
\hline $\begin{array}{c}\mathrm{Fe} \\
{ }^{56} \mathrm{Co}_{0}\end{array}$ & \multicolumn{6}{|l|}{$O(E)$} \\
\hline $\begin{array}{l}52 \mathrm{Mn} \\
55 \mathrm{Mn}\end{array}$ & $\mathbf{3}_{\mathrm{He}}^{0}$ & $\begin{array}{l}\text { NDG } \\
8.0+0\end{array}$ & $4.0+1$ & $\begin{array}{l}\text { CAL } \\
\text { TOK }\end{array}$ & $\begin{array}{l}\text { T Woosleyt } \\
\text { E Watanabiet }\end{array}$ & $\begin{array}{l}7509 \\
6250\end{array}$ \\
\hline$F_{0}$ & $p$ & $6.0+0$ & $5.2+1$ & OAV & E Sehoent & 1829 \\
\hline$F_{\theta}$ & $p$ & $7.1+0$ & $6.3+1$ & KFK & C Muenzel't & 1830 \\
\hline$F_{\theta}$ & p & $7.1+0$ & $6.3+1$ & ORL & E Wlllianst & $1831+$ \\
\hline $\mathrm{Fe}_{\mathrm{e}}$ & p & $8.0+0$ & $1.1+1$ & CCP & R Zatolokint & 1832 \\
\hline$F_{\theta}$ & $\mathrm{p}$ & $1.3+1$ & $4.4+1$ & KLN & Michel+ & .1833 \\
\hline$F_{0}$ & p & $5.9+2$ & & LAS & E Ortht & $1834+$ \\
\hline${ }^{53} \mathrm{Fe}$ & 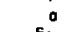 & & & CAL & I Woosleyt & 7542 \\
\hline${ }^{54} \mathrm{Fe}$ & ${ }^{6} \mathrm{LI}$ & $9.8+0$ & $9.4+1$ & INU & E Jastrzobshit & 9185 \\
\hline${ }^{55_{\mathrm{Fo}}}$ & $p$ & NDG & & CAL & $T$ Woosley+ & 1877 \\
\hline $56 \mathrm{Fe}_{0}$ & p & TR & $6.3+0$ & BRL & E Nillert & 1923 \\
\hline${ }^{56} \mathrm{Fe}_{\mathrm{F}}$ & p & TR & $1.2+1$ & CAL & T Woosleyt & 1924 \\
\hline${ }^{36} \mathrm{Fo}$ & p & TR & $6.4+0$ & KTY & E Duncont & 1925 \\
\hline${ }^{56} \mathrm{Fe}_{\mathrm{e}}$ & p & $4.0+0$ & $5.0+1$ & KLN & M Michelt & 1926 \\
\hline${ }^{36} \mathrm{Fe}$ & D & $4.8+0$ & $3.9+1$ & HAR & E Jenklnst & 1927 \\
\hline
\end{tabular}




\begin{tabular}{|c|c|c|c|c|c|c|}
\hline Target & $\operatorname{lnc}$ & $\begin{array}{l}E_{m i n} \\
\left(M_{\theta} V\right)\end{array}$ & $\begin{array}{l}\text { Emox } \\
(\operatorname{MeV})\end{array}$ & Lab & Author & No. \\
\hline \multicolumn{7}{|l|}{${ }^{56} \mathrm{Co}$} \\
\hline $56_{\mathrm{Fe}}$ & p & $4.8+0$ & $3.9+1$ & KFK & C Muenzelt & 1928 \\
\hline $56_{F E}$ & p & $5.0+0$ & $7.0+1$ & BNL. & T Divadecenam. & 1929 \\
\hline $56_{F e}$ & p & $5.0+0$ & $5.0+2$ & GSF & O Ramaly+ & 1930 \\
\hline${ }^{36} \mathrm{Fe}$ & p & $6.0+0$ & $1.4+1$ & TOK & E Tanahat & $1931+$ \\
\hline${ }^{56} \mathrm{Fe}_{\mathrm{e}}$ & $p$ & $1.1+1$ & $4.5+1$ & MIL & E Godiolit & $1932+$ \\
\hline${ }^{57} \mathrm{Fe}$ & p & $4.0+2$ & & NRL & T Silberbergt & 1960 \\
\hline${ }^{50} \mathrm{Fe}$ & p & $4.0+2$ & & NRL & T silberbergt & 1970 \\
\hline${ }^{59} \mathrm{Co}$ & p & $3.8+1$ & $6.3+1$ & DAV & E Schoent & 2059 \\
\hline${ }^{59} \mathrm{CO}$ & 0 & $2.7+1$ & $4.5+1$ & $\mathrm{KLN}$ & Michelt & 2055 \\
\hline${ }^{50} \mathrm{Co}$ & $p$ & $4.5+1$ & & NAL & T Pelerson. & 2056 \\
\hline${ }^{59} \mathrm{Co}$ & p & $3.5+1$ & & NFL & TPelerson. & 2057 \\
\hline${ }^{59} \mathrm{Co}$ & $p$ & $5 \cdot 3+1$ & & NRL & i Peterson. & 2062 \\
\hline${ }^{59} \mathrm{Co}_{0}$ & ${ }^{3} \mathrm{He}$ & $1.8+1$ & $4.0+1$ & TOK & E Murakamit & 6266 \\
\hline Ni & $p$ & $1.2+1$ & $4.4+1$ & $\mathrm{KLN}$ & E Michel+ & $2173+$ \\
\hline $\mathrm{NI}$ & p & $6.0+2$ & & MTR & E CIInet & $2174+$ \\
\hline $\mathrm{Ni}$ & a & $1.0+1$ & $4.0+1$ & TOK & E Muramatsut & 7637 \\
\hline${ }^{98_{\mathrm{NI}}}$ & p & $2.7+i$ & $5.6+1$ & KFK & C Muenzelt & 2281 \\
\hline $58_{\mathrm{NI}}$ & $p$ & $2.7+1$ & $5.6+1$ & TOK & E Tanahat & $2282+$ \\
\hline $58_{\mathrm{NI}}$ & p & & $8.0+1$ & NRL & TPoterson. & 2283 \\
\hline${ }^{58} \mathrm{NI}$ & p & $8.0+1$ & $1.6+2$ & INU & E Sadlert & 2317 \\
\hline $58_{\mathrm{Ni}}$ & $p$ & $8.0+1$ & $1.4+2$ & MIL & T Gadiolit & 2318 \\
\hline $58_{\mathrm{Ni}}$ & 0 & $2.0+2$ & & ANL & E Schiffer. & 2319 \\
\hline $60 \mathrm{NI}$ & $p$ & $1.4+2$ & & INU & E Sodlert & 2361 \\
\hline $60 \mathrm{Ni}$ & p & $1.4+2$ & & MIL & T Gadiolit & 2362 \\
\hline $60_{\mathrm{Ni}}$ & p & $2.0+2$ & & ANL & E Schiffer. & 2363 \\
\hline $62_{\mathrm{Ni}}$ & p & $1.4+2$ & & MIL & T Godiolit & 2412 \\
\hline cu & 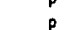 & $6.0+2$ & & MTR & E Clinet & $2537+$ \\
\hline${ }^{63} \mathrm{Cu}$ & ${ }^{3} \mathrm{He}$ & $3.9+1$ & $6.9+1$ & ANL & E Golchertt & $6301+$ \\
\hline${ }^{63} \mathrm{Cu}$ & ${ }^{3} \mathrm{He}$ & $3.9+1$ & $6.9+1$ & $\mathrm{KFK}$ & C Muenzelt & 6302 \\
\hline \multicolumn{7}{|l|}{${ }^{57} \mathrm{Co}$} \\
\hline${ }^{56} \mathrm{Fe}$ & a & $5.1+1$ & & KAZ & E Burtebaevt & 7558 \\
\hline \multicolumn{7}{|c|}{ dent $O(E)$} \\
\hline $238 \mathrm{U}$ & p & $1.0+3$ & $3.0+5$ & KFK & C Muenzel+ & 4906 \\
\hline${ }^{87} \mathrm{Co}$ & cumula & ve sps & .118110 & on or & (E) & \\
\hline
\end{tabular}

Cu p $2.0+25.9+2$ LAS E Ortht

\begin{tabular}{lllll}
\hline Torgel Inc & $\begin{array}{l}\text { Emln } \\
\text { IMeV) }\end{array}$ & $\begin{array}{l}\text { Imax Lab La } \\
\text { IMe }\end{array}$ & Author & No. \\
\hline
\end{tabular}

${ }^{57} \mathrm{Co}$ cumulative thick torget yiald.

${ }^{59} \mathrm{C}_{0} \quad \rho \quad 2.6+18.4+1$ LVN E Hasbroeht $2057+$

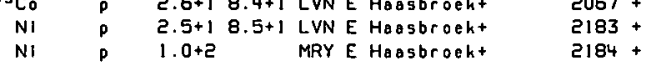

${ }^{27} C_{0} \quad \sigma(E)$

Fe 'Li $1.0+12.4+1$ HAR E Conlont. 9437

$59 \mathrm{Co} \quad 2.2+15.0+1$ KLN M MIchelt 2065

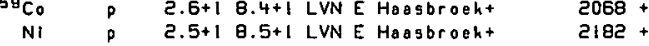

Cu $p$ 5.9+2 LASE Oriht

57 CO partial rav o(E)

${ }^{95} \mathrm{Fe}_{\mathrm{F}} \mathrm{P} 3.7+03.8+0$ LuQ E Rangacharyulut 1934

${ }^{37} \mathrm{Co}$ product ylald

${ }^{56} \mathrm{Fe}_{\mathrm{B}} \mathrm{p} \quad 1.2+0$ 3.0+0 RAM E Rahmant 1938

${ }^{37} \mathrm{CO}$ thick lorgot yleld

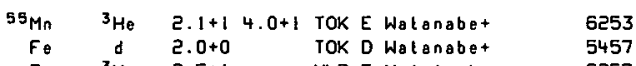

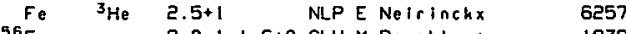

${ }^{56} \mathrm{Fe} P \quad 8.0-11.6+0$ CLU M Roughtont 1939

NI $\quad 1.8+15.5+1$ TOK O Walanabet 2185

$\begin{array}{rlllll}\mathrm{NI} & \mathrm{a} & 1.0+1 & 4.0+1 & \text { TOK E Muramalsut } & 7638 \\ { }_{58} & \mathrm{Ni} & 1.8+1 & 5.5+1 & \text { TOK D Wolanabe+ } & 2284\end{array}$

${ }^{57} \mathrm{CO}_{0}$ overago O(E)

${ }^{57} \mathrm{Fe} P \quad 1.6+01.9+0$ ORL E Johnsont 1958

$37 C_{0}$ relative o(E)

${ }^{56} \mathrm{~F}_{\mathrm{F}}{ }^{3} \mathrm{He} \quad 8.0+1 \quad$ NRL E singht 6263

$\begin{array}{lllll}5 B_{\mathrm{Nl}} & { }^{3} \mathrm{He} & 8.0+1 & \text { NRL E Singht } & 6278 \\ { }^{5 B_{\mathrm{NI}}} & 0 & 6.2+1 & \text { TOK E Hamagahit. } & 7663\end{array}$

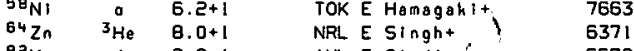

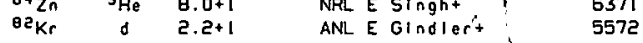

$37 \mathrm{Co}-0(E)=10010 \mathrm{r}$

${ }_{52} \mathrm{Cr} \quad{ }^{14} \mathrm{~N} \quad 7.1+1 \quad 1.0+2$ LYO T BIIIereyt 10399 


\begin{tabular}{|c|c|c|c|c|c|c|}
\hline Target & $\operatorname{lnc}$ & $\begin{array}{l}\text { Emin } \\
(\mathrm{MeV})\end{array}$ & $\begin{array}{l}\text { Emax } \\
(\mathrm{MeV})\end{array}$ & Lab & Author & No. \\
\hline${ }^{57} \mathrm{Co}$ & o(ミ) & & . & & & \\
\hline $53 \mathrm{Mn}$ & a & Nag & & CAL & T Woosleyt & 7511 \\
\hline $\mathrm{Fe}$ & p & $6.0+0$ & $3.0+1$ & DAV & E Schoent & 1837 \\
\hline $\mathrm{Fe}_{\mathrm{e}}$ & p & $1.3+1$ & $4.4+1$ & KLN & Michel+ & 1838 \\
\hline $\mathrm{Fe}$ & $p$ & $1.9+1$ & $4.4+1$ & RIC & E Waltont & 1839 \\
\hline & p & $5.9+2$ & & LAS & E Ortht & $1840+$ \\
\hline${ }^{54} \mathrm{Fe}$ & a & TR & $1.3+1$ & CAL & T Woosleyt & 7545 \\
\hline${ }^{54} \mathrm{Fe}$ & ${ }^{5} L 1$ & $9.8+0$ & $9.4+1$ & INU & E Jastrzebshit & 9185 \\
\hline${ }^{56} \mathrm{Fe}$ & $p$ & NDG & & CAL & T Woosleyt & 1935 \\
\hline${ }^{56} \mathrm{Fe}$ & p & 6.0-1 & $3.0+0$ & $\mid \mathrm{JI}$ & E Krivonosout & 1936 \\
\hline${ }^{56} \mathrm{Fe}$ & p & $8 \cdot 0-1$ & $1.6+0$ & CLU & E Roughiont & 1937 \\
\hline $57 \mathrm{Fe}$ & p & NDG & & KTY & E Sehharant. & 1952 \\
\hline $57 \mathrm{Fe}$ & p & IR & $1.2+1$ & CAL & T Woosleyt & 1953 \\
\hline${ }^{97} \mathrm{Fe}$ & p & TR & $5.5+0$ & ORL & E Johngont & $1954+$ \\
\hline${ }^{57} \mathrm{Fo}$ & p & $1.7+0$ & $1.4+1$ & KFK & C Muenzelt & 1955 \\
\hline $57 \mathrm{Fe}$ & $p$ & $2.0+0$ & $5.0+1$ & KLN & M Michel+ & 1955 \\
\hline${ }^{57} \mathrm{Fe}$ & p & $3.7+0$ & $1.4+1$ & TOK & E Tanakg+ & $1957+$ \\
\hline $57 \mathrm{Fe}$ & p & $4.0+2$ & & NRL & T sllberbergt & 1960 \\
\hline${ }^{58} F_{8}$ & p & $1.4+1$ & $5 \cdot 0+1$ & KLN & Michelt & 1966 \\
\hline $50^{\mathrm{Fe}}$ & D & $4.0+2$ & & NRL & T Silberbergt & 1970 \\
\hline${ }^{59} \mathrm{C}_{0}$ & p & $2.3+1$ & $6 \cdot 3+1$ & OAV & E Schoent & 2065 \\
\hline${ }^{59} \mathrm{Co}$ & p & $1.5+1$ & $4.5+1$ & $\mathrm{KLN}$ & Michel+ & 2063 \\
\hline${ }^{59} \mathrm{C}_{0}$ & p & $3.0+1$ & & NRL & T Peterson. & 2064 \\
\hline${ }^{59} \mathrm{Co}$ & $p$ & $3.5+1$ & & NRL & T Peterson. & 2069 \\
\hline${ }^{59} \mathrm{co}$ & ${ }^{3} \mathrm{He}$ & $1.8+1$ & $4.0+1$ & TOK & E Murahamit & 6266 \\
\hline NI & $p$ & $1.2+1$ & $4.4+1$ & $\mathrm{KLN}$ & E Michel+ & 2.79 \\
\hline $\mathrm{Ni}$ & p & $1.2+1$ & $4.5+1$ & MCG & E & $2180+$ \\
\hline $\mathrm{NI}$ & p & $6.0+2$ & & MTR & E Clinet & $2181+$ \\
\hline $\mathrm{Ni}$ & 0 & $1.0+1$ & $4.0+1$ & TOK & E Muramatsut & 7637 \\
\hline${ }^{5 \theta^{n i}}$ & p & $T R$ & $8.0+1$ & NRL & T Peterson. & 2285 \\
\hline${ }^{5 \theta_{\mathrm{NI}}}$ & p & $1.2+1$ & $2.2+1$ & KFK & C Muenzel+ & 2285 \\
\hline $58_{\mathrm{Ni}}$ & p & $1.2+1$ & $1.9+1$ & PTN & E Kaufmen. & $2287+$ \\
\hline${ }^{58} \mathrm{Ni}$ & p & $1.3+1$ & $5.7+1$ & IOK & E Tanghat & $2288+$ \\
\hline${ }^{58} \mathrm{Ni}$ & p & $2.2+1$ & & ORL & E Cohent & $2289+$ \\
\hline${ }^{58} \mathrm{Ni}$ & p & $8 \cdot 0+1$ & $1.6+2$ & INU & E Sadlert & 2317 \\
\hline${ }^{50} \mathrm{Ni}$ & p & $8 \cdot 0+1$ & $1.4+2$ & MIL & T Gadiolit & 2318 \\
\hline${ }^{5 \theta^{N i}}$ & p & $2.0+2$ & & ANL & E Schiffer. & 2319 \\
\hline${ }^{60} \mathrm{Ni}$ & p & $6.8+0$ & $5.5+1$ & KFK & C Muenzelt & 2340 \\
\hline${ }^{60} \mathrm{Ni}$ & p & $6.8+0$ & $5.5+1$ & TOK & E Tanahat & $2341+$ \\
\hline${ }^{60} \mathrm{Ni}$ & p & $1.0+0$ & $1.3+1$ & CAL & T Woosleyt & 2337 \\
\hline${ }^{60} \mathrm{Ni}$ & p & $7.4+0$ & $1.3+1$ & KFK & C Muenzeit & 2338 \\
\hline
\end{tabular}

\begin{tabular}{|c|c|c|c|c|c|c|}
\hline Target & $\ln c$ & $\begin{array}{l}\text { Emin } \\
(M e V)\end{array}$ & $\begin{array}{l}\text { EmBx } \\
(M e V)\end{array}$ & Lab W & Author & No. \\
\hline${ }^{57} \mathrm{Co}$ & $O(E)$ & & & & & \\
\hline${ }^{50} \mathrm{Ni}$ & p & $7.4+0$ & $1.3+1$ & PIN E & E Koufman. & 2339 \\
\hline${ }^{60} \mathrm{Ni}$ & p & $1.4+2$ & & INU E & E Sadlert & 2361 \\
\hline${ }^{60} \mathrm{Ni}$ & p & $1.4+2$ & & MIL T & T Gadiolit & 2362 \\
\hline $60_{\mathrm{Ni}}$ & p & $2.0+2$ & & ANL $E$ & Eschifler. & 2363 \\
\hline${ }^{62} \mathrm{Ni}$ & p & $1.4+2$ & & MIL T & T Gadiolit & 2412 \\
\hline${ }^{54} \mathrm{Ni}$ & p & $1.4+2$ & & INU E & E Sadlert+ & 2434 \\
\hline${ }^{64} \mathrm{Ni}$ & p & $1.4+2$ & & MIL T & I Gadiolit & 2435 \\
\hline Cu & p & $3.6+1$ & $5.8+1$ & KFK C & C Muenzelt & 2541 \\
\hline $\mathrm{Cu}$ & p & $3.6+1$ & $5.8+1$ & ORL E & E williamst & 2542 \\
\hline & & $6.0+2$ & & MIR E & E Clinet & 2543 \\
\hline${ }^{63} \mathrm{Cu}$ & ${ }^{3} \mathrm{He}$ & $1.2+1$ & $6.9+1$ & ANL $E$ & E. Golchert+ & 6303 \\
\hline${ }^{63} \mathrm{Cu}$ & ${ }^{3} \mathrm{He}$ & $1.2+1$ & $6.9+1$ & KFK C & C Muenzelt & $630 y$ \\
\hline
\end{tabular}

${ }^{8} \mathrm{CO}$ including metastable? O(E)

${ }^{59} \mathrm{Co} P$ P $2.2+1 \quad$ KFK C Muenzelt 2071

${ }^{59} \mathrm{CO} P$ P $2.2+1$ ORL E Cohent

$60 \mathrm{Ni} P 2.7+15.7+1$ KFK C Muenzel+ 2342

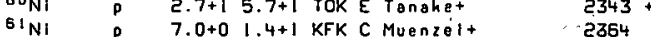

$5 . \mathrm{Ni}$ P $7.0+01.4+1$ TOK E Tanake+

${ }^{59} \mathrm{Co}_{0}$ indegendent including melastable o(E)

Cu $P 5.9+2$ LASE Ortht : jez

${ }^{50} \mathrm{Co}$ including metastable thich targat yield

${ }^{59} \mathrm{Co} P \quad 2.2+15.4+1$ LVN E Hasbrooht $5075+$

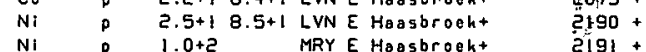

${ }^{58} \mathrm{C}_{0}$ including metastable o(E)

${ }^{59} \mathrm{Co} \quad 2.2+18.4+1$ LVN E HaAbroeht $2074+$

NI P $1.2+14.4+1$ KLNE Michel+

$\mathrm{Ni} \quad 2.5+18.5+1$ LVN E Hasbroekt $2187+$

Cu p $2.7+15.9+1$ KFK C Muenzelt 2545

Cu P $2.7+15.9+1$ ORL E Williamst $2546+$

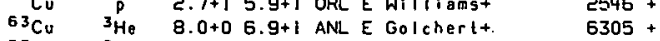

${ }_{63}{ }^{3} \mathrm{Cu} \quad{ }^{3} \mathrm{He} \quad 8.0+06.9+1$ KFK C Muenzelt 6306

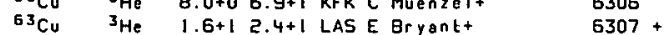




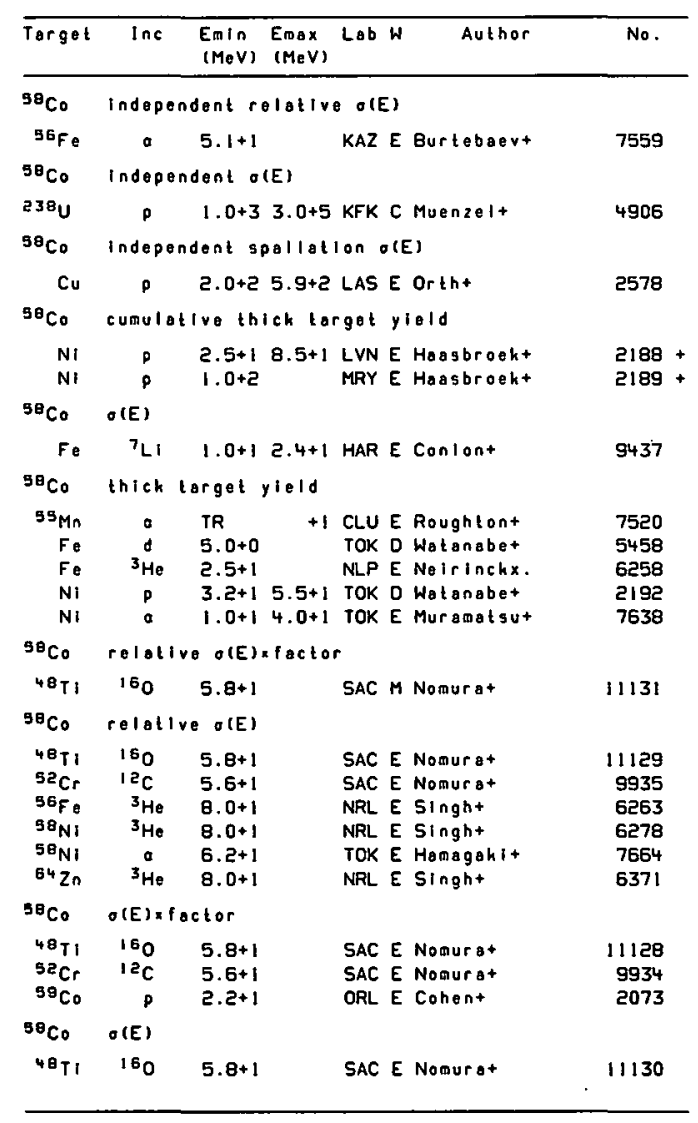

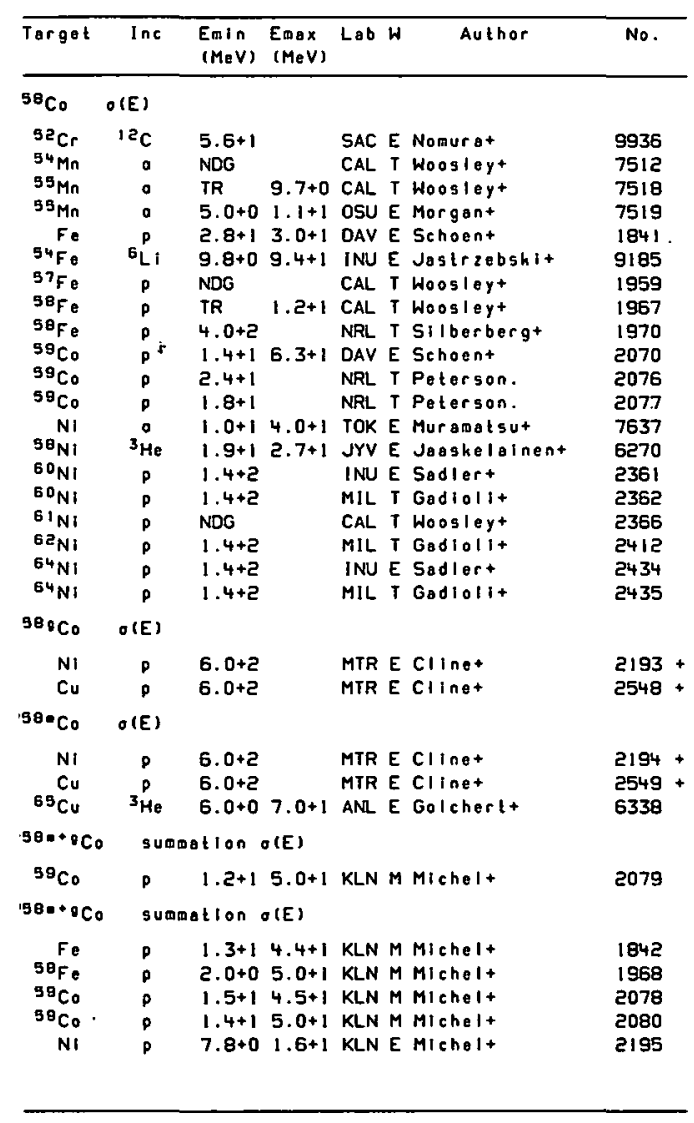




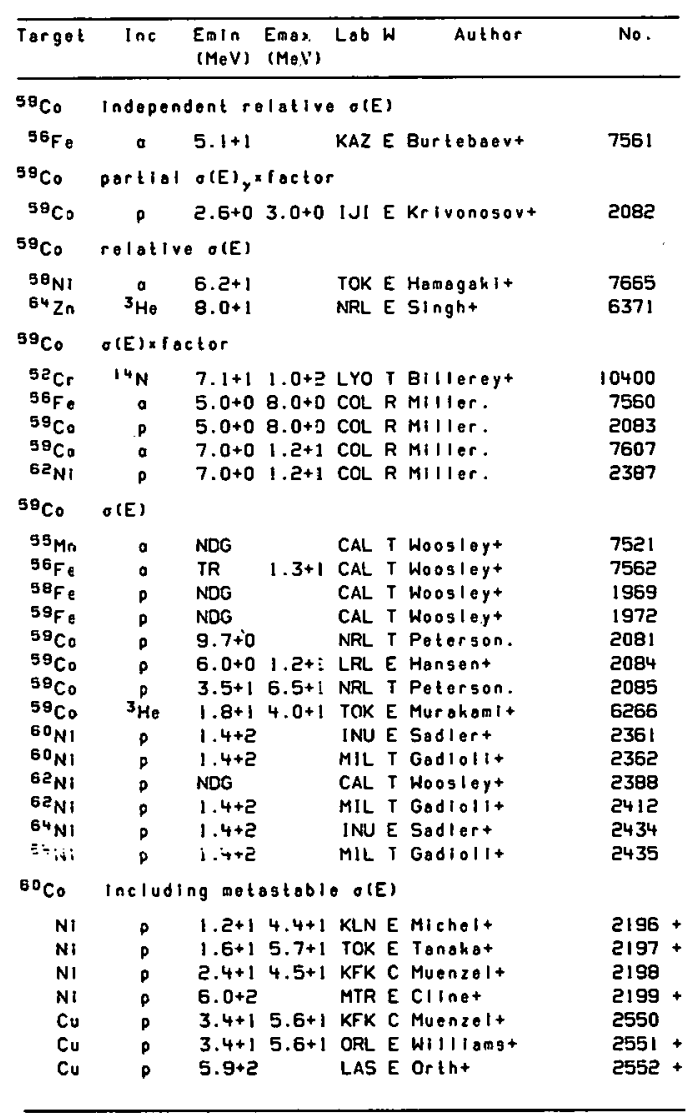

\begin{tabular}{lllll}
\hline Torget Ine $\begin{array}{c}\text { Enin } \\
(\mathrm{MeV})\end{array}$ & $\begin{array}{l}\text { Eax } \\
(\mathrm{MeV})\end{array}$ & Lab $W$ & Author & No. \\
\hline 60 & &
\end{tabular}

${ }^{\circ} \mathrm{Co}_{0}$ Incluoing metostable o(E)

$\begin{array}{rllll}\text { Cu } & p & 6.0+2 & \text { MTR E Clinet } & 2553+ \\ { }_{65} \mathrm{CU}^{3} & 3_{\mathrm{HO}} & \mathbf{8 . 1 + 0} 6.9+1 \text { ANL E Golchirt+ } & 6339+\end{array}$

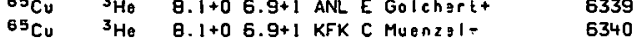

${ }^{60} \mathrm{Co}$ independent o(E)

$23 \mathrm{U}_{\mathrm{U}} \mathrm{P} \quad 1.0+33.0+5 \mathrm{KFKC}$ Muenzel+ 4906

${ }^{\circ} \mathrm{CO} O \quad \sigma(\mathrm{E})$

$56_{M n}$ a NDG . CAL I Woogleyt 7522

57Fe a TR $1.3+1$ CAL T Woosleyt 7570

$59 \mathrm{Fe}$ NDG CAL T Woosleyt
$62_{\mathrm{NI}}$

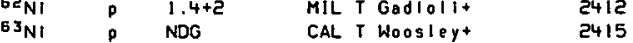

64 Ni $P$ P $1.4+2 \quad$ INIE Sodlert 2434

64 Ni P $1.4+2$ MIL TGodiolit

60A.9CO SUmation O(E)

$\mathrm{Ni} P$ NDG KLNE Michelit 2200

Gl Co cunulative spollation o(E)

Cu $p \quad 2.0+25.9+2$ LAS E Ortht 2577

${ }^{6} C_{0}$ cumulative o(E)

$\begin{array}{lllll}N 1 & p & 6.0+2 & \text { MTRE Clinet } & 2201 \\ \mathrm{Cu} & \mathrm{p} & 6.0+2 & \text { MTR E Clinet } & 2554\end{array}$

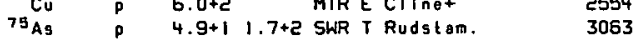

GICO $\sigma(E) \times$ foctor

${ }^{59} \mathrm{CO}$ - $2.3+13.4+1$ COL R Miller. 7608

$6 I^{\mathrm{N} 1} \mathrm{~N} 2.3+13.4+1$ COL R Miller. 5490

3.0+1 $4.5+1$ COL R Miller. 2389

6 'CO $O$ (E)

\begin{tabular}{|c|c|c|c|c|c|c|}
\hline $57 \mathrm{Mn}$ & व & NDG & & CAL T & T Wooslert & 7523 \\
\hline & $\theta$ & NDG & & CAL $T$ & T Wooslept & 7524 \\
\hline${ }^{58} \mathrm{Fe}$ & 。 & TR & $1.3+1$ & CAL $T$ & T Woosle, & 7576 \\
\hline $60^{\circ} \mathrm{Fo}$ & $p$ & NDG & & CAL & T Wooslert & 1975 \\
\hline${ }^{61} \mathrm{Fe}$ & p & NDG & & CAL $T$ & T Wooslegt & 1977 \\
\hline $\mathrm{BS}_{\mathrm{NI}}$ & p & $1.4+2$ & & MIL T & T Godolit & 2412 \\
\hline${ }^{64} \mathrm{NI}$ & $p$ & NDG & & CAL T & t Woosleyt & 2421 \\
\hline
\end{tabular}




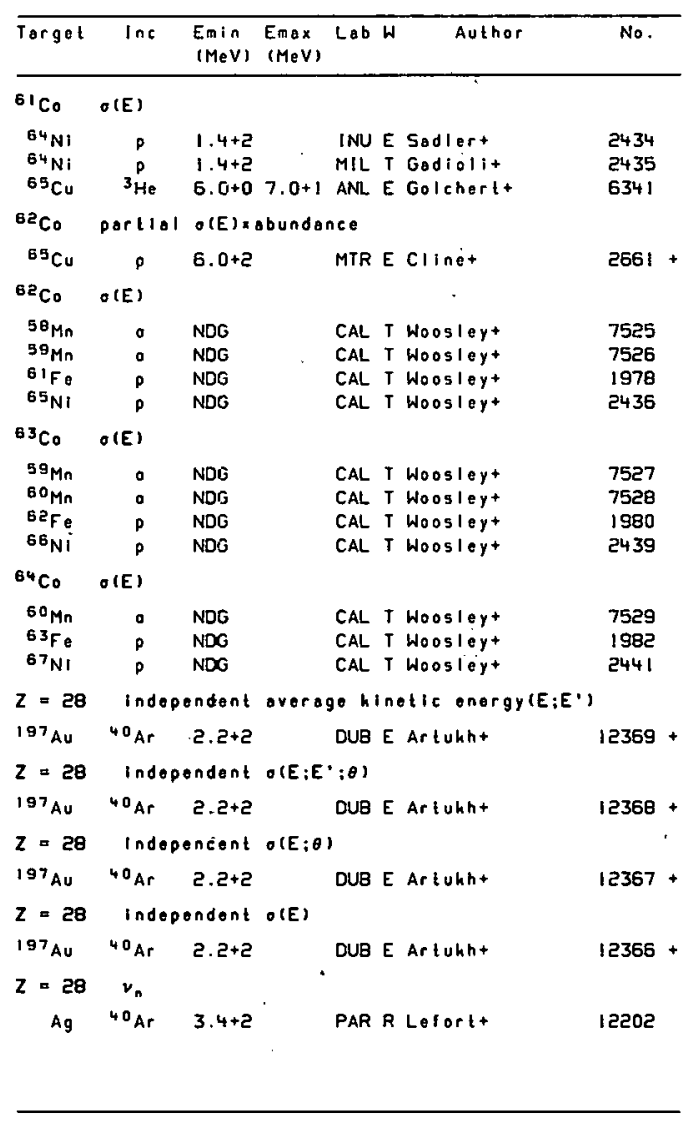

\begin{tabular}{|c|c|c|c|c|c|c|}
\hline Target & Inc & $\begin{array}{l}\text { Emin } \\
\text { (MeV) }\end{array}$ & $\begin{array}{l}\text { Emax } \\
(\operatorname{MeV})\end{array}$ & Lab & Author & No. \\
\hline${ }^{33} \mathrm{Ni}$ & $\cdot(E)$ & & & & & \\
\hline${ }^{40} \mathrm{CB}_{B}$ & 160 & $6.0+1$ & $6.5+1$ & BRK & Eviera+ & 11032 \\
\hline $5^{5} \mathrm{Ni}$ & relative & $\theta O(E)$ & & & & \\
\hline $5^{4} \mathrm{Fe}$ & ${ }^{3} \mathrm{He}$ & $1.3+1$ & $1.5+1$ & AAU E & E Hornsholt & 6262 \\
\hline${ }_{59} \mathrm{NI}$ & $\sigma(E)$ & & & & & \\
\hline $\begin{array}{l}51 \mathrm{Fe} \\
54 \mathrm{Fo} \\
52 \mathrm{Co} \\
5{ }^{4} \mathrm{Co}\end{array}$ & $\begin{array}{c}\stackrel{0}{\mathrm{He}} \\
0 \\
\mathrm{p}\end{array}$ & $\begin{array}{l}\text { NDG } \\
1.2+1 \\
\text { NDG } \\
\text { NDG }\end{array}$ & $1.5+1$ & $\begin{array}{l}\text { CAL } \\
\text { AAU } \\
\text { CAL. } \\
\text { CAL }\end{array}$ & $\begin{array}{l}\text { T Woosleyt } \\
\text { E Hornshojt } \\
\text { I Woosleyt } \\
\text { T Woosleyt }\end{array}$ & $\begin{array}{l}7539 \\
6261 \\
7588 \\
1983\end{array}$ \\
\hline${ }^{56} \mathrm{NI}$ & compoun & denucle & QUS OLE & & & \\
\hline${ }^{40} \mathrm{Ca}$ & 160 & $5.2+1$ & $2.1+2$ & ANL & E vigdort & 11033 \\
\hline${ }_{25} \mathrm{NI}$ & cumulat & Ive ele & & & & \\
\hline $\begin{array}{l}{ }^{5 \theta} \mathrm{NI} \\
{ }^{5 \theta_{N i}}\end{array}$ & p & $\begin{array}{l}2.7+1 \\
2.7+1\end{array}$ & $\begin{array}{l}5.7+1 \\
5.7+1\end{array}$ & $\begin{array}{l}\text { KFK } \\
\text { TOK }\end{array}$ & $\begin{array}{l}\text { C Muenzel+ } \\
\text { E Tanahat }\end{array}$ & $\begin{array}{l}2292 \\
2293+\end{array}$ \\
\hline${ }^{56} \mathrm{NI}$ & product & $y 101 d$ & & & & \\
\hline${ }^{56} C_{0}$ & $p$ & NDG & & ROS & E Beckert+ & 1985 \\
\hline${ }^{5 B} \mathrm{NI}$ & thick & orget y & $y 101 d$ & & & \\
\hline $\begin{array}{l}\mathrm{Fo}_{0} \\
59^{2} \mathrm{Co}\end{array}$ & ${ }^{3} \mathrm{He}$ & $\begin{array}{l}2.5+1 \\
4.9+1\end{array}$ & $8.4+1$ & $\begin{array}{l}\text { NLP } \\
\text { LVN }\end{array}$ & $\begin{array}{l}\text { E Neirinckx. } \\
\text { E Hoasbrookt }\end{array}$ & $\begin{array}{l}6259 \\
2092+\end{array}$ \\
\hline $\mathrm{Ni}$ & p & $3.0+i$ & $8.4+1$ & LVN & E Haosbroeht & $2205+$ \\
\hline NI & D & $3.0+1$ & $5.5+1$ & TOK & O Watanabet & 2206 \\
\hline $\begin{aligned} \mathrm{NI} \\
5 \theta_{\mathrm{NI}}\end{aligned}$ & 0 & $1.0+1$ & $4.0+1$ & TOK & E Muramatsut & 7638 \\
\hline $0 \mathrm{nI}$ & $D$ & $3.0+1$ & $5.5+1$ & TOK & D Watanabet & 2290 \\
\hline${ }^{56} \mathrm{NI}$ & $O(E) \times a b$ & undance & & & & \\
\hline $\begin{array}{l}59 \mathrm{Co} \\
{ }^{59}{ }_{\mathrm{NI}}\end{array}$ & $p$ & $\begin{array}{l}3.8+1 \\
1.2+1\end{array}$ & $\begin{array}{l}4.5+1 \\
4.4+1\end{array}$ & $\begin{array}{l}\text { KLN } \\
\text { KLN }\end{array}$ & $\begin{array}{l}\text { M Michel+ } \\
\text { E Michel+ }\end{array}$ & $\begin{array}{l}2086 \\
2291+\end{array}$ \\
\hline $58 \mathrm{NI}$ & $O(E)$ & & & & & \\
\hline${ }^{52} \mathrm{Fe}$ & 。 & NDG & & CAL & T Woosleyt & 7541 \\
\hline${ }_{54}^{54} \mathrm{Fe}$ & $6_{L I}$ & $9.8+0$ & $9.4+1$ & INU & E Jastrzebshit & 9185 \\
\hline${ }^{53} \mathrm{C}_{0}$ & 0 & NDE & & CAL & T Woosleyt & 7590 \\
\hline${ }^{55} \mathrm{Ca}$ & p & NOE & & CAL & T Woosleyt & 1984 \\
\hline${ }^{59} \mathrm{Co}$ & 0 & $4.4+1$ & $5.0+1$ & KLN & M Michel+ & 2087 \\
\hline${ }^{59} \mathrm{Co}_{0}$ & $p$ & $4.9+1$ & $8.4+1$ & LVN & E Hoogbroeht & $2088+$ \\
\hline
\end{tabular}




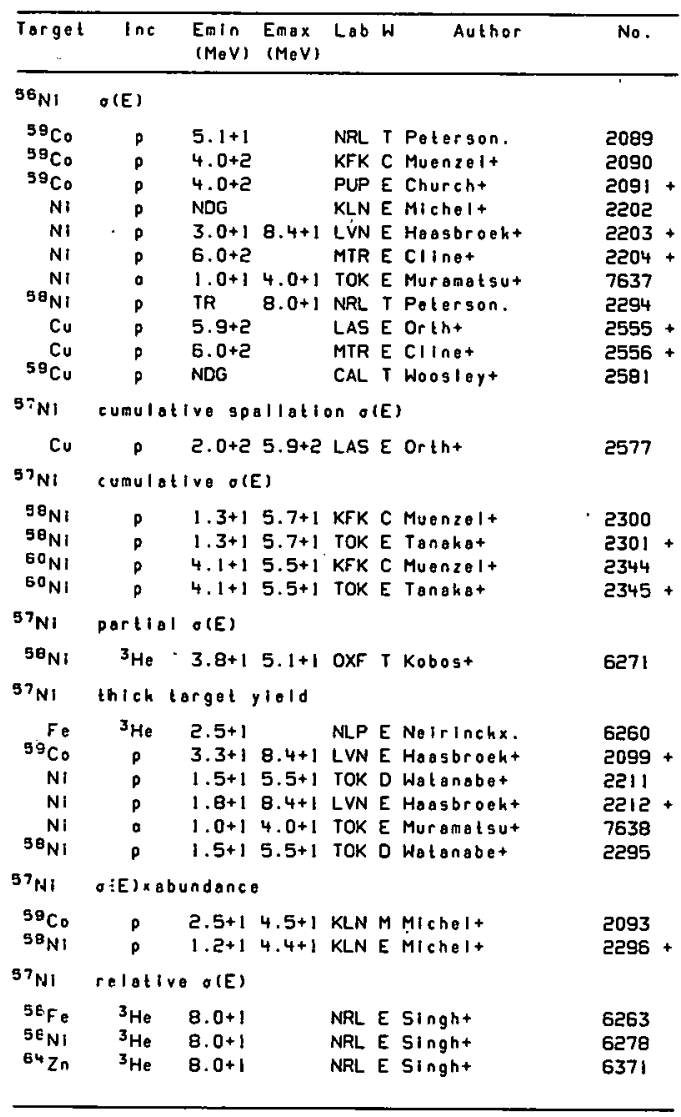

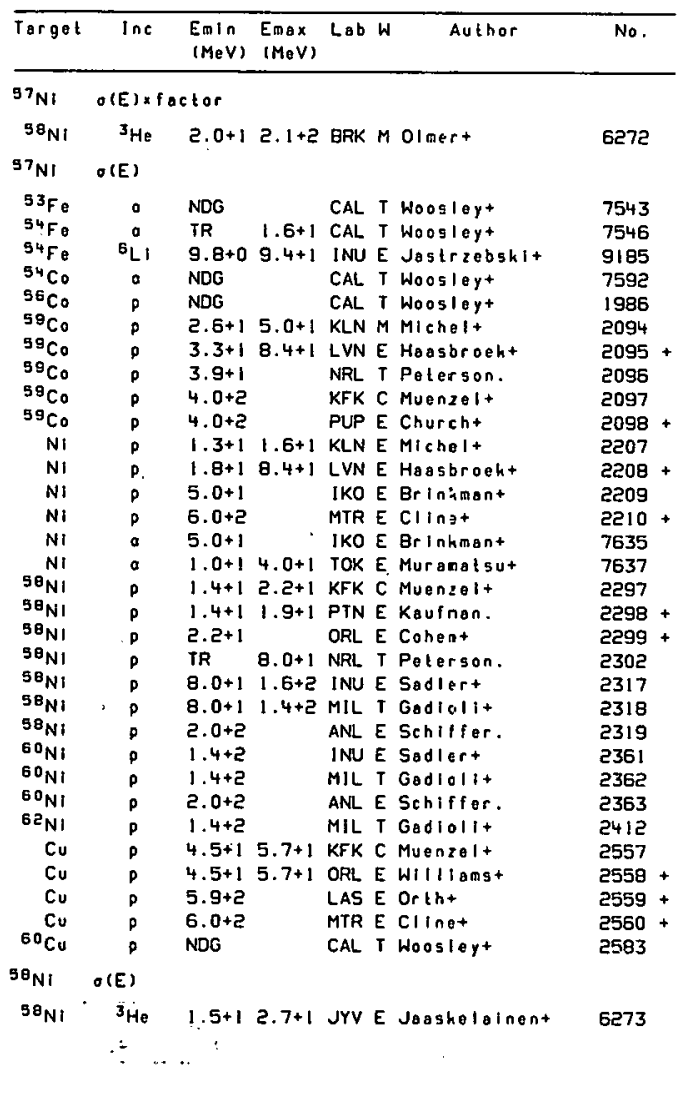




\begin{tabular}{|c|c|c|c|c|c|c|}
\hline Target & Inc & $\begin{array}{l}\text { Emin } \\
(M e V)\end{array}$ & $\begin{array}{l}\text { Emax } \\
(\operatorname{MeV})\end{array}$ & Lab & Author & No. \\
\hline${ }^{58} \mathrm{NI}$ & part|a| & relat & IVe OlE & & & \\
\hline $\begin{array}{l}{ }^{54} \mathrm{Fe} \\
{ }^{54} \mathrm{Fe}\end{array}$ & $\begin{array}{l}6 \mathrm{LI} \\
160\end{array}$ & $\begin{array}{l}3.4+1 \\
5.0+1\end{array}$ & & $\begin{array}{l}\text { LAS } \\
\text { LAS }\end{array}$ & $\begin{array}{l}M \text { Hansont } \\
M \text { Hansont }\end{array}$ & $\begin{array}{r}9184 \\
11156\end{array}$ \\
\hline${ }^{5 a_{N 1}}$ & portial & $O(E)$ & & & & \\
\hline $\begin{array}{l}{ }^{54} \mathrm{Fe} \\
{ }^{58} \mathrm{Ni}\end{array}$ & $\begin{array}{c}0 \\
\text { product }\end{array}$ & $\begin{array}{l}7.6+0 \\
\text { yield }\end{array}$ & $1.3+1$ & NWU & E Raghunathan. & 7547 \\
\hline $\begin{array}{l}{ }^{5 \theta_{N i}} \\
{ }^{5 \theta_{\mathrm{Ni}}}\end{array}$ & 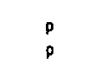 & $\begin{array}{l}3.1+0 \\
3.1+0\end{array}$ & $\begin{array}{l}4.2+0 \\
4.2+0\end{array}$ & $\begin{array}{l}\text { TNL } \\
\text { TNL }\end{array}$ & $\begin{array}{l}\text { E Flynn. } \\
\text { E Flynn. }\end{array}$ & $\begin{array}{l}2303 \\
2305\end{array}$ \\
\hline $58 \mathrm{Ni}$ & relative & e of(E), & afactor & & & \\
\hline${ }^{40} \mathrm{Ti}_{\mathrm{i}}$ & 160 & $5.8+1$ & & SAC & M Nomurat & 11131 \\
\hline $58_{\mathrm{NI}}$ & relative & e $O(E)$ & & & & \\
\hline $\begin{array}{l}{ }^{48} \mathrm{Ti} \\
5 \mathrm{C}^{\mathrm{C}} \\
56 \mathrm{Fe} \\
50_{\mathrm{Ni}} \\
58_{\mathrm{Ni}} \\
58_{\mathrm{Ni}} \\
50_{\mathrm{Ni}} \\
{ }^{64} \mathrm{Zn}\end{array}$ & $\begin{array}{c}16 \mathrm{O} \\
1 \mathrm{C}^{1} \mathrm{C} \\
{ }^{3} \mathrm{He} \\
{ }^{3} \mathrm{He} \\
a \\
a \\
a \\
{ }^{a} \mathrm{He} \\
{ }^{3} \mathrm{He}\end{array}$ & $\begin{array}{l}5 \cdot 8+1 \\
5 \cdot 6+1 \\
8 \cdot 0+1 \\
8 \cdot 0+1 \\
6 \cdot 2+1 \\
6 \cdot 2+1 \\
5 \cdot 2+1 \\
8 \cdot 0+1\end{array}$ & & $\begin{array}{l}\text { SAC } \\
\text { SAC } \\
\text { NRL } \\
\text { NRL } \\
\text { TOK } \\
\text { TOK } \\
\text { TOK } \\
\text { NRL }\end{array}$ & $\begin{array}{l}\text { E Nomurat } \\
\text { E Nomurat } \\
\text { E Singht } \\
\text { E Singht } \\
\text { E Hamgah It } \\
\text { E Hamagahit } \\
\text { E Hamagahit } \\
\text { E Singht }\end{array}$ & $\begin{array}{r}11129 \\
9935 \\
6263 \\
6278 \\
7666 \\
7667 \\
7668 \\
6371\end{array}$ \\
\hline${ }^{5 \theta^{N I}}$ & $O(E) \times f a$ & ctor & & & & \\
\hline $\begin{array}{l}{ }^{48} \mathrm{Ti} \\
5 \mathrm{Cr} \\
5 \mathrm{C}_{\mathrm{C}}\end{array}$ & $\begin{array}{r}150 \\
12 \mathrm{C} \\
0\end{array}$ & $\begin{array}{l}E .8+1 \\
E .6+1 \\
2.2+1\end{array}$ & & $\begin{array}{l}\text { SAC } \\
\text { SAC } \\
\text { ORL }\end{array}$ & $\begin{array}{l}\text { E Nomurat } \\
\text { E Nomurat } \\
\text { E Cohent }\end{array}$ & $\begin{array}{r}11128 \\
9934 \\
2100\end{array}$ \\
\hline${ }^{58_{N 1}}$ & O(E) & & & & & \\
\hline${ }^{48} \mathrm{Ti}$ & 160 & $5.8+1$ & & SAC & E Nomurat & 11130 \\
\hline${ }^{52} \mathrm{Cr}$ & $1^{2} \mathrm{C}$ & $5.6+1$ & & SAC & E Nomurat & 9936 \\
\hline${ }^{54} \mathrm{Fe}$ & $a$ & NDG & & $\mathrm{CAL}$ & T Woosleyt & 7548 \\
\hline${ }^{56} \mathrm{Fe}$ & ${ }^{6} \mathrm{Li}$ & $6.5+1$ & $8.5+1$ & INU & E Jastrzebsht+ & 9204 \\
\hline${ }^{57} \mathrm{Fe}$ & ${ }^{6} \mathrm{Li}$ & $6.5+1$ & $0.5+1$ & INU & E Singht & 9206 \\
\hline $5 \theta_{\mathrm{Fe}}$ & $5 \mathrm{Li}$ & $6.5+1$ & $8.5+1$ & INU & E Jastrzebskit & 9207 \\
\hline${ }^{55} \mathrm{Co}$ & $a$ & NDG & & CAL & T Woosleyt & 7594 \\
\hline 5 & p & NDG & & CAL & T Woosleyt & 1988 \\
\hline & $p$ & $2.4+1$ & & NRL & T Peterson. & 2101 \\
\hline${ }^{5 B_{N i}}$ & p & $4.8+1$ & $5.5+1$ & NRL & $T$ Peterson. & 2304 \\
\hline${ }^{50} \mathrm{Ni}$ & p & $3.0+1$ & $1.6+2$ & INU & E Sadlert & 2317 \\
\hline
\end{tabular}

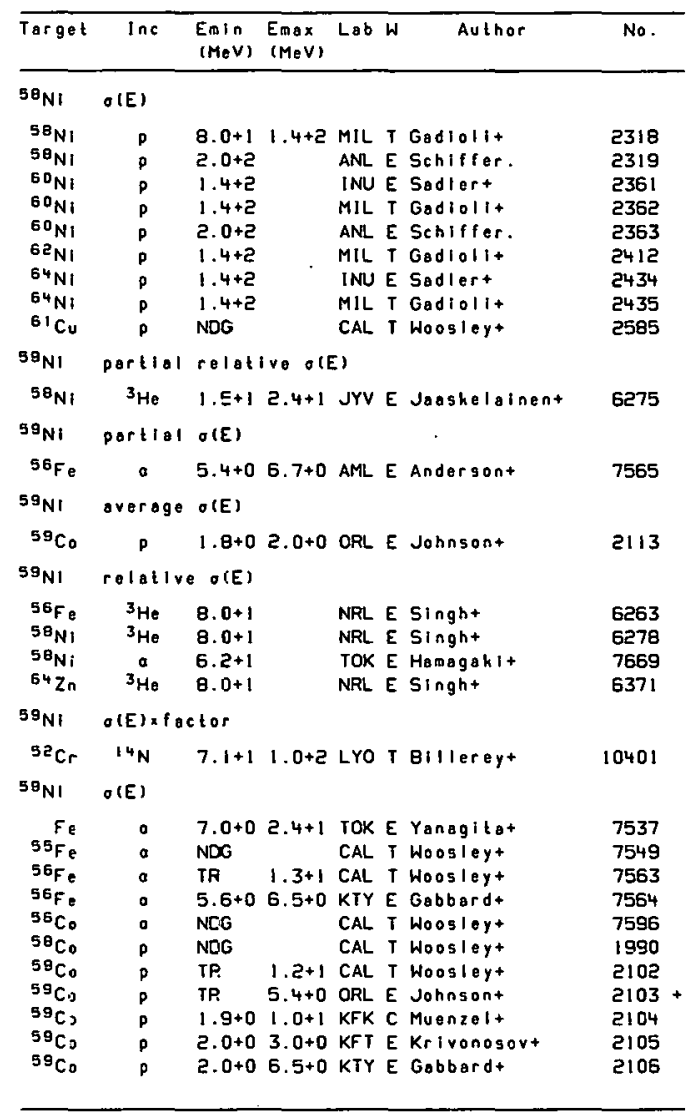




\begin{tabular}{|c|c|c|c|c|c|c|}
\hline Target & $\ln c$ & $\begin{array}{l}\text { Emin } \\
(\mathrm{MeV})\end{array}$ & $\begin{array}{l}E \max \\
(\mathrm{MeV})\end{array}$ & Lab & Author & No. \\
\hline $59 \mathrm{~N} 1$ & $O(E)$ & & & & & \\
\hline${ }^{59} \mathrm{Co}$ & $p$ & $2.4+0$ & $3.8+0$ & IJI & E Dryapachentot & 2107 \\
\hline${ }^{59} \mathrm{Co}_{0}$ & P & $3.0+0$ & $5.0+0$ & INU & Kallast & ह108 \\
\hline${ }^{59} \mathrm{Co}$ & $p$ & $4.0+0$ & $5.5+0$ & LRL & E Alberl. & $2109+$ \\
\hline${ }^{59} \mathrm{Co}$ & p & $4.5+0$ & $6.7+0$ & ROC & E Tahetanit & $2110+$ \\
\hline${ }^{59} \mathrm{Co}$ & $p$ & $5.0+0$ & $1.0+1$ & LRL & E Hangent & $2111+$ \\
\hline${ }^{59} \mathrm{Co}$ & $p$ & $1.1+1$ & & NRL & T Peterson. & 2112 \\
\hline${ }^{3 \theta_{\mathrm{Ni}}}$ & ${ }^{3} \mathrm{He}$ & $1.5+1$ & $2.7+1$ & JYV & E Jashelainent & 6274 \\
\hline $58^{\mathrm{NI}}$ & $a$ & $8.0+1$ & $1.6+2$ & MRY & E Wut & 7670 \\
\hline${ }^{60} \mathrm{NI}$ & p & $1.4+2$ & & INU & E Sadiert & $236 !$ \\
\hline${ }_{60} \mathrm{NI}$ & p & $1.4+2$ & & MIL & TGadiolit & 2362 \\
\hline $62 \mathrm{NI}$ & p. & $1.4+2$ & & MIL & T Gadiolit & 2412 \\
\hline${ }^{64} \mathrm{Ni}$ & p & $1.4+2$ & & INU & E Sadiert & 2434 \\
\hline${ }^{64} \mathrm{Ni}$ & $p$ & $1.4+2$ & & MIL & T Gadiolit & 2435 \\
\hline $\mathrm{Ba}^{2} \mathrm{Cu}$ & p & NDG & & CAL & T Woosleyt & 2587 \\
\hline${ }^{60} 0_{\mathrm{NI}}$ & partial & produc & et $y \in 01$ & & & \\
\hline${ }^{60} \mathrm{Ni}$ & $a$ & $8.0+0$ & $1.8+1$ & TNL & E Wellert & 7686 \\
\hline $\operatorname{san}_{\mathrm{Ni}}$ & partial & $O(E) \times$ & factor & & & \\
\hline $48_{T I}$ & 160 & $5 \cdot 8+1$ & & SAC & E Nomurat & 11127 \\
\hline${ }^{52} \mathrm{Cr}$ & $1{ }^{2} \mathrm{C}$ & $5.6+1$ & & $S A C$ & E Nomurat & 9933 \\
\hline${ }^{59} \mathrm{Co}$ & a & $2.7+1$ & & $\mid \mathrm{JI}$ & E Toharevshilit & 7611 \\
\hline${ }^{60} \mathrm{NI}$ & partial & $\sigma(E)$ & & & & , \\
\hline $\begin{array}{l}{ }^{56} \mathrm{Fe} \\
{ }^{59} \mathrm{Co} \\
{ }^{59} \mathrm{Co}\end{array}$ & a & $\begin{array}{l}4 \cdot 2+0 \\
2 \cdot 5+1 \\
2 \cdot 7+1\end{array}$ & $7.1+0$ & $\begin{array}{l}\text { AML } \\
\text { MOS } \\
\text { IJI }\end{array}$ & $\begin{array}{l}\text { E Andersont } \\
\text { E Lebedevt } \\
\text { E Toharevshilt }\end{array}$ & $\begin{array}{l}7566 \\
7609 \\
7610\end{array}$ \\
\hline${ }^{60} \mathrm{Ni}$ & product & yield & & & & \\
\hline $\begin{array}{l}60 \mathrm{Ni} \\
60^{6 i}\end{array}$ & $p$ & $\begin{array}{l}3.0+0 \\
3.0+0\end{array}$ & $\begin{array}{l}3.5+0 \\
3.5+0\end{array}$ & $\begin{array}{l}\text { TNL } \\
\text { TNL }\end{array}$ & $\begin{array}{l}\text { E Flynn. } \\
\text { E Flynn. }\end{array}$ & $\begin{array}{l}2346 \\
2347\end{array}$ \\
\hline${ }^{60} 0^{N I}$ & relative & e $\sigma(E)$ & xfactor & & & \\
\hline $48_{\mathrm{Ti}}$ & 160 & $5.8+1$ & & SAC & M Nomurat & 11131 \\
\hline $60_{\mathrm{NI}}$ & relative & O $\sigma(E)$ & & & & \\
\hline${ }^{46} \mathrm{Ti}$ & 160 & $2.8+1$ & $5.8+1$ & SAC & E Ballinit & 11111 \\
\hline${ }^{48} T i$ & 160 & $5.8+1$ & & SAC & E Nomurat & 11129 \\
\hline${ }^{50} \mathrm{Cr}$ & ${ }^{12} \mathrm{c}$ & $2.8+1$ & $5.8+1$ & SAC & E Ballinit & 9930 \\
\hline${ }^{52} \mathrm{Cr}$ & $12 \mathrm{C}$ & $5.6+1$ & & SAC & E Nomurat & 9935 \\
\hline
\end{tabular}

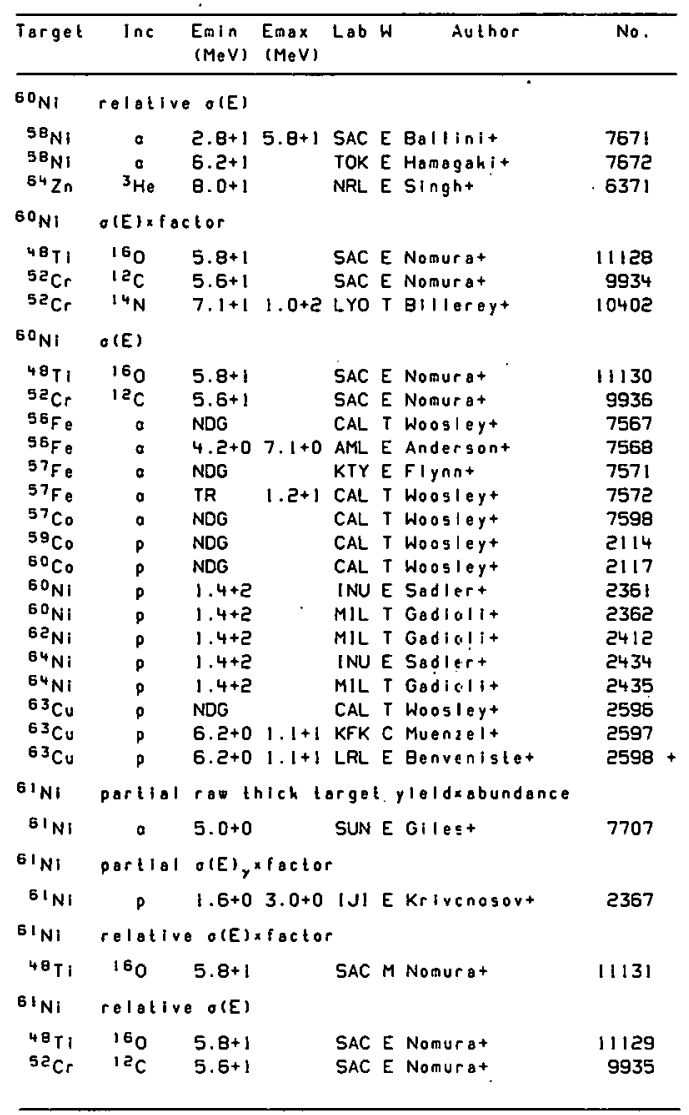




\begin{tabular}{|c|c|c|c|c|c|c|}
\hline Target & $\ln e$ & $\begin{array}{l}\text { Esin } \\
\text { (MeV) }\end{array}$ & $\begin{array}{l}E \max \\
(M e V)\end{array}$ & Lob $h$ & Author & No. \\
\hline $81^{N I}$ & \multicolumn{2}{|c|}{ O(E) a factor } & & & & \\
\hline $\begin{array}{l}{ }^{48} \mathrm{Ti} \\
52 \mathrm{Cr} \\
62 \mathrm{Ni}\end{array}$ & $\begin{array}{r}160 \\
12 \mathrm{C} \\
p\end{array}$ & $\begin{array}{l}5.8+1 \\
5.6+1 \\
2.2+1\end{array}$ & & $\begin{array}{l}\text { SAC } \\
\text { SAC } \\
\text { ORL }\end{array}$ & $\begin{array}{l}\text { E Nomurat } \\
\text { E Nomura+ } \\
\text { E Cohont }\end{array}$ & $\begin{array}{r}11128 \\
9934 \\
2390\end{array}$ \\
\hline${ }^{6} \mathrm{~N} 1$ & $O(E)$ & & & & & \\
\hline $\begin{array}{l}48 \mathrm{TI} \\
52 \mathrm{Cr} \\
57 \mathrm{Fe} \\
58 \mathrm{Fe} \\
60 \mathrm{Co} \\
61 \mathrm{Co} \\
62 \mathrm{Ni} \\
64 \mathrm{Ni} \\
64 \mathrm{NI} \\
64 \mathrm{Cu}\end{array}$ & $\begin{array}{r}150 \\
12 c \\
o \\
0 \\
p \\
p \\
p \\
p \\
p \\
p\end{array}$ & $\begin{array}{l}5.8+1 \\
5.6+1 \\
\text { NDG } \\
\text { TR } \\
\text { NDG } \\
\text { NDG } \\
1.4+2 \\
1.4+2 \\
1.4+2 \\
\text { NOG }\end{array}$ & $1.2+1$ & $\begin{array}{l}\text { SAC } \\
\text { SAC } \\
\text { CAL } \\
\text { CAL } \\
\text { CAL } \\
\text { CAL } \\
\text { MIL } \\
\text { INU E } \\
\text { MIL } \\
\text { CAL }\end{array}$ & $\begin{array}{l}\text { E Nomurat } \\
\text { E Nomurat } \\
T \text { Woosleyt } \\
T \text { Woosleyt } \\
T \text { Woosleyt } \\
T \text { Woosleyt } \\
T \text { Gadiolit } \\
\text { E Sadlert } \\
\text { T Gadiolit } \\
T \text { Woosleyt }\end{array}$ & $\begin{array}{r}11130 \\
9938 \\
7573 \\
7577 \\
2118 \\
2120 \\
2412 \\
2434 \\
2435 \\
2650\end{array}$ \\
\hline \multirow{16}{*}{ งั่ } & portlal & $\theta(E)_{y} x$ & x factor & & & \\
\hline & p & $2.0+0$ & $3.0+0$ & IJIE & E Krivonogort & 2392 \\
\hline & Dortiol & O(E)Y & & & & \\
\hline & P & $7.0+0$ & $2.6+1$ & LRL & E koopmont & 2394 \\
\hline & Dartlal & $O(E)$ & & & & \\
\hline & D & $1.1+0$ & $3.6+0$ & AML $\mathrm{r}$ & M Soilhooghit & 2662 \\
\hline & $t_{r}$ & & & & & \\
\hline & a & $1.0+1$ & & AML & E Kennedy* & 7613 \\
\hline & thleh to & argot y & yleld & & & \\
\hline & 160 & $3.9+1$ & $5.1+1$ & ORL & E Wellst & 11230 \\
\hline & rolotivo & - $O(E)$ & & & & \\
\hline & ${ }^{3} \mathrm{He}$ & $8.0+1$ & & NRL & E singht & 6371 \\
\hline & $O(E) \times$ foc & ctor & & & & \\
\hline & $\dot{p}$ & $\begin{array}{l}5.0+0 \\
5.0+0\end{array}$ & $\begin{array}{l}9.0+0 \\
8.0+0\end{array}$ & $\begin{array}{l}\mathrm{COL} F \\
\mathrm{COL} F\end{array}$ & $\begin{array}{l}\text { R Miller. } \\
\text { R miller. }\end{array}$ & $\begin{array}{l}7612 \\
2393\end{array}$ \\
\hline & $O(E)$ & & & & & \\
\hline & ${ }^{6} \mathrm{Li}$ & $6.5+1$ & $8.5+1$ & INU E & E Jastrzebshit & 9204 \\
\hline
\end{tabular}

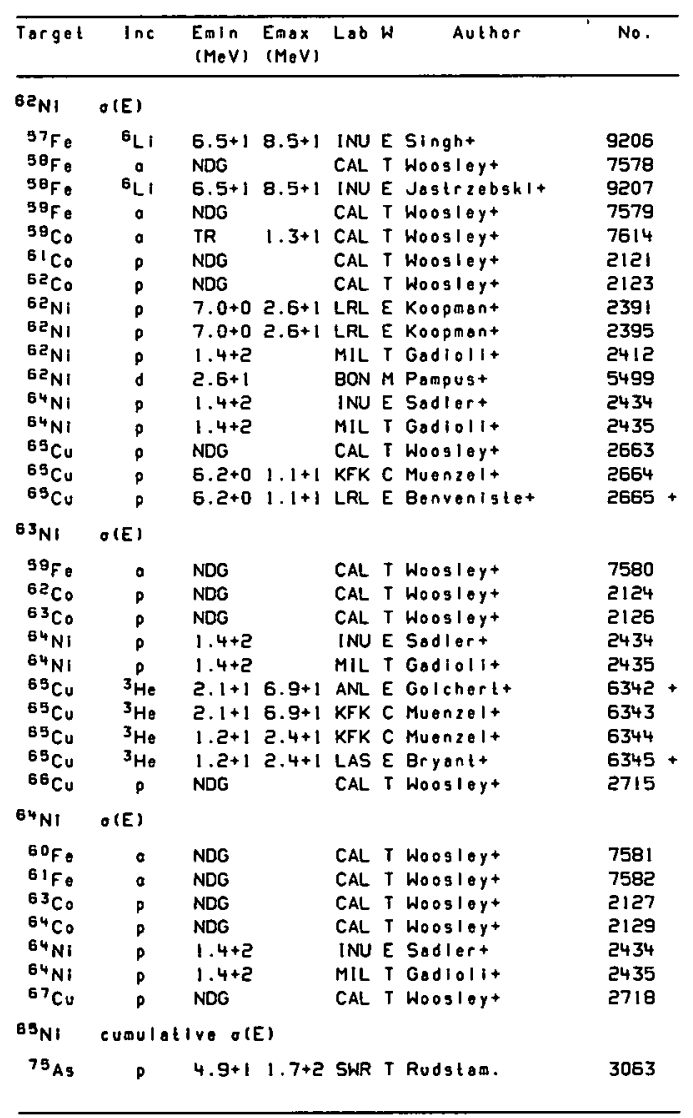




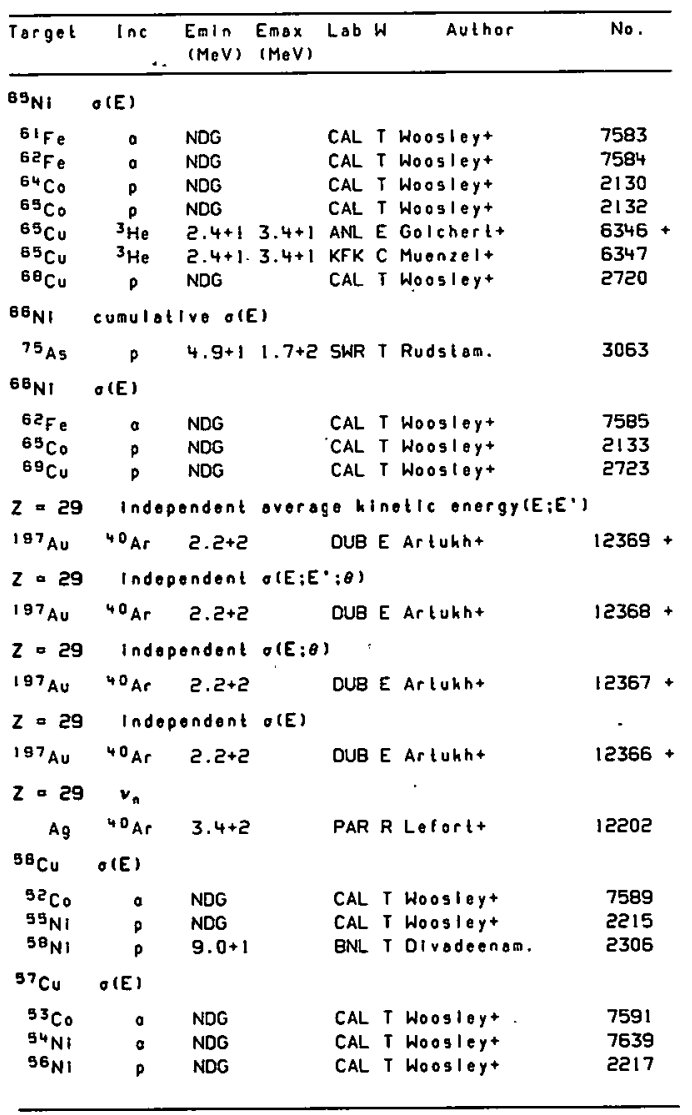

\begin{tabular}{|c|c|c|c|c|c|c|}
\hline Target & $\ln c$ & $\begin{array}{l}E m \ln \\
(M e V)\end{array}$ & $\begin{array}{l}\text { Emax } \\
(M e V)\end{array}$ & Lab $W$ & Author & No. \\
\hline${ }^{57} \mathrm{Cu}$ & $O(E)$ & & & & & \\
\hline $\begin{array}{l}58_{\mathrm{Ni}} \\
{ }^{63} \mathrm{Cu} \\
{ }^{6}{ }^{3} \mathrm{Cu}\end{array}$ & $\begin{array}{l}{ }^{\mathrm{p}} \\
{ }^{3} \mathrm{He} \\
{ }^{3} \mathrm{He}\end{array}$ & $\begin{array}{l}9.0+1 \\
5.4+1 \\
5.4+1\end{array}$ & $\begin{array}{l}6.9+1 \\
6.9+1\end{array}$ & $\begin{array}{l}\text { BNL T } \\
\text { ANL E } \\
\text { KFK C }\end{array}$ & $\begin{array}{l}\text { T Divadeenam. } \\
\text { E Golchert+ } \\
\text { C Muenzelt }\end{array}$ & $\begin{array}{l}2307 \\
6308+ \\
6309\end{array}$ \\
\hline${ }^{50} \mathrm{Cu}$ & product & yieldx & aloctor & & & \\
\hline${ }_{{ }^{58} \mathrm{Cu}}^{58}$ & $\begin{array}{r}\text { D } \\
O(E)\end{array}$ & $9.5+0$ & & WMU $M$ & M Bernstein. & 2308 \\
\hline $\begin{array}{l}{ }^{54} \mathrm{CO} \\
55_{\mathrm{Ni}} \\
57_{\mathrm{Ni}} \\
58_{\mathrm{Ni}} \\
{ }^{58_{\mathrm{Ni}}}\end{array}$ & $\begin{array}{l}: \\
: \\
p \\
p \\
p\end{array}$ & $\begin{array}{l}\text { NDG } \\
\text { NDG } \\
\text { NDG } \\
\text { TR } \\
\text { TR }\end{array}$ & $\begin{array}{l}1.2+1 \\
8.0+1\end{array}$ & $\begin{array}{l}\text { CAL T } \\
\text { CAL } \\
\text { CAL } \\
\text { CAL } \\
\text { NRL T }\end{array}$ & $\begin{array}{l}\text { T Woosleyt } \\
\text { T Woosley+ } \\
\text { T Woosleyt } \\
\text { T Woosley+ } \\
\text { T Peterson. }\end{array}$ & $\begin{array}{l}7593 \\
7641 \\
2218 \\
2309 \\
2310\end{array}$ \\
\hline${ }^{50} \mathrm{Cu}$ & $O(E)$ & & & & & \\
\hline${ }^{58} \mathrm{Ni}$ & $\begin{array}{c}{ }^{3} \mathrm{He} \\
\text { product }\end{array}$ & $\begin{array}{l}1.5+1 \\
y 1010\end{array}$ & $2.7+1$ & JYV E & E Jaaskelationt & 6276 \\
\hline${ }^{58} \mathrm{Ni}$ & $\begin{array}{c}p \\
\text { thlch to }\end{array}$ & $\begin{array}{l}\text { 8.0-1 } \\
\text { orget y }\end{array}$ & $\begin{array}{l}1.9+0 \\
y 1010\end{array}$ & $\operatorname{MOS} R$ & R Varlamove & 2311 \\
\hline${ }^{58} \mathrm{Ni}$ & relotive & $\begin{array}{r}1.3+0 \\
-O(E)\end{array}$ & $3: 4+0$ & CLU $M$ & M Roughtont & 2315 \\
\hline $\begin{array}{l}{ }^{5 \theta_{N 1}} \\
{ }^{64} \mathrm{Zn}\end{array}$ & $\begin{array}{l}{ }^{3} \mathrm{He} \\
{ }^{3} \mathrm{He}\end{array}$ & $\begin{array}{l}8.0+1 \\
8.0+1\end{array}$ & & $\begin{array}{l}\text { NRL E } \\
\text { NRL E }\end{array}$ & $\begin{array}{l}\text { E singht } \\
\text { E singht }\end{array}$ & $\begin{array}{l}6278 \\
6371\end{array}$ \\
\hline${ }^{59} \mathrm{Cu}$ & $O(E)$ & & & & & \\
\hline $\begin{array}{l}55 \mathrm{Co} \\
58_{\mathrm{Ni}} \\
58_{\mathrm{Ni}} \\
58_{\mathrm{Ni}} \\
58_{\mathrm{Ni}} \\
6 \mathrm{Zn}^{\mathrm{n}}\end{array}$ & $\begin{array}{l}\dot{p} \\
p \\
p \\
p \\
0 \\
p\end{array}$ & $\begin{array}{l}\text { NDG } \\
\text { NDG } \\
6.0-1 \\
1.3+0 \\
8.0+1 \\
\text { NDG }\end{array}$ & $\begin{array}{l}3.0+0 \\
3.4+0 \\
1.6+2\end{array}$ & $\begin{array}{l}\text { CAL T } \\
\text { CAL T } \\
\text { IJI E } \\
\text { CLU E } \\
\text { MRY E } \\
\text { CAL T }\end{array}$ & $\begin{array}{l}\text { T Woosleyt } \\
\text { T Woosleyt } \\
\text { E Krivonosoyt } \\
\text { E Roughtont } \\
\text { E Wut } \\
\text { T Woosleyt }\end{array}$ & $\begin{array}{l}7595 \\
2312 \\
2313 \\
2314 \\
7673 \\
2741\end{array}$ \\
\hline${ }^{60} \mathrm{Cu}$ & cumuloti & ivez ot & $(E)$ & & & \\
\hline $\begin{array}{l}{ }^{63} \mathrm{Cu} \\
{ }^{60} \mathrm{Cu}\end{array}$ & $\begin{array}{c}D \\
\text { cumulati }\end{array}$ & $\begin{array}{l}3.5+1 \\
\text { ive ole }\end{array}$ & (E) & $H I R E$ & E Nomst & $2599+$ \\
\hline $\mathrm{Cu}$ & p & $6.0+2$ & & MTR E & E clinet & $2561+$ \\
\hline
\end{tabular}




\begin{tabular}{|c|c|c|c|c|c|c|}
\hline Target & $\ln c$ & $\begin{array}{l}\text { Emin } \\
\text { (MeV) }\end{array}$ & $\begin{array}{l}\text { Emax } \\
(\operatorname{MeV})\end{array}$ & Lat W & Author & No. \\
\hline${ }^{60} \mathrm{Cu}$ & cumulat: & ive olE & & & & \\
\hline $\begin{array}{l}{ }^{75} \mathrm{As} \\
{ }^{60} \mathrm{Cu}\end{array}$ & $\begin{array}{c}P \\
\text { portial }\end{array}$ & $\begin{array}{l}4.9+1 \\
O(E)\end{array}$ & $1.7+2$ & SWR T & Rudstom. & 3063 \\
\hline${ }^{39} \theta_{\mathrm{NI}}$ & ${ }^{3} \mathrm{He}$ & $8.0+0$ & $1.5+1 F$ & PUP E & Leet & 6277 \\
\hline${ }^{60} \mathrm{Cu}$ & product & $y|\in| d x$ & * factor & & & \\
\hline $\begin{array}{l}{ }^{60} \mathrm{NI} \\
{ }^{60} \mathrm{Cu}\end{array}$ & thlck to & $\begin{array}{l}7.0+0 \\
\text { arget y }\end{array}$ & $y \mid 01 d$ & WMU M & Bernstein. & 2348 \\
\hline${ }_{{ }^{60}}^{\mathrm{NI}}$ & a & $\begin{array}{r}1.0+1 \\
\theta \sigma(E)\end{array}$ & $4.0+1$ & TOK: E & Muramelsut & 7638 \\
\hline $\begin{array}{l}{ }^{5 \theta_{N I}} \\
{ }^{5 \theta_{N I}} \\
6{ }^{6} Z_{n}\end{array}$ & $\begin{array}{l}{ }^{3} \mathrm{He} \\
{ }^{3} \\
{ }^{\mathrm{He}}\end{array}$ & $\begin{array}{l}8.0+1 \\
6.2+1 \\
8 \cdot 0+1\end{array}$ & & $\begin{array}{l}\text { NRL E } \\
\text { TOK: } E \\
\text { NRL E }\end{array}$ & $\begin{array}{l}\text { Singht } \\
\text { Hamagahit } \\
\text { Singht }\end{array}$ & $\begin{array}{l}6278 \\
7674 \\
6371\end{array}$ \\
\hline${ }^{60} \mathrm{Cu}$ & $O(E)$ & & & & & \\
\hline${ }^{56} \mathrm{Co}$ & 。 & NDG & & CAL T & Woosleyt & 7597 \\
\hline $\begin{array}{r}\mathrm{NI} \\
50_{\mathrm{NI}}\end{array}$ & $:$ & $\begin{array}{l}1.0+1 \\
8.0+1\end{array}$ & $\begin{array}{l}4.0+1 \\
1.6+2\end{array}$ & $\begin{array}{l}\text { TOK } E \\
\text { MRY E }\end{array}$ & $\begin{array}{l}\text { Muromsisut } \\
\text { Wut }\end{array}$ & $\begin{array}{l}7637 \\
7675\end{array}$ \\
\hline $59_{\mathrm{Ni}}$ & $\rho$ & NDG & & CAL T & Woosleyt & 2323 \\
\hline${ }^{60} \mathrm{NI}$ & $p$ & $T R$ & $1.3+1 c$ & CAL $T$ & Woosleyt & 2349 \\
\hline $60 \mathrm{Ni}$ & $\rho$ & $6.7+0$ & $1.5+1$ & TOK E & Tanakgt & $2350+$ \\
\hline${ }^{60} 0^{N I}$ & $p$ & $1.2+1$ & & KFK C & Muenzel+ & 2351 \\
\hline${ }^{60} \mathrm{NI}$ & p & $1.2+1$ & & ORL E & Blossert & $2352+$ \\
\hline${ }^{63} \mathrm{Cu}$ & ${ }^{3} \mathrm{He}$ & $2.1+1$ & $3.4+1$ & ANL E & Golchert* & $6310+$ \\
\hline${ }^{53} \mathrm{Cu}$ & ${ }^{3} \mathrm{He}$ & $2.1+1$ & $3.4+1 k$ & KFK: C & Muenzel+ & 6311 \\
\hline $632 \mathrm{n}$ & $\rho$ & NDG & & CAL T & Woosleyt & 2743 \\
\hline $\begin{array}{l}{ }^{64} \mathrm{Zn} \\
{ }^{64} \mathrm{Zn}\end{array}$ & $\begin{array}{l}p \\
p\end{array}$ & $\begin{array}{l}\text { TR } \\
1.8+1\end{array}$ & $\begin{array}{l}2.4+1 k \\
2.4+1 k\end{array}$ & $\begin{array}{l}\text { ORL E } \\
\text { KFK C }\end{array}$ & $\begin{array}{l}\text { Cohent } \\
\text { Muenzel+ }\end{array}$ & $\begin{array}{l}2750+ \\
2751\end{array}$ \\
\hline${ }^{61} \mathrm{C}_{U}$ & Independ & dentre & elative & e $\sigma(E)$ & & \\
\hline $60^{N 1}$ & a & $5.1+1$ & & $K A Z E$ & Burtebaev+ & 7687 \\
\hline${ }^{61} \mathrm{Cu}$ & cumulati & ive spo & ollation & on $O(E)$ & & \\
\hline $\begin{array}{c}\mathrm{Cu} \\
{ }^{6} \mathrm{Cu}\end{array}$ & $\frac{P}{\text { cumulati }}$ & $\begin{array}{l}2.0+5 \\
\text { ive ole }\end{array}$ & E) & LAS E & Ortht & 2577 \\
\hline $\begin{array}{l}\mathrm{Cu} \\
\mathrm{Cu}\end{array}$ & $p$ & $\begin{array}{l}2.0+1 \\
2.0+1\end{array}$ & $\begin{array}{l}5.8+1 k \\
5.8+1\end{array}$ & $\begin{array}{l}\text { KFK C } \\
\text { ORL E }\end{array}$ & $\begin{array}{l}\text { Muenzel+ } \\
\text { Williamst }\end{array}$ & $\begin{array}{l}2562 \\
2563+\end{array}$ \\
\hline
\end{tabular}

\begin{tabular}{llllll}
\hline Target Inc & $\begin{array}{l}\text { Emin } \\
(M e V)\end{array}$ & $\begin{array}{l}\text { Emax Lab } \\
(M e V)\end{array}$ & Author & No. \\
\hline
\end{tabular}

6.1 Cu cumulallve o(E)

Cu $P$ 6.0+5 MTR E CIInot

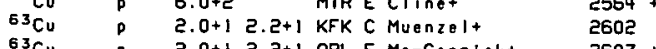

${ }_{63}{ }^{6} \mathrm{Cu} \quad 2.0+12.2+1$ ORL E Mc-Cormicht $2603+$

${ }_{63} \mathrm{Cu}^{3} \mathrm{Ho}$ 6.7+0 3.4+1 KFK C Muenzelt 6312

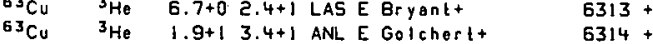

${ }^{75}$ As $P \quad 4.9+1 \quad 1.7+2$ SWR T Rudstam. 3063

$230 P$ i.e+4 ANL E Changt 4873

$230 P$ l.e+4 KFK C Muenzelt 4874

CICu partial rav o(E)

${ }^{60} \mathrm{NI} P \quad 1.7+01.9+0$ DUB E Kraflt 2359

${ }^{6}{ }^{6}$ Cu partiol relotive o(E)

${ }^{60} \mathrm{NI} P \quad 1.8+12.9+1$ ALB E Bohhareet 2353

GICu portlal o(E) xfactor

$\begin{array}{lllll}{ }^{64} \mathrm{Zn} P & 1.2+1 & \text { PUR E Luxt } & 2757\end{array}$

EICu partlat o(E)

$61 \mathrm{NI} 4.1+05.3+0$ WAS E Braga. 2375

sicu thick target yield

${ }^{59} \mathrm{Co}{ }^{3} \mathrm{He} \quad 1.8+14.0+1$ TOK E Murskamit 6264

${ }^{59} \mathrm{Co} a \quad 1.5+14.0+1$ TOK E Murakamit 7617

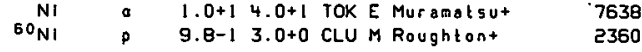

GCU raU olE

$61 \mathrm{Ni} P 2.9+03.4+0$ ORL E Johnsont 2376

${ }^{6} \mathrm{Cu}$ relative o(E) $\times$ factor

$48_{11} 160 \quad 5.8+1 \quad$ SAC M Nomurat 11131

${ }^{6} \mathrm{Cu}$ relative o(E)

$\begin{array}{llllr}{ }^{48} \mathrm{Ti} & 160 & 5.8+1 & \text { SAC E Nomurat } & 11129 \\ { }_{52} \mathrm{Cr} & 12 \mathrm{C} & 5.6+1 & \text { SAC E Nomurat } & 9935\end{array}$

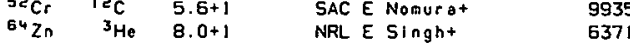




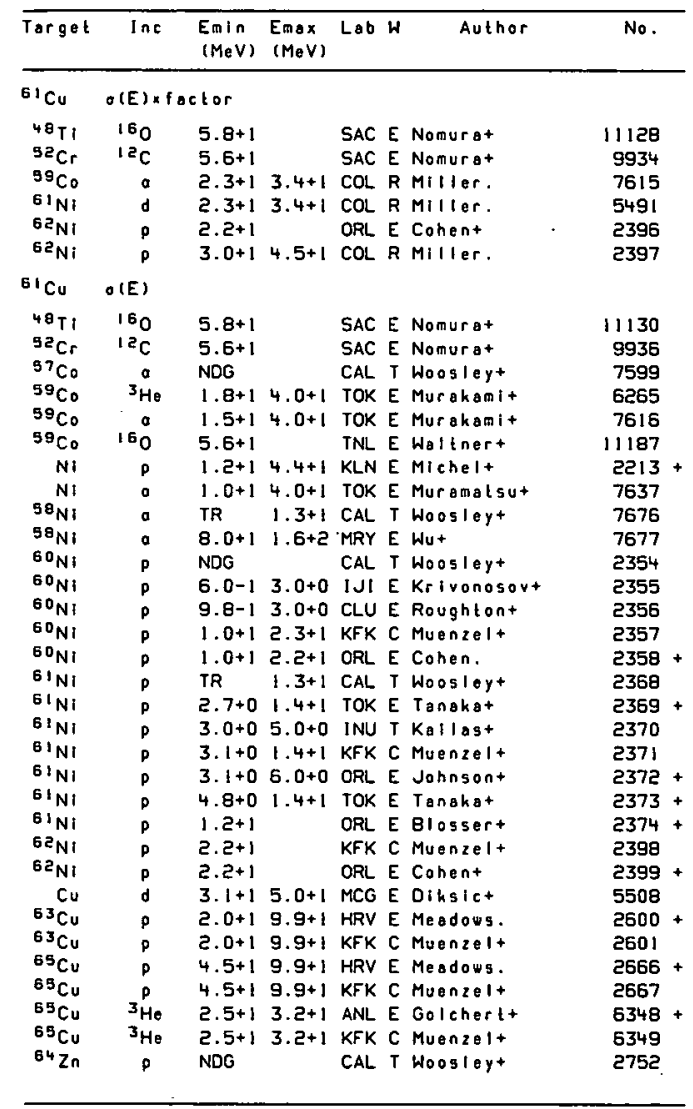

\begin{tabular}{|c|c|c|c|c|c|c|}
\hline Target & $\ln e$ & $\begin{array}{l}\text { Emin } \\
(M e V)\end{array}$ & $\begin{array}{l}\text { Emax } \\
(\mathrm{MeV})\end{array}$ & Lab & Author & No. \\
\hline${ }^{6} \mathrm{Cu}$ & $O(E)$ & & & & & \\
\hline $\begin{array}{l}64 \mathrm{Zn} \\
64 \mathrm{Zn} \\
64 \mathrm{Zn} \\
64 \mathrm{Zn} \\
64 \mathrm{Zn}\end{array}$ & $\begin{array}{l}p \\
p \\
p \\
p \\
d\end{array}$ & $\begin{array}{l}\text { TR } \\
\cdot 7.1+0 \\
1.2+1 \\
2.3+1 \\
8.4+0\end{array}$ & $\begin{array}{l}2.4+1 \\
2.4+1 \\
4.2+1 \\
1.4+1\end{array}$ & $\begin{array}{l}\text { ORL } \\
\text { KFK } \\
\text { PUR E } \\
\text { MIL E } \\
\text { KTO E }\end{array}$ & $\begin{array}{l}\text { E Cohent } \\
\text { C Muenzelt } \\
\text { E Luxt } \\
\text { E Guazzonit } \\
\text { E Night+ }\end{array}$ & $\begin{array}{l}2753+ \\
2754 \\
2755 \\
2755 \\
5535\end{array}$ \\
\hline${ }^{62} \mathrm{Cu}$ & indepen & ident re & elotive & e $O(E)$ & & \\
\hline${ }^{60^{6}} \mathrm{NI}$ & $\begin{array}{c}a \\
\text { portial }\end{array}$ & $\begin{array}{l}5.1+1 \\
O(E)\end{array}$ & & KAZ E & E Burtabaevt & 7692 \\
\hline $\begin{array}{l}60 \mathrm{NI} \\
{ }_{65} \mathrm{NI}\end{array}$ & $\underset{\mathrm{p}}{{ }^{3} \mathrm{He}}$ & $\begin{array}{l}8.0+0 \\
\text { NDG }\end{array}$ & $1.5+1$ & $\begin{array}{l}\text { PUP E } \\
\text { ORE E }\end{array}$ & $\begin{array}{l}\text { E Leet } \\
\text { E Ross. }\end{array}$ & $\begin{array}{l}6279 \\
2400\end{array}$ \\
\hline${ }^{62} \mathrm{Cu}$ & relativ & I. $\mathrm{I}_{\mathrm{y}}$. & & & & \\
\hline 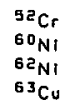 & $\begin{array}{r}14 \mathrm{~N} \\
\stackrel{9}{p} \\
\mathrm{P}\end{array}$ & $\begin{array}{l}5.0+1 \\
2.2+1 \\
7.5+0 \\
2.2+1\end{array}$ & $\begin{array}{l}7.5+1 \\
3.5+1 \\
2.8+1\end{array}$ & $\begin{array}{l}\text { GRE E } \\
\text { GRE E } \\
\text { GHE E } \\
\text { GRE E }\end{array}$ & $\begin{array}{l}\text { E Ung-Chant } \\
\text { E Ung-Chant } \\
\text { E Ung-Chant } \\
\text { E Ung-Chant }\end{array}$ & $\begin{array}{r}10403 \\
7689 \\
2401 \\
2609\end{array}$ \\
\hline${ }^{82} \mathrm{Cu}$ & product & yleld & & & & \\
\hline $61 \mathrm{NI}$ & $p$ & NDG & & LNDE & E Erionossont & 2380 \\
\hline${ }^{62} \mathrm{Cu}$ & thick t & target $y$ & yleld & & & \\
\hline $\begin{array}{l}6 I^{2} \mathrm{Ni} \\
6 I_{N I}\end{array}$ & $1{ }^{p}{ }_{0}$ & $\begin{array}{l}9.8-1 \\
3.9+1\end{array}$ & $\begin{array}{l}3 \cdot 5+0 \\
5 \cdot 1+1\end{array}$ & $\begin{array}{l}\text { CLU } \\
\text { ORL E }\end{array}$ & $\begin{array}{l}\text { M Roughtont } \\
\text { E Wellst }\end{array}$ & $\begin{array}{r}2381 \\
11231\end{array}$ \\
\hline${ }^{\mathrm{a}} \mathrm{Cu}$ & $O(E) \times f o$ & etor & & & & \\
\hline $\begin{array}{l}6{ }^{60} \mathrm{Ni} \\
{ }^{63} \mathrm{Cu} \\
{ }^{63} \mathrm{Cu}\end{array}$ & p & $\begin{array}{l}2.0+1 \\
2.2+1 \\
2 \cdot 1+1\end{array}$ & $\begin{array}{l}3.5+1 \\
3.3+1\end{array}$ & $\begin{array}{l}\text { COL F } \\
\text { ORL E } \\
\text { COL F }\end{array}$ & $\begin{array}{l}\text { R Miller. } \\
\text { E Cohent } \\
\text { R Miller. }\end{array}$ & $\begin{array}{l}7688 \\
2610 \\
2604\end{array}$ \\
\hline${ }^{82} \mathrm{Cu}$ & $O(E)$ & & & & . & \\
\hline $\begin{array}{l}58 \mathrm{Co} \\
{ }^{59} \mathrm{Co} \\
60 \mathrm{Ni}\end{array}$ & $a$ & $\begin{array}{l}\text { NDG } \\
\text { TR } \\
2.3+1\end{array}$ & $\begin{array}{l}9.4+0 \\
3.9+1\end{array}$ & $\begin{array}{l}\text { CAL T } \\
\text { CAL T } \\
\text { KFK }\end{array}$ & $\begin{array}{l}\text { T Woosleyt } \\
\text { T Woostey+ } \\
\text { C Muonzel+ }\end{array}$ & $\begin{array}{l}7600 \\
7618 \\
7690\end{array}$ \\
\hline $60^{N I}$ & $a$ & $2.3+1$ & $3.9+1$ & BRK E & E Ghosa!. & $7691+$ \\
\hline $61 \mathrm{NI}$ & $p$ & NDG & & CAL $T$ & T Woosleyt & 2377 \\
\hline $61 \mathrm{NI}$ & g & $6.0-1$ & $3.0+0$ & $|J| \varepsilon$ & E Krivonosovt & 2378 \\
\hline $\mathrm{BINI}^{2}$ & $\mathrm{p}$ & $9.8-1$ & $3.5+0$ & CLU E & E Roughlont & 2379 \\
\hline $\operatorname{SE}_{\mathrm{NI}}$ & p & $T R$ & $1.2+1$ & CAL 1 & T Woosleyt & 2402 \\
\hline
\end{tabular}




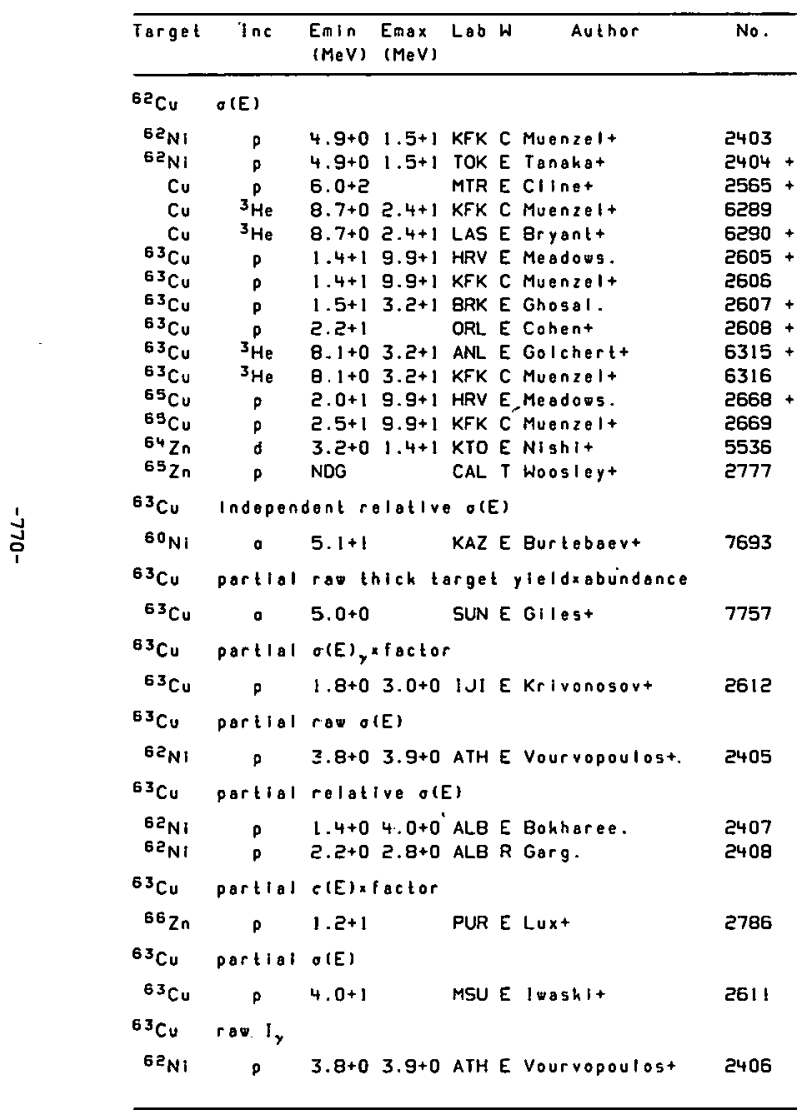

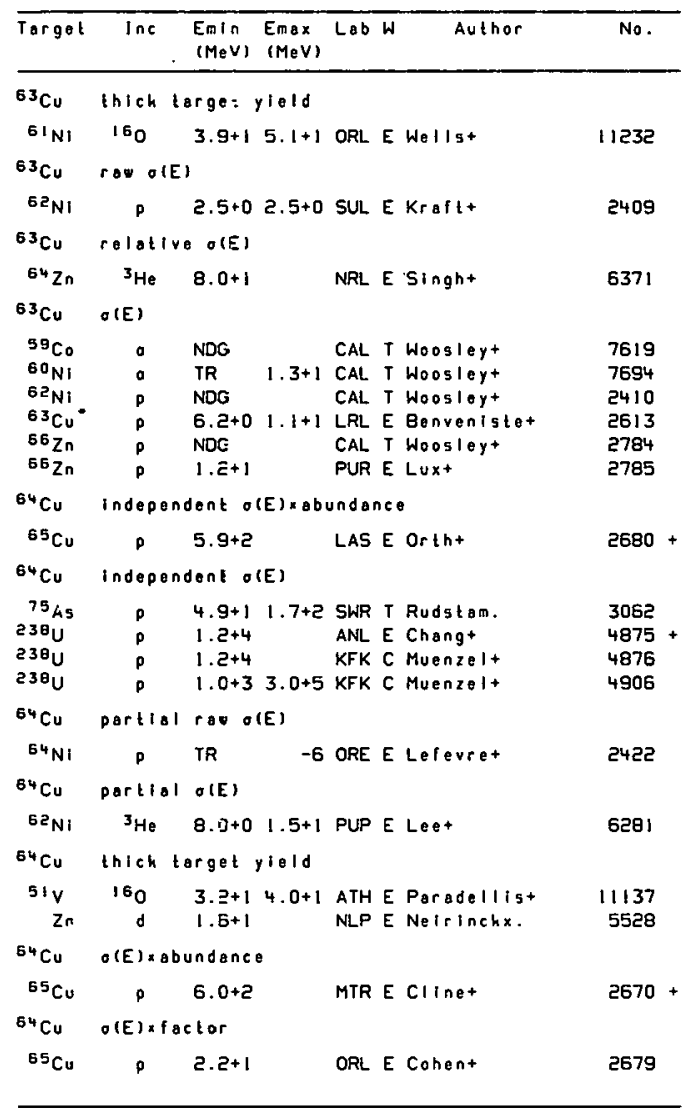




\begin{tabular}{|c|c|c|c|c|c|c|}
\hline Target & $\ln c$ & $\begin{array}{l}\text { Emin } \\
\text { (MeV) }\end{array}$ & $\begin{array}{l}\text { Emax } \\
\text { (MeV. }\end{array}$ & Lab & Author & No. \\
\hline${ }^{64} \mathrm{Cu}$ & $O(E)$ & & & & & \\
\hline${ }^{60} \mathrm{Co}$ & a & NDG & & CAL & T Woosleyt & 7621 \\
\hline$B I_{N i}$ & $a$ & TR & $1.3+1$ & CAL & T Woosleyt & 7708 \\
\hline${ }^{63} \mathrm{Ni}$ & $p$ & NDG & & CAL & T Woosleyt & 2416 \\
\hline $64 \mathrm{NI}$ & p & $T R$ & $1.2+1$ & CAL & T Woosleyt & 2423 \\
\hline${ }^{64} \mathrm{Ni}$ & p & $3.5+0$ & $1.5+1$ & TOK & E Tonakat & $2424+$ \\
\hline${ }^{64} \mathrm{Ni}$ & $p$ & $4.8+0$ & $1.4+1$ & KFK & C Muenzelt & 2425 \\
\hline${ }^{64} \mathrm{Ni}$ & p & $4.8+0$ & $1.4+i$ & TOK & E Tanakat & $2426+$ \\
\hline${ }^{84} \mathrm{Ni}$ & p & $1.2+1$ & & ORL & E Blossert & $2427+$ \\
\hline $\mathrm{Cu}$ & d & $5.8+0$ & $2.4+1$ & CRC & E Dinsict & 5509 \\
\hline $\mathrm{Cu}_{u}$ & d & $1.3+1$ & $5.0+1$ & MCG & E Diksict & 5510 \\
\hline${ }^{63} \mathrm{Cu}$ & d & $4.0+0$ & $1.1+1$ & KFK & C Muenzel+ & 5513 \\
\hline${ }^{63} \mathrm{C} u$ & g & $4.0+0$ & $1.1+i$ & OSA & E Ohamurat & $5514+$ \\
\hline${ }^{63} \mathrm{Cu}$ & $3_{\mathrm{He}}$ & $6.7+0$ & $3.4+1$ & KFK & C Muenzelt & 6317 \\
\hline${ }^{63} \mathrm{Cu}$ & ${ }^{3} \mathrm{He}$ & $6.7+0$ & $2.4+1$ & LAS & E Bryant+ & $6318+$ \\
\hline${ }^{63} \mathrm{Cu}$ & ${ }^{3} \mathrm{He}$ & $2.1+1$ & $3.4+1$ & ANL & E Golchert+ & $6319+$ \\
\hline${ }^{65} \mathrm{Cu}$ & $p$ & TR & $2.4+1$ & ORL & E Cohent & $2671+$ \\
\hline${ }^{65} \mathrm{Cu}$ & $p$ & $9.8+0$ & $9.9+1$ & HRV & E Meadows... & $2672+$ \\
\hline${ }^{65} \mathrm{Cu}$ & $p$ & $9.8+0$ & $9.9+1$ & $\mathrm{KFK}$ & C Muenzelt & $267 \overline{3}$ \\
\hline${ }^{65} \mathrm{Cu}$ & p & $1.9+1$ & $8.5+1$ & MCG & E Meghirt & $2674+$ \\
\hline${ }^{65} \mathrm{Cu}$ & p & $2.2+1$ & & ORL & E Cohent & $2675+$ \\
\hline${ }^{65} \mathrm{Cu}$ & p & $3.5+1$ & $8.5+1$ & MCG & E Goliniert & $2676+$ \\
\hline${ }^{65} \mathrm{Cu}$ & p & $1.3+1$ & $2.5+1$ & BNL & E Collit & $2677+$ \\
\hline${ }^{65} \mathrm{Cu}$ & $p$ & i. $5+1$ & $7.0+1$ & BNL & T Dlvadeenam. & 2678 \\
\hline${ }^{65} \mathrm{Cu}$ & ${ }^{3} \mathrm{He}$ & $1.9+1$ & $3.2+1$ & ANL & E Golchert+ & $6350+$ \\
\hline${ }^{65} \mathrm{Cu}$ & ${ }^{3} \mathrm{He}$ & $1.9+1$ & $3.2+1$ & $\mathrm{KFK}$ & C Muenzelt & 6351 \\
\hline${ }^{65} \mathrm{Cu}$ & 0 & $2.0+1$ & $2.5+1$ & KFK & C Muenzel+ & 7769 \\
\hline${ }^{65} \mathrm{Cu}$ & a & $2.0+1$ & $2.5+1$ & LAS & E Eryant+ & $7770+$ \\
\hline $67 \mathrm{Zn}$ & p & NDG & & CAL & I Woosleyt & 2799 \\
\hline${ }^{65} \mathrm{Cu}$ & partial & raw th & hick ta & arget & tyloldyabundance & \\
\hline${ }^{65} \mathrm{Cu}$ & a & $5.0+0$ & & SUN & E Gilest & 7772 \\
\hline${ }^{65} \mathrm{Cu}$ & partlal & $\sigma(E)_{y} x$ & x factor & & & \\
\hline${ }^{65} \mathrm{Cu}$ & p & $2.0+0$ & $3.0+0$ & IJIE & E Krivonosovt & 2681 \\
\hline${ }^{65} \mathrm{Cu}$ & partial & spectr & rum ave & eroge & - $O(E)$ & \\
\hline${ }^{65} \mathrm{Cu}$ & $a$ & $2.3+1$ & $2.7+1$ & MUU & E Eberhardt & 7771 \\
\hline${ }^{65} \mathrm{Cu}$ & portiol & tow ol & (E) & & & \\
\hline${ }^{64} \mathrm{NI}$ & p & $2.0+0$ & $2.6+0$ & LND & E Erlandssont & 2433 \\
\hline
\end{tabular}

\begin{tabular}{|c|c|c|c|c|c|c|}
\hline Target & $\ln c$ & $\begin{array}{l}E m \mid n \\
(M e V)\end{array}$ & $\begin{array}{l}\text { Emax } \\
(M e V)\end{array}$ & Lab W & Auther & No. \\
\hline${ }^{65} \mathrm{Cu}_{U}$ & partlal & $\sigma(E) \times f$ & factor & & & \\
\hline${ }^{88} \mathrm{Zn}$ & p & $1.2+1$ & & PUR E & $=\operatorname{Lux} x$ & 2821 \\
\hline${ }^{63} \mathrm{Cu}$ & relotive & 01, at & treson & nance & & \\
\hline${ }^{64} \mathrm{NI}$ & p & $3.2+0$ & & DUP E & Kraft+ & 2429 \\
\hline${ }^{63} \mathrm{Cu}$ & product & yield & & & & \\
\hline${ }^{64} \mathrm{NI}$ & p & $1.9+0$ & $3.0+0$ & LND E & Erlandsant & 2431 \\
\hline${ }^{65} \mathrm{Cu}$ & $\cos \theta(E)$ & & & & & \\
\hline${ }^{64} \mathrm{NI}$ & p & $3.1+0$ & $3.3+0$ & DUB E & Eraft+ & 2428 \\
\hline${ }^{6 S} \mathrm{Cu}$ & $\sigma(E) \times f$ ac & ctor & & & & \\
\hline $\begin{array}{l}{ }^{64} \mathrm{N1} \\
6 \mathrm{Bn}^{2}\end{array}$ & p & $\begin{array}{l}\text { NDG } \\
\text { NDG }\end{array}$ & & $\begin{array}{l}\text { CAL E } \\
\text { CAL E }\end{array}$ & $\begin{array}{l}\text { Zyskingt } \\
\text { Zyskindt }\end{array}$ & $\begin{array}{l}2430 \\
2820\end{array}$ \\
\hline${ }^{65} \mathrm{Cu}$ & $\sigma(E)$ & & & & & \\
\hline $\begin{array}{l}61 \mathrm{C}_{0} \\
65^{2}\end{array}$ & a & NDG & & CAL T & Woosleyt & 7622 \\
\hline 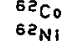 & a & $\begin{array}{l}\text { NDG } \\
\text { TR }\end{array}$ & $1.3+1$ & $\begin{array}{l}\text { CAL } \mathbf{T} \\
\text { CAL }\end{array}$ & $\begin{array}{l}\text { I Woosleyt } \\
\text { T Woosleyt }\end{array}$ & $\begin{array}{l}7623 \\
7714\end{array}$ \\
\hline $64 \mathrm{NI}$ & p & NDG & & CAL T & Toosle?t & 2432 \\
\hline $65_{\mathrm{NI}}$ & $p$ & NDG & & CAL $T$ & Woosle:- & 2437 \\
\hline${ }^{85} \mathrm{Cu}$ & p & $6.0+0$ & $1.2+1$ & LRL E & Hangent & 2682 \\
\hline${ }^{65} \mathrm{Cu}$, & p & $6.2+0$ & $1.1+1$ & LRL E & Benvenistet & 2683 \\
\hline${ }^{65} \mathrm{Cu}$ & p & $7.5+0$ & & MITE & Shoret & 2684 \\
\hline${ }^{68} \mathrm{Zn}$ & p & NDG & & CAL T & Woostest & 2817 \\
\hline${ }^{8} 2 \mathrm{Zn}$ & p & $3.4+0$ & $5.5+0$ & CAL E & Zyshinc. & 2818 \\
\hline $68 \mathrm{Zn}$ & p & $1.2+1$ & & PUR E & Luxt & 2819 \\
\hline${ }^{66} \mathrm{Cu}$ & portiol & $O(\varepsilon)$ & & & & \\
\hline${ }^{64} \mathrm{NI}$ & ${ }^{3} \mathrm{He}$ & $8.0+0$ & $1.5+1$ & PUP E & Leet & 6285 \\
\hline${ }^{66} \mathrm{Cu}$ & $\sigma(E)$ & & & & & \\
\hline${ }^{62} \mathrm{Co}$ & a & NDG & & CAL $T$ & I Woosleyt & 7624 \\
\hline $65_{\mathrm{Ni}}$ & p & NDG & & CAL T & Woosleyt & 2438 \\
\hline${ }^{65} \mathrm{Cu}$ & d & $3.4+0$ & $1 \cdot 1+1$ & KFK C & Muenzelt & 5519 \\
\hline${ }^{65} \mathrm{Cu}$ & $d$ & $3.4+0$ & $1.1+1$ & OSA E & Ohamurat & 5520 \\
\hline${ }^{65} \mathrm{Cu}$ & ${ }^{3} \mathrm{He}$ & $6.0+0$ & $7.0+1$ & ANL E & Golchert+ & 6352 \\
\hline${ }^{69} \mathrm{Zn}$ & $p$ & NDG & & CAL T & Toosleyt & 2857 \\
\hline
\end{tabular}




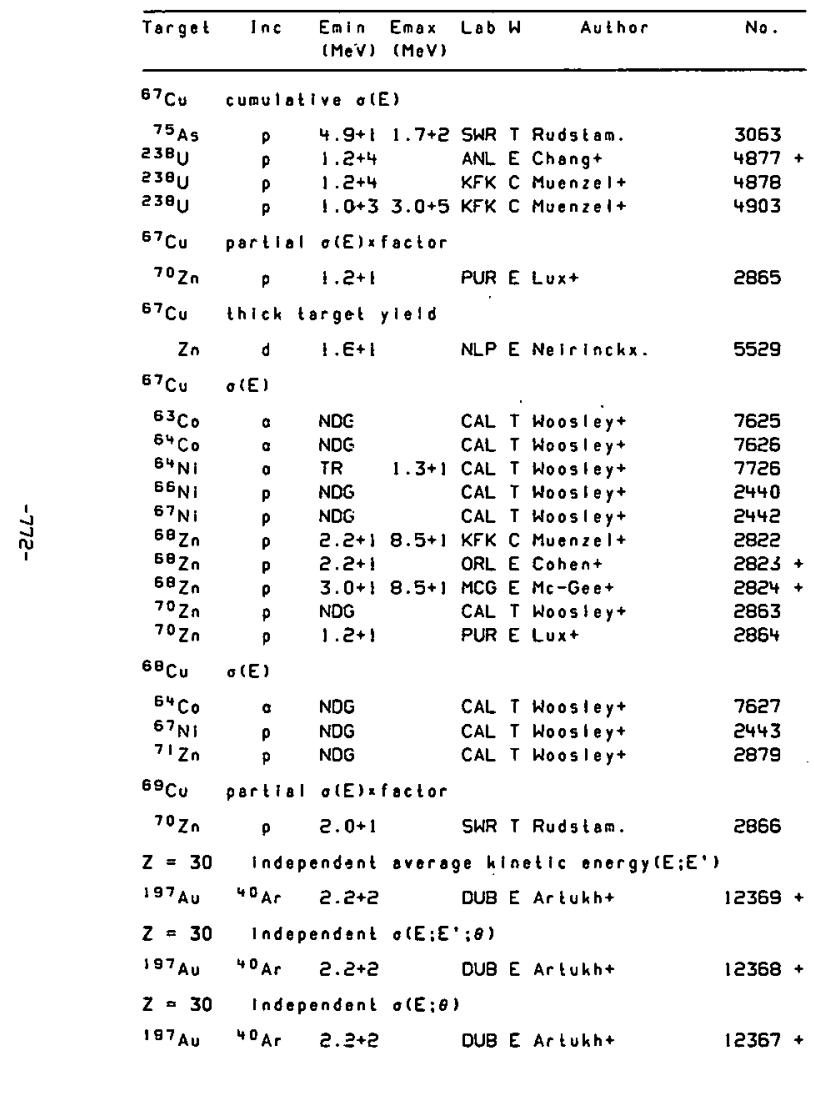

\begin{tabular}{|c|c|c|c|c|c|c|}
\hline Target & $\ln c$ & $\begin{array}{l}\text { Emin } \\
(\mathrm{MeV})\end{array}$ & $\begin{array}{l}\text { Emax } \\
(\operatorname{MeV})\end{array}$ & Lab & Author & No. \\
\hline$z=30$ & \multicolumn{2}{|c|}{ Independent } & $O(E)$ & & & \\
\hline $197 \mathrm{Au}$ & ${ }^{40} \mathrm{Ar}$ & $2.2+5$ & & OuB & E Artuht & $12366+$ \\
\hline$z=30$ & $v_{n}$ & & & & & \\
\hline $\mathrm{Ag}$ & ${ }^{40} \mathrm{Ar}$ & $3.4+2$ & & PAR & R Lefort+ & 12202 \\
\hline $57 \mathrm{Zn}$ & product & yleld & & & & \\
\hline${ }^{4}{ }^{\circ} \mathrm{Ca}$ & $20 \mathrm{Ne}$ & NDG & & BRK & R Cernyt & 11850 \\
\hline${ }^{37} \mathrm{Zn}$ & $\theta(E)$ & & & & & \\
\hline${ }^{40} \mathrm{Ca}$ & ${ }^{20} \mathrm{Ne}$ & NDG & & BRK & R Cerny. & 11849 \\
\hline $5 \theta 2 n$ & $O(E)$ & & & & & \\
\hline${ }^{54} \mathrm{NI}$ & a & NDG & & CAL & T Woosleyt & 7640 \\
\hline${ }^{57} \mathrm{Cu}$ & $p$ & NDG & & CAL & T Woosleyt & 2579 \\
\hline${ }^{59} \mathrm{Zn}$ & $O(E)$ & & & & & \\
\hline${ }^{55} \mathrm{Ni}$ & c & NDG & & CAL & T Woosleyt & 7642 \\
\hline${ }^{56} \mathrm{Cu}$ & : & NDG & & CAL & T Woosleyt & 7746 \\
\hline${ }^{30} \mathrm{Cu}$ & $p$ & NDG & & CAL & T Woosleyt & 2580 \\
\hline $60 \mathrm{Zn}$ & $\theta(E)$ & & & & & \\
\hline${ }^{56} \mathrm{NI}$ & a & NDG & & CAL & T Woosleyt & 764.4 \\
\hline $\mathrm{Cu}$ & o & $4.8+1$ & & HIR & E Yoshizavat & 2566 \\
\hline${ }^{57} \mathrm{Cu}$ & $\therefore$ & NOG & & CAL & $T$ Woosleyt & 7748 \\
\hline.$^{59} \mathrm{Cu}$ & $\rho$ & NDG & & CAL & T Woosley+ & 2582 \\
\hline${ }^{63} \mathrm{Cu}$ & 0 & $4.8+1$ & & HIR & $E$ Nomat & 2614 \\
\hline $63 \mathrm{Ga}$ & 0 & NDG & & CAL & T Woosleyt & 2885 \\
\hline $6 ! Z_{n}$ & $O(E) \times a b u$ & undance & exiacto & & & \\
\hline${ }^{63} \mathrm{Cu}$ & $D$ & $3.0+1$ & $5.2+1$ & HIR & E Nomat & $2616+$ \\
\hline $612 n$ & relallue & - $O(E)$ & & & & \\
\hline $\begin{array}{l}5 \theta_{N 1} \\
{ }^{64} \mathrm{Zn}\end{array}$ & $\begin{array}{l}{ }^{3} \mathrm{He} \\
{ }^{3} \mathrm{He}\end{array}$ & $\begin{array}{l}8.0+1 \\
8.0+1\end{array}$ & & $\begin{array}{l}\text { NRL } \\
\text { NRL }\end{array}$ & $\begin{array}{l}\text { E singht } \\
\text { E singht }\end{array}$ & $\begin{array}{l}6278 \\
6371\end{array}$ \\
\hline $6 ! \mathrm{Zn}$ & $\theta(E)$ & & & & & \\
\hline $57_{N I}$ & . & NDG & & CAL & T Woosleyt & 7645 \\
\hline${ }^{5 a^{N I}}$ & 。 & $\mathrm{IR}$ & $1.4+1$ & CAL & T Woosleyt & 7678 \\
\hline $\mathrm{Cu}$ & p & $3.0+1$ & $4.8+1$ & HIR & E Yoshizanat & 2567 \\
\hline${ }^{58} \mathrm{Cu}$ & : & NDG & & CAL & T Woosleyt & 7750 \\
\hline
\end{tabular}




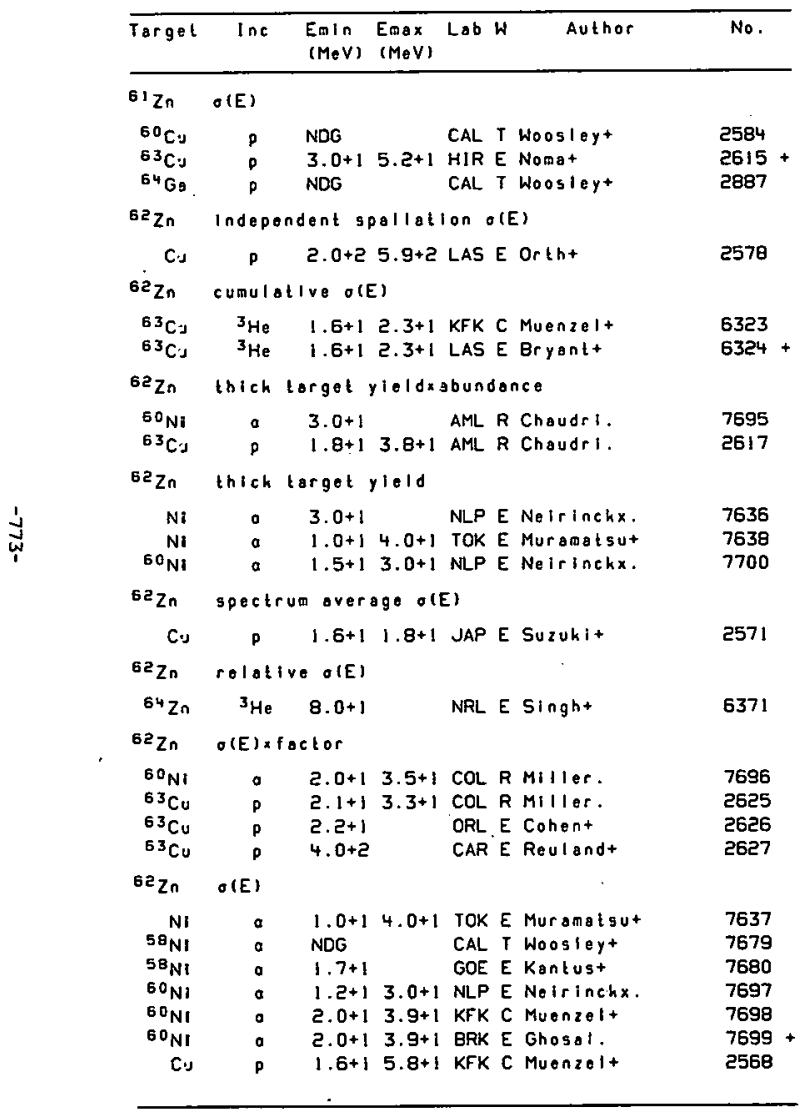

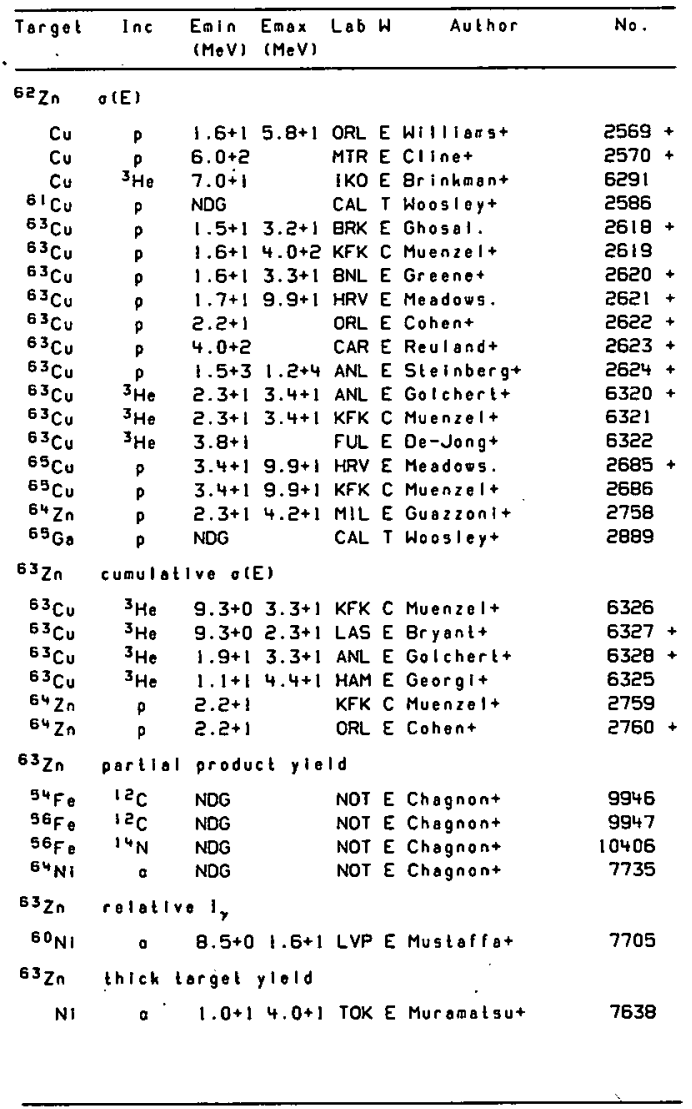




\begin{tabular}{|c|c|c|c|c|c|c|}
\hline Target & $\ln c$ & $\begin{array}{l}\text { Emin } \\
(\mathrm{MeV})\end{array}$ & $\begin{array}{l}E_{\max } \\
(\mathrm{MeV})\end{array}$ & Lab & Author & No. \\
\hline${ }^{63} \mathrm{Zn}$ & \multicolumn{6}{|c|}{ relotive $\sigma(E)$} \\
\hline${ }^{64} \mathrm{Zn}$ & ${ }^{3} \mathrm{He}$ & B. $0+1$ & & NRL & E singht & 6371 \\
\hline${ }^{63} \mathrm{Zn}$ & \multicolumn{6}{|c|}{ o(E) a factor } \\
\hline $\begin{array}{l}\mathrm{Cu}_{u} \\
{ }^{63} \mathrm{Cu}_{\mathrm{C}}\end{array}$ & $\begin{array}{l}p \\
p\end{array}$ & $\begin{array}{l}3.0+1 \\
4.0+2\end{array}$ & $5.2+1$ & $\begin{array}{l}\text { HIR } \\
\text { CAR }\end{array}$ & $\begin{array}{l}\text { E Nomat } \\
\text { E Reulandt }\end{array}$ & $\begin{array}{l}2573+ \\
2646\end{array}$ \\
\hline $63 \mathrm{Zn}$ & \multicolumn{6}{|l|}{$\sigma(E)$} \\
\hline NI & $a$ & $1.0+1$ & $4.0+1$ & TOK & E Muramatgut & 7637 \\
\hline${ }^{59} \mathrm{Ni}$ & $a$ & NOG & & CAL & T Woosleyt & 7684 \\
\hline $60 \mathrm{NI}$ & 0 & $T R$ & $1.3+1$ & CAL & T Woosleyt & 7701 \\
\hline $60 \mathrm{Ni}$ & $a$ & $1.3+1$ & $3.9+1$ & BRK & E Ghosal. & $7702+$ \\
\hline $60 \mathrm{Ni}$ & 0 & $1.9+1$ & $2.4+1$ & KFK & C Muenzelt & 7703 \\
\hline $60^{N I}$ & 0 & $1.9+1$ & $2.4+1$ & MUN & E Hillet & $7704+$ \\
\hline Cu & p & $6.0+5$ & & MTR & E clinet & $2572+$ \\
\hline & p & $2.5+1$ & $5.2+1$ & $H I R$ & E Yoshizang+ & 2574 \\
\hline${ }^{62} \mathrm{Cu}$ & p & NDG & & CAL & T Woosleyt & 2588 \\
\hline${ }^{63} \mathrm{Cu}$ & p & NDG & & BNL & R Goldberg. & 2628 \\
\hline${ }^{63} \mathrm{Cu}$ & p & $T R$ & $1.2+1$ & CAL & T Woosleyt & 2629 \\
\hline${ }^{63} \mathrm{Cu}$ & p & $T R$ & $5.8+0$ & ORL & E Johnsont & $2630+$ \\
\hline${ }^{63} \mathrm{Cu}$ & p & $3.9+0$ & $2.2+1$ & BRK & E Ghosal. & $2631+$ \\
\hline${ }^{63} \mathrm{Cu}$ & $p$ & $4.0+0$ & $2.5+1$ & BNL & E Collet & $2632+$ \\
\hline${ }^{63} \mathrm{Cu}$ & p & $4.2+0$ & $6 \cdot 3+0$ & ETH & E Blasert & $2633+$ \\
\hline${ }^{83} \mathrm{Cu}$ & p & $4.2+0$ & $9.9+1$ & KFK & C Muenzelt & 2634 \\
\hline${ }^{63} \mathrm{Cu}$ & p & $4.3+0$ & $6.6+0$ & ROC & E Toketonit & $2635+$ \\
\hline${ }^{63} \mathrm{Cu}$ & p & $4.5+0$ & $1.0+1$ & ANL & E Wingt & $2636+$ \\
\hline${ }^{63} \mathrm{Cu}$ & 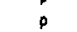 & $4.9+0$ & $1.0+1$ & CLA & E Howe. & $2637+$ \\
\hline${ }^{63} \mathrm{Cu}$ & $\rho$ & $5.0+0$ & $9.9+1$ & HRV & E Moodous. & $2638+$ \\
\hline${ }^{63} \mathrm{Cu}$ & p & $6.0+0$ & $1.1+1$ & LRL & E Hansent & $2639+$ \\
\hline${ }^{63} \mathrm{Cu}$ & p & $6.8+0$ & & osu & E Dellt & $2640+$ \\
\hline${ }^{B 3} \mathrm{Cu}$ & $p$ & $8.7+0$ & $1.6+1$ & MUN & E Hillet & $2641+$ \\
\hline${ }^{53} \mathrm{Cu}$ & $p$ & $9.3+0$ & & $B I R$ & E Chacheltt+ & $2642+$ \\
\hline${ }^{83} \mathrm{Cu}$ & p & $9.9+0$ & & HAR & E Jonest & $2643+$ \\
\hline${ }^{63} \mathrm{Cu}$ & p & $4.0+2$ & & CAR & E Roulandt & $2644+$ \\
\hline${ }^{63} \mathrm{Cu}$ & p & $1.5+3$ & $1.2+4$ & ANL & E Steinberg+ & $2645+$ \\
\hline${ }^{63} \mathrm{Cu}$ & d & $6.8+0$ & $1.1+1$ & KFK & C Muenzelto & 5515 \\
\hline${ }^{B}{ }^{3} C_{U}$ & d & $6.8+0$ & $1,1+1$ & OSA & E Ohamurat & 5516 \\
\hline${ }^{69} \mathrm{Cu}$ & $p$ & $3.0+1$ & $9.9+1$ & HRV & E Moodous. & 2687 \\
\hline${ }^{65} \mathrm{Cu}$ & $p$ & $3.0+1$ & $9.9+1$ & KFK & C Muenzelt & 2688 \\
\hline $\mathrm{Zn}$ & a & $1.0+1$ & $4.0+1$ & TOK & E Nagamet & 7800 \\
\hline${ }^{64} \mathrm{Zn}$ & p & $2.3+1$ & $4.2+1$ & MIL & E Guazzonit & $276 !$ \\
\hline
\end{tabular}

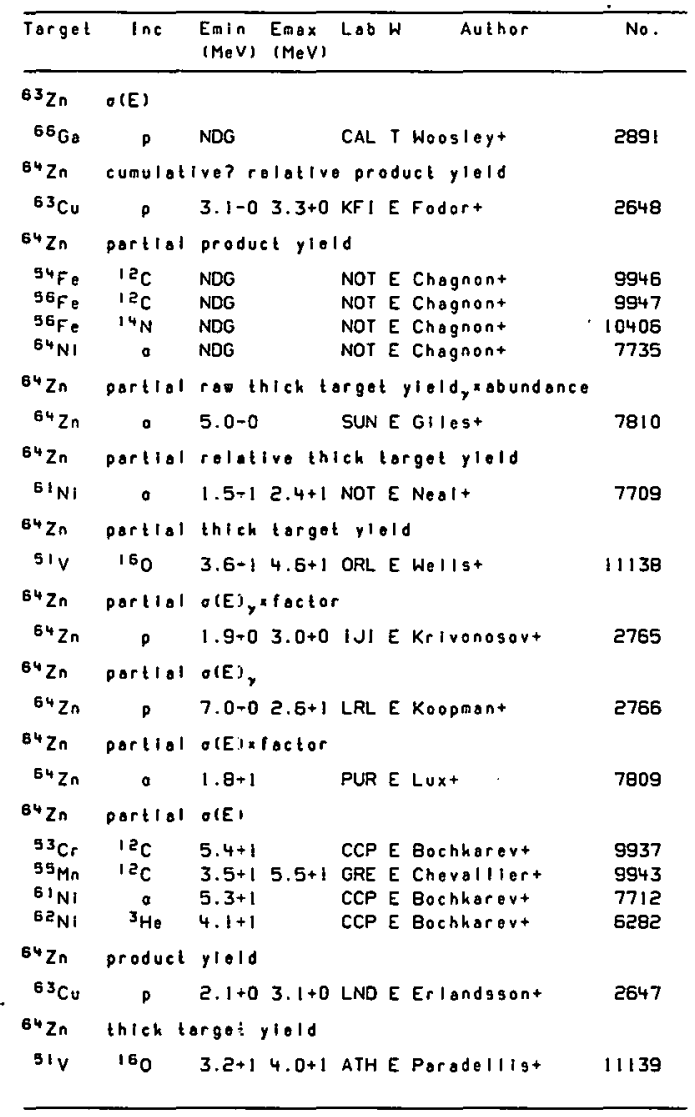




\begin{tabular}{|c|c|c|c|c|c|c|}
\hline Target & $\ln c$ & $\begin{array}{l}\text { Emin } \\
(M e V)\end{array}$ & $\begin{array}{l}\text { Emax } \\
(\operatorname{MeV})\end{array}$ & Lab & Author & No. \\
\hline${ }^{64} \mathrm{Zn}$ & \multicolumn{6}{|c|}{ relative o(E) } \\
\hline $64 \mathrm{Zn}$ & $\stackrel{\mathrm{He}}{\mathrm{He}}$ & $8.0+1$ & & NRL & E singht & $637 l$ \\
\hline${ }^{64} \mathrm{Zn}$ & \multicolumn{6}{|c|}{$\sigma(E) \times f a c t o r$} \\
\hline${ }^{65} \mathrm{Cu}$ & p & $2.2+1$ & & ORL & E Cohent & 2689 \\
\hline${ }^{64} \mathrm{Zn}$ & \multicolumn{6}{|l|}{$\theta(E)$} \\
\hline${ }^{53} \mathrm{Cr}$ & $12_{C}$ & $4 \cdot 1+1$ & & CCP & E Bochkarevt & 9938 \\
\hline $60^{\circ} \mathrm{NI}$ & 0 & NDG & & CAL & T Woosleyt & 7706 \\
\hline $6 I_{\mathrm{N}}$ & 0 & & $1.3+1$ & CAL & T Woosleyt & 7710 \\
\hline $61^{N i}$ & $a$ & $5 \cdot 3+1$ & & $\mathrm{CCP}$ & E Bochharevt & 7711 \\
\hline $62_{\mathrm{NI}}$ & ${ }^{3} \mathrm{He}$ & $4.1+1$ & & $\mathrm{CCP}$ & E Bochkarevt & 6283 \\
\hline${ }^{63} \mathrm{Cu}$ & p & NDG & & CAL & T Woosleyt & 2649 \\
\hline${ }^{63} \mathrm{Cu}_{\mathrm{u}}$ & $a$ & & $4.8+1$ & BRK & E Gonzalez-VIdal+ & 7758 \\
\hline${ }^{64} \mathrm{Zn}$ & $p$ & $7.0+0$ & $2.6+1$ & LRL & E koopmant & 2762 \\
\hline${ }^{64} \mathrm{Zn}$ & p & $7.0+0$ & $2.6+1$ & LRL & E koopmant & 2763 \\
\hline${ }^{64} \mathrm{Zn}$ & p & $1.3+1$ & & PUR & E Lux+ & 2764 \\
\hline${ }^{64} \mathrm{Zn}$ & $a$ & $1.8+1$ & & PUR & E Luxt+ & 7808 \\
\hline $67 \mathrm{Ga}$ & p & NDG & & CAL & Toosleyt & 2893 \\
\hline${ }^{85} \mathrm{Zn}$ & \multicolumn{6}{|c|}{ cumulative? $O(E)$} \\
\hline $\begin{array}{l}68 Z_{n} \\
6 Z_{n}\end{array}$ & $p$ & $\begin{array}{l}3.0+1 \\
4.0+1\end{array}$ & $\begin{array}{l}8.5+1 \\
8.5+1\end{array}$ & $\begin{array}{l}\text { MCG } \\
\text { KFK }\end{array}$ & $\begin{array}{l}\text { E Mc-Geet } \\
\text { C Muenzelt }\end{array}$ & $\begin{array}{l}2825+ \\
2826\end{array}$ \\
\hline${ }^{65} 2 \mathrm{n}$ & \multicolumn{6}{|c|}{ cumulative $O(E) \times$ abundance? } \\
\hline${ }^{63} \mathrm{Cu}$ & o. & $1.5+1$ & $2.3+1$ & sta & E Lint & $7759+$ \\
\hline${ }^{65} \mathrm{Zn}$ & \multicolumn{6}{|c|}{ cumulatlve $\sigma(E)$} \\
\hline $\begin{array}{l}66 \mathrm{Zn} \\
{ }^{66} \mathrm{Zn} \\
{ }^{79} \mathrm{As}\end{array}$ & $\begin{array}{l}p \\
p \\
p\end{array}$ & $\begin{array}{l}2.2+1 \\
2 \cdot 2+1 \\
4 \cdot 9+1\end{array}$ & $1.7+2$ & $\begin{array}{l}\text { KFK } \\
\text { ORL } \\
\text { SWR }\end{array}$ & $\begin{array}{l}\text { C Muenzel+ } \\
\text { E Cohent } \\
\text { T Rudgtam. }\end{array}$ & $\begin{array}{l}2787 \\
2788+ \\
3063\end{array}$ \\
\hline${ }^{85} \mathrm{Zn}$ & \multicolumn{6}{|c|}{ portlol product yiold } \\
\hline${ }^{54} \mathrm{Fe}$ & $12_{C}$ & NDG & & & E Chognont & \\
\hline${ }^{56} \mathrm{Fe}$ & $12_{c}$ & NOG & & NOT & E Chognont & 9947 \\
\hline${ }^{56} \mathrm{Fo}$ & ${ }^{14} \mathrm{~N}$ & NDG & & & E Chagnont & 10406 \\
\hline${ }^{64} \mathrm{NI}$ & 3 & NDG & & NOT & E Chognont & 7735 \\
\hline${ }^{63} \mathrm{Zn}$ & \multicolumn{6}{|c|}{ partlal relollve thich target yield } \\
\hline $\mathrm{BE}_{\mathrm{Ni}}$ & $=$ & $1.5+1$ & $2.4+1$ & NOT & E Neol+ & 7719 \\
\hline
\end{tabular}

\begin{tabular}{|c|c|c|c|c|c|c|}
\hline Target & Inc & $\begin{array}{l}\text { Ealn } \\
(\mathrm{MeV})\end{array}$ & $\begin{array}{l}\text { Emax } \\
(\operatorname{Mev})\end{array}$ & Lab & Author & No. \\
\hline${ }^{89} \mathrm{Zn}$ & partlol & rov of & (E) & & & \\
\hline${ }^{65} \mathrm{Cu}$ & p & $T R$ & +0 & ORE & E Leferret & 2690 \\
\hline${ }^{65} \mathrm{Zn}$ & relative & - $\mathrm{l}_{\gamma}$ & & & & \\
\hline $62_{\mathrm{Ni}}$ & $a$ & $8.0+0$ & $1.4+1$ & LVP & E Kogant & 7720 \\
\hline${ }^{65} \mathrm{Zn}$ & $\mathrm{I}_{y}$ & & & & & \\
\hline${ }_{65} \mathrm{Ni}^{1}$ & $a$ & $1.0+1$ & $1.2+1$ & LVP & E Jonest & 7718 \\
\hline${ }^{65} \mathrm{Zn}$ & product & $\sin 10 x$ & ifactor & & & \\
\hline${ }^{65} \mathrm{Cu}$ & $p$ & $2.2+0$ & & WMU I & M Bernsteln. & 2709 \\
\hline${ }^{65} \mathrm{Zn}$ & thlok to & $\operatorname{argat} y$ & $y / .19$ & & & \\
\hline $\begin{array}{l}51 \mathrm{~V} \\
\mathrm{NI}\end{array}$ & $\begin{array}{r}160 \\
a\end{array}$ & $\begin{array}{l}3.2+1 \\
1.0+1\end{array}$ & $\begin{array}{l}4.0+1 \\
4.0+1\end{array}$ & $\begin{array}{l}\text { ATH } \\
\text { TOK }\end{array}$ & $\begin{array}{l}\text { E Paradelliga } \\
\text { E Muramatsat }\end{array}$ & $\begin{array}{r}11140 \\
7638\end{array}$ \\
\hline${ }_{61}{ }^{N}$ & 160 & $4.1+1$ & $5 \cdot 1+1$ & ORL & E Wollst & 11233 \\
\hline$Z n$ & $a$ & $5.0+0$ & $3.8+1$ & TOK & E Nagamet & 7801 \\
\hline${ }^{63} \mathrm{Zn}$ & averago & $\sigma(E)$ & & & & \\
\hline${ }^{65} \mathrm{Cu}$ & $p$ & $2.0+0$ & $2.2+0$ & ORL & E Johnsont & $27 ! 1$ \\
\hline${ }^{55} \mathrm{Zn}$ & $O(E) \times f a c$ & ctor & & & & \\
\hline${ }^{65} \mathrm{Cu}$ & $p$ & $1.4+1$ & $2.2+1$ & ORL & E Willianst & 2710 \\
\hline${ }^{69} \mathrm{Zn}$ & $\sigma(E)$ & & & & & \\
\hline 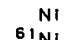 & $a$ & $1.0+1$ & $4.0+1$ & TOK & E Muramatsat & 7637 \\
\hline$R_{61} I_{1}$ & & NDO & & CAL & I Woo & 7713 \\
\hline $61 \mathrm{NI}$ & ${ }^{16} 0$ & $4.6+1$ & & ORL 1 & M Wellst & 11234 \\
\hline${ }^{62} \mathrm{Ni}$ & a & TR & $1.3+1$ & CAL & T Woöslept & 7 \\
\hline $62^{N I}$ & a & $4.6+0$ & $7.0+0$ & CAL & E Dayidsant & 7716 \\
\hline${ }^{6} \mathrm{NI}$ & $a$ & $6.9+0$ & $8.8+0$ & CAL & E Zyshindt & 7717 \\
\hline $\mathrm{Cu}$ & & $8.0+0$ & $1.1+1$ & CCP & R Zalolotint & 2575 \\
\hline${ }^{63} \mathrm{Cu}$ & ${ }^{3} \mathrm{He}$ & $7.0+0$ & $2.5+1$ & KFK & C Muenzel+ & \\
\hline${ }^{63} \mathrm{Cu}$ & ${ }^{3} \mathrm{He}$ & $7.0+0$ & $2.5+1$ & LAS & E Bryant+ & 63 \\
\hline${ }^{64} \mathrm{Cu}$ & p & NDG & & CAL & I Wooslest & \\
\hline${ }^{85} \mathrm{Cu}$ & P & TR & $1.3+1$ & CAL & I Woosleyt & 2691 \\
\hline${ }^{65} \mathrm{Cu}$ & $\mathrm{p}$ & $2.0+0$ & $4.6+11$ & MIL & T Gadialit & 2692 \\
\hline${ }^{65} \mathrm{Cu}$ & $p$ & $2.2+0$ & $3.4+1$ & KFK & C Muenzel+ & 2693 \\
\hline${ }^{65} \mathrm{Cu}$ & D & $2.2+0$ & $5.8+0$ & ORL & E Johnsor.t & 2694 \\
\hline${ }^{65} \mathrm{Cu}$ & $p$ & $2 . \theta+0$ & $6.3+0$ & ETH & E Blagert & 269 \\
\hline${ }^{83} \mathrm{Cu}$ & $p$ & $2.9+0$ & $2.5+1$ & BNL & E Collot & 2696 \\
\hline
\end{tabular}




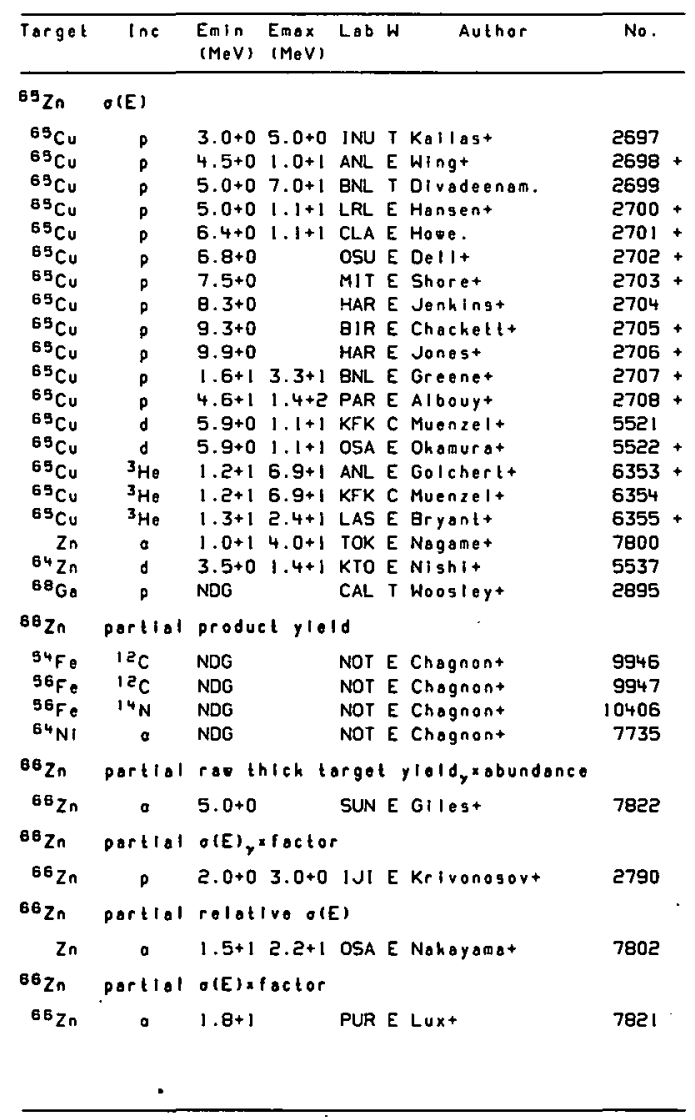

\begin{tabular}{|c|c|c|c|c|c|c|}
\hline Target & $\ln c$ & $\begin{array}{l}\text { Emin } \\
(\operatorname{MeV})\end{array}$ & $\begin{array}{l}\text { Emax } \\
(\mathrm{MeV})\end{array}$ & Lab & Author & No. \\
\hline${ }^{66} \mathrm{Zn}$ & relallue & - $\mathrm{I}_{y}$ & & & & \\
\hline $64 \mathrm{Ni}$ & 。 & $1.9+1$ & $2.5+1$ & NOT & E Neal- & 7727 \\
\hline${ }^{8 B} \mathrm{Zn}$ & product & yield & & & & \\
\hline${ }^{69} \mathrm{Cu}$ & $\rho$ & $2.2+0$ & $2.8+0$ & LND & E Erlandssont & 2714 \\
\hline${ }^{8 B} \mathrm{Zn}$ & $\theta(E)$ & & & & & \\
\hline $62_{\mathrm{Ni}}$ & 0 & NDG & & CAL & T Woosloyt & 7721 \\
\hline $62_{\mathrm{Ni}}$ & 0 & $4.8+0$ & $8.7+0$ & CAL & E Zyskindt & 7722 \\
\hline${ }^{63} \mathrm{Cu}$ & a & TR & $1.3+1$ & CAL & T Woosleyt & 7760 \\
\hline${ }^{65} \mathrm{Cu}$ & p & NDG & & CAL & $T$ Woosleyt & 2712 \\
\hline${ }^{55} \mathrm{Cu}$ & p & $1.1+0$ & $3.6+0$ & AML & M 5withooskit & 2713 \\
\hline${ }^{55} \mathrm{Cu}$ & 0 & TR & $4.8+1$ & BRK & E Gonzalez-VIdal + & 7773 \\
\hline${ }^{65} \mathrm{Cu}$ & 160 & $3.9+1$ & $5.1+1$ & ORL & M Wellst & $1 ! 297$ \\
\hline${ }^{66} \mathrm{Cu}$ & p & NDG & & CAL & T Woosleyt & 2716 \\
\hline $66 \mathrm{Zn}$ & $p$ & $1.3+1$ & & PUR & $E$ Luxt & 2789 \\
\hline $66 \mathrm{Zn}$ & 。 & $1.8+1$ & & PUR & E Luxt & 7820 \\
\hline $69 \mathrm{Ga}$ & $p$ & NDG & & CAL & T Woosleyt & 2898 \\
\hline${ }^{67} \mathrm{Zn}$ & relotive & - $I_{r}$ & & & & \\
\hline $64 \mathrm{NI}$ & ${ }^{6} \mathrm{LI}$ & NDG & & NOT & E Neol+ & 9211 \\
\hline $67 \mathrm{Zn}$ & portiol & produc & et ytel & & & \\
\hline${ }^{54} \mathrm{Fe}$ & ${ }^{12} \mathrm{C}$ & NDG & & NOT & E Chagnont & 9946 \\
\hline${ }^{56} \mathrm{Fe}$ & $\operatorname{lac}_{\mathrm{C}}$ & NDG & & NOT & E Chegnont & 9947 \\
\hline $56 \mathrm{Fe}$ & $14 \mathrm{~N}$ & NDG & & NOT & E Chognont & 10408 \\
\hline${ }^{64} \mathrm{Ni}$ & 0 & NOG & & NOT & E Chegnont & 7735 \\
\hline${ }^{87} \mathrm{Zn}$ & portlol & ros th & hich to & arget & tyleldyabundance & \\
\hline $67 Z n$ & 。 & $5.0+0$ & & SUN & E Gllest & 7827 \\
\hline $67 \mathrm{Zn}$ & portlal & $O(E)_{y}$ & foctor & & & \\
\hline${ }^{67} \mathrm{Zn}$ & p & $1.1 \rightarrow 0$ & $3.0+0$ & IJI & E Krivonosort & 2800 \\
\hline $872 n$ & rolotive & - $\mathrm{I}_{y}$ & & & & \\
\hline $\begin{array}{l}64 \mathrm{NI} \\
64 \mathrm{NI}\end{array}$ & : & $\begin{array}{l}6.5+0 \\
8.2+0\end{array}$ & $1.7+1$ & $\begin{array}{l}\text { LVP } \\
\text { LYO }\end{array}$ & $\begin{array}{l}\text { E Lorniet } \\
\text { E Duffalt. }\end{array}$ & $\begin{array}{l}7728 \\
7729\end{array}$ \\
\hline${ }^{64} \mathrm{NI}$ & 0 & $1.5+1$ & $2.3+1$ & NOT & E Neal+ & 7730 \\
\hline${ }^{67} \mathrm{Zn}$ & $O(E)$ I $A C$ & ctor & & & & \\
\hline${ }^{6 \theta} \mathrm{Zn}$ & p & $2.2+1$ & & ORL & E Cohent & 2827 \\
\hline
\end{tabular}




\begin{tabular}{|c|c|c|c|c|c|c|}
\hline Target & $\operatorname{lnc}$ & $\begin{array}{l}E m \mid n \\
(M \circ V)\end{array}$ & $\begin{array}{l}\text { Emax } L \\
(\mathrm{MeV})\end{array}$ & Lab $k$ & Author & No. \\
\hline${ }^{67} \mathrm{Zn}$ & $O(E)$ & & & & & \\
\hline${ }^{63} \mathrm{Ni}$ & 0 & NDG & & CAL T & T Woosleyt & 7724 \\
\hline $464 \mathrm{Ni}$ & a & $T R$ & $1.2+: 0$ & CAL $T$ & T Woosleyt & 7731 \\
\hline $64 \mathrm{Ni}$ & a & $5.4+0$ & $7.4+00$ & CAL E & E Zyshindt & 7732 \\
\hline${ }^{66} \mathrm{Cu}$ & p & NDG & & CAL T & T Woosleyt & 2717 \\
\hline${ }^{70} \mathrm{Ga}$ & 0 & NDG & & CAL T & T Woosleyt & 2913 \\
\hline${ }^{68} \mathrm{Zn}$ & portlal & row th & hick tar & argot & yieldyabundence & \\
\hline${ }^{6 \theta} \mathrm{Zn}$ & a & $5.0+0$ & & SUN E & E Griest & 7833 \\
\hline${ }^{60} \mathrm{Zn}$ & partlat & $\sigma(E)^{y} x$ & $x$ factor & & & \\
\hline${ }^{68} \mathrm{Zn}$ & $p$ & $2.0+0$ & $3.0+0$ & IJIE & E Krlvonosovt & 2829 \\
\hline${ }^{68} \mathrm{Zn}$ & partial & $O(E) \times 1$ & factor & & & \\
\hline${ }^{68} \mathrm{Zn}$ & 0 & $1.8+1$ & & PUR E & E Lux+ & 7832 \\
\hline${ }^{68} \mathrm{Zn}$ & $\theta(E)$ & & & & & \\
\hline $64 \mathrm{Ni}$ & a & NDG & & CAL T & T Woosleyt & 7733 \\
\hline${ }^{64} \mathrm{Ni}$ & a & $4.6+0$ & $7.2+0$ & CAL E & E Zyshindt & 7734 \\
\hline${ }^{65} \mathrm{Cu}$ & a & & $1.4+10$ & CAL $I$ & I Woosleyt & 7774 \\
\hline${ }^{67} \mathrm{Cu}$ & $p$ & NDG & & CAL $\mathrm{I}$ & I Woosleyt & 2719 \\
\hline${ }^{68} \mathrm{Cu}$ & p & NDG & & CAL $\mathrm{I}$ & I Woosley+ & 2721 \\
\hline${ }^{68} \mathrm{Zn}$ & D & $1.3+1$ & & PUR E & E Luxt & 2828 \\
\hline $68 \mathrm{Zn}$ & a & $1.8+1$ & & PUR E & E Luxt & 7831 \\
\hline 'IGá & p & NDG & & CAL T & T Woosleyt & 2917 \\
\hline $69 \mathrm{Zn}$ & cumulati & Ive ole & & & & \\
\hline${ }^{75} \mathrm{As}_{5}$ & p & $4.9+1$ & $1.7+2$ & SWR T & T Rudstom. & 3063 \\
\hline${ }^{69} \mathrm{Zn}$ & poritiol & $\sigma(E) \times r$ & factor & & & \\
\hline${ }^{70} \mathrm{Zn}$ & p & $2 \cdot 0+1$ & & SWR T & T Rudstam. & $286 ?$ \\
\hline${ }^{69} \mathrm{Zn}$ & $\sigma(E)$ & & & & & \\
\hline $65_{\mathrm{Ni}}$ & a & NDO & & CAL T & T Woosleyt & 7736 \\
\hline${ }^{68} \mathrm{Cu}$ & p & NDG & & CAL T & T Woosleyt & 2722 \\
\hline${ }^{69} \mathrm{Cu}$ & $p$ & NDG & & CAL $T$ & T Hoosleyt & 2724 \\
\hline${ }^{72} \mathrm{Ga}$ & p & NDG & & CAL T & T Woosloyt & 2932 \\
\hline $69: \mathrm{Zn}$ & $\theta(E)$ & & & & & \\
\hline $71 G_{0}$ & $\rho$ & $2.2+1$ & & KFK C & C Muenze1t & 2918 \\
\hline 7100 & D & $2.2+1$ & & ORLE & E Mc-Cormlcht & $2919+$ \\
\hline
\end{tabular}

\begin{tabular}{|c|c|c|c|c|c|c|}
\hline Target & Ine & $\begin{array}{l}E m \mid n \\
(M e V)\end{array}$ & $\begin{array}{l}\text { Emax } \\
(\operatorname{MeV})\end{array}$ & Lab W & Acthor & No. \\
\hline $69 \cdot \mathrm{Zn}$ & $\sigma(E)$ & & & & & \\
\hline $\begin{array}{l}71 G a \\
71 G a\end{array}$ & $p$ & $\begin{array}{l}2.2+1 \\
2.2+1\end{array}$ & & $\begin{array}{l}\text { KFK C } \\
\text { ÖRL E }\end{array}$ & $\begin{array}{l}\text { Muenzelt } \\
\text { Mc-Cormick: }\end{array}$ & $\begin{array}{l}2920 \\
2921+\end{array}$ \\
\hline $69 \cdot 1 \cdot 2 n$ & rotio & - $O(E)$ & & & & \\
\hline $\begin{array}{l}{ }^{70} \mathrm{Zn} \\
{ }^{70} \mathrm{Ga}\end{array}$ & 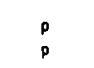 & $\begin{array}{l}2.5+1 \\
2.5+1\end{array}$ & $\begin{array}{l}1.5+2 \\
1.5+2\end{array}$ & $\begin{array}{l}\text { SWR T } \\
\text { SWR T }\end{array}$ & $\begin{array}{l}\text { Rudstam. } \\
\text { Rudstam. }\end{array}$ & $\begin{array}{l}2868 \\
2914\end{array}$ \\
\hline $70 \mathrm{Zn}$ & partial & $O(E)_{y} x$ & afactor & & & \\
\hline $70^{\circ} \mathrm{Zn}$ & 8 & $1.8+0$ & $3.0+0$ & IJIE & Krivonosort & 2871 \\
\hline $70 \mathrm{Zn}$ & partial & $\sigma(E)=1$ & factor & & & \\
\hline $\begin{array}{l}70 \mathrm{Zn} \\
70 \mathrm{Zn}\end{array}$ & $\begin{array}{l}p \\
q\end{array}$ & $\begin{array}{l}2.0+1 \\
1.8+1\end{array}$ & & $\begin{array}{l}\text { SWR T } \\
\text { PUR E }\end{array}$ & $\begin{array}{l}\text { Rudstan. } \\
\text { Luxt }\end{array}$ & $\begin{array}{l}2869 \\
7844\end{array}$ \\
\hline $70 \mathrm{Zn}$ & $\theta(E)$ & & & & & \\
\hline $\begin{array}{l}66 \mathrm{Ni} \\
{ }^{69} \mathrm{Cu} \\
70 \mathrm{Zn} \\
70 \mathrm{Zn} \\
73 \mathrm{Go}\end{array}$ & $\begin{array}{l}a \\
p \\
p \\
a \\
p\end{array}$ & $\begin{array}{l}\text { NDG } \\
\text { NDG } \\
1.3+1 \\
1.8+1 \\
\text { NDG }\end{array}$ & & $\begin{array}{ll}\text { CAL } & T \\
\text { CAL } & T \\
\text { PUR } & E \\
\text { PUR } & E \\
\text { CAL } & T\end{array}$ & $\begin{array}{l}\text { Toosleyt } \\
\text { Thosle:t } \\
\text { Wuxt } \\
\text { Luxt } \\
\text { Woosleyt }\end{array}$ & $\begin{array}{l}7737 \\
2725 \\
2870 \\
7843 \\
2935\end{array}$ \\
\hline $71 \mathrm{Zn}$ & $O(E)$ & & & & & \\
\hline${ }^{74} \mathrm{Ge}$ & $p$ & NOG & & CAL $\mathbf{T}$ & Toosleyt & 2938 \\
\hline $72 z_{n}$ & cumulot & Ive fis & sgion y & $y 1010$ & & \\
\hline $237_{\mathrm{Np}}$ & 0 & $2.0+1$ & $4.1+1$ & CCP R & R Loort & 9091 \\
\hline${ }^{12} \mathrm{Zn}$ & $\sigma(E)$ & & & & & \\
\hline${ }^{75} \mathrm{Ga}$ & p & NDG & & CAL $T$ & T Woosleyt & 2941 \\
\hline $7=31$ & Indepe & ondent & overag & $g e k \ln$ & notlc onergr(E;E') & \\
\hline $197 \mathrm{Au}$ & "OAr & $2.2+2$ & & DUP E & E Artuhht & $12369+$ \\
\hline$z=31$ & Indepe & ondent & OLE:E' & $\cdot: \theta 1$ & & \\
\hline $187 \mathrm{Au}$ & ${ }^{40} \mathrm{Ar}$ & $2.2+2$ & & DUB E & Ertukht & $12368+$ \\
\hline$z \cdot=31$ & Indepe & ondent & $\theta(E ; \theta)$ & & & \\
\hline $187 \mathrm{Au}$ & ${ }^{40} \mathrm{Ar}$ & $2.2+2$ & & DUB E & E Artuhht & $12367+$ \\
\hline
\end{tabular}




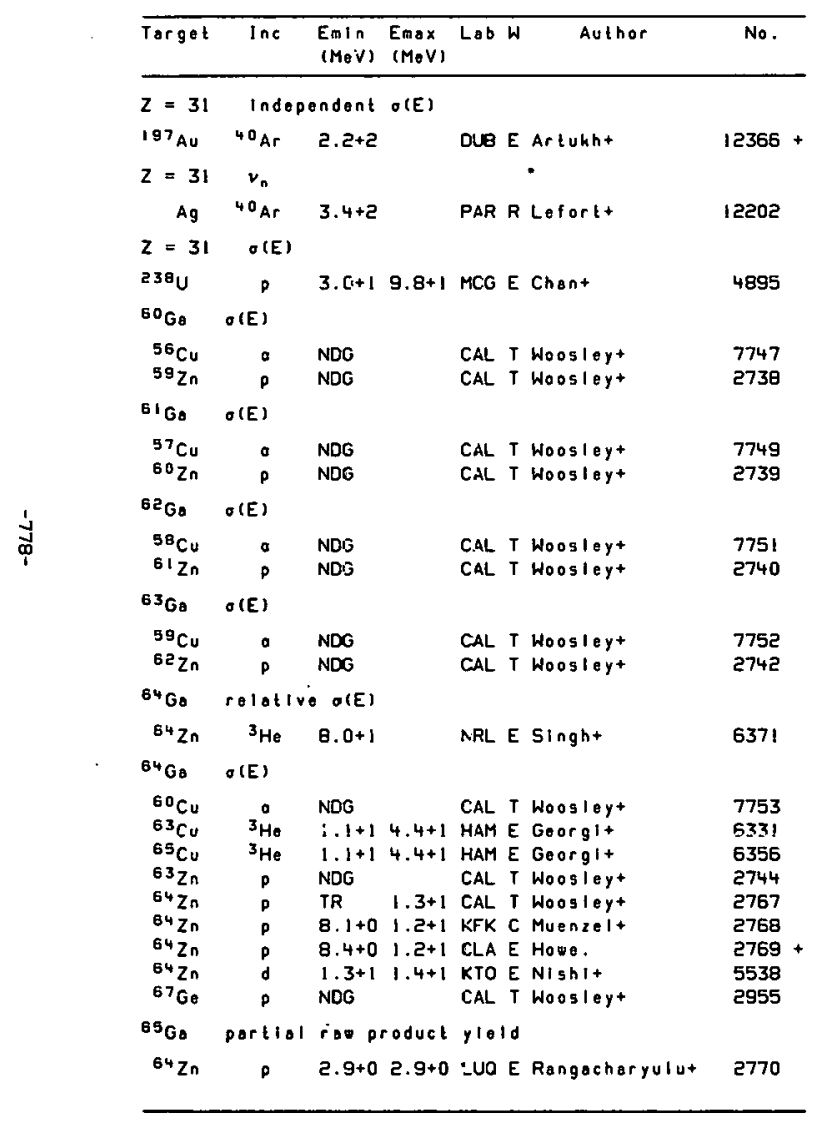

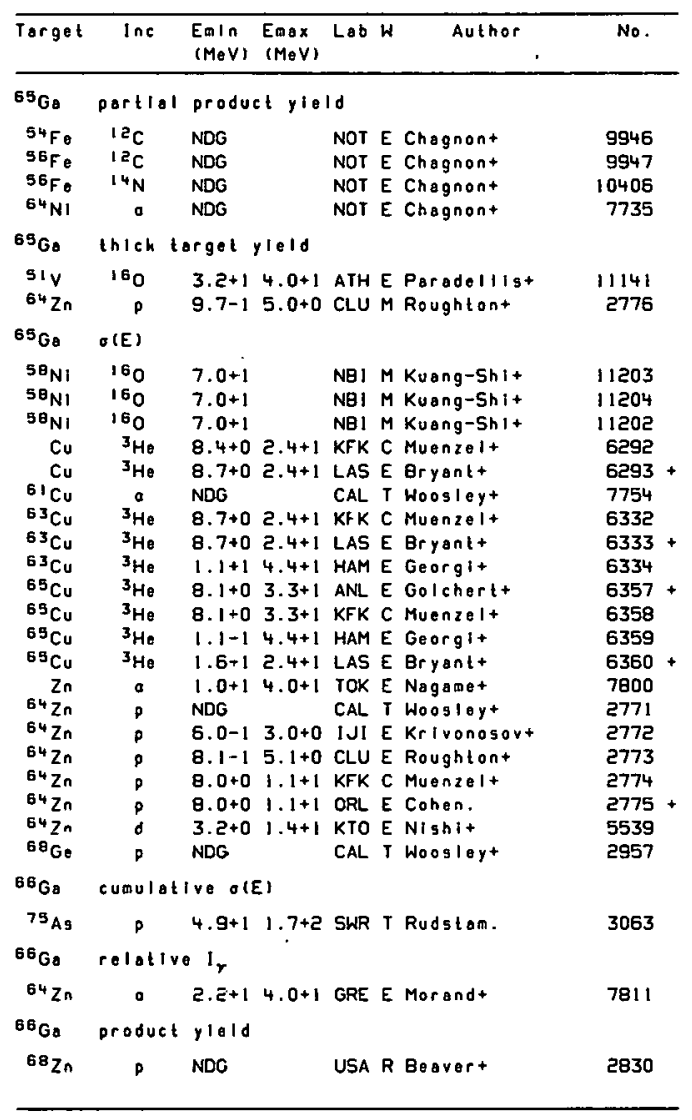




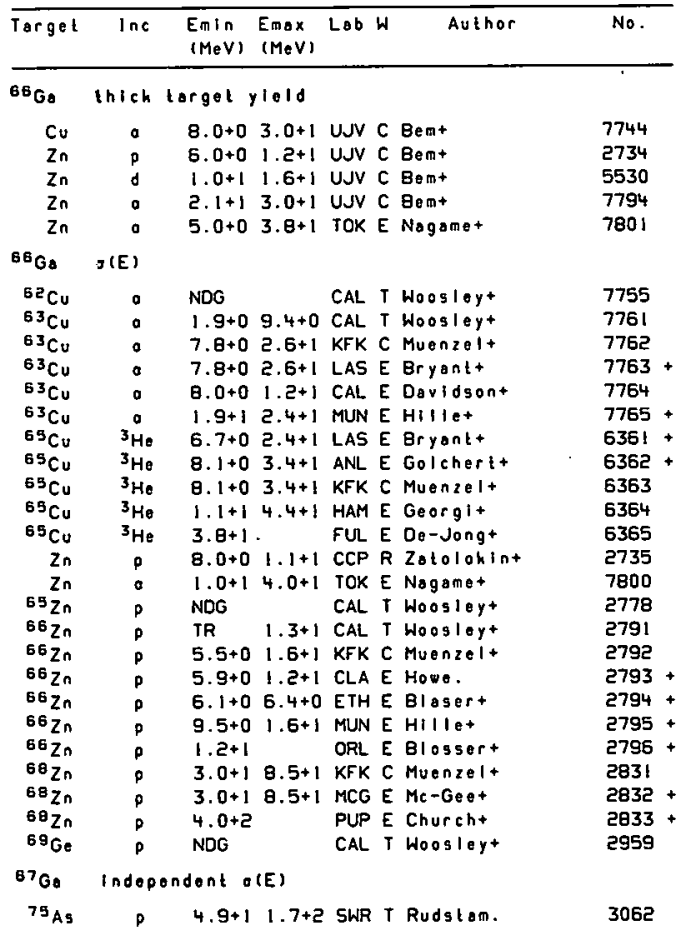

69Go cuavlotive thich torget yleld

Zn a 8.8+0 9.6+1 LVN E Hassbrookt 7797 + 67Go rolative o(E)

$57^{7} \mathrm{Fe} \quad{ }^{2} \mathrm{C} \quad 3.2+14.4+1 \mathrm{KLN} E$ Zobel+ 9948

\begin{tabular}{lllll}
\hline Target Inc Emin Emax Lab & Author \\
& (MeV) (MaV) & No. \\
\hline
\end{tabular}

\section{7ga cumulallive o(E)}

2n 8 8.8+0 9.6+1 LVN E Hasabroeht $7796+$

87Ga partlol ras product yleld

${ }^{66} Z$ n $P$ NDG LUO E Rançacharyulut 2797

67Go portiol product yield

$\begin{array}{llllr}{ }^{54} \mathrm{Fe} & 12 \mathrm{C} & \text { NDG } & \text { NOT E Chagnont } & 9946 \\ 56 \mathrm{Fe} & 12 \mathrm{C} & \text { NDG } & \text { NOT E Chagnont } & 9947 \\ 56 \mathrm{Fe} & 14 \mathrm{~N} & \text { NDG } & \text { NOT E Chagnont } & 10405\end{array}$

$\begin{array}{lrrrr} & & & \text { NOT E Chagnont } & 9947 \\ 56 \mathrm{Fe} & 14 \mathrm{~N} & \text { NDG } & \text { NOT E Chagnont } & 10406 \\ 54 \mathrm{NI} & \text { a } & \text { NDG } & \text { NOT E Chagnont } & 7735\end{array}$

87G. partiol o(E)

$67 \mathrm{Zn} P \quad 3.7+04.1+0$ ATH E Paradellis. 2801

67Ga 1 ,

${ }^{53} \mathrm{Cr} \quad{ }^{160} \quad 3.5+15.1+1$ LVP E Al-Nasert 11143

67Ga product yield

$\begin{array}{lllll}{ }^{66} \mathrm{Zn} & d & 1.6+1 & \text { NLPE Neirinixt } & 554 \\ 60 \mathrm{Zn} & P & \text { NDG } & \text { USA R Beavert }\end{array}$

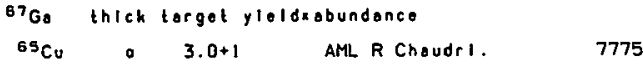

67go inlch largot yiold

${ }^{59} \mathrm{Co} 1604.2+15.2+1$ TNL E waltinert 11168

Cu $\quad 1.6+13.0+1$ UJV C Bemt $\quad 7745$

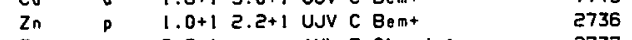

$2 n$ P $2.2+1 \quad$ AML R Chaujr:. 2737

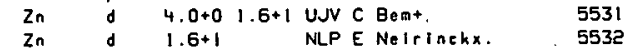

$\begin{array}{llll}2 n & 8.0+01.6+1 \text { AML } R \text { Chausri. } & 5533 \\ 2 n & & 7795\end{array}$

$2 n$ a $5.0+03.8+1$ TOK E Neganet 7801

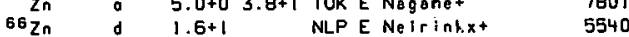

Q7G. overage o(E)

${ }^{87} \mathrm{Zn} \quad 1.8+01.9+0$ ORL E Johnsoret 2807 


\begin{tabular}{|c|c|c|c|c|c|c|}
\hline Targot & $\ln c$ & $\begin{array}{l}\text { Enin } \\
(\mathrm{MeV})\end{array}$ & $\begin{array}{l}\text { Emax } \\
(\mathrm{MeV})\end{array}$ & Lab & Author & No. \\
\hline${ }^{67} \mathrm{Go}$ & $\sigma(E) \times f o$ & octor & & & & \\
\hline${ }^{68} \mathrm{Zn}$ & $p$ & $2.2+1$ & & ORL & E Cohent & 2839 \\
\hline${ }^{67} \mathrm{Go}$ & $\sigma(E)$ & & & & & \\
\hline${ }^{39} \mathrm{Co}$ & 160 & $3.0+1$ & $5.6+1$ & TNL & E Wallnert & 11167 \\
\hline${ }^{53} \mathrm{Cu}$ & $a$ & NOG & & CAL & T Woosleyt & 7766 \\
\hline${ }^{53} \mathrm{Cu}$ & 160 & $4.0+1$ & $8.5+1$ & PAR & E Langerint & 11276 \\
\hline${ }^{65} \mathrm{Cu}$ & ${ }^{3} \mathrm{He}$ & $8.1+0$ & $6.9+1$ & ANL & E Golchert+ & $6366+$ \\
\hline${ }^{65} \mathrm{Cu}$ & ${ }^{3} \mathrm{He}$ & $8.1+0$ & $6.9+1$ & KFK & C Muenzel+ & 6367 \\
\hline${ }^{65} \mathrm{Cu}$ & ${ }^{3} \mathrm{He}$ & $8.4+0$ & $2.4+1$ & LAS & E Bryont+ & $6368+$ \\
\hline${ }^{65} \mathrm{Cu}$ & ${ }^{3} \mathrm{He}$ & $1.1+1$ & $4.4+1$ & HAM & E Georgit & 6369 \\
\hline${ }^{65} \mathrm{Cu}_{4}$ & $a$ & $1.1+1$ & $2.6+1$ & KFK & C Muenzel+ & 7778 \\
\hline${ }^{65} \mathrm{Cu}$ & 0 & $1.1+1$ & $2.6+1$ & LAS & E Bryontt & $7779+$ \\
\hline${ }^{65} \mathrm{Cu}$ & $a$ & $1.8+1$ & $6.0+1$ & KFK E & E Graft & $7776+$ \\
\hline${ }^{65} \mathrm{Cu}$ & a & $1.8+1$ & $6.0+1$ & KFK & C Muenzel+ & 7777 \\
\hline$z_{n}$ & : & $1.0+1$ & $4.0+1$ & TOK & E Nagame+ & 7800 \\
\hline${ }^{64} \mathrm{Zn}$ & a & TR & $1.4+1$ & CAL & T Woosleyt & 7812 \\
\hline${ }^{64} \mathrm{Zn}$ & a & $1.8+1$ & & PUR E & E Luxt & 7813 \\
\hline${ }^{66} Z_{n}$ & p & NOG & & CAL & T Woosley+ & 2798 \\
\hline $67 \mathrm{Zn}$ & $p$ & $T R$ & $1.3+:$ & CAL & T Woosleyt & 2802 \\
\hline $67 \mathrm{Zn}$ & p & $T R$ & $5 \cdot 3+0$ & ORL & E Johnsont & $2803+$ \\
\hline $672 \mathrm{n}$ & p & $1.8+0$ & $6.4+0$ & KFK & C Muenzel+ & 2804 \\
\hline $67 \mathrm{Zn}$ & p & $2.0+0$ & $3.0+0$ & KFT & E Krivonosort & 2805 \\
\hline $67 \mathrm{Zn}$ & 0 & $2.3+0$ & $6.4+0$ & ETH & E Blasert & $2806+$ \\
\hline${ }^{68} \mathrm{Zn}$ & p & $1.5+1$ & $8.5+1$ & KFK & C Muenzelt & 2835 \\
\hline${ }^{6 B} Z_{n}$ & p & $1.5+1$ & $8.5+1$ & $M C G$ & E Mc-Gee+ & $2836+$ \\
\hline${ }^{6} Z_{n}$ & 0 & $2.2+1$ & & ORL & E Cohent & $2837+$ \\
\hline $68 \mathrm{Zn}$ & 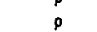 & $4.0+2$ & & PUP & E Churcht & $2838+$ \\
\hline${ }^{70} \mathrm{Ge}$ & p & NDG & & CAL & T Woosleyt & $296 !$ \\
\hline${ }^{6 \theta_{\mathrm{GB}}}$ & Indepen & njent of & (E) & & & \\
\hline${ }^{75} \mathrm{As}$ & م & $4 \cdot 9+1$ & $1.7+2$ & SWR & TRudstom." & 3062 \\
\hline${ }^{68} \mathrm{G}_{B}$ & thlck t & target $y$ & yield & & & \\
\hline${ }^{55} \mathrm{Mn}$ & 160 & $4.1+1$ & & ATH & E Costat & 11144 \\
\hline $2 n$ & 0 & $5.0+0$ & $3.8+1$ & TOK & E Nagame+ & $780 !$ \\
\hline${ }^{67} \mathrm{Zn}$ & $p$ & $9.7-1$ & $3.7+0$ & CLU $\mathrm{r}$ & M Roughtont & 2811 \\
\hline${ }^{68} \mathrm{Zn}$ & $p$ & $4.1+0$ & $5.0+0$ & $\mathrm{CLU} \mathrm{r}$ & M Roughtont & 2853 \\
\hline${ }^{6 \theta} \mathrm{Go}$ & COE OLE & & & & & \\
\hline $68 \mathrm{Zn}$ & $p$ & $3.6+0$ & $4.0+0$ & ORL & E Johnsont & 2852 \\
\hline
\end{tabular}

\begin{tabular}{|c|c|c|c|c|c|c|}
\hline Target & $\ln c$ & $\begin{array}{l}\text { Emin } \\
(\mathrm{MeV})\end{array}$ & $\begin{array}{l}\text { Emax } \\
(\mathrm{MeV})\end{array}$ & Lab & Author & No. \\
\hline${ }^{68} \mathrm{Ga}_{\mathrm{a}}$ & \multicolumn{6}{|c|}{ ofEI a factor } \\
\hline${ }^{68} \mathrm{Ga}$ & $p$ & $2.2+1$ & & ORL & E Cohent & 2901 \\
\hline${ }^{68} \mathrm{Ga}_{\mathrm{a}}$ & \multicolumn{6}{|l|}{$O(\varepsilon)$} \\
\hline${ }^{64} \mathrm{Cu}$ & a & NDG & & CAL & T Woosleyt & 7767 \\
\hline${ }^{65} \mathrm{Cu}$ & : & $1.4+0$ & $8.5+0$ & CAL & T Woosleyt & 7780 \\
\hline${ }^{65} \mathrm{Cu}$ & $a$ & $6.2+0$ & $1.2+1$ & CAL & E Oavidsont & 7781 \\
\hline${ }^{65} \mathrm{Cu}$ & $a$ & $7.8+0$ & $2.6+1$ & KFK & C Muenzel+ & 7782 \\
\hline${ }^{65} \mathrm{Cu}$ & $a$ & $7.8+0$ & $2.6+1$ & LAS & E Bryant+ & 7783 \\
\hline${ }^{69} \mathrm{Cu}$ & $a$ & $1.9+1$ & $2.4+1$ & MUN & E Hillot & 7784 \\
\hline $\mathrm{Zn}$ & o & $1.0+1$ & $4.0+1$ & TOK & E Nagamet & 7800 \\
\hline${ }^{7} \mathrm{Zn}$ & p & NDG & & CAL & T Woosleyt & 2808 \\
\hline${ }^{67} \mathrm{Zn}$ & p & $6.0-1$ & $3.0+0$ & IJI & E Krivonosort & 2809 \\
\hline $67 \mathrm{Zn}$ & p & $9.7-1$ & $3.7+0$ & CLU & E Roughtont & 2810 \\
\hline${ }^{60} \mathrm{Zn}$ & p & TR & $1.3+1$ & CAL & T Woosleyt & 2840 \\
\hline${ }^{68} \mathrm{Zn}$ & $p$ & T२ & $5.9+0$ & ORL & E Johnsont & 2841 \\
\hline${ }^{68} \mathrm{Zn}$ & p & $3.4+0$ & $6.4+0$ & ETH & E Blasert & 2842 \\
\hline${ }^{68} \mathrm{Zn}$ & $p$ & $3.4+0$ & $8.5+1$ & KFK & C Muenzelt & 2843 \\
\hline${ }^{68} \mathrm{Zn}$ & p & $3.8+0$ & $5.0+0$ & CAL & E Zyshind. & 2844 \\
\hline${ }^{68} \mathrm{Zn}$ & $p$ & $3.9+0$ & $5.0+0$ & CLU & E Roughtont & 2845 \\
\hline${ }^{68} Z_{n}$ & $p$ & $5.6+0$ & $1.2+1$ & CLA & E Howe. & 2846 \\
\hline $68 \mathrm{Zn}$ & $p$ & 8. $0+0$ & $0.0+2$ & NRL & R Silberbergt & 2847 \\
\hline${ }^{68} \mathrm{Zn}$ & $p$ & $9.5+0$ & $1.6+1$ & MUN & E HIIIO+ & 2848 \\
\hline $682 n$ & p & $1.0+1$ & $8.5+1$ & MCG & E Megeet & 2849 \\
\hline${ }^{68} \mathrm{Zn}$ & p & $1.2+1$ & & ORL & E glossert & 2850 \\
\hline${ }^{68} \mathrm{Zn}$ & $p$ & $4.0+2$ & & PUP & E Churcht & $285 !$ \\
\hline${ }^{69} \mathrm{Ga}$ & $p$ & c. $2+1$ & & KFK & C Muenzelt & 2899 \\
\hline${ }^{69} \mathrm{Ga}$ & 9 & c.2+1 & & ORL & E Cohent & 2900 \\
\hline${ }^{71 G e}$ & p & NOG & & CAL & T Woosleyt & 2968 \\
\hline${ }^{69} \mathrm{Ga}$ & \multicolumn{6}{|c|}{ porblal o(E), } \\
\hline${ }^{69} \mathrm{Ga}$ & p & $4.8+0$ & & ATH & E Paradellist & 2902 \\
\hline${ }^{69} \mathrm{Go}$ & \multicolumn{6}{|c|}{ portlal e(E)xfactor } \\
\hline $70 \mathrm{Zn}$ & $p$ & $2.0+1$ & & SWR & T Ruostam. & 2872 \\
\hline${ }^{69} \mathrm{Go}$ & \multicolumn{6}{|c|}{ Celative $O(E)$} \\
\hline${ }^{65} \mathrm{Cu}$ & 160 & $3.9+1$ & $5 \cdot 1+1$ & ORL & M Wellst & 11298 \\
\hline${ }^{69} \mathrm{Ga}$ & \multicolumn{6}{|c|}{ olErnfactor } \\
\hline${ }^{68} \mathrm{Zn}$ & p & NDG & & CAL & E Zyshindt & 2856 \\
\hline
\end{tabular}




\begin{tabular}{|c|c|c|c|c|c|c|}
\hline Target & $\ln c$ & $\begin{array}{l}\text { Emin } \\
(M e V)\end{array}$ & $\begin{array}{l}\text { Emax } \\
(M e V)\end{array}$ & Lab & Author & No. \\
\hline${ }^{69} \mathrm{Ga}$ & $\theta(E)$ & & & & & \\
\hline${ }^{59} \mathrm{Co}$ & 160 & $5.6+1$ & & TNL & E Waltnert & 11187 \\
\hline${ }^{65} \mathrm{Cu}$ & 0 & NDG & & CAL & T Woosleyt & 7785 \\
\hline${ }^{66} \mathrm{Zn}$ & $a$ & $T R$ & $1.4+1$ & CAL & T Woosleyt & 7823 \\
\hline $66 \mathrm{Zn}^{6}$ & 0 & $1.8+1$ & & PUR & $E$ Luxt & 7824 \\
\hline${ }^{60} \mathrm{Zn}$ & $p$ & NDG & & CAL & T Woosleyt & 2854 \\
\hline${ }^{68} \mathrm{Zn}$ & p & $1.7+0$ & $5 \cdot(1+0$ & CAL & E Zyshind. & 2855 \\
\hline${ }^{69} \mathrm{Zn}$ & p & NDG & & CAL & T Woosleyt & 2858 \\
\hline $72 \mathrm{Ge}$ & p & NDG & & CAL & I Woosley+ & 2970 \\
\hline${ }^{70} \mathrm{Ga}$ & Independ & dent & (E) & & & \\
\hline${ }^{75} \mathrm{As}$ & $p$ & $4.9+1$ & $1.7+2$ & SWR & I Rudstam. & 3062 \\
\hline${ }^{70} \mathrm{Gs}$ & portiol & relot & IYO OLE & & & \\
\hline${ }^{68} Z_{n}$ & 。 & $3 \cdot 1+1$ & & GRE & E Morandt & 7835 \\
\hline${ }^{70} \mathrm{Go}$ & portlol & $\sigma(E) \times$ & foctor & & & \\
\hline $702 n$ & p & $2.0+1$ & & SWR & T Rudsitam. & 2873 \\
\hline${ }^{70} \mathrm{Ga}$ & rolotive & - $\mathrm{I}_{y}$ & & & & \\
\hline${ }^{68} \mathrm{Zn}$ & $a$ & $2 \cdot 3+1$ & $4.0+1$ & GRE & E Morandt & 7834 \\
\hline${ }^{70} \mathrm{Go}$ & product & $y \mid e 1 d$ & In actor & & & \\
\hline $70 \mathrm{Zn}$ & p & $1.5+0$ & & WMU & M Bernstein. & 2874 \\
\hline${ }^{70} \mathrm{Go}$ & overago & $O(E)$ & & & & \\
\hline${ }^{70} \mathrm{Zn}$ & p & $1.5+0$ & $1.5+0$ & $\mathrm{ORL}$ & E Johnsont & 2877 \\
\hline $10^{\mathrm{Ga}}$ & $O(E) \times f a c$ & ictor & & & & - \\
\hline${ }^{71} \mathrm{Gg}$ & p & $2.2+1$ & & ORL & E Cohent & 2924 \\
\hline $70 \mathrm{Go}$ & $\theta(E)$ & & & & & \\
\hline${ }^{68} \mathrm{Cu}$ & a & NDG & & $\mathrm{CAL}$ & T Woosley+ & 7786 \\
\hline $67 \mathrm{Zn}$ & a & $T R$ & $1.4+1$ & $\mathrm{CAL}$ & Thoosleyt & 7828 \\
\hline $69 Z n$ & p & NDG & & CAL & T Woosleyt & 2859 \\
\hline${ }^{70} \mathrm{Zn}$ & $p$ & TR & $1.3+1$ & $\mathrm{CAL}$ & $T$ Woosleyt & 2875 \\
\hline $70 \mathrm{Zn}$ & p & $2.0+0$ & $3.0+0$ & $\mathrm{KFT}$ & E Krivonosort & 2876 \\
\hline${ }^{71} \mathrm{Go}$ & : & $2.2+1$ & & KFK & C Muenzel+ & 2922 \\
\hline 710 & $\mathrm{p}$ & $2.5+1$ & & ORL & E Cohent & $2923+$ \\
\hline${ }^{73} \mathrm{Ge}$ & $p$ & NDS & & CAL & T Woosleyt & 2984 \\
\hline
\end{tabular}

\begin{tabular}{|c|c|c|c|c|c|c|}
\hline Target & $\operatorname{lnc}$ & $\begin{array}{l}\text { EmIn } \\
(\mathrm{MeV})\end{array}$ & $\begin{array}{l}E \max \\
(M \in V)\end{array}$ & Lab & Author & No. \\
\hline $71 G a$ & $\sigma(E)$ & & & & & \\
\hline $\begin{array}{l}6^{67} \mathrm{Cu} \\
6^{68} \mathrm{Zn} \\
68 \mathrm{Zn} \\
7^{70} \mathrm{Zn} \\
71 \mathrm{Zn} \\
{ }^{74} \mathrm{Ge}\end{array}$ & $\begin{array}{l}a \\
a \\
a \\
p \\
p \\
p\end{array}$ & $\begin{array}{l}\text { NDG } \\
\text { TR } \\
1.8+1 \\
\text { NDG } \\
\text { NDG } \\
\text { NDG }\end{array}$ & $1.4+1$ & $\begin{array}{l}\text { CAL } \\
\text { CAL } \\
\text { PUR } \\
\text { CAL } \\
\text { CAL } \\
\text { CAL }\end{array}$ & $\begin{array}{l}\text { T Woosley+ } \\
\text { T Woosley+ } \\
\text { E Luxt } \\
\text { T Woosley+ } \\
\text { T Woosle } \\
\text { T Woosle } \\
\text { T }\end{array}$ & $\begin{array}{l}7787 \\
7836 \\
7837 \\
2878 \\
2880 \\
2987\end{array}$ \\
\hline $72 G 0$ & cumulo & ive olE & & & & \\
\hline $\begin{array}{l}75 \text { AS } \\
72_{\text {Ga }}\end{array}$ & $\begin{array}{r}p \\
O(E)\end{array}$ & $4.9+1$ & $1.7+2$ & $5 W R$ & T Rudstan. & 3063 \\
\hline $\begin{array}{l}{ }^{68} \mathrm{Cu} \\
{ }^{712 n} \\
{ }^{75} \mathrm{Ge}\end{array}$ & $\begin{array}{l}a \\
p \\
p\end{array}$ & $\begin{array}{l}\text { NDG } \\
\text { NDG } \\
\text { NDG }\end{array}$ & & $\begin{array}{l}\text { CAL } \\
\text { CAL } \\
\text { CAL }\end{array}$ & $\begin{array}{l}\text { T Woosieyt } \\
\text { T Woosleyt } \\
\text { T Woosleyt }\end{array}$ & $\begin{array}{l}7788 \\
2881 \\
2999\end{array}$ \\
\hline${ }^{73} \mathrm{Ga}_{\mathrm{a}}$ & cumulo & ive ole & & & & \\
\hline${ }^{75} \mathrm{As}$ & $\begin{array}{r}p \\
\sigma(E)^{\prime}\end{array}$ & $4.9+1$ & $1.7+2$ & SWR & T Rudstan. & 3063 \\
\hline $\begin{array}{l}70 \mathrm{Zn} \\
70 \mathrm{Zn} \\
76 \mathrm{Ge}\end{array}$ & a & $\begin{array}{l}\text { TR } \\
1.8+1 \\
\text { NDG }\end{array}$ & $1.4+1$ & $\begin{array}{l}\text { CAL } \\
\text { PUR } \\
\text { CAL }\end{array}$ & $\begin{array}{l}\text { T Woosleyt } \\
\text { E Lux+ } \\
\text { T Woosleyt }\end{array}$ & $\begin{array}{l}7845 \\
7846 \\
3002\end{array}$ \\
\hline${ }^{74} \mathrm{Gs}$ & cumulo & 1ve $11 \mathrm{~s}$ & ssion & yield & & \\
\hline $\begin{array}{l}238 \mathrm{U} \\
{ }^{74} \mathrm{Ga}\end{array}$ & o(E) & $3.0+1$ & $9.8+1$ & MCG & E Chan- & 4914 \\
\hline${ }^{77} \mathrm{Ge}$ & P & NDG & & $\mathrm{CAL}$ & T Woostest & 3008 \\
\hline${ }^{75} \mathrm{G}$ 。 & cusule & ive fis & sgion & yleld & & \\
\hline $23 \theta_{U}$ & $p$ & $3 \cdot 0+1$ & $9.8+1$ & MCG & E Chant & 4914 \\
\hline${ }^{75} \mathrm{Go}$ & $O(E)$ & & . & & $\vdots$ & \\
\hline${ }^{78} \mathrm{Ge}$ & d & $1.1+1$ & $1.4+1$ & $\mathrm{KTOO}$ & ENishit & 5544 \\
\hline${ }^{76} \mathrm{G}$ & cumule & Ive fis & ssion & yield & : & \\
\hline $23 \theta_{U}$ & . $p$ & $3.0+1$ & $9.8+1$ & MCG & E Chant & 4914 \\
\hline${ }^{76} \mathrm{G}$ & $\sigma(E)$ & & & & : & \\
\hline${ }^{78} \mathrm{Ge}$ & $d$ & $8.7+0$ & $1.4+1$ & KTO & E Nishit & 5545 \\
\hline
\end{tabular}




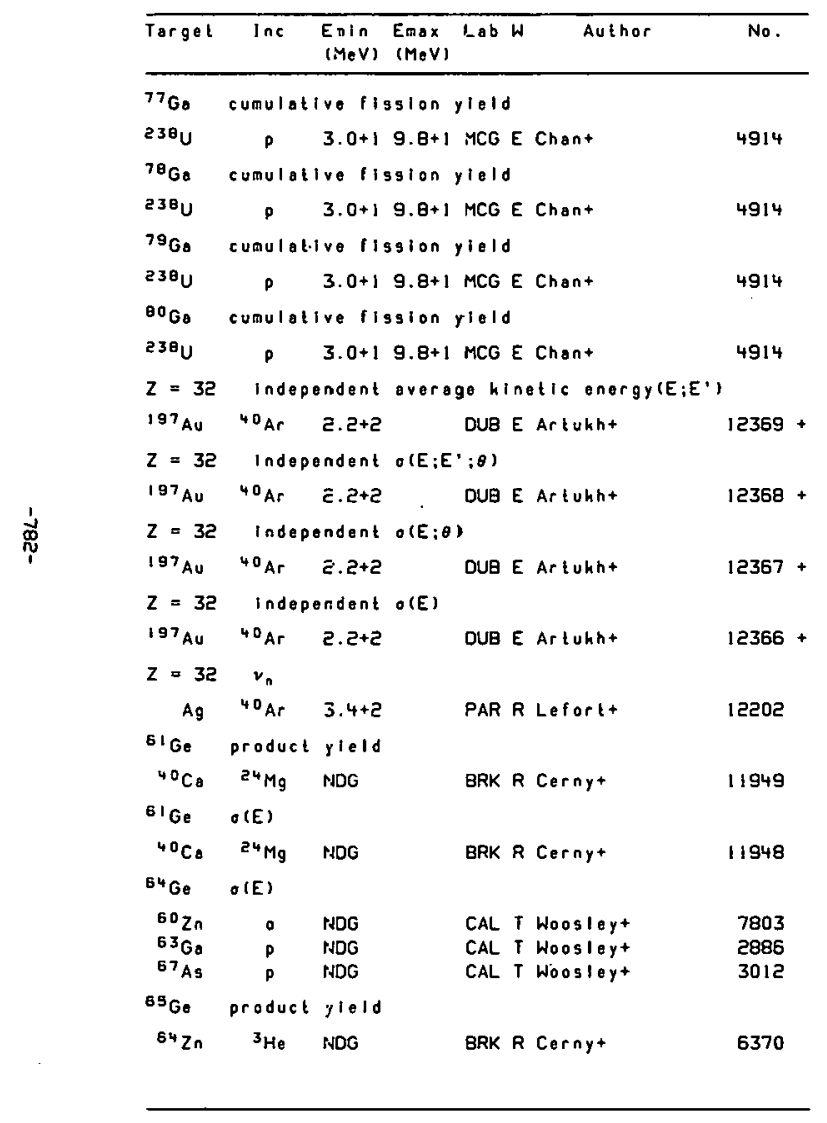

\begin{tabular}{|c|c|c|c|c|c|c|}
\hline Target & $\ln c$ & $\begin{array}{l}m \mid n \\
(M e V)\end{array}$ & $\begin{array}{l}\text { Emax } \\
\left(M_{\theta} V\right)\end{array}$ & Lob & Author & No. \\
\hline${ }^{65} \mathrm{Ge}$ & $\sigma(E)$ & & & & & \\
\hline $\begin{array}{l}61 \mathrm{Zn} \\
62 \mathrm{Ga} \\
64 \mathrm{Ga} \\
6 \theta_{\mathrm{AS}}\end{array}$ & $\begin{array}{l}0 \\
0 \\
p \\
p\end{array}$ & $\begin{array}{l}\text { NDG } \\
\text { NDG } \\
\text { NDG } \\
\text { NDG }\end{array}$ & . & $\begin{array}{l}\text { CAL } \\
\text { CAL } \\
\text { CAL } \\
\text { CAL }\end{array}$ & $\begin{array}{l}\text { T Woogleyt } \\
\text { T Woosleyt } \\
T \text { Woosleyt } \\
T \text { Woosleyt }\end{array}$ & $\begin{array}{l}7804 \\
7853 \\
2888 \\
3014 .\end{array}$ \\
\hline${ }^{66} \mathrm{Ge}$ & thick & arget y & yleld & & & \\
\hline $\mathrm{Bg}_{\mathrm{Ge}}^{\mathrm{Zn}}$ & $\begin{array}{r}0 \\
O(E)\end{array}$ & $5.0+0$ & $3.8+1$ & TOK & E Nagame+ & 7801 \\
\hline $\begin{array}{l}{ }^{59} \mathrm{Ni} \\
\mathrm{Zn} \\
62 \mathrm{Zn} \\
63 \mathrm{Gg} \\
65_{\mathrm{Ga}} \\
{ }^{6} 9_{\mathrm{As}}\end{array}$ & $\begin{array}{r}160 \\
0 \\
0 \\
0 \\
p \\
p\end{array}$ & $\begin{array}{l}7.0+1 \\
1.0+1 \\
\text { NDE } \\
\text { NDG } \\
\text { NDG } \\
\text { NDG }\end{array}$ & $4,0+1$ & $\begin{array}{l}\text { NBI } \\
\text { TOK } \\
\text { CAL } \\
\text { CAL } \\
\text { CAL } \\
\text { CAL }\end{array}$ & $\begin{array}{l}\text { M Kuang-Shit } \\
\text { E Nagamet } \\
\text { T Woosleyt } \\
\text { T Woosleyt } \\
\text { T Woosleyt } \\
\text { T Woosleyt }\end{array}$ & $\begin{array}{r}11205 \\
7800 \\
7805 \\
7855 \\
2890 \\
3016\end{array}$ \\
\hline $\begin{array}{l}{ }^{57} \mathrm{Ge} \\
{ }^{75} \mathrm{As}\end{array}$ & cumulat & $\begin{array}{l}\text { Hive ole } \\
4.9+1\end{array}$ & E) & SWR & T Rudstam. & 3063 \\
\hline $67 \mathrm{Ge}$ & partial & i relati & Ive ole & & & \\
\hline $67 \mathrm{Ge}$ & $a^{a}$ & $\begin{array}{l}1.5+1 \\
I_{r}\end{array}$ & $2.2+1$ & OSA & E Nahoyemat & 7802 \\
\hline $\begin{array}{l}{ }^{64} \mathrm{Zn} \\
{ }^{57} \mathrm{Ge}\end{array}$ & a pro & $\begin{array}{c}9.7+0 \\
\text { duct }\end{array}$ & $\begin{array}{l}1.0+1 \\
\text { ield }\end{array}$ & ANL & E Murphy+ & 7815 \\
\hline $\begin{array}{l}{ }^{64} \mathrm{Zn} \\
{ }^{67} \mathrm{Ge}\end{array}$ & $O(E)$ & $9.5+0$ & $9.8+0$ & LVP & E Al-Nasert & 7814 \\
\hline $\begin{array}{l}2 n \\
63 \mathrm{Zn} \\
64 \mathrm{Zn} \\
65_{\mathrm{GB}} \\
{ }^{0} 0_{\mathrm{AS}}\end{array}$ & $\begin{array}{l}: \\
a \\
0 \\
p \\
p\end{array}$ & $\begin{array}{l}1.0+1 \\
\text { NDG } \\
\text { TR } \\
\text { NDG } \\
\text { NDG }\end{array}$ & $\begin{array}{l}4.0+1 \\
1.3+1\end{array}$ & $\begin{array}{l}\text { TOK } \\
\text { CAL } \\
\text { CAL } \\
\text { CAL } \\
\text { CAL }\end{array}$ & $\begin{array}{l}\text { E Noganet } \\
\text { T Woosleyt } \\
\text { T Woosleyt } \\
\text { T Woosley+ } \\
\text { T Woosley+ }\end{array}$ & $\begin{array}{l}7800 \\
7806 \\
7816 \\
2892 \\
3018\end{array}$ \\
\hline${ }^{68} \mathrm{Ge}$ & compoun & do-nucle & QUS OLE & & & \\
\hline $\begin{array}{l}{ }^{5 \theta_{\mathrm{Ni}}} \\
{ }^{6 \theta_{\mathrm{Ge}}}\end{array}$ & $\begin{array}{l}160 \\
\text { cumulat }\end{array}$ & $\begin{array}{l}4.5+1 \\
1 v e 0(E\end{array}$ & $\begin{array}{l}7.7+1 \\
\text { E) }\end{array}$ & $\mathrm{NB} 1$ & E simpsont & 11206 \\
\hline${ }^{75} \mathrm{AS}$ & p & $4.9+1$ & $1.7+2$ & SWR & T Rudstam. & 3063 \\
\hline
\end{tabular}




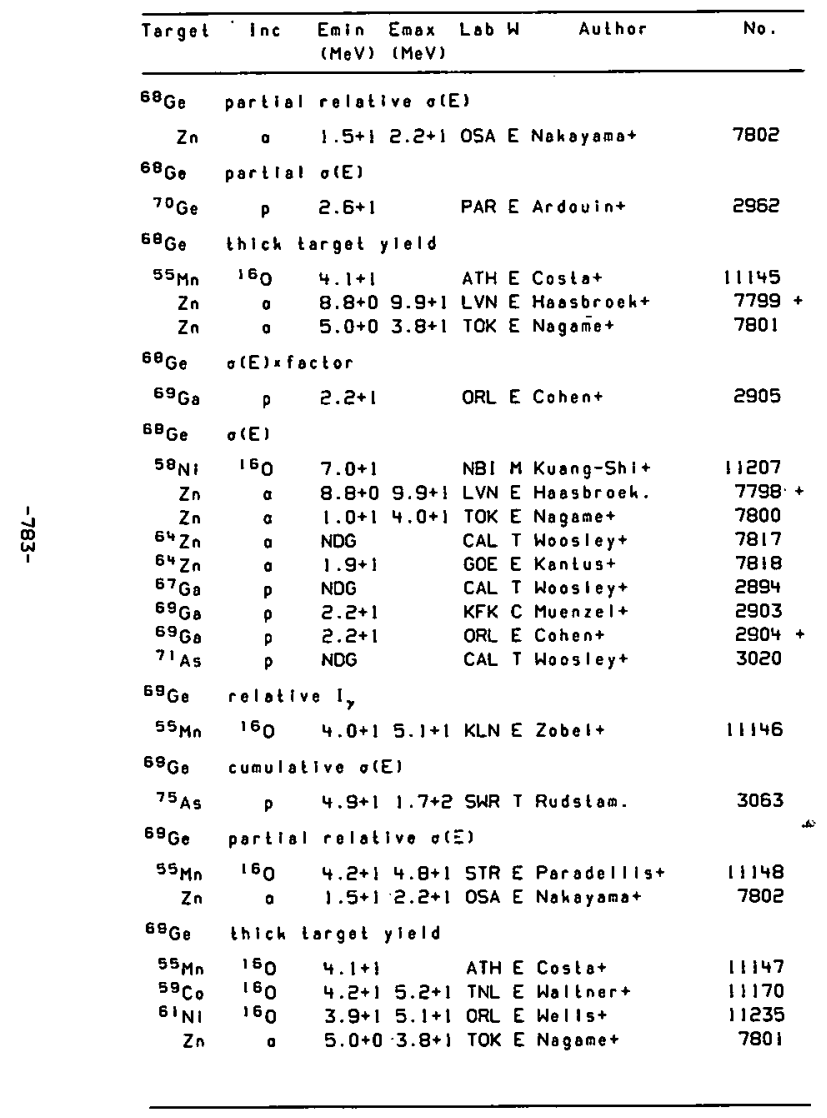

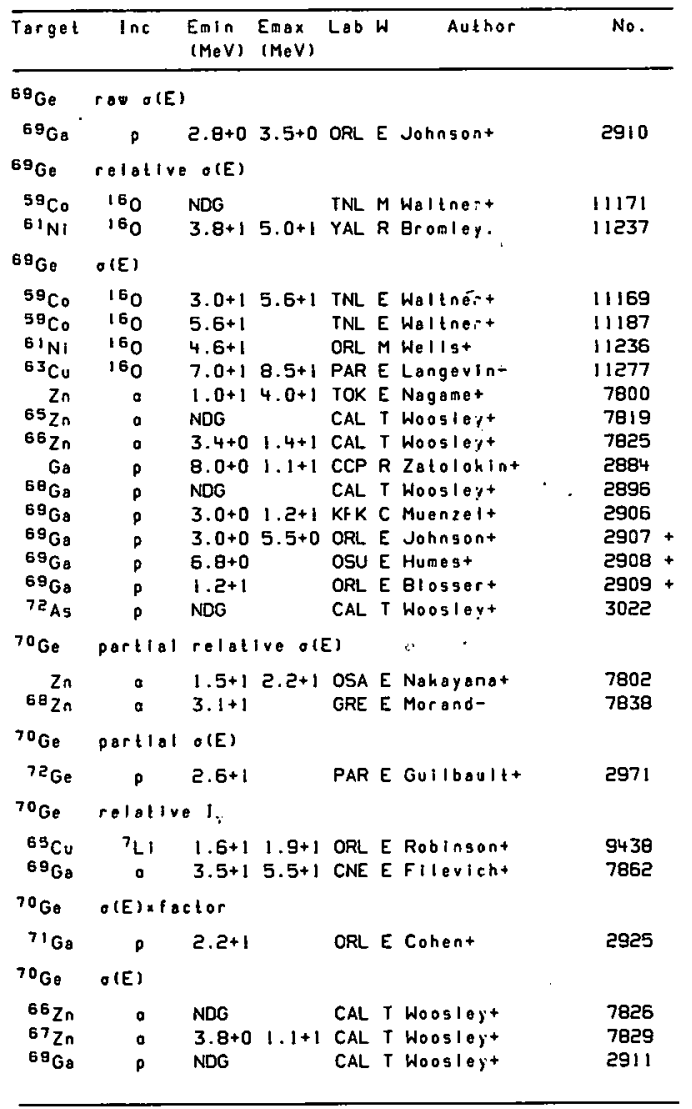




\begin{tabular}{|c|c|c|c|c|c|c|}
\hline Target & Ine & $\begin{array}{l}\text { EmIn } \\
(\mathrm{MeV})\end{array}$ & $\begin{array}{l}\text { Emax } \\
(M e V)\end{array}$ & Lab & Author & No. \\
\hline${ }^{70} \mathrm{Ge}$ & $\theta(E)$ & & & & & \\
\hline $\begin{array}{l}{ }^{70} \mathrm{Go} \\
{ }^{73} \mathrm{As}\end{array}$ & $\begin{array}{l}p \\
p\end{array}$ & $\begin{array}{l}\text { NDG } \\
\text { NDG }\end{array}$ & & $\begin{array}{l}\text { CAL } \\
\text { CAL }\end{array}$ & $\begin{array}{l}\text { T Woosleyt } \\
\text { T Woosleyt }\end{array}$ & $\begin{array}{l}2915 \\
3024\end{array}$ \\
\hline IGo. & Independ & sent of & (E) & & & \\
\hline${ }^{75} \mathrm{As}$ & $p$ & $4.9+1$ & $1.7+2$ & SWR & T Rudstam. & 3062 \\
\hline TIGO & portiol & rolati & ive ofE & & & \\
\hline $2 n$ & 0 & $1.5+1$ & $2.2+1$ & $O S A$ & E Nakayama+ & 2802 \\
\hline $71 G_{0}$ & average & $O(E)$ & & & & \\
\hline$M_{G}$ & $p$ & $1.0+0$ & $1.2+0$ & ORL & E Johnsont & 2930 \\
\hline $71 G 0$ & $O(E)$ & & & & & \\
\hline $\begin{array}{l}672 n \\
68 \mathrm{Zn} \\
70 \mathrm{Go} \\
71 \mathrm{Go} \\
71 \mathrm{Go} \\
71 \mathrm{Go} \\
71 \mathrm{Go} \\
74 \mathrm{As}\end{array}$ & $\begin{array}{l}\text { a } \\
\text { a } \\
p \\
p \\
p \\
p \\
p \\
\text { p } \\
p\end{array}$ & $\begin{array}{l}\text { NDG } \\
2.3+0 \\
\text { NDG } \\
\text { TR } \\
1.0+0 \\
3.0+0 \\
6.0+0 \\
\text { NDG }\end{array}$ & $\begin{array}{l}1.1+1 \\
5.7+0 \\
5.7+0 \\
5.0+0\end{array}$ & $\begin{array}{l}\text { CAL } \\
\text { CAL } \\
\text { CAL } \\
\text { ORL E } \\
\text { KFK } \\
\text { INU } \\
\text { OSU } \\
\text { CAL }\end{array}$ & $\begin{array}{l}\text { I Woosleyt } \\
\text { I Woosleyt } \\
\text { T Woosley+ } \\
\text { E Johnsont } \\
\text { C Muenzel+ } \\
\text { T Koilogt } \\
\text { E Humest } \\
\text { T Woosleyt }\end{array}$ & $\begin{array}{l}7830 \\
7839 \\
2916 \\
2926+ \\
2927 \\
2928 \\
2929+ \\
3026\end{array}$ \\
\hline $72 \mathrm{Ge}$ & portlol & reloti & Iro olE & & & \\
\hline${ }^{74} \mathrm{Ge}$ & $p$ & $2.0+1$ & $5.2+1$ & ORL & E Restert & 2989 \\
\hline $7 e_{G 0}$ & portiol & $O(E)$ & & & & \\
\hline${ }^{74} \mathrm{Ge}$ & p & $2.6+1$ & & PAR & E Guilbaultt & 2988 \\
\hline $72 \mathrm{Go}$ & $O(E) \times f O C$ & lor & & & & \\
\hline $68 \mathrm{Zn}$ & a & NDG & & CAL & E Zyshindt & 7840 \\
\hline${ }^{72} \mathrm{GO}$ & $\sigma(E)$ & & & & & \\
\hline $\begin{array}{l}{ }^{65} \mathrm{Cu} \\
68 \mathrm{Zn} \\
71 \mathrm{~GB} \\
72 \mathrm{~GB} \\
{ }^{75} \mathrm{As}\end{array}$ & $\begin{array}{r}160 \\
p \\
p \\
p \\
p\end{array}$ & $\begin{array}{l}4.6+1 \\
\text { NDG } \\
\text { NDC } \\
\text { NDG } \\
\text { NDC }\end{array}$ & & $\begin{array}{l}\text { SAC } \\
\text { CAL } \\
\text { CAL } \\
\text { CAL } \\
\text { CAL }\end{array}$ & $\begin{array}{l}\text { E Bollinl+ } \\
\text { T Woosleyt } \\
\text { T Woosleyt } \\
\text { T Woosleyt } \\
\text { T Woosleyt }\end{array}$ & $\begin{array}{r}11313 \\
7841 \\
2931 \\
2933 \\
3031\end{array}$ \\
\hline${ }^{73} \mathrm{Ge}$ & $\sigma(E)$ & & & & & \\
\hline${ }^{69} Z_{n}$ & . & NDE & & CAL & T Woogleyt & 7842 \\
\hline
\end{tabular}

\begin{tabular}{|c|c|c|c|c|c|c|}
\hline Target & $\operatorname{Inc}$ & $\begin{array}{l}\text { Emin } \\
(\mathrm{MeV})\end{array}$ & $\begin{array}{l}\text { Emax } \\
(M e V)\end{array}$ & Lab & Author & No. \\
\hline${ }^{73} \mathrm{Go}$ & $O(E)$ & & & & & \\
\hline 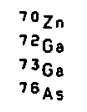 & $\begin{array}{l}\text { a } \\
\text { p } \\
p \\
p\end{array}$ & $\begin{array}{l}3.6+C \\
\text { NDG } \\
\text { NDG } \\
\text { NDG }\end{array}$ & $1 \cdot 1+1$ & $\begin{array}{l}\text { CAL } \\
\text { CAL } \\
\text { CAL } \\
\text { CAL }\end{array}$ & $\begin{array}{l}\text { T Woosley+ } \\
\text { T Woosley+ } \\
\text { T Woosley+ } \\
\text { I Woosleyt }\end{array}$ & $\begin{array}{l}7847 \\
2934 \\
2936 \\
3064\end{array}$ \\
\hline${ }^{74} \mathrm{Ge}$ & partial & relati & IVe ole & & & \\
\hline${ }^{76} \mathrm{Ge}$ & p & $2.0+1$ & $5.2+1$ & ORL E & E Restert & 3004 \\
\hline${ }^{74} \mathrm{Ge}$ & portiol & $\sigma(E)$ & & & & \\
\hline${ }^{78} \mathrm{Ge}$ & p & $2.6+1$ & & PAR E & E Gullbault & 3003 \\
\hline${ }^{74} \mathrm{Ge}$ & $\sigma(E)$ & & & & & \\
\hline $\begin{array}{l}70 \mathrm{Zn} \\
71 \mathrm{Zn} \\
73 \mathrm{Ga} \\
74 \mathrm{Go} \\
{ }^{77} \mathrm{As}\end{array}$ & $\begin{array}{l}a \\
a \\
p \\
p \\
p\end{array}$ & $\begin{array}{l}\text { NDG } \\
\text { NDG } \\
\text { NDG } \\
\text { NDG } \\
\text { NDG }\end{array}$ & & $\begin{array}{l}\text { CAL } \\
\text { CAL } \\
\text { CAL } \\
\text { CAL } \\
\text { CAL }\end{array}$ & $\begin{array}{l}\text { T Woosley+ } \\
\text { T Woosley+ } \\
\text { T Woosley+ } \\
\text { T Woosley+ } \\
\text { T Woosley+ }\end{array}$ & $\begin{array}{l}7848 \\
7849 \\
2937 \\
2939 \\
3067\end{array}$ \\
\hline${ }^{75} \mathrm{Go}$ & cumuloti & IVE OIE & & & & \\
\hline $\begin{array}{l}232 \mathrm{Th} \\
{ }^{75} \mathrm{Go}\end{array}$ & o(E) & $3.5+1$ & $8.5+1$ & MCG & E Diksict & 4783 \\
\hline $\begin{array}{l}74 \mathrm{Ga} \\
{ }^{75} \mathrm{Ga} \\
{ }^{78} \mathrm{As}\end{array}$ & $\begin{array}{l}p \\
p \\
p\end{array}$ & $\begin{array}{l}\text { NDG } \\
\text { NDG } \\
\text { NDG }\end{array}$ & & $\begin{array}{l}\text { CAL } \\
\text { CAL } \\
\text { CAL }\end{array}$ & $\begin{array}{l}\text { T Woosley+ } \\
\text { T Woosley+ } \\
\text { T Hoosleyt }\end{array}$ & $\begin{array}{l}2940 \\
2942 \\
3069\end{array}$ \\
\hline${ }^{76} \mathrm{Ge}$ & cumulati & IVO OLE & & & & \\
\hline $\begin{array}{l}23 \theta_{U} \\
{ }^{76} G_{0}\end{array}$ & $\begin{array}{r}p \\
O(E)\end{array}$ & $3.5+1$ & $8.5+1$ & MCOE & E Goliniert & 4916 \\
\hline $\begin{array}{l}{ }^{75} \mathrm{Ga} \\
{ }^{79} \mathrm{As}\end{array}$ & $p$ & $\begin{array}{l}\text { NDG } \\
\text { NDG }\end{array}$ & & $\begin{array}{l}\text { CAL } \\
\text { CAL }\end{array}$ & $\begin{array}{l}\text { T Woosleyt } \\
\text { T Woosleyt }\end{array}$ & $\begin{array}{l}2943 \\
3072\end{array}$ \\
\hline 770 & portiol & cumulo & olive & $O(E)$ & & \\
\hline $232 \mathrm{Th}$ & $p$ & $3.5+1$ & $0.5+1$ & MCG & E Diksict & 4784 \\
\hline${ }^{77} \mathrm{Go}$ & Indopend & dent $f 1$ & 193100 & $y 1010$ & & \\
\hline $209_{11}$ & ${ }^{12} \mathrm{C}$ & $7.5+1$ & $1.0+2$ & HAR & M Branquinhot & 10152 \\
\hline
\end{tabular}




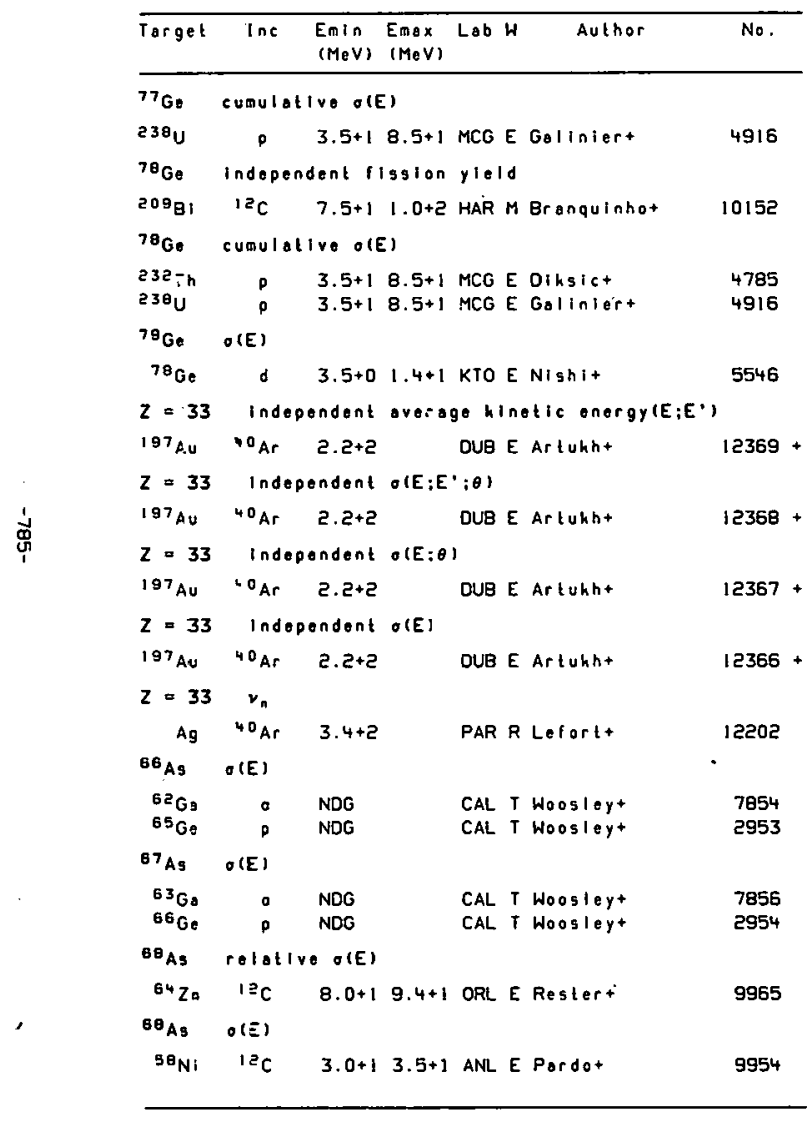

\begin{tabular}{|c|c|c|c|c|c|c|}
\hline Target & $\ln c$ & $\begin{array}{l}E_{m} \mid n \\
(M e V)\end{array}$ & $\begin{array}{l}\text { Emax } \\
(\operatorname{MeV})\end{array}$ & Lab $\mathrm{h}$ & Author & No. \\
\hline${ }^{80} \mathrm{As}^{\circ}$ & $O(E)$ & & & & & \\
\hline $\begin{array}{l}58 \mathrm{NI} \\
64 \mathrm{Ga} \\
67 \mathrm{Ge} \\
7150\end{array}$ & $\begin{array}{r}150 \\
0 \\
p \\
p\end{array}$ & $\begin{array}{l}7.0+1 \\
\text { NDG } \\
\text { NDG } \\
\text { NDG }\end{array}$ & & $\begin{array}{l}\text { NQI } \\
\text { CAL } \\
\text { CAL } \\
\text { CAL }\end{array}$ & $\begin{array}{l}\text { M Kuang-Shit } \\
T \text { Woosleyt } \\
T \text { Woosleyt } \\
T \text { Woosleyt }\end{array}$ & $\begin{array}{r}11208 \\
7857 \\
2956 \\
3084\end{array}$ \\
\hline${ }^{69} 9_{A 5}$ & compo & $d-n u$ & ous of & & & \\
\hline${ }^{50}{ }^{N I}$ & 160 & $4.5+1$ & $7.7+1$ & NEi & E Simpsont & 11210 \\
\hline
\end{tabular}

${ }^{60} \mathrm{As}$ relolive $\mathrm{I}_{\mathrm{r}}$

$54 \mathrm{Fe} 1804.5+15.5+1$ KLNE Hellneistert 11666

89 Ag Iniek largat yleld

$55 \mathrm{Mn} 160 \quad 4.1+1$ ATH E costet 11149

$60^{\text {As }}$ rolotivo o(E)

${ }^{64} \mathrm{Zn} \quad{ }^{12} \mathrm{C} \quad 7.2+19.4+1$ ORL E Restert 9966

$B B_{A 9} O(E)$

$38_{\mathrm{NI}} 160 \quad 7.0+1 \quad \mathrm{NBI} M$ Kuang-Sh1+ 11211

${ }_{65} 8^{N 1} 18007.0+1 \quad$ NBI M Kuang-Shit 11209

${ }^{65} \mathrm{~GB}^{5}$ a NDG CAL TWOOgleyt 7858

$\begin{array}{lllll}{ }^{58} \mathrm{Ge} & \text { P } & \text { NDG } & \text { CAL T Woogley+ } & 2958 \\ 72 \mathrm{Se} & p & \text { NDG } & \text { CAL T Woogley+ } & 3086\end{array}$

${ }^{70}$ As cumulative o(E)

${ }^{75} \mathrm{As} D 4.9+1 \quad 1.7+2$ SWR I Rudstam. 3063

$70^{\circ S}$ partiol relative o(E)

${ }^{70} \mathrm{Ge} D \quad 8.6+01.4+1$ FUL E TEn $8: \operatorname{lnht} 2963$

$10^{\circ S}$ rolative $I_{\gamma}$

${ }^{60} \mathrm{Ni}{ }^{2} \mathrm{C} \quad 5.0+1 \quad$ BNLE Filevieht 9959

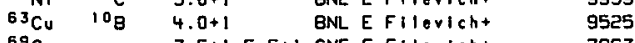

${ }^{69} \mathrm{Ga} a 3.5+15.5+1$ CNE E Filevicht 7863

$70 \mathrm{Ge} \quad 3.5+15.5+1$ CNE E fllevilht 7895

$70_{\text {AS }}$ relative O(E)

${ }^{64} \mathrm{Zn} \quad \mathrm{IIC}^{2} \mathrm{C} .3+19.4+1$ ORL E Restort 9967 


\begin{tabular}{|c|c|c|c|c|c|c|}
\hline Target & $\ln c$ & $\begin{array}{l}\text { Em:n} \\
(M e V)\end{array}$ & $\begin{array}{l}\text { Emax } \\
(M e V)\end{array}$ & Lab & Author & No. \\
\hline${ }^{70} \mathrm{As}$ & $\theta(E)$ & & & & & \\
\hline $\begin{array}{l}63 \mathrm{Cu} \\
{ }^{63} \mathrm{Ga} \\
69 \mathrm{Ge} \\
72^{2} \mathrm{Ge} \\
72 \mathrm{Ge} \\
{ }^{73} \mathrm{Ge}\end{array}$ & $\begin{array}{r}150 \\
p \\
p \\
p \\
p \\
p\end{array}$ & $\begin{array}{l}5.0+1 \\
\text { NDI } \\
\text { NDS } \\
1.0+2 \\
1.0+2 \\
\text { NDG }\end{array}$ & $\begin{array}{l}8.5+1 \\
4.0+2 \\
4.0+2\end{array}$ & $\begin{array}{l}\text { PAR } \\
\text { CAL } \\
\text { CAL } \\
\text { KFK } \\
\text { PUP } \\
\text { CAL }\end{array}$ & $\begin{array}{l}\text { E Langevint } \\
T \text { Woosleyt } \\
T \text { Woosleyt } \\
C \text { Muenzel+ } \\
\text { E Chureht } \\
T \text { Hoosleyt }\end{array}$ & $\begin{array}{r}11278 \\
7859 \\
2960 \\
2972 \\
2973+ \\
3088\end{array}$ \\
\hline${ }_{A 5}$ & compoun & nd-nucle & QUS OLE & & & \\
\hline $\begin{array}{l}58_{N 1} \\
71_{A g}\end{array}$ & $\begin{array}{l}160 \\
\text { cumulat }\end{array}$ & $\begin{array}{l}4.5+1 \\
\text { tive ole }\end{array}$ & $\begin{array}{l}7.7+1 \\
\text { E) }\end{array}$ & NB! & E simpsont & 11213 \\
\hline $\begin{array}{l}75 \mathrm{As} \\
{ }^{71} \mathrm{As}\end{array}$ & $\begin{array}{c}p \\
\text { partiol }\end{array}$ & $\begin{array}{l}4.9+1 \\
\text { overag }\end{array}$ & $\begin{array}{r}1.7+2 \\
g e .0(E)\end{array}$ & SWR & T Rudgtom. & 3063 \\
\hline${ }^{70} \mathrm{Ge}$ & $p$ & $2.0+0$ & $3.0+0$ & WWA & E Szeflingket & 2964 \\
\hline${ }^{71} \mathrm{As}$ & $\begin{array}{c}\text { relativ } \\
\text { a }\end{array}$ & $\begin{array}{l}\text { No } I_{y} \\
2.4+1\end{array}$ & & KLN & E Hellat & 7864 \\
\hline $71 \mathrm{As}$ & Inick & target $y$ & yield & & & \\
\hline $\begin{array}{l}{ }^{59} \mathrm{Co} \\
61 \mathrm{NI} \\
{ }^{70} \mathrm{Ge}\end{array}$ & $\begin{array}{r}160 \\
160 \\
p\end{array}$ & $\begin{array}{l}4.2+1 \\
3.9+1 \\
1.6+0\end{array}$ & $\begin{array}{l}5.2+1 \\
5.1+1 \\
5.0+0\end{array}$ & $\begin{array}{l}\text { TNL } \\
\text { ORL } \\
\text { CLU }\end{array}$ & $\begin{array}{l}\text { E Waltinert } \\
\text { E Wellst } \\
\text { M Roughtont }\end{array}$ & $\begin{array}{r}11173 \\
11238 \\
2967\end{array}$ \\
\hline "As & $O(E)$ & & & & & \\
\hline $\begin{array}{l}59 \mathrm{CO} \\
5 \theta_{\mathrm{NI}}\end{array}$ & $\begin{array}{l}150 \\
150\end{array}$ & $\begin{array}{l}3 \cdot 0+1 \\
7 \cdot 0+1\end{array}$ & $5.6+1$ & $\begin{array}{l}\text { TNL } \\
\text { NB1 }\end{array}$ & $\begin{array}{l}\text { E Wallnert } \\
\text { M Kuang-Shit }\end{array}$ & $\begin{array}{l}11172 \\
11212\end{array}$ \\
\hline $\mathrm{GINI}^{2}$ & 160 & $4.6+1$ & & ORL & M Wellst & 11239 \\
\hline${ }^{63} \mathrm{Cu}$ & 150 & $5.0+1$ & $0.5+1$ & PAR & E Langevint & 11279 \\
\hline $67 \mathrm{Go}$ & $a$ & NOG & & CAL & T Woosleyt & 7860 \\
\hline Ge & ${ }^{3} \mathrm{He}$ & +1 & $3.7+1$ & LIE & E Gulllaumet & 6372 \\
\hline $\mathrm{Ge}$ & a & +1 & $4.0+1$ & LIE & E Gulllaume + & $\begin{array}{l}7875 \\
2965\end{array}$ \\
\hline $\begin{array}{l}{ }^{70} \mathrm{Ge} \\
70 \mathrm{Gg}\end{array}$ & p & NDG & & CAL & T Woosleyt & $\begin{array}{l}2965 \\
2966\end{array}$ \\
\hline $\begin{array}{l}70 \mathrm{Ge} \\
72 \mathrm{Ge}\end{array}$ & $\begin{array}{l}p \\
p\end{array}$ & $\begin{array}{l}1.5+0 \\
1.0+2\end{array}$ & $\begin{array}{l}5.1+0 \\
4.0+2\end{array}$ & $\begin{array}{l}\text { CLU } \\
\text { KFK }\end{array}$ & $\begin{array}{l}\text { E Roughiont } \\
\text { C Muenzelt }\end{array}$ & $\begin{array}{l}2966 \\
2974\end{array}$ \\
\hline $72 \mathrm{Ge}$ & $p$ & $1.0+5$ & $4.0+2$ & PUP & E Churest & 2975 \\
\hline${ }^{72} \mathrm{Ge}$ & ${ }^{3} \mathrm{He}$ & +1 & $3.7+1$ & LIE & E Guillaumet & 6383 \\
\hline${ }^{72} \mathrm{Ge}_{\mathrm{e}}$ & a & +1 & $4.0+1$ & LIE & E Guillaumet & 7905 \\
\hline${ }^{74} \mathrm{Se}$ & p & NDG & & CAL & T Woosley+ & 3090 \\
\hline
\end{tabular}

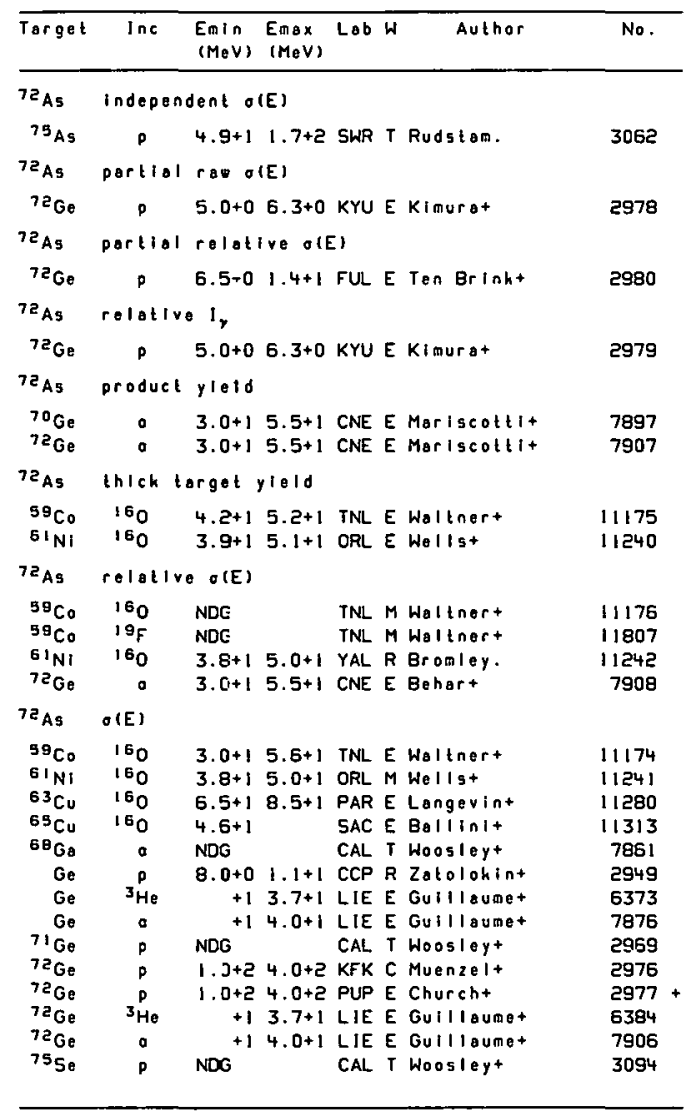




\begin{tabular}{|c|c|c|c|c|c|c|}
\hline Target & $\ln c$ & $\begin{array}{l}\text { Emin } \\
(M \theta V)\end{array}$ & $\begin{array}{l}E m \Delta x \\
(M e y)\end{array}$ & Lob & Author & No. \\
\hline $72 \bullet A .9$ & \multicolumn{6}{|c|}{ gartial celative product yleld } \\
\hline $59 \mathrm{CO}$ & $: 60$ & $4 \cdot 3+1$ & $5.6+1$ & BEL & E Raghavent & 11177 \\
\hline${ }^{73} \mathrm{As}$ & \multicolumn{6}{|c|}{ cumulatlye thlek arget yleld } \\
\hline $\mathrm{C} \cdot \mathrm{e}$ & a & $1.1+1$ & $4.4-1$ & FEI & E Denitrievt & $7877+$ \\
\hline${ }^{73} \mathrm{As}$ & partial & averag & ge O:El & & & \\
\hline $7 a_{\mathrm{ce}}$ & p & $2.0+0$ & $3.0-0$ & WWA & E Szeflingkat & 2981 \\
\hline${ }^{73}$ As & \multicolumn{6}{|c|}{ relative ty } \\
\hline $71 \mathrm{Ga}$ & o & $2.1+1$ & & KLN & E Heilst & 7867 \\
\hline${ }^{73} \mathrm{As}$ & product & yiald & & & & \\
\hline${ }^{72} \mathrm{Ge}$ & p & $3.0+0$ & $3.4+0$ & TRM & E Babat & 2982 \\
\hline${ }^{73} \mathrm{As}$ & \multicolumn{6}{|c|}{ thlch target yleldxabundance } \\
\hline${ }^{71} \mathrm{Ga}$ & $a$ & $1.9+1$ & $4.4+1$ & FEI & E Dmilrievt & $7868+$ \\
\hline${ }^{73} \mathrm{AS}$ & \multicolumn{6}{|c|}{ thich torget yielc } \\
\hline $\begin{array}{l}\mathrm{Ga} \\
\mathrm{Ge} \\
\mathrm{Ge}\end{array}$ & $\begin{array}{l}a \\
\dot{p} \\
d\end{array}$ & $\begin{array}{l}1.9+1 \\
7.0+0 \\
6.6+0\end{array}$ & $\begin{array}{l}4.4+1 \\
2.2+1 \\
2.2+1\end{array}$ & $\begin{array}{l}\text { FEI } \\
\text { FEI } \\
\text { FEI }\end{array}$ & $\begin{array}{l}\text { E Daitrievt } \\
\text { E Dmilrievt } \\
\text { E Dailrievt }\end{array}$ & $\begin{array}{l}7851+ \\
2950 \div \\
5542+\end{array}$ \\
\hline${ }^{73} \mathrm{As}$ & average & $\theta(E)$ & & & & \\
\hline${ }^{73} \mathrm{Ge}$ & p & $1.1+0$ & $1.4+0$ & ORL & E Johnsont & 2985 \\
\hline${ }^{73} \mathrm{As}$ & \multicolumn{6}{|c|}{ relalive o(E) } \\
\hline $72_{G S}$ & a & $3.0+1$ & $5.5+1$ & CNE & E Behart & 7909 \\
\hline${ }^{73} \mathrm{As}$ & \multicolumn{6}{|l|}{$O(E)$} \\
\hline $59 \mathrm{CO}_{0}$ & 160 & $5.6+1$ & & TNL & E Wallnert & 11187 \\
\hline $\begin{array}{l}65 \\
{ }^{65 u}\end{array}$ & 160 & $4.6+1$ & & SAC & E Ballinit & 11313 \\
\hline${ }_{79}^{69} \mathrm{Ga}$ & $a$ & NDG & & CAL & T Woosleyt & 7865 \\
\hline $\begin{array}{l}72 \mathrm{Ge} \\
76 \mathrm{Se}\end{array}$ & p & NDG & & CAL & T Woosleyt & 2983 \\
\hline${ }^{76} \mathrm{Se}$ & p & NOG & & CAL & T Woosleyt & 3096 \\
\hline${ }^{74} \mathrm{As}$ & \multicolumn{6}{|c|}{ Independent $O(E)$} \\
\hline${ }^{75} \mathrm{As}$ & p & $4.9+1$ & $1.7+2$ & SWR & T Rudstam. & 3062 \\
\hline${ }^{74} \mathrm{As}$ & portlal & relati & I ve olE & & & \\
\hline $7 a_{\mathrm{Ge}}$ & 。 & $3.0+1$ & $5.5+1$ & CNE & E Bermodez+ & 7910 \\
\hline
\end{tabular}

\begin{tabular}{|c|c|c|c|c|c|c|}
\hline Target & $\ln c$ & $\begin{array}{l}E(\mathrm{In} n \\
(\mathrm{MeV})\end{array}$ & $\begin{array}{l}\text { Emax } \\
(\operatorname{MeV})\end{array}$ & Lab b & futhor & No. \\
\hline${ }^{74} \mathrm{As}$ & partial & relati & IVe OLE & E) & & \\
\hline${ }^{74} \mathrm{Ge}$ & p & $T R$ & +0 & ORE E & E Leferret & 2990 \\
\hline${ }^{74} \mathrm{As}$ & partial & $\theta(E)$ & & & & \\
\hline${ }^{74} \mathrm{Ge}$ & $p$ & $3.4-1$ & & ORE E & E Rosst & 2991 \\
\hline${ }^{74} \mathrm{As}$ & thick to & orget $y$ & yieldxo & obundo & yence & \\
\hline 710 & 0 & $1.3+1$ & $4.4+1$ & FEI E & E Dmitrievt & $7869+$ \\
\hline${ }^{74} \mathrm{As}$ & thick to & argot $y$ & yiald & & & \\
\hline${ }^{6} \mathrm{Ni}$ & 160 & $3.9+1$ & $5.1+1$ & ORL E & E Wellst & 11243 \\
\hline Ga & a & $1.3+1$ & $4.4+1$ & FEI E & E Dmitriest & $7852+$ \\
\hline Ge & $p$ & $7.0+0$ & $2.2+1$ & FEI E & E Dmitriest & $2952+$ \\
\hline Ge & d & $6.6+0$ & $2.2+1$ & FE! E & E Dmitriest & $5543+$ \\
\hline$G e$ & o & $2.4+1$ & $4.4+1$ & FEI E & E Dallelert & $787 \theta+$ \\
\hline${ }^{74} \mathrm{Ge}$ & p & $3.5+0$ & $5.0+0$ & CLU $M$ & M Roughto.nt & 2994 \\
\hline${ }_{75} \mathrm{As}$ & p & $2.2+1$ & & FEI E & E Doliticiest & $3035+$ \\
\hline${ }^{79} \mathrm{As}$ & d & $2.2+1$ & & FEI E & Enitrieyt & $5549+$ \\
\hline${ }^{75} \mathrm{As}$ & a & $4.3+1$ & & FEI E & E Dalirient & $7938+$ \\
\hline $74 \mathrm{As}$ & $r a w o(E)$ & & & & & \\
\hline${ }^{74} \mathrm{Ge}$ & $p$ & $3.2+0$ & $3.7+0$ & ORL E & E Johnsint & 2995 \\
\hline${ }^{74} \mathrm{Ag}$ & relative & $10 O(E)$ & & & & \\
\hline $61 \mathrm{Ni}$ & 150 & $3.8+1$ & $5.0+1$ & YAL $P$ & R Bromlsy. & 11245 \\
\hline${ }^{74} \mathrm{As}$ & $O(E) \times f a C$ & actor & & & & \\
\hline${ }^{75} \mathrm{As}$ & $p$ & $2.2+1$ & & ORL E & E Cohent & 3034 \\
\hline${ }^{74} \mathrm{As}$ & $O(E)$ & & & & & \\
\hline${ }^{61} \mathrm{Ni}$ & 160 & $3.8+1$ & $5.0+1$ & ORL $M$ & $M$ Wells- & 11244 \\
\hline${ }^{70} \mathrm{G} B$ & a & & & CAL I & I Woosloyt & 7866 \\
\hline Ge & p & $8.0+0$ & $1.1+1$ & CCP $P$ & R Zatolokint & 2951 \\
\hline${ }^{73} \mathrm{Ge}$ & p & NDG & & CAL $\mathrm{T}$ & T Woosleyt & 2986 \\
\hline${ }^{74} \mathrm{Ge}$ & p & $3.4+0$ & $5.8+0$ & KFK O & C Muenzelt & 2992 \\
\hline${ }^{74} \mathrm{Ge}$ & $p$ & $3.4+0$ & $5.8+0$ & ORL E & E Johnsant & $2993+$ \\
\hline $75_{\mathrm{As}}$ & p & $2.2+1$ & & KFK O & C Muenzelt & 3032 \\
\hline${ }^{73} \mathrm{As}$ & p & $2.2+1$ & & ORL E & E Cohen- & $3033+$ \\
\hline${ }^{77} \mathrm{Se}$ & p & NDG & & CAL T & T Woosleyt & 3099 \\
\hline
\end{tabular}




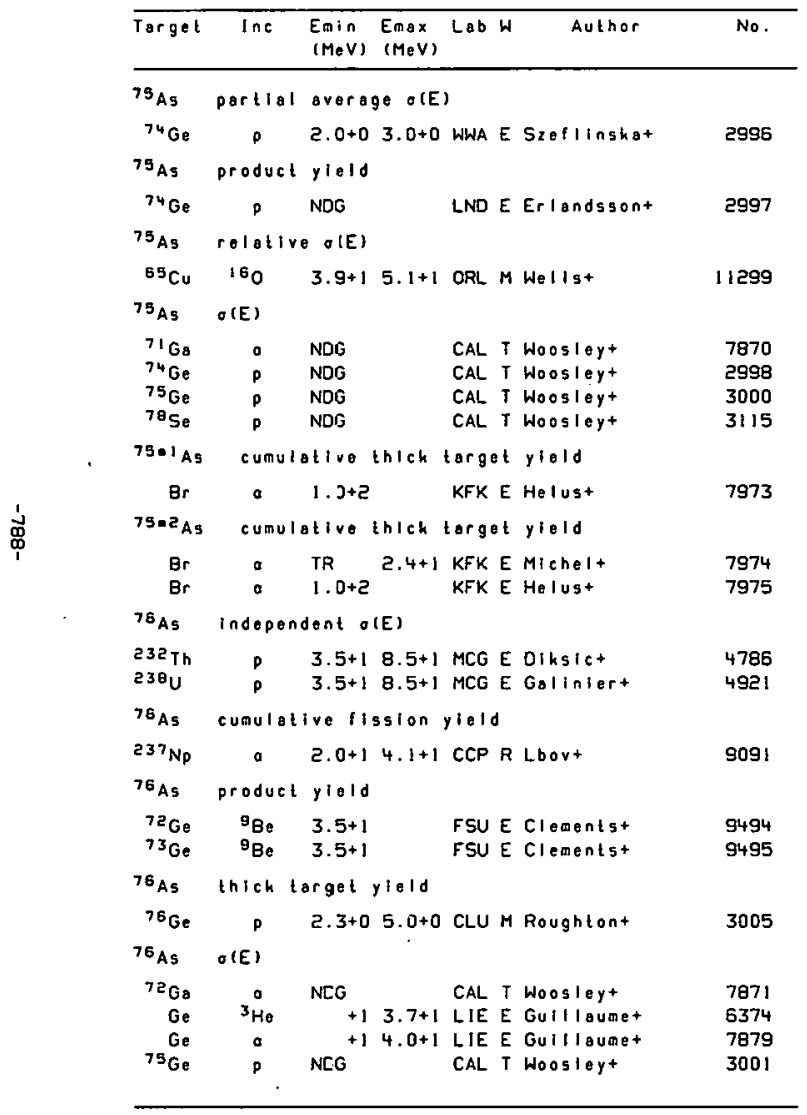

\begin{tabular}{|c|c|c|c|c|c|c|}
\hline Torget & $\ln c$ & $\begin{array}{l}\text { Emin } \\
(\mathrm{MeV})\end{array}$ & $\begin{array}{l}E \max \\
(\mathrm{MeV})\end{array}$ & Lab $W$ & Author & No. \\
\hline${ }^{76}$ Ag & $O(E)$ & & & & & \\
\hline $\begin{array}{l}{ }^{76} \mathrm{Ge} \\
{ }^{79} \mathrm{Se}\end{array}$ & $\begin{array}{l}p \\
p\end{array}$ & $\begin{array}{l}1.8+0 \\
N D G\end{array}$ & $5.1+0$ & $\begin{array}{l}\text { CLU E } \\
\text { CAL T }\end{array}$ & $\begin{array}{l}\text { E Roughtont } \\
\text { T Woogleyt }\end{array}$ & $\begin{array}{l}3006 \\
3118\end{array}$ \\
\hline $7^{77}$ & partial & cumula & ative o & $\sigma(E)$ & & \\
\hline $238_{U}$ & p & $3.5+1$ & $8.5+1$ & MCG E & E Galiniert & 4879 \\
\hline $77_{A S}$ & portlal & cumulo & attiveo & $\sigma(E)$ & & \\
\hline $232 \mathrm{Th}$ & $\mathbf{p}$ & $3.5+1$ & $8.5+1$ & MCG $\varepsilon$ & E Dihsict & 4787 \\
\hline${ }^{77}$ AS & cumuloti & Ive fls & sgion $y$ & yield & & \\
\hline $237^{\mathrm{Np}}$ & a & $2.0+1$ & $4.1+1$ & CCP R & R Lbovt & 9091 \\
\hline "TAS & $O(E)$ & & & & & \\
\hline $\begin{array}{l}{ }^{73} \mathrm{Go} \\
74 \mathrm{Go} \\
76 \mathrm{Ge} \\
77 \mathrm{Ge} \\
{ }^{80} \mathrm{Se}\end{array}$ & $\begin{array}{l}a \\
a \\
p \\
p \\
p\end{array}$ & $\begin{array}{l}\text { NDG } \\
\text { NDG } \\
\text { NDG } \\
\text { NDG } \\
\text { NDG }\end{array}$ & & $\begin{array}{l}\text { CAL T } \\
\text { CAL } \\
\text { CAL } \\
\text { CAL } \\
\text { CAL T }\end{array}$ & $\begin{array}{l}\text { T Woosley+ } \\
\text { T Woosleyt } \\
\text { T Wooley+ } \\
\text { T Woosley+ } \\
\text { T Woogley+ }\end{array}$ & $\begin{array}{l}7872 \\
7873 \\
3007 \\
3009 \\
3120\end{array}$ \\
\hline${ }^{7 \theta_{A S}}$ & independ & dent of & (E) & & & \\
\hline $\begin{array}{l}232 i \mathrm{ih} \\
23 \theta_{\mathrm{u}}\end{array}$ & p & $\begin{array}{l}3.5+1 \\
3.5-1\end{array}$ & $\begin{array}{l}8.5+1 \\
8.5+1\end{array}$ & $\begin{array}{l}\text { MCG E } \\
\text { MCG E }\end{array}$ & $\begin{array}{l}\text { E Diksict } \\
\text { E Galiniert }\end{array}$ & $\begin{array}{l}4788 \\
4921\end{array}$ \\
\hline${ }^{7 \theta_{A B}}$ & product & yield & & & & \\
\hline $\begin{array}{l}{ }^{72} \mathrm{Ge} \\
{ }^{7 B_{\mathrm{AS}}}\end{array}$ & $\begin{array}{l}{ }^{9} \mathrm{Be} \\
\mathrm{O}(\mathrm{E})\end{array}$ & $3.5-1$ & & FSU E & E clementst & 9494 \\
\hline $\begin{array}{l}74 \mathrm{Ge} \\
{ }^{77} \mathrm{Ge} \\
78_{\mathrm{Ge}} \\
81 \mathrm{Se}\end{array}$ & $\begin{array}{l}a \\
p \\
d \\
p\end{array}$ & $\begin{array}{l}\text { NDG } \\
\text { NDG } \\
3.5+0 \\
\text { NDG }\end{array}$ & $1.4+1$ & $\begin{array}{l}\text { CAL T } \\
\text { CAL T } \\
\text { KTO E } \\
\text { CAL T }\end{array}$ & $\begin{array}{l}\text { T Woosley+ } \\
\text { T Woosley+ } \\
\text { E Nishit } \\
\text { T Woosleyt }\end{array}$ & $\begin{array}{l}7874 \\
3010 \\
5547 \\
3129\end{array}$ \\
\hline${ }^{79} \mathrm{As}$ & cumulati & ive ste & & & & \\
\hline $\begin{array}{l}2354 h \\
238_{U}\end{array}$ & p & $\begin{array}{l}3.5+1 \\
3.5+1\end{array}$ & $\begin{array}{l}8.5+1 \\
8.5+1\end{array}$ & $\begin{array}{l}\text { MCG E } \\
\text { MCG E }\end{array}$ & $\begin{array}{l}\text { E Olhsict } \\
\text { E Galiniert }\end{array}$ & $\begin{array}{l}4789 \\
4916\end{array}$ \\
\hline${ }^{79} \mathrm{Ag}$ & $\sigma(E)$ & & & & & \\
\hline${ }^{78} \mathrm{Ge}_{\mathrm{e}}$ & d & $3.2+0$ & $1.4+1$ & КTO E & E Nighit & 5548 \\
\hline
\end{tabular}




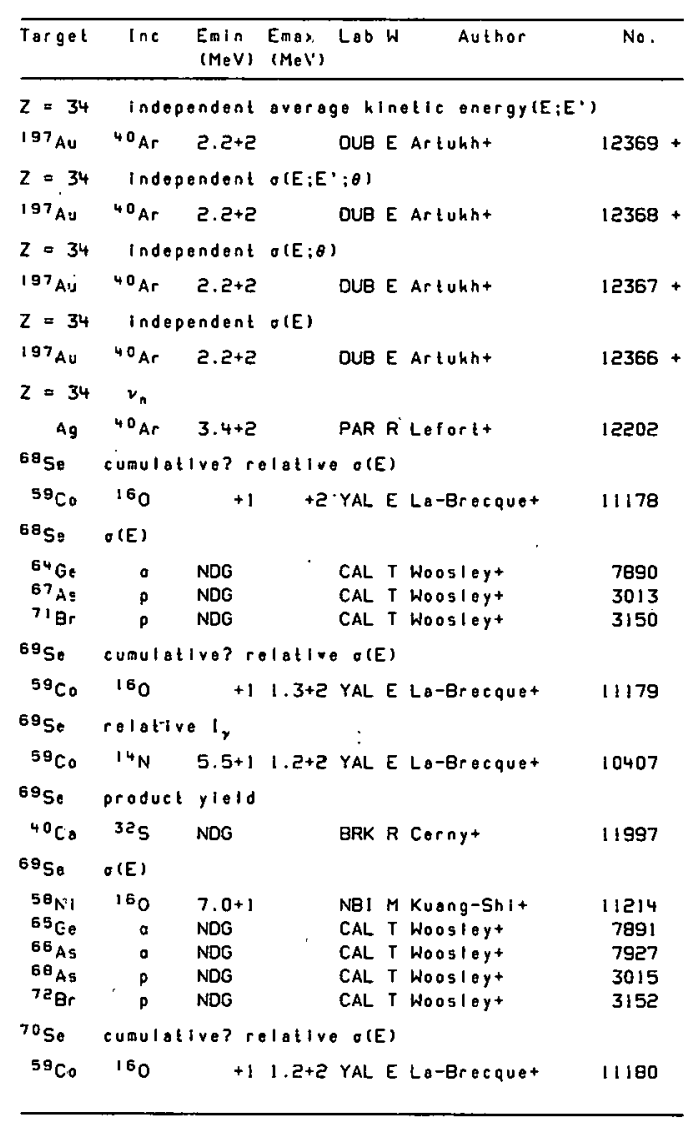

\begin{tabular}{|c|c|c|c|c|c|c|}
\hline Target & $\ln c$ & $\begin{array}{l}\text { Emin } \\
(\operatorname{MeV})\end{array}$ & $\begin{array}{l}\text { Emax } \\
(\operatorname{MeV})\end{array}$ & Lab & Author & No. \\
\hline${ }^{70} \mathrm{Se}$ & relotiv & $\theta O(E)$ & & & & \\
\hline $\begin{array}{l}59 \mathrm{Co}_{0} \\
{ }^{64} \mathrm{Zn}\end{array}$ & $\begin{array}{l}14 \mathrm{~N} \\
1{ }^{2} \mathrm{C}\end{array}$ & $\begin{array}{r}+1 \\
6.3+1\end{array}$ & $\begin{array}{l}1.2+2 \\
9.4+1\end{array}$ & $\begin{array}{l}\text { YAL } \\
\text { ORL }\end{array}$ & $\begin{array}{l}\text { E Lo-Brecquet } \\
\text { E Restert }\end{array}$ & $\begin{array}{r}10408 \\
9968\end{array}$ \\
\hline${ }^{70} \mathrm{Se}$ & $\sigma(E)$ & & & & & \\
\hline $\begin{array}{l}{ }^{66} \mathrm{Ge} \\
{ }^{67} \mathrm{As} \\
{ }^{69} \mathrm{As} \\
{ }^{73} \mathrm{Br}\end{array}$ & $\begin{array}{l}a \\
a \\
p \\
p\end{array}$ & $\begin{array}{l}\text { NDG } \\
\text { NDG } \\
\text { NDG } \\
\text { NDG }\end{array}$ & & $\begin{array}{l}\text { CAL } \\
\text { CAL } \\
\text { CAL } \\
\text { CAL }\end{array}$ & $\begin{array}{l}\text { T Wooslyy- } \\
\text { T Wooslay+ } \\
\text { T Woosligy+ } \\
\text { T Woosley+ }\end{array}$ & $\begin{array}{l}7892 \\
7929 \\
3017 \\
3154\end{array}$ \\
\hline $7 \mathrm{Se}$ & thich & $\operatorname{arget} y$ & yeld & & & \\
\hline$E_{N 1}$ & 160 & $3.9+1$ & $5.1+1$ & ORL & E Wells:- & 11246 \\
\hline $715 e$ & relotiv & $\theta O(E)$ & & & $\cdot$ & \\
\hline $\begin{array}{l}{ }^{84} \mathrm{Zn} \\
{ }^{70} \mathrm{Ge}\end{array}$ & ${ }_{0}^{12} \mathrm{C}$ & $\begin{array}{r}6.3+1 \\
+1\end{array}$ & $\begin{array}{l}9.4+1 \\
1.2+2\end{array}$ & ORL & $\begin{array}{l}\text { E Restert } \\
\text { E La-Brecquet }\end{array}$ & $\begin{array}{l}9969 \\
7898\end{array}$ \\
\hline 115 & $\sigma(E)$ & & & & & \\
\hline $\begin{array}{l}{ }^{58} \mathrm{Ni} \\
67 \mathrm{Ge} \\
{ }^{70} \mathrm{As} \\
{ }^{74} \mathrm{Br}\end{array}$ & $\begin{array}{r}160 \\
0 \\
p \\
p\end{array}$ & $\begin{array}{l}7.0+1 \\
\text { NDG } \\
\text { NDG } \\
\text { NDG }\end{array}$ & & $\begin{array}{l}\text { NBI } \\
\text { CAL } \\
\text { CAL } \\
\text { CAL }\end{array}$ & $\begin{array}{l}\text { M Kuong-Shit } \\
T \text { Woosleyt } \\
\text { T Woosleyt } \\
\text { T Woosleyt }\end{array}$ & $\begin{array}{r}11215 \\
7893 \\
3019 \\
3156\end{array}$ \\
\hline 7250 & cumulat & ive com & apound- & nucl & lous $O(E)$ & \\
\hline $\begin{array}{l}5 \theta_{\mathrm{NI}} \\
7 \mathrm{C}_{\mathrm{Se}}\end{array}$ & $\begin{array}{l}160 \\
\text { compoun }\end{array}$ & $\begin{array}{c}4.5+1 \\
\text { ndenucle }\end{array}$ & $\begin{array}{l}7.7+1 \\
\text { eus olE }\end{array}$ & $\begin{array}{l}\text { NBI } \\
\text { E) }\end{array}$ & E simpsant & 11216 \\
\hline $\begin{array}{l}{ }^{5 \theta_{\mathrm{Ni}}} \\
7 \mathrm{E}_{\mathrm{Se}}\end{array}$ & $\begin{array}{l}160 \\
\text { indopen }\end{array}$ & $\begin{array}{l}4.5+1 \\
\text { ident ol }\end{array}$ & $\begin{array}{l}7.7+1 \\
\text { (E) }\end{array}$ & NBI & E simpsont & 11220 \\
\hline${ }^{75} \mathrm{As}$ & p & $4.9+1$ & $1.7+2$ & SWR & T Rudstam. & 3062 \\
\hline${ }^{72} \mathrm{Se}$ & partiol & relati & ive otE & & & \\
\hline${ }^{58_{\mathrm{N} 1}}$ & 160 & $4.6+1$ & $5.8+1$ & KLN & E Hell meis :ert & $\mid 1221$ \\
\hline 7258 & relativ & $\theta l_{y}$ & & & & \\
\hline${ }^{58_{N 1}}$ & 160 & $4.0+1$ & $5.8+1$ & BNL & E Lieb+ & 11219 \\
\hline${ }^{72} \mathrm{Se}$ & thick t & arget $y$ & $y \mid e 1 d x e$ & Bbund & tance & \\
\hline${ }^{70} \mathrm{Ge}$ & a & $2.5+1$ & $4.4+1$ & FEI & E Dmiteiert & $7899+$ \\
\hline
\end{tabular}




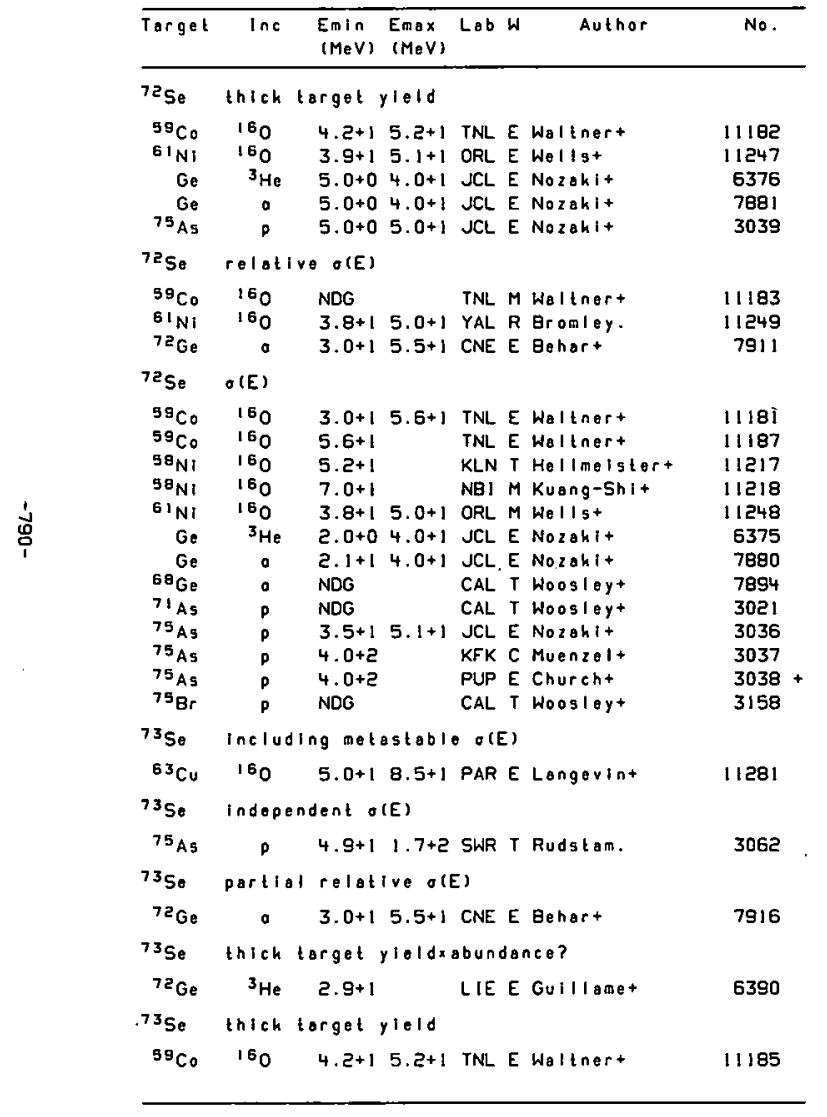

\begin{tabular}{|c|c|c|c|c|c|c|}
\hline Target & $\ln c$ & $\begin{array}{l}\text { Emln } \\
(\operatorname{MeV})\end{array}$ & $\begin{array}{l}\text { Emax } \\
(\operatorname{MeV})\end{array}$ & Lob & Author & No. \\
\hline${ }^{73} \mathrm{Se}$ & thick & target $y$ & ylold & & & \\
\hline $\begin{array}{r}61 \mathrm{NI} \\
\mathrm{Ge} \\
\mathrm{Ge} \\
\mathrm{Ge} \\
\mathrm{Ge} \\
{ }^{70} \mathrm{Ge} \\
7 \mathrm{Ge}^{\mathrm{Ge}} \\
7 \mathrm{C}_{\mathrm{Ge}} \\
7 \mathrm{Ge}_{\mathrm{Ge}} \\
0 \times 1 \mathrm{Ge} \\
0 \times 1 \mathrm{Ge} \\
75_{\mathrm{AS}}\end{array}$ & $\begin{array}{c}16 \mathrm{O} \\
{ }^{3} \mathrm{He} \\
{ }^{3} \mathrm{He} \\
a \\
a \\
0 \\
{ }^{3} \mathrm{He} \\
{ }^{3} \mathrm{He} \\
0 \\
{ }^{3} \mathrm{He} \\
0 \\
0\end{array}$ & $\begin{array}{l}3 \cdot 9+1 \\
5 \cdot 0+0 \\
2 \cdot 0+1 \\
5 \cdot 0+0 \\
1 \cdot 5+1 \\
T R \\
1 \cdot 7+1 \\
3 \cdot 7+1 \\
3 \cdot 2+1 \\
3 \cdot 7+1 \\
2 \cdot 6+1 \\
5 \cdot 0+0\end{array}$ & $\begin{array}{r}5 \cdot 1+1 \\
4 \cdot 0+1 \\
3 \cdot 5+1 \\
4 \cdot 0+1 \\
3 \cdot 7+1 \\
+1 \\
3 \cdot 7+1 \\
4.0+1 \\
4 \\
5 \cdot 0+1\end{array}$ & $\begin{array}{l}\text { ORL } \\
\text { JCL } \\
\text { ENL } \\
\text { JCL } \\
\text { ENL } \\
\text { CLU } \\
\text { ENL } \\
\text { JUL } \\
\text { BNL } \\
\text { LIE } \\
\text { LIE } \\
\text { JCL }\end{array}$ & $\begin{array}{l}\text { E Wellst } \\
\text { E Nozoklt } \\
\text { E Guillaumet } \\
\text { E Nozakit } \\
\text { E Gulllaumet } \\
\text { E Roughtont } \\
\text { E Guliloumet } \\
\text { R Oalmt } \\
\text { E Gulllaumet } \\
\text { E Gulllaumet } \\
\text { E Gulllaumet } \\
\text { E Nozakit }\end{array}$ & $\begin{array}{r}11250 \\
6379 \\
6380 \\
7884 \\
7885 \\
7902 \\
6388 \\
6389 \\
7915 \\
6394 \\
7926 \\
3042\end{array}$ \\
\hline${ }^{73} \mathrm{Se}$ & reloti & $v \theta O(E)$ & & & & \\
\hline $\begin{array}{l}{ }^{59} \mathrm{Co} \\
73 \mathrm{Se}\end{array}$ & $\begin{array}{l}160 \\
\sigma(E)\end{array}$ & NEG & & TNL & M Waltnert & 11188 \\
\hline $\begin{array}{l}{ }^{59} \mathrm{Co} \\
5^{59} \mathrm{CO} \\
61 \mathrm{Ni}\end{array}$ & $\begin{array}{l}160 \\
160 \\
160\end{array}$ & $\begin{array}{l}3 \cdot 0+1 \\
5 \cdot 6+1 \\
4.6+1\end{array}$ & $5.6+1$ & $\begin{array}{l}\text { TNL } \\
\text { TNL } \\
\text { ORL }\end{array}$ & $\begin{array}{l}\text { E Waltinert } \\
\text { E Waltinert } \\
\text { M Wellst }\end{array}$ & $\begin{array}{l}11184 \\
11187 \\
11251\end{array}$ \\
\hline $\mathrm{Ge}_{\mathrm{G}}$ & $\begin{array}{l}{ }^{3} \mathrm{Ho} \\
{ }^{3} \mathrm{He}\end{array}$ & $\begin{array}{r}1.0+1 \\
+1\end{array}$ & $\begin{array}{l}4 \cdot 0+1 \\
3 \cdot 7+1\end{array}$ & $\begin{array}{l}\text { JCL } \\
\text { LIE }\end{array}$ & $\begin{array}{l}\text { E Nozakit } \\
\text { E Guillaume+ }\end{array}$ & $\begin{array}{l}6377 \\
6378\end{array}$ \\
\hline $\mathrm{Ge}$ & $a$ & $+i$ & $4.0+1$ & LIE & E Guillaumet & 7882 \\
\hline $\begin{aligned} \mathrm{Ge} \\
69_{\mathrm{Ge}}\end{aligned}$ & a & $\begin{array}{l}1.3+1 \\
\text { NDG }\end{array}$ & $4.0+i$ & $\begin{array}{l}J C L \\
C A L\end{array}$ & $\begin{array}{l}\text { E Nozokit } \\
\text { T Woosleyt }\end{array}$ & $\begin{array}{l}7883 \\
7895\end{array}$ \\
\hline $70 \mathrm{Ge}$ & a & $1.3+1$ & $3.2+1$ & BNL & E Guillaume+ & 7900 \\
\hline${ }^{70} \mathrm{Ge}$ & 0 & $1.3+1$ & $3.2+1$ & JUL & $\mathrm{ROAimt}^{+}$ & 7901 \\
\hline${ }^{72} \mathrm{Ge}$ & ${ }^{3} \mathrm{He}$ & +1 & $3.7+1$ & LIE & E Guillaume+ & 6385 \\
\hline $72_{\mathrm{Ge}}$ & ${ }^{3} \mathrm{He}$ & $1.6+1$ & $3.6+1$ & BNL & E Guillaume+ & 6386 \\
\hline${ }^{72} \mathrm{Ge}$ & ${ }^{3} \mathrm{He}$ & $1.6+1$ & $3.6+1$ & JUL & $R a_{B} I_{m+}$ & 6307 \\
\hline${ }^{72} \mathrm{Ge}$ & $a$ & +1 & $4.0+1$ & LIE & E Guillaumet & 7912 \\
\hline${ }^{72} \mathrm{Ge}$ & a & $3.2+1$ & $4.0+1$ & $\mathrm{BNL}$ & E Guillaumet & 7913 \\
\hline $72_{\mathrm{Ge}}$ & & $3.3+1$ & $4.0+1$ & JUL & ROaint & 7914 \\
\hline${ }^{73} \mathrm{Ge}$ & ${ }^{3} \mathrm{He}$ & $3.2+1$ & $3.6+1$ & BNL & E Guillaumet & 6392 \\
\hline${ }^{73} \mathrm{Ge}$ & ${ }^{3} \mathrm{He}$ & $3.3+1$ & $3.6+1$ & JUL & $\mathrm{ROaim}^{+}$ & 6393 \\
\hline $72 \mathrm{As}$ & p & NDG & & CAL & T Woosleyt & 3023 \\
\hline $75 \mathrm{As}$ & p & $2.0+0$ & $1.5+1$ & $\mathrm{JCL}$ & ENozahit & 3040 \\
\hline${ }^{75} \mathrm{As}$ & p & $3.0+1$ & $8.0+1$ & MCG & M Brodoviteht & 3041 \\
\hline${ }^{76} \mathrm{Br}$ & p & NDG & & CAL & T Woosleyt & 3160 \\
\hline
\end{tabular}




\begin{tabular}{|c|c|c|c|c|c|c|}
\hline Target & Inc & $\begin{array}{l}\text { Emin } \\
(\mathrm{MeV})\end{array}$ & $\begin{array}{l}\text { Emax } \\
(\operatorname{MeV})\end{array}$ & Lab. & Author & No. \\
\hline 73950 & \multicolumn{2}{|c|}{ relative O(E) } & 1 & & & \\
\hline${ }^{64} \geq n$ & ${ }^{12} \mathrm{c}$ & $6 \cdot 3+1$ & $9.4+1$ & ORL & E Restert & 9970 \\
\hline 73.58 & $\sigma(E)$ & . & & & & \\
\hline $\begin{array}{l}{ }^{63} \mathrm{Cu}_{\mathrm{U}} \\
{ }^{75} \mathrm{Ag} \\
{ }_{79} \mathrm{AS}\end{array}$ & $\begin{array}{r}160 \\
p \\
p\end{array}$ & $\begin{array}{l}5.0+1 \\
4.0+2 \\
4.0+2\end{array}$ & $8.5+1$ & $\begin{array}{l}\text { PAR } \\
\text { KFK } \\
\text { PUP }\end{array}$ & $\begin{array}{l}\text { E Langevint } \\
\text { C Muenzel+ } \\
\text { E Chureht }\end{array}$ & $\begin{array}{l}11282 \\
3043 \\
3044\end{array}$ \\
\hline 73.58 & \multicolumn{3}{|c|}{ relative o(E) } & & & \\
\hline${ }^{64} \mathrm{zn}$ & ${ }^{12} \mathrm{C}$ & $6.3+1$ & $9.4+1$ & ORL & E Restert & 9971 \\
\hline $73.5 e$ & $\sigma(E)$ & & & & & \\
\hline $\begin{array}{l}63 \mathrm{Cu} \\
75 \mathrm{hs} \\
75 \mathrm{hs}\end{array}$ & $\begin{array}{r}160 \\
p \\
p\end{array}$ & $\begin{array}{l}5.0+1 \\
4.0+2 \\
4.0+2\end{array}$ & $8.5+1$ & $\begin{array}{l}\text { PAR } \\
\text { KFK } \\
\text { PUP }\end{array}$ & $\begin{array}{l}\text { E Langevint } \\
\text { C Muenzel+ } \\
\text { E Churcht }\end{array}$ & $\begin{array}{r}11283 \\
3045 \\
3046+\end{array}$ \\
\hline $73 m+95 e$ & \multicolumn{3}{|c|}{ Sumation $O(E)$} & & & \\
\hline $\begin{array}{l}75 \mathrm{As} \\
75 \mathrm{As}\end{array}$ & $\begin{array}{l}p \\
p\end{array}$ & $\begin{array}{l}4.0+2 \\
4.0+2\end{array}$ & & $\begin{array}{l}\text { KFK } \\
\text { PUP }\end{array}$ & $\begin{array}{l}\text { C Muenzelt } \\
\text { E Chureht+ }\end{array}$ & $\begin{array}{l}3047 \\
3048+\end{array}$ \\
\hline${ }^{74} \mathrm{Se}$ & \multicolumn{4}{|c|}{ partial o(E) $\times$ factor } & & \\
\hline${ }^{74} \mathrm{Se}$ & $p$ & $1.4+0$ & $3.0+0$ & IJIE & E Krivonosovt & 3091 \\
\hline${ }^{74} \mathrm{Se}$ & product & yield & & & & \\
\hline${ }^{70} \mathrm{Ge}$ & ${ }^{9} \mathrm{Be}$ & $2.5+1$ & & FSU & E Clementst & $9493^{\circ}$ \\
\hline${ }^{74} \mathrm{Se}$. & thich to & arget y & yield & & & ' \\
\hline $6{ }^{1} \mathrm{Ni}$ & ${ }^{16} 0$ & $3.9+1$ & $5 \cdot 1+1$ & ORL & E Wellst & 11252 \\
\hline${ }^{74} \mathrm{Se}$ & \multicolumn{2}{|c|}{ relalive o(E) } & & & & \\
\hline${ }^{72} \mathrm{Gg}$ & $a$ & $3.0+1$ & $5.5+1$ & CNE E & E Behart & 7917 \\
\hline${ }^{74} \mathrm{Se}$ & \multicolumn{2}{|c|}{ O(E) x foctor } & & & & \\
\hline${ }^{75} \mathrm{As}$ & $\rho$ & $2.2+1$ & & ORL & E Cohent & 3049 \\
\hline${ }^{74} \mathrm{Se}$ & $\theta(E)$ & & & & & \\
\hline 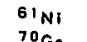 & 180 & $4.6+1$ & & ORL & M Wellst & 11253 \\
\hline $73_{\mathrm{AS}}$ & $\stackrel{a}{p}$ & $\begin{array}{l}\text { NDG } \\
\text { NDG }\end{array}$ & & $\begin{array}{l}\text { CAL } \\
\text { CAL }\end{array}$ & $\begin{array}{l}\text { T Woosleyt } \\
\text { T Woosleyt }\end{array}$ & $\begin{array}{l}7903 \\
3025\end{array}$ \\
\hline${ }^{74} \mathrm{As}$ & p & NOG & & CAL & T Woosleyt & 3027 \\
\hline${ }^{77} \mathrm{Br}$ & p & NDG & & CAL & T Woosleyt & 3162 \\
\hline
\end{tabular}

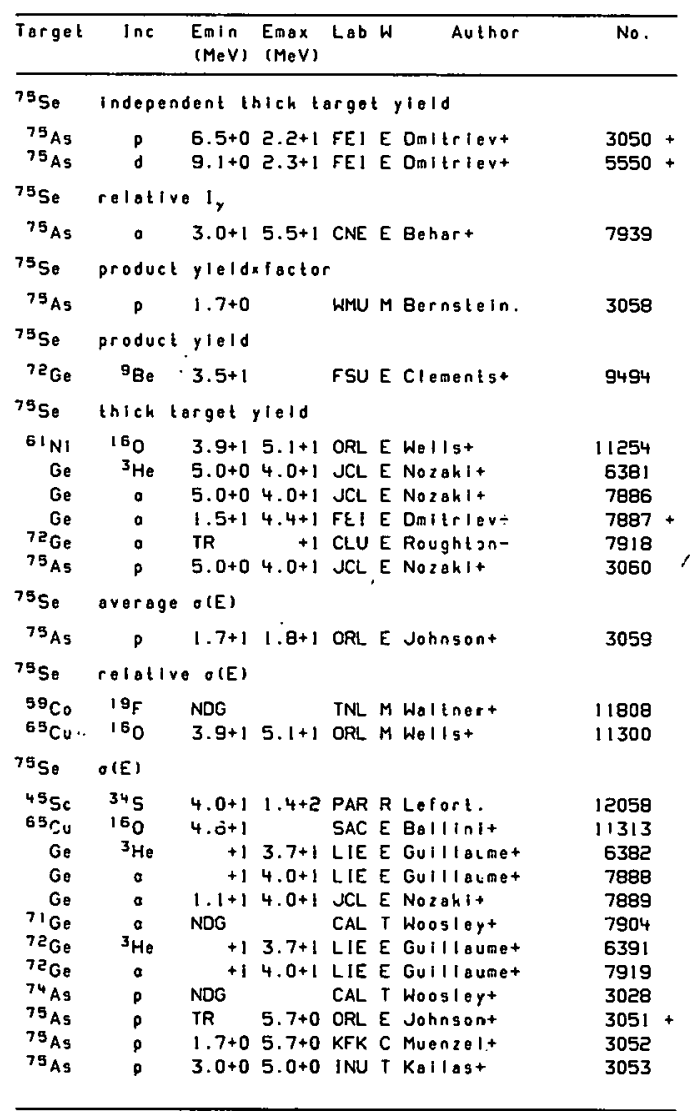




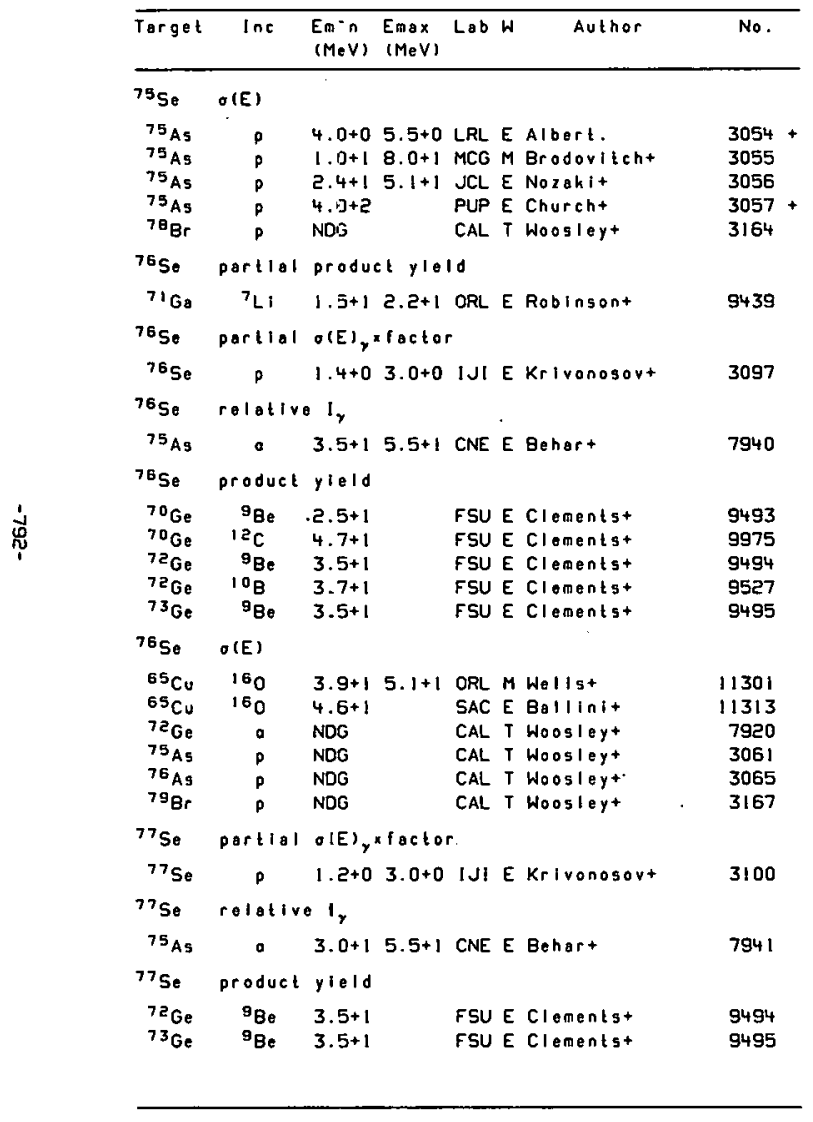

\begin{tabular}{ccccc}
\hline Torget & Inc Eqin Emax Lab W \\
(MeV) (MeV)
\end{tabular}




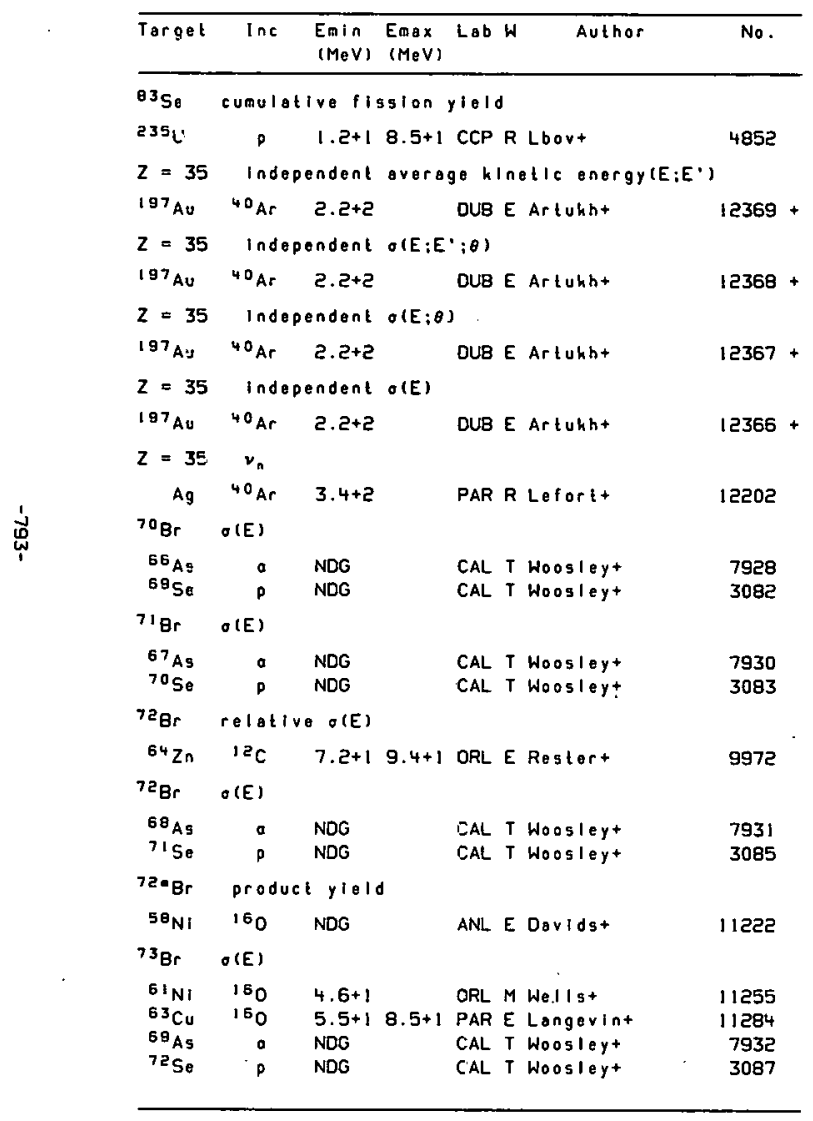

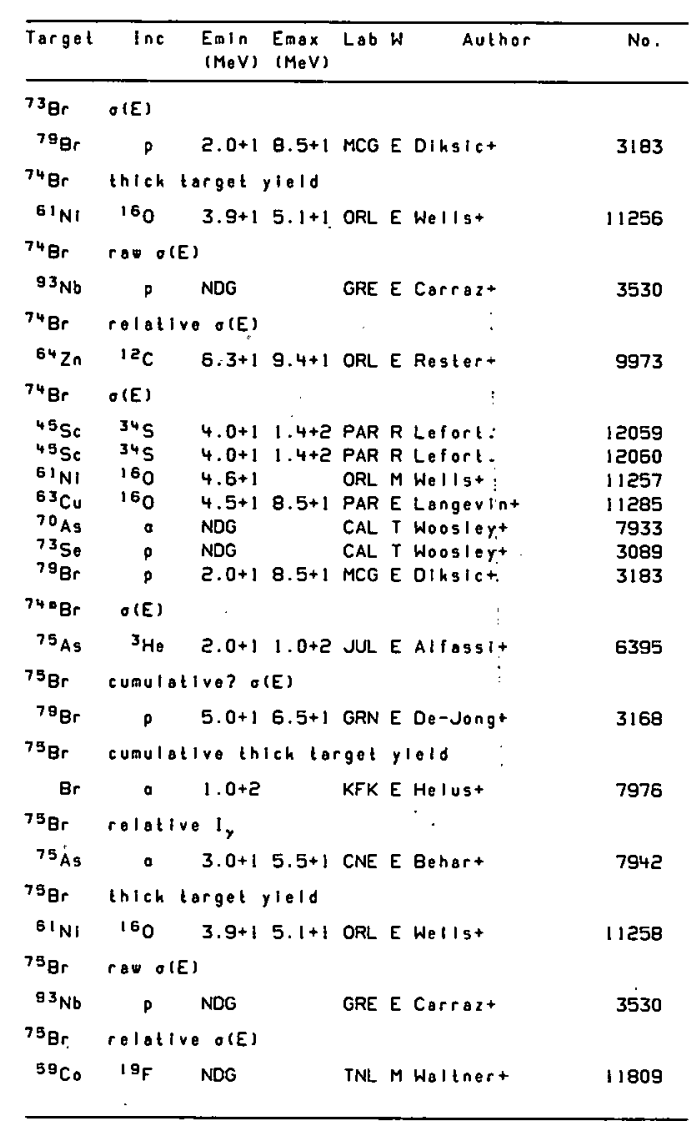




\begin{tabular}{|c|c|c|c|c|c|c|}
\hline Target & $\operatorname{lnc}$ & $\begin{array}{l}\text { Emin } \\
(M e v)\end{array}$ & $\begin{array}{l}\text { Emox } \\
(\operatorname{MeV})\end{array}$ & Lob & Author & No. \\
\hline${ }^{73} \mathrm{Br}$ & $\sigma(E)$ & & & & & \\
\hline $\begin{array}{l}{ }^{45} \mathrm{Sc} \\
{ }^{53} \mathrm{Cu} \\
69 \mathrm{Cu} \\
{ }^{61} \mathrm{As} \\
{ }^{75} \mathrm{As} \\
{ }^{74} \mathrm{Se} \\
{ }^{74} \mathrm{Se} \\
\mathrm{Br} \\
81 \mathrm{Br}\end{array}$ & $\begin{array}{l}345 \\
160 \\
160 \\
a \\
3 \text { He } \\
p \\
p \\
d \\
p\end{array}$ & $\begin{array}{l}4.0+1 \\
5.5+1 \\
4.6+1 \\
\text { NDG } \\
2.0-1 \\
\text { NDG } \\
6.0-1 \\
1.0+1 \\
1.5+1\end{array}$ & $\begin{array}{l}1.4+2 \\
8.5+1 \\
1.0+2 \\
3.0+0 \\
9.0+1 \\
8.5+1\end{array}$ & $\begin{array}{l}\text { PAR } \\
\text { PAR } \\
\text { SAC } \\
\text { CAL } \\
\text { JUL } \\
\text { CAL } \\
\text { IJI } \\
\text { JUL } \\
\text { MCG }\end{array}$ & $\begin{array}{l}\text { R Lofort. } \\
\text { E Langevint } \\
\text { E Ballinit } \\
\text { T Woosleyt } \\
\text { E Alfossit } \\
\text { T Woosley+ } \\
\text { E Krivonosort } \\
\text { R Slocklint } \\
\text { E Diksict }\end{array}$ & $\begin{array}{r}12061 \\
11286 \\
11313 \\
7934 \\
6396 \\
3092 \\
3093 \\
5552 \\
3195\end{array}$ \\
\hline${ }^{76} \mathrm{Br}$ & cusulati & ive thl & lck tor & rgot & yield & \\
\hline Br & - & $1.0+2$ & & KFK & E Helust & 7977 \\
\hline${ }^{78} \mathrm{Or}$ & partiol & $\cdot(E)$ & & & & \\
\hline $\begin{array}{l}{ }^{75} \mathrm{As} \\
{ }^{76} \mathrm{Br}\end{array}$ & $\begin{array}{c}a \\
\text { rolative }\end{array}$ & $\begin{array}{l}4.5+1 \\
.1 .\end{array}$ & & CNE & E Behart & 7946 \\
\hline $\begin{array}{l}{ }^{75} \mathrm{As} \\
{ }^{76} \mathrm{Br}\end{array}$ & $\begin{array}{c}0 \\
\text { product }\end{array}$ & $\begin{array}{l}3.5+1 \\
\text { yield }\end{array}$ & $5.5+1$ & CNE & E Behort & 7945 \\
\hline $\begin{array}{l}70_{\mathrm{Ge}} \\
72_{\mathrm{Ge}}\end{array}$ & $\begin{array}{l}90 \mathrm{Be} \\
{ }^{10} \mathrm{~B}\end{array}$ & $\begin{array}{l}2.5+1 \\
3.7+1\end{array}$ & & $\begin{array}{l}\text { FSU } \\
\text { FSU }\end{array}$ & $\begin{array}{l}\text { E Clementst } \\
\text { E Clementst }\end{array}$ & $\begin{array}{l}9493 \\
9527\end{array}$ \\
\hline${ }^{76} \mathrm{Br}$ & thlek to & orgat $y$ & $y 1010$ & & & \\
\hline $\begin{array}{r}B ! N i \\
7 I_{A S} \\
\text { Se } \\
50\end{array}$ & $\begin{array}{r}160 \\
a \\
p \\
a\end{array}$ & $\begin{array}{l}3 \cdot 9+1 \\
3 \cdot 0+1 \\
1.0+1 \\
2.0+1\end{array}$ & $\begin{array}{l}5 \cdot 1+1 \\
4 \cdot 0+1 \\
5 \cdot 0+1 \\
5 \cdot 0+1\end{array}$ & $\begin{array}{l}\mathrm{ORL} \\
\mathrm{JCL} \\
\mathrm{JCL} \\
\mathrm{JCL}\end{array}$ & $\begin{array}{l}\text { E Wellst } \\
\text { E Nozohit } \\
\text { E Nozahit } \\
\text { E Nozakl+ }\end{array}$ & $\begin{array}{r}11259 \\
7944 \\
3077 \\
7958\end{array}$ \\
\hline${ }^{78} \mathrm{Br}$ & $r \Delta v O(E)$ & & & & & \\
\hline${ }^{93} \mathrm{Nb}$ & $p$ & NDG & & GRE & E Carraz+ & 3530 \\
\hline${ }^{76} \mathrm{Br}$ & relative & - $\sigma(E)$ & & & & \\
\hline${ }^{59} \mathrm{Co}$ & $19 F$ & NEG & & TNL & M Wallnert & 11810 \\
\hline${ }^{78} \mathrm{Br}$ & O $(E)$. $f$ oc & ctor & & & & \\
\hline $\begin{array}{l}{ }^{77} \mathrm{Se} \\
76 \mathrm{Br}\end{array}$ & $\begin{array}{r}P \\
O(E)\end{array}$ & $4.0+2$ & & CAR & E Revilandt & 3103 \\
\hline${ }^{45} \mathrm{Se}$ & ${ }^{34} \mathrm{~s}$ & $4.0+1$ & $1.4+2$ & PAR & R Lefort. & 12062 \\
\hline
\end{tabular}

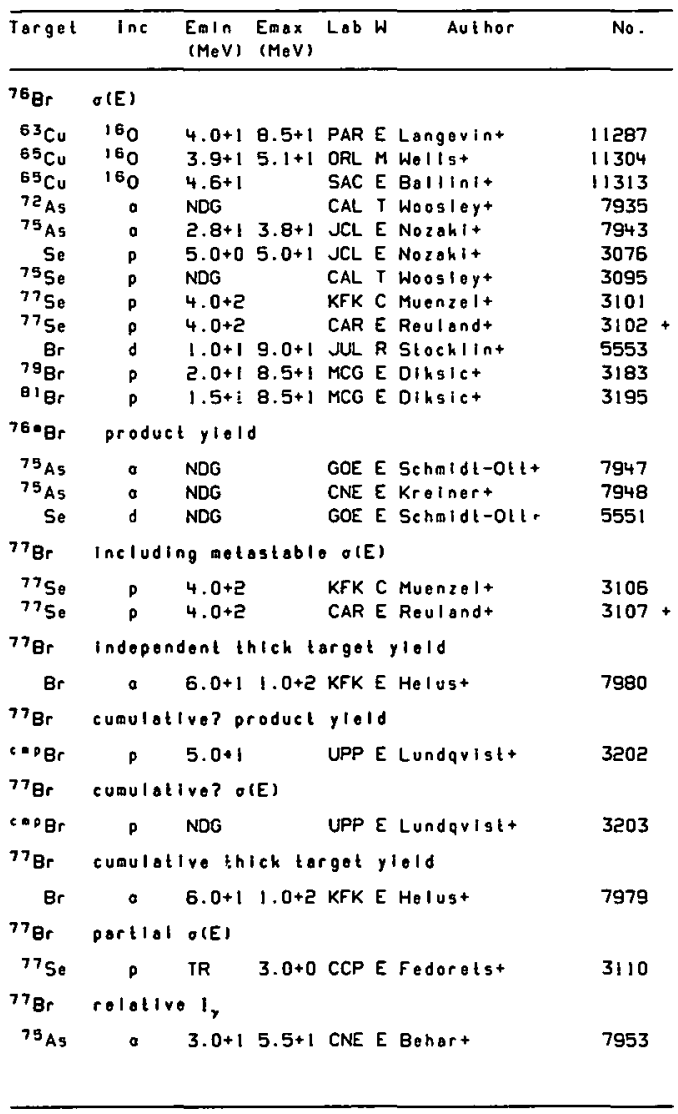




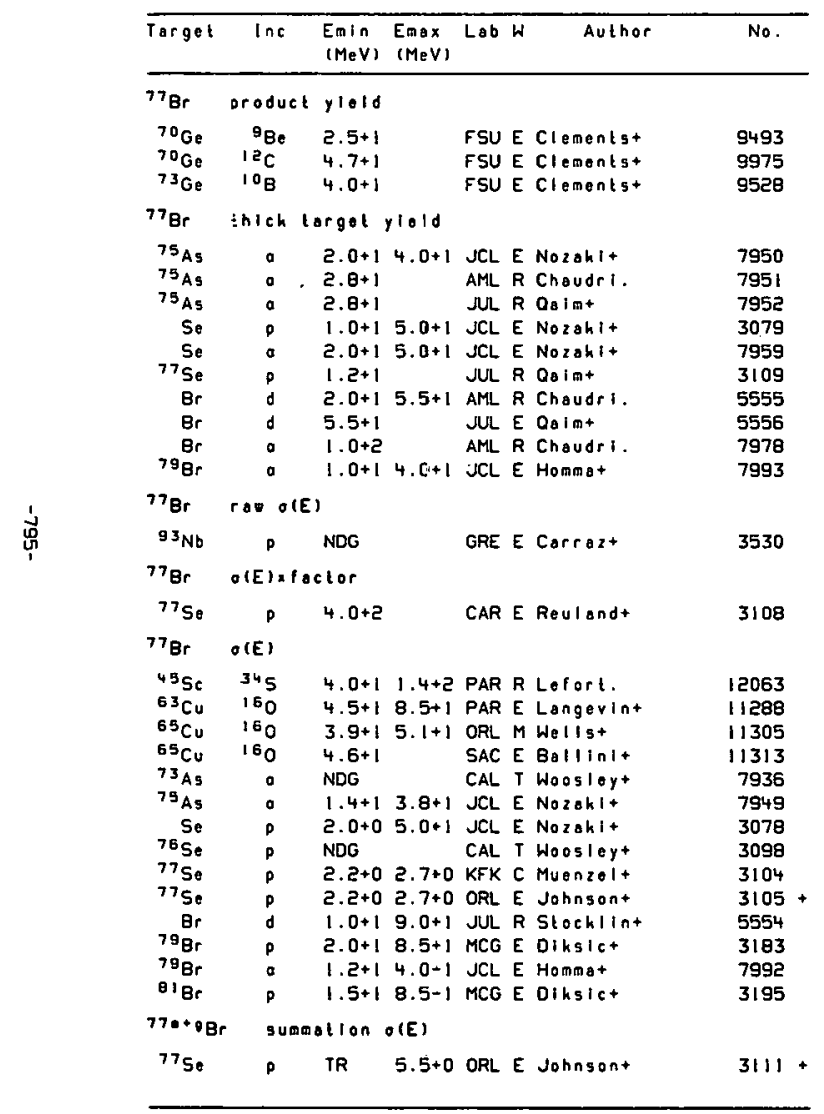

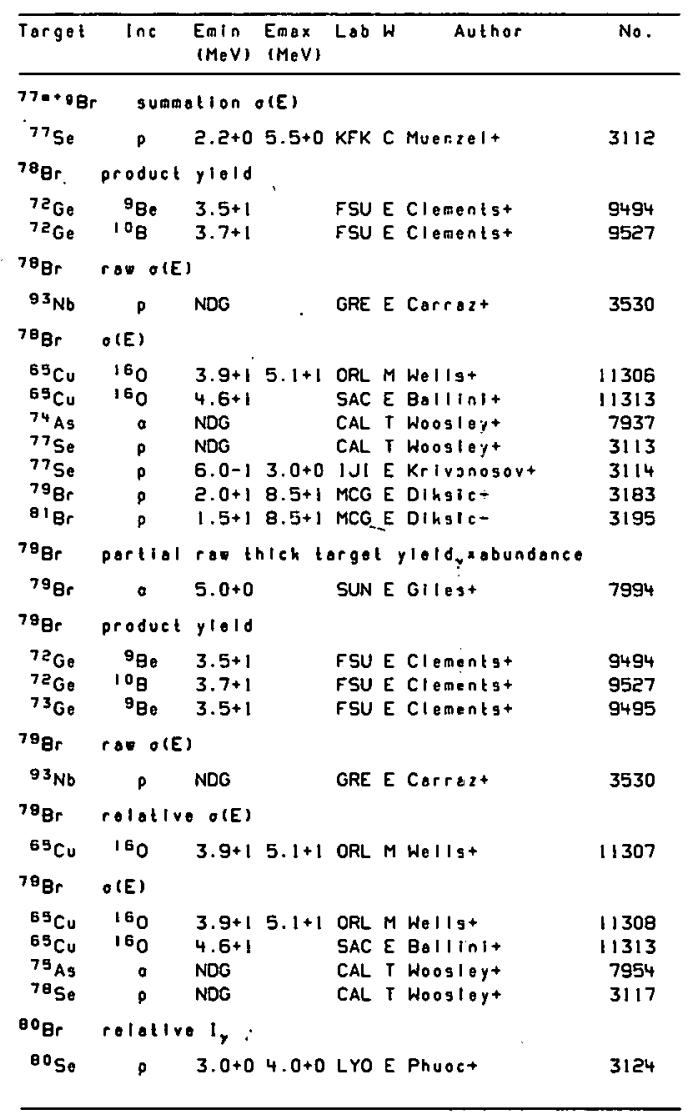




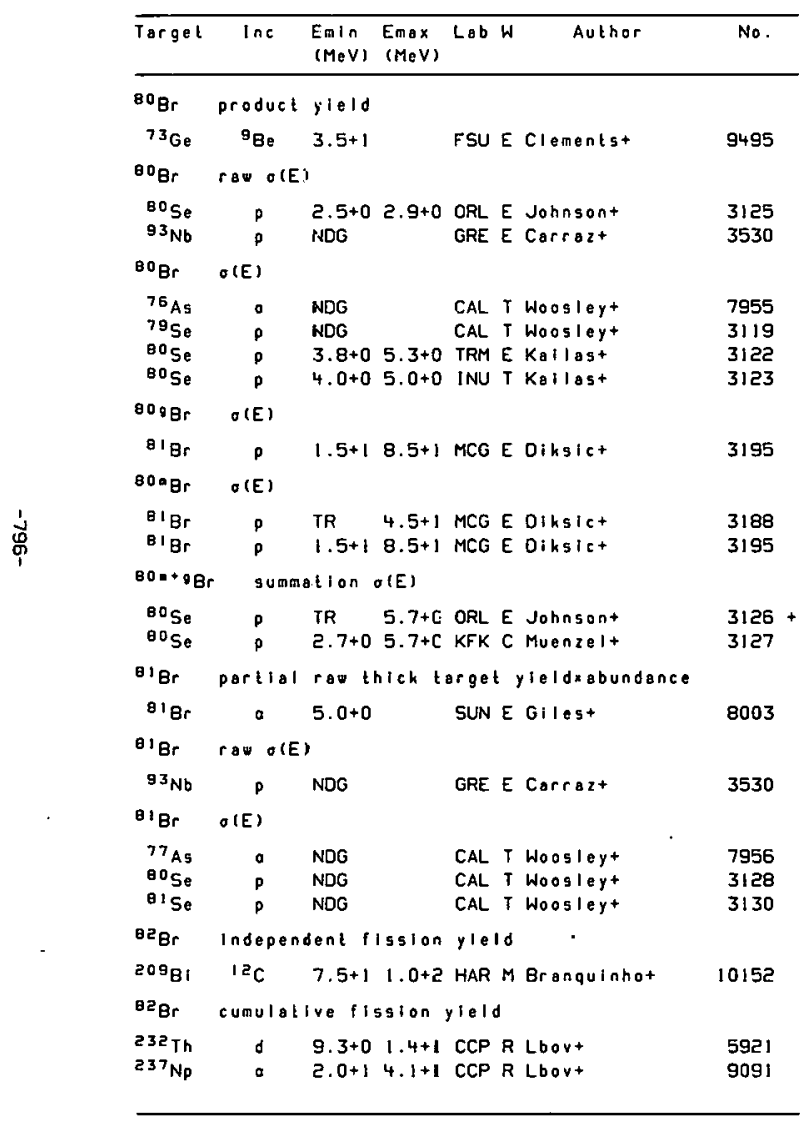

\begin{tabular}{|c|c|c|c|c|c|c|}
\hline Target & $\ln c$ & $\begin{array}{l}\text { Emin } \\
\text { (Mevi) }\end{array}$ & $\begin{array}{l}\text { Emax } \\
\text { (MeV) }\end{array}$ & Lab & Author & No. \\
\hline $82 \mathrm{Br}$ & relative & e 1 , & & & & \\
\hline $\begin{array}{l}8 \mathrm{se}_{\mathrm{se}} \\
8 \mathrm{gar}^{\mathrm{B}}\end{array}$ & inich ta & $\begin{array}{l}3.0+0 \\
\text { arget } y\end{array}$ & $\begin{array}{l}4.0+0 \\
y \in 10\end{array}$ & LYO & E Phuoct & 3132 \\
\hline $\begin{array}{l}\mathrm{Se} \\
\mathrm{Se}\end{array}$ & p & $\begin{array}{l}1 \cdot 0+1 \\
2 \cdot 0+1\end{array}$ & $\begin{array}{l}2 \cdot 5+1 \\
3 \cdot 0+1\end{array}$ & $\begin{array}{l}J C L \\
J C L\end{array}$ & $\begin{array}{l}\text { ENozahit } \\
\text { E Nozahit }\end{array}$ & $\begin{array}{l}3081 \\
7960\end{array}$ \\
\hline $8 a_{1}$ & raw o(E) & & & & & \\
\hline $93_{\mathrm{Nb}}$ & $p$ & NOG & & GRE & E Corrazt & 3530 \\
\hline${ }^{82} \mathrm{Br}$ & average & $\sigma(E)$ & & & & \\
\hline $\begin{array}{l}{ }_{82} \mathrm{Se} \\
{ }^{82} \mathrm{Br}\end{array}$ & $\begin{array}{r}p \\
o(E)\end{array}$ & $9.1-1$ & $1.2+0$ & ORL & E Johnsont & 3135 \\
\hline $\begin{array}{r}{ }^{7 \theta} \mathrm{As} \\
\mathrm{Se} \\
\mathrm{BISe} \\
\theta^{2} \mathrm{Se} \\
\mathrm{B}^{2} \mathrm{Se}\end{array}$ & $\begin{array}{l}a \\
p \\
p \\
p \\
p\end{array}$ & $\begin{array}{l}\text { NDG } \\
2.0+0 \\
\text { NDG } \\
9.3-1 \\
9.3-1\end{array}$ & $\begin{array}{l}5.0+1 \\
1.5+0 \\
1.5+0\end{array}$ & $\begin{array}{l}\text { CAL } \\
\text { JCL } \\
\text { CAL } \\
\text { KFK } \\
\text { ORL }\end{array}$ & $\begin{array}{l}\text { T Woosleyt } \\
\text { E Nozahit } \\
\text { T Woosleyt } \\
\text { C Muenzel+ } \\
\text { E Johnsont }\end{array}$ & $\begin{array}{l}7957 \\
3080 \\
3131 \\
3133 \\
3134\end{array}$ \\
\hline 82.180 & ratio & $0 O(E)$ & & & & \\
\hline $\begin{array}{l}200^{8 \mathrm{Bi}} \\
85.108 \mathrm{r}\end{array}$ & ${ }^{12} \mathrm{C}$ & $\begin{array}{r}8.5+1 \\
0 \quad J(E)\end{array}$ & $9.4+1$ & HAR & E Goodellt+ & 10114 \\
\hline $\begin{array}{l}204 \mathrm{~Pb} . \\
{ }^{83} \mathrm{Br}\end{array}$ & $\begin{array}{c}a \\
\text { cumulati }\end{array}$ & $\begin{array}{l}\text { NDG } \\
\text { ive fis }\end{array}$ & ssion $y$ & & E Goodallt & 8853 \\
\hline $\begin{array}{l}232 \mathrm{Th} \\
23 \mathrm{~S}_{\mathrm{U}}\end{array}$ & p & $\begin{array}{l}9.3+0 \\
1.2+1\end{array}$ & $\begin{array}{l}1.4+1 \\
8.5+1\end{array}$ & $\begin{array}{l}\mathrm{CCP} \\
\mathrm{CCP}\end{array}$ & $\begin{array}{l}\text { R Lbovt } \\
\text { R Lbovt }\end{array}$ & $\begin{array}{l}5921 \\
4852\end{array}$ \\
\hline${ }^{8} \mathrm{Br}_{\mathrm{r}}$ & $O(E) \times 800$ & undance & & & & \\
\hline $\begin{array}{l}{ }^{82} \mathrm{Se} \\
{ }^{63} \mathrm{Br}\end{array}$ & $\begin{array}{c}\rho \\
r a s\end{array}$ & $8.0+0$ & $1.6+1$ & $\mathrm{JCL}$ & E Nozahit & 3137 \\
\hline $\begin{array}{l}{ }^{93} \mathrm{Nb} \\
{ }^{83} \mathrm{Br}\end{array}$ & $\begin{array}{r}P \\
O(E)\end{array}$ & NDG & & GRE & E Carraz+ & 3530 \\
\hline $\begin{array}{c}82 \mathrm{Se} \\
83=19 \mathrm{Br}\end{array}$ & $\begin{array}{l}\text { p } \\
\text { ratio }\end{array}$ & $\begin{array}{r}E .0+1 \\
0 . O(E)\end{array}$ & & GOE & E Kantust & 3136 \\
\hline $209^{B i}$ & ${ }^{12} \mathrm{C}$ & $8.5+1$ & $9.4+1$ & HAR & $\varepsilon$ Goodall+ & 10115 \\
\hline
\end{tabular}




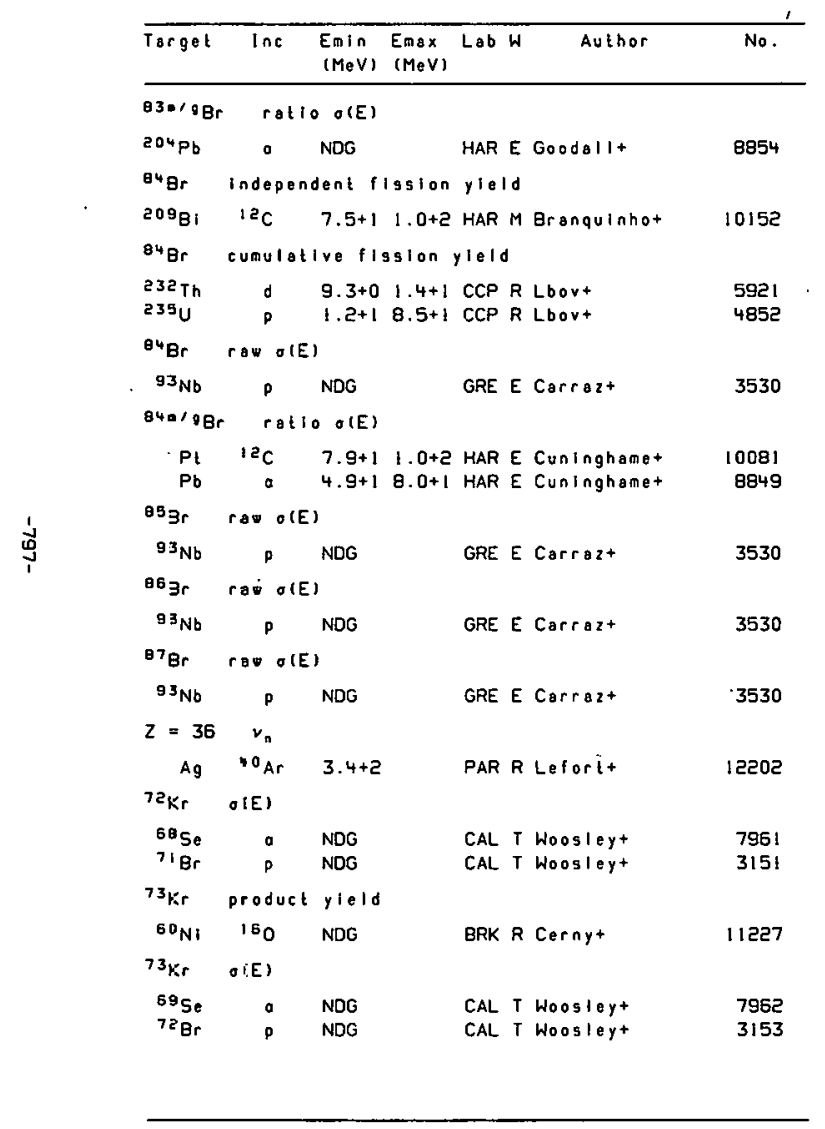

\begin{tabular}{|c|c|c|c|c|c|c|}
\hline Target & $\ln e$ & $\begin{array}{l}\text { Emin } \\
(\mathrm{MeV})\end{array}$ & $\begin{array}{l}\text { Emax } \\
(\operatorname{MeV})\end{array}$ & Lab & Author & No. \\
\hline $\begin{array}{l}{ }^{74} \mathrm{Kr} \\
{ }^{61} \mathrm{NI}\end{array}$ & $\begin{array}{l}\text { thich } \\
160\end{array}$ & $\begin{array}{c}\operatorname{target} y \\
4.2+1\end{array}$ & $\begin{array}{l}\text { yield } \\
5.0+1\end{array}$ & ORL & E Wellst & 11260 \\
\hline${ }^{74} \mathrm{Kr}$ & relati & ive o(E) & & & & \\
\hline $\begin{array}{l}{ }^{61} \mathrm{NI} \\
{ }^{74} \mathrm{Kr}\end{array}$ & $\begin{array}{l}160 \\
O(E)\end{array}$ & $4.2+1$ & $5.0+1$ & YAL & R Bromley. & 11262 \\
\hline $\begin{array}{l}{ }^{61} \mathrm{NI} \\
{ }^{70} \mathrm{Se} \\
{ }^{73} \mathrm{Br}\end{array}$ & $\begin{array}{r}160 \\
p \\
p\end{array}$ & $\begin{array}{l}4.6+1 \\
\text { NDG } \\
\text { NOG }\end{array}$ & & $\begin{array}{l}\text { ORL } \\
\text { CAL } \\
\text { CAL }\end{array}$ & $\begin{array}{l}\text { M Wellst } \\
\text { T Woos ey+ } \\
\text { T Woos ey+ }\end{array}$ & $\begin{array}{r}11261 \\
7963 \\
3155\end{array}$ \\
\hline${ }^{75} \mathrm{Kr}$ & thlch & target y & yiald & & & \\
\hline $\begin{array}{l}{ }^{61} \mathrm{NI} \\
{ }^{75} \mathrm{Kr}\end{array}$ & $\begin{array}{l}160 \\
\sigma(E)\end{array}$ & $3.9+1$ & $5.1+1$ & ORL & E Wellst & 11263 \\
\hline $\begin{array}{l}{ }^{45} \mathrm{Sc} \\
61 \mathrm{Ni} \\
63 \mathrm{Cu} \\
71 \mathrm{Se} \\
8 \mathrm{Br} \\
{ }^{74} \mathrm{Br}\end{array}$ & $\begin{array}{l}345 \\
150 \\
150 \\
0 \\
j \\
\rho\end{array}$ & $\begin{array}{l}4.0+1 \\
4.6+1 \\
6.5+1 \\
\text { NDG } \\
1.0+1 \\
\text { NDG }\end{array}$ & $\begin{array}{c}1.4+2 \\
8.5+1 \\
9.0+1\end{array}$ & $\begin{array}{l}\text { PAR } \\
\text { ORL } \\
\text { PAR } \\
\text { CAL } \\
\text { JUL } \\
\text { CAL }\end{array}$ & $\begin{array}{l}\text { R Lefort. } \\
M \text { Wellat } \\
\text { E Langevint } \\
\text { T Woosleyt } \\
R \text { Stochlint } \\
T \text { Woosleyt }\end{array}$ & $\begin{array}{r}12064 \\
11264 \\
11289 \\
7964 \\
5557 \\
3157\end{array}$ \\
\hline${ }^{76} \mathrm{Kr}$ & thlch & target y & $y=1 d x a$ & abund & dance? & \\
\hline $\begin{array}{l}{ }^{79} \mathrm{Br} \\
{ }^{76} \mathrm{Kr}\end{array}$ & $\begin{array}{c}D \\
\text { thick }\end{array}$ & $\begin{array}{l}6.5+1 \\
\text { target } y\end{array}$ & yleld & GRN & $E D_{e}-J_{e n g+}$ & 3170 \\
\hline $\begin{array}{r}6 I_{\mathrm{NI}} \\
\mathrm{Se} \\
\mathrm{Br} \\
\mathrm{Br}\end{array}$ & $\begin{array}{c}16 \mathrm{O} \\
{ }^{3} \mathrm{He} \\
p \\
d\end{array}$ & $\begin{array}{l}3 \cdot 9+1 \\
3 \cdot 8+1 \\
4 \cdot 0+1 \\
8 \cdot 0+1\end{array}$ & $\begin{array}{l}5.1+1 \\
5.0+1\end{array}$ & $\begin{array}{l}\text { ORL } \\
\text { FUL } \\
\text { JCL } \\
\text { JUL }\end{array}$ & $\begin{array}{l}\text { E Wellst } \\
\text { E Oe-Jangt } \\
\text { E Nozahit } \\
\text { E Oaimt }\end{array}$ & $\begin{array}{l}11265 \\
6397 \\
3140 \\
5559\end{array}$ \\
\hline${ }^{76} \mathrm{Kr}$ & $O(E)$ & & & & & \\
\hline $\begin{array}{l}{ }^{45} \mathrm{Sc} \\
63 \mathrm{Cu} \\
72 \mathrm{cu}\end{array}$ & $\begin{array}{l}345 \\
160\end{array}$ & $\begin{array}{l}4.0+1 \\
4.5+1\end{array}$ & $\begin{array}{l}1.4+2 \\
8.5+1\end{array}$ & $\begin{array}{l}\text { PAR } \\
\text { PAR }\end{array}$ & $\begin{array}{l}\text { R Lefort. } \\
\text { E Langevint }\end{array}$ & $\begin{array}{r}12065 \\
11290 \\
7065\end{array}$ \\
\hline $\begin{array}{r}72 \mathrm{Se} \\
\mathrm{Br}\end{array}$ & : & $\begin{array}{l}\text { NOG } \\
3.8+1\end{array}$ & $5.0+1$ & $\begin{array}{l}\text { CAL } \\
\text { JCL }\end{array}$ & $\begin{array}{l}\text { T Woosleyt } \\
\text { E Nozakit }\end{array}$ & $\begin{array}{l}7965 \\
3138\end{array}$ \\
\hline Br & $p$ & $4.0+1$ & $8.0+1$ & UPP & E Lundquistt & $\begin{array}{l}3139 \\
5559\end{array}$ \\
\hline $\begin{array}{r}\mathrm{Br} \\
{ }_{75} \mathrm{Br}\end{array}$ & $\int_{0}^{0}$ & $\begin{array}{l}1.0+1 \\
\text { NDG }\end{array}$ & $9.0+1$ & $\begin{array}{l}\text { JUL } \\
\text { CAL }\end{array}$ & $\begin{array}{l}\text { R Stochlint } \\
\text { T Woosteyt }\end{array}$ & $\begin{array}{l}5558 \\
3159\end{array}$ \\
\hline $\begin{array}{l}{ }^{79} \mathrm{Br} \\
{ }^{79} \mathrm{Br}\end{array}$ & p & $3.9+1$ & $6.5+1$ & GRN & E De-Jongt & $\begin{array}{l}3169 \\
3192\end{array}$ \\
\hline & & $1.0+1$ & $8.5+1$ & MCG & E Olksict & \\
\hline
\end{tabular}




\begin{tabular}{|c|c|c|c|c|c|c|}
\hline Target & $\ln c$ & $\begin{array}{l}\text { Emin } \\
(\mathrm{MeV})\end{array}$ & $\begin{array}{l}E \max \\
(\operatorname{MeV})\end{array}$ & Lob & Author & No. \\
\hline${ }^{76} \mathrm{Kr}$ & $O(E)$ & & & & & \\
\hline $\begin{array}{l}79 \mathrm{Br} \\
{ }^{8} \mathrm{Br} \\
{ }^{9} \mathrm{Br}\end{array}$ & $\begin{array}{l}d \\
p \\
d\end{array}$ & $\begin{array}{l}\text { NOG } \\
2.5+1 \\
\text { NDG }\end{array}$ & $8.5+1$ & $\begin{array}{l}\text { JUL E } \\
\text { MCG E } \\
\text { JUL E }\end{array}$ & $\begin{array}{l}\text { E Oalnt } \\
\text { E Dihsict } \\
\text { E Oaint }\end{array}$ & $\begin{array}{l}5564 \\
3194 \\
5566\end{array}$ \\
\hline${ }^{77} \mathrm{Kr}$ & cumulat & tivo? th & hick to & arget & $t y 1010$ & \\
\hline $\begin{array}{r}\mathrm{Br} \\
{ }^{\mathrm{Br}} \mathrm{Kr}\end{array}$ & $\begin{array}{c}p \\
\text { cumulat }\end{array}$ & $\begin{array}{r}4.0+1 \\
\text { tiro? }\end{array}$ & $4.5^{+1}$ & MCG & E Oiksict & $3: 46$ \\
\hline $\begin{array}{r}\mathrm{Br} \\
79 \mathrm{Br} \\
81 \mathrm{Br}\end{array}$ & $\begin{array}{l}p \\
p \\
p\end{array}$ & $\begin{array}{l}\text { TR } \\
\text { TR } \\
\text { TR }\end{array}$ & $\begin{array}{l}4 \cdot 5+1 \\
4 \cdot 5+1 \\
4 \cdot 5+1\end{array}$ & $\begin{array}{l}\text { MCG } \\
\text { MCG } \\
M C G E\end{array}$ & $\begin{array}{l}\text { E Dihsict } \\
\text { E Dihsict } \\
\text { E Diksict }\end{array}$ & $\begin{array}{l}3145 \\
3171 \\
3 ! 89\end{array}$ \\
\hline${ }^{1} \mathrm{Kr}$ & cumulat & tive thi & inch tor & orget & $y / 019$ & \\
\hline $\mathrm{Br}$ & a & $1.0+2$ & & KFK E & E Helust & 7981 \\
\hline${ }^{77} \mathrm{Kr}$ & product & t field & & & & \\
\hline $\begin{array}{l}{ }^{70} \mathrm{Ge} \\
{ }^{70} \mathrm{Ge} \\
\mathrm{Br} \\
81 \mathrm{Br}\end{array}$ & $\begin{array}{c}9 \mathrm{Be} \\
\operatorname{li}^{2} \mathrm{C} \\
p \\
d\end{array}$ & $\begin{array}{l}2.5+1 \\
4.7+1 \\
5.0+1 \\
7.8+1\end{array}$ & & $\begin{array}{l}\text { FSU } \\
\text { FSU } \\
\text { UPP E } \\
\text { JUL F }\end{array}$ & $\begin{array}{l}\text { E Clementst } \\
\text { E Clementst } \\
\text { E Lundquigt+ } \\
\text { R Kupfernagelt }\end{array}$ & $\begin{array}{l}9493 \\
9975 \\
3144 \\
5567\end{array}$ \\
\hline${ }^{77} \mathrm{Kr}$ & thich & torget $y$ & $y 101 d \times 0$ & abundo & dance? & , \\
\hline${ }^{79} \mathrm{Br}$ & p & $6.5+1$ & & GRN E & E De-Jongt & 3175 \\
\hline${ }^{7} \mathrm{Kr}$ & thich & torgot $r$ & yiold & & & \\
\hline $\begin{array}{r}\mathrm{Se} \\
\mathrm{Br} \\
\mathrm{Br} \\
\mathrm{Br} \\
\mathrm{Br} \\
\mathrm{Br} \\
\mathrm{Br}\end{array}$ & $\begin{array}{c}{ }^{3} \mathrm{He} \\
\mathrm{p} \\
d \\
d \\
\alpha \\
a \\
p\end{array}$ & $\begin{array}{l}3 \cdot 8+1 \\
2 \cdot 5+1 \\
2 \cdot 0+1 \\
5 \cdot 0+1 \\
2 \cdot 0+1 \\
\text { MOG } \\
3 \cdot 0+1\end{array}$ & $\begin{array}{l}5.0+1 \\
5.5+1 \\
5.0+1 \\
8.0+1\end{array}$ & $\begin{array}{l}\text { FUL } \\
\text { JCL } \\
\text { AML } \\
\text { JUL } \\
\text { JCL } \\
\text { AML F } \\
\text { UPP E }\end{array}$ & $\begin{array}{l}\text { E De-Jongt } \\
\text { E Nozakit } \\
R \text { Chaudri. } \\
\text { E Oolat } \\
\text { E Nozakit } \\
\text { R Chaudri. } \\
\text { E Lundquigt+ }\end{array}$ & $\begin{array}{l}6398 \\
3143 \\
5561 \\
5562 \\
7982 \\
7983 \\
3204\end{array}$ \\
\hline $7_{\mathrm{Kr}}$ & $O(E)$ & & & & & \\
\hline $\begin{array}{l}45 \mathrm{Sc} \\
63 \mathrm{Cu} \\
73 \mathrm{cos}\end{array}$ & $\begin{array}{l}345 \\
160\end{array}$ & $\begin{array}{l}4.0+1 \\
4.0+1\end{array}$ & $\begin{array}{l}1.4+2 \\
8.5+1\end{array}$ & $\begin{array}{l}\text { PAR } \\
\text { PAR }\end{array}$ & $\begin{array}{l}\text { R Lefort. } \\
\text { E Langevint }\end{array}$ & $\begin{array}{r}12066 \\
.11291 \\
.7296\end{array}$ \\
\hline $\begin{array}{r}{ }^{73} \mathrm{Se} \\
\mathrm{Br} \\
\mathrm{Br}\end{array}$ & $\begin{array}{l}a \\
p \\
p\end{array}$ & $\begin{array}{l}\text { NDG } \\
2.5+1 \\
2.8+1\end{array}$ & $\begin{array}{l}5.0+1 \\
8 \cdot 0+1\end{array}$ & $\begin{array}{l}\text { CAL } \\
\text { JCL } \\
\text { UPP E }\end{array}$ & $\begin{array}{l}\text { T Woosleyt } \\
\text { E Nozokit } \\
\text { E Lundquislt }\end{array}$ & $\begin{array}{l}7966 \\
3141 \\
3142\end{array}$ \\
\hline Br & d & $1.0+1$ & $9.0+1$ & JUL F & R Slocklint & 5560 \\
\hline
\end{tabular}

\begin{tabular}{|c|c|c|c|c|c|c|}
\hline Target & $\ln t$ & $\begin{array}{l}\text { Emin } \\
(M e V)\end{array}$ & $\begin{array}{l}\text { Emax } \\
(\operatorname{MeV})\end{array}$ & Lob W & Author & No. \\
\hline${ }^{77} \mathrm{Kr}$ & $O(E)$ & & & & & \\
\hline $\begin{array}{l}76 \mathrm{Br} \\
79 \mathrm{Br} \\
79 \mathrm{Br} \\
79 \mathrm{Br} \\
79 \mathrm{Br} \\
79 \mathrm{Br} \\
7{ }^{7} \mathrm{Br} \\
\mathrm{B}: \mathrm{Br} \\
\mathrm{B} ! \mathrm{Br}\end{array}$ & $\begin{array}{l}p \\
p \\
p \\
p \\
p \\
p \\
d \\
p \\
p \\
d\end{array}$ & $\begin{array}{l}\text { NDG } \\
\text { TR } \\
\text { TR } \\
2.5+1 \\
1.0+1 \\
\text { NDG } \\
\text { TR } \\
2.5+1 \\
\text { NDG }\end{array}$ & $\begin{array}{l}4.5+1 \\
5 \cdot 2+1 \\
6.5+1 \\
8.5+1 \\
4.5+1 \\
8.5+1\end{array}$ & $\begin{array}{l}\text { CAL T } \\
\text { JUL E } \\
\text { JCL E } \\
\text { GRN E } \\
\text { MCG E } \\
\text { JUL E } \\
\text { JUL E } \\
\text { MCG E } \\
\text { JUL E }\end{array}$ & $\begin{array}{l}\text { Woosleyt } \\
\text { Kniepert } \\
\text { Nozehit } \\
\text { De-Jongt } \\
\text { Dihsict } \\
\text { Oalmt } \\
\text { Kniepert } \\
\text { Dihsiet } \\
\text { Oolme }\end{array}$ & $\begin{array}{l}3161 \\
3172 \\
3173 \\
3174 \\
3182 \\
5565 \\
3190 \\
3194 \\
5568\end{array}$ \\
\hline${ }^{7 \theta_{\mathrm{Kr}}}$ & portiol & relati & IV ORE & & & \\
\hline $\begin{array}{l}{ }^{65} \mathrm{Cu} \\
{ }^{50} \mathrm{Zn}\end{array}$ & $\begin{array}{l}160 \\
12 \mathrm{C}\end{array}$ & $\begin{array}{l}4 \cdot 2+1 \\
3 \cdot 3+1\end{array}$ & $\begin{array}{l}5.8+1 \\
3.8+1\end{array}$ & $\begin{array}{l}\text { KLN E } \\
\text { ORL E }\end{array}$ & $\begin{array}{l}\text { Helimeistert } \\
\text { Robinsont }\end{array}$ & $\begin{array}{r}11309 \\
9974\end{array}$ \\
\hline${ }^{7 \theta_{\mathrm{Kr}}}$ & product & yield & & & & \\
\hline $\begin{array}{l}72_{\mathrm{Ge}} \\
72_{\mathrm{Ge}}\end{array}$ & $\begin{array}{l}98 \mathrm{Be} \\
10 \mathrm{~B}\end{array}$ & $\begin{array}{l}3.5+1 \\
3.7+1\end{array}$ & & $\begin{array}{l}\text { FSU E } \\
\text { FSU E }\end{array}$ & $\begin{array}{l}\text { Cloments } \\
\text { Cloments }\end{array}$ & $\begin{array}{l}9494 \\
9527\end{array}$ \\
\hline${ }^{7} \theta_{\mathrm{Kr}}$ & $O(E)$ & & & & $\cdot$ & \\
\hline $\begin{array}{l}{ }^{65} \mathrm{Cu} \\
{ }^{74} \mathrm{Se} \\
{ }^{77} \mathrm{Br}\end{array}$ & $\begin{array}{r}160 \\
0 \\
p\end{array}$ & $\begin{array}{l}4.5+1 \\
\text { NDS } \\
\text { NDG }\end{array}$ & & $\begin{array}{l}\text { SAC E } \\
\text { CAL T V } \\
\text { CAL T }\end{array}$ & $\begin{array}{l}\text { Ballinit } \\
\text { Woosleyt } \\
\text { Woosleyt }\end{array}$ & $\begin{array}{r}11313 \\
7967 \\
3163\end{array}$ \\
\hline $7 \theta_{\mathrm{Kr}}$ & includit & ng mote & eslable & $\theta-\theta(E)$ & & \\
\hline $\begin{array}{r}\mathrm{Br} \\
79 \mathrm{Br} \\
79 \mathrm{Br} \\
79^{79} \mathrm{Br} \\
79^{\circ} \mathrm{Br}\end{array}$ & $\begin{array}{l}p \\
p \\
p \\
p \\
p\end{array}$ & $\begin{array}{l}3.0+0 \\
3.0+0 \\
3.0+0 \\
6 . \theta+0 \\
1.2+1\end{array}$ & $\begin{array}{l}2.5+1 \\
2.5+1 \\
2.5+1\end{array}$ & $\begin{array}{l}\text { BNL E } \\
\text { KFK C : } \\
\text { BNL E } \\
\text { OSU E } \\
\text { ORL E }\end{array}$ & $\begin{array}{l}\text { Collet } \\
\text { Muenzelt } \\
\text { Collet } \\
\text { Humest } \\
\text { Blossert }\end{array}$ & $\begin{array}{l}3148 \\
3177 \\
3178+ \\
3179+ \\
3180+\end{array}$ \\
\hline $1 \theta_{\mathrm{Kr}}$ & cumulat & Ive? th & hleh ta & arget $y$ & yield & \\
\hline${ }_{7 \theta_{\mathrm{Kr}}}^{\mathrm{Br}}$ & cunulat & $\begin{array}{l}4.0+1 \\
\text { lvethl }\end{array}$ & $\begin{array}{l}4.5+1 \\
\text { lck tor }\end{array}$ & $\begin{array}{l}\text { MCG E } \\
\text { rgot yis }\end{array}$ & $\begin{array}{l}\text { Diksiet } \\
\text { ield }\end{array}$ & 3149 \\
\hline${ }^{78_{\mathrm{Kr}}}$ & $\mathrm{I}^{a}$ & $1.0+2$ & & KFK E r & Helugt & 7984 \\
\hline${ }^{79} \mathrm{Br}$ & a & $4.5+1$ & $5.5+1$ & CNE E & Behart & 7995 \\
\hline
\end{tabular}




\begin{tabular}{|c|c|c|c|c|c|c|}
\hline Target & $\ln c$ & $\begin{array}{l}\text { Emin } \\
\text { (MeV) }\end{array}$ & $\begin{array}{l}E_{\max } \\
(\operatorname{MeV})\end{array}$ & Lab & Author & No. \\
\hline${ }^{79} \mathrm{Kr}$ & product & $y|\in| d$ & & & & \\
\hline $\begin{array}{l}{ }^{70} \mathrm{Ge} \\
7^{72} \mathrm{Ge} \\
73 \mathrm{Ge} \\
{ }^{73} \mathrm{Ge}\end{array}$ & $\begin{array}{l}12 \mathrm{C} \\
10 \mathrm{~B} \\
9 \mathrm{Bo} \\
10_{\mathrm{B}}\end{array}$ & $\begin{array}{l}4 \cdot 7+1 \\
3 \cdot 7+1 \\
3 \cdot 5+1 \\
4.0+1\end{array}$ & & $\begin{array}{l}\text { FSU E } \\
\text { FSU E } \\
\text { FSU E } \\
\text { FSU E }\end{array}$ & $\begin{array}{l}\text { E Clementst } \\
\text { E Clementst } \\
\text { E Clementst } \\
\text { E Clementst }\end{array}$ & $\begin{array}{l}9975 \\
9527 \\
9495 \\
9528\end{array}$ \\
\hline $78 \mathrm{Kr}$ & thlck te & orget $y$ & yleld & & & \\
\hline${ }^{19} \mathrm{Kr}$ & $\begin{array}{l}{ }^{3} \mathrm{He} \\
O(E)\end{array}$ & $1.8+1$ & $3.8+1$ & JCL E & E Homma* & 6399 \\
\hline $\begin{array}{l}{ }^{65} \mathrm{Cu} \\
{ }^{65} \mathrm{Cu} \\
{ }^{75} \mathrm{Se} \\
\mathrm{Br} \\
\mathrm{Br} \\
\mathrm{Br}^{78} \mathrm{Br} \\
{ }^{79} \mathrm{Br} \\
{ }^{79} \mathrm{Br} \\
{ }^{79} \mathrm{Br} \\
{ }^{81} \mathrm{Br} \\
{ }^{1} 1 \mathrm{Br}\end{array}$ & $\begin{array}{c}160 \\
160 \\
0 \\
\rho \\
d \\
\rho \\
\rho \\
p \\
{ }^{2} \mathrm{He}_{\mathrm{H}} \\
\rho \\
\rho\end{array}$ & $\begin{array}{l}3.9+1 \\
4.6+1 \\
\text { NDG } \\
1.8+1 \\
1.0+1 \\
\text { NDG } \\
\text { TR } \\
1.0+1 \\
1.1+1 \\
\text { TR } \\
2.5+1\end{array}$ & $\begin{array}{l}5.1+1 \\
8.0+1 \\
9.0+1 \\
4.5+1 \\
8.5+1 \\
3.8+1 \\
4.5+1 \\
8.5+1\end{array}$ & $\begin{array}{l}\text { ORL N } \\
\text { SAC E } \\
\text { CAL } \\
\text { UPP E } \\
\text { JUL F } \\
\text { CAL } \\
\text { JUL E } \\
\text { MCG E } \\
\text { JCL E } \\
\text { JUL E } \\
\text { MCG E }\end{array}$ & $\begin{array}{l}\text { M Welist } \\
\text { E Ballinit } \\
\text { T Woosleyt } \\
\text { E Lundquist+ } \\
\text { R Stocklint } \\
\text { T Woosleyt } \\
\text { E Kniepert } \\
\text { E Diksict } \\
\text { E Homale } \\
\text { E Kniepert } \\
\text { E Oiksiet }\end{array}$ & $\begin{array}{r}11310 \\
11313 \\
7968 \\
3147 \\
5563 \\
3165 \\
3176 \\
3182 \\
6402 \\
3191 \\
3194\end{array}$ \\
\hline $80_{\mathrm{Kr}}$ & partlat & produc & ct $y 101$ & & & \\
\hline $\begin{array}{l}{ }^{78} \mathrm{Se} \\
{ }^{60} \mathrm{Kr}\end{array}$ & $\mathrm{I}^{\circ}$ & +1 & $2.7+1 F$ & ROS E & E Funket & 7971 \\
\hline $\begin{array}{l}{ }^{79} \mathrm{Br} \\
60 \mathrm{Kr}\end{array}$ & $\begin{array}{c}0 \\
\text { product }\end{array}$ & $\begin{array}{l}3.5+1 \\
y 1018\end{array}$ & $6.5+1$ & CNE E & E Behart & 7996 \\
\hline $\begin{array}{l}{ }^{70} \mathrm{Ge} \\
{ }^{73} \mathrm{Ge} \\
73 \mathrm{Ge}\end{array}$ & $\begin{array}{l}{ }^{12} \mathrm{C} \\
9^{9} \mathrm{Be} \\
10_{\mathrm{B}}\end{array}$ & $\begin{array}{l}4.7+1 \\
3.5+1 \\
4.0+1\end{array}$ & & $\begin{array}{l}\text { FSU } \\
\text { FSU E } \\
\text { FSU }\end{array}$ & $\begin{array}{l}\text { E Clementst } \\
\text { E Clementst } \\
\text { E Clementst }\end{array}$ & $\begin{array}{l}9975 \\
9495 \\
9528\end{array}$ \\
\hline $80_{\mathrm{Kr}}$ & $a(E)$ & & & & & \\
\hline $\begin{array}{l}{ }^{76} \mathrm{Se} \\
79 \mathrm{Br} \\
80 \mathrm{gr}\end{array}$ & $\begin{array}{l}o \\
p \\
p\end{array}$ & $\begin{array}{l}\text { NOG } \\
\text { NDG } \\
\text { NDG }\end{array}$ & & $\begin{array}{l}\text { CAL } \\
\text { CAL } \\
\text { CAL }\end{array}$ & $\begin{array}{l}\text { T Woosleyt } \\
\text { T Woosleyt } \\
\text { T Woosleyt }\end{array}$ & $\begin{array}{l}7969 \\
3181 \\
3185\end{array}$ \\
\hline$\theta 1 \mathrm{Kr}$ & portiol & $O(E)$ & & & & \\
\hline O1日r & p & $1.8+0$ & $5.5+0$ & KYU E & E Toyoshima. & 3192 \\
\hline
\end{tabular}

\begin{tabular}{|c|c|c|c|c|c|c|}
\hline Target & $\ln c$ & $\begin{array}{l}\text { Emin } \\
(M e V)\end{array}$ & $\begin{array}{l}\text { Emax } \\
(\operatorname{MeV})\end{array}$ & Lob W & Author & No. \\
\hline $81 \mathrm{kr}$ & $\mathrm{I}$, & & & & & \\
\hline${ }^{79} \mathrm{Br}$ & a & $3.0+1$ & $5.0+1$ & CNE E & E Behart & 7997 \\
\hline $\mathbf{B}_{\mathrm{Kr}}$ & $O(E)$ & & & & & \\
\hline $\begin{array}{l}{ }^{77} \mathrm{Se} \\
{ }^{80} \mathrm{gr}\end{array}$ & : & $\begin{array}{l}\text { NDG } \\
\text { NDG }\end{array}$ & & $\begin{array}{l}\text { CAL T } \\
\text { CAL T }\end{array}$ & $\begin{array}{l}\text { I Wooslest } \\
\text { T Woosle,t }\end{array}$ & $\begin{array}{l}7970 \\
3186\end{array}$ \\
\hline $81 \cdot \mathrm{kr}$ & $O(E)$ & & & & & \\
\hline$\because \bullet \mathrm{Br}$ & a & NOG & & IPS E & E Alterlingt & 8010 \\
\hline $82_{K r}$ & relative & e $1 y$ & & & & \\
\hline${ }^{76} \mathrm{Ge}$ & ${ }^{12} \mathrm{c}$ & $3.5+1$ & $4.5+1$ & BuC $\varepsilon$ & E Irascut & 9976 \\
\hline$\theta 2_{K r}$ & $O(E)$ & & & & & \\
\hline $\begin{array}{l}{ }^{78} \mathrm{Se} \\
0^{1} \mathrm{Br} \\
{ }^{2} \mathrm{Br}\end{array}$ & $\begin{array}{l}a \\
p \\
p\end{array}$ & $\begin{array}{l}\text { NDG } \\
\text { NDG } \\
\text { NDG }\end{array}$ & & $\begin{array}{ll}\text { CAL } & \text { T } \\
\text { CAL } & T \\
\text { CAL } & \text { T }\end{array}$ & $\begin{array}{l}\text { T Woosleyt } \\
\text { T Woosley+ } \\
\text { T Woosley+ }\end{array}$ & $\begin{array}{l}7972 \\
3193 \\
3197\end{array}$ \\
\hline $83 \mathrm{kr}$ & $O(E)$ & & & & & \\
\hline $\begin{array}{l}{ }^{82} \mathrm{Br} \\
{ }^{83} \mathrm{Br}\end{array}$ & $\begin{array}{l}\rho \\
\rho\end{array}$ & $\begin{array}{l}\text { NDG } \\
\text { NOG }\end{array}$ & & $\begin{array}{l}\text { CAL T } \\
\text { CAL T }\end{array}$ & $\begin{array}{l}\text { T Woosleyt } \\
\text { T Woosleyt }\end{array}$ & $\begin{array}{l}3198 \\
3200\end{array}$ \\
\hline${ }^{84} \mathrm{Kr}$ & o(E) & & & & & \\
\hline${ }^{83} \mathrm{Br}$ & $p$ & NDG & & CAL T & T Woosley+ & 3201 \\
\hline $85 \times \mathrm{Kr}$ & cumula & tive fl & ission & $y 1010$ & d & \\
\hline $\begin{array}{l}232 \mathrm{Th} \\
235 \mathrm{U}\end{array}$ & $\begin{array}{l}\text { d } \\
\text { d }\end{array}$ & $\begin{array}{l}9.3+0 \\
9.3+0\end{array}$ & $\begin{array}{l}1.4+1 \\
1.4+1\end{array}$ & $\begin{array}{l}\text { CCP R } \\
\text { CCP R }\end{array}$ & $\begin{array}{l}\text { R Lbovt } \\
R \text { Lbovt }\end{array}$ & $\begin{array}{l}5921 \\
5947\end{array}$ \\
\hline $85 . \mathrm{Kr}$ & lnlek & $\operatorname{largat}$ & $y=1 d x$ & Ixobund & dance & \\
\hline${ }^{B 4} \mathrm{Kr}$ & d & $1.5+1$ & & AML $R$ & R Chaudri. & 5576 \\
\hline${ }^{87} \mathrm{Kr}$ & Indepen & dent fl & igsion & yiold & & \\
\hline $\begin{array}{l}{ }^{209} \mathrm{gi} \\
87 \mathrm{Kr}\end{array}$ & $\begin{array}{l}12 \mathrm{C} \\
\text { cumulat }\end{array}$ & $\begin{array}{l}7.5+1 \\
\text { ive fls }\end{array}$ & $\begin{array}{l}1.0+2 \\
\text { gaton }\end{array}$ & $\begin{array}{l}\text { HAR M } \\
\text { yiold }\end{array}$ & M Branquinhot & 10152 \\
\hline $\begin{array}{l}232 \mathrm{Th} \\
23 \mathrm{U}_{\mathrm{U}}\end{array}$ & $d$ & $\begin{array}{l}9.3+0 \\
9.3+0\end{array}$ & $\begin{array}{l}1.4+1 \\
1.4+1\end{array}$ & $\begin{array}{l}\text { CCP R } \\
\text { CCP R }\end{array}$ & $\begin{array}{l}R \text { Lbovt } \\
R \text { Lbovt }\end{array}$ & $\begin{array}{l}592 ! \\
5947\end{array}$ \\
\hline${ }^{8 \theta_{\mathrm{Kr}}}$ & Indepen & dent 1 & Ission & yiold & & \\
\hline $209_{B 1}$ & $12 \mathrm{c}$ & $7.5+1$ & $1.0+2$ & HAR M & M Eranqulinot & 10152 \\
\hline
\end{tabular}




\begin{tabular}{|c|c|c|c|c|c|c|}
\hline Target & Inc & $\begin{array}{l}\text { Emin } \\
(\mathrm{Me} \%)\end{array}$ & $\begin{array}{l}\text { Emax } \\
(\operatorname{MeV})\end{array}$ & Lab & Author & No. \\
\hline $8 \theta_{\mathrm{Kr}}$ & \multicolumn{6}{|c|}{ cumulative flasion ylelo } \\
\hline $\begin{array}{l}232 \mathrm{Th} \\
23 \mathrm{U}^{\mathrm{U}}\end{array}$ & d & $\begin{array}{l}9.3+0 \\
9.3+0\end{array}$ & $\begin{array}{l}1.4+1 \\
1.4+1\end{array}$ & $\begin{array}{l}\text { CCP F } \\
\text { CCP F }\end{array}$ & $\begin{array}{l}\text { R Lbovt } \\
\text { R Lbove }\end{array}$ & $\begin{array}{l}5921 \\
5947\end{array}$ \\
\hline \multicolumn{7}{|l|}{$z=37$} \\
\hline Ag & $40 \mathrm{Ar}$ & \multirow{2}{*}{\multicolumn{2}{|c|}{$3.4+2$}} & \multirow{2}{*}{\multicolumn{2}{|c|}{ PAR R Lefort+ }} & \multirow[t]{2}{*}{12202} \\
\hline${ }^{77} \mathrm{Rb}$ & $\sigma(E)$ & & & & & \\
\hline $\begin{array}{l}{ }^{40} \mathrm{Co} \\
4^{45} \mathrm{Sc} \\
63 \mathrm{Cu}\end{array}$ & $\begin{array}{l}{ }^{40} \mathrm{Ca} \\
345 \\
160\end{array}$ & $\begin{array}{l}1.1+2 \\
4 \cdot 0+1 \\
4 \cdot 0+1\end{array}$ & $\begin{array}{l}1.5+2 \\
1.4+2 \\
8.5+1\end{array}$ & $\begin{array}{l}\text { PNL } \\
\text { PAR } \\
\text { PAR }\end{array}$ & $\begin{array}{l}\text { E Listert } \\
\text { R Lefort. } \\
\text { E Langevint }\end{array}$ & $\begin{array}{l}12428 \\
12067 \\
11292\end{array}$ \\
\hline${ }^{7 \theta_{R b}}$ & \multicolumn{6}{|l|}{$O(E)$} \\
\hline $\begin{array}{l}{ }^{40} \mathrm{Co} \\
{ }^{65} \mathrm{Cu}\end{array}$ & $\begin{array}{l}40 \mathrm{Co} \\
160\end{array}$ & $\begin{array}{l}1.1+2 \\
3 \cdot 9+1\end{array}$ & $\begin{array}{l}1.5+2 \\
5.1+1\end{array}$ & $\begin{array}{l}\text { BNL } \\
\text { ORL }\end{array}$ & $\begin{array}{l}\text { E Listert } \\
\text { M Wellst }\end{array}$ & $\begin{array}{l}12428 \\
11311\end{array}$ \\
\hline${ }^{79} \mathrm{Rb}$ & \multicolumn{6}{|c|}{ compound-nuclous o(E) } \\
\hline${ }^{63} \mathrm{Cu}$ & 160 & $4,0+1$ & $8.5+1$ & PAR E & E Langerint & 11293 \\
\hline${ }^{79} \mathrm{Rb}$ & produet & ylald & & & & \\
\hline $\begin{array}{l}{ }^{70} \mathrm{Ge} \\
{ }^{72} \mathrm{Ge} \\
{ }^{73} \mathrm{Ge}\end{array}$ & $\begin{array}{l}12 \mathrm{C} \\
10 \mathrm{~B} \\
10 \mathrm{~B}\end{array}$ & $\begin{array}{l}4.7+1 \\
3.7+1 \\
4.0+1\end{array}$ & & $\begin{array}{l}\text { FSU E } \\
\text { FSU } \\
\text { FSU }\end{array}$ & $\begin{array}{l}\text { E Clementst } \\
\text { E Clementst } \\
\text { E Clementst }\end{array}$ & $\begin{array}{l}9975 \\
9527 \\
9528\end{array}$ \\
\hline${ }^{79} \mathrm{RB}$ & \multicolumn{6}{|c|}{ relative thich target ylold } \\
\hline $\mathrm{Kr}$ & p & $3.2+1$ & & BNL & E Lambrecht+ & 3205 \\
\hline${ }^{7 \theta_{R B}}$ & \multicolumn{6}{|l|}{$\theta(E)$} \\
\hline $\begin{array}{l}{ }^{40} \mathrm{Ca} \\
{ }^{65} \mathrm{Cu} \\
{ }^{65} \mathrm{Cu}\end{array}$ & $\begin{array}{l}{ }^{40} \mathrm{Co} \\
180 \\
180\end{array}$ & $\begin{array}{l}1 \cdot 1+2 \\
3 \cdot 9+1 \\
4 \cdot 6+1\end{array}$ & $\begin{array}{l}1.5+2 \\
5.1+1\end{array}$ & $\begin{array}{l}\text { BNL } \\
\text { ORL } \\
\text { SAC }\end{array}$ & $\begin{array}{l}\text { E Listert } \\
\text { M Wellst } \\
\text { E Bailinit }\end{array}$ & $\begin{array}{l}12428 \\
11312 \\
11313\end{array}$ \\
\hline${ }^{80} \mathrm{Rb}$ & \multicolumn{6}{|c|}{ relative I, } \\
\hline${ }^{79} \mathrm{Br}$ & - & $3.5+1$ & $5.5+1$ & CNE & E Behert & 7998 \\
\hline${ }^{80} \mathrm{Rb}$ & product & $y 101 d$ & & & & \\
\hline $\begin{array}{l}{ }^{70} \mathrm{Ge} \\
{ }^{3} \mathrm{Ge}\end{array}$ & $\begin{array}{l}12 \mathrm{C} \\
10 \mathrm{~g}\end{array}$ & $\begin{array}{l}4.7+1 \\
4.0+1\end{array}$ & & FSU & $\begin{array}{l}\text { E Clementst } \\
\text { E Clementst }\end{array}$ & $\begin{array}{l}9975 \\
9528\end{array}$ \\
\hline$\theta^{\prime} \mathrm{Rb}$ & \multicolumn{6}{|c|}{ cumulative? spollation O(E) } \\
\hline${ }^{85} \mathrm{Rb}$ & p & $4.0+1$ & $1.6+2$ & HAR & E Syme+ & 3235 \\
\hline
\end{tabular}

\begin{tabular}{|c|c|c|c|c|}
\hline Target & $\ln c$ & $\begin{array}{ll}\text { Emln } & \text { Emax } \\
(\mathrm{MeV}) & (\mathrm{MeV})\end{array}$ & Lab W & No. \\
\hline $\mathbf{1}_{\mathrm{Rb}}$ & \multicolumn{4}{|c|}{ including potastable o(E) } \\
\hline $\begin{array}{l}90 Z r \\
81 R b\end{array}$ & \multicolumn{4}{|c|}{ cumulative spallation $O(E)$} \\
\hline $\mathrm{Rb}$ & $p$ & $7.0+i$ & HAR E Syme+ & 3222 \\
\hline 81 Ro & \multicolumn{4}{|c|}{ Indopendent spaliation $O(E)$} \\
\hline Rb & p & $7.0+1$ & HAR E Syme+ & 3223 \\
\hline$\theta \mathbf{I}_{\mathbf{R b}}$ & \multicolumn{4}{|c|}{ spollatlon product yield } \\
\hline $\mathrm{Rb}$ & p & $7.0+1$ & HAR E Symet & 3220 \\
\hline O'Ro & \multicolumn{4}{|c|}{ cumulative thick target yleld } \\
\hline Br & 。 & $1.0+2$ & KFK E Helus* & 7985 \\
\hline$\theta^{\prime} \mathbf{R b}$ & \multicolumn{4}{|l|}{ t, } \\
\hline${ }^{79} \mathrm{Br}$ & a & $3.0+14.5+1$ & CNE E Behart & 8002 \\
\hline $81_{R O}$ & \multicolumn{4}{|c|}{ relotive producl yleld } \\
\hline$c \triangle p B r$ & a & $3.0+1$ & BNL E Lambrecht+ & 8011 \\
\hline${ }^{\theta} \mathbf{R b}$ & \multicolumn{4}{|c|}{ product yleld } \\
\hline $\begin{array}{r}{ }^{82} \mathrm{Kr} \\
\mathrm{Rb}\end{array}$ & $\begin{array}{l}\text { p } \\
p\end{array}$ & $\begin{array}{l}\text { NDG } \\
2.0+1 \quad 1.6+2\end{array}$ & $\begin{array}{l}\text { USA R Beavert } \\
\text { HRV E Schneidert }\end{array}$ & $\begin{array}{l}3213 \\
3221\end{array}$ \\
\hline${ }^{B} \mathbf{R b}$ & \multicolumn{4}{|c|}{ thick torget yieldxabundance } \\
\hline $\begin{array}{l}79 \mathrm{Br} \\
81 \mathrm{Br}\end{array}$ & $3_{\mathrm{He}}^{0}$ & $\begin{array}{l}3 \cdot 0+15 \cdot 0+1 \\
2 \cdot 2+1\end{array}$ & $\begin{array}{l}\text { AML R Chaudri. } \\
\text { AML R Choudri. }\end{array}$ & $\begin{array}{l}7999 \\
6403\end{array}$ \\
\hline $\mathbf{B I}^{\prime} \mathbf{R b}$ & \multicolumn{4}{|c|}{ relotive thick lorget yield } \\
\hline kr & p & $3.2+1$ & BNL E Lambrecht+ & 3207 \\
\hline ' $\mathbf{R} \mathbf{R}$ & \multicolumn{4}{|c|}{ thich torget yleld } \\
\hline $\begin{array}{r}8 r \\
79 \mathrm{Br} \\
\mathrm{Kr}\end{array}$ & $\begin{array}{l}{ }^{3} \mathrm{He} \\
0 \\
\mathrm{p}\end{array}$ & 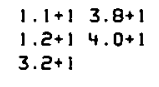 & $\begin{array}{l}\text { JCL E Hommat } \\
\text { JCL E Hommat } \\
\text { BNL E Lambrecht+ }\end{array}$ & $\begin{array}{l}6401 \\
8001 \\
3206\end{array}$ \\
\hline $\mathbf{P}^{\prime} \mathbf{R b}$ & \multicolumn{4}{|l|}{$O(E)$} \\
\hline $\begin{array}{r}\mathrm{Br} \\
79_{\mathrm{Br}} \\
{ }^{85} \mathrm{Rb}\end{array}$ & $\begin{array}{c}3 \mathrm{He} \\
0 \\
0\end{array}$ & $\begin{array}{l}2.6+14.0+1 \\
1.2+1 \\
6.8+1\end{array}$ & $\begin{array}{l}\text { JCL E Hommat } \\
\text { JCL E Hommat } \\
\text { HRV E Schneldert }\end{array}$ & $\begin{array}{l}6400 \\
6000 \\
3236\end{array}$ \\
\hline
\end{tabular}




\begin{tabular}{|c|c|c|c|c|c|c|}
\hline Target & Inc & $\begin{array}{l}\text { Emin } \\
\left(M_{\theta} V\right)\end{array}$ & $\begin{array}{l}\text { Emex } \\
(M \in V)\end{array}$ & Lab $\mathrm{H}$ & Author & No. \\
\hline$\theta^{\prime} \mathrm{Rb}$ & $O(E)$ & & & & & \\
\hline $90 \mathrm{Zr}$ & P & $6.0+1$ & $9 . E+1$ & MIL T & Tadiolit & 3423 \\
\hline $81 \mathrm{~GB}$ & cumula & tive of & (E) & & & \\
\hline${ }^{85} \mathrm{Rb}$ & $p$ & $4.5+1$ & $6.5+1$ & OSA E & E roshizovot & 3237 \\
\hline 8 I 9 Rb & relati & ve $\sigma(E)$ & & & & \\
\hline${ }^{82} \mathrm{Kr}$ & d & $2.2+1$ & & ANL E & E Gindlert & 5573 \\
\hline Q I "Rb & O(E) & & & & & \\
\hline${ }^{85} \mathrm{Rb}$ & D & $4.5+1$ & $6.5+1$ & OSA E & $=\operatorname{Yoshl} 2 \theta 08+$ & 3238 \\
\hline${ }^{82} \mathrm{Rb}$ & product & $y / e 19$ & & & & \\
\hline${ }^{\theta}{ }^{\mathrm{Kr}}$ & p & NDG & & USA R & Beavert & 3214 \\
\hline${ }^{\theta 2} \mathrm{Rb}$ & $\theta(E)$ & & & & & \\
\hline $90 \mathrm{Zr}$ & $\mathbf{p}$ & $4.0+1$ & $9.5+1$ & MIL T & T Gadioll+ & 3424 \\
\hline$\theta 5$ จRb & $O(E)$ & & & & & \\
\hline $90 \mathrm{Zr}$ & $p$ & $4.4+1$ & $8.6+1$ & MCG E & E Kantelot & 3425 \\
\hline$\theta 2 \bullet \mathrm{Rb}$ & cumula & tive? & spollat & $\operatorname{tion} \theta$ & $a(E)$ & \\
\hline $\begin{array}{r}R b \\
05 R b\end{array}$ & $\begin{array}{l}\mathrm{P} \\
\mathrm{P}\end{array}$ & $\begin{array}{l}7 \cdot 0+1 \\
4 \cdot 0+1\end{array}$ & $\begin{array}{l}1.0+2 \\
1.6+2\end{array}$ & $\begin{array}{l}\text { HAR E } \\
\text { HAR E }\end{array}$ & $\begin{array}{l}\text { E Symet } \\
\text { E Symet }\end{array}$ & $\begin{array}{l}3224 \\
3239\end{array}$ \\
\hline$\theta 2 \cdot \mathbf{R b}$ & eumula & live th & hick ta & arget & yield & \\
\hline $8 r$ & a & $1.0+2$ & & KFK E & E Helust & 7988 \\
\hline $\operatorname{\theta e} \bullet \mathbf{R b}$ & $r e l e t t$ & ive prod & duct yi & ield & & \\
\hline$c \oplus P B r$ & a & $3 \cdot 0+1$ & & BNL E & E Lambrecht+ & 8012 \\
\hline B $2 \bullet \mathbf{R b}$ & thich & target & $y 1010 x$ & $x$ abund & dance & \\
\hline${ }^{81} \mathrm{Br}$ & ${ }^{3} \mathrm{He}$ & $2.2+1$ & & AML $R$ & R Chaudrl. & 6404 \\
\hline $\mathbf{\theta} \mathbf{e} \mathbf{R b}$ & relatis & ive thic & ch torg & get $y^{\prime}$ & leld & \\
\hline $\mathrm{kr}$ & p & $3.2+1$ & & BNLE $E$ & E Lambrechl+ & 3208 \\
\hline$\theta$ ?・Rb & thick & targot & $y|\in| d$ & & & \\
\hline $\begin{array}{c}\mathrm{Br} \\
8{ }^{\mathrm{Br}}\end{array}$ & ${ }_{3}^{a} \mathrm{He}$. & $\begin{array}{l}1.2+1 \\
1.1+1\end{array}$ & $\begin{array}{l}4.0+1 \\
3.8+1\end{array}$ & $\begin{array}{l}\text { JCL E } \\
\text { JCL E }\end{array}$ & $\begin{array}{l}\text { E Hommat } \\
\text { E Hommat }\end{array}$ & $\begin{array}{l}7987 \\
6406\end{array}$ \\
\hline
\end{tabular}

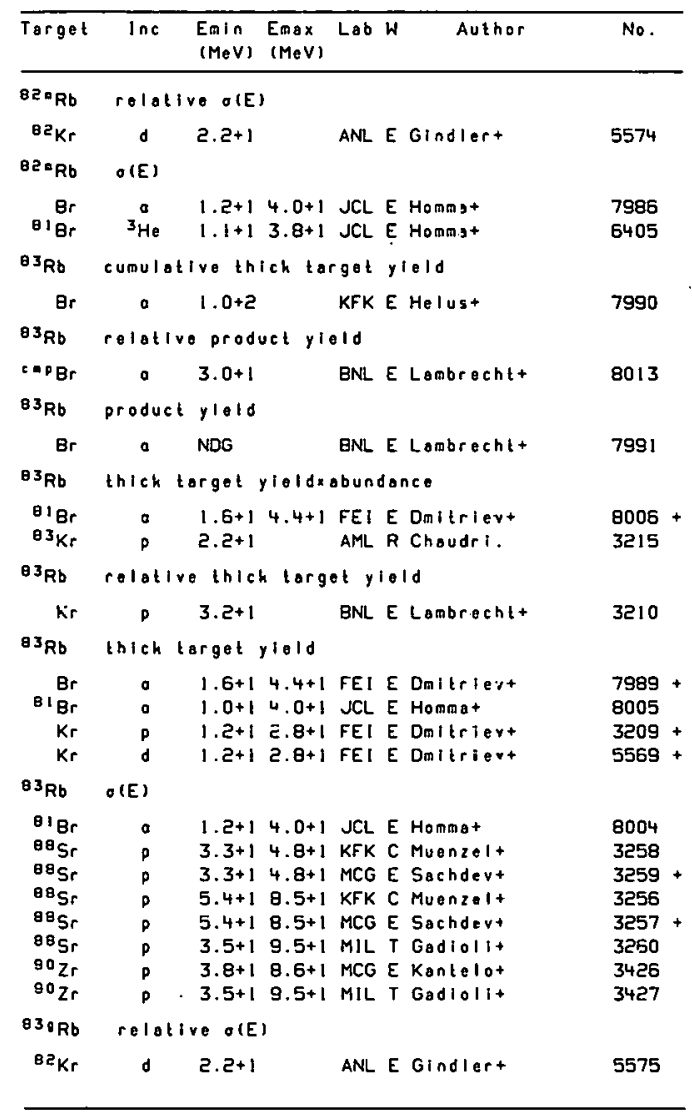




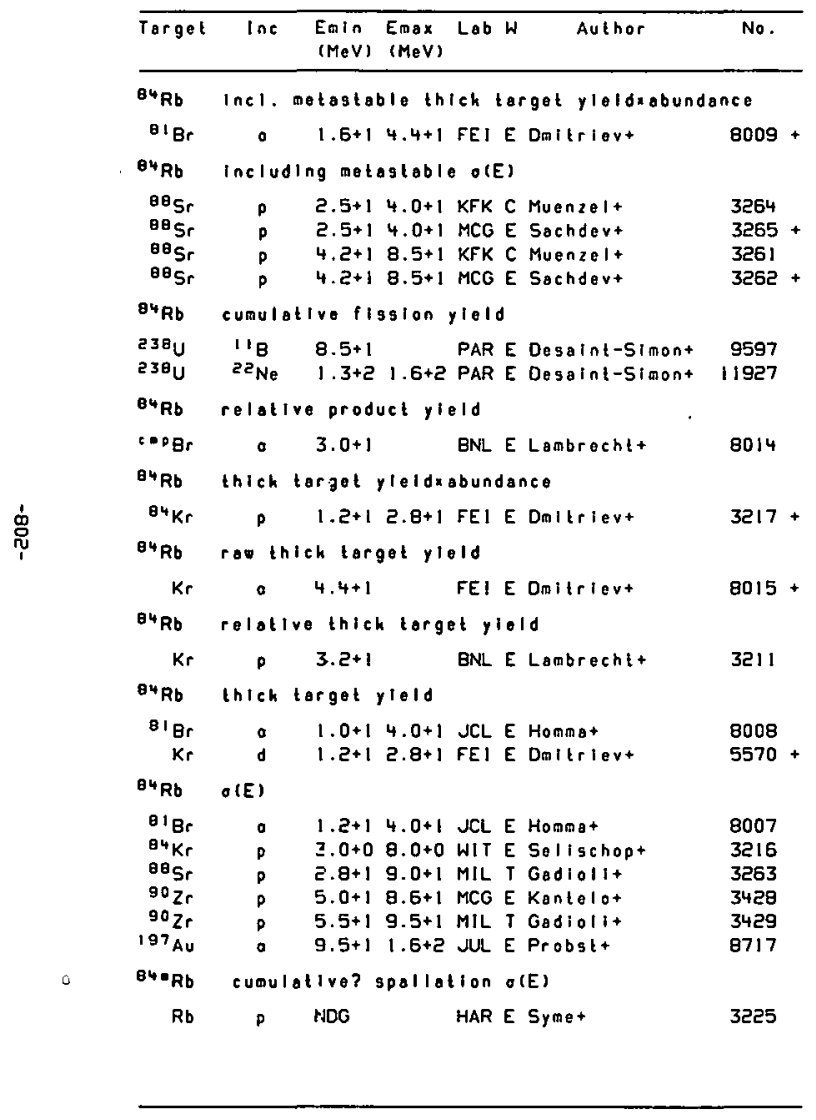

\begin{tabular}{|c|c|c|c|c|}
\hline Target & $\operatorname{lnc}$ & $\begin{array}{ll}\text { Enin } & \text { Emax } \\
\left(M e^{\prime} y\right) & (M e V) \\
\end{array}$ & Lab W & No. \\
\hline${ }^{89^{R b}}$ & cumule & trefission & yeld & \\
\hline $\begin{array}{l}-5 \\
23 \theta_{U} \\
23 \theta^{2}\end{array}$ & $\begin{array}{l}118 \\
22_{\mathrm{Ne}}\end{array}$ & $\begin{array}{l}8.5+1 \\
1.3+2 \quad 1.6+2\end{array}$ & $\begin{array}{l}\text { PAR E Desaint-simont } \\
\text { PAR E Desaint-simont }\end{array}$ & $\begin{array}{r}9597 \\
11927\end{array}$ \\
\hline${ }^{\mathbf{B s}_{\mathrm{Rb}}}$ & portlal & roothlck to & target yleldy abundance & \\
\hline${ }^{89} \mathrm{Rb}$ & . & $5.0+0$ & SUN E Gllest & 8020 \\
\hline${ }^{86_{R b}}$ & Includ & ng metastable & le $O(E)$ & \\
\hline $\begin{array}{l}{ }_{8 \theta}{ }^{8} \mathrm{Sr} \\
{ }^{8} \mathrm{Sr}\end{array}$ & $p$ & $\begin{array}{ll}3.3+1 & 8.5+1 \\
3.3+1 & 8.5+1\end{array}$ & $\begin{array}{l}\text { IKFK C Muenzel+ } \\
\text { I MCG E Sachdev+ }\end{array}$ & $\begin{array}{l}3267 \\
3258+\end{array}$ \\
\hline${ }^{86_{R b}}$ & Indeper & dent fission & $n$ yleld & \\
\hline $\begin{array}{l}238 \mathrm{U} \\
{ }^{23} 3 \mathrm{~B}^{2}\end{array}$ & 9 & $\begin{array}{l}5 \cdot 6+1 \\
5 \cdot 6+1\end{array}$ & $\begin{array}{l}\text { MCG E Clarat } \\
\text { MCG E Clarat }\end{array}$ & $\begin{array}{l}5962 \\
5962\end{array}$ \\
\hline${ }^{86} \mathrm{Rb}$ & indeper & dent $O(E)$ & & \\
\hline $\begin{array}{l}238 \mathrm{U} \\
238 \mathrm{U}\end{array}$ & $d$ & $\begin{array}{l}5.0+1 \\
5.0+1\end{array}$ & $\begin{array}{l}\text { MCG E Clara+ } \\
\text { MCG E Clara+ }\end{array}$ & $\begin{array}{l}5951 \\
5961\end{array}$ \\
\hline${ }^{86} \mathrm{Rb}$ & cumulal & ive rission & $y / \theta 1 d$ & \\
\hline $\begin{array}{l}23 \mathrm{Th}^{\mathrm{Th}} \\
23 \mathrm{C}_{\mathrm{U}} \\
23 \mathrm{U}_{\mathrm{U}} \\
237_{\mathrm{Np}}\end{array}$ & 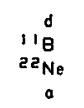 & 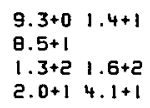 & $\begin{array}{l}\text { CCP R Lbovt } \\
\text { PAR E Desaini-simont } \\
\text { PAR E Desaint-5imont } \\
\text { I CCP R Loovt }\end{array}$ & $\begin{array}{r}5921 \\
9957 \\
11927 \\
9091\end{array}$ \\
\hline${ }^{86_{\mathrm{Rb}}}$ & thich & arget yleldxe & abundances & \\
\hline $\begin{array}{l}{ }^{86} \mathrm{Kr} \\
{ }^{86} \mathrm{Kr}\end{array}$ & $\stackrel{p}{d}$ & $\begin{array}{ll}1.2+1 & 2.8+1 \\
1.2+1 & 2.8+1\end{array}$ & $\begin{array}{l}1 \text { FEI E Dailtiert } \\
1 \text { FEI E Daltriert }\end{array}$ & $\begin{array}{l}3219+ \\
5577+\end{array}$ \\
\hline${ }^{86} \mathrm{Rb}$ & roo th & ch target yio & 1010 & \\
\hline $\mathrm{kr}$ & $\circ$ & $4.4+1$ & FEI E Dmitrievt & $8016+$ \\
\hline${ }^{85} \mathrm{Rb}$ & reloli & - thlek targe & get yiold & \\
\hline $\begin{aligned} \mathrm{Kr} \\
\mathrm{BE}_{\mathrm{Rb}}\end{aligned}$ & thick & $\begin{array}{l}3 . z+1 \\
\text { arget yield }\end{array}$ & BNL E Lambrecht+ & 3212 \\
\hline${ }^{86_{\mathrm{Rb}}} \mathrm{Kr}$ & $\begin{array}{r}0 \\
\text { O(E) }\end{array}$ & $1.2+12.8+1$ & I FEI E Daritrlevt & $5571+$ \\
\hline $\begin{array}{l}86 \mathrm{Kr} \\
{ }^{88} \mathrm{Sr}\end{array}$ & $p$ & 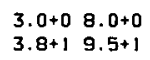 & $\begin{array}{l}\text { WIT E Sellischopt } \\
\text { MIL T Godiolit }\end{array}$ & $\begin{array}{l}3218 \\
3266\end{array}$ \\
\hline
\end{tabular}




\begin{tabular}{|c|c|c|c|c|c|}
\hline Target & $\ln s$ & $\begin{array}{ll}\text { Emin } & \text { Emax } \\
(M e V) & (M e V)\end{array}$ & Lab W & Author & No. \\
\hline${ }^{86_{R b}}$ & $\sigma(E)$ & & & & \\
\hline $197 \mathrm{Au}$ & 0 & $9.5+1 \quad 1.6+2$ & JUL E & Probstt & 8718 \\
\hline${ }^{87} \mathrm{Rb}$ & cunulat & ive flssion y & yleld & & \\
\hline $\begin{array}{l}238 u \\
230 u\end{array}$ & $\begin{array}{l}11 \mathrm{~B} \\
22_{\mathrm{Ne}}\end{array}$ & $\begin{array}{l}8.5+1 \\
1.3+2 \quad 1.6+2\end{array}$ & $\begin{array}{l}\text { PAR E } \\
\text { PAR E }\end{array}$ & $\begin{array}{l}\text { Desaint-Simont } \\
\text { Desaint-Simont }\end{array}$ & $\begin{array}{r}9597 \\
11927\end{array}$ \\
\hline $\mathbf{\theta \theta}_{\mathbf{R b}}$ & cumulat & ive flasion $y$ & yleld & & \\
\hline 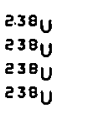 & $\begin{array}{l}118 \\
118 \\
22_{\mathrm{Ne}} \\
22_{\mathrm{Ne}}\end{array}$ & $\begin{array}{ll}8.5+1 & \\
8 \cdot 5+1 & \\
1.3+2 & 1.6+2 \\
1.3+2 & 1.6+2\end{array}$ & $\begin{array}{l}\text { PAR E } \\
\text { PAR E } \\
\text { PAR E } \\
\text { PAR E }\end{array}$ & $\begin{array}{l}\text { Desaint-5imont } \\
\text { Desaint-5imont } \\
\text { Desaint-Simont } \\
\text { Desaint-Simont }\end{array}$ & $\begin{array}{r}9597 \\
9597 \\
11927 \\
11927\end{array}$ \\
\hline $89_{R b}$ & Indepen & dent fission & yield & & \\
\hline $\begin{array}{l}20{ }^{2} \mathrm{BI} \\
238_{\mathrm{U}}\end{array}$ & $\begin{array}{r}12 \mathrm{C} \\
d\end{array}$ & $\begin{array}{l}7.5+1 \\
5.0+1\end{array}$ & $\begin{array}{l}\text { HAR M } \\
\text { MCG E }\end{array}$ & $\begin{array}{l}\text { Branquintiot } \\
\text { Clarat }\end{array}$ & $\begin{array}{r}10152 \\
5962\end{array}$ \\
\hline${ }^{89} \mathrm{Rb}$ & indepen & dent $O(E)$ & & & \\
\hline $238 \mathrm{U}$ & g & $5.0+1$ & MCG E & Clarst & 5961 \\
\hline${ }^{89_{R b}}$ & cumulat & ive fission & pleld & & \\
\hline $\begin{array}{l}238 \mathrm{U} \\
238 \mathrm{U}\end{array}$ & $\begin{array}{l}n{ }_{10} \\
2 a_{\mathrm{Ne}}\end{array}$ & $\begin{array}{l}8.5+1 \\
1.3+2\end{array}$ & $\begin{array}{l}\text { PAR E } \\
\text { PAR E }\end{array}$ & $\begin{array}{l}\text { Desaint-Simont } \\
\text { Desaint-Simont }\end{array}$ & $\begin{array}{r}9597 \\
11927\end{array}$ \\
\hline${ }^{90} \mathrm{Rb}$ & indepen & dent fission & yleld & & \\
\hline $23 \theta^{U}$ & d & $5.0+1$ & MCG E & $\mathrm{Clarat}$ & 5962 \\
\hline${ }^{80} \mathrm{Rb}$ & Indepen & dent $O(E)$ & & & \\
\hline $23 \theta^{U}$ & $d$ & $5.0+1$ & MCG E & $\mathrm{Clara+}$ & 5961 \\
\hline${ }^{90} 0_{\mathrm{Rb}}$ & cumulat & ive fission & pleld & & \\
\hline $\begin{array}{l}-4 \\
538^{4}\end{array}$ & $\begin{array}{l}118 \\
22_{\mathrm{Ne}}\end{array}$ & $\begin{array}{l}8.5+1 \\
1.3+2 \quad 1.6+2\end{array}$ & $\begin{array}{l}\text { PAR E } \\
\text { PAR E }\end{array}$ & $\begin{array}{l}\text { Desaint-Simont } \\
\text { Dosaint-Simont }\end{array}$ & $\begin{array}{r}9597 \\
11927\end{array}$ \\
\hline $91 \mathbf{R b}$ & indepen & dent lission & $y i e l d$ & & \\
\hline $23 \theta_{U}$ & d & $5.0+1$ & MCG E & Clarat & 5962 \\
\hline${ }^{9} !_{R b}$ & Indepen & dent $O(E)$ & & & \\
\hline $238^{2}$ & $d$ & $5.0+1$ & MCG E & Clarat & 5961 \\
\hline
\end{tabular}

\begin{tabular}{|c|c|c|c|c|c|}
\hline Target & $\ln e$ & $\begin{array}{ll}E_{\min } & E_{\max } \\
\left(M_{\theta} V\right) & \left(M_{\theta} V\right)\end{array}$ & Lab $\mathrm{W}$ & Author & No. \\
\hline $91_{R b}$ & \multicolumn{5}{|c|}{ cumulative fission yield } \\
\hline $\begin{array}{l}-4 \\
53 \theta^{u}\end{array}$ & $\begin{array}{l}11 \mathrm{~B} \\
22_{\mathrm{Ne}}\end{array}$ & $\begin{array}{l}8.5+1 \\
1.3+2 \quad 1.6+2\end{array}$ & $\begin{aligned} & \text { PAR E } \\
\text { PAR E } & \text { PAR }\end{aligned}$ & $\begin{array}{l}\text { Desain:-5imont } \\
\text { Desain:-Simant }\end{array}$ & $\begin{array}{r}9597 \\
11927\end{array}$ \\
\hline $92_{R b}$ & \multicolumn{2}{|c|}{ independont fisgion } & yleld & & \\
\hline $23 \theta^{4}$ & o & $5.0+1$ & MCG E & Clarat & 5962 \\
\hline${ }^{92_{R b}}$ & \multicolumn{5}{|c|}{ independont $\sigma(E)$} \\
\hline $23 \theta^{4}$ & d & $5.0+1$ & MCG E & Clarat & 5961 \\
\hline${ }^{92} \cdot R \mathbf{b}$ & \multicolumn{5}{|c|}{ cuanlative fission yleld } \\
\hline 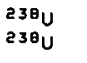 & $\begin{array}{l}118 \\
2{ }^{2} \mathrm{Ne}\end{array}$ & $\begin{array}{l}8.5+1 \\
1.3+2 \quad 1.6+2\end{array}$ & $\begin{array}{l}\text { PAR E } \\
\text { PAR E }\end{array}$ & $\begin{array}{l}\text { Desalnt-Simont } \\
\text { Desalnt-Simont }\end{array}$ & $\begin{array}{r}9597 \\
11927\end{array}$ \\
\hline $\mathbf{9 3}_{\mathrm{Rb}}$ & \multicolumn{2}{|c|}{ independent lission } & $y \mid e / d$ & & \\
\hline $23 \theta^{4}$ & d & $5.0+1$ & MCG E & Clarat & 5962 \\
\hline${ }^{93} \mathrm{Rb}$ & \multicolumn{5}{|c|}{ independent $O(E)$} \\
\hline $23 \theta^{U}$ & d & $5.0+1$ & MCG E & Clarat & $596 !$ \\
\hline${ }^{93} \mathrm{Rb}$ & \multicolumn{5}{|c|}{ cumulative figsion yield } \\
\hline $\begin{array}{l}-4 \\
53 \theta^{2} u \\
53 \theta^{2}\end{array}$ & $\begin{array}{l}118 \\
2 z_{\mathrm{Ne}}\end{array}$ & $\begin{array}{l}8.5+1 \\
1.3+2 \quad 1.6+2\end{array}$ & $\begin{aligned} & \text { PAR E } \\
& \text { P PAR E }\end{aligned}$ & $\begin{array}{l}\text { Desaint-simont } \\
\text { Desaint-simont }\end{array}$ & $\begin{array}{r}9597 \\
11927\end{array}$ \\
\hline${ }^{94} R b$ & \multicolumn{5}{|c|}{ independent fisston yleld } \\
\hline $23 \theta_{U}$ & d & $5.0+1$ & MCG E & Clarat & 5962 \\
\hline${ }^{94} R b$ & \multicolumn{5}{|c|}{ Independent $O(E)$} \\
\hline $23 \theta_{U}$ & d & $5.0+1$ & MCG E & Clarat & 5981 \\
\hline${ }^{84} R b$ & \multicolumn{5}{|c|}{ cumulative fisgion yield } \\
\hline 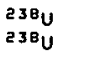 & $\begin{array}{l}118 \\
22_{\mathrm{Ne}}\end{array}$ & $\begin{array}{l}0.5+1 \\
1.3+2 \quad 1.6+2\end{array}$ & $\begin{array}{l}\text { PAR E } \\
\text { PAR E }\end{array}$ & $\begin{array}{l}\text { Desaint-Simont } \\
\text { Desaint-Simont }\end{array}$ & $\begin{array}{r}9597 \\
11927\end{array}$ \\
\hline${ }^{95} \mathrm{Rb}$ & \multicolumn{5}{|c|}{ independent fission yield } \\
\hline $238_{U}$ & $d$ & $5.0+1$ & MCG E & Clarat & 5952 \\
\hline${ }^{85_{R b}}$ & \multicolumn{5}{|c|}{ Independent $\sigma[E]$} \\
\hline $238^{2} \mathrm{U}$ & o & $5.0+1$ & MCG E & Clarat & 5961 \\
\hline
\end{tabular}




\begin{tabular}{|c|c|c|c|c|c|}
\hline Target & Inc & $\begin{array}{l}\text { Emin Emax } \\
\text { (MeV) (MeV) }\end{array}$ & Lab W & Author & No. \\
\hline${ }^{96} R \mathrm{~B}$ & \multicolumn{5}{|c|}{ Independent figsion ylold } \\
\hline $23 \theta^{U}$ & $d$ & $5.0+1$ & MCG E & Clorat & 5962 \\
\hline${ }^{96} \mathrm{Rb}$ & \multicolumn{5}{|c|}{ Independent o(E) } \\
\hline $23 \theta^{4}$ & d & $5.0+1$ & MCG E & Clorat & 5951 \\
\hline${ }^{97} \mathrm{Rb}$ & \multicolumn{5}{|c|}{ Indopendent fission yiold } \\
\hline $239^{2} \mathrm{U}$ & d & $5.0+1$ & MCG E & $\mathrm{Clarg}^{+}$ & 5962 \\
\hline $97_{R b}$ & \multicolumn{5}{|c|}{ indepondent $O(E)$} \\
\hline${ }^{23} \theta^{2} U$ & d & $5.0 \cdot 1$ & MCG E & Clarat & 5961 \\
\hline${ }^{\theta \theta_{R b}}$ & \multicolumn{5}{|c|}{ independent lission yield } \\
\hline $238_{U}$ & $d$ & $5.0+1$ & MCOE & Clarat & 5962 \\
\hline${ }^{8 \theta_{R b}}$ & \multicolumn{5}{|c|}{ Indepondent $\sigma(E)$} \\
\hline $23 \theta^{4}$ & d & $5.0+1$ & MCG E & Clarat & 5961 \\
\hline \multicolumn{6}{|l|}{$z=38$} \\
\hline $\mathrm{Ag}$ & $40 \mathrm{Ar}$ & $3.4+2$ & PAR R & Lefort+ & 12202 \\
\hline $79 \mathrm{Sr}$ & \multicolumn{5}{|c|}{ oroduct yiold } \\
\hline${ }^{40} \mathrm{Co}$ & ${ }^{40} \mathrm{Ca}$ & NDG & BRK $R$ & Cernyt & 12427 \\
\hline${ }^{80} \mathrm{Sr}$ & \multicolumn{5}{|c|}{ product yiald } \\
\hline${ }^{70} \mathrm{Ge}$ & ${ }^{12} \mathrm{C}$ & $4.7+1$ & FSU E & Clementst & 9975 \\
\hline OI $5 r$ & \multicolumn{5}{|c|}{ cumulative spatlation o(E) } \\
\hline $\begin{array}{l}{ }^{85} \mathrm{Rb} \\
{ }^{87} \mathrm{Rb}\end{array}$ & $\begin{array}{l}p \\
p\end{array}$ & $\begin{array}{l}\text { NDG } \\
\text { NDG }\end{array}$ & $\begin{array}{l}\text { HAR E } \\
\text { HAR E }\end{array}$ & $\begin{array}{l}\text { Woodt } \\
\text { Woodt }\end{array}$ & $\begin{array}{l}3240 \\
3246\end{array}$ \\
\hline OIsr & \multicolumn{5}{|c|}{ spolilotion product yield } \\
\hline Ro & $p$ & $7.0+1 \quad 1.0+2$ & HAR E & Symet & 3227 \\
\hline O'sr & \multicolumn{5}{|c|}{ Spollotion o(E) } \\
\hline Ro & $p$ & $7.0+1$ & HAR E & Symet & 3226 \\
\hline B'sr. & \multicolumn{5}{|c|}{ product yield } \\
\hline $\begin{array}{l}{ }^{85} \mathrm{Rb} \\
{ }^{85} \mathrm{Rb}\end{array}$ & D & $\begin{array}{l}6 . E+1 \\
1 . E+2\end{array}$ & $\begin{array}{l}\text { DAV E } \\
\text { GRN E }\end{array}$ & $\begin{array}{l}\text { Lagunag-Solart } \\
\text { Von Herkt }\end{array}$ & $\begin{array}{l}3241 \\
8021\end{array}$ \\
\hline
\end{tabular}

\begin{tabular}{|c|c|c|c|c|c|}
\hline Torget & $\ln c$ & $\begin{array}{l}\text { Emin } \\
(\mathrm{MeV})\end{array}$ & $\begin{array}{l}\text { Emax } \\
\text { (Mev) }\end{array}$ & Author & No. \\
\hline Ol & $O(E)$ & & & & \\
\hline $\begin{array}{l}{ }^{85} \mathrm{Rb} \\
{ }^{85} \mathrm{Rb} \\
{ }^{85} \mathrm{Rb} \\
{ }^{85} \mathrm{Rb}\end{array}$ & $\begin{array}{l}p \\
p \\
p \\
p\end{array}$ & $\begin{array}{l}4.5+1 \\
4.5+1 \\
5.4+1 \\
6.9+1\end{array}$ & $\begin{array}{l}6.5+1 \\
6.5+1 \\
6.9+1\end{array}$ & $\begin{array}{l}\text { GRN E Van Herkt } \\
\text { OSA E Yoshlzawat } \\
\text { WUR E Hegedugt } \\
\text { HRV E Sehneidert. }\end{array}$ & $\begin{array}{l}3242 \\
3243 \\
3244 \\
3245\end{array}$ \\
\hline$e^{8} \mathbf{s}$ & Incluo & $\operatorname{lng}$ meto & ostoble & $\theta(E)$ & \\
\hline $\begin{array}{l}90 \mathrm{Zr} \\
{ }^{92} \mathrm{Sr}\end{array}$ & $\stackrel{p}{\text { relat }}$ & $\begin{array}{l}8.0+1 \\
v \in I_{y}\end{array}$ & $8.6+1$ & MCG E Kantelo+ & 3430 \\
\hline${ }^{76} \mathrm{Se}$ & ${ }^{12 a} \mathrm{c}$ & $3.6+1$ & $5 \cdot 2+1$ & MUU E Korschinikt & 9981 \\
\hline$e^{2} \mathrm{sr}$ & thlck & torget $y$ & $y=1 d$ & & \\
\hline $\mathbf{R b}$ & $p$ & $4.8+1$ & & GRN E Buhshpant & 3228 \\
\hline$e_{2} 2 t$ & $O(E)$ & & & & \\
\hline $\begin{array}{l}90 \mathrm{Zr} \\
90 \mathrm{Zr}\end{array}$ & p & $\begin{array}{l}7.0+i \\
7.5+i\end{array}$ & $\begin{array}{l}9.0+1 \\
9.5+1\end{array}$ & $\begin{array}{l}\text { BNL T Divadoenam. } \\
\text { MIL I Gadiolit }\end{array}$ & $\begin{array}{l}3431 \\
3432\end{array}$ \\
\hline$e_{5 r}$ & incluo & $\operatorname{lng}$ mota & astablo & - $O(E)$ & \\
\hline $\begin{array}{l}90 \mathrm{Zr} \\
{ }^{83} \mathrm{gr}\end{array}$ & $\begin{array}{c}p \\
\text { cumulo }\end{array}$ & $\begin{array}{c}5.5+1 \\
\text { tive ran }\end{array}$ & $\begin{array}{l}8.6+11 \\
\text { thick }\end{array}$ & $\begin{array}{l}\text { MCG E kontelot } \\
\text { k torget ylold }\end{array}$ & 3433 \\
\hline $\begin{array}{l}\mathrm{Kr} \\
{ }^{3} \mathrm{Sr}\end{array}$ & $\begin{array}{c}a \\
\text { cumule }\end{array}$ & $\begin{array}{l}4.4+1 \\
\text { t1 }\end{array}$ & & FEI E Dmitrievt & $8017+$ \\
\hline $\begin{array}{l}238^{3} \mathrm{U} \\
e^{83} \mathrm{Sr}\end{array}$ & $\stackrel{p}{\text { rolat }}$ & $\begin{array}{l}1.0+3 \\
y=1\end{array}$ & $3.0+5$ & KFK C Muenzel+ & 4903 \\
\hline${ }^{76} \mathrm{Ge}$ & ${ }^{12} \mathrm{C}$ & $3.5+1$ & $4.5+1$ & BUC E Irascu+ & 9977 \\
\hline${ }^{03} \mathrm{sr}$ & $\theta(E)$ & & & & \\
\hline $\begin{array}{l}90 \mathrm{Zr} \\
90 \mathrm{Zr}\end{array}$ & p & $\begin{array}{l}6.0+1 \\
6.5+1\end{array}$ & $\begin{array}{l}9.0+1 \\
9.5+1\end{array}$ & $\begin{array}{l}\text { BNL T Divadecram. } \\
\text { MIL T Gadiolit }\end{array}$ & $\begin{array}{l}3434 \\
3435\end{array}$ \\
\hline${ }^{84} \mathrm{Sr}$ & rolati & ve $l$, & & & \\
\hline $\begin{array}{l}{ }^{76} \mathrm{Ge} \\
{ }^{85} \mathrm{Sr}\end{array}$ & $\begin{array}{l}12 c \mathrm{C} \\
\text { reloti }\end{array}$ & $\begin{array}{l}3.5+1 \\
v \in I_{y}\end{array}$ & $4.5+1$ & BUC E Irascut & 9978 \\
\hline $\begin{array}{l}{ }^{76} \mathrm{Ge} \\
\theta^{2} \mathrm{Kr}\end{array}$ & $\begin{array}{r}12 \mathrm{c} \\
\therefore\end{array}$ & $\begin{array}{l}3.5+1 \\
1.4+1\end{array}$ & $\begin{array}{l}4.4+1 \\
2.1+1\end{array}$ & $\begin{array}{l}\text { BUC E Ivascut } \\
\text { IPS E Arnell+ }\end{array}$ & $\begin{array}{l}9979 \\
8018\end{array}$ \\
\hline
\end{tabular}




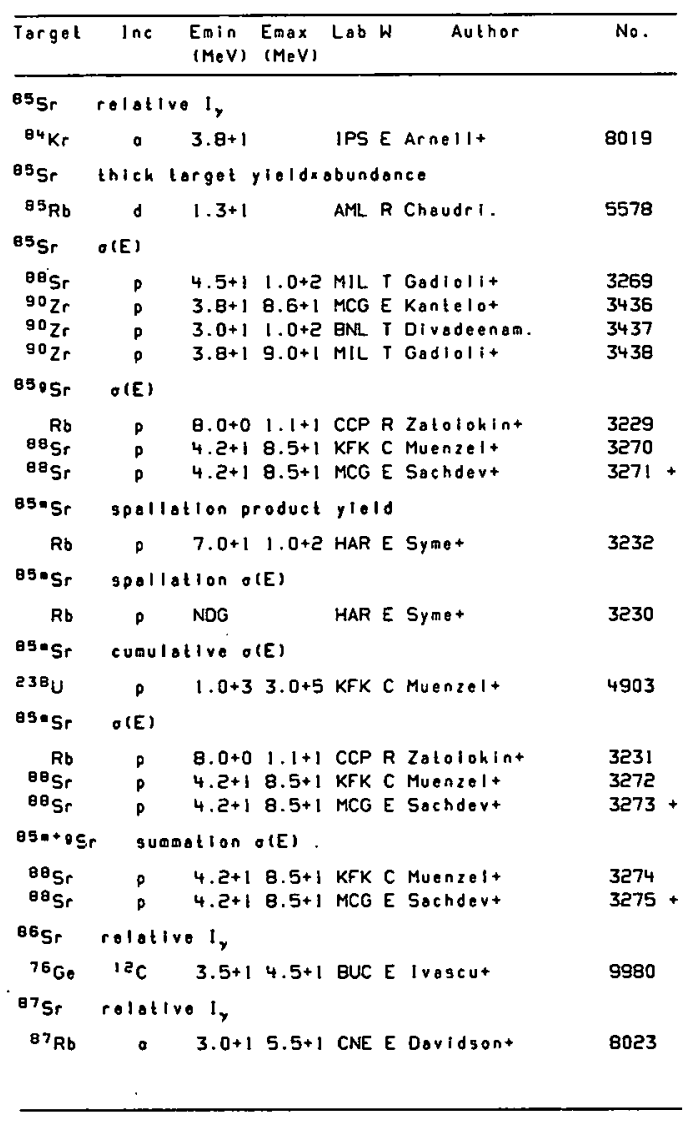

\begin{tabular}{|c|c|c|c|c|c|c|}
\hline Target & $\operatorname{lnc}$ & $\begin{array}{l}\text { Emin } \\
(M e V)\end{array}$ & $\begin{array}{l}\text { Emax } \\
(\operatorname{MeV})\end{array}$ & Lab W & Author & No. \\
\hline$\theta 7=5 r$ & $\theta(E)$ & & & & & \\
\hline $\begin{array}{r}R b \\
87_{R b} \\
97_{R b}\end{array}$ & $\begin{array}{l}p \\
p \\
p\end{array}$ & $\begin{array}{l}8.0+0 \\
1.2+1 \\
1.2+1\end{array}$ & $1.1+1$ & $\begin{array}{l}\text { CCP } \\
\text { KFK } \\
\text { ORL } \\
\text { O }\end{array}$ & $\begin{array}{l}\text { Zatoloitint } \\
\text { Muenzel+ } \\
\text { Blosso:t }\end{array}$ & $\begin{array}{l}3233 \\
3247 \\
3248+\end{array}$ \\
\hline $\begin{array}{l}{ }^{88} \mathrm{Sr} \\
{ }^{87} \mathrm{Rb}\end{array}$ & $\begin{array}{c}\text { rolative } \\
\text { a }\end{array}$ & $\begin{array}{l}I_{1} \\
3.0+1=\end{array}$ & $5.5+1$ & CNE E & Davidsont & 8024 \\
\hline${ }^{\theta \theta} \mathrm{Sr}$ & cumul ot I & Irefigs & sion $y$ & yield & & \\
\hline $\begin{array}{l}232 \mathrm{Th} \\
233 \mathrm{U} \\
237_{\mathrm{Np}}\end{array}$ & $\begin{array}{l}d \\
d \\
d\end{array}$ & $\begin{array}{l}9.3+0 \\
9.0+0 \\
2.0+1\end{array}$ & $\begin{array}{l}1.4+1 \\
2 \cdot 3+1 \\
4.1+1\end{array}$ & $\begin{array}{l}\text { CCP } R \\
\text { CCP } R \\
\text { CCP } R\end{array}$ & $\begin{array}{l}\text { Lbout } \\
\text { Lbout } \\
\text { Lbout }\end{array}$ & $\begin{array}{l}5921 \\
5936 \\
9091\end{array}$ \\
\hline${ }^{89} \mathrm{Sr}$ & cumulat 1 & IVe ole & & & & \\
\hline $\begin{array}{l}23 \theta^{20} \\
{ }^{89} \mathrm{Sr}\end{array}$ & $\begin{array}{c}p \\
\text { fission }\end{array}$ & $\begin{array}{l}1.0+3 \\
y 101 d=\end{array}$ & $\begin{aligned} & 3.0+5 \\
= & \text { foctor }\end{aligned}$ & KFK C & Muenze:t & 4903 \\
\hline $248 \mathrm{Cm}$ & - & $2.4+1$ & $3.4+1$ & KUR E & Lisint & 9117 \\
\hline $91 \mathrm{sr}$ & Independ & dent 11 & ission & $y / 01 d$ & & \\
\hline $\begin{array}{l}209 \mathrm{Bi} \\
8 \mathrm{I} \mathrm{Sr}\end{array}$ & 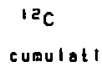 & $\begin{array}{l}7.5+1 \\
\text { Ive fis }\end{array}$ & $\begin{array}{l}1.0+2 \mid \\
\text { sion } y\end{array}$ & $\begin{array}{l}\text { HAR M } \\
\text { yiold }\end{array}$ & Branquintiot & 10152 \\
\hline $\begin{array}{l}232 \mathrm{Th} \\
233 \mathrm{U} \\
237_{\mathrm{NP}}\end{array}$ & $\begin{array}{l}9 \\
d \\
0\end{array}$ & $\begin{array}{l}9.3+0 \\
9.0+0 \\
2.0+1\end{array}$ & $\begin{array}{l}1.4+1 \\
2.3+1 \\
4.1+1\end{array}$ & $\begin{array}{l}\text { CCP R } \\
\text { CCP R } \\
\text { CCP R }\end{array}$ & $\begin{array}{l}\text { Lbovt } \\
\text { Lbovt } \\
\text { Lbovt }\end{array}$ & $\begin{array}{l}5921 \\
5936 \\
9091\end{array}$ \\
\hline $915 r$ & cumulall & Ive otE & & & & \\
\hline $238 \mathrm{U}$ & $p$ & $1.0+3$ & $3.0+5$ & $\mathrm{KFK} \mathrm{C}$ & Muenzelt & 4903 \\
\hline $915 r$ & flesion & $y i e l d x$ & afoctor & & & \\
\hline $\begin{array}{l}248 \mathrm{Cm} \\
815 r\end{array}$ & of(E) A fac & $\begin{array}{l}2.4+1 \\
\text { etor }\end{array}$ & $3.4+1$ & KUR E & LIsInt & 9117 \\
\hline $\begin{array}{l}232 \mathrm{Th} \\
\text { gisr }\end{array}$ & $\begin{array}{r}p \\
O(E)\end{array}$ & $1.0+1$ & $2.2+1$ & TOK E & Kudot & 4790 \\
\hline $\begin{array}{l}188 \mathrm{Hg} \\
199 \mathrm{Hg} \\
200 \mathrm{Hg} \\
201 \mathrm{Hg}^{2}\end{array}$ & $\begin{array}{l}a \\
a \\
0 \\
0\end{array}$ & $\begin{array}{l}4.1+1 \\
4.1+1 \\
4.1+1 \\
4.1+1\end{array}$ & $\begin{array}{l}6.5+1 \\
6.5+1\end{array}$ & $\begin{array}{l}\text { WWS E } \\
\text { WWS E } \\
\text { WWS E } \\
\text { WWS E }\end{array}$ & $\begin{array}{l}\text { Neuzil } \\
\text { Neuzil. } \\
\text { Neuzill. } \\
\text { Neuzil. }\end{array}$ & $\begin{array}{l}8801 \\
8806 \\
8811 \\
8815\end{array}$ \\
\hline
\end{tabular}




\begin{tabular}{|c|c|c|c|c|c|c|}
\hline Target & $\ln c$ & $\begin{array}{l}\text { Emin } \\
(M \otimes V)\end{array}$ & $\begin{array}{l}E \operatorname{mex} \\
(M e V)\end{array}$ & Lab & Author & No. \\
\hline $915 r$ & $\sigma(E)$ & & & & & \\
\hline $\begin{array}{l}20{ }^{2} \mathrm{Hg} \\
204 \mathrm{Hg}\end{array}$ & : & $\begin{array}{l}4.1+1 \\
4.1+1\end{array}$ & & $\begin{array}{l}\text { WWS } \\
\text { WWS }\end{array}$ & $\begin{array}{l}\text { E Neuz:ll. } \\
\text { E Neuzil. }\end{array}$ & $\begin{array}{l}8822 \\
8829\end{array}$ \\
\hline $92 \mathrm{Sr}$ & indepen & ndent it & isslon & $y 101$ & & \\
\hline $\begin{array}{l}209 \mathrm{Br} \\
92 \mathrm{Sr}\end{array}$ & $\begin{array}{l}{ }^{12} C \\
O(E)\end{array}$ & $7.5+1$ & $1.0+2$ & HAR & M Branquinhot & 10152 \\
\hline $\begin{array}{l}198_{\mathrm{Hg}} \\
199_{\mathrm{Hg}} \\
200 \mathrm{Hg} \\
201 \mathrm{Hg} \\
20{ }^{2} \mathrm{Hg} \\
204 \mathrm{Hg}\end{array}$ & $\begin{array}{l}a \\
a \\
a \\
\dot{a} \\
\dot{a} \\
\dot{a}\end{array}$ & $\begin{array}{l}4.1+1 \\
4.1+1 \\
4.1+1 \\
4.1+1 \\
4.1+1 \\
4.1+1\end{array}$ & $\begin{array}{l}6.5+1 \\
6.5+1 \\
\end{array}$ & $\begin{array}{l}\text { WWS } \\
\text { WWS } \\
\text { WWS } \\
\text { WWS } \\
\text { WWS } \\
\text { WWS }\end{array}$ & $\begin{array}{l}\text { E Neuzil. } \\
\text { E Neuzil. } \\
\text { E Neuzil. } \\
\text { E Neuzzi. } \\
\text { E Neuzil. } \\
\text { E Neuzil. }\end{array}$ & $\begin{array}{l}8801 \\
8806 \\
8811 \\
8815 \\
8822 \\
8829\end{array}$ \\
\hline$z=39$ & $v_{n}$ & & & & & \\
\hline${ }^{80 Y} Y^{A g}$. & $\begin{array}{l}40 \mathrm{Ar} \\
\text { O(E) }\end{array}$ & $3.4+2$ & & PAR & R Lefort+ & 12202 \\
\hline $\begin{array}{l}\mathrm{NI} \\
\mathrm{NI}\end{array}$ & $\begin{array}{l}24 \mathrm{Mg} \\
2 \theta_{\mathrm{Si}}\end{array}$ & $\begin{array}{l}9.5+1 \\
9.5+1\end{array}$ & & $\begin{array}{l}\text { BNL } \\
\text { ENL }\end{array}$ & $\begin{array}{l}\text { E Listert } \\
\text { E Listert }\end{array}$ & $\begin{array}{l}11950 \\
11974\end{array}$ \\
\hline${ }^{\theta 2}{ }^{B n}{ }^{1}$ & ${ }^{\mathrm{I}}{ }_{2 \mathrm{O}_{\mathrm{Ne}}}$ & NEG & & MOL & E Marolt & 11853 \\
\hline${ }^{83} Y$ & thich to & argat yi & ield & & & \\
\hline${ }^{86} \mathrm{Sr}$ & $\begin{array}{c}p \\
\text { partlat }\end{array}$ & $\begin{array}{l}5.5+1 \\
0(\varepsilon)\end{array}$ & & GRN & E Bukshpant & 3251 \\
\hline $\begin{array}{l}{ }^{80} S_{r} \\
0 \theta_{S} \\
09_{Y} \\
\log _{Y}\end{array}$ & $\begin{array}{l}p \\
p \\
p \\
p\end{array}$ & $\begin{array}{l}6.0+1 \\
6.0+1 \\
6.6+1 \\
6.6+1\end{array}$ & $\begin{array}{l}8.5+1 \\
8.5+1 \\
8.5+1 \\
8.5+1\end{array}$ & $\begin{array}{l}\text { KFK } \\
\text { MCG } \\
\text { KFK } \\
\text { MCG }\end{array}$ & $\begin{array}{l}\text { C Muenzel+ } \\
\text { E Sachdevt } \\
\text { C Muenzelt } \\
\text { M Sahat }\end{array}$ & $\begin{array}{l}3277 \\
3278+ \\
3315 \\
3316+\end{array}$ \\
\hline $84 Y$ & $O(E) \times f a c$ & ctor & & & & \\
\hline${ }^{93^{8}}{ }^{86} \quad 0$ & O(E) & $9.0+1$ & & BNL & T Divadeenam. & 3532 \\
\hline $\begin{array}{l}{ }^{88} \mathrm{Sr} \\
89 \mathrm{Y} \\
90 \mathrm{Zr}\end{array}$ & $\begin{array}{l}p \\
p \\
p\end{array}$ & $\begin{array}{l}5.0+1 \\
6.0+1 \\
4.5+1\end{array}$ & $\begin{array}{l}9.0+1 \\
1.0+2 \\
8 \cdot 0+1\end{array}$ & $\begin{array}{l}\text { MIL } \\
\text { MIL } \\
\text { BNL }\end{array}$ & $\begin{array}{l}\text { T Gadiolit } \\
\text { T Gadiolit } \\
\text { T Oivadeenam. }\end{array}$ & $\begin{array}{l}3276 \\
3314 \\
3439\end{array}$ \\
\hline
\end{tabular}

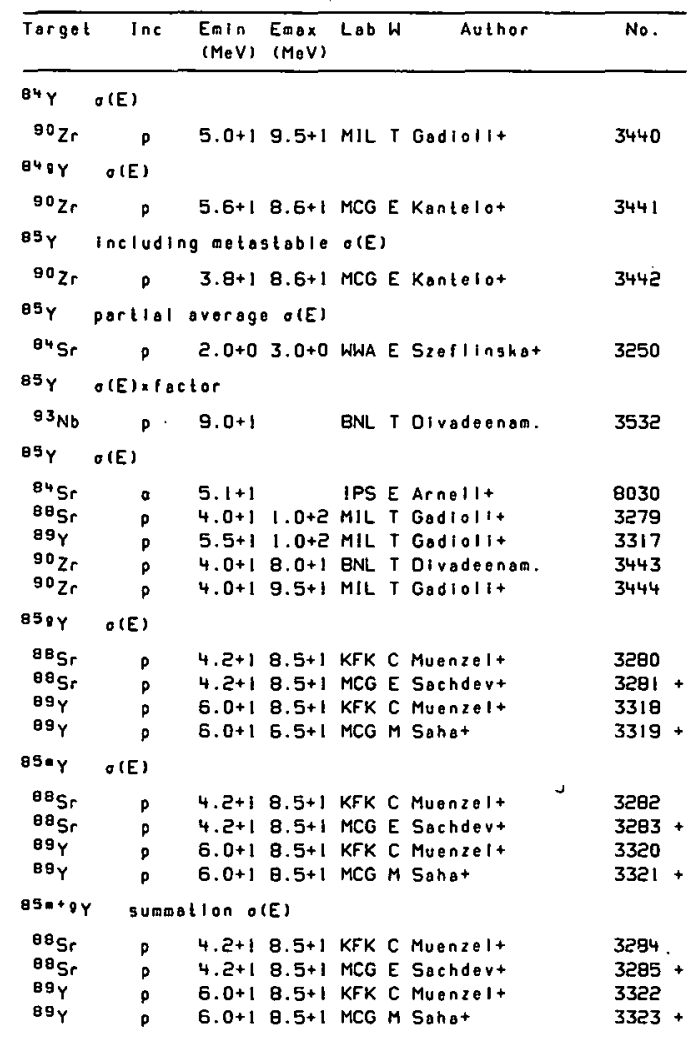




\begin{tabular}{|c|c|c|c|c|c|c|}
\hline Target & $\ln 6$ & $\begin{array}{l}\text { Em } 1 n \\
(\mathrm{MeV})\end{array}$ & $\begin{array}{l}\text { Emax } \\
(\mathrm{MeV})\end{array}$ & Lab W & futhor & No. \\
\hline
\end{tabular}

Q6019Y rallo O(E)

$\begin{array}{llll}\text { 99Y } & p & 4.5+18.5+1 \text { KFK C Muenze1+ } & 3333 \\ \text { B9Y } & P & 4.5+18.5+1 \text { MCG M Sahat } & 3334\end{array}$

87y including metestable $\sigma(E)$

$90 \mathrm{Zr} \quad 1.2+18.6+1$ MCG E Kantelo+ 3449

07y partial average o(E)

${ }^{86} 5$ r $P$ 2.0+0 3.0+0 WWA E Szellingkat 3252

Q7y relative 1 ,

${ }^{87} \mathrm{Rb}$ a $3.0+15.5+1$ CNE E Davidsont 8025

Q7y product yield

$90 \mathrm{Zr} P 3.5+1$ ZUR E Guyert 3453

Q7y thick target yieldabundance

85Rb a $3.2+1$ AML R Choudil. 8022

87y thlak torget yleld

${ }^{87} \mathrm{Sr} P \quad 2.6+0$ 5.7+0 CLU M Rough:orit 3253

Q7y O(E)infoctor

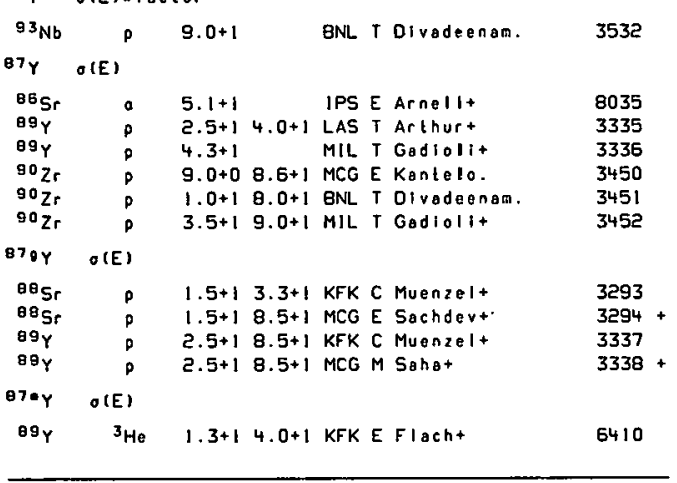




\begin{tabular}{|c|c|c|c|c|c|c|}
\hline Target & $\operatorname{lnc}$ & $\begin{array}{l}E m i n \\
(r e v)\end{array}$ & $\begin{array}{l}\text { Emax } \\
\text { (MeV) }\end{array}$ & Lab & Author & No. \\
\hline \multicolumn{7}{|c|}{ B7・Y O(E) } \\
\hline $\begin{array}{l}{ }^{8 \theta} \theta_{S r} \\
\theta_{S r} \\
\theta_{S} 9_{Y} \\
\theta_{Y} \\
\theta_{Y}\end{array}$ & $\begin{array}{c}p \\
p \\
p \\
p \\
p \\
3 \text { He }\end{array}$ & $\begin{array}{l}1.5+1 \\
1.5+1 \\
2.5+1 \\
2.5+1 \\
1.5+1\end{array}$ & $\begin{array}{l}3 \cdot 3+1 \\
8 \cdot 5+1 \\
8 \cdot 5+1 \\
8 \cdot 5+1 \\
4 \cdot 0+1\end{array}$ & $\begin{array}{l}\text { KFK } \\
\text { MCG } \\
\text { KFK } \\
\text { MCG } \\
\text { KFK }\end{array}$ & $\begin{array}{l}\text { C Muenzel+ } \\
\text { E Sachdert } \\
\text { C Muenzel+ } \\
\text { M Sahat } \\
\text { E Flacht }\end{array}$ & $\begin{array}{l}3295 \\
3295+ \\
3339 \\
3340+ \\
6409\end{array}$ \\
\hline \multicolumn{7}{|l|}{$87 \cdot 99$} \\
\hline $\begin{array}{l}{ }^{80} \mathrm{Sr} \\
{ }^{80} \mathrm{Sr} \\
{ }^{89 Y} \\
{ }^{89 Y}\end{array}$ & $\begin{array}{l}p \\
p \\
p \\
p\end{array}$ & $\begin{array}{l}\text { TR } \\
1.5+1 \\
2.5+1 \\
2.5+1\end{array}$ & $\begin{array}{l}8 \cdot 5+1 \\
3 \cdot 3+1 \\
8 \cdot 5+1 \\
8 \cdot 5+1\end{array}$ & $\begin{array}{l}\text { MCG } \\
\text { KFK } \\
\text { KFK } \\
\text { MCG }\end{array}$ & $\begin{array}{l}\text { E Sachdevt } \\
\text { C Muenzel+ } \\
\text { C Muenzelt } \\
\text { M Sahat }\end{array}$ & $\begin{array}{l}3297+ \\
3298 \\
3341 \\
3342+\end{array}$ \\
\hline \multicolumn{7}{|c|}{ at 10 OLE) } \\
\hline $\begin{array}{l}{ }^{89} Y \\
89 Y\end{array}$ & $p$ & $\begin{array}{l}2.5+1 \\
2.5+1\end{array}$ & $\begin{array}{l}8.5+1 \\
8.5+1\end{array}$ & $\begin{array}{l}\text { KFK } \\
\text { MCG }\end{array}$ & $\begin{array}{l}\text { C Muenzel+ } \\
\text { MSahat }\end{array}$ & $\begin{array}{l}3343 \\
3344\end{array}$ \\
\hline \multicolumn{7}{|c|}{ glativo l, } \\
\hline${ }^{87} \mathrm{Rb}$ & 。 & $3.0+1$ & $5.5+1$ & CNE & E Davidsont & 8026 \\
\hline \multicolumn{7}{|l|}{$88 Y$} \\
\hline $\begin{array}{l}87 \mathrm{Sr} \\
80 \mathrm{Sr}\end{array}$ & $\begin{array}{l}p \\
p\end{array}$ & $\begin{array}{l}2.6+0 \\
4.6+0\end{array}$ & $\begin{array}{l}4.3+0 \\
5.7+0\end{array}$ & $\begin{array}{l}\mathrm{CLU} \\
\mathrm{CLU}\end{array}$ & $\begin{array}{l}\text { M Roughtont } \\
\text { M Roughtont. }\end{array}$ & $\begin{array}{l}3254 \\
3304\end{array}$ \\
\hline \multicolumn{7}{|c|}{ (E) x foctor } \\
\hline${ }^{93} \mathrm{Nb}$ & 0 & c. $0+1$ & & BNL & T Divadeenam. & 3532 \\
\hline \multicolumn{7}{|l|}{$88_{Y}$} \\
\hline $885 r$ & D & +0 & $5.8+0$ & $\mathrm{KTY}$ & E Sehharant & 3299 \\
\hline${ }^{8} 85$ & p & $3.0+0$ & $8.0+0$ & WIT & E Selisehopt & 3300 \\
\hline${ }^{10} \mathrm{~S} r$ & p & $7.0+0$ & $0.5+1$ & KFK & C Muenzelt & 3301 \\
\hline${ }^{80} 5 r$ & p & $7.0+0$ & $8.5+1$ & MCG & E Sachdert & $3302+$ \\
\hline${ }^{10} \mathrm{sr}$ & p & $6.9+1$ & $1.4+2$ & PAR & E Albouyt & $3303+$ \\
\hline $099_{Y}$ & p & $1.5+1$ & $8.5+1$ & KFK & C Muenzel. & 3347 \\
\hline${ }^{89}{ }_{Y}$ & p & $1.5+1$ & $8.5+1$ & MCO & $M$ Sahat & $3348+$ \\
\hline $89 y_{Y}$ & p & $: .7+1$ & $4.0+1$ & LAS & T Arthurt & 3345 \\
\hline $89 Y$ & & $3.0+1$ & $9.0+1$ & MIL & T Gadiolit & 3346 \\
\hline $89 y$ & ${ }^{3} \mathrm{He}$ & +0 & $4.0+1$ & KFK & E Flacht & 6411 \\
\hline${ }^{90} \mathrm{Zr}$ & $\rho$ & $3.0+1$ & $8.6+1$ & MCG & E kontelot & 3454 \\
\hline $90 \mathrm{zr}$ & p & $3.0+1$ & $8.0+1$ & BNL & $T$ divodecnam. & 3455 \\
\hline $90 \mathrm{Zr}$ & p & $4.0+1$ & $9.5+1$ & MIL & T Gadiolit & 3456 \\
\hline
\end{tabular}

\begin{tabular}{|c|c|c|c|c|c|}
\hline Targal & $\ln x$ & $\begin{array}{l}\text { Emin } \quad \text { Emax } \\
(\operatorname{MeV}) \quad(\operatorname{MeV})\end{array}$ & Lab & Author & No. \\
\hline$\theta_{Y}$ & partial & spectrum aver & rage & $O(E)$ & \\
\hline${ }^{89} Y$ & 0 & $2.3+1 \quad 2.7+1$ & MUU & E Eberharot & 8043 \\
\hline $\mathbf{8 9}_{\mathbf{Y}}$ & portiol & overago o(E) & & & \\
\hline${ }^{8 \theta} \mathrm{Sr}$ & p & $2.0+0 \quad 3.0+0$ & WWA & E Szeflinghat & 3305 \\
\hline${ }^{89} Y$ & portlat & relative o(E) & & & \\
\hline${ }^{87} \mathrm{Rb}$ & a & $2.4+1 \quad 3.6+1$ & CLU & EFieldst & 8028 \\
\hline${ }^{89 y}$ & relative & - $I_{y}$ & & & \\
\hline${ }^{87} \mathrm{Rb}$ & 。 & $3.0+1 \quad 5.5+1$ & CNE & E Davidsont & 8027 \\
\hline 88Y & O(E) xfac & ctor & & & \\
\hline${ }^{93} \mathrm{Nb}$ & p & $9.0+1$ & $\mathrm{BNL}$ & T Divadeenam. & 3532 \\
\hline${ }^{89} \mathrm{Y}$ & $O(E)$ & & & & \\
\hline $\begin{array}{l}{ }_{B}{ }^{5} \mathrm{Sr} \\
{ }^{8} \mathrm{Sr}\end{array}$ & $\begin{array}{l}p \\
p\end{array}$ & $\begin{array}{l}\text { NOG } \\
3.0+0\end{array}$ & $\begin{array}{l}\text { OSA } \\
\text { LRL }\end{array}$ & $\begin{array}{l}\text { E Nakayamat } \\
\text { E Oietricht }\end{array}$ & $\begin{array}{l}3308 \\
3307\end{array}$ \\
\hline $90 Y$ & cumulat I & Ive fission yl & 1010 & & \\
\hline $\begin{array}{l}232 \mathrm{Th} \\
235 \mathrm{U}\end{array}$ & $d^{d}$ & $\begin{array}{ll}9.3+0 & 1.4+1 \\
1.2+1 & 8.5+1\end{array}$ & $\begin{array}{l}\mathrm{CCP} \\
\mathrm{CCP}\end{array}$ & $\begin{array}{l}\text { R Lbove } \\
\text { R Lbove }\end{array}$ & $\begin{array}{l}5921 \\
4852\end{array}$ \\
\hline${ }^{90} \mathrm{Y}$ & otEIAf ac & etor & & & \\
\hline $\begin{array}{l}912 r \\
93_{\mathrm{Nb}}\end{array}$ & t & $\begin{array}{l}1.1+1 \quad 1.8+1 \\
9.0+1\end{array}$ & $\begin{array}{l}\text { LAS } \\
\text { BNL }\end{array}$ & $\begin{array}{l}\text { E Britt+ } \\
\text { T Divadeenam. }\end{array}$ & $\begin{array}{l}6055 \\
3532\end{array}$ \\
\hline 90Y & $O(E)$ & & & & \\
\hline $\begin{array}{l}\text { 89y } \\
\text { 80.YY }\end{array}$ & $\begin{array}{c}0 \\
\text { cumulat }\end{array}$ & $\begin{array}{l}5.0+01.5+1 \\
\text { tive fission }\end{array}$ & $\begin{array}{l}\text { xTO } \\
y 1010\end{array}$ & M Ohamurat & 5579 \\
\hline $\begin{array}{l}-40.4 \\
90.4\end{array}$ & $\begin{array}{r}P \\
O(E)\end{array}$ & $1.2+18.5+1$ & $\mathrm{CCP}$ & R Lbovt & 4852 \\
\hline $\begin{array}{l}\text { 89Y } \\
\text { 89YY }\end{array}$ & ${ }_{3}^{d} \mathrm{He}$ & $\begin{array}{ll}5.0+0 & 1.5+1 \\
1.5+1 & 4.0+1\end{array}$ & $\begin{array}{l}\text { KTO } \\
\text { KFK }\end{array}$ & $\begin{array}{l}\text { M Ohamurat } \\
\text { E Flacht }\end{array}$ & $\begin{array}{l}5580 \\
6412\end{array}$ \\
\hline B'y & cunulati & ive fission yi & 1010 & & \\
\hline $\begin{array}{l}235 T h \\
235_{\mathrm{U}} \\
237_{\mathrm{Ng}}\end{array}$ & $\begin{array}{l}\text { j } \\
\text { p } \\
\text { p }\end{array}$ & $\begin{array}{ll}9.3+0 & 1.4+1 \\
1.2+1 & 8.5+1 \\
2.0+1 & 4.1+1\end{array}$ & $\begin{array}{l}\mathrm{CCP} \\
\mathrm{CCP} \\
\mathrm{CCP}\end{array}$ & $\begin{array}{l}R \text { Lbort } \\
R \text { Lbort } \\
R \text { Lbort }\end{array}$ & $\begin{array}{l}5921 \\
4852 \\
9091\end{array}$ \\
\hline
\end{tabular}




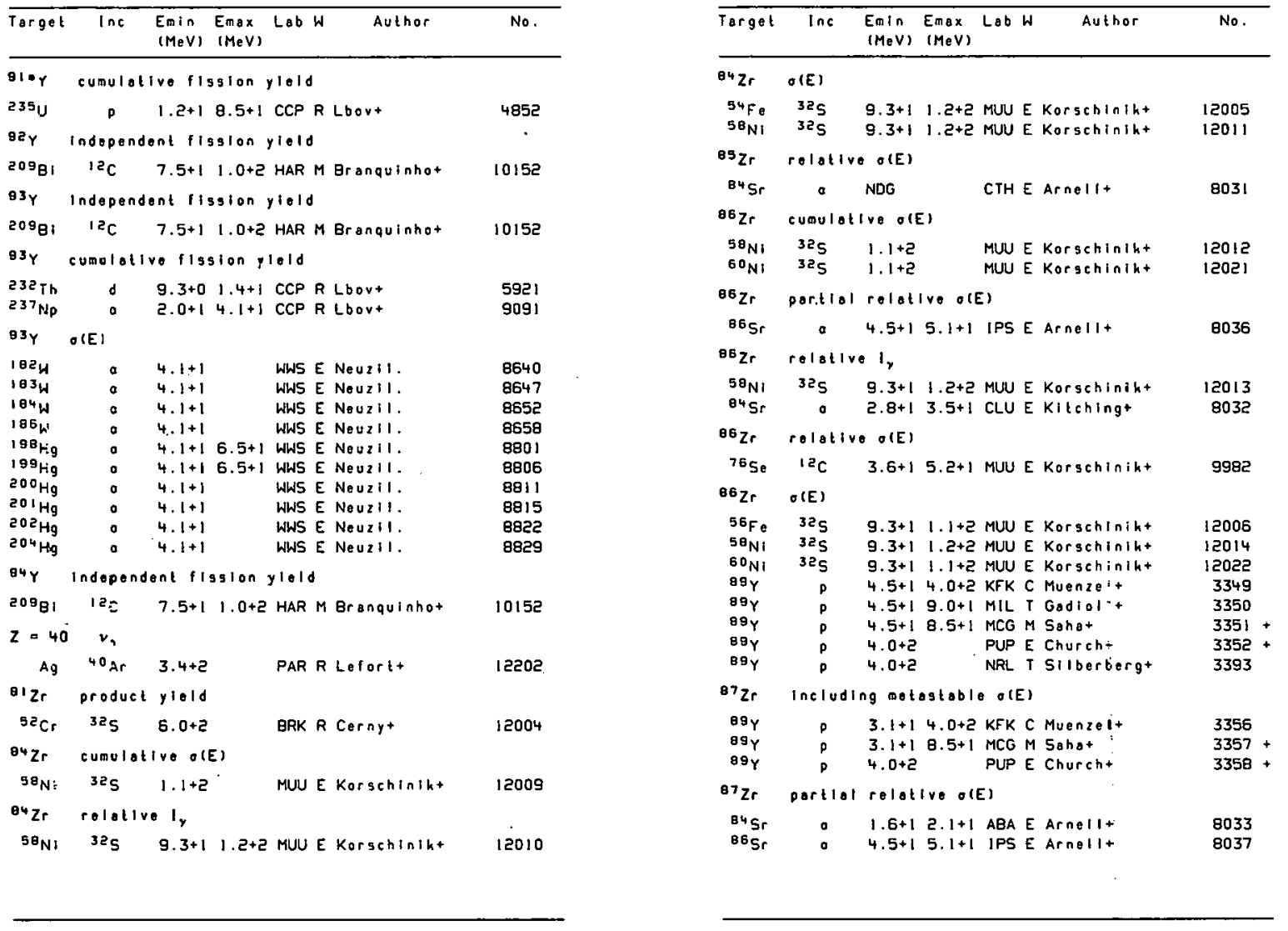




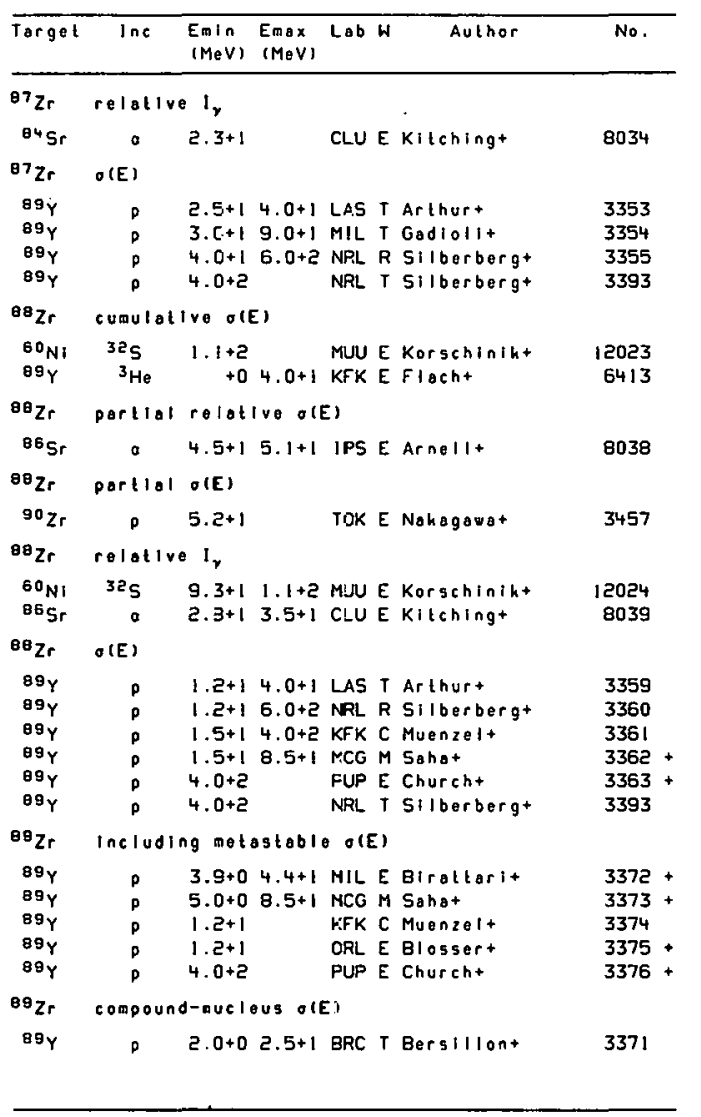

\begin{tabular}{|c|c|c|c|c|c|c|}
\hline Target & $\operatorname{lnc}$ & $\begin{array}{l}\text { Emin } \\
(M e V)\end{array}$ & $\begin{array}{l}\text { Emax } \\
\text { (MeV) }\end{array}$ & Lab $h$ & Author & No. \\
\hline${ }^{89} \mathrm{Zr}$ & $O(E)$ & & & & & \\
\hline ө9Y & ${ }^{3} \mathrm{He}$ & +0 & $4.0+1 k$ & KFK E & E Flacht & 6415 \\
\hline${ }^{89} \mathrm{Zr}$ & portlot & raw oll & (E) & & & \\
\hline${ }^{89} \mathrm{Y}$ & $p$ & $3.5+0$ & $4.8+0 \mathrm{C}$ & ORL & E Johnsont & 3379 \\
\hline${ }^{89} \mathrm{Zr}$ & partlal & relall & ive $\theta(E)$ & & & \\
\hline $\operatorname{89}_{Y}$ & $p$ & $3.5+0$ & $4.8+00$ & ORL E & E Johnsont & 3381 \\
\hline$\theta^{8} \mathrm{zr}$ & portiol & $O(E)$ & & & & \\
\hline B9 Y & $\rho$ & $1.0+1$ & $4.2+1 \mathrm{E}$ & BRC & T Bersillont & 3370 \\
\hline${ }^{89} \mathrm{Zr}$ & relative & $I_{y}$ & & & & \\
\hline $89 Y$ & ${ }^{3} \mathrm{He}$ & $2.0+1$ & $2.8+1<$ & UJV E & E Spalokt & 6414 \\
\hline${ }^{89} \mathrm{Zr}$ & relalive & $\sigma(E)$ & & & & \\
\hline 89Y & p & $1 . \theta+0$ & $5.8+00$ & ORL E & E Johnsont & 3380 \\
\hline${ }^{80} \mathrm{Zr}$ & $O(E) \times f a C$ & tor & & & & \\
\hline $\begin{array}{l}\text { 89y } \\
\text { 99y }\end{array}$ & $\begin{array}{l}p \\
p\end{array}$ & $\begin{array}{l}2.5+0 \\
3.5+0\end{array}$ & $\begin{array}{l}7.0+00 \\
6.5+0 \mathrm{C}\end{array}$ & $\begin{array}{l}\text { ORL. } \\
\text { ORL }\end{array}$ & $\begin{array}{l}\text { M Johnsont } \\
\text { E Johnsont }\end{array}$ & $\begin{array}{l}3377 \\
3378\end{array}$ \\
\hline${ }^{80} \mathrm{Zr}$ & $O(E)$ & & & & & \\
\hline $\begin{array}{l}\text { 89Y } \\
\text { 99Y }\end{array}$ & $\begin{array}{l}p \\
p\end{array}$ & $\begin{array}{l}2.0+0 \\
3.6+0\end{array}$ & $\begin{array}{l}4.2+1 \mathrm{E} \\
5.8+0 \mathrm{C}\end{array}$ & $\begin{array}{l}\text { BRC } \\
\text { ORL }\end{array}$ & $\begin{array}{l}\text { R Bersillont } \\
\text { E Johnsont }\end{array}$ & $\begin{array}{l}3364 \\
3365\end{array}$ \\
\hline $\mathrm{BS}^{\mathrm{B}}$ & $p$ & $4.0+0$ & $2.0+14$ & LAS T & T Arthur. & 3366 \\
\hline${ }^{89}{ }^{8}$ & p & $4.0+0$ & $6.0+21$ & NRL F & R Sllberbergt & 3367 \\
\hline $\begin{array}{l}\theta_{Y} \\
\theta_{Y}\end{array}$ & $\begin{array}{c}p \\
p \\
p\end{array}$ & $\begin{array}{l}0.0+0 \\
1.0+1\end{array}$ & $\begin{array}{l}1.1+10 \\
8 \cdot 0+1\end{array}$ & CCP F & $\begin{array}{l}\text { R Zalolohint. } \\
\text { T Gadlolit }\end{array}$ & $\begin{array}{l}3368 \\
3369\end{array}$ \\
\hline${ }^{89} Y$ & $p$ & $4.0+2$ & & NRL & T Silberbergt & 3393 \\
\hline 89. $\mathrm{zr}$ & $\sigma(E)$ & & & & & \\
\hline $\begin{array}{l}89_{Y} \\
899_{Y}\end{array}$ & p & $\begin{array}{l}1.2+1 \\
1.2+1\end{array}$ & & $\begin{array}{l}\text { KFK } \\
\text { ORL }\end{array}$ & $\begin{array}{l}\text { C Muenzelt } \\
\text { E Blossert }\end{array}$ & $\begin{array}{l}3382 \\
3383-\end{array}$ \\
\hline $98 \cdots+9 z$ & suma & ation $\sigma$ & $\sigma(E)$ & & & \\
\hline${ }^{89} \mathrm{Y}$ & p & $7.0+0$ & 8.1+0 L & LRL E & E Albert. & $3384+$ \\
\hline${ }^{80} \mathrm{Zr}$ & port|s| & $O(E)$ & & & & \\
\hline $90 \mathrm{Zr}$ & ${ }^{3} \mathrm{He}$ & $7.0+1$ & $9.0+1 c$ & OSA E & E Matsuokat & 6423 \\
\hline
\end{tabular}




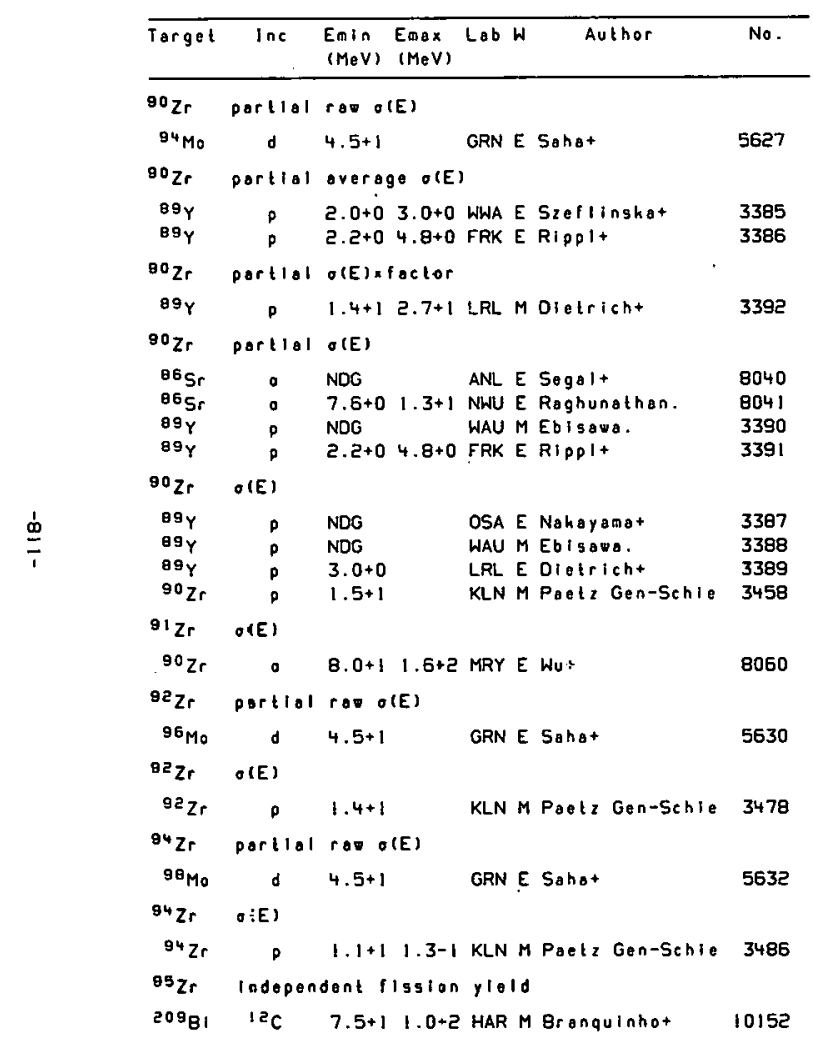

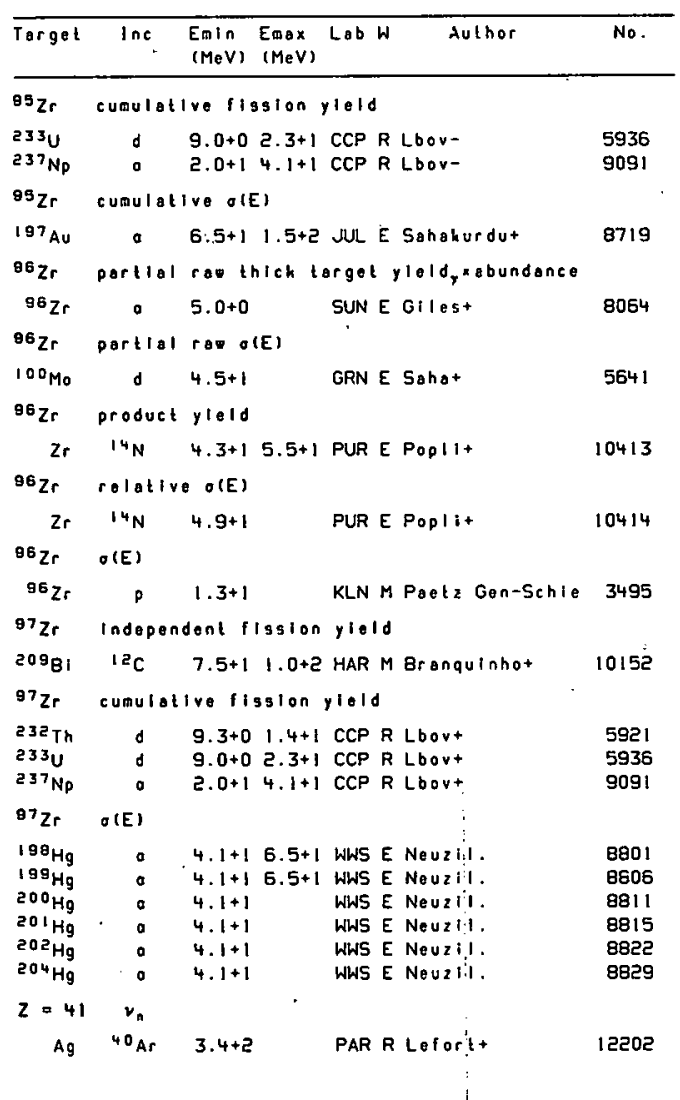




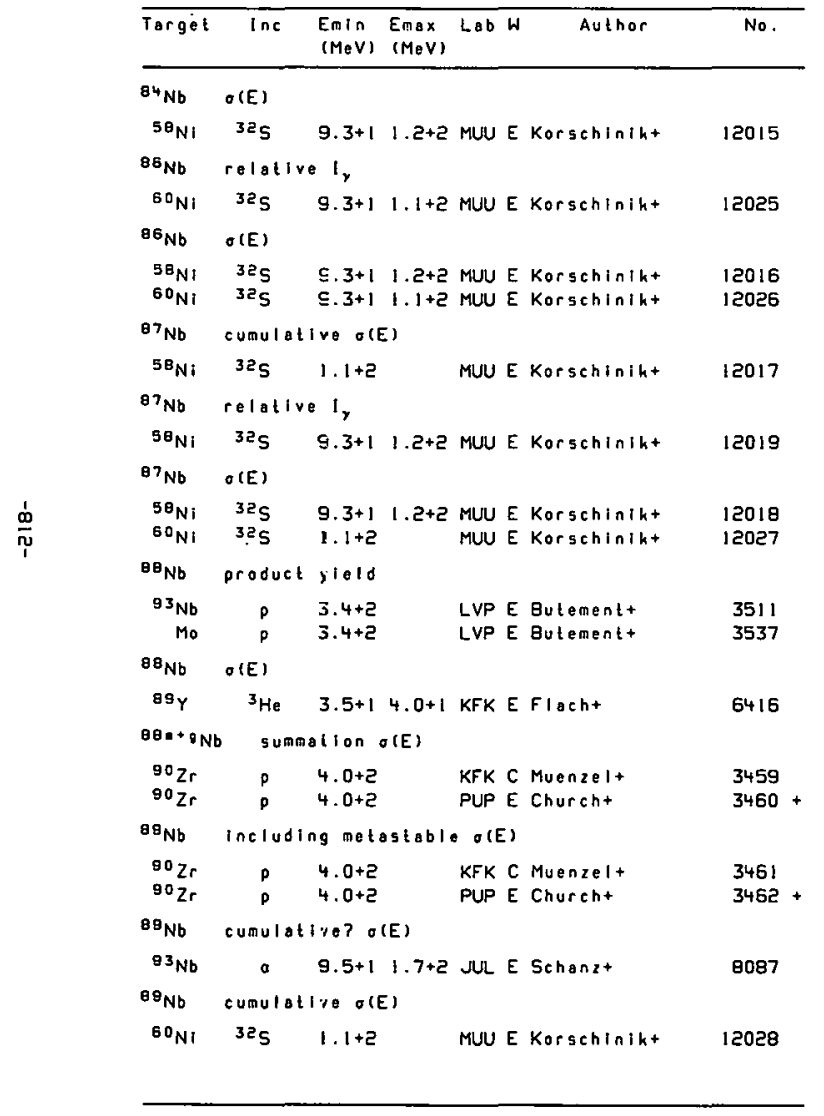

\begin{tabular}{|c|c|c|c|c|c|c|}
\hline Torget & $\ln c$ & $\begin{array}{l}\text { Emin } \\
(\mathrm{MeV})\end{array}$ & $\begin{array}{l}\text { Emax } \\
(\mathrm{MeV})\end{array}$ & Lab & Author & No. \\
\hline${ }^{89_{\mathrm{Nb}}}$ & \multicolumn{6}{|c|}{ relative $\mathrm{I}_{y}$} \\
\hline${ }^{89} \mathrm{Y}$ & ${ }^{3} \mathrm{He}$ & $2.0+1$ & $2.8+1$ & usv & E Spaleht & 6417 \\
\hline${ }^{8} \mathrm{Nb}$ & \multicolumn{6}{|l|}{$O(E)$} \\
\hline $\begin{array}{l}99 \mathrm{Y} \\
90 \mathrm{Zr}\end{array}$ & $\stackrel{3}{3} \mathrm{He}$ & $\begin{array}{l}2.0+1 \\
3 \cdot 0+1\end{array}$ & $\begin{array}{l}4.0+1 \\
1.0+2\end{array}$ & $\begin{array}{l}\text { KFK } \\
\text { MIL }\end{array}$ & $\begin{array}{l}\text { E Flacht } \\
\text { T Godiolit }\end{array}$ & $\begin{array}{l}6418 \\
3463\end{array}$ \\
\hline $89^{9} \mathrm{Nb}$ & \multicolumn{6}{|l|}{$\theta(E)$} \\
\hline${ }^{93} \mathrm{Nb}$ & 0 & $6.4+1$ & $7.2+1$ & MAN & E Eranquinhot & 8088 \\
\hline$\theta \theta^{\circ} \mathrm{Nb}$ & \multicolumn{6}{|c|}{ product yleld } \\
\hline $\begin{array}{r}93 \mathrm{Nb} \\
\mathrm{Mo}\end{array}$ & p & $\begin{array}{l}3.4+2 \\
3.4+2\end{array}$ & & $\begin{array}{l}\text { LVP } \\
\text { LVP }\end{array}$ & $\begin{array}{l}\text { E Butement+ } \\
\text { E Butement+ }\end{array}$ & $\begin{array}{l}3512 \\
3538\end{array}$ \\
\hline $89^{9} \mathrm{Nb}$ & \multicolumn{6}{|l|}{$O(E)$} \\
\hline $90 \mathrm{Zr}$ & d & $2.0+1$ & $2.7+1$ & CNE & E Ushert & 5601 \\
\hline $89 . \mathrm{Nb}$ & \multicolumn{6}{|l|}{$\theta(E)$} \\
\hline $93_{\mathrm{Nb}}$ & $a$ & $6.4+1$ & $7.2+1$ & MAN & E Eranquinhot & 8089 \\
\hline $89 \bullet^{\mathrm{Nb}}$ & \multicolumn{6}{|c|}{ product yield } \\
\hline $\begin{array}{r}93_{\mathrm{Nb}} \\
\mathrm{Mo}\end{array}$ & $p$ & $\begin{array}{l}3.4+2 \\
3.4+2\end{array}$ & & $\begin{array}{l}\text { LVP } \\
\text { LVP }\end{array}$ & $\begin{array}{l}\text { E Butement+ } \\
\text { E Butement+ }\end{array}$ & $\begin{array}{l}3513 \\
3539\end{array}$ \\
\hline $89 . \mathrm{Nb}$ & \multicolumn{6}{|l|}{$O(E)$} \\
\hline $90 \mathrm{Zr}$ & d & $2 \cdot 3+1$ & $2.7+1$ & CNE & E Ushert & 5602 \\
\hline$\theta 9 n+9 \mathrm{Nb}$ & \multicolumn{6}{|c|}{$\theta(E)$} \\
\hline${ }^{93} \mathrm{Nb}$ & a & $5 \div 4+1$ & $7 \cdot 2+1$ & MAN & E Branquinhot & 8090 \\
\hline $89=19 \mathrm{Nb}$ & \multicolumn{6}{|c|}{ rat10 o(E) } \\
\hline $90 \mathrm{Zr}$ & d & $2.3+1$ & $2.7+1$ & CNE & E Ushert & 5603 \\
\hline${ }^{80} \mathrm{Nb}$ & \multicolumn{6}{|c|}{ including melastable thick target yield } \\
\hline $\begin{array}{r}M_{0} \\
92_{M 0}\end{array}$ & d & $\begin{array}{l}3.4+0 \\
1.2+1\end{array}$ & $\begin{array}{l}1.3+1 \\
1.3+1\end{array}$ & UJV & $\begin{array}{l}\text { E Ronda- } \\
\text { E Rondat }\end{array}$ & $\begin{array}{l}5609+ \\
5626+\end{array}$ \\
\hline${ }^{90} \mathrm{Nb}$ & \multicolumn{6}{|c|}{ Including metastable $O(E)$} \\
\hline $\begin{array}{l}89_{Y} \\
90 \mathrm{Zr} \\
90 \mathrm{Zr}\end{array}$ & $\begin{array}{l}{ }^{3} \mathrm{He} . \\
\mathrm{p} \\
\mathrm{p}\end{array}$ & $\begin{array}{l}1.5+1 \\
1.2+1 \\
1.2+1\end{array}$ & $4.0+1$ & $\begin{array}{l}\text { KFK } \\
\text { KFK } \\
\text { ORL }\end{array}$ & $\begin{array}{l}\text { E Flacht } \\
\text { C Muenzelt } \\
\text { E Blosgert }\end{array}$ & $\begin{array}{l}6420 \\
3465 \\
3466+\end{array}$ \\
\hline
\end{tabular}




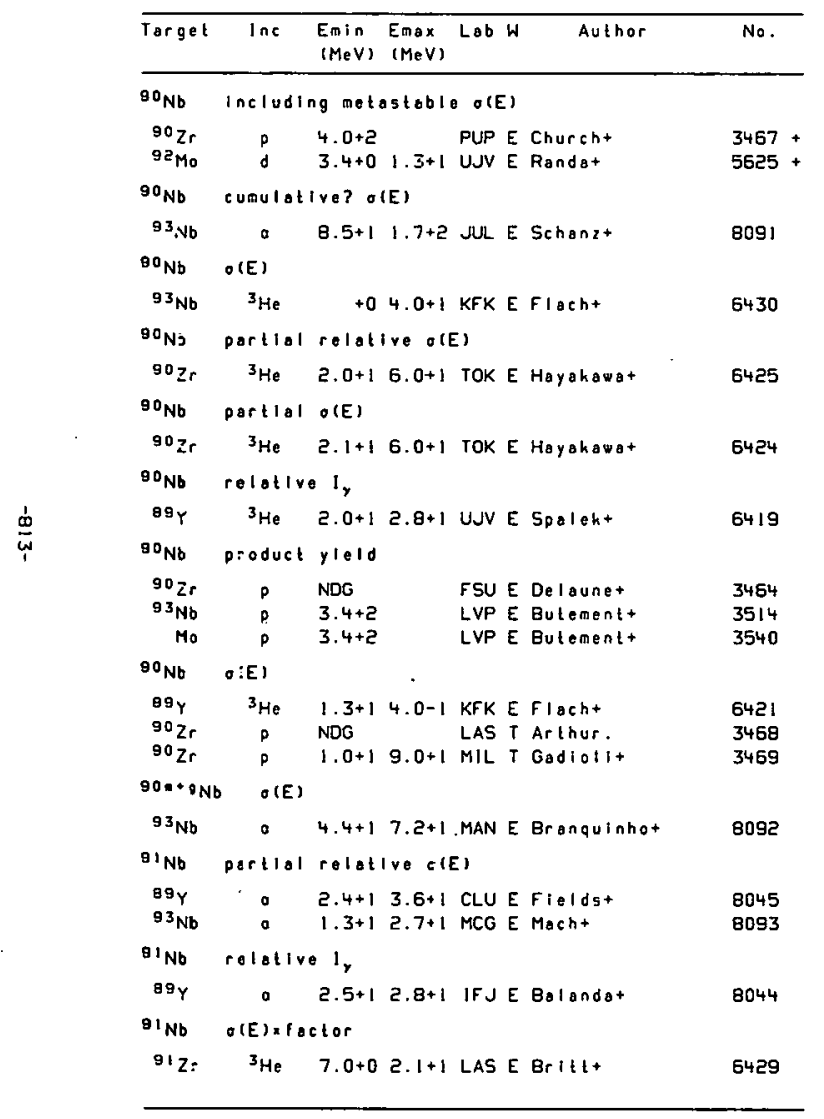

\begin{tabular}{lcccc}
\hline Target & Inc & $\begin{array}{l}\text { Emin } \\
\text { (MeV) }\end{array}$ \\
(MeV)
\end{tabular}




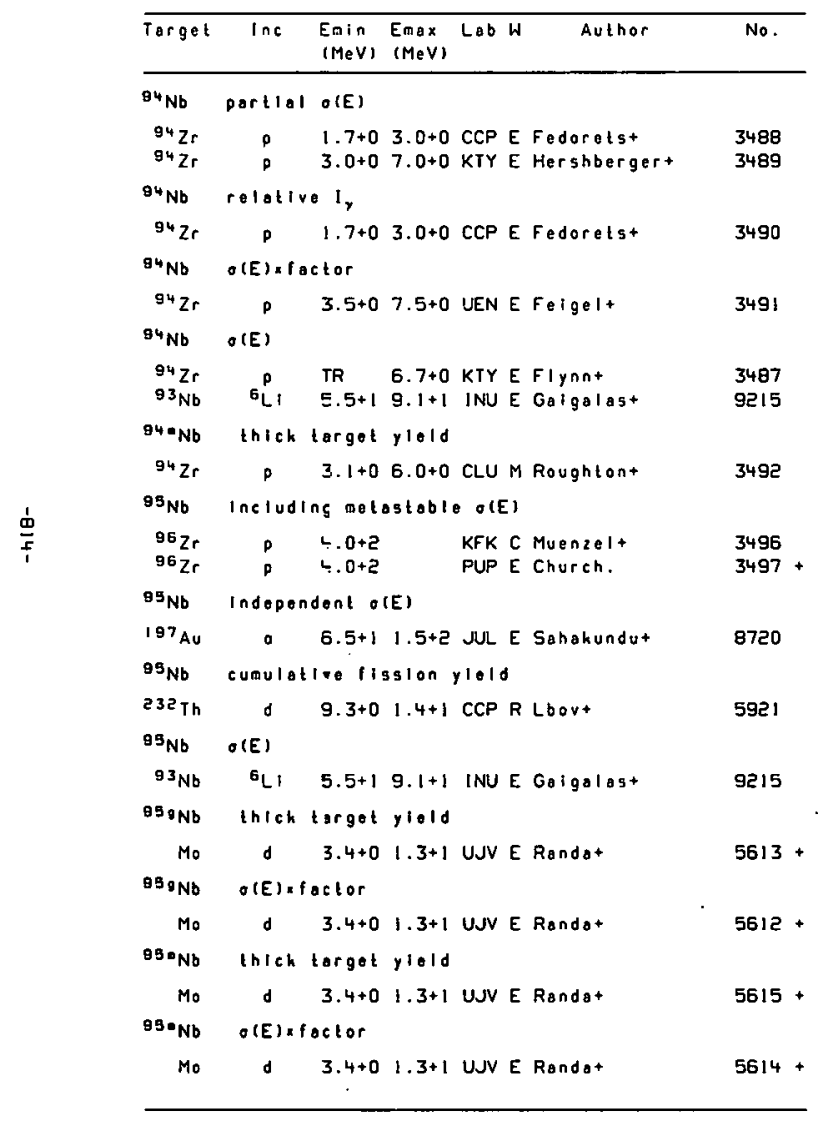

\begin{tabular}{|c|c|c|c|c|c|c|}
\hline Target & Inc & $\begin{array}{l}\text { Emin } \\
\text { (MeV) }\end{array}$ & $\begin{array}{l}\text { Emax } \\
(\operatorname{MeV})\end{array}$ & Lob $\mathrm{h}$ & Author & No. \\
\hline $95 \cdot \mathrm{Nb}$ & $O(E)$ & & & 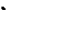 & & \\
\hline $\begin{array}{l}96 Z r \\
96 Z r\end{array}$ & p & $\begin{array}{l}4.0+2 \\
4.0+2\end{array}$ & & $\begin{array}{l}\text { KFK C } \\
\text { PUP E }\end{array}$ & $\begin{array}{l}\text { C Muenzel+ } \\
\text { E Church. }\end{array}$ & $\begin{array}{l}3498 \\
3499+\end{array}$ \\
\hline $95 \cdot+9 \mathrm{Nb}$ & sum & nationo & $O(E)$ & & & \\
\hline${ }^{93} \mathrm{Nb}$ & $\circ$ & $3.4+1$ & $4.4+1$ & MAN E & E Branquinhot & 8095 \\
\hline $95 . / 9 \mathrm{Nb}$ & rat & $100(E)$ & & & & \\
\hline $\begin{array}{l}96 \mathrm{Zr} \\
235 \mathrm{U}\end{array}$ & p & $\begin{array}{l}4.0+2 \\
1.0+1\end{array}$ & & $\begin{array}{l}\text { PUP E } \\
\text { LAS T }\end{array}$ & $\begin{array}{l}\text { E Church. } \\
T \text { Madiandt }\end{array}$ & $\begin{array}{l}3500 \\
4846\end{array}$ \\
\hline $96 \mathrm{Nb}$ & Indepe & dent II & ission & $y 1010$ & & \\
\hline $\begin{array}{l}209^{\circ i} \\
96 \mathrm{Nb}\end{array}$ & $\begin{array}{l}12 \mathrm{C} \\
\text { cumula }\end{array}$ & $\begin{array}{l}7.5+1 \\
\text { ive ils }\end{array}$ & $\begin{array}{l}1.0+2 \\
\text { ssion } y\end{array}$ & $\begin{array}{l}\text { HAR M } \\
y / 01 d\end{array}$ & M Branquinhot & 10152 \\
\hline $\begin{array}{l}232 T \mathrm{~h} \\
237 \mathrm{~Np}\end{array}$ & g & $\begin{array}{l}9.3+0 \\
2.0+1\end{array}$ & $\begin{array}{l}1.4+1 \\
4.1+1\end{array}$ & $\begin{array}{l}\text { CCP R } \\
\text { CCP R }\end{array}$ & $\begin{array}{l}R \text { Lbovt } \\
R \text { Lbout }\end{array}$ & $\begin{array}{l}5921 \\
\text { s091 }\end{array}$ \\
\hline $96 \mathrm{Nb}$ & thich & target y & $y 1010$ & & & \\
\hline $\begin{array}{l}96 \mathrm{Zr} \\
\mathrm{Mo}_{0}\end{array}$ & $\begin{array}{l}\text { d } \\
\text { p }\end{array}$ & $\begin{array}{l}3.1+0 \\
3.4+0\end{array}$ & $\begin{array}{l}6.0+0 \\
1.3+1\end{array}$ & $\begin{array}{l}\text { CLU M } \\
\text { UJV E }\end{array}$ & $\begin{array}{l}\text { M Roughtont } \\
\text { E Rondat }\end{array}$ & $\begin{array}{l}3505 \\
5616+\end{array}$ \\
\hline${ }^{86} \mathrm{Nb}$ & $\theta(E) \times 1$ & actor & & & & \\
\hline $\begin{array}{l}{ }^{96} \mathrm{Zr} \\
96 \mathrm{Nb}\end{array}$ & $\begin{array}{r}P \\
O(E)\end{array}$ & $3.5+0$ & $7.5+0$ & UEN E & E Felgel+ & 3504 \\
\hline $\begin{array}{l}96 \mathrm{Zr} \\
96 \mathrm{Zr} \\
96 \mathrm{Zr} \\
93 \mathrm{Nb} \\
9 \theta_{\mathrm{Mo}} \\
90_{\mathrm{Mo}}\end{array}$ & $\begin{array}{c}p \\
p \\
p \\
G L i \\
d \\
d\end{array}$ & $\begin{array}{l}3 \cdot 0+0 \\
4.0+2 \\
4.0+2 \\
5.5+1 \\
3.4+0 \\
6.0+0\end{array}$ & $\begin{array}{l}8.0+0 \\
9.1+1 \\
1.3+1 \\
1.2+1\end{array}$ & $\begin{array}{l}\text { WITE } \\
\text { XFK C } \\
\text { PUP E } \\
\text { INU E } \\
\text { UJV E } \\
\text { LPI E }\end{array}$ & $\begin{array}{l}\text { E Seligehopt } \\
\text { C Muenzelt } \\
\text { E Churcht } \\
\text { E Galgalast } \\
\text { E Randat } \\
\text { E Zarubint }\end{array}$ & $\begin{array}{l}3501 \\
3502 \\
3503+ \\
9215 \\
5633+ \\
5634\end{array}$ \\
\hline${ }^{97} \mathrm{Nb}$ & Indepe & ident $f$ & ission & $y \mid e 10$ & & \\
\hline $\begin{array}{l}{ }^{09} \mathrm{Bi} \\
97 \mathrm{Nb}\end{array}$ & $\begin{array}{l}{ }^{12} \mathrm{C} \\
\text { cumula }\end{array}$ & $\begin{array}{l}7.5+1 \\
\text { Ive } f 19\end{array}$ & $\begin{array}{l}1.0+2 \\
\text { gsion } y\end{array}$ & $\begin{array}{l}\text { HAR } \\
\text { YieId }\end{array}$ & M Branquinhot & 10152 \\
\hline $\begin{array}{l}232 \mathrm{Th} \\
87_{\mathrm{Nb}}\end{array}$ & $\begin{array}{r}d \\
O(E)\end{array}$ & $9.3+0$ & $1.4+1$ & CCP $F$ & R Lbovt & 5921 \\
\hline${ }^{93} \mathrm{Nb}$ & ${ }^{6} L_{i}$ & $5.5+1$ & $9.1+1$ & INUE & E Gaigalast & 9215 \\
\hline
\end{tabular}




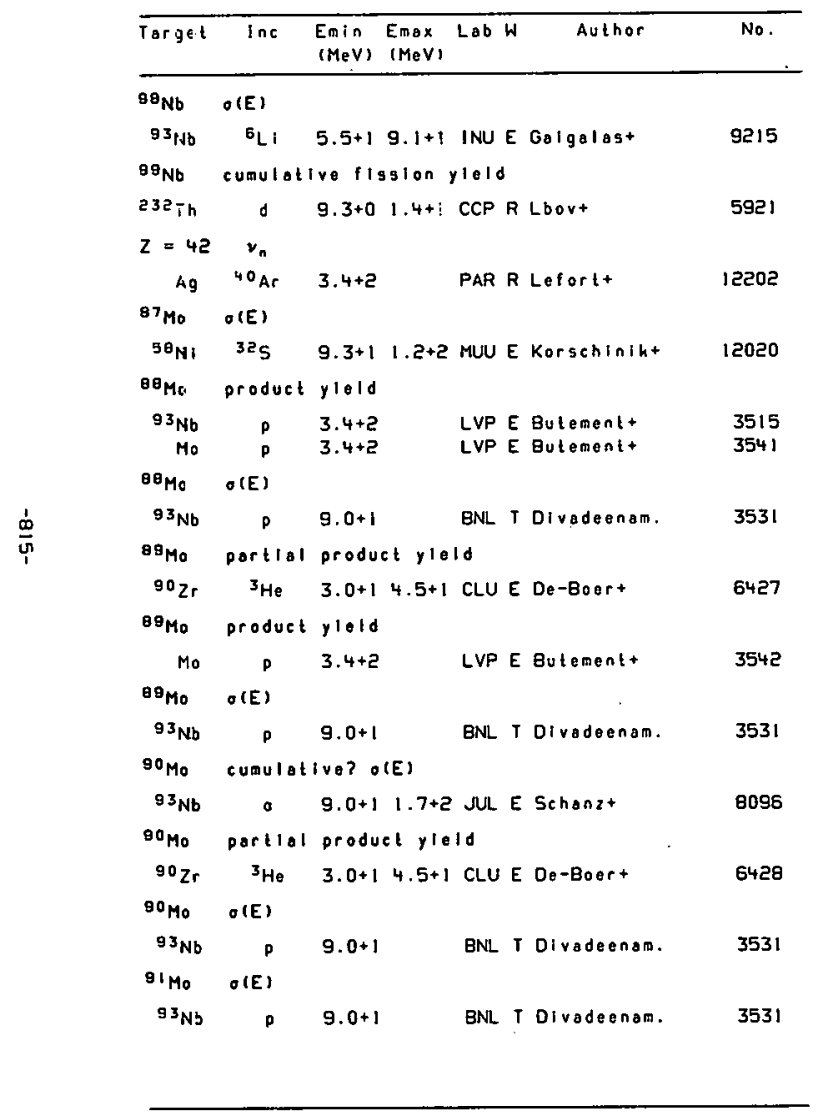

\begin{tabular}{|c|c|c|c|c|c|c|}
\hline Targot & $\ln c$ & $\begin{array}{l}E \min \\
(\mathrm{MeV})\end{array}$ & $\begin{array}{l}E \max \\
(\mathrm{MeV})\end{array}$ & Lab W & Althor & No. \\
\hline $92 \mathrm{Mo}$ & product & yield & & 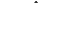 & & \\
\hline $92 M_{0}$ & $p$ & $7.0+0$ & $8.5+0$ & ATH E & E Papdopoulost & 3549 \\
\hline $92 \mathrm{Mo}$ & $\sigma(E)$ & & & & & \\
\hline${ }^{93} \mathrm{Nb}$ & $p$ & $9.0+1$ & & BNL T & T Divadeenam. & 3531 \\
\hline${ }^{93} \mathrm{Mo}_{0}$ & $\sigma(E)$ & & & & & \\
\hline${ }^{93} \mathrm{Nb}$ & ${ }^{3} \mathrm{He}$ & +0 & $4.0+1$ & KFK E & E flacht & 6431 \\
\hline${ }^{93} \mathrm{Mo}$ & partiol & $O(E) \times f$ & factor & & & \\
\hline${ }^{93} \mathrm{Nb}$ & D & NDG & & CCP T & I Guzhovshil. & 3516 \\
\hline $93 \mathrm{Mo}$ & raw $\mathrm{I}_{y}$ & & & & & \\
\hline${ }^{93} \mathrm{Nb}$ & $p$ & $3.0+0$ & $8.0+0$ & OSA E & E Tahahashit & 3523 \\
\hline${ }^{93} \mathrm{Mo}$ & thich to & $\operatorname{arget} y$ & $y / 010$ & & & \\
\hline${ }^{93} \mathrm{Nb}$ & $p$ & $1.3+0$ & $2.1+0$ & ORL $E$ & E Johnsont & 3520 \\
\hline${ }^{93} \mathrm{Mo}_{0}$ & average & $O(E)$ & & & & \\
\hline${ }^{93} \mathrm{Nb}$ & p & $1.3+0$ & $1.5+0$ & ORLE & E Johnsont & 3524 \\
\hline $93 \mathrm{Mo}$ & relotive & $O(E)$ & & & & \\
\hline${ }^{93} \mathrm{Nb}$ & p & $1.5+0$ & $4.9+0$ & ORL E & E Johnsolt & 3525 \\
\hline${ }^{93} \mathrm{Mo}$ & $\sigma(E) \times f \theta C$ & actor & & & & \\
\hline $\begin{array}{l}93 \mathrm{Nb} \\
{ }^{93} \mathrm{Nb}\end{array}$ & p & $\begin{array}{l}1.8+0 \\
2.1+0\end{array}$ & $\begin{array}{l}4.8+0 \\
6.0+0\end{array}$ & $\begin{array}{l}\text { ORL E } \\
\text { ORL E }\end{array}$ & $\begin{array}{l}\text { E Johnsont } \\
\text { E Johnsont }\end{array}$ & $\begin{array}{l}3521 \\
3522\end{array}$ \\
\hline${ }^{83} \mathrm{Mo}$ & $\theta(E)$ & & & & & \\
\hline $\begin{array}{l}93_{\mathrm{Nb}} \\
93_{\mathrm{Nb}} \\
9 \mathbf{3}_{\mathrm{Nb}} \\
9 \mathbf{3}_{\mathrm{Nb}}\end{array}$ & $\begin{array}{c}p \\
p \\
p \\
g_{L I}\end{array}$ & $\begin{array}{l}1.3+0 \\
1.3+0 \\
2.0+0 \\
5.5+1\end{array}$ & $\begin{array}{l}2.1+0 \\
2.1+0 \\
5.5+0 \\
9.1+1\end{array}$ & $\begin{array}{l}\text { KFK C } \\
\text { ORL E } \\
\text { ORL E } \\
\text { INU E }\end{array}$ & $\begin{array}{l}\text { C Muenzest } \\
\text { E Johngont } \\
\text { E Johngoget } \\
\text { E Galgalagt }\end{array}$ & $\begin{array}{l}3517 \\
3518 \\
3519 \\
9214\end{array}$ \\
\hline $93 \cdot \mathrm{MO}_{0}$ & $O(E)$ & & & & & \\
\hline $\begin{array}{l}93_{\mathrm{Nb}} \\
2 \mathbf{3 0}^{3} \mathrm{U}\end{array}$ & : & $\begin{array}{l}3.8+1 \\
1.0+3\end{array}$ & $\begin{array}{l}7.2+1 \\
3 \cdot 0+5\end{array}$ & $\begin{array}{l}\text { MAN E } \\
\text { KFK C }\end{array}$ & $\begin{array}{l}\text { E Branquinhot } \\
\text { C Muenzolt }\end{array}$ & $\begin{array}{l}8097 \\
4903\end{array}$ \\
\hline $93 \cdot \mathrm{Mo}_{0}$ & $O(E)$ & & & & & \\
\hline${ }^{93} \mathrm{Nb}$ & p & $8.0+0$ & $1.1+1$ & CCP F & R Zatolotint & 3526 \\
\hline
\end{tabular}




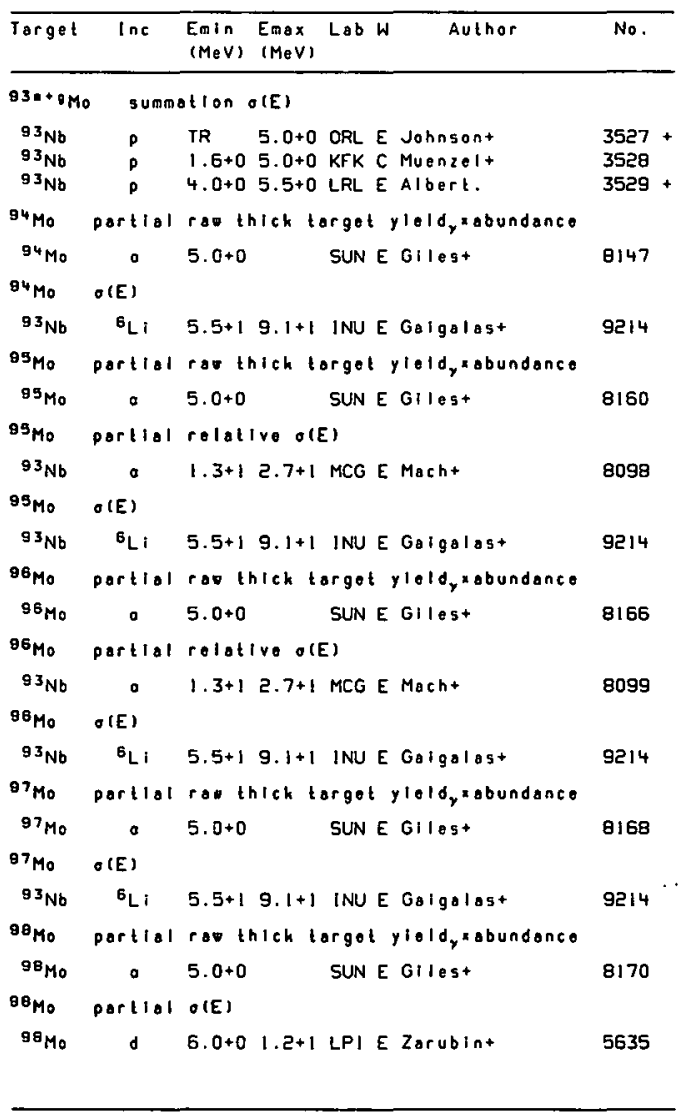

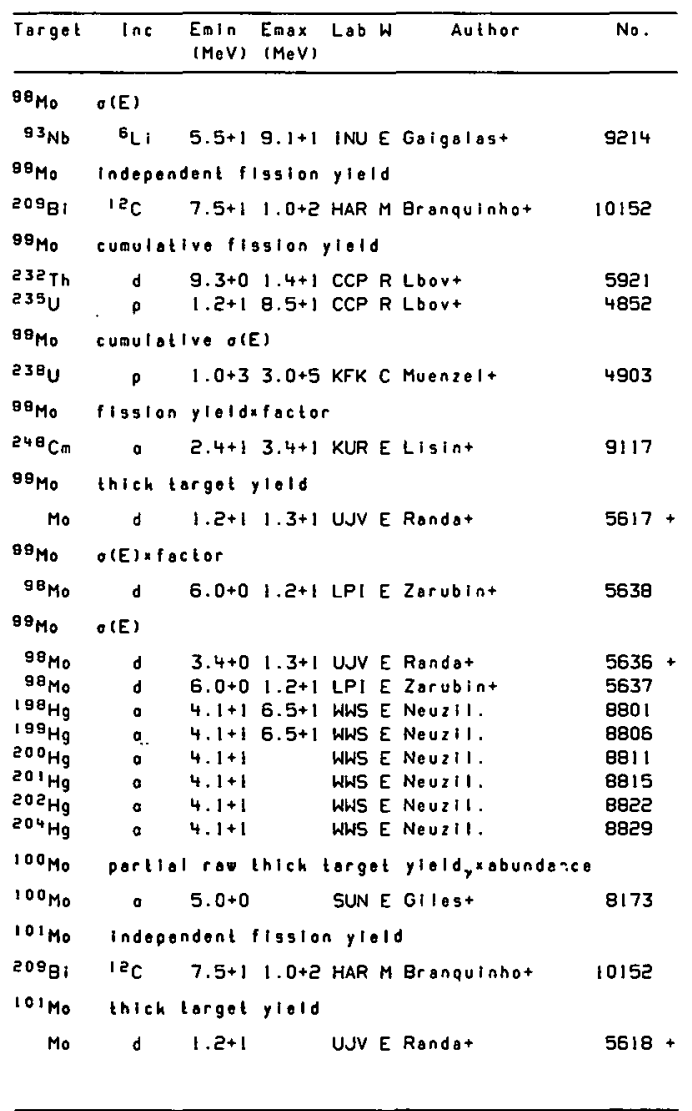




\begin{tabular}{|c|c|c|c|c|c|c|}
\hline Target & Inc & $\begin{array}{l}\text { Eqin } \\
(M e V)\end{array}$ & $\begin{array}{l}\text { Emax } \\
\text { (Mev) }\end{array}$ & Lab & Author & No. \\
\hline $101 \mathrm{Mo}$ & $\sigma(E)$ & & & & & \\
\hline $100 \mathrm{Mo}$ & d & $4.9+0$ & $1.2+1$ & UJV & E Randat & $5642+$ \\
\hline$z=43$ & $v_{n}$ & & & & & \\
\hline$A g$ & $40 \mathrm{Ar}$ & $3.4+2$. & & PAR & R Lefortt & 12202 \\
\hline $91 \bullet \pm \oplus T c$ & sumn & nationo & $O(E)$ & & & \\
\hline $\begin{array}{l}9 I_{\mathrm{Nb}} \\
91_{\mathrm{Nb}}\end{array}$ & $a$ & $\begin{array}{l}4.0+1 \\
4.4+1\end{array}$ & $\begin{array}{l}6.8+1 \\
7.2+1\end{array}$ & $\begin{array}{l}\text { MAN } \\
\text { HAR }\end{array}$ & $\begin{array}{l}\text { E Branquinho+ } \\
\text { E Branquinhot }\end{array}$ & $\begin{array}{l}8065 \\
8066\end{array}$ \\
\hline $91 \cdot / 9 T c$ & rot & $10 \sigma(E)$ & & & & \\
\hline $\begin{array}{l}91 \mathrm{Nb} \\
91 \mathrm{Nb}\end{array}$ & a & $\begin{array}{l}4.4+1 \\
5.6+1\end{array}$ & $7 \cdot 2+1$ & $\begin{array}{l}\text { HAR } \\
\text { MAN }\end{array}$ & $\begin{array}{l}\text { E Branquinhot } \\
\text { E Branquinho+ }\end{array}$ & $\begin{array}{l}8067 \\
8068\end{array}$ \\
\hline $82 \mathrm{TC}$ & $\circ(E)$ & & & & & \\
\hline $\begin{array}{l}93_{\mathrm{Nb}} \\
93_{\mathrm{Nb}} \\
9 \mathbf{3}_{\mathrm{Nb}}\end{array}$ & $\begin{array}{c}{ }^{3} \mathrm{He} \\
{ }^{3} \mathrm{He} \\
0\end{array}$ & $\begin{array}{r}+0 \\
1.1+1 \\
5.6+1\end{array}$ & $\begin{array}{l}4.0+1 \\
4.4+1 \\
7.2+1\end{array}$ & $\begin{array}{l}\text { KFK } \\
\text { HAM } \\
\text { MAN }\end{array}$ & $\begin{array}{l}\text { E Flacht } \\
\text { E Georgit } \\
\text { E Branquinhot }\end{array}$ & $\begin{array}{l}6432 \\
6433 \\
8100\end{array}$ \\
\hline $92 \cdot+9 \mathrm{TC}$ & sumet & nation. & $o(E)$. & 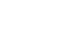 & & \\
\hline $\begin{array}{l}91 \mathrm{Nb} \\
91 \mathrm{Nb}\end{array}$ & : & $\begin{array}{l}2 . \theta+1 \\
2 . \theta+1\end{array}$ & $\begin{array}{l}7.2+1 \\
7.2+1\end{array}$ & $\begin{array}{l}\text { HAR } \\
\text { MAN }\end{array}$ & $\begin{array}{l}\text { E Branquinhot } \\
\text { E Branquinhot }\end{array}$ & $\begin{array}{l}8069 \\
8070\end{array}$ \\
\hline $82.19 \mathrm{Tc}$ & rat & $0 \sigma(E)$ & & & & \\
\hline $\begin{array}{l}9 I_{\mathrm{Nb}} \\
9 I_{\mathrm{Nb}}\end{array}$ & 0 & $\begin{array}{l}2 . \theta+1 \\
2 . \theta+1\end{array}$ & $\begin{array}{l}7.2+1 \\
5.6-1\end{array}$ & $\begin{array}{l}\text { HAR } \\
\text { MAN }\end{array}$ & $\begin{array}{l}\text { E Branquinhot } \\
\text { E Branquinhot }\end{array}$ & $\begin{array}{l}8071 \\
8072\end{array}$ \\
\hline${ }^{93} \mathrm{Te}$ & O(E) & & & & & \\
\hline $\begin{array}{l}93^{3} \mathrm{Nb} \\
93_{\mathrm{Nb}} \\
93^{\mathrm{Nb}} \\
93_{\mathrm{Nb}} \\
96 \mathrm{Mo}\end{array}$ & $\begin{array}{l}{ }^{3} \mathrm{He} \\
{ }^{3} \mathrm{He} \\
0 \\
{ }^{0} \mathrm{LI} \\
\mathrm{P}\end{array}$ & $\begin{array}{r}+0 \\
1.1+1 \\
3 \cdot 0+1 \\
5.5+1 \\
3.1+1\end{array}$ & $\begin{array}{l}4.0+1 \\
4.4+1 \\
1.7+2 \\
9.1+1 \\
8.0+1\end{array}$ & $\begin{array}{l}\text { KFK } \\
\text { HAM } \\
\text { JUL } \\
\text { INU } \\
\text { MCG }\end{array}$ & $\begin{array}{l}\text { E Flacht } \\
\text { E Goorgit } \\
\text { E Sehanz+ } \\
\text { E Gaigalast } \\
\text { T Brodovitcht }\end{array}$ & $\begin{array}{l}6434 \\
6435 \\
8119 \\
9213 \\
3564\end{array}$ \\
\hline $839 T C$ & thick & $\operatorname{target}$ & $y 1018$ & & & \\
\hline $\begin{array}{l}92_{M 0} \\
930 T c\end{array}$ & $\begin{array}{c}p \\
\sigma(E)\end{array}$ & $1.6+0$ & $5.0+0$ & CLU & M Roughton+ & 3550 \\
\hline $\begin{array}{l}92 \mathrm{MO}_{0} \\
92_{\mathrm{M}_{0}} \\
92_{\mathrm{MO}_{0}}\end{array}$ & $\begin{array}{l}p \\
q \\
q\end{array}$ & $\begin{array}{l}1.6+0 \\
2.6+1 \\
2.6+1\end{array}$ & $\begin{array}{l}5.0+1] \\
5.5+1 \\
5.5+1\end{array}$ & $\begin{array}{l}\text { CLU } \\
\text { KFK } \\
\text { KFK }\end{array}$ & $\begin{array}{l}\text { E Roughtont } \\
\text { E Graft } \\
\text { C Muenzelt }\end{array}$ & $\begin{array}{l}3551 \\
8130+ \\
8131\end{array}$ \\
\hline
\end{tabular}

\begin{tabular}{|c|c|c|c|c|c|c|}
\hline Torget & $\ln c$ & $\begin{array}{l}\text { Emin } \\
\text { (MeV) }\end{array}$ & $\begin{array}{l}\text { Emax } \\
(\operatorname{MeV})\end{array}$ & Lob W & Author & No. \\
\hline $939 \mathrm{Tc}$ & $\theta(E)$ & & & & & \\
\hline $\begin{array}{l}96 \mathrm{Mo} \\
{ }^{96} \mathrm{Mo}\end{array}$ & $p$ & $\begin{array}{l}3 \cdot 0+1 \\
3 \cdot 0+1\end{array}$ & $\begin{array}{l}6.5+1 \\
6.5+1\end{array}$ & $\begin{array}{l}\text { KFK C } \\
\text { MCG E }\end{array}$ & $\begin{array}{l}\text { C Muenzel+ } \\
\text { E Hogan. }\end{array}$ & $\begin{array}{l}3565 \\
3565\end{array}$ \\
\hline $93=T C$ & thick & torget & ylold & & & \\
\hline $\begin{array}{r}92 \mathrm{Mo} \\
93 . \mathrm{Tc}\end{array}$ & $\begin{array}{c}p \\
O(E)\end{array}$ & $1.6+0$ & $5.0+0$ & CLU M & M Roughtont & 3552 \\
\hline $\begin{array}{l}92 M_{0} \\
92 M_{0} \\
92 M_{0} \\
95 M_{0} \\
96 M_{0}\end{array}$ & $\begin{array}{l}p \\
a \\
o \\
p \\
p\end{array}$ & $\begin{array}{l}1 \cdot 5+0 \\
3 \cdot 0+1 \\
3 \cdot 0+1 \\
3 \cdot 0+1 \\
3 \cdot 0+1\end{array}$ & $\begin{array}{l}5 \cdot 1+0 \\
5 \cdot 5+1 \\
5 \cdot 5+1 \\
6 \cdot 5+1 \\
6 \cdot 5+1\end{array}$ & $\begin{array}{l}\text { CLU E } \\
\text { KFK E } \\
\text { KFK } \\
\text { KFK } \\
\text { MCG } \\
\text { MCG }\end{array}$ & $\begin{array}{l}\text { E Roughtont } \\
\text { E Groft } \\
\text { C Muenzel+ } \\
\text { C Muenzel+ } \\
\text { E Mogan. }\end{array}$ & $\begin{array}{l}3553 \\
8132+ \\
8133 \\
3567 \\
3568+\end{array}$ \\
\hline $830+0 T_{C}$ & sum & notion. & o(E) & & & \\
\hline $\begin{array}{l}91^{9} \mathrm{Nb} \\
91^{1} \mathrm{Nb} \\
93_{\mathrm{Nb}} \\
96_{\mathrm{Mb}} \\
{ }^{96} \mathrm{Mo}\end{array}$ & $\begin{array}{l}a \\
a \\
a \\
p \\
p\end{array}$ & $\begin{array}{l}1.5+1 \\
1.8+1 \\
4.4+1 \\
3.0+1 \\
3.0+1\end{array}$ & $\begin{array}{l}3 \cdot 2+1 \\
4 \cdot 8+1 \\
7 \cdot 2+1 \\
9 \cdot 0+1 \\
8 \cdot 0+1\end{array}$ & $\begin{array}{l}\text { MAN E } \\
\text { HAR E } \\
\text { HAR E } \\
\text { KFK C } \\
\text { MCG E }\end{array}$ & $\begin{array}{l}\text { E Branquinhot } \\
\text { E Branguinhot } \\
\text { E Branguinhot } \\
\text { C Muenzelt } \\
\text { E Hogan. }\end{array}$ & $\begin{array}{l}8073 \\
8074 \\
8101 \\
3569 \\
3570\end{array}$ \\
\hline $93 . / 9 T C$ & rat & $10 O(E)$ & & & & \\
\hline $\begin{array}{l}91_{\mathrm{Nb}} \\
91_{\mathrm{Nb}} \\
93_{\mathrm{Nb}} \\
95_{\mathrm{Mb}}\end{array}$ & $\begin{array}{l}a \\
\dot{a} \\
0 \\
0\end{array}$ & $\begin{array}{l}1.6+1 \\
1.8+1 \\
4.4+1 \\
3.0+1\end{array}$ & $\begin{array}{l}5 \cdot 0+1 \\
4.8+1 \\
7.2+1 \\
6.5+1\end{array}$ & $\begin{array}{l}\text { MAN E } \\
\text { HAR E } \\
\text { HAR E } \\
\text { MCG E }\end{array}$ & $\begin{array}{l}\text { Erangulnhot } \\
\text { Eranculnhot } \\
\text { E Branculnhot } \\
\text { E Hogan. }\end{array}$ & $\begin{array}{l}8075 \\
8076 \\
8102 \\
3571\end{array}$ \\
\hline${ }^{94} \mathrm{Tc}$ & $\sigma(E)$ & & & & & \\
\hline${ }^{93} \mathrm{Nb}$ & $-3^{\mathrm{He}}$ & +0 & $4.0+1$ & KFK E & E Flocht & 6436 \\
\hline $93 \mathrm{Nb}$ & ${ }^{3} \mathrm{He}$ & $1.1+1$ & $4.4+1$ & HAM E & E Georglt & 6437 \\
\hline${ }^{83} \mathrm{Nb}$ & $\circ$ & $3.0+1$ & $1.7+2$ & JUL E & Echanz+ & 8119 \\
\hline${ }^{93} \mathrm{Nb}$ & ${ }^{6} \mathrm{LI}$ & $5.5+1$ & $9.1+1$ & INU E & E Gaigalast & 9213 \\
\hline $94 \mathrm{Mo}$ & $\rho$ & $T R$ & $6.7+0$ & KTY E & Flynnt & 3554 \\
\hline $\begin{array}{l}{ }^{94} \mathrm{Mo}_{0} \\
96 \mathrm{Mo}\end{array}$ & d & $\begin{array}{l}8.0+0 \\
2.0+1\end{array}$ & $\begin{array}{l}1 \cdot 2+1 \\
8 \cdot 0+1\end{array}$ & $\begin{array}{l}\text { SUL E } \\
\text { MCG T }\end{array}$ & $\begin{array}{l}\text { E Aleksandrout } \\
\text { T Brodoziteht }\end{array}$ & $\begin{array}{l}5628+ \\
3572\end{array}$ \\
\hline $949 \mathrm{TC}$ & $\sigma(E)$ & & & & & \\
\hline $\begin{array}{l}92 M_{0} \\
92 M_{0} \\
96 M_{0} \\
96 M_{0}\end{array}$ & $\begin{array}{l}\dot{q} \\
\dot{p} \\
p \\
p\end{array}$ & $\begin{array}{l}1.8+1 \\
1.8+1 \\
2.0+1 \\
2.0+1\end{array}$ & $\begin{array}{l}4.2+1 \\
4.2+1 \\
6.5+1 \\
6.5+1\end{array}$ & $\begin{array}{l}\text { KFK } \\
\text { KFK } \\
\text { KFK } \\
\text { MCG } \\
\text { MC }\end{array}$ & $\begin{array}{l}\text { Eraft } \\
\text { C Muenzelt } \\
\text { C Muenzelt } \\
\text { E Hogan. }\end{array}$ & $\begin{array}{l}8134+ \\
8135 \\
3573 \\
3574+\end{array}$ \\
\hline
\end{tabular}




\begin{tabular}{|c|c|c|c|c|c|c|}
\hline Target & Inc & $\begin{array}{l}\text { Emin } \\
(\mathrm{MeV})\end{array}$ & $\begin{array}{l}E_{\max } \\
(\operatorname{MeV})\end{array}$ & Lab r & Author & No. \\
\hline $94 \cdot T C$ & $O(E)$ & & & & & \\
\hline $\begin{array}{l}92 \mathrm{Mo} \\
92 \mathrm{Mo} \\
96 \mathrm{Mo} \\
98 \mathrm{Mo}\end{array}$ & $\begin{array}{l}a \\
a \\
p \\
p\end{array}$ & $\begin{array}{l}2.0+1 \\
2 \cdot 0+1 \\
2.0+1 \\
2.0+1\end{array}$ & $\begin{array}{l}3 \cdot 7+1 \\
3 \cdot 7+1 \\
6 \cdot 5+1 \\
6 \cdot 5+1\end{array}$ & $\begin{array}{l}\text { KFK } \\
\text { KFK } \\
\text { KFK } \\
\text { MCG }\end{array}$ & $\begin{array}{l}\text { E Graft } \\
\text { C Muenzelt } \\
\text { C Muenzelt } \\
\text { E Hogan. }\end{array}$ & $\begin{array}{l}\text { 8136+ } \\
\text { 8137 } \\
3575 \\
3576+\end{array}$ \\
\hline $940+9 T_{c}$ & summe & pationo & $O(E)$ & & & \\
\hline $\begin{array}{l}91_{\mathrm{Nb}} \\
91_{\mathrm{Nb}} \\
93_{\mathrm{Nb}} \\
96 \mathrm{Mb} \\
96 \mathrm{Mb}\end{array}$ & $\begin{array}{l}a \\
a \\
a \\
p \\
p\end{array}$ & $\begin{array}{l}1.0+1 \\
1.5+1 \\
2.8+1 \\
2.0+1 \\
2.0+1\end{array}$ & $\begin{array}{l}2 \cdot 6+1 \\
2 \cdot 1+1 \\
7 \cdot 2+1 \\
8 \cdot 0+1 \\
8 \cdot 0+1\end{array}$ & $\begin{array}{l}\text { MAN } \\
\text { HAR } \\
\text { HAR E } \\
\text { KFK } \\
\text { MCG E }\end{array}$ & $\begin{array}{l}\text { E Branquinhot } \\
\text { E Branquinhot } \\
\text { E Branquinho+ } \\
\text { C Muenzelt } \\
\text { E Hogan. }\end{array}$ & $\begin{array}{l}8077 \\
8078 \\
8103 \\
3577 \\
3578+\end{array}$ \\
\hline $84.19 \mathrm{TC}$ & ratio & $0.0(E)$ & & & & \\
\hline $\begin{array}{l}91 \mathrm{Nb} \\
91 \mathrm{Nb} \\
93_{\mathrm{Nb}} \\
93_{\mathrm{Nb}} \\
96 \mathrm{Mo}\end{array}$ & $\begin{array}{l}a \\
a \\
a \\
a\end{array}$ & $\begin{array}{l}1.2+1 \\
1.5+1 \\
2 \cdot 8+1 \\
2.8+1 \\
\overline{\boldsymbol{c}} \cdot 0+1\end{array}$ & $\begin{array}{l}2.4+1 \\
2.1+1 \\
7.2+1 \\
3 \cdot 8+1 \\
6.5+1\end{array}$ & $\begin{array}{l}\text { MAN E } \\
\text { HAR } \\
\text { HAR } \\
\text { MAN E } \\
\text { MCG }\end{array}$ & $\begin{array}{l}\text { E Eranquinhot } \\
\text { E Branquinhot } \\
\text { E Branquinhot } \\
\text { E Branquinhot } \\
\text { E Hogan. }\end{array}$ & $\begin{array}{l}8079 \\
8080 \\
8104 \\
8105 \\
3579\end{array}$ \\
\hline${ }^{95} \mathrm{Tc}$ & portial & relati & IVO OLE & E) & & \\
\hline $\begin{array}{l}{ }^{93} \mathrm{Nb} \\
{ }^{85} \mathrm{TC}_{\mathrm{C}}\end{array}$ & $\begin{array}{c}0 \\
\text { relotive }\end{array}$ & $\begin{array}{l}1.3+1 \\
I_{y}\end{array}$ & $2.7+1$ & MCO & E Macht & 8107 \\
\hline $93_{\mathrm{Nb}}$ & a & $2.3+1$ & $2.7+1$ & ROS & E Wintert & 8105 \\
\hline${ }^{99} T_{C}$ & $O(E)$ & & & & & \\
\hline $\begin{array}{l}93 \mathrm{Nb} \\
93_{\mathrm{Nb}} \\
93^{3} \mathrm{Nb} \\
94 \mathrm{Mo} \\
95 \mathrm{Mo} \\
95 \mathrm{Mo} \\
95 \mathrm{Mo} \\
96 \mathrm{Mo}\end{array}$ & $\begin{array}{c}{ }^{3} \mathrm{He} \\
0 \\
{ }^{0} \mathrm{Li} \\
0 \\
p \\
p \\
p \\
p\end{array}$ & $\begin{array}{l}1.1+1 \\
3.0+1 \\
5.5+1 \\
5.1+0 \\
1 R \\
2.4+0 \\
1.5+1 \\
1.2+1\end{array}$ & $\begin{array}{l}4 \cdot 4+1 \\
1 \cdot 7+2 \\
9 \cdot 1+1 \\
1.2+1 \\
6 \cdot 7+0 \\
5 \cdot 3+0 \\
8 \cdot 0+1\end{array}$ & $\begin{array}{l}\text { HAM } \\
\text { UL E } \\
\text { INU E } \\
\text { SUL E } \\
\text { KTY E } \\
\text { CLU E } \\
\text { MIN E } \\
\text { MCG }\end{array}$ & $\begin{array}{l}\text { E Georgit } \\
\text { E Schanz+ } \\
\text { E Galgolast } \\
\text { E Aleksandrovt } \\
\text { E Flynnt } \\
\text { E Roughtont } \\
\text { E Flligeraldt } \\
\text { T Grodovitcht }\end{array}$ & $\begin{array}{l}6438 \\
8119 \\
9213 \\
5629+ \\
3555 \\
3556 \\
3557 \\
3580\end{array}$ \\
\hline $8507 c$ & thlch & target & yleld & & & \\
\hline $.95 \mathrm{Mo}$ & $p$ & $3.1+0$ & $4.7+0$ & CLU $\mathrm{r}$ & M Roughtont & 3558 \\
\hline $959_{\mathrm{TC}}$ & $\theta(E)$ & & & & & \\
\hline $92 M_{0}$ & o & $1.2+1$ & $\begin{array}{c}2.7+1 \\
r\end{array}$ & KFK E & E Graft & $8138+$ \\
\hline
\end{tabular}

\begin{tabular}{|c|c|c|c|c|c|c|}
\hline Target & $\ln c$ & $\begin{array}{l}\text { Enin } \\
(\mathrm{MeV})\end{array}$ & $\begin{array}{l}\text { Emax } \\
(\mathrm{MeV})\end{array}$ & Lab & Author & No. \\
\hline $95 \mathrm{O}^{\mathrm{TC}}$ & $O(E)$ & & & & & \\
\hline $\begin{array}{l}92 \mathrm{Mo} \\
94 \mathrm{Mo} \\
94 \mathrm{Mo} \\
96 \mathrm{Mo} \\
96 \mathrm{Mo}\end{array}$ & $\begin{array}{l}a \\
a \\
a \\
p \\
p\end{array}$ & $\begin{array}{l}1.2+1 \\
3 \cdot 0+1 \\
3 \cdot 0+1 \\
1.5+1 \\
1.5+1\end{array}$ & $\begin{array}{l}2.7+1 \\
5 \cdot 5+1 \\
5 \cdot 5+1 \\
6.5+1 \\
6.5+1\end{array}$ & $\begin{array}{l}\text { KFK } \\
\text { KFK } \\
\text { KFK } \\
\text { KFK } \\
\text { MCG }\end{array}$ & $\begin{array}{l}\text { C Muenzel+ } \\
\text { E Graft } \\
\text { C Muenzel+ } \\
\text { C Muenzel+ } \\
\text { E Mogan. }\end{array}$ & $\begin{array}{l}8139 \\
8148+ \\
8149 \\
3581 \\
3582 .\end{array}$ \\
\hline $95 \bullet^{\top} \mathrm{TC}$ & produ & yleld & & & & \\
\hline
\end{tabular}

$5 \bullet$ te product yleld

Mo o $1.8+1$ B!RE Oaim. 5521

Q5. Th thich torget yield

${ }^{93} \mathrm{Nb} \quad 1.8+14.3+1 F E I E$ Daltrievt

Mo $\quad 1,1+13 z+1$ FEl

Mo $\quad \mathrm{p} 1.1+12.2+1$ FEl E Omilrievt $3544+$

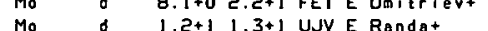

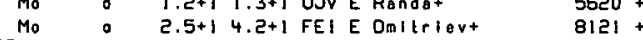

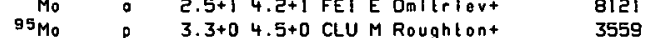

QS*TC O(E)

$\mathrm{Mo}$
$92 \mathrm{Mo}$

$92 \mathrm{Mo} \quad a \quad 1.3+13.2+1 \mathrm{KFK}$ E Graft $\quad 8140$

$92 \mathrm{Mo} \quad a \quad 1.3+13.2+1$ KFK C Muenzelt 8141

$94 \mathrm{Mo} a \quad 3.4+15.6+1$ KFK E Graft 8150

$94 \mathrm{Mo} a \quad 3.4+15.8+1$ KFK C Muenzelt 8151

$96 \mathrm{Mo} P 1.5+16.5+1$ KFK C Muenzel $\quad 3583$

96 Mo $p$ 1.5+1 $6.5+1$ MCG E Hogan. $3584+$

Q5e+9TC sumation $O(E)$

${ }^{93} \mathrm{Nb} a \mathrm{~N}^{2} 1.8+14.8+1$ HAR E Branquinhot 8109

$96 \mathrm{Mo}$ p $1.5+1$ 8.0+1 KFK C Muenzel+ 3585

85०19TC rollo O(E)

${ }_{93}^{93} \mathrm{Nb} \quad 1.6+17.2+1$ HAR E Goodal1+ 8110

${ }^{96} T$ in including metastoble thick target yield

$\begin{array}{rlllll}93_{\mathrm{Nb}} & \text { a } & 1.2+1 & 4.3+1 \text { FEI } E \text { Dmllirlevt } & 8111+ \\ \text { Mo } & \text { p } & 1.1+1 & 2.2+1 \text { FEI E Dmllrievt } & 3546+ \\ \text { Mo } & \text { d } & 8.1+0 & 2.2+1 \text { FEI E Dmliriert } & 5622+ \\ \text { Mo } & \text { d } & 1.2+1 & 1.3+1 \text { UJV E Randat } & 5623+\end{array}$

$5623+$ 


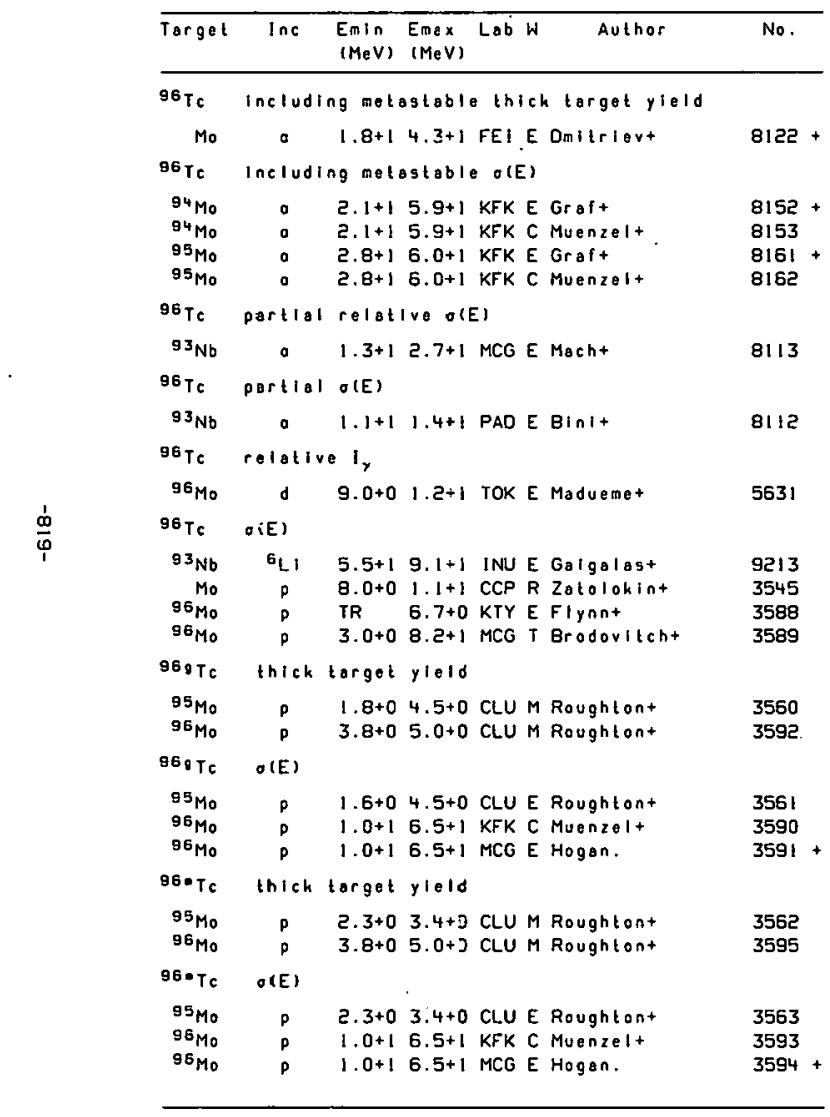

\begin{tabular}{|c|c|c|c|c|c|c|}
\hline Target & $\ln c$ & $\begin{array}{l}\text { Emln } \\
(M e V)\end{array}$ & $\begin{array}{l}\text { Emax } \\
(M e V)\end{array}$ & Lob W & Author & No. \\
\hline $980+9 \mathrm{TC}$ & summ & nation. & $0(E)$ & & & \\
\hline $\begin{array}{l}93 \mathrm{Nb} \\
96 \mathrm{Mo} \\
96 \mathrm{Mo}\end{array}$ & p & $\begin{array}{l}1.5+1 \\
1.0+1 \\
1.0+1\end{array}$ & $\begin{array}{l}2.1+1 \\
8 \cdot 0+1 k \\
8.0+1\end{array}$ & $\begin{array}{l}\text { HAR E } \\
\text { KFK C } \\
\text { MCG E }\end{array}$ & $\begin{array}{l}\text { Brangunhot } \\
\text { Muengol+ } \\
\text { Hogan. }\end{array}$ & $\begin{array}{l}8114 \\
3596 \\
3597\end{array}$ \\
\hline $960 \%$ OT & roll & $0 O(E)$ & . & & & \\
\hline $\begin{array}{l}93^{9} \mathrm{Nb} \\
93 \mathrm{Nb} \\
96 \mathrm{Mo}\end{array}$ & $a$ & $\begin{array}{l}1.5+1 \\
1.5+1 \\
1.0+1\end{array}$ & $\begin{array}{l}2.1+1 r \\
2 \cdot 3+1 r \\
6.5+1 r\end{array}$ & $\begin{array}{l}\text { HAR E } \\
\text { MAN } E \\
\text { MCG E }\end{array}$ & $\begin{array}{l}\text { Branquinhot } \\
\text { Branquinhot } \\
\text { Hogan. }\end{array}$ & $\begin{array}{l}8115 \\
8116 \\
3598\end{array}$ \\
\hline $97 \mathrm{Tc}$ & portial & relat & Ive $\sigma(E)$ & Exabun & undance & \\
\hline $97 \mathrm{Mo}$ & 。 & $1.5+0$ & $8.0+0$ & ATH E & Exenoclist & 3599 \\
\hline${ }^{87} \mathrm{TC}$ & $O(E)$ & & & & & \\
\hline $93 \mathrm{Nb}$ & $\mathrm{E}_{\mathrm{LI}}$ & $5.5+1$ & $9.1+1$ & INU E & Ealgalast & 9213 \\
\hline $97 . T_{c}$ & thick & targot & $y / e 1 d$ & & & \\
\hline $\begin{array}{l}\text { Mo } \\
\text { Mo }\end{array}$ & o & $\begin{array}{l}1.1+1 \\
8.1+0\end{array}$ & $\begin{array}{l}2.2+1 F \\
2.2+1 F\end{array}$ & $\begin{array}{l}\text { FEI E } \\
\text { FEI E }\end{array}$ & $\begin{array}{l}\text { Datirievt } \\
\text { Dmitrievt }\end{array}$ & $\begin{array}{l}3547+ \\
5624+\end{array}$ \\
\hline${ }^{98} \mathrm{TC}$ & $\cos \mathrm{I}_{y}$ & & & & & \\
\hline $9 \theta_{M 0}$ & p & $T R$ & $3.1+0 \mathrm{~K}$ & KTY E & E Miraclet & 3600 \\
\hline${ }^{88} \mathrm{Tc}$ & $\sigma(E)$ & & & & & \\
\hline $\begin{array}{l}{ }^{93} \mathrm{Nb} \\
{ }^{9} \mathrm{MO}_{\mathrm{M}}\end{array}$ & $\begin{array}{l}{ }^{6} \mathrm{LI} \\
\mathrm{P}\end{array}$ & $\begin{array}{l}5.5+1 \\
\text { TR }\end{array}$ & $\begin{array}{l}9.1+1 \\
6.7+0\end{array}$ & $\begin{array}{l}\text { INU E } \\
\text { KTY E }\end{array}$ & $\begin{array}{l}\text { Galgalast } \\
\text { Flynnt }\end{array}$ & $\begin{array}{l}9213 \\
3601\end{array}$ \\
\hline${ }^{80} \mathrm{Tc}$ & $\theta(E)$ & & & & & \\
\hline${ }^{98} \mathrm{Mo}_{0}$ & $p$ & $1.4+0$ & $5.3+00$ & CLUE & Eough!ont & 3602 \\
\hline $99=\mathrm{Tc}$ & thlch & target & yleld & & & \\
\hline${ }^{90} \mathrm{Mo}_{0}$ & $p$ & $1.6+0$ & $4.7+00$ & CLU M & 4 Rough: ort & 3603 \\
\hline $990 \mathrm{Te}$ & $O(E) \times f$ & lactor & & & & \\
\hline${ }^{99} \mathrm{Mo}_{0}$ & d & $6.0+0$ & $1.2+1$ & LPI E & Earubint & 5639 \\
\hline $99 . T C$ & $\sigma(E)$ & & & & & \\
\hline $\begin{array}{l}99_{M 0} \\
98_{M 0}\end{array}$ & p & $\begin{array}{l}1.6+0 \\
6.0+0\end{array}$ & $\begin{array}{l}4.7+0 \\
1.2+1\end{array}$ & $\begin{array}{l}\text { CLUE } \\
\text { LPIE }\end{array}$ & $\begin{array}{l}\text { Eoughtont } \\
\text { Earubint }\end{array}$ & $\begin{array}{l}3604 \\
5640\end{array}$ \\
\hline
\end{tabular}




\begin{tabular}{|c|c|c|c|c|c|c|}
\hline Target & $\ln 8$ & $\begin{array}{l}\text { Enin } \\
(M e V)\end{array}$ & $\begin{array}{l}\operatorname{Egax} \\
(\operatorname{MeV})\end{array}$ & Lab W & Author & No. \\
\hline $101 \mathrm{TC}$ & thich & torget & yield & & & \\
\hline $\begin{array}{l}100 \mathrm{Mo} \\
101 \mathrm{Tc}\end{array}$ & $\stackrel{p}{O(E)}$ & $1.9+0$ & $5.0+0$ & CLU M & M Roughtont & 3607 \\
\hline $\begin{array}{l}98 \mathrm{Mo} \\
99 \mathrm{Mo} \\
100 \mathrm{Mo} \\
100 \mathrm{Mo} \\
100 \mathrm{Mo} \\
100 \mathrm{Mo}\end{array}$ & $\begin{array}{l}\dot{a} \\
\dot{p} \\
p \\
p \\
a \\
a\end{array}$ & $\begin{array}{l}1.7+1 \\
1.7+1 \\
1.9+0 \\
2.0+1 \\
2.6+1 \\
2.6+1\end{array}$ & $\begin{array}{l}3 \cdot 8+1 \\
3 \cdot 8+1 \\
5 \cdot 0+0 \\
\\
5 \cdot 5+1 \\
\mathbf{5} \cdot 5+1\end{array}$ & $\begin{array}{l}\text { KFK E } \\
\text { KFK C } \\
\text { CLU E } \\
\text { GOE E } \\
\text { KFK E } \\
\text { KFK C }\end{array}$ & $\begin{array}{l}\text { E Graft } \\
\text { C Muenzel+ } \\
\text { E Roughtont } \\
\text { E Kantust } \\
\text { E Graft } \\
\text { C Muenzel+ }\end{array}$ & $\begin{array}{l}8171+ \\
8172 \\
3605 \\
3506 \\
8174+ \\
8175\end{array}$ \\
\hline $102 * T c$ & $O(E)$ & & & & & \\
\hline $\begin{array}{l}100 \mathrm{Mo} \\
100 \mathrm{Mo}\end{array}$ & $:$ & $\begin{array}{l}2.3+1 \\
2.3+1\end{array}$ & $\begin{array}{l}4.7+1 \\
4.7+1\end{array}$ & $\begin{array}{l}K=K E \\
K \bar{F} K \quad C\end{array}$ & $\begin{array}{l}\text { E Graft } \\
\text { C Muenzelt }\end{array}$ & $\begin{array}{l}8176+ \\
8177\end{array}$ \\
\hline${ }^{103} \mathrm{TC}$ & $O(E)$ & & & & & \\
\hline $\begin{array}{l}100 \mathrm{Mo} \\
100 \mathrm{Mo}\end{array}$ & $:$ & $\begin{array}{l}2.4+1 \\
2.4+1\end{array}$ & & $\begin{array}{l}\text { KFK E } \\
\text { KFK C }\end{array}$ & $\begin{array}{l}\text { E Graft } \\
\text { C Muenzelt }\end{array}$ & $\begin{array}{l}8178+ \\
8179\end{array}$ \\
\hline $104 \mathrm{Tc}$ & indepe & ondent $f$ & fisgion & $y 101$ & id & \\
\hline $\begin{array}{l}209_{B i} \\
Z=44\end{array}$ & $\begin{array}{l}1 a_{C} \\
v_{n}\end{array}$ & $7.5+1$ & $1.0+2$ & HAR $M$ & Mranquinhot & 10152 \\
\hline Ag & ${ }^{40} \mathrm{Ar}$ & $3.4+2$ & & PAR $R$ & R Lefort+ & 12202 \\
\hline${ }^{9} \mathbf{3}_{\mathrm{Ru}}$ & $O(E)$ & & & & & \\
\hline $93_{\mathrm{Nb}}$ & ${ }^{6} \mathrm{Li}$ & $5.5+1$ & $9.1+1$ & INU E & E Galgalast & 9212 \\
\hline${ }^{94} R u$ & $\theta(E)$ & & & & & \\
\hline $\begin{array}{l}93 \mathrm{Nb} \\
92 \mathrm{Mo} \\
92 \mathrm{Mo}\end{array}$ & $\begin{array}{l}\text { ELI } \\
\vdots \\
0\end{array}$ & $\begin{array}{l}5.5+1 \\
\vdots .9+1 \\
7.9+1\end{array}$ & $\begin{array}{l}9.1+1 \\
4 \cdot 1+1 \\
4.1+1\end{array}$ & $\begin{array}{l}\text { INU E } \\
\text { KFFK E } \\
\text { KFK C C }\end{array}$ & $\begin{array}{l}\text { E Gaigalast } \\
\text { E Graft } \\
\text { C Muenzelt }\end{array}$ & $\begin{array}{l}9212 \\
8142+ \\
8143\end{array}$ \\
\hline${ }^{89} \mathrm{Ru}$ & thick t & terget $y$ & $y 101 d$ & & & \\
\hline $\begin{array}{l}{ }^{92} \mathrm{Mo} \\
{ }^{95} \mathrm{Ru}\end{array}$ & o(E) & TR & +1 & CLU E & E Roughtont & 8146 \\
\hline $\begin{array}{l}93_{\mathrm{Nb}} \\
92 \mathrm{Mo} \\
92 \mathrm{Mo} \\
94 \mathrm{Mo}_{0}\end{array}$ & $\begin{array}{c}\text { ELi } \\
\vdots \\
\vdots\end{array}$ & $\begin{array}{l}5.5+1 \\
1.2+1 \\
1.2+1 \\
3.0+1\end{array}$ & $\begin{array}{l}9 \cdot 1+1 \\
3 \cdot 0+1 \\
3 \cdot 0+1 \\
5 \cdot 9+1\end{array}$ & $\begin{array}{l}\text { INU E } \\
\text { KFK E } \\
\text { KFK C } \\
\text { KFK E }\end{array}$ & $\begin{array}{l}\text { E Gaigalast } \\
\text { E Graft } \\
\text { C Muenzelt } \\
\text { E Graft }\end{array}$ & $\begin{array}{l}9212 \\
8144+ \\
8145 \\
8154+\end{array}$ \\
\hline
\end{tabular}

\begin{tabular}{|c|c|c|c|c|c|c|}
\hline Torget & $\ln c$ & $\begin{array}{l}\text { Em In } \\
(\mathrm{MeV})\end{array}$ & $\begin{array}{l}\text { Emax } L \\
(M \theta V)\end{array}$ & Lab & Author & No. \\
\hline${ }^{95} R_{u}$ & $O(E)$ & & & & & \\
\hline $94 \mathrm{Mo}$ & a & $3.0+1$ & $5.9+1 k$ & KFK C & C Muenzel+ & 8155 \\
\hline${ }^{96} R_{u}$ & portiol & relats & Ive o(E) & $\Leftrightarrow$ & & \\
\hline $\begin{array}{l}96 R u \\
95 R u \\
95_{R u}\end{array}$ & $\begin{array}{l}16 a \\
325\end{array}$ & $\begin{array}{l}8.5+0 \\
3.3+1 \\
4.8+1\end{array}$ & & $\begin{array}{l}\text { LVP E } \\
\text { LVP E } \\
\text { LVP E }\end{array}$ & $\begin{array}{l}\text { E Maynard+ } \\
\text { E Maynard } \\
\text { E Maynardt }\end{array}$ & $\begin{array}{r}8182 \\
11330 \\
12033\end{array}$ \\
\hline${ }^{96} R_{u}$ & $\theta(E)$ & & & & & \\
\hline${ }^{93} \mathrm{Nb}$ & ${ }^{6} \mathrm{LI}$ & $5.5+1$ & $9.1+11$ & INU E & E Goigalast & 9212 \\
\hline $97_{R u}$ & product & yleld & & & . & \\
\hline $\begin{array}{l}\text { Mo } \\
\text { Mo }\end{array}$ & ${ }_{0}^{3} \mathrm{He}$ & $\begin{array}{l}1.8+1 \\
1.6+1\end{array}$ & $\begin{array}{l}3.0+1 \mathrm{~J} \\
2.2+1 \mathrm{~J}\end{array}$ & $\begin{array}{l}\text { JUL R } \\
\text { JUL R }\end{array}$ & 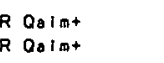 & $\begin{array}{l}6439 \\
8125\end{array}$ \\
\hline${ }^{97} \mathrm{Ru}$ & thich tor & orgot $r$ & yield & & & \\
\hline $\begin{array}{l}\text { Mo } \\
94 \mathrm{Mo}\end{array}$ & : & $\begin{array}{l}2.2+1 \\
\text { TR }\end{array}$ & $\begin{array}{r}3.0+1 \mathrm{G} \\
+1 \mathrm{C}\end{array}$ & $\begin{array}{l}\text { GER E } \\
\text { CLU E }\end{array}$ & $\begin{array}{l}\text { E Silvestert } \\
\text { E Roughtont }\end{array}$ & $\begin{array}{l}8125 \\
8159\end{array}$ \\
\hline${ }^{97} \mathbf{R u}_{\mathbf{u}}$ & $O(E) \times f O C$ & etor & & & & \\
\hline Mo & a & $4.0+1$ & & ALB E & E Kishoret & 8124 \\
\hline${ }^{87} \mathbf{R u}$ & $\mathscr{O}(E)$ & & & & & \\
\hline $9 \mathbf{3}_{\mathrm{Nb}}$ & $6_{L I}$ & $5.5+1$ & $9.1+1 \mathrm{I}$ & INU E & E Gaigalast & 9212 \\
\hline Mo & 0 & $4.0+1$ & & ALB E & E kighoret & 8123 \\
\hline $94 \mathrm{Mo}$ & a & $1.2+1$ & $4.6+1 \mathrm{~J}$ & JUL $R$ & R $Q_{a}$ m $^{+}$ & 8156 \\
\hline $94 \mathrm{Mo}$ & 0 & $1.2+1$ & $5.9+1 \mathrm{~K}$ & KFK E & E Graf- & 8157 . \\
\hline${ }^{94} \mathrm{Mo}$ & $a$ & $1.2+1$ & $5.9+1 \mathrm{~K}$ & KFK C & C Muenzelt+ & 8158 \\
\hline $95 \mathrm{MO}^{\circ}$ & $a$ & $1.8+1$ & $6.0+1$ & UR $R$ & R Darat & 8163 \\
\hline${ }^{95} \mathrm{Mo}$ & a & $1.8+1$ & $6.0+1 \mathrm{~K}$ & KFK E & E Graft & $8164+$ \\
\hline${ }^{95} \mathrm{Mo}$ & a & $1 . \theta+1$ & $6.0+1 \mathrm{~K}$ & KFK C & C Muenzelt & 8165 \\
\hline${ }^{98} \mathbf{R u}$ & partiol & $v_{y}$ & & & & \\
\hline${ }^{96} \mathrm{Mo}$ & . & $3.0+1$ & & WAS $\varepsilon$ & $\varepsilon$ Westerberg+ & 8167 \\
\hline$\theta^{8} \mathrm{Ru}$ & portiol & rolot & IVo o(E) & & . & \\
\hline $\begin{array}{l}{ }^{9 \theta} \mathrm{Ru} \\
{ }^{9} \mathrm{Ru} \\
{ }^{9} \theta_{\mathrm{Ru}}\end{array}$ & $\begin{array}{l}160 \\
325\end{array}$ & $\begin{array}{l}8 \cdot 5+0 \\
3 \cdot 3+1 \\
4 \cdot 8+1\end{array}$ & & $\begin{array}{l}\text { LVP E } \\
\text { LVP E } \\
\text { LVP E }\end{array}$ & $\begin{array}{l}\text { E Maynard+ } \\
\text { E Maynard+ } \\
\text { E Maynard+ }\end{array}$ & $\begin{array}{r}8183 \\
11331 \\
12035\end{array}$ \\
\hline
\end{tabular}




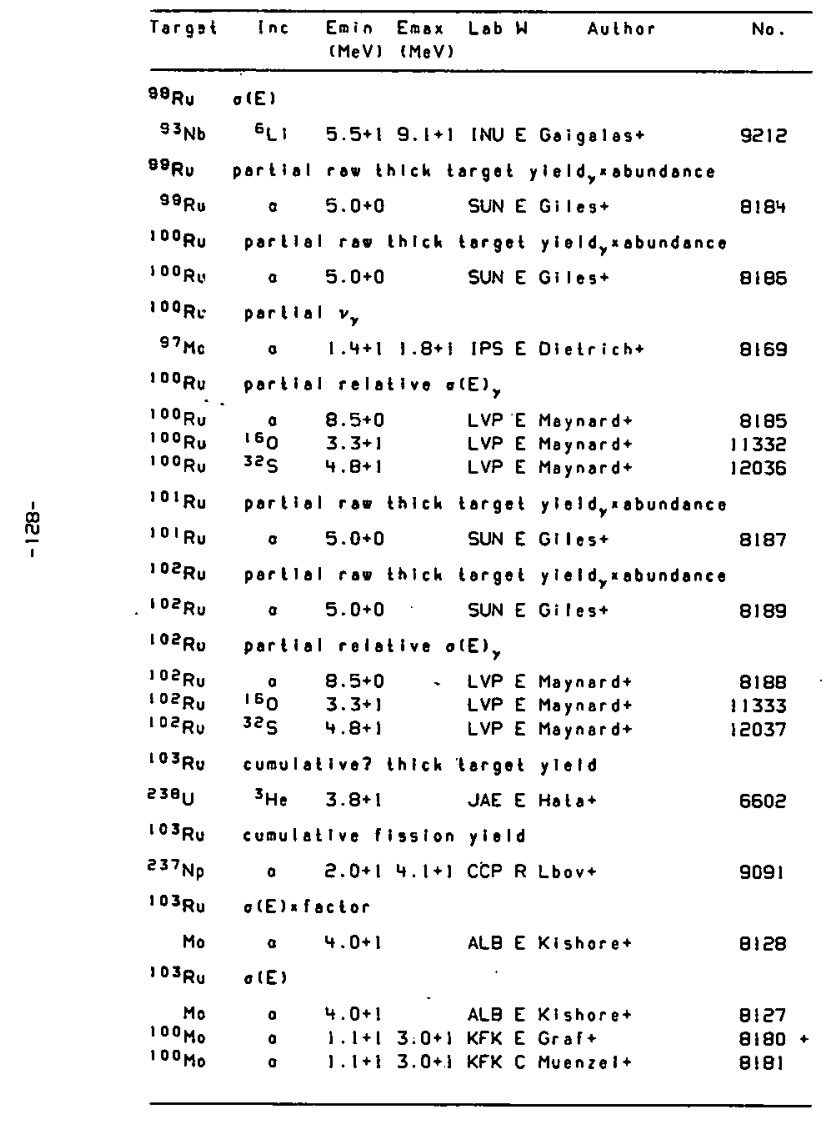

\begin{tabular}{|c|c|c|c|c|c|c|}
\hline Target & $\operatorname{lng}$ & $\begin{array}{l}E_{\min } \\
(\operatorname{MeV})\end{array}$ & $\begin{array}{l}\text { Emax } \\
(\operatorname{MeV})\end{array}$ & Lob W & Ajthor & No. \\
\hline $103 \mathrm{Ru}$ & $O(E)$ & & & & & \\
\hline $197 \mathrm{AU}$ & a & $6.5+1$ & $1.6+2$ & JUL $\varepsilon$ & Probs:+ & 8721 \\
\hline${ }^{104} \mathrm{Ru}$ & portis & l reot & thich t & torget & $y$ leldy abundance & \\
\hline $104 \mathrm{Ru}$ & a & $5.0+0$ & & SUN E & Giles- & 8191 \\
\hline${ }^{104} \mathrm{Ru}$ & portio & I relot & tive of & $(E)^{y}$ & & \\
\hline $\begin{array}{l}104 \mathrm{Ru} \\
104 \mathrm{Ru} \\
10^{4} \mathrm{Ru}\end{array}$ & $\begin{array}{l}10 \\
160 \\
325\end{array}$ & $\begin{array}{l}8 \cdot 5+0 \\
3 \cdot 3+1 \\
4.8+1\end{array}$ & & $\begin{array}{l}\text { LVP E } \\
\text { LVP E } \\
\text { LVP E }\end{array}$ & $\begin{array}{l}\text { Moynaed } \\
\text { Maynard+ } \\
\text { Maynard+ }\end{array}$ & $\begin{array}{r}8190 \\
11334 \\
12038\end{array}$ \\
\hline${ }^{105} \mathrm{Ru}$ & cumule & tive fl & 1ssion & $y 1010$ & & \\
\hline $\begin{array}{l}233_{\mathrm{U}} \\
237_{\mathrm{Np}}\end{array}$ & $\begin{array}{l}\text { d } \\
\text { a }\end{array}$ & $\begin{array}{l}9.0+0 \\
2.0+1\end{array}$ & $\begin{array}{l}2.3+1 \\
4.1+1\end{array}$ & $\begin{array}{l}\text { CCP R } \\
\text { CCP R }\end{array}$ & $\begin{array}{l}\text { Lbovt } \\
\text { Lbovt }\end{array}$ & $\begin{array}{l}5936 \\
9091\end{array}$ \\
\hline${ }^{106} R v$ & cumulo & tive it & ission & $y \operatorname{lold}$ & & \\
\hline $\begin{array}{l}23{ }^{3} N_{D} \\
2=45\end{array}$ & $v_{0}^{\circ}$ & $2.0+1$ & $4 \cdot 1+1$ & CCP R & Lbove & 9091 \\
\hline Ag & ${ }_{\mathrm{NO}} \mathrm{Ar}$ & $3.4+2$ & & PAR $R$ & Lefort+ & 12202 \\
\hline $99-R h$ & $O(E)$ & & & & & \\
\hline $\begin{array}{l}{ }^{99} \mathrm{Ru} \\
{ }^{9}{ }^{9} \mathrm{Ru}\end{array}$ & $\begin{array}{l}p \\
p\end{array}$ & $\begin{array}{l}1.2+1 \\
1.2+1\end{array}$ & & $\begin{array}{l}\text { KFK C } \\
\text { ORL E }\end{array}$ & $\begin{array}{l}\text { Muenzel+ } \\
\text { Blossert }\end{array}$ & $\begin{array}{l}3600 \\
3609+\end{array}$ \\
\hline $101-R h$ & cumul & ollues & groduct & 1 ylold & & \\
\hline Pd & $p$ & $6.3+1$ & & DAV E & Lagunas-Solart & 3628 \\
\hline $101 \cdot R h$ & cumul & ative $t$ & thick t & targot & $y 1010$ & \\
\hline Pd & $p$ & $4.0+1$ & $6.5+1$ & OAVE & Logunas-5olart & 3627 \\
\hline $101 \cdot R h$ & $\sigma(E)$ & - & & & : & \\
\hline $\begin{array}{l}101 \text { Ru } \\
101 \text { Ru }\end{array}$ & $p$ & $\begin{array}{l}1.2+1 \\
1.2+1\end{array}$ & & $\begin{array}{l}\text { KFK C } \\
\text { ORL E }\end{array}$ & $\begin{array}{l}\text { Muenzelt } \\
\text { Glossert }\end{array}$ & $\begin{array}{l}3610 \\
3611+\end{array}$ \\
\hline $10^{3} \mathrm{Rh}$ & portio & t rast & thick t & target & yield, sobundonce & \\
\hline $103 \mathrm{Rh}$ & o & $5.0+0$ & & SUN E & Gilest & 8194 \\
\hline${ }^{103} \mathrm{Rh}$ & produc & $t y 1010$ & & & : & \\
\hline $\mathrm{Zr}$ & $14 \mathrm{~N}$ & $4 \cdot 3+1$ & $5.5+1$ & PUR E & Poplit & 10413 \\
\hline
\end{tabular}




\begin{tabular}{|c|c|c|c|c|c|c|}
\hline Torget & $\ln c$ & $\begin{array}{l}\text { Emin } \\
(\mathrm{MeV})\end{array}$ & $\begin{array}{l}\text { Emax } \\
(\operatorname{MeV})\end{array}$ & Lab & Author & No. \\
\hline${ }^{103} \mathrm{Rh}$ & \multicolumn{6}{|c|}{ relalive o(E) } \\
\hline $2 r$ & ${ }^{14} \mathrm{~N}$ & $4.9+1$ & & \multicolumn{2}{|c|}{ PUR E Popl1+ } & 10414 \\
\hline$z=46$ & $v_{n}$ & & & & & \\
\hline Ag & $40 \mathrm{Ar}$ & $3.4+2$ & & \multicolumn{2}{|c|}{ PAR R Lefortt } & 12202 \\
\hline $25 p^{p d}$ & \multicolumn{6}{|c|}{ product yleld } \\
\hline $92 \mathrm{Zr}$ & 160 & $5.4+1$ & $6.6+1$ & PUR & E Popl1+ & 11324 \\
\hline $92 P d$ & \multicolumn{6}{|c|}{ relative,$(E)$} \\
\hline $92 \mathrm{Zr}$ & 160 & $6.0+1$ & & \multicolumn{2}{|c|}{ PUR E POPI1+ } & 11325 \\
\hline 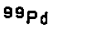 & \multicolumn{6}{|c|}{ Pelative $g(E)$} \\
\hline $\begin{array}{l}{ }^{70} \mathrm{Ge} \\
91 \mathrm{Zr}\end{array}$ & $\begin{array}{l}32_{5} \\
12 \mathrm{C}\end{array}$ & $\begin{array}{l}1 \cdot 1+2 \\
5 \cdot 6+1\end{array}$ & $\begin{array}{l}1.4+2 \\
6.6+1\end{array}$ & $\begin{array}{l}\mathrm{BNL} \\
\mathrm{BNL}\end{array}$ & $\begin{array}{l}\text { E Pleit } \\
\text { E Piel+ }\end{array}$ & $\begin{array}{r}12029 \\
9983\end{array}$ \\
\hline $100 \mathrm{pd}$ & \multicolumn{6}{|c|}{ cumulative $O(E)$} \\
\hline $238^{2}$ & $p$ & $1.0+3$ & $3.0+5$ & $\mathrm{KFK}$ & C Muenzelt & 4903 \\
\hline 10180 & \multicolumn{6}{|c|}{ cumulative thleh target yield } \\
\hline Pd & $p$ & $4.0+1$ & $6.5+1$ & DAV & E Lagunas-Solart & 3629 \\
\hline $101 \mathrm{Pd}$ & \multicolumn{6}{|c|}{ product yield } \\
\hline $\begin{array}{r}\mathrm{Zr} \\
103 \mathrm{Rh}\end{array}$ & ${ }^{14} \mathrm{~N}$ & $\begin{array}{l}4 \cdot 3+1 \\
5 \cdot 0+1\end{array}$ & $5.5+1$ & $\begin{array}{l}\text { PUR } \\
\text { DAV }\end{array}$ & $\begin{array}{l}\text { E Poplit } \\
\text { E Logunas-Solart }\end{array}$ & $\begin{array}{r}10413 \\
3615\end{array}$ \\
\hline $101 \mathrm{Pd}$ & \multicolumn{6}{|c|}{ relative $O(E)$} \\
\hline $\mathrm{Zr}$ & $14 \mathrm{~N}$ & $4.9+1$ & & PUR & E Popl1+ & 10414 \\
\hline $102 \mathrm{Pd}$ & \multicolumn{6}{|c|}{ partlal raw thich target yleldyabundance } \\
\hline $10 \mathrm{PPd}^{2}$ & a & $5.0+0$ & & SUN & E Gilest & 8203 \\
\hline $105 \mathrm{pq}$ & \multicolumn{6}{|c|}{ partiol relative o(E), } \\
\hline $\begin{array}{l}102 \mathrm{Pd} \\
102 \mathrm{Pd} \\
102 \mathrm{Pd}\end{array}$ & $\begin{array}{l}160 \\
325\end{array}$ & $\begin{array}{l}8.5+0 \\
3.3+1 \\
? .8+1\end{array}$ & & $\begin{array}{l}\text { LVP } \\
\text { LVP } \\
\text { LVP }\end{array}$ & $\begin{array}{l}\text { E Maynardt } \\
\text { E Maynardt } \\
\text { E Maynordt }\end{array}$ & $\begin{array}{r}8202 \\
11335 \\
12039\end{array}$ \\
\hline $102 \mathrm{Pd}$ & \multicolumn{6}{|c|}{ portial relative o(E) } \\
\hline $82 \mathrm{Zr}$ & ${ }^{13} \mathrm{C}$ & $5 \cdot 1+1$ & & PUR & E Graut & 10202 \\
\hline
\end{tabular}

\begin{tabular}{|c|c|c|c|c|c|c|c|}
\hline Target & $\ln c$ & $\begin{array}{l}\text { EnIn } \\
(\mathrm{MeV})\end{array}$ & $\begin{array}{l}\text { Emax } \\
(\operatorname{MeV})\end{array}$ & Lab & $W$ & Author & No. \\
\hline $102 \mathrm{pd}$ & \multicolumn{7}{|c|}{ product yield } \\
\hline $\begin{array}{r}\mathrm{Zr} \\
92 \mathrm{Zr}\end{array}$ & $\begin{array}{l}14 \mathrm{~N} \\
160\end{array}$ & $\begin{array}{l}4 \cdot 3+1 \\
5 \cdot 4+1\end{array}$ & $\begin{array}{l}5.5+1 \\
6.6+1\end{array}$ & $\begin{array}{l}\text { PUR } \\
\text { PUR }\end{array}$ & & $\begin{array}{l}\text { Poplit } \\
\text { Poplit }\end{array}$ & $\begin{array}{l}10413 \\
11324\end{array}$ \\
\hline $102 \mathrm{Pd}$ & \multicolumn{7}{|c|}{ Pelative $\sigma(E)$} \\
\hline${ }_{92}^{Z r}$ & $\begin{array}{l}14 \mathrm{~N} \\
160\end{array}$ & $\begin{array}{l}4.9+1 \\
6.0+1\end{array}$ & & $\begin{array}{l}\text { PUR } \\
\text { PUR }\end{array}$ & & $\begin{array}{l}\text { Poplit } \\
\text { Poplit }\end{array}$ & $\begin{array}{l}10414 \\
11325\end{array}$ \\
\hline $102 \mathrm{Pd}$ & $O(E)$ & & & & & & \\
\hline${ }^{103} \mathrm{Rh}$ & $\mathbf{p}$ & $1.4+1$ & & TOK & $\mathrm{E}$ & Sahait & 3616 \\
\hline $103 \mathrm{Pd}$ & \multicolumn{7}{|c|}{ cumulatlve o(E) } \\
\hline $\begin{array}{l}238 U \\
23 \theta_{U} \\
23 \theta_{U}\end{array}$ & $\begin{array}{l}p \\
p \\
p\end{array}$ & $\begin{array}{l}1.0+3 \\
4.5+2 \\
4.5+2\end{array}$ & $3.0+5$ & $\begin{array}{l}\text { KFK } \\
\text { KFK } \\
\text { CHI }\end{array}$ & $\begin{array}{l}c \\
c \\
\varepsilon\end{array}$ & $\begin{array}{l}\text { Muenzel+ } \\
\text { Muenzel+ } \\
\text { Panontint }\end{array}$ & $\begin{array}{l}4903 \\
4917 \\
4919+\end{array}$ \\
\hline${ }^{103} \mathrm{Pd}$ & \multicolumn{7}{|c|}{ product yield } \\
\hline $\begin{aligned} & 92 \mathrm{Zr} \\
& 23 \theta_{U}\end{aligned}$ & $\begin{array}{r}160 \\
p\end{array}$ & $\begin{array}{l}5 \cdot 4+1 \\
3 \cdot 0+5\end{array}$ & $6.6+1$ & $\begin{array}{l}\text { PUR } \\
\text { NAL }\end{array}$ & $E$ & $\begin{array}{l}\text { Poplit } \\
\text { Chongt }\end{array}$ & $\begin{array}{r}11324 \\
4880\end{array}$ \\
\hline $103 \mathrm{Pd}$ & \multicolumn{7}{|c|}{ relative o(E) } \\
\hline $92 \mathrm{Zr}$ & 160 & $6.0+1$ & & PUR & E & Poplit & 11325 \\
\hline $103 \mathrm{Pd}$ & \multicolumn{7}{|c|}{ o(E)xfactor } \\
\hline $\begin{array}{l}103 \mathrm{Rh} \\
103 \mathrm{Rh}\end{array}$ & p & $\begin{array}{l}2.5+0 \\
2.5+0\end{array}$ & $\begin{array}{l}7.0+0 \\
6.5+0\end{array}$ & $\begin{array}{l}\text { ORL } \\
\text { ORL }\end{array}$ & $M$ & $\begin{array}{l}\text { Johnsont } \\
\text { Johnsont }\end{array}$ & $\begin{array}{l}3622 \\
3623\end{array}$ \\
\hline $103 \mathrm{Pd}$ & \multicolumn{7}{|l|}{$\sigma(E)$} \\
\hline 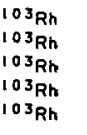 & $\begin{array}{l}p \\
p \\
p \\
p \\
p\end{array}$ & $\begin{array}{l}\text { TR } \\
2.3+0 \\
2.5+0 \\
4.0+0 \\
5.0+0\end{array}$ & $\begin{array}{l}5 \cdot 7+0 \\
1.0+1 \\
6 \cdot 0+0 \\
5 \cdot 5+0 \\
1.0+1\end{array}$ & $\begin{array}{l}\text { ORL } \\
\text { KFK } \\
\text { ORL } \\
\text { LRL } \\
\text { LRL }\end{array}$ & $\begin{array}{l}E \\
C \\
E \\
E \\
E\end{array}$ & $\begin{array}{l}\text { Johnsont } \\
\text { Muenzelt } \\
\text { Johnsont } \\
\text { Albert. } \\
\text { Honsent }\end{array}$ & $\begin{array}{l}3617+ \\
3618 \\
3619 \\
3620+ \\
3621+\end{array}$ \\
\hline $104 \mathrm{Pd}$ & \multicolumn{7}{|c|}{ independent relative $O(E)$} \\
\hline${ }^{103} \mathrm{Rh}$ & a & $5 \cdot 1+1$ & & KAZ & $E$ & Burtebaevt & 8195 \\
\hline $104 \mathrm{Pd}$ & \multicolumn{7}{|c|}{ partial raw thlek targot yieldyo } \\
\hline $104 \mathrm{Pc}$ & a & $5.0+0$ & & SUN & E & Gilest & 8208 \\
\hline
\end{tabular}




\begin{tabular}{|c|c|c|c|c|}
\hline Target & $\ln c$ & $\begin{array}{ll}\text { Emin } & \text { Emox } \\
(M e V) & (M e v) \\
\end{array}$ & Lab W & No. \\
\hline $104 \mathrm{Pd}$ & \multicolumn{4}{|c|}{ jortial relative o(E), } \\
\hline $\begin{array}{l}104 \mathrm{Pd} \\
104 \mathrm{Pd} \\
104 \mathrm{Pd}\end{array}$ & $\begin{array}{l}180^{\circ} \\
325\end{array}$ & $\begin{array}{l}8.5+0 \\
3.3+1 \\
4.8+1\end{array}$ & $\begin{array}{l}\text { LVP E Maynardt } \\
\text { LVP E Maynardt } \\
\text { LVP E Maynardt }\end{array}$ & $\begin{array}{r}8207 \\
11336 \\
12042\end{array}$ \\
\hline $104 \mathrm{Pd}$ & \multicolumn{4}{|c|}{ partial relative o(E) } \\
\hline $94 \mathrm{Zr}$ & ${ }^{13} \mathrm{c}$ & $4.7+1$ & PUR E Grout & 10203 \\
\hline $104 \mathrm{Pd}$ & \multicolumn{4}{|c|}{ product yield } \\
\hline $\begin{array}{l}\mathrm{zr} \\
\cdot 92 \mathrm{zr} \\
\operatorname{gezr}\end{array}$ & $\begin{array}{l}14 \mathrm{~N} \\
160 \\
160\end{array}$ & 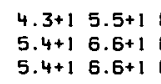 & $\begin{array}{l}\text { PUR E Poplit } \\
\text { PUR E Poplit } \\
\text { PUR E Poplit }\end{array}$ & $\begin{array}{l}10413 \\
11324 \\
11324\end{array}$ \\
\hline $104 \mathrm{pd}$ & \multicolumn{4}{|c|}{ relative o(E) } \\
\hline $\begin{array}{l}2 r \\
92 \mathrm{zr} \\
92 \mathrm{zr}\end{array}$ & $\begin{array}{l}14 \mathrm{~N} \\
160 \\
160\end{array}$ & $\begin{array}{l}4.9+1 \\
6.0+1 \\
6.0+1\end{array}$ & $\begin{array}{l}\text { PUR E Popllt+ } \\
\text { PUR E Poplit } \\
\text { PUR E Poplit }\end{array}$ & $\begin{array}{l}10414 \\
11325 \\
11325\end{array}$ \\
\hline $104 \mathrm{Pd}$ & $o(E)$ & & & \\
\hline $107 \mathrm{Ag}$ & $\rho$ & $2.3+1$ & MIL E Coll1t & 3699 \\
\hline $105 \mathrm{pd}$ & \multicolumn{4}{|c|}{ Independent relative o(E) } \\
\hline $103_{R h}$ & $a$ & $5.1+1$ & KAZ E Burtebaevt & 8196 \\
\hline${ }_{105} \mathrm{Pd}$ & \multicolumn{4}{|c|}{ partial product yleld } \\
\hline${ }^{96} \mathrm{Zr}$ & $12 \mathrm{c}$ & $4.5+1$ & PUR E Grau. & 9984 \\
\hline${ }^{105} \mathrm{Pd}$ & \multicolumn{4}{|c|}{ partial rav thich target yield, abundance } \\
\hline${ }^{105} \mathrm{Pd}$ & $\circ$ & $5.0+0$ & SUN E Gilest & 8213 \\
\hline $105 \mathrm{Pd}$ & \multicolumn{4}{|c|}{ Polative $\mathrm{I}_{y}$} \\
\hline $104 \mathrm{Ru}$ & a & $3.2+1$ & SUL E Grigorevt & 8192 \\
\hline $106 \mathrm{pd}$ & \multicolumn{4}{|c|}{ Independent relative o(E) } \\
\hline $103 \mathrm{Rh}$ & . & $5.1+1$ & KAZ E Burteboevt & 8197 \\
\hline${ }^{106} \mathrm{Pd}$ & \multicolumn{4}{|c|}{ partial relative o(E) } \\
\hline${ }^{98} \mathrm{zr}$ & ${ }^{23} \mathrm{C}$ & $4.5+1$ & PUR E Graut & 10204 \\
\hline${ }^{108} \mathrm{Pd}$ & \multicolumn{4}{|c|}{ product ylold } \\
\hline $\mathrm{zr}$ & $14 \mathrm{~N}$ & $4.3+15.5+1$ & PUR E Popll* & 10413 \\
\hline
\end{tabular}

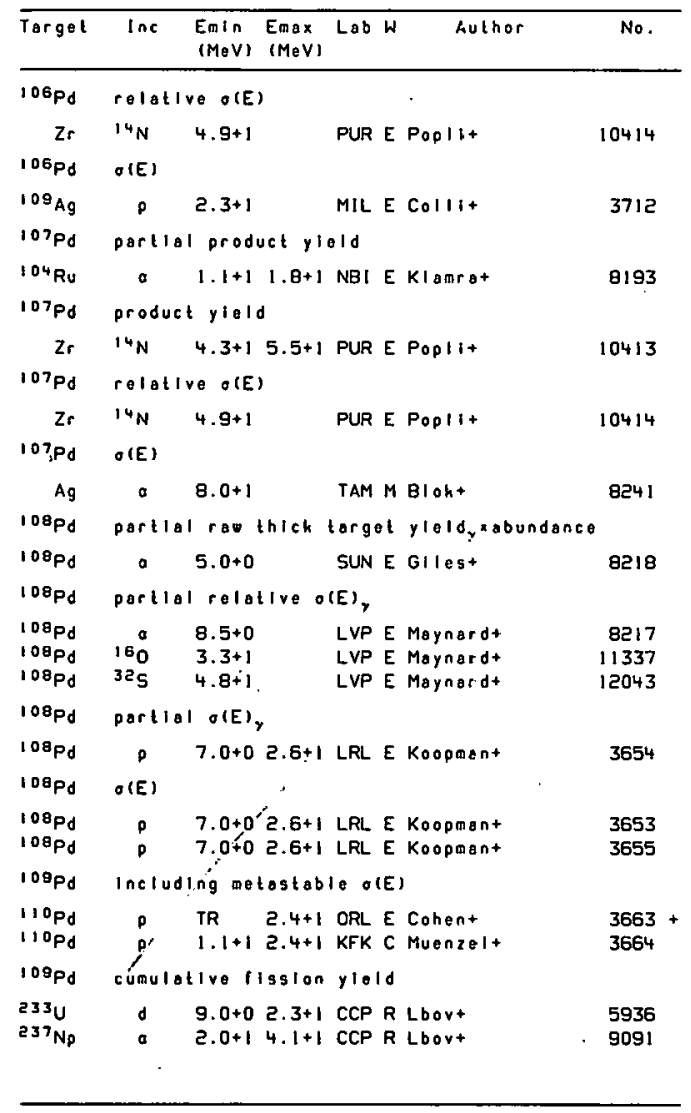




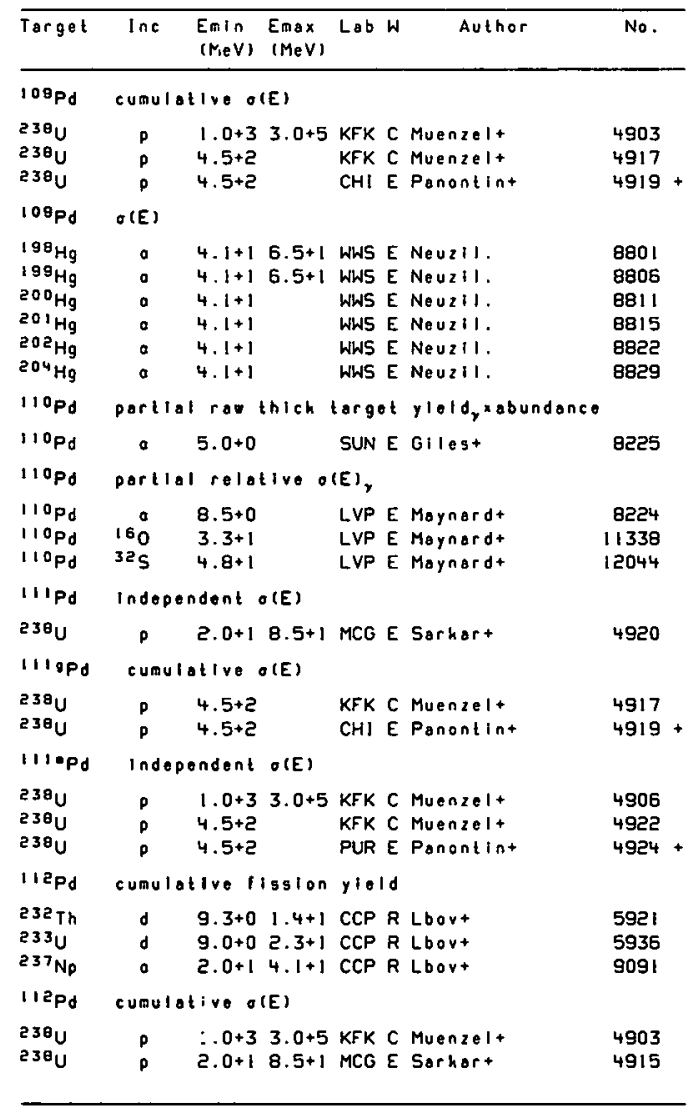

\begin{tabular}{|c|c|c|c|c|c|c|}
\hline Target & Inc & $\begin{array}{l}\text { Emin } \\
(\mathrm{MeV})\end{array}$ & $\begin{array}{l}\text { Emsx } \\
(M e V)\end{array}$ & Lab & Author & No. \\
\hline $112 \mathrm{pd}$ & \multicolumn{6}{|c|}{ cumulative o(E) } \\
\hline $\begin{array}{l}238 \mathrm{U} \\
238^{2} \mathrm{U}\end{array}$ & $p$ & $\begin{array}{l}4.5+2 \\
4.5+2\end{array}$ & & $\begin{array}{l}\mathrm{KFK} \\
\mathrm{CHI}\end{array}$ & $\begin{array}{l}\text { C Muenzel+ } \\
\text { E Panontint }\end{array}$ & $\begin{array}{l}4917 \\
4919\end{array}$ \\
\hline$\| \varepsilon^{p d}$ & \multicolumn{6}{|c|}{ fission yleldxfoctor } \\
\hline $\begin{array}{l}248 \mathrm{Cm} \\
112 \mathrm{Pd}\end{array}$ & \multicolumn{5}{|l|}{$O(E)$} & $9: 17$ \\
\hline $\begin{array}{l}198 \mathrm{Hg} \\
199 \mathrm{Hg} \\
200 \mathrm{Hg} \\
201 \mathrm{Hg} \\
202 \mathrm{Hg} \\
204 \mathrm{Hg}\end{array}$ & $\begin{array}{l}a \\
a \\
a \\
a \\
a \\
a\end{array}$ & $\begin{array}{l}4 \cdot 1+1 \\
4.1+1 \\
4 \cdot 1+1 \\
4.1+1 \\
4 \cdot 1+1 \\
4.1+1\end{array}$ & $\begin{array}{l}6.5+1 \\
6.5+1\end{array}$ & $\begin{array}{l}\text { WWS } \\
\text { WWS } \\
\text { WWS } \\
\text { WWS } \\
\text { WWS } \\
\text { WWS }\end{array}$ & $\begin{array}{l}\text { E Neuzil. } \\
\text { E Nevzil. } \\
\text { E Neuzil. } \\
\text { E Nevzil. } \\
\text { E Nevili. } \\
\text { E Nevzil. }\end{array}$ & $\begin{array}{l}8801 \\
8806 \\
8811 \\
8815 \\
8822 \\
8829\end{array}$ \\
\hline$z=47$ & $v_{n}$ & & & & & \\
\hline Ag & $40 \mathrm{Ar}$ & $3.4+2$ & & PAR & R Lefortt & 12202 \\
\hline $101 \mathrm{Ag}$ & \multicolumn{6}{|c|}{ product yleld } \\
\hline $\begin{array}{l}\text { Pd } \\
\text { IOI }\end{array}$ & $\begin{array}{c}P \\
\sigma(E)\end{array}$ & $6.6+1$ & & DAV & E Lagunag-Solart & 3630 \\
\hline $\begin{array}{l}104 \mathrm{Pd} \\
106 \mathrm{Pd}\end{array}$ & $\begin{array}{l}\sigma_{L i} \\
\sigma_{L} ;\end{array}$ & $\begin{array}{l}5.4+1 \\
5.4+1\end{array}$ & $\begin{array}{l}9.9+1 \\
9.9+1\end{array}$ & $\begin{array}{l}\text { INU } \\
\text { INU }\end{array}$ & $\begin{array}{l}\text { M Castanedat } \\
\text { M Costanedat }\end{array}$ & $\begin{array}{l}9220 \\
9239\end{array}$ \\
\hline $102 \mathrm{Ag}$ & $\sigma(E)$ & & & & & \\
\hline $\begin{array}{l}104 \mathrm{Pd} \\
104 \mathrm{Pd} \\
106 \mathrm{Pd}\end{array}$ & $\begin{array}{l}d \\
\sigma_{L ;} \\
\sigma_{L i}\end{array}$ & $\begin{array}{l}1.6+2 \\
5 \cdot 4+1 \\
5 \cdot 4+1\end{array}$ & $\begin{array}{l}9.9+1 \\
9.9+1\end{array}$ & $\begin{array}{l}\text { INU } \\
\text { INU } \\
\text { INU }\end{array}$ & $\begin{array}{l}\text { M Castanedat } \\
\text { M Costanedat } \\
\text { M Costanedat }\end{array}$ & $\begin{array}{l}5644 \\
9220 \\
9239\end{array}$ \\
\hline $103 \mathrm{Ag}$ & thich & torget & yiold & & & \\
\hline $\begin{array}{l}102 \mathrm{Pd} \\
103 \mathrm{Ag}\end{array}$ & $\begin{array}{c}P \\
O(E)\end{array}$ & $2.6+0$ & $6.8+0$ & $\mathrm{CLU}$ & M Roughtont & 3631 \\
\hline $\begin{array}{l}104 \mathrm{Pd} \\
104 \mathrm{Pd} \\
104 \mathrm{Pd} \\
108 \mathrm{Pd}\end{array}$ & $\begin{array}{c}0 \\
0 \\
\sigma_{L i} \\
6 L i\end{array}$ & $\begin{array}{l}1 \cdot 6+2 \\
8 \cdot 1+1 \\
5 \cdot 4+1 \\
5 \cdot 4+1\end{array}$ & $\begin{array}{l}9.9+1 \\
9.9+1\end{array}$ & $\begin{array}{l}\text { INU } \\
\text { INU } \\
\text { INU } \\
\text { INU }\end{array}$ & $\begin{array}{l}M \text { Castanedat } \\
M \text { Castanedat } \\
M \text { Costanedat } \\
M \text { Castanedat }\end{array}$ & $\begin{array}{l}5644 \\
8212 \\
9220 \\
9239\end{array}$ \\
\hline $104 \mathrm{Ag}$ & partio & 01 O(E) & & & & \\
\hline${ }^{103} \mathrm{Rh}$ & : & NDG & & GRN & E Ludziejewshit & 8198 \\
\hline
\end{tabular}




\begin{tabular}{|c|c|c|c|c|c|c|}
\hline Target & Inc & $\begin{array}{l}\text { Emln } \\
(\mathrm{MeV})\end{array}$ & $\begin{array}{l}\text { Emax } \\
(\operatorname{me} 2)\end{array}$ & Lab & Author & No. \\
\hline $104 \mathrm{Ag}$ & \multicolumn{6}{|c|}{ product yield } \\
\hline $\begin{array}{r}2 r \\
92 Z r\end{array}$ & $\begin{array}{l}14 \mathrm{~N} \\
160\end{array}$ & $\begin{array}{l}4.3+1 \\
5 \cdot 4+1\end{array}$ & $\begin{array}{l}5.5-1 \\
6.6-1\end{array}$ & $\begin{array}{l}\text { PUR } \\
\text { PUR }\end{array}$ & $\begin{array}{l}\text { E Popli+ } \\
\text { E Popli+ }\end{array}$ & $\begin{array}{l}10413 \\
11324\end{array}$ \\
\hline $104 \mathrm{Ag}$ & \multicolumn{6}{|c|}{ thlch torget yield } \\
\hline $104 \mathrm{Pd}$ & $p$ & $5.2+0$ & $6.0+0$ & CLU & M Roughtont & 3632 \\
\hline${ }^{104} \mathrm{Ag}$ & \multicolumn{6}{|c|}{ rolalive o(E) } \\
\hline $\begin{array}{rl}Z z & z r \\
& \end{array}$ & $\begin{array}{l}14 \mathrm{~N} \\
160\end{array}$ & $\begin{array}{l}4.9+1 \\
6.0+1\end{array}$ & & $\begin{array}{l}\text { PUR } \\
\text { PUR }\end{array}$ & $\begin{array}{l}\text { E Poplit } \\
\text { E Poplit }\end{array}$ & $\begin{array}{l}10414 \\
11325\end{array}$ \\
\hline $104 \mathrm{Ag}$ & \multicolumn{6}{|l|}{$\theta(\varepsilon)$} \\
\hline $\begin{array}{l}104 \mathrm{Pd} \\
104 \mathrm{Pd} \\
104 \mathrm{Pd} \\
106 \mathrm{Pd} \\
105 \mathrm{Pd}\end{array}$ & $\begin{array}{c}d \\
0 \\
\sigma_{L i} \\
d \\
\sigma_{L i}\end{array}$ & $\begin{array}{l}1.6+2 \\
8 \cdot 1+1 \\
5 \cdot 4+1 \\
1.6+2 \\
5.4+1\end{array}$ & $9.9+1$ & $\begin{array}{l}\text { INU } \\
\text { INU } \\
\text { INU } \\
\text { INU } \\
\text { INU }\end{array}$ & $\begin{array}{l}\text { M Castanedat } \\
\text { M Castanedat } \\
\text { M Cagtanedat } \\
\text { M Castanedat } \\
\text { M Castanedat }\end{array}$ & $\begin{array}{l}5644 \\
8212 \\
9220 \\
5545 \\
9239\end{array}$ \\
\hline $105 \mathrm{Ag}$ & \multicolumn{6}{|c|}{ cumulativo $O(E)$} \\
\hline $\begin{array}{l}238 U \\
238 U \\
238 U\end{array}$ & $\begin{array}{l}p \\
p \\
p\end{array}$ & $\begin{array}{l}1.0+3 \\
4.5+2 \\
4.5+2\end{array}$ & $3.0+5$ & $\begin{array}{l}\text { KFK } \\
\text { KFK } \\
\text { CHI }\end{array}$ & $\begin{array}{l}\text { C Muenzel+ } \\
\text { C Muenzelt } \\
\text { E Panontint }\end{array}$ & $\begin{array}{l}4903 \\
4917 \\
4918+\end{array}$ \\
\hline${ }^{105} \mathrm{Ag}$ & \multicolumn{6}{|c|}{ partlal relative $E(E)$} \\
\hline $105 p d$ & $p$ & $6.0+0$ & & $\mathrm{CCP}$ & E Mihut & 3638 \\
\hline${ }^{105} \mathrm{Ag}$ & \multicolumn{6}{|c|}{ relative $t_{y}$} \\
\hline $\begin{array}{l}103 \mathrm{Rh} \\
103 \mathrm{Rh} \\
105 \mathrm{Pd}\end{array}$ & $\begin{array}{l}a \\
a \\
p\end{array}$ & $\begin{array}{l}1.7+1 \\
1.9+1 \\
6.0+0\end{array}$ & $\begin{array}{l}3.2+1 \\
2.5+1\end{array}$ & $\begin{array}{l}\text { FUL } \\
\text { KLN } \\
\text { CCP }\end{array}$ & $\begin{array}{l}\text { E Kalshovent } \\
\text { E Hippet } \\
\text { E Mihut }\end{array}$ & $\begin{array}{l}8199 \\
8200 \\
3637\end{array}$ \\
\hline $105 \mathrm{Ag}$ & \multicolumn{6}{|c|}{ product yield } \\
\hline $\begin{array}{r}\mathrm{Zr} \\
92 \mathrm{Zr} \\
23 \mathrm{gu}^{-}\end{array}$ & $\begin{array}{r}14 \mathrm{~N} \\
160 \\
P\end{array}$ & $\begin{array}{l}4.3+1 \\
5.4+1 \\
3.0+5\end{array}$ & $\begin{array}{l}5.5+1 \\
6.6+1\end{array}$ & $\begin{array}{l}\text { PUR } \\
\text { PUR } \\
\text { NAL }\end{array}$ & $\begin{array}{l}\text { E Poplit } \\
\text { E Poplit } \\
\text { E Changt }\end{array}$ & $\begin{array}{r}10413 \\
11324 \\
4881\end{array}$ \\
\hline${ }^{105} \mathrm{Ag}$ & \multicolumn{6}{|c|}{ thlck target yleld } \\
\hline${ }^{105} \mathrm{Pd}$ & $p$ & $3.6+0$ & $6.0+0$ & $\mathrm{CLU}$ & M Roughtont & 3640 \\
\hline${ }^{105} \mathrm{Ag}$ & \multicolumn{6}{|c|}{ relotive of(E) } \\
\hline $\mathrm{Zr}$ & ${ }^{14} \mathrm{~N}$ & $4.9+1$ & & PUR & E Poplit & 10414 \\
\hline
\end{tabular}

\begin{tabular}{|c|c|c|c|c|c|c|}
\hline Targat & Inc & $\begin{array}{l}E_{m \mid n} \\
(M e V)\end{array}$ & $\begin{array}{l}\text { Emax } \\
(M e V)\end{array}$ & Lab h & Author & No. \\
\hline${ }^{105} \mathrm{Ag}$ & \multicolumn{6}{|c|}{ relative o(E) } \\
\hline $\begin{array}{r}92 \mathrm{Zr} \\
105 \mathrm{Pd}\end{array}$ & $\begin{array}{r}160 \\
p\end{array}$ & $\begin{array}{l}5.0+1 \\
2.1+0\end{array}$ & $5.7+0$ & $\begin{array}{l}\text { PUR E } \\
\text { ORL E }\end{array}$ & $\begin{array}{l}\text { E Poplit } \\
\text { E Johnsont }\end{array}$ & $\begin{array}{r}11325 \\
3636\end{array}$ \\
\hline${ }^{105} \mathrm{Ag}$ & \multicolumn{5}{|c|}{ O(E) Xf actor } & \\
\hline $\begin{array}{l}105 \mathrm{Pd} \\
105 \mathrm{Pd}\end{array}$ & $p$ & $\begin{array}{l}2.0+0 \\
2.5+0\end{array}$ & $\begin{array}{l}5.6+0 \\
7.0+0\end{array}$ & $\begin{array}{l}\text { ORL } \\
\text { ORL }\end{array}$ & $\begin{array}{l}\text { E Johnsont } \\
\text { M Johnsont }\end{array}$ & $\begin{array}{l}3634 \\
3635\end{array}$ \\
\hline${ }^{105} \mathrm{Ag}$ & $O(E)$ & & & & & \\
\hline $\begin{array}{l}104 \mathrm{Pd} \\
104 \mathrm{Pd} \\
104 \mathrm{Pd} \\
105 \mathrm{Pd} \\
106 \mathrm{Pd} \\
106 \mathrm{Pd}\end{array}$ & $\begin{array}{c}d \\
d \\
0 \\
{ }^{6} L i \\
p \\
d \\
{ }^{6} L_{i}\end{array}$ & $\begin{array}{l}1.6+2 \\
8 \cdot 1+1 \\
5 \cdot 4+1 \\
2.0+0 \\
1.6+2 \\
5 \cdot 4+1\end{array}$ & $\begin{array}{l}9.9+1 \\
5.6+0 \\
9.9+1\end{array}$ & $\begin{array}{l}\text { INU M } \\
\text { INU M } \\
\text { INU M } \\
\text { ORL E } \\
\text { INU M } \\
\text { INU M }\end{array}$ & $\begin{array}{l}\text { M Castanedat } \\
\text { M Castaredat } \\
M \text { Castaredat } \\
\text { E Johnsont } \\
\text { M Castanedat } \\
\text { M Castanecat }\end{array}$ & $\begin{array}{l}5644 \\
8212 \\
9220 \\
3639 \\
5645 \\
9239\end{array}$ \\
\hline \multicolumn{7}{|c|}{$105++g \mathrm{Ag}$, summation $O(E) \times$ factor } \\
\hline $\begin{array}{l}105 \mathrm{Pd} \\
105 \mathrm{Pd}\end{array}$ & $\begin{array}{l}\mathbf{p} \\
\mathbf{p}\end{array}$ & $\begin{array}{l}\text { TR } \\
2.1+0\end{array}$ & $\begin{array}{l}5.7+0 \\
5.7+0\end{array}$ & $\begin{array}{l}\text { ORL } \\
\text { KFK C }\end{array}$ & $\begin{array}{l}\text { E Johnsent } \\
\text { C Muenzelt }\end{array}$ & $\begin{array}{l}3641+ \\
3642\end{array}$ \\
\hline $106 \mathrm{Ag}$ & \multicolumn{6}{|c|}{ partial relative o(E) } \\
\hline${ }^{106} \mathrm{Pd}$ & $\rho$ & $6.5+0$ & & OHOE & E Devant & 3647 \\
\hline $106 \mathrm{Ag}$ & \multicolumn{6}{|c|}{ product yiold } \\
\hline $\begin{array}{r}2 r \\
106 \mathrm{Ag}\end{array}$ & \multicolumn{6}{|c|}{ ras o(E) } \\
\hline $\begin{array}{l}106 \mathrm{Pd} \\
106 \mathrm{Ag}\end{array}$ & $\stackrel{p}{\text { relst }}$ & \multicolumn{4}{|c|}{ relstive o(E) } & 3646 \\
\hline $\begin{array}{r}\mathrm{Zr} \\
106 \mathrm{Pd}\end{array}$ & ${ }^{14} \mathrm{~N}$ & $\begin{array}{l}4.9+1 \\
3.8+0\end{array}$ & $5.7+0$ & $\begin{array}{l}\text { PUR E } \\
\text { ORL E }\end{array}$ & $\begin{array}{l}\text { E Poplit } \\
\text { E Johnsoot }\end{array}$ & $\begin{array}{r}10414 \\
3644\end{array}$ \\
\hline $106 \mathrm{Ag}$ & \multicolumn{6}{|c|}{ O(E)xfoctor } \\
\hline $\begin{array}{l}106 \mathrm{Pd} \\
106 \mathrm{Ag}\end{array}$ & $\begin{array}{c}P \\
O(E)\end{array}$ & $2.0+0$ & $5.6+0$ & ORL E & E Johnsort & 3645 \\
\hline $\begin{array}{l}104 \mathrm{Pd} \\
104 \mathrm{Pd} \\
104 \mathrm{Pd} \\
106 \mathrm{Pd}\end{array}$ & $\begin{array}{c}d \\
0 \\
E_{L i} \\
d\end{array}$ & $\begin{array}{l}1.6+2 \\
8.1+1 \\
5.4+1 \\
1.6+2\end{array}$ & $9.9+1$ & $\begin{array}{l}\text { INU Y } \\
\text { INU Y } \\
\text { INU Y } \\
\text { INU T }\end{array}$ & $\begin{array}{l}\text { M Castanedat } \\
M \text { Castanedat } \\
M \text { Castaneda+ } \\
M \text { Castanedat }\end{array}$ & $\begin{array}{l}5644 \\
8212 \\
9220 \\
5645\end{array}$ \\
\hline
\end{tabular}




\begin{tabular}{|c|c|c|c|c|c|c|}
\hline Targel & $\ln c$ & $\begin{array}{l}\text { Emin } \\
(M e v) ~\end{array}$ & $\begin{array}{l}\text { Emax } \\
(\operatorname{MeV})\end{array}$ & Lab $h$ & Author & No. \\
\hline $106 \mathrm{Ag}$ & O(E) & & & & & \\
\hline $\begin{array}{l}106 \mathrm{Pd} \\
106 \mathrm{Pd} \\
107 \mathrm{Ag}\end{array}$ & $\underset{d}{6_{L I}^{a}}$ & $\begin{array}{l}8.1+1 \\
5.4+19 \\
T R\end{array}$ & $\begin{array}{l}9.9+1 \\
2.4+1\end{array}$ & $\begin{array}{l}\text { INU M } \\
\text { INU M } \\
\text { BRK E }\end{array}$ & $\begin{array}{l}\text { M Castonedat } \\
\text { M Castanedat } \\
\text { E Gonzalez-Vidalt }\end{array}$ & $\begin{array}{l}8216 \\
9239 \\
5651\end{array}$ \\
\hline $1069 A_{9}$ & lhlck & $\operatorname{target}$ & $y=10$ & & & \\
\hline $\begin{array}{l}106 \mathrm{Pd} \\
107 \mathrm{Ag}\end{array}$ & $\begin{array}{l}\text { p } \\
\text { d }\end{array}$ & $\begin{array}{l}4.0+0= \\
1.1+1=2\end{array}$ & $\begin{array}{l}6.0+0 \\
2.7+1\end{array}$ & $\begin{array}{l}\text { CLU M } \\
\text { CNE E }\end{array}$ & $\begin{array}{l}\text { M Roughiont } \\
\text { E Lallit }\end{array}$ & $\begin{array}{l}3648 \\
5653\end{array}$ \\
\hline $1060 \mathrm{Ag}$ & $O(E)$ & & & & & \\
\hline $\begin{array}{l}107 \mathrm{Ag} \\
107 \mathrm{Ag} \\
107 \mathrm{Ag}\end{array}$ & $\begin{array}{l}p \\
p \\
d\end{array}$ & $\begin{array}{ll}\text { TR } \\
1.3+1 \\
1.1+1\end{array}$ & $\begin{array}{l}2.4+1 \\
2 \cdot 4+1 \\
2 \cdot 7+1\end{array}$ & $\begin{array}{l}\text { ORL E } \\
\text { KFK C } \\
\text { CNE E }\end{array}$ & $\begin{array}{l}\text { E Cohent } \\
\text { C Muenzelt } \\
\text { E Lallit }\end{array}$ & $\begin{array}{l}3700+ \\
3701 \\
5652\end{array}$ \\
\hline $106 \cdot A 9$ & indepe & ondent. & $O(E)$ & & & \\
\hline $\begin{array}{l}53 \theta^{\prime} \\
238 U \\
238 U\end{array}$ & p & $\begin{array}{l}1.0+33 \\
4.5+2 \\
4.5+2\end{array}$ & $3.0+5$ & $\begin{array}{l}\text { KFK } \\
\text { KFK } \\
C H I \\
C H I\end{array}$ & $\begin{array}{l}\text { C Muenzel+ } \\
\text { C Muenzel+ } \\
\text { E Panontint }\end{array}$ & $\begin{array}{l}4906 \\
4922 \\
4923+\end{array}$ \\
\hline $105 . \mathrm{Ag}$ & produc & ct yold & & & & \\
\hline 2380 & p & $3.0+5$ & & NAL E & E Changt & 4882 \\
\hline 106.Ag & thick & torgot & $y 1010$ & & & \\
\hline $\begin{array}{l}106 \mathrm{Pd} \\
106 . \mathrm{Ag}\end{array}$ & $\begin{array}{c}p \\
\sigma(E)\end{array}$ & $4.0+0 \sigma$ & $6.0+0$ & CLU M & M Roughlont & 3649 \\
\hline $\begin{array}{l}107 \mathrm{Ag} \\
106 \%+\text { PAg }^{\circ}\end{array}$ & o & $\begin{array}{l}1.1+12 \\
\text { motton }\end{array}$ & $\begin{array}{l}2.7+1 \\
0(E) \times 1\end{array}$ & $\begin{array}{l}\text { CNE E } \\
\text { factor }\end{array}$ & E Lallit & 5654 \\
\hline 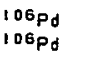 & p & $\begin{array}{ll}1.25 & 5 \\
3.8+0 & 5\end{array}$ & $\begin{array}{l}5.7+0 \\
5.7+0\end{array}$ & $\begin{array}{l}\text { ORL } \varepsilon \\
\text { KFK C }\end{array}$ & $\begin{array}{l}\text { E Johnsont } \\
\text { C Muenzolt }\end{array}$ & $\begin{array}{l}3650+ \\
3651\end{array}$ \\
\hline \multicolumn{7}{|c|}{ 108./OAg ratlo $\sigma(E)$} \\
\hline $107 \mathrm{Ag}$ & d & $1,1+1=$ & $2.7+1$ & CNE E & E LoIII+ & 5655 \\
\hline $107 \mathrm{Ag}$ & portial & i row th & thlck t & target & t yleldyobundonce & \\
\hline $107 \mathrm{Ag}$ & a & $5.0+0$ & & SUN E & E Gilest & 8242 \\
\hline $107 \mathrm{Ag}$ & portial & I relatt & tive ol & (E) & & \\
\hline $104 \mathrm{Ru}$ & ${ }^{6} L_{1}$ & $2.6+13$ & $3.4+1$ & KLN E & E Schuht & 9216 \\
\hline
\end{tabular}

\begin{tabular}{|c|c|c|c|c|c|c|}
\hline Torget & Inc & $\begin{array}{l}\text { Emin } \\
(M e V)\end{array}$ & $\begin{array}{l}\text { Emox } \\
(M e V)\end{array}$ & Lab & Author & No. \\
\hline $07 \mathrm{Ag}$ & \multicolumn{6}{|c|}{ product yiold } \\
\hline $2 r$ & $14 \mathrm{~N}$ & $4.3+1$ & $5.5+1$ & PUR E & E Poplit & 10413 \\
\hline $107 \mathrm{Ag}$ & \multicolumn{6}{|c|}{ relative o(E) } \\
\hline $\mathrm{zr}$ & ${ }^{14} \mathrm{~N}$ & $4.9+1$ & & PUR E & E Popl1- & 10414 \\
\hline $107 \mathrm{Ag}$ & \multicolumn{6}{|l|}{$O(E)$} \\
\hline $104 \mathrm{Pd}$ & $\therefore$ & $8.1+1$ & & INU M & M Costanedat & Bele \\
\hline${ }^{14} \mathrm{Pd}$ & ${ }^{6} \mathrm{LI}$ & $5.4+1$ & $9.9+1$ & INU $M$ & M Castanedat & 9220 \\
\hline${ }_{6}^{6} \mathrm{Pd}$ & d & $1,6+2$ & & INU $\mathrm{M}$ & M Castonedat & 5645 \\
\hline${ }_{0}^{6} \mathrm{Pd}$ & 0 & $8 \cdot 1+1$ & & INU $M$ & M Castanedat & 8216 \\
\hline${ }^{105} \mathrm{Pd}$ & ${ }^{6} \mathrm{LI}$ & $5.4+1$ & $9.9+1$ & INU $\mathrm{M}$ & M Costanedat & 9239 \\
\hline $\begin{array}{r}A g \\
110 \mathrm{Co}\end{array}$ & $\dot{p}$ & $\begin{array}{l}8.0+1 \\
1.6+1\end{array}$ & & $\begin{array}{l}\text { TAM M } \\
\text { PUR E }\end{array}$ & $\begin{array}{l}\text { M Eloht } \\
\text { E Luxt }\end{array}$ & $\begin{array}{l}8241 \\
3738\end{array}$ \\
\hline $108 \mathrm{Ag}$ & \multicolumn{6}{|c|}{ portiol o(E) } \\
\hline $108 \mathrm{Pd}$ & p & $2.6+0$ & $4.0+0$ & TITE & E Hottorit & 3857 \\
\hline${ }^{108} \mathrm{Ag}$ & \multicolumn{6}{|c|}{$\operatorname{rav} O(E)$} \\
\hline${ }^{108 \mathrm{Pd}}$ & $p$ & $2.5+0$ & $3.2+0$ & ORL E & E Johnsont & 3659 \\
\hline $10 \theta_{A g}$ & \multicolumn{6}{|c|}{ Pelative o(E) } \\
\hline $100 \mathrm{Pd}$ & $p$ & $2.4+0$ & $5.7+0$ & ORL E & E Johnsont & 3656 \\
\hline${ }^{100} \mathrm{Ag}$ & \multicolumn{6}{|c|}{ olEinfactor } \\
\hline${ }^{108} \mathbf{P d}$ & p & $2.0+0$ & $5.6+0$ & ORL & E Johnsont & 3658 \\
\hline $108 \mathrm{Ag}$ & \multicolumn{6}{|l|}{$O(E)$} \\
\hline $104 \mathrm{Pd}$ & ${ }^{6} \mathrm{LI}$ & $5.4+1$ & $9.9+1$ & INU $M$ & MCestanedat & 9220 \\
\hline${ }_{60}$ & d & $1.6+2$ & & INU $\mathrm{M}$ & $M$ Costanedat & 5645 \\
\hline $105 \mathrm{Pd}$ & $a$ & $8.1+1$ & & INU $M$ & M Castanedat & 8216 \\
\hline $105 \mathrm{Pd}$ & ${ }^{6} L_{1}$ & $5.4+1$ & $9.9+1$ & INU $\mathrm{F}$ & M Castanedat & 9239 \\
\hline Ag & a & $8.0+1$ & & TAM $M$ & $M$ Bloht & $8 \approx 41$ \\
\hline & d & TR & $2.4+1$ & BRK E & E Gonzalez- & 5656 \\
\hline $108 n+0 A_{9}$ & \multicolumn{2}{|c|}{9 gumation } & $O(E) \times 1$ & ractor & & \\
\hline $\begin{array}{l}108_{P d} \\
100 \mathrm{Pd}\end{array}$ & $p$ & $\begin{array}{l}\text { TR } \\
2.4+0\end{array}$ & $\begin{array}{l}5.7+0 \\
5.7+0\end{array}$ & $\begin{array}{l}\text { ORL } \\
\text { KFK }\end{array}$ & $\begin{array}{l}\text { E Johnsont } \\
\text { C Muenzel+ }\end{array}$ & $\begin{array}{l}3660+ \\
3661\end{array}$ \\
\hline $109_{A S}$ & \multicolumn{6}{|c|}{ portlal rav thlch target yleldy" } \\
\hline${ }^{109} \mathrm{Ag}$ & - & $5.0+0$ & & SUN E & E Gilest & 8247 \\
\hline
\end{tabular}




\begin{tabular}{|c|c|c|c|c|c|c|}
\hline Target & Inc & $\begin{array}{l}\mathrm{Em} / \mathrm{n} \\
(\mathrm{MeV})\end{array}$ & $\begin{array}{l}E \max \\
(\mathrm{MeV})\end{array}$ & Lab & Author & No. \\
\hline $100_{\mathrm{Ag}}$ & $O(E)$ & & & & & \\
\hline $\begin{array}{r}106 \mathrm{Pd} \\
{ }^{106} \mathrm{Pd} \\
\mathrm{Ag}\end{array}$ & ${ }_{a}^{c} L_{1}$ & $\begin{array}{l}8 \cdot 1+1 \\
5 \cdot 4+1 \\
8 \cdot 0+1\end{array}$ & $9.9+1$ & $\begin{array}{l}\text { INU I } \\
\text { INU I } \\
\text { TAM I }\end{array}$ & $\begin{array}{l}\text { M Castaneda+ } \\
\text { M Castaneda+ } \\
\text { M Bloht }\end{array}$ & $\begin{array}{l}8216 \\
9239 \\
8241\end{array}$ \\
\hline $110^{\circ} \mathrm{g}$ & partl & $\mid \sigma(E)$ & & & & \\
\hline $110 \mathrm{Pd}$ & D & $1.6+0$ & $4.0+0$ & TIT & E Hattorit & 3666 \\
\hline $110_{\mathrm{Ag}}$ & $\sigma(\varepsilon) \times$ & actor & & & & \\
\hline $\begin{array}{l}110 \mathrm{Pd} \\
110_{\mathrm{Ag}}\end{array}$ & $\begin{array}{c}P \\
O(E)\end{array}$ & $2.0+0$ & $5.6+0$ & ORL & E Johnsont. & 3665 \\
\hline $\begin{array}{r}106 \mathrm{Pd} \\
106 \mathrm{Pd} \\
110 \mathrm{Pd} \\
\mathrm{Ag}\end{array}$ & $\begin{array}{c}a \\
{ }^{a} L_{1} \\
p \\
a\end{array}$ & $\begin{array}{l}8 \cdot 1+1 \\
5 \cdot 4+1 \\
2.5+0 \\
8.0+1\end{array}$ & $\begin{array}{l}9.9+1 \\
6.0+0\end{array}$ & $\begin{array}{l}\text { INU I } \\
\text { INU I } \\
\text { ORL } \\
\text { TAM I }\end{array}$ & $\begin{array}{l}\text { M Castanedat } \\
\text { M Castanedat } \\
\text { E Johnsont } \\
\text { M Bloht }\end{array}$ & $\begin{array}{l}8216 \\
9239 \\
3667 \\
8241\end{array}$ \\
\hline $1100_{A g}$ & inde & endent & $O(E)$ & & & \\
\hline $\begin{array}{l}539^{4} \\
23 \theta^{4}\end{array}$ & $\begin{array}{l}p \\
p\end{array}$ & $\begin{array}{l}4.5+2 \\
4.5+2\end{array}$ & & $\begin{array}{l}\text { KFK } \\
\mathrm{CHI}\end{array}$ & $\begin{array}{l}\text { C Muenzel+ } \\
\text { E Panontint }\end{array}$ & $\begin{array}{l}4922 \\
4923+\end{array}$ \\
\hline $1100 \mathrm{Ag}$ & inde & endent & $\sigma(E)$ & & & \\
\hline $\begin{array}{l}230^{\mathrm{U}} \\
1100 \mathrm{Ag}\end{array}$ & $\begin{array}{c}p \\
O(E)\end{array}$ & $1.0+3$ & $3.0+5$ & KFK & C Muenzelt & 4906 \\
\hline $\begin{array}{l}197 \mathrm{AU} \\
1100.0 \mathrm{Ag}\end{array}$ & 9 & $\begin{array}{l}9.0+1 \\
\text { mation }\end{array}$ & $\begin{array}{l}1.6+2 \\
O(E) \times f\end{array}$ & $\begin{array}{l}\text { JUL } \\
\text { factor }\end{array}$ & $\begin{array}{l}\text { E Probst+ } \\
\text { or }\end{array}$ & 8722 \\
\hline $\begin{array}{l}110 \mathrm{Pd} \\
110 \mathrm{Pd}\end{array}$ & $\begin{array}{l}p \\
p\end{array}$ & $\begin{array}{l}T R \\
2.1+0\end{array}$ & $\begin{array}{l}5.7+0 \\
5.7+C\end{array}$ & $\begin{array}{l}\text { ORL } \\
\text { KFK }\end{array}$ & $\begin{array}{l}\text { E Johnsont } \\
\text { C Muenzelt }\end{array}$ & $\begin{array}{l}3670+ \\
3671\end{array}$ \\
\hline $1100.8 \mathrm{Ag}$ & suc & Ination & $O(E)$ & & & \\
\hline $\begin{array}{l}110 \mathrm{Pd} \\
110 \mathrm{Pd}\end{array}$ & $p$ & $\begin{array}{l}\text { TR } \\
2.1+0\end{array}$ & $\begin{array}{l}5.7+0 \\
5.7+0\end{array}$ & $\begin{array}{l}\text { ORL } \\
\text { KFK }\end{array}$ & $\begin{array}{l}\text { E Johngont } \\
\text { C Muenzel+ }\end{array}$ & $\begin{array}{l}3668+ \\
3669\end{array}$ \\
\hline $111 \mathrm{Ag}$ & Indep & ndento & o(E) & & & \\
\hline $\begin{array}{l}23 \mathrm{~B}^{\mathrm{U}} \\
111 \mathrm{Ag}\end{array}$ & $\begin{array}{c}P \\
\text { cumul }\end{array}$ & $\begin{array}{l}2.0+1 \\
\text { the } f 1\end{array}$ & $\begin{array}{l}\text { 8.5+1 } \\
\text { Ission }\end{array}$ & $\begin{array}{l}\text { MCG } \\
\text { yiel }\end{array}$ & $\begin{array}{l}\text { E Sorkart } \\
\text { Id }\end{array}$ & 4920 \\
\hline $\begin{array}{l}232 \mathrm{Th} \\
237 \mathrm{~Np}\end{array}$ & d & $\begin{array}{l}9.3+0 \\
2.0+1\end{array}$ & $\begin{array}{l}1.4+1 \\
4.1+1\end{array}$ & $\begin{array}{l}\text { CCP F } \\
\text { CCP F }\end{array}$ & $\begin{array}{l}\text { R Lbovt } \\
R \text { Lbove }\end{array}$ & $\begin{array}{l}5921 \\
9091\end{array}$ \\
\hline
\end{tabular}

\begin{tabular}{|c|c|c|c|c|c|c|}
\hline Tafget & $\ln c$ & $\begin{array}{l}E \min \\
(\mathrm{MeV})\end{array}$ & $\begin{array}{l}\text { max } \\
(\mathrm{MeV})\end{array}$ & Lab & Gu:hor & No. \\
\hline $111 \mathrm{Ag}$ & \multicolumn{6}{|c|}{ cumulative o(E). } \\
\hline $\begin{array}{l}238 \mathrm{U} \\
238 \mathrm{U} \\
23 \theta_{\mathrm{U}} \\
238_{\mathrm{U}}\end{array}$ & $\begin{array}{l}p \\
p \\
p \\
p\end{array}$ & $\begin{array}{l}1.0+3 \\
2.0+1 \\
4.5+2 \\
4.5+2\end{array}$ & $\begin{array}{l}3.0+5 \\
8.5+1\end{array}$ & $\begin{array}{l}\text { KFK } \\
\text { MCG } \\
\text { KFK } \\
\text { CH! }\end{array}$ & $\begin{array}{l}\text { C Muenzel+ } \\
\text { E Sarkirt+ } \\
\text { C Muentel+ } \\
\text { E Panontint }\end{array}$ & $\begin{array}{l}4903 \\
4915 \\
4917 \\
4919+\end{array}$ \\
\hline $111_{A g}$ & \multicolumn{6}{|c|}{ product yiold } \\
\hline $23 \theta_{0}$ & $p$ & $3.0+5$ & & NAL & E Chang* & 4883 \\
\hline $111 \mathrm{Ag}$ & \multicolumn{6}{|c|}{ Hasion ylaldxioctor } \\
\hline $248 \mathrm{Cm}$ & $a$ & $2.4+1$ & $3.4+1$ & KUR & E Lisint & 9117 \\
\hline $111_{A g}$ & \multicolumn{6}{|l|}{$O(E)$} \\
\hline $\begin{array}{l}190_{\mathrm{Hg}} \\
199 \mathrm{Hg} \\
200 \mathrm{Hg} \\
201 \mathrm{Hg} \\
202 \mathrm{Hg} \\
204 \mathrm{Hg}\end{array}$ & $\begin{array}{l}\dot{0} \\
0 \\
0 \\
0 \\
0 \\
0\end{array}$ & $\begin{array}{l}4.1+1 \\
4.1+1 \\
4.1+1 \\
4.1+1 \\
4.1+1 \\
4.1+1\end{array}$ & $\begin{array}{l}6.5+1 \\
6.5+1\end{array}$ & $\begin{array}{l}\text { WWS } \\
\text { WWS } \\
\text { WWS } \\
\text { WWS } \\
\text { WWS } \\
\text { WWS }\end{array}$ & $\begin{array}{l}\text { E Neuzil. } \\
\text { E Neuzil. } \\
\text { E Neuzil. } \\
\text { E Neuzil. } \\
\text { E Neuzil. } \\
\text { E Neuzil. }\end{array}$ & $\begin{array}{l}8801 \\
8806 \\
8811 \\
8815 \\
8822 \\
8829\end{array}$ \\
\hline $112 \mathrm{Ag}$ & \multicolumn{6}{|c|}{ independent fission yield } \\
\hline $209_{B i}$ & ${ }^{12} \mathrm{C}$ & $7.5+1$ & $1.0+2$ & HAR & M Branquinhot & 10152 \\
\hline $112 \mathrm{Ag}$ & \multicolumn{6}{|c|}{ Independent $\sigma(E)$} \\
\hline 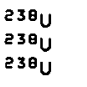 & $\begin{array}{l}p \\
p \\
p\end{array}$ & $\begin{array}{l}2.0+1 \\
4.5+2 \\
4.5+2\end{array}$ & 8.5+1 & $\begin{array}{l}\text { MCG } \\
\text { KFK } \\
\text { PUR }\end{array}$ & $\begin{array}{l}\text { E Sarkart } \\
\text { C Muenzel+ } \\
\text { E Panontint }\end{array}$ & $\begin{array}{l}4920 \\
4922 \\
4924\end{array}$ \\
\hline $11 a_{A g}$ & \multicolumn{6}{|c|}{ cumulative flssion yield } \\
\hline $\begin{array}{l}232 \mathrm{Th} \\
23^{3} \mathrm{~Np}\end{array}$ & d & $\begin{array}{l}9.3+0 \\
2.0+1\end{array}$ & $\begin{array}{l}1.4+1 \\
4.1+1\end{array}$ & $\begin{array}{l}\text { CCP } \\
\text { CCP }\end{array}$ & $\begin{array}{l}R \text { Lbovt } \\
R \text { Lbovt }\end{array}$ & $\begin{array}{l}5921 \\
9091\end{array}$ \\
\hline $113 \mathrm{Ag}$ & \multicolumn{6}{|c|}{ Independent fission yield } \\
\hline $\begin{array}{l}209_{B i} \\
113_{A g}\end{array}$ & \multicolumn{6}{|c|}{ cumulative lisslon ylold } \\
\hline $\begin{array}{l}232 \mathrm{Th} \\
237_{\mathrm{Ng}}\end{array}$ & d & $\begin{array}{l}9.3+0 \\
2.0+1\end{array}$ & $\begin{array}{l}1.4+1 \\
4.1+1\end{array}$ & $\begin{array}{l}\mathrm{CCP} \\
\mathrm{CCP}\end{array}$ & $\begin{array}{l}R \text { Lbovt } \\
R \text { Lbovt }\end{array}$ & $\begin{array}{l}5921 \\
9091\end{array}$ \\
\hline $113 \mathrm{Ag}$ & \multicolumn{6}{|c|}{ cumulative $O(E)$} \\
\hline $238^{4}$ & ค & $2.0+1$ & $8.5+1$ & MCG & E Sarkan-t & 4915 \\
\hline
\end{tabular}




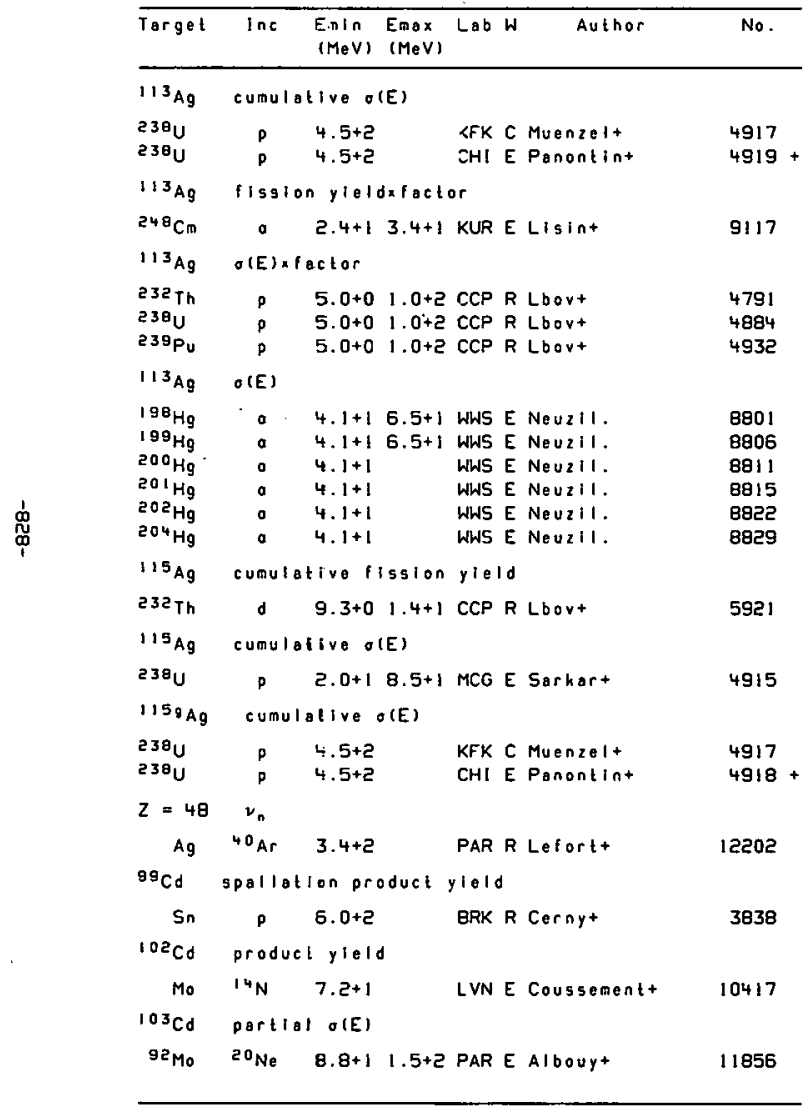

\begin{tabular}{|c|c|c|c|c|c|c|}
\hline Target & $\ln c$ & $\begin{array}{l}\text { Emin } \\
(\mathrm{MeV})\end{array}$ & $\begin{array}{l}\text { Emax } \\
(\operatorname{MeV})\end{array}$ & Lab & Author & No. \\
\hline${ }^{103} \mathrm{Cs}$ & \multicolumn{6}{|c|}{ produci yleld } \\
\hline Mo & $14 N$ & $7.2+1$ & & LVN & E Coussement+ & 10417 \\
\hline${ }^{103} \mathrm{Co}$ & $\theta(E)$ & & & & & \\
\hline $\begin{array}{l}104 \mathrm{Pd} \\
106 \mathrm{Pd}\end{array}$ & $\begin{array}{l}6_{L i} \\
{ }^{6} L i\end{array}$ & $\begin{array}{l}5.4+1 \\
5.4+1\end{array}$ & $\begin{array}{l}9.9+1 \\
9.9+1\end{array}$ & $\begin{array}{l}\text { INU } \\
\text { INU }\end{array}$ & $\begin{array}{l}\text { MCastanedat } \\
\text { M Castanedat }\end{array}$ & $\begin{array}{l}9219 \\
9238\end{array}$ \\
\hline $104 \mathrm{Cd}$ & \multicolumn{6}{|c|}{ partial product yield } \\
\hline $104 \mathrm{Ag}$ & $p$ & TR & $1.4+0$ & OHO & E Devan. & 3695 \\
\hline${ }^{104} \mathrm{Cd}$ & \multicolumn{6}{|c|}{ partial o(E) } \\
\hline $\begin{array}{r}92 \mathrm{Mo} \\
104 \mathrm{Pd}\end{array}$ & $\begin{array}{r}20 \mathrm{Ne} \\
{ }^{3} \mathrm{He}\end{array}$ & $\begin{array}{l}8.5+1 \\
2.6+1\end{array}$ & $\begin{array}{l}1.5+2 \\
4.6+1\end{array}$ & $\begin{array}{l}\text { PAR } \\
\text { JCL }\end{array}$ & $\begin{array}{l}\text { E Albouyt } \\
\text { E Hashizumet }\end{array}$ & $\begin{array}{r}11857 \\
6440\end{array}$ \\
\hline${ }^{104} \mathrm{Cd}$ & \multicolumn{6}{|c|}{ celotive 1 , } \\
\hline $102 \mathrm{Pd}$ & $a$ & $3.3+1$ & & GRE & E Genevey-Riviert & 8204 \\
\hline${ }^{104} \mathrm{Cd}$ & \multicolumn{6}{|c|}{ product ylald } \\
\hline${ }^{92} \mathrm{Zr}$ & 150 & $5.4+1$ & $6.6+1$ & PUR & E Poplit & 11324 \\
\hline $104 \mathrm{Cd}$ & \multicolumn{6}{|c|}{ relative o(E) } \\
\hline $92_{2} r$ & 160 & $6.0+1$ & & PUR & E Poplit & 11325 \\
\hline $104 \mathrm{Cd}$ & \multicolumn{6}{|l|}{$O(E)$} \\
\hline $\begin{array}{l}104 \mathrm{Pd} \\
104 \mathrm{Pd} \\
106 \mathrm{Pd}\end{array}$ & $\begin{array}{l}\sigma_{L I}^{0} \\
\sigma_{L I}\end{array}$ & $\begin{array}{l}8.1+1 \\
5.4+1 \\
5.4+1\end{array}$ & $\begin{array}{l}9.9+1 \\
5.9+1\end{array}$ & $\begin{array}{l}\text { INU } \\
\text { INU } \\
\text { INU }\end{array}$ & $\begin{array}{l}\text { M Castanedat } \\
\text { M Castanedat } \\
\text { M Castanedat }\end{array}$ & $\begin{array}{l}8211 \\
9219 \\
9238\end{array}$ \\
\hline${ }^{105} \mathrm{Cd}$ & \multicolumn{6}{|c|}{ partlal o(E) } \\
\hline $\begin{array}{l}92 \mathrm{Mo} \\
{ }^{105} \mathrm{Cd}\end{array}$ & $\begin{array}{l}20 \mathrm{Ne} \\
\text { relot }\end{array}$ & \multicolumn{5}{|c|}{ relotive I, } \\
\hline $\begin{array}{l}102 \mathrm{Pd} \\
104 \mathrm{Pd}\end{array}$ & $\therefore$ & $\begin{array}{l}2.4+1 \\
3.7+1\end{array}$ & $4.8+1$ & $\begin{array}{l}\text { GRE } \\
\text { GRE }\end{array}$ & $\begin{array}{l}\text { E Genevey-Riviert } \\
\text { E Genevey-Riviert }\end{array}$ & $\begin{array}{l}8205 \\
8209\end{array}$ \\
\hline${ }^{105} \mathrm{Cd}$ & \multicolumn{6}{|c|}{ oroducl yield } \\
\hline $92 \mathrm{Zr}$ & 150 & $5.4+i$ & $6.6+1$ & PUR & E Poplit & 11324 \\
\hline${ }^{105} \mathrm{Cd}$ & \multicolumn{6}{|c|}{ relotive ofE } \\
\hline $82 \mathrm{Zr}$ & ${ }^{150}$ & $6.0+1$ & & PUR & E Poplit & 11325 \\
\hline
\end{tabular}




\begin{tabular}{|c|c|c|c|c|c|c|}
\hline Target & $\operatorname{lnc}$ & $\begin{array}{l}\text { Emin } \\
(\mathrm{MeV})\end{array}$ & $\begin{array}{l}E \max \\
(\operatorname{MeV})\end{array}$ & Lab W & Author & No. \\
\hline${ }^{105} \mathrm{Cd}$ & $\sigma(E)$ & & & & & \\
\hline $\begin{array}{l}104 \mathrm{Pd} \\
104 \mathrm{Pd} \\
106 \mathrm{Pd}\end{array}$ & $\begin{array}{l}\sigma_{L i} \\
\sigma_{L i}\end{array}$ & $\begin{array}{l}8 \cdot 1+1 \\
5 \cdot 4+1 \\
5 \cdot 4+1\end{array}$ & $\begin{array}{l}9.9+1 \\
9.9+1\end{array}$ & $\begin{array}{l}\text { INU M } \\
\text { INU M } \\
\text { INU M }\end{array}$ & $\begin{array}{l}\text { M Castanedat } \\
\text { M Castanedat } \\
\text { M Castanedat }\end{array}$ & $\begin{array}{l}9211 \\
9219 \\
9238\end{array}$ \\
\hline${ }^{106} \mathrm{Cd}$ & \multicolumn{6}{|c|}{ partial product yletd } \\
\hline $\begin{array}{l}{ }^{106} \mathrm{Ag} \\
{ }^{106} \mathrm{Cd}\end{array}$ & $\begin{array}{c}p \\
\text { parll }\end{array}$ & \multicolumn{4}{|c|}{ partlal relative o(E) } & 3696 \\
\hline $\begin{array}{l}105 \mathrm{Cd} \\
106 \mathrm{Cd} \\
106 \mathrm{Cd}\end{array}$ & $\begin{array}{l}150 \\
325\end{array}$ & $\begin{array}{l}8.5+0 \\
3.3+1 \\
4.8+1\end{array}$ & & $\begin{array}{l}\text { LVP E } \\
\text { LVP E } \\
\text { LVP E }\end{array}$ & $\begin{array}{l}\text { E Maynard+ } \\
\text { Eaynard+ } \\
\text { E Maynard+ }\end{array}$ & $\begin{array}{r}8257 \\
11339 \\
12045\end{array}$ \\
\hline${ }^{106} \mathrm{Cd}$ & \multicolumn{6}{|c|}{ partlal relotive o(E) } \\
\hline $\begin{array}{l}104 \mathrm{Pd} \\
{ }^{108} \mathrm{Cd}\end{array}$ & \multicolumn{6}{|c|}{ relotive $t_{y}$} \\
\hline $\begin{array}{l}102 \mathrm{Pd} \\
106 \mathrm{Cd}\end{array}$ & $I_{y}^{0}$ & \multicolumn{4}{|c|}{$\mathbf{I}_{y}$} & 8206 \\
\hline $\begin{array}{l}94 \mathrm{Zr} \\
96 \mathrm{Mo} \\
97 \mathrm{Mo}\end{array}$ & $\begin{array}{l}160 \\
13 \mathrm{C} \\
12 \mathrm{C}\end{array}$ & $\begin{array}{l}6 \cdot 3+1 \\
3 \cdot 7+1 \\
3 \cdot 7+1\end{array}$ & $\begin{array}{l}4.7+1 \\
4.7+1\end{array}$ & $\begin{array}{l}\text { PUR E } \\
\text { PUR E } \\
\text { PUR E }\end{array}$ & $\begin{array}{l}\text { E Samuelsont } \\
\text { E Samuelsont } \\
\text { E Samuelsont }\end{array}$ & $\begin{array}{r}11326 \\
10205 \\
9985\end{array}$ \\
\hline${ }^{106} \mathrm{Cd}$ & \multicolumn{6}{|c|}{ product ylald } \\
\hline $\begin{array}{l}92 \mathrm{Zr} \\
{ }^{106} \mathrm{Cd}\end{array}$ & $\begin{array}{l}: 60 \\
\text { relat }\end{array}$ & \multicolumn{4}{|c|}{ relative o(E) } & 11324 \\
\hline $\begin{array}{r}82 \mathrm{Zr} \\
{ }^{108} \mathrm{Cd}\end{array}$ & $\begin{array}{r}180 \\
0\end{array}$ & $\begin{array}{l}6.0+1 \\
5.0+1\end{array}$ & & $\begin{array}{l}\text { PUR E } \\
\text { TOK E }\end{array}$ & $\begin{array}{l}\text { E Poplit } \\
\text { E Hashimolot }\end{array}$ & $\begin{array}{r}11325 \\
8260\end{array}$ \\
\hline${ }^{108} \mathrm{Cd}$ & $e(E)$ & . & & & & \\
\hline $\begin{array}{l}104 \mathrm{Pd} \\
104 \mathrm{Pd} \\
106 \mathrm{Pd} \\
106 \mathrm{Pd}\end{array}$ & $\begin{array}{c}\sigma_{L i}^{0} \\
0 \\
\delta_{L i}\end{array}$ & $\begin{array}{l}8 \cdot 1+1 \\
5 \cdot 4+1 \\
8 \cdot 1+1 \\
5 \cdot 4+1\end{array}$ & $\begin{array}{l}9.9+1 \\
9.9+1\end{array}$ & $\begin{array}{l}\text { INUM } \\
\text { INUM M } \\
\text { INU M } \\
\text { INU M }\end{array}$ & $\begin{array}{l}\text { M Castaneda+ } \\
M \text { Castaneda+ } \\
\text { M Costaneda+ } \\
\text { M Castaneda+ }\end{array}$ & $\begin{array}{l}8211 \\
9219 \\
8215 \\
9238\end{array}$ \\
\hline $107 \mathrm{Cd}$ & \multicolumn{6}{|c|}{ portiol relativo $\sigma(E)$} \\
\hline${ }^{106} \mathrm{Pd}$ & $a$ & $3.5+1$ & $4.4+1$ & TOK E & E Ohyat & 8214 \\
\hline
\end{tabular}

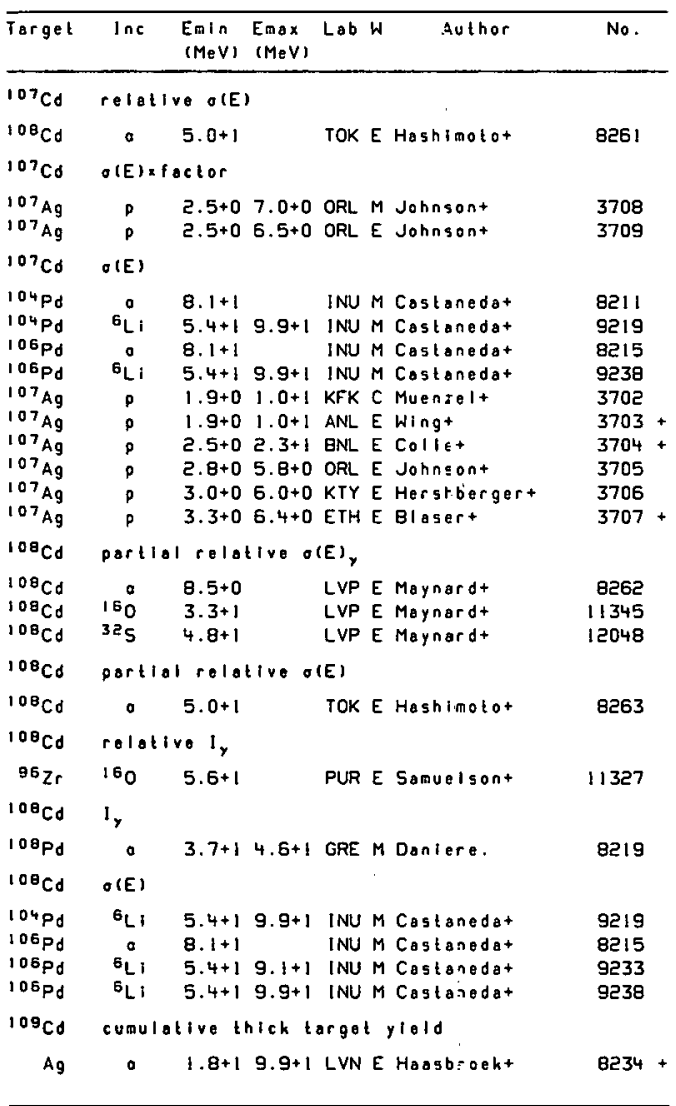




\begin{tabular}{|c|c|c|c|c|c|c|}
\hline Target & $\ln c$ & $\begin{array}{l}E \cap I n \\
(M \otimes V)\end{array}$ & $\begin{array}{l}\text { Emax } \\
(M \theta V)\end{array}$ & Lab & Author & No. \\
\hline${ }^{109} \mathrm{Cd}$ & \multicolumn{6}{|c|}{ cumulative o(E) } \\
\hline $\begin{array}{r}\mathrm{Ag} \\
{ }_{109} \mathrm{Cd}\end{array}$ & \multicolumn{5}{|c|}{ partial relotive o(E) } & $8233+$ \\
\hline $\begin{array}{l}{ }_{100} \mathrm{Cd} \\
100 \mathrm{Pd}\end{array}$ & $a$ & $\begin{array}{l}3 \cdot 5+1 \\
5 \cdot 0+1\end{array}$ & $4.4+1$ & $\begin{array}{l}\text { TOK } \\
\text { TOK }\end{array}$ & $\begin{array}{l}\text { E Ohyat } \\
\text { E Hashimotot }\end{array}$ & $\begin{array}{l}8221 \\
8264\end{array}$ \\
\hline${ }^{109} \mathrm{Cd}$ & \multicolumn{6}{|c|}{ relative ly } \\
\hline $\begin{array}{l}100 \mathrm{Pd} \\
{ }^{109} \mathrm{Cd}\end{array}$ & \multicolumn{6}{|c|}{ product yield } \\
\hline${ }^{109} \mathrm{Ag}$ & $p$ & NDG & & ROS & E Beckert+ & 3720 \\
\hline${ }^{109} \mathrm{Cd}$ & \multicolumn{6}{|c|}{$\sigma(E) \times$ tactor } \\
\hline $\begin{array}{l}\log _{A g} \\
\log _{A g}\end{array}$ & $\begin{array}{l}p \\
p\end{array}$ & $\begin{array}{l}2.5+0 \\
2.5+0\end{array}$ & $\begin{array}{l}7.0+0 \\
6.5+0\end{array}$ & ORL & $\begin{array}{l}\text { M Johnsont } \\
\text { E Johnsont }\end{array}$ & $\begin{array}{l}3721 \\
3722\end{array}$ \\
\hline${ }^{109} \mathrm{Cd}$ & \multicolumn{6}{|l|}{$\sigma(E)$} \\
\hline $\begin{array}{l}104 \mathrm{Pd} \\
106 \mathrm{Pd} \\
106 \mathrm{Pd}\end{array}$ & $\begin{array}{l}\sigma_{L I} \\
\sigma_{L I}^{0}\end{array}$ & $\begin{array}{l}5.4+1 \\
\text { 8. } 1+1 \\
\text { E. } 4+1\end{array}$ & $\begin{array}{l}9.9+1 \\
9.9+1\end{array}$ & $\begin{array}{l}\text { INU } \\
\text { INU } \\
\text { INU }\end{array}$ & $\begin{array}{l}M \text { Costonedat } \\
M \text { Castonedat } \\
M \text { Castonedat }\end{array}$ & $\begin{array}{l}9219 \\
8215 \\
9238\end{array}$ \\
\hline $\mathrm{Ag}$ & 0 & $\varepsilon .0+1$ & & TAM & M Bloht & 8241 \\
\hline $\log _{\mathrm{Ag}}$ & $p$ & TR & $5.8+0$ & ORL & E Johnsont & $3713+$ \\
\hline${ }^{109} \mathrm{Ag}$ & $p$ & $\bar{c} .5+0$ & $1.0+1$ & KFK & C Muenzel+ & 3714 \\
\hline${ }^{109} \mathrm{Ag}$ & $p$ & $\bar{c} .8+0$ & $5.8+0$ & ORL & E Johnsont & 3715 \\
\hline $\log _{A} 9$ & $p$ & $3.0+0$ & $6.0+0$ & $\mathrm{KTY}$ & E Hershbergert & 3716 \\
\hline $109_{A g}$ & $p$ & $4.2+0$ & $6.4+0$ & ETH & E Blasert & $3717+$ \\
\hline $\begin{array}{l}109 \mathrm{Ag} \\
109_{\mathrm{Ag}}\end{array}$ & $p$ & $\begin{array}{l}5.0+0 \\
6.4+0\end{array}$ & $\begin{array}{l}1.0+1 \\
6.8+0\end{array}$ & ANL & $\begin{array}{l}\text { E Wingt } \\
\text { E Lovehlhovet }\end{array}$ & $\begin{array}{l}3718+ \\
3719\end{array}$ \\
\hline $110 \mathrm{Cd}$ & \multicolumn{6}{|c|}{ compound-nucleus OIE) } \\
\hline $110^{\circ} \mathrm{Cd}$ & D & $1.6+1$ & & PUR & E Lux + & 3741 \\
\hline $110^{\circ} \mathrm{Cd}$ & \multicolumn{6}{|c|}{ partial raw thick torget yieldyabundance } \\
\hline $110^{\circ} \mathrm{Co}$ & $a$ & $5.0+0$ & & SUN & E Gllest & 8269 \\
\hline $110 \mathrm{Cd}$ & \multicolumn{6}{|c|}{ portiol rolative otely } \\
\hline $\begin{array}{l}110 \mathrm{Cd} \\
110^{\circ} \mathrm{Cd} \\
110^{\circ} \mathrm{Cd}\end{array}$ & $\begin{array}{l}180^{\circ} \\
325\end{array}$ & $\begin{array}{l}8.5+0 \\
3.3+1 \\
4.8+1\end{array}$ & & $\begin{array}{l}\text { LVP } \\
\text { LVP } \\
\text { LVP }\end{array}$ & $\begin{array}{l}\text { E Maynardt } \\
\text { E Maynardt } \\
\text { E Maynard+ }\end{array}$ & $\begin{array}{r}8268 \\
11350 \\
12049\end{array}$ \\
\hline
\end{tabular}

\begin{tabular}{|c|c|c|c|c|c|c|}
\hline Target & $\operatorname{lnc}$ & $\begin{array}{l}E_{R} \mid n \\
\left(M_{0} V\right)\end{array}$ & $\begin{array}{l}\text { Emex } \\
\left(M_{e} V\right)\end{array}$ & Lab W & Author & No. \\
\hline $110^{\circ} \mathrm{Cd}$ & \multicolumn{6}{|c|}{ portial o(E), } \\
\hline $110 \mathrm{Cd}$ & p & $7.0+0$ & $2.6+1$ & L LRL E & E koopmant & 3743 \\
\hline $110^{\circ} \mathrm{Cd}$ & \multicolumn{6}{|c|}{ portiol oleixfoctor } \\
\hline${ }^{110} \mathrm{Cd}$ & p & $6.1+0$ & $8.1+0$ & TOK E & E Sogot & 3742 \\
\hline $110^{\circ} \mathrm{Cd}$ & \multicolumn{6}{|c|}{ relollve Ir } \\
\hline $108 \mathrm{Pd}$ & a & $3.7+1$ & $4.6+1$ & I GRE M & M Oentere. & өз22 \\
\hline $110 \mathrm{Cd}$ & \multicolumn{6}{|c|}{ O(E) \& f actor } \\
\hline $110^{\circ} \mathrm{Cd}$ & $p$ & $5.8+0$ & $8.1+0$ & O TOK E & E Sogot & 3744 \\
\hline $110 \mathrm{Cd}$ & \multicolumn{6}{|l|}{$O(E)$} \\
\hline $\begin{array}{r}\mathrm{Ag} \\
107 \mathrm{Ag} \\
110^{\circ} \mathrm{Cd} \\
110^{\circ} \mathrm{Cd}\end{array}$ & $\begin{array}{l}a \\
a \\
p \\
p\end{array}$ & $\begin{array}{l}8.0+1 \\
2.0+1 \\
7.0+0 \\
1.6+1\end{array}$ & $2.6+1$ & $\begin{array}{l}\text { TAM M } \\
\text { PUR E } \\
\text { I LRL E } \\
\text { PUR E }\end{array}$ & $\begin{array}{l}\text { M Oloht } \\
\text { E Luxt } \\
\text { E Koopmant } \\
\text { E Luxt }\end{array}$ & $\begin{array}{l}8241 \\
8243 \\
3739 \\
3740\end{array}$ \\
\hline $111 \mathrm{Cd}$ & \multicolumn{6}{|c|}{ portlol rea thick target y $101 d_{2}$ xabundonce } \\
\hline $111 \mathrm{Cd}$ & - & $5.0+0$ & & SUN E & E Gilest & 8272 \\
\hline $111 \mathrm{Cd}$ & \multicolumn{6}{|c|}{ portlal relative o(E) } \\
\hline $110 \mathrm{Pd}$ & a & $3.5+1$ & $4.4+1$ & I TOK E & E Ohyot & 8226 \\
\hline "WCd & \multicolumn{6}{|c|}{ relative Ir } \\
\hline $108 \mathrm{Pd}$ & a & $3.7+1$ & $4.6+1$ & I GRE $M$ & M Doniere. & 8223 \\
\hline $111 \mathrm{Cd}$ & \multicolumn{6}{|l|}{$O(\varepsilon)$} \\
\hline $\mathrm{Ag}$ & - & 8.0+1 & & TAM M & M Bloht & 8241 \\
\hline$\|^{2} \mathrm{Cd}$ & \multicolumn{6}{|c|}{ partlal row thich largol yleldyabundance } \\
\hline $112 \mathrm{Cd}$ & - & $5.0+0$ & & SUN E & E Gilest & 8274 \\
\hline${ }^{\prime \prime 2} \mathrm{Cd}$ & \multicolumn{6}{|c|}{ partlal relative o(E), } \\
\hline $\begin{array}{l}\| 12 \mathrm{Cd} \\
112 \mathrm{Cd} \\
112 \mathrm{Cd}\end{array}$ & $\begin{array}{l}160 \\
325\end{array}$ & $\begin{array}{l}8.5+0 \\
3.3+1 \\
4.8+1\end{array}$ & & $\begin{array}{l}\text { LVP E } \\
\text { LVP E } \\
\text { LVP E }\end{array}$ & $\begin{array}{l}\text { E Maynard+ } \\
\text { E Maynard+ } \\
\text { E Maynord+ }\end{array}$ & $\begin{array}{r}8273 \\
11353 \\
12050\end{array}$ \\
\hline${ }^{113} \mathrm{Cd}$ & \multicolumn{6}{|c|}{ partlal rov thlck target yield xabundance } \\
\hline $113 \mathrm{Cd}$ & - & $5.0+0$ & & SUN E & E Gilest & 8277 \\
\hline
\end{tabular}




\begin{tabular}{|c|c|c|c|c|c|c|}
\hline Targel & $\operatorname{Inc}$ & $\begin{array}{l}\text { Emin } \\
(\mathrm{MeV})\end{array}$ & $\begin{array}{l}\text { Emax } \\
(\mathrm{MeV})\end{array}$ & Lab W & Author & No. \\
\hline 11400 & \multicolumn{6}{|c|}{ partial row thich target yleldy $x$ abundance } \\
\hline $114 \mathrm{Cd}$ & o & $5 \cdot 0+0$ & & SUN E & E Gilest & 8279 \\
\hline $114 \mathrm{Co}$ & \multicolumn{6}{|c|}{ Dortiol relative o(E), } \\
\hline $\begin{array}{l}114 \mathrm{Cc} \\
114 \mathrm{Cd} \\
11{ }^{4} \mathrm{Cd}\end{array}$ & $\begin{array}{l}10^{\circ} \\
325\end{array}$ & $\begin{array}{l}8.5+0 \\
3.3+1 \\
4.8+1\end{array}$ & & $\begin{array}{l}\text { LVP E } \\
\text { LVP E } \\
\text { LVP E }\end{array}$ & $\begin{array}{l}\text { Maynard+ } \\
\text { Maynard+ } \\
\text { Maynardt }\end{array}$ & $\begin{array}{r}8278 \\
11354 \\
12051\end{array}$ \\
\hline${ }^{114} \mathrm{Cd}$ & \multicolumn{6}{|c|}{ partial o(E), } \\
\hline $114 \mathrm{Cd}$ & P & $7.0+0$ & $2.6+1$ & I LRL E & E Koopmant & 3787 \\
\hline $114 \mathrm{Cd}$ & $\sigma(E)$ & & & & & \\
\hline $\begin{array}{l}1144 \mathrm{Cd} \\
114 \mathrm{Cd}\end{array}$ & p & $\begin{array}{l}7.0+0 \\
7.0+0\end{array}$ & $\begin{array}{l}2.6+1 \\
2.6+1\end{array}$ & $\begin{array}{l}\text { LRL E } \\
\text { LRL E }\end{array}$ & $\begin{array}{l}\text { Koopmant } \\
\text { Koopmant }\end{array}$ & $\begin{array}{l}3786 \\
3788\end{array}$ \\
\hline $115 \mathrm{Co}$ & \multicolumn{6}{|c|}{ Independent o(E) } \\
\hline $\begin{array}{l}238 \mathrm{U} \\
115 \mathrm{Cd}\end{array}$ & \multicolumn{6}{|c|}{ cumulative lisgion yleld } \\
\hline $\begin{array}{l}232 \mathrm{Th} \\
233 \mathrm{U}\end{array}$ & $\begin{array}{l}d \\
d\end{array}$ & $\begin{array}{l}9.3+0 \\
9.0+0\end{array}$ & $\begin{array}{l}1.4+1 \\
2.3+1\end{array}$ & $\begin{array}{l}\text { CCP } R \\
\text { CCP } R\end{array}$ & $\begin{array}{l}R \text { Lbovt } \\
R \text { Lbovt }\end{array}$ & $\begin{array}{l}5921 \\
5936\end{array}$ \\
\hline${ }^{115} \mathrm{Cd}$ & \multicolumn{6}{|c|}{ fission ytaldefoctor } \\
\hline${ }^{24} \mathrm{C}_{\mathrm{ar}}$ & a & $2.4+1$ & $3.4+1$ & KUR E & E Lisint & 9117 \\
\hline $118 \mathrm{Cd}$ & \multicolumn{6}{|c|}{ olE)afoctor } \\
\hline $\begin{array}{l}232 \mathrm{Th} \\
1150 \mathrm{Cd}\end{array}$ & \multicolumn{6}{|c|}{ cunulative fission yield } \\
\hline $\begin{array}{l}232 \mathrm{th} \\
233 \mathrm{U}\end{array}$ & $d$ & $\begin{array}{l}9.3+0 \\
9.0+0\end{array}$ & $\begin{array}{l}1.4+1 \\
2.3+1\end{array}$ & $\begin{array}{l}\text { CCP R } \\
\text { CCP R }\end{array}$ & $\begin{array}{l}R \text { Lbovt } \\
R \text { Lbovt }\end{array}$ & $\begin{array}{l}5921 \\
5936\end{array}$ \\
\hline $1150 \mathrm{cod}$ & \multicolumn{6}{|c|}{ cumulative $\sigma(E)$} \\
\hline $\begin{array}{l}23 \theta_{U} \\
238_{U}\end{array}$ & $p$ & $\begin{array}{l}4.5+2 \\
4.5+2\end{array}$ & & $\begin{array}{l}\text { KFK C } \\
\mathrm{CHI} \mathrm{E}\end{array}$ & $\begin{array}{l}\text { C Muenzelt } \\
\text { E Panontint }\end{array}$ & $\begin{array}{l}4917 \\
4918+\end{array}$ \\
\hline $1150 \mathrm{Cd}$ & \multicolumn{6}{|c|}{ o(E) af actor } \\
\hline $\begin{array}{r}\mathrm{Cd} \\
114 \mathrm{Cd} \\
116 \mathrm{Cd}\end{array}$ & $\begin{array}{l}\text { d } \\
\text { d }\end{array}$ & $\begin{array}{l}8 \cdot 0+0 \\
8 \cdot 0+0 \\
1.5+1\end{array}$ & $\begin{array}{l}2 . \theta+1 \\
2.8+1 \\
2 . \theta+1\end{array}$ & $\begin{array}{l}\text { CNE E } \\
\text { CNE E } \\
\text { CNE E }\end{array}$ & $\begin{array}{l}\text { E Ushert } \\
E \text { Ushert } \\
E \text { Ushert }\end{array}$ & $\begin{array}{l}5661 \\
5695 \\
5698\end{array}$ \\
\hline
\end{tabular}

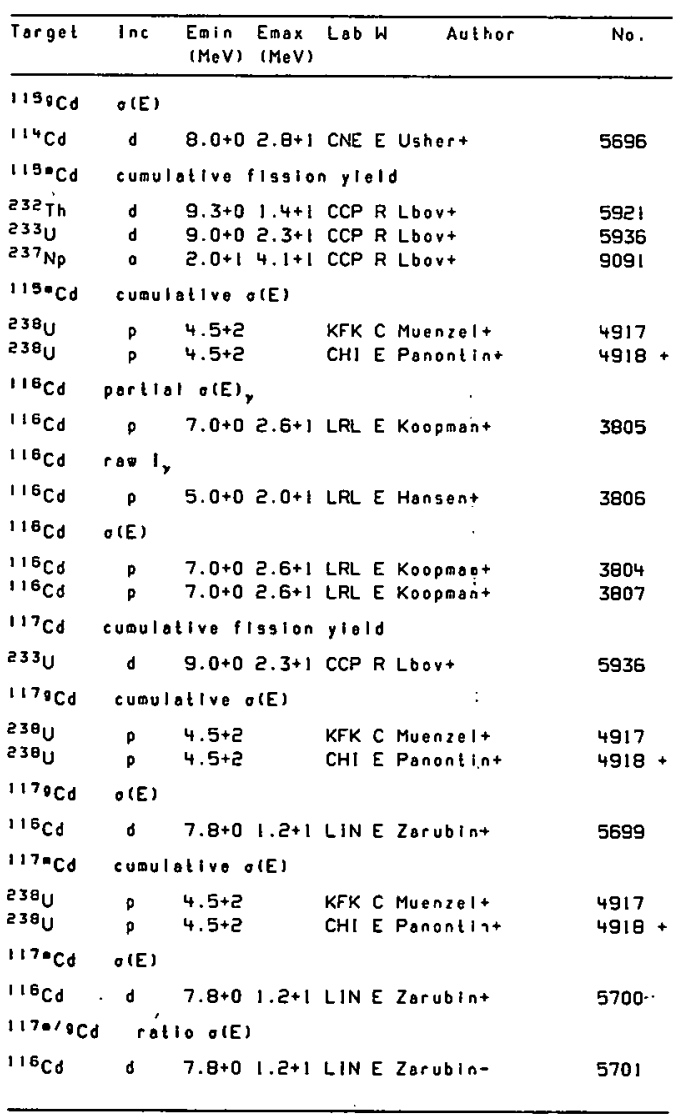




\begin{tabular}{|c|c|c|c|c|c|c|}
\hline Target & $\ln c$ & $\begin{array}{l}\text { Emin } \\
(\mathrm{MeV})\end{array}$ & $\begin{array}{l}\text { Emax } \\
(\mathrm{MeV})\end{array}$ & Lab & Author & No. \\
\hline$z=49$ & $v_{n}$ & & & & & \\
\hline Ag & ${ }^{40} \mathrm{Ar}$ & $3.4+2$ & & PAR & R Lefort+ & 12202 \\
\hline$z=49$ & OIE) & & & & & \\
\hline $238 \mathrm{U}$ & P & $3.0+1$ & $9.8+1$ & MCG & E Chont & 4895 \\
\hline $\log _{\mathrm{In}}$ & ras ote & & & & & \\
\hline${ }^{106} \mathrm{Cd}$ & ${ }^{3} \mathrm{He}$ & $3.2+1$ & $5.9+1$ & KAZ & E Burminshiti & 6442 \\
\hline 10510 & $\sigma(E)$ & & & & & \\
\hline $\begin{array}{l}104 \mathrm{Pd} \\
106 \mathrm{Pd}\end{array}$ & $\begin{array}{l}{ }^{6} L i \\
{ }^{6} L i\end{array}$ & $\begin{array}{l}5.4+1 \\
5.4+1\end{array}$ & $\begin{array}{l}9.9+1 \\
9.9+1\end{array}$ & $\begin{array}{l}\text { INU } \\
\text { INU }\end{array}$ & $\begin{array}{l}\text { M Castanedat } \\
\text { M Castanedat }\end{array}$ & $\begin{array}{l}9211 \\
9237\end{array}$ \\
\hline $\log _{0} \ln$ & produc & ct & & & & \\
\hline${ }^{106} \mathrm{Cd}$ & d & $1.9+1$ & $3 \cdot 1+1$ & GRE & E Riviert & 5674 \\
\hline $\log \theta \ln$ & produc & ct : le & & & & \\
\hline${ }^{106} \mathrm{Cd}$ & d & $1.9+1$ & $3.1+1$ & GRE & E Riviert & 5675 \\
\hline $108 / n$ & partls & I rsw & $a(E)$ & & & \\
\hline${ }^{106} \mathrm{Cd}$ & ${ }^{3} \mathrm{He}$ & $2.1+1$ & $5.9+1$ & KAZ & E Burminskit+ & 6443 \\
\hline${ }^{108}$ In & produc & $t y \mid 010$ & & & & \\
\hline Mo & $14 \mathrm{~N}$ & $7.2+1$ & & LVN & E Coussement+ & 10417 \\
\hline $108 \mathrm{ln}$ & $\sigma(E)$ & & & & & \\
\hline $\begin{array}{l}104 \mathrm{Pd} \\
106 \mathrm{Pd}\end{array}$ & $\begin{array}{l}{ }^{6} L: \\
{ }^{6} L ;\end{array}$ & $\begin{array}{l}5.4+1 \\
5.4+1\end{array}$ & $\begin{array}{l}9.9+1 \\
9.9+1\end{array}$ & $\begin{array}{l}\text { INU } \\
\text { INU }\end{array}$ & $\begin{array}{l}\text { M Castanedat } \\
M \text { Castanedat }\end{array}$ & $\begin{array}{l}9218 \\
9237\end{array}$ \\
\hline $107 / n$ & product & I yield & & & & \\
\hline Mo & $14 \mathrm{~N}$ & $7.2+1$ & & LVN & E Coussementt & 10417 \\
\hline $107 \ln$ & $\ln \mid c k$ & torget & yield & & & \\
\hline${ }^{106} \mathrm{Cd}$ & $p$ & $3.0+0$ & $5.0+0$ & $\mathrm{CLU}$ & Mroughtont & 3736 \\
\hline $107 \mathrm{ln}$ & rovole & & & & & \\
\hline${ }^{106} \mathrm{Cos}$ & ${ }^{3} \mathrm{He}$ & $2 \cdot 1+1$ & $5.9+1$ & KAZ & E Burminshit & 6444 \\
\hline $107 \ln$ & $O(E)$ & & & & & \\
\hline $\begin{array}{l}104 \mathrm{Pd} \\
106 \mathrm{Pd}\end{array}$ & $\begin{array}{l}6_{L I} \\
6_{L I}\end{array}$ & $\begin{array}{l}5.4+1 \\
5.4+1\end{array}$ & $\begin{array}{l}9.9+1 \\
9.9+1\end{array}$ & $\begin{array}{l}\text { INU } \\
\text { INU }\end{array}$ & $\begin{array}{l}\text { M Castanedat } \\
\text { M Castanedat }\end{array}$ & $\begin{array}{l}9218 \\
9237\end{array}$ \\
\hline
\end{tabular}

\begin{tabular}{|c|c|c|c|c|c|c|}
\hline Target & $\operatorname{lnc}$ & $\begin{array}{l}\mathrm{Emin} \\
(\mathrm{MeV})\end{array}$ & $\begin{array}{l}\text { Emax } \\
(\operatorname{MeV})\end{array}$ & Lob & Author & No. \\
\hline $107 . \mathrm{In}$ & \multicolumn{6}{|c|}{ row $O(E)$} \\
\hline${ }^{106} \mathrm{Cd}$ & ${ }^{3} \mathrm{He}$ & $2.1+1$ & $4.5+1$ & KAZ & E Burminshit+ & 6445 \\
\hline $100 \ln$ & \multicolumn{6}{|c|}{ product yield } \\
\hline Mo & $14 \mathrm{~N}$ & $7.2+!$ & & LVN & E Coussement t & 10417 \\
\hline $108 \ln$ & $O(E)$ & & & & & \\
\hline $\begin{array}{l}104 \mathrm{Pd} \\
106 \mathrm{Pd} \\
106 \mathrm{Pd}\end{array}$ & $\begin{array}{l}\sigma_{L I} \\
E_{L I} \\
\sigma_{L I}\end{array}$ & $\begin{array}{l}5.4+1 \\
5.4+1 \\
5.4+1\end{array}$ & $\begin{array}{l}9.9+1 \\
9.9+1 \\
9.9+1\end{array}$ & $\begin{array}{l}\text { INU } \\
\text { INU } \\
\text { INU }\end{array}$ & $\begin{array}{l}\text { M Castanedat } \\
\text { M Castanedat } \\
\text { M Castanedat }\end{array}$ & $\begin{array}{l}9218 \\
9234 \\
9237\end{array}$ \\
\hline $\log _{\text {In }}$ & \multicolumn{6}{|c|}{ Including metastable o(E) } \\
\hline $110^{\circ} \mathrm{Cd}$ & $p$ & $1.4+1$ & $2.1+1$ & UFT & E Shakunt & $3745+$ \\
\hline 10910 & \multicolumn{6}{|c|}{ Cumulalive $O(E)$} \\
\hline $\begin{array}{l}238^{2} \\
23 \theta_{U}\end{array}$ & $\begin{array}{l}\rho \\
\rho\end{array}$ & $\begin{array}{l}4.5+2 \\
4.5+2\end{array}$ & & $\begin{array}{l}\mathrm{KFK} \\
\mathrm{CHI}\end{array}$ & $\begin{array}{l}\text { C Muenzelt } \\
\text { E Panontint }\end{array}$ & $\begin{array}{l}4917 \\
4918+\end{array}$ \\
\hline $1081 n$ & \multicolumn{6}{|c|}{ partlal pelallve o(E) } \\
\hline $\begin{array}{l}107 \mathrm{Ag} \\
108 \mathrm{Cd}\end{array}$ & $a$ & $\begin{array}{l}2 \cdot 2+1 \\
5 \cdot 0+1\end{array}$ & $3.0+1$ & $\begin{array}{l}\text { FUL } \\
\text { TOK }\end{array}$ & $\begin{array}{l}\text { E Van Poelgeest+ } \\
\text { E Hashimotot }\end{array}$ & $\begin{array}{l}8244 \\
8265\end{array}$ \\
\hline $10 \theta_{1 n}$ & \multicolumn{6}{|c|}{ product yiold } \\
\hline Mo & $14 \mathrm{~N}$ & $7.2-1$ & & LVN & E Coussement+ & 10417 \\
\hline $109 / n$ & \multicolumn{6}{|c|}{ Inlck torgot yield } \\
\hline${ }_{100}^{A g}$ & 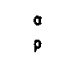 & $\begin{array}{l}1.7+1 \\
3.0+0\end{array}$ & $\begin{array}{l}3.0+1 \\
5.0+0\end{array}$ & $\begin{array}{l}\text { UJV } \\
\text { CLU }\end{array}$ & $\begin{array}{l}\text { C Bemt } \\
\text { M Roughtont }\end{array}$ & $\begin{array}{l}8235 \\
3737\end{array}$ \\
\hline $109 / n$ & \multicolumn{6}{|l|}{$O(E)$} \\
\hline $\begin{array}{r}93 \mathrm{Nb} \\
{ }_{106} \mathrm{Pd}\end{array}$ & $\begin{array}{l}160 \\
6 \mathrm{LI}\end{array}$ & $\begin{array}{l}5 \cdot 0+1 \\
5 \cdot 4+1\end{array}$ & $\begin{array}{l}6.5+1 \\
9.9+1\end{array}$ & $\begin{array}{l}\text { MIT } \\
\text { INU }\end{array}$ & $\begin{array}{l}\text { M Branfordt } \\
\text { M Castanedat }\end{array}$ & $\begin{array}{r}11328 \\
9237\end{array}$ \\
\hline $109 \ln$. & \multicolumn{6}{|c|}{ of(E) a foctor } \\
\hline $\begin{array}{l}\mathrm{Cd} \\
108 \mathrm{Cd} \\
110 \mathrm{Cd}\end{array}$ & $\begin{array}{l}9 \\
d \\
d\end{array}$ & $\begin{array}{l}8.0+0 \\
8.0+0 \\
1 . E+1\end{array}$ & $\begin{array}{l}2.8+1 \\
2.8+1 \\
2.8+1\end{array}$ & $\begin{array}{l}\text { CNE } \\
\text { CNE } \\
\text { CNE }\end{array}$ & $\begin{array}{l}\text { E Ushert } \\
\text { E Ushert } \\
\text { E Ushert }\end{array}$ & $\begin{array}{l}5662 \\
5676 \\
5677\end{array}$ \\
\hline $110 \mathrm{ln}$ & \multicolumn{6}{|c|}{ partlal product yiold } \\
\hline $\begin{array}{l}108 \mathrm{Pd} \\
107 \mathrm{Ag}\end{array}$ & $e_{L i}$ & $\begin{array}{l}\text { NOG } \\
\text { NDG }\end{array}$ & & $\begin{array}{l}\text { SUB } \\
\text { SUB }\end{array}$ & $\begin{array}{l}\text { E Eibert+ } \\
\text { E Eibert+ }\end{array}$ & $\begin{array}{l}9240 \\
8245\end{array}$ \\
\hline
\end{tabular}




\begin{tabular}{|c|c|c|c|c|c|c|}
\hline Target & $\ln c$ & $\begin{array}{l}\text { Emln } \\
(\mathrm{MeV})\end{array}$ & $\begin{array}{l}\text { Emax } \\
(\operatorname{MeV})\end{array}$ & Lab & Author & No. \\
\hline $1101 \mathrm{n}$ & produc & A yiold & & & & \\
\hline Mo & ${ }^{14} \mathrm{~N}$ & $7.2+1$ & & LVN & E Coussement+ & 10417 \\
\hline $\operatorname{ltc} \ln$ & thlck & target & $y / e 1 d$ & & & \\
\hline $\mathrm{Ag}$ & $a$ & $1.3+1=$ & $3.0+1$ & UJV & C Bemt & 8236 \\
\hline $110^{I n}$ & $e(E)$ & & & & & \\
\hline $\begin{array}{l}106 \mathrm{Pd} \\
\mathrm{Ag} \\
110 \mathrm{CJ} \\
110 \mathrm{CJ}\end{array}$ & $\begin{array}{l}{ }^{6} L I \\
a \\
p \\
p\end{array}$ & $\begin{array}{l}5 \cdot 4+1 \\
8 \cdot 0+1 \\
2 \cdot 5+0 \\
6 \cdot 3+0\end{array}$ & $\begin{array}{l}9.9+1 \\
6.0+0 \\
7.3+0\end{array}$ & $\begin{array}{l}\text { INU } \\
\text { TAM } \\
\text { ORL } \\
\text { CCP }\end{array}$ & $\begin{array}{l}\text { M Castanedat } \\
\text { M Blokt } \\
\text { E Johnsont } \\
\text { E Abranovicht }\end{array}$ & $\begin{array}{l}9237 \\
8241 \\
3746 \\
3747\end{array}$ \\
\hline $11001 n$ & Indep & pendent & $O(E)$ & & & \\
\hline $\begin{array}{l}23 \theta_{U} \\
23 \theta_{1}\end{array}$ & $\begin{array}{l}p \\
p\end{array}$ & $\begin{array}{l}4.5+2 \\
4.5+2\end{array}$ & & $\begin{array}{l}\mathrm{KFK} \\
\mathrm{CHI}\end{array}$ & $\begin{array}{l}\text { C Muenzel+ } \\
\text { E Panontint }\end{array}$ & $\begin{array}{l}4922 \\
4923+\end{array}$ \\
\hline $1100: n$ & $\sigma(E):$ & factor & & & & \\
\hline $\begin{array}{r}\mathrm{Cd} \\
110 \mathrm{Cd} \\
111 \mathrm{Cd}\end{array}$ & $\begin{array}{l}d \\
d \\
d\end{array}$ & $\begin{array}{l}1.1+1 \\
1.0+1 \\
1.7+1\end{array}$ & $\begin{array}{l}2.7+1 \\
2.8+1 \\
2.8+1\end{array}$ & $\begin{array}{l}\text { CNE } \\
\text { CNE } \\
\text { CNE }\end{array}$ & $\begin{array}{l}\text { E. Ushert } \\
\text { E Ushert } \\
\text { E Ushert }\end{array}$ & $\begin{array}{l}5663 \\
5678 \\
5685\end{array}$ \\
\hline $110,1 n$ & $g(E)$ & & & & & \\
\hline $\begin{array}{l}110 \mathrm{Cd} \\
1100 \mathrm{Cd} \\
110^{\circ} \mathrm{Cd} \\
111 \mathrm{Cd}\end{array}$ & $\begin{array}{l}p \\
p \\
d \\
d\end{array}$ & $\begin{array}{l}4.5+0 \\
4.5+0.8 \\
1.1+1 \\
1.8+1\end{array}$ & $\begin{array}{l}6.4+0 \\
6.4+0 \\
2.4+! \\
2.7+i\end{array}$ & $\begin{array}{l}\text { ETH } \\
\text { KFK } \\
\text { CNE } \\
\text { CNE }\end{array}$ & $\begin{array}{l}\text { E Blasert } \\
\text { C Muenzel+ } \\
\text { EUshert } \\
\text { E Ushert }\end{array}$ & $\begin{array}{l}3748+ \\
3749 \\
5679 \\
5686\end{array}$ \\
\hline 110.10 & indep & iondent & $O(E)$ & & & \\
\hline $\begin{array}{l}238 u \\
238 u\end{array}$ & p & $\begin{array}{l}4.5+2 \\
4.5+2\end{array}$ & & $\begin{array}{l}\text { KFK } \\
\mathrm{CH}]\end{array}$ & $\begin{array}{l}\text { C Muenzelt } \\
\text { E Panontint }\end{array}$ & $\begin{array}{l}4922 \\
4923+\end{array}$ \\
\hline $1100 \cdot \ln$ & $O(E) \times$ & factor & & & & \\
\hline $\begin{array}{r}\mathrm{Cd} \\
1110 \mathrm{Cd} \\
111 \mathrm{Cd}\end{array}$ & $\begin{array}{l}d \\
d \\
d\end{array}$ & $\begin{array}{l}1.1+1 \\
1.0+1 \\
1.7+1\end{array}$ & $\begin{array}{l}2.7+1 \\
2.8+1 \\
2.8+1\end{array}$ & $\begin{array}{l}\text { CNE } \\
\text { CNE } \\
\text { CNE }\end{array}$ & $\begin{array}{l}\text { E Ushert } \\
\text { E Ushert } \\
\text { E Ushert }\end{array}$ & $\begin{array}{l}5664 \\
5680 \\
5687\end{array}$ \\
\hline $11001 n$ & $\sigma(E)$ & & & & & \\
\hline $\begin{array}{l}\because 0 \mathrm{Cd} \\
\because 1 \mathrm{Cd}\end{array}$ & d & $\begin{array}{l}1.1+1= \\
1.8+1\end{array}$ & $\begin{array}{l}2.4+1 \\
2.7+1\end{array}$ & $\begin{array}{l}\text { CNE } \\
\text { CNE }\end{array}$ & $\begin{array}{l}\text { E Ushert } \\
\text { E Ushert }\end{array}$ & $\begin{array}{l}5681 \\
5688\end{array}$ \\
\hline
\end{tabular}

\begin{tabular}{|c|c|c|c|c|c|}
\hline Target & $\ln c$ & $\begin{array}{l}E m \mid n \\
(M e V)\end{array}$ & $\begin{array}{l}\text { Emax } \\
(\operatorname{MeV})\end{array}$ & Author & No. \\
\hline $1100+0 \ln$ & sum & amation & $O(E)$ & & . \\
\hline $\begin{array}{l}110 \mathrm{Cd} \\
111 \mathrm{Cd}\end{array}$ & p & $\begin{array}{l}6.5+0 \\
1.2+1\end{array}$ & $\begin{array}{l}2.1+1 \\
2.1+1\end{array}$ & $\begin{array}{l}\text { UFT E Skahunt } \\
\text { UFT E Shahunt }\end{array}$ & $\begin{array}{l}3750+ \\
3753+\end{array}$ \\
\hline \multicolumn{6}{|c|}{ 110.1\%gIn ratio o(E) } \\
\hline $\begin{array}{l}110 \mathrm{Cd} \\
110 \mathrm{Cd} \\
111 \mathrm{Cd}\end{array}$ & $\begin{array}{l}p \\
d \\
d\end{array}$ & $\begin{array}{l}6.0+0 \\
1.1+1 \\
2.1+1\end{array}$ & $\begin{array}{l}2.0+1 \\
2 \cdot 4+1 \\
2 \cdot 7+1\end{array}$ & $\begin{array}{l}\text { UFT T Shahunt } \\
\text { CNE E Ushert } \\
\text { CNE E Ushert }\end{array}$ & $\begin{array}{l}3751 \\
5682 \\
5689\end{array}$ \\
\hline $\begin{array}{l}111 \mathrm{In} \\
\mathrm{Ag}\end{array}$ & includ & $\begin{array}{l}\operatorname{ling} \text { met } \\
1.2+1\end{array}$ & $\begin{array}{r}\text { tastab } \\
9.4+1\end{array}$ & $\begin{array}{l}\text { le thlch targe: yleld } \\
\text { LVNE Hasb-oekt }\end{array}$ & $8239+$ \\
\hline $11 \ln$ & Inctud & ding met & tastob & le o(E) & \\
\hline 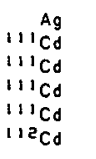 & $\begin{array}{l}q \\
p \\
p \\
p \\
p \\
p\end{array}$ & $\begin{array}{l}1.2+1 \\
2.1+0 \\
2.1+0 \\
3.0+0 \\
1.2+1 \\
1.3+1\end{array}$ & $\begin{array}{l}9.4+1 \\
1.2+1 \\
1.0+1 \\
6.4+0 \\
2.1+1\end{array}$ & $\begin{array}{l}\text { LVN E Haasbroekt } \\
\text { KFK C Muenzel+ } \\
\text { ANL E Wingt } \\
\text { ETH E Blasert } \\
\text { ORL E Blossert } \\
\text { UFT E Shakunt }\end{array}$ & $\begin{array}{l}9238+ \\
3755 \\
3757+ \\
3758+ \\
3759+ \\
3768+\end{array}$ \\
\hline $11 \ln$ & Indepe & ondent $r$ & relati & vo o(E) & \\
\hline $\begin{array}{l}\| 1 \mathrm{Cd} \\
\| 1 \mathrm{ln}\end{array}$ & $\begin{array}{c}p \\
\text { cumula }\end{array}$ & $\begin{array}{l}6.0+0 \\
\text { tive or }\end{array}$ & $\begin{array}{l}1.1+1 \\
\text { (E) }\end{array}$ & UPP E Diatrieht & 3762 \\
\hline $\begin{array}{l}-4 \\
53 \theta^{u} \\
53 \theta^{4} \\
5\end{array}$ & $\begin{array}{l}p \\
p\end{array}$ & $\begin{array}{l}4.5+2 \\
4.5+2\end{array}$ & & $\begin{array}{l}\text { KFK C Muenzel+ } \\
\text { CHI E Panonlint }\end{array}$ & $\begin{array}{l}4917 \\
4919+\end{array}$ \\
\hline$\because 1 \ln$ & relati & ive $I_{y}$ & & & \\
\hline $1 / \mathrm{Ca}$ & p & $6.0+0$ & & SUL E Zarubint & 3755 \\
\hline $111 n$ & produe & t ylald & & & \\
\hline $\begin{array}{l}11 \mathrm{Cdd}^{\mathrm{Cd}} \\
\end{array}$ & $\begin{array}{l}p \\
p\end{array}$ & $\begin{array}{l}4.5+1 \\
\text { NDG }\end{array}$ & & $\begin{array}{l}\text { UPP E Lundavist+ } \\
\text { USA R Beavert }\end{array}$ & $\begin{array}{l}3731 \\
3766\end{array}$ \\
\hline $1111 n$ & thich & larget & $y 1 \in 1 d x$ & xabundance & \\
\hline $\begin{array}{l}109 \mathrm{Ag} \\
110 \mathrm{Cd} \\
112 \mathrm{Cd}\end{array}$ & $\begin{array}{l}\text { a } \\
\text { d } \\
\text { p }\end{array}$ & $\begin{array}{l}3.0+1 \\
1.2+1 \\
2.2+1\end{array}$ & & $\begin{array}{l}\text { AML } R \text { Chaudri. } \\
\text { AML } R \text { Chaudri. } \\
\text { AML } R \text { Chaudri. }\end{array}$ & $\begin{array}{l}8248 \\
5683 \\
3767\end{array}$ \\
\hline $111 \mathrm{ln}$ & thlch & target & $y \in e l d$ & & \\
\hline Ag & $\circ$ & $1.6+1$ & $3.0+1$ & UJV C Bemt & 8237 \\
\hline
\end{tabular}




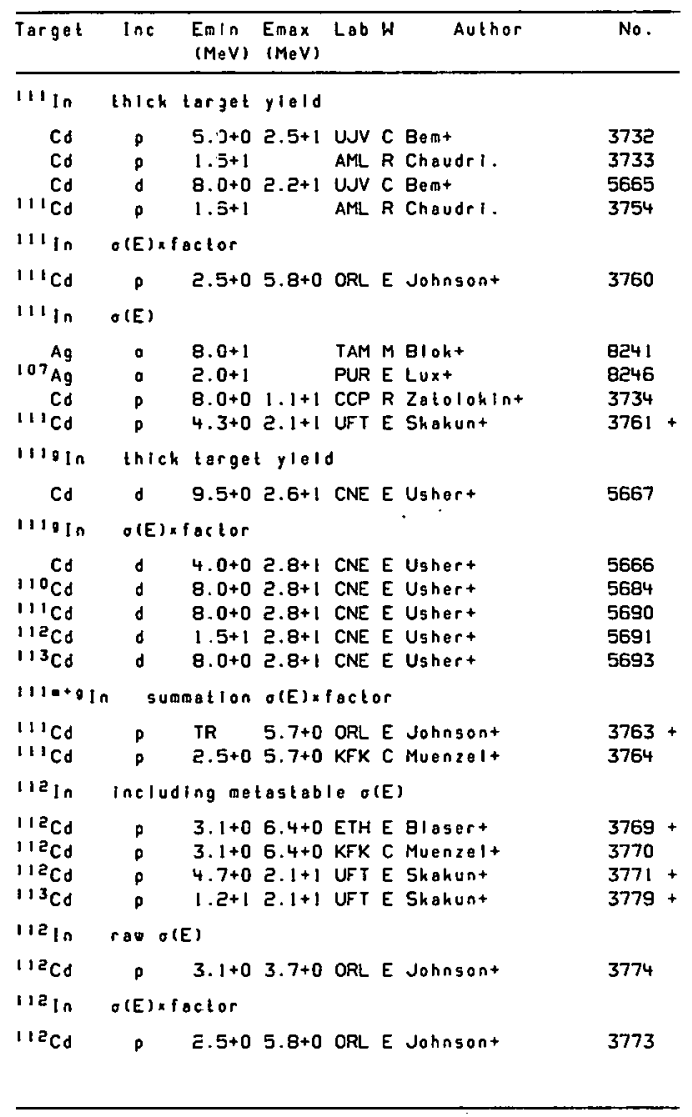

\begin{tabular}{|c|c|c|c|c|c|}
\hline Target & Inc & $\begin{array}{l}\text { Emin } \\
(\mathrm{MeV})\end{array}$ & $\begin{array}{l}\text { Eiax } \\
(\mathrm{MeV})\end{array}$ & Lob W & No. \\
\hline \multicolumn{6}{|c|}{$112_{1 n} \quad O(E)$} \\
\hline $\begin{aligned} & \mathrm{Ag} \\
& 1 \mathrm{a}_{\mathrm{Cd}}\end{aligned}$ & $a$ & $\begin{array}{l}8.0+1 \\
6.6+0\end{array}$ & $7.8+0$ & $\begin{array}{l}\text { TAM M Bloht } \\
\text { CCP E Abramovicht }\end{array}$ & $\begin{array}{l}8241 \\
3772\end{array}$ \\
\hline \multicolumn{6}{|c|}{ Summation o(E)afactor } \\
\hline $\begin{array}{l}115 \mathrm{Cd} \\
115 \mathrm{Cd}\end{array}$ & $\begin{array}{l}p \\
p\end{array}$ & $\begin{array}{l}\mathrm{TR} \\
2.5+0\end{array}$ & $\begin{array}{l}5.7+0 \\
5.7+0\end{array}$ & $\begin{array}{l}\text { ORL E Johnsont } \\
\text { KFK C Muenzelt }\end{array}$ & $\begin{array}{l}3775+ \\
3776\end{array}$ \\
\hline \multicolumn{6}{|c|}{$112-11010$ rotio o(E) } \\
\hline $115 \mathrm{~cd}$ & p & $4.0+0$ & $2.0+1$ & UFT T Shakunt & 3777 \\
\hline \multicolumn{6}{|c|}{ Independent relative o(E) } \\
\hline $113 \mathrm{Cd}$ & p & $6.0+0$ & $1.1+1$ & UPP E Dielricht & 3781 \\
\hline \multicolumn{6}{|c|}{ cumulative fission yield } \\
\hline \multirow{2}{*}{$\begin{array}{l}23 \theta_{0} \\
1131 n\end{array}$} & $\mathbf{p}$ & $3.0+1$ & $9.8+1$ & MCG E Chant & 4914 \\
\hline & $O(E) x$ & actor & & & \\
\hline $113 \mathrm{Cd}$ & $\rho$ & $2.5+0$ & $5.8+0$ & ORL E Johnsont & 3780 \\
\hline \multicolumn{6}{|c|}{ Indopendent $O(E)$} \\
\hline $\begin{array}{l}113 \mathrm{Cd} \\
114 \mathrm{Cd} \\
23 \theta_{U} \\
230_{U}\end{array}$ & $\begin{array}{l}p \\
p \\
p \\
p\end{array}$ & $\begin{array}{l}4.7+0 \\
9.8+0 \\
4.5+2 \\
4.5+2\end{array}$ & $\begin{array}{l}2.1+1 \\
2.1+1\end{array}$ & $\begin{array}{l}\text { UFT E Shohunt } \\
\text { UFT E Skohunt } \\
\text { KFK C Muenzel+ } \\
\text { CHI E Panontint }\end{array}$ & $\begin{array}{l}3782+ \\
3789+ \\
4922 \\
4923+\end{array}$ \\
\hline \multicolumn{6}{|c|}{ o(E) x actor } \\
\hline $\begin{array}{l}C d \\
1112 \mathrm{Cd} \\
113 \mathrm{Cd} \\
114 \mathrm{Cd}\end{array}$ & $\begin{array}{l}d \\
d \\
d \\
d\end{array}$ & $\begin{array}{l}8.0+0 \\
8.0+0 \\
8.0+0 \\
1.5+1\end{array}$ & $\begin{array}{l}2.8+1 \\
2.8+1 \\
2.8+1 \\
2.8+1\end{array}$ & $\begin{array}{l}\text { CNE E Ushert } \\
\text { CNE E Ushert } \\
\text { CNE E Ushert } \\
\text { CNE E Ushert }\end{array}$ & $\begin{array}{l}5668 \\
5692 \\
5694 \\
5697\end{array}$ \\
\hline \multicolumn{6}{|c|}{$1130+\theta / n$ Sumaation $O(E) \times$ factor } \\
\hline $\begin{array}{l}113 \mathrm{Cd} \\
113 \mathrm{Cd}\end{array}$ & $\begin{array}{l}p \\
p\end{array}$ & $\begin{array}{l}\text { TR } \\
2.5+0\end{array}$ & $\begin{array}{l}5.7+0 \\
5.7+0\end{array}$ & $\begin{array}{l}\text { ORL E Jornsont } \\
\text { KFK C Muenzel+ }\end{array}$ & $\begin{array}{l}3783+ \\
3784\end{array}$ \\
\hline \multicolumn{6}{|c|}{ cumulative figston yleld } \\
\hline $23 \theta_{U}$ & p & $3 \cdot 0+1$ & $9.8+1$ & MCG E Chant & 4914 \\
\hline \multicolumn{6}{|c|}{ partial product yield } \\
\hline $110 \mathrm{Pd}$ & ${ }^{7} L_{1}$ & NDG & & SUB E Elbert+ & 9442 \\
\hline
\end{tabular}




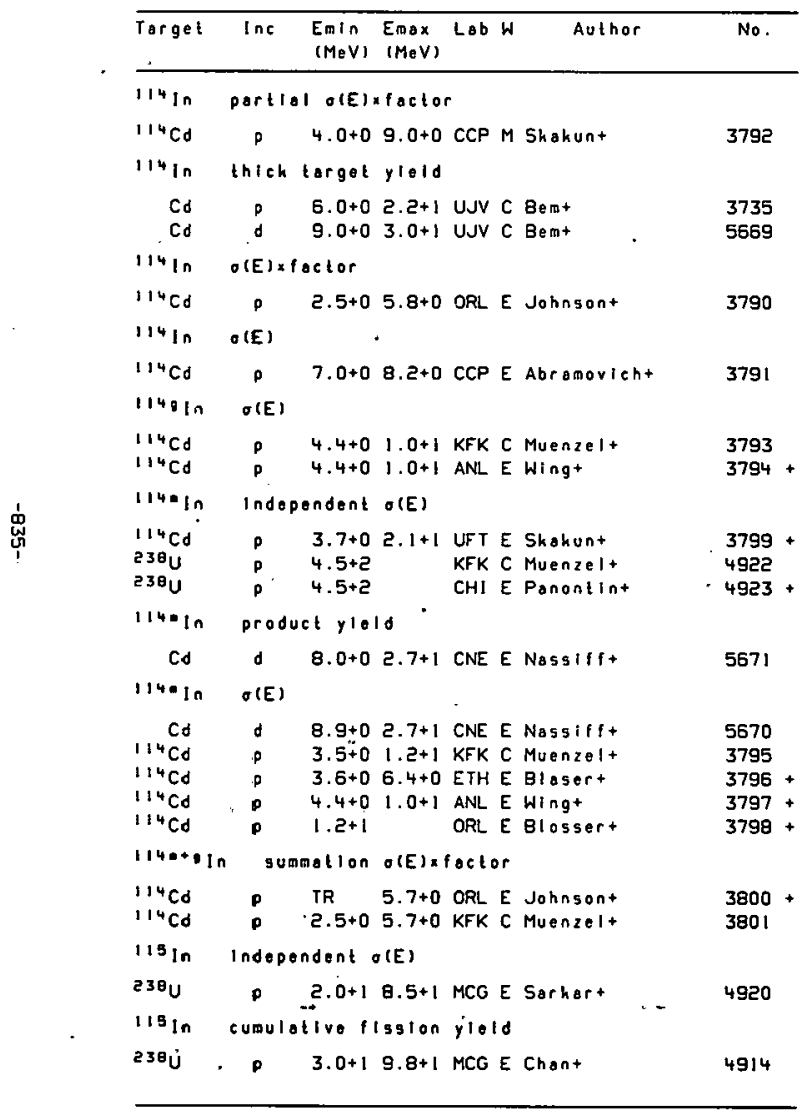

\begin{tabular}{|c|c|c|c|c|c|c|}
\hline Target & $\ln c$ & $\begin{array}{l}\text { Em } \ln \\
(\mathrm{MeV})\end{array}$ & $\begin{array}{l}\text { Emax } \\
(\operatorname{MeV})\end{array}$ & Lab & Author & No. \\
\hline $115 \cdot 1 \mathrm{n}$ & indepe & endent & $\sigma(E)$ & & & \\
\hline $\begin{array}{l}238 \mathrm{U} \\
238 \mathrm{U}\end{array}$ & $p$ & $\begin{array}{l}4.5+2 \\
4.5+2\end{array}$ & & $\begin{array}{l}\text { KFK } \\
\mathrm{CHI}\end{array}$ & $\begin{array}{l}\text { C Muenzelt } \\
\text { E Panontin- }\end{array}$ & $\begin{array}{l}4922 \\
4923+\end{array}$ \\
\hline $115 \cdot 1 n$ & ' thlch & torget & $t y|\theta| d$ & & & \\
\hline $\begin{array}{l}114 \mathrm{Cd} \\
115 \cdot \mathrm{ln}\end{array}$ & $\begin{array}{l}\dot{P} \\
O(E)\end{array}$ & $3.0+0=$ & $5.0+0$ & CLU & M Roughton- & 3802 \\
\hline $\begin{array}{l}116 \mathrm{Cd} \\
116 \mathrm{In}\end{array}$ & cumula & $\begin{array}{l}1.2+1 \\
\text { the }\end{array}$ & $\begin{array}{l}2.1+1 \\
19310 n\end{array}$ & $\begin{array}{l}\text { UFT } \\
y \mid 01\end{array}$ & $\begin{array}{l}\text { E Shatiunt } \\
\text { Id }\end{array}$ & $3808+$ \\
\hline $\begin{array}{l}23 \theta_{0} \\
118 \ln \end{array}$ & $\begin{array}{c}9 \\
\text { parlio }\end{array}$ & $\begin{array}{l}3.0+15 \\
1.0(E) \times 1\end{array}$ & $\begin{array}{l}9.8+1 \\
\text { foctor }\end{array}$ & MCG & E Chant & 4914 \\
\hline $\begin{array}{l}116 \mathrm{Cd} \\
110 \mathrm{ln}\end{array}$ & $\stackrel{p}{O(E) \times f}$ & $\begin{array}{l}5.0+0 \\
\text { actor }\end{array}$ & $9.0+0$ & CCP & M Shahunt & 3812 \\
\hline $\begin{array}{l}116 \mathrm{Cd} \\
116 \mathrm{in}\end{array}$ & $\begin{array}{c}P \\
\sigma(E)\end{array}$ & $2.5+0$ & $6.5+0$ & ORL & E Johnsont & 3809 \\
\hline $\begin{array}{l}116 \mathrm{Cd} \\
116 \mathrm{Cd} \\
116 \mathrm{Cd}\end{array}$ & $\begin{array}{l}p \\
p \\
d\end{array}$ & $\begin{array}{l}2.5+0= \\
7.3+0 \\
7.8+0\end{array}$ & $\begin{array}{l}5.8+0 \\
0.5+0 \\
1.2+1\end{array}$ & $\begin{array}{l}\text { ORL } \\
\text { CCP } \\
\text { LIN }\end{array}$ & $\begin{array}{l}\text { E Johnsont } \\
\text { E Abromorlisht } \\
\text { E Zorubint }\end{array}$ & $\begin{array}{l}3810 \\
381 ! \\
5702\end{array}$ \\
\hline $116 \cdot 1 \ln$ & incle & uding ae & netoste & .0610 & product pleld & \\
\hline $\begin{array}{l}\text { Cod } \\
118.1 \mathrm{In}\end{array}$ & $\begin{array}{l}d \\
\text { incle }\end{array}$ & $\begin{array}{l}\theta .0+0= \\
\text { uding as }\end{array}$ & $\begin{array}{l}2.7+1 \\
\text { nolosto }\end{array}$ & $\begin{array}{l}\text { CNE } \\
.010\end{array}$ & $\begin{array}{l}\text { E Nossif:t } \\
\text { o(E) }\end{array}$ & 5673 \\
\hline $\begin{array}{r}C d \\
1: 6_{C d}\end{array}$ & d & $\begin{array}{l}9.2+0= \\
4.5+0=\end{array}$ & $\begin{array}{l}2.3+1 \\
2.1+1\end{array}$ & $\begin{array}{l}\text { CNE } \\
\text { UFT }\end{array}$ & $\begin{array}{l}\text { E Naggif:- } \\
\text { E Shakun- }\end{array}$ & $\begin{array}{l}5672 \\
3813+\end{array}$ \\
\hline $116 \cdot \ln$ & indepe & endent & $O(E)$ & · & & \\
\hline 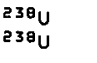 & $\begin{array}{l}\rho \\
\rho\end{array}$ & $\begin{array}{l}4.5+2 \\
4.5+2\end{array}$ & & $\begin{array}{l}\text { KFK } \\
\mathrm{CHI}\end{array}$ & $\begin{array}{l}\text { C Muenze't } \\
\text { E Ponont'nt }\end{array}$ & $\begin{array}{l}4922 \\
4923+\end{array}$ \\
\hline $\begin{array}{l}118 \cdot \ln \\
116 \mathrm{Cd}\end{array}$ & thlck & $\begin{array}{l}\text { tergot } \\
3.0+0=\end{array}$ & $\begin{array}{l}=1010 \\
5.0+0\end{array}$ & CLU & M Roughtont & 3814 \\
\hline $116 \cdot 1+02$ & $e+9 \ln$ & summoll & llon ol & (E) $\times f$ & factor & \\
\hline $\begin{array}{l}116 \mathrm{Cd} \\
11 \mathrm{Cdd}\end{array}$ & p & $\begin{array}{l}\text { TR } \\
2.5+0\end{array}$ & $\begin{array}{l}5.8+0 \\
5.7+0\end{array}$ & $\begin{array}{l}\text { ORL } \\
\text { KFK }\end{array}$ & $\begin{array}{l}\text { E Johnsost } \\
\text { C Muonzeit }\end{array}$ & $\begin{array}{l}3815+ \\
3816\end{array}$ \\
\hline
\end{tabular}




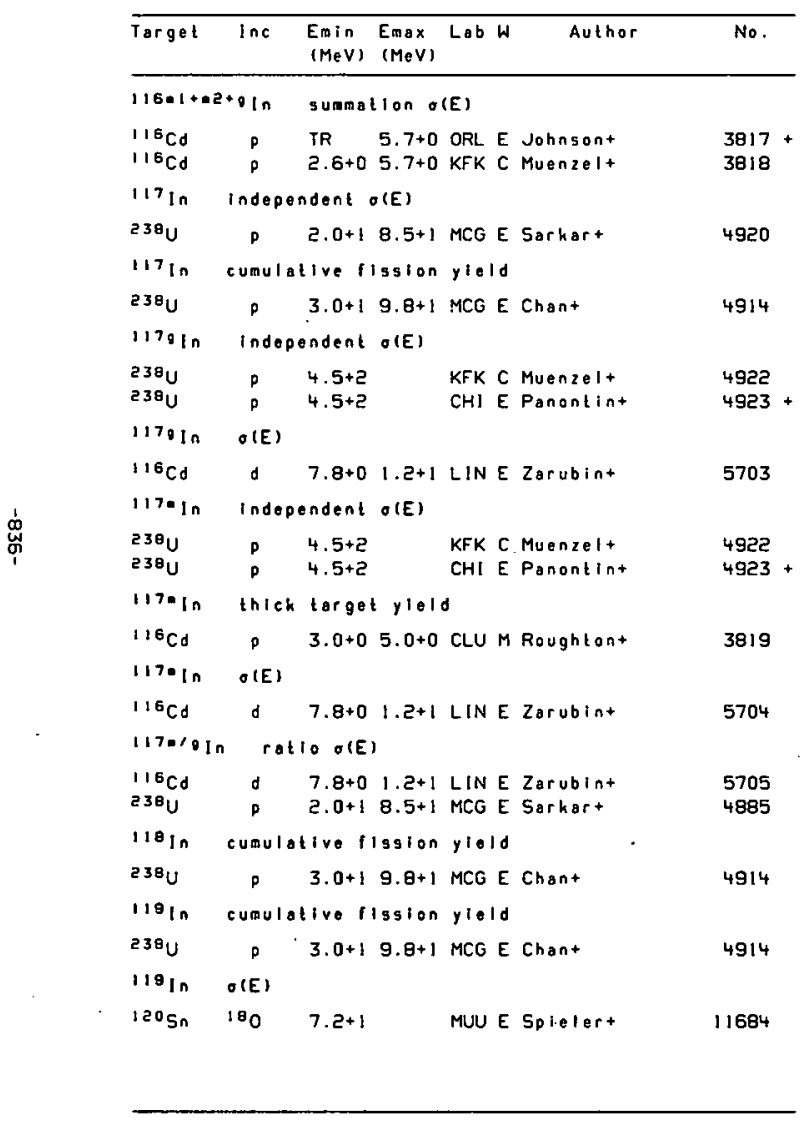

\begin{tabular}{|c|c|c|c|c|}
\hline Target & $\operatorname{Inc}$ & $\begin{array}{l}\text { Emin } E_{m a x} \\
(M e V)(M e V)\end{array}$ & Author & No. \\
\hline 12010 & cumul & tive fission & ylold & \\
\hline $238_{U}$ & $p$ & $3.0+19.8+1$ & MCG E Chant & 4914 \\
\hline I20.In & prod & ict yield & & \\
\hline $238_{U}$ & p & $1.0+2$ & MCG E Cheung+ & 4886 \\
\hline $121 / n$ & cumul & tive fission & $y 1018$ & \\
\hline $238_{U}$ & p & $3.0+19.8+1$ & MCG E Chant & 4914 \\
\hline $122 \ln$ & cumul & tive figsion & $y 101 d$ & \\
\hline $23 \theta_{U}$ & $p$ & $3.0+19.8+1$ & MCG E Chant & 4914 \\
\hline $122 \cdot \ln$ & prod & lat yleld & & \\
\hline $238 \mathrm{U}$ & $p$ & $1.0+2$ & MCG E Cheung+ & 4887 \\
\hline $123 \ln$ & cumul & tive figsion & yiels & \\
\hline $23 \theta_{U}$ & p & $3.0+19.8+1$ & MCG E Chant & 4914 \\
\hline $124 \ln$ & cumul & tlue fission & $y+01 d$ & \\
\hline $238_{U}$ & $p$ & $3.0+19.8+1$ & MCG E Chant & 4914 \\
\hline $124=\ln$ & prod & let ylold & & \\
\hline $23 \theta_{U}$ & $\rho$ & $1.0+2$ & MCG E Cheungt & 4888 \\
\hline $125 / n$ & cumul 1 & tive tigston & ylald & \\
\hline $23 \theta_{U}$ & $\rho$ & $3.0+19.8+1$ & MCG E Chont & 4914 \\
\hline $126 \mathrm{In}$ & cumul & tive ligston & $y 1010$ & \\
\hline $23 \theta_{U}$ & $p$ & $3.0+1 \quad 9.8+1$ & MCG E Chant & 4914 \\
\hline $127 \ln$ & cunul & tive figston & yield & \\
\hline $23 \theta_{U}$ & p & $3.0+19.8+1$ & MCG E Chant & $49 ! 4$ \\
\hline $128 \mathrm{ln}$ & cumul & tive ligston & $y 1010$ & \\
\hline $23 \theta_{U}$ & $p$ & $3.0+19.8+1$ & MCO E Chant & 4914 \\
\hline $129 \ln$ & cumul & tive lission & $y / 01 d$ & \\
\hline $230 \mathrm{U}$ & $p$ & $3.0+1 \quad 9.8+1$ & MCG E Chont & 4914 \\
\hline$z=50$ & $v_{n}$ & & & \\
\hline Ag & ${ }^{40} \mathrm{Ar}$ & $3.4+2$ & PAR R Lefort+ & 12202 \\
\hline
\end{tabular}




\begin{tabular}{|c|c|c|c|c|c|c|}
\hline Target & $\operatorname{lnc}$ & $\begin{array}{l}\text { Emin } \\
(\mathrm{MeV})\end{array}$ & $\begin{array}{l}E \max \\
(\operatorname{MeV})\end{array}$ & Lab h & Author & No. \\
\hline $1085 n$ & \multicolumn{6}{|c|}{$\cos O(E)$} \\
\hline${ }^{106} \mathrm{Cd}$ & $3_{\mathrm{He}}$ & $2.1+1$ & $5.9+1$ & KAZ E & E Burminshit & 6446 \\
\hline $1085 n$ & $\sigma(E)$ & & & & & : \\
\hline $102 \mathrm{Cd}$ & 。 & $1.8+1$ & & GOE E & E Kantust & 8256 \\
\hline $107 \mathrm{Sn}$ & \multicolumn{6}{|c|}{ pọrtial o(E) } \\
\hline $92 M_{0}$ & ${ }^{20} \mathrm{Ne}$ & $8.5+1$ & $1.4+2$ & PAR E & E Albouyt & 11859 \\
\hline${ }^{107} \mathrm{Sn}$ & \multicolumn{6}{|c|}{$r a w o(E)$} \\
\hline${ }^{106} \mathrm{Cd}$ & ${ }^{3} \mathrm{He}$ & $2.1+1$ & $4.7+1$ & KAZ E & E Burminshlit & 6447 \\
\hline${ }^{10 \theta} S_{n}$ & \multicolumn{6}{|c|}{ portiol row o(E) } \\
\hline $106 \mathrm{Co}$ & a & $2.1+1$ & $3.8+1$ & MAN E & E Varleyt & 8258 \\
\hline $1005 n$ & \multicolumn{6}{|c|}{ portlat o(E) } \\
\hline $92 \mathrm{Mo}$ & $23_{\mathrm{Ne}}$ & $8.0+1$ & $1.4+2$ & PAR & E Albouyt & 11860 \\
\hline${ }^{109} S_{n}$ & \multicolumn{6}{|c|}{ portial rolotive $g(E)$} \\
\hline${ }^{100} \mathrm{Cd}$ & a & $5 \cdot 0+1$ & & TOK E & E Heshimotot & 8266 \\
\hline $108 \mathrm{sn}$ & \multicolumn{6}{|c|}{ porthal o(E) } \\
\hline $92 \mathrm{Mo}$ & $20 \mathrm{Ne}$ & 8. $0+1$ & $1.2+2$ & PAR 8 & E Albouyt & 11861 \\
\hline $110 \mathrm{sn}$ & \multicolumn{6}{|c|}{ portlol o(E) } \\
\hline $92 \dot{m o}$ & ${ }^{20} \mathrm{Ne}$ & $8.8+1$ & $1: 0+2$ & PAR & EAloouyt & 11862 \\
\hline $1125 n$ & \multicolumn{6}{|c|}{ relative $I^{2}$} \\
\hline $110 \mathrm{Cd}$ & - & $1.7+1$ & $3.3+!$ & FUL & E Bront & 8270 \\
\hline $113 \mathrm{sn}$ & \multicolumn{6}{|c|}{ Including motastoblo? thich torget ylold } \\
\hline $\begin{array}{l}\text { Cd } \\
\text { in } \\
\text { ln }\end{array}$ & $\begin{array}{l}0 \\
\text { p } \\
\text { d }\end{array}$ & $\begin{array}{l}1.6+1 \\
6.0+0 \\
7.0+0\end{array}$ & $\begin{array}{l}4.4+1 \\
2.2+1 \\
2.2+1\end{array}$ & $\begin{array}{l}\text { FEI } \\
\text { FEI. } \\
\text { FEI }\end{array}$ & $\begin{array}{l}\text { E Dmilrievt } \\
\text { E Dmitrievt } \\
\text { E Dmitrievt }\end{array}$ & $\begin{array}{l}8254+ \\
3826+ \\
5706+\end{array}$ \\
\hline $113 \mathrm{sn}$ & \multicolumn{6}{|c|}{ cumulotive o(E) } \\
\hline $\begin{array}{l}538^{U} \\
538^{4} \\
5\end{array}$ & p & $\begin{array}{l}4.5+2 \\
4.5+2\end{array}$ & & $\begin{array}{l}\text { KFK } \\
\mathrm{CHI}\end{array}$ & $\begin{array}{l}\text { C Muenzelt } \\
\text { E Panonlint }\end{array}$ & $\begin{array}{l}4917 \\
4918+\end{array}$ \\
\hline $1135 n$ & \multicolumn{6}{|c|}{ relotive Iy } \\
\hline $114 \mathrm{Cd}$ & 。 & $3.0+1$ & $6.7+1$ & TOK & E Hashimotot & 8281 \\
\hline
\end{tabular}

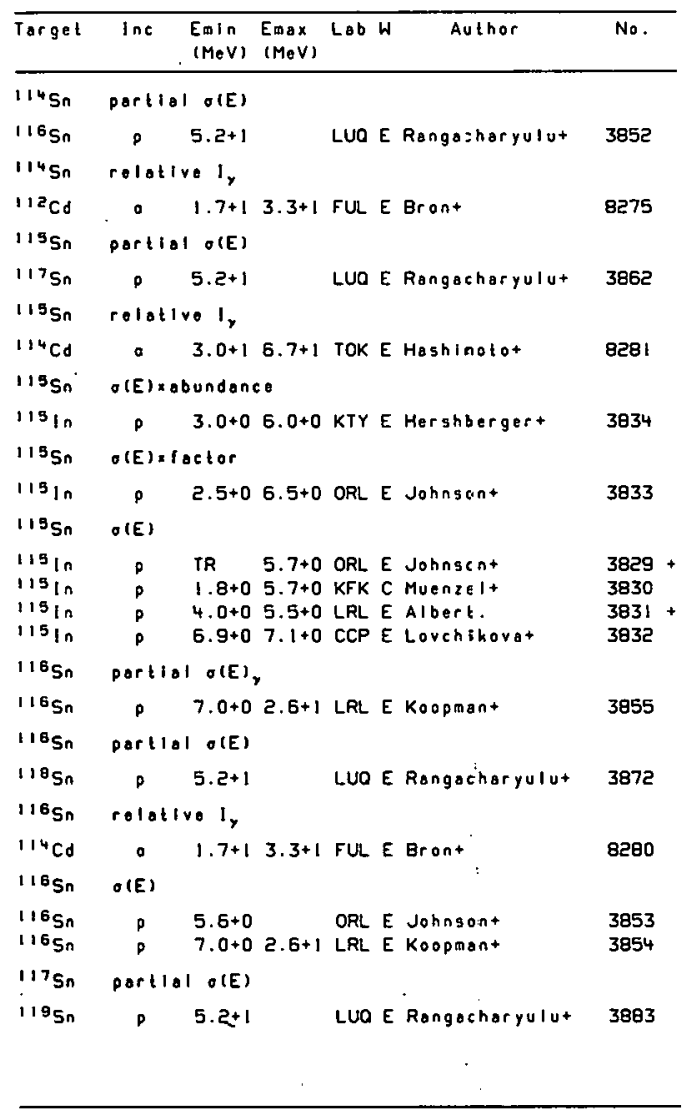




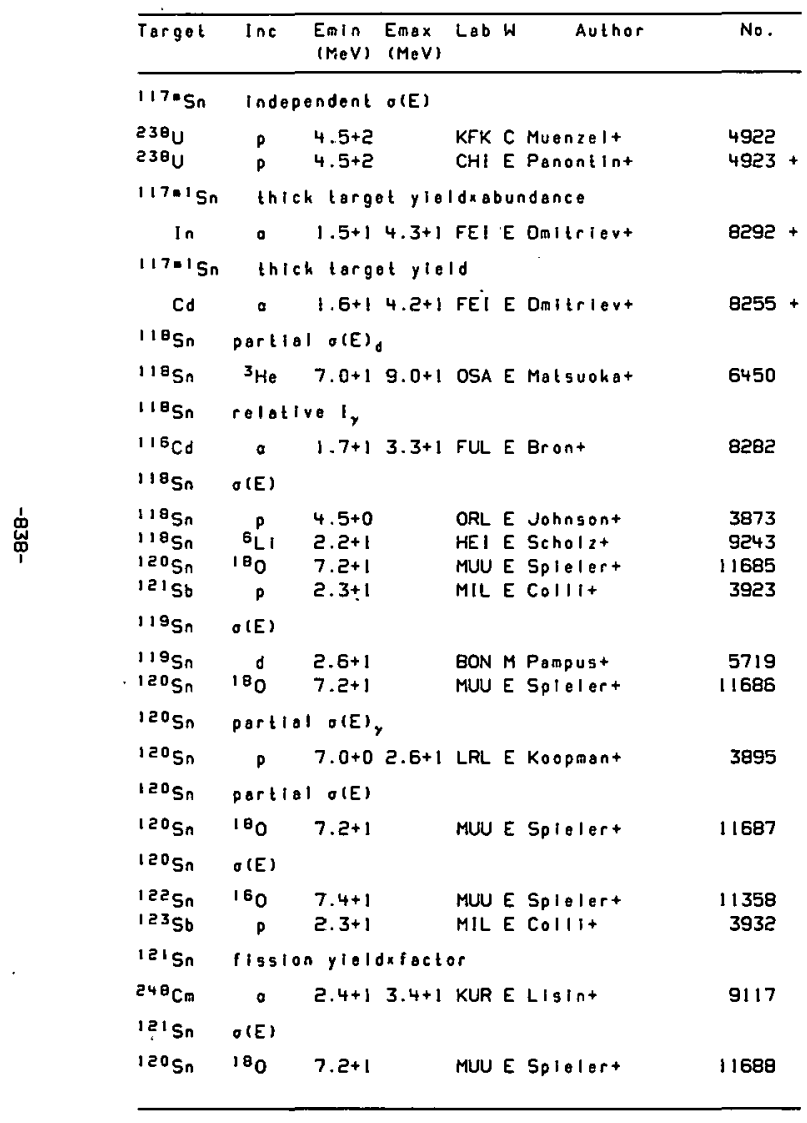

\begin{tabular}{|c|c|c|c|c|c|c|}
\hline Target & $\ln c$ & $\begin{array}{l}E_{m i n} \\
(\mathrm{MeV})\end{array}$ & $\begin{array}{l}\text { Emax } \\
\text { (MeV) }\end{array}$ & Lab & Author & No. \\
\hline $1215 n$ & $O(E)$ & & & & & \\
\hline $1225 n$ & 160 & $7.4+1$ & & MUU & E Spielert & 11359 \\
\hline $1225 n$ & parti & $\sigma(E)$ & & & & \\
\hline $1225 n$ & 160 & $7.4+1$ & & MUU & E Splelert & 11360 \\
\hline $1225 n$ & $\theta(E)$ & & & & & \\
\hline $120 \mathrm{sn}$ & $10_{0}$ & $7.2+1$ & & MUU & E Splelert & 11689 \\
\hline $1235 n$ & $\sigma(E)$ & & & & & \\
\hline $1255 n$ & 160 & $7.4+1$ & & MUU & E Splelert & 11361 \\
\hline $12305 n$ & cumu & ative & $O(E)$ & & & \\
\hline $\begin{array}{l}238 U \\
238 u\end{array}$ & $\begin{array}{l}p \\
p\end{array}$ & $\begin{array}{l}4.5+2 \\
4.5+2\end{array}$ & & $\begin{array}{l}\mathrm{KFK} \\
\mathrm{CHI}\end{array}$ & $\begin{array}{l}\text { C Muenzelt } \\
\text { E Panonlint }\end{array}$ & $\begin{array}{l}4917 \\
4918+\end{array}$ \\
\hline $123 \cdot 5 n$ & cumu & alive & $O(E)$ & & & \\
\hline $\begin{array}{l}53 \theta^{4} \\
53 \theta^{4} \\
53\end{array}$ & $\begin{array}{l}p \\
p\end{array}$ & $\begin{array}{l}4.5+2 \\
4.5+2\end{array}$ & & $\begin{array}{l}\mathrm{KFK} \\
\mathrm{CHI}\end{array}$ & $\begin{array}{l}\text { C Muenzelt } \\
\text { E Panontint }\end{array}$ & $\begin{array}{l}4917 \\
4918+\end{array}$ \\
\hline $1255 n$ & $f \mid s g 1$ & in yielc & $d \times f a c t$ & & & \\
\hline $\begin{array}{l}24{ }^{24} \mathrm{Cm} \\
1250 \mathrm{Sn}\end{array}$ & $\begin{array}{c}a \\
\text { cumu }\end{array}$ & $\begin{array}{l}2.4+1 \\
\text { ative }\end{array}$ & $\begin{array}{l}3.4+1 \\
O(E)\end{array}$ & KUR & E LISint & 9117 \\
\hline 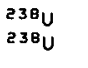 & $\begin{array}{l}p \\
p\end{array}$ & $\begin{array}{l}4.5+2 \\
4.5+2\end{array}$ & & $\begin{array}{l}\text { KFK } \\
\text { CHI }\end{array}$ & $\begin{array}{l}\text { C Muenzelt } \\
\text { E Ponantint }\end{array}$ & $\begin{array}{l}4917 \\
4918+\end{array}$ \\
\hline $127 \cdot 5 n$ & cums & ative & $O(E)$ & & & \\
\hline $\begin{array}{l}238 \mathrm{U} \\
238 \mathrm{U}\end{array}$ & $\begin{array}{l}\mathbf{p} \\
\mathbf{p}\end{array}$ & $\begin{array}{l}4.5+2 \\
4.5+2\end{array}$ & & $\begin{array}{l}\mathrm{KFK} \\
\mathrm{CHI}\end{array}$ & $\begin{array}{l}\text { C Muenzelt } \\
\text { E Panonlint }\end{array}$ & $\begin{array}{l}4917 \\
4918+\end{array}$ \\
\hline $128 \mathrm{Sn}$ & cumul & theo & (E) & & & \\
\hline $\begin{array}{l}238_{U} \\
238_{U}\end{array}$ & $\begin{array}{l}p \\
p\end{array}$ & $\begin{array}{l}4.5+2 \\
4.5+2\end{array}$ & & $\begin{array}{l}\mathrm{KFK} \\
\mathrm{CHI}\end{array}$ & $\begin{array}{l}\text { C Muenzelt } \\
\text { E Panonlint }\end{array}$ & $\begin{array}{l}4917 \\
4918+\end{array}$ \\
\hline $129.5 n$ & cumu & Ative & $O(E)$ & & & \\
\hline 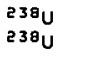 & $\stackrel{p}{p}$ & $\begin{array}{l}4.5+2 \\
4.5+2\end{array}$ & & $\begin{array}{l}\mathrm{KFK} \\
\mathrm{CHI}\end{array}$ & $\begin{array}{l}\text { C Muenzelt } \\
\text { E Panonlint }\end{array}$ & $\begin{array}{l}4917 \\
4918+\end{array}$ \\
\hline$z=51$ & $v_{n}$ & & & & & \\
\hline Ag & ${ }^{40} \mathrm{Ar}$ & $3.4+2$ & & PAR & R Lefort+ & 12202 \\
\hline
\end{tabular}




\begin{tabular}{|c|c|c|c|c|c|}
\hline Target & $\operatorname{lnc}$ & $\begin{array}{l}\text { Emin } \\
(\mathrm{MeV})\end{array}$ & 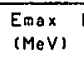 & Lab W & No. \\
\hline $1135 j$ & thick & target & $y 1010$ & & \\
\hline 1125. & م & $3.0+0$ & $4.0+0$ & CLU M Roughtont & 3845 \\
\hline $115 \mathrm{Sb}$ & cumuls & alive of & (E) & & \\
\hline 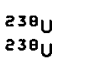 & $p$ & $\begin{array}{l}4.5+2 \\
4.5+2\end{array}$ & & $\begin{array}{l}\text { KFK C Muenzelt } \\
\text { CHI E Panontint }\end{array}$ & $\begin{array}{l}4917 \\
4918 \text {. }\end{array}$ \\
\hline 11956 & relet & ive $I_{y}$ & & & \\
\hline 11310 & o & $2.0+1$ & $3.0+1$ & FUL $E$ Bront & 8294 \\
\hline $119 \mathrm{sb}$ & thick & targot & ylold & & \\
\hline $1155 n$ & م & $4.7+0$ & $6.0+0$ & CLU M Roughtont & 3846 \\
\hline $116 \mathrm{sb}$ & parli & a 1 O(E) & & & \\
\hline $1165 n$ & p & $5.5+0$ & $5.7+0$ & ORL $E$ Johnsont & 3857 \\
\hline $116 \mathrm{st}$ & $r g \otimes t$ & hick tor & rget yi & ield & \\
\hline $116 \mathrm{sr}$ & . & $5.4+0$ & $5.7+0$ & ORL E Johnsont & 3856 \\
\hline 1165 & thich & target & yiald & & \\
\hline $1165 \mathrm{n}$ & p & $5.4+0$ & $5.7+0$ & ORL E Johnsont & 3858 \\
\hline $1189 \subseteq b$ & inder & pendent & $O(E)$ & & \\
\hline $\begin{array}{l}238 \mathrm{U} \\
238 \mathrm{U}\end{array}$ & $p$ & $\begin{array}{l}4.5+2 \\
4.5+2\end{array}$ & & $\begin{array}{l}\text { KFK C Muenzel+ } \\
\text { CHI E Panontlint }\end{array}$ & $\begin{array}{l}4922 \\
4923+\end{array}$ \\
\hline $116.5 b$ & inder & pondent & $O(E)$ & & \\
\hline 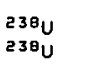 & p & $\begin{array}{l}4.5+2 \\
4.5+2\end{array}$ & & $\begin{array}{l}\text { KFK C Muenzel+ } \\
\text { CHI E Panontint }\end{array}$ & $\begin{array}{l}4922 \\
4923+\end{array}$ \\
\hline 116.195 & $b \quad r a$ & 110 O(E) & & & \\
\hline $1151 n$ & a & $1.7+1$ & $3.8+1$ & MAN E Grant+ & 8295 \\
\hline 11750 & indep & endent. & O(E) & & \\
\hline $\begin{array}{l}23 \theta^{2} \\
23 \theta^{2}\end{array}$ & $p$ & $\begin{array}{l}4.5+2 \\
4.5+2\end{array}$ & & $\begin{array}{l}\text { KFK C Muenzolt } \\
\text { CHI E Ponontint }\end{array}$ & $\begin{array}{l}4922 \\
4923+\end{array}$ \\
\hline $117 \mathrm{sb}$ & thich & target & y/eld & & \\
\hline $1175 n$ & p & $3.0+0$ & $6.0+0$ & CLU M Roughtont & 3858 \\
\hline
\end{tabular}

\begin{tabular}{|c|c|c|c|c|c|}
\hline Target & $\operatorname{lnc}$ & $\begin{array}{l}E m \ln \\
(M e V)\end{array}$ & $\begin{array}{l}E_{\max } \\
(\operatorname{MeV})\end{array}$ & Lab W & No. \\
\hline $1175 b \quad 0$ & \multicolumn{5}{|c|}{ o(E) x factor } \\
\hline $1175 n$ & $\mathrm{p}$ & $2.6+0$ & $5.4+0$ & ORL E Johns,ont & 3867 \\
\hline $117_{\mathrm{Sb}} \sigma$ & \multicolumn{5}{|l|}{$\sigma(E)$} \\
\hline $\begin{array}{l}5 n \\
1155 n \\
1175 n \\
1175 n \\
1175 n \\
1175 n\end{array}$ & $\begin{array}{l}p \\
p \\
p \\
p \\
p \\
p \\
p\end{array}$ & $\begin{array}{l}8.0+0 \\
5.6+0 \\
T R \\
3.0+0 \\
3.7+0 \\
3.7+0\end{array}$ & $\begin{array}{l}1.1+1 \\
7.1+0 \\
5.4+0 \\
6.4+0 \\
6.4+0\end{array}$ & $\begin{array}{l}\text { CCP R Zatolohint } \\
\text { ORL E Johnont } \\
\text { ORL E Johnsont } \\
\text { ORL E Johnsont } \\
\text { ETH E Blasert } \\
\text { KFK C Muenzelt }\end{array}$ & $\begin{array}{l}3839 \\
3359 \\
3863 \\
3884 \\
38655 \\
3866\end{array}$ \\
\hline $11 \theta_{S b} p$ & \multicolumn{5}{|c|}{ partial o(E) } \\
\hline $118_{5 n}$ & p & $4.5+0$ & $4.6+0$ & ORL E Johnsent & 3875 \\
\hline $118 \mathrm{sb} r$ & \multicolumn{5}{|c|}{ row thlch target yleld } \\
\hline $1185 n$ & $p$ & $4.4+0$ & $4.6+0$ & ORL E Johnsent & 3874 \\
\hline $118_{5 b} t$ & \multicolumn{5}{|c|}{ thick target yield } \\
\hline $1185 n$ & $p$ & $4.4+0=$ & $4.6+0$ & ORL E Johnsant & 3879 \\
\hline $11 \theta_{5 b} 0$ & \multicolumn{5}{|c|}{ otE $\times$ factor } \\
\hline $118_{5 n}$ & p & $4.5+0=$ & $5.5+0$ & ORL E Johnsant & 3878 \\
\hline $110_{5 b} 0$ & \multicolumn{5}{|l|}{$\sigma(E)$} \\
\hline $\begin{array}{l}1185 n \\
1185 n\end{array}$ & p & $\begin{array}{l}\text { TR } \\
4.6+0\end{array}$ & $\begin{array}{l}7.1+0 \\
5.3+0\end{array}$ & $\begin{array}{l}\text { ORL E Johnsont } \\
\text { ORL E Johnsont }\end{array}$ & $\begin{array}{l}3876 \\
3877\end{array}$ \\
\hline $1180 \mathrm{sb}$ & \multicolumn{5}{|c|}{ Independent $O(E)$} \\
\hline $\begin{array}{l}238 \mathrm{U} \\
238^{\prime}\end{array}$ & $p$ & $\begin{array}{l}4.5+2 . \\
4.5+2\end{array}$ & & $\begin{array}{l}\text { KFK C Muenzalt } \\
\text { CHI E Panontint }\end{array}$ & $\begin{array}{l}4922 \\
4923+\end{array}$ \\
\hline $118.15 b$ & \multicolumn{5}{|c|}{$\sigma(E)$} \\
\hline $\begin{array}{c}5 n \\
1180 / 05 b\end{array}$ & \multicolumn{5}{|c|}{ ratloo(E) } \\
\hline $115 / n$ & 0 & $1.7+12$ & $2.1+1$ & MAN E Grant+ & 8296 \\
\hline $119 \mathrm{sb}$ & \multicolumn{5}{|c|}{ partlal o(E) } \\
\hline $1185 n$ & : & $\mid .6+1$ & $1.8+1$ & MIN E Fitzge-aldt & 3890 \\
\hline
\end{tabular}




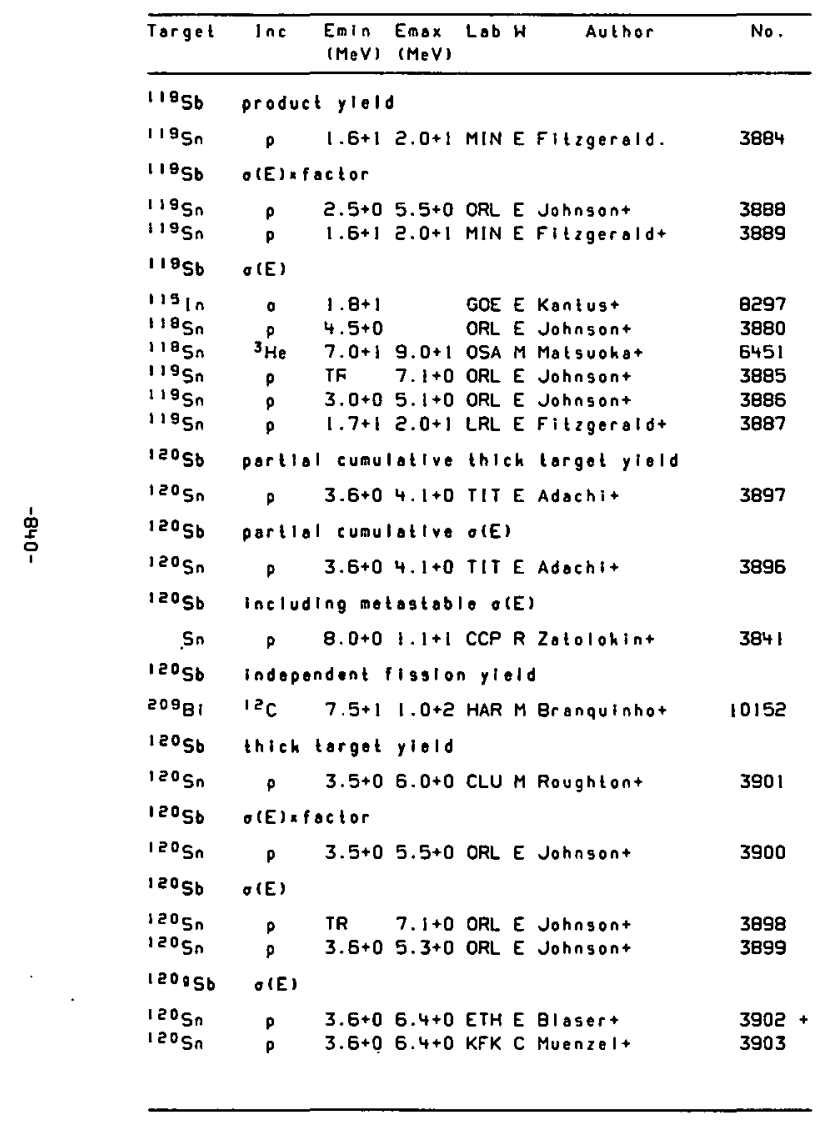

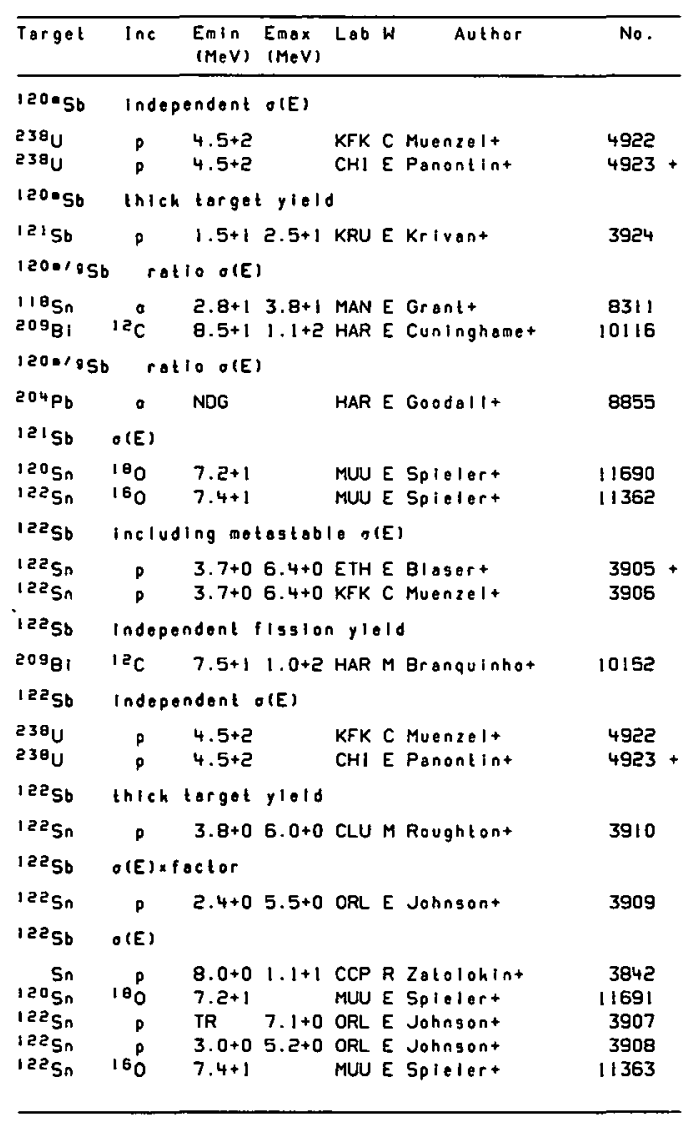




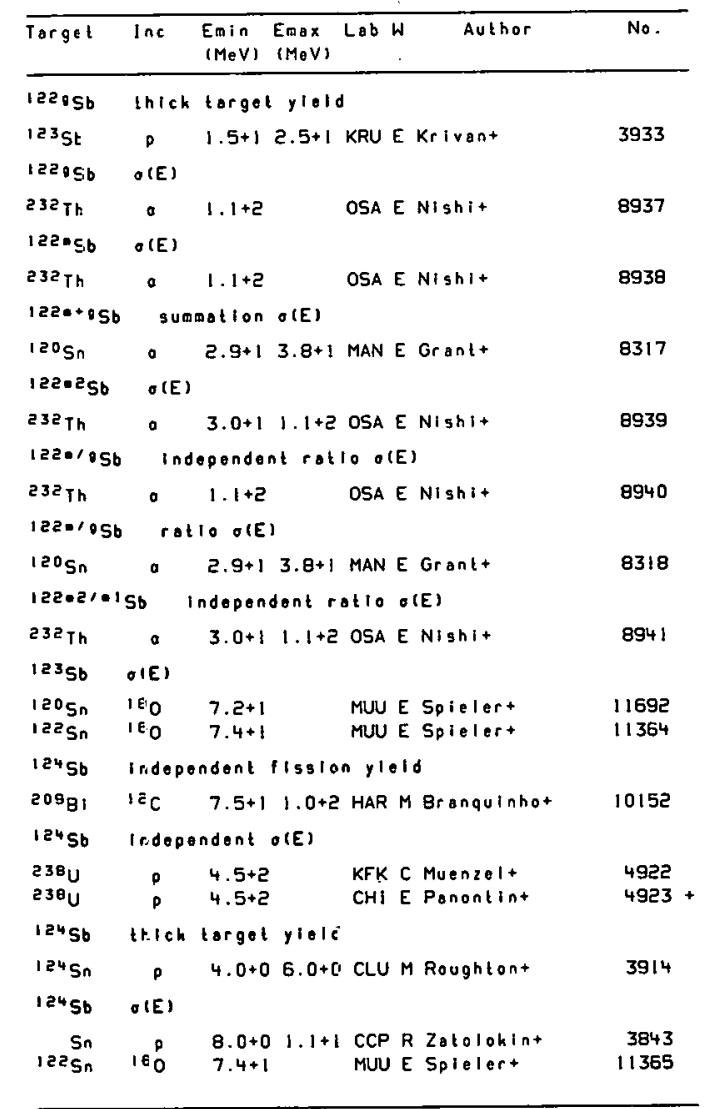

\begin{tabular}{|c|c|c|c|c|c|c|}
\hline Target & $\ln c$ & $\begin{array}{l}\text { Emin } \\
(M e V)\end{array}$ & $\begin{array}{l}\text { Emax } \\
(\mathrm{MeV})\end{array}$ & Lab W & Althor & No. \\
\hline $124 \mathrm{Sb}$ & $O(E)$ & & & & & \\
\hline $\begin{array}{l}12^{4} \mathrm{Sn} \\
12^{4} \mathrm{Sn}\end{array}$ & $p$ & $\begin{array}{l}\text { TR } \\
2.5+0\end{array}$ & $\begin{array}{l}7.1+0 \\
5.5+0\end{array}$ & $\begin{array}{l}\text { ORL E } \\
\text { ORL E }\end{array}$ & $\begin{array}{l}\text { Johnsent } \\
\text { Johnsent }\end{array}$ & $\begin{array}{l}3912 \\
3913\end{array}$ \\
\hline 124056 & Indep & endent & $O(E)$ & & & \\
\hline $\begin{array}{l}238 \mathrm{U} \\
238 \mathrm{U}\end{array}$ & $\begin{array}{l}p \\
P\end{array}$ & $\begin{array}{l}4.5+2 \\
4.5+2\end{array}$ & & $\begin{array}{l}\text { KFK C } \\
\text { CHI E }\end{array}$ & $\begin{array}{l}\text { Muenzel+ } \\
\text { Panontint }\end{array}$ & $\begin{array}{l}4922 \\
4923\end{array}$ \\
\hline \multicolumn{7}{|c|}{$124=+0$ Sb ratlo $\theta(E)$} \\
\hline $1225 n$ & 0 & $2.5+1$ & $3.8+1$ & MAN E & Grant* & 8320 \\
\hline${ }^{125} \mathrm{sb}$ & Indeper & ndent o & o(E) & & & \\
\hline $\begin{array}{l}238_{U} \\
238 U\end{array}$ & p & $\begin{array}{l}4.5+2 \\
4.5+2\end{array}$ & & $\begin{array}{l}\text { KFK C } \\
\text { CHI E }\end{array}$ & $\begin{array}{l}\text { Muenzelt } \\
\text { Panontint }\end{array}$ & $\begin{array}{l}4922 \\
4923+\end{array}$ \\
\hline $125 \mathrm{sb}$ & cumulat & tive or & (E) & & & \\
\hline $\begin{array}{l}238 \mathrm{U} \\
238_{U}\end{array}$ & p & $\begin{array}{l}4.5+2 \\
4.5+2\end{array}$ & & $\begin{array}{l}\text { KFK C } \\
\text { CHI E }\end{array}$ & $\begin{array}{l}\text { Muenzel+ } \\
\text { Ponontint }\end{array}$ & $\begin{array}{l}4917 \\
4918+\end{array}$ \\
\hline 12856 & & indent $f$ & fission & n yield & & \\
\hline $209^{\mathrm{Bi}}$ & ${ }^{12} \mathrm{C}$ & $7.5+1$ & $1.0+2+$ & HAR M & Bronquinhot & 10152 \\
\hline $126.5 b$ & ladepe & endent & $O(E)$ & & & \\
\hline $\begin{array}{l}53 \theta^{2} \\
23 \theta^{\prime}\end{array}$ & p & $\begin{array}{l}4.5+2 \\
4.5+2\end{array}$ & & $\begin{array}{l}\text { KFK C } \\
\mathrm{CH} 1 \mathrm{E}\end{array}$ & $\begin{array}{l}\text { Muenzel+ } \\
\text { Pononlinat }\end{array}$ & $\begin{array}{l}4922 \\
4923+\end{array}$ \\
\hline 126.50 & $\sigma(E)$ & & & & & \\
\hline $232 \mathrm{Th}$ & 0 & $3.0+1$ & $1.1+20$ & OSA E & $\mathrm{Nighit}$ & 8942 \\
\hline $126 \cdot 5 b$ & Indepe & endent & $O(E)$ & & & \\
\hline $\begin{array}{l}238 \mathrm{U} \\
23 \theta^{2} \mathrm{U}\end{array}$ & p & $\begin{array}{l}4.5+2 \\
4.5+2\end{array}$ & & $\begin{array}{l}\text { KFK C } \\
\text { CHI E }\end{array}$ & $\begin{array}{l}\text { Muenzelt } \\
\text { Panontina }\end{array}$ & $\begin{array}{l}4922 \\
4923+\end{array}$ \\
\hline $126-5 b$ & $\sigma(E)$ & & & & & \\
\hline $\begin{array}{l}232 \mathrm{Th} \\
126=+8 \mathrm{Sb}\end{array}$ & o suma & $\begin{array}{l}3.0+1 \\
\text { mation }\end{array}$ & $\begin{array}{l}1.1+20 \\
O(E)\end{array}$ & OSA E & Nishit & 8943 \\
\hline $\begin{array}{l}1245 n \\
126=25 b\end{array}$ & $\begin{array}{l}0 \\
O(E)\end{array}$ & $2.9+1$ & $3.8+1 r$ & MAN E & Grant & 8321 \\
\hline $232 \mathrm{Th}$ & $a$ & $3.0+1$ & $1.1+20$ & OSA E & Nishit & 8944 \\
\hline
\end{tabular}




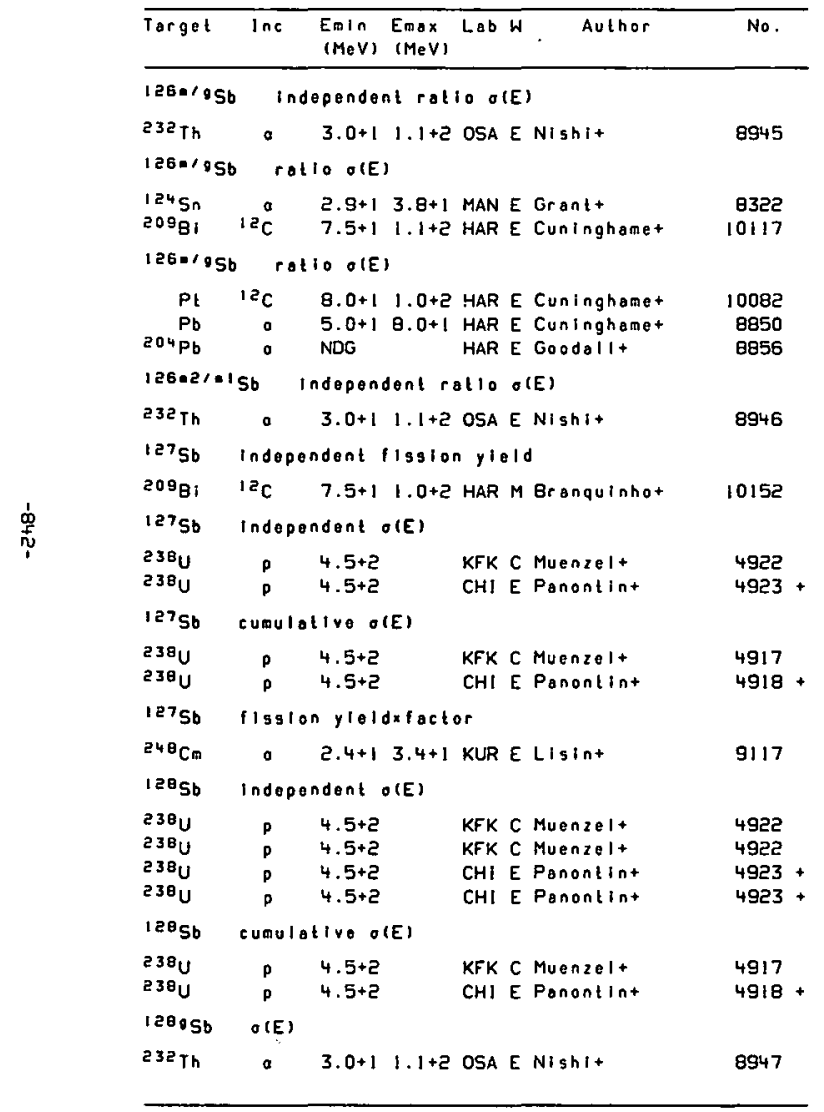

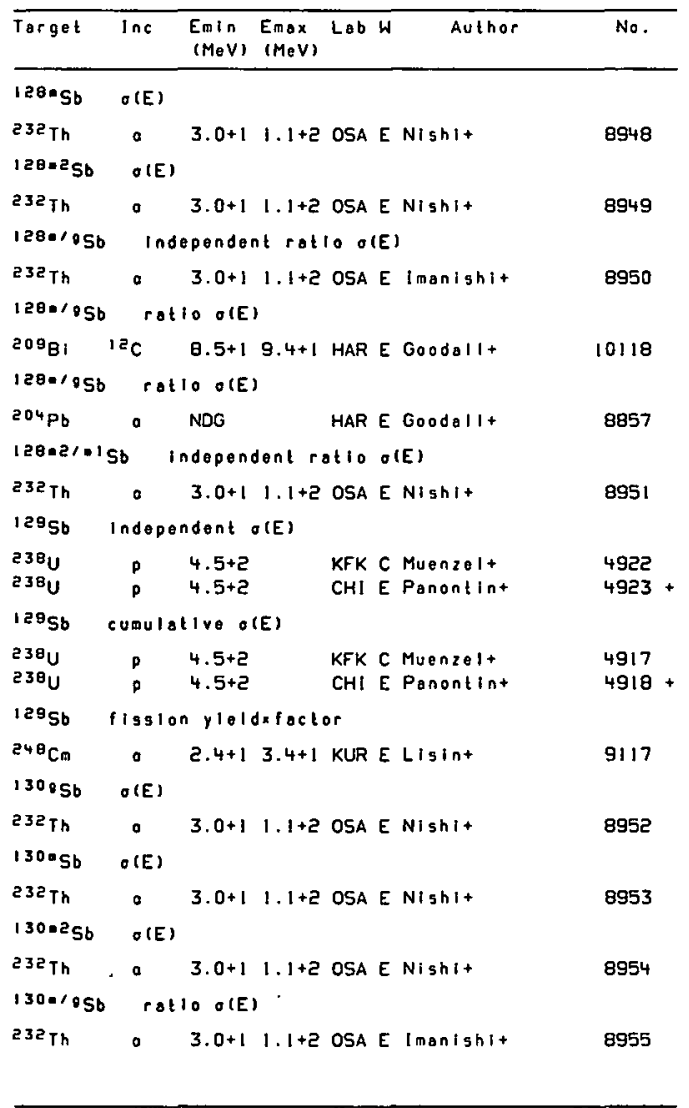




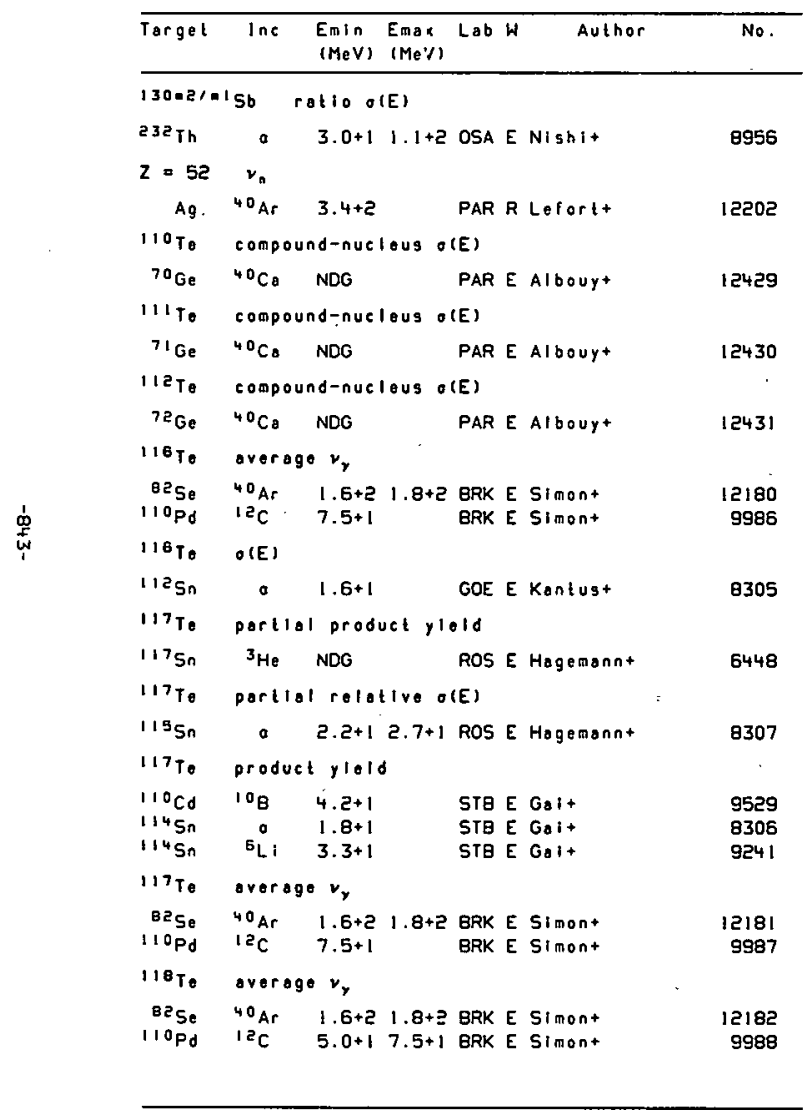

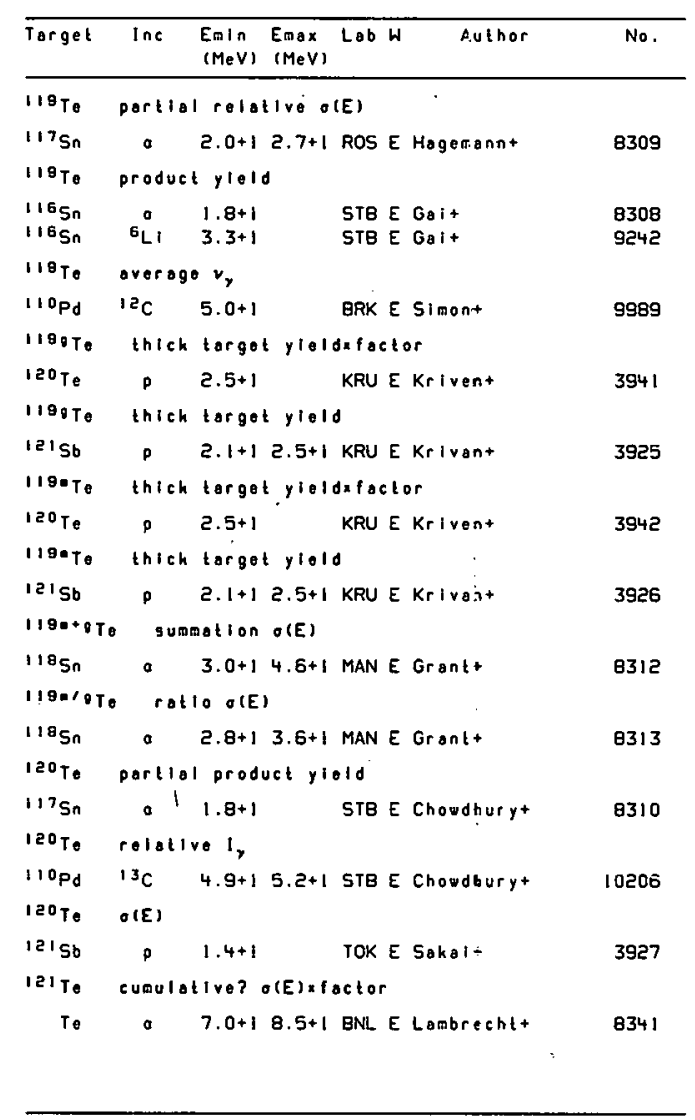




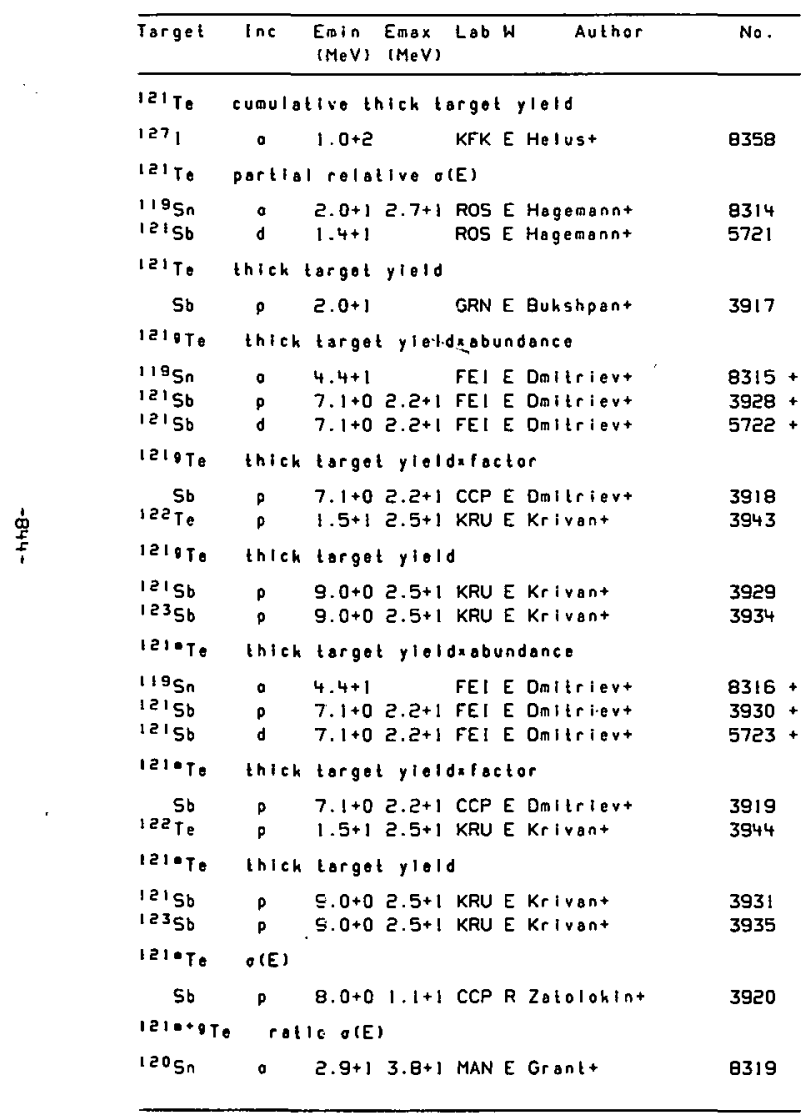

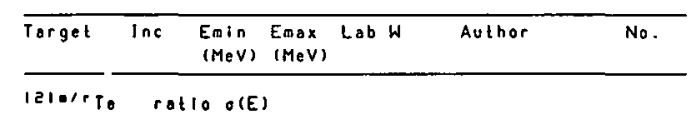

Sb O $1.0+12.2+1$ FEI E Dnitrievt 5720

$125 \mathrm{~T}^{\circ} O\left(\mathrm{C}^{2}\right)$

$1205018007.5+1 \quad$ MUN E Spielert

le3sb p $1.4+1$ TOKE Sahait 3936

123 to o(E) afoctor

$120 \mathrm{Sn} T_{\mathrm{Li}} 3.5+1$ ROS E Hagemannt 9443

$123 \mathrm{Te} O(E)$

$1205 n 180 \quad 7.2+1 \quad$ MUU E Spielert 11694

$1225 n 1607.4+1$ MUUE Spielert 11366

123. Te thich torget yleldrabundence

$1235 \mathrm{~b} P \quad 7.1+02.2+1$ FEI E Dmitrievt $3937+$

$1235 \mathrm{Sb} 7.1+02.2+1$ FEI E Omitrievt $5724+$

123.Te thich torget yieldafactor

$5 b$ P $7.1+02.2+1$ CCP E Omilrievt 3921

$\begin{array}{llll}124 \mathrm{Te} & p & 1.5+12.5+1 \text { KRU E Krivant } & 3954 \\ 1271 & \rho & 1.5+12.5+1 \text { KRU E Krivant } & 4025\end{array}$

123.Te thich terget ylold

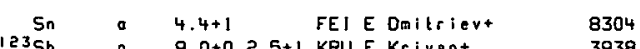

$9.0+02.5+1$ KRU E Krivant 3938

123. T⿱

Sb $P \quad 8.0+01.1+1$ CCP R Zatolohint 3922

$124 \mathrm{~T}^{\mathrm{S}} \mathrm{O}(\mathrm{E})$

$\begin{array}{lllll}1205 n & 180 & 7.2+1 & \text { MUU E Spielert } & 11695 \\ 1225 n & 160 & 7.4+1 & \text { MUU E Spielert } & 11367\end{array}$

${ }^{125} \mathrm{~T}$ O $O$ (E)

1205 n $180 \quad 7.2+1$ MUU E Spielert

IEs 160 7.t+1 MUUE Spielert 11368

150 TE $O(E)$

$\begin{array}{lllll}120 & & \\ 1205 n & 180 & 7.2+1 & \text { MUUE Spielert } & 11697 \\ 1255 & 160 & 7 . t+1 & \text { MUUE Spielert } & 11369 \\ & & & \end{array}$ 


\begin{tabular}{|c|c|c|c|c|}
\hline Target & $\ln c$ & $\begin{array}{ll}\text { Emin } & \text { Emax } \\
(\operatorname{MeV}) & (\operatorname{Me} v)\end{array}$ & Author & No. \\
\hline $131 \mathrm{Te}$ & $\nabla(E)$ & & & \\
\hline${ }^{130} \mathrm{Te}$ & d & $4.3+0 \quad 1.4+1$ & КTO M Ohamura+ & 5729 \\
\hline 131. To & $O(E)$ & & & \\
\hline${ }^{130} \mathrm{Te}$ & d & $4.3+0 \quad 1.4+1$ & KTO M Okamura+ & 5730 \\
\hline $131018 \mathrm{Te}$ & lo inde & -pendene rati & $100(E)$ & \\
\hline $2327 \mathrm{~h}$ & 。 & $3.0+1 \quad 1.1-2$ & OSAE imanishit & 8957 \\
\hline 133.100 & le inde & opendent rati & Io $O(E)$ & \\
\hline 232 in & a & $3.0+1 \quad 1.1-2$ & OSA E Imanishit & 8958 \\
\hline$z=53$ & $v_{n}$ & & & \\
\hline Ag & ${ }^{2} \mathrm{O}_{\mathrm{Ar}}$ & $3.4+2$ & PAR R Lefort+ & 12202 \\
\hline 113 & groduct & yield & & \\
\hline $127 i$ & D & $3.4+2$ & LVP E Butement* & 4026 \\
\hline 1181 & product & yiald & & \\
\hline 127 & $p$ & $3.4+2$ & LVP E Butement+ & 4027 \\
\hline 1191 & cumulati & ive? o(E) & & \\
\hline$|27|$ & d & $8.5+19.0+1$ & JUL E Weinricht & 5731 \\
\hline $119_{1}$ & producl & yield & & \\
\hline $127 \mid$ & $p$ & $3.4+2$ & LVP E Butementt & 4028 \\
\hline $120_{1}$ & cumulall & ive? o(E) & & \\
\hline 127 & d & $8.0+19.0+1$ & JUL E Weinricht & 5732 \\
\hline 1211 & cumulali & ive thick tar & rgot yloldxfoctor & \\
\hline $\operatorname{cop} 1$ & $p$ & $4.8+17.8+1$ & HAR E Syme+ & 4072 \\
\hline 1211 & cumulatl & lve thick lar & rgot ylold & \\
\hline$|27|$ & 。 & $1.0+2$ & KFK E Helus+ & 8359 \\
\hline 1211 & product & $y 101 d$ & & \\
\hline$|27|$ & d & $7.8+1$ & JUL E Weinricht & 5763 \\
\hline $\mid 21$ & thlek to & orgol yleld & & \\
\hline Sb & ${ }^{3} \mathrm{He}$ & $1.2+12.6+1$ & UJV C Bemt & 6452 \\
\hline
\end{tabular}

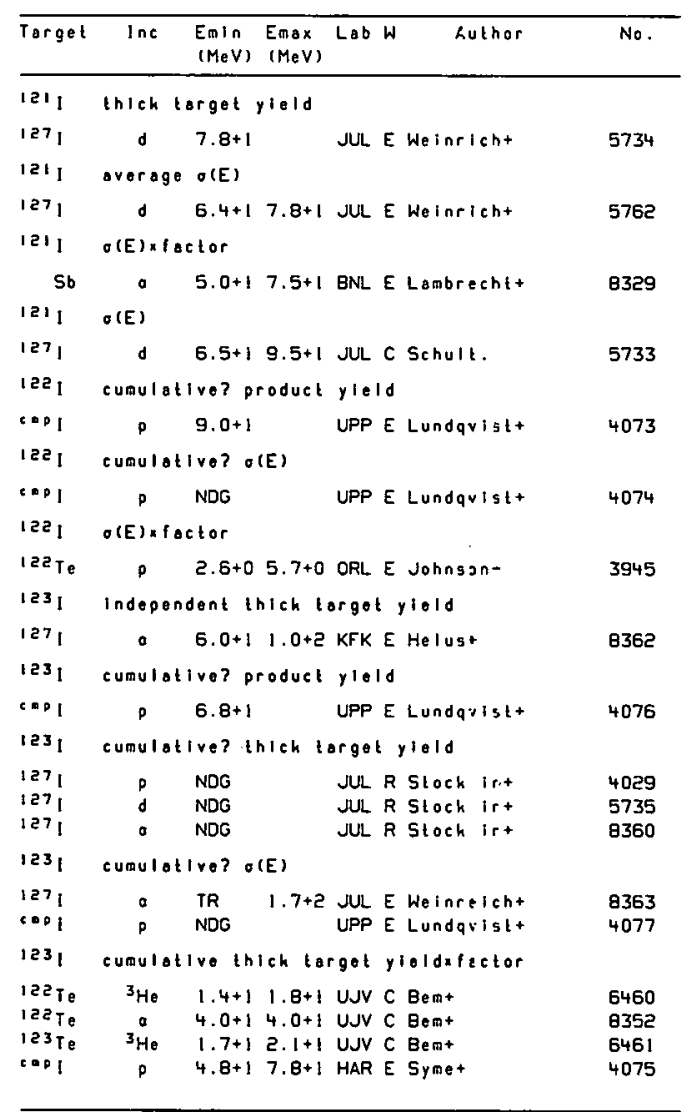




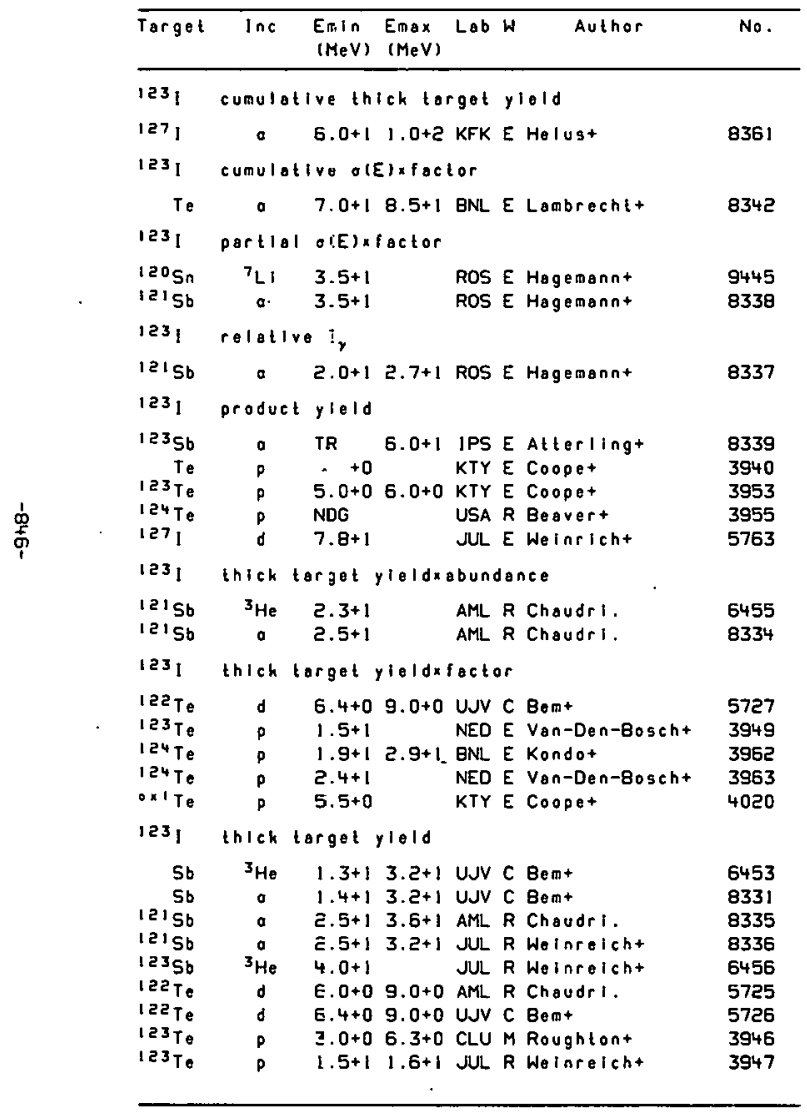

\begin{tabular}{|c|c|c|c|c|c|c|}
\hline Targat & $\ln c$ & $\begin{array}{l}\text { Emin } \\
(\mathrm{MeV})\end{array}$ & $\begin{array}{l}\text { Emax } \\
(\mathrm{MeV})\end{array}$ & Lab & Author & No. \\
\hline $123 \mathrm{I}$ & thich to & argot $y$ & yiold & & & \\
\hline $\begin{array}{l}124 \mathrm{Te} \\
124 \mathrm{Te} \\
124 \mathrm{Te}\end{array}$ & $\begin{array}{l}p \\
p \\
p\end{array}$ & $\begin{array}{l}2.6+1 \\
2.8+1 \\
3.0+1\end{array}$ & $3.0+1$ & $\begin{array}{l}\text { BNL } \\
\text { JUL } \\
\text { AML }\end{array}$ & $\begin{array}{l}\text { E Kondot } \\
R \text { Weinrelcht } \\
R \text { Chaudri. }\end{array}$ & $\begin{array}{l}3956 \\
3957 \\
3958\end{array}$ \\
\hline 1231 & overoge & $\sigma(E)$ & & & & \\
\hline $\begin{array}{l}127_{1} \\
123_{1}\end{array}$ & $\frac{d}{d(E) \times f_{a}}$ & $\begin{array}{l}6.4+1 \\
\text { actor }\end{array}$ & $7.8+1$ & JUL & E Weinricht & 5762 \\
\hline $\begin{array}{r}120 \mathrm{Sn} \\
5 \mathrm{~s} \\
123 \mathrm{Te} \\
124 \mathrm{Te} \\
124 \mathrm{Te} \\
124 \mathrm{Te}\end{array}$ & $\begin{array}{c}{ }^{7} L I \\
0 \\
P \\
p \\
p \\
P\end{array}$ & $\begin{array}{l}3 \cdot 5+1 \\
5 \cdot 0+1 \\
1.0+1 \\
9.0+0 \\
1.9+1 \\
4.0+2\end{array}$ & $\begin{array}{l}7.5+1 \\
1.9+1 \\
2.3+1 \\
2.9+1\end{array}$ & $\begin{array}{l}\text { ROS } \\
\text { BNL } \\
\text { NED } \\
\text { NEO } \\
\text { BNL } \\
\text { CAR }\end{array}$ & $\begin{array}{l}\text { E Hagemannt } \\
\text { E Lambrecht+ } \\
\text { E Van-Den-Boseht } \\
\text { E Van-Den-Boscht } \\
\text { E Kondot } \\
\text { E Revlandt }\end{array}$ & $\begin{array}{l}9444 \\
8330 \\
3948 \\
3959 \\
3960 \\
3961\end{array}$ \\
\hline 1231 & O(E) & & & & & \\
\hline $\begin{array}{l}123 \mathrm{Te} \\
124 \mathrm{Te} \\
124 \mathrm{Te} \\
124 \mathrm{Te} \\
124 \mathrm{Te} \\
125 \mathrm{Te} \\
125 \mathrm{Te} \\
126 \mathrm{Te} \\
126 \mathrm{Te} \\
127 \mathrm{~J}\end{array}$ & $\begin{array}{l}p \\
p \\
p \\
p \\
p \\
p \\
p \\
p \\
p \\
d\end{array}$ & $\begin{array}{l}1.5+1 \\
1.2+1 \\
2.8+1 \\
4.0+2 \\
4.0+2 \\
4.0+2 \\
4.0+2 \\
4.0+2 \\
4.0+2 \\
5.2+1\end{array}$ & $\begin{array}{l}2.8+1 \\
2 \\
9.5+1\end{array}$ & $\begin{array}{l}\text { JUL } \\
\text { BNL } \\
\text { JUL } \\
\text { KFK } \\
\text { CAR } \\
\text { KFK } \\
\text { PUP } \\
\text { KFK } \\
\text { PUP } \\
\text { JUL }\end{array}$ & $\begin{array}{l}\text { C Schult. } \\
\text { E Kondot } \\
\text { C Schult. } \\
\text { C Muenzelt } \\
\text { E Reulandt } \\
\text { C Muenzel+ } \\
\text { E Churcht } \\
\text { C Muenzel+ } \\
\text { E Churcht } \\
\text { C Schult. }\end{array}$ & $\begin{array}{l}3950 \\
3964 \\
3965 \\
3966 \\
3967 \\
3980 \\
3981 \\
3990 \\
3991 \\
5736\end{array}$ \\
\hline 1241 & indopens & dent $f$ & insion & $y \mid 01$ & 10 & \\
\hline $\begin{array}{l}209_{B i} \\
124 \mathrm{I}\end{array}$ & $\begin{array}{l}\operatorname{la}_{\mathrm{C}} \mathrm{C} \\
\text { cunulat }\end{array}$ & $\begin{array}{l}7.5+1 \\
\text { tve? th }\end{array}$ & $\begin{array}{l}1.0+2 \\
\text { hlch }\end{array}$ & $\begin{array}{l}\text { HAR } \\
\text { orget }\end{array}$ & $\begin{array}{l}M \text { Branquinhot } \\
\text { yleldxfactor }\end{array}$ & 10152 \\
\hline $\begin{array}{l}122 \mathrm{Te} \\
124 \mathrm{I}\end{array}$ & $\begin{array}{c}0 \\
\text { cumulat }\end{array}$ & $\begin{array}{l}4.0+1 \\
\text { tive }|\mathrm{h}|\end{array}$ & $\begin{array}{l}4.0+1 \\
1 \text { ch to }\end{array}$ & $\begin{array}{l}\text { UJV } \\
\text { aget }\end{array}$ & $\begin{array}{l}C \text { Bemt } \\
y \mid 010\end{array}$ & 8353 \\
\hline $\begin{array}{l}1271 \\
1241\end{array}$ & $\begin{array}{c}0 \\
\text { product }\end{array}$ & $\begin{array}{l}1.0+2 \\
y 1010\end{array}$ & & KFK & E Helust & 8364 \\
\hline $\begin{array}{l}123 \mathrm{Te} \\
124 \mathrm{Te} \\
1271\end{array}$ & $\begin{array}{l}p \\
p \\
d\end{array}$ & $\begin{array}{l}5.0+0 \\
\text { NDG } \\
7.8+1\end{array}$ & $6.0+0$ & $\begin{array}{l}\text { KTY } \\
\text { USA } \\
\text { JUL }\end{array}$ & $\begin{array}{l}\text { E Coopet } \\
R \text { Boavert } \\
\text { E Weinrlent }\end{array}$ & $\begin{array}{l}3953 \\
3968 \\
5763\end{array}$ \\
\hline
\end{tabular}




\begin{tabular}{|c|c|c|c|c|c|c|}
\hline Terget & $\ln c$ & $\begin{array}{l}\text { Emin } \\
\text { (MeV) }\end{array}$ & $\begin{array}{l}E \operatorname{mex} \\
(\mathrm{MeV})\end{array}$ & Lab W & Author & No. \\
\hline 1241 & thich 1 & target & $y \operatorname{lol} \mid d x i$ & foctor & & \\
\hline $\begin{array}{l}122 \mathrm{Te} \\
124 \mathrm{Te} \\
0 \times 1 \mathrm{Te}\end{array}$ & $\begin{array}{l}0 \\
p \\
p\end{array}$ & $\begin{array}{l}6.4+0 \\
1.9+1 \\
5.5+0\end{array}$ & 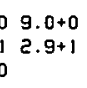 & $\begin{array}{l}\text { UUV C } \\
\text { BNL E } \\
\text { XTY E }\end{array}$ & $\begin{array}{l}\text { C Bemt } \\
\text { E Kondot } \\
\text { E Coopet }\end{array}$ & $\begin{array}{l}5728 \\
3978 \\
402 !\end{array}$ \\
\hline \multicolumn{7}{|c|}{ thich torget ylete } \\
\hline $\begin{array}{r}S b \\
12+\frac{S b}{\mathrm{~T} e}\end{array}$ & $\begin{array}{c}{ }^{3} \mathrm{He} \\
0 \\
0\end{array}$ & $\begin{array}{l}1.5+1 \\
8.0+0 \\
1.2+1\end{array}$ & $\begin{array}{l}3.1+1 \\
3.2+1 \\
1\end{array}$ & $\begin{array}{l}\text { UUV C } \\
\text { UJV C } \\
\text { SNL E }\end{array}$ & $\begin{array}{l}\text { C Bent } \\
\text { C Beat } \\
\text { E Kondot }\end{array}$ & $\begin{array}{l}6454 \\
8333 \\
3977\end{array}$ \\
\hline $12+i$ & overage & $\theta \sigma(E)$ & & & & \\
\hline$|23|$ & d & $6.4+1$ & $7.8+1$ & UL E & E Weinrleht & 5762 \\
\hline $12 v_{1}$ & $\sigma(E) \times f$ & octor & & & & . \\
\hline 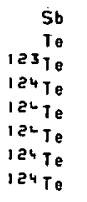 & $\begin{array}{l}0 \\
\dot{0} \\
p \\
p \\
p \\
p \\
p \\
p\end{array}$ & $\begin{array}{l}5.0+1 \\
7.0+1 \\
1.0+1 \\
2.6+0 \\
9.0+0 \\
1.8+1 \\
1.9+1 \\
4.0+2\end{array}$ & $\begin{array}{l}7.5+1 \\
1 \quad 8.5+1 \\
1.9-1 \\
5.7-0 \\
0.3-1 \\
12.9+1 \\
12.9+1 \\
2\end{array}$ & $\begin{array}{l}\text { BNL E } \\
\text { BNL E } \\
\text { NED E } \\
\text { ORL E } \\
\text { NED E } \\
\text { JUL R } \\
\text { BNL E } \\
\text { CAR E }\end{array}$ & $\begin{array}{l}\text { E Lambrecht, } \\
\text { E Lambrecht+ } \\
\text { E Van-Den-Boscht } \\
\text { E Johnsont } \\
\text { E Van-Den-Boscht } \\
\text { R Stochlint } \\
\text { E Kondot } \\
\text { E Reulandt }\end{array}$ & $\begin{array}{l}8332 \\
8343 \\
3951 \\
3969 \\
3970 \\
3971 \\
3972+ \\
3973\end{array}$ \\
\hline $124 \mathrm{I}$ & $\sigma(E)$ & & & & & \\
\hline $124 \mathrm{Te}$ & $\rho$ & $1.0+1$ & $12.8+1$ & BNL E & E Kondot & 3974 \\
\hline $124 \mathrm{Te}$ & p & $4.0+2$ & & KFK C & C Muenzelt & 3975 \\
\hline $12^{4} \mathrm{Te}$ & p & $4.0+2$ & & CAR E & E Reulandt & $3976+$ \\
\hline${ }^{125} \mathrm{Te}$ & p & $4.0+2$ & & KFK C & C Muenzel+ & 3982 \\
\hline $125 \mathrm{Tg}$ & p & $4.0+2$ & & PUP E & E Churcht & $3983+$ \\
\hline $126 \mathrm{Tg}$ & $p$ & $4.0+2$ & & KFK C & C Muenzelt & 3992 \\
\hline $126 \mathrm{Ts}$ & p & $4.0+2$ & & PUP E & E churcht & $3993+$ \\
\hline 1271 & p & $3.0+1$ & $8.5+1$ & MCG E & E Diksict & $4030+$ \\
\hline $127 i$ & d & $4.1+1$ & $9.5+1$ & JUL & C Schult. & 5737 \\
\hline $125 \mathrm{I}$ & cumulat & lve? pr & groduct & ylold & & \\
\hline $\operatorname{cop}_{1}$ & p & $4.0+1$ & & UPP E & E Lundquist + & 4079 \\
\hline $125 \mathrm{I}$ & cumulat & Iye? th & thich to & orget & yleldxfoctor & \\
\hline $122_{\mathrm{Te}}$ & - & $4.0+1$ & $4.0+1$ & UJV C & C Bemt & 8354 \\
\hline
\end{tabular}

\begin{tabular}{|c|c|c|c|c|c|}
\hline Target & $\ln c$ & $\begin{array}{ll}\text { Emin } & E_{\max } \\
(\mathrm{MeV}) & (\mathrm{MeV})\end{array}$ & Lab & Author & No. \\
\hline $129_{1}$ & \multicolumn{5}{|c|}{ Cunulatlve? o(E) } \\
\hline c口p & $\rho$ & NDG & \multicolumn{2}{|c|}{ UPP E Lundarist+ } & 4080 \\
\hline $\operatorname{lag}_{1}$ & \multicolumn{4}{|c|}{ cumulative thich torget yleldatactor } & \\
\hline$c=P_{1}$ & $p$ & $4.8+17.8+1$ & HAR & E Syme+ & 4078 \\
\hline $125_{1}$ & \multicolumn{4}{|c|}{ cumulative thlch torget yleld } & \\
\hline 1271 & o & $1.0+2$ & KFK & E Helust & 8365 \\
\hline $123_{1}$ & \multicolumn{4}{|c|}{ cumulative o(E) af actor } & \\
\hline Te & $\circ$ & $7.0+18.5+1$ & BNL & E Lombrecht+ & 8344 \\
\hline 1251 & \multicolumn{4}{|c|}{ colative ty } & \\
\hline $1235 b$ & a & $2.0+1 \quad 2.7+1$ & ROS & E Hagenannt & 8340 \\
\hline as! & \multicolumn{4}{|c|}{ thick torgot yioldafoctor } & \\
\hline $122 \mathrm{Te}$ & d & $6.4+09.0+0$ & usv & C Bemt & 5728 \\
\hline I25, & \multicolumn{4}{|c|}{ otEl Xfoctor } & \\
\hline $125 \mathrm{Te}$ & p & $2.6+0 \quad 5.7+0$ & ORL & E Johniont & 3986 \\
\hline 1251 & \multicolumn{4}{|l|}{$O(E)$} & \\
\hline $\begin{array}{l}125 \mathrm{Te} \\
125 \mathrm{Te} \\
126 \mathrm{Te} \\
126 \mathrm{Te}\end{array}$ & $\begin{array}{l}p \\
p \\
p \\
p\end{array}$ & $\begin{array}{l}4.0+2 \\
4.0+2 \\
4.0+2 \\
4.0+2\end{array}$ & $\begin{array}{l}\text { KFK } \\
\text { PUP } \\
\text { KFK } \\
\text { PUP }\end{array}$ & $\begin{array}{l}\text { C Muenzel+ } \\
\text { E Churcht } \\
\text { C Muenzel+ } \\
\text { E Churest }\end{array}$ & $\begin{array}{l}3984 \\
3985+ \\
3994 \\
3995+\end{array}$ \\
\hline $\mid 250.91$ & \multicolumn{4}{|c|}{ Sumation $O(E)$ afoctor } & \\
\hline $\begin{array}{l}125 \\
152 \mathrm{Te} \\
156\end{array}$ & p & $\begin{array}{lr}\text { TR } & 5.7+0 \\
3.0+0 & 5.7+0\end{array}$ & $\begin{array}{l}\text { ORL } \\
\text { KFK }\end{array}$ & $\begin{array}{l}\text { E Johns,snt } \\
\text { C Muenzolt }\end{array}$ & $\begin{array}{l}3987+ \\
3988\end{array}$ \\
\hline 1261 & \multicolumn{2}{|c|}{ independent lission } & $y 101$ & & \\
\hline $209^{B i}$ & ${ }^{12} \mathrm{C}$ & $7.5+1: .0+2$ & HAR & M Bronguinhot & $1015 \vec{c}$ \\
\hline 126$\}$ & \multicolumn{2}{|c|}{ Independent o(E) } & & & \\
\hline 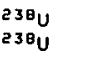 & p & $\begin{array}{l}1 \cdot 0+33 \cdot 0+5 \\
1.2+3\end{array}$ & $\begin{array}{l}\text { KFK } \\
\text { BNL }\end{array}$ & $\begin{array}{l}\text { C Muenze1+ } \\
\text { E Yut }\end{array}$ & $\begin{array}{l}4906 \\
4907+\end{array}$ \\
\hline $12 \mathbf{B}_{1}$ & \multirow{2}{*}{\multicolumn{4}{|c|}{$\begin{array}{l}\text { cumulative thich targot ylold } \\
\text { KFK E Helugt }\end{array}$}} & \\
\hline 1271 & & & & E Helust & 8366 \\
\hline
\end{tabular}




\begin{tabular}{|c|c|c|c|c|c|c|}
\hline Target & $\ln x$ & $\begin{array}{l}\mathrm{Em} / \mathrm{m} \\
(\mathrm{MeV})\end{array}$ & $\begin{array}{l}\text { Emax } \\
(\operatorname{MeV})\end{array}$ & Lab h & Author & No. \\
\hline $1266_{1}$ & product & yiold & & & & \\
\hline $\begin{array}{l}123 \mathrm{Te} \\
127 \mathrm{I}\end{array}$ & $\begin{array}{l}\text { d } \\
\text { p }\end{array}$ & $\begin{array}{l}5.0+0 \\
7 . \theta+1\end{array}$ & $6.0+0$ & $\begin{array}{l}\text { XTY E } \\
\text { JUL E }\end{array}$ & $\begin{array}{l}\text { E Coopet } \\
\text { E Welnelicht }\end{array}$ & $\begin{array}{l}3953 \\
5763\end{array}$ \\
\hline$|26|$ & thick to & orset y & yieldxi & factor & & \\
\hline $\begin{array}{l}152 \mathrm{Te} \\
125 \mathrm{Te} \\
0 \times 1 \mathrm{Te}\end{array}$ & $\begin{array}{l}0 \\
0 \\
0\end{array}$ & $\begin{array}{l}6.4+0 \\
4.0+1 \\
5.5+0\end{array}$ & $\begin{array}{l}9.0+0 \\
4.0+1\end{array}$ & $\begin{array}{l}\text { UJV } \\
\text { UJV } \\
\text { KTY E }\end{array}$ & $\begin{array}{l}C \text { Bent } \\
\text { C Bent } \\
\text { E Coopet }\end{array}$ & $\begin{array}{l}5728 \\
8355 \\
4022\end{array}$ \\
\hline 1261 & thick to & orget y & $y / 01 d$ & & & \\
\hline $\begin{array}{l}{ }_{125} 5_{\mathrm{S}} \\
126_{\mathrm{I}}\end{array}$ & $\begin{array}{c}p \\
\text { overage }\end{array}$ & $\begin{array}{l}3.0+0 \\
O(E)\end{array}$ & $6.3+0$ & CLU $\mathrm{r}$ & M Roughtont & 3999 \\
\hline $127 \mid$ & $d$ & $6.4+1$ & $7.8+1$ & JuL E & E Weinricht & 5762 \\
\hline $1266_{I}$ & $O(E) \times f o c$ & ctor & & & & \\
\hline $\begin{array}{r}\mathrm{Te} \\
123 \mathrm{Te} \\
126 \mathrm{Te}\end{array}$ & $\begin{array}{l}\dot{0} \\
\dot{p} \\
p\end{array}$ & $\begin{array}{l}7.0+1 \\
1.0+1 \\
2.6+0\end{array}$ & $\begin{array}{l}8 \cdot 5+1 \\
1.8+1 \\
5 \cdot 7+0\end{array}$ & $\begin{array}{l}\text { BNL } \\
\text { NED E } \\
\text { ORL E }\end{array}$ & $\begin{array}{l}\text { E Lambrecht+ } \\
\text { E Van-Den-Boseht } \\
\text { E Johnsont }\end{array}$ & $\begin{array}{l}8345 \\
3952 \\
3998\end{array}$ \\
\hline $125_{1}$ & $O(E)$ & & & & & \\
\hline $\begin{array}{l}126 \mathrm{Te} \\
126 \mathrm{Te} \\
126 \mathrm{Te} \\
127 \mathrm{I} \\
127 \mathrm{I}\end{array}$ & $\begin{array}{c}p \\
p \\
3_{H e} \\
p \\
d\end{array}$ & $\begin{array}{l}4.0+2 \\
4.0+2 \\
2.0+1 \\
3.0+1 \\
1.4+1\end{array}$ & $\begin{array}{l}2.7+1 \\
8.5+1 \\
9.5+1\end{array}$ & $\begin{array}{l}\text { KFK } \\
\text { PUP E } \\
\text { JYV E } \\
\text { MCG E } \\
\text { JUL C }\end{array}$ & $\begin{array}{l}\text { C Muenzelt } \\
\text { E Churcht } \\
\text { E Hatlulat } \\
\text { E Dihsict } \\
\text { C Schult. }\end{array}$ & $\begin{array}{l}3996 \\
3997+ \\
6466 \\
4031+ \\
5738\end{array}$ \\
\hline $125=+81$ & I summe & ations & $O(E) \times f O$ & octor & & \\
\hline $\begin{array}{l}126 \mathrm{Te} \\
126 \mathrm{Te}\end{array}$ & $p$ & $\begin{array}{l}\text { IR } \\
2.9+0\end{array}$ & $\begin{array}{l}5.7+0 \\
5.7+0\end{array}$ & $\begin{array}{l}\text { ORL } \\
\text { KFK }\end{array}$ & $\begin{array}{l}\text { E Johnsont } \\
\text { C Muenzelt }\end{array}$ & $\begin{array}{l}4000+ \\
4001\end{array}$ \\
\hline 1271 & partial & specte & rum ave & erage & - $O(E)$ & \\
\hline$|27|$ & $a$ & $2 \cdot 3+1$ & $2.7+1$ & MUU E & E Eberhordt & 8367 \\
\hline 1271 & $O(E)$ & & & & & \\
\hline $\begin{array}{l}125 \mathrm{Sn} \\
126 \mathrm{~T}_{0} \\
127 \mathrm{I}\end{array}$ & $\begin{array}{c}160 \\
{ }^{3} \mathrm{He} \\
p\end{array}$ & $\begin{array}{l}7.4+1 \\
2.0+1 \\
5.0+0\end{array}$ & $\begin{array}{l}2.7+1 \\
5.0+1\end{array}$ & $\begin{array}{l}\text { MUU E } \\
\text { JYV E } \\
\text { MNA }\end{array}$ & $\begin{array}{l}\text { E Splelert } \\
\text { E Hattulat } \\
\text { M Sourihest }\end{array}$ & $\begin{array}{r}11370 \\
6467 \\
4032\end{array}$ \\
\hline $12 \theta_{1}$ & independ & idont il & ission & yiold & & \\
\hline $20 \theta_{B i}$ & ${ }^{12} \mathrm{C}$ & $7.5+1$ & $1.0+2$ & HAR N & M Branquinhot & 10152 \\
\hline
\end{tabular}

\begin{tabular}{|c|c|c|c|c|c|c|}
\hline Target & $\cdot \ln c$ & $\begin{array}{l}\text { Emin } \\
(M e V)\end{array}$ & $\begin{array}{l}\text { Emax } \\
(\operatorname{MeV})\end{array}$ & Lab & Author & No. \\
\hline $12 \theta_{1}$ & thlek t & torget & yield & & & \\
\hline $\begin{array}{l}12 \mathrm{~T}_{\mathrm{e}} \\
128^{1}\end{array}$ & $\begin{array}{c}P \\
O(E), f_{0}\end{array}$ & $\begin{array}{l}2.8+0 \\
\text { octor }\end{array}$ & $6.3+0$ & CLU $\mathrm{Y}$ & M Roughtont & 4004 \\
\hline $\begin{array}{l}12 \theta_{\mathrm{Te}} \\
128 \cdot \mathrm{I}\end{array}$ & raw of & $\begin{array}{l}2.6+0 \\
\text { (E) }\end{array}$ & $5.7+0$ & ORL E & E Johnsont & 4003 \\
\hline $\begin{array}{l}128 \mathrm{Te} \\
23 \mathrm{Th}^{\mathrm{Th}} \\
53 \mathrm{~B}_{\mathrm{U}}\end{array}$ & $\begin{array}{l}p \\
p \\
p\end{array}$ & $\begin{array}{l}1.7+1 \\
1.7+1 \\
1.7+1\end{array}$ & & $\begin{array}{l}\text { MCG E } \\
\text { MCG E } \\
\text { MCG E }\end{array}$ & $\begin{array}{l}\text { E Diksict } \\
\text { E Oiksict } \\
\text { E Oiksict }\end{array}$ & $\begin{array}{l}4005 \\
4793 \\
4889\end{array}$ \\
\hline $12 \theta \bullet+01$ & Summ & motion & $\sigma(E) \times f a$ & actor & & \\
\hline $\begin{array}{l}128 \mathrm{Te} \\
128 \mathrm{Te}\end{array}$ & $\begin{array}{l}p \\
P\end{array}$ & $\begin{array}{l}T R \\
3.0+0\end{array}$ & $\begin{array}{l}5.7+0 \\
5.7+0\end{array}$ & $\begin{array}{l}\text { ORL } \\
\text { KFK }\end{array}$ & $\begin{array}{l}\text { E Johnsont } \\
\text { C Muenzelt }\end{array}$ & $\begin{array}{l}4005+ \\
4007\end{array}$ \\
\hline 1301 & indepen & ndento & o(E) & & & \\
\hline $\begin{array}{l}23 \theta_{U} \\
23 \theta_{U}\end{array}$ & $p$ & $\begin{array}{l}1.0+3 \\
1.2+3\end{array}$ & $3^{3} 3.0+5$ & $\begin{array}{l}\text { KFK } \\
\text { BNL }\end{array}$ & $\begin{array}{l}\text { C Muenzel+ } \\
\text { E Yut }\end{array}$ & $\begin{array}{l}4906 \\
4907+\end{array}$ \\
\hline 1301 & cumulat & tive $f$ & ission y & y1010 & & \\
\hline $\begin{array}{l}237 \mathrm{~Np} \\
130 !\end{array}$ & $\begin{array}{c}0 \\
\text { product }\end{array}$ & $\begin{array}{r}2.0+1 \\
t y 1010\end{array}$ & $4.1+1$ & $\mathrm{CCP} F$ & R Lbovt & 9091 \\
\hline $\begin{array}{l}123 \mathrm{Te} \\
1301\end{array}$ & p & $\begin{array}{l}5.0+0 \\
\text { torgel }\end{array}$ & $\begin{array}{l}6.0+0 \\
y 101 d x f\end{array}$ & $\begin{array}{l}\text { KTY E } \\
\text { foctor }\end{array}$ & $\begin{array}{l}\text { E Coopet } \\
\text { or }\end{array}$ & 3953 \\
\hline $\begin{array}{l}122 \mathrm{Te} \\
0 \times 1 \mathrm{Te}\end{array}$ & d & $\begin{array}{l}6.4+0 \\
5.5+0\end{array}$ & $9.0+0$ & UJV & $\begin{array}{l}\text { C Bemt } \\
\text { E Coopet }\end{array}$ & $\begin{array}{l}5728 \\
4023\end{array}$ \\
\hline 1301 & $\sigma(\varepsilon) \times f$ & actor & & & & \\
\hline $\begin{array}{l}130 \mathrm{Te} \\
130 \mathrm{Te} \\
130 \mathrm{Te}\end{array}$ & $\begin{array}{l}p \\
p \\
p\end{array}$ & $\begin{array}{l}2.5+0 \\
2.6+0 \\
1.0+1\end{array}$ & $\begin{array}{l}7.0+0 \\
5.7+0 \\
11.9+1\end{array}$ & $\begin{array}{l}\text { ORL } \\
\text { ORL } \\
\text { NED E }\end{array}$ & $\begin{array}{l}\text { M Johnsont } \\
\text { E Johnsont } \\
\text { E Van-Den-8oscht }\end{array}$ & $\begin{array}{l}4009 \\
4010 \\
4011\end{array}$ \\
\hline $130_{1}$ & $O(E)$ & & & & & \\
\hline $\begin{array}{l}130 \mathrm{Te} \\
130 \mathrm{Te}\end{array}$ & ${ }^{3} \mathrm{He}$ & $\begin{array}{l}2.5+0 \\
2.0+1\end{array}$ & $\begin{array}{l}6.0+0 \\
2.7+1\end{array}$ & $\begin{array}{l}\text { ORL E } \\
\text { JYV E }\end{array}$ & $\begin{array}{l}\text { E Johnsont } \\
\text { E Hattulat }\end{array}$ & $\begin{array}{l}4012 \\
6470\end{array}$ \\
\hline 13001 & thlck & targot & tyield & & & \\
\hline $130 \mathrm{Te}$ & $\circ$ & $2.8+0$ & $6.3+0$ & CLU $\mathrm{r}$ & M Roughtont & 4013 \\
\hline
\end{tabular}




\begin{tabular}{|c|c|c|c|c|c|c|}
\hline Target & Inc & $\begin{array}{l}\text { Emln } \\
\text { (MeV) }\end{array}$ & $\begin{array}{l}\text { Embx } \\
(\operatorname{MeV})\end{array}$ & Lab W & Author & No. \\
\hline $130 \mathrm{n}_{1}$ & \multicolumn{6}{|c|}{ thick torget yleld } \\
\hline $130 \mathrm{Te}$ & p & $2.8+0$ & $6.3+0$ & O CLUMF & Roughiont & 4014 \\
\hline 1300.81 & \multicolumn{6}{|c|}{ Summation $O(E) \times f$ actor } \\
\hline $\begin{array}{l}\begin{array}{l}130 \mathrm{Te} \\
130 \mathrm{Te}\end{array} \\
{ }^{30} \mathrm{~T}\end{array}$ & p & $\begin{array}{l}\text { TR } \\
2.7+0\end{array}$ & $\begin{array}{l}5.7+0 \\
5.7+0\end{array}$ & $\begin{array}{l}0 \text { ORL E } \\
\text { O KFK C, }\end{array}$ & $\begin{array}{l}\text { Johnsont } \\
\text { Muenzelt }\end{array}$ & $\begin{array}{l}4017 \\
4018\end{array}$ \\
\hline $130 \cdots \cdot 01$ & \multicolumn{6}{|c|}{ Summation $O(E)$} \\
\hline $\begin{array}{l}130 \mathrm{Te} \\
130 \mathrm{Te}\end{array}$ & $p$ & $\begin{array}{l}\text { IR } \\
2.7+0\end{array}$ & $\begin{array}{l}5.7+0 \\
5.7+0\end{array}$ & $\begin{array}{l}0 \text { ORL E } \\
\text { O KFK C }\end{array}$ & $\begin{array}{l}\text { Johnsont } \\
\text { Muenzel }\end{array}$ & $\begin{array}{l}4015 \\
4016\end{array}$ \\
\hline 1311 & \multicolumn{6}{|c|}{ Independent ission yield } \\
\hline $209_{9 ;}$ & $12 \mathrm{c}$ & $7.5+1$ & $1.0+2$ & HAR ME & Branquintot & 10152 \\
\hline 1311 & \multicolumn{6}{|c|}{ cumulative figsion ylela } \\
\hline $\begin{array}{l}{ }^{232} \mathrm{Th} \\
{ }^{237} \mathrm{~Np}\end{array}$ & d & $\begin{array}{l}9.3+0 \\
2.0+1\end{array}$ & $\begin{array}{l}1.4+1 \\
4.1+1\end{array}$ & $\begin{array}{l}\text { CCP R } \\
\text { CCP R }\end{array}$ & $\begin{array}{l}\text { Lbout } \\
\text { Lbovt }\end{array}$ & $\begin{array}{l}5921 \\
9091\end{array}$ \\
\hline $131 \mathrm{I}$ & \multicolumn{6}{|c|}{ thick lorget yioldxfactor } \\
\hline I'25Te & 0 & $6.4+0^{\circ}$ & $9.0+0$ & D UJV C E & Bent & 5728 \\
\hline 1311 & \multicolumn{5}{|c|}{$O(E) \times$ factor } & \\
\hline Te & a & $7.0+1$ & $8.5+1$ & BNLE E & Lambrechtt & 8346 \\
\hline $131 \mathrm{I}$ & \multicolumn{5}{|l|}{$\sigma(E)$} & \\
\hline${ }^{130} \mathrm{Te}$ & p & $2.1+1$ & & $\operatorname{GOE} E \mathrm{~K}$ & Kantust & 4019 \\
\hline $132_{1}$ & \multicolumn{6}{|c|}{ cunulative fission yield } \\
\hline${ }^{232} \mathrm{Th}$ & d & $9.3+0$ & $1.4+:$ & CCPRL & Lbovt & 5921 \\
\hline 132.101 & \multicolumn{6}{|c|}{ independent ratio o(E) } \\
\hline $232 \mathrm{Th}$ & . & $3.0+1$ & $1.1+2$ & OSAE & $\operatorname{lman} \mid \operatorname{shi} i^{+}$ & 8959 \\
\hline $133 \mathrm{I}$ & \multicolumn{6}{|c|}{ cunulative fission yleld } \\
\hline $232 \mathrm{Th}$ & d & $9.3+0$ & $1.4+1$ & $1 C C P R L$ & Lbout & 5921 \\
\hline $233 \mathrm{U}$ & 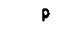 & $2.0+1$ & $8.5+1$ & CCPR $R$ & Lbout & 4838 \\
\hline 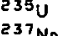 & p & $1.2+1$ & $8.5+1$ & $1 \mathrm{CCP} R$ & Loovt & 4852 \\
\hline${ }^{237} \mathrm{~Np}$ & a & $2.0+1$ & $4.1+1$ & I CCP R L & Loovt & so9ı \\
\hline $133_{1}$ & \multicolumn{6}{|c|}{ cumulative $O(E)$} \\
\hline $238^{2}$ & p & $1.0+3$ & $3.0+5$ & $\mathrm{KFK} \mathrm{C}$ & Muenzel+ & 4903 \\
\hline
\end{tabular}

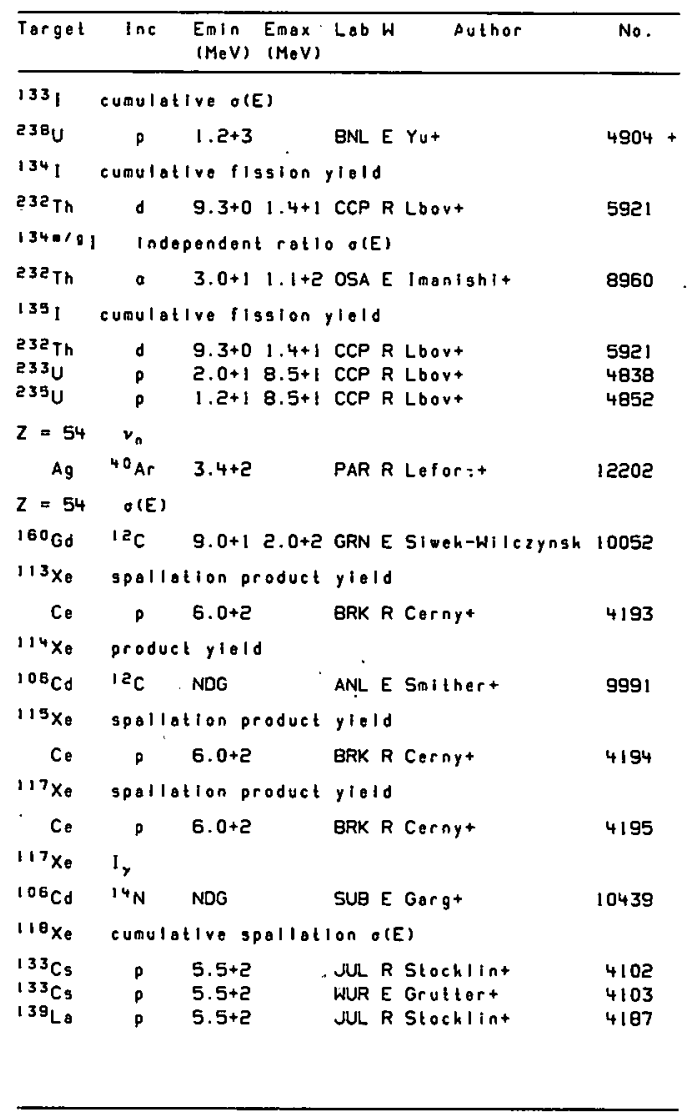




\begin{tabular}{|c|c|c|c|c|}
\hline Target & Inc & $\begin{array}{ll}\text { EmIn } & \text { Emax } \\
(\mathrm{MeV}) & (\mathrm{MeV})\end{array}$ & Lab W & No. \\
\hline $118 x_{0}$ & \multicolumn{4}{|c|}{ spallallon o(E) } \\
\hline $\begin{array}{l}133 \mathrm{Cs} \\
139_{\mathrm{La}} \\
139_{\mathrm{La}}\end{array}$ & $\begin{array}{l}p \\
p \\
p\end{array}$ & $\begin{array}{l}5.5+2 \\
5.5+2 \\
5.5+2\end{array}$ & $\begin{array}{l}\text { WUR E Grultert } \\
\text { JUL R Slocklint } \\
\text { WUR E Grultert }\end{array}$ & $\begin{array}{l}4107 \\
4190 \\
419 !\end{array}$ \\
\hline $110 \times x_{0}$ & \multicolumn{4}{|c|}{ cumulatlve $O(E)$} \\
\hline $\begin{array}{l}1271 \\
118 \times 0\end{array}$ & \multicolumn{4}{|c|}{ Independent relative o(E) } \\
\hline $\begin{array}{l}106 \mathrm{~cd} \\
119 \times 0\end{array}$ & \multicolumn{4}{|c|}{ cumulative spollation o(E) } \\
\hline $\begin{array}{l}133 \mathrm{Cs} \\
133 \mathrm{Cs} \\
139 \mathrm{Ls}\end{array}$ & $\begin{array}{l}p \\
p \\
p\end{array}$ & $\begin{array}{l}5.5+2 \\
5.5+2 \\
5.5+2\end{array}$ & $\begin{array}{l}\text { JUL R Stocklint } \\
\text { WUR E Grultert } \\
\text { JUL R Slocklint }\end{array}$ & $\begin{array}{l}4102 \\
4103 \\
4187\end{array}$ \\
\hline $118 \times 0$ & \multicolumn{4}{|c|}{ Spollation O(E) } \\
\hline $\begin{array}{l}133 \mathrm{Cs} \\
139 \mathrm{LO} \\
139 \mathrm{LO}\end{array}$ & $\begin{array}{l}p \\
p \\
p\end{array}$ & $\begin{array}{l}5.5+2 \\
5.5+2 \\
5.5+2\end{array}$ & $\begin{array}{l}\text { WUR E Grultert } \\
\text { JUL R Stocklint } \\
\text { WUR E Gruttert }\end{array}$ & $\begin{array}{l}4107 \\
4190 \\
4191\end{array}$ \\
\hline $120 \times 0$ & \multicolumn{4}{|c|}{ Independent relotive o(E) } \\
\hline $\begin{array}{l}106 \mathrm{Cd} \\
120 \times 0\end{array}$ & \multicolumn{4}{|c|}{ cumulatlve spallatlon o(E) } \\
\hline $\begin{array}{l}133 \mathrm{Cs} \\
133 \mathrm{Cs} \\
139 \mathrm{Lo}\end{array}$ & $\begin{array}{l}p \\
p \\
p\end{array}$ & $\begin{array}{l}5.5+2 \\
5.5+2 \\
5.5+2\end{array}$ & $\begin{array}{l}\text { JUL R Stochlint } \\
\text { WUR E Gruttert } \\
\text { JUL R Stochlint }\end{array}$ & $\begin{array}{l}4102 \\
4103 \\
4187\end{array}$ \\
\hline $120 \times 0$ & \multicolumn{4}{|c|}{ Spallation o(E) } \\
\hline $\begin{array}{l}133 \mathrm{Cs} \\
139 \mathrm{LB} \\
139 \mathrm{Lo}\end{array}$ & $\begin{array}{l}p \\
p \\
p\end{array}$ & $\begin{array}{l}5 \cdot 5+2 \\
5.5+2 \\
5 \cdot 5+2\end{array}$ & $\begin{array}{l}\text { WUR E Grutlert } \\
\text { JUR R Stochlint } \\
\text { WUR E Gruttert }\end{array}$ & $\begin{array}{l}4107 \\
4190 \\
4191\end{array}$ \\
\hline $120 \times 0$ & \multicolumn{4}{|c|}{ cumulative o(E) } \\
\hline $127 \mid$ & d & $8.0+19.5+1$ & JUL C Schult. & 5740 \\
\hline $120 \times 0$ & \multicolumn{4}{|c|}{ relotive I, } \\
\hline $110 \mathrm{Cd}$ & ${ }^{13} \mathrm{C}$ & $4.9+15.2+1$ & STB E Chowdhuryt & 10207 \\
\hline
\end{tabular}

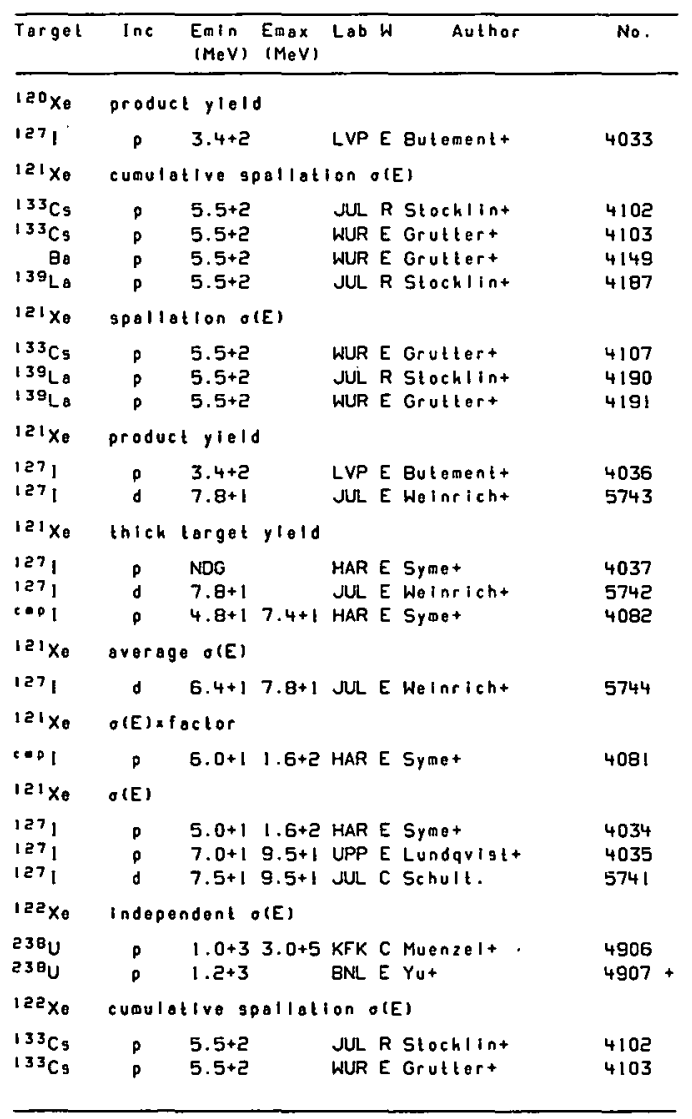




\begin{tabular}{|c|c|c|c|c|}
\hline Targat & Inc & $\begin{array}{ll}\text { EmIn } & \text { Emax } \\
(\mathrm{MeV}) & (\mathrm{MeV})\end{array}$ & Author & No. \\
\hline $122 \times 3$ & \multicolumn{4}{|c|}{ eumulative spallation o(E) } \\
\hline $\begin{array}{r}\mathrm{Bg} \\
139_{\mathrm{Lg}}\end{array}$ & $\begin{array}{l}p \\
p\end{array}$ & $\begin{array}{l}5 \cdot 5+2 \\
5 \cdot 5+2\end{array}$ & $\begin{array}{l}\text { WUR.E Grutlert } \\
\text { JUL.R Stochlint }\end{array}$ & $\begin{array}{l}4149 \\
4.187\end{array}$ \\
\hline $12 e_{x}$ & \multicolumn{4}{|c|}{ spallation o(E) } \\
\hline $\begin{array}{l}133 \mathrm{Cs} \\
139 \mathrm{Lg} \\
139 \mathrm{Lg}\end{array}$ & $\begin{array}{l}p \\
p \\
p\end{array}$ & $\begin{array}{l}5.5+2 \\
5.5+2 \\
5.5+2\end{array}$ & $\begin{array}{l}\text { WUR E Gruttert } \\
\text { JUL R Stochlint } \\
\text { WUR E Gruttert }\end{array}$ & $\begin{array}{l}4107 \\
4190 \\
4191\end{array}$ \\
\hline $122 \times$ & \multicolumn{3}{|c|}{ cunulatlve $O(E)$} & \\
\hline $\begin{array}{l}238_{U} \\
23 \theta_{U}\end{array}$ & $p$ & $\begin{array}{l}1.0+3 \\
1.2+3\end{array}$ & $\begin{array}{l}\text { KFK C Muenzel+ } \\
\text { ENL E Yut }\end{array}$ & $\begin{array}{l}4903 \\
4904+\end{array}$ \\
\hline $122 x$ & \multicolumn{4}{|c|}{ product yleld } \\
\hline $\begin{array}{l}1571 \\
1571\end{array}$ & p & $\begin{array}{l}9 \cdot 0+1 \\
7.8+1\end{array}$ & $\begin{array}{l}\text { UPP E Lundaulstt } \\
\text { JUL E Weinricht }\end{array}$ & $\begin{array}{l}4040 \\
5746\end{array}$ \\
\hline $125 x^{4}$ & \multicolumn{4}{|c|}{ avorage $O(E)$} \\
\hline $\begin{array}{l}|27| \\
122 \times 0\end{array}$ & $\begin{array}{c}0 \\
\sigma(E)\end{array}$ & $6.4+17.8+1$ & JUL E Weinrlaht & 5747 \\
\hline $\begin{array}{l}1271 \\
1271 \\
1271\end{array}$ & $\begin{array}{l}\text { p } \\
p \\
\text { d }\end{array}$ & 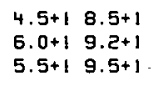 & $\begin{array}{l}\text { MCG E Diksict } \\
\text { UPP E Lundaristt } \\
\text { JUL C Schult. }\end{array}$ & $\begin{array}{l}4038+ \\
4039 \\
5745\end{array}$ \\
\hline $123 \times e$ & \multicolumn{4}{|c|}{ cumulative spallation o(E) } \\
\hline $\begin{array}{r}133 \mathrm{Cs} \\
133 \mathrm{Cs} \\
8 \mathrm{Ba} \\
139 \mathrm{La}\end{array}$ & $\begin{array}{l}p \\
p \\
p \\
p \\
p\end{array}$ & $\begin{array}{l}5 \cdot 5+2 \\
5 \cdot 5+2 \\
5 \cdot 5+2 \\
5 \cdot 5+2\end{array}$ & $\begin{array}{l}\text { JUL R Stochlint } \\
\text { WUR } E \text { Gruttert } \\
\text { WUR } E \text { Gruttert } \\
\text { JUL R Stochlint }\end{array}$ & $\begin{array}{l}4102 \\
4103 \\
4149 \\
4187\end{array}$ \\
\hline $123 x e$ & \multicolumn{4}{|c|}{ Independont spollation $O(E)$} \\
\hline $\begin{array}{r}133=s \\
133=5 \\
3 a \\
\exists a \\
139.8 \\
139.8\end{array}$ & $\begin{array}{l}p \\
p \\
p \\
p \\
p \\
p\end{array}$ & $\begin{array}{l}5 \cdot 5+2 \\
5 \cdot 5+2 \\
5 \cdot 5+2 \\
5 \cdot 5+2 \\
5 \cdot 5+2 \\
5 \cdot 5+2\end{array}$ & $\begin{array}{l}\text { JUL R Stockllnt } \\
\text { WUR E Gruttert } \\
\text { JUL R Stocklint } \\
\text { WUR E Gruttert } \\
\text { JUL R Stochlint } \\
\text { WUR E Gruttert }\end{array}$ & $\begin{array}{l}4104 \\
4105 \\
4150 \\
4151 \\
4188 \\
4189\end{array}$ \\
\hline $123 \times e$ & \multicolumn{4}{|c|}{ spallation o(E) } \\
\hline $133 \mathrm{cs}$ & $p$ & $5.5+2$ & WUR E Grutter+ & 4107 \\
\hline
\end{tabular}

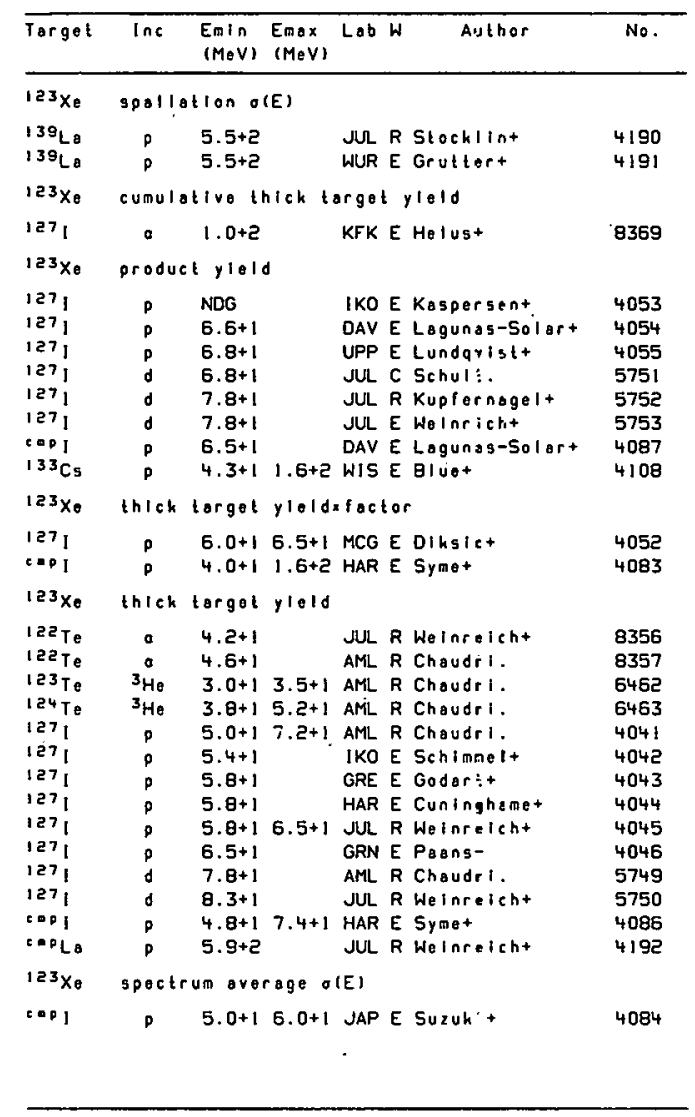




\begin{tabular}{|c|c|c|c|c|c|c|}
\hline Torget & $\operatorname{lnc}$ & $\begin{array}{l}\text { Emin } \\
\text { (MeV) }\end{array}$ & $\begin{array}{l}\text { Emax } \\
(\operatorname{MeV})\end{array}$ & Lab & Author & No. \\
\hline $123 \times 0$ & overa & ge $O(E)$ & & & & \\
\hline $\begin{array}{l}1271 \\
123 \times 0\end{array}$ & $\underset{O(E) \times}{d}$ & $\begin{array}{l}6.4+1 \\
\text { ioclor }\end{array}$ & $7.8+1$ & JUL E & E Weinricht & 5754 \\
\hline $\begin{array}{l}\text { To } \\
127 ! \\
c \in p i\end{array}$ & $\dot{a}$ & $\begin{array}{l}7.0+1 \\
9.0+1 \\
4.0+1\end{array}$ & $\begin{array}{l}8.5+1 \\
1.0+2 \\
1.6+2\end{array}$ & $\begin{array}{l}\text { BNL E } \\
\text { BNL E } \\
\text { HAR E }\end{array}$ & $\begin{array}{l}\text { E Lambrecht+ } \\
\text { E Lambrecht+ } \\
\text { E Symet }\end{array}$ & $\begin{array}{l}8347 \\
8368 \\
4085\end{array}$ \\
\hline $123 \times 8$ & $\sigma(E)$ & & & & & \\
\hline $\begin{array}{l}1271 \\
1271 \\
1271 \\
1271 \\
1271 \\
1271 \\
1271\end{array}$ & $\begin{array}{l}p \\
p \\
p \\
p \\
p \\
d \\
a\end{array}$ & $\begin{array}{l}2.0+1 \\
4 \cdot 3+1 \\
4 \cdot 4+1 \\
5 \cdot 0+1 \\
5 \cdot 5+1 \\
5 \cdot 8+1 \\
8 \cdot 9+1\end{array}$ & $\begin{array}{l}1.6+2 \\
6.6+1 \\
6.8+1 \\
9.8+1 \\
8.5+1 \\
8.9+1 \\
1.1+2\end{array}$ & $\begin{array}{l}\text { HAR E } \\
\text { GRN E } \\
\text { JUL R } \\
\text { UPP E } \\
\text { MCG E } \\
\text { JUL R } \\
\text { UL R }\end{array}$ & $\begin{array}{l}\text { E Symet } \\
\text { E Paanst } \\
R \text { Stochlint } \\
\text { E Lundavistt } \\
\text { E Diksict } \\
\text { R Stocklint } \\
\text { R Stocklint }\end{array}$ & $\begin{array}{l}4047 \\
4048 \\
4049 \\
4050 \\
4051 \\
5748 \\
8370\end{array}$ \\
\hline $124 \times 0$ & indep & indent. & $O(E)$ & & & \\
\hline $\begin{array}{l}238_{U} \\
238^{U}\end{array}$ & $\begin{array}{l}\rho \\
\rho\end{array}$ & $\begin{array}{l}1.0+3 \\
1.2+3\end{array}$ & $3.0+5$ & $\begin{array}{l}\text { KFK C } \\
\text { BNL E }\end{array}$ & $\begin{array}{l}\text { C Muenzel+ } \\
\text { E Yut }\end{array}$ & $\begin{array}{l}4906 \\
4907+\end{array}$ \\
\hline $\begin{array}{r}124 \times 0 \\
8 a\end{array}$ & cumule & $\begin{array}{l}\text { tive? } \\
6.0+2\end{array}$ & thich t & $\begin{array}{l}\text { torget } \\
\text { KLN E }\end{array}$ & $\begin{array}{l}\text { yleldxfoctor } \\
\text { E Kalser. }\end{array}$ & $41 ! 1$ \\
\hline $124 \times$ & cumule & tive? s & spectro & UI ove & gerage $O(E) \times$ foctor & \\
\hline $\begin{array}{r}\theta a \\
124 \times 0\end{array}$ & $\begin{array}{c}p \\
\text { cumul }\end{array}$ & $\begin{array}{l}\text { GCR } \\
\text { tive? }\end{array}$ & rolativ & $\begin{array}{l}\text { KLN E } \\
\text { ve OCE }\end{array}$ & $\begin{array}{l}\text { E Kalsor. } \\
\text { (E) }\end{array}$ & 4113 \\
\hline $\begin{array}{l}1{ }^{130} \mathrm{Ba} \\
124 \mathrm{xe}\end{array}$ & $\begin{array}{c}p \\
\text { cumul }\end{array}$ & $\begin{array}{l}\text { 1.0+2 } \\
\text { tive? }\end{array}$ & $O(E) \times f$ & $\begin{array}{l}\text { KLN E } \\
\text { octor }\end{array}$ & E kaiser. & 4155 \\
\hline $\begin{array}{r}8 a \\
124 \times 0\end{array}$ & $\begin{array}{c}p \\
\text { cumul }\end{array}$ & $\begin{array}{l}3.8+1 \\
\text { tive? }\end{array}$ & $\begin{array}{l}6.0+2 \\
\sigma(E)\end{array}$ & KLN E & E Kalser. & 4110 \\
\hline $\begin{array}{r}1271 \\
\quad 8 .\end{array}$ & 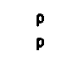 & $\begin{array}{l}2.0+1 \\
1.0+2\end{array}$ & $\begin{array}{l}8.5+1 \\
6.0+2\end{array}$ & $\begin{array}{l}\text { MCG E } \\
\text { KLN E }\end{array}$ & $\begin{array}{l}\text { E Diksict } \\
\text { E Koiser. }\end{array}$ & $\begin{array}{l}4056 \\
4112\end{array}$ \\
\hline $124 \times$ & cumule & tive ot & $(E)$ & & & \\
\hline $\begin{array}{l}238_{U} \\
238_{U}\end{array}$ & $p$ & $\begin{array}{l}1.0+3 \\
1.2+3\end{array}$ & $3.0+5$ & $\begin{array}{l}\text { KFK C } \\
\text { BNL E }\end{array}$ & $\begin{array}{l}\text { C Muenzelt } \\
\text { E Yut }\end{array}$ & $\begin{array}{l}4903 \\
4904+\end{array}$ \\
\hline
\end{tabular}

\begin{tabular}{|c|c|c|c|c|}
\hline Target & $\ln c$ & $\begin{array}{l}\text { Emin Emax } \\
(M e V) \quad(M \circ V)\end{array}$ & Lab W Author & No. \\
\hline${ }^{124} \times e$ & $\theta(E)$ & & & \\
\hline 1271 & p & $4.5+1 \quad 8.5+1$ & MCG E Diksict & 4057 \\
\hline $125 \times 0$ & $\operatorname{lnclu}$ & $\operatorname{lng}$ metagtabl & te o(E) & \\
\hline 1271 & p & $2.0+1 \quad 8.5+1$ & MCG $E$ Diksict & $4061+$ \\
\hline $125 \times 0$ & indep & indent o(E) & & \\
\hline $\begin{array}{l}23 \theta_{U} \\
23 \theta_{U}\end{array}$ & p & $\begin{array}{l}1.0+3 \quad 3.0+5 \\
1.2+3\end{array}$ & $\begin{array}{l}\text { KFK C Muenze1+ } \\
\text { BNL } E \text { Yut }\end{array}$ & $\begin{array}{l}4906 \\
4907+\end{array}$ \\
\hline $125 \times 0$ & cumul 1 & tive spatlat: & $\operatorname{lon} \sigma(E)$ & \\
\hline $\begin{array}{r}133 \mathrm{Cs} \\
133 \mathrm{Cs} \\
\mathrm{Ba} \\
\mathrm{Ba} \\
139 \mathrm{La}\end{array}$ & $\begin{array}{l}p \\
p \\
p \\
p \\
p\end{array}$ & $\begin{array}{l}5 \cdot 5+2 \\
5 \cdot 5+2 \\
5 \cdot 5+2 \\
5 \cdot 5+2 \\
5 \cdot 5+2\end{array}$ & $\begin{array}{l}\text { JUL R Stocklint } \\
\text { WUR E Gruttert } \\
\text { JUL R Stocklint } \\
\text { WUR E Gruttert } \\
\text { JUL R Stocklint }\end{array}$ & $\begin{array}{l}4102 \\
4103 \\
4148 \\
4149 \\
4187\end{array}$ \\
\hline $125 \times 0$ & indep & indent spollat & tion $O(E)$ & \\
\hline $\begin{array}{r}133 \mathrm{Cs} \\
133 \mathrm{Cs} \\
80 \\
88 \\
139 \mathrm{Lo} \\
139 \mathrm{Ls}\end{array}$ & $\begin{array}{l}p \\
p \\
p \\
p \\
p \\
p\end{array}$ & $\begin{array}{l}5.5+2 \\
5.5+2 \\
5.5+2 \\
5.5+2 \\
5.5+2 \\
5.5+2\end{array}$ & $\begin{array}{l}\text { JUL R Stocklint } \\
\text { WUR E Grultert } \\
\text { JUL R Stocklint } \\
\text { WUR E Grultert } \\
\text { JUL R Stocklint } \\
\text { WUR E Gruttert }\end{array}$ & $\begin{array}{l}4104 \\
4105 \\
4150 \\
4151 \\
4188 \\
4189\end{array}$ \\
\hline $125 \times$ & Spoll & tion $O(E)$ & & \\
\hline $\begin{array}{l}133 \mathrm{Cs} \\
139 \mathrm{LB} \\
13 \mathrm{LCO}^{\mathrm{Ca}}\end{array}$ & $\begin{array}{l}p \\
p \\
p\end{array}$ & $\begin{array}{l}5.5+2 \\
5.5+2 \\
5.5+2\end{array}$ & $\begin{array}{l}\text { WUR E Grultert } \\
\text { JUL R Stocklint } \\
\text { WUR E Gruttert }\end{array}$ & $\begin{array}{l}4107 \\
4190 \\
419 !\end{array}$ \\
\hline $125 x_{e}$ & cumul & the thich to & orget yiold & \\
\hline $127 !$ & 0 & $1.0+2$ & KFK E Helust & 8372 \\
\hline $125 x_{0}$ & cumul & Hive $O(E) \times f o c$ & ctor & \\
\hline $\begin{array}{l}127 \mid \\
125 \times 0\end{array}$ & $\begin{array}{c}a \\
\text { cumul }\end{array}$ & $\begin{array}{l}7.6+1 \quad 1.0+2 \\
\text { live o(E) }\end{array}$ & BNL E Lambrecht+ & 0371 \\
\hline $\begin{array}{l}23 \theta_{0} \\
2380\end{array}$ & $p$ & $\begin{array}{l}1.0+3 \\
1.2+3\end{array} 3.0+5$ & $\begin{array}{l}\text { KFK C Muenzel+ } \\
\text { BNLE } \mathrm{Yu}^{+}\end{array}$ & $\begin{array}{l}4903 \\
4904\end{array}$ \\
\hline
\end{tabular}




\begin{tabular}{|c|c|c|c|c|c|c|}
\hline Tarscet & Ine & $\begin{array}{l}E \min \\
(\mathrm{MeV})\end{array}$ & $\begin{array}{l}\text { Emax } \\
(M e y)\end{array}$ & Lab & Author & No. \\
\hline $125 \times x_{0}$ & partia & al relat & tive or & $(E)$ & & \\
\hline $\begin{array}{l}125 T_{0} \\
125 \times 0\end{array}$ & $\begin{array}{l}{ }^{3} \mathrm{He} \\
\text { produc }\end{array}$ & $\begin{array}{l}2.0+1 \\
\text { ct } y+e 10\end{array}$ & $2.7+1$ & JYV & E Helppit & 6464 \\
\hline $\begin{array}{l}133 \mathrm{Cs} \\
1231 \\
1521 \\
157 \\
131\end{array}$ & $\begin{array}{l}p \\
p \\
d \\
p\end{array}$ & $\begin{array}{l}4 \cdot 0+1 \\
4 \cdot 5+1 \\
7 \cdot 8+1 \\
4.3+1\end{array}$ & $1.6-2$ & $\begin{array}{l}\text { UPP } \\
\text { DAV } \\
\text { JUL } \\
\text { WIS }\end{array}$ & $\begin{array}{l}\text { E Lundarlstt } \\
\text { E Logunas-Solart } \\
\text { E Weinrieht } \\
\text { E Bluet }\end{array}$ & $\begin{array}{l}4062 \\
4063 \\
5756 \\
4108\end{array}$ \\
\hline $125 \times 0$ & thlck & torget & $y|0| d x$ & $\ln f \Delta c t$ & tor & \\
\hline cas I & p & $2.0+1$ & $1.6+2$ & HAR & E Symet & 4088 \\
\hline $125 \times x_{\theta}$ & lhick & targot & $y|e| d$ & & & \\
\hline $\begin{aligned} T e \\
T e \\
1271 \\
1271 \\
\operatorname{cop} 1\end{aligned}$ & $\begin{array}{l}{ }^{3} \mathrm{He} \\
\dot{p} \\
p \\
p \\
p\end{array}$ & $\begin{array}{l}8.0+0 \\
1.0+1 \\
\text { NDO } \\
6.5+1 \\
4.8+1\end{array}$ & $\begin{array}{l}4 \cdot 0+1 \\
4 \cdot 0+1 \\
7.4+1\end{array}$ & $\begin{array}{l}\text { TOK } \\
\text { TOK } \\
\text { HAR } \\
\text { GRN } \\
\text { HAR }\end{array}$ & $\begin{array}{l}\text { E Hommat } \\
\text { E Hommat } \\
\text { E Symet } \\
\text { E Paenst } \\
\text { E Symet }\end{array}$ & $\begin{array}{l}6459 \\
8350 \\
4064 \\
4065 \\
4090\end{array}$ \\
\hline $125 \times 0$ & overag & $g e$ o(E) & & & & \\
\hline $127 \mathrm{I}$ & $d$ & $6.4+1$ & $7.8+1$ & JUL & E Weinricht & 5757 \\
\hline $125 \times$ & $O(E) \times f$ & factor & & & & \\
\hline 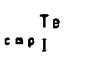 & a & $\begin{array}{l}7.0+1 \\
2.0+1\end{array}$ & $\begin{array}{l}8.5+1 \\
1.6+2\end{array}$ & $\begin{array}{l}\text { BNL } \\
\text { HAR }\end{array}$ & $\begin{array}{l}\text { E Lembrecht+ } \\
\text { E Symet }\end{array}$ & $\begin{array}{l}8348 \\
4089\end{array}$ \\
\hline $125 \times=$ & $O(E)$ & & & & & \\
\hline 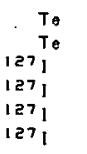 & $\begin{array}{c}{ }^{3} \mathrm{He} \\
a \\
p \\
p \\
p \\
d\end{array}$ & $\begin{array}{l}8 \cdot 0+0 \\
1 \cdot 0+1 \\
2 \cdot 0+1 \\
4 \cdot 0+1 \\
4 \cdot 3+1 \\
2 \cdot 5+1\end{array}$ & $\begin{array}{l}4.0+1 \\
4.0+1 \\
1.5+2 \\
9.8+1 \\
6 \cdot 5+1 \\
9 \cdot 5+1\end{array}$ & $\begin{array}{l}\text { TOK } \\
\text { TOK } \\
\text { HAR } \\
\text { UPP } \\
\text { GRN } \\
\text { JUL }\end{array}$ & $\begin{array}{l}\text { E Hommat } \\
\text { E Hommat } \\
\text { E Symet } \\
\text { E Lundqutst+ } \\
E \text { Poanst } \\
C \text { Schult. }\end{array}$ & $\begin{array}{l}6458 \\
8349 \\
4058 \\
4059 \\
4060 \\
5755\end{array}$ \\
\hline $126 \times 0$ & indepe & endent. & $\sigma(E)$ & & & \\
\hline $\begin{array}{l}53 \theta_{y} \\
23 \theta^{2}\end{array}$ & p & $\begin{array}{l}1.0+3 \\
1.2+3\end{array}$ & $3.0+5$ & $\begin{array}{l}\text { KFK } \\
\text { BNL. }\end{array}$ & $\begin{array}{l}\text { C Muenzel+ } \\
\text { E Yut }\end{array}$ & $\begin{array}{l}4905 \\
4907+\end{array}$ \\
\hline $126 \times e$ & cumula & ative? & thlck & $\operatorname{targe}$ & et yloldxfactor & \\
\hline$\exists a$ & p & $6.0+2$ & & KLN & E Kaiser. & 4116 \\
\hline
\end{tabular}

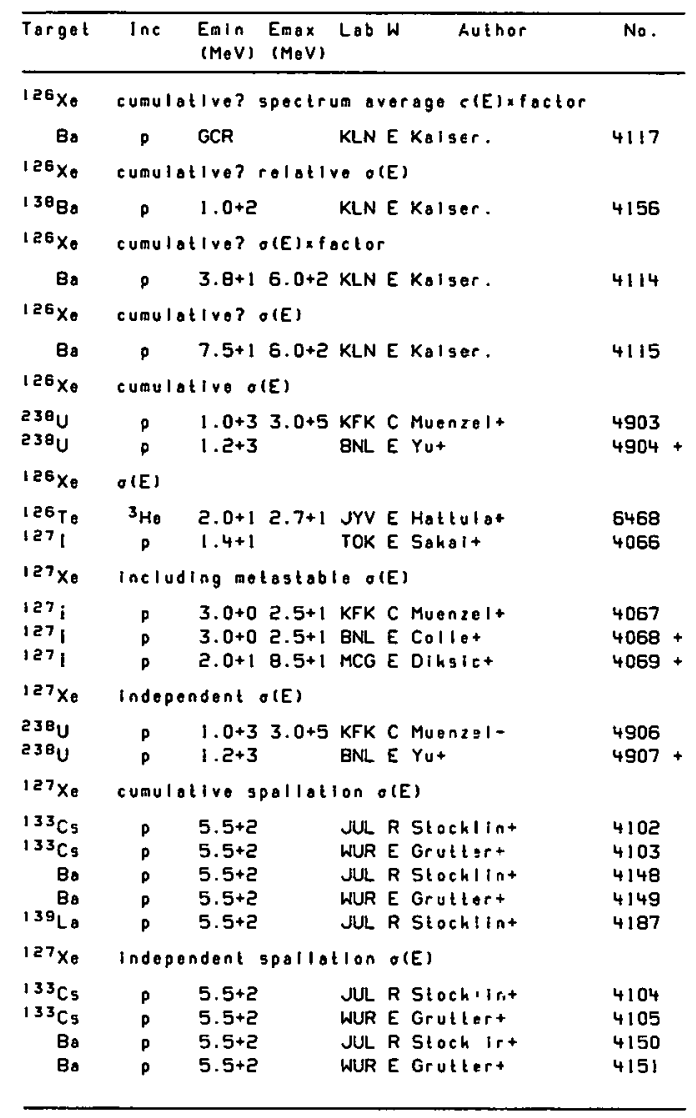




\begin{tabular}{|c|c|c|c|c|}
\hline Torgel & $\operatorname{lnc}$ & $\begin{array}{ll}\text { Emin } & \text { Emax } \\
(\operatorname{MeV}) & (\mathrm{MeV})\end{array}$ & Author & No. \\
\hline $127 \times 0$ & \multicolumn{4}{|c|}{ Independent spallotion o(E) } \\
\hline $\begin{array}{l}139 \mathrm{LO} \\
139 \mathrm{LO}\end{array}$ & $p$ & $\begin{array}{l}5.5+2 \\
5.5+2\end{array}$ & $\begin{array}{l}\text { JUL R Stochlint } \\
\text { WUR E Grubtert }\end{array}$ & $\begin{array}{l}4188 \\
4189\end{array}$ \\
\hline $127 x_{0}$ & \multicolumn{4}{|c|}{ spollation o(E) } \\
\hline $\begin{array}{l}133 \mathrm{Cg} \\
139 \mathrm{La} \\
139 \mathrm{La}\end{array}$ & $\begin{array}{l}p \\
p \\
p\end{array}$ & $\begin{array}{l}5.5+2 \\
5.5+2 \\
5.5+2\end{array}$ & $\begin{array}{l}\text { WUR E Gruttert } \\
\text { JUL R Stochlint } \\
\text { WUR E Gruttert }\end{array}$ & $\begin{array}{l}4107 \\
4190 \\
4191\end{array}$ \\
\hline $127 \times 0$ & \multicolumn{4}{|c|}{ cumulatlve? relatlive o(E) } \\
\hline${ }^{138} \mathrm{Ba}$ & p & $1.0+2$ & KLN E Kalser. & 4157 \\
\hline $127 \times e$ & \multicolumn{4}{|c|}{ cumulative? o(E)afoctor } \\
\hline $\begin{array}{r}80 \\
127 \times 0\end{array}$ & \multicolumn{4}{|c|}{ cumulative? o(E) } \\
\hline $\begin{array}{r}B 0 \\
127 X_{0}\end{array}$ & \multicolumn{4}{|c|}{ cumulative thlck tergot ylold } \\
\hline 1271 & 0 & $1.0+2$ & KFK E Helugt & 8373 \\
\hline $127 x_{0}$ & \multicolumn{4}{|c|}{ cusulative of } \\
\hline 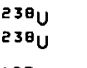 & P & $\begin{array}{l}1.0+3 \\
1.2+3\end{array} 3.0+5$ & $\begin{array}{l}\text { KFK C Muenzel+ } \\
\text { BNLE Yut }\end{array}$ & $\begin{array}{l}4903 \\
4904+\end{array}$ \\
\hline $127 \times 0$ & \multicolumn{4}{|c|}{ product yleld } \\
\hline 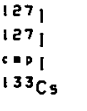 & $\begin{array}{l}p \\
p \\
p \\
p\end{array}$ & $\begin{array}{l}2.0+1 \\
7.8+1 \\
2.0+1 \\
4.3+1 \quad 1.6+2\end{array}$ & $\begin{array}{l}\text { UPP E Lundaviglt } \\
\text { JUL E Weinricht } \\
\text { UPP E Lundarist+ } \\
\text { WIS E Bluet }\end{array}$ & $\begin{array}{l}4070 \\
5760 \\
4091 \\
4108\end{array}$ \\
\hline $127 \times 0$ & \multicolumn{4}{|c|}{ thich tergot ylold } \\
\hline 1271 & d & $2.6+1$ & IKO E Schimmel+ & 5758 \\
\hline $127 \times 0$ & \multicolumn{4}{|c|}{ overoge of $(E)$} \\
\hline $\begin{array}{l}1271 \\
127 \times 0\end{array}$ & $\begin{array}{c}o(E) \\
9\end{array}$ & $6.4+17.8+1$ & JUL E Weinricht & 5761 \\
\hline $\begin{array}{l}128 \mathrm{~T} \\
1271 \\
1271\end{array}$ & $\begin{array}{c}{ }_{3}^{3} \mathrm{He} \\
\mathrm{p} \\
9\end{array}$ & 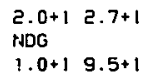 & $\begin{array}{l}\text { JYV E Hattulat } \\
\text { UPP E Lundavist+ } \\
\text { JUL C Schull. }\end{array}$ & $\begin{array}{l}6469 \\
4071 \\
5759\end{array}$ \\
\hline
\end{tabular}

\begin{tabular}{|c|c|c|c|c|c|c|}
\hline Torge: & $\operatorname{Inc}$ & $\begin{array}{l}\text { Emin } \\
(M 0 V)\end{array}$ & $\begin{array}{l}\text { Emax } \\
(\operatorname{MeV})\end{array}$ & Lab W & Author & No. \\
\hline${ }^{27} x_{e}$ & $\sigma(E)$ & & $\cdot$ & & & \\
\hline$\because D_{1}$ & $p$ & NDG & & UPP E & Lundquist & 4092 \\
\hline $12 \theta_{0}$ & Indop & ndent. & $O(E)$ & & & \\
\hline $\begin{array}{l}23 \theta^{2} \\
230 \mathrm{U}\end{array}$ & $p$ & $\begin{array}{l}1.0+3 \\
1.2+3\end{array}$ & $3.0+5$ & $\begin{array}{l}\text { KFK C } \\
\text { BNL E }\end{array}$ & $\begin{array}{l}\text { Muenzel+ } \\
\text { Yu+ }\end{array}$ & $\begin{array}{l}4908 \\
4907+\end{array}$ \\
\hline $128 \times 0$ & cumul & liver & thick & tergot & yieldafoctor & \\
\hline $\mathrm{Ba}$ & $p$ & $6.0+2$ & & KLN E & Kalser. & 4121 \\
\hline $128 \times 0$ & cumul & tive? & spoctri & un aver & roge o(E) xfactor & \\
\hline $\mathrm{Ba}$ & $p$ & GCR & & KLN E & Kalser. & 4123 \\
\hline $128 \times 0$ & cusul & tiver & relolis & ve ef(E) & & \\
\hline $138^{88}$ & ip & $1.0+2$ & & KLN E & Kaiser. & 4158 \\
\hline $128 \times$ & cunul & Hue? & $O(E) \times f$ & actor & & \\
\hline $\mathrm{Ba}$ & D & $3.8+1$ & $6.0+2$ & KLN E & Kalser. & 4120 \\
\hline $12 x_{0}$ & cumul & tiver & $O(E)$ & & s & \\
\hline $\mathrm{Ba}$ & P & $6 \cdot 0+1$ & $6.0+2$ & KLN E & Kalser. & 4122 \\
\hline $128 \times 0$ & cumul & tive o & (E) & & & \\
\hline $\begin{array}{l}23 \theta^{2} \\
23 \theta^{2}\end{array}$ & p & $\begin{array}{l}1.0+3 \\
1.2+3\end{array}$ & $3.0+5$ & $\begin{array}{l}\text { KFK C } \\
\text { BNL E }\end{array}$ & $\begin{array}{l}\text { Muenzel+ } \\
\text { Yut }\end{array}$ & $\begin{array}{l}4903 \\
4904+\end{array}$ \\
\hline $129 \times 0$ & indep & odent o & $O(E)$ & & & \\
\hline $\begin{array}{l}238 \mathrm{U} \\
238 \mathrm{U}\end{array}$ & p & $\begin{array}{l}1.0+3 \\
1.2+3\end{array}$ & $3.0+5$ & $\begin{array}{l}\text { KFK C } \\
\text { BNL E }\end{array}$ & $\begin{array}{l}\text { Muenzel+ } \\
\text { Yut }\end{array}$ & $\begin{array}{l}4906 \\
4907 .\end{array}$ \\
\hline $129 x_{0}$ & cumul & tive? & thlek & target & yleidafactor & \\
\hline$\theta e$ & p & $6.0+2$ & & KLN E & Kalser. & 4125 \\
\hline $129 x_{6}$ & cunul & tive? $=$ & spectro & un over & rage $O(E) \times$ foctor & \\
\hline $\begin{array}{r}80 \\
129 \times 0\end{array}$ & $\begin{array}{c}p \\
\text { cumul }\end{array}$ & $\begin{array}{l}\text { GCR } \\
\text { tivo? }\end{array}$ & relotir & $\begin{array}{l}\text { KLN E } \\
\text { ve O(E) }\end{array}$ & $\begin{array}{l}\text { Kaiser. } \\
\Rightarrow\end{array}$ & 4127 \\
\hline $\begin{array}{l}138 \mathrm{Bo} \\
128 \mathrm{Xo}_{0}\end{array}$ & $\begin{array}{c}P \\
\text { cumul }\end{array}$ & $\begin{array}{l}1.0+2 \\
\text { tive? }\end{array}$ & $\sigma(E) \times f$ & $\begin{array}{l}\text { KLN E } \\
\text { octor }\end{array}$ & Kaiser. & 4159 \\
\hline $\mathrm{Ba}$ & p & $3.8+1$ & $6.0+2$ & KLN E & Kalser. & 4124 \\
\hline
\end{tabular}




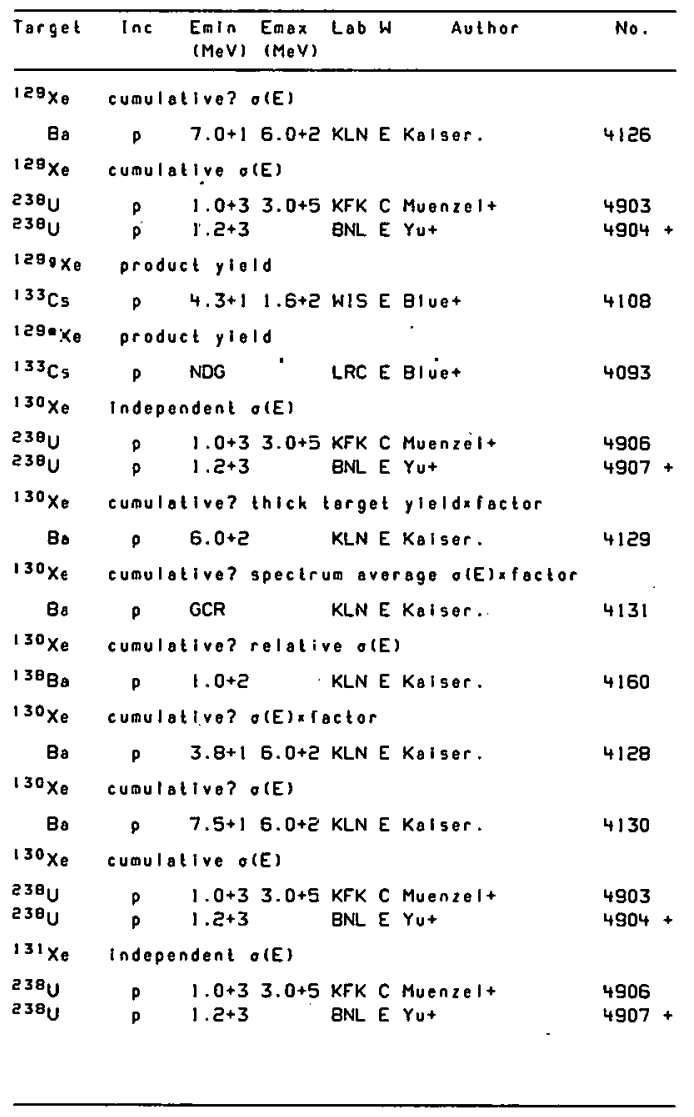

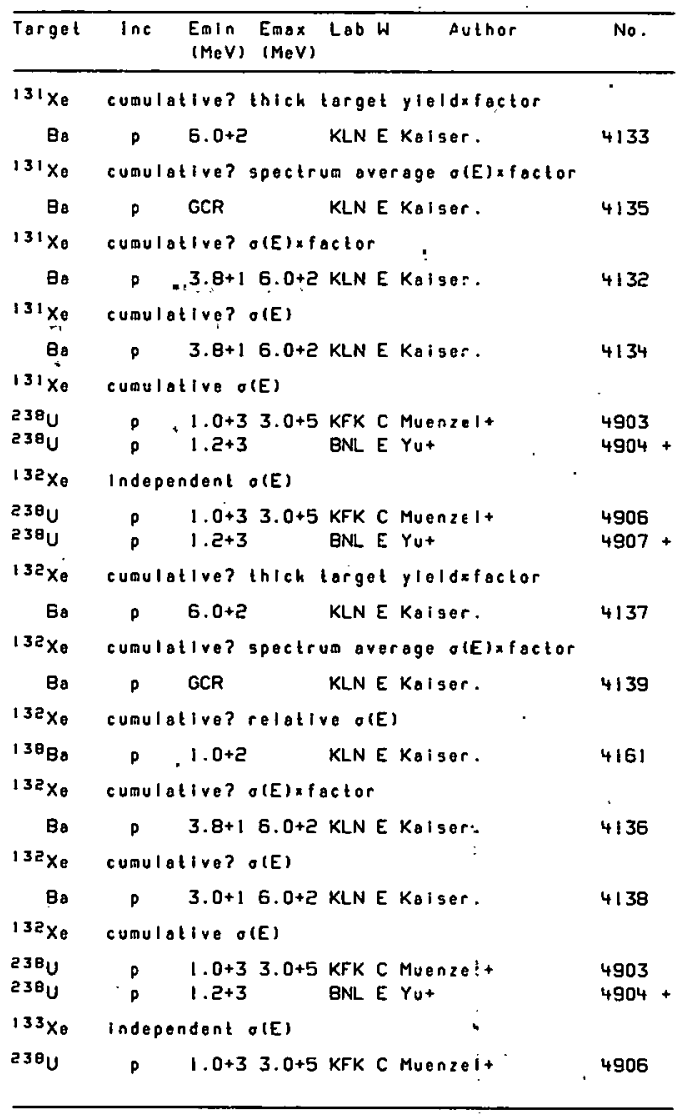




\begin{tabular}{|c|c|c|c|c|}
\hline Targe! & $\ln c$ & $\begin{array}{ll}\text { Emin } & \text { Emax } \\
(M e V) & (M e V)\end{array}$ & Lab W & No. \\
\hline $133 \times e$ & Indep & andont $O(E)$ & & \\
\hline $238_{\mathrm{U}}$ & p & $1.2+3$ & BNL E Yu+ & $4907+$ \\
\hline $133 \times 6$ & cumul & Aive lission & yield & \\
\hline $\begin{array}{l}233 \mathrm{U} \\
133 \mathrm{Xe}\end{array}$ & $\begin{array}{c}p \\
\text { cumul }\end{array}$ & $\begin{array}{l}2.0+18.5+1 \\
\text { live } \sigma(E)\end{array}$ & CCP R Lbovt & 4838 \\
\hline $\begin{array}{l}23 \theta^{U} \\
23 \theta^{3}\end{array}$ & p & $\begin{array}{l}1.0+3 \quad 3.0+5 \\
1.2+3\end{array}$ & $\begin{array}{l}\text { KFK C Muenzel+ } \\
\text { BNLE Yu+ }\end{array}$ & $\begin{array}{l}4903 \\
4904+\end{array}$ \\
\hline $1330 \times 0$ & cume & lotive fission & n yleld & \\
\hline $\begin{array}{l}233 \mathrm{U} \\
235_{\mathrm{U}}\end{array}$ & p & $\begin{array}{ll}2.0+1 & 8.5+1 \\
1.2+1 & 8.5+1\end{array}$ & $\begin{array}{l}\text { CCP R Lbovt } \\
\text { CCP R Lbovt }\end{array}$ & $\begin{array}{l}4838 \\
4852\end{array}$ \\
\hline $133 . x_{0}$ & cume & lative fission & $n$ yleld & \\
\hline $\begin{array}{l}233 u \\
235 u\end{array}$ & p & $\begin{array}{ll}2.0+1 & 8.5+1 \\
1.2+1 & 8.5+1\end{array}$ & $\begin{array}{l}\text { CCP R Lbovt } \\
\text { CCP R Lbovt }\end{array}$ & $\begin{array}{l}4838 \\
4852\end{array}$ \\
\hline $134 x_{0}$ & indep & ondent $O(E)$ & & \\
\hline $\begin{array}{l}23 \theta_{U} \\
23 \theta_{U}\end{array}$ & $\begin{array}{l}p \\
p\end{array}$ & $\begin{array}{l}1.0+3 \\
1 \cdot 2+3\end{array} 3 \cdot 0+5$ & $\begin{array}{l}\text { KFKC Muenzel+ } \\
\text { BNLE Yu+ }\end{array}$ & $\begin{array}{l}4906 \\
4907+\end{array}$ \\
\hline $134 x_{0}$ & cumul & tive? thlch t & target yleldxfactor & \\
\hline $134 \times 0$ & cumul & $\begin{array}{l}6.0+2 \\
\text { ative? spectru }\end{array}$ & $\begin{array}{l}\text { KLN E Kaiser. } \\
\text { um average o(E) afoctor }\end{array}$ & 4141 \\
\hline $\begin{array}{r}80 \\
134 \times 0\end{array}$ & $\stackrel{p}{\text { cumul }}$ & $\begin{array}{l}\text { GCR } \\
\text { ative? relativ }\end{array}$ & $\begin{array}{l}\text { KLN E Kalser. } \\
\text { vo O(E) }\end{array}$ & 4143 \\
\hline $\begin{array}{l}13 \theta_{\mathrm{Ba}} \\
134 \mathrm{Xe}\end{array}$ & $\stackrel{p}{\text { cumul }}$ & $\begin{array}{l}1.0+2 \\
\text { ative? } O(E) \times f 0\end{array}$ & $\begin{array}{l}\text { KLNE Kalser. } \\
\text { actor }\end{array}$ & 4162 \\
\hline $\begin{array}{r}80 \\
134 \times 0\end{array}$ & $\stackrel{p}{\text { cumul }}$ & $\begin{array}{l}3.8+16.0+2 \\
\text { livo? } \sigma(E)\end{array}$ & KLN E Kaiser. & 4140 \\
\hline $\begin{array}{r}B a \\
134 \times 0\end{array}$ & $\stackrel{p}{\text { cumul }}$ & $\begin{array}{l}9.5+16.0+2 \\
\text { tive } \sigma(E)\end{array}$ & KLN E Kaiser. & 4142 \\
\hline $\begin{array}{l}53 \theta^{2} \\
23 \theta^{2}\end{array}$ & p & $\begin{array}{l}1.0+3 \\
1.2+3\end{array}$ & $\begin{array}{l}\text { KFK C Muenzol+ } \\
\text { BNL E Yut }\end{array}$ & $\begin{array}{l}4903 \\
4904+\end{array}$ \\
\hline
\end{tabular}

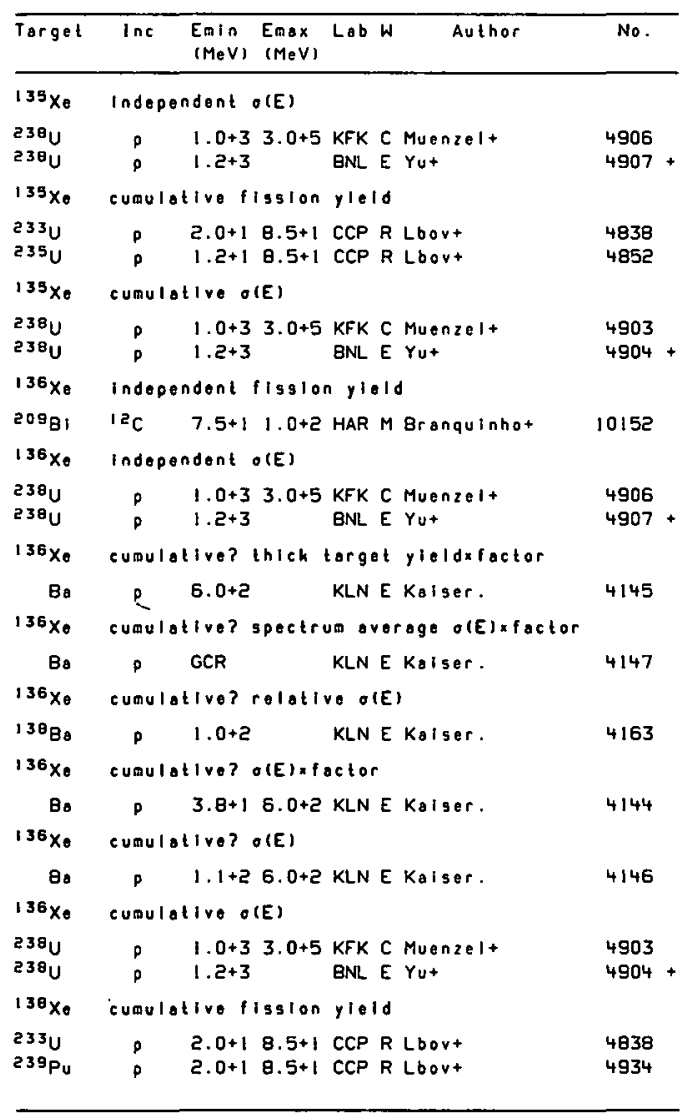




\begin{tabular}{|c|c|c|c|c|}
\hline Target & $\ln c$ & $\begin{array}{ll}\text { Emin } & \text { Emax } \\
(M e V) & (M e V)\end{array}$ & Author & No. \\
\hline$z=55$ & $v_{n}$ & & & \\
\hline Ag & ${ }^{40} \mathrm{Ar}$ & $3.4+2$ & PAR R Lefort+ & 12202 \\
\hline $11+C_{s}$ & \multicolumn{2}{|c|}{ spallation product } & $y|\theta| d$ & \\
\hline Lө & p & $6.0+2$ & BRK R Cerny+ & 4165 \\
\hline $115 \mathrm{Cs}$ & \multicolumn{2}{|c|}{ spollation product } & yield & \\
\hline Lo & $p$ & $6.0+2$ & BRK R Cerny+ & 4166 \\
\hline $\mid I E_{i} C_{s}$ & \multicolumn{2}{|c|}{ spallation prodect } & yiold & \\
\hline La & p & $6: 0+2$ & BRK R Cerny+ & 4167 \\
\hline $138 \mathrm{Cs}$ & \multicolumn{3}{|c|}{ partlal product ylold } & \\
\hline $106 \mathrm{Cd}$ & ${ }^{16} 0$ & NDG & STE E Garg. & 11342 \\
\hline $120 \mathrm{Cs}$ & \multicolumn{2}{|c|}{ spallation prodoct } & $y 1010$ & \\
\hline La & $p$ & $6.0+2$ & BRK R Cernyt & 4168 \\
\hline $121 \mathrm{cs}$ & \multicolumn{3}{|c|}{ partial product yleld } & \\
\hline $110 \mathrm{Cd}$ & ${ }^{14} \mathrm{~N}$ & NDG & STB E Garg. & 10440 \\
\hline $1=\mathrm{CCs}_{\mathrm{s}}$ & \multicolumn{3}{|c|}{ partial product yiold } & \\
\hline $11 \mathrm{Cd}$ & $14 \mathrm{~N}$ & NDG & STB E Garg. & $1044 !$ \\
\hline $123=9$ & \multicolumn{3}{|c|}{ cumulative spallation $a(E)$} & \\
\hline $\begin{array}{r}133 \mathrm{Cs} \\
133 \mathrm{Cs} \\
\mathrm{Ba} \\
\mathrm{Ba} \\
139 \mathrm{La}\end{array}$ & $\begin{array}{l}p \\
p \\
p \\
p \\
p\end{array}$ & $\begin{array}{l}5.5+2 \\
5 \cdot 5+2 \\
5 \cdot 5+2 \\
5 \cdot 5+2 \\
5 \cdot 5+2\end{array}$ & $\begin{array}{l}\text { JUL R Stocklint } \\
\text { WUR E Gruttert } \\
\text { JUL R Stocklint } \\
\text { WUR E Gruttert } \\
\text { JUL R Stocklint }\end{array}$ & $\begin{array}{l}4102 \\
4103 \\
4148 \\
4149 \\
4187\end{array}$ \\
\hline${ }^{123} \mathrm{Cs}_{5}$ & \multicolumn{3}{|c|}{ Spallotion o(E) } & \\
\hline $\begin{array}{l}133 \mathrm{Cs} \\
13 \mathrm{C}_{\mathrm{LB}} \\
13 \mathrm{O}_{\mathrm{La}}\end{array}$ & $\begin{array}{l}p \\
p \\
p\end{array}$ & $\begin{array}{l}5 \cdot 5+2 \\
5 \cdot 5+2 \\
5 \cdot 5+2\end{array}$ & $\begin{array}{l}\text { WUR E Gruttert } \\
\text { JUL R Stochlint } \\
\text { WUR E Gruttert }\end{array}$ & $\begin{array}{l}4107 \\
4190 \\
4191\end{array}$ \\
\hline $123 \mathrm{Cs}$ & \multicolumn{3}{|c|}{ partlal product yiold } & \\
\hline $\begin{array}{l}112 \mathrm{Cd} \\
1155 \mathrm{sn}\end{array}$ & $\begin{array}{l}14 \mathrm{~N} \\
10_{B}\end{array}$ & $\begin{array}{l}\text { NDG } \\
\text { NDG }\end{array}$ & $\begin{array}{l}\text { STB E Garg. } \\
\text { STB E Garg. }\end{array}$ & $\begin{array}{r}10442 \\
9530\end{array}$ \\
\hline
\end{tabular}

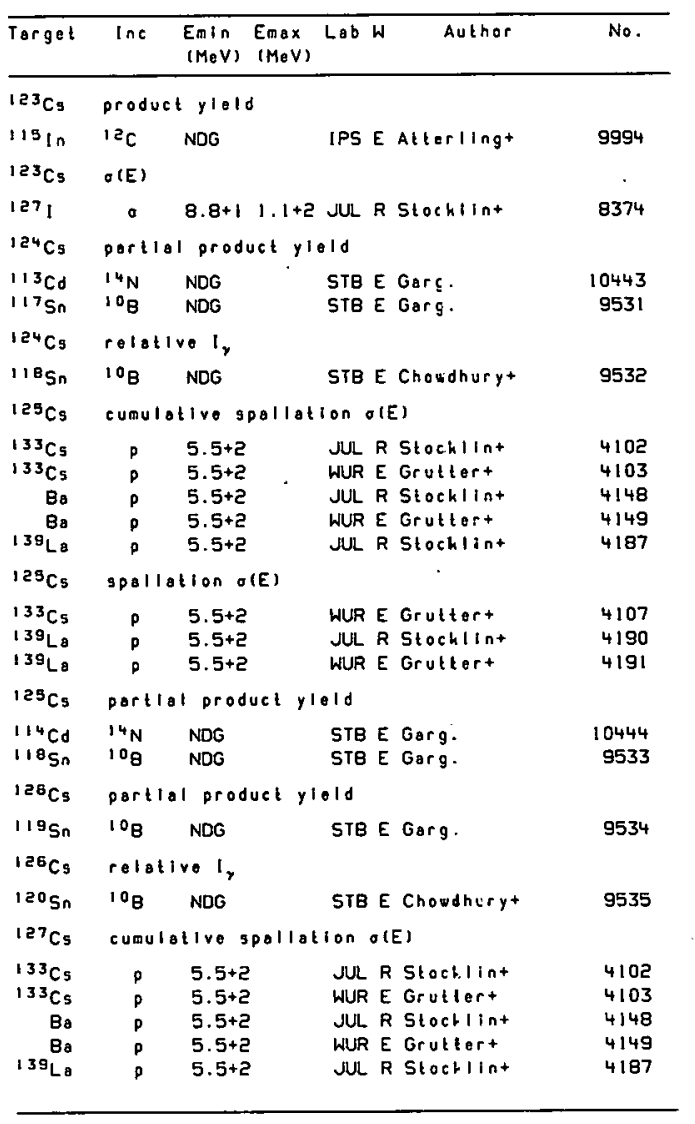




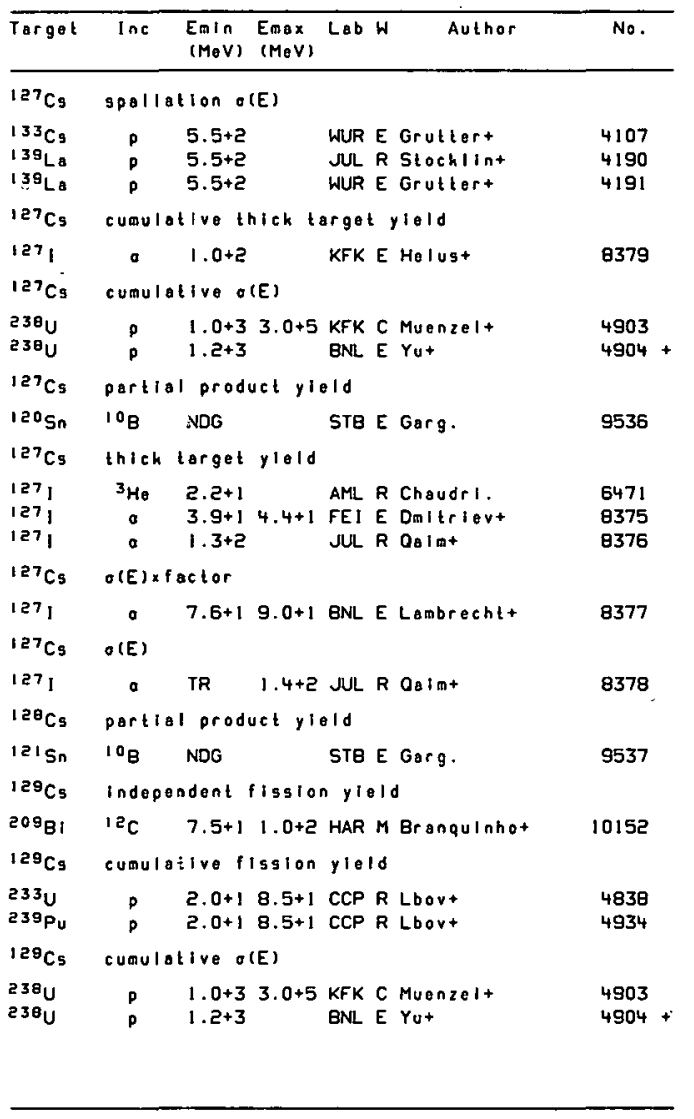

\begin{tabular}{|c|c|c|c|c|c|c|}
\hline Targot & $\ln c$ & $\begin{array}{l}\text { EnIn } \\
(M \circ V)\end{array}$ & $\begin{array}{l}\text { Enax } \\
(\mathrm{MoV})\end{array}$ & Lab $h$ & Author & No. \\
\hline${ }^{129} \mathrm{Cs}$ & \multicolumn{6}{|c|}{ partlol product yiald } \\
\hline $\begin{array}{l}122 \mathrm{Sn} \\
126 \mathrm{~T}^{\circ} \\
127 \mathrm{l}\end{array}$ & $\begin{array}{c}{ }_{10 B}^{10} \\
{ }^{6} L_{1} \\
0\end{array}$ & $\begin{array}{l}\text { NDG } \\
\text { NDG } \\
\text { NDG }\end{array}$ & & $\begin{array}{l}\text { STB E } \\
\text { STB E } \\
\text { STB E }\end{array}$ & $\begin{array}{l}\text { E Garg. } \\
\text { E Garg. } \\
\text { E Garg. }\end{array}$ & $\begin{array}{l}9538 \\
9244 \\
8384\end{array}$ \\
\hline $129 \mathrm{cs}$ & thlck & torget & yiold & & & \\
\hline $\begin{array}{l}1271 \\
1271 \\
1271 \\
127 !\end{array}$ & $\begin{array}{l}0 \\
0 \\
0 \\
0\end{array}$ & $\begin{array}{l}2.0+1 \\
3.0+1 \\
4.5+1 \\
1.0+2\end{array}$ & $3.6+1$ & $\begin{array}{l}\text { FEI E } \\
\text { AML } \\
\text { GRN E } \\
\text { JUL F }\end{array}$ & $\begin{array}{l}\text { E Omitriout } \\
\text { R Choudri. } \\
\text { E Bukshpant } \\
\text { R Ooimt }\end{array}$ & $\begin{array}{l}8380 \\
8381 \\
8382 \\
8383\end{array}$ \\
\hline $129 \mathrm{Cg}$ & \multicolumn{6}{|c|}{ of $(E) \times 1$ actor } \\
\hline $\begin{array}{l}127 \mathrm{l} \\
129 \mathrm{cg}\end{array}$ & $\begin{array}{c}0 \\
O(E)\end{array}$ & $7.6+1$ & $9.0+1$ & BNL E & E Lambrecht+ & 8385 \\
\hline $\begin{array}{l}127 \mathrm{l} \\
130 \mathrm{cs}\end{array}$ & $\begin{array}{c}\circ \\
\text { cumule }\end{array}$ & \multicolumn{2}{|c|}{ cumulative fission $y$} & $\begin{array}{l}\text { an } \\
y \mid 010\end{array}$ & $\begin{array}{l}\text { R Oolat } \\
\text { d }\end{array}$ & 8388 \\
\hline $\begin{array}{l}233 \mathrm{U} \\
23 \theta_{\mathrm{U}} \\
23 \mathrm{O}^{\mathrm{U}} \\
239 \mathrm{Pu}\end{array}$ & $\begin{array}{c}p \\
118 \\
22_{\mathrm{Ne}} \\
p\end{array}$ & $\begin{array}{l}2.0+1 \\
8 \cdot 5+1 \\
1.3+2 \\
2.0+1\end{array}$ & $\begin{array}{l}8.5+1 \\
1.6+2 \\
8.5+1\end{array}$ & $\begin{array}{l}\text { CCP R } \\
\text { PAR E } \\
\text { PAR E } \\
\text { CCP R }\end{array}$ & $\begin{array}{l}R \text { Lbovt } \\
\text { E Desaint-Simont } \\
\text { E Desaint-Simont } \\
R \text { Lbovt }\end{array}$ & $\begin{array}{r}4838 \\
9597 \\
11927 \\
4934\end{array}$ \\
\hline $130 \mathrm{Cs}$ & \multicolumn{6}{|c|}{ portlol product ylold } \\
\hline $\begin{array}{l}123 \mathrm{Sn} \\
127 \mathrm{Te}\end{array}$ & $\begin{array}{c}{ }^{10} B \\
{ }^{6} L I\end{array}$ & $\begin{array}{l}\text { NDG } \\
\text { NDG }\end{array}$ & & $\begin{array}{l}\text { STB E } \\
\text { STB E }\end{array}$ & $\begin{array}{l}\text { E Garg. } \\
\text { E Garg. }\end{array}$ & $\begin{array}{l}9539 \\
9245\end{array}$ \\
\hline $130 \mathrm{Cg}$ & \multicolumn{6}{|c|}{ relotive $I_{y}$} \\
\hline $128 \mathrm{Te}_{\mathrm{\theta}}$ & $6_{\mathrm{L}}$ & NDG & & STB E & E Choodhury+ & 9246 \\
\hline $131 C_{9}$ & \multicolumn{6}{|c|}{ cumulative lission yield } \\
\hline $\begin{array}{l}233 \mathrm{U} \\
238^{\mathrm{U}} \\
238_{\mathrm{U}} \\
23 \mathrm{Pu}^{\mathrm{Pu}}\end{array}$ & $\begin{array}{c}p \\
2118 \\
118 \\
\mathrm{Ne}\end{array}$ & $\begin{array}{l}2.0+1 \\
8.5+1 \\
1.3+2 \\
2.0+1\end{array}$ & $\begin{array}{l}8.5+1 \\
1.6+2 \\
8.5+1\end{array}$ & $\begin{array}{l}\text { CCP R } \\
\text { PAR E } \\
\text { PAR E } \\
\text { CCP R }\end{array}$ & $\begin{array}{l}\text { R Lbovt } \\
\text { E Desaint-Simont } \\
\text { E Desoint-simont } \\
\text { R Lbovt }\end{array}$ & $\begin{array}{r}4938 \\
9597 \\
11927 \\
4934\end{array}$ \\
\hline $131 \mathrm{Cg}_{9}$ & \multicolumn{6}{|c|}{ partiol product yiold } \\
\hline $\begin{array}{l}124 \mathrm{Sn} \\
128 \mathrm{Te}\end{array}$ & $\begin{array}{c}10_{B} \\
6 \mathrm{LI}\end{array}$ & $\begin{array}{l}\text { NDG } \\
\text { NDG }\end{array}$ & & $\begin{array}{l}\text { STR E } \\
\text { STB E }\end{array}$ & $\begin{array}{l}\text { E Garg. } \\
\text { E Garg. }\end{array}$ & $\begin{array}{l}9540 \\
9247\end{array}$ \\
\hline
\end{tabular}




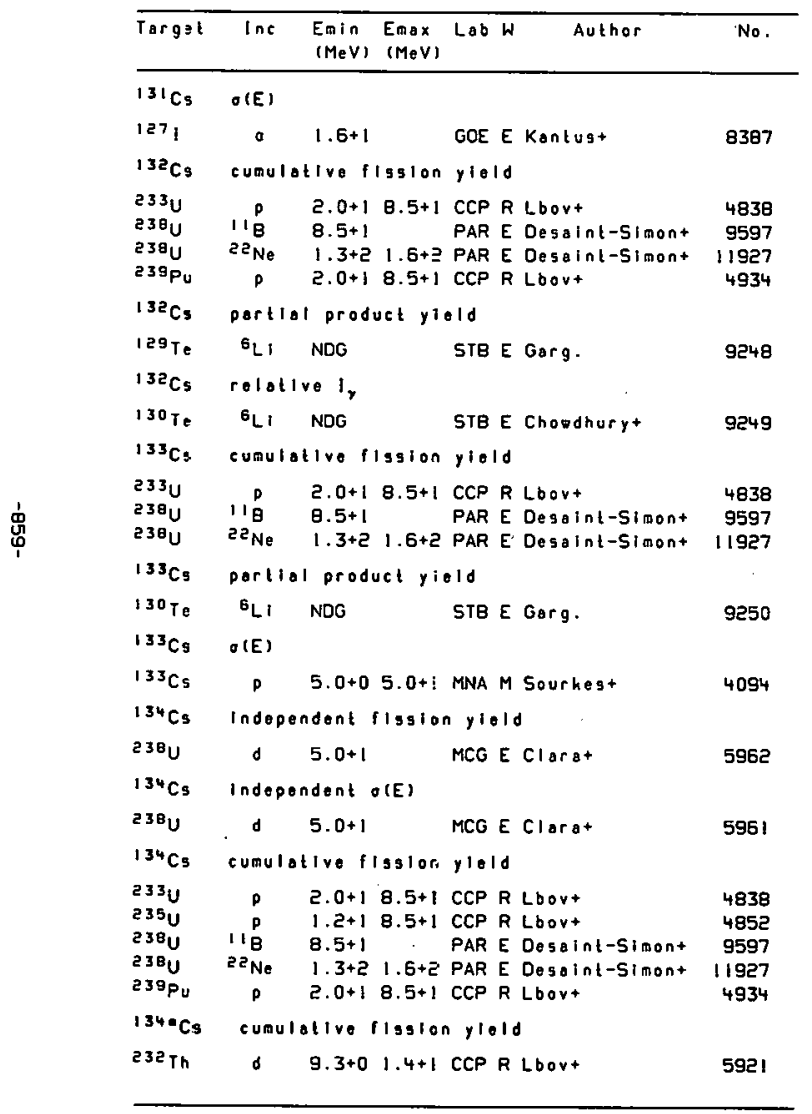

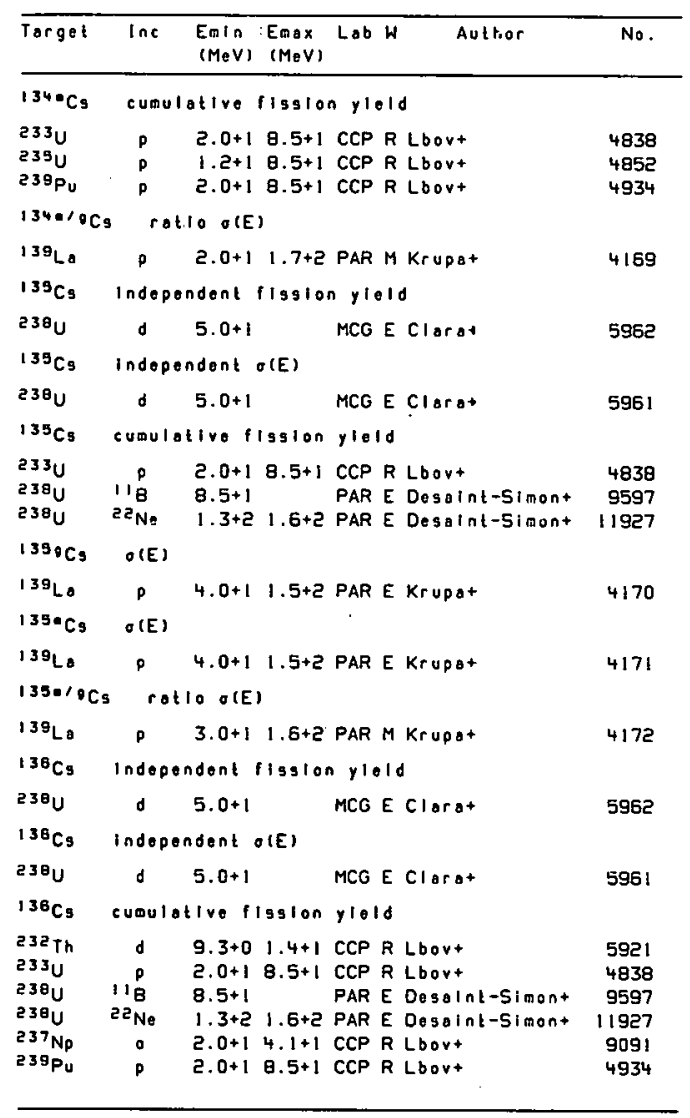




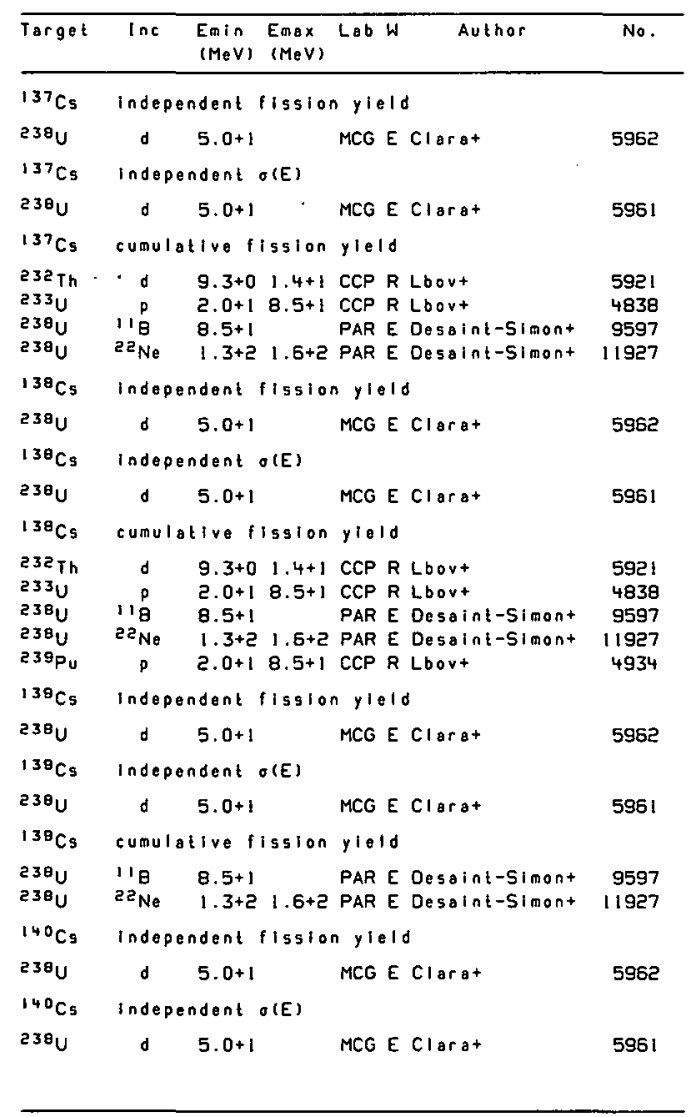

\begin{tabular}{|c|c|c|c|c|c|}
\hline Target & Inc & $\begin{array}{ll}\text { Emin } & \text { Emax } \\
(M e V) & (M e V)\end{array}$ & Lab W & Author & No. \\
\hline${ }^{140} \mathrm{Cs}$ & \multicolumn{5}{|c|}{ cumulative fission yleld } \\
\hline $\begin{array}{l}23 \theta_{U} \\
23 \theta_{U}\end{array}$ & $\begin{array}{l}11 \mathrm{~B} \\
22_{\mathrm{Ne}}\end{array}$ & $\begin{array}{ll}8.5+1 & \\
1.3+2 & 1.6+2\end{array}$ & $\begin{array}{l}\text { PAR E } \\
\text { PAR E }\end{array}$ & $\begin{array}{l}\text { Degaint-Simant } \\
\text { Desaint-Simon+ }\end{array}$ & $\begin{array}{r}9597 \\
11927\end{array}$ \\
\hline${ }^{141} \mathrm{Cs}$ & \multicolumn{5}{|c|}{ Independent fission yleld } \\
\hline $238^{4}$ & d & $5.0+1$ & MCG E & Clarat & 5962 \\
\hline $141 \mathrm{Cs}$ & \multicolumn{5}{|c|}{ Independent o(E) } \\
\hline 2380 & d & $5 \cdot 0+1$ & MCG E & Clarat & 5961 \\
\hline $14 \mathrm{ECs}_{3}$ & \multicolumn{5}{|c|}{ independent fission yield } \\
\hline $23 \theta_{U}$ & d & $5 \cdot 0+1$ & MCG E & $\mathrm{Clarat}^{+}$ & 5962 \\
\hline $142 \mathrm{cs}$ & \multicolumn{5}{|c|}{ Independent $O(E)$} \\
\hline $23 \theta^{2}$ & d & $5.0+1$ & MCG E & Clarat & 5961 \\
\hline $14 \mathrm{a}_{\mathrm{Cs}}$ & \multicolumn{5}{|c|}{ cumulative fission yield } \\
\hline $\begin{array}{l}23 \theta_{U} \\
23 \theta_{U} \\
238_{U} \\
23 \theta_{U}\end{array}$ & $\begin{array}{l}118 \\
11 \mathrm{~B} \\
22^{\mathrm{Ne}} \\
22^{\mathrm{Ne}}\end{array}$ & $\begin{array}{ll}8.5+1 & \\
8.5+1 & \\
1.3+2 & 1.6+2 \\
1.3+2 & 1.6+2\end{array}$ & $\begin{array}{l}\text { PAR E } \\
\text { PARE } \\
\text { PAR E } \\
\text { PAR E }\end{array}$ & $\begin{array}{l}\text { Desaint-Simont } \\
\text { Desaint-Simont } \\
\text { Desaint-Simont } \\
\text { Desaint-Simont }\end{array}$ & $\begin{array}{r}9597 \\
9597 \\
11927 \\
11927\end{array}$ \\
\hline $1+3 \mathrm{Cs}$ & \multicolumn{5}{|c|}{ independent fission yield } \\
\hline $23 \theta_{U}$ & d & $5.0+1$ & MCG E & Clarat & 5962 \\
\hline${ }^{143} \mathrm{Cs}$ & \multicolumn{5}{|c|}{ Independent $O(E)$} \\
\hline $23 \theta_{U}$ & $d$ & $5.0+1$ & MCG E & Clarat & 5961 \\
\hline $144 \mathrm{Cs}$ & \multicolumn{5}{|c|}{ Independent fissian yield } \\
\hline $238 \mathrm{U}$ & d & $5 \cdot 0+1$ & MCG E & Clarat & 5962 \\
\hline $144 \mathrm{Cs}$ & \multicolumn{5}{|c|}{ Independent o(E) } \\
\hline $23 \mathbf{B}_{\mathrm{u}}$ & d & $5.0+1$ & MCG E & Clarat & 5961 \\
\hline${ }^{145} \mathrm{Cs}$ & \multicolumn{5}{|c|}{ independent fission yleld } \\
\hline $238_{U}$ & d & $5.0+1$ & MCG $\varepsilon$ & Clarat & 5962 \\
\hline $14^{49} \mathrm{Cs}$ & \multicolumn{5}{|c|}{ independent $O(E)$} \\
\hline $23 \theta_{U}$ & d & $5.0+1$ & MCG E & Clarat & 5961 \\
\hline
\end{tabular}




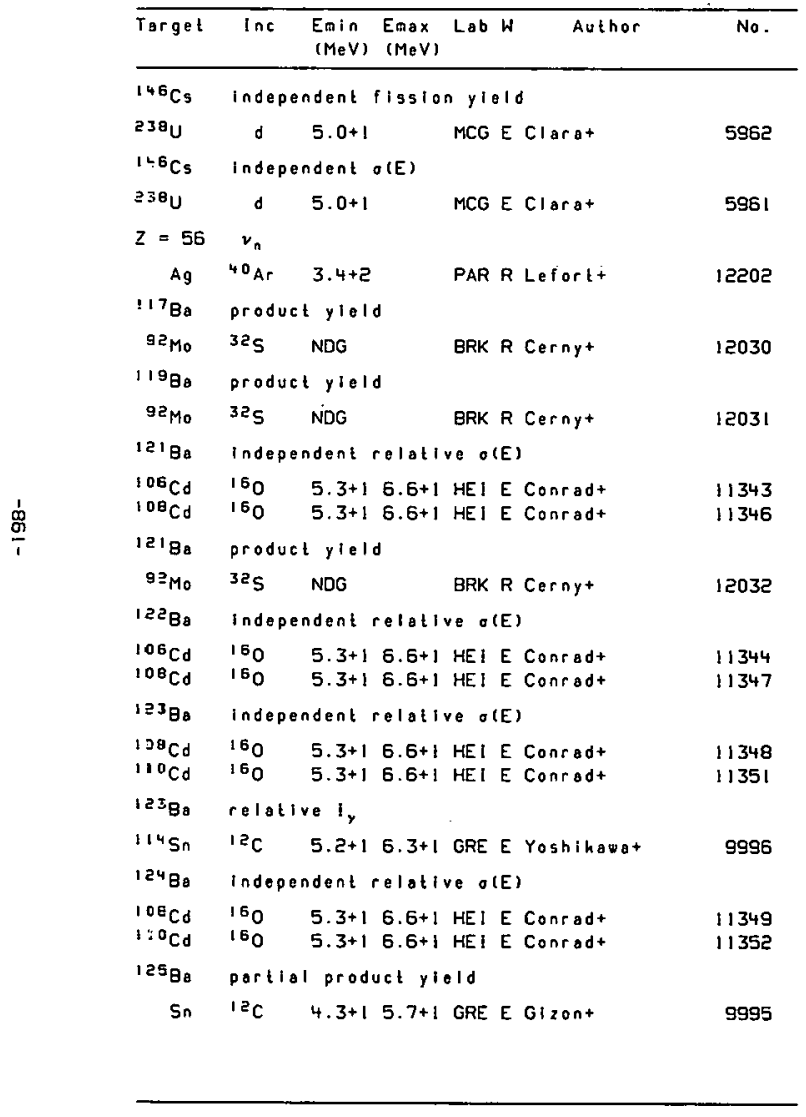

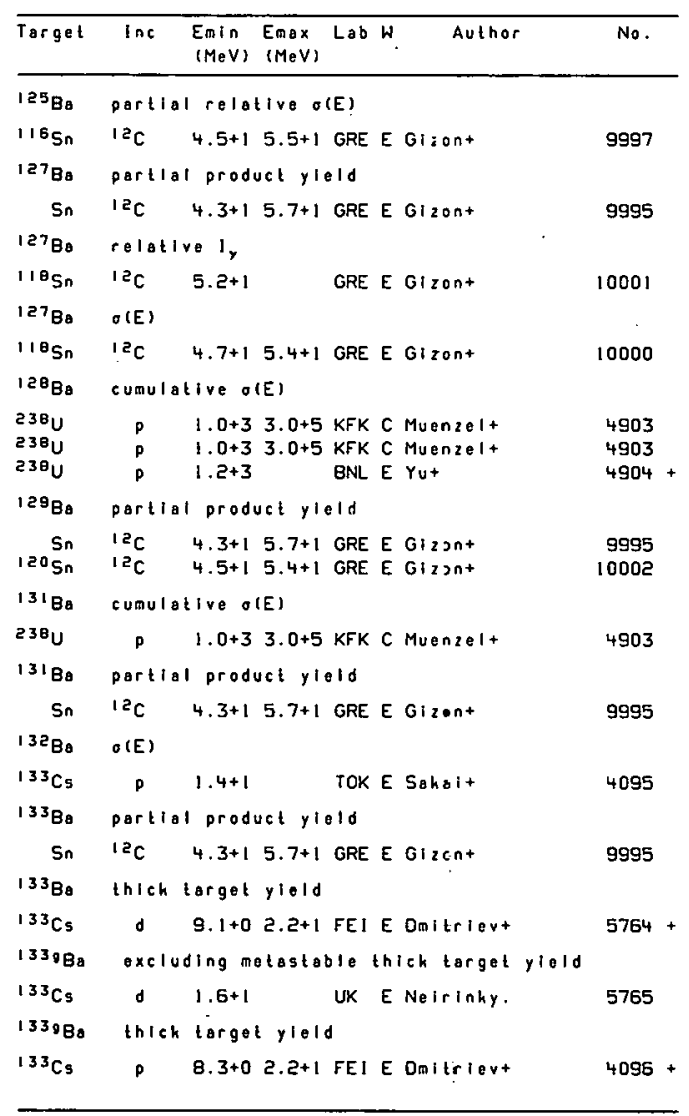




\begin{tabular}{|c|c|c|c|c|c|c|}
\hline Target: & $\ln c$ & $\begin{array}{l}\text { Emin } \\
(M e V)\end{array}$ & $\begin{array}{l}E_{\max } \\
(\mathrm{MeV})\end{array}$ & Lab & Author & No. \\
\hline 133090 & Indepen & endent & $O(E)$ & & & \\
\hline $\begin{array}{l}23 \theta^{4} \\
133 \cdot 80\end{array}$ & p thick & $\begin{array}{l}1.0+3 \\
\text { terget }\end{array}$ & $\begin{array}{l}3.0+5 \\
t \text { yield }\end{array}$ & $\begin{array}{l}\text { KFK C } \\
\text { dxfoct }\end{array}$ & $\begin{array}{l}\text { C Muenzelt } \\
\text { tor }\end{array}$ & 4906 \\
\hline $\begin{array}{l}133 \mathrm{Cs} \\
133 \mathrm{msa}^{3}\end{array}$ & d & $\begin{array}{l}1.6+1 \\
\text { torget }\end{array}$ & iyleld & UK E & E Neirinky. & 5766 \\
\hline $\begin{array}{l}133 \mathrm{Cs} \\
133 \mathrm{Cs} \\
133 \mathrm{Bs}\end{array}$ & $\begin{array}{c}p \\
d(E)\end{array}$ & $\begin{array}{l}8.3+0 \\
9.1+0\end{array}$ & $\begin{array}{l}2.2+1 \\
2.2+1\end{array}$ & $\begin{array}{l}\text { FE! } \\
\text { FEI }\end{array}$ & $\begin{array}{l}\text { E Omitrlev+ } \\
\text { E Dmitritevt }\end{array}$ & $\begin{array}{l}4100+ \\
5767+\end{array}$ \\
\hline $\begin{array}{l}133 \mathrm{Cs} \\
133 \mathrm{Cs} \\
133 \mathrm{Cs}\end{array}$ & $\begin{array}{ll}p & 8 \\
p & 1 \\
p & 1\end{array}$ & $\begin{array}{l}8 \cdot 0+0 \\
1.2+1 \\
1.2+1\end{array}$ & $1.1+1$ & $\begin{array}{l}\text { CCP F } \\
\text { KFK } \\
\text { ORL }\end{array}$ & $\begin{array}{l}\text { R Zatolohint } \\
\text { C Muenzel+ } \\
\text { E Blossert }\end{array}$ & $\begin{array}{l}4097 \\
4098 \\
4099\end{array}$ \\
\hline 133.1980 & - ratio & $O \sigma(E)$ & & & & \\
\hline $\begin{array}{l}133 \mathrm{Cs} \\
133 \mathrm{Cs}\end{array}$ & $\begin{array}{lll}\mathbf{p} & \mathbf{E} \\
\mathbf{d} & \mathrm{S} & \end{array}$ & $\begin{array}{l}8.3+0 \\
9.1+0\end{array}$ & $\begin{array}{l}2.2+1 \\
2.2+1\end{array}$ & $\begin{array}{l}\text { FEI } \\
\text { FEI }\end{array}$ & $\begin{array}{l}\text { E Daitrievt } \\
\text { E Daitrievt }\end{array}$ & $\begin{array}{l}4101 \\
5768\end{array}$ \\
\hline 135.80 & Indeper & indent & $O(E)$ & & & \\
\hline $\begin{array}{l}238_{0} \\
135.80\end{array}$ & thich & $\begin{array}{l}1.0+3 \\
\text { torgot }\end{array}$ & $\begin{array}{l}3.0+5 \\
t y=1 d\end{array}$ & KFK C & C Muenzelt+ & 4906 \\
\hline $\begin{array}{l}133 \mathrm{Cs} \\
139 \mathrm{La}\end{array}$ & $\stackrel{0}{\circ}$ & $\begin{array}{l}1.1+1 \\
1.4+1\end{array}$ & $\begin{array}{l}2.1+1 \\
2.2+1\end{array}$ & FEI & $\begin{array}{l}\text { E Omilrient } \\
\text { E Domitrievt }\end{array}$ & $\begin{array}{l}8389 \\
4174\end{array}$ \\
\hline 135000 & lhlch & target & t yield & & & \\
\hline $139 \mathrm{La}$ & p & $1.4+1$ & $2.2+1$ & $\mathrm{CCP}$ & E Omitrlyevt & 4173 \\
\hline${ }^{136} \mathrm{Ba}$ & direct-1 & -intero & oction & O(E) & & \\
\hline $139 \mathrm{La}$ & p & $2.0+1$ & & MIL $r$ & M MIlazzo-Coll1+ & 4177 \\
\hline${ }^{136} \mathrm{Ba}$ & portial & $O(E)$ & & & & \\
\hline $\begin{array}{l}139 \mathrm{La} \\
136 \mathrm{Ba}\end{array}$ & $\stackrel{p}{\rho(E)}$ & $2.0+1$ & & MIL $N$ & M Milszzo-Coll1+ & 4176 \\
\hline $\begin{array}{l}139_{\mathrm{La}} \\
138_{\mathrm{Ba}}\end{array}$ & cumulat & $\begin{array}{l}\dot{2} .0+1 \\
\text { ive } 11\end{array}$ & ission. & $\begin{array}{l}\text { MIL } \\
y 1010\end{array}$ & M Milazzo-Collit & 4175 \\
\hline $\begin{array}{l}232 \mathrm{Ih} \\
233_{\mathrm{U}}\end{array}$ & d & $\begin{array}{l}9.3+0 \\
9.0+0\end{array}$ & $\begin{array}{l}1.4+1 \\
2.3+1\end{array}$ & $\begin{array}{l}\text { CCP F } \\
\text { CCP P }\end{array}$ & $\begin{array}{l}R \text { Lbout } \\
R \text { Lbout }\end{array}$ & $\begin{array}{l}5921 \\
5936\end{array}$ \\
\hline
\end{tabular}

\begin{tabular}{|c|c|c|c|c|c|c|}
\hline Target & $\operatorname{lnc}$ & $\begin{array}{l}\text { Emin } \\
\text { (MeV) }\end{array}$ & $\begin{array}{l}\text { Emax } \\
(M e V)\end{array}$ & Lab W & Author & No. \\
\hline $13^{39} \mathrm{Ba}$ & $O(E) x$ & factor & & & & \\
\hline $\begin{array}{l}232 \mathrm{Th} \\
23 \theta_{\mathrm{U}} \\
239 \mathrm{Pu}\end{array}$ & $\begin{array}{l}p \\
p \\
p\end{array}$ & $\begin{array}{l}5 \cdot 0+0 \\
5 \cdot 0+0 \\
5 \cdot 0+0\end{array}$ & $\begin{array}{l}1.0+2 \\
1.0+2 \\
1.0+2\end{array}$ & $\begin{array}{l}\text { CCP } R \\
\text { CCP R } \\
\text { CCP } R\end{array}$ & $\begin{array}{l}R \text { Lbovt } \\
R \text { Lbovt } \\
R \text { Lbout }\end{array}$ & $\begin{array}{l}4794 \\
4890 \\
4933\end{array}$ \\
\hline $140 \mathrm{Bo}$ & cumu 1 & ative fl & ission & $y+010$ & & \\
\hline $\begin{array}{l}232 \mathrm{Th} \\
233 \mathrm{U} \\
23 \mathrm{NP}\end{array}$ & $\begin{array}{l}\text { d } \\
\text { d }\end{array}$ & $\begin{array}{l}9.3+0 \\
9 \cdot 0+0 \\
2 \cdot 0+1\end{array}$ & $\begin{array}{l}1.4+1 \\
2.3+1 \\
4.1+1\end{array}$ & $\begin{array}{l}\text { CCP R } \\
\text { CCP R } \\
\text { CCP R }\end{array}$ & $\begin{array}{l}R \text { Lbovt } \\
R \text { Lbovt } \\
R \text { Lbovt }\end{array}$ & $\begin{array}{l}5921 \\
5938 \\
9091\end{array}$ \\
\hline 1400 & cumul & ottive ol & (E) & & & \\
\hline $\begin{array}{l}230^{\circ} \\
14^{0} \mathrm{Oo}\end{array}$ & $\begin{array}{c}p \\
O(E)\end{array}$ & $1.0+3$ & $3.0+5$ & KFK C & C Muenzel+ & 4903 \\
\hline $\begin{array}{l}136 \times \mathrm{e} \\
z=57\end{array}$ & $v_{n}^{0}$ & $2.0+1$ & & GOE E & E Kantust & 8388 \\
\hline $\begin{array}{r}\text { Ag } \\
129_{\mathrm{LO}}\end{array}$ & $\begin{array}{l}40_{A C} \\
\text { produ }\end{array}$ & $\begin{array}{l}3.4+2 \\
\text { cet } y 1010\end{array}$ & & PAR $R$ & R Lefortt & 12202 \\
\hline $\begin{array}{l}1{ }^{10} \mathrm{Ba} \\
13{ }^{13} \mathrm{La}\end{array}$ & $\begin{array}{c}\text { p } \\
\text { produ }\end{array}$ & $\begin{array}{l}5.0+0 \\
\text { ct } y 1010\end{array}$ & $3.0+1$ & MCG E & E Brodeurt & 4152 \\
\hline $\begin{array}{l}133 \mathrm{Cs} \\
134 \mathrm{Lo}\end{array}$ & $\begin{array}{c}{ }^{3} \mathrm{He} \\
\text { thlck }\end{array}$ & $\begin{array}{l}\text { NDG } \\
\text { targot }\end{array}$ & $y / 01 d$ & TOK E & E Chibat & 5472 \\
\hline $\begin{array}{l}134 \mathrm{Bo} \\
136 \mathrm{Lo}\end{array}$ & $\begin{array}{c}p \\
\text { thlck }\end{array}$ & $\begin{array}{r}4.5+0 \\
\text { torgot }\end{array}$ & $\begin{array}{l}6.3+0 \\
\text { yield }\end{array}$ & CLU M & M Roughtont & 4153 \\
\hline $\begin{array}{l}13608 \\
137 \mathrm{Lo}\end{array}$ & $\begin{array}{c}\text { p } \\
\text { direc }\end{array}$ & $\begin{array}{r}3.9+0 \\
t-1 \text { ntera }\end{array}$ & $\begin{array}{l}6.3+0 \\
\text { action }\end{array}$ & $\begin{array}{l}\text { CLU M } \\
O(E)\end{array}$ & M Roughtont & 4154 \\
\hline $\begin{array}{l}140 \mathrm{Ce} \\
137 \mathrm{LO}\end{array}$ & $\begin{array}{c}p \\
\text { porti }\end{array}$ & $\begin{array}{r}2.0+1 \\
.1 \quad 0(E)\end{array}$ & & MIL M & M Milozzo-Collit+ & 4201 \\
\hline $\begin{array}{l}140 \mathrm{Ce} \\
137 \mathrm{Lo}\end{array}$ & $\begin{array}{c}p \\
\sigma(E)\end{array}$ & $2.0+1$ & 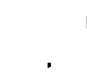 & MIL M & Milazzo-Collit & 4200 \\
\hline $14^{\circ} \mathrm{Co}$ & $p$ & $2.0+1$ & & MIL M & M Milozzo-Collit & 4199 \\
\hline
\end{tabular}




\begin{tabular}{|c|c|c|c|c|c|c|}
\hline Target & $\begin{array}{r}\ln c \\
\quad c \\
\end{array}$ & $\begin{array}{l}\text { Emin } \\
(\mathrm{MeV})\end{array}$ & $\begin{array}{l}\text { Emax } \\
\text { (MeV) }\end{array}$ & Lab W & Author & No. \\
\hline $139_{L}$ & \multicolumn{6}{|c|}{ partlal thich target yleldy } \\
\hline $139_{L a}$ & $p$ & $4.0+0=$ & $5.5+3$ & INDE & Kulharnit & 4178 \\
\hline $140 \mathrm{Ls}$ & \multicolumn{6}{|c|}{ compound-nucleus $g(E)$} \\
\hline $138 \mathrm{Bt}$ & d & $5.0+0$ & $1.6+1$ & \multicolumn{2}{|c|}{ GOE E Kantus+ } & \multirow[t]{2}{*}{5770} \\
\hline $140 \mathrm{Lo}$ & \multicolumn{3}{|c|}{ cunviative figsion } & yield & & \\
\hline $237_{\mathrm{Np}}$ & $a$ & $2.0+14$ & $4.1+10$ & CCP R & Lbovt & 9091 \\
\hline $140 \mathrm{Lg}$ & \multicolumn{6}{|l|}{$O(E)$} \\
\hline $13 \theta_{\mathrm{Ba}}$ & d & $8.0+0$ & $2.6+i c$ & GOE E & Kontust & 5769 \\
\hline$z=58$ & \multicolumn{6}{|l|}{$v_{n}$} \\
\hline hg & $40 \mathrm{Ar}$ & $3.4+2$ & - $F$ & PAR R & Lefortt & 12202 \\
\hline$z=50$ & \multicolumn{6}{|l|}{$\sigma(E)$} \\
\hline $160 \mathrm{gd}$ & ${ }^{12} \mathrm{C}$ & $9.0+1$ & $2.0+2$ & GRN $E$ & Sloek-WIIczyngh & 10051 \\
\hline $129 \mathrm{Co}$ & \multicolumn{6}{|c|}{ relative 1 , } \\
\hline $116 \varepsilon_{n}$ & 160 & $5.5+1 \varepsilon$ & $8.5+1$ & BRK E & Gizont & 11355 \\
\hline $131 \mathrm{Co}$ & \multicolumn{6}{|c|}{ relative 1 , } \\
\hline $1185 n$ & 160 & $5.5+1 \varepsilon$ & $8.5+1$ & BRK E & Gizont & 11358 \\
\hline${ }^{131} \mathrm{Ce}$ & \multicolumn{6}{|l|}{ O(E) } \\
\hline $130 \mathrm{Te}$ & ${ }^{13} c$ & $1.3+2$ & $1.4+2$ & IPS $R$ & Johnson. & 10208 \\
\hline $132 c_{\theta}$ & \multicolumn{6}{|l|}{$O(E)$} \\
\hline $\begin{array}{l}130 \mathrm{Te} \\
139 \mathrm{Lg}\end{array}$ & $\begin{array}{r}{ }^{13} \mathrm{C} \\
\mathrm{p}\end{array}$ & $\begin{array}{l}1.2+2 \\
\text { NDG }\end{array}$ & $1.4+2$ & $\begin{array}{l}\text { IPS R } \\
\text { CER E }\end{array}$ & $\begin{array}{l}\text { Johnson. } \\
\text { Ravnt }\end{array}$ & $\begin{array}{r}10209 \\
4179\end{array}$ \\
\hline${ }^{133} \mathrm{Ce}$ & \multicolumn{6}{|l|}{$O(E)$} \\
\hline $130 \mathrm{To}$ & ${ }^{13} \mathrm{C}$ & $1.0+2$ & $1.4+2$ & JPS R & Johnson. & 10210 \\
\hline${ }^{134} \mathrm{Co}$ & \multicolumn{6}{|l|}{$\sigma(E)$} \\
\hline $139 \mathrm{Lo}$ & p & NDG & & CER E & Ravnt & 4180 \\
\hline${ }^{135} \mathrm{C}$ & \multicolumn{6}{|l|}{$\theta(E)$} \\
\hline $130 \mathrm{~T}$ & ${ }^{13} \mathrm{C}$ & $7.8+1$ & $1.4+2$ & IPS R & Johnson. & 10211 \\
\hline
\end{tabular}

\begin{tabular}{|c|c|c|c|c|c|c|}
\hline Target & Inc & $\begin{array}{l}\text { Emin } \\
(\mathrm{MeV})\end{array}$ & $\begin{array}{l}\text { Emax } L \\
(\mathrm{MeV})\end{array}$ & Lab W & Author & No. \\
\hline${ }^{136} \mathrm{Ce}$ & \multicolumn{6}{|c|}{ relotive $I_{y}$} \\
\hline $136 \mathrm{Ba}$ & a & $6.1+1$ & & JUL E & Muller-lieggiant & 8391 \\
\hline${ }^{136} \mathrm{C}_{\theta}$ & \multicolumn{6}{|l|}{$\sigma(E)$} \\
\hline $139 \mathrm{La}$ & p & NDG & & CER E & Raynt & 4181 \\
\hline${ }^{137} \mathrm{Ce}$ & \multicolumn{6}{|c|}{ relative $I_{y}$} \\
\hline $\begin{array}{l}13 \theta_{\mathrm{Ba}} \\
138_{8 \mathrm{a}} \\
138_{\mathrm{Ba}}\end{array}$ & $\begin{array}{l}{ }^{3} \mathrm{He} \\
a \\
0\end{array}$ & $\begin{array}{l}3.8+1 \\
4.9+1 \\
7.0+1\end{array}$ & $5.9+1$ & $\begin{array}{l}\text { IKOE } \\
\text { IKO E } \\
\text { JUL E }\end{array}$ & $\begin{array}{l}\text { Ludzlejeoshit } \\
\text { Ludziejeughit } \\
\text { Muller-Veggiant }\end{array}$ & $\begin{array}{l}6473 \\
8392 \\
8393\end{array}$ \\
\hline${ }^{137} \mathrm{Ce}_{\theta}$ & \multicolumn{6}{|l|}{$O(E)$} \\
\hline $\begin{array}{l}130 \mathrm{Je} \\
130 \mathrm{Ce}\end{array}$ & \multicolumn{5}{|c|}{ relative Ir } & 10212 \\
\hline $\begin{array}{l}13 \theta_{\mathrm{Ba}} \\
13 \theta_{\mathrm{Ba}}\end{array}$ & $a$ & $\begin{array}{l}4.5+1 \\
7.0+1\end{array}$ & $5.9+1$ & $\begin{array}{l}\text { IKO E } \\
\text { JUL E }\end{array}$ & $\begin{array}{l}\text { Ludzielenshit } \\
\text { Euller-Vgggiant }\end{array}$ & $\begin{array}{l}8394 \\
8395\end{array}$ \\
\hline${ }^{139} \mathrm{Ce}$ & \multicolumn{6}{|c|}{ Including molostable o(E) } \\
\hline $\begin{array}{l}139 \mathrm{La} \\
139 \mathrm{La} \\
139 \mathrm{La}\end{array}$ & $\begin{array}{l}p \\
p \\
p\end{array}$ & $\begin{array}{l}4.5+0 \\
4.5+0 \\
3.5+1\end{array}$ & $\begin{array}{l}1.0+1 \\
1.0+1 \\
1.3+2\end{array}$ & $\begin{array}{l}\text { ANLE } \\
\text { KFK E } \\
\text { PAR E }\end{array}$ & $\begin{array}{l}\text { E Wingt } \\
\text { E Muenzedt } \\
\text { E Albouyt }\end{array}$ & $\begin{array}{l}4183+ \\
4184 \\
4185 .\end{array}$ \\
\hline $139 \mathrm{Ce}$ & \multicolumn{6}{|c|}{ reletive I, } \\
\hline $\begin{array}{l}138_{\mathrm{Ba}} \\
139 \mathrm{Ce}\end{array}$ & $\begin{array}{c}a \\
O(E)\end{array}$ & $4.5+1$ & $5.9+1$ & IKO E & E ludzieieoshit & 8398 \\
\hline $\begin{array}{l}130 \mathrm{Te} \\
139 \mathrm{La}\end{array}$ & $\begin{array}{r}{ }^{13} \mathrm{c} \\
\mathrm{p}\end{array}$ & $\begin{array}{l}4 \cdot 0+1 \\
8 \cdot 0+0\end{array}$ & $\begin{array}{l}0.5+1 \\
3.7+1\end{array}$ & $\begin{array}{l}\text { IPS R } \\
\text { COL R }\end{array}$ & $\begin{array}{l}\text { R Johnsos. } \\
\text { R Miller. }\end{array}$ & $\begin{array}{r}10213 \\
4182\end{array}$ \\
\hline $140 \mathrm{Co}$ & \multicolumn{6}{|c|}{ partiai relative o(E) } \\
\hline $\begin{array}{l}138 \mathrm{Bo} \\
141 \mathrm{Ce}\end{array}$ & $\begin{array}{c}a \\
\text { cuoul }\end{array}$ & $\begin{array}{l}1.6+1 \\
\text { tive f } 1\end{array}$ & \multicolumn{4}{|c|}{ cunoulative figsion yield } \\
\hline $\begin{array}{l}232 \mathrm{Th} \\
237_{\mathrm{Np}}\end{array}$ & d & $\begin{array}{l}9.3+0 \\
2.0+1\end{array}$ & $\begin{array}{l}1.4+1 \\
4.1+1\end{array}$ & $\begin{array}{l}\mathrm{CCP} R \\
\mathrm{CCP} R\end{array}$ & $\begin{array}{l}R \text { Lbovt } \\
R \text { Lbovt }\end{array}$ & $\begin{array}{l}5921 \\
9091\end{array}$ \\
\hline $141 \mathrm{Co}$ & \multicolumn{6}{|c|}{ Dortlal relalive o(E) } \\
\hline $13 \theta_{B a}$ & $\circ$ & $1.6+1$ & $2.1+1$ & JYV E & E Kownackit & 8398 \\
\hline
\end{tabular}




\begin{tabular}{|c|c|c|c|c|c|}
\hline Target & Inc & $\begin{array}{ll}\text { Emin } & \text { Emax } \\
(M e V) & (M e V)\end{array}$ & Lab & Author & No. \\
\hline $141 \mathrm{Ce}$ & \multicolumn{5}{|c|}{ flssion yioldafoctor } \\
\hline $2{ }^{48} \mathrm{Cm}$ & $a$ & $2.4+1 \quad 3.4+1$ & KUR & E Lisint & 9117 \\
\hline $141 \mathrm{Ce}$ & \multicolumn{5}{|l|}{$O(E)$} \\
\hline $139 \mathrm{LB}$ & d & $2.3+1$ & \multicolumn{2}{|c|}{ GOE E Kantust } & \multirow[t]{2}{*}{5771} \\
\hline $143 \mathrm{Co}$ & \multicolumn{2}{|c|}{ cumulative flgalon } & $y|e|$ & & \\
\hline $\begin{array}{l}232 \mathrm{Th} \\
237_{\mathrm{Np}}\end{array}$ & d & $\begin{array}{ll}9.3+0 & 1.4+1 \\
2.0+1 & 4.1+1\end{array}$ & $\begin{array}{l}\text { CCP } \\
\text { CCP }\end{array}$ & $\begin{array}{l}\text { R Lbovt } \\
R \text { Lbovt }\end{array}$ & $\begin{array}{l}5921 \\
9091\end{array}$ \\
\hline $14^{3} \mathrm{Ce}$ & \multicolumn{5}{|c|}{ fission yieldxfoctor } \\
\hline $24{ }^{8} \mathrm{Cm}$ & a & $2.4+13.4+1$ & KUR & E Lisint & 9117 \\
\hline${ }^{144} \mathrm{Co}$ & \multicolumn{2}{|c|}{ cumulative figsion } & \multicolumn{2}{|c|}{ yield } & \\
\hline $232 \mathrm{Th}$ & d & $9.3+0 \quad 1.4+1$ & $\mathrm{CCP}$ & R Lbovt & 5921 \\
\hline${ }^{144} \mathrm{Ce}$ & \multicolumn{5}{|c|}{ fission yleldxfactor } \\
\hline $\begin{array}{l}2{ }^{48} \mathrm{Cm} \\
1{ }^{46} \mathrm{Ce}\end{array}$ & curaul & \multicolumn{3}{|c|}{ cunalative $O(E)$} & 9117 \\
\hline $23 \theta_{U}$ & p & $3 . \overline{5}+18.5+1$ & MCG & E Galiniert & 4916 \\
\hline Ia'pr & \multicolumn{5}{|c|}{ product yleld } \\
\hline${ }^{96} \mathrm{Ru}$ & 325 & NDG & BRK & R Cerny+ & 12034 \\
\hline $138 \mathrm{pr}$ & \multicolumn{5}{|c|}{ partiol product yield } \\
\hline $139 \mathrm{LB}$ & $a$ & NEG & MSU & E Aryaelnejadt & 8400 \\
\hline $14: \mathrm{Pr}$ & \multicolumn{5}{|c|}{ relative Iy } \\
\hline $139 \mathrm{L \theta}$ & $a$ & $2.4+1$ & OSA & E Nagait & 8401 \\
\hline $141 \mathrm{Pr}$ & \multicolumn{5}{|l|}{$O(E)$} \\
\hline $141 \mathrm{Pr}$ & ${ }^{12} \mathrm{C}$ & $7.5+1 \quad 1.0+2$ & GRE & E Jastrzebshit & 10009 \\
\hline $142 \mathrm{Pr}$ & \multicolumn{5}{|c|}{ Including metastable? o(E) } \\
\hline $\begin{array}{l}142 \mathrm{Ce} \\
142 \mathrm{C \theta}\end{array}$ & $p$ & $\begin{array}{l}1.2+1 \\
1.2+1\end{array}$ & $\begin{array}{l}\text { KFK } \\
\text { ORL }\end{array}$ & $\begin{array}{l}\text { C Muenzelt } \\
\text { E Blossert }\end{array}$ & $\begin{array}{l}4202 \\
4203+\end{array}$ \\
\hline $142 \mathrm{pr}$ & \multicolumn{5}{|c|}{ cumulatize fission yleld } \\
\hline $237_{\mathrm{Np}}$ & $a$ & $2 \cdot 0+1 \quad 4 \cdot 1+1$ & CCP & R Lbovt & 9091 \\
\hline
\end{tabular}

\begin{tabular}{|c|c|c|c|c|c|c|}
\hline$\overline{\text { Target }}$ & $\operatorname{Inc}$ & $\begin{array}{l}\text { Emin } \\
(\mathrm{MeV})\end{array}$ & $\begin{array}{l}\text { Emax } \\
(\operatorname{MeV})\end{array}$ & Lob & Author & No. \\
\hline$+{ }^{3} \mathrm{Pr}$ & $O(E)$ & & & & & \\
\hline $\begin{array}{l}139 \mathrm{La} \\
142 \mathrm{Ce}\end{array}$ & a & $\begin{array}{l}1.6+1 \\
2.0+1\end{array}$ & & $\begin{array}{l}\text { GOE } \\
\text { GOE }\end{array}$ & $\begin{array}{l}\text { E Kantust } \\
\text { E Kantust }\end{array}$ & $\begin{array}{l}8402 \\
4204\end{array}$ \\
\hline $145 \mathrm{Pr}$ & cumule & tive $f$ & ission & yiol & & \\
\hline $\begin{array}{l}237 \mathrm{~Np} \\
146 \mathrm{Pr}\end{array}$ & indepe & $\begin{array}{l}2.0+1 \\
\text { indent }\end{array}$ & $\begin{array}{l}4.1+1 \\
0(E)\end{array}$ & $\mathrm{CCP}$ & R Lbovt & 9091 \\
\hline $\begin{array}{l}238 \mathrm{U} \\
146 \mathrm{Pr}\end{array}$ & $\begin{array}{c}p \\
\text { cumule }\end{array}$ & $\begin{array}{l}3.5+1 \\
\text { tive f }\end{array}$ & $\begin{array}{l}8.5+1 \\
155 \operatorname{lon}\end{array}$ & $\begin{array}{l}\text { MCG } \\
y|\in|\end{array}$ & $\begin{array}{l}\text { E Galiniert } \\
\text { Id }\end{array}$ & 4921 \\
\hline $\begin{array}{l}238 \mathrm{U} \\
146 \mathrm{Pr}\end{array}$ & $\underset{\text { cumul }}{p}$ & $\begin{array}{l}2.0+1 \\
\text { tive o }\end{array}$ & $\begin{array}{l}8.5+1 \\
(E)\end{array}$ & MCG & E Galiniert & 4912 \\
\hline $\begin{array}{l}238_{\mathrm{U}} \\
147 \mathrm{Pr}\end{array}$ & $\begin{array}{c}p \\
O(E)\end{array}$ & $3 \cdot 5+1$ & $8.5+1$ & MCG & E Galiniert & 4916 \\
\hline $\begin{array}{l}150 \mathrm{NJ} \\
150_{\mathrm{Nd}}\end{array}$ & $\begin{array}{l}\mathbf{p} \\
\mathbf{p}\end{array}$ & $\begin{array}{l}1.8+1 \\
1.8+1\end{array}$ & & $\begin{array}{l}\text { KFK } \\
\text { MIL }\end{array}$ & $\begin{array}{l}\text { C Muenzel+ } \\
\text { M Milazzo-Collit }\end{array}$ & $\begin{array}{l}4248 \\
4249+\end{array}$ \\
\hline $129_{\mathrm{Nd}}$ & produc & $t y \in 01$ & & & & \\
\hline $102 \mathrm{Pd}$ & 325 & NDG & & BRK & R Cernyt & 12040 \\
\hline $131 \mathrm{Nd}$ & produc & i yiel & & & & \\
\hline $\begin{array}{l}102 P d d \\
138_{\mathrm{Nd}}\end{array}$ & $\begin{array}{l}32 S \\
\sigma(E)\end{array}$ & NDG & & BRK & R Cernyt & 12041 \\
\hline $\begin{array}{l}141 \mathrm{Pr} \\
141 \mathrm{Pr}\end{array}$ & $\begin{array}{l}p \\
p\end{array}$ & $\begin{array}{l}3.8+1 \\
3.8+1\end{array}$ & $\begin{array}{l}8.5+1 \\
8.5+1\end{array}$ & $\begin{array}{l}\text { KFK } \\
\text { MCG }\end{array}$ & $\begin{array}{l}\text { C Muenzel+ } \\
\text { M Hogon. }\end{array}$ & $\begin{array}{l}4206 \\
4207+\end{array}$ \\
\hline $139_{\mathrm{Nd}}$ & relot & ve $l_{y}$ & & & & \\
\hline $\begin{array}{l}128 \mathrm{Te} \\
140 \mathrm{Ce} \\
140 \mathrm{Ce}\end{array}$ & $\begin{array}{c}16 \mathrm{O} \\
{ }^{3} \mathrm{He} \\
a\end{array}$ & $\begin{array}{l}9.0+1 \\
3.8+1 \\
5.2+1\end{array}$ & $5.9+1$ & $\begin{array}{l}\text { GRE } \\
\text { IKO } \\
\text { IKO }\end{array}$ & $\begin{array}{l}\text { E Gizont } \\
\text { E Ludzlejeoshit } \\
\text { E Ludzlejeyshit }\end{array}$ & $\begin{array}{r}11371 \\
6474 \\
8407\end{array}$ \\
\hline $1389 \mathrm{Nd}$ & $\sigma(E)$ & & & & & \\
\hline $\begin{array}{l}141 \mathrm{Pr} \\
141 \mathrm{Pr}\end{array}$ & $\begin{array}{l}p \\
p\end{array}$ & $\begin{array}{l}2.5+1 \\
2.5+1\end{array}$ & $\begin{array}{l}8.5+1 \\
8.5+1\end{array}$ & $\begin{array}{l}\text { KFK } \\
\text { MCG }\end{array}$ & $\begin{array}{l}\text { C Muenzel+ } \\
\text { M Hogan. }\end{array}$ & $\begin{array}{l}4208 \\
4209+\end{array}$ \\
\hline $139 . \mathrm{Nd}$ & $\sigma(E)$ & & & & & \\
\hline $110 \mathrm{Pd}$ & ${ }^{40}{ }_{\mathrm{Ar}}$ & $1.9+2$ & $2.0+2$ & PAR & E Della-Negrat & $12 ! 84$ \\
\hline
\end{tabular}




\begin{tabular}{|c|c|c|c|c|c|c|}
\hline Target & $\ln c$ & $\begin{array}{l}\text { Emin } \\
(M e V)\end{array}$ & $\begin{array}{l}\text { Emax } \\
(\mathrm{MeV})\end{array}$ & Lab r & Author & No. \\
\hline $39 \cdot \mathrm{Nd}$ & $\sigma(E)$ & & & & & \\
\hline $\begin{array}{l}134 \mathrm{Ba} \\
141 \mathrm{Pr} \\
141 \mathrm{Pr}\end{array}$ & $\begin{array}{r}160 \\
p \\
p\end{array}$ & $\begin{array}{l}1.0+2 \\
2.5+18 \\
2.5+18\end{array}$ & $\begin{array}{l}1.2+2 \\
8.5+1 \\
8.5+1\end{array}$ & $\begin{array}{l}\text { PAR } \\
\text { KFK } \\
\text { MCG }\end{array}$ & $\begin{array}{l}\text { E Della-Negrat } \\
\text { C Muenzelt } \\
\text { M Hogan. }\end{array}$ & $\begin{array}{l}11373 \\
4210 \\
4211\end{array}$ \\
\hline $1390.9 \mathrm{~N}$ & sum & matlon & $O(E)$ & & & \\
\hline $\begin{array}{l}141 \mathrm{Pr} \\
141 \mathrm{Pr}\end{array}$ & p & $\begin{array}{l}2.5+18 \\
2.5+1 \varepsilon\end{array}$ & $\begin{array}{l}8.5+1 \\
8 \cdot 5+1\end{array}$ & $\begin{array}{l}\text { KFK } \\
\text { MCG }\end{array}$ & $\begin{array}{l}\text { C Muenzel+ } \\
\text { M Hogan. }\end{array}$ & $\begin{array}{l}4212 \\
4213+\end{array}$ \\
\hline \multicolumn{7}{|c|}{ 139.19Nd rotlo O(E) } \\
\hline $\begin{array}{l}141 \mathrm{Pr} \\
141 \mathrm{Pr}\end{array}$ & $p$ & $\begin{array}{l}2.5+18 \\
2.5+18\end{array}$ & $\begin{array}{l}8.5+1 \\
8.5+1\end{array}$ & $\begin{array}{l}\text { KFK } \\
\text { MCG }\end{array}$ & $\begin{array}{l}\text { C Muenzel+ } \\
\text { M Hogan. }\end{array}$ & $\begin{array}{l}4214 \\
4215+\end{array}$ \\
\hline $140 \mathrm{Nd}$ & reloti & vo I, & & & & \\
\hline $\begin{array}{l}1{ }^{100} \mathrm{Ce} \\
1{ }^{140} \mathrm{Nd}\end{array}$ & $\begin{array}{c}0 \\
O(E)\end{array}$ & $4.5+1=$ & $5.9+1$ & IKO E & E Lodziejexshit & 408 \\
\hline $\begin{array}{l}141 \mathrm{Pr} \\
141 \mathrm{Pr}\end{array}$ & $\begin{array}{l}p \\
p\end{array}$ & $\begin{array}{l}9.0+0 \\
9.0+0\end{array}$ & $\begin{array}{l}8.5+1 \\
8.5+1\end{array}$ & $\begin{array}{l}\text { KFK } \\
\text { MCG }\end{array}$ & $\begin{array}{l}\text { C Muenzel+ } \\
\text { M Hogan. }\end{array}$ & $\begin{array}{l}4216 \\
4217+\end{array}$ \\
\hline $141 \mathrm{Nd}$ & incluod & $\operatorname{lng}$ meto & lastobl & 10018 & (E) & \\
\hline $\begin{array}{l}141 \mathrm{Pr} \\
141 \mathrm{Pr}\end{array}$ & $\begin{array}{l}\mathbf{p} \\
\mathbf{p}\end{array}$ & $\begin{array}{l}9.0+0 \\
9.0+0\end{array}$ & $\begin{array}{l}3 \cdot 8+1 \\
3 \cdot 8+1\end{array}$ & $\begin{array}{l}\text { KFK } \\
\text { MCG }\end{array}$ & $\begin{array}{l}\text { C Muenzel+ } \\
\text { M Hogon. }\end{array}$ & $\begin{array}{l}4218 \\
4219 .\end{array}$ \\
\hline $141 \mathrm{Nd}$ & rolatl & ve Iy & & & & \\
\hline $\begin{array}{l}140 \mathrm{Ce} \\
140 \mathrm{Ce} \\
142 \mathrm{Ce}\end{array}$ & $\begin{array}{l}\circ \\
\vdots \\
\circ\end{array}$ & $\begin{array}{l}2.8+1 \\
4.5+15 \\
4.5+1\end{array}$ & $\begin{array}{l}4 \cdot 0+1 \\
5 \cdot 9+1 \\
5 \cdot 9+1\end{array}$ & $\begin{array}{l}\text { IPS } \\
\text { IKO } \\
\text { IKO }\end{array}$ & $\begin{array}{l}\text { E Ludzlejewshit } \\
\text { ELdziejeoshit } \\
\text { E Ludzlejeoshit }\end{array}$ & $\begin{array}{l}8409 \\
8410 \\
8413\end{array}$ \\
\hline $141 . \mathrm{Nd}$ & produ & ct $y 1010$ & & & & \\
\hline $\begin{array}{l}142_{N d} \\
142_{N d}\end{array}$ & $\stackrel{p}{r 01011}$ & $\begin{array}{l}3.5+1 \text { e } \\
\text { ve I, }\end{array}$ & $6.0+1$ & MCG E & E Deslaurierst & 4221 \\
\hline $\begin{array}{l}140 \mathrm{Ce} \\
140 \mathrm{Ce}\end{array}$ & $a$ & $\begin{array}{l}2.8+1 \\
4.5+1\end{array}$ & $\begin{array}{l}4.0+1 \\
5.9+1\end{array}$ & IPS & $\begin{array}{l}\text { E Ludzlejeuskit } \\
\text { E Ludziejeuskit }\end{array}$ & $\begin{array}{l}841 ! \\
8412\end{array}$ \\
\hline $144 \mathrm{No}$ & reloti & ve $I_{y}$ & & & & \\
\hline $\begin{array}{l}142 \mathrm{Ce} \\
147 \mathrm{Nd}\end{array}$ & $\begin{array}{c}a \\
\text { cumula }\end{array}$ & $\begin{array}{l}2.2+1 \\
t i v e f 1\end{array}$ & $\begin{array}{l}2.7+1 \\
155100\end{array}$ & $\begin{array}{l}\text { ROS } \\
y / e 1\end{array}$ & $\begin{array}{l}\text { E Berzint } \\
\text { Id }\end{array}$ & 8414 \\
\hline $233 \mathrm{U}$ & 8 & $9.0+0$ & $2 \cdot 3+1$ & $\operatorname{CCP}$ & R Lbove & 5936 \\
\hline
\end{tabular}

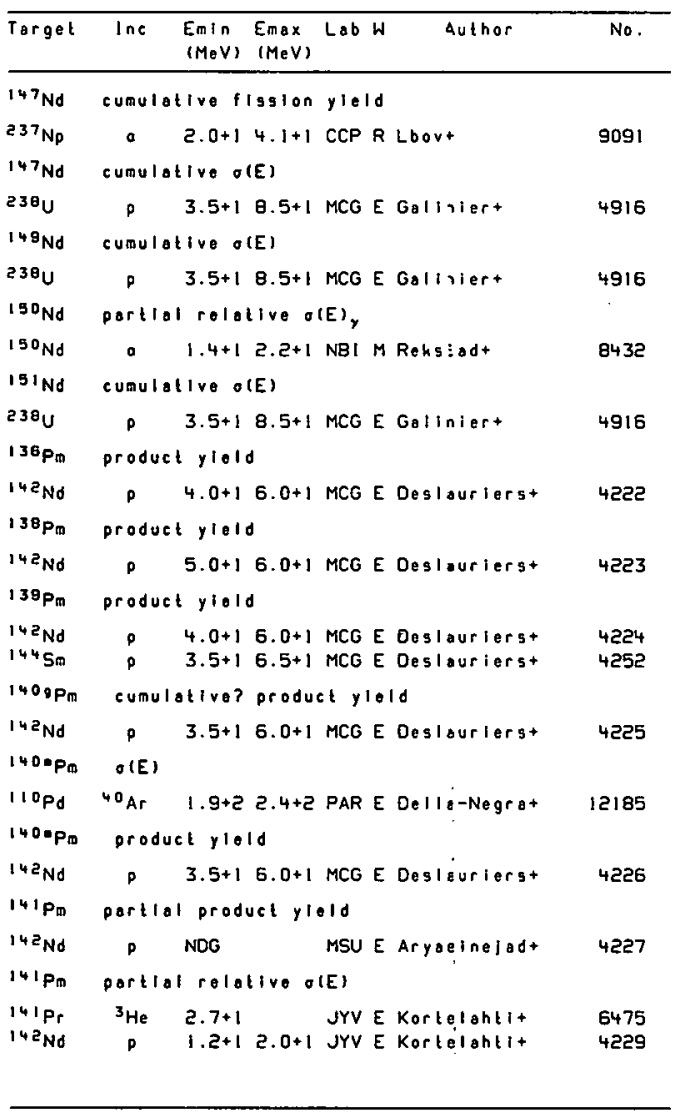




\begin{tabular}{|c|c|c|c|c|c|c|}
\hline Target & $\ln c$ & $\begin{array}{l}\text { Emin } \\
(\operatorname{MeV})\end{array}$ & $\begin{array}{l}\text { Emax } \\
(M e V)\end{array}$ & Lab & Author & No. \\
\hline $141 \mathrm{Pm}$ & \multicolumn{6}{|c|}{ product yleld } \\
\hline $142 \mathrm{Nd}$ & $p$ & $3.5+1$ & $6.0+1$ & MCG & E Deslaurierst & 4228 \\
\hline $142 \mathrm{Pm}$ & \multicolumn{6}{|c|}{ reletive 1 y } \\
\hline $\begin{array}{l}145 \mathrm{Nd} \\
14 \mathrm{a}^{\mathrm{Nd}}\end{array}$ & $\begin{array}{l}\text { d } \\
\text { p }\end{array}$ & $\begin{array}{l}5.0+0 \\
1.4+1\end{array}$ & $1.0+1$ & $\begin{array}{l}\text { ROS } \\
\text { ROS }\end{array}$ & $\begin{array}{l}\text { E Funket } \\
\text { E Funket }\end{array}$ & $\begin{array}{l}4230 \\
5772\end{array}$ \\
\hline $1{ }^{43} \mathrm{Pm}$ & \multicolumn{6}{|c|}{ portiol relative o(E) } \\
\hline $\begin{array}{l}141 \mathrm{Pr} \\
143 \mathrm{Nd} \\
144 \mathrm{Nd} \\
145 \mathrm{Nd}\end{array}$ & $\begin{array}{l}0 \\
d \\
p \\
p\end{array}$ & $\begin{array}{l}\bar{E} .2+1 \\
1.4+1 \\
1.6+1 \\
1.2+1\end{array}$ & $\begin{array}{l}2.7+1 \\
2.0+1 \\
2.0+1\end{array}$ & $\begin{array}{l}\text { ROS } \\
\text { ROS } \\
\text { JYY } \\
\text { JYV }\end{array}$ & $\begin{array}{l}\text { E Pradet } \\
\text { E Pradet } \\
\text { E Kortelahti+ } \\
\text { E Kortelahti+ }\end{array}$ & $\begin{array}{l}8417 \\
5773 \\
4232 \\
4234\end{array}$ \\
\hline $143 \mathrm{Pm}$ & \multicolumn{6}{|c|}{ relative $I_{y}$} \\
\hline $141 \mathrm{Pr}$ & - & $e .4+1$ & & OSA & E Nagait & 8416 \\
\hline $143 \mathrm{Pm}$ & \multicolumn{6}{|c|}{ thick target yteld } \\
\hline $1{ }^{43} \mathrm{Nd}$ & $p$ & $4.7+0$ & $6.0+0$ & CLU & M Rougtiont & 4231 \\
\hline $144 \mathrm{~Pa}$ & \multicolumn{6}{|c|}{ relative $I_{y}$} \\
\hline $141 \mathrm{Pr}$ & a & $2.4+1$ & & OSA & E Nagait & 8418 \\
\hline $144 \mathrm{Pm}$ & \multicolumn{6}{|c|}{ thlck terget yield } \\
\hline $\begin{array}{l}144 \mathrm{Nd} \\
144 \mathrm{Pm}\end{array}$ & \multicolumn{6}{|l|}{$O(E)$} \\
\hline $\begin{array}{l}147 \mathrm{Sm} \\
147 \mathrm{Sm}\end{array}$ & $\begin{array}{l}p \\
p\end{array}$ & $\begin{array}{l}1.8+1 \\
1.8+1\end{array}$ & & $\begin{array}{l}\text { KFK } \\
\text { MIL }\end{array}$ & $\begin{array}{l}\text { C Muenzel+ } \\
\text { M Milazzo-Coll1+ }\end{array}$ & $\begin{array}{l}4264 \\
4265 .\end{array}$ \\
\hline $1{ }^{45} P \mathbb{m}$ & \multicolumn{6}{|c|}{ pertlal relatlue o(E) } \\
\hline $\begin{array}{l}146 \mathrm{Nd} \\
145 \mathrm{Pm}\end{array}$ & $\stackrel{p}{r e \mid a t l}$ & \multicolumn{4}{|c|}{ relative $I_{y}$} & 4236 \\
\hline $145 \mathrm{Nd}$ & $p$ & $8.0+0$ & & OSA & E NagBi+ & 4235 \\
\hline${ }^{146} \mathrm{Pm}_{\mathrm{D}}$ & lhick & target & $y$ yeld & & & \\
\hline $146 \mathrm{Nd}$ & $p$ & $3.9+0$ & $6.0+0$ & CLU & M Roughtont & 4237 \\
\hline $147 \mathrm{Pm}$ & \multicolumn{3}{|c|}{ diract-interaction } & $O(E)$ & & \\
\hline $150 \mathrm{Sm}$ & p & $2.0+1$ & & MIL & M Milazzo-Collit & 4273 \\
\hline
\end{tabular}

\begin{tabular}{|c|c|c|c|c|c|c|}
\hline Target & $\ln c$ & $\begin{array}{l}\text { Eoin } \\
\text { (MeV) }\end{array}$ & $\begin{array}{l}E_{\max } \\
(\mathrm{MeV})\end{array}$ & Lab & Author & No. \\
\hline $147 \mathrm{Pm}$ & \multicolumn{6}{|c|}{ Dartlal relative o(E) } \\
\hline $14 \mathrm{~B}_{\mathrm{Nd}}$ & ? & $1.2+1$ & $2.0+1$ & JYV E & E Kortelahtit & 4239 \\
\hline $147 \mathrm{Pr}$ & \multicolumn{6}{|c|}{ partial o(E) } \\
\hline $150 \mathrm{Sm}$ & p & $2.0+1$ & & MIL $M$ & M Milazzo-Collit & 4272 \\
\hline $147 P_{\text {田 }}$ & \multicolumn{6}{|c|}{ relolive $l_{y}$} \\
\hline $14 \theta_{N d}$ & p & $1.2+1$ & $2.0+1$ & JYV E & E Korlelahtit & 4238 \\
\hline $147 \mathrm{Pm}$ & \multicolumn{6}{|l|}{$\theta(E)$} \\
\hline $1505 \mathrm{~m}$ & $p$ & $2.0+1$ & & MIL $\mathrm{r}$ & M Milazzo-Collit & 4271 \\
\hline $14 \theta_{\mathrm{Pm}}$ & \multicolumn{6}{|c|}{ eumulative fission ylold } \\
\hline $238^{U}$ & $\rho$ & $2.0+1$ & $8.5+1$ & MCG E & E Galiniert & 4912 \\
\hline $148 \mathrm{Pm}$ & \multicolumn{6}{|c|}{ o(E) a factor } \\
\hline $232 \mathrm{Th}$ & ${ }^{3} \mathrm{He}$ & $2.6+1$ & $4.1+1$ & MUN M & M Aumannt & 6583 \\
\hline $1480 \mathrm{Pm}$ & \multicolumn{6}{|c|}{ independent o(E) } \\
\hline $\begin{array}{l}232 \mathrm{Th} \\
23 \theta_{\mathrm{U}}\end{array}$ & $\stackrel{a}{p}$ & $\begin{array}{l}2.6+1 \\
3.5+1\end{array}$ & $\begin{array}{l}4 \cdot 1+1 \\
8 \cdot 5+1\end{array}$ & $\begin{array}{l}\text { MUN E } \\
\text { MCG E }\end{array}$ & $\begin{array}{l}\text { E Aumannt } \\
\text { E Galiniert }\end{array}$ & $\begin{array}{l}8961+ \\
4921\end{array}$ \\
\hline $1489 \mathrm{Pm}$ & \multicolumn{6}{|c|}{ thich target yleld } \\
\hline $148 \mathrm{Nd}$ & p & $3.9+0$ & $6.0+0$ & CLU $\mathrm{r}$ & M Roughtont & 4242 \\
\hline $1480 \mathrm{~Pa}$ & \multicolumn{6}{|l|}{$O(E)$} \\
\hline $\begin{array}{l}146 \mathrm{Nd} \\
148_{\mathrm{Nd}} \\
148_{\mathrm{Nd}} \\
14 \theta_{\mathrm{Nd}} \\
14 \theta_{\mathrm{Nd}}\end{array}$ & $\begin{array}{l}a \\
p \\
p \\
p \\
d \\
a\end{array}$ & $\begin{array}{l}2.9+1 \\
9.0+0 \\
1.1+1 \\
5.5+0 \\
2.5+1\end{array}$ & $\begin{array}{l}5.9+1 \\
4 \cdot 5+1 \\
4.6+1 \\
5 \cdot 4+1 \\
5 \cdot 7+1\end{array}$ & $\begin{array}{l}\text { MUN E } \\
\text { JUL E } \\
\text { MUN E } \\
\text { MUN E } \\
\text { MUN E }\end{array}$ & $\begin{array}{l}\text { E Aumannt } \\
\text { E Guchelt } \\
\text { E Aumannt } \\
\text { E Aumannt } \\
\text { E Aumannt }\end{array}$ & $\begin{array}{l}8421+ \\
4240 \\
4241+ \\
5774+ \\
8428\end{array}$ \\
\hline $1400 \mathrm{Pm}$ & \multicolumn{6}{|c|}{ Independent $O(E)$} \\
\hline $\begin{array}{l}232 \mathrm{Th} \\
148 . \mathrm{P}_{\mathrm{mg}}\end{array}$ & $\begin{array}{c}\text { a } \\
\text { thick }\end{array}$ & $\begin{array}{l}2.6+1 \\
\operatorname{target}\end{array}$ & $\begin{array}{l}4.1+1 \\
t y 1 e 1 d\end{array}$ & MUN E & E Aumannt & $8962+$ \\
\hline $148_{\mathrm{Nd}}$ & $p$ & $3.9+0$ & $6.0+0$ & CLU $M$ & M Roughtont & 4245 \\
\hline $148 . \mathrm{Pm}$ & $\sigma(E)$ & & & & & \\
\hline $\begin{array}{l}146 \mathrm{Nd} \\
148_{\mathrm{Nd}}\end{array}$ & : & $\begin{array}{l}2.9+1 \\
9.0+0\end{array}$ & $\begin{array}{l}5.9+1 \\
4.5+1\end{array}$ & $\begin{array}{l}\text { MUN E } \\
\text { JU E }\end{array}$ & $\begin{array}{l}\text { E Aumanat } \\
\text { E Guckel+ }\end{array}$ & $\begin{array}{l}8422+ \\
4243\end{array}$ \\
\hline
\end{tabular}




\begin{tabular}{|c|c|c|c|c|c|c|}
\hline Target & $\operatorname{Inc}$ & $\begin{array}{l}\text { Emin } \\
(M e V)\end{array}$ & $\begin{array}{l}\text { Emax } \\
(\mathrm{MeV})\end{array}$ & Lab W & Author & No. \\
\hline $148=P_{m}$ & $O(E)$ & & & & & \\
\hline $\begin{array}{l}14 \theta_{\mathrm{Nd}} \\
148_{\mathrm{Nd}} \\
148_{\mathrm{Nd}}\end{array}$ & $\begin{array}{l}p \\
d \\
\text { d }\end{array}$ & $\begin{array}{l}1.1+1 \\
5.5+0 \\
2.5+1\end{array}$ & $\begin{array}{ll}4.6+1 & 1 \\
5.4+1 & 1 \\
5.7+1 & 1\end{array}$ & $\begin{array}{l}\text { MUN E } \\
\text { MUN E } \\
\text { MUN E }\end{array}$ & $\begin{array}{l}\text { Aumannt } \\
\text { Aumannt } \\
\text { Aumannt }\end{array}$ & $\begin{array}{l}4244+ \\
5775+ \\
8429\end{array}$ \\
\hline $148.19 \mathrm{Pm}$ & ind & dependen & nt rati & $100(E)$ & & \\
\hline $\begin{array}{l}232 \mathrm{Th} \\
148.19 \mathrm{Pm}\end{array}$ & $a^{a}$ rot & $\begin{array}{l}2.6+1 \\
10 \sigma(E)\end{array}$ & $4.1+11$ & MUN E & Aumannt & $8963+$ \\
\hline $\begin{array}{l}146_{\mathrm{Nd}} \\
148_{\mathrm{Nd}} \\
148_{\mathrm{Nd}} \\
148_{\mathrm{Nd}} \\
232 \mathrm{Th}\end{array}$ & $\begin{array}{c}a \\
p \\
p \\
p \\
d \\
{ }^{3} \mathrm{He}\end{array}$ & $\begin{array}{l}2.9+1 \\
8 \cdot 0+0 \\
9.0+0 \\
9.0+0 \\
2.6+1\end{array}$ & $\begin{array}{l}5.9+1 \\
2.2+1 \\
4.5+1 \\
1.8+1 \\
4.1+1\end{array}$ & $\begin{array}{l}\text { MUN E } \\
\text { MUN E } \\
\text { JUL E } \\
\text { MUN E } \\
\text { MUN M }\end{array}$ & $\begin{array}{l}\text { Aumannt } \\
\text { Aumannt } \\
\text { Guckelt } \\
\text { Aumbnnt } \\
\text { Aumannt }\end{array}$ & $\begin{array}{l}8423+ \\
4246+ \\
4247 \\
5776+ \\
6584\end{array}$ \\
\hline $149 \mathrm{Pm}$ & Indepe & indent o & $\sigma(E)$ & & & \\
\hline $\begin{array}{l}238 \mathrm{U} \\
149 \mathrm{Pm}\end{array}$ & $\begin{array}{c}\text { p } \\
\text { cumula }\end{array}$ & $\begin{array}{l}3.5+1 \\
\text { tive } f 1\end{array}$ & $\begin{array}{l}8.5+11 \\
\mid \operatorname{ssion}\end{array}$ & $\begin{array}{l}\text { MCG E } \\
\text { yield }\end{array}$ & Galinlert & 4921 \\
\hline $\begin{array}{l}230 \mathrm{U} \\
149 \mathrm{Pz}\end{array}$ & $\begin{array}{c}\text { p } \\
\text { cumula }\end{array}$ & $\begin{array}{l}\text { eे. } 0+1 \\
\text { tive oll }\end{array}$ & $\begin{array}{l}8.5+11 \\
\text { (E) }\end{array}$ & MCG E & Galinlert & 4912 \\
\hline $\begin{array}{l}230^{2} \mathrm{U} \\
149 \mathrm{Pm}\end{array}$ & $\begin{array}{c}p \\
\text { partia }\end{array}$ & $\begin{array}{l}3.5+1 \\
\text { i relat }\end{array}$ & $\begin{array}{l}8.5+11 \\
\text { tive oll }\end{array}$ & $\begin{array}{l}\text { MCG E } \\
\text { (E) }\end{array}$ & Goliniert & 4916 \\
\hline $\begin{array}{l}150 \mathrm{Ng} \\
149 \mathrm{Pm}\end{array}$ & $\begin{array}{c}p \\
\text { O(E) }\end{array}$ & $1.2+1$ & $1.6+1$ & JYVE & Kortelahtit & 4250 \\
\hline $\begin{array}{l}152 \mathrm{Sn} \\
152 \mathrm{Sn}\end{array}$ & p & $\begin{array}{l}1.8+1 \\
1.8+1\end{array}$ & & $\begin{array}{l}\text { KFK } \\
\text { MIL } \\
M\end{array}$ & $\begin{array}{l}\text { Muenzol+ } \\
\text { Milazzo-Collit }\end{array}$ & $\begin{array}{l}4279 \\
4279+\end{array}$ \\
\hline $190 \mathrm{pn}$ & Indepe & indent 0 & $\sigma(E)$ & & & \\
\hline $\begin{array}{l}230^{2} \mathrm{U} \\
150 \mathrm{Pm}\end{array}$ & $\begin{array}{c}\text { p } \\
\text { cumula }\end{array}$ & $\begin{array}{l}3.5+1 \\
\text { tive fi }\end{array}$ & $\begin{array}{l}8.5+11 \\
\text { ission }\end{array}$ & $\begin{array}{l}\text { MCG E } \\
\text { yleld }\end{array}$ & Galiniert & 4921 \\
\hline${ }^{238} \mathrm{U}$ & $p$ & $2.0+1$ & $8.5+11$ & MCOE & Galinlert & 4912 \\
\hline $150 \mathrm{Pr}$ & thick & target & $y / 010$ & & & \\
\hline $150 \mathrm{Nd}$ & $p$ & $3.0+0$ & $6.0+0$ & CLU M & Roughton+ & 4251 \\
\hline
\end{tabular}

\begin{tabular}{|c|c|c|c|c|c|}
\hline Target & $\operatorname{lnc}$ & $\begin{array}{ll}\text { EmIn } & \text { Emax } \\
\text { (MeV) } & \text { (MeV) }\end{array}$ & Lob W & A. Author & No. \\
\hline $151 \mathrm{Pm}$ & indepe & ondont $\sigma(E)$ & & & \\
\hline $238_{U}$ & $p$ & $3.5+1 \quad 8.5+1$ & MCG E & Ealinigrt & 4921 \\
\hline $151 \mathrm{Pm}$ & cumula & ative fission & $y 101 d$ & & \\
\hline $238_{4}$ & $p$ & $2.0+18.5+1$ & MCG $\varepsilon$ & Ealintert & 4912 \\
\hline $151 \mathrm{Pm}$ & cumula & alive $O(E)$ & & & \\
\hline $238 \mathrm{U}$ & $p$ & $3.5+1 \quad 8.5+1$ & MCG $\varepsilon$ & Eolintert & 4916 \\
\hline${ }^{151} \mathrm{Pm}$ & $\theta(E)$ & & & & \\
\hline $\begin{array}{l}154 \mathrm{5m} \\
154 \mathrm{Sm}\end{array}$ & p & $\begin{array}{l}1.8+1 \\
1.8+1\end{array}$ & $\begin{array}{l}\text { KFK C } \\
\text { MIL M }\end{array}$ & $\begin{array}{l}\text { Muenze:+ } \\
\text { Yilezzo-Coll1t+ }\end{array}$ & $\begin{array}{l}4284 \\
4285+\end{array}$ \\
\hline$z=62$ & parti & $|\theta| O(E)$ & & & \\
\hline $\begin{array}{l}110 \mathrm{Pd} \\
134 \mathrm{ga}\end{array}$ & $\begin{array}{l}{ }^{40} \mathrm{Ar} \\
10^{0}\end{array}$ & $\begin{array}{ll}1.4+2 & 2.4+2 \\
6.3+1 & 1.2+2\end{array}$ & $\begin{array}{l}\text { PAR E } \\
\text { PAR E }\end{array}$ & $\begin{array}{l}\text { Della-Megrat } \\
\text { Della-Meçat }\end{array}$ & $\begin{array}{l}12199 \\
11388\end{array}$ \\
\hline${ }^{133} \mathrm{Sm}$ & produc & ct yield & & & \\
\hline${ }^{106} \mathrm{Cd}$ & ${ }^{32} \mathrm{~s}$ & NDG & BRK $R$ & Pernyt & 12045 \\
\hline $135 \mathrm{sm}$ & produc & ct yield & & & \\
\hline${ }^{106} \mathrm{Cd}$ & 325 & NDG & BRK $R$ & Cernyt & 12047 \\
\hline $139 \mathrm{sm}$ & $\sigma(E)$ & & & & \\
\hline $\begin{array}{l}142_{\mathrm{Nd}} \\
142_{\mathrm{Nd}}\end{array}$ & ${ }^{a} L_{i}$ & $\begin{array}{ll}5.4+1 & 9.9+1 \\
5.4+1 & 9.9+1\end{array}$ & $\begin{array}{l}\text { INU M } \\
\text { INU M }\end{array}$ & $\begin{array}{l}\text { 1Fleissriert } \\
1 \text { Fleisgrert }\end{array}$ & $\begin{array}{l}8420 \\
9254\end{array}$ \\
\hline $140 \mathrm{Sm}$ & produc & et yield & & & \\
\hline $144 \mathrm{sm}$ & $p$ & $3.5+16.5+1$ & MCG E & Deslaurlerst & 4253 \\
\hline $140 \mathrm{sm}$ & $\sigma(E)$ & & & : & . \\
\hline $\begin{array}{l}142_{\mathrm{Nd}} \\
142_{\mathrm{Nd}}\end{array}$ & ${ }^{6} \mathrm{Li}$ & 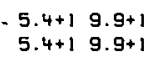 & $\begin{array}{l}\text { INU M } \\
\text { INU M }\end{array}$ & $\begin{array}{l}\text { IFleissreet } \\
\text { I Fleissrert }\end{array}$ & $\begin{array}{l}8420 \\
9254\end{array}$ \\
\hline $141 \mathrm{Sm}$ & produc & et ylold & & $;$ & \\
\hline $144 \mathrm{sm}$ & $p$ & $3.5+16.5+1$ & MCG E & Deslaurierst & 4254 \\
\hline $141 \mathrm{Sm}$ & $\theta(E)$ & & & . & \\
\hline $\begin{array}{l}142 \mathrm{Nd} \\
14 \mathrm{e}_{\mathrm{Nd}}\end{array}$ & ${ }^{a} L_{1}$ & $\begin{array}{ll}5.4+1 & 9.9+1 \\
5.4+1 & 9.9+1\end{array}$ & $\begin{array}{l}\text { INU M } \\
\text { INU M }\end{array}$ & $\begin{array}{l}\text { M Fleissrert } \\
\text { M fleisgnert }\end{array}$ & $\begin{array}{l}8420 \\
9254\end{array}$ \\
\hline
\end{tabular}




\begin{tabular}{|c|c|c|c|c|c|c|}
\hline Target & $\ln c$ & $\begin{array}{l}\text { Em In } \\
(M e V)\end{array}$ & $\begin{array}{l}\text { Emax } \\
(\operatorname{MeV})\end{array}$ & Lab & Author & No. \\
\hline $141.5 \%$ & $\theta(E)$ & & & & & \\
\hline $\begin{array}{l}110 \mathrm{Pd} \\
141-\mathrm{Sm}\end{array}$ & $\begin{array}{l}{ }^{40} \mathrm{Ar} \\
\text { O(E) }\end{array}$ & $1.6+2$ & $2.4+2$ & PAR & E Della-Negrat & 12186 \\
\hline $\begin{array}{l}134 \mathrm{Ba} \\
1410105\end{array}$ & $\mathrm{~m}^{160} \mathrm{rat}$ & $\begin{array}{l}1.0+2 \\
10.0(E)\end{array}$ & $1.2+2$ & PAR & E Della-Negrat & 11374 \\
\hline $\begin{array}{l}144 \mathrm{Sm} \\
142 \mathrm{Sm}\end{array}$ & $p_{\text {relati }}^{p}$ & $\begin{array}{l}3.0+1 \\
\text { ve } I_{y}\end{array}$ & $6.0+1$ & MCG & E Kennedy+ & 4255 \\
\hline $\begin{array}{l}14^{44} \mathrm{Sm} \\
1425 m\end{array}$ & $\stackrel{a}{O(E)}$ & $7.8+1$ & $1.2+2$ & JUL & $E$ Mariscottit & 8450 \\
\hline $\begin{array}{l}142_{\mathrm{Nd}} \\
142_{\mathrm{Nd}}\end{array}$ & ${ }^{6}{ }_{L i}^{a}$ & $\begin{array}{l}5.4+1 \\
5.4+1\end{array}$ & $\begin{array}{l}9.9+1 \\
9.9+1\end{array}$ & $\begin{array}{l}\text { INU } \\
\text { INU }\end{array}$ & $\begin{array}{l}\text { MFleisgnert } \\
\text { MFleisgnert }\end{array}$ & $\begin{array}{l}8420 \\
9254\end{array}$ \\
\hline 14350 & $\theta(E)$ & & & & & \\
\hline $\begin{array}{l}14 E_{N d} \\
142 \mathrm{Nd} \\
145 \mathrm{Nd} \\
145 \mathrm{Nd}\end{array}$ & $\begin{array}{c}\sigma_{L i}^{a} \\
{ }^{a} \\
{ }^{a} L i\end{array}$ & $\begin{array}{l}5 \cdot 4+1 \\
5 \cdot 4+1 \\
5 \cdot 4+1 \\
5 \cdot 4+1\end{array}$ & $\begin{array}{l}9.9+1 \\
9.9+1 \\
9.9+1 \\
9.9+1\end{array}$ & $\begin{array}{l}\text { INU } \\
\text { INU } \\
\text { INU } \\
\text { INU }\end{array}$ & $\begin{array}{l}\text { MFleissnert } \\
M F l e i s g n e r+ \\
M F l e i s g n e r+ \\
M \text { Fleissnert }\end{array}$ & $\begin{array}{l}8420 \\
9254 \\
8427 \\
9260\end{array}$ \\
\hline $143 \cdot 5 \pi$ & $\sigma(E)$ & & & & & \\
\hline $\begin{array}{l}110 \mathrm{Pd} \\
143.5 \mathrm{~m}\end{array}$ & $\begin{array}{l}40_{A r} \\
\sigma(E)\end{array}$ & $1.4+2$ & $2.1+2$ & PAR & E Della-Negrat & 12187 \\
\hline $\begin{array}{l}13480 \\
14458\end{array}$ & $\begin{array}{l}160 \\
\text { pertio }\end{array}$ & $\begin{array}{r}7.9+1 \\
101 E 1\end{array}$ & $1.1+2$ & PAR & E Della-Negrat & 11375 \\
\hline $\begin{array}{l}144 \mathrm{Sm} \\
1445 m\end{array}$ & $\begin{array}{c}P \\
O(\varepsilon)\end{array}$ & $2.6+1$ & & LRL & E Wongt & 4256 \\
\hline $\begin{array}{l}142_{\text {Nd }} \\
142_{\text {Nd }} \\
146_{\mathrm{Nd}} \\
146_{\mathrm{Nd}}\end{array}$ & $\begin{array}{l}\sigma_{L i}^{\alpha} \\
\sigma_{L i}^{\alpha}\end{array}$ & $\begin{array}{l}5 \cdot 4+1 \\
5.4+1 \\
5.4+1 \\
5.4+1\end{array}$ & $\begin{array}{l}9.9+1 \\
9.9+1 \\
9.9+1 \\
9.9+1\end{array}$ & $\begin{array}{l}\text { INU } \\
\text { INU } \\
\text { INU } \\
\text { INU }\end{array}$ & $\begin{array}{l}\text { MFleissnert } \\
\text { MFleissnert } \\
\text { MFleissnert } \\
\text { MFleissnert }\end{array}$ & $\begin{array}{l}8420 \\
9254 \\
8427 \\
9260\end{array}$ \\
\hline $1455 \mathrm{~m}$ & $O(E)$ & & & & & \\
\hline $\begin{array}{l}146 \mathrm{Nd} \\
146 \mathrm{Nd}\end{array}$ & $\sigma_{L i}^{a}$ & $\begin{array}{l}5.4+1 \\
5.4+1\end{array}$ & $\begin{array}{l}9.9+1 \\
9.9+1\end{array}$ & $\begin{array}{l}\text { INU } \\
\text { INU }\end{array}$ & $\begin{array}{l}\text { MFleissnert } \\
\text { MFleissnert }\end{array}$ & $\begin{array}{l}8427 \\
9260\end{array}$ \\
\hline
\end{tabular}

\begin{tabular}{|c|c|c|c|c|c|c|}
\hline Target & $\operatorname{lnc}$ & $\begin{array}{l}\text { Emin } \\
(\mathrm{MeV})\end{array}$ & $\begin{array}{l}\text { Emax } \\
(\operatorname{MeV})\end{array}$ & Lob $W$ & Author & No. \\
\hline
\end{tabular}

$146 \mathrm{Nd}{ }^{3} \mathrm{He} 1.9+12.7+1$ JYV E Hammarent 6476

$146 \mathrm{Nd} \quad 4.0+15.0+1$ MSU E Brount 8424

148 Sm $O(E)$

$146 \mathrm{Nd} \quad 5.4+19.9+1$ INU M Fleissnert 8427

$146 \mathrm{Nd} \mathrm{B}_{\mathrm{L}} 5.4+19.9+1$ INU M Fleissnert 9250

1475 partlal relative o(E)

$146 \mathrm{Nd} \quad 2.0+13.7+1$ UST E Hammarent 8425

$148^{\mathrm{Nd}}{ }^{3} \mathrm{He} \quad 1.9+12.7+1$ JYV E Hammarent $\quad 6477$

$147 \mathrm{Sm} \quad O(\mathrm{E})$

$146 \mathrm{Nd} \quad 0.4+19.9+1$ INU M Fleissnert 8427

$146 \mathrm{Nd} \quad 6_{L 1} \quad 5.4+19.9+1$ INU M Fleissnert 9250

${ }^{150} \mathrm{Nd}{ }^{6} \mathrm{Ll} \quad 5.4+19.9+1$ INU MFleissnert. 9263

$140 \mathrm{Sm}$ Dartlal o(E),

$1485 \mathrm{~m} \quad 2.6+1$ LRL E Wongt 4267

1485 martlal relative ofE

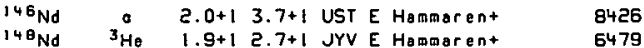

${ }^{140}{ }^{14}$ m partial o(E)

$148 \mathrm{Nd}{ }^{3} \mathrm{He} \quad 2.2+12.7+1$ JYV E Hammorent 6478

$148 \mathrm{Sm} \quad O(E)$

148 Nd $\quad 5.4+19.9+1$ INU MFleissnert 8427

$146 \mathrm{Nd} \quad 6 \mathrm{Ll} \quad 5.4+19.9+1$ INU M Fleissnert 9260

150 Nd Li $5.4+19.9+1$ INU M Fleissnert 9263

$14 \mathrm{\theta}^{\mathrm{Nd}}$ a $2.2+13.7+1$ UST E Hammarent 8430

$150 \mathrm{Nd}{ }^{3} \mathrm{He} 2.2+12.7+1$ JYV E Hammarent

$149 \mathrm{Sm}$ partiol o(E)

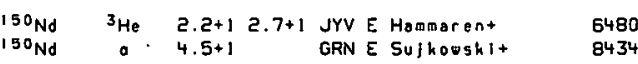




\begin{tabular}{|c|c|c|c|c|c|c|}
\hline Target & $\ln c$ & $\begin{array}{l}\text { Emin } \\
(M e V)\end{array}$ & $\begin{array}{l}\text { Emax } \\
(\operatorname{MeV})\end{array}$ & Lab h & Author & No. \\
\hline 1495 & \multicolumn{6}{|c|}{ rolative 1 , } \\
\hline $150 \mathrm{Nd}$ & a & $4.5+1$ & & GRN E & E Sujhouskit & 8433 \\
\hline $1495 a$ & $\sigma(E)$ & & & & & $\therefore$ \\
\hline $\begin{array}{l}150 \mathrm{No} \\
150 \mathrm{Nd} \\
150 \mathrm{So}\end{array}$ & $\begin{array}{l}{ }_{6}^{a} \\
{ }^{6} \mathrm{Ll} \\
{ }^{\mathrm{He}}\end{array}$ & $\begin{array}{l}4.5+1 \\
5.4+1 \\
1.9+1\end{array}$ & $\begin{array}{l}9.9+1 \\
2.7+1\end{array}$ & $\begin{array}{l}\text { GRN E } \\
\text { INU } \\
\text { JYV }\end{array}$ & $\begin{array}{l}\text { E Sujkooshit } \\
\text { M Fleissnert } \\
\text { E Hammerent }\end{array}$ & $\begin{array}{l}8435 \\
9263 \\
6483\end{array}$ \\
\hline $1905 m$ & \multicolumn{6}{|c|}{ partial o(E), } \\
\hline $150 \mathrm{Sa}$ & $p$ & $2.6+1$ & & LRL & E Wong+ & 4274 \\
\hline $1505 n$ & \multicolumn{6}{|c|}{ portiol o(E) } \\
\hline $\begin{array}{l}150 \mathrm{Ne} \\
150 \mathrm{Ne}\end{array}$ & $\stackrel{{ }^{3} \mathrm{He}}{0}$ & $\begin{array}{l}2.2+1 \\
4.5+1\end{array}$ & $2.7+1$ & $\begin{array}{l}\text { JYV } \\
\text { GRN }\end{array}$ & $\begin{array}{l}\text { E Hamarent } \\
\text { E Sujkoushit }\end{array}$ & $\begin{array}{l}6482 \\
8438\end{array}$ \\
\hline${ }^{150} 5 \pi$ & \multicolumn{6}{|c|}{ relative I, } \\
\hline $\begin{array}{l}150 \mathrm{Nd} \\
190 \mathrm{Sog}\end{array}$ & \multicolumn{6}{|c|}{ averoge $v_{y}$} \\
\hline $\begin{array}{l}150 \mathrm{Nd} \\
150 \mathrm{Som}\end{array}$ & \multicolumn{6}{|l|}{$\theta(E)$} \\
\hline $\begin{array}{l}150 \mathrm{Nd} \\
150 \mathrm{Nd}\end{array}$ & $\sigma_{L i}^{a}$ & $\begin{array}{l}4.5+1 \\
5.4+1\end{array}$ & $9.9+1$ & $\begin{array}{l}\text { GRN } \\
\text { INU }\end{array}$ & $\begin{array}{l}\text { E Sulkowskl+ } \\
\text { M Floissnert }\end{array}$ & $\begin{array}{l}9439 \\
9263\end{array}$ \\
\hline $131 \mathrm{sm}$ & \multicolumn{6}{|c|}{ pertlel relotive $C(E)$} \\
\hline $\begin{array}{l}150 \mathrm{sm} \\
151 \mathrm{sm}\end{array}$ & \multicolumn{5}{|c|}{ partlal o(E) } & 6485 \\
\hline $150 \mathrm{Nd}$ & - & $4.5+1$ & & GRN & E Sulkowshit & 3441 \\
\hline 15150 & \multicolumn{6}{|c|}{ relotive Ir } \\
\hline $\begin{array}{l}150 \mathrm{Nd} \\
1515 \mathrm{~m}\end{array}$ & \multicolumn{5}{|l|}{$O(E)$} & 8440 \\
\hline $\begin{array}{l}150 \mathrm{Nd} \\
150 \mathrm{Nd} \\
150 \mathrm{Sm}\end{array}$ & $\begin{array}{l}{ }^{5} \mathrm{Li} \\
{ }^{3} \mathrm{He}\end{array}$ & $\begin{array}{l}4.5+1 \\
5 \cdot 4+1 \\
2.2+1\end{array}$ & $\begin{array}{l}9.9+1 \\
2.7+1\end{array}$ & $\begin{array}{l}\text { GRN } \\
\text { INU } \\
\text { JYV }\end{array}$ & $\begin{array}{l}\text { E Suihowshit } \\
\text { M Fleissnert } \\
\text { E Hommarent }\end{array}$ & $\begin{array}{l}8442 \\
9263 \\
6484\end{array}$ \\
\hline $152 \mathrm{sm}$ & \multicolumn{6}{|c|}{ partial o(E), } \\
\hline $1925 m$ & $p$ & $2.6+1$ & & LRL & E Wongt & 4280 \\
\hline
\end{tabular}

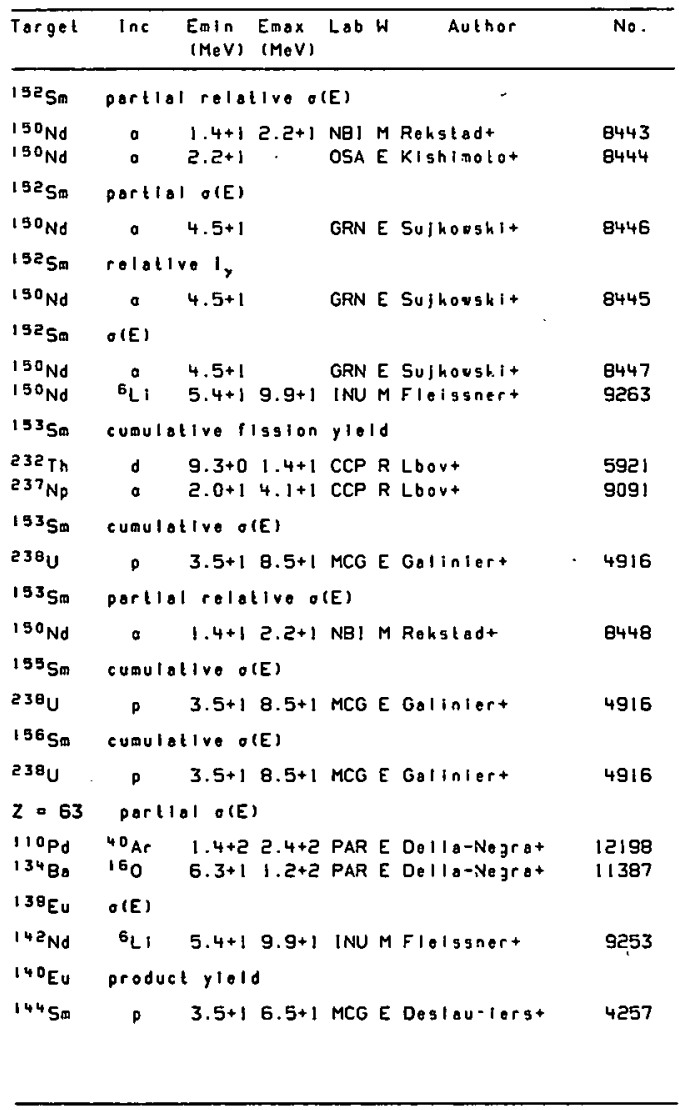




\begin{tabular}{|c|c|c|c|c|c|}
\hline Target & $\operatorname{lnc}$ & $\begin{array}{l}\text { Emin } \\
\text { (MeV) }\end{array}$ & 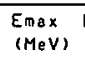 & Lab W & No. \\
\hline $140 E_{U}$ & $O(E)$ & & & & \\
\hline $14 a_{\mathrm{Nd}}$ & ${ }^{6} \mathrm{Li}$ & $5.4+1$ & $9.9+1$ & INU M Fleissnert & 9253 \\
\hline $141 \mathrm{Ev}$ & $o(E)$ & & & & \\
\hline $142 \mathrm{No}$ & $s_{\mathrm{Li}}$ & $5.4+1$ & $9.9+1$ & INU MFieissnert & .9253 \\
\hline $1418 \mathrm{Eu}$ & prod & Iet yiel & & & \\
\hline $1445 \mathrm{~m}$ & p & $3.5+1$ & $6.5+1$ & MCG E Destaurierst & .4258 \\
\hline $141-E_{v}$ & prod & ict yiel & & & \\
\hline $1445 \mathrm{~m}$ & p & $3.5+1$ & $6.5+1$ & MCG E Deslauriers* & 4259 \\
\hline $142 \mathrm{Eu}$ & $O(E)$ & & & & \\
\hline $14{ }^{2} \mathrm{Nd}$ & $\mathrm{E}_{\mathrm{LI}}$ & $5.4+1$ & $9.9+1$ & INU MFleissnert & 9253 \\
\hline${ }^{143} \varepsilon_{u}$ & part 1 & I produ & uct yle & eld & \\
\hline $1445 \mathrm{~m}$ & p & NDG & & MSU E Aryaeinejadt & 4250 \\
\hline${ }^{143} \mathrm{Ev}_{0}$ & produ & it yield & & & \\
\hline $14 \Sigma_{\mathrm{Nd}}$ & ${ }^{6} \mathrm{LI}$ & $6.5+1$ & & NOT E Rakel. & 9251 \\
\hline${ }^{143} \mathrm{Eu}_{u}$ & $O(E)$ & & & & \\
\hline $\begin{array}{l}142_{\mathrm{Nd}} \\
146_{\mathrm{Nd}}\end{array}$ & $\begin{array}{l}{ }_{6}^{6}{ }^{6} \\
{ }^{6} L_{1}\end{array}$ & $\begin{array}{l}5.4+1 \\
5.4+1\end{array}$ & $\begin{array}{r}9.9+1 \\
9.9+1\end{array}$ & $\begin{array}{l}\text { INU M Fleissnert } \\
\text { INU M Fleigsnert }\end{array}$ & $\begin{array}{l}9253 \\
9259\end{array}$ \\
\hline${ }^{144} \mathrm{Ev}$ & parti & $10(E)$ & & & \\
\hline $1445 \mathrm{~m}$ & p & $2.6+1$ & & LRL E Wongt & 4261 \\
\hline $144 E_{U}$ & relat & ive $I_{y}$ & & & \\
\hline $\begin{array}{l}1445 \mathrm{~m} \\
144 \mathrm{sm}\end{array}$ & $\begin{array}{l}\text { p } \\
\text { d }\end{array}$ & $\begin{array}{l}5.0+0 \\
1.4+1\end{array}$ & $1.0+1$ & $\begin{array}{l}\text { ROS E Funket } \\
\text { ROS E Funket }\end{array}$ & $\begin{array}{l}4262 \\
5777\end{array}$ \\
\hline${ }^{144} \mathrm{Eu}^{-}$ & $O(E)$ & & & & \\
\hline $\begin{array}{l}142_{\mathrm{Nd}} \\
146_{\mathrm{Nd}}\end{array}$ & $\begin{array}{l}6 \mathrm{bL} \\
{ }^{6} \mathrm{LI}\end{array}$ & $\begin{array}{l}5.4+1 \\
5.4+1\end{array}$ & $\begin{array}{l}9.9+1 \\
9.9+1\end{array}$ & $\begin{array}{l}\text { INU M Fleissnert } \\
\text { INU MFleissnert }\end{array}$ & $\begin{array}{l}9253 \\
9259\end{array}$ \\
\hline $149 \mathrm{Eu}$ & cumul & the of & & & \\
\hline $\begin{array}{l}110 \mathrm{Pd} \\
134 \mathrm{Ba}\end{array}$ & $\begin{array}{l}40 \mathrm{Ar} \\
160\end{array}$ & $\begin{array}{l}1.5+2 \\
7.4+1\end{array}$ & $\begin{array}{l}2.0+2 \\
1.2+2\end{array}$ & $\begin{array}{l}\text { PAR E Della-Negrat } \\
\text { PAR E Della-Negrat }\end{array}$ & $\begin{array}{l}12188 \\
11376\end{array}$ \\
\hline
\end{tabular}

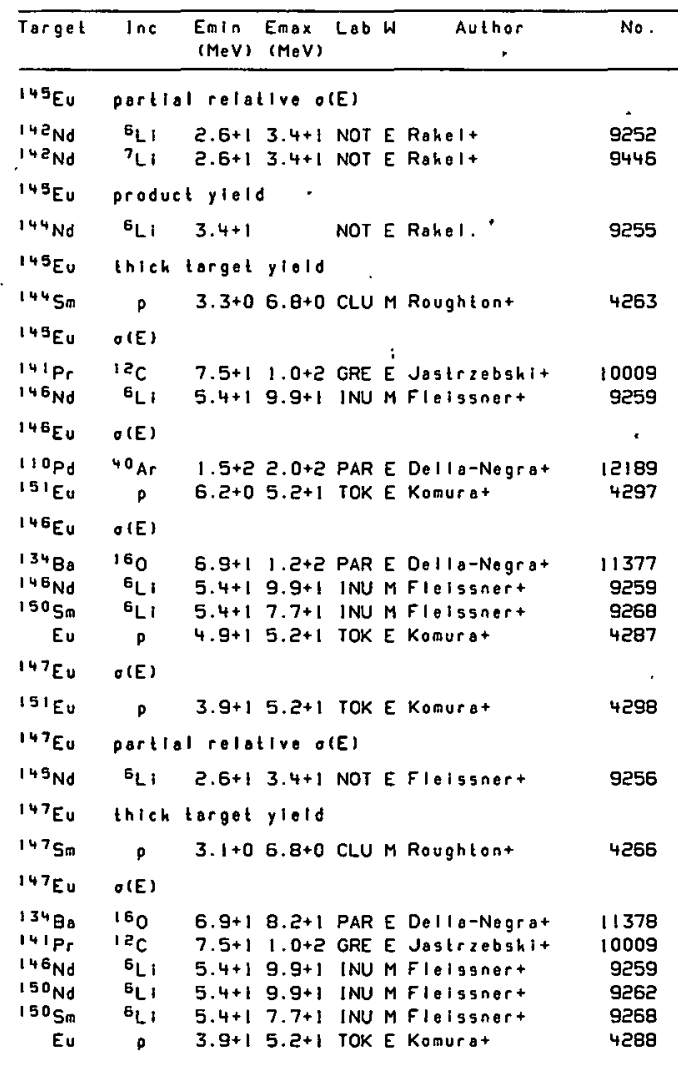




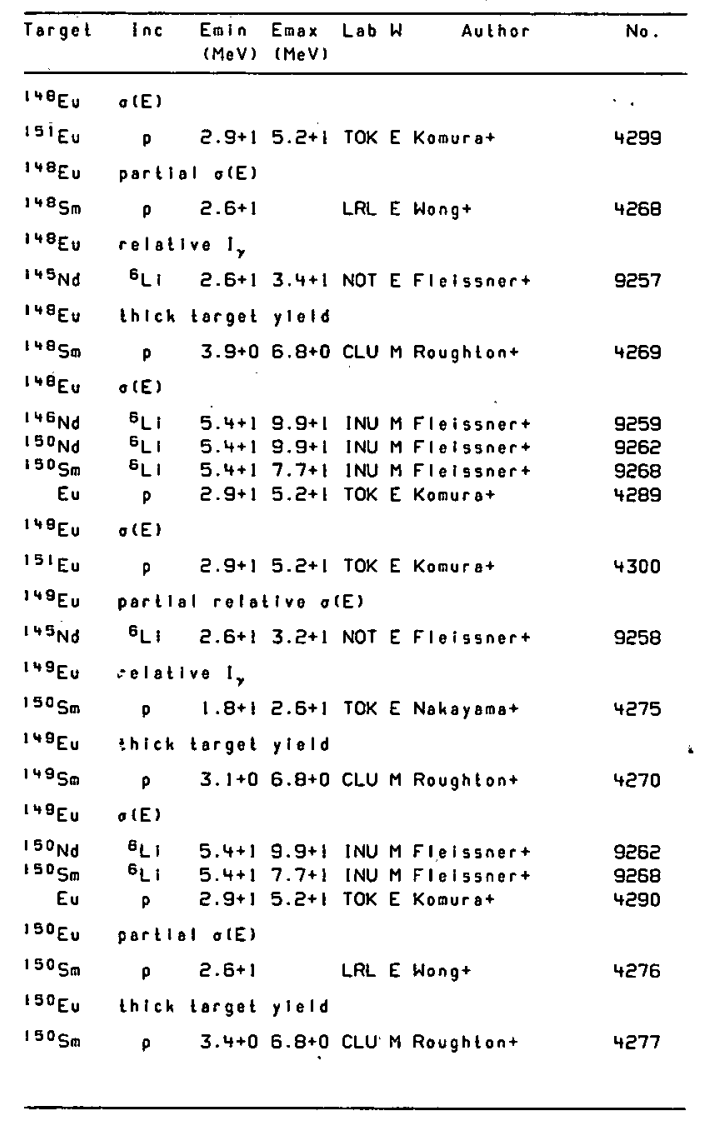

\begin{tabular}{|c|c|c|c|c|c|c|}
\hline Target & $\operatorname{lnc}$ & $\begin{array}{l}\text { Emin } \\
(\mathrm{MeV})\end{array}$ & $\begin{array}{l}E_{\max } \\
(\operatorname{MeV})\end{array}$ & Lab h & Author & No. \\
\hline $150 \mathrm{Eu}$ & $\sigma(\varepsilon)$ & & & & & \\
\hline $\begin{array}{l}150 \mathrm{Nd} \\
150 \mathrm{Sm}\end{array}$ & $\begin{array}{l}\sigma_{L I} \\
\sigma_{L i}\end{array}$ & $\begin{array}{l}5 \cdot 4+1 \\
5.4+1\end{array}$ & $\begin{array}{l}9.9+1 \\
7.7+1\end{array}$ & $\begin{array}{l}\text { INU } \\
\text { INU }\end{array}$ & $\begin{array}{l}\text { MFleissnert } \\
\text { MFleissnert }\end{array}$ & $\begin{array}{l}9262 \\
9268\end{array}$ \\
\hline $151 E_{U}$ & \multicolumn{6}{|c|}{ partial product yleld } \\
\hline $\begin{array}{l}152 \mathrm{Sm} \\
151 \mathrm{Eu}\end{array}$ & $\begin{array}{c}p \\
o(E)\end{array}$ & $1.9+1$ & $2.8+1$ & MIL E & E Biancot & 4281 \\
\hline $\begin{array}{l}150 \mathrm{Nd} \\
150 \mathrm{Sm}\end{array}$ & $\begin{array}{l}{ }^{6} L i \\
{ }^{6} L i\end{array}$ & $\begin{array}{l}5.4+1 \\
5.4+1\end{array}$ & $\begin{array}{l}9.9+1 \\
7.7+1\end{array}$ & $\begin{array}{l}\text { INU } \\
\text { INU }\end{array}$ & $\begin{array}{l}\text { MFleissnert } \\
\text { MFleigsnert }\end{array}$ & $\begin{array}{l}9262 \\
9268\end{array}$ \\
\hline $152 \mathrm{Eu}$ & \multicolumn{6}{|c|}{ partiol o(E) } \\
\hline $1525 \mathrm{~m}$ & D & $2.6+1$ & & LRL E & E Wongt & 4282 \\
\hline $152 \mathrm{Eu}$ & \multicolumn{6}{|c|}{ thlch target yleld } \\
\hline $1525 m$ & $p$ & $3.0+0$ & $6.8+0$ & CLU $M$ & M Rougitont & 4283 \\
\hline${ }^{193} \mathrm{Eu}$ & \multicolumn{3}{|c|}{ direct-interaction } & \\
\hline $158 \mathrm{Gd}$ & p & $2.0+1$ & & & M Milazzo-Collit & 4315 \\
\hline${ }^{153} \mathrm{Eu}$ & \multicolumn{5}{|c|}{ portlal o(E) } & . \\
\hline $155 \mathrm{Go}$ & p & $2.0+1$ & & MIL M & M Milazzo-Collit & 4314 \\
\hline${ }^{153} \mathrm{Eu}$ & \multicolumn{6}{|l|}{$\sigma(E)$} \\
\hline${ }^{156} \mathrm{Gd}$ & $p$ & $2.0+1$ & & MIL M & M Milazzo-Collit & 4313 \\
\hline${ }^{156} E_{v}$ & \multicolumn{6}{|c|}{ cumulative fission yield } \\
\hline $232 \mathrm{Th}$ & d & $9.3+0$ & $1.4+1$ & CCP F & $R$ ibovt & 5921 \\
\hline${ }^{157} \mathrm{EU}_{\mathrm{U}}$ & \multicolumn{3}{|c|}{ cumulative flasion } & \multicolumn{3}{|c|}{ yield } \\
\hline $\begin{array}{l}233 \mathrm{U} \\
237 \mathrm{~Np}\end{array}$ & d & $\begin{array}{l}9.0+0 \\
2.0+1\end{array}$ & $\begin{array}{l}2.3+1 \\
4.1+1\end{array}$ & $\begin{array}{l}\text { CCP } F \\
\text { CCP F }\end{array}$ & $\begin{array}{l}R \text { Lbovt } \\
R \text { Lbovt }\end{array}$ & $\begin{array}{l}5936 \\
9091\end{array}$ \\
\hline $157 \mathrm{Eu}$ & \multicolumn{6}{|c|}{ cugulative o(E) } \\
\hline $23 \theta_{U}$ & i & $3.5+1$ & $8.5+1$ & MCG E & E Galiniert & 4916 \\
\hline$z=64$ & \multicolumn{6}{|c|}{ portlol $\sigma(E)$} \\
\hline $\begin{array}{l}110 \mathrm{Pd} \\
134 \mathrm{Ba}\end{array}$ & $\begin{array}{l}{ }^{40} \mathrm{Ar} \\
16 \mathrm{O}\end{array}$ & $\begin{array}{l}1.4+2 \\
6.3+1\end{array}$ & $\begin{array}{l}2.4+2 \\
1.2+2\end{array}$ & $\begin{array}{l}\text { PAR E } \\
\text { PAR E }\end{array}$ & $\begin{array}{l}\text { E Della-Negrat } \\
\text { E Oella-Negrat }\end{array}$ & $\begin{array}{l}12197 \\
11386\end{array}$ \\
\hline
\end{tabular}




\begin{tabular}{|c|c|c|c|c|c|c|}
\hline Target & $\ln c$ & $\begin{array}{l}\text { Emln } \\
(M e V)\end{array}$ & $\begin{array}{l}\text { Emax } \\
(\operatorname{MeV})\end{array}$ & Lab & Author & No. \\
\hline $142 \mathrm{Gd}$ & \multicolumn{6}{|c|}{ pelative $I_{r}$} \\
\hline $144 \mathrm{sm}$ & 0 & $9.2+1$ & $1.3+2$ & JUL & E Mariscottit & 8451 \\
\hline $144 \mathrm{Gd}$ & \multicolumn{6}{|c|}{ relative 1 , } \\
\hline $14^{4} \mathrm{Sm}$ & $a$ & $6.0+1$ & $9.0+1$ & JUL & E Mariscottit & 8452 \\
\hline $145 \mathrm{Gd}$ & \multicolumn{6}{|c|}{ relotive $I_{y}$} \\
\hline $1445 m$ & a & E. $0+1$ & $6.7+1$ & JUL & E Mariscottit & 8453 \\
\hline $145 \mathrm{Gd}$ & \multicolumn{6}{|c|}{$\sigma(E) \times$ factor } \\
\hline $\begin{array}{l}110 \mathrm{Pd} \\
134 \mathrm{Ba}\end{array}$ & $\begin{array}{l}40 \mathrm{Ar} \\
16 \mathrm{O}\end{array}$ & $\begin{array}{l}1.5+2 \\
8.1+1\end{array}$ & $\begin{array}{l}2.1+2 \\
1.2+2\end{array}$ & $\begin{array}{l}\text { PAR. } \\
\text { PAR }\end{array}$ & $\begin{array}{l}\text { E Della-Negra+ } \\
\text { E Della-Negra+ }\end{array}$ & $\begin{array}{l}12190 \\
11379\end{array}$ \\
\hline 145800 & \multicolumn{6}{|l|}{$\theta(E)$} \\
\hline $\begin{array}{l}110 \mathrm{Pd} \\
134 \mathrm{Bg}\end{array}$ & $\begin{array}{l}40 \mathrm{Ar} \\
160\end{array}$ & $\begin{array}{l}1.4+2 \\
8.1+1\end{array}$ & $\begin{array}{l}2.0+2 \\
1.2+2\end{array}$ & $\begin{array}{l}\text { PAR } \\
\text { PAR }\end{array}$ & $\begin{array}{l}\text { E Della-Negrat } \\
\text { E Della-Negrat }\end{array}$ & $\begin{array}{l}12191 \\
11380\end{array}$ \\
\hline $145.6 \mathrm{~d}$ & \multicolumn{6}{|c|}{ 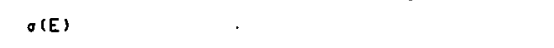 } \\
\hline $\begin{array}{l}110 \mathrm{Pd} \\
134 \mathrm{Bo}\end{array}$ & $\begin{array}{l}40 \mathrm{Ar} \\
160\end{array}$ & $\begin{array}{l}1.4+2 \\
7.9+1\end{array}$ & $\begin{array}{l}2.1+2 \\
1.2+2\end{array}$ & $\begin{array}{l}\text { PAR } \\
\text { PAR }\end{array}$ & $\begin{array}{l}\text { E Della-Negrat } \\
\text { E Della-Negrat }\end{array}$ & $\begin{array}{l}12192 \\
11381\end{array}$ \\
\hline \multicolumn{7}{|c|}{$1459+\cdots a d$ cumuletive $O(E)$} \\
\hline $\begin{array}{l}110 \mathrm{Pd} \\
134 \mathrm{Ba}\end{array}$ & $\begin{array}{l}40 \mathrm{Ar} \\
10^{\circ}\end{array}$ & $\begin{array}{l}1.5+2 \\
9.1+1\end{array}$ & $\begin{array}{l}2.1+2 \\
1.2+5\end{array}$ & $\begin{array}{l}\text { PAR } \\
\text { PAR }\end{array}$ & $\begin{array}{l}\text { E Della-Negrat } \\
\text { E Della-Negrat }\end{array}$ & $\begin{array}{l}12193 \\
11382\end{array}$ \\
\hline $145 \mathrm{Gd}$ & \multicolumn{6}{|c|}{ partiol product ylald } \\
\hline${ }^{148} 5 \mathrm{~m}$ & $a$ & $7.0+1$ & $9.0+1$ & IPS & E Klelnhelnzt & 8457 \\
\hline${ }^{148} \mathrm{Gd}$ & \multicolumn{6}{|c|}{ relative $l_{r}$} \\
\hline $1445 m$ & 。 & $2.2+1$ & $2.5+1$ & UL & E Ogava+ & 8454 \\
\hline $146 \mathrm{Gd}$ & \multicolumn{6}{|l|}{$v_{y}$} \\
\hline 15050 & 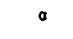 & $9.0+1$ & $1.4+\varepsilon$ & JUL & E Ogawa+ & 8464 \\
\hline $148 \mathrm{Gd}$ & \multicolumn{6}{|c|}{$\sigma(E) \times f a c t o r$} \\
\hline $\begin{array}{l}1100 \mathrm{Pd} \\
134 \mathrm{Bg}\end{array}$ & $\begin{array}{l}40 \mathrm{Ar} \\
160\end{array}$ & $\begin{array}{l}1.5+2 \\
8.1+1\end{array}$ & $\begin{array}{l}2.1+2 \\
1.2+5\end{array}$ & $\begin{array}{l}\text { PAR } \\
\text { PAR }\end{array}$ & $\begin{array}{l}\text { E Della-Negra+ } \\
\text { E Dello-Negra+ }\end{array}$ & $\begin{array}{l}12195 \\
11384\end{array}$ \\
\hline $146 \mathrm{Gd}$ & $\sigma(E)$ & & & & & \\
\hline $\begin{array}{l}110 \mathrm{pd} \\
134 \mathrm{Bd}\end{array}$ & $\begin{array}{l}40 \mathrm{Ar} \\
160\end{array}$ & $\begin{array}{l}1.4+2 \\
6.9+1\end{array}$ & $\begin{array}{l}2.1+2 \\
1.2+5\end{array}$ & $\begin{array}{l}\text { PAR } \\
\text { PAR }\end{array}$ & $\begin{array}{l}\text { E Della-Negrat } \\
\text { E Della-Negrat }\end{array}$ & $\begin{array}{l}12194 \\
11383\end{array}$ \\
\hline
\end{tabular}

\begin{tabular}{|c|c|c|c|c|c|c|}
\hline Target & $\ln c$ & $\begin{array}{l}\text { Emin } \\
(M e V)\end{array}$ & $\begin{array}{l}\text { Emax } \\
(\operatorname{MeV})\end{array}$ & Lob & Author & No. \\
\hline $146 \mathrm{Gd}$ & $O(E)$ & & & & & \\
\hline $\begin{array}{l}\text { Eu } \\
\text { ISIEu }\end{array}$ & $\begin{array}{l}p \\
p\end{array}$ & $\begin{array}{l}4.6+1 \\
4.6+1\end{array}$ & $\begin{array}{l}5.2+1 \\
5.2+1\end{array}$ & $\begin{array}{l}\text { TOK E } \\
\text { TOK E }\end{array}$ & $\begin{array}{l}\text { E Komurot } \\
\text { E Komurat }\end{array}$ & $\begin{array}{l}4291 \\
4301\end{array}$ \\
\hline $147 \mathrm{Gd}$ & portl & I prod & luet yie & eld & & \\
\hline $\begin{array}{l}148 \mathrm{Sm} \\
149 \mathrm{Sm}\end{array}$ & a & $\begin{array}{l}7.0+1 \\
7.0+1\end{array}$ & $\begin{array}{l}9 \cdot 0+1 \\
9 \cdot 0+1\end{array}$ & $\begin{array}{l}\text { IPS E } \\
\text { IPS E }\end{array}$ & $\begin{array}{l}\text { E Kleinheinzt } \\
\text { E Kleinhelazt }\end{array}$ & $\begin{array}{l}8458 \\
8460\end{array}$ \\
\hline $147 \mathrm{Gd}$ & relat & $v e J_{y}$ & & & & \\
\hline $\begin{array}{l}144 \mathrm{Gm} \\
147 \mathrm{Gd}\end{array}$ & $v_{y}^{a}$ & $2.2+1$ & $2.5+1$ & JUL E & E Ogawat & 8455 \\
\hline $\begin{array}{l}150 \mathrm{Sm} \\
147 \mathrm{Gd}\end{array}$ & $\begin{array}{c}a \\
O(E)\end{array}$ & $7.5+1$ & $1.4+2$ & JUL E & E Ogawat & 8465 \\
\hline $\begin{array}{l}110 \mathrm{Pd} \\
134 \mathrm{Bo} \\
141 \mathrm{Pr} \\
\mathrm{Eu} \\
151 \mathrm{Eu}\end{array}$ & $\begin{array}{l}40 \mathrm{Ar} \\
160 \\
12 \mathrm{C} \\
p \\
P\end{array}$ & $\begin{array}{l}1.4+2 \\
6.3+1 \\
7.5+1 \\
3.7+1 \\
3.7+1\end{array}$ & $\begin{array}{l}1.9+2 \\
1.9+2 \\
1.0+2 \\
1.0+2 \\
5.2+1 \\
2.5+1\end{array}$ & $\begin{array}{l}\text { PAR E } \\
\text { PAR E } \\
\text { GRE E } \\
\text { TOK E } \\
\text { TOK E }\end{array}$ & $\begin{array}{l}\text { E Dolla-Negrat } \\
\text { E Della-Negrat } \\
\text { E Jastrzebskit } \\
\text { E Komurat } \\
\text { E Komurat }\end{array}$ & $\begin{array}{r}12196 \\
11385 \\
10009 \\
4292 \\
4302\end{array}$ \\
\hline $14 \theta_{\mathrm{Gd}}$ & portl & 1 prod & luet yio & .19 & & \\
\hline $\begin{array}{l}14 \theta_{\mathrm{m}} \\
1{ }^{14 \theta_{\mathrm{Gd}}}\end{array}$ & $\begin{array}{c}a \\
\text { partl }\end{array}$ & $\begin{array}{l}5.1+1 \\
1 \quad O(E)\end{array}$ & & MUU E & E Kleinheinzt & 8459 \\
\hline $\begin{array}{l}154 \mathrm{Gd} \\
14 \theta^{\circ} \mathrm{d}\end{array}$ & $v^{a}$ & $5.0+1$ & $1.2+2$ & GRN E & E Chmieleuskat & 8493 \\
\hline $\begin{array}{l}150 \mathrm{Sm} \\
140_{\mathrm{Gd}}\end{array}$ & $\begin{array}{c}a \\
O(E)\end{array}$ & $6.5+1$ & $1.4+2$ & JUL E & E Oganat & 8466 \\
\hline $\begin{array}{r}\text { Eu } \\
151 E u\end{array}$ & $p$ & $\begin{array}{l}2.6+1 \\
2.6+1\end{array}$ & $\begin{array}{l}5 \cdot 2+1 \\
5 \cdot 2+1\end{array}$ & $\begin{array}{l}\text { TOK E } \\
\text { TOK E }\end{array}$ & $\begin{array}{l}\text { E Komurat } \\
\text { E Komurat }\end{array}$ & $\begin{array}{l}4293 \\
4303\end{array}$ \\
\hline $149 \mathrm{Gd}$ & partl & 1 prod & luct yie & .10 & & \\
\hline $\begin{array}{l}149 \mathrm{Sm} \\
149_{\mathrm{Gd}}\end{array}$ & parti & $\begin{array}{r}3.9+1 \\
1 \text { O(E) }\end{array}$ & $6.0+1$ & IPS E & E Hjortht & $846 !$ \\
\hline $190 \mathrm{Sm}$ & a & $3.9+1$ & $6.0+1$ & IPS E & E Hiortht & 8468 \\
\hline
\end{tabular}




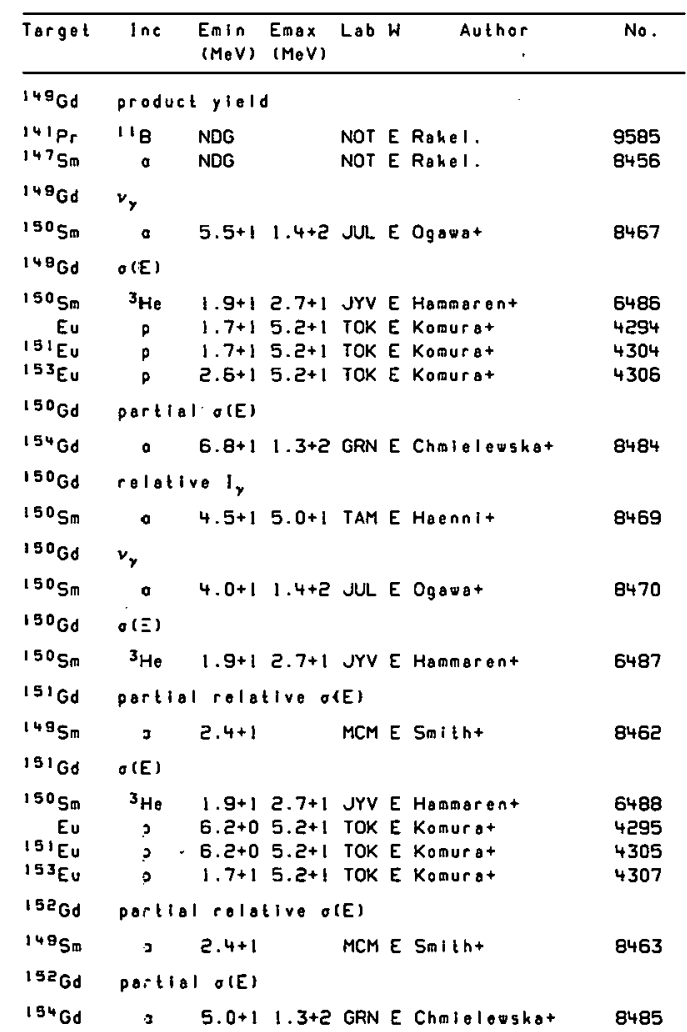

\begin{tabular}{lccc}
\hline Target & IaC & $\begin{array}{l}\text { Emin } \\
\text { (MeV) }\end{array}$ \\
(MeV)
\end{tabular}




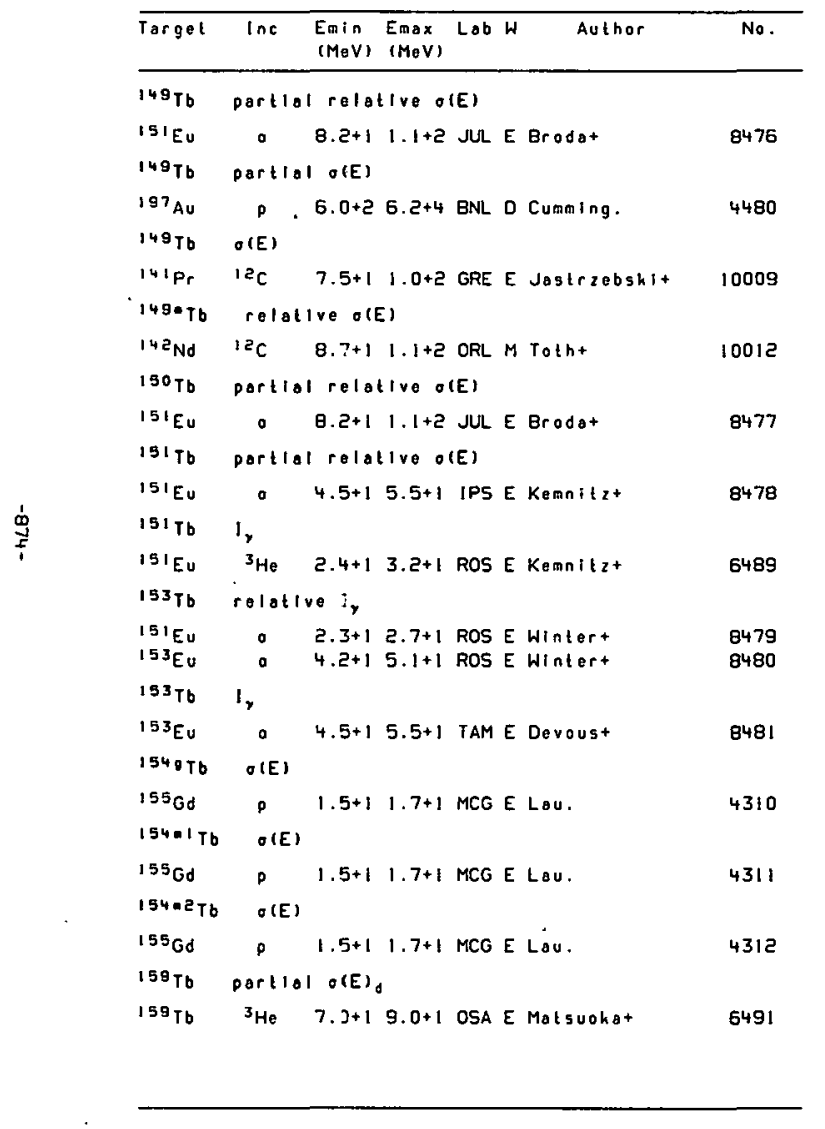

\begin{tabular}{|c|c|c|c|c|c|c|}
\hline Target & $\ln c$ & $\begin{array}{l}E m I n \\
(M e V)\end{array}$ & $\begin{array}{l}\text { Emax } \\
(M e V)\end{array}$ & Lob $\mathrm{W}$ & Author & No. \\
\hline $160 \mathrm{~Tb}$ & cumula & tive ti & ission & yield & & \\
\hline $\begin{array}{l}237 \mathrm{~Np} \\
160 \mathrm{~Tb}\end{array}$ & $\begin{array}{c}a \\
O(E)\end{array}$ & $2.0+1$ & $4.1+1$ & $C C P R$ & Lbout & 9091 \\
\hline $\begin{array}{l}160 \mathrm{Gd} \\
160 \mathrm{Gd}\end{array}$ & p & $\begin{array}{l}4.3+0 \\
4.3+0\end{array}$ & $\begin{array}{l}4.4+1 \\
4.4+1\end{array}$ & $\begin{array}{l}\text { KFK C } \\
\text { MIL E }\end{array}$ & $\begin{array}{l}\text { Muenzel+ } \\
\text { Birattarit }\end{array}$ & $\begin{array}{l}4316 \\
4317 .\end{array}$ \\
\hline $161 \mathrm{~Tb}$ & cumulo & tive fI & ission & yleld & & \\
\hline $\begin{array}{l}23^{7} \mathrm{~Np} \\
Z=66\end{array}$ & $\begin{array}{c}a \\
\sigma(E)\end{array}$ & $2.0+1$ & $4.1+1$ & CCP R & Lbovt & 9091 \\
\hline $\begin{array}{l}160 \mathrm{Gd} \\
140^{\circ y}\end{array}$ & $\begin{array}{l}12 C \\
r o 10 t 1\end{array}$ & $\begin{array}{l}9.0+1 \\
\text { ve } O(E)\end{array}$ & $2.0+2$ & GRN E & Sigek-Wilczynsh & 10050 \\
\hline $\begin{array}{l}142_{\mathrm{Nd}} \\
190 \mathrm{Oy}\end{array}$ & $\begin{array}{l}1{ }^{2} \mathrm{C} \\
\text { portlo }\end{array}$ & $\begin{array}{l}8.7+1 \\
\text { orodu }\end{array}$ & $\begin{array}{l}1.1+2 \\
\text { uct } y / \theta\end{array}$ & ORL $M$ & 4 Totht & 10013 \\
\hline $\begin{array}{l}15{ }^{2} \mathrm{Gd} \\
150 \mathrm{Oy}\end{array}$ & $\begin{array}{c}\text { o } \\
\text { portia }\end{array}$ & $\begin{array}{r}8.6+1 \\
O(E)\end{array}$ & & MUU E & Klelnheinz+ & 8482 \\
\hline $\begin{array}{l}154 \mathrm{Gd} \\
150 \mathrm{Oy}\end{array}$ & $v_{y}^{a}$ & $9.0+1$ & $1,3+2$ & GRN E & Chareleoshat & 8486 \\
\hline $\begin{array}{l}144^{4} \mathrm{Nd} \\
{ }^{150} \mathrm{Dy}\end{array}$ & $\begin{array}{l}{ }^{12} \mathrm{C} \\
\nu_{n}\end{array}$ & NDG & & LND E & Corlent & 10015 \\
\hline $\begin{array}{l}14{ }^{4} \mathrm{Nd} \\
130 \mathrm{Dy}\end{array}$ & $\begin{array}{l}{ }^{2} \mathrm{C} \\
\text { relot }\end{array}$ & $\begin{array}{l}\text { NDG } \\
\text { ve } g(E)\end{array}$ & & LND E & Cerlent & 10014 \\
\hline $\begin{array}{l}141 \mathrm{Pr} \\
190 \mathrm{Or}\end{array}$ & $\begin{array}{l}{ }^{14} N \\
O(E)\end{array}$ & $5.8+1$ & $9.8+1$ & ORL $M$ & $4 \mathrm{Schmidt-Ott+}$ & 10446 \\
\hline $\begin{array}{l}141 \mathrm{pr} \\
154 \mathrm{Gd}\end{array}$ & $\begin{array}{r}14 \mathrm{~N} \\
0\end{array}$ & $\begin{array}{l}9.2+1 \\
6.7+1\end{array}$ & $1 \cdot 3+2$ & $\begin{array}{l}\text { ORL M } \\
\text { GRN E }\end{array}$ & $\begin{array}{l}1 \text { Schmidt-Oltt } \\
\text { Chmieloushat }\end{array}$ & $\begin{array}{r}10445 \\
8487\end{array}$ \\
\hline $1510 y$ & portlo & I relat & tive or & (E) & & \\
\hline $\begin{array}{l}\text { IazSn } \\
1910 y\end{array}$ & $\begin{array}{l}325 \\
\text { relot }\end{array}$ & $\begin{array}{c}1.2+2 \\
\text { vo } O(E)\end{array}$ & $1.6+2$ & ROC E & Listert & 12055 \\
\hline $141 \mathrm{Pr}$ & $14 \mathrm{~N}$ & $5.8+1$ & $9.8+1$ & ORL $M$ & 4 Schmidt-Olt+ & 10448 \\
\hline
\end{tabular}




\begin{tabular}{|c|c|c|c|c|c|c|}
\hline Target & $\ln c$ & $\begin{array}{l}\text { Emin } \\
(M e V)\end{array}$ & $\begin{array}{l}\text { Emax } \\
(M e V)\end{array}$ & Lob W & Author & No. \\
\hline $1510 y$ & $\sigma(E)$ & & & & & \\
\hline $\begin{array}{l}141 \mathrm{Pr} \\
154 \mathrm{Gd}\end{array}$ & $\begin{array}{r}14 \mathrm{~N} \\
0\end{array}$ & $\begin{array}{l}9.2+1 \\
8.0+1\end{array}$ & $1.3+2$ & $\begin{array}{l}\text { ORL M } \\
\text { GRN } \stackrel{E}{E}\end{array}$ & $\begin{array}{l}\text { M Schridt-Ott+ } \\
\text { E Chraidershat }\end{array}$ & $\begin{array}{r}10447 \\
8488\end{array}$ \\
\hline${ }^{152} \mathrm{Dy}$ & portl & I relat & tive ol & (E) & & \\
\hline $\begin{array}{l}146 \mathrm{Nd} \\
154 \mathrm{Gd}\end{array}$ & $\begin{array}{r}{ }_{12} \mathrm{c} \\
0\end{array}$ & $\begin{array}{l}1.0+2 \\
8 \cdot 0+1\end{array}$ & & $\begin{array}{l}\text { GRN E } \\
\text { GRN E }\end{array}$ & $\begin{array}{l}\text { E Jansent } \\
\text { E Jangent }\end{array}$ & $\begin{array}{r}10016 \\
8490\end{array}$ \\
\hline $1320 y$ & portl & I $\sigma(E)$ & & & & \\
\hline $\begin{array}{l}154 \mathrm{Gd} \\
152 \mathrm{Dy}\end{array}$ & $\begin{array}{c}\text { O } \\
\text { overo }\end{array}$ & $\begin{array}{l}6.7+1 \\
\text { e } v_{y}\end{array}$ & $1.0+2$ & GRN E & E Jensent & 8489 \\
\hline $\begin{array}{l}190 \mathrm{Nd} \\
19 \mathrm{EO}\end{array}$ & $\begin{array}{l}{ }^{12} \mathrm{C} \\
v_{y}\end{array}$ & $1.0+2$ & $1.2+5$ & GRN E & E Ochelst & 10020 \\
\hline $145 \mathrm{No}$ & $12 \mathrm{C}$ & NDG & & LND E & E Cartent & 10018 \\
\hline${ }^{152} \mathrm{Dy}$ & $v_{n}$ & & & & & \\
\hline $146 \mathrm{Nd}$ & ${ }^{12} \mathrm{c}$ & NDG & & LND E & E Carlent & 10017 \\
\hline $1520 y$ & $O(E)$ & & & & & \\
\hline $\begin{array}{l}154 \mathrm{Gd} \\
153 \mathrm{Oy}\end{array}$ & $\begin{array}{c}a \\
\text { portl }\end{array}$ & $\begin{array}{l}5.7+1 \\
1 \text { O(E) }\end{array}$ & $1.3+2$ & GRN $E$ & E Chaleleushat & 8491 \\
\hline $\begin{array}{l}154 \mathrm{Gd} \\
155 \mathrm{Gd}\end{array}$ & : & $\begin{array}{l}6.7+1 \\
6.7+1\end{array}$ & $\begin{array}{l}1.0+2 \\
1.0+2\end{array}$ & $\begin{array}{l}\text { GRN E } \\
\text { GRN E }\end{array}$ & $\begin{array}{l}\text { E Jonsent } \\
\text { E Jangent }\end{array}$ & $\begin{array}{l}8492 \\
8498\end{array}$ \\
\hline $1530 y$ & avera & e $v_{y}$ & & & & \\
\hline $\begin{array}{l}150 \mathrm{Nd} \\
153_{\mathrm{Dy}}\end{array}$ & $\begin{array}{l}{ }^{12} C \\
O(E)\end{array}$ & $1.0+2$ & $1.2+2$ & GRN E & E Ochelst & 10020 \\
\hline $\begin{array}{l}154 \mathrm{Gd} \\
154 \mathrm{OY}\end{array}$ & $\begin{array}{c}0 \\
\text { partl }\end{array}$ & $\begin{array}{l}5.6+1 \\
1 \sigma(E)\end{array}$ & $8.4+1$ & GRN E & E Chrieleushat & 8493 \\
\hline $\begin{array}{l}154 \mathrm{Gd} \\
156 \mathrm{Gd}\end{array}$ & : & $\begin{array}{l}5 \cdot 0+1 \\
6 \cdot 7+1\end{array}$ & $\begin{array}{l}1.0+2 \\
1.0+2\end{array}$ & $\begin{array}{l}\text { GRN E } \\
\text { GRN E }\end{array}$ & $\begin{array}{l}\text { E Chmieleoshot } \\
\text { E Jonsent }\end{array}$ & $\begin{array}{l}8494 \\
8500\end{array}$ \\
\hline $1540 y$ & ovore & e $v_{y}$ & & & & \\
\hline $150 \mathrm{Nd}$ & ${ }^{12} \mathrm{c}$ & $1.0+2$ & $1.2+2$ & GRN E & E Ockelst & 10020 \\
\hline
\end{tabular}

\begin{tabular}{|c|c|c|c|c|c|c|}
\hline Target & Inc & $\begin{array}{l}\text { Emin } \\
(\mathrm{MeV})\end{array}$ & $\begin{array}{l}\text { Emax } \\
(\operatorname{MeV})\end{array}$ & Lab & Author & No. \\
\hline${ }^{154} \mathrm{Dy}$ & $O(E)$ & & & & & \\
\hline $154 \mathrm{Gd}$ & 0 & $4.7+1$ & $1.1+2$ & GRN & E Chalelewshat & 8495 \\
\hline${ }^{155} \mathrm{Dy}$ & $O(E)$ & & & & & \\
\hline $162 \mathrm{Dy}$ & 。 & $1.2+2$ & & OSA & E Elirit & 8537 \\
\hline $1550 y$ & partlo & $O(E)$ & & & & \\
\hline $\begin{array}{l}154 \mathrm{Gd} \\
160 \mathrm{Gd}\end{array}$ & $:$ & $\begin{array}{l}4.7+1 \\
5.0+1\end{array}$ & $\begin{array}{l}1.1+2 \\
1.3+2\end{array}$ & $\begin{array}{l}\text { GRN } \\
\text { GRN }\end{array}$ & $\begin{array}{l}\text { E Chmielewshat } \\
\text { E Chmielewshat }\end{array}$ & $\begin{array}{l}8497 \\
8506\end{array}$ \\
\hline${ }^{155} \mathrm{Dy}$ & overag & ge $v_{y}$ & & & & \\
\hline $150 \mathrm{Nd}$ & ${ }^{12} \mathrm{C}$ & $1.0+2$ & $1.2+2$ & GRN & E Ockelst & 10020 \\
\hline${ }^{155} \mathrm{Oy}$ & $\sigma(E)$ & & & & & \\
\hline${ }^{154} \mathrm{Gd}$ & a & $4 \cdot 7+1$ & $1.1+2$ & GRN & E Chaielewshat & 8496 \\
\hline${ }^{156} \mathrm{Dy}$ & $\sigma(E)$ & & & & & \\
\hline $1620 y$ & : & $1.2+2$ & & OSA & E Elirit & 8537 \\
\hline${ }^{156} \mathrm{Dy}$ & partlo & l overa & s ge $v_{y}$ & & & \\
\hline $180 \mathrm{Gd}$ & a & $7.5+1$ & $1.1+2$ & GRN & E De-Voigt+ & 8507 \\
\hline${ }^{136} \mathrm{Dy}$ & partio & $10(E)$ & & & & \\
\hline $\begin{array}{l}160 \mathrm{Gd} \\
150^{\circ}\end{array}$ & a & $\begin{array}{l}7.2+1 \\
3.0+1\end{array}$ & $8.0+1$ & $\begin{array}{l}\text { GRN } \\
\text { BNL }\end{array}$ & $\begin{array}{l}\text { E Chalelewshat } \\
\text { E Kolata+ }\end{array}$ & $\begin{array}{l}8508 \\
4323\end{array}$ \\
\hline${ }^{156} \mathrm{Oy}$ & reloti & ive $I_{\text {, }}$ & & & & \\
\hline $\begin{array}{l}155 \mathrm{Gd} \\
159 \mathrm{~Tb}\end{array}$ & a & $\begin{array}{l}4.5+1 \\
4.4+1\end{array}$ & & $\begin{array}{l}\text { IKO } \\
\text { IKO }\end{array}$ & $\begin{array}{l}\text { E De-Boert } \\
\text { E De-Boort }\end{array}$ & $\begin{array}{l}8501 \\
4320\end{array}$ \\
\hline${ }^{156} \mathrm{Dy}$ & overos & $v_{y}$ & & & & \\
\hline $\begin{array}{l}150 \mathrm{Nd} \\
158 \mathrm{OY}\end{array}$ & $\begin{array}{l}\text { Ia } C \\
O(E)\end{array}$ & $1.0+2$ & $1.2+2$ & GRN & E Ockelst & 10020 \\
\hline $\begin{array}{l}160 \mathrm{Gd} \\
160 \mathrm{Gd}\end{array}$ & $:$ & $\begin{array}{l}8 \cdot 0+1 \\
4.0+1\end{array}$ & $\begin{array}{l}1.3+2 \\
1.2+2\end{array}$ & $\begin{array}{l}\text { GRN } \\
\text { GRN }\end{array}$ & $\begin{array}{l}\text { E Chmieleoshat } \\
\text { E Ochelst }\end{array}$ & $\begin{array}{l}8509 \\
8523\end{array}$ \\
\hline${ }^{157} \mathrm{Dr}$ & $O(E)$ & & & & & \\
\hline $1820 y$ & $a$ & $1.2+2$ & & OSA & E EJifi+ & 8537 \\
\hline
\end{tabular}




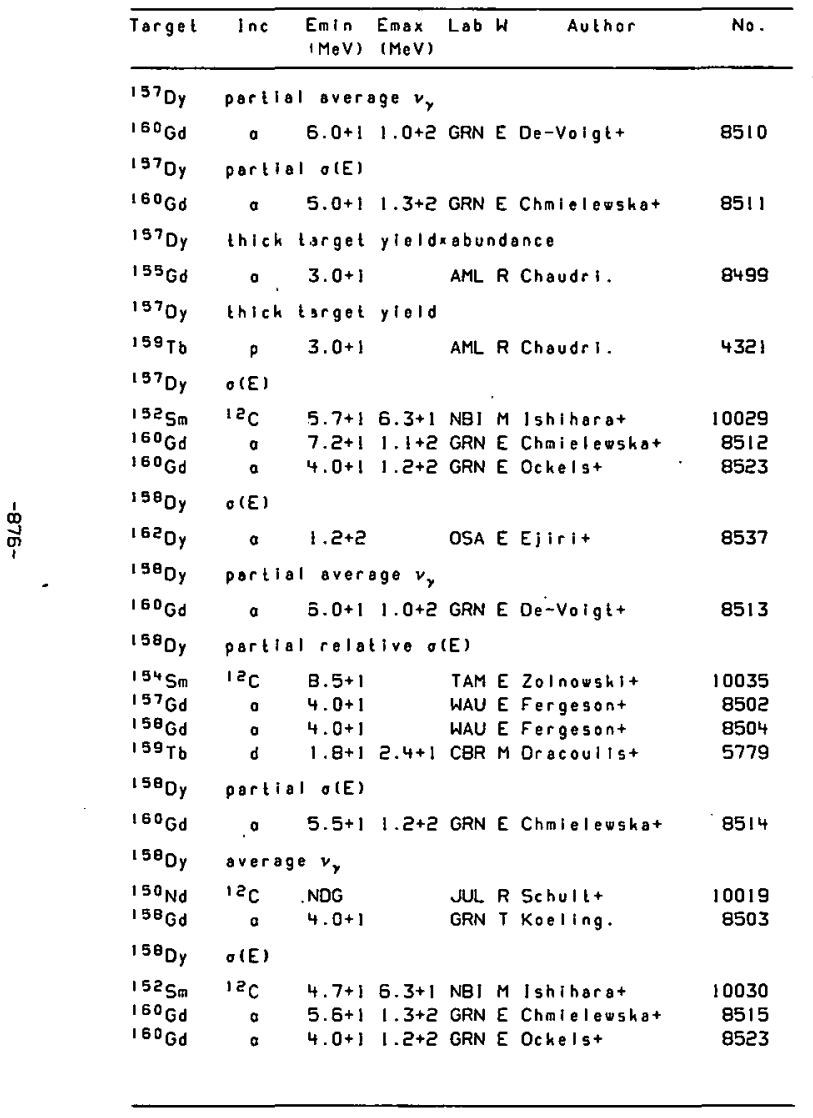

\begin{tabular}{|c|c|c|c|c|c|c|}
\hline Target & Inc & $\begin{array}{l}\text { Emin } \\
(M e V)\end{array}$ & $\begin{array}{l}\text { Emax } \\
(\operatorname{MeV})\end{array}$ & Lab & Author & No. \\
\hline${ }^{159} \mathrm{Dy}$ & $O(E)$ & & & & & \\
\hline $182 \mathrm{Dy}$ & a & $1.2+2$ & & OSA & E Elirit & 8537 \\
\hline${ }^{15} \theta^{\circ y}$ & porti & 1 avera & age $v_{y}$ & & & \\
\hline $160 \mathrm{Gd}$ & 。 & $4.5+1$ & $7.0+1$ & GRN & E De-Volglt & 8516 \\
\hline${ }^{159} \mathrm{Dy}$ & portl & I O E & & & & \\
\hline $150 \mathrm{Gd}$ & 0 & $4.7+1$ & $1.1+2$ & GRN & E Chmielewshat & 8518 \\
\hline${ }^{159} \mathrm{Dy}$ & $\sigma(E)$ & & & & & \\
\hline $\begin{array}{l}160 \mathrm{Gd} \\
150 \mathrm{Gd} \\
159 \mathrm{~Tb}\end{array}$ & $\begin{array}{l}0 \\
7 \mathrm{Li}\end{array}$ & $\begin{array}{l}4.7+1 \\
4.0+1 \\
2.5+1\end{array}$ & $\begin{array}{l}1.1+2 \\
1.2+2 \\
3.5+1\end{array}$ & $\begin{array}{l}\text { GRN } \\
\text { GRN } \\
\text { NB! }\end{array}$ & $\begin{array}{l}\text { E Choreleasiat } \\
\text { E Ochelst } \\
\text { M Ishihara+ }\end{array}$ & $\begin{array}{l}8517 \\
8523 \\
9452\end{array}$ \\
\hline $1500 y$ & $O(E)$ & & & & & \\
\hline${ }^{162} \mathrm{Dy}$ & a & $1.2+2$ & & OSA & E Ejirlt & 8537 \\
\hline${ }^{160} D_{y}$ & porti & 1 avere & age $v_{y}$ & & & \\
\hline $160 \mathrm{Gd}$ & - & $4.0+1$ & $8.0+1$ & GRN & E De-Voiglt & 8520 \\
\hline $1600 y$ & perti & $1 O(E)$ & & & & \\
\hline $160 \mathrm{Gd}$ & . & $5.0+1$ & $1.3+2$ & GRN & E Charielewshat & 8521 \\
\hline $1600 y$ & avera & $\theta v_{y}$ & & & & \\
\hline $160 \mathrm{Gd}$ & a & $4.0+1$ & $4.9+1$ & GRN & T Koeling. & 8519 \\
\hline $1600 y$ & $O(E)$ & & & & & \\
\hline $\begin{array}{l}160 \mathrm{Gd} \\
160 \mathrm{Gd} \\
159 \mathrm{~Tb} \\
159 \mathrm{~Tb}\end{array}$ & $\begin{array}{c}a \\
a \\
{ }^{3} \mathrm{He} \\
{ }^{7} \mathrm{Li}\end{array}$ & $\begin{array}{l}4.7+1 \\
4 \cdot 0+1 \\
7.0+1 \\
2.4+1\end{array}$ & $\begin{array}{l}5.6+1 \\
1.2+2 \\
9.0+1 \\
3 \cdot 5+1\end{array}$ & $\begin{array}{l}\text { GRN } \\
\text { GRN } \\
\text { OSA } \\
\text { NBI }\end{array}$ & $\begin{array}{l}\text { E Chmielewskat } \\
\text { E Ochelst } \\
\text { M Matsuohat } \\
\text { M Ishiharat }\end{array}$ & $\begin{array}{l}8522 \\
8523 \\
6492 \\
9453\end{array}$ \\
\hline $162_{D y}$ & parti & I rela & tive of & $(E)_{y}$ & & \\
\hline $1620 y$ & 160 & $4.9+1$ & $7.2+1$ & CRL & E Guidryt & 11439 \\
\hline${ }^{162} \mathrm{DY}$ & parti & I $O(E)$ & & & & \\
\hline $165 \mathrm{Ho}$ & $p$ & $6.0+1$ & & OSA & E Ejirit & 4330 \\
\hline $1620 y$ & $\theta(E)$ & & & & & \\
\hline $164 \mathrm{Dy}$ & $a$ & $1.2+2$ & & OSA & EEjirit & 8554 \\
\hline
\end{tabular}




\begin{tabular}{|c|c|c|c|c|c|c|}
\hline Target & $\ln c$ & $\begin{array}{l}\text { Emin } \\
(M e V)\end{array}$ & $\begin{array}{l}\text { Emax } \\
(\operatorname{MeV})\end{array}$ & Lab & Author & No. \\
\hline${ }^{163} \mathrm{Dy}$ & $\sigma(E)$ & & & & & \\
\hline $164 \mathrm{Dy}$ & $a$ & $1.2+2$ & & OSA & E Eilrit & 8554 \\
\hline $164 \mathrm{Dy}$ & $O(E)$ & & & & & \\
\hline $1640 y$ & a & $1.2+2$ & & OSA & E Eirit & 8554 \\
\hline${ }^{165} \mathrm{Dy}_{\mathrm{y}}$ & $\sigma(E)$ & & & & & \\
\hline $164 \mathrm{Dy}$ & a & $1.2+2$ & & OSA & E Eirit & 8554 \\
\hline $1660 y$ & $O(E)$ & & & & & \\
\hline${ }^{164} \mathrm{Dy}$ & a & $1.2+2$ & & OSA & E Ejiri+ & 8554 \\
\hline $14 \mathrm{\theta}_{\mathrm{HO}}$ & produc & tyleld & & & & \\
\hline $142 \mathrm{sm}$ & ${ }^{10} \mathrm{~B}$ & $8.5+1$ & $9.6+1$ & TAM & E Totht & 9543 \\
\hline $149_{\mathrm{Ho}}$ & produc & t yield & & & & \\
\hline $1425 m$ & ${ }^{10} \mathrm{~B}$ & $8.5+1$ & $9.6+1$ & TAM & E Totht & 9544 \\
\hline $191 \mathrm{HO}$ & partia & $10(E)$ & & & & \\
\hline $\begin{array}{l}1185 n \\
1185 n \\
1215 b \\
1215 b\end{array}$ & $\begin{array}{l}40 \mathrm{Ar} \\
40 \mathrm{Ar} \\
40 \mathrm{Ar} \\
40_{\mathrm{Ar}}\end{array}$ & $\begin{array}{l}1.5+2 \\
2.0+2 \\
1.5+2 \\
2.3+2\end{array}$ & $\begin{array}{l}2.8+2 \\
2.6+2 \\
2.8+2 \\
2.9+2\end{array}$ & $\begin{array}{l}\text { PAR } \\
\text { KFK } \\
\text { PAR } \\
\text { KFK }\end{array}$ & $\begin{array}{l}\text { E Gaurint } \\
\text { C Muenzel+ } \\
\text { E Gauvint } \\
\text { C Muenzel+ }\end{array}$ & $\begin{array}{l}12245+ \\
12246 \\
12282+ \\
12283\end{array}+$ \\
\hline $1519 \mathrm{Ho}$ & relot & ive olE & & & & \\
\hline $\begin{array}{l}142_{\mathrm{NO}} \\
1519 \mathrm{HO}\end{array}$ & $\begin{array}{l}{ }^{14} \mathrm{~N} \\
O(E)\end{array}$ & $5.8+1$ & $9 . E+1$ & ORL & MSehmidt-Ott+ & 10449 \\
\hline $\begin{array}{l}142 \mathrm{Nd} \\
151 \mathrm{CO}^{\circ}\end{array}$ & $\begin{array}{l}{ }^{14} \mathrm{~N} \\
\text { relat }\end{array}$ & $\begin{array}{l}7.3+1 \\
\text { ive } \sigma<E\end{array}$ & E) & ORL & MSchmidt-Ott+ & 10450 \\
\hline $\begin{array}{l}142_{\mathrm{No}} \\
151 \mathrm{a} \mathrm{Ho}\end{array}$ & $\begin{array}{l}{ }^{14} N \\
O(E)\end{array}$ & $5.8+1$ & $9.8+1$ & ORL & MSchmidt-Ott+ & 10451 \\
\hline $\begin{array}{l}142_{\mathrm{Nd}} \\
152_{\mathrm{Ho}}\end{array}$ & $\begin{array}{l}{ }^{14} \mathrm{~N} \\
O(E)\end{array}$ & $7.3+1$ & $7.9+1$ & ORL & MSthmidt-Ott+ & 10452 \\
\hline $\begin{array}{l}116 \mathrm{Sn} \\
152_{\mathrm{Ho}}\end{array}$ & $\begin{array}{l}40 \mathrm{Ar} \\
\text { port } 1 \mathrm{a}\end{array}$ & $\begin{array}{l}5.0+1 \\
1 \text { OIE) }\end{array}$ & $1.2+2$ & PAR & E Gaurint & 12234 \\
\hline $118_{5 n}$ & ${ }^{+O} \mathrm{Ar}$ & $1.5+2$ & $2.8+2$ & PAR & E Gauvint & $12248+$ \\
\hline
\end{tabular}

\begin{tabular}{|c|c|c|c|c|c|c|}
\hline Target & $\operatorname{linc}$ & $\begin{array}{l}E_{\min } \\
(\mathrm{MeV})\end{array}$ & $\begin{array}{l}\text { Emax } \\
(\operatorname{MeV})\end{array}$ & Lab & Author & No. \\
\hline${ }^{152} \mathrm{Ho}_{\mathrm{O}}$ & partia & $1 \sigma(E)$ & & & & \\
\hline $\begin{array}{l}1185 n \\
1215 b \\
1215 b\end{array}$ & $\begin{array}{l}40 \mathrm{Ar} \\
40 \mathrm{Ar} \\
40 \mathrm{Ar}\end{array}$ & $\begin{array}{l}1.7+2 \\
1.5+2 \\
2.3+2\end{array}$ & $\begin{array}{l}2.5+2 \\
2.8+2 \\
2.9+2\end{array}$ & $\begin{array}{l}\text { KFK } \\
\text { PAR } \\
\text { KFK }\end{array}$ & $\begin{array}{l}\text { C Muerzel+ } \\
\text { E Gausint } \\
\text { C Muerzel+ }\end{array}$ & $\begin{array}{l}12249 \\
12284 \\
12285\end{array}+$ \\
\hline $15 \mathrm{H}_{\mathrm{HO}}$ & $\sigma(E)$ & & & & & \\
\hline $\begin{array}{l}1185 \mathrm{5n} \\
1528 \mathrm{Ho}\end{array}$ & $\begin{array}{l}{ }^{40} \mathrm{Ar} \\
\mathrm{relat}\end{array}$ & $\begin{array}{l}5.0+1 \\
\text { lve } 018\end{array}$ & E) & PAR & E Gauvint & 12247 \\
\hline $\begin{array}{l}14 \Sigma_{\mathrm{Nd}} \\
1529 \mathrm{HO}\end{array}$ & $\begin{array}{l}14 N \\
O(E)\end{array}$ & $5.8+1$ & $9.8+1$ & ORL & M Schaidt-Ott+ & 10453 \\
\hline $\begin{array}{l}142_{\mathrm{Nd}} \\
152 \mathrm{O}_{\mathrm{Ho}}\end{array}$ & $\begin{array}{l}14 \mathrm{~N} \\
\text { relat }\end{array}$ & $\begin{array}{l}7.3+1 \\
\text { ive ole }\end{array}$ & E) $7.5+1$ & ORL & M Schaidt-Ot t+ & 10454 \\
\hline $\begin{array}{l}142^{\mathrm{NO}} \\
152 \square_{\mathrm{HO}}\end{array}$ & $\begin{array}{l}{ }^{14} \mathrm{~N} \\
O(E)\end{array}$ & $5.8+1$ & $9.8+1$ & ORL & M Schaidl-Otlt & 10455 \\
\hline $\begin{array}{l}142 \mathrm{Nd} \\
153_{\mathrm{Ho}}\end{array}$ & $\begin{array}{l}14 \mathrm{~N} \\
\text { partia }\end{array}$ & $\begin{array}{l}7.3+1 \\
1 \text { prod }\end{array}$ & $\begin{array}{l}7.9+1 \\
\text { uel y } 10\end{array}$ & $\begin{array}{l}\text { ORL } \\
\text { eld }\end{array}$ & MSchmidt-Ott+ & 10456 \\
\hline $\begin{array}{l}122 \mathrm{Sn} \\
141 \mathrm{Pr}\end{array}$ & $\begin{array}{l}35 \mathrm{Cl} \\
150\end{array}$ & $\begin{array}{l}\text { NDG } \\
\text { NDG }\end{array}$ & & $\begin{array}{l}\text { YAL } \\
\text { YAL }\end{array}$ & $\begin{array}{l}\text { E Radford+ } \\
\text { E Radford+ }\end{array}$ & $\begin{array}{l}12139 \\
11389\end{array}$ \\
\hline${ }^{155} \mathrm{Ho}$ & partla & I relat & trve ol & (E) & & \\
\hline $\begin{array}{l}145 \mathrm{Nd} \\
155 \mathrm{Ho}\end{array}$ & ${ }_{i_{\gamma}}^{14} \mathrm{~N}$ & $6.5+1$ & $8.6+1$ & GRE & E Foint & 10457 \\
\hline $\begin{array}{l}150 \mathrm{Sm} \\
157 \mathrm{Ho}\end{array}$ & $\begin{array}{l}10 \theta \\
\sigma(E)\end{array}$ & $6.0+1$ & $7.0+1$ & TAM & E Devoust & 9545 \\
\hline $\begin{array}{l}16 \mathrm{E}_{\mathrm{y}} \\
15 \mathrm{~B}_{\mathrm{Ho}}\end{array}$ & $a(E)$ & $1.2+2$ & & OSA & E Ejir + & 8543 \\
\hline $\begin{array}{l}162 \mathrm{OY} \\
199_{\mathrm{Ho}}\end{array}$ & $\sigma(E)$ & $1.2+2$ & & OSA & E Ejirit & 8543 \\
\hline $\begin{array}{l}16 e_{0 y} \\
160 \mathrm{Ho}\end{array}$ & $\begin{array}{c}a \\
\text { partia }\end{array}$ & $\begin{array}{l}1.2+2 \\
1.0(E)\end{array}$ & & OSA & E Ejirit & 8543 \\
\hline $165 \mathrm{Ho}_{0}$ & $p$ & $6.0+1$ & & OSA & EEjirit & 4330 \\
\hline
\end{tabular}




\begin{tabular}{|c|c|c|c|c|c|c|}
\hline Targat & $\ln c$ & $\begin{array}{l}\text { Emin } \\
\vdots \mathrm{MeV}\}\end{array}$ & $\begin{array}{l}E \max \\
(\operatorname{MeV})\end{array}$ & Lab & Author & No. \\
\hline $160 \mathrm{Ho}$ & \multicolumn{6}{|c|}{ colative o(E) } \\
\hline${ }^{154} \mathrm{Dy}$ & ${ }^{3} \mathrm{He}$ & $6.5+1$ & & OSA & E Sahait & 6495 \\
\hline $160^{\circ} \mathrm{O}$ & \multicolumn{6}{|l|}{$O(E)$} \\
\hline $162 D_{y}$ & व & $1.2+2$ & & OSA & E Elirit & 8543 \\
\hline $161 \mathrm{Ho}_{0}$ & \multicolumn{6}{|c|}{ partial o(E) } \\
\hline${ }^{165} \mathrm{Ho}$ & p & $6.0+1$ & & OSA & E Eilrit & 4330 \\
\hline $161 \mathrm{HO}$ & \multicolumn{6}{|c|}{ relatlve o(E) } \\
\hline $164 \mathrm{Dy}$ & ${ }^{3} \mathrm{He}$ & $5.5+1$ & & OSA & E Sahalt & 6495 \\
\hline $161 \mathrm{Ho}$ & \multicolumn{6}{|l|}{$O(E)$} \\
\hline${ }^{162} \mathrm{O}_{y}$ & 。 & $1.2+2$ & & $O S A$ & E Ejiri+ & 8543 \\
\hline $16 \mathrm{H}_{\mathrm{O}}$ & \multicolumn{6}{|c|}{ partial o(E) } \\
\hline $165 \mathrm{Ho}$ & $p$ & $6.0+1$ & & OSA & E Ejirit & 4330 \\
\hline $162_{\mathrm{Ho}}$ & \multicolumn{6}{|c|}{ relative $\sigma(E)$} \\
\hline${ }^{164} \mathrm{Oy}$ & ${ }^{3} \mathrm{He}$ & $6.5+1$ & & OSA & E Sohait & 6495 \\
\hline $163 \mathrm{Ho}$ & \multicolumn{6}{|c|}{ partlal o(E) } \\
\hline${ }^{165} \mathrm{Ho}$ & $p$ & $6.0+1$ & & OSA & E Eltrit & 4330 \\
\hline $183 \mathrm{Ho}$ & \multicolumn{6}{|c|}{ relative o(E) } \\
\hline${ }^{164} \mathrm{Dy}$ & ${ }^{3} \mathrm{He}$ & $6.5+1$ & & $O S A$ & E Sohait & 6495 \\
\hline $165_{\mathrm{Ho}}$ & \multicolumn{6}{|c|}{ portial $\sigma(E)$} \\
\hline $165 \mathrm{Ho}$ & $p$ & $6.0+1$ & & OSA & EEliti+ & 4330 \\
\hline${ }^{185} \mathrm{Ho}$ & \multicolumn{6}{|c|}{ rolative o(E) } \\
\hline $164 \mathrm{DY}$ & ${ }^{3} \mathrm{He}$ & $6.5+1$ & & OSA & E Sakait & 6495 \\
\hline$z=68$ & \multicolumn{6}{|c|}{ relstive o(E) } \\
\hline $\begin{array}{l}1620 y \\
1640 y\end{array}$ & $a$ & $\begin{array}{l}5 \cdot 0+1 \\
5 \cdot 0+1\end{array}$ & $\begin{array}{l}1.5+5 \\
1.2+5\end{array}$ & $\begin{array}{l}\text { OSA } \\
\text { OSA }\end{array}$ & $\begin{array}{l}\text { EElirit } \\
\text { EEjirit }\end{array}$ & $\begin{array}{l}8536 \\
8553\end{array}$ \\
\hline $151 \mathrm{Er}$ & \multicolumn{6}{|c|}{ relative o(E) } \\
\hline $1640 y$ & $a$ & $5.0+1$ & $1.2+2$ & OSA & E Ejirit & 8558 \\
\hline
\end{tabular}

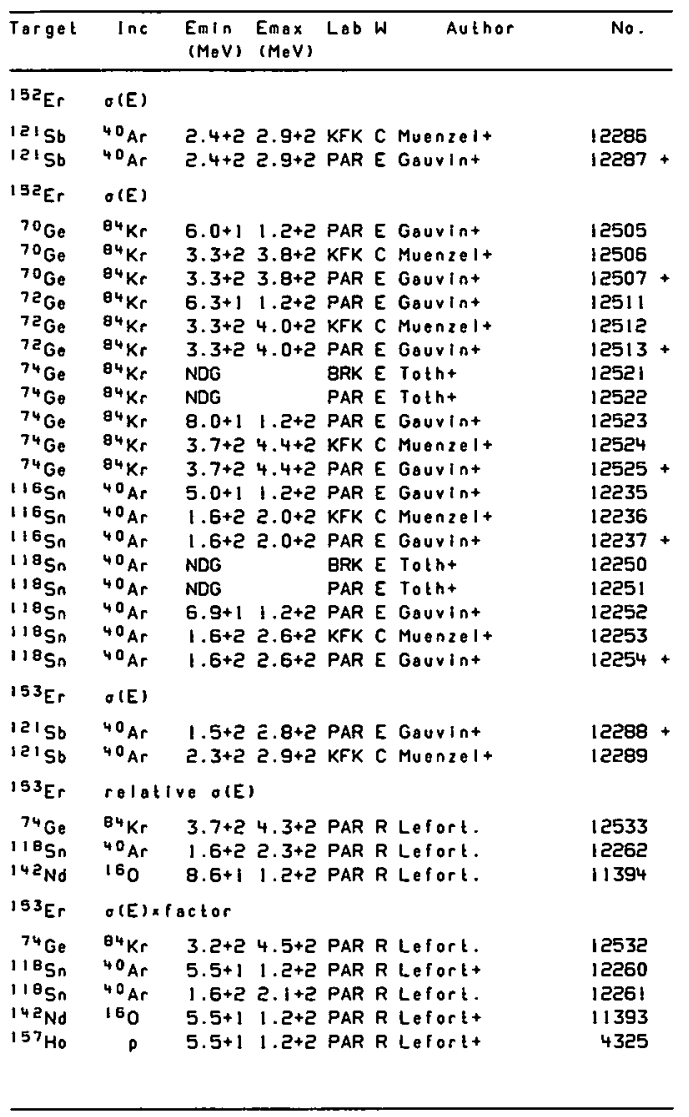




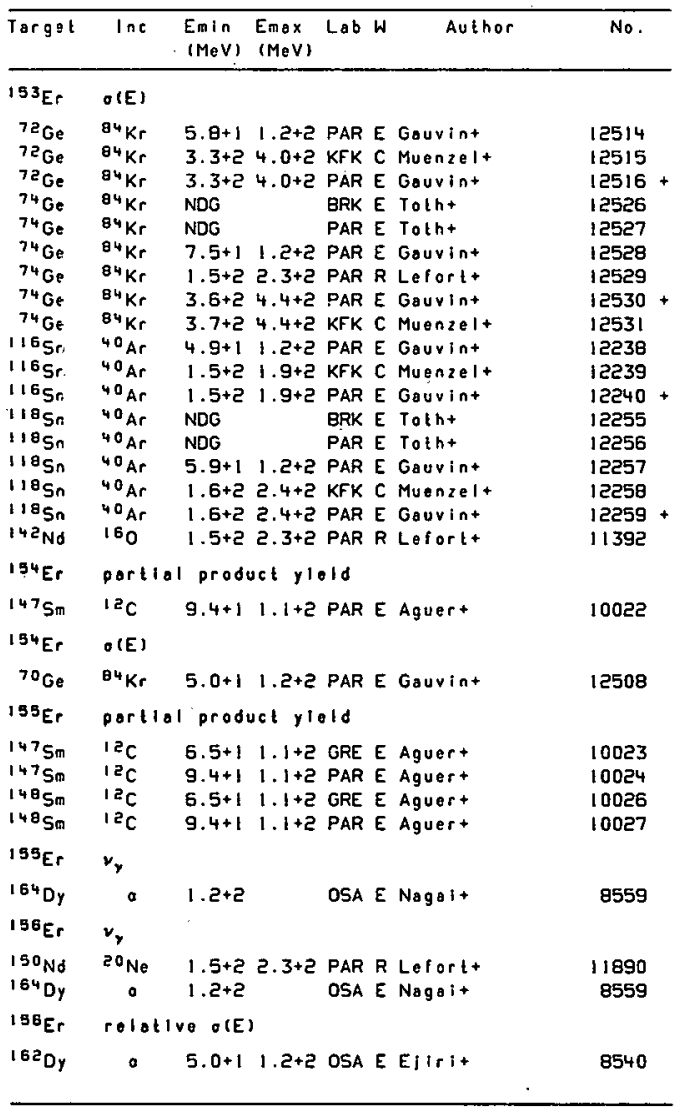

\begin{tabular}{|c|c|c|c|c|c|c|}
\hline Target & $\ln c$ & $\begin{array}{l}\text { Emin } \\
(M e V)\end{array}$ & $\begin{array}{l}\text { Emax } \\
\text { (MeV) }\end{array}$ & Lab & Asthor & No. \\
\hline${ }^{156} \mathrm{Er}$ & $O(E)$ & & & & & \\
\hline $1620 y$ & a & $7.0+1$ & $9.0+1$ & OSA & E EJirl+ & 8539 \\
\hline${ }^{157} \mathrm{Er}$ & par : 1 & I relat & tive ol & (E) & & \\
\hline $1620 y$ & a & $9.0+1$ & & OSA & E Ejiri- & 8525 \\
\hline $157 \mathrm{Er}$ & $v_{y}$ & & & & & \\
\hline $\begin{array}{l}150 \mathrm{Nd} \\
162^{\mathrm{Dy}} \\
154 \mathrm{Dy}\end{array}$ & $\begin{array}{c}20 \mathrm{Ne} \\
a \\
a\end{array}$ & $\begin{array}{l}1.5+2 \\
9.0+1 \\
1.2+2\end{array}$ & $2.3+2$ & $\begin{array}{l}\text { PAR } \\
\text { OSA } \\
\text { OSA }\end{array}$ & $\begin{array}{l}\text { R Lefort+ } \\
\text { EEjirl+ } \\
\text { E Nagal+ }\end{array}$ & $\begin{array}{r}11890 \\
8526 \\
8559\end{array}$ \\
\hline${ }^{157} \mathrm{Er}$ & relot & ve $\sigma(E)$ & & & & \\
\hline $1620 y$ & a & $5.0+1$ & $1.2+2$ & OSA & E Ejirit & 8540 \\
\hline $157 \mathrm{Er}$ & $O(E)$ & & & & & ' \\
\hline $\begin{array}{l}1620 y \\
1640 y\end{array}$ & : & $\begin{array}{l}7.0+1 \\
1.2+2\end{array}$ & $9.0+1$ & $\begin{array}{l}\text { OSA } \\
\text { OSA }\end{array}$ & $\begin{array}{l}\text { E Ejirit } \\
\text { E Ejiri+ }\end{array}$ & $\begin{array}{l}8539 \\
8557\end{array}$ \\
\hline${ }^{150} \mathrm{Er}$ & $v_{y}$ & & & & & \\
\hline $\begin{array}{l}150 \mathrm{Nd} \\
164 \mathrm{Oy}\end{array}$ & ${ }_{0}^{20} \mathrm{Ne}$ & $\begin{array}{l}1.5+2 \\
1.2+2\end{array}$ & $2.3+2$ & $\begin{array}{l}\text { PAR } \\
\text { OSA }\end{array}$ & $\begin{array}{l}\text { R Lefor:t } \\
\text { E Nagal+ }\end{array}$ & $\begin{array}{r}11890 \\
8559\end{array}$ \\
\hline${ }^{158} \mathrm{Er}$ & colot & vo $O(E)$ & & & & \\
\hline $\begin{array}{l}162 \mathrm{Oy} \\
164 \mathrm{Oy} \\
169 \mathrm{Ho}\end{array}$ & $\underset{3}{a}$ & $\begin{array}{l}5.0+1 \\
5.0+1 \\
1.0+2\end{array}$ & $\begin{array}{l}1.2+2 \\
1.2+2\end{array}$ & $\begin{array}{l}\text { OSA } \\
\text { OSA } \\
\text { OSA }\end{array}$ & $\begin{array}{l}\text { E Ejirit } \\
\text { E Ejirit } \\
\text { E Sohoit }\end{array}$ & $\begin{array}{l}8540 \\
8558 \\
6498\end{array}$ \\
\hline${ }^{15 \theta} \mathrm{Er}$ & $O(E)$ & & & & & \\
\hline $\begin{array}{l}152 \mathrm{Dy} \\
164 \mathrm{Dy}\end{array}$ & : & $\begin{array}{l}7.0+1 \\
1.2+2\end{array}$ & $9.0+1$ & $\begin{array}{l}\text { OSA } \\
\text { OSA }\end{array}$ & $\begin{array}{l}\text { E Ejirit } \\
\text { E Ejirit }\end{array}$ & $\begin{array}{l}8539 \\
8557\end{array}$ \\
\hline${ }^{158} \mathrm{Er}$ & porti & I relot & theo & (E) & & \\
\hline $\begin{array}{l}162 \mathrm{Dy} \\
150^{\mathrm{Er}}\end{array}$ & $v_{y}^{0}$ & $9.0+1$ & & OSA & E Elirl+ & 8527 \\
\hline $\begin{array}{l}150 \mathrm{Nd} \\
1620 \mathrm{Dy} \\
164 \mathrm{Dy}\end{array}$ & $\begin{array}{c}20 \mathrm{Ne} \\
a \\
a\end{array}$ & $\begin{array}{l}1.5+2 \\
9.0+1 \\
1.2+2\end{array}$ & $2.3+2$ & $\begin{array}{l}\text { PAR } \\
\text { OSA } \\
\text { OSA }\end{array}$ & $\begin{array}{l}\text { R Lefort- } \\
\text { E Elirit } \\
\text { E Nagolt }\end{array}$ & $\begin{array}{r}11890 \\
8528 \\
8559\end{array}$ \\
\hline${ }^{159} \mathrm{Er}$ & relot & ve $O(E)$ & & & & \\
\hline $1620 y$ & $a$ & $5.0+1$ & $1.2+2$ & OSA & $E E_{j}|r|+$ & 8540 \\
\hline
\end{tabular}




\begin{tabular}{|c|c|c|c|c|c|c|}
\hline Target & $\operatorname{lnc}$ & $\begin{array}{l}\text { Emin } \\
\left(M_{\theta} V\right)\end{array}$ & $\begin{array}{l}\text { Emax } \\
(\operatorname{MeV})\end{array}$ & Lab & Author & No. \\
\hline${ }^{139} \mathrm{Er}$ & \multicolumn{3}{|c|}{ relalive e(E) } & \multicolumn{2}{|r|}{ • } & \\
\hline $\begin{array}{l}154 \mathrm{OY} \\
159 \mathrm{Er}\end{array}$ & $\begin{array}{c}a \\
O(E)\end{array}$ & $5.0+1$ & $1.2+2$ & OSA & E Ejirit & 8558 \\
\hline $\begin{array}{l}148_{\mathrm{Nd}} \\
162 \mathrm{Oy} \\
164 \mathrm{Oy}\end{array}$ & $\begin{array}{r}160 \\
a \\
a\end{array}$ & $\begin{array}{l}6.8+1 \\
7.0+1 \\
1.2+2\end{array}$ & $\begin{array}{l}8.4+1 \\
9.0+1\end{array}$ & $\begin{array}{l}\text { NBI } \\
\text { OSA } \\
\text { OSA }\end{array}$ & $\begin{array}{l}\text { R Hershind. } \\
\text { E Ejirit } \\
\text { E Ejirit }\end{array}$ & $\begin{array}{r}11398 \\
8539 \\
8557\end{array}$ \\
\hline $160 \mathrm{Er}$ & parti & 1 ayera & $\operatorname{ag\theta } v_{y}$ & & & \\
\hline $\begin{array}{l}150 \mathrm{Nd} \\
166 \mathrm{Er}\end{array}$ & ${ }^{20} \mathrm{Ne}$ & $\begin{array}{l}9 \cdot 4+1 \\
6 \cdot \theta+1\end{array}$ & $\begin{array}{l}1.1+2 \\
9.5+1\end{array}$ & $\begin{array}{l}\text { ORL } \\
\text { ORL }\end{array}$ & $\begin{array}{l}\text { E Sarantitest } \\
\text { E Sarantitest }\end{array}$ & $\begin{array}{r}11889 \\
8579\end{array}$ \\
\hline $180 \mathrm{Er}$ & part 1 & $1 v_{y}$ & & & & \\
\hline $165 \mathrm{Ho}$ & $p$ & $5.0+1$ & & OSA & E Ejirit & 4327 \\
\hline $160 \mathrm{Er}$ & parti & I relat & tive of & (E) & & \\
\hline $1620 y$ & a & $9.10+1$ & & OSA & E Elirit & 8529 \\
\hline${ }^{160} \mathrm{Er}$ & portl & I O(E) & & & & \\
\hline $\begin{array}{l}150 \mathrm{Nd} \\
166 \mathrm{Er}\end{array}$ & ${ }^{20} \mathrm{Ne}$ & $\begin{array}{l}1.1+2 \\
6.3+1\end{array}$ & $9.5+1$ & $\begin{array}{l}\text { ORL } \\
\text { ORL }\end{array}$ & $\begin{array}{l}\text { E Sarantlitest } \\
\text { E Sorantitest }\end{array}$ & $\begin{array}{r}11888 \\
8578\end{array}$ \\
\hline${ }^{160} \mathrm{Er}$ & overe & - $v_{v}$ & & & & \\
\hline $\begin{array}{l}145 \mathrm{Nd} \\
193 \mathrm{Sm}\end{array}$ & $\begin{array}{l}180 \\
120\end{array}$ & $\begin{array}{l}\text { NOG } \\
\text { NDG }\end{array}$ & & JUL & $\begin{array}{l}\text { R Schultt } \\
\text { R Sctioltt }\end{array}$ & $\begin{array}{l}11698 \\
10034\end{array}$ \\
\hline $180 \mathrm{Er}$ & $v_{y}$ & & & $=$ & & \\
\hline $\begin{array}{l}1620 y \\
1640 y\end{array}$ & $:$ & $\begin{array}{l}5.0+1 \\
1.2+2\end{array}$ & $1.2+2$ & OSA & $\begin{array}{l}\text { E Nagoit } \\
\text { E Nagoit }\end{array}$ & $\begin{array}{l}8530 \\
8559\end{array}$ \\
\hline${ }^{160} \mathrm{Er}$ & relat & vo $O(E)$ & & & & \\
\hline $\begin{array}{l}162 \mathrm{Dy} \\
164 \mathrm{Dy} \\
164 \mathrm{DY} \\
165 \mathrm{Ho}\end{array}$ & $\begin{array}{l}3^{\circ} \\
{ }^{3} \mathrm{He} \\
3^{3} \mathrm{He}\end{array}$ & $\begin{array}{l}5 \cdot 0+1 \\
6 \cdot 5+1 \\
5 \cdot 0+1 \\
1.0+2\end{array}$ & $\begin{array}{l}1.5+5 \\
1.5+5\end{array}$ & $\begin{array}{l}\text { OSA } \\
\text { OSA } \\
\text { OSA } \\
\text { OSA }\end{array}$ & $\begin{array}{l}\text { E Ejirit } \\
\text { E Sahoit } \\
\text { E Ejirit } \\
\text { E Sahait }\end{array}$ & $\begin{array}{l}8540 \\
5494 \\
8558 \\
6498\end{array}$ \\
\hline $160 \mathrm{Er}$ & O(E) & & & & & \\
\hline $\begin{array}{l}148 \mathrm{Nd} \\
152 \mathrm{Sm} \\
162 \mathrm{OH} \\
164 \mathrm{Oy}\end{array}$ & $\begin{array}{r}160 \\
120 \\
a \\
a\end{array}$ & $\begin{array}{l}5.6+1 \\
4.7+1 \\
7.0+1 \\
1.2+2\end{array}$ & $\begin{array}{l}0.4+1 \\
6.3+1 \\
9.0+1\end{array}$ & $\begin{array}{l}\text { NBI } \\
\text { NBI } \\
\text { OSA } \\
\text { OSA }\end{array}$ & $\begin{array}{l}\text { R Herskind. } \\
M \text { lshiharat } \\
\text { E EjIrit } \\
E \text { Ejtrit }\end{array}$ & $\begin{array}{r}11399 \\
10031 \\
8539 \\
8557\end{array}$ \\
\hline
\end{tabular}

\begin{tabular}{|c|c|c|c|c|c|c|}
\hline Target & $\operatorname{Inc}$ & $\begin{array}{l}\text { EmIn } \\
(M e V)\end{array}$ & $\begin{array}{l}\text { Emax } \\
(M e V)\end{array}$ & Lab & Author & No. \\
\hline${ }^{160} \mathrm{Er}$ & $O(E)$ & & & & & \\
\hline $165 \mathrm{Ho}$ & p & $6.0+1$ & & OSA & E Ejiri+ & 4331 \\
\hline $161 \mathrm{Er}$ & \multicolumn{6}{|c|}{ compound-nucleus $O(E)$} \\
\hline $\begin{array}{l}14 \mathrm{~B}_{\mathrm{Nd}} \\
150 \mathrm{Nd}\end{array}$ & $\begin{array}{l}180 \\
160\end{array}$ & $\begin{array}{l}6.9+1 \\
6.9+1\end{array}$ & $\begin{array}{l}7.8+1 \\
7.8+1\end{array}$ & $\begin{array}{l}\text { NQ1 } \\
\text { NE1 }\end{array}$ & $\begin{array}{l}\text { M Andersent } \\
\text { M Andersent }\end{array}$ & $\begin{array}{l}11702 \\
11405\end{array}$ \\
\hline $161 \mathrm{Er}$ & \multicolumn{6}{|c|}{ portlot compound-nucleus $O(E)$} \\
\hline $\begin{array}{l}14 \mathrm{~B}_{\mathrm{Nd}} \\
150_{\mathrm{Nd}}\end{array}$ & $\begin{array}{l}180 \\
160\end{array}$ & $\begin{array}{l}7.9+1 \\
7.8+1\end{array}$ & & $\begin{array}{l}\mathrm{NBI} \\
\mathrm{NBI}\end{array}$ & $\begin{array}{l}\text { M Andersent } \\
\text { MAndersent }\end{array}$ & $\begin{array}{l}11704 \\
11407\end{array}$ \\
\hline $181 \mathrm{Er}$ & \multicolumn{6}{|c|}{ portlal overogo $v_{y}$} \\
\hline $\begin{array}{l}150 \mathrm{Nd} \\
166 \mathrm{Er}\end{array}$ & $\underset{a}{20} \mathrm{Ne}$ & $\begin{array}{l}9.4+1 \\
6.8+1\end{array}$ & $\begin{array}{l}1.1+2 \\
9.5+1\end{array}$ & $\begin{array}{l}\text { ORL } \\
\text { ORL }\end{array}$ & $\begin{array}{l}\text { E Sarantitest } \\
\text { E Sarantitest }\end{array}$ & $\begin{array}{r}11889 \\
8579\end{array}$ \\
\hline $161 \mathrm{Er}$ & \multicolumn{6}{|c|}{ portlol $v_{y}$} \\
\hline $\begin{array}{l}148_{N d} \\
150 N d\end{array}$ & $\begin{array}{l}180 \\
160\end{array}$ & $\begin{array}{l}6.9+1 \\
6.9+1\end{array}$ & $\begin{array}{l}7.8+1 \\
7.8+1\end{array}$ & $\begin{array}{l}\text { NBI } \\
\text { NQ1 }\end{array}$ & $\begin{array}{l}M \text { Andersent } \\
M \text { Andersent }\end{array}$ & $\begin{array}{l}11703 \\
11406\end{array}$ \\
\hline $161 \mathrm{Er}$ & \multicolumn{6}{|c|}{ portlot of(E) } \\
\hline $\begin{array}{l}150 \mathrm{Nd} \\
165 \mathrm{Ho} \\
165 \mathrm{Er}\end{array}$ & $\begin{array}{c}20 \\
{ }^{20} \mathrm{Ne} \\
\mathrm{\rho}\end{array}$ & $\begin{array}{l}1 \cdot 1+2 \\
6 \cdot 0+1 \\
6 \cdot 8+1\end{array}$ & $9.5+1$ & $\begin{array}{l}\text { ORL } \\
\text { OSA } \\
\text { ORL }\end{array}$ & $\begin{array}{l}\text { E Saranitilest } \\
\text { E Ejirit } \\
\text { E Sarantiltast }\end{array}$ & $\begin{array}{r}11888 \\
4330 \\
8578\end{array}$ \\
\hline $161 \mathrm{Er}$ & \multicolumn{6}{|l|}{$v_{y}$} \\
\hline $\begin{array}{l}150 \mathrm{NO} \\
162 \mathrm{DY}\end{array}$ & ${ }^{20} \mathrm{Ne}$ & $\begin{array}{l}1.5+2 \\
9.0+1\end{array}$ & $2.3+2$ & $\begin{array}{l}\text { PAR } \\
\text { OSA }\end{array}$ & $\begin{array}{l}\text { R Lefor: } \\
\text { EEjirit }\end{array}$ & $\begin{array}{r}11890 \\
8532\end{array}$ \\
\hline $161 \mathrm{Er}$ & \multicolumn{6}{|c|}{ rolative o(E) } \\
\hline $\begin{array}{l}162 \mathrm{Dy} \\
162 \mathrm{Dy} \\
164 \mathrm{Dy} \\
165 \mathrm{Ho}\end{array}$ & 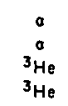 & $\begin{array}{l}9.0+1 \\
5.0+1 \\
6.5+1 \\
1.0+2\end{array}$ & $1.2+2$ & $\begin{array}{l}\text { OSA } \\
\text { OSA } \\
\text { OSA } \\
\text { OSA }\end{array}$ & $\begin{array}{l}\text { E Ejirit } \\
\text { E Ejirit } \\
\text { E Sakai+ } \\
\text { E Sakal+ }\end{array}$ & $\begin{array}{l}8531 \\
8540 \\
6494 \\
6498\end{array}$ \\
\hline $161 \mathrm{Er}$ & \multicolumn{6}{|l|}{$O(\varepsilon)$} \\
\hline $\begin{array}{l}150 \mathrm{Nd} \\
152 \mathrm{Sm} \\
162 \mathrm{Dy} \\
164 \mathrm{Dy} \\
165 \mathrm{Ho}\end{array}$ & $\begin{array}{r}160 \\
12 \mathrm{c} \\
a \\
a \\
p\end{array}$ & $\begin{array}{l}6.8+1 \\
4.7+1 \\
7.0+1 \\
1.2+2 \\
6.0+1\end{array}$ & $\begin{array}{l}8.4+1 \\
6.3+1 \\
9.0+1\end{array}$ & $\begin{array}{l}\text { NB! } \\
\text { NBi } \\
\text { OSA } \\
\text { OSA } \\
\text { OSA }\end{array}$ & $\begin{array}{l}\text { R Herghind. } \\
\text { M Ishiharat } \\
\text { E Ejirit } \\
\text { E Ejiri+ } \\
\text { E Ejiri+ }\end{array}$ & $\begin{array}{r}11408 \\
10032 \\
8539 \\
8557 \\
4331\end{array}$ \\
\hline
\end{tabular}




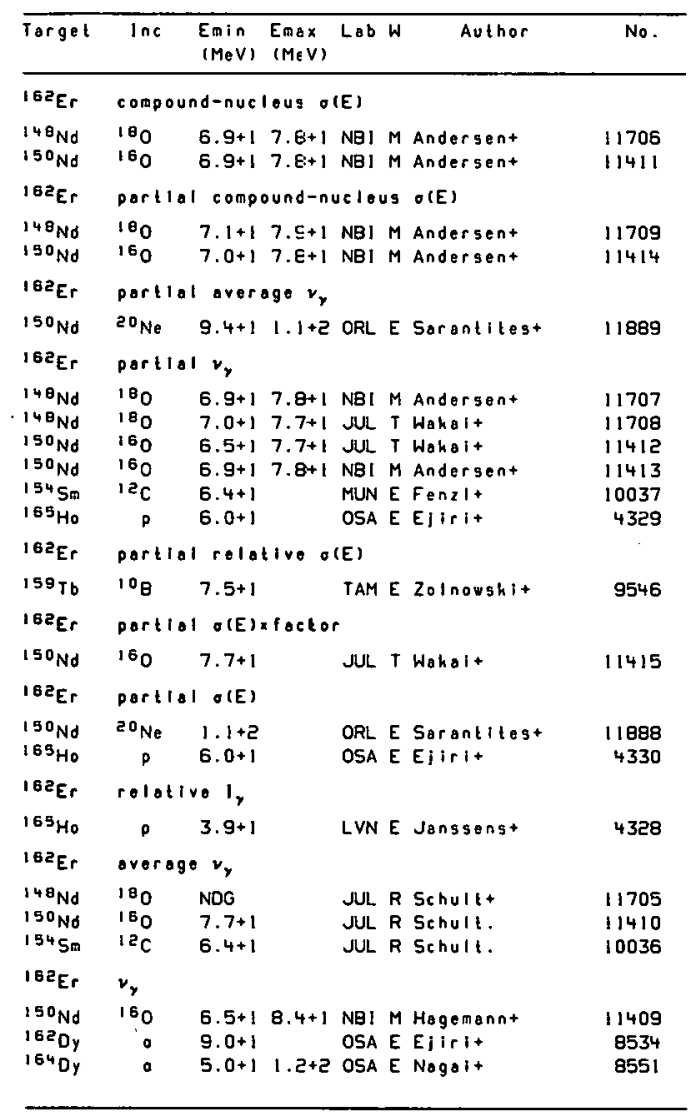

\begin{tabular}{|c|c|c|c|c|c|c|}
\hline Target & $\ln c$ & $\begin{array}{l}\text { Emin } \\
(\mathrm{MeV})\end{array}$ & $\begin{array}{l}\text { Emax } \\
(\operatorname{MeV})\end{array}$ & Lob & Author & No. \\
\hline \multicolumn{7}{|l|}{$162 \mathrm{Er}$} \\
\hline $164 \mathrm{Dy}$ & 0 & $1.2+2$ & & OSA E & E Nagai+ & 8559 \\
\hline $162_{\mathrm{Er}}$ & \multicolumn{6}{|c|}{ relalive $\sigma(E)$} \\
\hline $\begin{array}{l}1620 y \\
1620 y \\
1640 y \\
1640 y \\
165 \mathrm{Ho}\end{array}$ & $\begin{array}{c}0 \\
\stackrel{0}{a} \\
{ }^{3} \mathrm{He} \\
{ }^{\circ} \\
{ }^{3} \mathrm{He}\end{array}$ & $\begin{array}{l}9 \cdot 0+1 \\
5 \cdot 0+1 \\
6 \cdot 5+1 \\
5 \cdot 0+1 \\
1.0+2\end{array}$ & $\begin{array}{l}1.2+2 \\
1.2+2\end{array}$ & $\begin{array}{l}\text { OSA } \\
\text { OSA } \\
\text { OSA E } \\
\text { OSA } \\
\text { OSA }\end{array}$ & $\begin{array}{l}\text { E Elirit } \\
\text { E Ejirit } \\
\text { E Sahait } \\
\text { E Ejirit } \\
\text { E Sahalt }\end{array}$ & $\begin{array}{l}8533 \\
8540 \\
6494 \\
8558 \\
6498\end{array}$ \\
\hline${ }^{162} \mathrm{Er}$ & \multicolumn{6}{|l|}{$O(E)$} \\
\hline $\begin{array}{l}150 \mathrm{Nd} \\
152 \mathrm{Sm} \\
159 \mathrm{~Tb} \\
162 \mathrm{Dy} \\
164 \mathrm{Dy} \\
165 \mathrm{Ho}\end{array}$ & $\begin{array}{c}160 \\
1{ }^{16} \mathrm{C} \\
7_{\mathrm{L}} \\
a \\
0 \\
0 \\
0\end{array}$ & $\begin{array}{l}5.5+1 \\
4.7+1 \\
2.4+1 \\
7.0+1 \\
1.2+2 \\
6.0+1\end{array}$ & $\begin{array}{l}8.4+1 \\
5.4+1 \\
3.5+1 \\
9.0+1\end{array}$ & $\begin{array}{l}\text { NBI } \\
\text { NBI } \\
\text { NBI } \\
\text { OSA } \\
\text { OSA } \\
\text { OSA }\end{array}$ & 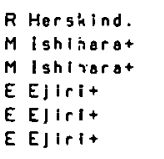 & $\begin{array}{r}11416 \\
10033 \\
9454 \\
8539 \\
8557 \\
4331\end{array}$ \\
\hline${ }^{183} \mathrm{Er}$ & \multicolumn{6}{|c|}{ portlol o(E) } \\
\hline${ }^{165} \mathrm{Ho}$ & $p$ & $6.0+1$ & & OSA & E EjIrit & 4330 \\
\hline${ }^{163} \mathrm{Er}$ & \multicolumn{6}{|c|}{ relative o(E) } \\
\hline $\begin{array}{l}1620 y \\
154 D_{y} \\
164 D y\end{array}$ & ${ }_{0}^{0} \mathrm{He}$ & $\begin{array}{l}5.0+1 \\
6.5+1 \\
5.0+1\end{array}$ & $\begin{array}{l}1.2+2 \\
1.2+2\end{array}$ & $\begin{array}{l}\text { OSA } \\
\text { OSA } \\
\text { OSA }\end{array}$ & $\begin{array}{l}\text { E Ejir:t } \\
\text { E Soha:- } \\
\text { E Elir:- }\end{array}$ & $\begin{array}{l}8540 \\
6494 \\
8558\end{array}$ \\
\hline${ }^{163} \mathrm{Er}$ & \multicolumn{6}{|l|}{$\sigma(E)$} \\
\hline $\begin{array}{l}159 \mathrm{~Tb} \\
162 \mathrm{Dy} \\
164 \mathrm{Dy} \\
169 \mathrm{Ho}\end{array}$ & $\begin{array}{c}{ }^{7} \mathrm{LI} \\
0 \\
0 \\
0\end{array}$ & $\begin{array}{l}2 \cdot 4+1 \\
7 \cdot 0+1 \\
1.2+2 \\
6.0+1\end{array}$ & $\begin{array}{l}3.5+1 \\
9.0+1\end{array}$ & $\begin{array}{l}\text { NBI } \\
\text { OSA } \\
\text { OSA } \\
\text { OSA }\end{array}$ & $\begin{array}{l}\text { M Jshiharat } \\
\text { E Ejirit } \\
\text { E Ejirit } \\
\text { E Ejirit }\end{array}$ & $\begin{array}{l}9455 \\
8539 \\
8557 \\
4331\end{array}$ \\
\hline${ }^{184} \mathrm{Er}$ & \multicolumn{6}{|c|}{ parllol relotivo o(E) } \\
\hline $\begin{array}{l}162 \mathrm{Dy} \\
163 \mathrm{Dy} \\
164 \mathrm{Dy} \\
164 \mathrm{Dy} \\
165 \mathrm{Ho}\end{array}$ & $\begin{array}{c}: \\
{ }^{3} \mathrm{He} \\
0 \\
0\end{array}$ & $\begin{array}{l}2.2+1 \\
4 \cdot 0+1 \\
2 \cdot 5+1 \\
4 \cdot 0+1 \\
1.8+1\end{array}$ & $2.4+1$ & $\begin{array}{l}\text { OSA } \\
\text { WAU E } \\
\text { OSA } \\
\text { WAU E } \\
\text { CBR }\end{array}$ & $\begin{array}{l}\text { E Kishimotot } \\
\text { E Fergesont } \\
\text { E Kishimoto+ } \\
\text { E Fergesont } \\
\text { M Drocoulist }\end{array}$ & $\begin{array}{l}8535 \\
8544 \\
6493 \\
8552 \\
5780\end{array}$ \\
\hline $164 \mathrm{Er}$ & \multicolumn{6}{|c|}{ porllol o(E) } \\
\hline $165 \mathrm{HO}_{0}$ & P & $6.0+1$ & & OSA E & E Ejirlt & 4330 \\
\hline
\end{tabular}




\begin{tabular}{|c|c|c|c|c|c|}
\hline$\overline{T a r g e t}$ & $\operatorname{lnc}$ & $\begin{array}{l}\text { Emin } \\
(\mathrm{MeV})\end{array}$ & $\begin{array}{l}E_{\max } \\
(\mathrm{MeV})\end{array}$ & Lab W & No. \\
\hline${ }^{164} \mathrm{Er}$ & \multicolumn{5}{|c|}{ relative $\mathrm{l}_{y}$} \\
\hline $\begin{array}{l}165 \mathrm{Ho} \\
165 \mathrm{Ho}\end{array}$ & ${ }^{3} \mathrm{He}$ & $\begin{array}{l}4.4+1 \\
7.3+1\end{array}$ & & $\begin{array}{l}\text { GRE E Foint } \\
\text { GRE E Foint }\end{array}$ & $\begin{array}{l}6496 \\
8561\end{array}$ \\
\hline $164 \mathrm{Er}$ & \multicolumn{5}{|c|}{ average $v_{y}$} \\
\hline $150^{\mathrm{Nd}}$ & 180 & NoG & & \multirow{2}{*}{$\begin{array}{l}\text { JUL R Schult+ } \\
\text { JUL R Schult+ }\end{array}$} & 11711 \\
\hline${ }^{156} \mathrm{Sm}$ & ${ }^{12} \mathrm{c}$ & NOG & & & 10038 \\
\hline $164 \mathrm{Er}$ & \multicolumn{5}{|c|}{ relative $O(E)$} \\
\hline $\begin{array}{l}164 \mathrm{Dy} \\
185 \mathrm{Ho}\end{array}$ & $-3_{\mathrm{He}}^{0}$ & $\begin{array}{l}5.0+1 \\
1.0+2\end{array}$ & $1.2+2$ & $\begin{array}{l}\text { OSA E Elirit } \\
\text { OSA E Sahait }\end{array}$ & $\begin{array}{l}8558 \\
6498\end{array}$ \\
\hline $184 \mathrm{Er}$ & \multicolumn{5}{|l|}{$O(E)$} \\
\hline $164 \mathrm{Oy}$ & a & $1.2+2$ & & \multirow{2}{*}{$\begin{array}{l}\text { OSA E Elirit } \\
\text { OSA E Elirit }\end{array}$} & 8557 \\
\hline $165 \mathrm{Ho}_{\mathrm{O}}$ & ' & $6.0+1$ & & & 4331 \\
\hline${ }^{165} \mathrm{Er}$ & \multicolumn{4}{|l|}{ O(E) } & \\
\hline $164 \mathrm{Dy}$ & a & $1.2+2$ & & OSA E Ejirit & 8557 \\
\hline $166 \mathrm{Er}$ & \multicolumn{5}{|c|}{ partial raw thich targot yieldy } \\
\hline $166_{\mathrm{Er}}$ & . & $1.4+1$ & & ORL E Mc-Gowant & 8574 \\
\hline${ }^{166} \mathrm{Er}$ & \multicolumn{4}{|c|}{ partial rav thich target yieldabundance } & \\
\hline $166_{E r}$ & a & $5.0+0$ & & SUN E Gilest & 8573 \\
\hline${ }^{166} \mathrm{Er}$ & \multicolumn{5}{|c|}{ relative o(E) } \\
\hline${ }^{165} \mathrm{Ho}_{\mathrm{O}}$ & ${ }^{3} \mathrm{He}$ & $: .0+2$ & & OSA E Sahait & 6498 \\
\hline $167 \mathrm{Er}$ & \multicolumn{5}{|c|}{ partial ras thich arget yieldyabundance } \\
\hline 167 Er & a & $5.0+0$ & & SUN E Gllost & 8583 \\
\hline $16 \theta_{E r}$ & \multicolumn{3}{|c|}{ partlal raw thlch } & Larget yieldy*abundance & \\
\hline $16 \theta_{\mathrm{Er}}$ & ${ }^{\prime}$ & $5.0+0$ & & SUN E Gllest & 8586 \\
\hline $\begin{array}{l}16 \theta_{\mathrm{Er}} \\
168_{\mathrm{Er}}\end{array}$ & \multicolumn{3}{|c|}{ partlal raw thick } & $\begin{array}{l}\text { torget yieldy } \\
\text { ORLE Mc-Gowant }\end{array}$ & 8585 \\
\hline $170 \mathrm{Er}$ & \multicolumn{3}{|c|}{ partial rav thich } & Larget yieldyabundance & \\
\hline $170_{\mathrm{Er}}$ & 0 & $5.0+0$ & & SUN E Gllest & 8589 \\
\hline
\end{tabular}

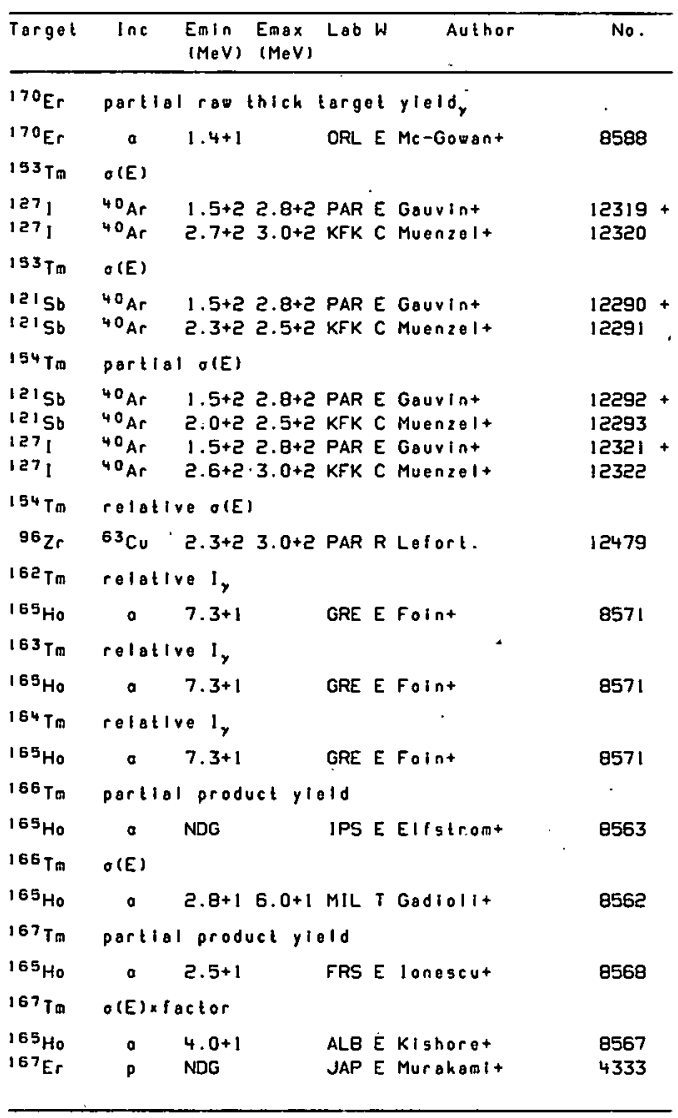




\begin{tabular}{|c|c|c|c|c|c|}
\hline Target & $\operatorname{lnc}$ & $\begin{array}{l}\text { Emin } \\
(\mathrm{MeV})\end{array}$ & $\begin{array}{l}E \max \\
(\operatorname{MaV})\end{array}$ & LabW. & No. \\
\hline $167 \mathrm{Tm}$ & $O(E)$ & & & & \\
\hline $\begin{array}{l}165 \mathrm{Ho} \\
163_{\mathrm{Ho}} \\
163_{\mathrm{Ho}} \\
0 \times 1 \mathrm{Er}\end{array}$ & $\begin{array}{l}a \\
a \\
a \\
p\end{array}$ & $\begin{array}{l}\text { NDG } \\
1.8+1 \\
4.0+1 \\
\text { NDG }\end{array}$ & $5.8+1$ & $\begin{array}{l}\text { JAP E Murahami+ } \\
\text { MLL T Gadiollt } \\
\text { ALB E Kishoret } \\
\text { JAP E Murahamit }\end{array}$ & $\begin{array}{l}8554 \\
8555 \\
8556 \\
4334\end{array}$ \\
\hline $168 \mathrm{Tm}$ & direc & -intero & action & $O(E)$ & \\
\hline $171 \mathrm{Yb}$ & p & $2.0+1$ & & MIL MMIIazzo-Collit & 4351 \\
\hline $188_{\mathrm{Tm}}$ & parti & of $O(E)$ & & & \\
\hline $171 Y \mathrm{Yb}$ & p & $2.0+1$ & & MIL M Milazzo-Colllit & 4350 \\
\hline $16 \theta_{\mathrm{Tm}}$ & $\sigma(E) \times$ & rector & & & \\
\hline $169 \mathrm{Ho}$ & 。 & $4.0+1$ & & ALB E kishoret & 8570 \\
\hline $168 \mathrm{Tm}$ & $O(E)$ & & & & \\
\hline $\begin{array}{l}165 \mathrm{Ho} \\
171 \mathrm{Yb}^{2}\end{array}$ & $\dot{p}$ & $\begin{array}{l}4 \cdot 0+1 \\
2.0+1\end{array}$ & & $\begin{array}{l}\text { ALB E Kighoret. } \\
\text { MIL Milaizo-Callit }\end{array}$ & 8569 \\
\hline $169_{\mathrm{Tm}}$ & rolat & ive $\mathrm{l}_{y}$ & & . & \\
\hline${ }^{169} \mathrm{Tm}$ & ${ }^{3} \mathrm{He}$ & $4.2+1$ & $5.7+1$ & I GRE E Barneoudt & 6500 \\
\hline $173 \mathrm{Tm}$ & $O(E)$ & & & & . \\
\hline $\begin{array}{l}176 \mathrm{Yb} \\
176 \mathrm{Yb}\end{array}$ & $?$ & $\begin{array}{l}1.8+1 \\
1.8+1\end{array}$ & & $\begin{array}{l}\text { KFK C Muenzelt } \\
\text { MIL M Milazzo-Collit }\end{array}$ & $\begin{array}{l}4352 \\
4353+\end{array}$ \\
\hline $153 \mathrm{Yb}$ & row o & & & & \\
\hline $181 \mathrm{Ta}$ & p & NDG & & GRE E Carrazt & 4410 \\
\hline $154 \mathrm{Yb}$ & raso & & & & \\
\hline $181 \mathrm{Ta}$ & p & NOG & & GRE E Carrat+ & 4410 \\
\hline${ }^{155} \mathrm{Yb}$ & $\theta(E)$ & & & & \\
\hline 1271 & ${ }^{40} \mathrm{Ar}$ & $1.5+2$ & $2.8+2$ & ? PAR E Gaurint & 12323 \\
\hline${ }^{155} \mathrm{Yb}$ & rovo & (E) & & & \\
\hline $101 \mathrm{TO}$ & g & NDG & & GRE $\varepsilon$ Carrazt & 4410 \\
\hline $156 \mathrm{Yb}$ & $\theta(E)$ & & & & \\
\hline 1271 & ${ }^{40} \mathrm{Ar}$ & $1.5+2$ & $2.8+2$ & PAR E Gauvint & 12324 \\
\hline
\end{tabular}

\begin{tabular}{|c|c|c|c|c|c|c|}
\hline Target & $\ln c$ & $\begin{array}{l}\text { Emin } \\
(\mathrm{MeV})\end{array}$ & $\begin{array}{l}\text { Emax } \\
(\mathrm{MeV})\end{array}$ & Lab & Author & No. \\
\hline $156 \mathrm{Yb}_{\mathrm{b}}$ & \multicolumn{6}{|c|}{ ras o(E) } \\
\hline $18 t_{T a}$ & $\rho$ & NDG & & GRE & E Carrait & 4410 \\
\hline $157 \mathrm{Yb}$ & \multicolumn{6}{|c|}{ rovo(E) } \\
\hline $181 \mathrm{Ta}$ & $\rho$ & NDG & & GRE & E Corrait & 4410 \\
\hline $15 \theta_{Y b}$ & \multicolumn{6}{|c|}{ rav o(E) } \\
\hline $18 L_{\mathrm{T}}^{\mathrm{T}} \mathrm{a}$ & $p$ & NDG & & GRE & E Carrait & 4410 \\
\hline $159 \mathrm{Yb}$ & \multicolumn{6}{|l|}{$v_{r}$} \\
\hline $\begin{array}{l}150 \mathrm{Nd} \\
150^{\circ}\end{array}$ & $\begin{array}{l}20 \mathrm{Ne} \\
{ }^{2} \mathrm{C}\end{array}$ & $\begin{array}{l}1.3+2 \\
1.5+2\end{array}$ & $\begin{array}{l}1.6+2 \\
2.3+2\end{array}$ & ORL & $\begin{array}{l}\text { R Stokslead. } \\
\text { R Leforlt }\end{array}$ & $\begin{array}{l}11893 \\
10044\end{array}$ \\
\hline $159 \mathrm{Yb}$ & \multicolumn{6}{|c|}{$\operatorname{rov} G(E)$} \\
\hline $181 \mathrm{Ta}$ & $p$ & NDG & & GRE & E Carraz+ & 4410 \\
\hline $159_{Y b}$ & \multicolumn{6}{|l|}{$O(E)$} \\
\hline $\begin{array}{l}150_{\mathrm{Nd}} \\
150_{\mathrm{Gd}}\end{array}$ & $\begin{array}{l}20 \mathrm{Ne} \\
1{ }^{2} \mathrm{C}\end{array}$ & $\begin{array}{l}1.3+2 \\
1.5+2\end{array}$ & $\begin{array}{l}1.6+2 \\
2.3+2\end{array}$ & ORL & $\begin{array}{l}\text { R Stohsteod. } \\
\text { R Leforlt }\end{array}$ & $\begin{array}{l}11891 \\
10042\end{array}$ \\
\hline $160 \mathrm{Yb}$ & \multicolumn{6}{|c|}{ portial relative $\sigma(E)$} \\
\hline $\begin{array}{l}144 \mathrm{Nd} \\
160 \mathrm{Yb}\end{array}$ & \multicolumn{6}{|c|}{ relative $I_{y}$} \\
\hline $1485 m$ & ${ }^{150} 0$ & $9.5+1$ & & STR & E Beck+ & 11421 \\
\hline $160 \mathrm{Yb}$ & \multicolumn{6}{|c|}{ overage $v_{y}$} \\
\hline $\begin{array}{l}126 \mathrm{Te} \\
150 \mathrm{YO}\end{array}$ & \multicolumn{6}{|l|}{$v_{y}$} \\
\hline $\begin{array}{l}150 \mathrm{Nd} \\
150 \mathrm{Nd} \\
150_{\mathrm{Nd}} \\
150_{\mathrm{Gd}}\end{array}$ & $\begin{array}{l}20 \mathrm{Ne} \\
20 \mathrm{Ne} \\
20 \mathrm{Ne} \\
12 \mathrm{C}\end{array}$ & $\begin{array}{l}1.5+2 \\
1.3+2 \\
1.5+2 \\
1.5+2\end{array}$ & $\begin{array}{l}2 \cdot 3+2 \\
1.6+2 \\
2.3+2 \\
2.3+2\end{array}$ & $\begin{array}{l}\text { PAR } \\
\text { ORL } \\
\text { PAR } \\
\text { PAR }\end{array}$ & $\begin{array}{l}\text { R Lefortt } \\
R \text { Stohstoad. } \\
R \text { Lefortt } \\
R \text { Lefortt }\end{array}$ & $\begin{array}{l}11890 \\
11893 \\
11894 \\
10044\end{array}$ \\
\hline $160 \mathrm{Yb}$ & \multicolumn{6}{|c|}{ roo $\sigma(E)$} \\
\hline $\begin{array}{l}18 ! \mathrm{Ta} \\
160 \mathrm{Yb}\end{array}$ & $\begin{array}{c}p \\
O(E)\end{array}$ & NDG & & GRE & E Carraz+ & 4410 \\
\hline $\begin{array}{l}126 \mathrm{Te} \\
150_{\mathrm{Nd}}\end{array}$ & $\begin{array}{l}{ }^{40} \mathrm{Ar} \\
20 \mathrm{Ne}\end{array}$ & $\begin{array}{l}1.8+2 \\
1.3+2\end{array}$ & $1.6+2$ & $\begin{array}{l}\text { BRK } \\
\text { ORL }\end{array}$ & $\begin{array}{l}\text { E Simont } \\
\text { R Stohstead. }\end{array}$ & $\begin{array}{l}12294 \\
11891\end{array}$ \\
\hline
\end{tabular}




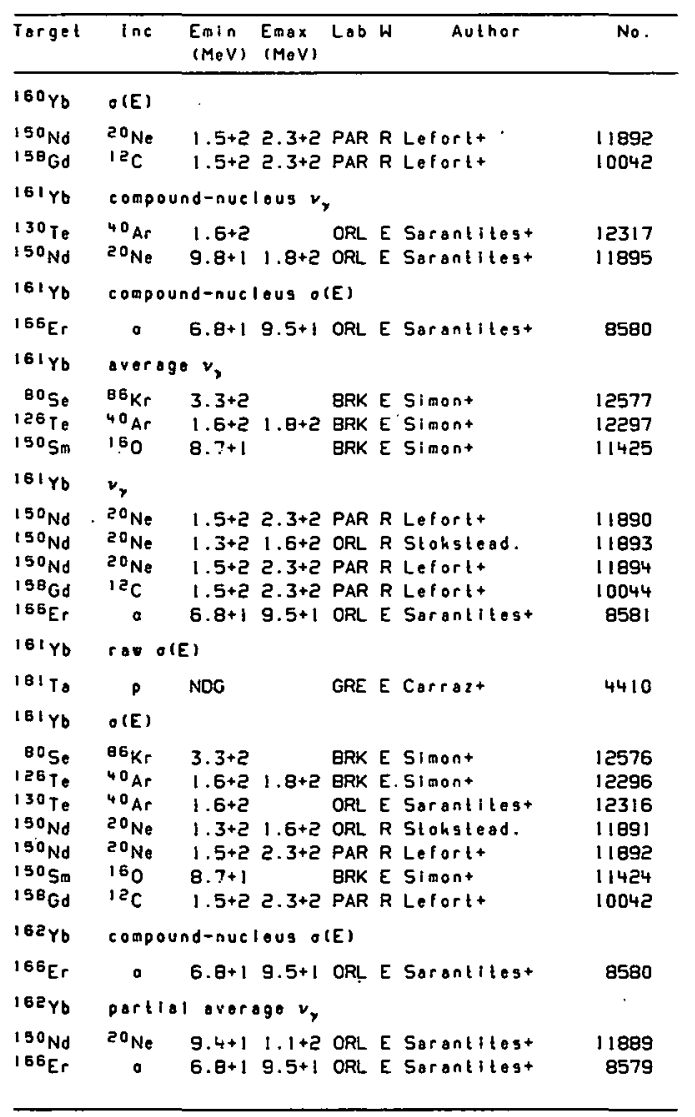

\begin{tabular}{|c|c|c|c|c|c|c|}
\hline Target & $\operatorname{Inc}$ & $\begin{array}{l}\text { Emin } \\
(M e V)\end{array}$ & $\begin{array}{l}E_{m \Delta x} \\
(M \in V)\end{array}$ & Lob & Author & No. \\
\hline $162_{Y b}$ & \multicolumn{6}{|c|}{ partla: o(E) } \\
\hline $\begin{array}{l}150 \mathrm{Nd} \\
165 \mathrm{Er}\end{array}$ & $\underset{\mathrm{a}}{20} \mathrm{Ne}$ & $\begin{array}{l}1.1+2 \\
6.8+1\end{array}$ & $9.5+1$ & $\begin{array}{l}\text { ORL } \\
\text { ORL }\end{array}$ & $\begin{array}{l}\text { E Sarantilest } \\
\text { E Sarantitest }\end{array}$ & $\begin{array}{r}11888 \\
8578\end{array}$ \\
\hline $182 \mathrm{Yb}$ & \multicolumn{6}{|c|}{ overoge $v_{y}$} \\
\hline $\begin{array}{l}80 \mathrm{Se} \\
126 \mathrm{Te} \\
150 \mathrm{Sm}\end{array}$ & $\begin{array}{l}86 \mathrm{Kr} \\
40 \mathrm{Ar} \\
16 \mathrm{O}\end{array}$ & $\begin{array}{l}3 \cdot 1+2 \\
1.6+2 \\
8 \cdot 7+1\end{array}$ & $\begin{array}{l}3.3+2 \\
1.8+2\end{array}$ & $\begin{array}{l}\text { BRK } \\
\text { BRK } \\
\text { BRK }\end{array}$ & $\begin{array}{l}\text { E simont } \\
\text { E Simont } \\
\text { E Simont }\end{array}$ & $\begin{array}{l}12579 \\
12299 \\
11427\end{array}$ \\
\hline $162 Y \mathrm{Y}$ & \multicolumn{6}{|l|}{$v_{\gamma}$} \\
\hline $\begin{array}{l}150 \mathrm{Nd} \\
150 \mathrm{Nd} \\
150 \mathrm{Nd} \\
150 \mathrm{Gd} \\
156 \mathrm{Er}\end{array}$ & $\begin{array}{l}20 \mathrm{Ne} \\
20 \mathrm{Ne} \\
20 \mathrm{Ne} \\
12 \mathrm{C} \\
0\end{array}$ & $\begin{array}{l}1.5+2 \\
1.3+2 \\
1.5+2 \\
1.5+2 \\
6.8+1\end{array}$ & $\begin{array}{l}2.3+2 \\
1.6+2 \\
2.3+2 \\
2 \cdot 3+2 \\
9 \cdot 5+1\end{array}$ & $\begin{array}{l}\text { PAR } \\
\text { ORL } \\
\text { PAR } \\
\text { PAR } \\
\text { ORL }\end{array}$ & $\begin{array}{l}\text { R Lefortt } \\
R \text { Stokstead. } \\
R \text { Lefortt } \\
R \text { Lefortt } \\
\text { E Sarantitest }\end{array}$ & $\begin{array}{r}11890 \\
11893 \\
11894 \\
10044 \\
8581\end{array}$ \\
\hline $162 Y 0$ & \multicolumn{6}{|c|}{ row $O(E)$} \\
\hline $181 \mathrm{Ta}$ & $p$ & NDG & & GRE & E Carrazt & 4410 \\
\hline $162 y_{0}$ & \multicolumn{6}{|l|}{$O(E)$} \\
\hline $\begin{array}{l}80 \mathrm{Se} \\
126 \mathrm{Te} \\
130 \mathrm{Te} \\
150 \mathrm{No} \\
190 \mathrm{Nd} \\
150 \mathrm{Sm} \\
150 \mathrm{Gd}\end{array}$ & $\begin{array}{l}866_{\mathrm{Kr}} \\
40_{\mathrm{Ar}} \\
40_{\mathrm{Ar}} \\
20 \mathrm{Ne} \\
20 \mathrm{Ne} \\
160 \\
12 \mathrm{C}\end{array}$ & $\begin{array}{l}3.1+2 \\
1.6+2 \\
1.6+2 \\
1.3+2 \\
1.5+2 \\
8.7+1 \\
1.5+2\end{array}$ & $\begin{array}{l}3 \cdot 3+2 \\
1.8+2 \\
1 \cdot 6+2 \\
2 \cdot 3+2 \\
2 \cdot 3+2\end{array}$ & $\begin{array}{l}\text { BRK } \\
\text { BRK } \\
\text { ORL } \\
\text { ORL } \\
\text { PAR } \\
\text { BRK } \\
\text { PAR }\end{array}$ & $\begin{array}{l}\text { E Simont } \\
\text { E Simant } \\
\text { E Sarantilest } \\
R \text { Stohsteod. } \\
\text { R Lefortt } \\
\text { E Simont } \\
\text { R Lefortt }\end{array}$ & $\begin{array}{l}12578 \\
12298 \\
12316 \\
11891 \\
11892 \\
11426 \\
10042\end{array}$ \\
\hline $163 \mathrm{Yb}$ & \multicolumn{6}{|c|}{ compound-nueleus $v_{y}$} \\
\hline $\begin{array}{l}130 \mathrm{Te} \\
150 \mathrm{Nd}\end{array}$ & $\begin{array}{l}{ }^{40} \mathrm{Ar} \\
20 \mathrm{Ne}\end{array}$ & $\begin{array}{l}1.6+2 \\
9.8+1\end{array}$ & $1.8+2$ & $\begin{array}{l}\text { ORL E } \\
\text { ORL E }\end{array}$ & $\begin{array}{l}\text { E Sorantilest } \\
\text { E Sorantilest }\end{array}$ & $\begin{array}{l}12317 \\
11895\end{array}$ \\
\hline $163 Y_{b}$ & \multicolumn{6}{|c|}{ compound-nucleus o(E) } \\
\hline $\begin{array}{l}186 \mathrm{Er} \\
163 \mathrm{Yb}\end{array}$ & \multicolumn{6}{|c|}{ partial average $v_{y}$} \\
\hline $\begin{array}{l}150 \mathrm{No} \\
168 \mathrm{Er}\end{array}$ & $\underset{0}{20} \mathrm{Ne}$ & $\begin{array}{l}9.4+1 \\
6.8+1\end{array}$ & $\begin{array}{l}1.1+2 \\
9 \cdot 5+1\end{array}$ & $\begin{array}{l}\text { ORL E } \\
\text { ORL }\end{array}$ & $\begin{array}{l}\text { E Sarantitest } \\
\text { E Sarantitest }\end{array}$ & $\begin{array}{r}11809 \\
8579\end{array}$ \\
\hline $183 \mathrm{Yb}$ & \multicolumn{6}{|c|}{ partlal o(E) } \\
\hline $150 \mathrm{Nd}$ & ${ }^{20} \mathrm{Ne}$ & $1.1+2$ & & ORL & E Serantitest & 11888 \\
\hline
\end{tabular}




\begin{tabular}{|c|c|c|c|c|c|c|}
\hline Tor,get & $\ln c$ & $\begin{array}{l}\text { Emin } \\
(\operatorname{MeV})\end{array}$ & $\begin{array}{l}\text { Emax } \\
\left(M_{\theta} V\right)\end{array}$ & Lob h & Author & No. \\
\hline $163 \mathrm{YO}_{0}$ & \multicolumn{6}{|c|}{ pertial ofE) } \\
\hline $\begin{array}{l}165 \mathrm{Er} \\
163 \mathrm{Yb}\end{array}$ & $\begin{array}{c}\text { a } \\
\text { averag }\end{array}$ & \multicolumn{3}{|c|}{ average vy } & E Serantitest & 8578 \\
\hline $\begin{array}{l}60 \mathrm{Se} \\
82 \mathrm{Se} \\
126 \mathrm{To} \\
130 \mathrm{Te} \\
150 \mathrm{Sm}\end{array}$ & $\begin{array}{l}96_{\mathrm{Kr}} \\
66_{\mathrm{Kr}} \\
40 \mathrm{Ar} \\
40 \mathrm{Ar} \\
16_{\mathrm{O}}\end{array}$ & $\begin{array}{l}3.1+2 \\
3.3+2 \\
1.6+2 \\
1.8+2 \\
8.7+1\end{array}$ & $\begin{array}{l}3.3+\bar{e} \\
1.8+\bar{\varepsilon}\end{array}$ & $\begin{array}{l}\text { BRK E } \\
\text { BRK E } \\
\text { BRK E } \\
\text { BRK E } \\
\text { BRK E }\end{array}$ & $\begin{array}{l}\text { E Simont } \\
\text { E simont } \\
\text { E Simont } \\
\text { E Simont } \\
\text { E Simont }\end{array}$ & $\begin{array}{l}12581 \\
12585 \\
12301 \\
12303 \\
11429\end{array}$ \\
\hline $163 \mathrm{Yb}$ & $v_{y}$ & & & & & \\
\hline $\begin{array}{l}150 \mathrm{Nd} \\
150 \mathrm{Nd} \\
150 \mathrm{Nd} \\
158 \mathrm{Gd} \\
166 \mathrm{Er}\end{array}$ & $\begin{array}{l}20 \mathrm{Ne} \\
20 \mathrm{Ne} \\
20 \mathrm{Ne} \\
12 \mathrm{C} \\
a\end{array}$ & $\begin{array}{l}1.5+2 \\
1.3+2 \\
1.5+2 \\
1.5+2 \\
6.8+1\end{array}$ & $\begin{array}{l}2.3+\bar{E} \\
1.6+\bar{E} \\
2 \cdot 3+\bar{c} \\
2.3+\bar{z} \\
9.5+1\end{array}$ & $\begin{array}{l}\text { PAR F } \\
\text { ORL F } \\
\text { PAR F } \\
\text { PAR F } \\
\text { ORL E }\end{array}$ & $\begin{array}{l}\text { R Lefortt } \\
R \text { Stohstead. } \\
R \text { Lefortt } \\
R \text { Lefortt } \\
\text { E Sarantitest }\end{array}$ & $\begin{array}{r}11890 \\
11893 \\
11894 \\
10044 \\
8581\end{array}$ \\
\hline${ }^{163} \mathrm{Yb}$ & \multicolumn{6}{|c|}{$r \otimes v^{\prime}(E)$} \\
\hline $\begin{array}{l}181 \mathrm{Ta} \\
163 \mathrm{Yb}\end{array}$ & \multicolumn{6}{|l|}{$O(E)$} \\
\hline $\begin{array}{l}20 \mathrm{Se} \\
82 \mathrm{Se} \\
126 \mathrm{Te} \\
130 \mathrm{Te} \\
130 \mathrm{Te} \\
150 \mathrm{Nd} \\
150 \mathrm{Nd} \\
150 \mathrm{Sd} \\
150 \mathrm{Gd}\end{array}$ & $\begin{array}{l}06 \mathrm{Kr} \\
06 \mathrm{Kr} \\
40 \mathrm{Ar} \\
40 \mathrm{Ar} \\
40 \mathrm{Ar} \\
20 \mathrm{Ne} \\
20 \mathrm{Ne} \\
16 \mathrm{O} \\
12 \mathrm{C}\end{array}$ & $\begin{array}{l}3.1+2 \\
3.3+2 \\
1.6+2 \\
1.8+2 \\
1.6+2 \\
1.3+2 \\
1.5+2 \\
8.7+1 \\
1.5+2\end{array}$ & $\begin{array}{l}3.3+2 \\
1.8+2 \\
1.6+2 \\
2.3+2 \\
2.3+2\end{array}$ & $\begin{array}{l}\text { ERK E } \\
\text { ERK E } \\
\text { ERK E } \\
\text { BRK E } \\
\text { ORL E } \\
\text { ORL F } \\
\text { PAR F } \\
\text { ERK E } \\
\text { PAR F }\end{array}$ & $\begin{array}{l}\text { E Simont } \\
\text { E Simont } \\
\text { E Simont } \\
\text { E Simont } \\
\text { E Sorantitest } \\
R \text { Stokstead. } \\
\text { R Lefortt } \\
\text { E Simont } \\
\text { R Lefortt }\end{array}$ & $\begin{array}{l}12580 \\
12584 \\
12300 \\
12302 \\
12316 \\
11891 \\
11892 \\
11420 \\
10042\end{array}$ \\
\hline $164 \mathrm{Yb}$ & \multicolumn{6}{|c|}{ compound-nucleus $v_{y}$} \\
\hline $\begin{array}{l}130 \mathrm{Te} \\
150 \mathrm{Nd}\end{array}$ & $\begin{array}{l}{ }^{40} \mathrm{Ar} \\
20 \mathrm{Ne}\end{array}$ & $\begin{array}{l}1.6+2 \\
9.8+1\end{array}$ & $1.8+2$ & $\begin{array}{l}\text { ORL E } \\
\text { ORL }\end{array}$ & $\begin{array}{l}\text { E Sarantitest } \\
\text { E Sarantilest }\end{array}$ & $\begin{array}{l}12317 \\
11895\end{array}$ \\
\hline $164 \mathrm{rb}$ & \multicolumn{6}{|c|}{ compound-nucleus o(E) } \\
\hline $\begin{array}{l}166 \mathrm{Er} \\
164 \mathrm{Yb}\end{array}$ & portlo & $\begin{array}{l}6.8+1 \\
1 \text { compo }\end{array}$ & $\begin{array}{l}9.5+1 \\
\text { ound-au }\end{array}$ & $\begin{array}{l}\text { ORL E } \\
\text { ucleus }\end{array}$ & $\begin{array}{l}\text { E Sarantitest } \\
\text { us relative o(E) }\end{array}$ & 8580 \\
\hline $159 \mathrm{~Tb}$ & $14 \mathrm{~N}$ & $9.4+1$ & & JOL E & E Inamurat & 10463 \\
\hline
\end{tabular}

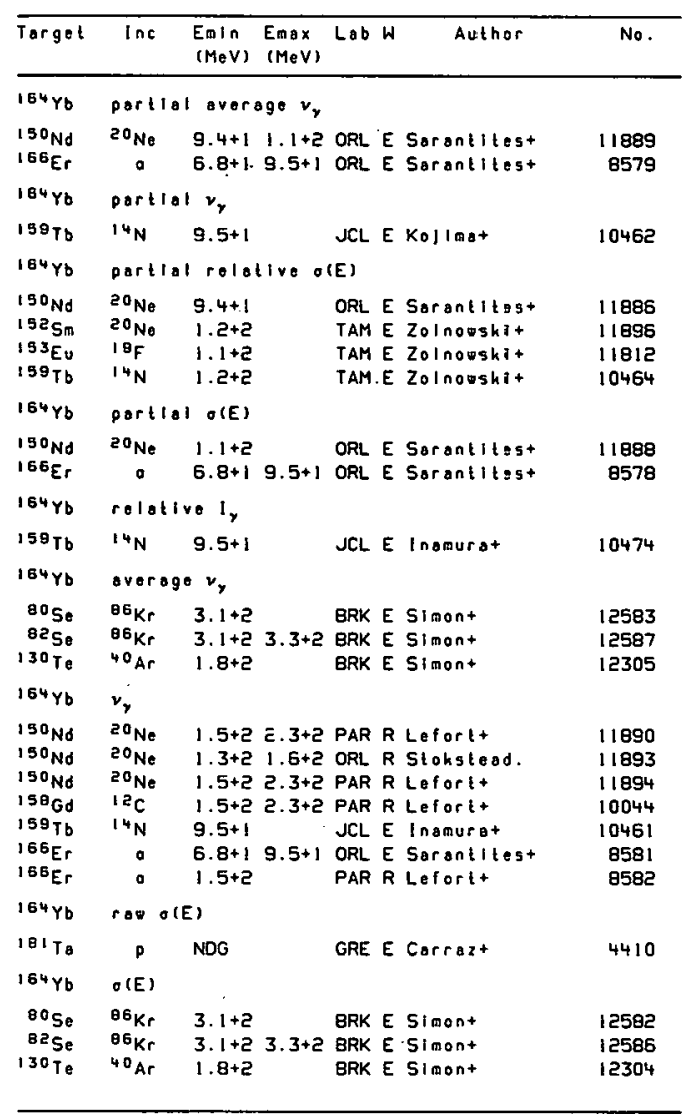




\begin{tabular}{|c|c|c|c|c|c|c|}
\hline Target & $\operatorname{lnc}$ & $\begin{array}{l}\text { Emin } \\
(\mathrm{MeV})\end{array}$ & $\begin{array}{l}\text { Emex } \\
(\mathrm{MeV})\end{array}$ & Lab $h$ & Author & No. \\
\hline $184 \mathrm{Yb}$ & $O(E)$ & & & & & \\
\hline $\begin{array}{l}130 \mathrm{Te} \\
150 \mathrm{Nd} \\
150 \mathrm{Nd} \\
150^{\mathrm{Gd}}\end{array}$ & $\begin{array}{l}{ }^{40} \mathrm{Ar} \\
20 \mathrm{Ne} \\
20 \mathrm{Ne} \\
12 \mathrm{C}\end{array}$ & $\begin{array}{l}1.6+2 \\
1.3+2 \\
1.5+2 \\
1.5+2\end{array}$ & $\begin{array}{l}1.6+2 \\
2.3+2 \\
2.3+2\end{array}$ & $\begin{array}{l}\text { ORL } \\
\text { ORL } \\
\text { PAR F } \\
\text { PAR F }\end{array}$ & $\begin{array}{l}\text { E Sarantilest } \\
R \text { Stokstead. } \\
\text { R Lefortt } \\
\text { R Lefortt }\end{array}$ & $\begin{array}{l}12316 \\
11891 \\
11892 \\
10042\end{array}$ \\
\hline $165 \mathrm{Yb}$ & compor & nd-nucl & $1003 v_{\gamma}$ & & & \\
\hline $\begin{array}{l}130 \mathrm{Te} \\
150 \mathrm{Nd}\end{array}$ & $\begin{array}{l}40 \mathrm{Ar} \\
20 \mathrm{Ne}\end{array}$ & $\begin{array}{l}1.6+2 \\
9.8+1\end{array}$ & $1.8+2$ & $\begin{array}{l}\text { ORL E } \\
\text { ORL E }\end{array}$ & $\begin{array}{l}\text { E Sarantitest } \\
\text { E Sarantitest }\end{array}$ & $\begin{array}{l}12317 \\
11895\end{array}$ \\
\hline $165 \mathrm{Yb}$ & compor & nd-nucl & leus ol & $(E)$ & 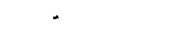 & \\
\hline $\begin{array}{l}155 \mathrm{Er} \\
165 \mathrm{YO}\end{array}$ & porti & $\begin{array}{l}6.8+1 \\
1 \text { compo }\end{array}$ & $\begin{array}{l}9.5+1 \\
\text { ound-nu }\end{array}$ & $\begin{array}{l}\text { ORL } \\
\text { ucleus }\end{array}$ & $\begin{array}{l}\text { E Sarantitest } \\
\text { us relative o(E) }\end{array}$ & 8580 \\
\hline${ }^{159} \mathrm{~Tb}$ & ${ }^{14} \mathrm{~N}$ & $9.4+1$ & & $J C L E$ & E Inamurat & 10468 \\
\hline $165 \mathrm{Yb}$ & partlo & I direc & $c t-\ln t e$ & ersett & llon relative ole & \\
\hline${ }^{159} \mathrm{~Tb}$ & $14 \mathrm{~N}$ & $9.4+1$ & & $J C L E$ & $E$ Inamurat & 10467 \\
\hline $165 \mathrm{Yb}$ & pertlo & 1 avero & $\operatorname{\theta g} \theta v_{y}$ & & & \\
\hline $\begin{array}{l}130 \mathrm{Te} \\
150 \mathrm{Nd} \\
166 \mathrm{Er}\end{array}$ & $\begin{array}{l}40 \mathrm{Ar} \\
20 \mathrm{Ne} \\
0\end{array}$ & $\begin{array}{l}1.5+2 \\
9.4+1 \\
6 \cdot 8+1\end{array}$ & $\begin{array}{l}1.1+2 \\
9.5+1\end{array}$ & $\begin{array}{l}\text { ORL E } \\
\text { ORL E } \\
\text { ORL E }\end{array}$ & $\begin{array}{l}\text { E Sarantitest } \\
\text { E Sarantitest } \\
\text { E Sarantitest }\end{array}$ & $\begin{array}{r}12309 \\
11889 \\
8579\end{array}$ \\
\hline $165 \mathrm{Yb}$ & partio & $1 v_{y}$ & & & & \\
\hline $159 \mathrm{~Tb}$ & ${ }^{14} \mathrm{~N}$ & $9.5+1$ & & $J C L E$ & E Kojimat & 10466 \\
\hline $165 Y b$ & partis & 1 relat & tive of & (E) & & \\
\hline $\begin{array}{l}130 \mathrm{Te} \\
150 \mathrm{Nd}\end{array}$ & $\begin{array}{l}40 \mathrm{Ar} \\
20 \mathrm{Ne}\end{array}$ & $\begin{array}{l}1.5+2 \\
9.4+1\end{array}$ & & $\begin{array}{l}\text { ORL E } \\
\text { ORL E }\end{array}$ & $\begin{array}{l}\text { E Sarantitest } \\
\text { E Sarantiest }\end{array}$ & $\begin{array}{l}12308 \\
11887\end{array}$ \\
\hline $165 Y_{b}$ & partio & I $\cdot(E)$ & & & & \\
\hline $\begin{array}{l}150 \mathrm{Nd} \\
166 \mathrm{Er}\end{array}$ & ${ }_{0}^{20} \mathrm{Ne}$ & $\begin{array}{l}1.1+2 \\
6.8+1\end{array}$ & $9.5+1$ & $\begin{array}{l}\text { ORL E } \\
\text { ORL E }\end{array}$ & $\begin{array}{l}\text { E Sarantitest } \\
\text { E Sarantitest }\end{array}$ & $\begin{array}{r}11888 \\
8578\end{array}$ \\
\hline $165 \mathrm{Yb}$ & relati & ve $I_{y}$ & & & & \\
\hline $\begin{array}{l}159 \mathrm{~Tb} \\
165 \mathrm{Yb}\end{array}$ & $\begin{array}{l}14 \mathrm{~N} \\
\text { averag }\end{array}$ & $\begin{array}{l}9.5+1 \\
v_{y}\end{array}$ & & $J C L E$ & E Inamurat & 10474 \\
\hline $\begin{array}{r}825 e \\
130 \mathrm{Te}\end{array}$ & $\begin{array}{l}86 \mathrm{Kr} \\
{ }^{40} \mathrm{Ar}\end{array}$ & $\begin{array}{l}3.1+2 \\
1.8+2\end{array}$ & $3.3+2$ & $\begin{array}{l}\text { BRK E } \\
\text { BRK E }\end{array}$ & $\begin{array}{l}\text { E Simont } \\
\text { E Simant }\end{array}$ & $\begin{array}{l}12589 \\
12307\end{array}$ \\
\hline
\end{tabular}

\begin{tabular}{|c|c|c|c|c|c|c|}
\hline Target & $\ln c$ & $\begin{array}{l}E m \mid n \\
(M e V)\end{array}$ & $\begin{array}{l}\text { Emax } \\
(\mathrm{MeV})\end{array}$ & Lab & Author & No. \\
\hline $165 \mathrm{Yb}$ & $v_{y}$ & & & & & \\
\hline $\begin{array}{l}150 \mathrm{Nd} \\
150 \mathrm{Nd} \\
150 \mathrm{Nd} \\
150_{\mathrm{Gd}} \\
159 \mathrm{~Tb} \\
166 \mathrm{Er} \\
166 \mathrm{Er}\end{array}$ & $\begin{array}{l}20 \\
20 \\
20 \\
20 \\
20 \\
12 \mathrm{Ce} \\
14 \mathrm{~N} \\
0 \\
0\end{array}$ & $\begin{array}{l}1.5+2 \\
1.3+2 \\
1.5+2 \\
1.5+2 \\
9.5+1 \\
6.8+1 \\
1.5+2\end{array}$ & $\begin{array}{l}2.3+2 \\
1 \cdot 6+2 \\
2 \cdot 3+2 \\
2 \cdot 3+2 \\
9 \cdot 5+1\end{array}$ & $\begin{array}{l}\text { PAR } \\
\text { ORL } \\
\text { PAR } \\
\text { PAR } \\
\text { JCL } \\
\text { ORL } \\
\text { PAR }\end{array}$ & $\begin{array}{l}\text { R Lefort+ } \\
R \text { Stohstead. } \\
R \text { Lefort+ } \\
R \text { Lefort+ } \\
\text { E Inamurat } \\
\text { E Sarantitest } \\
\text { R Lefort+ }\end{array}$ & $\begin{array}{r}11890 \\
11893 \\
11894 \\
10044 \\
10465 \\
8581 \\
8582\end{array}$ \\
\hline.$^{169} \mathrm{Yb}_{\mathrm{b}}$ & \multicolumn{6}{|c|}{$\operatorname{row} O(E)$} \\
\hline $\begin{array}{l}181 \mathrm{Ta} \\
165 \mathrm{Yb}\end{array}$ & $\begin{array}{c}p \\
O(E)\end{array}$ & NDG & & GRE & E Carraz+ & 4410 \\
\hline $\begin{array}{l}82 \mathrm{Se} \\
130 \mathrm{Te} \\
130 \% \mathrm{e} \\
150 \mathrm{Nd} \\
150 \mathrm{Nd} \\
158 \mathrm{Gd} \\
165 \mathrm{Tm}\end{array}$ & $\begin{array}{l}86 \mathrm{Kr} \\
{ }^{40} \mathrm{Ar} \\
40_{\mathrm{Ar}} \\
20 \mathrm{Ne} \\
20 \\
20_{\mathrm{Ne}} \\
1{ }^{2} \mathrm{C} \\
\mathrm{P}\end{array}$ & $\begin{array}{l}3.1+2 \\
1.8+2 \\
1.6+2 \\
1.3+2 \\
1.5+2 \\
1.5+2 \\
1.0+1\end{array}$ & $\begin{array}{l}3 \cdot 3+2 \\
1.6+2 \\
2.3+2 \\
2.3+2 \\
5 \cdot 0+1\end{array}$ & $\begin{array}{l}\text { BRK } \\
\text { BRK } \\
\text { ORL } \\
\text { ORL } \\
\text { PAR } \\
\text { PAR } \\
\text { TUD }\end{array}$ & $\begin{array}{l}\text { E Simont } \\
\text { E Simont } \\
\text { E Sarantilest } \\
\text { R Stokstead. } \\
\text { R Lefort+ } \\
\text { R Lefort+ } \\
\text { R Seidel+ }\end{array}$ & $\begin{array}{r}12588 \\
12306 \\
12316 \\
11891 \\
11892 \\
10042 \\
4335\end{array}$ \\
\hline $166 \mathrm{Yb}$ & \multicolumn{6}{|c|}{ compound-nucleus $v_{y}$} \\
\hline $\begin{array}{l}130 \mathrm{Te} \\
150^{\circ} \mathrm{Nd}\end{array}$ & $\begin{array}{l}{ }^{40} \mathrm{Ar} \\
20 \mathrm{Ne}\end{array}$ & $\begin{array}{l}1.6+2 \\
9.8+1\end{array}$ & $1.8+2$ & $\begin{array}{l}\text { ORL } \\
\text { ORL }\end{array}$ & $\begin{array}{l}\text { E Sarantitest } \\
\text { E Sarantilest }\end{array}$ & $\begin{array}{l}12317 \\
11895\end{array}$ \\
\hline $166 Y_{b}$ & \multicolumn{6}{|c|}{ compound-nucleus o(E) } \\
\hline $\begin{array}{l}166 \mathrm{Er} \\
186 \mathrm{Yb}\end{array}$ & $\begin{array}{c}0 \\
\text { partio }\end{array}$ & \multicolumn{4}{|c|}{ partial compound-nuclous relative $\sigma(E)$} & 8580 \\
\hline $159 \mathrm{~Tb}$ & $14 \mathrm{~N}$ & $9.4+1$ & & $\mathrm{JCL}$ & E Inamurat & 10472 \\
\hline $156 \mathrm{Yb}$ & \multicolumn{6}{|c|}{ partial direct-interaction relative $\sigma(E)$. } \\
\hline $\begin{array}{l}159 \mathrm{~Tb} \\
165 \mathrm{Yb}\end{array}$ & \multicolumn{6}{|c|}{ portial average $v_{y}$} \\
\hline $\begin{array}{l}130 \mathrm{Te} \\
150_{\mathrm{Nd}} \\
166_{\mathrm{Er}}\end{array}$ & $\begin{array}{c}40 \mathrm{Ar} \\
20_{\mathrm{Ne}} \\
0\end{array}$ & $\begin{array}{l}1.5+2 \\
9.4+1 \\
6.8+1\end{array}$ & $\begin{array}{l}1.1+2 \\
9.5+1\end{array}$ & $\begin{array}{l}\text { ORL } \\
\text { ORL } \\
\text { ORL }\end{array}$ & $\begin{array}{l}\text { E Sarantitest } \\
\text { E Sarantitest } \\
\text { E Sarantitest }\end{array}$ & $\begin{array}{r}12313 \\
11889 \\
-\quad 8579\end{array}$ \\
\hline $166 \mathrm{Yb}$ & \multicolumn{6}{|c|}{ partial vy } \\
\hline $159 \mathrm{~Tb}$ & ${ }^{14} \mathrm{~N}$ & $9.5+1$ & & $\mathrm{JCL}$ & E Kojimat & 10470 \\
\hline
\end{tabular}




\begin{tabular}{|c|c|c|c|c|c|c|}
\hline Target & Inc & $\begin{array}{l}\text { Emin } \\
(M e V)\end{array}$ & $\begin{array}{l}\text { Emax } \\
(\operatorname{MeV})\end{array}$ & Lab h & Author & No. \\
\hline $166 \mathrm{Yb}$ & \multicolumn{6}{|c|}{ partial $v_{y}$} \\
\hline${ }^{166} \mathrm{Er}$ & a & $5 \cdot 1+1$ & . & IPS E & E kereht & 8577 \\
\hline $188 \mathrm{Yb}$ & \multicolumn{6}{|c|}{ partial relative o(E) } \\
\hline $130 \mathrm{Te}$ & $40 \mathrm{Ar}$ & $1.5+2$ & & ORL E & E Sarantilest & 12312 \\
\hline $166 \mathrm{Yb}$ & \multicolumn{6}{|c|}{ partlal o(E) } \\
\hline $\begin{array}{l}150 \mathrm{Nd} \\
166 \mathrm{Er}\end{array}$ & $\underset{\mathrm{a}}{20} \mathrm{Ne}$ & $\begin{array}{l}1.1+2 \\
6.8+1\end{array}$ & $9.5+1$ & $\begin{array}{l}\text { ORL E } \\
\text { ORL E }\end{array}$ & $\begin{array}{l}\text { E Sarantlitest } \\
\text { E Saranlitest }\end{array}$ & $\begin{array}{r}11888 \\
8578\end{array}$ \\
\hline $166 \mathrm{Yb}$ & \multicolumn{3}{|c|}{ relative ly } & & & \\
\hline $\begin{array}{l}139 \mathrm{~Tb} \\
166 \mathrm{Yb}\end{array}$ & \multicolumn{6}{|c|}{ overage $v_{Y}$} \\
\hline $\begin{array}{l}82 \mathrm{Se} \\
130 \mathrm{Te}\end{array}$ & $\begin{array}{l}{ }^{B B_{\mathrm{Kr}}} \\
{ }^{\circ}{ }_{\mathrm{Ar}}\end{array}$ & $\begin{array}{l}3.1+2 \\
1.8+2\end{array}$ & & $\begin{array}{l}\text { BRK E } \\
\text { BRK E }\end{array}$ & $\begin{array}{l}\text { E Simont } \\
\text { E Simont }\end{array}$ & $\begin{array}{l}12591 \\
12311\end{array}$ \\
\hline $166 \mathrm{Yb}$ & \multicolumn{6}{|l|}{$\therefore$} \\
\hline $\begin{array}{l}159 \mathrm{~Tb} \\
166 \mathrm{Er} \\
166 \mathrm{Er} \\
166 \mathrm{Er}\end{array}$ & $\begin{array}{r}: 4 \mathrm{~N} \\
a \\
a \\
a\end{array}$ & $\begin{array}{l}9.5+1 \\
\text { NOG } \\
6.8+1 \\
1.5+2\end{array}$ & $9.5+1$ & $\begin{array}{l}\text { JCL E } \\
\text { LND E } \\
\text { ORL E } \\
\text { PAR F }\end{array}$ & $\begin{array}{l}\text { E lnamurat } \\
\text { E Carlent } \\
\text { E Sarantlitegt } \\
\text { R Lefortt }\end{array}$ & $\begin{array}{r}10469 \\
8576 \\
8581 \\
8582\end{array}$ \\
\hline${ }^{168} \mathrm{Yb}$ & \multicolumn{6}{|l|}{$r_{n}$} \\
\hline${ }^{166} \mathrm{Er}$ & a & NDG & & LNDE & E Carlont & 8575 \\
\hline $166 \mathrm{Yb}$ & \multicolumn{6}{|c|}{$\operatorname{ros} \theta(E)$} \\
\hline $181 \mathrm{Ta}$ & p & NDG & & GRE $E$ & E Carrazt & 4410 \\
\hline${ }^{166} \mathrm{Yb}_{\mathrm{b}}$ & \multicolumn{6}{|l|}{$O(E)$} \\
\hline 825 & $86 K_{r}$ & $3.1+2$ & & BRK E & E Simont & 12590 \\
\hline $\begin{array}{l}130 \mathrm{Te} \\
130 \mathrm{~T}\end{array}$ & ${ }_{40}^{40} \mathrm{Ar}$ & $1.8+2$ & & BRK E & E simont & 12310 \\
\hline${ }^{130} \mathrm{Te}$ & ${ }^{40} \mathrm{Ar}$. & $1.6+2$ & & ORL E & E Sarantitest & 12316 \\
\hline $169 \mathrm{Tm}$ & p & $3.0+1$ & $4.0+1$ & LAS I & I Arthurt & 4337 \\
\hline $169 \mathrm{Tm}$ & $p$ & $3 \cdot 1+1$ & $4.4+1$ & KFK C & C Muenzel+ & 4338 \\
\hline $169 \mathrm{Tm}$ & p & $3.1+1$ & $4.4+1$ & MIL E & E Birattarit & $4339+$ \\
\hline $167 \mathrm{Yb}$ & \multicolumn{6}{|c|}{ coopound-nucleus $x_{y}$} \\
\hline $\begin{array}{l}130 \mathrm{Te} \\
150 \mathrm{Nd}\end{array}$ & $\begin{array}{l}40 \mathrm{Ar} \\
20 \mathrm{Ne}\end{array}$ & $\begin{array}{l}1.6+2 \\
9.8+1\end{array}$ & $1.8+?$ & $\begin{array}{l}\text { ORLE } \\
\text { ORL E }\end{array}$ & $\begin{array}{l}\text { E Sorantitest } \\
\text { E Sarantitest }\end{array}$ & $\begin{array}{l}12317 \\
11895\end{array}$ \\
\hline
\end{tabular}

\begin{tabular}{|c|c|c|c|c|c|c|}
\hline Targe! & $\operatorname{Inc}$ & $\begin{array}{l}E m i n \\
(M e V)\end{array}$ & $\begin{array}{l}E \text { Eax } \\
(M e V)\end{array}$ & Lab & quthor & No. \\
\hline${ }^{167} \mathrm{Yb}$ & \multicolumn{6}{|c|}{ compound-nuclous o(E) } \\
\hline $\begin{array}{l}165 \mathrm{Er} \\
167 \mathrm{Yb}\end{array}$ & \multicolumn{6}{|c|}{ partial average $v_{y}$} \\
\hline $\begin{array}{l}130 \mathrm{Te} \\
150 \mathrm{Nd} \\
166 \mathrm{Er}\end{array}$ & $\begin{array}{c}40 \mathrm{Ar} \\
20 \mathrm{Ne} \\
0\end{array}$ & $\begin{array}{l}1.5+2 \\
9.4+1 \\
6.8+1\end{array}$ & $\begin{array}{l}1.1+2 \\
9.5+1\end{array}$ & $\begin{array}{l}\text { ORL } \\
\text { ORL E } \\
\text { ORL E }\end{array}$ & $\begin{array}{l}\text { E Sarantlliegt } \\
\text { E Sorantitest } \\
\text { E Sarantitest }\end{array}$ & $\begin{array}{r}12315 \\
11889 \\
8579\end{array}$ \\
\hline $167 \mathrm{Yb}$ & \multicolumn{6}{|c|}{ partlol relative o(E) } \\
\hline $\begin{array}{l}130 \mathrm{Te} \\
167 \mathrm{Yb}\end{array}$ & $\begin{array}{l}40 \mathrm{Ar} \\
\text { parti }\end{array}$ & \multicolumn{5}{|c|}{ partiol of(E) } \\
\hline $\begin{array}{l}150 \mathrm{Nd} \\
168 \mathrm{Er} \\
167 \mathrm{Yb}\end{array}$ & $\begin{array}{c}20 \mathrm{Ne} \\
0 \\
v_{\gamma}\end{array}$ & $\begin{array}{l}1.1+2 \\
6.8+1\end{array}$ & $9.5+1$ & $\begin{array}{l}\text { ORL E } \\
\text { ORL E }\end{array}$ & $\begin{array}{l}\text { E Sararititest } \\
\text { E Sarartitest }\end{array}$ & $\begin{array}{r}11888 \\
8578\end{array}$ \\
\hline $\begin{array}{l}130 \mathrm{Te} \\
166 \mathrm{Er} \\
186 \mathrm{Er}\end{array}$ & $\begin{array}{c}{ }^{40} \mathrm{Ar} \\
a \\
0\end{array}$ & $\begin{array}{l}1.5+2 \\
6.8+1 \\
1.5+2\end{array}$ & $9.5+1$ & $\begin{array}{l}\text { PAR } \\
\text { ORL E } \\
\text { PAR }\end{array}$ & $\begin{array}{l}\text { R Lefortt } \\
\text { E Sarantitest } \\
\text { R Lefortt }\end{array}$ & $\begin{array}{r}12318 \\
8581 \\
8582\end{array}$ \\
\hline $167 \mathrm{Yb}$ & \multicolumn{6}{|c|}{ row $O(E)$} \\
\hline $\begin{array}{l}181 \mathrm{Ta} \\
167 \mathrm{Yb}\end{array}$ & $\begin{array}{c}P \\
O(E)\end{array}$ & \multicolumn{4}{|c|}{$O(E)$} & 4410 \\
\hline $\begin{array}{l}130 \mathrm{Te} \\
169 \mathrm{Tm} \\
169 \mathrm{Tm} \\
169 \mathrm{Tm}\end{array}$ & $\begin{array}{c}{ }^{40} \mathrm{Ar} \\
p \\
p \\
P\end{array}$ & $\begin{array}{l}1.6+2 \\
1.9+1 \\
2.0+1 \\
2.0+1\end{array}$ & $\begin{array}{l}4.0+1 \\
4.2+1 \\
4.2+1\end{array}$ & $\begin{array}{l}\text { ORL E } \\
\text { LAS T } \\
\text { KFK } \\
\text { MIL E }\end{array}$ & $\begin{array}{l}\text { E Sarantitest } \\
\text { T Arthurt } \\
\text { C Muenzel+ } \\
\text { E Birattart+ }\end{array}$ & $\begin{array}{r}12316 \\
4340 \\
4341 \\
4342\end{array}$ \\
\hline $18 \theta_{\mathrm{Yb}}$ & \multicolumn{6}{|c|}{ compound-nucleus $v_{y}$} \\
\hline $\begin{array}{l}130 \mathrm{Te} \\
150 \mathrm{Nd}\end{array}$ & $\begin{array}{l}{ }^{40} \mathrm{Ar} \\
20 \mathrm{Ne}\end{array}$ & $\begin{array}{l}1.6+2 \\
9.8+1\end{array}$ & $1.8+2$ & $\begin{array}{l}\text { ORL E } \\
\text { ORL E }\end{array}$ & $\begin{array}{l}\text { E Sarantitest } \\
\text { E Sarantitest }\end{array}$ & $\begin{array}{l}12317 \\
11895\end{array}$ \\
\hline $160 \mathrm{Yb}$. & \multicolumn{6}{|c|}{ compound-nucleus o(E) } \\
\hline $\begin{array}{l}168 \mathrm{Er} \\
1.68 \mathrm{YO}\end{array}$ & \multicolumn{6}{|c|}{ portial overage $v_{y}$} \\
\hline $\begin{array}{l}150 \mathrm{Nd} \\
166 \mathrm{Er}\end{array}$ & $\underset{0}{20} \mathrm{Ne}$ & $\begin{array}{l}9.4+1 \\
6.8+1\end{array}$ & $\begin{array}{l}1.1+2 \\
9.5+1\end{array}$ & $\begin{array}{l}\text { ORL E } \\
\text { ORL E }\end{array}$ & $\begin{array}{l}\text { E Sorantitest } \\
\text { E Sorantitest }\end{array}$ & $\begin{array}{r}11889 \\
8579\end{array}$ \\
\hline
\end{tabular}




\begin{tabular}{|c|c|c|c|c|}
\hline Target & $\operatorname{lnc}$ & $\begin{array}{l}\text { Eimin Emax } \\
\text { (MeV) }\end{array}$ & Lab $\mathrm{W}$ & No. \\
\hline $16 \theta_{\mathrm{Yb}}$ & \multicolumn{4}{|c|}{ portial relative o(E) } \\
\hline $\begin{array}{l}167 \mathrm{Er} \\
168 \mathrm{Er} \\
169 \mathrm{Tm}\end{array}$ & $\begin{array}{l}0 \\
0 \\
0\end{array}$ & $\begin{array}{l}4.0+1 \\
4.0+1 \\
1.8+12.4+1\end{array}$ & $\begin{array}{l}\text { WAU E Fergesont } \\
\text { WAU E Fergesont } \\
\text { CBR M Oracoulist }\end{array}$ & $\begin{array}{l}8584 \\
8587 \\
5781\end{array}$ \\
\hline $160_{\mathrm{Yb}}$ & \multicolumn{3}{|c|}{ portial o(E) } & \\
\hline $\begin{array}{l}190 \mathrm{No} \\
165 \mathrm{Er}\end{array}$ & ${ }_{a}^{20} \mathrm{Ne}$ & $\begin{array}{l}1.1+2 \\
6.8+19.5+1\end{array}$ & $\begin{array}{l}\text { ORL E Sarantitest } \\
\text { ORL E Sarantilest }\end{array}$ & $\begin{array}{r}11888 \\
8578\end{array}$ \\
\hline $160_{\mathrm{Yb}}$ & \multicolumn{3}{|c|}{ relotive $1_{r}$} & \\
\hline $169_{\mathrm{Tm}}$ & ${ }^{3} \mathrm{He}$ & $4.2+1 \quad 5.7+1$ & GRE E Barneoudt & 6500 \\
\hline $160_{\mathrm{Yb}}$ & \multicolumn{2}{|l|}{$v_{\nu}$} & & \\
\hline $\begin{array}{l}130 \mathrm{Te} \\
165 \mathrm{Er} \\
166 \mathrm{Er}\end{array}$ & $\begin{array}{l}40 \mathrm{Ar} \\
:\end{array}$ & $\begin{array}{l}1.5+2 \\
E .8+19.5+1 \\
1.5+2\end{array}$ & $\begin{array}{l}\text { PAR R Leforlt } \\
\text { ORL E Sorantilest } \\
\text { PAR R Lefort+ }\end{array}$ & $\begin{array}{r}12318 \\
8581 \\
8582\end{array}$ \\
\hline $168_{\mathrm{Yb}}$ & \multicolumn{2}{|c|}{ row o(E) } & & \\
\hline $181 \mathrm{Ta}$ & p & NDO & GRE E Carraz+ & 4410 \\
\hline $180_{\mathrm{Yb}}$ & \multicolumn{2}{|l|}{ O(E) } & & \\
\hline $\begin{array}{l}130 \mathrm{Te} \\
169 \mathrm{Jm}\end{array}$ & ${ }^{40} \mathrm{Ar}$ & $\begin{array}{l}1.6+2 \\
1.0+1 \quad 4.0+1\end{array}$ & $\begin{array}{l}\text { ORL E Saronbitest } \\
\text { LAS T Arthurt }\end{array}$ & $\begin{array}{r}12316 \\
4343\end{array}$ \\
\hline $160 \mathrm{Yb}$ & \multicolumn{3}{|c|}{ including molostable (E) } & \\
\hline $\begin{array}{l}169 \mathrm{Tm} \\
169 \mathrm{Tm}\end{array}$ & $p$ & $\begin{array}{ll}3.3+0 & 4 \cdot 4+1 \\
3 \cdot 3+0 & 4 \cdot 4+1\end{array}$ & $\begin{array}{l}\text { KFK C Muenzel+ } \\
\text { MIL E Birattarit }\end{array}$ & $\begin{array}{l}4345 \\
4346\end{array}$ \\
\hline $180 \mathrm{Yb}$ & \multicolumn{2}{|l|}{$v_{y}$} & & \\
\hline $130 \mathrm{Te}$ & ${ }^{40} \mathrm{Ar}$ & $: .5+2$ & PAR R Lefort+ & 12318 \\
\hline $160_{\mathrm{Yb}}$ & \multicolumn{2}{|c|}{ rON OLE) } & & \\
\hline $181 \mathrm{To}$ & P & NOG & GRE E Carrazt+ & 4410 \\
\hline $169_{\mathrm{Yb}}$ & \multicolumn{2}{|l|}{$O(E)$} & & \\
\hline $169 \mathrm{Tm}$ & $p$ & $5.0+0 \quad 2.0+1$ & LAS I Arthur. & 4344 \\
\hline $170 \mathrm{Yb}$ & \multicolumn{2}{|c|}{ row $\sigma(E)$} & & \\
\hline $181 \mathrm{Ta}$ & p & NDG & GRE E Carrazt & 4410 \\
\hline
\end{tabular}

\begin{tabular}{|c|c|c|c|c|c|}
\hline Target & $\ln c$ & $\begin{array}{l}E \mathrm{Em} / \mathrm{n} \\
(\mathrm{MeV})\end{array}$ & $\begin{array}{l}\text { Emox } \\
(M \mathrm{MeV})\end{array}$ & Author & No. \\
\hline $171 \mathrm{Yb}$ & relat & ve $\mathrm{l}_{\text {y }}$ & & & . \\
\hline $169 \mathrm{Tm}$ & ${ }^{3} \mathrm{He}$ & $4 \cdot 2+1$ & $5.7+1$ & GRE E Barneoudt & 6500 \\
\hline $171 Y \mathrm{~b}$ & roso & & & & \\
\hline $181 \mathrm{Ta}$ & p & NDG & & GRE E Carraz+ & 4410 \\
\hline $172_{\mathrm{Yb}}$ & ravo & & & & \\
\hline $181 \mathrm{Ta}$ & p & NOG & & GRE E Carraz+ & 4410 \\
\hline $173 \mathrm{Yb}$ & rawo & & & & \\
\hline $181 \mathrm{To}$ & p & NDG & & GRE E Carraz+ & 4410 \\
\hline $174 \mathrm{Yb}$ & ravo & & & & \\
\hline 181 to & p & NDG & & GRE E Carrozt+ & 4410 \\
\hline $175 \mathrm{Yb}$ & rave & & & . & \\
\hline $181 \mathrm{Ta}$ & p & NDG & & GRE E Carraz+ & 4410 \\
\hline $156 \mathrm{Lu}$ & $O(E)$ & & & & \\
\hline 1271 & ${ }^{40} \mathrm{Ar}$ & $1.5+2$ & $2.8+2$ & PAR E Gauvint & 12325 \\
\hline $186_{\mathrm{Lu}}$ & rolol & vo $I_{\gamma}$ & & & \\
\hline $169 \mathrm{Tm}$ & ${ }^{3} \mathrm{He}$ & $4.2+1$ & $5.7+1$ & GRE E Barneoudt & 6500 \\
\hline $167 \mathrm{Lu}$ & relat & ve $I_{y}$ & & & \\
\hline $\begin{array}{l}169 \mathrm{Tm} \\
169 \mathrm{~mm} \\
169 \mathrm{gm} \\
170 \mathrm{Ym}\end{array}$ & $\begin{array}{l}{ }_{3}^{3} \mathrm{He} \\
{ }^{3} \mathrm{He} \\
0\end{array}$ & $\begin{array}{l}4.5+1 \\
4.2+1 \\
7.2+1\end{array}$ & $5.7+1$ & $\begin{array}{l}\text { GRE E Barneoudt } \\
\text { GRE E Barneoudt } \\
\text { GRE E Barneoudt }\end{array}$ & $\begin{array}{l}6499 \\
6500 \\
8591\end{array}$ \\
\hline & p & $4.0+1$ & & GRE E Barneoudt & 4348 \\
\hline${ }^{16 \theta_{\mathrm{Lu}}}$ & relot & ve 1 , & & & \\
\hline $169 \mathrm{Tm}$ & ${ }^{3} \mathrm{He}$ & $4.2+1$ & $5.7+1$ & GRE E Barneoudt & 6500 \\
\hline $189 \mathrm{Lv}$ & relat & ve Iy & & & \\
\hline $169 \mathrm{~T}$ & ${ }^{3} \mathrm{He}$ & $4.2+1$ & $5.7+1$ & GRE E Borneoudt & 6500 \\
\hline $171 \mathrm{Lu}$ & produ & 1 yleld & & & \\
\hline $169 \mathrm{Tm}$ & 。 & NDG & & YAL $E$ Sent & 8592 \\
\hline $177 \mathrm{Lu}$ & $\sigma(E)$ & & & & \\
\hline $176 \mathrm{Yb}$ & p & $6.0+0$ & $2.4+1$ & UPP $E$ Bergqurista & 4354 \\
\hline
\end{tabular}




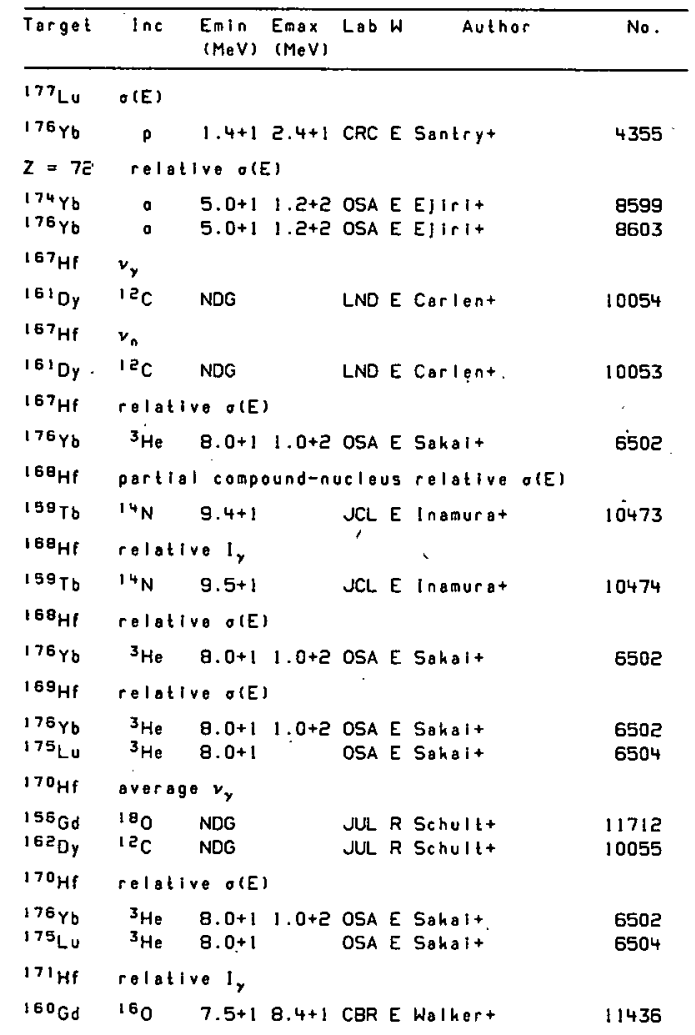

\begin{tabular}{|c|c|c|c|c|c|c|}
\hline Target & Inc & $\begin{array}{l}\text { Emin } \\
(\mathrm{MeV})\end{array}$ & $\begin{array}{l}\text { Emax } \\
(\operatorname{MeV})\end{array}$ & Lab & Author & No. \\
\hline $17 I_{\mathrm{HF}}$ & \multicolumn{6}{|c|}{ relative o(E) } \\
\hline $\begin{array}{l}175 \mathrm{Yb} \\
175 \mathrm{LU} \\
175 \mathrm{LU}\end{array}$ & $\begin{array}{l}{ }^{3} \mathrm{He} \\
{ }^{\mathrm{P}} \\
{ }^{3} \mathrm{He}\end{array}$ & $\begin{array}{l}8.0+1 \\
4.0+1 \\
8.0+1\end{array}$ & $1.0+2$ & $\begin{array}{l}\text { OSA } \\
\text { OSA } \\
\text { OSA }\end{array}$ & $\begin{array}{l}\text { E Sohalt } \\
\text { M Sahalt } \\
\text { E Sahalt }\end{array}$ & $\begin{array}{l}6502 \\
4360 \\
6504\end{array}$ \\
\hline $17 Z_{H f}$ & \multicolumn{6}{|c|}{ relative $\mathrm{t}_{y}$} \\
\hline $\begin{array}{l}160 \mathrm{Go} \\
172_{\mathrm{Hf}}\end{array}$ & \multicolumn{6}{|c|}{ product yleld } \\
\hline $\begin{array}{l}175_{\mathrm{LU}} \\
172_{\mathrm{HF}}\end{array}$ & \multicolumn{6}{|c|}{ overage $v_{y}$} \\
\hline $\begin{array}{l}158 \mathrm{Gd} \\
164 \mathrm{Dy}\end{array}$ & $\begin{array}{l}18_{0} \\
12 \mathrm{C}\end{array}$ & $\begin{array}{l}\text { NDG } \\
\text { NDG }\end{array}$ & & $\begin{array}{l}J U L \\
J L L\end{array}$ & $\begin{array}{l}R \text { Schultt } \\
R \text { Schultt }\end{array}$ & $\begin{array}{l}11713 \\
10056\end{array}$ \\
\hline $172_{\mathrm{HF}}$ & \multicolumn{6}{|c|}{ relotive o(E) } \\
\hline $\begin{array}{l}176 \mathrm{YU} \\
175 \mathrm{LU} \\
175 \mathrm{LU}\end{array}$ & $\begin{array}{l}{ }^{3} \mathrm{He} \\
{ }^{p} \mathrm{He}\end{array}$ & $\begin{array}{l}8.0+1 \\
4.0+1 \\
8.0+1\end{array}$ & $1.0+2$ & $\begin{array}{l}\text { OSA } \\
\text { OSA } \\
\text { OSA }\end{array}$ & $\begin{array}{l}\text { E Sakait } \\
\text { M Sakait } \\
\text { E Sakalt }\end{array}$ & $\begin{array}{l}6502 \\
4360 \\
6504\end{array}$ \\
\hline $173_{\mathrm{Hf}}$ & \multicolumn{6}{|c|}{ relative $O(E)$} \\
\hline $\begin{array}{l}176 \mathrm{Yb} \\
175 \mathrm{Lu} \\
175 \mathrm{LU} \\
173 \mathrm{HF}\end{array}$ & $\begin{array}{l}{ }^{3} \mathrm{He} \\
{ }^{p} \\
{ }^{p} \mathrm{He} \\
\sigma(E)\end{array}$ & $\begin{array}{l}8 \cdot 0+1 \\
4 \cdot 0+1 \\
8 \cdot 0+1\end{array}$ & $1.0+2$ & $\begin{array}{l}\text { OSA } \\
\text { OSA } \\
\text { OSA }\end{array}$ & $\begin{array}{l}\text { E Sakai+ } \\
\text { M Sakai+ } \\
\text { E Sakai+ }\end{array}$ & $\begin{array}{l}6502 \\
4360 \\
6504\end{array}$ \\
\hline $\begin{array}{l}181 \mathrm{Ta} \\
181 \mathrm{Ta}\end{array}$ & $\begin{array}{l}d \\
d\end{array}$ & $\begin{array}{l}6 \cdot 0+1 \\
6 \cdot 3+1\end{array}$ & $\begin{array}{l}1 \cdot 0+2 \\
8 \cdot 0+1\end{array}$ & $\begin{array}{l}\text { BNL } \\
\text { JUL }\end{array}$ & $\begin{array}{l}\text { T Divadeenam. } \\
\text { E Bisplirghofit }\end{array}$ & $\begin{array}{l}5791 \\
5792\end{array}$ \\
\hline${ }^{174} \mathrm{HF}$ & \multicolumn{6}{|c|}{ partlal relative o(E) } \\
\hline $\begin{array}{l}172 Y \mathrm{Yb} \\
173 \mathrm{Yb} \\
174 \mathrm{Yb} \\
174 \mathrm{Yb}\end{array}$ & 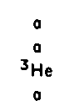 & $\begin{array}{l}2.2+1 \\
4.0+1 \\
2.5+1 \\
4.0+1\end{array}$ & & $\begin{array}{l}\text { OSA } \\
\text { WAU } \\
\text { OSA } \\
\text { WAU }\end{array}$ & $\begin{array}{l}\text { E Kishimeto+ } \\
\text { E Fergesent } \\
\text { E Kishimcto+ } \\
\text { E Fergesent }\end{array}$ & $\begin{array}{l}8594 \\
8597 \\
6501 \\
8598\end{array}$ \\
\hline $174 \mathrm{HF}$ & \multicolumn{6}{|c|}{ partlat o(E) x factor } \\
\hline $\begin{array}{l}171 Y \mathrm{Yb} \\
172 \mathrm{Yb} \\
175 \mathrm{Lu}\end{array}$ & $\begin{array}{l}a \\
0 \\
p\end{array}$ & $\begin{array}{l}2 \cdot 0+1 \\
2 \cdot 0+1 \\
4 \cdot 0+1\end{array}$ & $\begin{array}{l}2.8+1 \\
2.8+1\end{array}$ & $\begin{array}{l}\text { NBI } \\
\text { NBI } \\
\text { OSA }\end{array}$ & $\begin{array}{l}\text { EEjirit } \\
\text { E Ejrit } \\
\text { M Sahait }\end{array}$ & $\begin{array}{l}8593 \\
8595 \\
4357\end{array}$ \\
\hline
\end{tabular}




\begin{tabular}{|c|c|c|c|c|c|c|}
\hline Target & $\operatorname{lnc}$ & $\begin{array}{l}\text { Emin } \\
(\mathrm{MeV})\end{array}$ & $\begin{array}{l}\text { Emax } \\
(\operatorname{MeV})\end{array}$ & Lab & Author & No. \\
\hline $174 \mathrm{HF}$ & \multicolumn{6}{|c|}{ relative o(E) } \\
\hline $\begin{array}{l}176 \mathrm{Yb} \\
175 \mathrm{Lu} \\
175 \mathrm{Lu}\end{array}$ & $\begin{array}{l}{ }^{3} \mathrm{He} \\
{ }^{p} \mathrm{He}\end{array}$ & $\begin{array}{l}8.0+1 \\
4.0+1 \\
8.0+1\end{array}$ & $1.0+2$ & $\begin{array}{l}\text { OSA } \\
\text { OSA } \\
\text { OSA }\end{array}$ & $\begin{array}{l}\text { E Sahalt } \\
\text { M Sahait } \\
\text { E Sahait }\end{array}$ & $\begin{array}{l}6502 \\
4360 \\
6504\end{array}$ \\
\hline $174 \mathrm{HF}$ & $O(E)$ & & & & 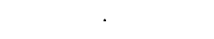 & \\
\hline $\begin{array}{l}165 \mathrm{Ho} \\
175 \mathrm{Hf}\end{array}$ & $\begin{array}{l}\text { "B } \\
\text { cumul }\end{array}$ & $\begin{array}{l}5.9+1 \\
\text { tive th }\end{array}$ & $\begin{array}{l}7.5+1 \\
\text { hich to }\end{array}$ & $\begin{array}{l}\text { JPS } \\
\text { orget }\end{array}$ & $\begin{array}{l}\text { R Johnson. } \\
\text { yleld }\end{array}$ & 9588 \\
\hline $\begin{array}{l}181 \mathrm{Ta} \\
181 \mathrm{Ta}\end{array}$ & $\begin{array}{l}p \\
p\end{array}$ & $\begin{array}{l}5.5+1 \\
1.0+2\end{array}$ & $8.5+1$ & $\begin{array}{l}\text { LVN } \\
\text { MRY }\end{array}$ & $\begin{array}{l}\text { E Haasbroekt } \\
\text { E Hasbroek+ }\end{array}$ & $\begin{array}{l}4384+ \\
4385+\end{array}$ \\
\hline${ }^{179} \mathrm{HI}$ & \multicolumn{6}{|c|}{ cumulativo $O(E)$} \\
\hline IQIT: & p & $5.5+1$ & $8.5+1$ & LVN & E Hasbroeht & $4383+$ \\
\hline $175_{\mathrm{Hf}}$ & thick & target & yield & & & \\
\hline $\mathrm{Ta}$ & $\rho$ & $5.5+1$ & $1.0+2$ & LVN & E Hoosbroekt & 4377 \\
\hline $175 \mathrm{HF}$ & \multicolumn{6}{|c|}{ relative o(E) } \\
\hline $\begin{array}{l}175 \mathrm{Lu} \\
175 \mathrm{Hf}\end{array}$ & \multicolumn{6}{|l|}{$O(E)$} \\
\hline $\begin{array}{l}{ }^{175} \mathrm{Lu} \\
\mathrm{Ta} \\
181 \mathrm{Ta}\end{array}$ & $\begin{array}{l}p \\
p \\
p\end{array}$ & $\begin{array}{l}\text { NDG } \\
5.5+1 \\
4.6+1\end{array}$ & $\begin{array}{l}1.0+2 \\
6.1+1\end{array}$ & $\begin{array}{l}\text { LAS } \\
\text { LVN } \\
\text { JUL }\end{array}$ & $\begin{array}{l}\text { T Arthur. } \\
\text { E Hassbroekt } \\
\text { E Bisplingholft }\end{array}$ & $\begin{array}{l}4358 \\
4376 \\
5793\end{array}$ \\
\hline${ }^{176} \mathrm{Hf}$ & \multicolumn{6}{|c|}{ portial rolative o(E) } \\
\hline $176 \mathrm{Yb}$ & 0 & $4.0+1$ & & WAU & E Fergesont & 8602 \\
\hline${ }^{176} \mathrm{HF}$ & \multicolumn{6}{|c|}{ rolative o(E) } \\
\hline $175 \mathrm{Lu}$ & ${ }^{3} \mathrm{He}$ & $8.0+1$ & & OSA & E Sakait & 6504 \\
\hline $177_{\mathrm{HF}}$ & \multicolumn{6}{|c|}{ portlal row thich larget yleldabundanco } \\
\hline $177_{\mathrm{Hf}}$ & $\circ$ & $5.0+0$ & & SUN & E Gilest & 8609 \\
\hline $178_{\mathrm{HF}}$ & \multicolumn{6}{|c|}{ partial raw thich torget yleldrabundanco } \\
\hline $178_{\mathrm{Hf}}$ & a & $5.0+0$ & & SUN & E Gilest & 8612 \\
\hline${ }^{179} \mathrm{HF}$ & \multicolumn{6}{|c|}{ portlol row thich terget yleldat } \\
\hline $179_{\mathrm{HI}}$ & 0 & $5.0+0$ & & SUN & E Gilest & 8613 \\
\hline
\end{tabular}

\begin{tabular}{|c|c|c|c|c|c|c|}
\hline Target & Inc & $\begin{array}{l}\text { Emin } \\
(\mathrm{MeV})\end{array}$ & $\begin{array}{l}\text { Emax } \\
(\operatorname{mev})\end{array}$ & Lab W & Author & No. \\
\hline $80_{\mathrm{HF}}$ & partlat & $1 \log 1$ & thlek t & target & yieldxabundance & 冫 \\
\hline $180 \mathrm{HF}$ & a & $5.0+0$ & & SUN E & Gilest & 8616 \\
\hline${ }_{181} \mathrm{HI}$ & $a(E)$ & & & & & \\
\hline $181 \mathrm{Ta}$ & d & $3.8+1$ & $7.9+1$ & JUL E & Eisplinghofft & 5794 \\
\hline${ }^{177} \mathrm{To}$ & $O(E)$ & & & & & \\
\hline $181 T 8$ & p & $4.0+1$ & $1.0+2$ & BNL T & Divadeenam. & 4386 \\
\hline${ }^{178} \mathrm{Ta}$ & portiol & I $O(E)$ & & & & \\
\hline $\begin{array}{l}181 \mathrm{Ta} \\
181 \mathrm{Ta}\end{array}$ & $p$ & $\begin{array}{l}2.9+1 \\
2.9+1\end{array}$ & $\begin{array}{l}4.4+1 \\
4.4+1\end{array}$ & $\begin{array}{l}\text { KFK C } \\
\text { MIL E }\end{array}$ & $\begin{array}{l}\text { Muenzelt } \\
\text { Birattarit }\end{array}$ & $\begin{array}{l}4388 \\
4389+\end{array}$ \\
\hline $17 \theta_{\mathrm{Ta}}$ & relotir & $v e I_{y}$ & & & & \\
\hline $\begin{array}{l}176 \mathrm{Lu} \\
178_{\mathrm{HF}} \\
178_{\mathrm{Hf}}\end{array}$ & $\begin{array}{l}a \\
p \\
d\end{array}$ & $\begin{array}{l}2.3+1 \\
6.7+0 \\
1.4+1\end{array}$ & $2.7+1$ & $\begin{array}{l}\text { ROS E } \\
\text { ROS E } \\
\text { ROS E }\end{array}$ & $\begin{array}{l}\text { Dubberst } \\
\text { Dubberst } \\
\text { Dubberst }\end{array}$ & $\begin{array}{l}8606 \\
4362 \\
5782\end{array}$ \\
\hline${ }^{178} \theta_{\mathrm{T}}$ & $O(E)$ & & & & & \\
\hline $\begin{array}{l}181 T_{\theta} \\
181 T_{\theta} \\
181 T_{\theta}\end{array}$ & $\begin{array}{l}\text { p } \\
\text { d } \\
\text { d }\end{array}$ & $\begin{array}{l}3 \cdot 5+1 \\
4 \cdot 0+1 \\
4 \cdot 2+1\end{array}$ & $\begin{array}{l}1.0+2 \\
1.0+2 \\
8.0+1\end{array}$ & $\begin{array}{l}\text { BNL T } \\
\text { BNL T } \\
\text { JUL E }\end{array}$ & $\begin{array}{l}\text { Divadeenam. } \\
\text { Divadeenam. } \\
\text { Bisplinghofit }\end{array}$ & $\begin{array}{l}4387 \\
5795 \\
5796\end{array}$ \\
\hline $178 \mathrm{TO}$ & $O(E)$ & & & & & \\
\hline $\begin{array}{l}181 \mathrm{Ta} \\
180 \mathrm{Ta}\end{array}$ & $\begin{array}{c}p \\
\sigma(E)\end{array}$ & $2.5+1$ & $1.0+2$ & BNL $T$ & Divadeanam. & 4390 \\
\hline $\begin{array}{l}180 \mathrm{HF} \\
181 \mathrm{~TB}\end{array}$ & $\begin{array}{l}p \\
p\end{array}$ & $\begin{array}{l}\text { NDG } \\
2.0+1\end{array}$ & $1.0+2$ & $\begin{array}{l}\text { ANL E } \\
\text { BNL T }\end{array}$ & Divadeenam. & $\begin{array}{l}4363 \\
4391\end{array}$ \\
\hline $1800 \mathrm{Ta}$ & thlck & targot & $t y 1010$ & & & \\
\hline $\begin{array}{l}180 \mathrm{HF} \\
1800 \mathrm{To}\end{array}$ & $\begin{array}{c}p \\
g(E)\end{array}$ & $8.0+0$ & $9.0+0$ & ANL E & Normont & 4365 \\
\hline${ }^{180} \mathrm{HF}$ & p & $8.0+0$ & $9.0+0$ & ANL E & Normant & 4364 \\
\hline $100 \cdot \mathrm{T}_{0}$ & thlch & torget & tylelo & & & \\
\hline $\begin{array}{l}180^{H F} \\
180 \cdot T_{0}\end{array}$ & $\begin{array}{c}P \\
O(E)\end{array}$ & $0.0+0$ & $9.0+0$ & ANL E & Normant & 4366 \\
\hline $181 \mathrm{To}$ & p & TR & $2.4+1$ & ORL E & Cohent & $4392+$ \\
\hline
\end{tabular}




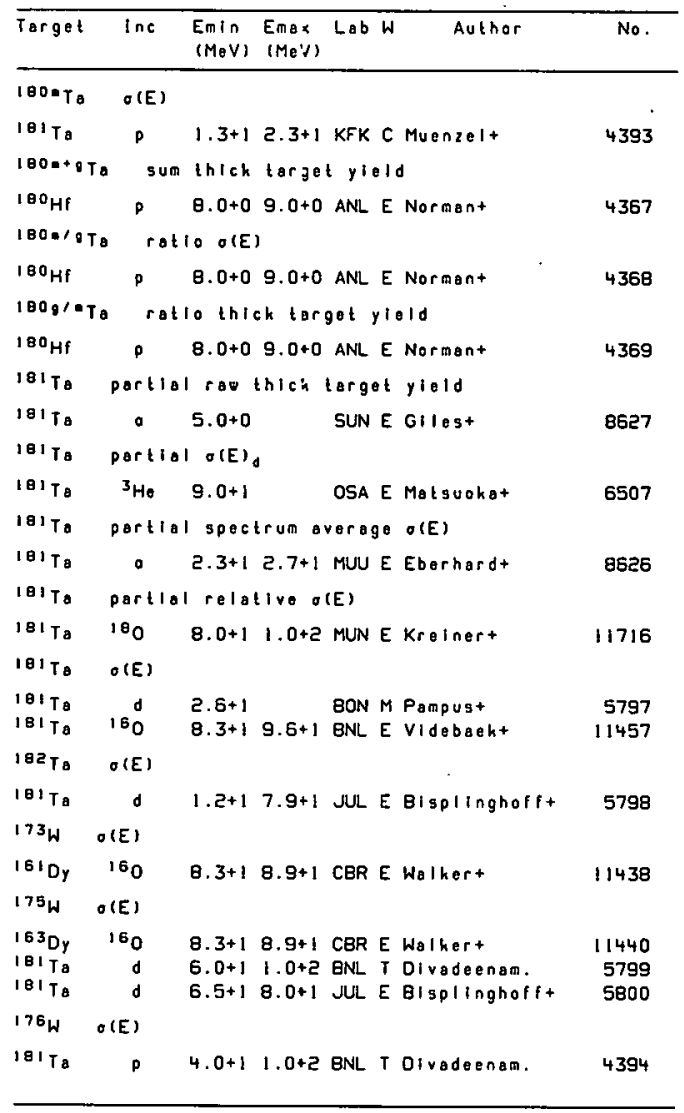

\begin{tabular}{|c|c|c|c|c|c|}
\hline Target & $\operatorname{lnc} c$ & $\begin{array}{l}E_{m}(n) \\
\left(M_{e} V\right)\end{array}$ & $\begin{array}{l}\text { Emax } \\
(\operatorname{MeV})\end{array}$ & Puthor & $\mathrm{Na}$. \\
\hline $176 \mathrm{~W}$ & $O(E)$ & & & & \\
\hline $\begin{array}{l}181 T 0 \\
181 T 0 \\
181 T 0 \\
177 \mathrm{~W}\end{array}$ & $\begin{array}{r}\begin{array}{r}p \\
d \\
d \\
O(E)\end{array} \\
0\end{array}$ & $\begin{array}{l}7.0+2 \\
4.0+1 \\
5.5+1\end{array}$ & $\begin{array}{l}6.0+3 \\
1.0+2 \\
8.0+1\end{array}$ & $\begin{array}{l}\text { NRL T Silberberg+ } \\
\text { BNL T Oivaceenam. } \\
\text { JUL E Bisplinghofft }\end{array}$ & $\begin{array}{l}4395 \\
5801 \\
5802\end{array}$ \\
\hline $\begin{array}{l}18178 \\
181 T_{8} \\
181 \mathrm{~T}_{8} \\
181 \mathrm{To}\end{array}$ & $\begin{array}{l}p \\
p \\
d \\
d\end{array}$ & $\begin{array}{l}3 \cdot 0+1 \\
8 \cdot 0+2 \\
4 \cdot 0+1 \\
4 \cdot 4+1\end{array}$ & $\begin{array}{l}1.0+2 \\
1.5+3 \\
1.0+2 \\
8.0+1\end{array}$ & $\begin{array}{l}\text { BNL T Divaceenam. } \\
\text { NRL R Silberbergt } \\
\text { BNL T Divadeenam. } \\
\text { JUL E Bisplinghofft }\end{array}$ & $\begin{array}{l}4396 \\
4397 \\
5803 \\
5804\end{array}$ \\
\hline $\begin{array}{l}178_{W} \\
180 \mathrm{~W}\end{array}$ & $\begin{array}{c}\text { partial } \\
p\end{array}$ & $\begin{array}{l}\text { roloti } \\
2.1+1\end{array}$ & ive pro & $\begin{array}{l}\text { oduct yield } \\
\text { YAL E Mortensen. }\end{array}$ & 4418 \\
\hline $178_{W}$ & relative & e Iy & & & \\
\hline $\begin{array}{l}177_{\mathrm{Hf}} \\
178_{\mathrm{W}}\end{array}$ & $\sigma(E)$ & $3.4+1$ & $4.6+1$ & MSU E Dorst & 8610 \\
\hline $\begin{array}{l}181 T 0 \\
181 \% 8 \\
181 \% 8 \\
18178 \\
181 T_{0}\end{array}$ & $\begin{array}{l}p \\
p \\
p \\
d \\
d\end{array}$ & $\begin{array}{l}2 \cdot 0+1 \\
2 \cdot 8+1 \\
2 \cdot 8+1 \\
2 \cdot 5+1 \\
3 \cdot 8+1\end{array}$ & $\begin{array}{l}1.0+2 \\
4.4+1 \\
4.4+1 \\
1.0+2 \\
7.9+1\end{array}$ & $\begin{array}{l}\text { BNL T Divacieanam. } \\
\text { KFK C Muenzelt } \\
\text { MIL E Birattarit } \\
\text { BNL T Diraceanam. } \\
\text { JUL E Bisplinghoift }\end{array}$ & $\begin{array}{l}4398 \\
4399 \\
4400 \\
5805 \\
5806\end{array}$ \\
\hline $179 \mathrm{~W}$ & Includin & ing wets & ostable & $? \sigma(E)$ & \\
\hline $\begin{array}{l}181 \mathrm{Ta} \\
181 \mathrm{To} \\
179_{\mathrm{W}}\end{array}$ & $\begin{array}{r}p \\
0(E)\end{array}$ & $\begin{array}{l}1.8+1 \\
1.8+1\end{array}$ & $\begin{array}{l}4.4+11 \\
4.4+11\end{array}$ & $\begin{array}{l}\text { KFK C Muenzel+ } \\
\text { MIL E Biratiarl+ }\end{array}$ & $\begin{array}{l}4402 \\
4403\end{array}$ \\
\hline $\begin{array}{l}181 \mathrm{Tg} \\
180 \mathrm{~W}\end{array}$ & partiol & $\begin{array}{l}2.0+1 \\
\text { relati }\end{array}$ & $\begin{array}{l}1.0+2 \\
\text { twe pro }\end{array}$ & $\begin{array}{l}\text { BNL T Divaceenam. } \\
\text { duct yield. }\end{array}$ & 4401 \\
\hline $\begin{array}{l}182_{W} \\
180_{W}\end{array}$ & parlial & $\begin{array}{l}2.1+1 \\
\text { reloti }\end{array}$ & Ive olE & $\begin{array}{l}\text { YAL E Mortensen. } \\
\text { E) }\end{array}$ & 4419 \\
\hline $\begin{array}{l}179 \mathrm{HF} \\
100 \mathrm{HF}\end{array}$ & $a$ & $\begin{array}{l}4 \cdot 0+1 \\
4 \cdot 0+1\end{array}$ & & $\begin{array}{l}\text { WAU E Fergesont } \\
\text { WAU E Fergesont }\end{array}$ & $\begin{array}{l}8614 \\
8617\end{array}$ \\
\hline $180 \mathrm{~W}$ & partlol & $\sigma(E)$ & & & \\
\hline $181 T_{0}$ & $p$ & $1.0+1$ & f & FEI E Birythovt & 4406 \\
\hline
\end{tabular}




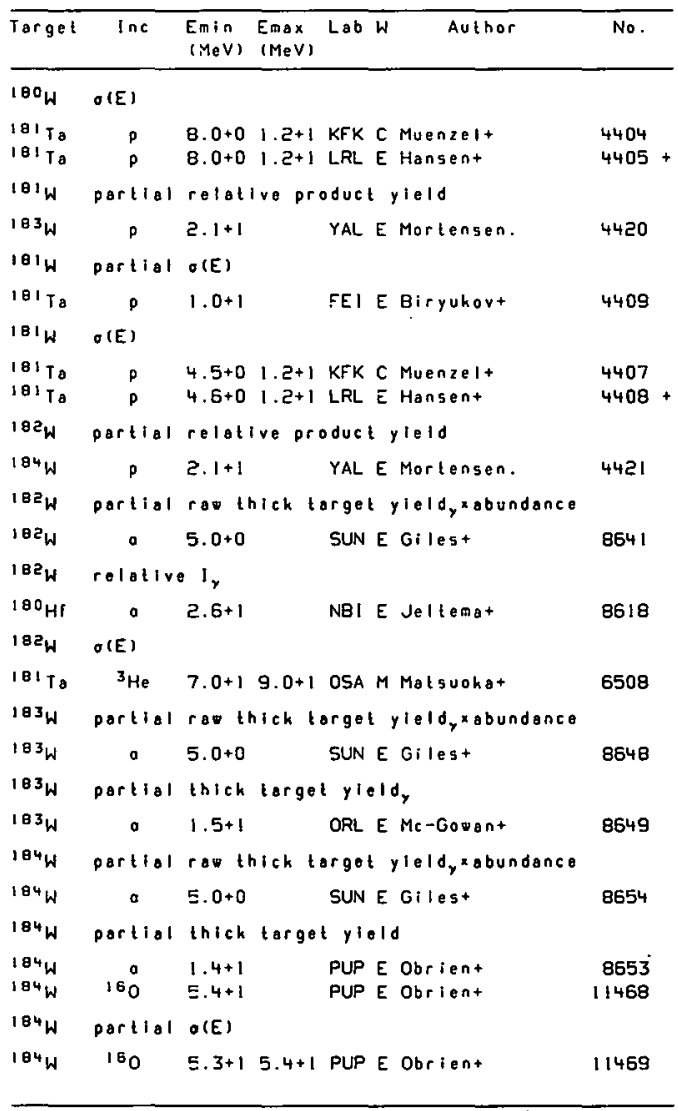

\begin{tabular}{|c|c|c|c|c|c|c|}
\hline Target & $\ln c$ & $\begin{array}{l}\text { Emin } \\
(M e V)\end{array}$ & $\begin{array}{l}\text { Emax } \\
(\operatorname{MeV})\end{array}$ & Lab h & Author & No. \\
\hline $186 w$ & partial & rawth & hick ta & arget & yieldyabundance & \\
\hline $186_{W}$ & 。 & $5 \cdot 0+0$ & & SUN E & E Gulest & 8660 \\
\hline $186_{W}$ & portiol & thick & target & $t$ yiel & 19 & \\
\hline $\begin{array}{l}186_{W} \\
186_{W}\end{array}$ & $15_{0}^{\circ}$ & $\begin{array}{l}1.4+1 \\
5.4+1\end{array}$ & & $\begin{array}{l}\text { PUP E } \\
\text { PUP E }\end{array}$ & $\begin{array}{l}\text { E Obrient } \\
\text { E Obrient }\end{array}$ & $\begin{array}{r}8659 \\
11470\end{array}$ \\
\hline $186_{W}$ & portiol & O(E) & & & & \\
\hline $\begin{array}{l}186 \mathrm{~W} \\
177_{R e}\end{array}$ & $\begin{array}{l}{ }^{16} 0 \\
\sigma(E)\end{array}$ & $5.3+1$ & $5.4+1$ & PUP E & E Obrient & 11471 \\
\hline $\begin{array}{l}181 \mathrm{Ta} \\
181 \mathrm{Ta} \\
181 \mathrm{Ta}\end{array}$ & $\begin{array}{c}{ }^{3} \mathrm{He} \\
{ }^{3} \mathrm{He} \\
0\end{array}$ & $\begin{array}{l}6.1+1 \\
6.1+1 \\
8.6+1\end{array}$ & $\begin{array}{l}7.4+1 \\
7.4+1 \\
1.0+2\end{array}$ & $\begin{array}{l}\text { IKO E } \\
\text { KFK C } \\
\text { KFK E }\end{array}$ & $\begin{array}{l}\text { E Hermest } \\
\text { C Muenzel+ } \\
\text { E Hermest }\end{array}$ & $\begin{array}{l}6509+ \\
6510 \\
8628\end{array}$ \\
\hline $17 \theta_{\mathrm{Re}}$ & spaltat & tion of & (E) & & & \\
\hline $\begin{array}{l}209_{B i} \\
178_{R e}\end{array}$ & $\begin{array}{c}P \\
\sigma(E)\end{array}$ & $3.8+2$ & & NRL $R$ & R Silberbergt & 4762 \\
\hline $\begin{array}{l}181 \mathrm{Ta} \\
181 \mathrm{Ta} \\
181 \mathrm{Tg}\end{array}$ & $\begin{array}{l}{ }^{3} \mathrm{He} \\
{ }^{3} \mathrm{He} \\
a\end{array}$ & $\begin{array}{l}5 \cdot 0+1 \\
5 \cdot 0+1 \\
7 \cdot 5+1\end{array}$ & $\begin{array}{l}7.4+1 \\
7.4+1 \\
9.9+1\end{array}$ & $\begin{array}{l}\text { jKO E } \\
\text { KFK O } \\
\text { KFK E }\end{array}$ & $\begin{array}{l}\text { E Hermest } \\
\text { C Muenzel+ } \\
\text { E Hermegt }\end{array}$ & $\begin{array}{l}6511+ \\
6512 \\
8629\end{array}$ \\
\hline${ }^{179} R_{0}$ & spallot & tion of & $(E)$ & & & \\
\hline $\begin{array}{l}{ }^{209} \mathrm{Bi} \\
179_{\mathrm{Re}}\end{array}$ & $\begin{array}{c}P \\
O(E)\end{array}$ & $3.8+2$ & & NRL $R$ & R Silberberg* & 4762 \\
\hline $\begin{array}{l}181 \mathrm{Ta} \\
181 \mathrm{Ta} \\
181 \mathrm{Ta}\end{array}$ & $\begin{array}{l}{ }^{3} \mathrm{He} \\
{ }^{3} \mathrm{He} \\
0\end{array}$ & $\begin{array}{l}3 \cdot 9+1 \\
3 \cdot 9+1 \\
6 \cdot 3+1\end{array}$ & $\begin{array}{l}7.4+1 \\
7.4+1 \\
9.2+1\end{array}$ & $\begin{array}{l}\text { IKO E } \\
\text { KFK O } \\
\text { KFK E }\end{array}$ & $\begin{array}{l}\text { E Hermest } \\
\text { C Muenzel+ } \\
\text { E Hermest }\end{array}$ & $\begin{array}{l}6513+ \\
6514 \\
8630\end{array}$ \\
\hline${ }^{180} \mathrm{Re}$ & & tion of & $(E)$ & & & \\
\hline $\begin{array}{l}2098 i \\
100 \mathrm{R}\end{array}$ & $\begin{array}{c}P \\
\sigma(E)\end{array}$ & $3.8+2$ & & NRL $F$ & R Silberbergt & 4762 \\
\hline $\begin{array}{l}181 \mathrm{Ta} \\
181 \mathrm{Ta} \\
181 \mathrm{Ta}\end{array}$ & $\begin{array}{l}{ }^{3} \mathrm{He} \\
{ }^{3} \mathrm{He} \\
0\end{array}$ & $\begin{array}{l}3 \cdot 0+1 \\
3 \cdot 0+1 \\
5 \cdot 0+1\end{array}$ & $\begin{array}{l}6 \cdot 3+1 \\
6 \cdot 3+1 \\
7.7+1\end{array}$ & $\begin{array}{l}\text { IKO E } \\
\text { KFK O } \\
\text { KFK E }\end{array}$ & $\begin{array}{l}\text { E Hermest } \\
\text { C Muenze I+ } \\
\text { E Hermest }\end{array}$ & $\begin{array}{l}6515+ \\
6516 \\
8631\end{array}$ \\
\hline $1 \theta_{\mathrm{R} \theta}$ & spollot & tion ol & $(E)$ & & & \\
\hline${ }^{209} \theta i$ & $p$ & $3.8+2$ & & NRL F & R Silberberg+ & 4762 \\
\hline
\end{tabular}




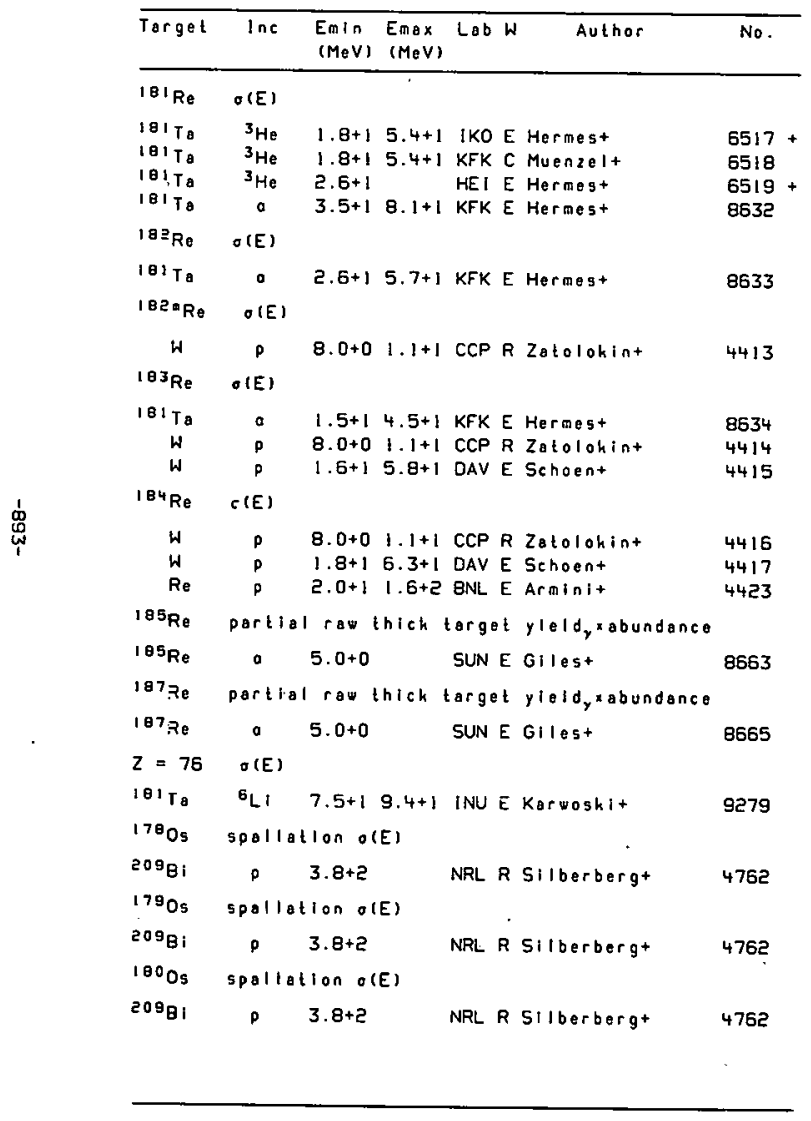

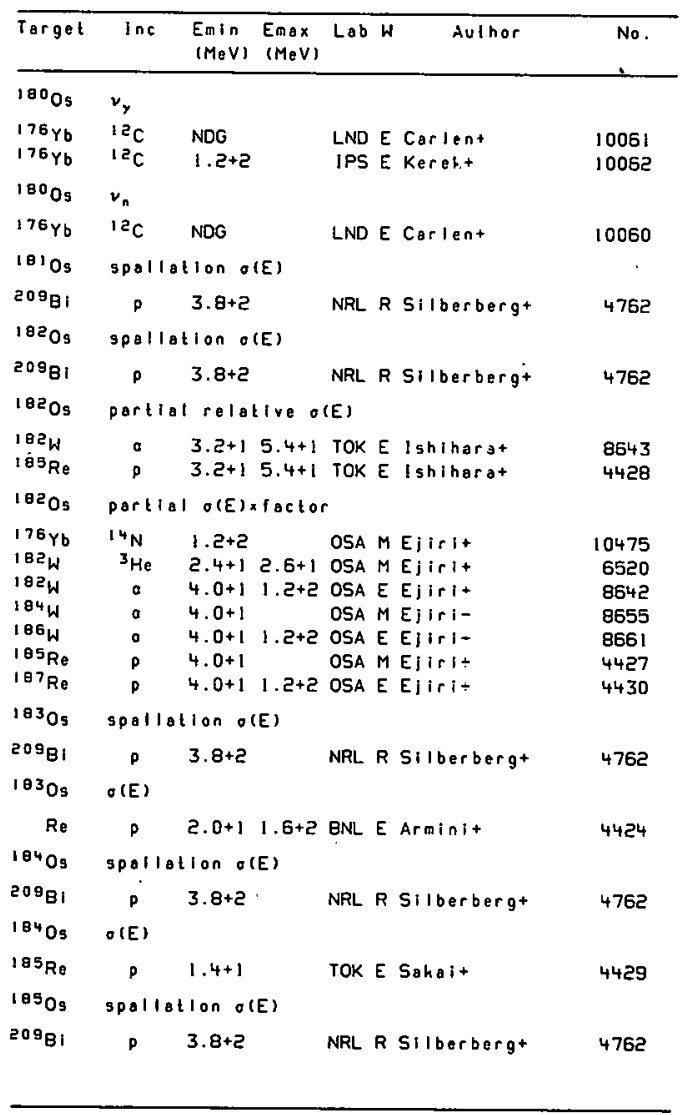




\begin{tabular}{|c|c|c|c|c|c|c|}
\hline Target & $\ln c$ & $\begin{array}{l}\text { Emin } \\
(\mathrm{MeV})\end{array}$ & $\begin{array}{l}\text { Emax } \\
(\mathrm{MeV})\end{array}$ & Lab & Author & No. \\
\hline 1850 & $O(E)$ & & & & & \\
\hline $\begin{array}{r}\mathrm{Re} \\
18\rangle_{\mathrm{Re}}\end{array}$ & $p$ & $\begin{array}{l}2.0+1 \\
2.0+1\end{array}$ & $\begin{array}{l}1.6+2 \\
1.6+2\end{array}$ & BNL & $\begin{array}{l}\text { E Arminit } \\
\text { E Arminit }\end{array}$ & $\begin{array}{l}4425 \\
4431\end{array}$ \\
\hline $1860 \mathrm{~s}$ & spoll & ticn of & (E) & & & \\
\hline $209 \mathrm{Bi}$ & $\rho$ & $3.8+2$ & & NRL & R Sitberberg* & 4762 \\
\hline $186 \mathrm{Og}$ & $O(E)$ & & & & 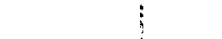 & \\
\hline $\begin{array}{l}174 \mathrm{Yb} \\
107 \mathrm{Re}\end{array}$ & $\begin{array}{r}12 \mathrm{C} \\
p\end{array}$ & $\begin{array}{r}+1 \\
1.4+1\end{array}$ & +2 & $\begin{array}{l}\text { STB } \\
\text { TOK }\end{array}$ & $\begin{array}{l}\text { T Delagranget } \\
\text { E Sakait }\end{array}$ & $\begin{array}{r}10059 \\
4432\end{array}$ \\
\hline 10709 & spoll & tion of & (E) & & & \\
\hline $209^{B r}$ & P & $3.8+2$ & & NRL & R silberbergt & 4762 \\
\hline${ }^{180} 0 \mathrm{~s}$ & spoll & llon of & (E) & & & \\
\hline $209^{B i}$ & $p$ & $3.8+2$ & & NRL & R Sliberberg+ & 4762 \\
\hline $1090 \mathrm{~g}$ & spoll & tion of & (E) & & & \\
\hline $209^{8 i}$ & $p$ & $3.8+2$ & & NRL & R Silberberg+ & 4762 \\
\hline $1890 \mathrm{~s}$ & $\sigma(E)$ & & & & & \\
\hline${ }^{187} \mathrm{Re}$ & ${ }^{7} \mathrm{Li}$ & $3.5+1$ & & ROS & E Hagemannt & 9456 \\
\hline 19209 & $O(E)$ & & & & & \\
\hline $1920 \mathrm{~s}$ & 150 & NOG & & HAR & E Cuninghamet & 11475 \\
\hline $193 \mathrm{Og}$ & porti & $10(E) \times$ & x factor & & . & \\
\hline $1920 \mathrm{~s}$ & d & $1.2+1$ & & FSU & E Bensont & 5808 \\
\hline $179 \mathrm{Ir}$ & spoll & tion of & (E) & & & \\
\hline $209^{\circ} \mathrm{I}$ & $p$ & $3.8+2$ & & NRL & R Silberbergt & 4762 \\
\hline $100 \mathrm{Ir}$ & spoll & tlon ol & (E) & & . & \\
\hline $200^{B i}$ & P & $3.8+2$ & & NRL & R Silberberg+ & 4762 \\
\hline 181 If & spoll & tion of & (E) & & & \\
\hline $209_{B 1}$ & $p$ & $3.8+2$ & & NRL & R Silberberg+ & 4762 \\
\hline $181 \mathrm{Ir}$ & produ & t yiold & & & & \\
\hline $169 \mathrm{Tm}$ & 150 & $7.0+1$ & $1.3+2$ & YAL & E Ladenbaver-Bel1 & 11443 \\
\hline
\end{tabular}

\begin{tabular}{|c|c|c|c|c|c|}
\hline Target & $\ln c$ & $\begin{array}{ll}\text { Emin } & \text { Emax } \\
(M e V) & (M e V)\end{array}$ & Lab h & Author & No. \\
\hline $102 \mathrm{Ir}$ & \multicolumn{5}{|c|}{ Spallotion o(E) } \\
\hline $209^{B 1}$ & P & $3.8+2$ & NRL & R silberbergt & 4762 \\
\hline $103 \mathrm{Ir}$ & \multicolumn{5}{|c|}{ spollotion o(E) } \\
\hline $\operatorname{sos}^{81}$ & $p$ & $3.8+2$ & NRL $F$ & R silberberg+ & 4762 \\
\hline $1041 r$ & \multicolumn{5}{|c|}{ Spollistlon $O(E)$} \\
\hline $209_{\mathrm{Bi}}$ & $p$ & $3.8+2$ & NRL $F$ & R Silberberg+ & 4762 \\
\hline $185 \mathrm{Ir}$ & \multicolumn{5}{|c|}{ Spollation o(E) } \\
\hline $209_{B 1}$ & p & $3.8+2$ & NRL $F$ & R silberberg+ & 4762 \\
\hline $\log _{\mathrm{l}} \mathrm{t}$ & \multicolumn{5}{|c|}{ portlal rolative o(E) } \\
\hline $\begin{array}{l}{ }^{109} R_{\theta} \\
107 \mathrm{Re}\end{array}$ & $:$ & $\begin{array}{ll}4 \cdot 1+1 & 5 \cdot 6+1 \\
7 \cdot 2+1 & 7 \cdot 7+1\end{array}$ & $\begin{array}{l}\text { GRE E } \\
\text { GRE E }\end{array}$ & $\begin{array}{l}\text { E Androt } \\
\text { E Andret }\end{array}$ & $\begin{array}{l}8664 \\
8665\end{array}$ \\
\hline $188_{1 r}$ & \multicolumn{5}{|c|}{ Spallation o(E) } \\
\hline${ }^{209} \mathrm{Bi}$ & $p$ & $3.8+2$ & ṆRL F & R Silberborgt & 4762 \\
\hline $187 \mathrm{Ir}$ & \multicolumn{5}{|c|}{ spollotion $\sigma^{\prime}(E)$} \\
\hline $209^{B 1}$ & $\dot{p}$ & $3.8+2$ & NRL F & R silberberg* & 4762 \\
\hline $187 \mathrm{ir}$ & \multicolumn{5}{|l|}{$\sigma(E)$} \\
\hline $175 \mathrm{Lu}$ & $1{ }^{12} \mathrm{C}$ & +1 & Sta $T$ & $T$ Delogranget & 10064 \\
\hline $108 \mathrm{ir}$ & \multicolumn{5}{|c|}{ SPOIlotION O(E) } \\
\hline $209_{B i}$ & p & $3.8+2$ & NRL F & R silberborgt & 4762 \\
\hline $189_{16}$ & \multicolumn{5}{|c|}{ Spollation o(E) } \\
\hline${ }^{209} \mathrm{BI}$ & $p$ & $3.8+2$ & NRL F & R silberberg+ & 4762 \\
\hline $189 \mathrm{ir}$ & \multicolumn{5}{|c|}{ portlal $\sigma(E)$} \\
\hline 19118 & $p$ & $3.5+1$ & PTN E & E Strublet & 4439 \\
\hline $1800 \mathrm{Ir}$ & \multicolumn{5}{|l|}{$\sigma(E)$} \\
\hline $\begin{array}{l}191 \mathrm{Ir} \\
100.1 \mathrm{Ir}\end{array}$ & $\begin{array}{l}P \\
\sigma(E)\end{array}$ & $1.5+1 \quad 1.7+1$ & MCOE & E Lau. & 4440 \\
\hline 18118 & D & $1.5+1 \quad 1.7+1$ & MCG E & E Lav. & 4441 \\
\hline
\end{tabular}




\begin{tabular}{|c|c|c|c|c|c|}
\hline Target & $\ln c$ & $\begin{array}{l}\text { Emin } \text { Emax } \\
(\mathrm{MeV})(\mathrm{MeV})\end{array}$ & Lab r & Author & No. \\
\hline $180.2 \mathrm{Ir}$ & O(E) & 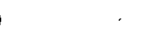 & & & \\
\hline $\begin{array}{l}1911 \mathrm{lr} \\
180019 \mathrm{gr}\end{array}$ & $P^{p}$ rall & $\begin{array}{l}1.5+1 \quad 1.7+1 \\
10 \cdot(E)\end{array}$ & MCG E & E Lau. & 4442 \\
\hline $1900 \mathrm{~s}$ & d & $1.2+1 \quad 1.4+1$ & Buc $F$ & R Poenaru. & 5807 \\
\hline$[\theta 1 \mid r$ & Dorlial & I row thlck t & target & it yleldyabundance & \\
\hline 191/r & $a$ & $5.0+0$ & SUN E & E Gilest & 8678 \\
\hline $191 \mathrm{Ir}$ & partlo & $1 \sigma(E)$ & & & \\
\hline 19318 & $p$ & $3.5+1$ & PTN E & E Strublet & 4447 \\
\hline 1818 & rolotil & ve 1 , & & & \\
\hline $\begin{array}{l}192 \mathrm{Og} \\
1920 \mathrm{~s}\end{array}$ & $\begin{array}{l}p \\
d\end{array}$ & 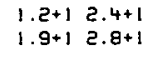 & $\begin{array}{l}\text { IBJ E } \\
1 B J E\end{array}$ & $\begin{array}{l}\text { E Luhasiaht } \\
\text { E Luhasiaht }\end{array}$ & $\begin{array}{l}4434 \\
5809\end{array}$ \\
\hline $1931 r$ & partio & l rasthlch & targot & t yiold $x$ abundonce & \\
\hline 19318 & a & $5.0+0$ & SUN E & E Gllest & 8679 \\
\hline $180 \mathrm{Pt}$ & spallot & tion o(E) & & . & \\
\hline $209 \mathrm{Bi}$ & P & $3.8+2$ & NRL $P$ & R Silberberg+ & 4762 \\
\hline$\{\theta \mid P t$ & spollat & tlon $O(E)$ & & & \\
\hline $20{ }^{0} \mathrm{BI}$ & p & $3.8+2$ & NRL & R Silberbergt & 4762 \\
\hline $181 \mathrm{Pt}$ & produc & $t y 1010$ & & & \\
\hline $\begin{array}{l}169 \mathrm{Tm} \\
18 \mathrm{PPt}^{\mathrm{P}}\end{array}$ & $\begin{array}{l}19 \mathrm{~F} \\
\text { spollal }\end{array}$ & $\begin{array}{l}1.0+21.3+2 \\
\text { llon } g(E)\end{array}$ & YAL & E Ladenbaver-8e11 & 11813 \\
\hline $\begin{array}{l}{ }^{20 \theta_{B 1}} \\
183 \mathrm{PI}\end{array}$ & spalla & $\begin{array}{l}3.8+2 \\
\text { tlon } \theta(E)\end{array}$ & NRL F & R Silberbergt & 4762 \\
\hline $\begin{array}{l}200_{\mathrm{Bl}} \\
184 \mathrm{Pl}\end{array}$ & spolla & $\begin{array}{l}3.8+2 \\
\operatorname{lton} o(E)\end{array}$ & NRL $F$ & R Sllberberg* & 4762 \\
\hline $\begin{array}{l}200^{B t} \\
104 \mathrm{Pt}\end{array}$ & product & $\begin{array}{l}3.8+2 \\
1 \quad y 1010\end{array}$ & NRL $F$ & R silberbergt & 4762 \\
\hline $181 \mathrm{~T}$ & $14 N$ & $1.4+2$ & MAN & E Oaim. & 10476 \\
\hline
\end{tabular}

\begin{tabular}{|c|c|c|c|c|c|}
\hline Target & $\ln c$ & $\begin{array}{ll}\text { Emin } & \text { Emsx } \\
(\mathrm{MeV}) & (\mathrm{MeV})\end{array}$ & Lab W & Author & No. \\
\hline${ }^{105} \mathrm{Pt}$ & spoll & thon $\sigma(E)$ & & & \\
\hline $\begin{array}{l}209^{\circ} \mathrm{Bt} \\
105 \mathrm{Pt}\end{array}$ & $\begin{array}{c}\text { p } \\
\text { produ }\end{array}$ & $\begin{array}{l}3.8+2 \\
t y i \theta l d\end{array}$ & NRL $R$ & R Silberbergt & 4762 \\
\hline $\begin{array}{l}181 \mathrm{Ta} \\
186 \mathrm{pt}\end{array}$ & $\begin{array}{l}14 \mathrm{~N} \\
\text { spoll }\end{array}$ & $\begin{array}{l}1.4+2 \\
110 n \text { o(E) }\end{array}$ & MAN E & E $O_{a 1 \mathrm{~m}}$. & 10477 \\
\hline $\begin{array}{l}209^{\circ} \mathrm{Bi} \\
186 \mathrm{Pt}\end{array}$ & $\begin{array}{c}\text { p } \\
\text { produ }\end{array}$ & $\begin{array}{l}3.8+2 \\
t \quad y i e l d\end{array}$ & NRL $R$ & R Silberberg+ & 4762 \\
\hline $\begin{array}{l}181 \mathrm{~TB} \\
187 \mathrm{PI}\end{array}$ & $\begin{array}{l}14 \mathrm{~N} \\
\text { spall }\end{array}$ & $\begin{array}{l}1.4+2 \\
\text { tion } 0(E)\end{array}$ & MAN E & E Doim. & 10478 \\
\hline $\begin{array}{l}209 \mathrm{Bl} \\
187 \mathrm{Pt}\end{array}$ & $p^{p}$ & $\begin{array}{l}3.8+2 \\
t \quad y i 01 d\end{array}$ & NRL $R$ & R Silberberg+ & 4762 \\
\hline $\begin{array}{l}181 \mathrm{Ta} \\
188 \mathrm{Pl}\end{array}$ & $\begin{array}{l}14 \mathrm{~N} \\
\text { gpalI }\end{array}$ & $\begin{array}{l}1.4+2 \\
110 n \text { O(E) }\end{array}$ & MAN $\varepsilon$ & E $O_{\theta} \mathrm{im}$. & 10479 \\
\hline $\begin{array}{l}200^{\circ i} \\
108 \mathrm{Pt}\end{array}$ & $p_{\text {pelet }}^{p}$ & $\begin{array}{l}3.8+2 \\
\text { ve } I_{y}\end{array}$ & NRL $R$ & R Silberberg+ & 4762 \\
\hline $\begin{array}{l}191 \mathrm{Ir} \\
189 \mathrm{Pt}\end{array}$ & $\begin{array}{c}p \\
\text { gpoll }\end{array}$ & $\begin{array}{l}3.8+1 \\
110 n \text { o(E) }\end{array}$ & TOK E & E Numas+ & 4443 \\
\hline $\begin{array}{l}200^{8 i} \\
189 \mathrm{Pt}\end{array}$ & $\begin{array}{c}\text { p } \\
\text { produ }\end{array}$ & $\begin{array}{l}3.8+2 \\
t \quad y i=1 d\end{array}$ & NRL R & R Silbarberg+ & 4762 \\
\hline $\begin{array}{l}181 \mathrm{Ta} \\
190 \mathrm{Pl}\end{array}$ & $\begin{array}{l}14 \mathrm{~N} \\
\text { Spoll }\end{array}$ & $\begin{array}{l}1.4+2 \\
110 n \text { o(E) }\end{array}$ & MAN E & $E a_{3} \mathrm{im}$. & 10480 \\
\hline $\begin{array}{l}20{ }^{\circ} \mathrm{Bi} \\
190 \mathrm{Pt}\end{array}$ & $\begin{array}{c}\text { p } \\
\text { partl }\end{array}$ & $\begin{array}{l}3.8+2 \\
\text { O(E) } \times \text { foctor }\end{array}$ & NRL R & R Silberberg+ & 4762 \\
\hline $\begin{array}{l}182 \mathrm{~W} \\
190 \mathrm{Pl}\end{array}$ & $\begin{array}{l}12 \mathrm{C} \\
O(E) x\end{array}$ & $\begin{array}{l}1.2+2 \\
\text { octor }\end{array}$ & BOR T & T Delajranget & 10074 \\
\hline $\begin{array}{l}18 \mathrm{E}_{\mathrm{W}} \\
180 \mathrm{Pt}\end{array}$ & $\begin{array}{l}I^{2} C \\
O(E)\end{array}$ & $7.0+1 \quad 1,3+2$ & BOR T & T Delagranget & 10073 \\
\hline $182_{W}$ & $12 \mathrm{C}$ & $7.0+1 \quad 1.3+2$ & BOR T & T Delagranget & 10072 \\
\hline
\end{tabular}




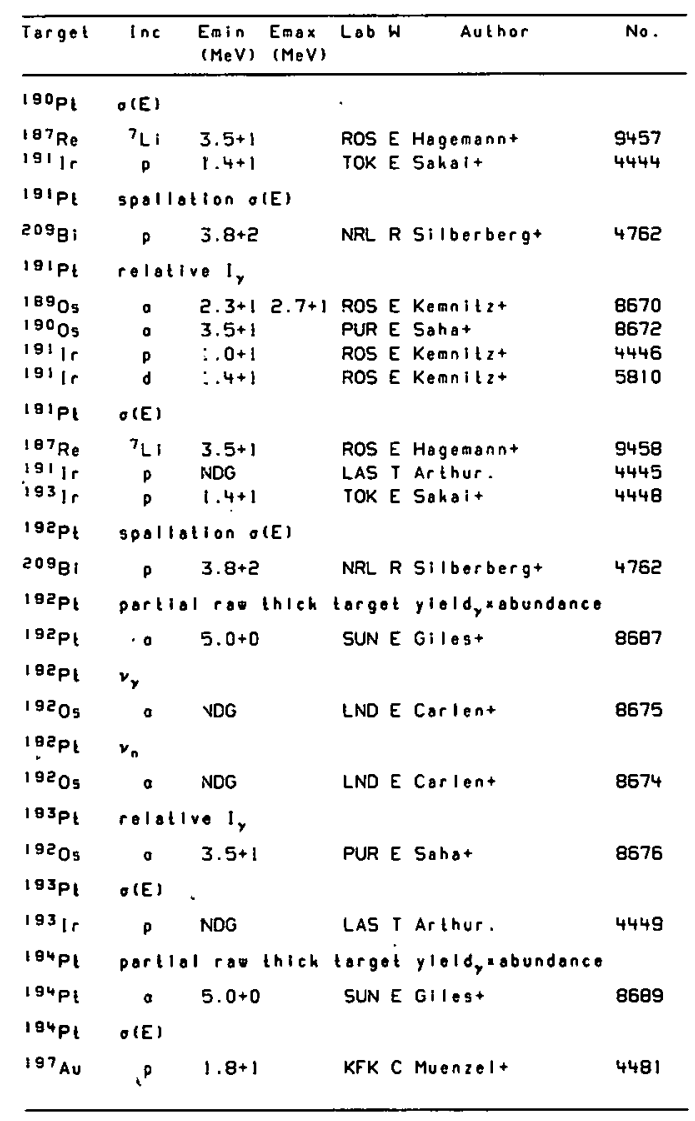

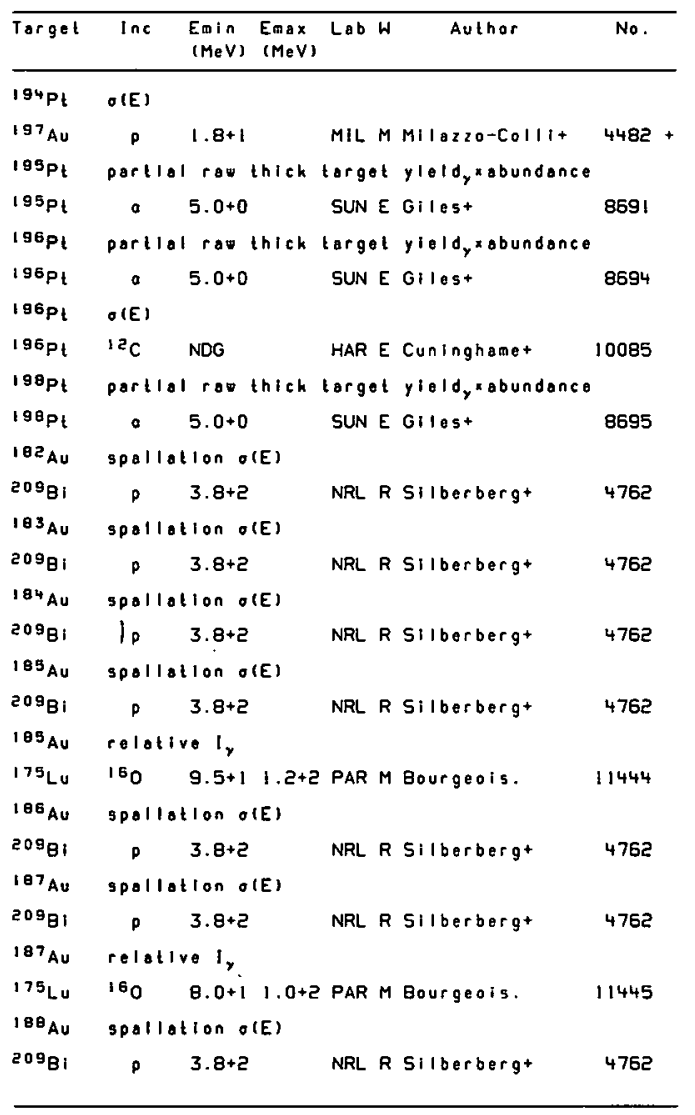




\begin{tabular}{|c|c|c|c|c|c|c|}
\hline Target & $\ln c$ & $\begin{array}{l}\text { Emin } \\
(\operatorname{MeV})\end{array}$ & $\begin{array}{l}\text { Emax } \\
(M e V)\end{array}$ & Lab & Author & No. \\
\hline${ }^{189} \mathrm{Au}$ & spall & tion ol & (E) & & & \\
\hline $\begin{array}{l}209^{8} \\
189_{A u}\end{array}$ & $\begin{array}{c}p \\
o(E)\end{array}$ & $3.8+2$ & & NRL & R Silberbergt & 4762 \\
\hline $\begin{array}{l}181 \mathrm{To} \\
190 \mathrm{At}\end{array}$ & $\begin{array}{l}{ }_{40} \mathrm{Ar} \\
\text { spall }\end{array}$ & $\begin{array}{l}\text { NOG } \\
\text { tion of }\end{array}$ & (E) & DUB & R Flerov. & 12355 \\
\hline $\begin{array}{l}209_{8 i} \\
190 \\
A v\end{array}$ & $\begin{array}{c}p \\
\sigma(E)\end{array}$ & $3.8+2$ & & NRL & R Silberbergt & 4762 \\
\hline $\begin{array}{l}181 \mathrm{Ta} \\
181 \mathrm{Au}\end{array}$ & $\begin{array}{l}{ }^{40} \mathrm{Ar} \\
\text { spall }\end{array}$ & $\begin{array}{l}\text { NDG } \\
\text { tion } 0\end{array}$ & (E) & OUB & R Flerov. & 12355 \\
\hline $\begin{array}{l}209^{B i} \\
191_{A U}\end{array}$ & $\begin{array}{c}p \\
\sigma(E)\end{array}$ & $3.8+2$ & & NRL & R Sillberbergt & 4762 \\
\hline $\begin{array}{l}18: \mathrm{Ta} \\
18: \mathrm{Ta} \\
\mathrm{Ir} \\
19: \mathrm{Ir} \\
193 \mathrm{Ir}\end{array}$ & 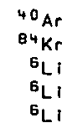 & $\begin{array}{l}\text { NDG } \\
\text { NDG } \\
6.2+1 \\
4.8+1 \\
4.8+1\end{array}$ & $\begin{array}{l}1.6+2 \\
1.6+2 \\
1.6+2\end{array}$ & $\begin{array}{l}\text { OUB } \\
\text { DUB } \\
\text { KFK } \\
\text { KFK } \\
\text { KFK }\end{array}$ & $\begin{array}{l}\text { R Fleroo. } \\
R \text { Flerov. } \\
\text { M Kroppt } \\
\text { M Kroppt } \\
M \text { Kroppt }\end{array}$ & $\begin{array}{r}12355 \\
12539 \\
9280 \\
9290 \\
9299\end{array}$ \\
\hline $192 \mathrm{Au}$ & indep & ndent & $\sigma(E)$ & & & \\
\hline $\begin{array}{l}197 \mathrm{Au} \\
197 \mathrm{Au}\end{array}$ & $\begin{array}{l}12 \mathrm{C} \\
160\end{array}$ & $\begin{array}{l}1.3+2 \\
1.4+2\end{array}$ & $1.7+2$ & $\begin{array}{l}\text { KFK } \\
\text { KFK }\end{array}$ & $\begin{array}{l}\text { C Muenzel+ } \\
\text { C Muenzel+ }\end{array}$ & $\begin{array}{l}10097 \\
11483\end{array}$ \\
\hline $192 \mathrm{Au}$ & spall & tion of & (E) & & & \\
\hline $\begin{array}{l}209_{\mathrm{Bl}} \\
182 \mathrm{AU}\end{array}$ & $\begin{array}{c}p \\
\text { cumut }\end{array}$ & $\begin{array}{l}3.8+2 \\
\text { tive of }\end{array}$ & (E) & NRL & R Silberbergt & 4762 \\
\hline $\begin{array}{l}197 \mathrm{Au} \\
197 \mathrm{Au} \\
202 \mathrm{Hg}\end{array}$ & $\begin{array}{r}{ }_{12} \mathrm{C} \\
{ }^{15} 0 \\
p\end{array}$ & $\begin{array}{l}1.3+2 \\
1.4+2 \\
1.2+1\end{array}$ & $\begin{array}{l}1.7+2 \\
8.6+1\end{array}$ & $\begin{array}{l}\text { KFK } \\
\text { KFK } \\
\text { MCG }\end{array}$ & $\begin{array}{l}\text { C Muenzel+ } \\
\text { C Muenzel+ } \\
\text { E Kantelot }\end{array}$ & $\begin{array}{c}10099 \\
11485 \\
4517+\end{array}$ \\
\hline $192 \mathrm{Au}$ & $\theta(E)$ & & & & & \\
\hline $\begin{array}{l}181 \mathrm{Ta} \\
181 \mathrm{Ta} \\
181 \mathrm{Ta} \\
202 \mathrm{Hg}\end{array}$ & $\begin{array}{c}4^{40} \mathrm{Ar} \\
\mathrm{B}^{4} \mathrm{Kr} \\
1{ }^{36} \mathrm{Xe} \\
\mathrm{p}\end{array}$ & $\begin{array}{l}\text { NDG } \\
\text { NDG } \\
\text { NDG } \\
7.4+1\end{array}$ & $0.5+1$ & $\begin{array}{l}\text { DUB } \\
\text { DUB } \\
\text { DUB } \\
\text { MIL }\end{array}$ & $\begin{array}{l}\text { R Flerov. } \\
\text { R Flerov. } \\
\text { R Flerov. } \\
\text { T Gadiolit }\end{array}$ & $\begin{array}{c}12355 \\
12539 \\
12608 \\
4518\end{array}$ \\
\hline
\end{tabular}

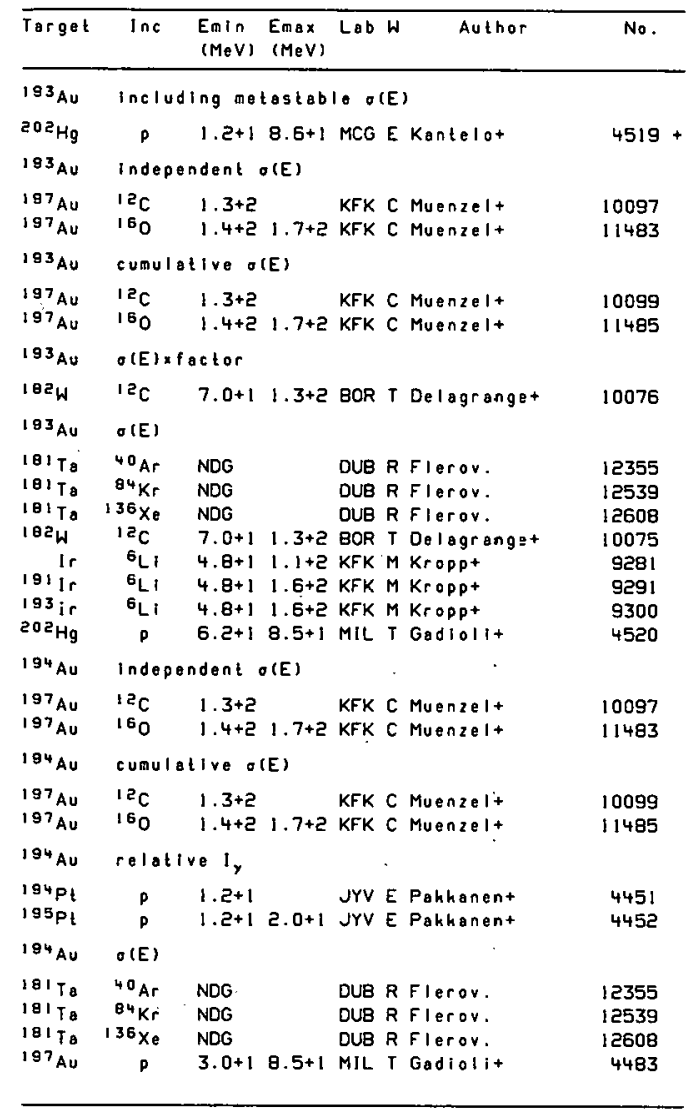




\begin{tabular}{|c|c|c|c|c|c|c|}
\hline Target & Inc & $\begin{array}{l}\text { Emin } \\
\text { (MeV) }\end{array}$ & $\begin{array}{l}\text { Emax } \\
(\operatorname{MeV})\end{array}$ & Lob & Author & No. \\
\hline${ }^{94} \mathrm{Au}$ & o(E) & & & & & \\
\hline $\begin{array}{l}197 \mathrm{Au} \\
197 \mathrm{Au} \\
197 \mathrm{Au} \\
197 \mathrm{Au} \\
197 \mathrm{Au} \\
202_{\mathrm{Hg}} \\
202_{\mathrm{Hg}}\end{array}$ & $\begin{array}{c}p \\
p \\
3_{\mathrm{He}} \\
0 \\
0 \\
p \\
\mathrm{p}\end{array}$ & $\begin{array}{l}3 \cdot 0+1 \\
3 \cdot 8+1 \\
2 \cdot 0+1 \\
5 \cdot 1+1 \\
5 \cdot 1+1 \\
1 \cdot 2+1 \\
5 \cdot 3+1\end{array}$ & $\begin{array}{l}7 \cdot 0+1 \\
9 \cdot 0+1 \\
1 \cdot 3+2 \\
9 \cdot 9+1 \\
9 \cdot 9+1 \\
8 \cdot 6+1 \\
8 \cdot 8+1\end{array}$ & $\begin{array}{l}\text { MIL T } \\
\text { MIL T } \\
\text { JUL } \\
\text { KFK C } \\
\text { ROC E } \\
\text { MCG E } \\
\text { MIL T }\end{array}$ & $\begin{array}{l}\text { T Gadiolit } \\
\text { T Gadiolit } \\
\text { T Aldersteint } \\
\text { C Muenzel+ } \\
\text { E Lanzafamet } \\
\text { E Kantelo+ } \\
\text { T Gadiolit }\end{array}$ & $\begin{array}{l}4484 \\
4485 \\
6522 \\
8723 \\
8724+ \\
4522+ \\
4521\end{array}$ \\
\hline $195 \mathrm{Au}$ & \multicolumn{6}{|c|}{ Independent $O(E)$} \\
\hline $\begin{array}{l}197 \mathrm{AU} \\
197 \mathrm{AU}\end{array}$ & $\begin{array}{l}12 \mathrm{C} \\
160\end{array}$ & $\begin{array}{l}1.3+2 \\
1.4+2\end{array}$ & $1.7+2$ & $\begin{array}{l}\text { KFK } \\
\text { KFK } 0\end{array}$ & $\begin{array}{l}\text { C Muenzel+ } \\
\text { C Muenzel+ }\end{array}$ & $\begin{array}{l}10097 \\
11483\end{array}$ \\
\hline $195_{A u}$ & \multicolumn{6}{|c|}{ cumulative $O(E)$} \\
\hline $\begin{array}{l}197 \mathrm{Au} \\
197_{\mathrm{AU}}\end{array}$ & $\begin{array}{l}a \\
a\end{array}$ & $\begin{array}{l}3.9+1 \\
3.9+1\end{array}$ & $\begin{array}{l}9.5+1 \\
9.5+1\end{array}$ & $\begin{array}{l}\text { KFK O } \\
\text { ROC E }\end{array}$ & $\begin{array}{l}\text { C Muenzel+ } \\
\text { E Lanzafomet }\end{array}$ & $\begin{array}{l}8725 \\
8726+\end{array}$ \\
\hline $185_{A U}$ & $\sigma(E)$ & & & & & \\
\hline $\begin{array}{l}181 \mathrm{TO} \\
181 \mathrm{TO} \\
197 \mathrm{AU} \\
197 \mathrm{Au} \\
197 \mathrm{AU} \\
20 \mathrm{Hg}^{\mathrm{H}}\end{array}$ & $\begin{array}{c}84 \mathrm{Kr} \\
136 \times \mathrm{Xe} \\
p \\
p \\
p \\
p\end{array}$ & $\begin{array}{l}\text { NDG } \\
\text { NDG } \\
2.8+1 \\
2.8+1 \\
\text { TR } \\
1.2+1\end{array}$ & $\begin{array}{l} \\
9.0+1 \\
9.0+1 \\
3.2+1 \\
8.6+1\end{array}$ & $\begin{array}{l}\text { DUA F } \\
\text { DUA } \\
\text { MIL T } \\
\text { MIL T } \\
\text { ERK E } \\
\text { MCG E }\end{array}$ & $\begin{array}{l}\text { R flerav. } \\
\text { R flerav. } \\
\text { T Gadiolit } \\
\text { T Gadiolit } \\
\text { E Gonzalez-Vidalt } \\
\text { E Kantelot }\end{array}$ & $\begin{array}{r}12539 \\
12608 \\
4486 \\
4487 \\
4488 \\
4523\end{array}$ \\
\hline $196 \mathrm{Au}$ & \multicolumn{6}{|c|}{ incluoing metastable? o(E) } \\
\hline $\begin{array}{l}197 \mathrm{Au} \\
197_{\mathrm{AU}}\end{array}$ & a & $\begin{array}{l}2 \cdot 5+1 \\
2 \cdot 5+1\end{array}$ & $\begin{array}{l}9.9+1 \\
9.9+1\end{array}$ & $\begin{array}{l}\text { KFK O } \\
\text { ROC E }\end{array}$ & $\begin{array}{l}\text { C Muenzel+ } \\
\text { E Lanzafamet }\end{array}$ & $\begin{array}{l}8727 \\
8728+\end{array}$ \\
\hline $196 \mathrm{Au}$ & \multicolumn{6}{|l|}{$O(E)$} \\
\hline $\begin{array}{l}181 \mathrm{Ta} \\
101 \mathrm{Ta} \\
187 \mathrm{Au} \\
197 \mathrm{Au} \\
197 \mathrm{Au} \\
197 \mathrm{Au} \\
202 \mathrm{Hg}\end{array}$ & $\begin{array}{c}{ }^{84} \mathrm{Kr} \\
136 \mathrm{Xe} \\
\mathrm{p} \\
\rho \\
{ }^{\mathrm{p}} \\
{ }^{3 \mathrm{He}} \\
\mathrm{p}\end{array}$ & $\begin{array}{l}\text { NDG } \\
\text { NDG } \\
2 \cdot 0+1 \\
2 \cdot 0+1 \\
3 \cdot 0+1 \\
2 \cdot 0+1 \\
3 \cdot 2+1\end{array}$ & $\begin{array}{l}8 \cdot 0+1 \\
8 \cdot 0+1 \\
7 \cdot 0+1 \\
1.3+2 \\
8 \cdot 5+1\end{array}$ & $\begin{array}{l}\text { DUQ F } \\
\text { OU日 F } \\
\text { MIL T } \\
\text { MIL T } \\
\text { MIL T } \\
\text { JUL E } \\
\text { MIL T }\end{array}$ & $\begin{array}{l}\text { R Flerov. } \\
\text { R Flerov. } \\
\text { T Gadiolit } \\
\text { T Gadiolit } \\
\text { T Gadiolit } \\
\text { E Aldersteint } \\
\text { T Gadiolit }\end{array}$ & $\begin{array}{r}12539 \\
12608 \\
4489 \\
4490 \\
4491 \\
6523 \\
4524\end{array}$ \\
\hline $1969 \mathrm{Av}$ & \multicolumn{6}{|c|}{ Independent $O(E)$} \\
\hline $\begin{array}{l}197 \mathrm{Au} \\
197_{\mathrm{Au}}\end{array}$ & $\begin{array}{l}12 \mathrm{C} \\
160\end{array}$ & $\begin{array}{l}1.3+2 \\
1.4+2\end{array}$ & $1.7+2$ & $\begin{array}{l}\text { KFK } \\
\text { KFK C }\end{array}$ & $\begin{array}{l}\text { C Muenzel+ } \\
\text { C Muenzel+ }\end{array}$ & $\begin{array}{l}10097 \\
11483\end{array}$ \\
\hline
\end{tabular}

\begin{tabular}{|c|c|c|c|c|c|c|}
\hline Target & $\operatorname{lnc}$ & $\begin{array}{l}E m \mid n \\
(M e V)\end{array}$ & $\begin{array}{l}E \max \\
(\operatorname{MeV})\end{array}$ & Lab & Author & No. \\
\hline $196 . \mathrm{AU}$ & \multicolumn{6}{|c|}{ independent $O(E)$} \\
\hline $\begin{array}{l}197 \mathrm{Au} \\
197 \mathrm{Au} \\
186.1+-2\end{array}$ & $\begin{array}{r}12 \mathrm{C} \\
16 \mathrm{O} \\
2+9 \mathrm{Au}\end{array}$ & $\begin{array}{l}1.3+2 \\
1.4+2 \\
\text { suamat }\end{array}$ & $\begin{array}{l}1.7+2 \\
\text { llon of }\end{array}$ & $\begin{array}{l}\text { KFK } \\
\text { KFK } \\
\text { (E) }\end{array}$ & $\begin{array}{l}\text { C Muenzel- } \\
\text { C Muenzel+ }\end{array}$ & $\begin{array}{l}10097 \\
11483\end{array}$ \\
\hline $\begin{array}{l}202_{\mathrm{Hg}} \\
186.19 \mathrm{AU}\end{array}$ & $\begin{array}{l}\mathrm{p} \\
\mathrm{ra}\end{array}$ & $\begin{array}{l}1.2+1 \\
10 \quad 0(E)\end{array}$ & $8.6+1$ & MCG & E Kantelo+ & $4525+$ \\
\hline $\begin{array}{l}196 \mathrm{Pt} \\
195 \mathrm{Pt}\end{array}$ & $\begin{array}{l}\text { d } \\
\text { p }\end{array}$ & $\begin{array}{l}7.0+0 \\
1.0+1\end{array}$ & $\begin{array}{l}1.1+1 \\
1.4+1\end{array}$ & $\begin{array}{l}\text { BUC } \\
\text { BUC }\end{array}$ & $\begin{array}{l}\text { R Poenaru. } \\
\text { R Poenaru. }\end{array}$ & $\begin{array}{l}4453 \\
581 !\end{array}$ \\
\hline $\begin{array}{l}197 \mathrm{AU} \\
197 \mathrm{AU}\end{array}$ & \multicolumn{4}{|c|}{ portial row thick tars } & $\begin{array}{l}\text { et yieldy } \\
\text { E Gilest }\end{array}$ & 8730 \\
\hline $197 \mathrm{AU}$ & \multicolumn{3}{|c|}{ portiol o(E)d } & & & \\
\hline $\begin{array}{l}197 \mathrm{Au} \\
197 \mathrm{Au}\end{array}$ & \multicolumn{5}{|c|}{ partial spectrum average o(E) } & 6524 \\
\hline $\begin{array}{l}197 \mathrm{AU} \\
197_{\mathrm{AU}}\end{array}$ & \multicolumn{2}{|l|}{$O(E)$} & $2.7+1$ & MUU & E Eberha & 8729 \\
\hline $\begin{array}{l}181 \mathrm{Ta} \\
181 \mathrm{Ta},\end{array}$ & $\begin{array}{l}{ }^{84} \mathrm{Kr} \\
136 \mathrm{Xe}\end{array}$ & $\begin{array}{l}\text { NDG } \\
\text { NDG }\end{array}$ & & $\begin{array}{l}\text { OUB } \\
\text { OUB }\end{array}$ & $\begin{array}{l}\text { R Flerov. } \\
\text { R flerov. }\end{array}$ & $\begin{array}{l}12539 \\
12608\end{array}$ \\
\hline${ }^{198}{ }_{A U}$ & \multicolumn{5}{|c|}{ Including metastable? $\sigma(E)$} & \\
\hline $\begin{array}{l}197 \mathrm{Au} \\
197_{\mathrm{Au}}\end{array}$ & : & $\begin{array}{l}3.7+1 \\
3.7+1\end{array}$ & $\begin{array}{l}9.5+1 \\
9.5+1\end{array}$ & $\begin{array}{l}\text { KFK } \\
\text { ROC }\end{array}$ & $\begin{array}{l}\text { C Muenzelt } \\
\text { E Lanzafamet }\end{array}$ & $\begin{array}{l}8731 \\
8732+\end{array}$ \\
\hline $18 \mathbf{A U}_{\mathrm{AU}}$ & \multicolumn{3}{|c|}{ independent $O(E)$} & & & \\
\hline $\begin{array}{l}197 \mathrm{Au} \\
197 \mathrm{Au}\end{array}$ & $\begin{array}{l}1{ }^{12} \mathrm{C} \\
150\end{array}$ & $\begin{array}{l}1.3+2 \\
1.4+2\end{array}$ & $1.7+2$ & $\begin{array}{l}\text { KFK } \\
\text { KFK }\end{array}$ & $\begin{array}{l}\text { C Muenzel+ } \\
\text { C Muenzel+ }\end{array}$ & $\begin{array}{l}10097 \\
11483\end{array}$ \\
\hline $198 \mathrm{Au}$ & \multicolumn{2}{|l|}{$O(E)$} & & & & \\
\hline $\begin{array}{l}181 \mathrm{Ta} \\
101 \mathrm{Ta} \\
197 \mathrm{Au} \\
202 \mathrm{Hg}\end{array}$ & $\begin{array}{r}{ }^{84} \mathrm{Kr} \\
136 \times e \\
{ }^{3} \mathrm{He} \\
p\end{array}$ & $\begin{array}{l}\text { NDG } \\
\text { NDG } \\
2.0+1 \\
2.0+1\end{array}$ & $\begin{array}{l}1.3+2 \\
8.5+1\end{array}$ & $\begin{array}{l}\text { DUB } \\
\text { DUB } \\
\text { JUL } \\
\text { MIL }\end{array}$ & $\begin{array}{l}\text { R Flerov. } \\
\text { R Flerov. } \\
\text { T Aldersielnt } \\
\text { T Gediolit }\end{array}$ & $\begin{array}{r}12539 \\
12608 \\
6525 \\
4526\end{array}$ \\
\hline $198 .+a_{A C}$ & su & mation. & $c(E)$ & & & \\
\hline $202 \mathrm{Hg}$ & p & $1.2+1$ & $8.6+1$ & MCG & E Kantelot & $4527+$ \\
\hline
\end{tabular}




\begin{tabular}{|c|c|c|c|c|c|c|}
\hline Target & $\operatorname{lnc}$ & $\begin{array}{l}\text { Emin } \\
(M e V)\end{array}$ & $\begin{array}{l}\text { Emax } \\
(M e V)\end{array}$ & Lab W & Author & No. \\
\hline${ }^{189} \mathrm{Au}$ & Indep & ndont. & $O(E)$ & & & \\
\hline $\begin{array}{l}197 \mathrm{Au} \\
197 \mathrm{Au}\end{array}$ & $\begin{array}{l}12 \mathrm{C} \\
160\end{array}$ & $\begin{array}{l}1.3+2 \\
1.4+2\end{array}$ & $1.7+2$ & $\begin{array}{l}\text { KFK C } \\
\text { KFK C }\end{array}$ & $\begin{array}{l}\text { C Muenzel+ } \\
\text { C Muenzel+ }\end{array}$ & $\begin{array}{l}10097 \\
11483\end{array}$ \\
\hline $199_{A u}$ & $\theta(E)$ & & & & & \\
\hline $\begin{array}{l}181 \mathrm{To} \\
197 \mathrm{Au} \\
202 \mathrm{Hg} \\
202 \mathrm{Hg}\end{array}$ & $\begin{array}{c}136 \times e \\
a \\
p \\
p\end{array}$ & $\begin{array}{l}\text { NDG } \\
4.0+1 \\
1.2+1 \\
2.0+1\end{array}$ & $\begin{array}{l}1.1+2 \\
8.6+1 \\
8.5+1\end{array}$ & $\begin{array}{l}\text { DUB R } \\
\text { TOK E } \\
\text { MCG E } \\
\text { MIL T }\end{array}$ & $\begin{array}{l}\text { R Flerov. } \\
\text { E Hashimolo+ } \\
\text { E Kantelo+ } \\
\text { T Gadiolit }\end{array}$ & $\begin{array}{l}12608 \\
8733 \\
4529+ \\
4528\end{array}$ \\
\hline $200 \mathrm{Au}$ & $O(E)$ & & & & & \\
\hline $\begin{array}{l}202_{\mathrm{Hg}} \\
2000+0 \mathrm{AU}\end{array}$ & p & $\begin{array}{l}3.0+1 \\
\text { mation }\end{array}$ & $\begin{array}{l}8.8+1 \\
\sigma(\varepsilon)\end{array}$ & MIL T & T Godiolit & 4530 \\
\hline $\begin{array}{l}202_{\mathrm{Hg}} \\
201 \mathrm{AU}\end{array}$ & $\begin{array}{c}p \\
O(\varepsilon)\end{array}$ & $1.2+1$ & $8.6+1$ & MCG E & E Kantelot & $4531+$ \\
\hline $\begin{array}{l}202 \mathrm{Hg} \\
202^{\mathrm{Hg}}\end{array}$ & p & $\begin{array}{l}1 \cdot 2+1 \\
3 \cdot 2+1\end{array}$ & $\begin{array}{l}0.6+1 \\
8.5+1\end{array}$ & $\begin{array}{l}\text { MCG } \varepsilon \\
\text { MIL T }\end{array}$ & $\begin{array}{l}\text { E Kantelot } \\
\text { T Gadiolit }\end{array}$ & $\begin{array}{l}4532+ \\
4533\end{array}$ \\
\hline$z=80$ & port & $010(E)$ & & & & \\
\hline $197 \mathrm{Au}$ & p & $8.0+0$ & $1.7+1$ & BuC $E$ & E Aleurat & 4508 \\
\hline $179 \mathrm{Hg}$ & spall & tion pr & roduct & $y 1010$ & & \\
\hline $\mathrm{Pb}$ & p & $6.0+2$ & & BRK $R$ & R Cernyt & 4618 \\
\hline $101 \mathrm{Hg}$ & spall & tion pr & roduet & yield & & \\
\hline $\mathrm{Pb}$ & $p$ & $6.0+2$ & & BRK R & R Cernyt & $46 ! 9$ \\
\hline${ }^{183} \mathrm{Hg}_{9}$ & spall & tion pr & roduet & yiold & & \\
\hline $\begin{array}{r}\mathrm{Pb} \\
183_{\mathrm{Hg}}\end{array}$ & $\begin{array}{c}p \\
\text { spall }\end{array}$ & $\begin{array}{l}6.0+2 \\
\text { tion of }\end{array}$ & (E) & BRK $R$ & R Cernyt & 4620 \\
\hline $\begin{array}{l}{ }^{209} \mathrm{Bl} \\
10^{4} \mathrm{Hg}\end{array}$ & $\begin{array}{c}p \\
\text { spall }\end{array}$ & $\begin{array}{l}3.8+2 \\
\text { tion ol }\end{array}$ & (E) & NRL $R$ & R Silberberg+ & 4762 \\
\hline $\begin{array}{l}{ }^{209} \mathrm{Bl} \\
{ }^{189} \mathrm{Hg}\end{array}$ & $\begin{array}{c}p \\
\text { spoll }\end{array}$ & $\begin{array}{l}3.8+2 \\
\text { tion of }\end{array}$ & (E) & NRL $R$ & R Silberberg+ & 4762 \\
\hline $209_{B 1}$ & 0 & $3.8+2$ & & NRL R & R Silberbergt & 4762 \\
\hline
\end{tabular}

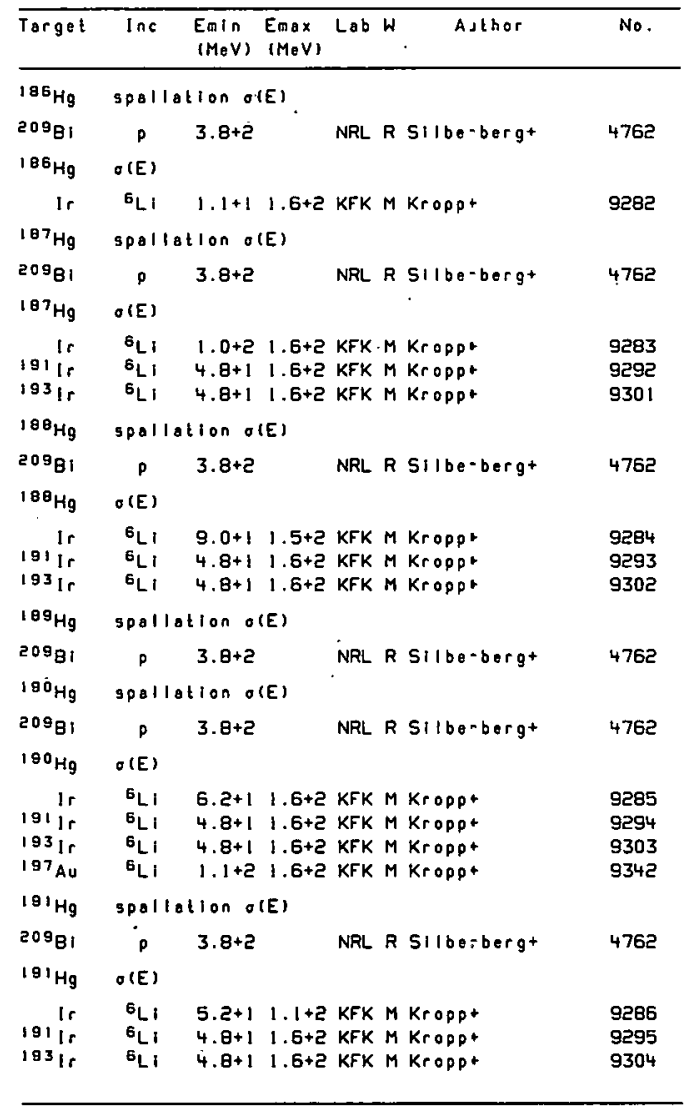




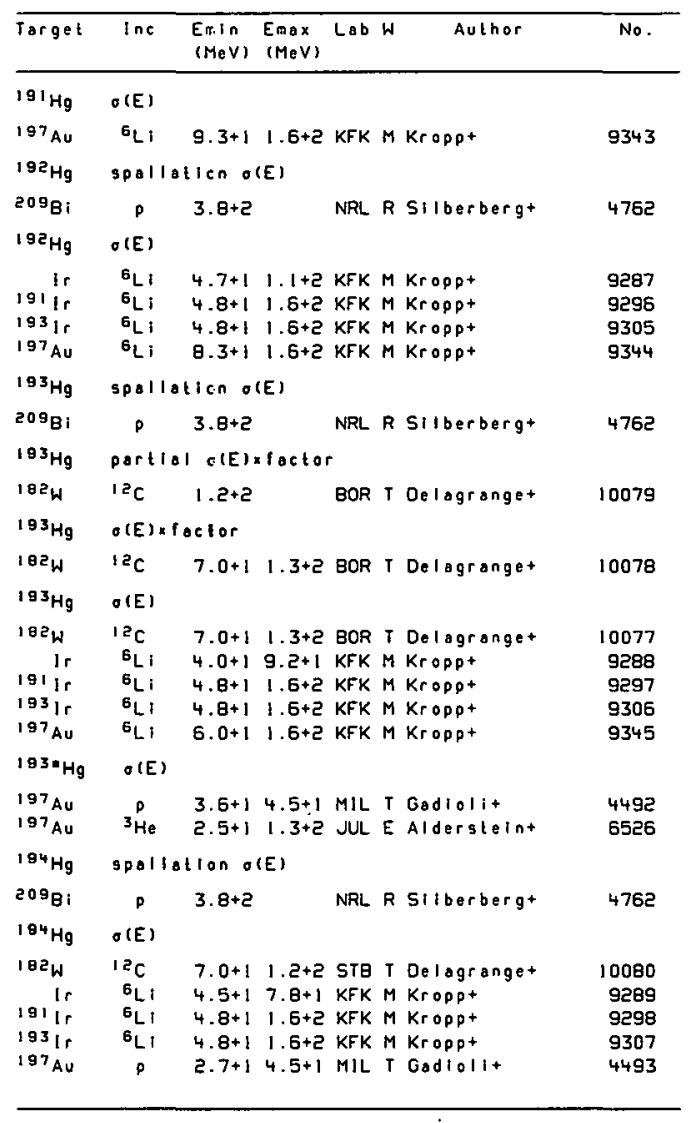

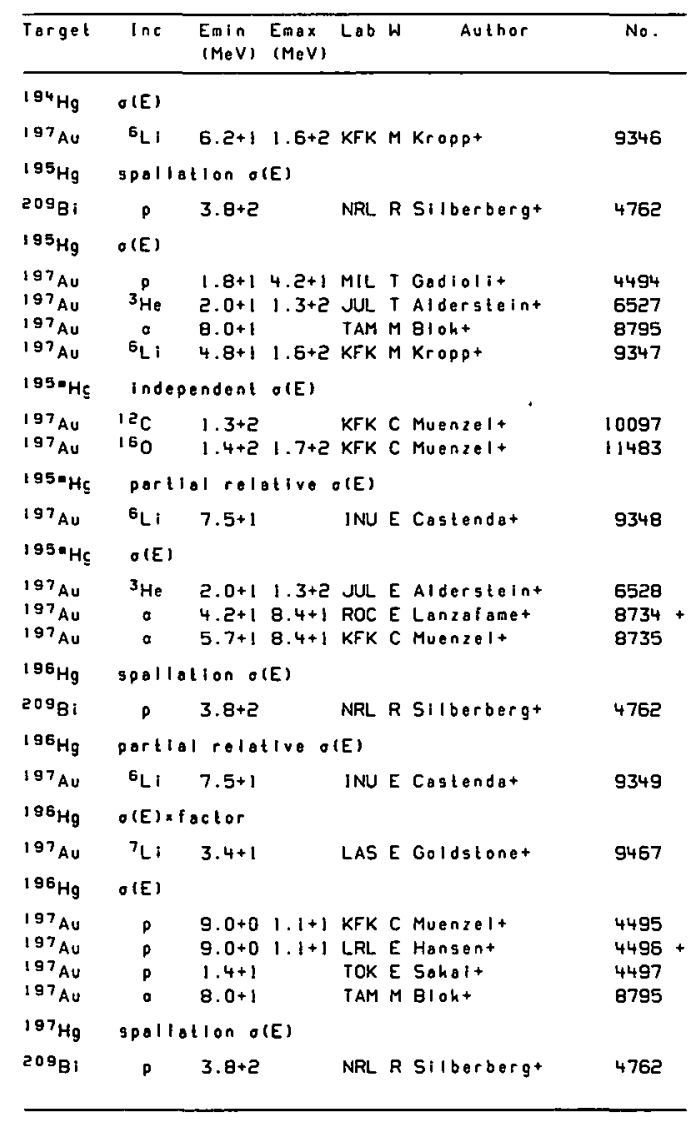




\begin{tabular}{|c|c|c|c|c|c|c|}
\hline Target & $\ln c$ & $\begin{array}{l}\text { Emin } \\
(M e V)\end{array}$ & $\begin{array}{l}\text { Emax } \\
(M e V)\end{array}$ & Lab & Author & No. \\
\hline $197 \mathrm{Hg}$ & \multicolumn{6}{|c|}{ relotive $I_{y}$} \\
\hline $\begin{array}{l}195 \mathrm{Pt} \\
198 \mathrm{Pt}\end{array}$ & : & $\begin{array}{l}2 \cdot 3+1 \\
5 \cdot 5+1\end{array}$ & $2.7+15$ & $\begin{array}{l}\text { ROS } \\
\text { BON }\end{array}$ & $\begin{array}{l}\text { E Kemnitz+ } \\
\text { E Mertinnt }\end{array}$ & $\begin{array}{l}8692 \\
8695\end{array}$ \\
\hline${ }^{197} \mathrm{Hg}$ & \multicolumn{6}{|c|}{ thick target yleld } \\
\hline $197 \mathrm{AU}$ & $p$ & $1.2+1$ & & AML F & R Cheudri. & 4498 \\
\hline $197 \mathrm{Hg}$ & \multicolumn{6}{|c|}{ o(E) xfactor } \\
\hline $\begin{array}{l}197_{\mathrm{AU}} \\
197_{\mathrm{Hg}}\end{array}$ & \multicolumn{6}{|l|}{$\sigma(E)$} \\
\hline $\begin{array}{l}197 \mathrm{Au} \\
197 \mathrm{Au} \\
197 \mathrm{Au}\end{array}$ & $\stackrel{p}{p}{ }_{a}^{\mathrm{He}}$ & $\begin{array}{l}6.0+0 \\
2.0+1 \\
8.0+1\end{array}$ & $\begin{array}{l}1.6+1 \\
1.3+2\end{array}$ & $\begin{array}{l}\text { LAS } \\
\text { JUL } \\
\text { TAM }\end{array}$ & $\begin{array}{l}\text { T Arthur. } \\
\text { T Aldersteint } \\
\text { M Blokt. }\end{array}$ & $\begin{array}{l}4499 \\
6529 \\
8795\end{array}$ \\
\hline $1970 \mathrm{Hg}$ & \multicolumn{6}{|l|}{$\theta(E)$} \\
\hline $\begin{array}{l}197 \mathrm{Au} \\
197 \mathrm{Au}\end{array}$ & p & $\begin{array}{l}5.0+0 \\
5.0+0\end{array}$ & $\begin{array}{l}1.3+1 \\
1.3+1\end{array}$ & $\begin{array}{l}\text { KFK } \\
\text { LRL }\end{array}$ & $\begin{array}{l}\text { C Muenzel+ } \\
\text { E Honsent }\end{array}$ & $\begin{array}{l}4500 \\
4501+\end{array}$ \\
\hline $197 \bullet \mathrm{Hg}$ & \multicolumn{6}{|c|}{ Independent $O(E)$} \\
\hline $\begin{array}{l}197 \mathrm{Au} \\
197 \mathrm{Au}\end{array}$ & $\begin{array}{l}12 c \\
160\end{array}$ & $\begin{array}{l}1.3+2 \\
1.4+2\end{array}$ & $1.7+2$ & $\begin{array}{l}\text { KFK } \\
\text { KFK }\end{array}$ & $\begin{array}{l}\text { C Muenzelt } \\
\text { C Muenzelt }\end{array}$ & $\begin{array}{l}10097 \\
11483\end{array}$ \\
\hline $197 . \mathrm{Hg}$ & \multicolumn{6}{|c|}{ partial relative o(E) } \\
\hline $\begin{array}{l}197 \mathrm{Au} \\
197 \bullet_{\mathrm{Hg}}\end{array}$ & $\begin{array}{l}{ }^{6} \text { Li } \\
\text { thlck }\end{array}$ & $\begin{array}{l}7.5+1 \\
\text { target }\end{array}$ & tyleid & INU & E Castendat & 9350 \\
\hline $\begin{array}{l}197 \mathrm{Au} \\
197 . \mathrm{Hg}\end{array}$ & $\begin{array}{c}p \\
O(E)\end{array}$ & $1: 2+1$ & & AML & R Choudel. & 4502 \\
\hline $\begin{array}{l}197 \mathrm{Au} \\
197 \mathrm{Au} \\
197 \mathrm{Au} \\
197 \mathrm{Au} \\
197 \mathrm{Au} \\
197 \mathrm{Au}\end{array}$ & $\begin{array}{c}\mathrm{p} \\
{ }^{p} \\
{ }^{3} \mathrm{He} \\
0 \\
a \\
a\end{array}$ & $\begin{array}{l}5 \cdot 0+0 \\
5 \cdot 0+0 \\
1.8+1 \\
3 \cdot 8+1 \\
3 \cdot 9+1 \\
4.2+1\end{array}$ & $\begin{array}{l}1.3+1 \\
1.3+1 \\
1.3+2 \\
7.7+1 \\
8.6+1 \\
7.7+1\end{array}$ & $\begin{array}{l}\text { KFK } \\
\text { LRL } \\
\text { JUL } \\
\text { ROC } \\
\text { BON } \\
\text { KFK }\end{array}$ & $\begin{array}{l}\text { C Muenzel+ } \\
\text { E Hansent } \\
\text { E Alderstelnt } \\
\text { E Lonzafomet } \\
\text { E Kurz+ } \\
\text { C Muenzel+ }\end{array}$ & $\begin{array}{l}4503 \\
4504+ \\
6530 \\
8736+ \\
8737+ \\
8738\end{array}$ \\
\hline $197 .+9 \mathrm{He}_{\mathrm{S}}$ & sum & mation & $O(E)$ & & & \\
\hline $\begin{array}{l}197 \mathrm{Au} \\
197_{\mathrm{Au}}\end{array}$ & $p$ & $\begin{array}{l}4 . \theta+0 \\
5 \cdot 0+0\end{array}$ & $\begin{array}{l}1.3+1 \\
1.3+1\end{array}$ & $\begin{array}{l}\text { LRL } \\
\text { KFK }\end{array}$ & $\begin{array}{l}\text { E Hansent } \\
\text { C Muenzel+ }\end{array}$ & $\begin{array}{l}4505+ \\
4506\end{array}$ \\
\hline
\end{tabular}

\begin{tabular}{|c|c|c|c|c|c|c|}
\hline Target & $\operatorname{lng}$ & $\begin{array}{l}\text { Emin } \\
(M e V)\end{array}$ & $\begin{array}{l}\text { Emax } \\
(\mathrm{MeV})\end{array}$ & Lab W & Author & No. \\
\hline${ }^{19 \theta_{\mathrm{Hg}}}$ & \multicolumn{6}{|c|}{ independent relative o(E) } \\
\hline $197 \mathrm{AU}$ & a & $5 \cdot 1+1$ & & KAZ E & Bur = ebsevt & 8740 \\
\hline $198_{\mathrm{Hg}}$ & \multicolumn{6}{|c|}{ Spallation o(E) } \\
\hline 20981 & P & $3.8+2$ & & NRL $R$ & Silberberg+ & 4762 \\
\hline $198_{\mathrm{Hg}}$ & \multicolumn{6}{|c|}{ partlal row thich target yle:dyabundonce } \\
\hline${ }^{198} \mathrm{Hg}$ & a & $5.0+0$ & & SUN E & Gilest & 8799 \\
\hline${ }^{19 \theta_{\mathrm{Hg}}}$ & \multicolumn{6}{|c|}{ relative Iy } \\
\hline${ }^{198} \mathrm{Pt}$ & ๑ & $4.8+1$ & & BON E & Gunthert & 8697 \\
\hline $198_{\mathrm{Hg}}$ & \multicolumn{6}{|c|}{$\sigma(E) \times f$ actor } \\
\hline $\begin{array}{l}198 \mathrm{Pt} \\
187 \mathrm{AU}\end{array}$ & $i_{L I}^{0}$ & $\begin{array}{l}4 \cdot 0+1 \\
3 \cdot 4+1\end{array}$ & & $\begin{array}{l}\text { BON E } \\
\text { LAS E }\end{array}$ & $\begin{array}{l}\text { Merlint } \\
\text { Golcstonet }\end{array}$ & $\begin{array}{l}8698 \\
9467\end{array}$ \\
\hline${ }^{19} \theta_{\mathrm{Hg}}$ & \multicolumn{6}{|l|}{$O(E)$} \\
\hline $\begin{array}{l}197 \mathrm{AU} \\
197 \mathrm{AU} \\
187 \mathrm{AU} \\
197_{\mathrm{AU}}\end{array}$ & $\begin{array}{c}{ }_{3}^{p} \mathrm{He}_{0} \\
a \\
a\end{array}$ & $\begin{array}{l}4.0+0 \\
7.0+1 \\
I R \\
8.0+1\end{array}$ & $\begin{array}{l}9.0+1 \\
4.0+1\end{array}$ & & $\begin{array}{l}\text { Sahat } \\
\text { Matguohat } \\
\text { Gonzalez-vidalt } \\
\text { Bloht }\end{array}$ & $\begin{array}{l}4507 \\
6531 \\
8739 \\
6795\end{array}$ \\
\hline${ }^{189} \mathrm{Hg}$ & \multicolumn{6}{|c|}{ independent relative o(E) } \\
\hline $197 \mathrm{Au}$ & a & $5.1+1$ & & KAZ E & Burtebaevt & 8741 \\
\hline $199_{\mathrm{Hg}}$ & \multicolumn{6}{|c|}{ partial raw thich target yletidyabundance } \\
\hline $199_{\mathrm{Hg}}$ & a & $5.0+0$ & & SUN E & Gllest & 8804 \\
\hline $199_{\mathrm{Hg}}$ & \multicolumn{6}{|c|}{ relative $I_{y}$} \\
\hline $19 \theta_{\mathrm{Pt}}$ & - & $3.1+1$ & $3.9+1$ & BONE & Mertint & 8699 \\
\hline $199_{\mathrm{Hg}}$ & \multicolumn{6}{|c|}{ otE)xifactor } \\
\hline $\begin{array}{l}198 \mathrm{Pt} \\
197 \mathrm{Au}\end{array}$ & $7_{L I}^{a}$ & $\begin{array}{l}4 \cdot 0+1 \\
3 \cdot 4+1\end{array}$ & & $\begin{array}{l}\text { BON E } \\
\text { LAS E }\end{array}$ & $\begin{array}{l}\text { Mertint } \\
\text { Goldstonet }\end{array}$ & $\begin{array}{l}8700 \\
9467\end{array}$ \\
\hline $199_{\mathrm{Hg}}$ & $O(E)$ & & & & & \\
\hline $197 \mathrm{Au}$ & a & $8.0+1$ & & TAM ME & Bloht & 8795 \\
\hline $199 a_{\mathrm{Hg}}$ & $\sigma(E)$ & & & & & \\
\hline${ }^{197} \mathrm{Au}$ & d & $5.0+0$ & $1.6+1$ & GOE E & Kantust & 5826 \\
\hline
\end{tabular}




\begin{tabular}{|c|c|c|c|c|c|}
\hline Target & $\operatorname{lnc}$ & $\begin{array}{l}\text { Emin } \\
(\mathrm{MeV})\end{array}$ & $\begin{array}{l}E \max \\
(M e V)\end{array}$ & Lab W & No. \\
\hline $189 .+9 \mathrm{Hg}$ & \multicolumn{5}{|c|}{ compound-nucleus sumation $O(E)$} \\
\hline $\begin{array}{l}197 \mathrm{Au} \\
189 .+9 \mathrm{Hg}\end{array}$ & $g^{d}$ sum & \multicolumn{3}{|c|}{ Sumation $O(E)$} & 5828 \\
\hline${ }^{197} \mathrm{Au}$ & d & $5.0+0$ & $1.5+1$ & GOE E Kantus+ & 5827 \\
\hline $200 \mathrm{Hg}_{\mathrm{g}}$ & \multicolumn{5}{|c|}{ independent relative o(E) } \\
\hline $197 \mathrm{Au}$ & a & $5.1+1$ & & KAZ E Burtebaevt & 8742 \\
\hline $200_{H_{9}}$ & \multicolumn{5}{|c|}{ direct-intoraction o(E) } \\
\hline $203 \mathrm{TI}$ & p & $2.0+1$ & & MIL M Milazzo-Collit & 4576 \\
\hline $200 \mathrm{Hg}$ & \multicolumn{5}{|c|}{ portiol roo thlck torget yleldyeabundonce } \\
\hline $200 \mathrm{Hg}$ & o & $5.0+0$ & & SUN E Gilegt & 8809 \\
\hline $200 \mathrm{Hg}$ & \multicolumn{5}{|c|}{ portial o(E). } \\
\hline $203 \mathrm{TI}$ & p & $2.0+1$ & & MIL M Milazzo-Collit & 4575 \\
\hline $200 \mathrm{Hg}$ & \multicolumn{5}{|c|}{ relative I, } \\
\hline $\begin{array}{l}190 \mathrm{PL} \\
200 \mathrm{Hg}\end{array}$ & \multicolumn{4}{|c|}{$O(E) \times$ factor } & 8701 \\
\hline $\begin{array}{l}197 \mathrm{Au} \\
200 \mathrm{Hg}\end{array}$ & \multicolumn{4}{|l|}{$O(E)$} & 9462 \\
\hline $\begin{array}{l}197 \mathrm{Au} \\
197 \mathrm{Au} \\
203 \mathrm{JI}\end{array}$ & $\underset{P}{7_{L i}^{a}}$ & $\begin{array}{l}8 \cdot 0+1 \\
3 \cdot 2+1 \\
2 \cdot 0+1\end{array}$ & & $\begin{array}{l}\text { TAM M Bloht } \\
\text { HEI E Quebert+ } \\
\text { MIL M Milazzo-Collit }\end{array}$ & $\begin{array}{l}8795 \\
9461 \\
4574\end{array}$ \\
\hline $201 \mathrm{Hg}$ & \multicolumn{5}{|l|}{$\sigma(E)$} \\
\hline $187 \mathrm{Au}$ & ${ }^{7} \mathrm{Li}$ & $3.2+1$ & & HEI E Quebert+ & 9463 \\
\hline $202_{H g}$ & \multicolumn{5}{|c|}{ diroct-intoraction $O(E)$} \\
\hline $205 \mathrm{Tl}$ & p & $2.0+1$ & & MIL M MIIOzzo-Collit & 4590 \\
\hline $202_{H_{9}}$ & \multicolumn{5}{|c|}{ portlol raw thick torget yleldxobundance } \\
\hline $202_{\mathrm{Hg}}$ & a & $5.0+0$ & & SUN E Gllest & 8818 \\
\hline $202^{\mathrm{Hg}}$ & \multicolumn{5}{|c|}{ porllol o(E) } \\
\hline $205 \mathrm{TI}$ & $p$ & $2.0+1$ & & MIL M MIIazzo-Collit & 4589 \\
\hline
\end{tabular}

\begin{tabular}{|c|c|c|c|c|c|c|}
\hline Target & $\ln c$ & $\begin{array}{l}\text { Emin } \\
(\operatorname{MeV})\end{array}$ & $\begin{array}{l}\text { Emax } \\
(M e V)\end{array}$ & Lab W & Author & No. \\
\hline${ }^{202} \mathrm{Hg}$ & $O(E)$ & & & & & \\
\hline $\cos _{\mathrm{TI}}$ & p & $2.0+1$ & & MIL M & M Milezzo-Collit+ & 4588 \\
\hline${ }^{206} \mathrm{Hg}$ & \multicolumn{6}{|c|}{ olE $\times$ foclor } \\
\hline $\begin{array}{l}197 \mathrm{AU} \\
197 \mathrm{AU}\end{array}$ & $\begin{array}{l}\sigma_{L} \\
P_{L i}\end{array}$ & $\begin{array}{l}3.9+1 \\
3.9+1\end{array}$ & & $\begin{array}{l}\text { LAS E } \\
\text { LAS E }\end{array}$ & $\begin{array}{l}\text { E Brittt+ } \\
\text { E Britt+ }\end{array}$ & $\begin{array}{l}9360 \\
9468\end{array}$ \\
\hline $207 \mathrm{Hg}$ & \multicolumn{6}{|c|}{ o(E) × foctor } \\
\hline $\begin{array}{l}197 \mathrm{AU} \\
197 \mathrm{Au}\end{array}$ & $\begin{array}{l}6_{L} ; \\
7_{L}:\end{array}$ & $\begin{array}{l}3.9+1 \\
3.9+1\end{array}$ & & $\begin{array}{l}\text { LAS E } \\
\text { LAS E }\end{array}$ & $\begin{array}{l}\text { E Britt+ } \\
\text { E Britt+ }\end{array}$ & $\begin{array}{l}9360 \\
9468\end{array}$ \\
\hline${ }^{200} \mathrm{Hg}$ & \multicolumn{6}{|c|}{$\sigma(E) \times$ factor } \\
\hline $\begin{array}{l}197 \mathrm{AU} \\
197_{\mathrm{AU}}\end{array}$ & $\begin{array}{l}6_{L 1} \\
7_{L i}\end{array}$ & $\begin{array}{l}3.9+1 \\
3.9+1\end{array}$ & & $\begin{array}{l}\text { LAS E } \\
\text { LAS E }\end{array}$ & $\begin{array}{l}\text { E Brittt+ } \\
\text { E Brittt+ }\end{array}$ & $\begin{array}{l}9360 \\
9468\end{array}$ \\
\hline${ }^{209} \mathrm{Hg}$ & \multicolumn{6}{|c|}{$O(E) \times$ faclor } \\
\hline $\begin{array}{l}197 \mathrm{Au} \\
197_{\mathrm{Au}}\end{array}$ & $\begin{array}{l}6_{L 1} \\
7_{L i}\end{array}$ & $\begin{array}{l}3.9+1 \\
3.9+1\end{array}$ & & $\begin{array}{l}\text { LAS E } \\
\text { LAS E }\end{array}$ & $\begin{array}{l}\text { E Briltt+ } \\
\text { E Brittt+ }\end{array}$ & $\begin{array}{l}9360 \\
9468\end{array}$ \\
\hline $210 \mathrm{Hg}$ & \multicolumn{6}{|c|}{$\sigma(E) \times$ foctor } \\
\hline $\begin{array}{l}197 \mathrm{AU} \\
Z=81\end{array}$ & $\begin{array}{l}{ }^{7} L I \\
O(E)\end{array}$ & $3.9+1$ & & \multicolumn{3}{|c|}{$O(E)$} \\
\hline $\begin{array}{l}194 \mathrm{Pt} \\
198 \mathrm{Pt}\end{array}$ & $\begin{array}{l}6_{L i} \\
{ }^{6} L i\end{array}$ & $\begin{array}{l}8 \cdot 5+1 \\
7 \cdot 5+1\end{array}$ & $\begin{array}{l}9.4+1 \\
9.4+1\end{array}$ & $\begin{array}{l}\text { INU E } \\
\text { INU E }\end{array}$ & $\begin{array}{l}\text { E Karwoshit } \\
\text { E Karvoshit }\end{array}$ & $\begin{array}{l}9313 \\
9319\end{array}$ \\
\hline $185 \mathrm{TI}$ & \multicolumn{6}{|c|}{ spollotion $O(E)$} \\
\hline $20{ }^{\circ} \mathrm{i}$ & $p$ & $3.8+2$ & & NRL $R$ & R Silberbergt & 4762 \\
\hline $186 \mathrm{TI}$ & \multicolumn{6}{|c|}{ Spoliation o(E) } \\
\hline $\begin{array}{l}20{ }^{2} \mathrm{Bi} \\
187 \mathrm{TI} .\end{array}$ & \multicolumn{5}{|c|}{ spollotion o(E) } & 4762 \\
\hline $\operatorname{209}^{01}$ & $p$ & $3.8+2$ & & NRL $R$ & R silberbergt & 4762 \\
\hline${ }_{18} 0^{T}$ & \multicolumn{6}{|c|}{ spollation o(E) } \\
\hline $209_{B i}$ & $p$ & $3.8+2$ & & NFL $R$ & R Silberbergt & 4762 \\
\hline $189_{\mathrm{TI}}$ & \multicolumn{6}{|c|}{ Spollotion o(E) } \\
\hline 20981 & p & $3.8+2$ & & NRL $R$ & R Silberbergt & 4762 \\
\hline
\end{tabular}




\begin{tabular}{|c|c|c|c|c|c|c|}
\hline Target & $\ln c$ & $\begin{array}{l}\text { Emin } \\
(\mathrm{MeV})\end{array}$ & $\begin{array}{l}\text { Emax } \\
(\mathrm{MeV})\end{array}$ & Lab & Author & No. \\
\hline$|90 \mathrm{~T}|$ & \multicolumn{4}{|c|}{ Spariation $\theta(E)$} & & \\
\hline $\begin{array}{l}2.09 \mathrm{~B} \mid \\
190 \mathrm{~T} \mid\end{array}$ & $\begin{array}{c}p \\
o(E)\end{array}$ & \multicolumn{2}{|l|}{$3.8+2$} & NRL & R Silberberg+ & 4762 \\
\hline $\begin{array}{l}197 \mathrm{Au} \\
191 \mathrm{TI}\end{array}$ & $\begin{array}{l}{ }^{3} \mathrm{He} \\
\text { spall }\end{array}$ & \multicolumn{2}{|c|}{$\begin{array}{l}1.0+21.3+2 \\
\text { lion } \sigma(E)\end{array}$} & JUL & E Aldersteint & 6532 \\
\hline $\begin{array}{l}{ }^{209} \mathrm{~B} \\
181 \mathrm{~T}\end{array}$ & $\stackrel{p}{\sigma(E)}$ & \multicolumn{2}{|l|}{$3.8+2$} & \multicolumn{2}{|c|}{ NRL R silberberg+ } & 4762 \\
\hline $\begin{array}{l}197 \mathrm{Au} \\
197 \mathrm{Au}\end{array}$ & $\begin{array}{l}{ }^{3} \mathrm{He} \\
6 \mathrm{Li}\end{array}$ & \multicolumn{2}{|c|}{$\begin{array}{ll}9.0+1 & 1.3+2 \\
1.4+2 & 1.6+2\end{array}$} & $\begin{array}{l}\text { JUL } \\
\text { KFK }\end{array}$ & $\begin{array}{l}\text { E Aldersteint } \\
\text { M Kroppt }\end{array}$ & \multirow[t]{2}{*}{$\begin{array}{l}6533 \\
9351\end{array}$} \\
\hline$|92 \mathrm{~T}|$ & \multicolumn{5}{|c|}{ spallation $O(E)$} & \\
\hline $\begin{array}{l}209_{\mathrm{Bi}} \\
192 \mathrm{Ti}\end{array}$ & \multicolumn{4}{|c|}{ partial relative o(E) } & R Silberbergt & 4762 \\
\hline $181 \mathrm{Ta}$ & 150 & $1 \cdot 0+2$ & & CNE & E Kreinert & 11460 \\
\hline $192 \mathrm{~T}$ & \multicolumn{3}{|c|}{ product yield } & \multicolumn{2}{|r|}{. } & \multirow{3}{*}{$\begin{array}{l}11459 \\
11714\end{array}$} \\
\hline $\begin{array}{l}181 \mathrm{Ta} \\
181 \mathrm{Ta}\end{array}$ & $\begin{array}{l}160 \\
180\end{array}$ & \multirow{2}{*}{\multicolumn{2}{|c|}{$\begin{array}{ll}9.5+1 & 1.0+2 \\
1.0+2 & 1.2+2\end{array}$}} & \multirow{2}{*}{\multicolumn{2}{|c|}{$\begin{array}{l}\text { CNE E Kreinert } \\
\text { CNE E Kreinert }\end{array}$}} & \\
\hline $192 \mathrm{~T}$ & $\sigma(E)$ & & & & & \\
\hline $\begin{array}{l}181 \mathrm{Ta} \\
197 \mathrm{Au}\end{array}$ & $\begin{array}{l}15_{0} \\
{ }^{3} \mathrm{He}\end{array}$ & $\begin{array}{l}9.3+1 \\
7.0+1\end{array}$ & $\begin{array}{l}1.8+2 \\
1.3+2\end{array}$ & $\begin{array}{l}\text { PAR } \\
\text { JUL }\end{array}$ & $\begin{array}{l}\text { M Rouleit } \\
\text { E Alderstelnt }\end{array}$ & $\begin{array}{r}11458 \\
6534\end{array}$ \\
\hline $193 \mathrm{TI}$ & \multicolumn{5}{|c|}{ Including metastable o(E) } & \\
\hline $\begin{array}{l}197 \mathrm{AU} \\
197 \mathrm{AU} \\
197_{\mathrm{AU}} \\
197_{\mathrm{AU}}\end{array}$ & $\begin{array}{c}{ }^{3} \mathrm{He} \\
{ }^{3} \mathrm{He} \\
a \\
a\end{array}$ & $\begin{array}{l}6.5+1 \\
6.5+1 \\
8.8+1 \\
8.8+1\end{array}$ & $\begin{array}{l}7.5+1 \\
7.5+1 \\
1.0+2 \\
1.0+2\end{array}$ & $\begin{array}{l}\text { IKO } \\
\text { KF́K } \\
\text { KFK } \\
\text { BON }\end{array}$ & $\begin{array}{l}\text { E Hermest } \\
\text { C Muenzel+ } \\
\text { C Muenzel+ } \\
\text { E Kurz+ }\end{array}$ & $\begin{array}{l}6535+ \\
6536 \\
8743 \\
8744+\end{array}$ \\
\hline $193 \mathrm{TI}$ & \multicolumn{5}{|c|}{ Spa!lation o(E) } & \\
\hline $\begin{array}{l}20{ }^{20} \mathrm{Bi} \\
193 \mathrm{TI}\end{array}$ & \multicolumn{5}{|c|}{ partial relative o(E) } & $476 ?$ \\
\hline $\begin{array}{l}181 \mathrm{Ta} \\
193 \mathrm{TI}\end{array}$ & $\begin{array}{l}1 \theta_{0} \\
O(E)\end{array}$ & \multicolumn{2}{|c|}{$8.0+1 \quad 1.0+2$} & \multicolumn{2}{|c|}{ MUN E Krelnert } & 11716 \\
\hline $181 \mathrm{~T}^{\circ}$ & .160 & $9.0+1$ & $1.6+2$ & PAR & M Roulet+ & 11461 \\
\hline
\end{tabular}

\begin{tabular}{|c|c|c|c|c|c|c|}
\hline Target & $\ln c$ & $\begin{array}{l}E_{\min } \\
(\mathrm{MeV})\end{array}$ & $\begin{array}{l}\text { Emax } \\
(M e V)\end{array}$ & Lab & Auther & No. \\
\hline $33_{11}$ & $a(E)$ & & & & & \\
\hline $\begin{array}{l}197 \mathrm{Au} \\
197 \mathrm{Au} \\
197 \mathrm{Au}\end{array}$ & $\begin{array}{l}{ }^{3} \mathrm{He} \\
{ }_{6}^{a} \\
{ }_{\mathrm{Li}}\end{array}$ & $\begin{array}{l}5.5+1 \\
T R \\
1.1+2\end{array}$ & $\begin{array}{l}1.3+2 \\
1.0+2 \\
1.6+2\end{array}$ & $\begin{array}{l}\text { JUL } \\
\text { KFK } \\
\text { KFK }\end{array}$ & $\begin{array}{l}\text { E Aldersleint } \\
\text { M Kurzt } \\
\text { M Kroppt }\end{array}$ & $\begin{array}{l}6537 \\
8796 \\
9352\end{array}$ \\
\hline $194 \pi 1$ & \multicolumn{6}{|c|}{ Spallation o(E) } \\
\hline $\begin{array}{l}209^{81} \\
19471\end{array}$ & \multicolumn{5}{|c|}{ partlal relative o(E) } & 4762 \\
\hline $\begin{array}{l}181 \mathrm{Ta} \\
181 \mathrm{Tg}\end{array}$ & $\begin{array}{l}180 \\
180\end{array}$ & $\begin{array}{l}9.5+1 \\
8.0+1\end{array}$ & $1.0+2$ & MUN & $\begin{array}{l}\text { E Kreinert } \\
\text { E Kreinert }\end{array}$ & $\begin{array}{l}11715 \\
11716\end{array}$ \\
\hline $194 \mathrm{TI}$ & $\sigma(E)$ & . & & & & \\
\hline $\begin{array}{l}181 \mathrm{To} \\
197 \mathrm{Au} \\
197 \mathrm{Au}\end{array}$ & $\begin{array}{c}150 \\
{ }^{15} \mathrm{He} \\
0\end{array}$ & $\begin{array}{l}8 \cdot 8+1 \\
2 \cdot 0+1 \\
8 \cdot 0+1\end{array}$ & $\begin{array}{l}1.4+2 \\
1.3+2\end{array}$ & $\begin{array}{l}\text { PAR } \\
\text { JUL } \\
\text { TAM }\end{array}$ & $\begin{array}{l}M \text { Roulet- } \\
\text { T Aldersteint } \\
\text { M Blokt }\end{array}$ & $\begin{array}{r}11462 \\
6538 \\
8795\end{array}$ \\
\hline 184aTा & $\sigma(E)$ & & & & & \\
\hline $\begin{array}{l}197 \mathrm{Au} \\
197 \mathrm{Au} \\
197 \mathrm{Au} \\
197 \mathrm{Au}\end{array}$ & $\begin{array}{c}{ }_{3}^{3} \mathrm{He} \\
{ }^{3} \mathrm{He} \\
0 \\
0 \\
a\end{array}$ & $\begin{array}{l}5 \cdot 0+1 \\
5 \cdot 0+1 \\
7.4+1 \\
7.4+1\end{array}$ & $\begin{array}{l}7.5+1 \\
7.5+1 \\
1.0+2 \\
1.0+2\end{array}$ & $\begin{array}{l}\text { IKO } \\
\text { KFK } \\
\text { KFK } \\
\text { BON }\end{array}$ & $\begin{array}{l}\text { E Hermes- } \\
\text { C Muenze-+ } \\
\text { C Muenze:+ } \\
\text { E Kurz+ }\end{array}$ & $\begin{array}{l}6539+ \\
6540 \\
8745 \\
8746+\end{array}$ \\
\hline${ }^{195} \mathrm{~T}$ & \multicolumn{6}{|c|}{ including metastable o(E) } \\
\hline $\begin{array}{l}197 \mathrm{Au} \\
197 \mathrm{Au} \\
197 \mathrm{Au} \\
197_{\mathrm{Au}} \\
197_{\mathrm{Au}}\end{array}$ & $\begin{array}{c}{ }_{3}^{3} \mathrm{He} \\
{ }^{3} \mathrm{He} \\
a \\
a \\
a\end{array}$ & $\begin{array}{l}3.7+1 \\
3.7+1 \\
6.0+1 \\
6 \cdot 5+1 \\
6.5+1\end{array}$ & $\begin{array}{l}7.5+1 \\
7.5+1 \\
8.6+1 \\
7.7+1 \\
7.7+1\end{array}$ & $\begin{array}{l}\text { IKO } \\
\text { KFK } \\
\text { EON } \\
\text { KFK } \\
\text { ROC }\end{array}$ & $\begin{array}{l}\text { E Hermest } \\
\text { C Muenzei+ } \\
\text { E Kurz+ } \\
\text { C Muenze:+ } \\
\text { E Lanzefamet }\end{array}$ & $\begin{array}{l}6541+ \\
6542 \\
8747+ \\
8748 \\
8749+\end{array}$ \\
\hline${ }^{195} \mathrm{TI}$ & \multicolumn{6}{|c|}{ independent $\sigma(E)$} \\
\hline $\begin{array}{l}197 \mathrm{Au} \\
197_{\mathrm{Au}}\end{array}$ & $\begin{array}{l}12 c \\
160\end{array}$ & $\begin{array}{l}1.3+2 \\
1.4+2\end{array}$ & $1.7+2$ & $\begin{array}{l}\text { KFK } \\
\text { KFK }\end{array}$ & $\begin{array}{l}\text { C Muenze + } \\
\text { C Muenze + }\end{array}$ & $\begin{array}{l}10097 \\
11483\end{array}$ \\
\hline${ }^{185} \mathrm{TI}$ & \multicolumn{6}{|c|}{ spallation o(E) } \\
\hline $\begin{array}{l}{ }^{209} \mathrm{Bl} \\
\mid 95 \mathrm{TI}\end{array}$ & \multicolumn{5}{|c|}{ cumulative o(E) } & 4762 \\
\hline $\begin{array}{l}197 \mathrm{Au} \\
197_{\mathrm{Au}}\end{array}$ & $\begin{array}{l}{ }^{12} \mathrm{C} \\
160\end{array}$ & $\begin{array}{l}1.3+2 \\
1.4+2\end{array}$ & $1.7+2$ & $\begin{array}{l}\text { KFK } \\
\text { KFK }\end{array}$ & $\begin{array}{l}\text { C Muenze:- } \\
\text { C Muenze:+ }\end{array}$ & $\begin{array}{l}10099 \\
11485\end{array}$ \\
\hline
\end{tabular}




\begin{tabular}{|c|c|c|c|c|c|c|}
\hline Torget & $\operatorname{linc}$ & $\begin{array}{l}\text { Enin } \\
(\mathrm{MeV})\end{array}$ & $\begin{array}{l}\text { Emax } \\
\text { (MeV) }\end{array}$ & Leb & Author & No. \\
\hline${ }^{195} \mathrm{Tl}$ & \multicolumn{6}{|c|}{ partial rolative o(E) } \\
\hline $\begin{array}{l}181 T a \\
185 T 1\end{array}$ & $\begin{array}{l}100 \\
\text { relat }\end{array}$ & $\begin{array}{l}8.0+1 \\
\text { ve } i_{y}\end{array}$ & $1.0+2$ & \multicolumn{2}{|c|}{ relablue is } & 11716 \\
\hline $\begin{array}{l}197 \mathrm{Au} \\
195 \mathrm{TI}\end{array}$ & \multicolumn{6}{|c|}{ product yiold } \\
\hline $\begin{array}{r}\mathrm{Ta} \\
199 \mathrm{TI}\end{array}$ & $\begin{array}{l}16_{0} \\
v_{y}\end{array}$ & NDG & & ORL & E Weil+ & 11446 \\
\hline $\begin{array}{l}{ }^{198} \mathrm{Pt} \\
195 \mathrm{TI}\end{array}$ & $\begin{array}{r}\sigma_{L 1} \\
0(E)\end{array}$ & $8.5+1$ & & INU & E Karvoshi+ & 9320 \\
\hline $\begin{array}{l}181 \mathrm{Ta} \\
197 \mathrm{Au} \\
197 \mathrm{Au} \\
197 \mathrm{Au} \\
197 \mathrm{Au}\end{array}$ & $\begin{array}{c}160 \\
{ }^{16} \mathrm{He} \\
0 \\
0 \\
{ }^{6} \mathrm{LI}\end{array}$ & $\begin{array}{l}9 \cdot 1+1 \\
3 \cdot 2+1 \\
9.0+1 \\
T R \\
7 \cdot 3+1\end{array}$ & $\begin{array}{l}1.1+2 \\
1.0+2 \\
1.0+2 \\
1.6+2\end{array}$ & $\begin{array}{l}\text { PAR } \\
\text { JUL } \\
\text { TAM } \\
\text { KFK } \\
\text { KFK }\end{array}$ & $\begin{array}{l}\text { M Roulelt } \\
\text { E Alderstelnt } \\
\text { M Bloht } \\
\text { M Kurzt } \\
\text { M Kroppt }\end{array}$ & $\begin{array}{r}11463 \\
6543 \\
6795 \\
8796 \\
9353\end{array}$ \\
\hline${ }^{196} \mathrm{TI}$ & spall & tion of & (E) & & & \\
\hline $\begin{array}{l}209_{B 1} \\
196 \mathrm{TI}\end{array}$ & $\stackrel{p}{\text { relat }}$ & $\begin{array}{l}3.8+2 \\
\text { ve } I_{r}\end{array}$ & & NRL & R Silberberg* & 4762 \\
\hline $\begin{array}{l}197 \mathrm{Au} \\
196 \mathrm{TI}\end{array}$ & $v_{y}^{a}$ & $5.5+1$ & $6.5+1$ & MUN & E Kreinert & $875 !$ \\
\hline $\begin{array}{l}{ }^{198} \mathrm{Pt} \\
196_{\mathrm{TI}}\end{array}$ & $\begin{array}{r}6_{L I} \\
\sigma(E)\end{array}$ & $8.5+1$ & & INU & E Karwoski+ & 9320 \\
\hline $\begin{array}{l}197 \mathrm{Au} \\
197_{\mathrm{Au}} \\
197_{\mathrm{Au}}\end{array}$ & $\begin{array}{c}{ }^{3} \mathrm{He} \\
: \\
:\end{array}$ & $\begin{array}{l}2.0+1 \\
8.0+1 \\
T R\end{array}$ & $\begin{array}{l}1.3+2 \\
1.0+2\end{array}$ & $\begin{array}{l}\text { JUL } \\
\text { TAM } \\
\text { KFK }\end{array}$ & $\begin{array}{l}\text { T Aldersteint } \\
\text { M Blokt } \\
\text { M Kurz+ }\end{array}$ & $\begin{array}{l}6544 \\
8795 \\
8796\end{array}$ \\
\hline $1966_{0}$ & Inde & endent & $O(E)$ & & & \\
\hline $\begin{array}{l}187 \mathrm{Au} \\
197_{\mathrm{Au}}\end{array}$ & $\begin{array}{l}12 c \\
150\end{array}$ & $\begin{array}{l}1.3+2 \\
1.4+2\end{array}$ & $1.7+2$ & $\begin{array}{l}\text { KFK } \\
\text { KFK }\end{array}$ & $\begin{array}{l}\text { C Muenzelt } \\
\text { C Muenzel+ }\end{array}$ & $\begin{array}{l}10097 \\
11483\end{array}$ \\
\hline $1960 \mathrm{TI}$ & $\theta(E)$ & & & & & \\
\hline $\begin{array}{l}197 \mathrm{Au} \\
197 \mathrm{Au} \\
197 \mathrm{Au}\end{array}$ & $\begin{array}{l}{ }^{3} \mathrm{He} \\
{ }^{3} \mathrm{He} \\
a\end{array}$ & $\begin{array}{l}2.5+1 \\
2.5+1 \\
4.6+1\end{array}$ & $\begin{array}{l}5.0+1 \\
7.2+1 \\
8.6+1\end{array}$ & $\begin{array}{l}\text { IKO } \\
\text { KFK } \\
\text { KFK }\end{array}$ & $\begin{array}{l}\text { E Hermest } \\
\text { C Muenzelt } \\
\text { C Muenzel+ }\end{array}$ & $\begin{array}{l}6545+ \\
6546 \\
8752\end{array}$ \\
\hline
\end{tabular}

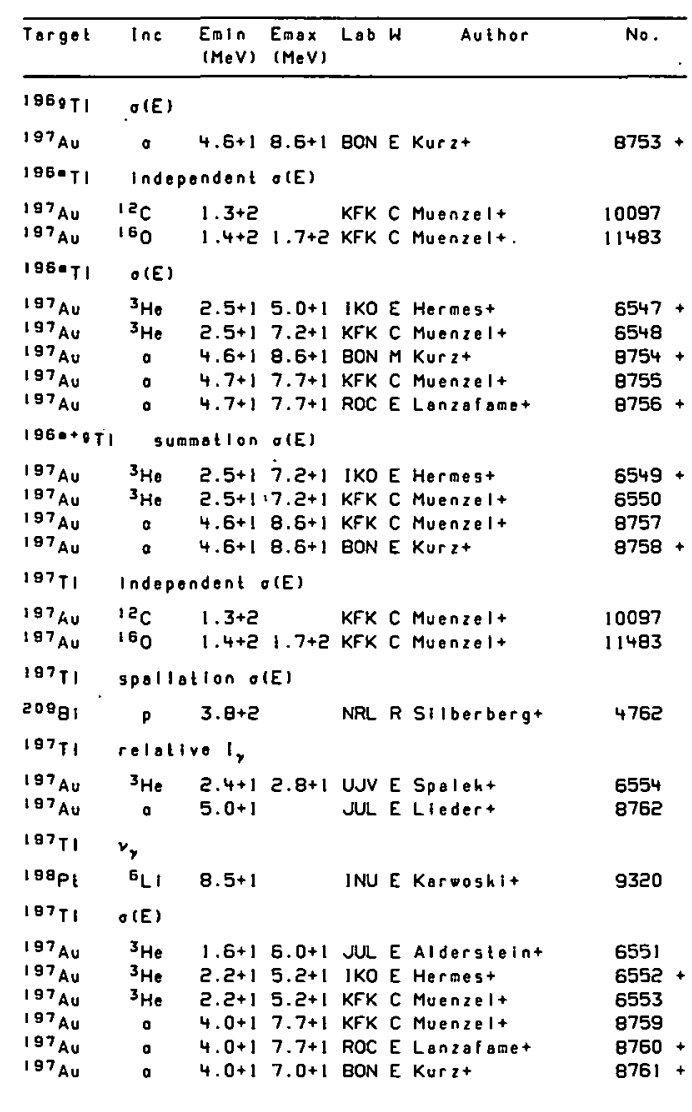




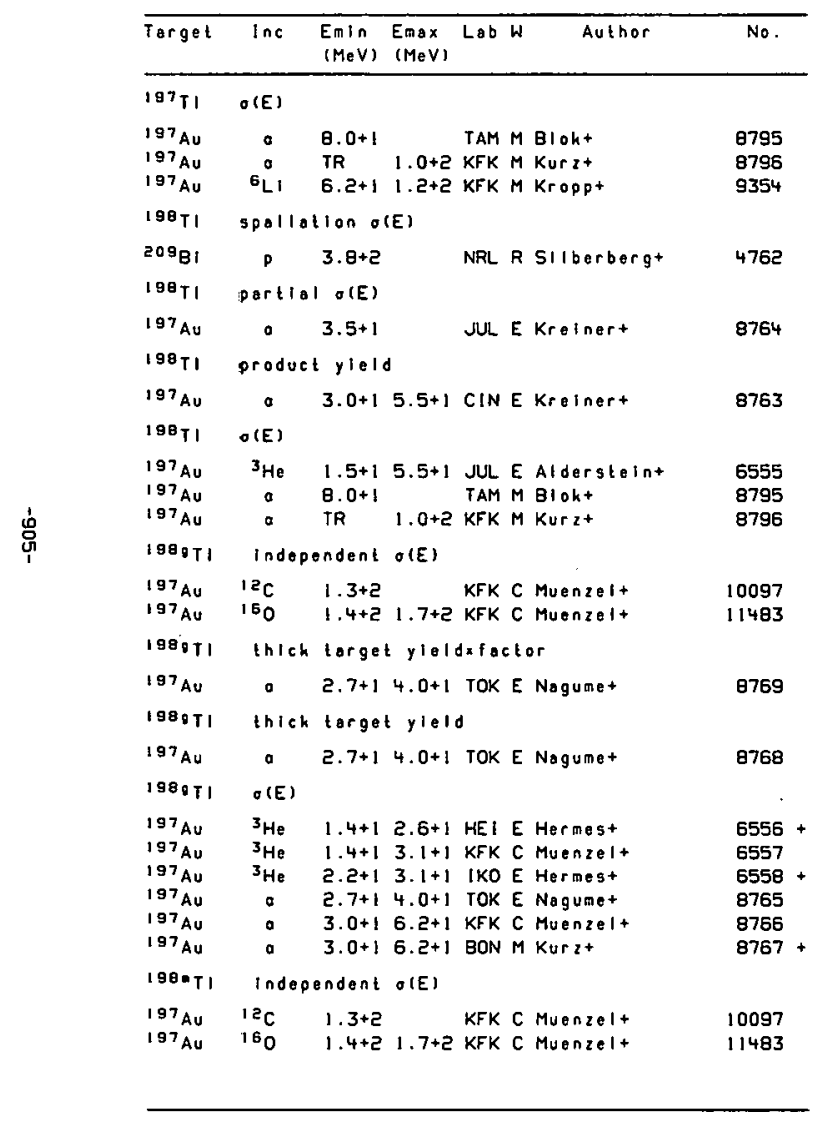

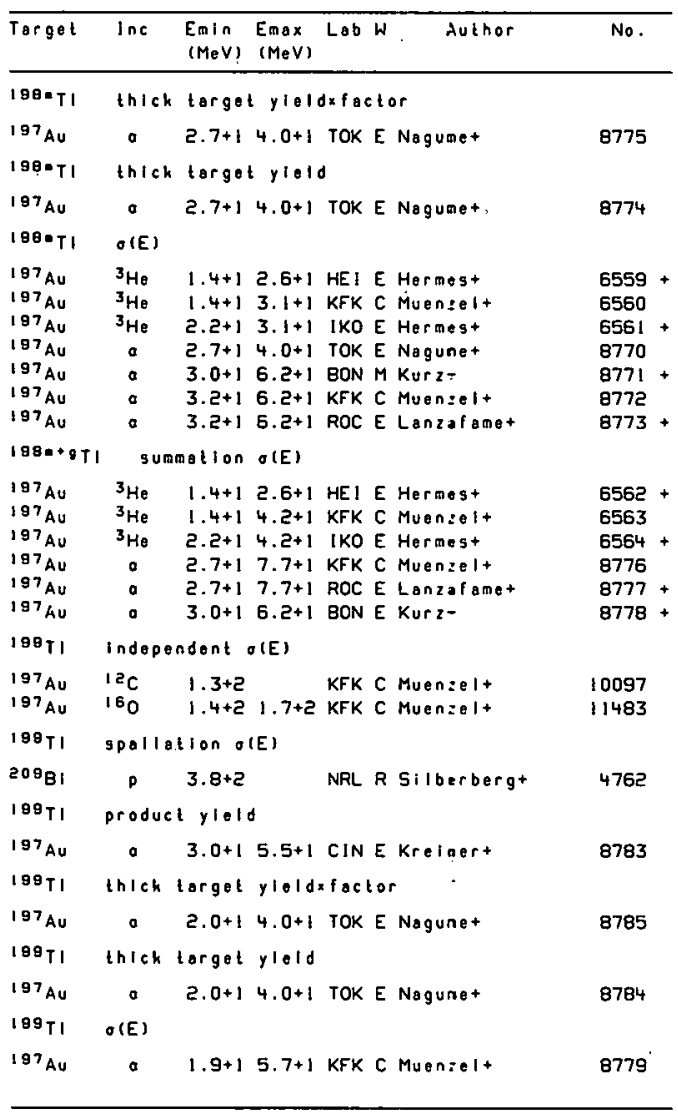




\begin{tabular}{|c|c|c|c|c|c|c|}
\hline Targel & $\ln c$ & $\begin{array}{l}\text { Emin } \\
(\mathrm{MeV})\end{array}$ & $\begin{array}{l}\text { Emax } \\
(\operatorname{MeV})\end{array}$ & Lab $W$ & Author & No. \\
\hline $199 \mathrm{Tl}$ & $O(E)$ & & & & & \\
\hline $\begin{array}{l}197 \mathrm{Au} \\
197 \mathrm{Au} \\
197 \mathrm{Au} \\
197 \mathrm{Au} \\
197 \mathrm{Au}\end{array}$ & $\begin{array}{l}a \\
a \\
a \\
a \\
0\end{array}$ & $\begin{array}{l}1.9+1 \\
1.9+1 \\
1.9+1 \\
8.0+1 \\
\text { TR }\end{array}$ & $\begin{array}{l}5 \cdot 7+1 \\
5 \cdot 2+1 \\
4 \cdot 0+1 \\
1 \cdot 0+2\end{array}$ & $\begin{array}{l}\text { ROC E } \\
\text { BON E } \\
\text { TOK E } \\
\text { TAM M } \\
\text { KFK M }\end{array}$ & $\begin{array}{l}\text { E Lanzatamet } \\
\text { E Kurz+ } \\
\text { E Nagumet } \\
\text { M Bloht } \\
\text { M Kurz+ }\end{array}$ & $\begin{array}{l}8780+ \\
8781+ \\
8782 \\
8795 \\
8796\end{array}$ \\
\hline $200 \mathrm{TI}$ & Indepe & ondento & $\sigma(E)$ & & & \\
\hline $\begin{array}{l}197 \mathrm{AU} \\
197 \mathrm{AU}\end{array}$ & $\begin{array}{l}12 \mathrm{C} \\
160\end{array}$ & $\begin{array}{l}1.3+2 \\
1.4+2\end{array}$ & $1.7+2$ & $\begin{array}{l}\text { KFK C } \\
\text { KFK C }\end{array}$ & $\begin{array}{l}\text { C Muenzel+ } \\
\text { C Muenzel+ }\end{array}$ & $\begin{array}{l}10097 \\
11483\end{array}$ \\
\hline I & spalle & ation ol & (E) & & & \\
\hline $\begin{array}{l}200^{8 i} \\
200 \mathrm{Ti}\end{array}$ & cumul. & $\begin{array}{l}3.8+2 \\
\text { ative? }\end{array}$ & product & $\begin{array}{l}\text { NRL } R \\
t y \mid 1\end{array}$ & $\begin{array}{l}\text { R Silberberg+ } \\
\text { idatactor }\end{array}$ & 4762 \\
\hline $\begin{array}{r}500 \mathrm{TI} \\
\mathrm{TI}\end{array}$ & cumula & $\begin{array}{l}\text { c. } 7+1 \\
\text { ative pr }\end{array}$ & $\begin{array}{l}4.5+1 \\
\text { roduct }\end{array}$ & $\begin{array}{l}\text { JUL E } \\
\text { yield }\end{array}$ & $\begin{array}{l}\text { E Oaimt } \\
\text { dxfoctor }\end{array}$ & 4540 \\
\hline $\begin{array}{l}205 \mathrm{TI} \\
200 \mathrm{TI}\end{array}$ & p & $\begin{array}{l}3.7+1 \\
\text { ct } y 1010\end{array}$ & $5 \cdot 0+1$ & DAVE & E Logunas-Solart & 4591 \\
\hline $\begin{array}{l}197 \mathrm{Au} \\
200 \mathrm{TI}\end{array}$ & $\begin{array}{c}a \\
t h \mid c k\end{array}$ & $\begin{array}{l}2.0+1 \\
\text { torget }\end{array}$ & $\begin{array}{l}5.5+1 \\
y 10 \mid d x\end{array}$ & $\begin{array}{l}\text { CINE } \\
\text { a facto }\end{array}$ & $\begin{array}{l}\text { E Kreinert } \\
\text { or }\end{array}$ & 8786 \\
\hline $\begin{array}{l}197 \mathrm{AU} \\
200 \mathrm{TI}\end{array}$ & $\begin{array}{c}a \\
t h \mid c k\end{array}$ & $\begin{array}{l}2.0+1 \\
\text { torget }\end{array}$ & $\begin{array}{l}4.0+1 \\
y i \in 1 d\end{array}$ & TOK E & E Nogumet & 8792 \\
\hline $\begin{array}{r}197 \mathrm{Au} \\
\mathrm{Hg} \\
\mathrm{Hg}\end{array}$ & $\begin{array}{l}a \\
p \\
d\end{array}$ & $\begin{array}{l}1.9+1 \\
1.0+1 \\
1.0+1\end{array}$ & $\begin{array}{l}3.5+1 \\
2.2+1 \\
2.3+1\end{array}$ & $\begin{array}{l}\text { TOK E } \\
\text { FEI E } \\
\text { FEI E }\end{array}$ & $\begin{array}{l}\text { E Nagumet } \\
\text { E Omitriert } \\
\text { E Omitriert }\end{array}$ & $\begin{array}{l}8791 \\
4509+ \\
5829+\end{array}$ \\
\hline 20001 & $O(E)$ & & & & & \\
\hline $\begin{array}{l}197 \mathrm{AU} \\
197 \mathrm{AU} \\
197 \mathrm{AU} \\
197 \mathrm{AU} \\
197_{\mathrm{AU}} \\
197_{\mathrm{AU}}\end{array}$ & $\begin{array}{l}a \\
a \\
a \\
a \\
a \\
a\end{array}$ & $\begin{array}{l}1.6+1 \\
1.9+1 \\
1.9+1 \\
1.9+1 \\
8.0+1 \\
\text { IR }\end{array}$ & $\begin{array}{l}4 \cdot 3+1 \\
9 \cdot 4+1 \\
9 \cdot 4+1 \\
3 \cdot 0+1 \\
1.0+2\end{array}$ & $\begin{array}{l}\text { BON E } \\
\text { KFK C } \\
\text { ROC E } \\
\text { TOK E } \\
\text { TAM M } \\
\text { KFK M }\end{array}$ & $\begin{array}{l}\text { E Kurz+ } \\
\text { C Muenzel+ } \\
\text { E Lanzafamet } \\
\text { E Nagumet } \\
\text { M Bloht } \\
\text { M Kurz+ }\end{array}$ & $\begin{array}{l}8787+ \\
8788 \\
8789+ \\
8790 \\
8795 \\
8796\end{array}$ \\
\hline $201 \mathrm{TI}$ & Indepe & oncent. & o(E) & & & \\
\hline $197 \mathrm{AU}$ & $12 \mathrm{C}$ & $1.3+2$ & & KFK C & C Muenzel+ & 10097 \\
\hline
\end{tabular}

\begin{tabular}{|c|c|c|c|c|}
\hline Target & $\operatorname{lnc}$ & $\begin{array}{ll}\text { Emin } & \text { Emax } \\
(M e V) & (\operatorname{MeV})\end{array}$ & Author & No. \\
\hline $201 \mathrm{TI}$ & Indepe & andent $O(E)$ & & \\
\hline $\begin{array}{l}197 \mathrm{Au} \\
201 \mathrm{TI}\end{array}$ & $\begin{array}{l}160 \\
\text { spalla }\end{array}$ & $\begin{array}{l}1.4+21.7+2 \\
\text { ation } O(E)\end{array}$ & KFK C Muenze1+ & 11483 \\
\hline $209_{B i}$ & $p$ & $3.8+2$ & NRL R Silberbergt & 4762 \\
\hline $\begin{array}{l}11 \\
50111\end{array}$ & $\begin{array}{c}\text { cumula } \\
p\end{array}$ & $\begin{array}{l}\text { Alve? product } \\
2.0+14.5+1\end{array}$ & $\begin{array}{l}\text { ylald } \\
\text { JUL E OAImt }\end{array}$ & 4542 \\
\hline $201 \mathrm{TI}$ & cumula & attue product & yleldxfoctor & \\
\hline $\begin{array}{l}205 \mathrm{TI} \\
201 \mathrm{TI}\end{array}$ & $\stackrel{\text { p }}{\text { cumula }}$ & $\begin{array}{l}3.7+15.0+1 \\
\text { ative product }\end{array}$ & $\begin{array}{l}\text { DAV E Lagunas-Solart } \\
\text { yleld }\end{array}$ & 4593 \\
\hline 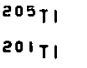 & $\begin{array}{c}p \\
\text { cumuls }\end{array}$ & $\begin{array}{l}3.7+15.0+1 \\
\text { dive thlek tor }\end{array}$ & $\begin{array}{l}\text { DAV E Lagunas-Solort } \\
\text { orget yiold }\end{array}$ & 4592 \\
\hline TI & d & $3.3+1$ & TAME BIUe+ & 5836 \\
\hline $201 \mathrm{Tl}$ & cololi & ive $I_{y}$ & & \\
\hline $202 \mathrm{Hg}$ & d & $1.8+12.5+1$ & CAN E Slocombet & 5833 \\
\hline $201 \mathrm{TI}$ & thlck & $\operatorname{targot} y 1018 x$ & xabundanco & \\
\hline $203 \mathrm{TI}$ & p & $3.0+1$ & AML R Chaudel. & 4577 \\
\hline $201 \mathrm{Tl}$ & thlch & target yleld & & \\
\hline $\begin{array}{l}\mathrm{Hg} \\
\mathrm{Hg} \\
\mathrm{Hg}\end{array}$ & $\begin{array}{l}\text { p } \\
\text { p } \\
\text { d }\end{array}$ & $\begin{array}{ll}1.0+1 & 2.2+1 \\
1.4+1 & 2 \cdot 0+1 \\
1.0+1 & 2 \cdot 3+1\end{array}$ & $\begin{array}{l}\text { FEI E Dalloliout } \\
\text { AML R Chaudri. } \\
\text { FEI } E \text { Daliriout }\end{array}$ & $\begin{array}{l}4510+ \\
4511 \\
5830+\end{array}$ \\
\hline $201 \mathrm{TI}$ & $\sigma(E)$ & & & \\
\hline $\begin{array}{r}197 \mathrm{Au} \\
197 \mathrm{Au} \\
202 \mathrm{Hg} \\
202^{\mathrm{Hg}} \\
\mathrm{TI}\end{array}$ & $\begin{array}{c}\gamma_{L \prime}^{0} \\
7^{\prime} \\
p \\
p \\
p\end{array}$ & $\begin{array}{l}8.0+1 \\
3.2+1 \\
4.0+2 \\
4.0+2 \\
1.1+14.5+1\end{array}$ & $\begin{array}{l}\text { TAM M Blokt } \\
\text { HEI E Quabert+ } \\
\text { KFK C Muenzel+ } \\
\text { PUP E Churcht } \\
\text { JUL E Oaint }\end{array}$ & $\begin{array}{l}8795 \\
9464 \\
4534 \\
4535+ \\
4541\end{array}$ \\
\hline $205 \mathrm{TI}$ & spalla & atlon o(E) & & \\
\hline $209_{B 1}$ & $p$ & $3.8+2$ & NRL R silberberg* & 4762 \\
\hline $202 \mathrm{TI}$ & cumuls & ollve produet & yloloxfactor & \\
\hline $205 \mathrm{TI}$ & p & $3.7+15.0+1$ & DAV E Lagunag-Solart & 4594 \\
\hline
\end{tabular}




\begin{tabular}{|c|c|c|c|c|c|c|}
\hline Target & $\ln c$ & $\begin{array}{l}\text { Emin } \\
(\mathrm{MeV})\end{array}$ & $\begin{array}{l}\text { Emax } \\
(\operatorname{MeV})\end{array}$ & Lab h & Author & No. \\
\hline $202 \mathrm{TI}$ & produc & ct yield & & & & \\
\hline $203 \mathrm{TI}$ & $p$ & NDG & & USA $F$ & R Beavert & 4578 \\
\hline $205 T$ & thick & target & $y i e l d$ & & & \\
\hline $\begin{array}{l}\mathrm{Hg} \\
\mathrm{Hg}\end{array}$ & p & $\begin{array}{l}1.0+1 \\
1.0+1\end{array}$ & $\begin{array}{l}2 \cdot 2+1 \\
2 \cdot 3+1\end{array}$ & $\begin{array}{l}\text { FEI } \\
\text { FEI E }\end{array}$ & $\begin{array}{l}\text { E Dmitrievt } \\
\text { E Dmitrievt }\end{array}$ & $\begin{array}{l}4512+ \\
5831+\end{array}$ \\
\hline $202 \mathrm{TI}$ & $\hat{\sigma}(E)$ & & & & & \\
\hline $\begin{array}{r}197 \mathrm{Au} \\
202^{\mathrm{Hg}} \\
202 \mathrm{Hg} \\
\mathrm{TI}\end{array}$ & $\begin{array}{c}P L i \\
p \\
p \\
p\end{array}$ & $\begin{array}{l}3 \cdot 2+1 \\
4 \cdot 0+2 \\
4 \cdot 0+2 \\
2.0+1\end{array}$ & $4.5+1$ & $\begin{array}{l}\text { HE 1 E } \\
\text { KFK C } \\
\text { PUP E } \\
\text { JUL E }\end{array}$ & $\begin{array}{l}\text { E Quebert+ } \\
\text { C Muenzelt } \\
\text { E Chureht } \\
\text { E Qaimt }\end{array}$ & $\begin{array}{l}9465 \\
4536 \\
4537+ \\
4543\end{array}$ \\
\hline $203 \mathrm{TI}$ & soalla & ation ol & (E) & & & \\
\hline $209 \mathrm{Bi}$ & p & $3.8+2$ & & NRL F & R Silberberg+ & 4762 \\
\hline $203 \mathrm{Tl}$ & direct & t-intera & action & $\sigma(E)$ & & \\
\hline${ }^{206} \mathrm{~Pb}$ & $p$ & $2.0+1$ & & MIL $r$ & M Milazzo-Collit+ & 4636 \\
\hline $203 \mathrm{TI}$ & partia & al $O(E)$ & & & . & \\
\hline $206 \mathrm{~Pb}$ & $p$ & $2.0+1$ & & MIL $r$ & M Milazzo-Collit & 4635 \\
\hline $203 T 1$ & $r=1$ at 1 & ive $I_{y}$ & & & & \\
\hline $\begin{array}{l}20^{4} \mathrm{Hg} \\
203 \mathrm{TI}\end{array}$ & $\begin{array}{c}d \\
\sigma(E)\end{array}$ & $1.8+1$ & $2.5+1$ & CAN E & E slocombet & 5834 \\
\hline $\begin{array}{l}197 \mathrm{Au} \\
206 \mathrm{~Pb}\end{array}$ & $\begin{array}{l}{ }^{7} L i \\
p\end{array}$ & $\begin{array}{l}3.2+1 \\
2.0+1\end{array}$ & & $\begin{array}{l}\text { HEI } \\
\text { MIL }\end{array}$ & $\begin{array}{l}\text { E Queberti } \\
\text { M Milazzo-Collit }\end{array}$ & $\begin{array}{l}9466 \\
4634\end{array}$ \\
\hline $204 \mathrm{TI}$ & sjolla & ation or & (E) & & & \\
\hline${ }^{209} \mathrm{Bi}$ & $p$ & $3.8+2$ & & NRL F & R Silberberg+ & 4762 \\
\hline $204 \mathrm{TI}$ & diroct & $t$-intera & action & $\sigma(E)$ & & \\
\hline $\begin{array}{l}207 \mathrm{~Pb} \\
204 \mathrm{TI}\end{array}$ & $\underset{\text { pertia }}{\text { p }}$ & $\begin{array}{r}2.0+1 \\
a 1 \quad O(E)\end{array}$ & $3.0+1$ & MIL N & M MIlazzo-Collit+ & 4644 \\
\hline $\begin{array}{l}207 \mathrm{~Pb} \\
204 \mathrm{TI}\end{array}$ & $\begin{array}{c}p \\
\text { thich }\end{array}$ & $\begin{array}{l}2.0+1 \\
\text { target }\end{array}$ & $\begin{array}{l}3.0+1 \\
y|0| d x\end{array}$ & $\begin{array}{l}\text { MIL } \\
\text { xabund }\end{array}$ & $\begin{array}{l}\text { Milazzo-Collit } \\
\text { dance }\end{array}$ & 4643 \\
\hline $\begin{array}{l}204 \mathrm{Hg} \\
204 \mathrm{Hg}\end{array}$ & $\begin{array}{l}\text { p } \\
\text { d }\end{array}$ & $\begin{array}{l}1.0+1 \\
1.0+1\end{array}$ & $\begin{array}{l}2.2+1 \\
2.2+1\end{array}$ & $\begin{array}{l}\text { FEI } \\
\text { FEI }\end{array}$ & $\begin{array}{l}\text { E Dmlitrievt } \\
\text { E Doletrievt }\end{array}$ & $\begin{array}{l}4538+ \\
5835+\end{array}$ \\
\hline
\end{tabular}

\begin{tabular}{|c|c|c|c|c|c|c|}
\hline Target & $\ln c$ & $\begin{array}{l}\text { Emin } \\
(\mathrm{MeV})\end{array}$ & $\begin{array}{l}\operatorname{Emax}_{\max } \\
(\mathrm{MeV})\end{array}$ & Lab W & Author & No. \\
\hline $204 \mathrm{Ti}$ & thick & target & yield & & & \\
\hline $\begin{array}{l}\mathrm{Hg} \\
\mathrm{Hg}\end{array}$ & o & $\begin{array}{l}1.0+1 \\
1.0+1\end{array}$ & $\begin{array}{l}2.2+1 \\
2.3+1\end{array}$ & $\begin{array}{l}\text { FEI E } \\
\text { FEI E }\end{array}$ & $\begin{array}{l}\text { E Dmitrievt } \\
\text { E Dmitrieut }\end{array}$ & $\begin{array}{l}4513 \\
5832+\end{array}$ \\
\hline $204 \mathrm{TI}$ & $\sigma(E)$ & & & & & \\
\hline $\begin{array}{l}207 \mathrm{~Pb} \\
205 \mathrm{TI}\end{array}$ & $\begin{array}{c}p \\
\text { direct }\end{array}$ & $\begin{array}{l}2.0+1 \\
\text {-intero }\end{array}$ & $\begin{array}{r}3.0+1 \\
\text { action }\end{array}$ & $\begin{array}{l}M: L M \\
O(E)\end{array}$ & M Milazzo-Collit & 4642 \\
\hline $\begin{array}{l}209_{\mathrm{Pb}} \\
205 \mathrm{Tt}\end{array}$ & $\begin{array}{c}p \\
\text { partio }\end{array}$ & $\begin{array}{r}2.0+1 \\
1 \quad O(E)\end{array}$ & $3.0+1$ & MIL M & M Milazzo-Collit & 4653 \\
\hline $\begin{array}{l}208 \mathrm{~Pb} \\
205 \mathrm{Tl}^{2}\end{array}$ & $\begin{array}{c}P \\
\sigma(E)\end{array}$ & $2.0+1$ & $3.0+1$ & MIL M & M Milazzo-Co11it & 4652 \\
\hline $\begin{array}{l}208 \mathrm{~Pb} \\
207 \mathrm{TI}\end{array}$ & $\begin{array}{c}p \\
\text { partia }\end{array}$ & $\begin{array}{r}2.0+1 \\
1 \quad \sigma(E)\end{array}$ & $3.0+1$ & MIL M & M Milazzo-Collit & 4651 \\
\hline 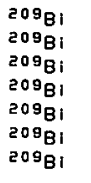 & $\begin{array}{l}14 \mathrm{~N} \\
16 \mathrm{O} \\
19 \mathrm{~F} \\
40 \mathrm{Ar} \\
40 \mathrm{Ca} \\
56 \mathrm{Fe} \\
6{ }^{3} \mathrm{Cu}\end{array}$ & $\begin{array}{l}6.0+1 \\
6.6+1 \\
7.0+1 \\
1.5+2 \\
1.5+2 \\
2.0+2 \\
2.2+2\end{array}$ & $\begin{array}{l}7.0+1 \\
8.0+1 \\
0.8+1 \\
1.7+2 \\
1.7+2 \\
2.2+2 \\
2.5+2\end{array}$ & $\begin{array}{l}\text { PAR T } \\
\text { PAR } \\
\text { PAR T } \\
\text { PAR T } \\
\text { PAR T } \\
\text { PAR T } \\
\text { PAR T }\end{array}$ & $\begin{array}{l}\text { T Blmbot+ } \\
\text { T BImbot+ } \\
\text { T Bimbott+ } \\
\text { T BImbot+ } \\
\text { T BImbot+ } \\
\text { T BImbot+ } \\
\text { T Bimbot+ }\end{array}$ & $\begin{array}{l}10498 \\
11543 \\
11818 \\
12377 \\
12435 \\
12464 \\
12485\end{array}$ \\
\hline $\begin{array}{l}z=82 \\
197_{A U}\end{array}$ & $\begin{array}{l}\sigma(E) \\
\sigma_{L I}\end{array}$ & $7.5+1$ & $9.4+1$ & INUE & E Karwoshit & 9358 \\
\hline${ }^{188} \mathrm{~Pb}$ & Sogile & ation of & (E) & & & \\
\hline $\begin{array}{l}189^{\mathrm{Pb}} \\
{ }_{009} \mathrm{Bl}\end{array}$ & $\begin{array}{c}\text { Do } \\
\text { spalla }\end{array}$ & $\begin{array}{l}3.8+2 \\
\text { tion ot }\end{array}$ & & NRL $F$ & R Silberbergt & $47 E 2$ \\
\hline $\begin{array}{l}200^{\circ} \mathrm{Bl} \\
190 \mathrm{~Pb}\end{array}$ & $\begin{array}{c}p \\
\text { spolla }\end{array}$ & $\begin{array}{l}3.8+2 \\
\text { ation of }\end{array}$ & (E) & NRL $R$ & R Silberberg+ & 4762 \\
\hline $\begin{array}{l}209 \mathrm{Bi} \\
191 \mathrm{~Pb}\end{array}$ & $\begin{array}{c}P \\
\text { spolla }\end{array}$ & $\begin{array}{l}3.8+2 \\
\text { tion of }\end{array}$ & & NRL $F$ & R Silberberg+ & 4762 \\
\hline $209_{B i}$ & p & $3.8+2$ & & NRL $\mathrm{F}$ & R silberbergt & 4762 \\
\hline
\end{tabular}




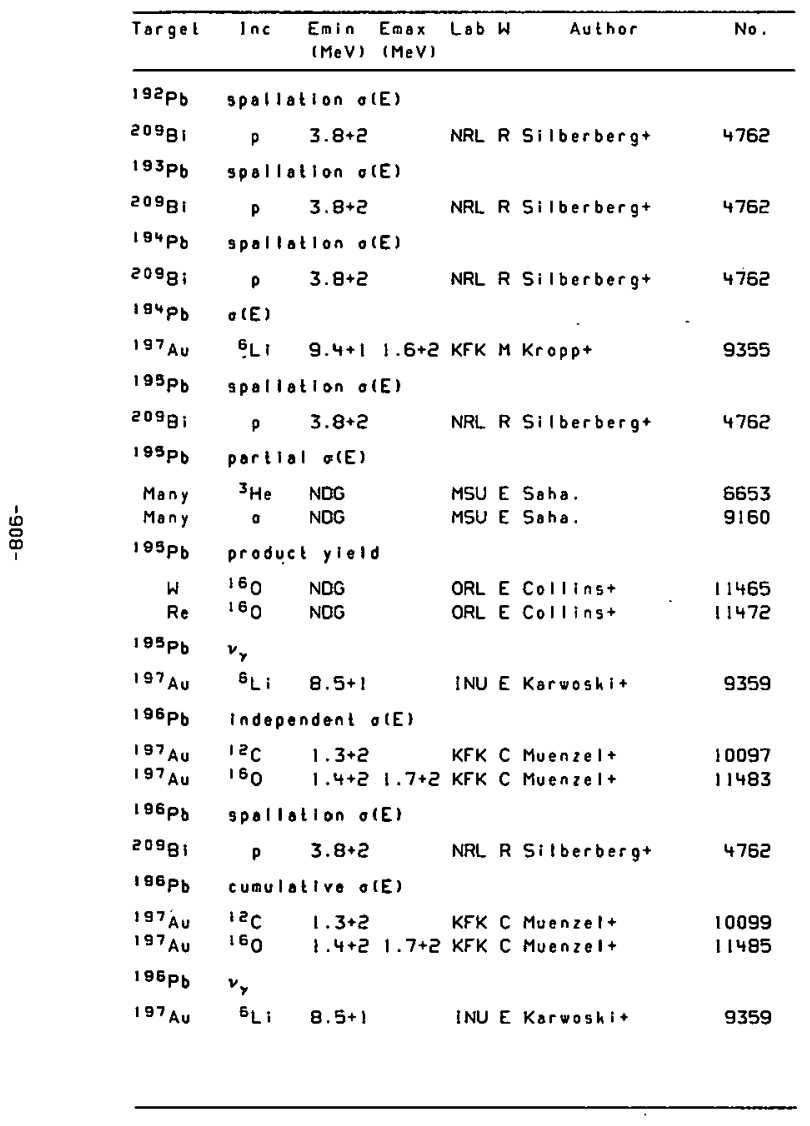

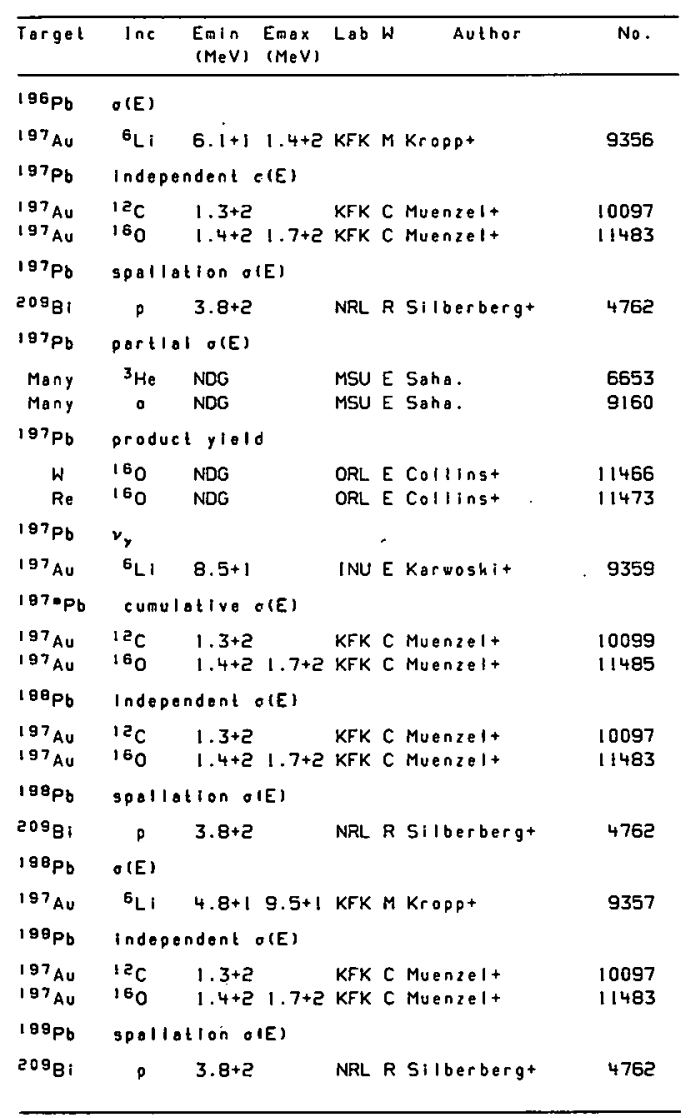




\begin{tabular}{|c|c|c|c|c|c|c|}
\hline Target & $\ln c$ & $\begin{array}{l}\text { Emin } \\
(\mathrm{MeV})\end{array}$ & $\begin{array}{l}\text { Emax } \\
(\mathrm{MeV})\end{array}$ & Lab $W$ & Author & No. \\
\hline $193 \mathrm{~Pb}$ & cumula & ative po & product & yielo & & \\
\hline 31 & $p$ & $3.7+1$ & $4.2+1$ & JUL $E$ & $=0 a \mathrm{Im}^{+}$ & 4546 \\
\hline $199 \mathrm{~Pb}$ & portis & $\theta 1 \sigma(E)$ & & & & \\
\hline $\begin{array}{l}\text { Many } \\
\text { Many }\end{array}$ & $\begin{array}{c}{ }^{3} \mathrm{He} \\
0\end{array}$ & $\begin{array}{l}\text { NOG } \\
\text { NOG }\end{array}$ & & $\begin{array}{l}\text { MSU E } \\
\text { MSU E }\end{array}$ & $\begin{array}{l}\text { Saha. } \\
=\text { Satia. }\end{array}$ & $\begin{array}{l}6653 \\
9160\end{array}$ \\
\hline $199 \mathrm{~Pb}$ & thich & torget & yielo & & & \\
\hline TI & $p$ & $4.5+1$ & & JUL E & E Qaim+ & 4545 \\
\hline${ }^{199} \mathrm{~Pb}$ & $g(E)$ & & & & & \\
\hline II & p & $3.8+1$ & $4.5+1$ & JUL E & $=Q_{a} \mid \mathrm{m}+$ & 4544 \\
\hline $200 \mathrm{pb}$ & indepe & endent & o(E) & & & \\
\hline $\begin{array}{l}197 \mathrm{Au} \\
197 \mathrm{A.U}\end{array}$ & $\begin{array}{l}: 2 c \\
160\end{array}$ & $\begin{array}{l}1.3+2 \\
1.4+2\end{array}$ & $1.7+2$ & $\begin{array}{l}\text { KFK C } \\
\text { KFK C }\end{array}$ & $\begin{array}{l}\text { CMuenzel+ } \\
\text { C Muenzel+ }\end{array}$ & $\begin{array}{l}10097 \\
11483\end{array}$ \\
\hline $200 \mathrm{Fb}$ & spalla & ation of & $(E)$ & & & \\
\hline${ }^{200} \mathrm{E}_{1}$ & p & $3.8+2$ & & NRL $R$ & R Silberbergt & 4762 \\
\hline $200_{\mathrm{Fb}}$ & cumula & ative po & roduct & yield & & \\
\hline TI & $p$ & $2.8+1$ & $4.2+1$ & JUL E & $=\mathrm{Oa}_{\mathrm{a} m \mathrm{~m}}$ & 4550 \\
\hline $200 \mathrm{Fb}$ & thick & target & yieldx & a $a_{a c}$ & & \\
\hline TI & p & $2.8+1$ & $3.3+1$ & DAV E & E Lagunas-Solart & 4552 \\
\hline $200 \mathrm{pb}$ & thick & target & yield & & & \\
\hline TI & p & $4.5+1$ & & J E E & E Orimt & 4549 \\
\hline $20 \mathrm{C}_{\mathrm{Pb}}$ & $\operatorname{sen} 06$ & & & & & \\
\hline TI & p & $2.9+1$ & $6.0+1$ & DAV E & E Lagunas-Solart & 4551 \\
\hline $20 \mathrm{cpb}$ & $e(E)$ & . & & & & \\
\hline TI & p & TR & $5.1+1$ & IKO E & E Leurst & 4547 \\
\hline$T_{1}$ & p & $2.6+1$ & $4.5+1$ & JUL E & $E Q_{a 1 m+}$ & 4548 \\
\hline 2031 & $\mathbf{p}$ & $2.8+1$ & $3.6+1$ & TAM E & E Blue* & 4579 \\
\hline 2031 & p & $2.9+1$ & $4.7+1$ & DAV E & E Lagunas-Solart & 4580 \\
\hline 2031 & d & $3.3+1$ & $4.8+1$ & TAM E & E Bluet & 5837 \\
\hline $20 E_{T 1}$ & p & $3.5+1$ & $5.8+1$ & DAVE & E Lagunas-Solart & 4595 \\
\hline
\end{tabular}

\begin{tabular}{|c|c|c|c|c|c|c|}
\hline Target & $\ln c$ & $\begin{array}{l}E_{m i n} \\
(M e V)\end{array}$ & $\begin{array}{l}\text { Emax } \\
(M e V)\end{array}$ & Lab W & $\begin{array}{r}\text { Ruthor } \\
\text {; }\end{array}$ & No. \\
\hline $201 \mathrm{~Pb}$ & \multicolumn{3}{|c|}{ Independent o(E) } & & & \\
\hline $\begin{array}{l}197 \mathrm{Au} \\
197 \mathrm{Au}\end{array}$ & $\begin{array}{l}12 \mathrm{C} \\
160\end{array}$ & $\begin{array}{l}1.3+2 \\
1.4+2\end{array}$ & $1.7+2$ & $\begin{array}{l}\text { KFK C } \\
\text { KFK C }\end{array}$ & Muenzel+ & $\begin{array}{l}10097 \\
11483\end{array}$ \\
\hline $201 \mathrm{~Pb}$ & \multicolumn{3}{|c|}{ spallation ofEl } & & & \\
\hline${ }^{209} \mathrm{~Pb}$ & $\begin{array}{l}p \\
p\end{array}$ & $\begin{array}{l}3.0+0 \\
3.8+2\end{array}$ & $6.5+0$ & $\begin{array}{l}\text { HAR E } \\
\text { NRL R }\end{array}$ & $\begin{array}{l}\text { Symet } \\
\text { Silberbergt }\end{array}$ & $\begin{array}{l}462 ! \\
476 !\end{array}$ \\
\hline $201 \mathrm{~Pb}$ & \multicolumn{3}{|c|}{ cumulative product } & $y i e l d$ & & \\
\hline $\begin{array}{r}\mathrm{TI} \\
201 \mathrm{~Pb}\end{array}$ & \multicolumn{3}{|c|}{ partiol o(E) } & JUL E & $=0 \mathrm{aim}+$ & 4557 \\
\hline $\begin{array}{l}\text { Many } \\
\text { Many }\end{array}$ & $\begin{array}{c}{ }^{3} \mathrm{He} \\
a\end{array}$ & $\begin{array}{l}\text { NDG } \\
\text { NDG }\end{array}$ & & $\begin{array}{l}\text { MSU E } \\
\text { MSU E }\end{array}$ & $\begin{array}{l}\text { Saha. } \\
\text { Saha. }\end{array}$ & $\begin{array}{l}5653 \\
9160\end{array}$ \\
\hline $201 \mathrm{pb}$ & \multicolumn{3}{|c|}{ product yleld } & & & \\
\hline $\begin{array}{l}\mathrm{TI} \\
203 \mathrm{TI} \\
203 \mathrm{TI}\end{array}$ & $\begin{array}{l}p \\
p \\
p\end{array}$ & $\begin{array}{l}\text { NDG } \\
\text { NDG } \\
2.9+1\end{array}$ & & $\begin{array}{l}\text { UPP E } \\
\text { USA R } \\
\text { DAV E }\end{array}$ & $\begin{array}{l}\text { Lundavislt } \\
\text { Beavert } \\
\text { Lagunas-Solart }\end{array}$ & $\begin{array}{l}4558 \\
4581 \\
4582\end{array}$ \\
\hline $201 \mathrm{~Pb}$ & \multicolumn{3}{|c|}{ thick ta } & x factor & & \\
\hline$T_{1}$ & $p$ & $2.8+1$ & $3.3+1$ & OAV E & Eagunas-Solart & 4560 \\
\hline $201 p o b^{2}$ & thick & $\operatorname{target}$ & yield & & & \\
\hline TI & $p$ & $2.4+1$ & $4.5+1$ & JUL E & 0 aimt & 4555 \\
\hline $201 \mathrm{~Pb}$ & \multicolumn{2}{|c|}{ rov $a(E)$} & & & & \\
\hline $\begin{array}{r}\mathrm{TI} \\
201 \mathrm{~Pb}\end{array}$ & $\begin{array}{c}D \\
O(E)\end{array}$ & $1.5+1$ & $6.0+1$ & DAV E & Lagunas-Solart & 4559 \\
\hline $\begin{array}{l}\text { TI } \\
\text { TI }\end{array}$ & $p$ & $\begin{array}{l}\text { NDG } \\
\text { TR }\end{array}$ & $5.1+1$ & $\begin{array}{l}\text { UPP E } \\
\text { IKO E }\end{array}$ & $\begin{array}{l}\text { Eundavista } \\
\text { Leurst }\end{array}$ & $\begin{array}{l}4553 \\
4554\end{array}$ \\
\hline TI & p & $1.5+1$ & $4.5+1$ & JUL E & Qaim+ & 4555 \\
\hline $203 \mathrm{TI}$ & p & $1.8+1$ & $4 \cdot 1+1$ & DAV E & Lagunas-Solart & 458 \\
\hline $203 \mathrm{TI}$ & p & $1.8+1$ & $4.4+1$ & TAM E & Eluet & 45 \\
\hline $203 \mathrm{TI}$ & d & $2.3+1$ & $4.8+1$ & TAM $\bar{E}$ & Bluet & 583 \\
\hline $209 \mathrm{TI}$ & $p$ & $3.4+1$ & $5.9+1$ & DAV E & Lagunas-Solart & 4596 \\
\hline $205 \mathrm{TI}$ & $d$ & $4.0+1$ & $4.8+1$ & TAME & Bluet & 5841 \\
\hline $202 \mathrm{pb}$ & \multicolumn{3}{|c|}{ spallation o(E) } & & & \\
\hline $\mathrm{Pb}$ & $p$ & $3.0+0$ & $6.5+0$ & HAR E & Syme+ & 4622 \\
\hline
\end{tabular}




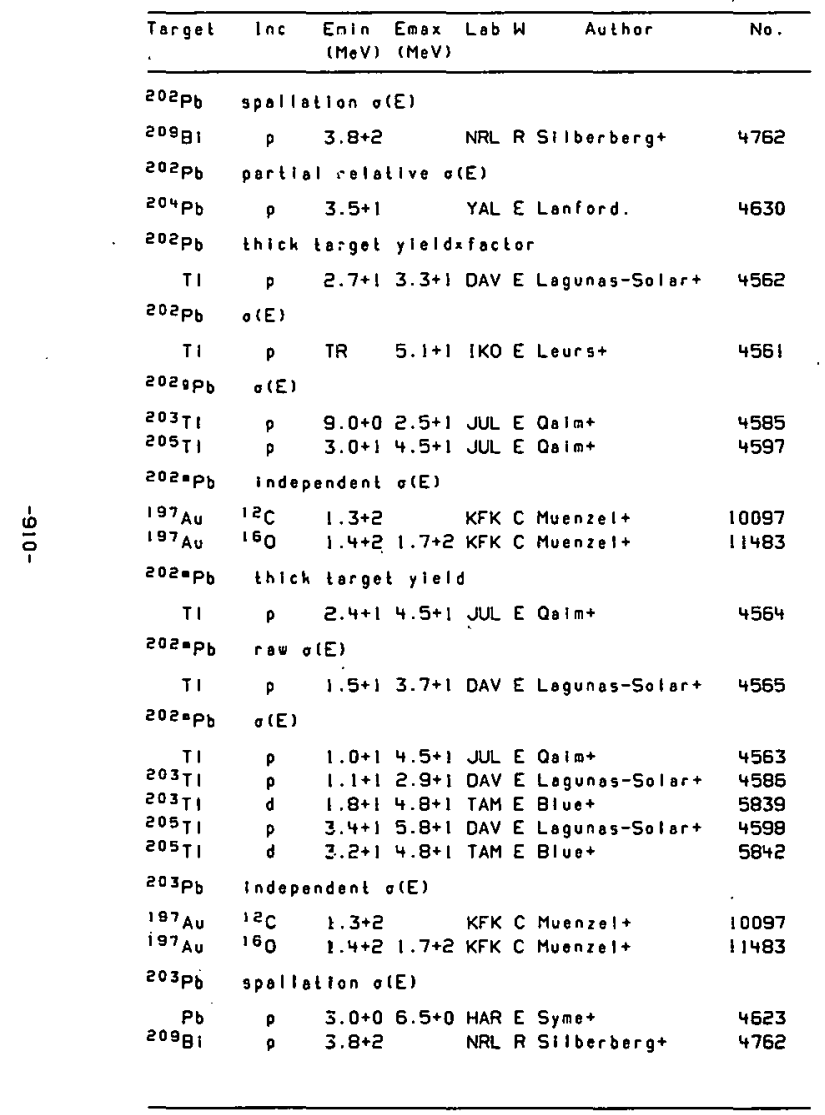

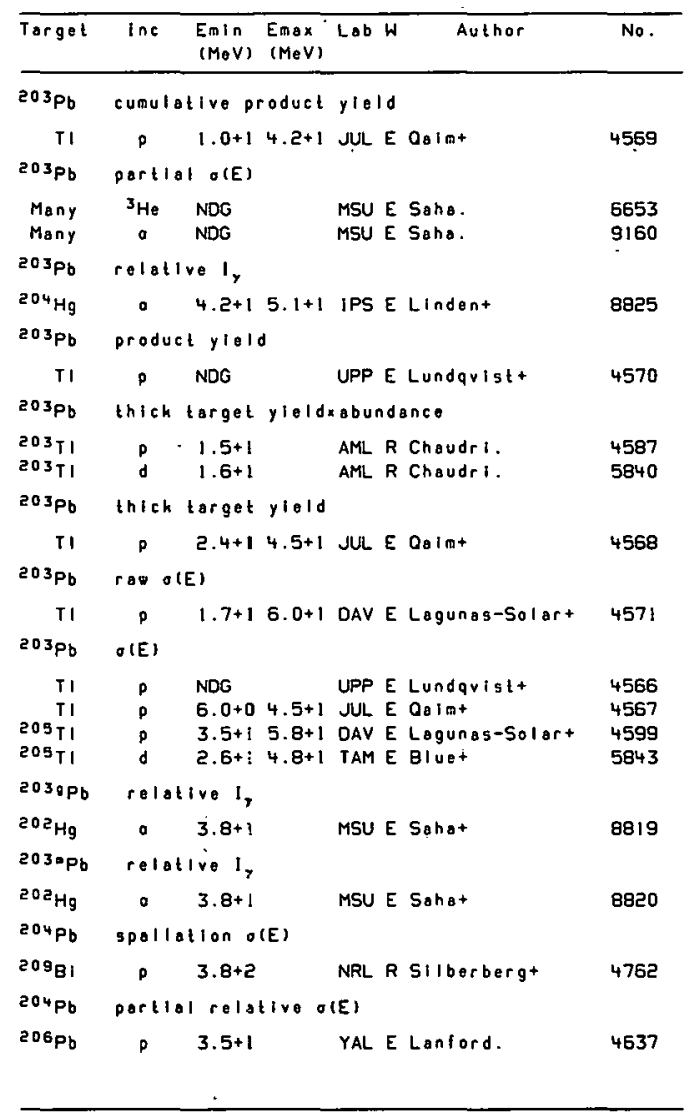




\begin{tabular}{|c|c|c|c|c|c|c|}
\hline Target & Inc & $\begin{array}{l}\text { Emin } \\
(\operatorname{MeV})\end{array}$ & $\begin{array}{l}\text { Emsx } \\
(\operatorname{MeV})\end{array}$ & Lab & Author & No. \\
\hline $204 \mathrm{~Pb}$ & \multicolumn{6}{|c|}{ rolative Iy } \\
\hline $204 \mathrm{Hg}$ & a & $4 \cdot 2+1$ & $5.1+1$ & IPS E & E Lindent & 8826 \\
\hline $204 \mathrm{~Pb}$ & $O(E)$ & & & $\therefore$ & . & \\
\hline $204 \mathrm{~Pb}$ & " & NDG & & HAR & E Cuninghamet & 8858 \\
\hline $2040 \mathrm{~Pb}$ & $O(E)$ & & & & & \\
\hline $\operatorname{sos}$ & D & $\theta .0+0$ & $4.5+1$ & $\mathrm{JU}$ & E Ooimt & 4600 \\
\hline${ }_{48}^{204} \mathrm{~Pb}$ & thick & torget & t yiolo & & & \\
\hline TI & D & $2.4+1$ & $4.5+1$ & JUL E & E Ooimt & 4572 \\
\hline $204 \cdot \mathrm{Pb}$ & covo & $(E)$. & & & & \\
\hline $\begin{array}{c}\text { II } \\
204=\mathrm{Pb}\end{array}$ & $\begin{array}{c}P \\
Q(E)\end{array}$ & $1.2+1$ & $3.7+1$ & DAV & E Lagunas-Solart & 4573 \\
\hline 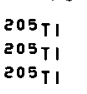 & $\begin{array}{l}p \\
p \\
0\end{array}$ & $\begin{array}{l}1.2+1 \\
3.5+1 \\
1.9+1\end{array}$ & $\begin{array}{l}4.5+1 \\
5.8+1 \\
3.6+1\end{array}$ & $\begin{array}{l}\text { JUL } \\
\text { DAV } \\
\text { TAM }\end{array}$ & $\begin{array}{l}\text { E Oaim" } \\
\text { E Lagunas-Solart } \\
\text { E Bluet }\end{array}$ & $\begin{array}{l}4601 \\
4602 \\
5844\end{array}$ \\
\hline${ }^{205} \mathrm{~Pb}$ & spollo & ation of & (E) & $\cdot$ & & \\
\hline $\operatorname{cog}_{\mathrm{Bi}}$ & p. & $3.8+2$ & & NRL & R silberbergt & 4762 \\
\hline${ }^{205} \mathrm{~Pb}$ & relotis & ive $I_{y}$ & & · & & \\
\hline${ }^{204} \mathrm{Hg}$ & a & $4.2+1$ & $5 \cdot 1+1$ & IPS & E Lindent & 8827 \\
\hline $206 \mathrm{pb}$ & spollo & tion ol & (E) & & & : \\
\hline $209^{B i}$ & D & $3.8+2$ & & NRL & R Silberberg* & 4762 \\
\hline $206 \mathrm{~Pb}$ & direct & - Intero & octlon & $\sigma(E)$ & & \\
\hline $209_{B i}^{\prime}$ & 。 & $2.0+1$ & & MIL I & M Milazzo-Collit & 4713 \\
\hline $208 \mathrm{~Pb}$ & portiol & $O(E)_{y}$ & & & & \\
\hline $206 \mathrm{~Pb}$ & $p$ & $7.0+0$ & $2.6+1$ & LRL & E koopmant & 4638 \\
\hline $208 \mathrm{~Pb}$ & partio & ol relat & tive of & (E) & & \\
\hline $\begin{array}{l}200 \mathrm{~Pb} \\
200 \mathrm{~Pb}\end{array}$ & $p$ & $\begin{array}{l}3.5+1 \\
3.5+1\end{array}$ & & $\begin{array}{l}\text { IPS } \\
\text { YAL }\end{array}$ & $\begin{array}{l}\text { T Pomart } \\
\text { E Lanford. }\end{array}$ & $\begin{array}{l}4655 \\
4656\end{array}$ \\
\hline $208 \mathrm{pb}$ & partial & 1 O(E) & & & & \\
\hline $205 \mathrm{TI}$ & $p$ & NDG & & WAU I & MEOIsowa. & 4604 \\
\hline
\end{tabular}

\begin{tabular}{|c|c|c|c|c|c|c|}
\hline Target & $\operatorname{lng}$ & $\begin{array}{l}\text { Emin } \\
(\mathrm{MeV})\end{array}$ & $\begin{array}{l}\text { Emax } \\
(M e V)\end{array}$ & Lab & Author & No. \\
\hline $206 \mathrm{~Pb}$ & \multicolumn{6}{|c|}{ partiol o(E) } \\
\hline $\begin{array}{l}206 \mathrm{~Pb} \\
208 \mathrm{~Pb} \\
208 \mathrm{~Pb} \\
20{ }^{0} \mathrm{Bi}\end{array}$ & $\begin{array}{l}p \\
p \\
p \\
p\end{array}$ & $\begin{array}{l}2.0+0 \\
4.4+1 \\
1.0+1 \\
2.0+1\end{array}$ & $\begin{array}{l}7.5+0 \\
8.0+1\end{array}$ & $\begin{array}{l}\text { GHT } \\
\text { BRK } \\
\text { JUL } \\
\text { MIL }\end{array}$ & $\begin{array}{l}\text { R Heydgt } \\
\text { E Rasmassont } \\
\text { T Tohir } \\
\text { M Milazzo-Collit }\end{array}$ & $\begin{array}{l}4639 \\
4654 \\
4657 \\
4712\end{array}$ \\
\hline${ }^{206} \mathrm{po}$ & \multicolumn{6}{|l|}{$o(E)$} \\
\hline $\begin{array}{l}205 \mathrm{TI} \\
209_{\mathrm{Bi}}\end{array}$ & p & $\begin{array}{l}\mathrm{NDG} \\
2.0+1\end{array}$ & & $\begin{array}{l}\text { WAU, } \\
\text { MIL, }\end{array}$ & $\begin{array}{l}\text { M Ebisswa. } \\
\text { M Milazzo-Collit }\end{array}$ & $\begin{array}{l}4603 \\
4711\end{array}$ \\
\hline $206 \bullet \mathrm{Pb}_{\mathrm{b}}$ & \multicolumn{6}{|c|}{ Independent (E) } \\
\hline $\begin{array}{l}208 \mathrm{~Pb} \\
206 \cdot \mathrm{Pb}\end{array}$ & $\begin{array}{c}D \\
O(E)\end{array}$ & $2.4+1$ & $5.2+1$ & rok & M Kawahamit & 4658 \\
\hline $\begin{array}{l}200 \mathrm{~Pb} \\
207 \mathrm{~Pb}\end{array}$ & $\begin{array}{c}p \\
\text { spelle }\end{array}$ & \multicolumn{5}{|c|}{ Spallation o(E) } \\
\hline $\cos _{B t}$ & $p$ & $3.8+2$ & & NRL & R Silberbergt & 4762 \\
\hline $207 \mathrm{~Pb}$ & \multicolumn{6}{|c|}{ portlol $O(E) \times$ foctor } \\
\hline $\begin{array}{l}208 \mathrm{pb} \\
208 \mathrm{pb} \\
209 \mathrm{pb}\end{array}$ & $\begin{array}{l}13 \mathrm{C} \\
160 \\
160\end{array}$ & $\begin{array}{l}5.2+1 \\
6.9+1 \\
1.0+2\end{array}$ & $\begin{array}{l}5.4+1 \\
7.3+1 \\
2.2+2\end{array}$ & $\begin{array}{l}\text { MIN } \\
\text { MIN } \\
\text { BRK }\end{array}$ & $\begin{array}{l}\text { M Froney+ } \\
\text { M Froney+ } \\
\text { M Piepert }\end{array}$ & $\begin{array}{l}10219 \\
11515 \\
11516\end{array}$ \\
\hline $207 \mathrm{~Pb}$ & \multicolumn{6}{|c|}{ portlal $\sigma(E)$. } \\
\hline $200 P_{b}$ & 150 & $1.0+2$ & $2.5+2$ & BRK & MPlopert & 11517 \\
\hline $207 \mathrm{~Pb}$ & \multicolumn{6}{|c|}{ o(E) afactor } \\
\hline $\begin{array}{l}208 \mathrm{~Pb} \\
207 \mathrm{~Pb}\end{array}$ & \multicolumn{6}{|l|}{$\theta(E)$} \\
\hline $\begin{array}{l}208 \mathrm{pb} \\
207 \mathrm{~Pb}\end{array}$ & \multicolumn{6}{|c|}{$O(E)$} \\
\hline $208 \mathrm{pb}$ & $p$ & $4.4+1$ & & BRK & E Rasmusgant & 4650 \\
\hline $207 \cdot \mathrm{Pb}$ & \multicolumn{6}{|c|}{ Indepondont o(E) } \\
\hline $\begin{array}{l}208_{\mathrm{Pb}} \\
207 \cdot \mathrm{Pb}\end{array}$ & $\begin{array}{l}\cdot p \\
O(E)\end{array}$ & $2.4+1$ & $5.2+1$ & TOK & M Kawakomit & 4661 \\
\hline $208 \mathrm{pb}$ & p & $2.4+1$ & $5.2+1$ & BRK & E Rasmusgant & 4662 \\
\hline
\end{tabular}




\begin{tabular}{|c|c|c|c|c|c|c|}
\hline Target & $\operatorname{lnc}$ & $\begin{array}{l}\text { Emin } \\
(M e V)\end{array}$ & $\begin{array}{l}E_{\max } \\
(\mathrm{MeV})\end{array}$ & Lab & Author & No. \\
\hline $200 \mathrm{~Pb}$ & \multicolumn{6}{|c|}{ Spallation o(E) } \\
\hline $209_{\mathrm{Bi}}$ & $p$ & $3.8+2$ & & NRL & R silberbergt & 4762 \\
\hline $200 \mathrm{~Pb}$ & \multicolumn{6}{|c|}{ Dartial o(E) } \\
\hline $200 \mathrm{~Pb}$ & p & $2.0+1$ & $1.5+2$ & JUL & T Wambacht & 4664 \\
\hline $200 \mathrm{pb}$ & a & $6.0+1$ & $1.6+5$ & BNL F & R Sandarfit & 8873 \\
\hline $200 \mathrm{pb}$ & $a$ & $6.5+1$ & & NBI & T Brogliat & 8874 \\
\hline $208 \mathrm{~Pb}$ & ${ }^{6} \mathrm{Li}$ & $9.0+1$ & $2.1+2$ & BNL & R Sandarfit & 9370 \\
\hline $208_{\mathrm{Pb}}$ & $12 \mathrm{c}$ & $1.1+2$ & $4.8+2=$ & SNL & R Sandorfit & 10102 \\
\hline $20 \theta_{\mathrm{Pb}}$ & 150 & $1.3+2$ & $2.8+2$ & BNL & R Sandorfit & 11521 \\
\hline $209^{B 1}$ & $14 \mathrm{~N}$ & $5.6+1$ & $7.0+1$ & PAR & T Bimbot+ & 10499 \\
\hline 20981 & 160 & $7.2+1$ & $8.0+1$ & PAR & T Bimbot+ & 11544 \\
\hline $209_{\mathrm{Bi}}$ & $19 \mathrm{~F}$ & $7.0+1$ & $9.0+1$ & PAR & T Bimbot+ & 11819 \\
\hline $209 \mathrm{Bi}$ & $40 \mathrm{Ar}$ & $1.5+2$ & $1.7+2$ & PAR & T Bimbot+ & 12378 \\
\hline $209 \mathrm{Bi}$ & ${ }^{40} \mathrm{CO}$ & $1.6+2$ & $1.7+2$ & PAR & T Bimbolt & 12436 \\
\hline $20 \theta^{\circ i}$ & ${ }^{56} \mathrm{Fe}$ & $2.1+2$ & $2.3+2$ & PAR & T Bimbot+ & 12465 \\
\hline $209^{\circ}$ & ${ }^{63} \mathrm{Cu}$ & $5.5+5$ & $2.5+2$ & PAR & T Bimbolt & 12486 \\
\hline $200 \mathrm{~Pb}$ & \multicolumn{6}{|l|}{$O(E)$} \\
\hline $204 \mathrm{Hg}$ & ${ }^{7} \mathrm{LI}$ & $3.5+1$ & & ROS & E Hagemannt & 9469 \\
\hline $208 \mathrm{pb}$ & $p$ & $7.0+0$ & $2.6+1$ & LRL & E Koopment & 4663 \\
\hline $208 \mathrm{~Pb}$ & p & $7.0+0$ & $2.6+1$ & LRL & E koopmant & 4665 \\
\hline $200 \mathrm{~Pb}$ & g & $6.0+0$ & $1.2+1$ & JUL & R Baur+ & 5876 \\
\hline $200 \mathrm{~Pb}$ & 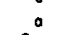 & $2.2+1$ & & SUR & T Jacksont & 8875 \\
\hline $208 \mathrm{pb}$ & ${ }^{6} \mathrm{Li}$ & $2.3+1$ & & HEI & E Scholzt & 9371 \\
\hline $208 \mathrm{pb}$ & 150 & $8.0+1$ & $1.0+2$ & BNL & E Videbaeht & 11520 \\
\hline $209 \mathrm{~Pb}$ & \multicolumn{6}{|c|}{ partiol o(E) a foctor } \\
\hline $208 \mathrm{pb}$ & ${ }^{13} \mathrm{C}$ & $5 \cdot 2+1$ & $5.4+11$ & MIN & M Franeyt & 10220 \\
\hline $208 \mathrm{pb}$ & 150 & $1.5+2$ & $3.0+2$ & BRK 1 & $M$ Olmert & 11524 \\
\hline $208 \mathrm{~Pb}$ & 170 & $6.7+1$ & & MIN I & M Froneyt & 11599 \\
\hline $209_{\mathrm{Pb}}$ & \multicolumn{3}{|c|}{ portlal o(E) } & & & . \\
\hline $208 \mathrm{pb}$ & 180 & $1.5+2$ & $3.0+2 E$ & BRK & Molmert & 11523 \\
\hline $209 \mathrm{~Pb}$ & \multicolumn{6}{|l|}{$\sigma(E)$} \\
\hline $200 \mathrm{~Pb}$ & 160 & $6.8+1$ & $3.1+2$ & ANL & T Mac-Farlanet & 11522 \\
\hline $180_{B i}$ & \multicolumn{6}{|c|}{ Spollotion ofE) } \\
\hline $209_{B i}$ & p & $3.8+2$ & & NRL & R silberbergt & 4762 \\
\hline
\end{tabular}

\begin{tabular}{|c|c|c|c|c|c|}
\hline Target & $\ln c$ & $\begin{array}{l}\text { Emin } \text { Emax } \\
(M e V) \quad(M e V)\end{array}$ & Lab & Author & No. \\
\hline $190 \mathrm{BI}$ & \multicolumn{5}{|c|}{ spollotion $\theta(E)$} \\
\hline $209 \mathrm{BI}$ & $p$ & $3.8+2$ & NRL & R silberbergt & 4762 \\
\hline 19181 & \multicolumn{5}{|c|}{ Spallatlon $O(E)$} \\
\hline $200^{\circ}$ & p & $3.8+2$ & NRL & R Sillberbergt & 4762 \\
\hline $192{ }_{B 1}$ & \multicolumn{5}{|c|}{ Spollotion $\sigma(E)$} \\
\hline $200^{8 i}$ & P & $3.8+2$ & NRL & R Sillberbergt & 4762 \\
\hline${ }^{193} \mathrm{BI}$ & \multicolumn{5}{|c|}{ spallation $\sigma(E)$} \\
\hline $200^{B i}$ & $p$ & $3.8+2$ & NRL & R Silberbergt & 4762 \\
\hline 1948 & \multicolumn{5}{|c|}{ Spollation o(E) } \\
\hline 20981 & p & $3.8+2$ & NRL & R Silberbergt & 4762 \\
\hline${ }^{195} \mathrm{BI}$ & \multicolumn{5}{|c|}{ Spollotion o(E) } \\
\hline $209 \mathrm{BI}$ & $\rho$ & $3.8+2$ & NRL & R Silberbergt & 4762 \\
\hline${ }^{186} \mathrm{BI}$ & \multicolumn{5}{|c|}{ Spollotion $\sigma(E)$} \\
\hline${ }^{209} \mathrm{Bi}$ & $p$ & $3.8+2$ & NRL & R silberberg* & 4762 \\
\hline 19781 & \multicolumn{5}{|c|}{ spollotion o(E) } \\
\hline $200 \mathrm{Bi}$ & $p$ & $3.8+2$ & NRL & R Silberbergt & 4762 \\
\hline $198_{\mathrm{BI}}$ & \multicolumn{5}{|c|}{ Indopendont $O(E)$} \\
\hline $\begin{array}{l}197 \mathrm{AU} \\
197_{\mathrm{AU}}\end{array}$ & $\begin{array}{l}12 \mathrm{C} \\
160\end{array}$ & $\begin{array}{l}1.3+2 \\
1.4+2 \quad 1.7+2\end{array}$ & $\begin{array}{l}\text { KFK } \\
\text { KFK }\end{array}$ & $\begin{array}{l}\text { C Muenzelt } \\
\text { C Muenzel+ }\end{array}$ & $\begin{array}{l}10097 \\
11483\end{array}$ \\
\hline $19 \theta_{\mathrm{BI}}$ & \multicolumn{5}{|c|}{ spollotion $O(E)$} \\
\hline $\begin{array}{l}209^{81} \\
198^{81}\end{array}$ & \multicolumn{4}{|c|}{ cumulative ofE } & 4762 \\
\hline $\begin{array}{l}197 \mathrm{AU} \\
197 \mathrm{AU}\end{array}$ & $\begin{array}{l}12 \mathrm{C} \\
160\end{array}$ & $\begin{array}{l}1.3+2 \\
1.4+2 \quad 1.7+2\end{array}$ & $\begin{array}{l}\text { KFK } \\
\text { KFK }\end{array}$ & $\begin{array}{l}\text { C Muenzelt } \\
\text { C Muenzelt }\end{array}$ & $\begin{array}{l}10099 \\
11485\end{array}$ \\
\hline $180_{B 1}$ & \multicolumn{5}{|c|}{ Independent $O(E)$} \\
\hline $\begin{array}{l}197 \mathrm{Au} \\
.197_{\mathrm{Au}}\end{array}$ & $\begin{array}{l}12 \mathrm{C} \\
160\end{array}$ & $\begin{array}{l}1.3+2 \\
1.4+2 \quad 1.7+2\end{array}$ & $\begin{array}{l}\text { KFK } \\
\text { KFK }\end{array}$ & $\begin{array}{l}\text { C Muenzelt } \\
\text { C Muenzelt }\end{array}$ & $\begin{array}{l}10097 \\
11483\end{array}$ \\
\hline $1890 i$ & \multicolumn{5}{|c|}{ spollotion o(E) } \\
\hline${ }^{209} \mathrm{Bi}$ & $p$ & $3.8+2$ & NRL & R Silberbergt & 4762 \\
\hline
\end{tabular}




\begin{tabular}{|c|c|c|c|c|}
\hline Torget & $\ln c$ & $\begin{array}{ll}\text { Emin } & \text { Emax } \\
(\operatorname{MeV}) & (\operatorname{MeV}) \\
\end{array}$ & Author & No. \\
\hline $19^{19}$ & \multicolumn{4}{|c|}{ Cumulative $O(E)$} \\
\hline $\begin{array}{l}197 \mathrm{Au} \\
197 \mathrm{Au}\end{array}$ & $\begin{array}{l}12 \mathrm{C} \\
160\end{array}$ & $\begin{array}{ll}1.3+2 & 1.41 \\
1.4+2 & 1.7+2\end{array}$ & $\begin{array}{l}\text { KFK C Muenzel+ } \\
\text { KFK C Muenzel+ }\end{array}$ & $\begin{array}{l}10099 \\
11485\end{array}$ \\
\hline $200_{\mathrm{BI}}$ & \multicolumn{4}{|c|}{ independent $\sigma(E)$} \\
\hline $\begin{array}{l}197 \mathrm{Au} \\
197 \mathrm{Au}\end{array}$ & $\begin{array}{l}12 \mathrm{C} \\
180\end{array}$ & 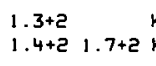 & $\begin{array}{l}\text { KFK C Muenzelt } \\
\text { KFK C Muenzelt }\end{array}$ & $\begin{array}{l}10097 \\
11483\end{array}$ \\
\hline $200_{\mathrm{BI}}$ & \multicolumn{4}{|c|}{ spallatlon $\theta(E)$} \\
\hline $\begin{array}{l}209_{\mathrm{B1}} \\
200_{\mathrm{B1}}\end{array}$ & \multicolumn{4}{|c|}{ cunulative $O(E)$} \\
\hline $\begin{array}{l}197_{A u} \\
197_{A u}\end{array}$ & $\begin{array}{l}12 \mathrm{c} \\
160\end{array}$ & $\begin{array}{ll}\begin{array}{l}1.3+2 \\
1.4+2\end{array} \quad 1.7+2 & 1\end{array}$ & $\begin{array}{l}\text { KFK C Muenzel+ } \\
\text { KFK C Muenzel+ }\end{array}$ & $\begin{array}{l}10099 \\
11485\end{array}$ \\
\hline 20181 & \multicolumn{4}{|c|}{ independent $\sigma(E)$} \\
\hline $\begin{array}{l}197 \mathrm{Au} \\
19{ }^{\mathrm{Au}}\end{array}$ & $\begin{array}{l}12 c \\
150\end{array}$ & $\begin{array}{ll}1.3+2 & \\
1.4+2 & 1.7+2\end{array}$ & $\begin{array}{l}\text { KFK C Muenze1t } \\
\text { KFK C Muenze1t }\end{array}$ & $\begin{array}{l}10097 \\
11483\end{array}$ \\
\hline 20181 & \multicolumn{4}{|c|}{ Spallation $O(\varepsilon)$} \\
\hline${ }^{209} \mathrm{Bi}$ & $\rho$ & $3.8+2$ & NRL R Silberberg+ & 4762 \\
\hline 20181 & \multicolumn{4}{|c|}{ cumulative $O(E)$} \\
\hline $\begin{array}{l}197 \mathrm{Au} \\
197 \mathrm{Au}\end{array}$ & $\begin{array}{l}-12 c \\
150\end{array}$ & $\begin{array}{ll}1.3+2 & \\
1.4+2 & 1.7+2\end{array}$ & $\begin{array}{l}\text { KFK C Muenzelt } \\
\text { KFK C Muenzelt }\end{array}$ & $\begin{array}{l}10099 \\
11485\end{array}$ \\
\hline $202_{B 1}$ & \multicolumn{4}{|c|}{ Independent $O(E)$} \\
\hline $\begin{array}{l}197 \mathrm{Au} \\
197 \mathrm{Au}\end{array}$ & $\begin{array}{l}12 c \\
160\end{array}$ & 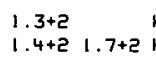 & $\begin{array}{l}\text { KFK C Muenzelt } \\
\text { KFK C Muenzelt }\end{array}$ & $\begin{array}{l}10097 \\
11483\end{array}$ \\
\hline $202_{81}$ & \multicolumn{4}{|c|}{ Spaliation $\sigma(E)$} \\
\hline $\begin{array}{r}\mathrm{Pb} \\
{ }^{209} \mathrm{gBl}_{\mathrm{Bl}}\end{array}$ & $\begin{array}{l}p \\
p\end{array}$ & $\begin{array}{l}3.0+06.5+0 \\
3.8+2\end{array}$ & $\begin{array}{l}\text { HAR E Symet } \\
\text { NRL R Sllberbergt }\end{array}$ & $\begin{array}{l}4624 \\
4762\end{array}$ \\
\hline $203_{9 i}$ & \multicolumn{4}{|c|}{ independent $\sigma(E)$} \\
\hline $\begin{array}{l}197 \mathrm{Au} \\
197 \mathrm{Au}\end{array}$ & $\begin{array}{l}12 \mathrm{C} \\
160\end{array}$ & 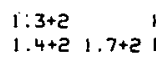 & $\begin{array}{l}\text { KFK C Muenzelt } \\
\text { KFK C Muenzel+ }\end{array}$ & $\begin{array}{l}10097 \\
11483\end{array}$ \\
\hline $203_{81}$ & \multicolumn{4}{|c|}{ Spallation o(E) } \\
\hline $\begin{array}{l}\mathrm{Pb} \\
{ }_{209}{ }^{81}\end{array}$ & $p_{p}^{p}$ & $\begin{array}{l}3.0+06.5+0 \\
3.8+2\end{array}$ & $\begin{array}{l}\text { HAR E Symet } \\
\text { NRL R Silberbergt }\end{array}$ & $\begin{array}{l}4625 \\
4762\end{array}$ \\
\hline
\end{tabular}

\begin{tabular}{|c|c|c|c|c|c|}
\hline Target & $\operatorname{lnc}$ & $\begin{array}{l}\text { Emln } \\
(M e V)\end{array}$ & $\begin{array}{l}\text { Emax } \\
(M e v)\end{array}$ & Author & No. \\
\hline $203_{B 1}$ & \multicolumn{5}{|c|}{ Celative Iy } \\
\hline $203 T_{11}$ & $a$ & $4.6+1$ & $5.5+1$ & \multirow[t]{2}{*}{ BON E Hubel+ } & \multirow[t]{2}{*}{8831} \\
\hline 20381 & $O(E)$ & & & & \\
\hline $206 \mathrm{pb}$ & ${ }^{3} \mathrm{He}$ & $8.5+1$ & $1.7+2$ & PAR E Andret & 6568 \\
\hline 20481 & \multicolumn{4}{|c|}{ spallation o(E) } & \\
\hline $\begin{array}{r}\mathrm{Pb} \\
209_{\mathrm{Bl}}\end{array}$ & p & $\begin{array}{l}3.0+0 \\
3.8+2\end{array}$ & $6.5+0$ & $\begin{array}{l}\text { HAR E Symet } \\
\text { NRL R Silberbe:gt }\end{array}$ & $\begin{array}{l}4626 \\
4762\end{array}$ \\
\hline 20481 & \multicolumn{5}{|c|}{ pertlat product ytolo } \\
\hline $\begin{array}{l}203 \mathrm{TI} \\
205 \mathrm{TI}\end{array}$ & $:$ & $\begin{array}{l}\text { NDG } \\
\text { NOG }\end{array}$ & & $\begin{array}{l}\text { IPS E Lonrotht } \\
\text { IPS E Lonrotht }\end{array}$ & $\begin{array}{l}8832 \\
8834\end{array}$ \\
\hline 20481 & \multicolumn{5}{|c|}{ pertlal celative o(E) } \\
\hline${ }^{209_{\mathrm{B} 1}}$ & ${ }^{10} \mathrm{~B}$ & $7.8+0$ & $8.8+1$ & YAL E Rolcht & 9551 \\
\hline 20481 & \multicolumn{5}{|c|}{ thich target yleldnobundance } \\
\hline $206 \mathrm{pb}$ & p & $3.2+1$ & & AML R Choudri. & 4640 \\
\hline $2048 i$ & \multicolumn{5}{|l|}{$\sigma(E)$} \\
\hline $209_{\mathbf{B i}}$ & $\mathrm{p}$ & $T R$ & $8.0+1$ & NRL T Peterson. & 4714 \\
\hline${ }^{209_{B 1}}$ & \multicolumn{5}{|c|}{ spallation o(E) } \\
\hline${ }^{209} 81$ & p & $3.8+2$ & & NRL R silberbergt & 4762 \\
\hline${ }^{205_{B 1}}$ & \multicolumn{5}{|c|}{ rolotive 1 , } \\
\hline $\begin{array}{l}205 \pi 1 \\
20571\end{array}$ & $:$ & $\begin{array}{r}3.5+1 \\
4.6+1\end{array}$ & $\begin{array}{l}5.1+1 \\
5.5+1\end{array}$ & $\begin{array}{l}\text { IPS E Lonnrotht } \\
\text { BON E Hubelt }\end{array}$ & $\begin{array}{l}8835 \\
8836\end{array}$ \\
\hline $203_{8 i}$ & \multicolumn{5}{|l|}{$O(E)$} \\
\hline $\begin{array}{l}206 \mathrm{~Pb} \\
209_{\mathrm{Bl}}\end{array}$ & ${ }^{3} \mathrm{He}$ & ${ }_{\mathrm{TR}}^{8.5+1}$ & $\begin{array}{l}1.7+2 \\
8.0+1\end{array}$ & $\begin{array}{l}\text { PAR E Andret } \\
\text { NRL T Petergon. }\end{array}$ & $\begin{array}{l}6568 \\
4715\end{array}$ \\
\hline 20681 & \multicolumn{5}{|c|}{ Spallation o(E) } \\
\hline $\begin{array}{r}\mathrm{Pb} \\
209_{\mathrm{Bl}}\end{array}$ & $\begin{array}{l}p \\
p\end{array}$ & $\begin{array}{l}3.0+0 \\
3.8+2\end{array}$ & $6.5+0$ & $\begin{array}{l}\text { HAR E Symet } \\
\text { NRL R Silberbergt }\end{array}$ & $\begin{array}{l}4827 \\
4752\end{array}$ \\
\hline $206_{8 i}$ & \multicolumn{5}{|c|}{ partlal o(E) } \\
\hline $205^{41}$ & 。 & $3.5+1$ & $4 \cdot 3+1$ & IPS E Lonnrolht & 8838 \\
\hline
\end{tabular}




\begin{tabular}{llll}
\hline Target Inc Emin Emax Lob $W$ & Author \\
$(M e V)(M e V)$ & No. \\
\hline
\end{tabular}

$206 \mathrm{Bi}$ relative $\mathrm{I}_{\mathrm{V}}$

$205 \mathrm{TI} a \quad 3.5+15.1+1$ IPSE Lonnrotht 8837

${ }^{208}{ }^{8}$ thich target yieldrabundance

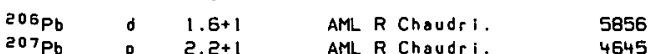

$20 \theta^{B} 1$ O(E)

$\begin{array}{rrrrrr}\mathrm{Pb} & \mathrm{P} & 8.0+0 & 1.1+1 & \mathrm{CCP} R \text { 2atolohint } & 4628 \\ 206 \mathrm{~Pb} & { }^{3} \mathrm{He} & 8.5+1 & 1.7+2 \text { PAR E Andret } & 6568\end{array}$

$209 \mathrm{~Pb} \quad \mathrm{He} \quad 8.5+11.7+2$ PCR E Andret

$209^{8} \mathrm{P} \quad 3.1+15.2+1$ TOK E Miranot 4716

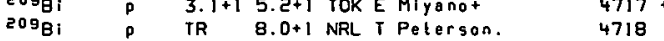

207 Bi spallation olEl

${ }^{209} \mathrm{Bi}$ P $3.8+2$ NRL R silberbergt 4762

$207 \mathrm{Bi}$ relative 1 ,

205 II a $3.0+14.3+1$ UST E Lonnroth. 8839

$205 \mathrm{Tl}$ a $3: 5+15.1+1$ IPS E Lonnrotht 8840

$200 \mathrm{~Pb} d 2.5+1$ UST E Lonnroth. 5877

$207_{B 1} \quad O(E)$

$2{ }^{09} \mathrm{Bi} P$ TR $\quad$ Q.5+1 NRL T Peterson. 4719

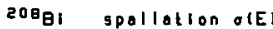

${ }^{209} \mathrm{gi} \quad p \quad 3.8+2 \quad$ NRL R Silberberg+ 4762

$20 \theta^{B} 1$ O(E)

$209_{1} P$ TR 8.0+1 NRL T Peterson. 4720

$200^{B 1}$ gpallation O(E)

$20{ }^{\circ} \mathrm{Bi} P \quad 3.8+2$ NRL R Silberberg+ 4762

$20 \theta^{\circ}$ i porllal o(E),

${ }^{209} \mathrm{gl}^{3} \mathrm{He} \quad 7.0+19.0+1$ OSA E Matsuohat 6572

$20 \theta_{B 1}$ portlal relative o(E)

$200_{\mathrm{Pb}} P \quad 1.0+14.0+1 \mathrm{BOL} T$ Longot 4668

68

\begin{tabular}{|c|c|c|c|c|c|c|}
\hline Target & $\ln c$ & $\begin{array}{l}E \ln n \\
(M e V)\end{array}$ & $\begin{array}{l}\text { Emax } \\
(\operatorname{MeV})\end{array}$ & Lab & Author & No. \\
\hline $208_{B 1}$ & \multicolumn{5}{|c|}{ partial o(E) xfactor } & , \\
\hline $\begin{array}{l}200 \mathrm{~Pb} \\
200 \mathrm{~Pb} \\
200 \mathrm{~Pb}\end{array}$ & $\begin{array}{l}{ }^{3} \mathrm{He} \\
160 \\
10_{0}\end{array}$ & $\begin{array}{l}5.1+1 \\
7.0+1 \\
1.0+2\end{array}$ & $\begin{array}{l}2.5+5 \\
5.5+5\end{array}$ & $\begin{array}{l}\text { BRK } \\
\text { BRK } \\
\text { BRK }\end{array}$ & $\begin{array}{l}\text { M Plepert } \\
\text { M Plepert } \\
\text { M Piepert }\end{array}$ & $\begin{array}{r}6570 \\
11533 \\
11535\end{array}$ \\
\hline $209_{\mathrm{Bi}}$ & \multicolumn{6}{|c|}{ partial o(E) } \\
\hline $\begin{array}{l}200 \mathrm{~Pb} \\
200 \mathrm{~Pb} \\
200 \mathrm{~Pb} \\
200 \mathrm{~Pb} \\
200 \mathrm{~Pb} \\
200 \mathrm{~Pb} \\
200 \mathrm{~Pb} \\
200 \mathrm{~Pb}\end{array}$ & $\begin{array}{l}p \\
15 p \\
150 \\
150 \\
160 \\
160 \\
160 \\
150\end{array}$ & $\begin{array}{l}\text { NDG } \\
8.0+0 \\
6.7+0 \\
6.9+1 \\
7.5+1 \\
7.5+1 \\
1.0+2 \\
1.0+2\end{array}$ & $\begin{array}{l}3 \cdot 0+1 \\
6.3+2 \\
3.1+2 \\
3.2+2 \\
3.2+2 \\
2.2+2\end{array}$ & $\begin{array}{l}\text { WAU } \\
\text { NJS } \\
\text { ANL } \\
\text { BRK } \\
\text { ANL } \\
\text { MSU } \\
\text { MAN } \\
\text { BRK }\end{array}$ & $\begin{array}{l}\text { M Ebisada. } \\
\text { T Lihart } \\
\text { R Maciarlane. } \\
\text { M Olmert } \\
\text { R Henning. } \\
\text { R Gelbke. } \\
\text { R Phillips. } \\
\text { M Piepert }\end{array}$ & $\begin{array}{r}4666 \\
4667 \\
11527 \\
11528 \\
11529 \\
11530 \\
11531 \\
11534\end{array}$ \\
\hline $209^{8}$ & \multicolumn{6}{|c|}{$O(E) \times$ foctor } \\
\hline $\begin{array}{l}20 \theta_{\mathrm{Pb}} \\
209_{\mathrm{Bl}}\end{array}$ & $\begin{array}{r}18.0 \\
0\end{array}$ & $\begin{array}{l}7.0+1 \\
7.0+0\end{array}$ & $\begin{array}{l}1.5+1 \\
2.5+2\end{array}$ & $\begin{array}{l}\text { BRK } \\
\text { COL }\end{array}$ & $\begin{array}{l}\text { M Piepert } \\
\text { R Miller. }\end{array}$ & $\begin{array}{r}11532 \\
4721\end{array}$ \\
\hline $200^{0} i$ & \multicolumn{6}{|l|}{$g(E)$} \\
\hline $\begin{array}{l}208 \mathrm{~Pb} \\
200 \mathrm{~Pb} \\
200 \mathrm{~Pb} \\
209 \mathrm{gi}\end{array}$ & $\begin{array}{r}1 p_{0}^{p} \\
160 \\
p\end{array}$ & $\begin{array}{l}\text { NDG } \\
6.8+1 \\
7.0+1 \\
T R\end{array}$ & $\begin{array}{l}3 \cdot 1+2 \\
2.2+2 \\
8.0+1\end{array}$ & $\begin{array}{l}\text { WAU } \\
\text { ANL } \\
\text { BRK } \\
\text { NRL }\end{array}$ & $\begin{array}{l}\text { M Ebisawa. } \\
\text { T Mac-Farianet } \\
\text { M Piepert } \\
\text { T Peterson. }\end{array}$ & $\begin{array}{r}4669 \\
11525 \\
11526 \\
4722\end{array}$ \\
\hline $210_{B i}$ & \multicolumn{6}{|l|}{$O(E)$} \\
\hline $\begin{array}{l}209 \mathrm{Bi} \\
\log _{\mathrm{Bi}} \\
209 \mathrm{gi} \\
20 \operatorname{li}_{\mathrm{Bi}}\end{array}$ & 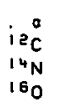 & $\begin{array}{l}8.0+1 . \\
6.8+1 \\
7 \cdot 7+1 \\
8.3+1\end{array}$ & $\begin{array}{l}1.6+2 \\
8.7+1 \\
9.7+1 \\
9.9+1\end{array}$ & $\begin{array}{l}\text { MRY } \\
\text { PAR } \\
\text { PAR } \\
\text { PAR }\end{array}$ & $\begin{array}{l}\text { E Wut } \\
\text { E Gardest } \\
\text { E Gardest } \\
\text { E Gardest }\end{array}$ & $\begin{array}{r}8911 \\
10119 \\
10500 \\
11545\end{array}$ \\
\hline $2\left|I_{B}\right|$ & \multicolumn{6}{|l|}{$O(E)$} \\
\hline $209_{B 1}$ & 180 & $8: 3+1$ & & MUU & E Mc-Gratht & 11718 \\
\hline 212.1日। & \multicolumn{6}{|c|}{ product yleld } \\
\hline $\begin{array}{l}23 \theta^{2} \\
24 \theta^{\circ} \mathrm{Cm}\end{array}$ & $\begin{array}{l}{ }^{40} \mathrm{Ar} \\
{ }^{48} \mathrm{CB}\end{array}$ & $\begin{array}{l}\text { NDG } \\
\text { NDG }\end{array}$ & & $\begin{array}{l}\text { BRK } \\
\text { BRK }\end{array}$ & $\begin{array}{l}\text { E Baisdent } \\
\text { E goisdent }\end{array}$ & $\begin{array}{l}12408 \\
12453\end{array}$ \\
\hline $2 \ln 2 \ln _{1}$ & \multicolumn{3}{|c|}{ product ylold } & & & \\
\hline $\begin{array}{l}208 \mathrm{~Pb} \\
230 \mathrm{U}\end{array}$ & $\begin{array}{l}{ }^{40} \mathrm{Ar} \\
{ }^{40} \mathrm{Ar}\end{array}$ & $\begin{array}{l}\text { NDG } \\
\text { NDG }\end{array}$ & & $\begin{array}{l}\text { BRK } \\
\text { BRK }\end{array}$ & $\begin{array}{l}\text { E Baisdent } \\
\text { E Baisdent. }\end{array}$ & $\begin{array}{l}12374 \\
12409\end{array}$ \\
\hline
\end{tabular}




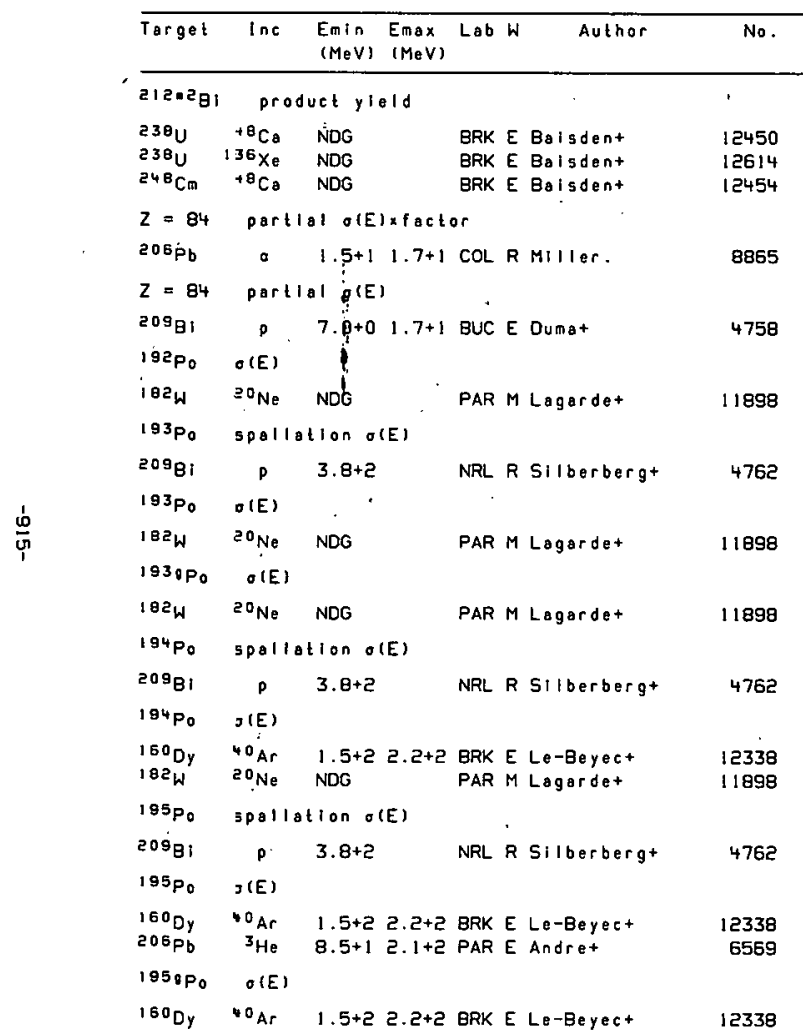

\begin{tabular}{|c|c|c|c|c|c|c|}
\hline Targat & $\ln c$ & $\begin{array}{l}\text { Emin } \\
(\mathrm{MeV})\end{array}$ & $\begin{array}{l}\text { Emax } \\
(\operatorname{MeV})\end{array}$ & Lab & Author & No. \\
\hline $95=P_{0}$ & $O(E)$ & & & & & \\
\hline $\begin{array}{l}160 \mathrm{Dy} \\
154 \mathrm{Dy} \\
206 \mathrm{~Pb}\end{array}$ & $\begin{array}{l}{ }^{40} \mathrm{Ar} \\
{ }^{40} \mathrm{Ar} \\
{ }^{3} \mathrm{He}\end{array}$ & $\begin{array}{l}1.5+2 \\
1.6+2 \\
8.5+1\end{array}$ & $\begin{array}{l}2.2+2 \\
2.6+2 \\
2.1+2\end{array}$ & $\begin{array}{l}\text { BRK E } \\
\text { BRK E } \\
\text { PAR E }\end{array}$ & $\begin{array}{l}\text { E Le-8eyect } \\
\text { E Le-Beyect } \\
\text { E Andret }\end{array}$ & $\begin{array}{r}12330 \\
12345 \\
6569\end{array}$ \\
\hline $196 \mathrm{Po}$ & spallo & trono & $(E)$ & . & & \\
\hline $\begin{array}{l}209 B_{1} \\
186 \ddot{p_{0}}\end{array}$ & $\begin{array}{c}P \\
O(E)\end{array}$ & $3.8+2$ & & NRL R & R Silderberg+ & 4762 \\
\hline $\begin{array}{l}160 \mathrm{Oy} \\
164 \mathrm{Oy} \\
206 \mathrm{~Pb}\end{array}$ & $\begin{array}{l}{ }^{40} \mathrm{Ar} \\
{ }^{40} \mathrm{Ar} \\
{ }^{3} \mathrm{He}\end{array}$ & $\begin{array}{l}1.5+2 \\
1.6+2 \\
8.5+1\end{array}$ & $\begin{array}{l}5.12 \\
2.5+5 \\
2.1+2\end{array}$ & $\begin{array}{l}\text { ERK E } \\
\text { BRK E } \\
\text { PAR E }\end{array}$ & $\begin{array}{l}\text { E Le-Beyect } \\
\text { E Le-Beyect } \\
\text { E Andret }\end{array}$ & $\begin{array}{r}12338 \\
12345 \\
6569\end{array}$ \\
\hline $197 \mathrm{Po}$ & spalia & tion or & (E) & & & \\
\hline $\begin{array}{l}209 \mathrm{Bi} \\
197 \mathrm{Po}^{2}\end{array}$ & $\begin{array}{c}p \\
o(E)\end{array}$ & $3.8+2$ & & NRL $R$ & R silberberg+ & 4762 \\
\hline $\begin{array}{l}160 \mathrm{Dy} \\
154 \mathrm{Dy} \\
206 \mathrm{~Pb}\end{array}$ & $\begin{array}{l}{ }^{40} \mathrm{Ar} \\
{ }^{40 \mathrm{Ar}} \\
{ }^{3} \mathrm{He}\end{array}$ & $\begin{array}{l}1.5+2 \\
1.6+2 \\
8.5+1\end{array}$ & $\begin{array}{l}2.2+2 \\
2.6+2 \\
2.1+2\end{array}$ & $\begin{array}{l}\text { BRK E } \\
\text { BRK E } \\
\text { PAR E }\end{array}$ & $\begin{array}{l}\text { E Le-Beyect } \\
\text { E Le-Beyect } \\
\text { E Andret }\end{array}$ & $\begin{array}{r}12338 \\
12345 \\
6569\end{array}$ \\
\hline $197 \mathrm{PPO}$ & $\sigma(E)$ & & & & & \\
\hline $\begin{array}{l}1600 y \\
197 . P_{0}\end{array}$ & $\begin{array}{l}{ }^{40} \mathrm{Ar} \\
O(E)\end{array}$ & $1.5+2$ & $2.2+2$ & BRK E & E Le-Beyect & 12338 \\
\hline $\begin{array}{l}160 \mathrm{Dy} \\
206 \mathrm{~Pb}\end{array}$ & $\begin{array}{l}{ }^{40} \mathrm{Ar} \\
{ }^{3} \mathrm{He}\end{array}$ & $\begin{array}{l}1.5+2 \\
8.5+1\end{array}$ & $\begin{array}{l}2.5+5 \\
z \cdot 1+5\end{array}$ & $\begin{array}{l}\text { BRK E } \\
\text { PAR E }\end{array}$ & $\begin{array}{l}\text { E Le-Beyect } \\
\text { E Andre+ }\end{array}$ & $\begin{array}{r}12338 \\
6569\end{array}$ \\
\hline $19 \theta \mathrm{Po}$ & gpallat & $\operatorname{tion} a t$ & (E) & & & \\
\hline $\begin{array}{l}209 \mathrm{Bi} \\
198 \mathrm{Po}\end{array}$ & $\begin{array}{c}p \\
O(E)\end{array}$ & $3.8+2$ & & NRL R & R silberbergt & 4762 \\
\hline $\begin{array}{l}164 \mathrm{Oy} \\
206 \mathrm{~Pb} \\
209^{\circ} \mathrm{Bi} \\
209^{\circ} \mathrm{Bi}\end{array}$ & $\begin{array}{c}40_{\mathrm{Ar}} \\
{ }^{3} \mathrm{He} \\
0 \\
0\end{array}$ & $\begin{array}{l}1.6+2 \\
8 \cdot 5+1 \\
1.2+2 \\
1.0+1\end{array}$ & $\begin{array}{l}2.6+2 \\
2.1+2 \\
1.3+2 \\
8.0+2\end{array}$ & $\begin{array}{l}\text { BRK E } \\
\text { PAR E } \\
\text { NRL T } \\
\text { NRL R }\end{array}$ & $\begin{array}{l}\text { E Le-Beyect } \\
\text { E Andret } \\
\text { T Silberbergt } \\
\text { R Silberbergt }\end{array}$ & $\begin{array}{r}12345 \\
6569 \\
4723 \\
4753\end{array}$ \\
\hline${ }^{199} \mathrm{Po}$ & spallat & tlon of & (E) & & & \\
\hline
\end{tabular}

${ }^{150} \mathrm{OY}$ Ar $1.5+2$ 2. $2+2$ GRK E Le-Beyect

${ }^{200^{\circ}} \mathrm{Bi} \quad 3.8+2$ NRL R Silberbergt 4762 


\begin{tabular}{|c|c|c|c|c|c|c|}
\hline Torget & $\operatorname{lnc}$ & $\begin{array}{l}\text { Emin } \\
(M e V)\end{array}$ & $\begin{array}{l}E_{\max } \\
(M \otimes V)\end{array}$ & Lob $W$ & Author & No. \\
\hline $199 p_{0}$ & rolot & ve $O(E)$ & & & & \\
\hline $\begin{array}{l}197 \mathrm{Au} \\
199 \mathrm{PO}\end{array}$ & $\begin{array}{l}{ }^{10} B \\
O(E)\end{array}$ & $7.8+1 \quad 1$ & $1.0+2 h$ & WWA E & E Kormant & 9547 \\
\hline $\begin{array}{l}206 \mathrm{~Pb} \\
20{ }^{20} \mathrm{BI}\end{array}$ & $\begin{array}{l}3 \mathrm{He} \\
\mathrm{P}\end{array}$ & $\begin{array}{l}E .5+1 \\
1.0+18\end{array}$ & $\begin{array}{l}1.7+2 \\
8.0+2\end{array}$ & $\begin{array}{l}\text { PAR E } \\
\text { NRL R }\end{array}$ & $\begin{array}{l}\text { Endret } \\
\text { R Silberberg+ }\end{array}$ & $\begin{array}{l}6568 \\
4763\end{array}$ \\
\hline $199 . \mathrm{Po}$ & $O(E)$ & & & & & \\
\hline $\begin{array}{l}164 \mathrm{Py} \\
205 \mathrm{~Pb}\end{array}$ & $\begin{array}{r}{ }^{40} \mathrm{Ar} \\
{ }^{3} \mathrm{He}\end{array}$ & $\begin{array}{l}1.6+2 \\
\varepsilon .5+1\end{array}$ & $\begin{array}{l}2.6+2 \\
1.7+2\end{array}$ & $\begin{array}{l}\text { SRK E } \\
\text { PAR E }\end{array}$ & $\begin{array}{l}\text { E Le-Beyect } \\
\text { E Andre+ }\end{array}$ & $\begin{array}{r}12345 \\
6568\end{array}$ \\
\hline $200 \mathrm{Po}$ & $3 p 011$ & $\operatorname{tin} \theta t$ & (E) & & & \\
\hline $\begin{array}{l}209 \mathrm{Bi} \\
200 \mathrm{Po}\end{array}$ & $p_{\text {relat }}^{p}$ & $\begin{array}{l}3.8+2 \\
\text { ve } O(E)\end{array}$ & & NRL $R$ & R Silberberg* & 4762 \\
\hline $197 \mathrm{Au}$ & $10 \mathrm{~B}$ & $7.8+1$ & $1.0+2$ & WWA E & E Kormant & 9548 \\
\hline $200 \mathrm{Po}$ & $O(E)$ & & & & & \\
\hline $\begin{array}{l}164 \mathrm{Dy} \\
206 \mathrm{~Pb} \\
209 \mathrm{Bi} \\
209_{\mathrm{Bi}}\end{array}$ & $\begin{array}{c}{ }^{40} \mathrm{Ar} \\
{ }^{3} \mathrm{He} \\
\mathrm{P} \\
\mathrm{P}\end{array}$ & $\begin{array}{l}1.6+2 \\
8 \cdot 5+1 \\
1.0+2 \\
1.0+1\end{array}$ & $\begin{array}{l}2.6+2 \\
1.7+5 \\
1.2+5 \\
8.0+5\end{array}$ & $\begin{array}{l}\text { ERK E } \\
\text { PAR E } \\
\text { NRL T } \\
\text { NRL R }\end{array}$ & $\begin{array}{l}\text { E Le-Beyect } \\
\text { E Andret } \\
\text { T Silberberg* } \\
\text { R Silberberg* }\end{array}$ & $\begin{array}{r}12345 \\
6568 \\
4724 \\
4763\end{array}$ \\
\hline $201 P_{0}$ & spall & tion olt & (E) & & & \\
\hline $209 \mathrm{Bi}$ & $p$ & $3.8+2$ & & NRL R & R Silberberg* & 4762 \\
\hline $201 \mathrm{Po}$ & relot & ve $O(E)$ & & & & \\
\hline $\begin{array}{l}197 \mathrm{Au} \\
197_{\mathrm{Au}}\end{array}$ & $\begin{array}{l}10 \mathrm{~B} \\
11 \mathrm{~B}\end{array}$ & $\begin{array}{l}? .8+1 \\
8.5+1\end{array}$ & $\begin{array}{l}1.0+21 \\
9.1+1\end{array}$ & $\begin{array}{l}\text { WWA E } \\
\text { WWA E }\end{array}$ & $\begin{array}{l}\text { E Kormant } \\
\text { E Kormant }\end{array}$ & $\begin{array}{l}9549 \\
9589\end{array}$ \\
\hline $201 \mathrm{Po}$ & $O(E)$ & & & & & \\
\hline $\begin{array}{l}164 \mathrm{Oy} \\
206 \mathrm{~Pb} \\
209 \mathrm{Bi}\end{array}$ & $\begin{array}{c}{ }^{40} \mathrm{Ar} \\
{ }^{3} \mathrm{He} \\
\mathrm{P}\end{array}$ & $\begin{array}{l}: .6+2 \\
8.5+1 \\
1.0+1\end{array}$ & $\begin{array}{l}2.8+2 \\
1.7+2 \\
8.0+2\end{array}$ & $\begin{array}{l}\text { PAR M } \\
\text { PAR E } \\
\text { NRL R }\end{array}$ & $\begin{array}{l}\text { M Lagardet } \\
\text { E Andret } \\
R \text { SIlberberg* }\end{array}$ & $\begin{array}{r}12346 \\
6568 \\
4763\end{array}$ \\
\hline $201 \mathrm{OPO}$ & prod & et $y|\theta|$ & & & & \\
\hline Ir & $14 \mathrm{~N}$ & NDG & & ORL E & E Westernt & 10481 \\
\hline $201-p_{0}$ & prod & ct yiel & & & & \\
\hline Ir. & ${ }^{14} \mathrm{~N}$ & NDG & & ORL E & E Westernt & 10482 \\
\hline
\end{tabular}

\begin{tabular}{|c|c|c|c|c|c|c|}
\hline Target & $\ln c$ & $\begin{array}{l}\text { Emin } \\
(\mathrm{MeV})\end{array}$ & $\begin{array}{l}E_{\max } \\
(\operatorname{MeV})\end{array}$ & Lab & Author & No. \\
\hline $201 \cdot p_{0}$ & $O(E)$ & & & & & \\
\hline $206 \mathrm{~Pb}$ & ${ }^{3} \mathrm{He}$ & $8.5+1$ & $1.7+2$ & PAR & E Andret & 6568 \\
\hline $202 P_{0}$ & indep & ndonto & o(E) & & & \\
\hline $\begin{array}{l}197 \mathrm{Au} \\
197 \mathrm{Au}\end{array}$ & $\begin{array}{l}12 \mathrm{C} \\
160\end{array}$ & $\begin{array}{l}1.3+2 \\
1.4+2\end{array}$ & $1.7+2$ & $\begin{array}{l}\text { KFK } \\
\text { KFK }\end{array}$ & $\begin{array}{l}\text { C Muenze1+ } \\
\text { C Muenzel+ }\end{array}$ & $\begin{array}{l}10097 \\
11483\end{array}$ \\
\hline $202 \mathrm{Po}$ & spalla & lion ol & (E) & & & \\
\hline $\begin{array}{l}209 \mathrm{Bi} \\
202 \mathrm{Po}\end{array}$ & $\begin{array}{c}\rho \\
\text { eumulo }\end{array}$ & $\begin{array}{l}3.8+2 \\
\text { tive ot }\end{array}$ & & NRL & R Silberberg+ & 4762 \\
\hline $\begin{array}{l}197 \mathrm{Au} \\
197_{\mathrm{Au}}\end{array}$ & $\begin{array}{l}12 \mathrm{C} \\
150\end{array}$ & $\begin{array}{l}1.3+2 \\
1.4+2\end{array}$ & $1.7+2$ & $\begin{array}{l}\text { KFK } \\
\text { KFK }\end{array}$ & $\begin{array}{l}\text { C Muenzelt } \\
\text { C Muenzel+ }\end{array}$ & $\begin{array}{l}10099 \\
11485\end{array}$ \\
\hline $202 \mathrm{po}$ & portic & $10(E)$ & & & & \\
\hline $\begin{array}{l}196 \mathrm{Pt} \\
204 \mathrm{~Pb}\end{array}$ & $\begin{array}{r}12 \mathrm{C} \\
0\end{array}$ & $\begin{array}{l}\text { NDG } \\
7.0+1\end{array}$ & $8.0+1$ & $\begin{array}{l}\text { HAR } \\
\text { HAR }\end{array}$ & $\begin{array}{l}\text { E Cuninghanet } \\
\text { E Cuninghame }\end{array}$ & $\begin{array}{r}10086 \\
8859\end{array}$ \\
\hline $202 \mathrm{Po}$ & relot & ve o(E) & & & & \\
\hline $\begin{array}{l}197_{\mathrm{AU}} \\
197_{\mathrm{AU}}\end{array}$ & $\begin{array}{l}10 \mathrm{~B} \\
118\end{array}$ & $\begin{array}{l}7.8+1 \\
7.4+1\end{array}$ & $\begin{array}{l}1.0+2 \\
9.1+1\end{array}$ & $\begin{array}{l}\text { WWA } \\
\text { WWA }\end{array}$ & $\begin{array}{l}\text { E Kormant } \\
\text { E Kormant }\end{array}$ & $\begin{array}{l}9550 \\
9590\end{array}$ \\
\hline${ }^{202} p_{0}$ & $\sigma(E)$ & & & & & \\
\hline $\begin{array}{l}164 \mathrm{Oy} \\
206 \mathrm{~Pb} \\
209 \mathrm{Bi} \\
209 \mathrm{Bi} \\
209 \mathrm{Bi}\end{array}$ & $\begin{array}{c}{ }^{40} \mathrm{Ar} \\
{ }^{3} \mathrm{He} \\
\mathrm{p} \\
\mathrm{p} \\
\mathrm{p}\end{array}$ & $\begin{array}{l}1.6+2 \\
8.5+1 \\
\text { TR } \\
9.0+1 \\
1.0+1\end{array}$ & $\begin{array}{l}2 . \theta+2 \\
1.7+2 \\
8 \cdot 5+1 \\
1.1+2 \\
8 \cdot 0+2\end{array}$ & $\begin{array}{l}\text { PAR } \\
\text { PAR } \\
\text { NRL } \\
\text { NRL } \\
\text { NRL }\end{array}$ & $\begin{array}{l}\text { M Logardet } \\
\text { E Andret } \\
\text { I Pelerson. } \\
\text { T Silberbergt } \\
\text { R Silberberg }\end{array}$ & $\begin{array}{r}12346 \\
6568 \\
4725 \\
4726 \\
4763\end{array}$ \\
\hline $20{ }^{3} \mathrm{Po}$ & Indep & indent. & $\sigma(E)$ & & & \\
\hline $\begin{array}{l}197 \mathrm{AU} \\
197 \mathrm{AU}\end{array}$ & $\begin{array}{l}12 \mathrm{C} \\
160\end{array}$ & $\begin{array}{l}1.3+2 \\
1.4+2\end{array}$ & $1.7+2$ & $\begin{array}{l}\text { KFK } \\
\text { KFK }\end{array}$ & $\begin{array}{l}\text { C Muenzelt } \\
\text { C Muenzelt }\end{array}$ & $\begin{array}{l}10097 \\
11483\end{array}$ \\
\hline $203 \mathrm{Po}$ & spalle & tion ol & (E) & & & \\
\hline $\begin{array}{l}209_{\mathrm{Bi}} \\
20 \mathrm{PO}_{\mathrm{O}}\end{array}$ & $\begin{array}{c}p \\
\text { cumul }\end{array}$ & $\begin{array}{l}3.8+2 \\
\text { tlve of }\end{array}$ & (E) & NRL & R Sllberbergt & 4762 \\
\hline $\begin{array}{l}197 \mathrm{Au} \\
203 \mathrm{Po}\end{array}$ & $\begin{array}{l}160 \\
\text { rolot }\end{array}$ & $\begin{array}{l}1.4+2 \\
\text { ve }\end{array}$ & $1.7+2$ & KFK & C Muenzel- & 11485 \\
\hline $197_{\mathrm{AU}}$ & $M_{B}$ & $6.0+1$ & $8.6+1$ & WWA & E Kormant & 9591 \\
\hline
\end{tabular}




\begin{tabular}{|c|c|c|c|c|c|c|}
\hline Target & Inc & $\begin{array}{l}E \operatorname{Emin} \\
(\mathrm{MeV})\end{array}$ & $\begin{array}{l}\text { Emax } \\
(\operatorname{MeV})\end{array}$ & Lab & Author & No. \\
\hline $203_{\mathrm{Po}}$ & $O(E)$ & & & & & \\
\hline $\begin{array}{l}164 \mathrm{Dy} \\
205 \mathrm{~Pb} \\
209 \mathrm{Bi} \\
209 \mathrm{Bi}\end{array}$ & $\begin{array}{c}{ }^{40} \mathrm{Ar} \\
{ }^{3} \mathrm{He} \\
p \\
0\end{array}$ & $\begin{array}{l}1.6+2 \\
8.5+1 \\
T R \\
1.0+1\end{array}$ & $\begin{array}{l}2.8+2 \\
1.7+2 \\
8.5+1 \\
8.0+2\end{array}$ & $\begin{array}{l}\text { PAR } \\
\text { PAR } \\
\text { NRL } \\
\text { NRL }\end{array}$ & $\begin{array}{l}M \text { Lagardet } \\
\text { E Andret } \\
T \text { Poterson. } \\
R \text { Silberbergt }\end{array}$ & $\begin{array}{r}12346 \\
6568 \\
4727 \\
4763\end{array}$ \\
\hline${ }^{204} \mathrm{Po}$ & \multicolumn{6}{|c|}{ Independent $O(E)$} \\
\hline $\begin{array}{l}197 \mathrm{Au} \\
197_{\mathrm{Au}}\end{array}$ & $\begin{array}{l}12 c \\
160\end{array}$ & $\begin{array}{l}1.3+2 \\
1.4+2\end{array}$ & $1.7+2$ & $\begin{array}{l}\text { KFK } \\
\text { KFK }\end{array}$ & $\begin{array}{l}\text { C Muenzel+ } \\
\text { C Muenzel+ }\end{array}$ & $\begin{array}{l}10097 \\
11483\end{array}$ \\
\hline $204 \mathrm{Po}$ & \multicolumn{6}{|c|}{ Spallation o(E) } \\
\hline $209_{B i}$ & p & $3.8+2$ & & NRL & R silberberg+ & 4762 \\
\hline $204 \mathrm{po}$ & \multicolumn{6}{|c|}{ cumulative $O(E)$} \\
\hline $\begin{array}{l}197 \mathrm{Au} \\
197 \mathrm{Au}\end{array}$ & $\begin{array}{l}12 \mathrm{c} \\
160\end{array}$ & $\begin{array}{l}1.3+2 \\
1.4+2\end{array}$ & $1.7+2$ & $\begin{array}{l}\mathrm{KFK} \\
\mathrm{KFK}\end{array}$ & $\begin{array}{l}\text { C Muenzelt } \\
\text { C Muenzelt }\end{array}$ & $\begin{array}{l}10099 \\
11485\end{array}$ \\
\hline $204 \mathrm{Po}$ & \multicolumn{6}{|c|}{ partial o(E) } \\
\hline $\begin{array}{l}196 \mathrm{pt} \\
204 \mathrm{~Pb}\end{array}$ & $\begin{array}{r}12 \mathrm{C} \\
0\end{array}$ & $\begin{array}{l}\text { NDG } \\
5.0+1\end{array}$ & $7.0+1$ & $\begin{array}{l}\text { HAR } \\
\text { HAR }\end{array}$ & $\begin{array}{l}\text { E Cuninghamet } \\
\text { E Cuninghamet }\end{array}$ & $\begin{array}{r}10087 \\
8860\end{array}$ \\
\hline $204 \mathrm{Po}$ & \multicolumn{6}{|c|}{ relative $O(E)$} \\
\hline $\begin{array}{l}197 \mathrm{Au} \\
204 \mathrm{PO}\end{array}$ & \multicolumn{6}{|l|}{$\sigma(E)$} \\
\hline $\begin{array}{l}164 \mathrm{Dy} \\
164 \mathrm{Dy} \\
206 \mathrm{~Pb} \\
209 \mathrm{Bi} \\
209 \mathrm{Bi} \\
209 \mathrm{Bi}\end{array}$ & $\begin{array}{l}{ }^{40} \mathrm{Ar} \\
{ }^{40} \mathrm{Ar} \\
{ }^{3} \mathrm{He} \\
\mathrm{P} \\
\mathrm{P} \\
\mathrm{P}\end{array}$ & $\begin{array}{l}1.6+2 \\
1.6+2 \\
8.5+1 \\
\text { IR } \\
7.0-5 \\
1.0+1\end{array}$ & $\begin{array}{l}2 \cdot 2+2 \\
2 \cdot 8+2 \\
1.7+2 \\
8 \cdot 5+1 \\
7 \cdot 8-4 \\
8 \cdot 0+2\end{array}$ & $\begin{array}{l}\text { PAR } \\
\text { PAR } \\
\text { PAR } \\
\text { NRL } \\
\text { NRL } \\
\text { NRL }\end{array}$ & $\begin{array}{l}\text { M Lagordat } \\
\text { M Lagardet } \\
\text { E Andret } \\
\text { T Peterson. } \\
\text { T Silberbergt } \\
\text { R Stiberbergt }\end{array}$ & $\begin{array}{r}12344 \\
12346 \\
6568 \\
4728 \\
4729 \\
4763\end{array}$ \\
\hline${ }^{205} \mathrm{po}_{0}$ & \multicolumn{6}{|c|}{ independent o(E) } \\
\hline $\begin{array}{l}197 \mathrm{Au} \\
197 \mathrm{Au}\end{array}$ & $\begin{array}{l}12 \mathrm{C} \\
160\end{array}$ & $\begin{array}{l}1.3+2 \\
1.4+2\end{array}$ & $1.7+2$ & $\begin{array}{l}K F K \\
K F K\end{array}$ & $\begin{array}{l}\text { C Muenzelt } \\
\text { C Muenzelt }\end{array}$ & $\begin{array}{l}10097 \\
11483\end{array}$ \\
\hline $205 p_{0}$ & \multicolumn{6}{|c|}{ Spallotion ofE } \\
\hline $\begin{array}{l}209 \mathrm{Bi} \\
205 \mathrm{Po}\end{array}$ & \multicolumn{6}{|c|}{ Cumulative $O(E)$} \\
\hline $197 \mathrm{Av}$ & ${ }^{12} \mathrm{C}$ & $1.3+2$ & & KFK & C Muenzelt & 10099 \\
\hline
\end{tabular}

\begin{tabular}{|c|c|c|c|c|c|c|}
\hline Target & $\ln c$ & $\begin{array}{l}\text { Em } \ln \\
(\mathrm{MeV})\end{array}$ & $\begin{array}{l}\text { Emax } \\
(M e V)\end{array}$ & Lab & Author & No. \\
\hline${ }^{205} \mathrm{Po}$ & \multicolumn{6}{|c|}{ cumulative $O(E)$} \\
\hline $\begin{array}{l}197 \mathrm{Au} \\
205 \mathrm{Po}\end{array}$ & $\begin{array}{l}160 \\
O(E)\end{array}$ & $1.4+2$ & $1.7+2$ & KFK & C Muenzelt+ & 11485 \\
\hline $\begin{array}{l}164 \mathrm{Dy} \\
206 \mathrm{~Pb} \\
209 \mathrm{Bi} \\
209 \mathrm{Bi}\end{array}$ & $\begin{array}{c}{ }^{40} \mathrm{Ar} \\
{ }^{3} \mathrm{He} \\
p \\
p\end{array}$ & $\begin{array}{l}1.6+2 \\
8.5+1 \\
T R \\
1.0+1\end{array}$ & $\begin{array}{l}2.8+2 \\
1.7+2 \\
8.5+1 \\
8.0+2\end{array}$ & $\begin{array}{l}\text { PAR } \\
\text { PAR } \\
\text { NRL } \\
\text { NRL }\end{array}$ & $\begin{array}{l}\text { M Lagardet } \\
\text { E Andiet } \\
\text { T Peterson. } \\
\text { R Sliberbergt }\end{array}$ & $\begin{array}{r}12346 \\
6568 \\
4730 \\
4763\end{array}$ \\
\hline $206 \mathrm{Po}_{0}$ & \multicolumn{6}{|c|}{ Spallotion o(E) } \\
\hline $\begin{array}{l}{ }^{209} \mathrm{Bi} \\
206 \mathrm{Po}\end{array}$ & \multicolumn{5}{|c|}{ portial rolative o(E) } & 4762 \\
\hline $\begin{array}{l}200^{01} \\
206 \mathrm{Po}\end{array}$ & \multicolumn{5}{|l|}{$O(E)$} & 9552 \\
\hline 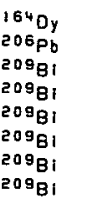 & $\begin{array}{c}{ }^{40} 0_{\mathrm{Ar}} \\
{ }^{3} \mathrm{He} \\
p \\
p \\
p \\
p \\
p \\
p\end{array}$ & $\begin{array}{l}1.6+2 \\
8 \cdot 5+1 \\
T R \\
2.6+1 \\
3.0+1 \\
3.0+1 \\
4.0+1 \\
1.0+1\end{array}$ & $\begin{array}{l}2.8+2 \\
1 \cdot 7+2 \\
8 \cdot 5+1 \\
5 \cdot 2+1 \\
4 \cdot 4+1 \\
4 \cdot 4+1 \\
1.0+2 \\
8 \cdot 0+2\end{array}$ & $\begin{array}{l}\text { PAR F } \\
\text { PAR } \\
\text { NRL } \\
\text { TOK E } \\
\text { KFK C } \\
\text { MIL E } \\
\text { NRL } \\
\text { NRL }\end{array}$ & $\begin{array}{l}\text { M Lagardet } \\
\text { E Andret } \\
\text { T Peterson. } \\
\text { E Migenot } \\
\text { C Muerizelt } \\
\text { E Birattarit } \\
\text { T Silberbergt } \\
\text { R Silterbergt }\end{array}$ & $\begin{array}{l}12346 \\
6568 \\
4731 \\
4732+ \\
4733 \\
4734+ \\
4735 \\
4763\end{array}$ \\
\hline $207 \mathrm{Po}$ & \multicolumn{6}{|c|}{ Including metostable o(E) } \\
\hline $\begin{array}{l}\operatorname{209}^{201} \\
209 \mathrm{BI} \\
209 \mathrm{BI}\end{array}$ & $\begin{array}{l}p \\
p \\
p\end{array}$ & $\begin{array}{l}1.8+1 \\
2.0+1 \\
2.0+1\end{array}$ & $\begin{array}{l}5.2+1 \\
4.0+1 \\
4.0+1\end{array}$ & $\begin{array}{l}\text { TOK E } \\
\text { KFK C } \\
\text { MIL E }\end{array}$ & $\begin{array}{l}\text { E Miyanot } \\
\text { C Muenzelt } \\
\text { E Girallarit }\end{array}$ & $\begin{array}{l}4736+ \\
4737 \\
4738+\end{array}$ \\
\hline $207 \mathrm{Po}$ & \multicolumn{6}{|c|}{ spollation o(E) } \\
\hline $\begin{array}{l}209 \mathrm{BI} \\
207 \mathrm{PO}\end{array}$ & produ & $\begin{array}{l}3.8+2 \\
t y i \in I^{\prime}\end{array}$ & & product yield & R Silberberg* & 4762 \\
\hline $\begin{array}{r}\mathrm{Pb} \\
207 \mathrm{Po}\end{array}$ & $\begin{array}{l}{ }^{3} \mathrm{He} \\
O(E)\end{array}$ & NDG & & TEH E & E Parsat & 6565 \\
\hline $\begin{array}{l}164 \mathrm{DY} \\
20 \mathrm{Bi}^{2} \\
200_{\mathrm{Bi}}\end{array}$ & $\begin{array}{c}{ }^{40} \mathrm{Ar} \\
\mathrm{P} \\
\mathrm{P}\end{array}$ & $\begin{array}{l}1.6+2 \\
T R \\
1.0+1\end{array}$ & $\begin{array}{l}2.8+2 \\
8.5+1 \\
8 \cdot 0+2\end{array}$ & $\begin{array}{l}\text { PAR } \\
\text { NRL } \\
\text { NRL }\end{array}$ & $\begin{array}{l}\text { M Lagardet } \\
\text { T Polerson. } \\
\text { R Sillberbergt }\end{array}$ & $\begin{array}{r}12346 \\
4739 \\
4763\end{array}$ \\
\hline
\end{tabular}




\begin{tabular}{|c|c|c|c|c|c|c|}
\hline Target & $\ln e$ & $\begin{array}{l}\text { Emin } \\
(M 0 V)\end{array}$ & $\begin{array}{l}\text { Emax } \\
(\operatorname{MeV})\end{array}$ & Lab & Author & No. \\
\hline $200 \mathrm{Po}$ & \multicolumn{3}{|c|}{ spallation o(E) } & & & \\
\hline $209^{B i}$ & p & $3.8+2$ & & NRL & R Silborberg+ & 4762 \\
\hline $200 \mathrm{Po}$ & \multicolumn{3}{|c|}{ relative $I_{y}$} & & & \\
\hline $208 \mathrm{pb}$ & a & $4.2+1$ & $5.1+1$ & UST & E Bergstromt & 8875 \\
\hline $200 \mathrm{Po}$ & \multicolumn{2}{|c|}{ o(E) af actor } & & & & \\
\hline $\begin{array}{l}206 \mathrm{~Pb} \\
209 \mathrm{Bi}\end{array}$ & p & $\begin{array}{l}1.5+1 \\
9.7+0\end{array}$ & $\begin{array}{l}1.7+1 \\
1.1+1\end{array}$ & $\begin{array}{l}\text { COL } \\
\text { ANL }\end{array}$ & $\begin{array}{l}\text { R Miller. } \\
\text { E Andret }\end{array}$ & $\begin{array}{l}8864 \\
4744\end{array}$ \\
\hline $20 \mathrm{PO}_{\mathrm{Po}}$ & \multicolumn{2}{|l|}{$O(E)$} & & & & \\
\hline $\begin{array}{l}1640 \mathrm{Oy} \\
209^{\mathrm{B}}\end{array}$ & ${ }_{\mathrm{P}}^{{ }_{\mathrm{H}}^{\mathrm{O}} \mathrm{Ar}}$ & $\frac{1.6+2}{T R}$ & $\begin{array}{l}2.8+2 \\
8.0+1\end{array}$ & $\begin{array}{l}\text { PAR } \\
\text { NRL }\end{array}$ & $\begin{array}{l}\text { M Lagardet } \\
T \text { Petergon. }\end{array}$ & $\begin{array}{r}12346 \\
4740\end{array}$ \\
\hline $209 \mathrm{Bi}$ & p & $9.7+0$ & $1.1+1$ & ANL & E Andret & 4741 \\
\hline $209_{B i}$ & p & $1.1+1$ & $9.0+1$ & NRL & T Sllberbergt & 4742 \\
\hline $209_{B i}$ & p & $1.8+1$ & $6.4+1$ & TOK & M Mlyonot & 4743 \\
\hline $209_{B i}$ & $p$ & $1.0+1$ & $8.0+2$ & NRL & R siliberbergt & 4763 \\
\hline $208 \mathrm{Po}_{0}$ & \multicolumn{5}{|c|}{ compound-nucleus $\sigma(E)$} & \\
\hline $209_{81}$ & $p$ & $1.8+1$ & $6.4+1$ & TOK & M Miyonot & 4751 \\
\hline $209 \mathrm{Po}$ & \multicolumn{3}{|c|}{ spallation o(E) } & & & · \\
\hline $20.9 \mathrm{Bi}$ & p & $3.8+2$ & & NRL & R silberberg+ & 4762 \\
\hline $209 \mathrm{Po}$ & \multicolumn{3}{|c|}{ diract-interaction } & $g(E)$ & & \\
\hline${ }^{209_{B 1}}$ & p & $1.8+1$ & $6.4+1$ & TOK & M Miyanot & 4752 \\
\hline $20{ }^{9} \mathrm{Po}$ & \multicolumn{3}{|c|}{ portial o(E) } & & & \\
\hline $209_{\mathrm{BI}}$ & p & $1 . \theta+1$ & $6.4+1$ & TOK & $M$ Miyonot & 4750 \\
\hline $20{ }^{9} \mathrm{Po}$ & \multicolumn{3}{|c|}{ a(E)xiactor } & & & \\
\hline $209_{B 1}$ & p & $5.0+0$ & $8.0+0$ & $\mathrm{COL}$ & R Miller. & 4745 \\
\hline${ }^{209} \mathrm{Po}$ & \multicolumn{2}{|l|}{$a(E)$} & & & & \\
\hline $\begin{array}{l}209_{B 1} \\
209_{B 1}\end{array}$ & $p$ & $\begin{array}{l}\text { TR } \\
9.8+0\end{array}$ & $\begin{array}{l}8.5+1 \\
1.0+1\end{array}$ & $\begin{array}{l}\text { NRL } \\
\text { KFK }\end{array}$ & $\begin{array}{l}\text { T Poterson. } \\
\text { C Muenzel+ }\end{array}$ & $\begin{array}{l}4746 \\
4747\end{array}$ \\
\hline $209_{B I}$ & p & $9.8+0$ & $1.0+1$ & ANL & E Wingt & $4748+$ \\
\hline 20981 & p & $1.8+1$ & $6.4+1$ & TOK & M Miyanot & 4749 \\
\hline $209_{81}$ & $p$ & $1.0+1$ & $8.0+2$ & NRL & R Siliberberg+ & 4763 \\
\hline
\end{tabular}

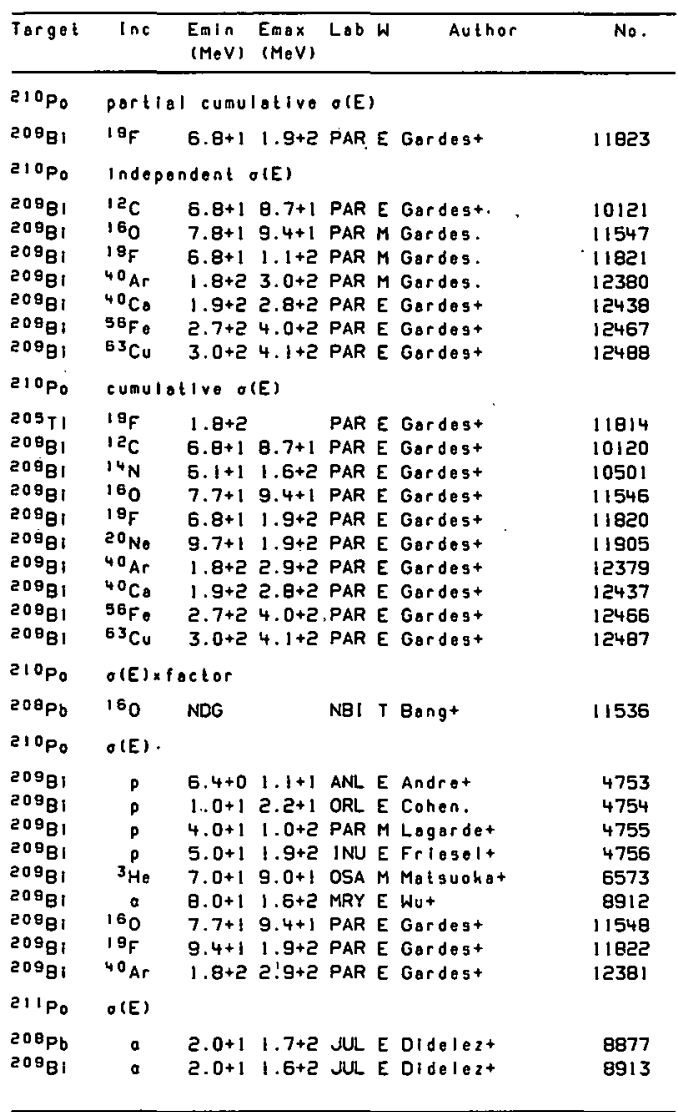




\begin{tabular}{|c|c|c|c|c|c|c|}
\hline Target & $\operatorname{lnc}$ & $\begin{array}{l}\text { Emin } \\
(M e V) 1\end{array}$ & $\begin{array}{l}\text { Emax } \\
(M e v)\end{array}$ & Lab & Author & No. \\
\hline EIPo & OCE) & & & & & \\
\hline $209^{\circ 1}$ & $a$ & $0.0+1 \quad 1$ & $1.6+2 r$ & MRY & E Wut & 6914 \\
\hline 2119po & $\theta(E)$ & & & & & \\
\hline $\begin{array}{l}208 \mathrm{~Pb} \\
20 \mathrm{Bl}^{\circ}\end{array}$ & $a$ & $\begin{array}{ll}2.0+1 & 1 \\
4.0+1 & 1\end{array}$ & $\begin{array}{l}1.8+2 \\
1.7+2\end{array}$ & JUL & $\begin{array}{l}\text { E Oidelezt } \\
\text { E Liedert }\end{array}$ & $\begin{array}{l}8878 \\
8915\end{array}$ \\
\hline $211 \cdot \bullet P_{0}$ & $\theta(E)$ & & & & & \\
\hline $\begin{array}{l}200 \mathrm{~Pb} \\
200 \mathrm{~Pb} \\
200 \mathrm{~Pb} \\
209 \mathrm{gi} \\
209 \mathrm{gi} \\
209 \mathrm{Bi} \\
209 \mathrm{Bi}\end{array}$ & $\begin{array}{c}a \\
E_{L i}^{a} \\
d \\
a \\
E_{L I}^{a}\end{array}$ & $\begin{array}{ll}2.0+1 & 1 \\
2 \cdot 0+1 & 1 \\
6.0+1 & 1 \\
7.5+0 & 1 \\
1.0+2 \\
4 \cdot 0+1 \\
6.0+1\end{array}$ & $\begin{array}{l}1.8+2 \\
1.3+25 \\
1.8+2 k \\
1.2+1 \\
1.7+2 \\
1.8+2\end{array}$ & $\begin{array}{l}\text { JUL } \\
\text { KFK } \\
\text { KFK } \\
\text { UEN } \\
\text { KFK } \\
\text { JUL } \\
\text { KFK }\end{array}$ & $\begin{array}{l}\text { E Didelezt } \\
\text { E Neumannt } \\
\text { E Neumannt } \\
\text { E Zenlot } \\
\text { E Neumanat } \\
\text { E Liedert } \\
\text { E Neumannt }\end{array}$ & $\begin{array}{l}8879 \\
8880 \\
9372 \\
5887 \\
8917 \\
8916 \\
9374\end{array}$ \\
\hline \multicolumn{7}{|c|}{ etin/gPo rollo o.(E) } \\
\hline $\begin{array}{l}200 \mathrm{~Pb} \\
200^{\circ} \mathrm{Bl}\end{array}$ & $a$ & $\begin{array}{ll}4.0+1 & 1 \\
4.0+1 & 1\end{array}$ & $\begin{array}{l}1.7+2 \\
1.7+2\end{array}$ & JUL & $\begin{array}{l}\text { E Liedert } \\
\text { E Lledert }\end{array}$ & $\begin{array}{l}8881 \\
8918\end{array}$ \\
\hline $2 I^{2} P_{0}$ & \multicolumn{6}{|c|}{ portial product yleld } \\
\hline $209_{B 1}$ & - & $4.5+1$ & & JUL & E Liedert & 8921 \\
\hline 2! 2po & $O(E)$ & & & & & \\
\hline $\begin{array}{l}209_{\mathrm{Bi}} \\
200_{\mathrm{Bi}}\end{array}$ & $:$ & \begin{tabular}{l|}
$2.0+1$ \\
$8 \cdot 0+1$
\end{tabular} & $\begin{array}{l}1.7+2 \\
1.6+2\end{array}$ & MULY & $\begin{array}{l}\text { E Didelez+ } \\
\text { E Wut }\end{array}$ & $\begin{array}{l}8919 \\
8920\end{array}$ \\
\hline aleqp, & $\theta(E)$ & & & & & \\
\hline $209_{B i}$ & - & $4.0+1 \quad 1$ & $1.7+2$ & JUL & E Liedert & 8922 \\
\hline 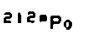 & $\sigma(E)$ & & & & & \\
\hline $\begin{array}{l}209 \mathrm{Bi} \\
20{ }^{8} \mathrm{Bi} \\
20{ }^{8} \mathrm{Bi}\end{array}$ & $\begin{array}{c}a \\
a \\
\sigma_{\mathrm{L}}\end{array}$ & $\begin{array}{l}4.0+1 \\
1.0+2 \\
1.6+2\end{array}$ & $1.7+2$ & $\begin{array}{l}\text { JUL } \\
\text { KFK } \\
\text { KFK }\end{array}$ & $\begin{array}{l}\text { E Liedert } \\
\text { E Neumannt } \\
\text { E Neumannt }\end{array}$ & $\begin{array}{l}8923 \\
8924 \\
9375\end{array}$ \\
\hline \multicolumn{7}{|c|}{ 212019P0 ratlo o(E) } \\
\hline $209_{\mathrm{Bi}}$ & a & $4.0+1$ & $1.7+2$ & JuL & E Liedert & 8925 \\
\hline$z=85$ & thick & target & $y 1010$ & & & \\
\hline $209^{8 i}$ & $p$ & $2.0+2$ & , & LAS & E Exp. & 4757 \\
\hline
\end{tabular}

\begin{tabular}{|c|c|c|c|c|c|c|}
\hline Target & $\ln c$ & $\begin{array}{l}\text { Emin } \\
(\mathrm{MeV})\end{array}$ & $\begin{array}{l}\text { Emox } \\
(\mathrm{MeV})\end{array}$ & Lob & Acthor & No. \\
\hline$z=85$ & $O(E)$ & & & & & \\
\hline $200 \mathrm{~Pb}$ & ${ }^{6} \mathrm{LI}$ & $7.5+1$ & $9.4+1$ & INU & E Karvoshit & 9373 \\
\hline${ }^{20 S_{A t}}$ & portlo & $1 O(E)$ & & & & \\
\hline $197 \mathrm{AU}$ & ${ }^{12} \mathrm{C}$ & NDG & & STB & E sjoroen. & 10094 \\
\hline $207 \mathrm{At}$ & portle & $I O(E)$ & & & & \\
\hline $204 \mathrm{Po}$ & ${ }^{6} \mathrm{LI}_{1}$ & NOG & & STE & E sjoreen. & 9361 \\
\hline $207 \mathrm{At}$ & $\sigma(E)$ & & & & & \\
\hline $\begin{array}{l}209_{\mathrm{BI}} \\
209_{\mathrm{BI}} \\
209_{\mathrm{BI}}\end{array}$ & $\begin{array}{l}19 \mathrm{~F} \\
40 \mathrm{Ar} \\
{ }^{40} \mathrm{Co}\end{array}$ & $\begin{array}{l}1.9+2 \\
2.1+2 \\
2.2+2\end{array}$ & $\begin{array}{l}2.9+2 \\
2.8+2\end{array}$ & $\begin{array}{l}\text { PAR } \\
\text { PAR } \\
\text { PAR }\end{array}$ & $\begin{array}{l}\text { E Gardegt } \\
\text { E Gardest } \\
\text { E Gardest }\end{array}$ & $\begin{array}{l}11824 \\
12382 \\
12439\end{array}$ \\
\hline $20 \dot{\theta}_{A t}$ & $o(E)$ & & & & & \\
\hline 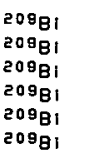 & $\begin{array}{l}198 \\
20 \mathrm{Ne} \\
40 \mathrm{Ar} \\
{ }^{40} \mathrm{Co} \\
56 \mathrm{Fe} \\
63 \mathrm{Cu}\end{array}$ & $\begin{array}{l}1.9+2 \\
2.0+2 \\
2.1+2 \\
2.0+2 \\
3.0+2 \\
3.3+2\end{array}$ & $\begin{array}{r} \\
2.9+2 \\
2.8+2 \\
3.7+2 \\
4.1+2\end{array}$ & $\begin{array}{l}\text { PAR } \\
\text { PAR } \\
\text { PAR } \\
\text { PAR } \\
\text { PAR } \\
\text { PAR }\end{array}$ & $\begin{array}{l}\text { E Gardest } \\
\text { E Gardest } \\
\text { E Gardagt } \\
\text { E Gardest } \\
\text { E Gardest } \\
\text { E Gardest }\end{array}$ & $\begin{array}{l}11825 \\
11906 \\
12383 \\
12440 \\
12468 \\
12489\end{array}$ \\
\hline $209_{A l}$ & cunule & tive? & $O(E)$ & & & \\
\hline $\begin{array}{l}200_{B t} \\
209_{A t}\end{array}$ & $\begin{array}{l}{ }^{12} \mathrm{C} \\
\text { poirtio }\end{array}$ & $\begin{array}{l}6.2+1 \\
1 \text { produ }\end{array}$ & $\begin{array}{l}7.2+1 \\
\text { uct yio }\end{array}$ & $\begin{array}{l}\text { IMP } \\
.10\end{array}$ & E Jun-Shengt & $10: 23$ \\
\hline $\begin{array}{l}209_{\mathrm{Bl}} \\
209_{\mathrm{Al}}\end{array}$ & $\begin{array}{l}{ }^{3} \mathrm{He} \\
\text { partlo }\end{array}$ & $\begin{array}{l}1.9+1 \\
1 \text { relat }\end{array}$ & $\begin{array}{l}E .2+1 \\
\text { tivo ol }\end{array}$ & $\begin{array}{l}\text { JYV } \\
\text { (E) }\end{array}$ & E Rahhonan. & 6574 \\
\hline $209_{\mathrm{Bl}}$ & 100. & $6.8+1$ & $8.8+1$ & YAL & E Ralicht & 9553 \\
\hline $200 \mathrm{At}$ & partio & I $\sigma(E)$ & & & & \\
\hline $206 \mathrm{~Pb}$ & $B_{L I}$ & NOG & & Бтв & E Sjoreen. & 9362 \\
\hline $20{ }^{A t}$ & $\theta(E)$ & & & & & \\
\hline $\begin{array}{l}197 \mathrm{AU} \\
200^{\circ} \mathrm{I}\end{array}$ & $\begin{array}{l}160^{\circ} \\
12 \mathrm{c}\end{array}$ & $\begin{array}{l}9 \cdot 3+1 \\
6 \cdot 6+1\end{array}$ & 8. $8+1$ & $\begin{array}{l}\text { BNL } \\
\text { PAR }\end{array}$ & $\begin{array}{l}\text { E Wegner- } \\
\text { E Gardes- }\end{array}$ & $\begin{array}{l}11480 \\
10122\end{array}$ \\
\hline $209^{\circ}$ & $19 \mathrm{~F}$ & $1.9+2$ & & PAR & E Gardes- & 11826 \\
\hline 20881 & $20 \mathrm{Ne}$ & $2.0+2$ & & PAR & E Gardos- & 11907 \\
\hline 20981 & $40 \mathrm{Ar}$ & $1.9+2$ & $2.9+2$ & PAR & E Gardes- & 12394 \\
\hline 20981 & ${ }^{40} \mathrm{Co}$ & $2.0+2$ & $2.8+2$ & PAR & E Gardes- & 12441 \\
\hline
\end{tabular}




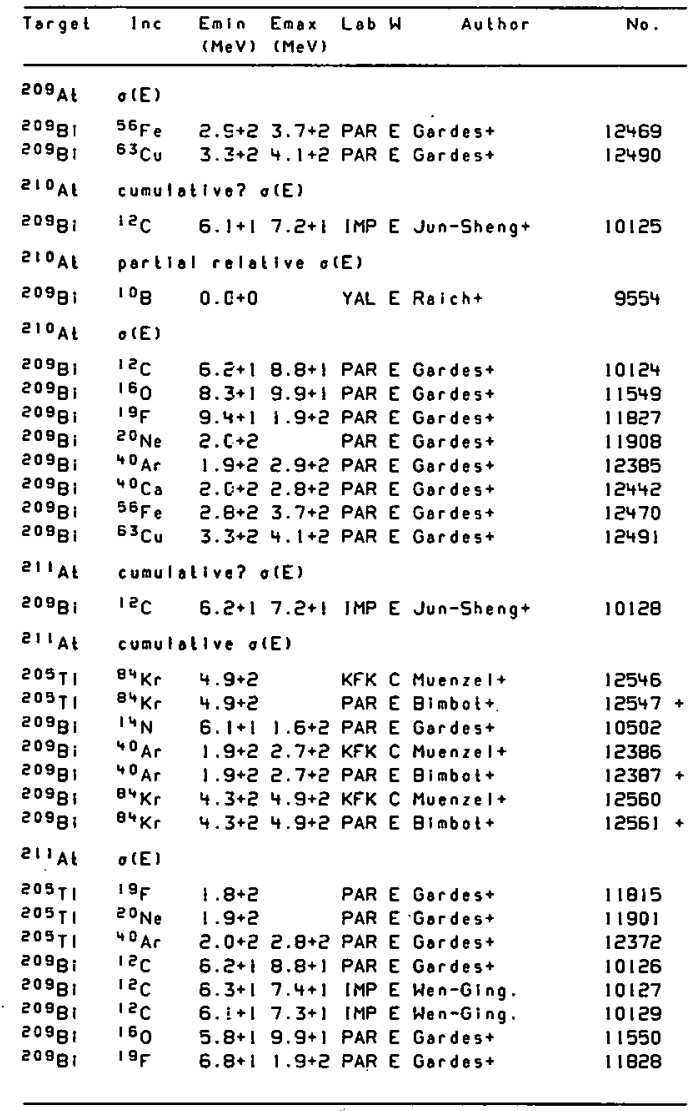

\begin{tabular}{|c|c|c|c|c|c|c|}
\hline Torget & $\ln e$ & $\begin{array}{l}\text { Emin } \\
(M e V)\end{array}$ & $\begin{array}{l}\text { Emox } \\
(\mathrm{MeV})\end{array}$ & Lab & Aulthor & No. \\
\hline $211_{A l}$ & $O(E)$ & & & & & \\
\hline $\begin{array}{l}209_{\mathrm{Bi}} \\
209_{\mathrm{Bi}} \\
209_{\mathrm{Bi}} \\
209_{\mathrm{Bi}} \\
209_{\mathrm{Bi}}\end{array}$ & $\begin{array}{l}20 \mathrm{Ne} \\
{ }^{20} \mathrm{Ar} \\
{ }^{40} \mathrm{Co} \\
56 \mathrm{Fe} \\
{ }^{63} \mathrm{Cu}\end{array}$ & $\begin{array}{l}9.7+1 \\
1.8+2 \\
1.7+2 \\
2.6+2 \\
3.0+2\end{array}$ & $\begin{array}{l}2.0+2 \\
2.9+2 \\
2.8+2 \\
4.0+2 \\
4.1+2\end{array}$ & $\begin{array}{l}\text { PAR } \\
\text { PAR } \\
\text { PAR } \\
\text { PAR } \\
\text { PAR }\end{array}$ & $\begin{array}{l}\text { E Gardest } \\
E \text { Gardest } \\
E \text { Gardest } \\
\text { E Gardest } \\
\text { E Gardest }\end{array}$ & $\begin{array}{l}11909 \\
12388 \\
12443 \\
12471 \\
12492\end{array}$ \\
\hline $2 \mid a_{A t}$ & \multicolumn{6}{|c|}{ rolotive o(E) } \\
\hline $208 \mathrm{~Pb}$ & ${ }^{7} \mathrm{Li}$ & $3.2+1$ & $4.4+1$ & STB & E sjoreent & 9470 \\
\hline $21 z_{A t}$ & $\theta(E)$ & & & & & \\
\hline $209_{B i}$ & 0 & $2.0+1$ & $1.7+2$ & JUL & E Didelezt & 8926 \\
\hline $213_{A t}$ & \multicolumn{6}{|c|}{ portiol $\sigma(E)$} \\
\hline $200 \mathrm{~Pb}$ & ${ }^{7} \mathrm{LI}$ & NDG & & STB & E SJoreen. & 9471 \\
\hline $213^{3} \mathrm{Al}$ & $\theta(E)$ & & & & & \\
\hline $209_{B i}$ & ${ }^{12} \mathrm{C}$ & $1.1+2$ & $1.3+2$ & YAL & E Gought & 10130 \\
\hline $214 \mathrm{Al}$ & $\theta(E)$ & & & & & \\
\hline $\begin{array}{l}209_{8 i} i \\
209_{8 i}\end{array}$ & $\begin{array}{l}12 c \\
12 c\end{array}$ & $\begin{array}{l}9.2+1 \\
9.5+1\end{array}$ & $\begin{array}{l}1.2+2 \\
1.3+2\end{array}$ & $\begin{array}{l}\text { MAN } \\
\text { YAL }\end{array}$ & $\begin{array}{l}\text { E Bell+ } \\
\text { E Gought }\end{array}$ & $\begin{array}{l}10131 \\
10132\end{array}$ \\
\hline $215_{\mathrm{Al}}$ & $\theta(E)$ & & & & & \\
\hline $\begin{array}{l}209_{B i} i \\
209_{B i}\end{array}$ & $\begin{array}{l}{ }^{12} \mathrm{C} \\
{ }^{12} \mathrm{C}\end{array}$ & $\begin{array}{l}7.9+1 \\
8.4+1\end{array}$ & $\begin{array}{l}1.2+2 \\
1.3+2\end{array}$ & $\begin{array}{l}\text { MAN } \\
\text { YAL }\end{array}$ & $\begin{array}{l}\text { E Bell+ } \\
\text { E Gought }\end{array}$ & $\begin{array}{l}10133 \\
10134\end{array}$ \\
\hline $216 \mathrm{At}$ & $\theta(E)$ & & & & & \\
\hline $\begin{array}{l}2098 i \\
2098 ;\end{array}$ & $\begin{array}{l}12 \mathrm{C} \\
{ }^{12} \mathrm{C}\end{array}$ & $\begin{array}{l}6.0+1 \\
7.5+1\end{array}$ & $\begin{array}{l}1.1+2 \\
1.3+2\end{array}$ & $\begin{array}{l}\text { MAN } \\
\text { YAL }\end{array}$ & $\begin{array}{l}\text { E Bellt+ } \\
\text { E Gought }\end{array}$ & $\begin{array}{l}10135 \\
10136\end{array}$ \\
\hline $217 \mathrm{Al}$ & $\sigma(E)$ & & & & & \\
\hline $\begin{array}{l}2099 i \\
2090 i\end{array}$ & $\begin{array}{l}{ }^{12} \mathrm{C} \\
{ }^{12} \mathrm{C}\end{array}$ & $\begin{array}{l}6.0+1 \\
7 \cdot 0+1\end{array}$ & $\begin{array}{l}9.0+1 \\
8 \cdot 5+1\end{array}$ & $\begin{array}{l}\text { MAN } \\
\text { YAL }\end{array}$ & $\begin{array}{l}\text { E Bell+ } \\
\text { E Gought }\end{array}$ & $\begin{array}{l}10137 \\
10138\end{array}$ \\
\hline $21 \theta_{A t}$ & $O(E)$ & & & & & \\
\hline $\begin{array}{l}209^{8 i} \\
209 \mathrm{Bi}\end{array}$ & $\begin{array}{l}12 c \\
12 c\end{array}$ & $\begin{array}{l}5.0+1 \\
6.5+1\end{array}$ & $\begin{array}{l}7.8+1 \\
8.5+1\end{array}$ & $\begin{array}{l}\text { MAN } \\
\text { YAL }\end{array}$ & $\begin{array}{l}\text { E Bell+ } \\
\text { E Gought }\end{array}$ & $\begin{array}{l}10139 \\
10140\end{array}$ \\
\hline$z=86$ & $O(E)$ & & & & & \\
\hline $209^{B i}$ & $14 N$ & $8.5+1$ & $9.5+1$ & JCL & E Nomur B+ & 10509 \\
\hline
\end{tabular}




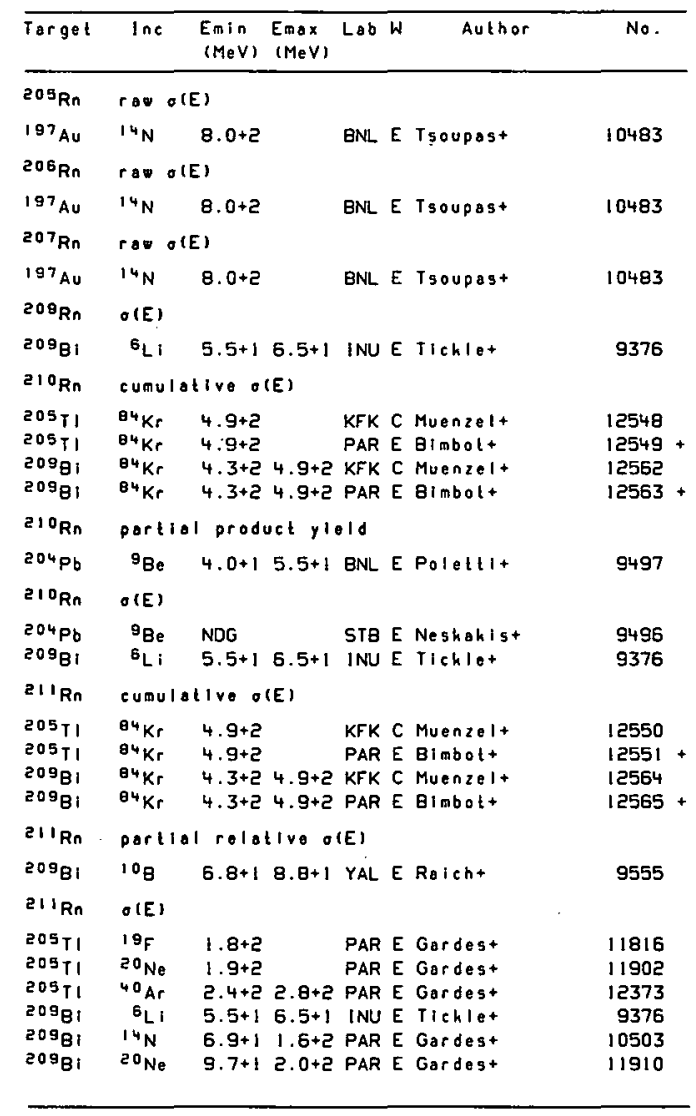

\begin{tabular}{|c|c|c|c|c|c|c|}
\hline Target & $\operatorname{lnc}$ & $\begin{array}{l}\text { Emin } \\
\text { (MeV) }\end{array}$ & $\begin{array}{l}\text { Emax } \\
(\operatorname{MeV})\end{array}$ & Lab W & Author & No. \\
\hline $2 I^{\prime} R n$ & $O(E)$ & & & & & \\
\hline $\begin{array}{l}209^{8 i} \\
200^{B i} \\
209^{8 i} \\
200^{B i}\end{array}$ & $\begin{array}{l}{ }^{40} \mathrm{Ar} \\
{ }^{40} \mathrm{Ca} \\
56 \mathrm{Fe} \\
{ }^{53} \mathrm{Cu}\end{array}$ & $\begin{array}{l}1.9+2 \\
2.0+2 \\
2.9+2 \\
3.1+2\end{array}$ & $\begin{array}{l}2.9+2 \\
2.8+2 \\
3.7+2 \\
4.1+2\end{array}$ & $\begin{array}{l}\text { PAR E } \\
\text { PAR E } \\
\text { PAR E } \\
\text { PAR E }\end{array}$ & $\begin{array}{l}\text { Gardest } \\
\text { Gardest } \\
\text { Gardest } \\
\text { Gardest }\end{array}$ & $\begin{array}{l}12389 \\
12444 \\
12472 \\
12493\end{array}$ \\
\hline $212_{R n}$ & prompt & produe & et y & & & \\
\hline $204 \mathrm{Hg}$ & ${ }^{13} \mathrm{C}$ & $7.2+1$ & $8.5+1$ & CRC E & Hornt & 10215 \\
\hline $212_{\mathrm{Rn}}$ & delaye & d grodu & luct yle & eld & & \\
\hline $204 \mathrm{Hg}$ & ${ }^{13} \mathrm{c}$ & $7.2+1$ & $8.5+1$ & CRC E & Hornt & 10216 \\
\hline $21 e_{R n}$ & produc & $t y i 010$ & & & & \\
\hline $204 \mathrm{Hg}$ & ${ }^{13} \mathrm{C}$ & NDG & & CRC E & Hornt & 10214 \\
\hline 21 $2 \mathrm{Rn}$ & $O(E)$ & & & & & \\
\hline $\begin{array}{l}209^{B i} \\
209_{B i}\end{array}$ & $\begin{array}{l}{ }^{6} \mathrm{Li} \\
{ }^{14} \mathrm{~N}\end{array}$ & $\begin{array}{l}5.5+1 \\
6.9+1\end{array}$ & $\begin{array}{l}6.5+1 \\
1.6+2\end{array}$ & $\begin{array}{l}\text { INU E } \\
\text { PARE }\end{array}$ & $\begin{array}{l}\text { Ticklet } \\
\text { Gardest }\end{array}$ & $\begin{array}{r}9376 \\
10504\end{array}$ \\
\hline $213 \mathrm{Rn}$ & $O(E)$ & & & & & \\
\hline $200^{\circ} \mathrm{B}$ & ${ }^{B} \mathrm{LI}$ & $5.5+1$ & $6.5+1$ & INU E & Tlaklet & 9376 \\
\hline $214 \mathrm{Rn}$ & $O(E)$ & & & & & \\
\hline $209_{B i}$ & ${ }^{6} \mathrm{LI}$ & $5.5+1$ & $5.5+1$ & INU E & Tlehl $\geqslant+$ & 9376 \\
\hline $22 I_{R n}$ & spollal & tion pr & iroduct & yield & & \\
\hline $232 \mathrm{th}$ & D & $3.7+2$ & & LVP E & Butement+ & 4795 \\
\hline $205_{\mathrm{Fr}}$ & $\sigma(E)$ & & & & & \\
\hline $174 \mathrm{Yb}$ & ${ }^{40} \mathrm{Ar}$ & $1.6+2$ & $2.4+2$ & ERK E & Le-Beyect & 12354 \\
\hline${ }^{206} \mathrm{Fr}$ & $O(E)$ & & & & & \\
\hline $174 \mathrm{Yb}$ & ${ }^{40} \mathrm{Ar}$ & $1.6+2$ & $2.4+2$ & BRK E & Le-Beyect & 12354 \\
\hline $207 \mathrm{Fr}$ & $O(E)$ & & & & & \\
\hline $174 \mathrm{Yb}$ & ${ }^{40} \mathrm{Ar}$ & $1.6+2$ & $2.4+2$ & BRK $\varepsilon$ & Le-Beyect & 12354 \\
\hline $200^{F r}$ & $\sigma(E)$ & & & & & \\
\hline $174 \mathrm{Yb}$ & ${ }^{40} \mathrm{Ar}$ & $1.6+2$ & $2.4+2$ & BRK E & Le-Beyect & 12354 \\
\hline
\end{tabular}




\begin{tabular}{|c|c|c|c|c|c|c|}
\hline Target & Inc & $\begin{array}{l}\text { Emin } \\
\text { (MeV) }\end{array}$ & $\begin{array}{l}\text { Emax } \\
(\operatorname{MeV})\end{array}$ & Lab & Author & No. \\
\hline $200^{F r}$ & $O(E)$ & & & & & \\
\hline $\begin{array}{l}174 \mathrm{Yb} \\
210 \mathrm{Fr}\end{array}$ & $\begin{array}{l}{ }^{40} \mathrm{Ar} \\
{ }^{\circ}(E)\end{array}$ & $1.6+2$ & $2.4+2$ & BRK E & E Lo-Beyect & 12354 \\
\hline $174 \mathrm{Yb}$ & ${ }^{40} \mathrm{Ar}$ & $1.6+2$ & $2.4+2$ & BRK E & E Le-Beyect & 12354 \\
\hline $21 I_{F r}$ & $O(E)$ & & & & & \\
\hline${ }_{x}^{174} \times 6$ & ${ }^{40} \mathrm{Ar}$ & $1.6+2$ & $2.4+2$ & ERK E & E Le-Beyect & 12354 \\
\hline $212 \mathrm{Fr}$ & $O(E)$ & & & & '. & \\
\hline $209_{81}$ & $12 c$ & $6.0+1$ & $1.2+2$ & MAN E & E Bell+ & 10141 \\
\hline $213 \mathrm{Fr}$ & $O(E)$ & & & & & \\
\hline $200_{\mathrm{Bi}}$ & ${ }^{12} \mathrm{C}$ & $6.6+1$ & $6.9+1$ & IMP E & E Jun-Shengt & 10142 \\
\hline $213 \mathrm{Fr}$ & portlel & I $a(E)$ & & & & \\
\hline $\begin{array}{l}205 \mathrm{TI} \\
21{ }^{4} \mathrm{Fr}\end{array}$ & $\begin{array}{l}{ }^{13} \mathrm{C}= \\
\mathrm{O}(\mathrm{E})\end{array}$ & $7.2+1$ & $8.6+1$ & CRC E & E Hornt & 10217 \\
\hline $209_{B i}$ & ${ }^{12} \mathrm{C}$ & $6.1+1$ & $6.9+1$ & $I M P E$ & E Jun-Shengt & 10143 \\
\hline $214 . \mathrm{Fr}$ & $O(E)$ & & & & & \\
\hline $209_{\mathrm{BI}}$ & ${ }^{12} \mathrm{C}$ & $6.2+1$ & $6.9+1$ & IMP & E Jun-Shongt & 10144 \\
\hline $220 \mathrm{Fr}$ & $O(E)$ & & & & & \\
\hline $200^{\circ} \mathrm{i}$ & ${ }^{14} \mathrm{C}$ & $6.1+1$ & $7.4+1$ & MUU E & E Hickst & 10227 \\
\hline $2=88$ & $O(E)$ & & & & & \\
\hline $209^{B i}$ & ${ }^{14} \mathrm{~N}$ & $8.5+1$ & $9.5+1$ & $\mathrm{JCL}$ & E Nomurst & 10508 \\
\hline${ }^{207} R_{0}$ & $O(E)$ & & & & & \\
\hline $174 \mathrm{Yb}$ & $40 \mathrm{Ar}$ & $1.6+2$ & $2.4+2$ & BRK & E Lo-Beyect & 12354 \\
\hline $208_{\mathrm{R}}$ & $O(E)$ & & & & & \\
\hline $174 \mathrm{Yb}$ & ${ }^{40} \mathrm{Ar}$ & $1.6+2$ & $2.4+2$ & BRK & E Le-Beyect & 12354 \\
\hline $20{ }^{\circ} \mathrm{Ro}$ & $\sigma(E)$ & & & & & \\
\hline $174 \mathrm{Yb}$ & ${ }^{40} \mathrm{Ar}$ & $1.5+2$ & $2.4+2$ & ERK & E Le-Beyect & 12354 \\
\hline $210 \mathrm{Ro}$ & $O(E)$ & & & & & \\
\hline $174 \mathrm{rb}^{\circ}$ & ${ }^{40} \mathrm{Ar}$ & $1.5+2$ & $2.4+2$ & BRK & E Le-Beyect & 12354 \\
\hline
\end{tabular}

\begin{tabular}{|c|c|c|c|c|c|c|}
\hline Target & $\ln c$ & $\begin{array}{l}\text { Enln } \\
(M e V)\end{array}$ & $\begin{array}{l}\text { Emax } \\
(\operatorname{MeV})\end{array}$ & Lab & Author & No. \\
\hline $211 R_{0}$ & $O(E)$ & & & & & \\
\hline $174 \mathrm{Yb}$ & $40 \mathrm{Ar}$ & $1.6+2$ & $2.4+2$ & BRK & E Le-Beyect & 12354 \\
\hline $213=\mathrm{RA}$ & rolo & Ive $I_{\text {, }}$ & & & & \\
\hline $209_{B i}$ & $10 \mathrm{~B}$ & $6.8+1$ & $8.8+1$ & YAL & E Rolcht & 9556 \\
\hline $214 \mathrm{Ro}$ & porti & $1 \sigma(E)$ & & & & \\
\hline $206 \mathrm{~Pb}_{\mathrm{b}}$ & ${ }^{13} \mathrm{C}$ & $7.2+1$ & $8.6+1$ & $\mathrm{CRC}$ & E Hornt & 10218 \\
\hline $215_{R}$ & $O(E)$ & & & & & \\
\hline $200 \mathrm{~Pb}$ & ${ }^{12} \mathrm{C}$ & $6.5+1$ & $9.6+1$ & SCL & E Nomurat & 10104 \\
\hline $216 \mathrm{Ra}$ & $\sigma(E)$ & & & & & \\
\hline $208 \mathrm{~Pb}_{\mathrm{b}}$ & $1^{12} \mathrm{C}$ & $6.5+1$ & $9.6+1$ & JCL & E Nomurat & 10104 \\
\hline $217 \mathrm{Ra}$ & $\sigma(E)$ & & & & & \\
\hline $208 \mathrm{~Pb}$ & $1^{2} \mathrm{C}$ & $6.5+1$ & $9.6+1$ & $\mathrm{JCL}$ & E Nomurat & 10104 \\
\hline $22 I_{R}$ & $O(E)$ & & & & & \\
\hline $209_{B i}$ & ${ }^{14} \mathrm{C}$ & $6.1+1$ & $7.4+1$ & MUU & E Hichst & 10228 \\
\hline $222_{R_{0}}$ & $O(E)$ & & & & & \\
\hline $209_{B i}$ & ${ }^{14} \mathrm{C}$ & $6.1+1$ & $7.4+1$ & MuU & E Hicht & 10229 \\
\hline $23^{3} \mathrm{Ra}_{\mathrm{a}}$ & $O(E)$ & & & & & \\
\hline $232 \mathrm{Th}$ & p & $5.1+1$ & $9.0+1$ & MCG & E Hogont & 4819 \\
\hline $22{ }^{4} \mathrm{Ro}$ & $O(E)$ & & & & & \\
\hline $\begin{array}{l}232 \mathrm{Th} \\
232 \mathrm{Th}\end{array}$ & p & $\begin{array}{l}5 \cdot 1+1 \\
5 \cdot 0+1\end{array}$ & $\begin{array}{l}9.0+1 \\
1.5+2\end{array}$ & $\begin{array}{l}\text { MCG } \\
\text { JUL }\end{array}$ & $\begin{array}{l}\text { E Hogant } \\
\text { E Scholzt }\end{array}$ & $\begin{array}{l}4819 \\
8969\end{array}$ \\
\hline $225 \mathrm{Rg}$ & $\sigma(E)$ & & & & & \\
\hline $232 \mathrm{Th}$ & p & $5.1+1$ & $9.0+1$ & MCG & E Hogant & 4819 \\
\hline $216_{A C}$ & $O(E)$ & & & & & \\
\hline $209^{\mathrm{Bi}}$ & ${ }^{12} \mathrm{C}$ & $7.5+1$ & $9.1+1$ & IMP & T Shu-Weit+ & 10145 \\
\hline $217_{A C}$ & $O(E)$ & & & & & \\
\hline $207 \mathrm{~Pb}$ & $14 \mathrm{~N}$ & $7.0+1$ & $9.6+1$ & $\mathrm{JCL}$ & E Nonurat & 10487 \\
\hline $\begin{array}{l}209 \mathrm{Bi}_{1} \\
20 \mathrm{Bi}^{2}\end{array}$ & $12 \mathrm{C}$ & $6.0+1$ & $7.9+1$ & IMP & E Jun-Shengt & 10146 \\
\hline $209^{81}$ & ${ }^{12} \mathrm{c}$ & $6.5+1$ & $9.0+1$ & IMP & T Shu-We It & 10147 \\
\hline
\end{tabular}




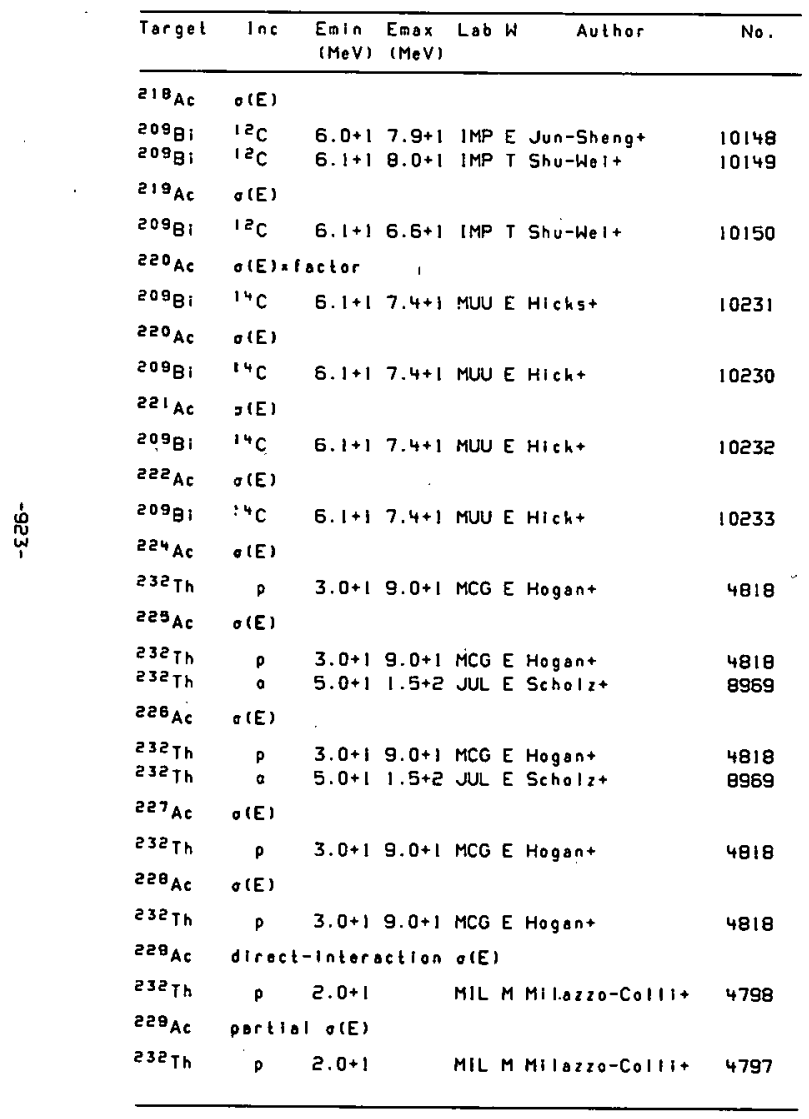

\begin{tabular}{|c|c|c|c|c|c|c|}
\hline Target & $\ln c$ & $\begin{array}{l}\text { Emin } \\
(\mathrm{MeV})\end{array}$ & $\begin{array}{l}\text { Emax } \\
(\operatorname{MeV})\end{array}$ & Lab I & Author & No. \\
\hline $229_{A C}$ & $\theta(E)$ & & & & & \\
\hline $232 \mathrm{Th}$ & p & $2.0+1$ & & MIL & M Milazzo-Collit & 4796 \\
\hline$z=90$ & $O(E)$ & & & & & \\
\hline $209_{81}$ & $14 \mathrm{~N}$ & $8.5+1$ & $9.5+1$ & JCL & E Nomurat & 10507 \\
\hline$e^{1 \theta} \mathrm{Th}$ & relati & ve prod & duct y' & reld & & \\
\hline $209 \mathrm{Br}$ & $14 \mathrm{~N}$ & $9.1+1$ & & JCL & E Nomurat & 10506 \\
\hline $218 \mathrm{Th}$ & $O\{E\}$ & & & & . & \\
\hline $209_{B I}$ & $14 \mathrm{~N}$ & $7.0+1$ & $9.6+1$ & JCL & E Nomura+ & 10505 \\
\hline $225 \mathrm{Th}$ & $O(E)$ & & & & & \\
\hline $232 \mathrm{Th}$ & 。 & $3.0+1$ & $1.1+2$ & OSA & ENishit & 8972 \\
\hline $229 \mathrm{Th}$ & $O(E)$ & & & & · & \\
\hline $\begin{array}{l}232 \mathrm{Th} \\
232 \mathrm{Th}\end{array}$ & : & $\begin{array}{l}3.0+1 \\
5 \cdot 0+1\end{array}$ & $\begin{array}{l}1.1+2 \\
1.5+2\end{array}$ & $\begin{array}{l}\text { OSA } \\
\text { JUL }\end{array}$ & $\begin{array}{l}\text { E Nighi,+ } \\
\text { E Seholiz+ }\end{array}$ & $\begin{array}{l}8968 \\
8969\end{array}$ \\
\hline $226 \mathrm{Th}$ & $\sigma(E)$ & & & & & \\
\hline $232 \mathrm{Th}$ & a & $3 \cdot 0+1$ & $1.1+2$ & OSA & ENishit & 8972 \\
\hline $228 \mathrm{Th}$ & $\theta(E)$ & & & & & \\
\hline $\begin{array}{l}232 \mathrm{Th} \\
232 \mathrm{Th}\end{array}$ & p & $\begin{array}{l}1.3+1 \\
3 \cdot 0+1\end{array}$ & $\begin{array}{l}9.0+1 \\
1.1+2\end{array}$ & MCG & $\begin{array}{l}\text { E Hogont } \\
\text { E Nishit }\end{array}$ & $\begin{array}{l}4817 \\
8968\end{array}$ \\
\hline $232 \mathrm{Th}$ & a & $5.0+1$ & $1.5+2$ & JUL & E Scholz+ & 8969 \\
\hline $22^{7} \mathrm{Th}$ & $\theta(E)$ & & & & & \\
\hline $232 \mathrm{Th}$ & a & $3.0+1$ & $1.1+2$ & OSA & E Nighit & 8972 \\
\hline $227 \mathrm{Th}$ & $O(E)$ & & & & & \\
\hline $\begin{array}{l}232 \mathrm{Th} \\
232 \mathrm{Th}\end{array}$ & $\begin{array}{l}p \\
a\end{array}$ & $\begin{array}{l}1.3+1 \\
3 \cdot 0+1\end{array}$ & $\begin{array}{l}9.0+1 \\
1.1+2\end{array}$ & $\begin{array}{l}\text { MCG } \\
\text { OSA }\end{array}$ & $\begin{array}{l}\text { E Hogon- } \\
\text { E Nishi- }\end{array}$ & $\begin{array}{l}4817 \\
8960\end{array}$ \\
\hline $232 \mathrm{Th}$ & a & $5.0+1$ & $1.5+2$ & JUL & E Sehol:- & 8969 \\
\hline $231 \mathrm{Th}$ & $O(E)$ & & & & & \\
\hline $\begin{array}{l}232 \mathrm{Th} \\
232 \mathrm{Th}\end{array}$ & p & $\begin{array}{l}1.6+1 \\
1.3+1\end{array}$ & $\begin{array}{l}2.0+1 \\
9.0+1\end{array}$ & $\begin{array}{l}\text { JYV } \\
\text { MCG }\end{array}$ & $\begin{array}{l}\text { E Cellert } \\
\text { E Hogont }\end{array}$ & $\begin{array}{l}4799 \\
4817\end{array}$ \\
\hline $232 \mathrm{Th}$ & pertla & l relat & blue ol & (E), & & \\
\hline $232 \mathrm{Th}$ & ${ }^{86} \mathrm{Kr}$ & $3.5+2$ & $4.6+2$ & ORL & E Guidrs+ & 12599 \\
\hline
\end{tabular}




\begin{tabular}{|c|c|c|c|c|c|c|}
\hline Target & $\ln c$ & $\begin{array}{l}\text { Emin } \\
(\text { reev) }\end{array}$ & $\begin{array}{l}\text { Emax } \\
(\operatorname{MeV})\end{array}$ & Lab & Author & No. \\
\hline $232 \mathrm{Th}$ & \multicolumn{6}{|c|}{ relative $O(E)$} \\
\hline $230 \mathrm{Th}$ & $t$ & NDG & & NTA & T Ragnarssont & 6070 \\
\hline $232 \mathrm{Th}$ & $O(E)$ & & & & & \\
\hline $232 \mathrm{Th}$ & $p$ & $5,0+0$ & $1.9+1$ & JYV & E Cellert & 4800 \\
\hline $226 \mathrm{~PB}_{\mathrm{B}}$ & $O(E)$ & & & & & \\
\hline $\begin{array}{l}232 \mathrm{Th} \\
232 \mathrm{Th} \\
232 \mathrm{Th}\end{array}$ & p & $\begin{array}{l}5 \cdot 0+1 \\
5 \cdot 0+1 \\
1.0+1\end{array}$ & $\begin{array}{l}1.0+2 \\
1.0+2 \\
9.0+1\end{array}$ & $\begin{array}{l}\text { KFK } \\
\text { MCG } \\
\text { MCG }\end{array}$ & $\begin{array}{l}\text { C Muenzel+ } \\
\text { E Suht } \\
\text { E Hogant }\end{array}$ & $\begin{array}{l}4801 \\
4802+ \\
4816\end{array}$ \\
\hline $227 \mathrm{~Pa}_{\mathrm{a}}$ & $O(E)$ & & & & & \\
\hline $\begin{array}{l}232 T h \\
232 T h \\
232 T h \\
232 T h\end{array}$ & $\begin{array}{l}p \\
p \\
p \\
o\end{array}$ & $\begin{array}{l}3.9+1 \\
3 \cdot 9+1 \\
1.0+1 \\
5.0+1\end{array}$ & $\begin{array}{l}1.0+2 \\
1.0+2 \\
9.0+1 \\
1.5+2\end{array}$ & $\begin{array}{l}\text { KFK } \\
\text { MCG } \\
\text { MCG } \\
\text { JUL }\end{array}$ & $\begin{array}{l}\text { C Muenzelt } \\
\text { E Sukt } \\
\text { E Hogant } \\
\text { E Scholzt }\end{array}$ & $\begin{array}{l}4803 \\
4804 \\
4816 \\
8969\end{array}$ \\
\hline $228 \mathrm{~Pa}$ & $\theta(E)$ & & & & & \\
\hline 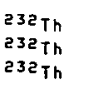 & $\begin{array}{l}p \\
a \\
a\end{array}$ & $\begin{array}{l}1.0+1 \\
1.1+2 \\
3.0+1\end{array}$ & $\begin{array}{l}9.0+1 \\
1.1+2\end{array}$ & $\begin{array}{l}\text { MCG } \\
\text { OSA } \\
\text { OSA }\end{array}$ & $\begin{array}{l}\text { E Hogant } \\
\text { E Nishit } \\
\text { E Nishit }\end{array}$ & $\begin{array}{l}4816 \\
8964 \\
8968\end{array}$ \\
\hline $230 \mathrm{~Pa}$ & $O(E) \times$ & actor & & & & \\
\hline $\begin{array}{l}232 \mathrm{Th} \\
232 \mathrm{Th}\end{array}$ & $\begin{array}{l}{ }^{6} L I \\
{ }^{\prime} L i\end{array}$ & $\begin{array}{l}3.9+1 \\
3.9+1\end{array}$ & & $\begin{array}{l}\text { LAS } \\
\text { LAS }\end{array}$ & $\begin{array}{l}\text { E Britt+ } \\
\text { E Britt+ }\end{array}$ & $\begin{array}{l}9378 \\
9476\end{array}$ \\
\hline $230 \mathrm{~PB}_{\mathrm{B}}$ & $O(E)$ & & & & & \\
\hline $\begin{array}{l}232 \mathrm{Th} \\
232 \mathrm{Th} \\
232 \mathrm{Th} \\
232 \mathrm{Th} \\
232 \mathrm{Th}\end{array}$ & $\begin{array}{l}p \\
p \\
p \\
d \\
q\end{array}$ & $\begin{array}{l}1.0+1 \\
1.5+1 \\
1.0+1 \\
1.9+1 \\
5.0+1\end{array}$ & $\begin{array}{l}2.2+1 \\
2 \cdot 0+1 \\
9 \cdot 0+1 \\
2.6+1 \\
1.5+2\end{array}$ & $\begin{array}{l}\text { TOK } \\
\text { JYV } \\
\text { MCG } \\
\text { EON } \\
\text { JUL }\end{array}$ & $\begin{array}{l}\text { E Kudot } \\
\text { E Cellert } \\
\text { E Hogant } \\
\text { E Raot } \\
\text { E Scholzt }\end{array}$ & $\begin{array}{l}4805 \\
4806 \\
4816 \\
5919 \\
8969\end{array}$ \\
\hline $23 I_{\mathrm{PB}}$ & $o(E) \times$ & actor & & & & \\
\hline $\begin{array}{l}232 \mathrm{Th} \\
232 \mathrm{Th}\end{array}$ & $\begin{array}{l}{ }^{6} L i \\
{ }^{7} L i\end{array}$ & $\begin{array}{l}3.9+1 \\
3.9+1\end{array}$ & & $\begin{array}{l}\text { LAS } \\
\text { LAS }\end{array}$ & $\begin{array}{l}E B r i t t+ \\
\text { E Britt+ }\end{array}$ & $\begin{array}{l}9378 \\
9476\end{array}$ \\
\hline $231 \mathrm{~Pa}$ & $O(E)$ & & & & & \\
\hline $\begin{array}{l}232 \mathrm{Th} \\
232 \mathrm{Th}\end{array}$ & p & $7.0+0$ & $\begin{array}{l}5.0+1 \\
5.3+1\end{array}$ & JYV & E Cellert & $\begin{array}{l}4807 \\
4808\end{array}$ \\
\hline
\end{tabular}

\begin{tabular}{|c|c|c|c|c|c|c|}
\hline Torget & $\ln c$ & $\begin{array}{l}\text { Emin } \\
(\mathrm{MeV})\end{array}$ & $\begin{array}{l}\text { Emax } \\
(M e V)\end{array}$ & Lab W & Author & No. \\
\hline $23{ }^{2} \mathrm{Pg}$ & \multicolumn{2}{|c|}{$\sigma(E) \times f$ actor } & & & & \\
\hline $\begin{array}{l}232 \mathrm{Th} \\
232 \mathrm{Th}\end{array}$ & $\begin{array}{l}{ }_{6}{ }^{2} 1 \\
{ }^{L}-1\end{array}$ & $\begin{array}{l}3 \cdot 9+1 \\
3 \cdot 9+1\end{array}$ & & $\begin{array}{l}\text { LAS E } \\
\text { LAS E }\end{array}$ & $\begin{array}{l}\text { E Brittt+ } \\
\text { E Britt+ }\end{array}$ & $\begin{array}{l}9378 \\
9476\end{array}$ \\
\hline $2{ }^{2} \mathrm{P}_{\mathrm{B}}$ & \multicolumn{2}{|l|}{$\theta(E)$} & & & & \\
\hline $\begin{array}{l}232 T h \\
232 T h \\
232 T h \\
232 T h\end{array}$ & $\begin{array}{l}p \\
p \\
p \\
d\end{array}$ & $\begin{array}{l}7.0+0 \\
1.0+1 \\
1.0+1 \\
9.0+0\end{array}$ & $\begin{array}{l}2.0+1 \\
2.2+1 \\
9.0+1 \\
2.6+1\end{array}$ & $\begin{array}{l}\text { JYV E } \\
\text { TOK E } \\
\text { MCG E } \\
\text { BON E }\end{array}$ & $\begin{array}{l}\text { E Collert } \\
\text { E Kudot } \\
\text { E Hogant } \\
\text { E Raot }\end{array}$ & $\begin{array}{l}4809 \\
4810 \\
4816 \\
5920\end{array}$ \\
\hline $233 \mathrm{po}$ & \multicolumn{2}{|c|}{ o(E) af actor } & & & & \\
\hline $\begin{array}{l}232 \mathrm{Th} \\
232 \mathrm{Th}\end{array}$ & $\begin{array}{l}{ }^{6} L_{1} \\
{ }^{2}{ }_{1}\end{array}$ & $\begin{array}{l}3.9+1 \\
3.9+1\end{array}$ & & $\begin{array}{l}\text { LAS E } \\
\text { LAS E }\end{array}$ & $\begin{array}{l}\text { E Brittt+ } \\
\text { E Brittt }\end{array}$ & $\begin{array}{l}9378 \\
9476\end{array}$ \\
\hline $234 \mathrm{~PB}_{\mathrm{B}}$ & \multicolumn{2}{|c|}{ o(E) xfactor } & & & & \\
\hline $232 \mathrm{Th}$ & ${ }^{7} \mathrm{Li}$ & $3.9+1$ & & LAS E & E Britt+ & 9476 \\
\hline${ }^{235} \mathrm{~PB}$ & \multicolumn{2}{|c|}{ o(E) x factor } & & & & \\
\hline $\begin{array}{l}232 \mathrm{Th} \\
228_{\mathrm{U}}\end{array}$ & \multicolumn{2}{|l|}{$\sigma(E)$} & & LAS E & E Goldstonet & 9474 \\
\hline $\begin{array}{l}232 \mathrm{Th} \\
232 \mathrm{Th}\end{array}$ & a & $\begin{array}{l}3 \cdot 0+1 \\
3 \cdot 0+1\end{array}$ & $\begin{array}{l}1,1+2 \\
1.1+2\end{array}$ & $\begin{array}{l}\text { OSA E } \\
\text { OSA E }\end{array}$ & $\begin{array}{l}\text { E Nishi+ } \\
\text { E Nishit }\end{array}$ & $\begin{array}{l}8968 \\
8973\end{array}$ \\
\hline 2594 & \multicolumn{2}{|l|}{$\sigma(E)$} & & & & \\
\hline $\begin{array}{l}232 \mathrm{Th} \\
23 \mathrm{Th}\end{array}$ & : & $\begin{array}{l}3 \cdot 0+1 \\
3 \cdot 0+1\end{array}$ & $\begin{array}{l}1.1+2 \\
1.1+2\end{array}$ & $\begin{array}{l}\text { OSA E } \\
\text { OSA E }\end{array}$ & $\begin{array}{l}\text { E Nishit } \\
\text { E Nishit }\end{array}$ & $\begin{array}{l}8958 \\
8973\end{array}$ \\
\hline $230 \mathrm{U}$ & \multicolumn{2}{|l|}{$\sigma(E)$} & & & & \\
\hline $\begin{array}{l}232 T h \\
232 T h \\
232 T h\end{array}$ & $\begin{array}{l}a \\
0 \\
0\end{array}$ & $\begin{array}{l}3 \cdot 0+1 \\
5 \cdot 0+1 \\
3 \cdot 0+1\end{array}$ & $\begin{array}{l}1.1+2 \\
1.5+2 \\
1.1+2\end{array}$ & $\begin{array}{l}\text { OSA E } \\
\text { JUL E } \\
\text { OSA E }\end{array}$ & $\begin{array}{l}\text { E Nishl+ } \\
\text { E Scholz } \\
\text { E Nishit }\end{array}$ & $\begin{array}{l}8968 \\
8959 \\
8973\end{array}$ \\
\hline $232 u$ & \multicolumn{3}{|c|}{ product yield } & & & \\
\hline $\begin{array}{l}232 \mathrm{Th} \\
234 \mathrm{U}\end{array}$ & \multicolumn{3}{|c|}{ relative $O(E)$} & BRK E & E Scotto & 9593 \\
\hline $232 u$ & $\mathrm{t}$ & NDG & & NTA T & T Ragnerssont & 6071 \\
\hline $235 \mathrm{U}$ & \multicolumn{2}{|l|}{$r a w a(E)$} & & & & \\
\hline $235 \mathrm{U}$ & $p$ & $1.4+1$ & $1.4+1$ & BUC E & E Borgeat & 4847 \\
\hline
\end{tabular}




\begin{tabular}{|c|c|c|c|c|c|}
\hline Torget & inc & $\begin{array}{l}\text { Emin } \\
(M e V)\end{array}$ & $\begin{array}{l}\text { Emax } \\
(\mathrm{MeV})\end{array}$ & Author & No. \\
\hline $236 \mathrm{U}$ & parlial & $\sigma(E) \times f o$ & factor & & \\
\hline 2350 & d & $1.1+1$ & & LVP E Butlert & 5944 \\
\hline $236 \mathrm{U}$ & \multicolumn{4}{|c|}{ spectrum average product yieldafactor } & \\
\hline$\because \triangle P U$ & p & SCR & & RiC T Wallont & 4926 \\
\hline $23 \theta^{\circ}$ & \multicolumn{4}{|c|}{ partlal relative o(E), } & \\
\hline $23 \theta^{2}$ & ${ }^{40} \mathrm{Ar}$ & $1.4+2$. & $2.2+5$ & ORL E Guidry+ & 12410 \\
\hline $23 \theta^{2}$ & \multicolumn{4}{|c|}{ partiol o(E)xfactor } & \\
\hline $238_{0}$ & 150 & $1.0+2=$ & $2.0+3$ & BRK T Bertscht & 11558 \\
\hline$z=93$ & \multicolumn{3}{|c|}{ cumulative? o(E) } & & \\
\hline $209^{8}$ & ${ }^{2}{ }^{\mathrm{N}} \mathrm{Ne}$ & $1.2+2$ & & BRK $E$ Somervillet & 11911 \\
\hline $235 \mathrm{~Np}$ & \multicolumn{3}{|c|}{ o(E)afactor } & & \\
\hline $232 \mathrm{Th}$ & ${ }^{3} \mathrm{Li}$ & $3.4+1$ & & LAS E Golostonet & 9475 \\
\hline $236 \mathrm{~Np}$ & \multicolumn{3}{|c|}{ orelufactor } & & \\
\hline $232 \mathrm{Th}$ & ${ }^{7} \mathrm{Lt}$ & $3.4+1$ & & LAS $E$ Goldstonet & 9475 \\
\hline $237^{\mathrm{NF}}$ & \multicolumn{3}{|c|}{ dr(E) nfoctor } & & \\
\hline $535 \mathrm{Tt}$ & \multirow{2}{*}{$\begin{array}{l}{ }^{7} L i \\
O(E)\end{array}$} & $3.4+1$ & & LASE Goldstonet & 9475 \\
\hline $237^{\mathrm{Np}}$ & & & & & \\
\hline $238_{U}$ & \multirow{2}{*}{\multicolumn{3}{|c|}{ 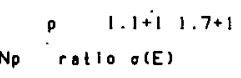 }} & FSU E Kandil. & 4891 \\
\hline $237 \cdot 9 \mathrm{gp}$ & & & & & \\
\hline $238^{2}$ & \multirow{2}{*}{$p_{\text {thick }}^{p}$} & $9.8+0$ & $1.2+1$ & MIL M Mignecot & 4892 \\
\hline $23 \theta_{\mathrm{Np}}$ & & target $y$ & yeidox & * factor & \\
\hline 2380 & ${ }^{\mathrm{T}} \mathrm{He}$ & $3.8+1$ & & JAE E Hala+ & 6603 \\
\hline $238_{\mathrm{Np}}$ & \multicolumn{3}{|c|}{ Celolive o(E) } & & \\
\hline $238^{2}$ & \multirow{2}{*}{\multicolumn{2}{|c|}{ o(E) a factor }} & $2.9+1$ & BIRE Oaim. & 6604 \\
\hline $238_{\mathrm{Np}}$ & & & & & \\
\hline $232 \mathrm{Th}$ & 'Li & $3.4+1$ & & LAS E Goldstonet & 947.5 \\
\hline $23 \theta_{\mathrm{Np}}$ & $\theta(E)$ & & & & \\
\hline $238^{U}$ & p. & $\mid .2+1$ & $1.8+1 \mathrm{f}$ & FSU E Kandil. & 4893 \\
\hline
\end{tabular}

\begin{tabular}{|c|c|c|c|c|c|c|}
\hline Target & $\operatorname{lnc}$ & $\begin{array}{l}\text { Emin } \\
\text { (MeV) }\end{array}$ & $\begin{array}{l}\text { Emax } \\
(\operatorname{MeV})\end{array}$ & Lab & Asthor & No. \\
\hline $2{ }^{3 \theta} \mathrm{Np}$ & $O(E)$ & & & & & \\
\hline $\begin{array}{l}238 \mathrm{U} \\
239^{\mathrm{Np}}\end{array}$ & $\begin{array}{c}\sigma \\
\text { cunule }\end{array}$ & $\begin{array}{l}\text { i.e+1 } \\
\text { ative ol }\end{array}$ & (E) & FSU & E kandil. & 5958 \\
\hline $\begin{array}{l}539^{n p} \\
538^{2}\end{array}$ & $\begin{array}{c}d \\
\text { thick }\end{array}$ & $\begin{array}{l}1.2+1 \\
\operatorname{target}\end{array}$ & $\begin{array}{l}1.6+1 \\
y i e 1 d x\end{array}$ & FSu & $\begin{array}{l}\text { E Kandil. } \\
\text { lor }\end{array}$ & 5959 \\
\hline $\begin{array}{l}238^{3} \mathrm{U} \\
239_{\mathrm{Np}}\end{array}$ & $\begin{array}{l}{ }^{3} \mathrm{He} \\
\text { relat }\end{array}$ & $\begin{array}{r}3.8+1 \\
\text { ive o(E) }\end{array}$ & 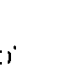 & JAE & E Hatat & 6605 \\
\hline $\begin{array}{l}23{ }^{2} \mathrm{U} \\
239^{\mathrm{NP}}\end{array}$ & $\begin{array}{l}{ }^{3} \mathrm{He} \\
\sigma(E) \times\end{array}$ & $\begin{array}{l}1.5+1 \\
\text { factor }\end{array}$ & $2.9+1$ & BIR & E Oaita. & 6606 \\
\hline $\begin{array}{l}236 \mathrm{U} \\
240 \mathrm{~Np}\end{array}$ & $\begin{array}{l}\text { PLi } \\
\text { relat }\end{array}$ & $\begin{array}{r}3.4+1 \\
\mid \text { | Ve O(E) }\end{array}$ & & LAS & E Goldslonet & 9479 \\
\hline $\begin{array}{l}230^{\circ} \mathrm{U} \\
240^{\circ} \mathrm{Np}\end{array}$ & $\begin{array}{r}{ }^{3} \mathrm{He} \\
o(E)\end{array}$ & $1.5+1$ & $2.9+1$ & BIR & E Oaim. & 6607 \\
\hline $\begin{array}{l}538^{\prime} \mathrm{U} \\
540.19 \mathrm{~N}\end{array}$ & $d x$ & $\begin{array}{r}2.4+1 \\
t 10 \quad 0(E)\end{array}$ & & GOE & E Kantugt & 5960 \\
\hline $\begin{array}{l}238^{2} \\
241 \mathrm{~Np}\end{array}$ & $\begin{array}{l}{ }^{3} \mathrm{He} \\
\text { pertic }\end{array}$ & $\begin{array}{l}1.5+1 \\
.1 \text { prods }\end{array}$ & $\begin{array}{l}2.9+1 \\
\text { fuct yio }\end{array}$ & & E Oala. & 6608 \\
\hline $\begin{array}{l}238 \mathrm{U} \\
241-\mathrm{Np}^{2}\end{array}$ & o & $\begin{array}{l}4.0+1 \\
\text { uct } y 10\end{array}$ & & BIR & E Qain. & 9044 \\
\hline $\begin{array}{l}23{ }^{8} \mathrm{U} \\
23^{4} \mathrm{Pu}\end{array}$ & $\begin{array}{c}0 \\
0(\varepsilon)\end{array}$ & $4.0+1$ & & BIR & E Gain. & 9045 \\
\hline $\begin{array}{l}233 \mathrm{U} \\
23{ }^{4} \mathrm{U} \\
23{ }^{4} \mathrm{U}\end{array}$ & $\underset{a}{{ }_{3}^{a} \mathrm{He}}$ & $\begin{array}{l}2.9+1 \\
2.2+1 \\
4.0+1\end{array}$ & $\begin{array}{l}4.0+1 \\
2.9+1 \\
4.4+1\end{array}$ & $\begin{array}{l}\text { STB } \\
\text { BRK } \\
\text { STB }\end{array}$ & $\begin{array}{l}\text { E Delagranget } \\
\text { E Somerviliet } \\
\text { E Delagranget }\end{array}$ & $\begin{array}{l}8989 \\
6591 \\
9003\end{array}$ \\
\hline $235 \mathrm{Pu}$ & portl & $.10(E)$ & & & & \\
\hline $\begin{array}{l}233 \mathrm{U} \\
233^{3} \mathrm{U}\end{array}$ & $:$ & $\begin{array}{l}2.2+1 \\
3.6+1\end{array}$ & $2.8+1$ & $\begin{array}{l}\text { STB } \\
\text { BRK }\end{array}$ & $\begin{array}{l}\text { TFleury+ } \\
\text { E Somerville+ }\end{array}$ & $\begin{array}{l}8991 \\
8992\end{array}$ \\
\hline $2{ }^{25} \mathrm{Pu}$ & $O(E)$ & & & & & \\
\hline $233 \mathrm{U}$ & 。 & $2.1+1$ & $3.0+1$ & STB & E Delagrange+ & 8990 \\
\hline
\end{tabular}




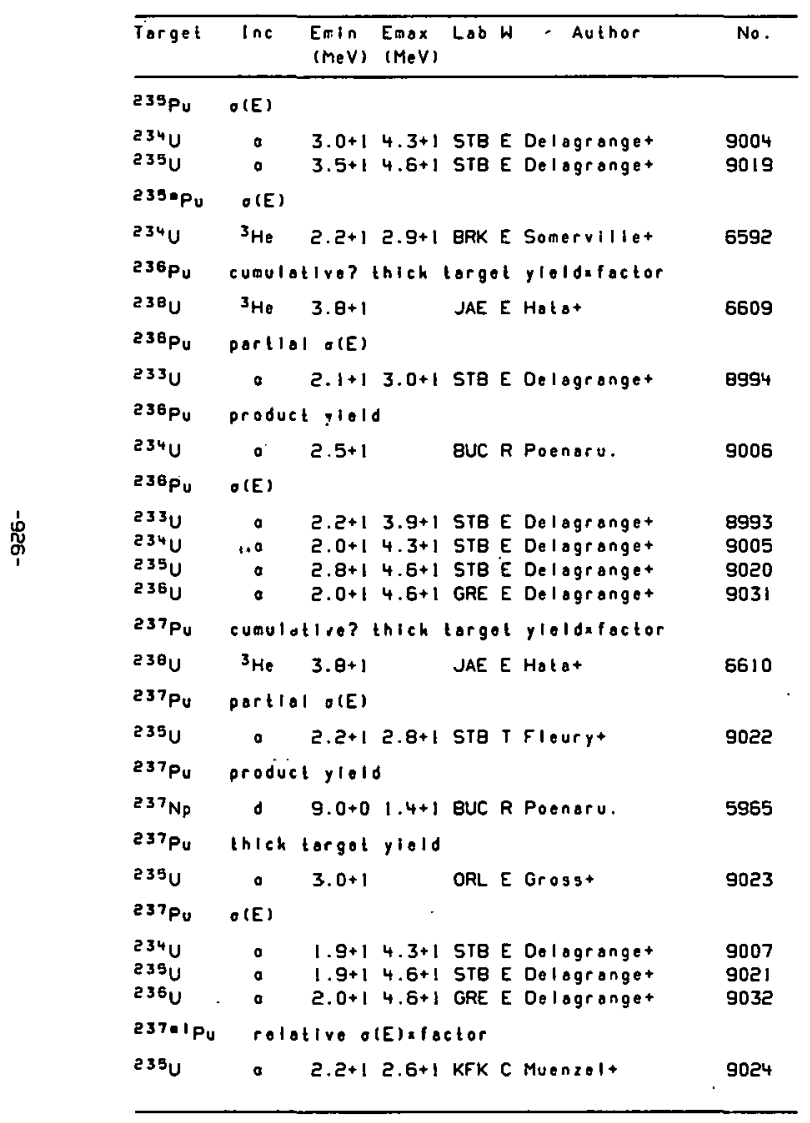

\begin{tabular}{|c|c|c|c|c|c|}
\hline Target & $\operatorname{lnc}$ & $\begin{array}{l}\text { Emin } \\
\text { (MeV) }\end{array}$ & $\begin{array}{l}\text { Emax } \\
(M e V)\end{array}$ & Lab W & No. \\
\hline $37.1 P u$ & $r e 10$ & otive ost & $(E) \times f \circ C$ & ctor & \\
\hline $\begin{array}{l}235 \mathrm{U} \\
237 \cdot \mathrm{Pu}\end{array}$ & $\stackrel{0}{\sigma(E)}$ & $2.2+1$ & $2.6+1$ & WAU $E$ Vandenboscht & $9025+$ \\
\hline $\begin{array}{l}234 \mathrm{U} \\
237.2 P_{\mathrm{Pu}}\end{array}$ & rolo & $\begin{array}{l}2.1+1 \\
\text { otive ott }\end{array}$ & $\begin{array}{l}3.0+1 \\
(E) \times f \text { oc }\end{array}$ & $\begin{array}{l}\text { STB E Delagranget } \\
\text { ictor }\end{array}$ & 9008 \\
\hline 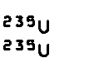 & $:$ & $\begin{array}{l}2.2+1 \\
2.2+1\end{array}$ & $\begin{array}{l}2.6+1 \\
2.6+1\end{array}$ & $\begin{array}{l}\text { KFK C Muenzel+ } \\
\text { WAU E Vandenboscht }\end{array}$ & $\begin{array}{l}9026 \\
9027+\end{array}$ \\
\hline${ }^{28} \mathrm{Pu}$ & produc & at ylold & & & \\
\hline $\begin{array}{l}236 \mathrm{U} \\
23 \mathrm{P}_{\mathrm{Pu}}\end{array}$ & $\stackrel{\circ}{\text { inleh }}$ & $\begin{array}{l}2.0+1 \\
\text { torgot }\end{array}$ & $\begin{array}{l}2.8+1 \\
y=10 x\end{array}$ & $\begin{array}{l}\text { BUC R Poenaru. } \\
\text { af actor }\end{array}$ & 9034 \\
\hline 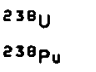 & $\begin{array}{l}{ }_{3}^{3} \mathrm{He} \\
O(E)\end{array}$ & $3.8+1$ & & JAE E Hatat & 6611 \\
\hline $\begin{array}{l}5 \\
236 \mathrm{U} \\
241 \mathrm{Pu}\end{array}$ & a & $\begin{array}{l}2.0+1 \\
\text { ct ylold }\end{array}$ & $d^{4.6+1}$ & GRE E, Delagranget & 9033 \\
\hline $\begin{array}{l}{ }^{240} \mathrm{Pu} \\
24 \mathrm{Pu}^{\mathrm{Pu}}\end{array}$ & $\stackrel{d}{\text { produc }}$ & $\begin{array}{l}1.3+1 \\
\text { ct } y \text { yeld }\end{array}$ & & BUC R Poenaru. & 5973 \\
\hline $\begin{array}{l}242 \mathrm{Pu}_{\mathrm{u}} \\
242 \mathrm{Pu}\end{array}$ & relati & $\begin{array}{l}\text { NDG } \\
\text { ive o(E) }\end{array}$ & & BUC R Poonaru. & 5974 \\
\hline 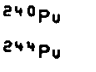 & rolot & $\begin{array}{l}\text { NDG } \\
\text { ivo } \sigma(E)\end{array}$ & & NTA T Ragnarsgont & 6074 \\
\hline $\begin{array}{l}{ }^{242} \mathrm{Pu} \\
{ }^{234} \mathrm{Am}\end{array}$ & $\stackrel{1}{\text { cumule }}$ & $\begin{array}{l}\text { NDG } \\
\text { otivo? }\end{array}$ & $O(E)$ & NTA T Ragnarssont & 6075 \\
\hline $\begin{array}{l}\begin{array}{l}233 \mathrm{U} \\
2378 \mathrm{Am}\end{array}\end{array}$ & $\begin{array}{l}{ }^{10} \theta \\
0(E)\end{array}$ & $5.5+1$ & $0.5+1$ & BRK E Somervillot & 9557 \\
\hline $\begin{array}{l}237_{\mathrm{Np}} \\
237 \cdot \mathrm{Am}\end{array}$ & $\begin{array}{l}0 \\
0(E)\end{array}$ & $\begin{array}{r}3.9+1 \\
\times \text { foctor }\end{array}$ & & STB E Fleury+ & $9067+$ \\
\hline $23 \mathrm{BPu}_{\mathrm{Pu}}$ & p & $3.0+0$ & $5.0+0$ & BUC R Poeniarut & 4931 \\
\hline
\end{tabular}




\begin{tabular}{|c|c|c|c|c|c|c|}
\hline Targe: & $\operatorname{lnc}$ & $\begin{array}{l}\text { Emin } \\
(M e V)\end{array}$ & $\begin{array}{l}\max \\
(\operatorname{MeV})\end{array}$ & Lab W & Author & No. \\
\hline $23 \theta_{A m}$ & \multicolumn{6}{|c|}{ cumulative? o(E) } \\
\hline $233_{U}$ & ' 'B & $6.0+1$ & & BRK E & E Willianst & 9595 \\
\hline $23 \theta_{A m}$ & \multicolumn{6}{|c|}{ partlal $\sigma(E)$} \\
\hline $237_{\mathrm{Np}}$ & - & $3.0+1$ & $3.7+1$ & ENL T & Tollatt & 9070 \\
\hline $2{ }^{3} \theta_{\mathrm{An}}$ & \multicolumn{6}{|c|}{ o(E) x foctor } \\
\hline $2{ }^{37} \mathrm{~Np}$ & $\circ$ & $1.9+1$ & $2.6+1$ & BNL T & T Gilott & 9069 \\
\hline $2{ }^{3} \theta_{A m}$ & \multicolumn{6}{|l|}{$\sigma(E)$} \\
\hline $237^{2} \mathrm{~Np}$ & $\circ$ & $1.9+1$ & $2.6+1$ & BNL T & T Gilott & 9068 \\
\hline $239_{9 A m}$ & \multicolumn{6}{|l|}{$c(E)$} \\
\hline $237 \mathrm{ND}$ & $\cdot$ & $2.9+1$ & & STB E & Eleury* & $9071+$ \\
\hline $230 \cdot \mathrm{Ann}$ & \multicolumn{6}{|c|}{$C(E)=$ factor } \\
\hline $237 \mathrm{~Np}$ & - & $3.0+1$ & $4.5+1$ & STB E & Eleury+ & $9073+$ \\
\hline 230.Am & \multicolumn{6}{|l|}{ - (E) } \\
\hline $237 \mathrm{~Np}$ & $\cdot$ & $3.0+1$ & $3.5+1$ & STB $E$ & Fleuryt & $9072+$ \\
\hline $23 \theta_{\mathrm{Am}}$ & \multicolumn{6}{|c|}{ Eumulatlve? o(E) } \\
\hline 2350 & 118. & $6.0+1$ & & BRK E & Willianst & 9595 \\
\hline $239_{\mathrm{Am}}$ & \multicolumn{6}{|c|}{ portlal of(E) a foctor } \\
\hline $237 \mathrm{~Np}$ & $\circ$ & $2.2+1$ & $2.8+1$ & STE T & T Fleuryt & 9078 \\
\hline $239_{A a}$ & \multicolumn{6}{|c|}{ portlal o(E) } \\
\hline $237 \mathrm{~Np}$ & a & $2.2+1$ & $3.7+1$ & BNL T & T G110t+ & 9077 \\
\hline $239 \mathrm{Ar}$ & \multicolumn{6}{|c|}{ olE) = foctor } \\
\hline $\begin{array}{l}235 \mathrm{U} \\
237 \mathrm{NF} \\
237 \mathrm{NF}\end{array}$ & $\begin{array}{l}\text { TLI } \\
\vdots \\
\vdots\end{array}$ & $\begin{array}{l}3.4+1 \\
\text { NDG } \\
2.0+1\end{array}$ & $3.5+1$ & $\begin{array}{l}\text { LAS E } \\
\text { STB T } \\
\text { ENL T }\end{array}$ & $\begin{array}{l}\text { Goldgtonet } \\
\text { Toliggranget } \\
\text { Ollot+ }\end{array}$ & $\begin{array}{l}9480 \\
9074 \\
9076\end{array}$ \\
\hline $239 \mathrm{An}$ & \multicolumn{6}{|l|}{$O(E)$} \\
\hline $2{ }^{37} \mathrm{~Np}$ & - & $2.0+1$ & $3.5+1$ & BNL T & T Gilatt & 9075 \\
\hline 239 Am & \multicolumn{6}{|l|}{$\theta(E)$} \\
\hline $\begin{array}{l}237 \mathrm{~Np}^{2} \\
237 \mathrm{~Np}\end{array}$ & $:$ & $\begin{array}{l}1.9+1 \\
2.0+1\end{array}$ & $2.3+1$ & $\begin{array}{l}\text { STB E } \\
\text { STB E }\end{array}$ & $\begin{array}{l}\text { Lint } \\
\text { Eleuryt }\end{array}$ & $\begin{array}{l}9079+ \\
9080+\end{array}$ \\
\hline
\end{tabular}

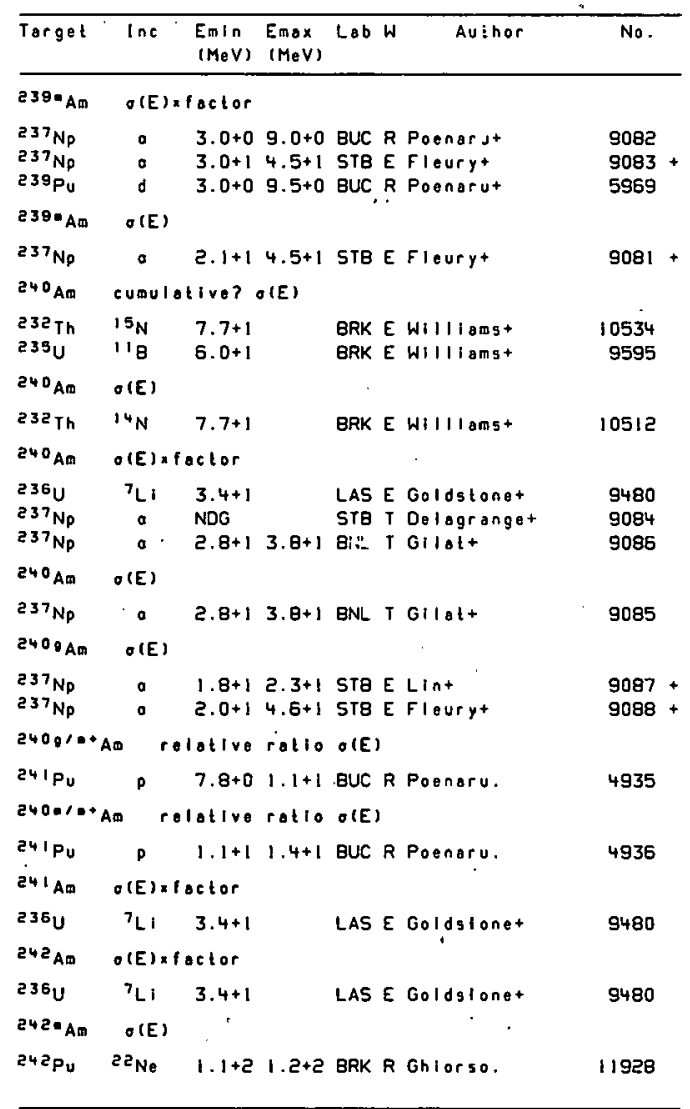




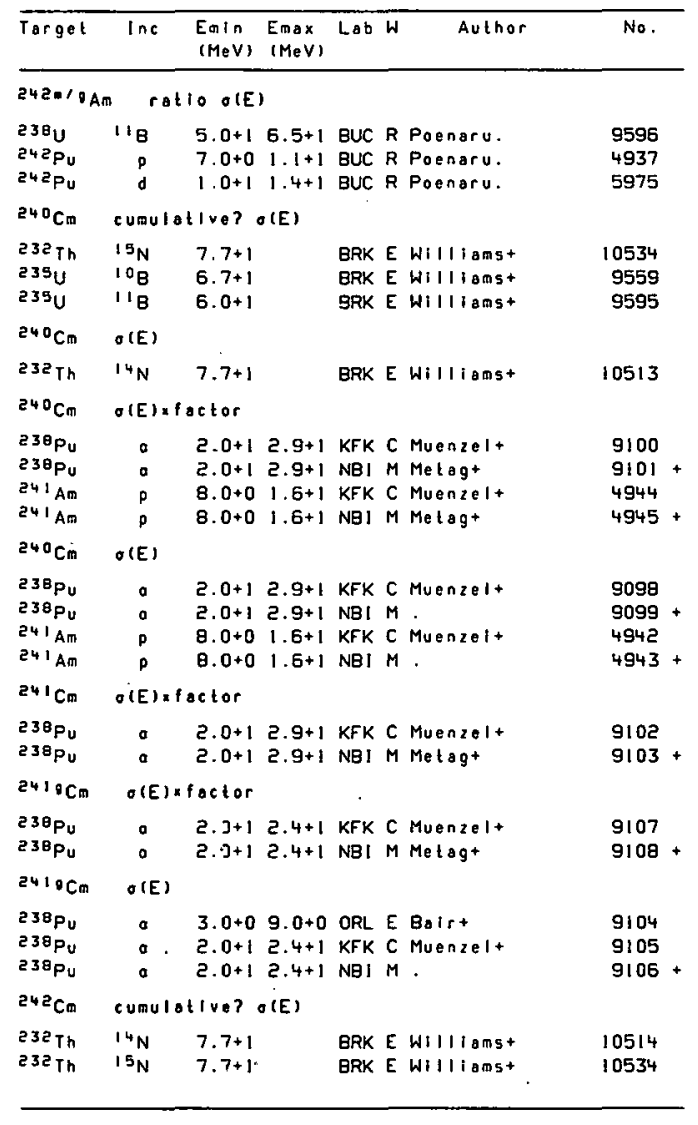

\begin{tabular}{|c|c|c|c|c|c|c|}
\hline Target & $\ln c$ & $\begin{array}{l}\text { Emln } \\
(\mathrm{MeV})\end{array}$ & $\begin{array}{l}\text { Emax } \\
(\operatorname{MeV})\end{array}$ & Lab & Author & No. \\
\hline $242 \mathrm{Cm}$ & \multicolumn{6}{|c|}{ cumulatlve? o(E) } \\
\hline $\begin{array}{l}235 \mathrm{U} \\
235 \mathrm{U}\end{array}$ & $\begin{array}{l}10 \mathrm{~B} \\
11 \mathrm{~B}\end{array}$ & $\begin{array}{l}6 \cdot 7+1 \\
6 \cdot 0+1\end{array}$ & & $\begin{array}{l}\text { BRK } \\
\text { BRK }\end{array}$ & $\begin{array}{l}\text { E Williamst } \\
\text { E Williamst }\end{array}$ & $\begin{array}{l}9559 \\
9595\end{array}$ \\
\hline $248 \mathrm{~cm}$ & \multicolumn{6}{|c|}{ relative o(E) } \\
\hline $\begin{array}{l}245 \mathrm{Cm} \\
241 \mathrm{gk}\end{array}$ & $\begin{array}{c}t \\
\sigma(E)\end{array}$ & NOG & & NTA & T Ragnarssont & 6078 \\
\hline $\begin{array}{l}235 \mathrm{U} \\
2428 k\end{array}$ & \multicolumn{6}{|c|}{ cumulative? $O(E)$} \\
\hline $\begin{array}{l}232 \mathrm{Th} \\
235_{U} \\
235_{U}\end{array}$ & $\begin{array}{l}15 \mathrm{~N} \\
10 \mathrm{~B} \\
11 \mathrm{~B}\end{array}$ & $\begin{array}{l}7 \cdot 7+1 \\
6 \cdot 7+1 \\
6 \cdot 0+1\end{array}$ & & $\begin{array}{l}\text { BRK } \\
\text { BRK } \\
\text { BRK E }\end{array}$ & $\begin{array}{l}\text { E Williamst } \\
\text { E Williamst } \\
\text { E Williamst }\end{array}$ & $\begin{array}{r}10534 \\
9559 \\
9595\end{array}$ \\
\hline $242_{B k}$ & \multicolumn{6}{|c|}{ product yiold } \\
\hline $\begin{array}{l}241 \mathrm{Am} \\
242_{\mathrm{Bk}}\end{array}$ & \multicolumn{6}{|l|}{$O(E)$} \\
\hline $\begin{array}{l}232 \mathrm{Th} \\
232 \mathrm{Th} \\
235 \mathrm{U}\end{array}$ & $\begin{array}{l}14 \mathrm{~N} \\
15 \mathrm{~N} \\
11 \mathrm{~B}\end{array}$ & $\begin{array}{l}7.7+1 \\
7.6+1 \\
5 \cdot 5+1\end{array}$ & $\begin{array}{l}9.1+1 \\
6.5+1\end{array}$ & $\begin{array}{l}\text { BRK } \\
\text { BRK } \\
\text { BRK E }\end{array}$ & $\begin{array}{l}\text { E Williamst } \\
\text { E Williamst } \\
\text { E Williamst }\end{array}$ & $\begin{array}{r}10515 \\
10533 \\
9594\end{array}$ \\
\hline $243 \mathrm{Bh}$ & \multicolumn{6}{|c|}{ cumulative? of(E) } \\
\hline $\begin{array}{l}235 \mathrm{U} \\
24{ }^{4} \mathrm{Cf}\end{array}$ & $\begin{array}{l}\text { "'o } \\
\text { compo }\end{array}$ & \multicolumn{5}{|c|}{ compound-nucleus o(E) } \\
\hline $\begin{array}{l}239 \mathrm{Pu} \\
239 \mathrm{Pu}\end{array}$ & $\begin{array}{l}14 \mathrm{~N} \\
20 \mathrm{Ne}\end{array}$ & $\begin{array}{l}\text { NDG } \\
\text { NDG }\end{array}$ & & $\begin{array}{l}\text { ORL } \\
\text { ORL }\end{array}$ & $\begin{array}{l}\text { E Hohnt } \\
\text { E Hahnt }\end{array}$ & $\begin{array}{l}10516 \\
11919\end{array}$ \\
\hline $2+4 \mathrm{Cf}$ & \multicolumn{6}{|l|}{$\theta(E)$} \\
\hline $\begin{array}{l}239 \mathrm{Pu} \\
239 \mathrm{Pu} \\
239 \mathrm{Pu} \\
239 \mathrm{Pu}\end{array}$ & $\begin{array}{l}12 \mathrm{C} \\
14 \mathrm{~N} \\
150 \\
20 \mathrm{Ne}\end{array}$ & $\begin{array}{l}\text { NDG } \\
\text { NDG } \\
\text { NDG } \\
\text { NDG }\end{array}$ & & $\begin{array}{l}\text { ORL } \\
\text { ORL } \\
\text { ORL } \\
\text { ORL }\end{array}$ & $\begin{array}{l}\text { E Hahnt } \\
\text { E Hahnt } \\
\text { E Hahnt } \\
\text { E Hahnt }\end{array}$ & $\begin{array}{l}10158 \\
10517 \\
11559 \\
11918\end{array}$ \\
\hline $245 \mathrm{Cf}$ & \multicolumn{6}{|c|}{ compound-nucleus o(E) } \\
\hline $\begin{array}{l}239 \mathrm{Pu}_{\mathrm{u}} \\
239 \mathrm{Pu}\end{array}$ & $\begin{array}{l}14 \mathrm{~N} \\
20 \mathrm{Ne}\end{array}$ & $\begin{array}{l}\text { NDG } \\
\text { NDG }\end{array}$ & & $\begin{array}{l}\text { ORL } \\
\text { ORL }\end{array}$ & $\begin{array}{l}\text { E Hehnt } \\
\text { E Hahnt }\end{array}$ & $\begin{array}{l}10518 \\
11921\end{array}$ \\
\hline
\end{tabular}




\begin{tabular}{|c|c|c|c|c|c|}
\hline Target & $\ln c$ & $\begin{array}{ll}\text { Emin } & \text { Emax } \\
\text { (MeV) } & (\mathrm{MeV})\end{array}$ & Lab & Author & No. \\
\hline $249 \mathrm{Cf}$ & $O(E)$ & & & & \\
\hline $\begin{array}{l}239 \mathrm{Pu} \\
239 \mathrm{Pu} \\
239 \mathrm{Pu} \\
239 \mathrm{Pu}\end{array}$ & $\begin{array}{l}12 \mathrm{C} \\
14 \mathrm{~N} \\
160 \\
20 \mathrm{Ne}\end{array}$ & $\begin{array}{l}\text { NDG } \\
\text { NOG } \\
\text { NDG } \\
\text { NDG }\end{array}$ & $\begin{array}{l}\text { ORL E } \\
\text { ORL, } \\
\text { ORL E } \\
\text { ORL E }\end{array}$ & $\begin{array}{l}\text { E Hahnt } \\
\text { E Hahnt } \\
\text { E Hahnt } \\
\text { E Hahnt }\end{array}$ & $\begin{array}{l}10159 \\
10519 \\
11560 \\
11920\end{array}$ \\
\hline $253 \mathrm{Cf}$ & relati & ve $\dot{o}(E)$ & & & \\
\hline $\begin{array}{l}254 \mathrm{Es} \\
256 \mathrm{Cf}\end{array}$ & $\begin{array}{c}t \\
\text { produc }\end{array}$ & $\begin{array}{l}1.6+1 \\
y / 010\end{array}$ & LAS E & E Lougheedt & 6085 \\
\hline $254 \mathrm{Cf}$ & $\mathfrak{l}$ & $1.6+1$ & LAS E & E Hoffmant & 6082 \\
\hline $234^{\circ} \mathrm{Eg}$ & relot & ive o(E) & & & \\
\hline $\begin{array}{l}254 \mathrm{Es} \\
25{ }^{255}\end{array}$ & $\begin{array}{c}t \\
\text { relat }\end{array}$ & $\begin{array}{c}1.6+1 \\
\text { ve } o(E)\end{array}$ & LAS E & E Lougheedt & 6085 \\
\hline $\begin{array}{l}254 \mathrm{Es} \\
256 \mathrm{Es}\end{array}$ & $\begin{array}{c}t \\
0(E)\end{array}$ & $1.6+1$ & LAS E & E Lougheed+ & 6086 \\
\hline $\begin{array}{l}254^{4} \text { s } \\
2360 \text { Es }\end{array}$ & $\begin{array}{c}\cdot 1 \\
\text { relat }\end{array}$ & $\begin{array}{l}1.6+1 \\
\text { Iye o(E) }\end{array}$ & LAS E & E Holfimant & 6084 \\
\hline $\begin{array}{l}254 \mathrm{Eg} \\
256 . \mathrm{Eg}\end{array}$ & t & $\begin{array}{l}1.6+1 \\
\text { ive o(E) }\end{array}$ & LAS E & E Lougheedt & 6086 \\
\hline $\begin{array}{l}254 \mathrm{Es}^{25} \\
25 \mathrm{C}^{2}\end{array}$ & $\begin{array}{c}t \\
\sigma(E)\end{array}$ & $1.6+1$ & LASE & E Lougheedt & 6086 \\
\hline $\begin{array}{l}257 F_{m} \\
254 \\
F m\end{array}$ & $\begin{array}{c}t \\
\text { reloti }\end{array}$ & $\begin{array}{c}1.6+1 \\
v 0(E)\end{array}$ & LAS E & E Hoffmant & 6087 \\
\hline $\begin{array}{l}25{ }^{4} \mathrm{Es} \\
255_{\mathrm{Fm}}\end{array}$ & $\operatorname{lil}_{\text {reti }}$ & $\begin{array}{c}1.6+1 \\
\text { vo } \sigma(E)\end{array}$ & LAS E & E Lougheedt & 6086 \\
\hline $\begin{array}{l}254 \mathrm{Es}_{\mathrm{s}} \\
256 \mathrm{Fm}\end{array}$ & $\begin{array}{c}\text { comul: } \\
\text { com }\end{array}$ & $\begin{array}{l}\text { l.6+1 } \\
\text { live o(E) }\end{array}$ & LAS E & Lougheedt & 5086 \\
\hline $\begin{array}{l}25 \mathrm{Cf} \\
253 \mathrm{Es}\end{array}$ & $\begin{array}{l}15 c \\
118\end{array}$ & 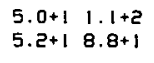 & $\begin{array}{l}\text { BRK E } \\
\text { BRK E }\end{array}$ & $\begin{array}{l}\text { Somervillet } \\
\text { Somervillet }\end{array}$ & $\begin{array}{r}10161 \\
9598\end{array}$ \\
\hline
\end{tabular}

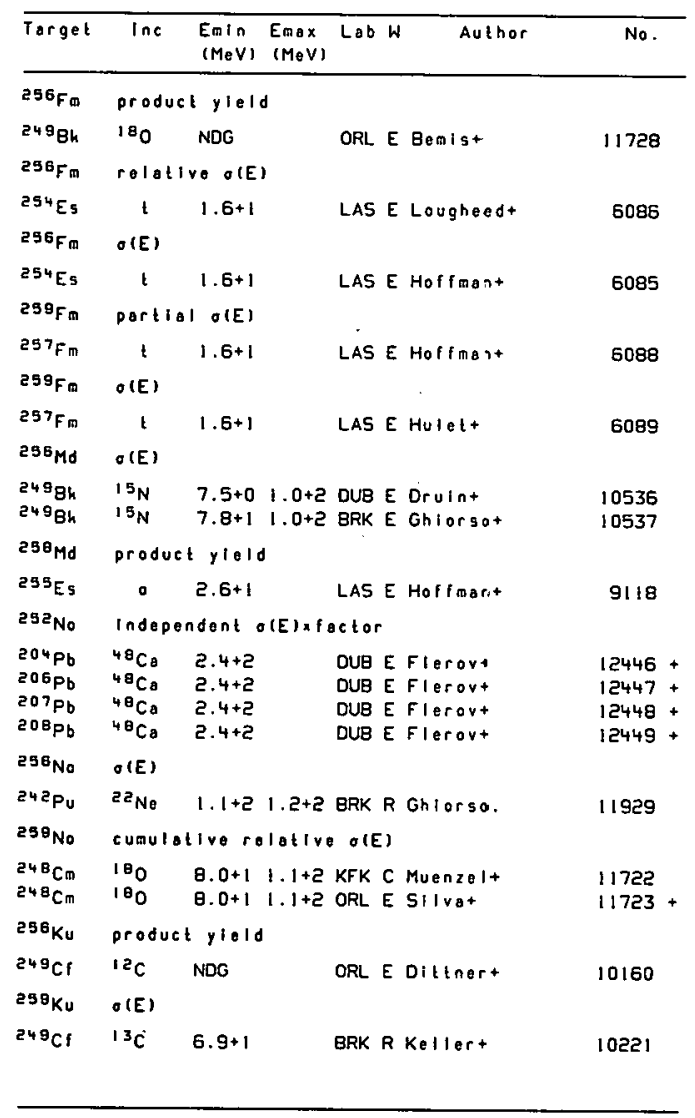




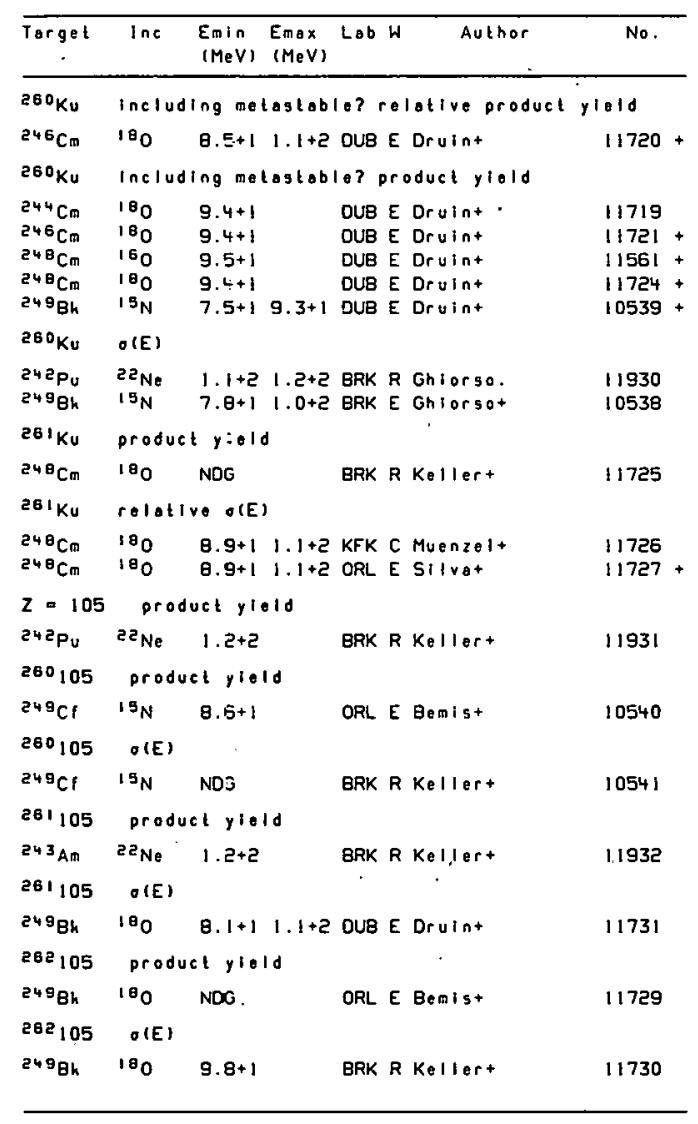

\begin{tabular}{|c|c|c|c|c|c|c|}
\hline Target & $\operatorname{lnc}$ & $\begin{array}{l}\text { Emin } \\
(\mathrm{MeV})\end{array}$ & $\begin{array}{l}\text { Emax } \\
(M e V)\end{array}$ & Lab W & Author & No. \\
\hline 262105 & $O(E)$ & & & & & \\
\hline $\begin{array}{l}249 \mathrm{gk} \\
263105\end{array}$ & $\begin{array}{l}10^{10} \\
O(E)\end{array}$ & $8 \cdot 1+1$ & $1.1+2$ & DUB E & Orulnt & 11731 \\
\hline $\begin{array}{l}249 \mathrm{gh} \\
284105\end{array}$ & $\begin{array}{l}1 \theta_{0} \\
\sigma(E)\end{array}$ & $8.1+1$ & $1.1+2$ & OUB E & Oruint & $1173 \mid$ \\
\hline $\begin{array}{l}249^{8 k} \\
299_{106}\end{array}$ & $\begin{array}{l}10_{0} \\
\text { produ }\end{array}$ & $\begin{array}{l}8.1+1 \\
c t y 1.01\end{array}$ & $1.1+2$ & DUB E & Oruint & 11731 \\
\hline $\begin{array}{l}207 \mathrm{~Pb} \\
20 \mathrm{~B}^{\mathrm{Pb}} \\
282106\end{array}$ & $\begin{array}{c}{ }^{54} \mathrm{Cr} \\
{ }^{54} \mathrm{Cr} \\
{ }_{0}(E)\end{array}$ & $\begin{array}{l}\text { NDG } \\
\text { NDG }\end{array}$ & & $\begin{array}{l}\text { BRK } R \\
\text { BRK } R\end{array}$ & $\begin{array}{l}\text { Kellert } \\
\text { Kellert }\end{array}$ & $\begin{array}{l}12460 \\
12461\end{array}$ \\
\hline $\begin{array}{l}249^{9} \mathrm{ff} \\
283108\end{array}$ & $\begin{array}{l}180 \\
\text { produ }\end{array}$ & $\begin{array}{l}8.1+1 \\
\text { ct } y 101\end{array}$ & $1.1+2$ & OUB E & Druint & 11733 \\
\hline $\begin{array}{l}24{ }^{9} \mathrm{Cf} \\
263106\end{array}$ & $\begin{array}{l}180 \\
\sigma(E)\end{array}$ & $9.5+1$ & & BRK $R$ & Kellert & 11732 \\
\hline $\begin{array}{l}24{ }^{9} \mathrm{Cf} \\
284106\end{array}$ & $\begin{array}{l}10^{\circ} \\
O(E)\end{array}$ & $8 \cdot 1+1$ & $1.1+2$ & DUB E & Drulnt & 11733 \\
\hline $\begin{array}{l}249 \mathrm{Cf} \\
294116\end{array}$ & $\begin{array}{l}180 \\
\text { eompo }\end{array}$ & $\begin{array}{l}8.1+1 \\
\text { und-nuc }\end{array}$ & $\begin{array}{l}1.1+2 \\
\text { cleus }\end{array}$ & $\begin{array}{l}\text { DUB E } \\
\sigma(E)\end{array}$ & Druint & 11733 \\
\hline $2{ }^{48} \mathrm{Ca}$ & ${ }^{48} \mathrm{Co}$ & NDG & & BRK $R$ & Kellert & 12455 \\
\hline
\end{tabular}


APPENDIX A--ABBREVIATIONS FOR MOLECULES AND MIXTURES

$t \mathrm{np}$

Compound. Informotion given in comment field.

$0 \times \mathrm{i}$

oxide.

vir Water. 
1. REFERENCE FIELD ORGANIZATION

The reference field in the section is composed of a reference type, on abbreviation of the reference. and publication date. The reference type is the first character in the field and is explained below. The abbeviation of the reference differs for references found in published periodicals, conference contributions and proceedings. books. numbered eports, and unpublished material (see next paragraph for the organization of the refeence). The fublication date is a three character code at the end of the field. Tne first character is the month the numbers 1 through 9 for the months january through September and the letters 0 . N. and $D$ for the months of October. November. and Decemberl. The last two characters are the last two numbers of the year of publicaticn (e.e., 1976=76).

2. REFERENCE (see beloo for expansions)

Generally speaking the sources of information fall into five catagories which are treated differently.

a. Published periodicals

b. Conference contributions and proceedings and books

c. Numbered reports

d. Unpublished material and private commutcations

e. Numerical data in computer filles

a. Published periodicals. Here the reference usually conglsts of a tuo to four letter abreviation of the pertadical, followed by the volume number and the page number.

EXAMPLE NP/A $274 \quad 202$

Nucl. Phys. A274, 202

b. Conference contributions and proceedings and books. In almost all cases, conferences are identified by the last two digits of the conference year. followed by an abreviation of its location. Books are indentilied by an abbreviation of their title. In both cases the code is, at most, B characters long.

EXAMPLE 65ANTWER

The International Conference on the Siudy of Nuclear Structure with Neutrons. Antwerp(1965).

c. Numbered reports. The customary report abbreviation is entered with the report number. The page is also ineluded if there is room in the field lsee comment field for page number in other cases).

\section{EXAMPLE OAP-42?}

Orange Aid Preprints-422. Cal. Inst. of Tech.

d. Unpublished material and private communications. Theses, private communications. preprinls. and unnumbered annual reports received by the center hove also been included, as well as similar information received by centers compiling charged-particle data into the internotional exchange format(EXFOR). Private communications cited by authors in a published paper have also been included.

Such material is entered with the author's name, with information about who cited or recelved the private communication. or other information such as TO BE PUBLISHED. ANNUAL REPORT, or TO BE COMPLETE in the reference fleld.

e. Numerical data in computer files. Numerical data stored in generally available computer libraries are indexed by a codedefining the library and an accession number. by which the data sets may be identified when requesting them fron a data center.

EXAMPLE EXFORBO004.002

Data available from EXFOR library.

Accession number is 80004.002

- At the present time only the EXFOR library is generally available. 


\title{
REFERENCE TYPE CODES
}

\author{
A Abstract \\ B Book \\ C Conference \\ J Journal \\ P Progress Report \\ R Report - Other than Projress \\ $S$ Conference pub. Ished as a Repo-t \\ $T$ Thesis or Dissertation \\ W Private Comaunication \\ 4 Data in Computerized Form \\ REFERENCE CODES
}

CONFERENCES, BOOKS, REPORTS, JOURNALS

63HOUSTO

7I ALBANY

73TRIEST

74NASH

74PETTEN

75MRYLND

75WASH

75ZUERI

76ANL-2

76ŁOWELL

$77 \mathrm{BNL}$

77 ANL -2

$77 \mathrm{NBS}$

77TENN

77TOKYO

T TRGRAZ

78HARWEL

79KNOX

FAST N.PH

PH-DAT

FAST NEUTRON PHYSICS CONF . . HOUSTON 1963

INTERNATIONAL CONFERENCE ON FAST NEUTRON PHYSICS

RICE UNIVERSITY, HOUSTON, TEXAS, 26-28 FEB 1963.

PROCEEDINGS PUBL ISHED AS PROGRESS IN FAST NEUTRON PHYSICS' BY UNIVERSITY OF CHIGAO PRESS, I963

INT. CONF. ON STATISTICAL PROPERTIES OF NUCLEI, ALBANY, 23-27 AUG.. 1971, PROC. BY PLENUM PRESS. NEW YORK, 1972 HEAVY-ION, HIGH SPIN STATES, AND NUCLEAR STRUCTURE INTERNAT IONAL EXIENDED SEMINAR ON NIICLEAR PHYSICS. TRIESTE, 17 SEPT - 21 DEC 1973

[NT.CONF. ON REACT IONS BETWEEN COMPLEX NUCLEI.

NASHVILLE, TENNESSEE, 10-14 JUNE 1974

2. INT .SYMP. ON NEUTRON CAPTURE GAMMA RAY SPECTROSCOPY AND RELATED TOPICS. PETTEN, 2-6 SEPT. I974

2. INT . CONF. ON CLUSTERING PHENOMENA IN NUCLEI COLLEGE PARK, MARYLAND, USA, 21-25 APRIL 1975 CONF. ON NUCLEAR CROSS-SECTIONS AND TECHNOLOGY. WASHINGION, D.C. 3-7 MARCH 1975

7. INT.CONF. ON CYCLOTRONS AND THEIR APPLICATIONS. ZURICH, 19-22 AUG, 1975. PROCEEDINGS PUBLISHEO IN EXPERIENTIA SUPPL. 24 (1975)

SYMP. ON MACROSCOPIC REACTIONS OF HEAVY ION COLLISIONS, ARGONNE NATIONAL LABORATORY, I-3 APRIL 1976

[NT . CONF. ON INTERACT IONS OF NEUTRONS WITH NUCLE] LOWELL, MASSACHUSETTS, 6-9 JULY 1976

SYMP. ON NEUTRON CROSS-SECTIONS FROM 10 TO $40 \mathrm{MEV}$, BROOKHAVEN NAT. LAB. NEW YORK, 3-5 MAY 1977. PROCEEDINGS PUBL ISHED AS BNL-NC5-50681 INFORMATION MEETING ON ACCELERATOR BREEDING, BROOKHAVEN NAT, LAB, 18-19 JANUARY 1977 SYMP. ON NEUTRON STANDARDS AND APPL ICATIONS. NAT.BUREAU OF STANDARDS, GAITHERSBURG, USA, 28-31 MARCH 1977 TOPICAL CONF. ON HEAVY ION COLLISIONS, FALLS CREEK, FALLS STATE PARK. TENNESSEE, 13-17 JUNE 1977 INT . CONF, ON NUCLEAR STRUCTURE, TOKYO, 5-10 SEPT . 1977 FEW BODY PROBLEMS AND NUCLEAR FORCES, 11 , GRAZ, GERMANY INT.CONF. ON NEUTRON PHYSICS AND NUCLEAR DATA FOR REACTORS AND OTHER APPLIED PURPOSES, AERE HARWELL, U.K. 25-29 SEPT. 1978 NUCLEAR CROSS SECTIONS FOR TECHNOLOGY, KNOXVILLE, TENN

FAST NEUTRON PHYSICS. EDITED BY MARION AND FOWLER.

[NTERSC IENCE, N.Y. (1960)

RUSSI AN TRANSLATION

rIZIKA BYGTRYH NEJTRONOV MOSKVA COSATOMIZDAT. I963

PHYSIK DATEN / PHYSICS DATA. COMPILATION SERIES

PUBL ISHED BY FACHINFORMAT IONSZENTRUM ENERGIE, PHYSIK, MATHEMAT IK, KARL SRUHE 
A-ETH-

A-IKO-

A-INS-

A-IPS-

$A-J C L-$

A-JYV-

A-LVP-

A-PAR-

A-RCNP-

A- TNL -

A-WAU-

AERE-PR/NP

AERE-R-

ANL -

ANL-NDM-

BLM-JB-

BNL -

$B N L-N C S-$

CEA-N-

CERN-

CONF -

COO-

CSIR-FIS-

DOE-NDC -

ERDA-NDC -

GS I -

IAEA-

INDC (CCP) -

INDC (GER) -

INDC (IRN) -

INDC ( SAF) -

INDC (SEC) -

INDC (SWD) -

INDC (UK) -

INIS-MF -

IPNO-PHN-

IPNO-RC-

ISN-

JINR-

JINR-E7-

JINR-P7-

JU-RR-

JUEL-

JUEL-SPEZ -

KDK-

KFK-

KFKI -

KHFT I -

LA-

LA-UR-

LAP-

LBL-

LSNN-

LU-NP-

LYCEN-

NASA-TM-

NEANDC (E) -

NEANDC $\{J\}-$

NEANDC IOR

NFL-

NRCC-

OAP-

ORNL-

ORNL-TM-

ORO-

OULNS-

PHOTONUCLE

SUN I -
EIDGENOSSISCHE TECHNISCHE HOCHSCHULE ANNUAL REP. INST. VOOR KERNPHYSISCH ONDERZOEK ANNUAL REPORT

U. GRENOBLE, INST SCIENCES NUCL. ANNUAL REPORT RES. INST. OF PHYSICS ANNUAL REPORT

CYCLOTRON LAB. . INST. OF PHYSICAL AND CHEMICAL

JYVAESKYLAE UNIVERSITY ANNUAL REPORT

UNIVERSITY OF LIVERPOOL ANNUAL REPORT

UNICERSITY OF PARIS (INCLUDIN:; ORSAYI ANNUAL REP.

RES.CENTRE FOR NUCL. PHYS. , OSAKA, ANNUAL REPORTS

TRIANGLE UNIVERSTIES NUCLEAR LAB. ANNUAL REPORT

UNIV. OF WASH!NGTON, SEATTLE, ANNUAL, PROG. REPORT

AERE-HARWELL REPORTS

AERE-HARWELL REPORTS

ARGONNE NATIONAL LABORATCRY REPORT SERIES

ARGONNE NATIONAL LABORATCRY REPORTS

ANHIUAL REFORT, OESCHILCUNIGERLABORATORIUH OER

BROOKHAVEN NAT IONAL LABORATORY REPORT SERIES

BROOKHAVEN NATIONAL LABORATORY REPORT SERIES

CENTRE D'ETUDES NUCLEAIRES, SACLAY, NOTE

CERN EUROP.ORG. FOR NUCL. RES. REPORTS

CONFERENCE PROCEEDINGS ISSUED AS REPORTS

CHICAGO OPERATIONS OFF ICE. A.EC, CONTRACT PEPORTS

COUNCIL FOR SCIENT. AND INDUST.RES. ,PRETORIA, REP

US DOE NUCL. OATA COMMITTEE REPORT SERIES

US ERDA NUCLEAR DATA COMMITTEE REPORT SERIES

GESELLSCHAFT FUER SCHWERIONENFORSCHUNG REPORTS

TECHNICAL REPORTS PUBLISHED BY IAEA. VIENNA

USSR REPORTS TO THE INT'L NUCL. DATA COMMITTEE

GERM.FED. REP. REPTS. TO THE INT'L NUCL.DATA COMM.

IRAN REPORTS TO THE INT'L NUCL. DATA COMMITTEE

SOUTH-AFRICAN REPORTS TO THE INT'L NUC.DATA COM.

INTERN . NUCL . DATA COMMITTEE, REPORT SERIES

SWEDISH REPORTS TO THE INT'L NUCL. DATA COMM.

BRITISH REPORTS TO THE INT 'L NUCL.DATA COMMITTEE

INIS MICROF ICHES. IDENTIFICATION ASSIGNED BY

INST.DE PHYS.NUCLEAIRE. ORSAY, PROGRESS REPORTS

INST. DE PHYS. NUCLEAIRE, ORSAY, REPORTS

U.DE GRENOBLE, INST.DES SCIENCES NUCLEAIRES RPIS

JOINT INST FOR NUCL. RES , DUBNA REPORTS

JOINT INST,FOR NUCL. RES. .OUBNA,REPORT-SERIES E7

JOINT INST,FOR NUCL.RES, OUBNA,REPORT-SERIES P7

UNIV. OF JYVAESKYLAE, DEPT. OF PHYSICS, REPORTS

KERNF ORSCHUNGSANLAGE. JUELICH, REPORTS

KERNFORSCHUNGSANLAGE JUEL ICH, PROGRESS REPORTS

SWEOISH NUCL. DATA COMMIITEE REPORTS

KERNFORSCHUNGSZENTRUM KARLSRUHE REPORT SERIES

CENTRAL RESEARCH INST. OF PHYSICS, HUNGARIAN

UKRA INSK, FIZ, TEKHIN, INST , KHARKOV REPORTS

LOS ALAMOS SCIENTIFIC LAB.REPORT SERIES

LOS ALAMOS SCIENTIFIC LAB.REPORT SERIES

CAL IF. INST . OF TECHNOLOGY LEMON AID PREPRINTS

LAWRENCE BERKELEY LAB REPORTS

INTERNAL REPT, INST. PHYSIOUE. UNIV. DE NANTES

LUND UNIV. REPORTS

UNIV. OF LYON REPORTS

NASA TECHINICAL NOTE

REPTS FROM CEC-COUNTRIES AND CEC TO NEANDC

JAPANESE REPORTS TO NEANDC

REPTS FROM MISC. OECD COUNTRIES TO NEANDC

STUDSVIK SCIENCE RES.LAB. REPORTS

NAT'L RES, COUNCIL OF CANADA, OTTAWA, REPORTS

CAL!F. INST. OF TECHNOLOGY, ORANGE AID PREPRINTS

OAK RIOGE NATIONAL LAB.REPORT SERIES

OAK RIDGE NATIONAL LAB. TECHNICAL MEMOS

OAK RIDGE OPERATIONS OFF ICE CONTRACT REPORTS

OSAKA UNIV. . LAB. OF NUCLEAR STUDIES ANNUAL REP.

PHOTONUCLEAR DATA, INST, OF NUCLEAR PHYSICS

REPTS BY SOUTHERN UNIVERSITIES NUCLEAR INST.
SWI TZERLAND

NETHERL ANDS

FRANCE

SWEDEN

JAPAN

F INL AND

U.K.

FRANCE

JAPAN

U.S.A.

U.S.A.

U.K.

U.K.

U.S.A.

U.S.A.

GERMANY, FR

U. 5.A.

U. 5.

FPANCE

U.S.A.

U.S.A.

SOUTH AFRICA

U.5.A.

U.S.A

GERMANY, FR

FRANCE

FRANCE

FRANCE

F INLAND

GERMANY,FR

GERMANY, FR

SWEDEN

GERMANY,FR

HUNGARY

U.S.S.R.

U. S.A.

U.S.A.

U.S.A.

U. S.A.

FRANCE

SWEDEN

FRANCE

U.S.A.

SWEDEN

CANADA

U.S.A.

U.S.A.

U.S.A.

U. S.A.

JAPAN

U.S.5.R.

SOUTH AFRICA 
TID-

U/KTY-

UCRL-

UJF -

UKNDC-P-

UWFDM-

YK-

ZFK-
OIV. OF TECHN. INF . .USAEC REPORTS

UNIVERSITY OF KENTUCKY REPORTS

LAWRENCE RAD. LAB. (BOTH BERKELEY AND LIVERMORE)

USTAV JAD.FYZIKY (INST.NUCL. PHYS.) REPORTS

PROGRESS REPORT FROM UKNDC

UNIV. OF WISCONSIN FUSION ENGINEERING REPORTS

JADERNYE KONSTANTY

ZENTRAL INST .F . KERNF ORSCHUNG ; ROSSENDORF , REPORTS
U.S.A.

U.S.A.

U.S.A.

CZECHOSLOV

U.K.

U.S.A.

U.S.S.R.

GERMANY,DR 


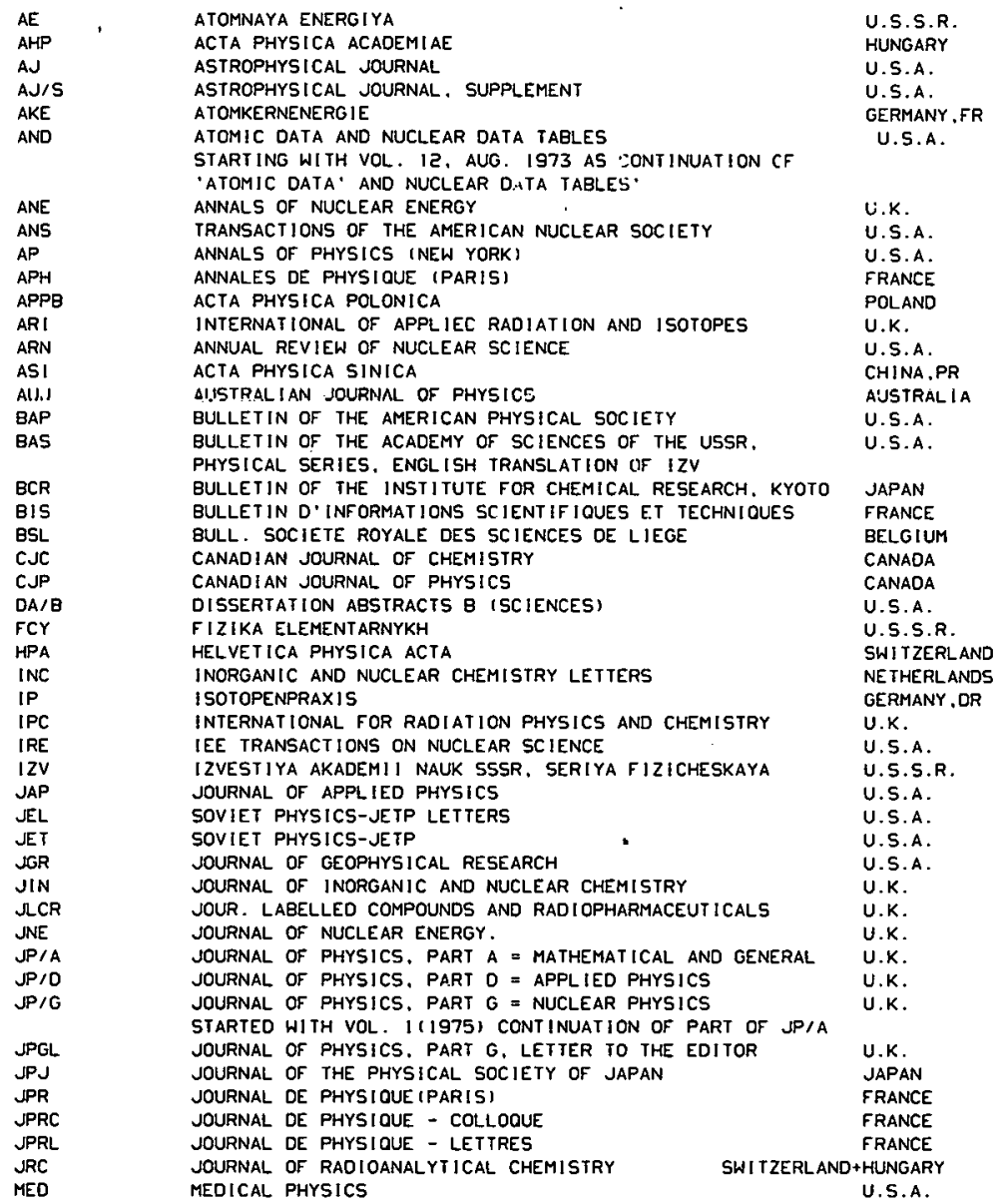




\begin{tabular}{|c|c|c|}
\hline \multirow{3}{*}{$\begin{array}{l}\text { NC } \\
\text { NC/A }\end{array}$} & NUUVO CIMENTO & ITALY \\
\hline & NUOVO CIMENTO SECTION A & ITALY \\
\hline & STARTING WITH VOL. 40 NO. 1 NOV. (1965) & \\
\hline $\mathrm{NCL}$ & LETTERE AL NUOVO CIMENTO & ITALY \\
\hline NCR & RIVISTA DEL NUOVO CIMENTO & ITALY \\
\hline NIM & NUCLEAR INSTRUMENTS AND METHODS & NETHERLANDS \\
\hline NP & NUCLEAR PHYSICS. UNTIL VOL. 89, DEC:66). & NEIHERLANDS \\
\hline NP/A & NUCLEAR PHYSICS, SECTION A. & NETHERLANDS \\
\hline NSE & NUCLEAR SCIENCE AND ENGINEERING & U.S.A. \\
\hline NUK & NUKLEONIK & GERMANY,FR \\
\hline PC & PHYSICS IN CANADA & CANADA \\
\hline PHE & PHYSICA ENERGIAE FORTIS & CHINA,PR \\
\hline PL & PHYSICS LETTERS. UNTIL VOL:. 23, DEC $(66)$. & NETHERLANDS \\
\hline $\mathrm{PL} / \theta$ & PHYSICS LETTERS. SECTION B. & NETHERLANDS \\
\hline $\mathrm{PL} / \mathrm{C}$ & PHYSICS REPORTS. A REVIEW SECTION OF PHYSICS LETTERS & NETHERLANDS \\
\hline PPS & $\begin{array}{l}\text { PROCEEOINGS OF THE PHYSICAL SOCIETY, LONDON } \\
\text { ENDS WITH VOL. } 92 \text { DEC. } 1967 \text {. CONTINUED AS JOUR. PHYSICS }\end{array}$ & U.K. \\
\hline PPSA & $\begin{array}{l}\text { PROCEEDINGS OF THE PHYSICAL SOCIETY (LONDON). SECTION A } \\
\text { EXISTING VOL. 92 OEC. } 1947-\text { VOL. 70 DEC. } 1957\end{array}$ & U.K. - \\
\hline PR & $\begin{array}{l}\text { PHYSICAL REVIEW } \\
\text { UNTIL VOL. 132(1953) AND FROM VOL. 14)(1966) }\end{array}$ & U.S.A. \\
\hline PR/A & $\begin{array}{l}\text { PHYSICAL REVIEW, PART A } \\
\text { EXISTING VOL. I33 JAN. } 1961 \text { - VOL. } 140 \text { DEC. I965 } \\
\text { STARTED WITH VOL. I IN } 1970\end{array}$ & U.S.A. \\
\hline PR/B & $\begin{array}{l}\text { PHYSICAL REVIEW, PART B } \\
\text { EXISTING VOL. I33 JAN. } 1961 \text { - VOL. } 140 \text { DEC. } 1965 \\
\text { STARTED WITH VOL. I IN } 1970\end{array}$ & U.S.A. \\
\hline$P R / C$ & $\begin{array}{l}\text { PHYSICAL REVIEW, PART C, NUCLEAR PHYSICS } \\
\text { STARTED WITH VOL. I IN } 1970\end{array}$ & U.S.A. \\
\hline PRL & PHYSICAL REVIEW LETTERS & U.S.A. \\
\hline PS & PHYSICA SCRIPIA & SWEDEN \\
\hline PTP & PROGRESS OF THEORETICAL PHYSICS & JAPAN \\
\hline RCA & RADIOCHIMICA ACTA & GERMANY,FR \\
\hline RPA & REVUE DE PHYSIQUE APPLIOUE & FRANCE \\
\hline RPP & REPORTS ON PROGRESS IN PHYSICS & U.K. \\
\hline RRP & REVUE ROUMAINE DE PHYSIOUE & ROMANIA \\
\hline RSI & REVIEW OF SCIENTIFIC INSTRUMENTS & U.S.A. \\
\hline SJA & SOVIET ATOMIC ENERGY, ENGLISH IRANSLATION OF AE & U.S.A. \\
\hline SJPN & $\begin{array}{l}\text { SOVIET JOURNAL OF PARTICLES AND NUCLEI, } \\
\text { ENGL ISH TRANSLATION OF FCY }\end{array}$ & U.S.A. \\
\hline SNP & $\begin{array}{l}\text { SOVIET JOURNAL OF NUCLEAR PHYSICS. } \\
\text { ENGLISH TRANSLATION OF YF }\end{array}$ & U.S.A. \\
\hline TAL & TALANTA & U.K. \\
\hline TRSA & PHILOSOPHICAL TRNASACTIIONS/SERIES A. ROYAL SOCIETY LONDON & NU.K. \\
\hline UFZ & UKRAINS'KII FIZICHNII ZHURNAL & U.S.S.R. \\
\hline UPJ & UKRAINIAN PHYSICS JOURNAL. ENGLISH TRANSLATION OF UFZ & U.S.A. \\
\hline YF & YADERNAYA FIZIKA & U.s.s.R. \\
\hline ZEP & $\begin{array}{l}\text { ZHURNAL EKSPERIMENTAL 'NOI I TEORETICHESKOI FIZIKI, } \\
\text { PISMA } \checkmark \text { REDAKTSIYU }\end{array}$ & U.S.S.R. \\
\hline ZET & ZHURNAL EKSPERIMENTAL'NOI I TEORETICHESKOI FIZIK! & U.S.S.R. \\
\hline ZN/A & ZEITSCHRIFT FUER NA.TURFORSCHUNG, SECIION A FROM VOL. 2 & GERMANY,FR \\
\hline $\mathrm{ZP}$ & ZEITSCHRIFT FUER PHYSIK & GERMANY,FR \\
\hline$Z P / A$ & $\begin{array}{l}\text { ZE ITSCHRIFT FUER PHYSIK, SECTION A = ATOMS AND NUCLE I } \\
\text { START ING WITH VOL. Z72 (JAN. 1975) }\end{array}$ & GERMANY.FR \\
\hline
\end{tabular}


ABBREVIATIONS FOR LABORATORIES AND INSTITUTIONS WHICH ARE ENIERED IN THE 'LAB' FIELD.

\begin{tabular}{|c|c|c|}
\hline AAU & AARHUS UNIVERSITY. AARHUS & DENMARK \\
\hline ABA & PHYSICS DEPT. ABO AKAOEMI. ABO & F INLAND \\
\hline AE & STUDSVIK ENERGITEKNIK AB & SWEDEN \\
\hline AIN & A. I.N.S.E. LUCAS HEIGHTS.NSW & AUSTRAL IA \\
\hline ALA & UNIVERSITY OF ALBERTA, EDMONTON, ALBERTA & CANADA \\
\hline ALB & STATE UNIVERSITY OF NEW YORK, ALBANY, N.Y. & U.S.A. \\
\hline ALG & ALGERIA & ALGER IA \\
\hline AML & U. OF MELBOURNE, MELBOURNE & AUSIRAL IA \\
\hline ANL & ARGONNE NATIONAL LAB, ARGONNE, ILLINOIS & U.S.A. \\
\hline ATH & NRC DEMOKRITOS, ATHENS & GREECE \\
\hline AUA & AAEC RES.EST . LUCAS HE IGHTS, NSW & AUSTRAL IA \\
\hline AUB & AUBURN UNIVERSITY, ALABAMA & U.S.A. \\
\hline AUL & AUSTRAL IA & AUSTRAL IA \\
\hline ECL & BELL TELEPHONE LABORATORIES, MIJRRAY HILI , N..I. & U.S.A. \\
\hline BER & HAHN-ME ITNER-INST . BERL IN & GSRMANY . FR \\
\hline BIR & U.OF BIRMINGHAM.ENGLAND & U.K. \\
\hline BKB & INST.BORIS KIDRICH, VINCA & YLGOSLAVIA \\
\hline BLN & BROOKLYN COLLEGE, THE CITY U. OF N.Y., BRUOKLYN, N.Y. & U.S.A. \\
\hline ENL & BROOKHAVEN NATIONAL LABORATORY, UPTON, N.Y. & U.S.A. \\
\hline $\mathrm{BOC}$ & BOCHUM, UNIV. & GERMANY, FR \\
\hline BOL & BOLOGNA, CNEN & ITALY \\
\hline BON & U.OF BONN & GERMANY.FR \\
\hline BOR & BORDEAUX, UNIV. + C.E.A. & FRANCE \\
\hline BRC & CEN BRUYERE LE CHATEL & FRANCE \\
\hline BRK & UNIV.OF CALIF. .LAWRENCE BERKELEY LAB.BFRKELEY.CALIF. & U.S.A. \\
\hline BRL & BALLISTIC RES.LABS, ABERDEEN, PROVING GROUNDS, MD & U.S.A. \\
\hline BuC & INSTITUTUL DE FIZICA SI INGINERIE NUCLEARA, BUCHAREST & ROMANIA \\
\hline EYu & BRIGHAM YOUNG UNIV. . PROVO. UTAH & U.S.A. \\
\hline BZL & BRAZIL & BRAZIL \\
\hline CAL & CAL IF. INSTITUTE OF TECHNOLOGY, PASADENA, CAL IF. & U. S.A. \\
\hline CAN & CANADA & CANADA \\
\hline CAR & CARNEGIE INSTIT. OF TECHNOLOGY,PITISBURGH,PA. & U.S.A. \\
\hline CBR & AUSTRAL I AN NATL. UNIV. ,CANBERRA & AUSTRAL IA \\
\hline $\mathrm{CCP}$ & UNION OF SOV.SOCIAL.REPUBLICS & U.S.S.R. \\
\hline CER & CERN, GENEVA, SWI TZERLAND & \\
\hline $\mathrm{CHI}$ & UNIV. OF CHICAGO, ILLINOIS & U.S.A. \\
\hline CIN & CENTRO NACIONAL DE INVEST!GACION, BUENOS AIRES & ARGENT INA \\
\hline CLA & UNIV. OF CAL IFORNIA.LOS ANGELES, CALIF. & U.S.A. \\
\hline CLU & COLORADO UNIV. . BOULOER, COLORADO & U.S.A. \\
\hline CNE & COMISION NACIONAL DE ENERGIA ATOMICA, BUENOS AIRES & ARGENT INA \\
\hline COL & COLUMBIA UNIV. NEW YORK.N.Y. & U.S.A. \\
\hline CRC & A.E.C.L., CHALK RIVER, ONIARIO & CANADA \\
\hline CSE & CASE WESTERN RESERVE UNIV. .CLEVELAND, OHIO & U.S.A. \\
\hline CTH & CHALMERS U.OF TECH. .GOTHENBURG & SWEDEN \\
\hline CUA & CATHOL IC UNIV. OF AMERICA, WASHINGION,O.C. & U.S.A. \\
\hline DAV & UNIV. OF CALIFORNIA, DAVIS, CAL IF. & U.S.A. \\
\hline DEB & ATOMKI, DEBRECEN & HUNGARY \\
\hline DUB & JOINT INST.FOR NUCL.RES. , DUBNA & \\
\hline EOG & U.OF EOINGURGH, SCOTLAND & U.K. \\
\hline ETH & EIDGENOSSISCHE TECHNISCHE HOCHSCHULE, ZURICH & SWI TZERLAND \\
\hline FE I & FIZ]KO-ENERGETICHESKIJ INST, , OBNINSK & U.S.S.R. \\
\hline FLA & UNIV. OF FLORIDA,GAINESVILLE,FLA. & U.S.A. \\
\hline FRI & CENTRAL FUEL RESEARCH INST, FRI, BIHAR & INDIA \\
\hline FRK & J.H.GOETHE UNIV. ,FRANKFURT & GERMANY.FR \\
\hline FRS & U. OF FRIBOURG & SWI TZERLAND \\
\hline FSU & FLORIDA STATE UNIV. . TALLAHASSEE, FLA. & U.S.A. \\
\hline FUL & VRI JE UNIVERSITEIT TE AMSTERDAM, DE BOELELAAN & NE THERLANDS \\
\hline GEL & BCMN GEEL & \\
\hline GEO & UNIV. OF GEORGIA , ATHENS, GEORGIA & U.S.A. \\
\hline GER & GERMANY. FED.REP. & GERMANY, FR \\
\hline GHI & UNIV, OF GHENT, GHENT & BELGIUM \\
\hline GOE & U.OF GOETTINGEN & GERMANY, FR \\
\hline GRA & CENTRE D.ETUDES NUCLEAIRES DE BORDEAUX-GRADIGNAN. & FRANCE \\
\hline GRE & CEA AND UNIV. GRENOBLE, ISERE & FRANCE \\
\hline GRN & GRONINTIFN & NE THERLANDS \\
\hline GSF & GODDARD SPACE FLJGHT CENTER & U.S.A. \\
\hline CS1 & GESELLSCHAF T FUER SCHWERI ONENFORSCHUNG. DARMSTADT & GERMANY, FR \\
\hline GVE & U. OF R.F.NFVA & SWITZERLAND \\
\hline HAM & HAMBURG, UNIVERSITAET & GERMANY,FR \\
\hline HAR & AERE, HARWELL, BERKS, ENGLAND & U.K. \\
\hline HE i & HE IDELBERG. UNI VERSITAET & GERMANY, FR \\
\hline HFA & TECHNION HAIFA & ISRAEL \\
\hline
\end{tabular}


HIROSHIMA, UNIVERSITY OF HIROSHIMA

U. OF HELSINKI, HELSINKI.

HARVARD UNIV. CAMBRIDGE, MASS.

SWIERK+ WARSZAWA, INST , BADAN JADR

INST. OF CHEMICAL PHYS. ,MOSCOW

INST ITUTO OE ENERGIA ATOMICA, SAO PALLO

KRAKOW, INST . F!Z.JADR.

INST. FIZICHESKIKH PROBLEM. MOS:_OW

INST.FUER STRAHLENPHYSIK, STUTTGART

INST. JADERNYKH ISSLEDOVANIJ A.N. UKRAINSKOI SSR, KIEV

INST. VOOR KERNPHYSISCH ONDERZOEK. AMSTERDAM

INST . OF MODERN PHYSICS, PEOPLE'S REP. OF CHINA INDIA

INDI ANA U. . INDIANA

INST . POL I TEC. NAC. , MEXICO CITY

RES. INST . OF PHYSICS IFORM.NOBEL INST.), STOCKHOLM

IMP , COLL. OF SCI + +TECHIN. LONDON

IND. INST, OF TECHNOL. .KANPUR

COMMISSARIAT A L'ENERGIE ATOMIOUE, CENTRE D.ETUDES DE

ITALY

JAERI, TOKA!

JAPAN

CYCLOTRON LAB., INST. OF PHYSICAL AND CHEMICAL RESEARCH,

JOHNS HOPKINS UNIV., BALTIMORE, MARYLAND

JUSTUS LIEBIG-UNIV. GIESSEN

KERNFORSCHUNGSANLAGE JUELICH

JYVAESKYLAE 、 UNIVERSITY

INST. JADERNOI FIZIKI, ALMA-ATA, KAZAKHSTAN

KFKI , EUDAPEST

KERNFORSCHUNGSZENTRUM. KARLSRUHE

KHARKOVSKIJ FIZIKO-TEKHN]CHESK IJ INST., KHARKOV

UNIVERS ITAET KOELN

KOSSUTH UNIV. DEBRECEN

KINGSTON, OUEEN'S UNIVERSITY

KARLSRUHE, UNIV.

KANSAS STATE UNIV. .MANHATTAN. KANSAS

KYOTO UNIV.

UNIV OF KENTUCKY LEXINGTON, KY

INST . AT.EN. 1.V.KURCHATOVA, MOSKVA

KYUSHU UNIV, DEPT OF NUCL ENG . FUKUOKA

LOS ALAMOS SC.LAB. NEW MEXICO

UNIV. OF LIEGE. LIEGE

LENINGRAD INST.NUCL. PHYS, ,USSR ACAD.SCI. , GATCHINA

LUND UNIV + TECH. UNIV.

LENINGRADSKIJ POL ITEKHNICHESKIJ INST

NASA-LEWIS RESEARCH CENTER, CLEVELAND, OHIO

UNIV. OF CALIF, LAWRENCE LIVERMORE LAB.LIVERMORE, CAL.

LAVAL UNIVERSITY, QUEBEC

U. OF LOUVAIN, LOUVAIN

U. OF L IVERPOOL ENGLAND

U. OF LYON, VILLEURBANNE, RHONE

MALAYSIA

U. OF MANCHESTER, ENGLAND

U. OF MARBURG

MCGILL UNIVERSITY, MONTREAL, OUEBEC

MCMASTER UNIVERSITY, HAMILTON, ONTARIO

U. OF MILAN

UNIV. OF MINNESOTA, MINNEAPOL IS, MINN

MASSACHUSETTS INST. OF TECH. CAMBRIDGE, MASS.

UNIV. OF MANITOBA, CYCLOTRON LAB. , WINNIPEG. MANI TOBA

U. OF MAINZ

C.E.N. MOL

UNIV, OF MONTREAL, OUEBEC

MOSCOW STATE UNIV. NUCLEAR PHYSICS INST., MOSCOW

MAX-PLANCK-INST I TUT FUER KERNPHYSIK, HEIDELBERO

NATIONAL RESEARCH COUNCIL. MONTRAL LAB

MARYLAND UNIV., COLLEGE PARK,MD

U. OF MUENSTER

MICHIGAN STATE UNIV. E.LANSING.MICH.

IDAHO NUCL, CORP. . IDAHO FALLS

MUSL IM UNIV. AL IGARH

TECHNISCHE UNIVERSITAET MUENCHEN

U. OF MUNICH.MUNICH

UNIV. OF NAGOYA

FERMI NATIONAL LAB., BATAVIA, ILLINOIS
JAPAN

F INLAND

U.S.A.

POLAND

U.S.S.R.

BRAZIL

POL AND

U.S.S.R.

GERMANY, FR

U.S.S.R.

NE THERLANDS

CHINA, PR

INDIA

U.S.A.

MEXICO

SWEOEN

U.K.

INDIA

FRANCE

1 TALY

JAPAN

JAPAN

JAPAN

U.S.A.

GERMANY,FR

GERMANY, FR

FINLAND

U.S.S.R.

HUNGARY

GERMANY.FR

U.S.S.R.

GERMANY FR

HUNGARY

CANADA

GERMANY,FR

U.S.A.

JAPAN

U.S.A.

U.S.S.R.

JAPAN

U.S.A.

BELGIUM

U.S.S.R.

SWEDEN

U.5.5.R.

U.S.A.

U.S.A.

CANADA

BELGIUM

U.K.

FRANCE

MALAYSIA

U.K.

GERMANY,FR

CANADA

CANADA

I TALY

U. S.A.

U.S.A.

CANADA

GERMANY,FR

BELGIUM

CANADA

U.S.S.R.

GERMANY, FR

CANADA

U.S.A.

GERMANY, FR

U.S.A.

U.S.A.

INDIA

GERMANY,FR

GERMANY,FR

JAPAN

U.S.A 
NIELS BOHR INST., COPENHAGEN

NATL. BUREAU OF STANDARDS. WASHINGTON.D.C.

N.CAROL INA STATE COLL., RALEIGH.N.C. NE THERLANDS

NI IGATA UNIV. . NIIGATA

INST. JOZEF STEFAN, LJUEL JANA

NATIONAL PHYSICAL RESEARCH LAB. . PRETORI.I

UNIV. OF NEW MEXICO.ALBUQUERQUE, iJ.M

UNIV.OF NOTRE DAME,S.BEND, INDIANA

US NAVAL RES.LAB., WASHINGTON.D.C.

UNIV. OF NEW SOUTH WALES

NORDI TA, DENMARK

NORTHWESTERN UNIV. ,EVANSTON, ILL.

U. OF AUCKLAND, AUCKLAND

OAK RIDGE ASSOCIATED UNIVERSITIES, OAK RIDGE, TENN.

OHIO UNIVERSITY, A THENS, OHIO

UNIV. UH UHEGUN, EULENE, OREGON

OAK RIDGE NATIONAL LAB.. TENN

OREGON SIATE UNIV., CORVALLIS, ORE.

OSAKA UNIV. OSAKA

OHIO STATE UNIVERSITY. COLUMBUS.OHIO

NAT . RESEARCH COUNCIL, OTTAWA, CNTARIO

U.OF OXFORD, OXFORO,ENGLAND

PADUA. UNI VERSITY AND LAB.NAT.LEGNARO

PAKISTAN

U. OF PARIS, (INCL. ORSAY), PARIS

UNIV. OF PENNSYLVANIA. PHILADELPHIA.PA.

$U$. OF POTCHEF STROM, POTCHEFSTROM

PRINCETON UNIVERSITY, PRINCETON. N.J.

UNIV.OF PITTSBURGH,PENNSYLVANIA

PURDUE UNIV. LAFAYETIE, INDIANA

DACCA, ATOMIC ENERGY CENTRE, RAMNA

RADIATION DYNAMICS. INC. WESTBURY. NEW YORK

RICE INST., HOUSTON, TEXAS

UNIV.OF ROCHESTER,N.Y

ZENTRAL INST , F . KERNF ORSCHUNG , ROSSENDORF

RUTGERS - THE STATE UNIV. NEW BRUNSWICK, N.J.

C.E.N. SACLAY, ESSONNE

SIMON FRAZER UNIVERSITY, BURNABY, B.C.

INST IT. OF NUCLEAR ENERGY RESEARCH, LUNG TAN SINGAPORE

STATE UNIVERSITY OF NEW YCRK. STONY BROOK, N.Y.

STANFORD UNIV. STANFORD, CALIF.

NUCL . RES. CENTRE. STRASBOURG

STATE UNIV. OF NEW YORK. BINGHAMPTON

STATE UNIVERSITY OF IOWA, IOWA CITY. IOWA

GOSUDARSTVENNYJ UNIV. ISTATE UNIV. I, LENINGRAD

NUCLEAR INST., SOUTHERN UNIV., FAURE, CAPE PROV.

U. OF SURREY, GUILFORD

STUDSVIK SCIENCE RESEARCH LABORATORY

THAILAND

TEXAS A.AND M. COLLEGE, TEXAS

TEHRAN UNIVERSITY NUCLEAR CENTRE, TEHRAN

TEL AVIV UNIVERSITY

UNIV. OF TEXAS, AUST IN, TEXAS

TECH. HOCHSCHULE, DARMST ADT

NATIONAL TSING HUA UNIV. HSIN-CHU

LEN INGRADSK IJ TEKHNOLOGICHESKIJ INST. IM. LENSOVETA

TOKYO INST.OF TECHNOLOGY, TOKYO

TRIANGLE UNIVERSITIES NUCL. LAB. ,DURHAM, NORTH CAROL INA

TOHOKU UNIV. . SENDAI

TOKYO UNIV. , TOKYO

UNIV. OF TORONTO, ONTARIO

TOMSK IJ POL ITEKHNICHESK!J INST ., TOMSK

BHABHA ATOM.RES. CENTRE, TROMBAY

UNIV, OF TSUKUBA, IBARAKI, JAPAN

DRESDEN, TECHN.UNIV

UNIVERSI TAET TUEBINÜEN

UNIV. OF BRITISH COLUMBIA, VANCOUVER,B.C.

BERN. UNIV.

U. OF ERLANGEN-NUERNBERO

UKRAINSKIJ FIZIKO-TEKHNICHESKIJ INST., KHARKOV

UNIV. OF ILLINOIS

KRAKOW. JAGELLONIAN UNIV

REZ U PRAHY
DENMARK

U.S.A.

U.S.A.

NETHERLANDS

JAPAN

YUGOSLAVIA

SOLTH AFRICA

U.S.A.

L.S.A.

U.S.A.

AUSIRAL IA

DENMARK

U.S.A.

NEW ZEALAND

U.S.A.

U.S.A.

U.S.A.

U.S.A.

U.S.A.

JAPAN

U.S.A.

CANADA

U.K.

I TALY

PAKISTAN

FRANCE

U.5. A.

SOUTH AFRICA

U.S.A.

U.S.A.

U.5.A.

BANGLA DESH

U.S.A.

U.S.A.

U.S.A.

GERMANY, DR

U.S.A.

FRANCE

CANADA

TAIWAN

SINAGAPORE

U.S.A.

U.S.A.

FRANCE

U.S.A.

U.S.A.

U.S.S.R.

SOUTH AFRICA

U.K.

SWEOEN

THA ILAND

U.S.A.

IRAN

ISRAEL

U.S.A.

GERMANY, FR

TAIWAN

U.S.S.R.

JAPAN

U.S.A.

JAPAN

JAPAN

CANADA

U.S.S.R.

INDIA

JAPAN

GERMANY.OR

GERMANY,FR

CANADA

SWITZERLAND

GEKMANY,FR

U.S.S.R.

U.S.A.

PULANU

CZECHOSLOV. 


\begin{tabular}{|c|c|}
\hline UK & UNITED KINGDOM \\
\hline UPP & U. OF UPPSALA \\
\hline USA & UNITED STATES OF AMERICA \\
\hline USP & UNIV.DE SAO PAULO, SAO PAULO \\
\hline UST & STOCKHOLM UNIVERSITY \\
\hline USU & UTAH STATE UNIVERSITY \\
\hline UTR & U. OF UTRECHT \\
\hline VIP & VIRGINIA POLYTECHNIC \\
\hline VIR & UNIV. OF VIRGINIA.CHARLOTTESVILLE, VA. \\
\hline VNV & CENTRE D'ETUDES DE LIMEIL, VILLENEUVE-SAINT-GEORGES \\
\hline WAS & WASHINGTON UNIV., ST.LOUIS, MISSOURI \\
\hline WAU & UNIV. OF WASHINGTON, SEATTLE, WASH. \\
\hline WIS & UNIV. OF WISCONSIN, MADISON, WIS. \\
\hline WIT & UNIV. OF WITWATERSRAND, JOHANRESBURG \\
\hline WMC & PHYSICS DEPT.COLLEGE OF WILLIAM AND MARY, \\
\hline WMU & WEST MICHIGAN UNIVERSITY \\
\hline WUR & EIDGENOSS ISCHES INST . FUER REAKT .FORSCH. ,WUERENL INGEN \\
\hline WWA & WARSZAWA, UNIVERSITY \\
\hline WWS & WESTERN WASHINGTON STATE COLLEGE, BELLINGHAM, WASH. \\
\hline WZ! & WEIZMANN INST., REHOVOTH \\
\hline YAL. & YALE UNIV. .NEW HAVEN, CONNECTICUT \\
\hline ZUR & ZURICH \\
\hline
\end{tabular}

U.K. SWEDEN

U.S.A.

BRAZIL

SWEDEN

U. 5. A.

NE THERLANDS

U.S.A.

U.S.A.

FRANCE :

U.S.A.

U.S.A.

U.S.A.

SOUTH AFRICA

U.S.A.

U.S.A.

SWITZERLAND

POLAND

U.S.A.

I SRAEL

U.S.A.

SWI T ZERLAND 
APPENDIX O --- SOME ABBREVIATIONS USED IN 'COMMENTS'

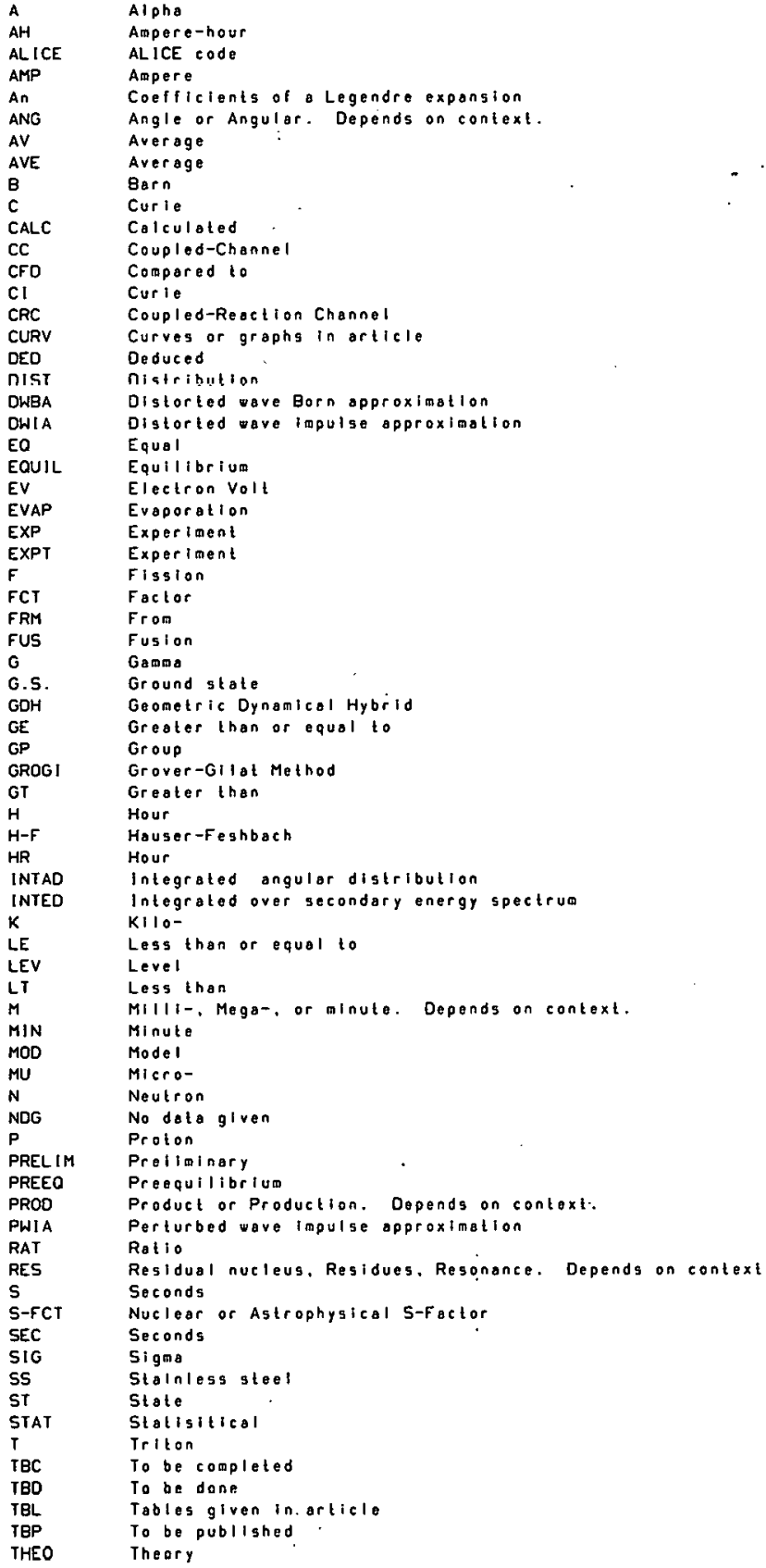

HABNDNG The reader is cautioned to refer to the orginal articles and not to any doto quoted in the coments nf this publication. 
In addition to the representation of on energy in a standard fivecharacter format (e.g. 1.7+1 means 17. Mevi, several aboreviations are employed. These are

GCR

NDG

SCR

TR

From the information given, the scanner has been able to obtain only ough estimate of the energy le.g.. +0 implies that the energy is in the Mav rongel.

Galactic Cosmic Ray Spectrum

No data given

Solar Cosmic Ray Spectrun.

Threshold 
APPENDIX F -- SYSTEMATIC STUDIES

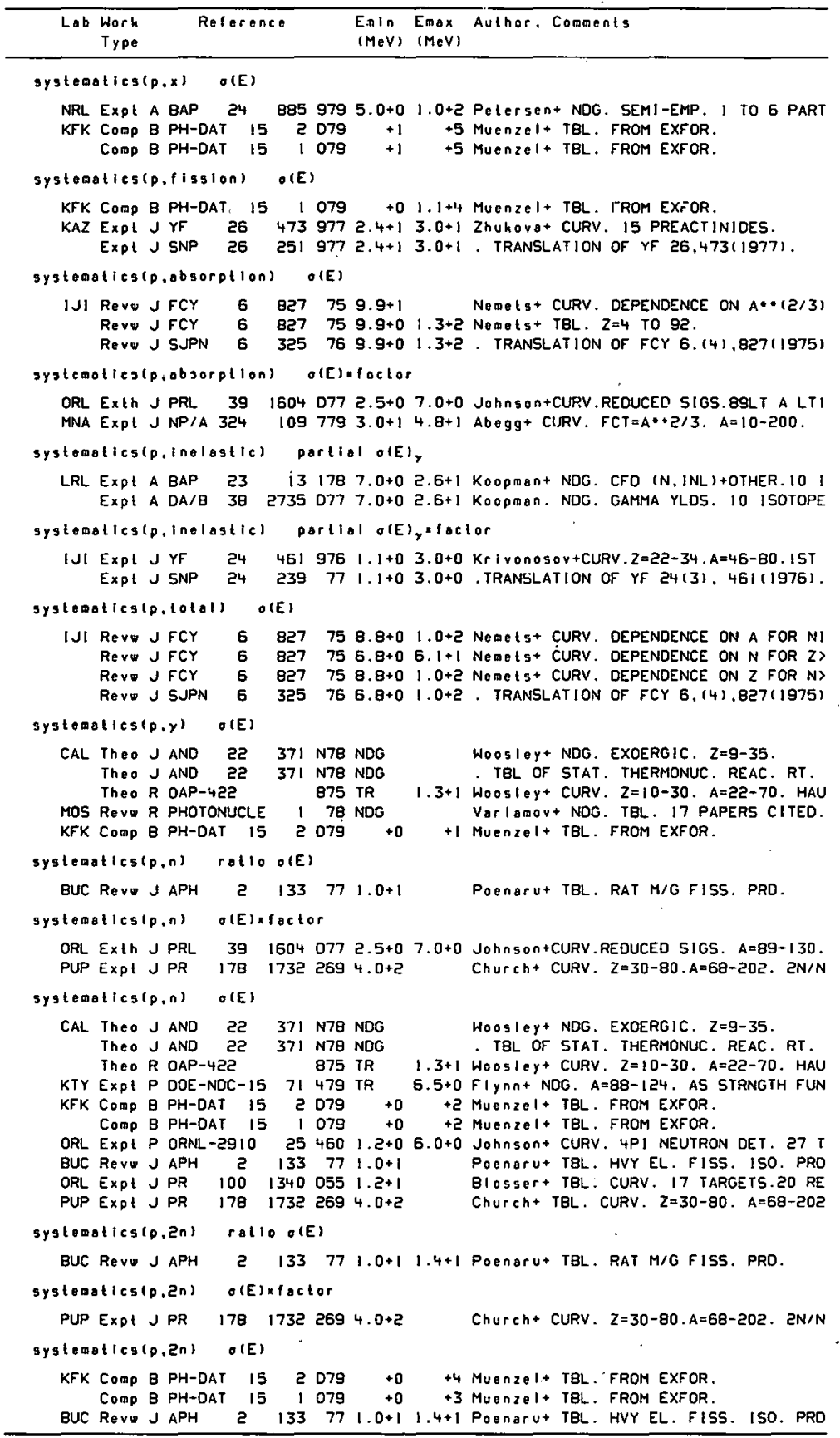


APPENDIX F -- SYSTEMATIC STUDIESICOnt)

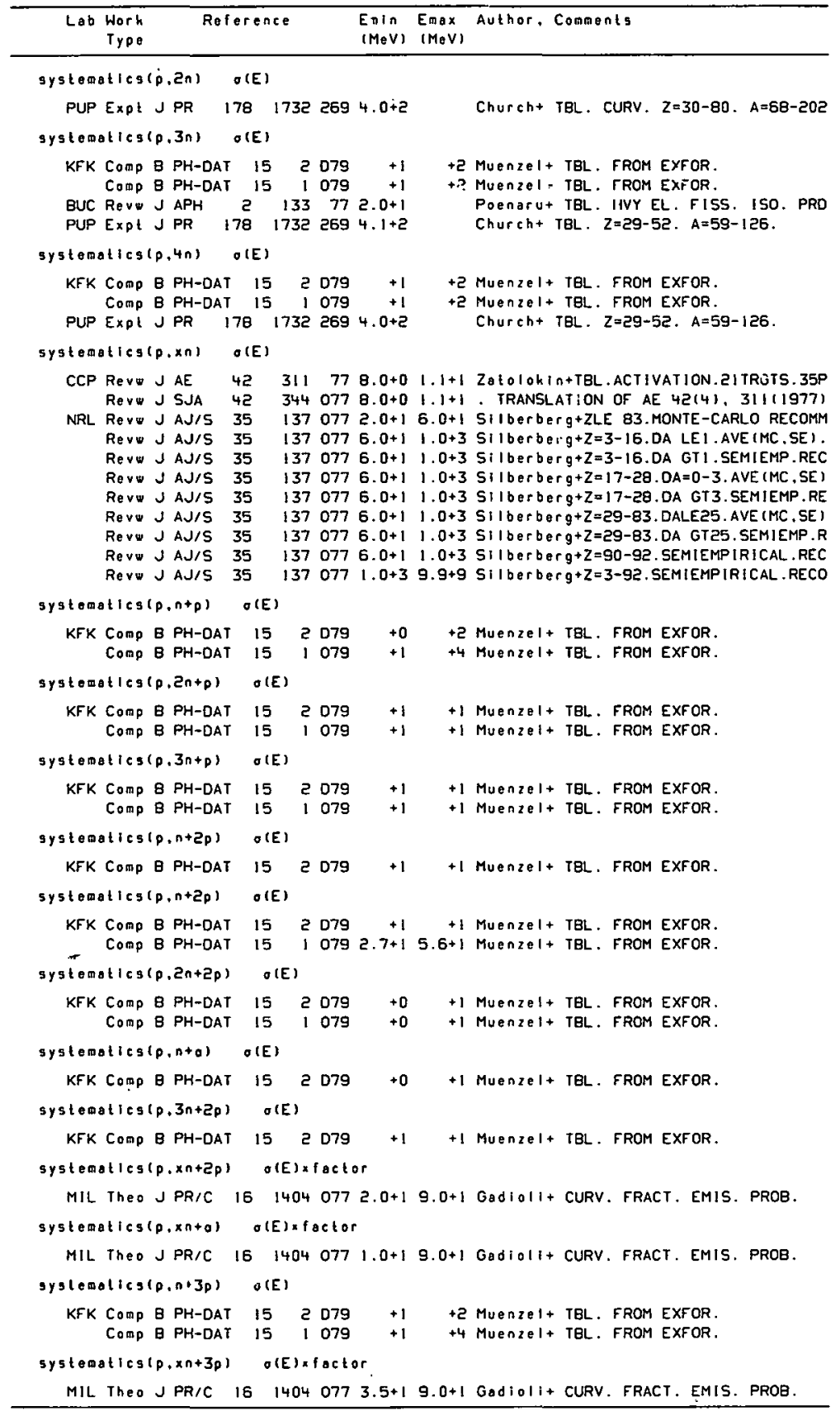




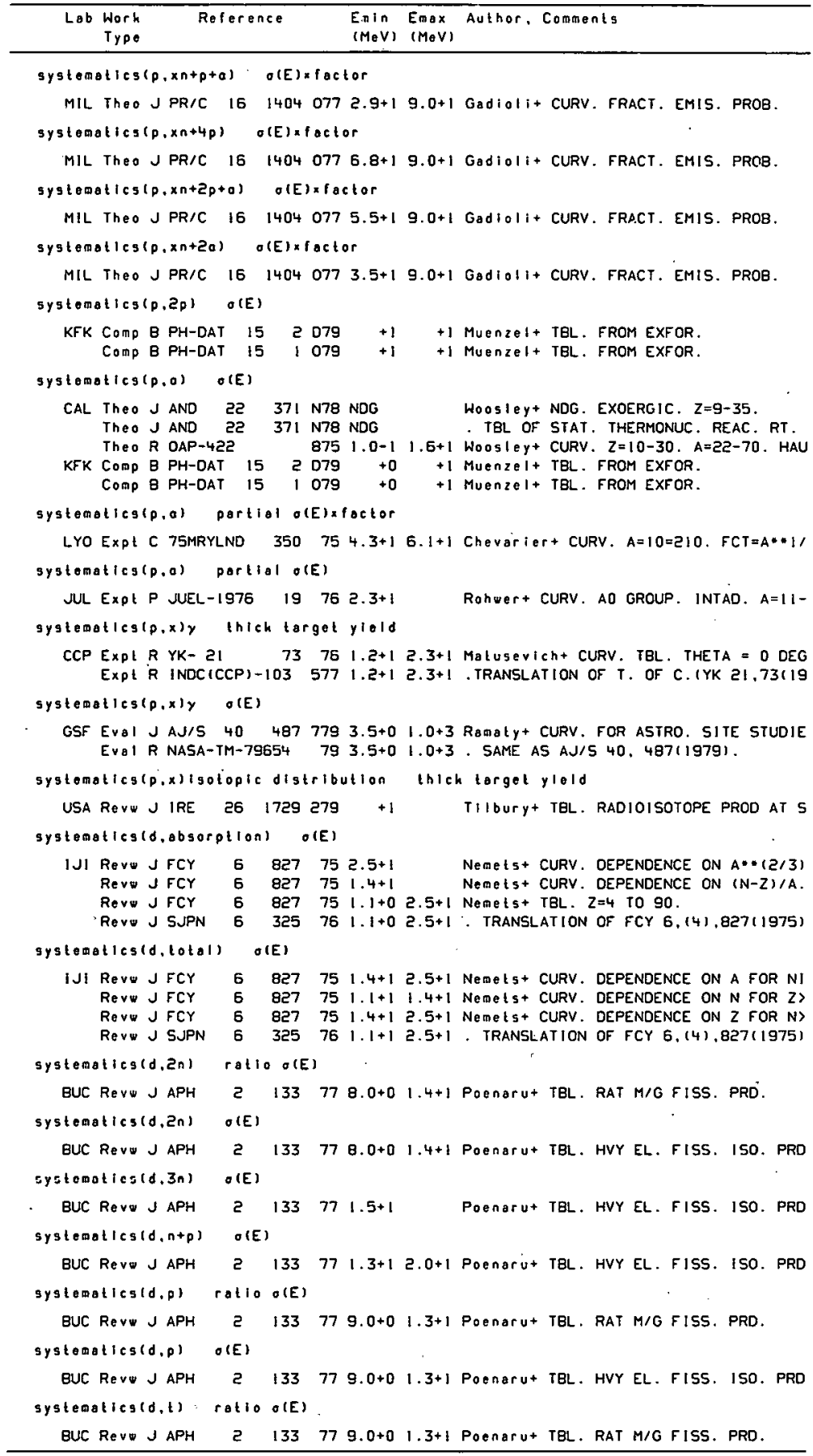




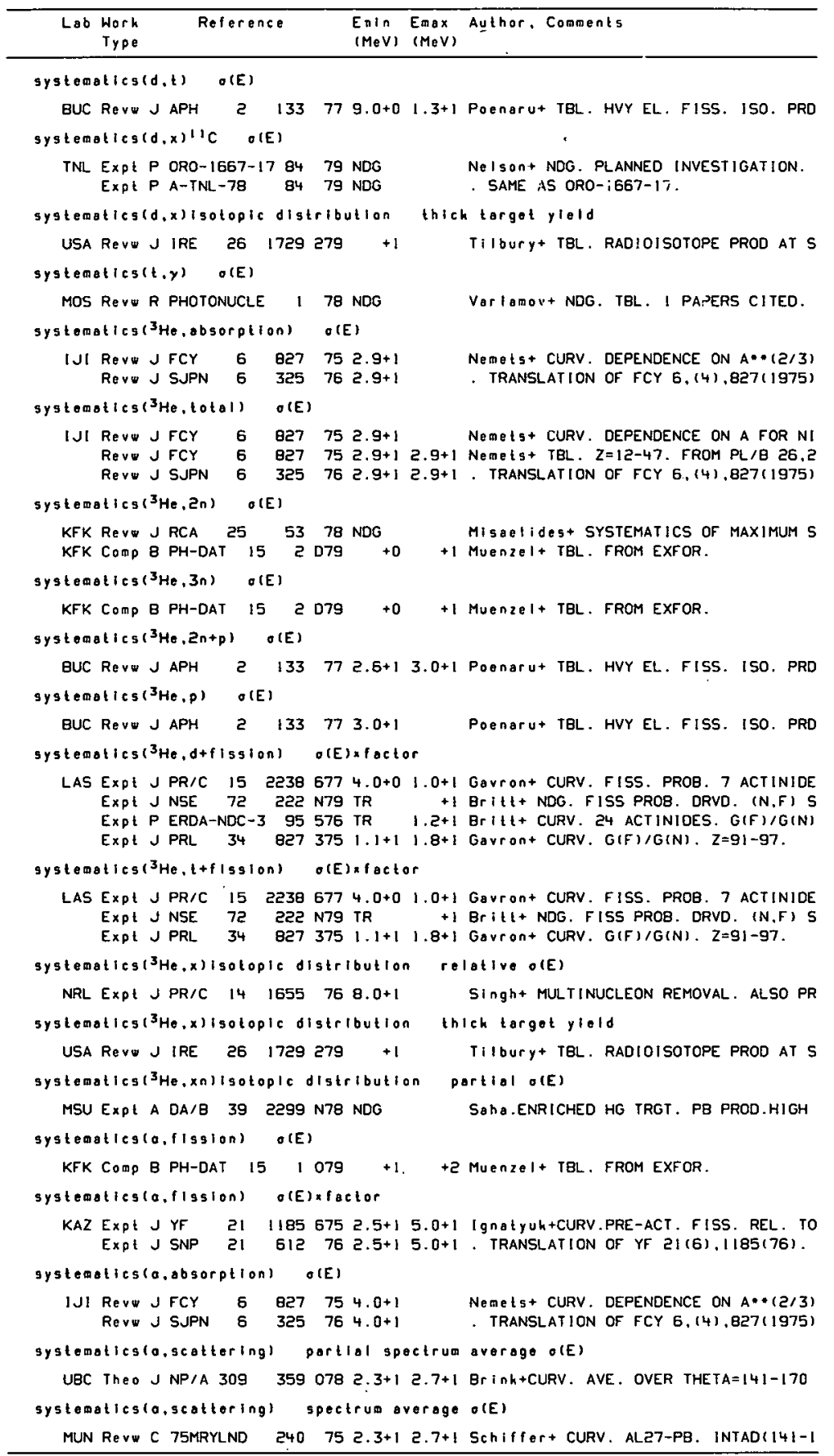


APPENDIX F --- SYSTEMATIC STUDIES(CONA)

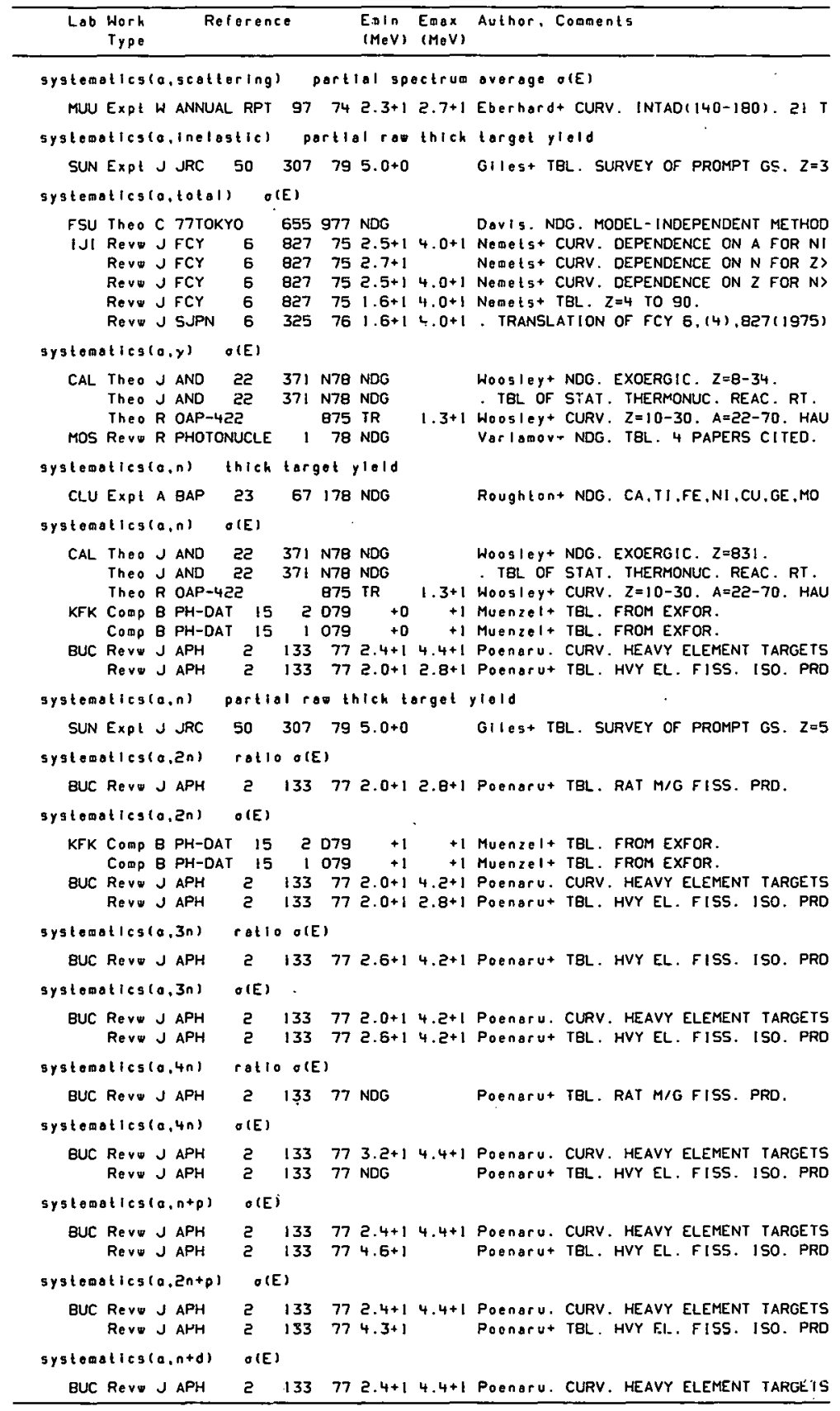


APPENDIX F --- SYSTEMATIC STUDIES(cONE)

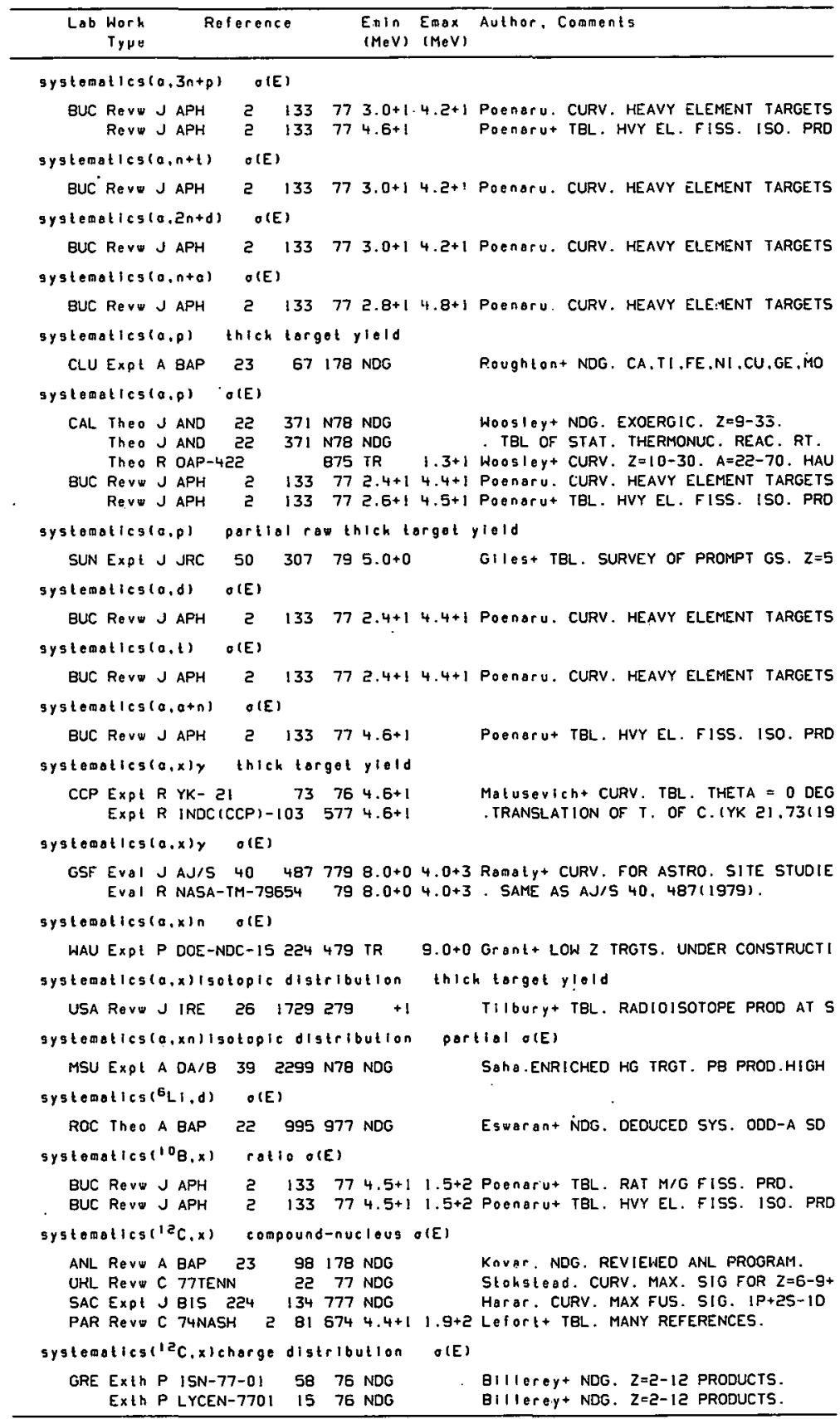


APPENDIX F --- SYSTEMATIC STUDIES (cont)

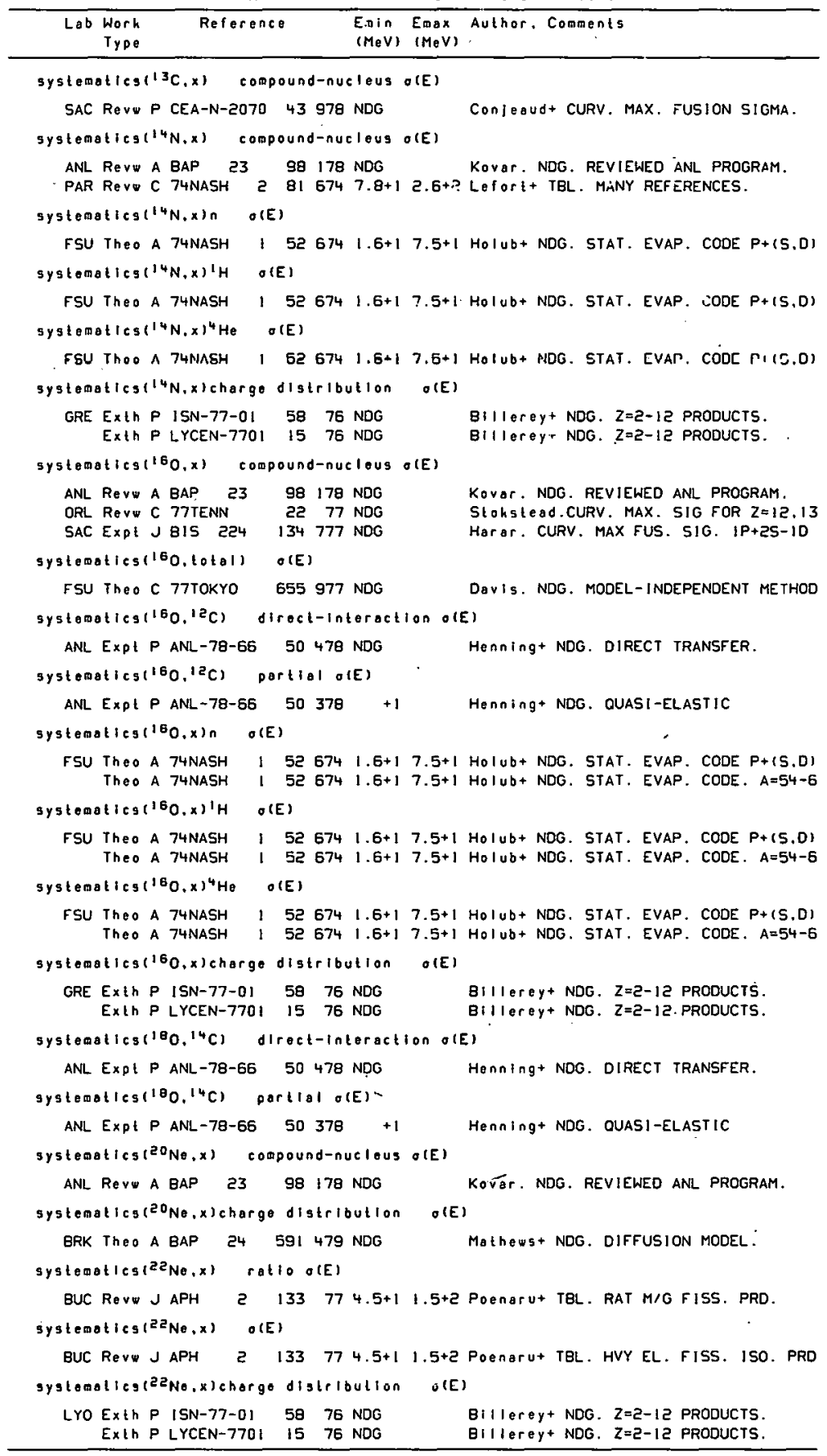


APPENDIX F --- SYSTEMATIC STUDIES(cont)

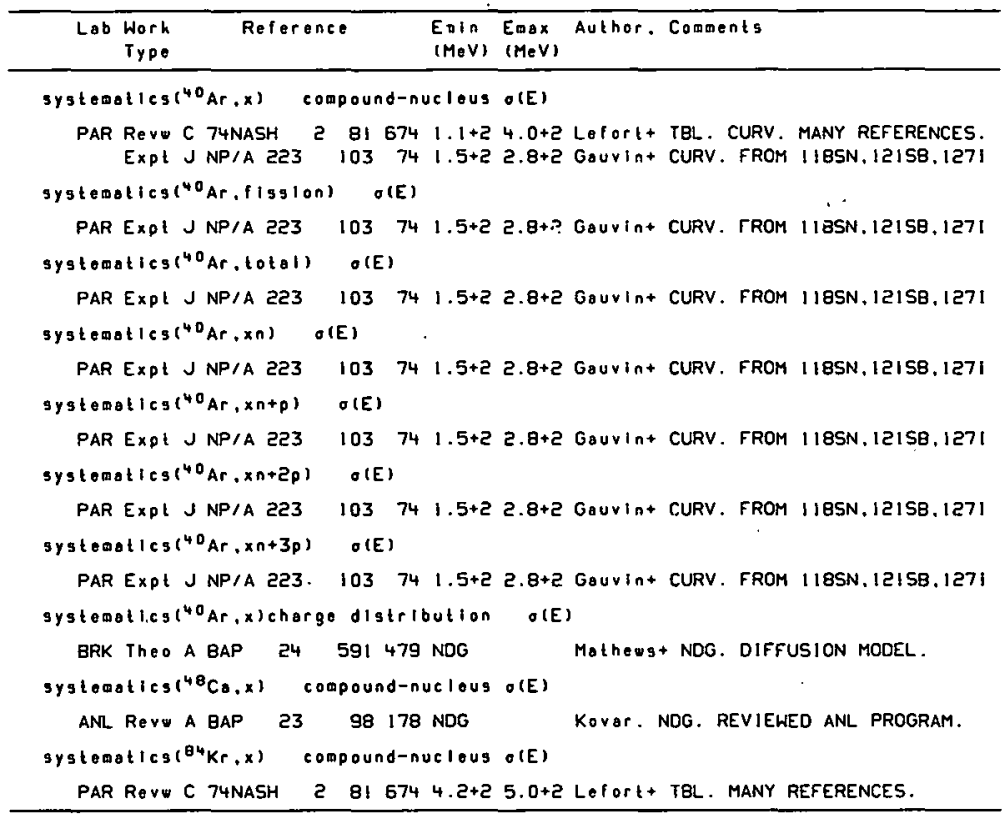




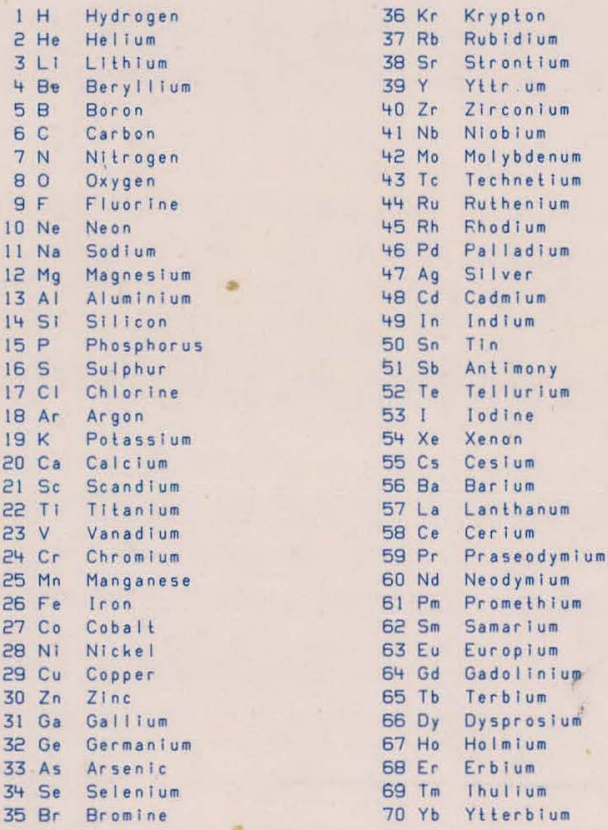

$71 \mathrm{Lu}$ Lutetium
$72 \mathrm{Hf}$ Hafnium
$72 \mathrm{Ta}$ Tantalum
$74 \mathrm{~W}$ Tungsten
$75 \mathrm{Re}$ Rhenium
$76 \mathrm{Os}$ Osmium
$77 \mathrm{Ir}$ Iridium
$78 \mathrm{Pt}$ Platinum
$79 \mathrm{Au}$ Gold
$80 \mathrm{Hg}$ Mercury
$81 \mathrm{TI}$ Thallium
$82 \mathrm{~Pb}$ Lead
$83 \mathrm{Bi}$ Bismuth
$84 \mathrm{Po}$ Polonium
$85 \mathrm{At}$ Astatine
$86 \mathrm{Rn}$ Radon
$87 \mathrm{Fr}$ Franclum
$88 \mathrm{Ra}$ Radium
$89 \mathrm{Ac}$ Actinium
$90 \mathrm{Th}$ Thorium
$91 \mathrm{~Pa}$ Protactinium
$92 \mathrm{U}$ Uranium
$93 \mathrm{~Np}$ Neptunium
$94 \mathrm{Pu}$ Plutonium
$95 \mathrm{Am}$ Americium
$96 \mathrm{Cm}$ Curium
$97 \mathrm{Bk}$ Berkelium
$98 \mathrm{Cf}$ Californium
$99 \mathrm{Es}$ Einsteinium
$100 \mathrm{Fm}$ Fermium
$101 \mathrm{Md}$ Mendelevium
$102 \mathrm{No}$ Nobelium
$103 \mathrm{Lr}$ Lawrencium
$104 \mathrm{Ku}$ Kurchatovium

